\title{
AFIP-7 Irradiation Summary Report
}

D. M. Perez

J. W. Nielsen

G. S. Chang

G. A. Roth

N. E. Woolstenhulme

September 2012

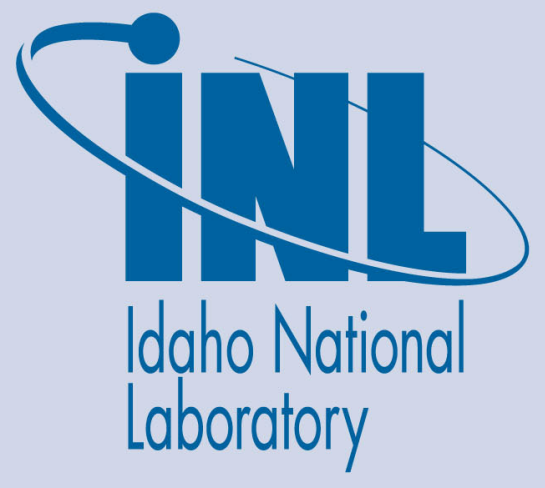

The INL is a U.S. Department of Energy National Laboratory operated by Battelle Energy Alliance 
INL/EXT-12-25915

\title{
AFIP-7 Irradiation Summary Report
}

\author{
D. M. Perez \\ J. W. Nielsen \\ G. S. Chang \\ G. A. Roth \\ N. E. Woolstenhulme
}

September 2012

\section{Idaho National Laboratory \\ Idaho Falls, Idaho 83415}

http://www.inl.gov

\author{
Prepared for the
}

U.S. Department of Energy

Office of National Nuclear Security Administration

Under DOE Idaho Operations Office

Contract DE-AC07-05ID14517 


\section{DISCLAIMER}

This information was prepared as an account of work sponsored by an agency of the U.S. Government. Neither the U.S. Government nor any agency thereof, nor any of their employees, makes any warranty, expressed or implied, or assumes any legal liability or responsibility for the accuracy, completeness, or usefulness, of any information, apparatus, product, or process disclosed, or represents that its use would not infringe privately owned rights. References herein to any specific commercial product, process, or service by trade name, trade mark, manufacturer, or otherwise, does not necessarily constitute or imply its endorsement, recommendation, or favoring by the U.S. Government or any agency thereof. The views and opinions of authors expressed herein do not necessarily state or reflect those of the U.S. Government or any agency thereof. 


\title{
AFIP-7 Irradiation Summary Report
}

\author{
INL/EXT-12-25915
}

September 2012

Approved by:

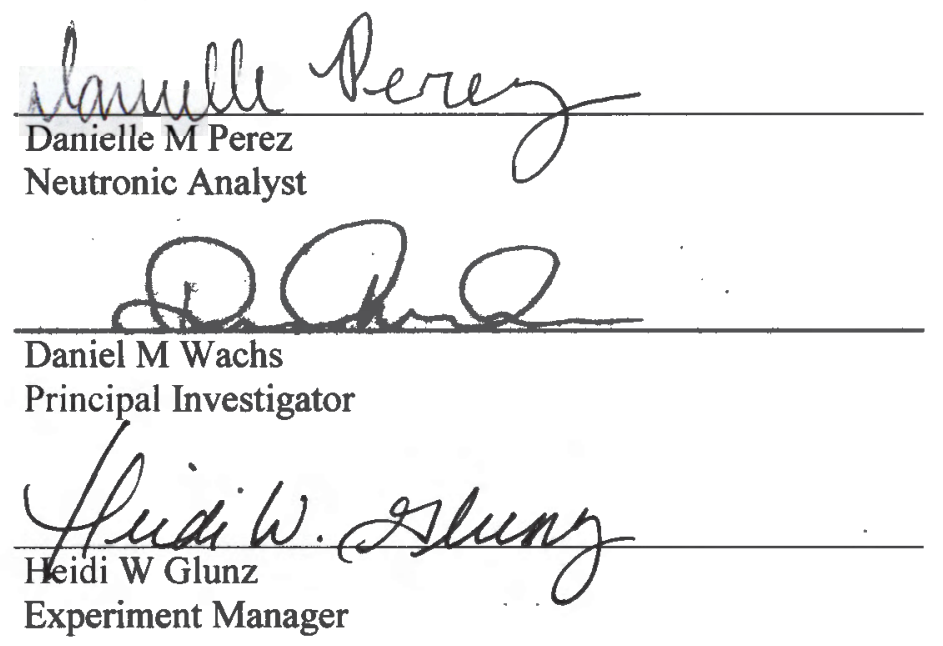

9/10/2012

Date

$9 / 10 / 2012$

Date

$9 / 10 / 2012$

Date 


\section{SUMMARY}

The Advanced Test Reactor (ATR) Full size plate In center flux trap Position (AFIP) experiment AFIP-7 was designed to evaluate the performance of monolithic uranium-molybdenum (U-Mo) fuels in geometries which are prototypic of US high power research reactor (HPRR) fuel plates ${ }^{1}$.

The following report summarizes the life of the AFIP-7 experiment through end of irradiation, including a brief description of the safety analysis, as-run neutronic analysis results, hydraulic testing results, and thermal analysis results. 


\section{CONTENTS}

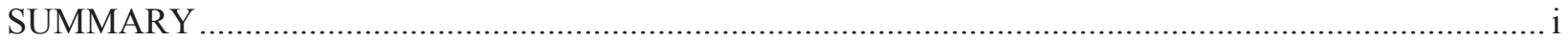

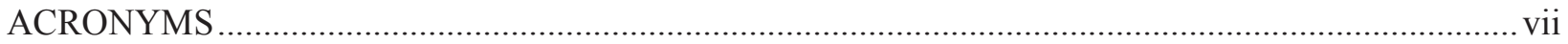

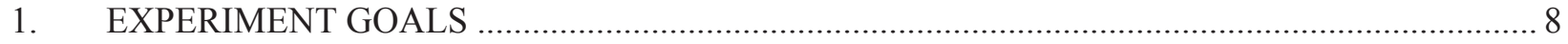

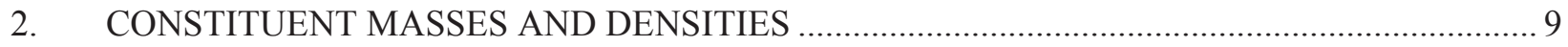

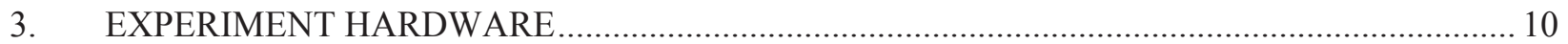

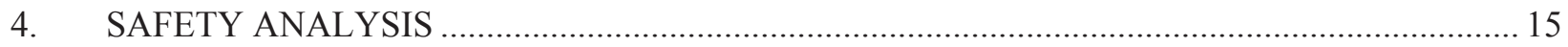

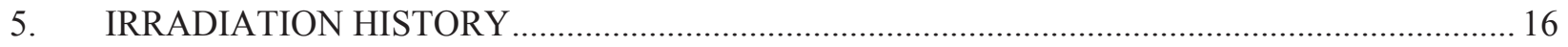

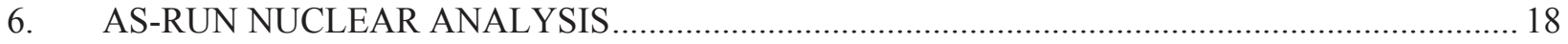

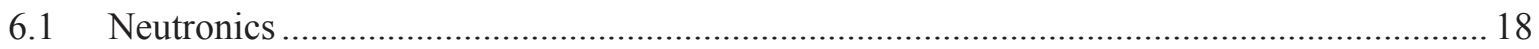

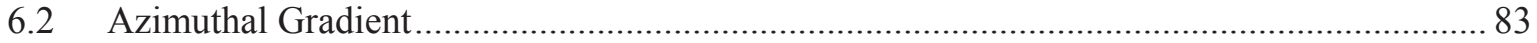

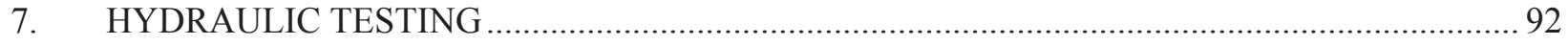

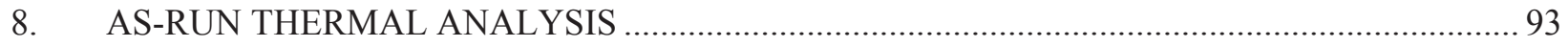

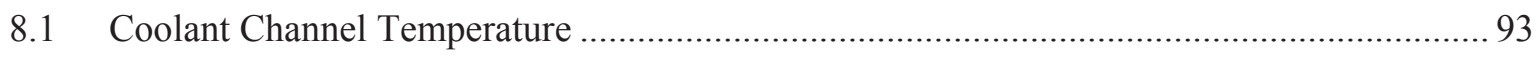

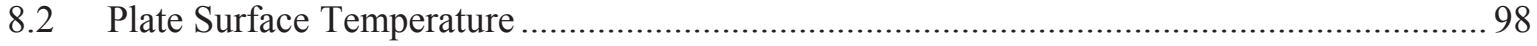

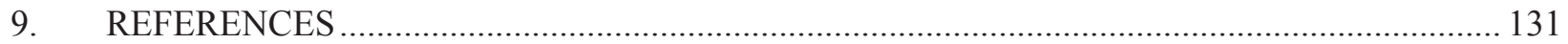

Appendix A: Individual Plate Power and Fission Density Plots....................................................... 132

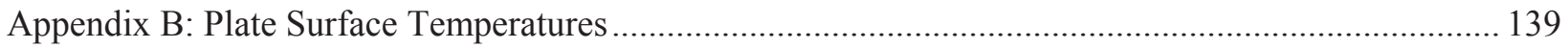




\section{FIGURES}

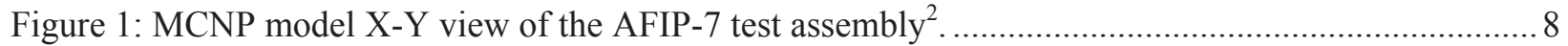

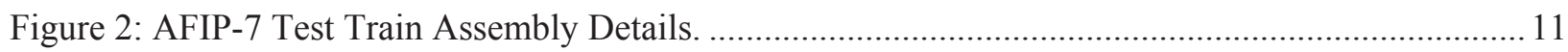

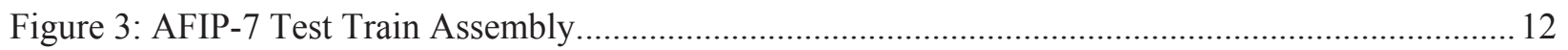

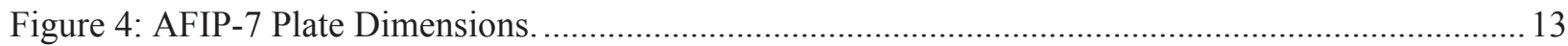

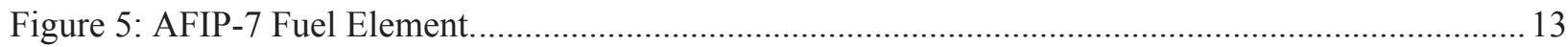

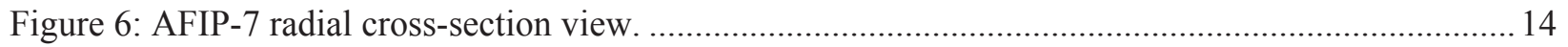

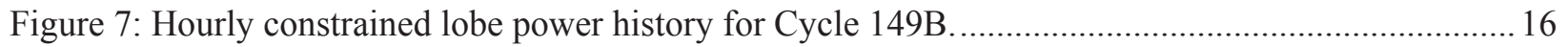

Figure 8: Hourly constrained lobe power history for Cycle 150B ...................................................... 17

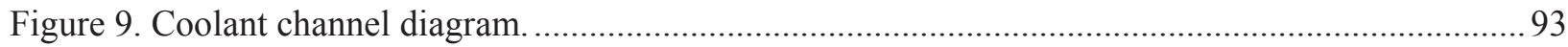

Figure 10: Coolant channel temperatures as a function of location along the AFIP-7 test assembly

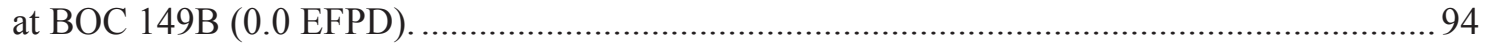

Figure 11: Coolant channel temperatures as a function of location along the AFIP-7 test assembly at MOC1 149B (15.0 EFPD).

Figure 12: Coolant channel temperatures as a function of location along the AFIP-7 test assembly at MOC2 149B (31.0 EFPD).

Figure 13: Coolant channel temperatures as a function of location along the AFIP-7 test assembly at EOC 149B (53.6 EFPD).

Figure 14: Coolant channel temperatures as a function of location along the AFIP-7 test assembly at BOC 150B (0 EFPD, cumulative 53.6 EFPD).

Figure 15: Coolant channel temperatures as a function of location along the AFIP-7 test assembly at MOC1 150B (18 EFPD, cumulative 71.6 EFPD).

Figure 16: Coolant channel temperatures as a function of location along the AFIP-7 test assembly at MOC2 150B (31 EFPD, cumulative 84.6 EFPD).

Figure 17: Coolant channel temperatures as a function of location along the AFIP-7 test assembly at EOC 150B (42.0 EFPD, cumulative 95.6 EFPD). 


\section{TABLES}

Table 1: Experiment matrix for AFIP-7 with as-built enrichments. ....................................................... 9

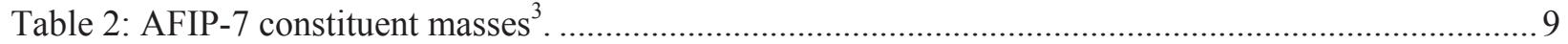

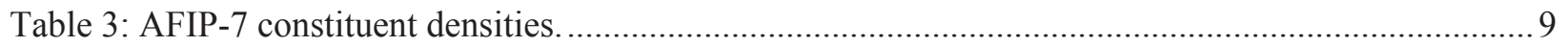

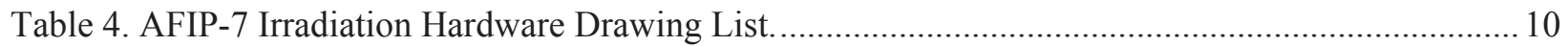

Table 5: Summary table of the safety analyses done for the AFIP-7 experiment..................................... 15

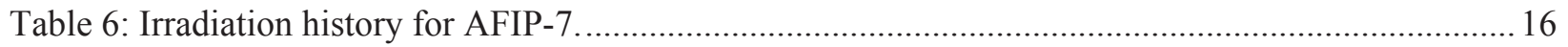

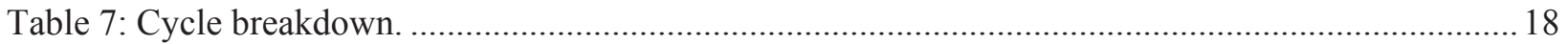

Table 8: Cycle 149B, MCNP-Calculated HGRs and Neutron Flux for AFIP-7, Plate Position 1, 0 EFPD (BOC) Average Center Lobe Power at 24.2 MW. ${ }^{2}$

Table 9: Cycle 149B, MCNP-Calculated HGRs, Neutron Flux, Depletion and Fission Density for AFIP-7, Plate Position 1, 15 EFPD (MOC1) Average Center Lobe Power at 24.2 MW. ${ }^{2}$........ 21

Table 10: Cycle 149B, MCNP-Calculated HGRs, Neutron Flux, Depletion and Fission Density for AFIP-7, Plate Position 1, 31 EFPD (MOC2) Average Center Lobe Power at 24.2 MW. ${ }^{2}$

Table 11: Cycle 149B, MCNP-Calculated HGRs, Neutron Flux, Depletion and Fission Density for AFIP-7, Plate Position 1, 53.6 EFPD (EOC) Average Center Lobe Power at 24.2 MW. ${ }^{2}$

Table 12: Cycle 149B, MCNP-Calculated HGRs and Neutron Flux for AFIP-7, Plate Position 2, 0 EFPD (BOC) Average Center Lobe Power at 24.2 MW. ${ }^{2}$

Table 13: Cycle 149B, MCNP-Calculated HGRs, Neutron Flux, Depletion and Fission Density for AFIP-7, Plate Position 2, 15 EFPD (MOC1) Average Center Lobe Power at 24.2 MW. ${ }^{2}$

Table 14: Cycle 149B, MCNP-Calculated HGRs, Neutron Flux, Depletion and Fission Density for AFIP-7, Plate Position 2, 31 EFPD (MOC2) Average Center Lobe Power at 24.2 MW. ${ }^{2}$

Table 15: Cycle 149B, MCNP-Calculated HGRs, Neutron Flux, Depletion and Fission Density for AFIP-7, Plate Position 2, 53.6 EFPD (EOC) Average Center Lobe Power at 24.2 MW. ${ }^{2}$

Table 16: Cycle 149B, MCNP-Calculated HGRs and Neutron Flux for AFIP-7, Plate Position 3, 0 EFPD (BOC) Average Center Lobe Power at 24.2 MW. ${ }^{2}$

Table 17: Cycle 149B, MCNP-Calculated HGRs, Neutron Flux, Depletion and Fission Density for AFIP-7, Plate Position 3, 15 EFPD (MOC1) Average Center Lobe Power at 24.2 MW. ${ }^{2}$

Table 18: Cycle 149B, MCNP-Calculated HGRs, Neutron Flux, Depletion and Fission Density for AFIP-7, Plate Position 3, 31 EFPD (MOC2) Average Center Lobe Power at 24.2 MW. ${ }^{2}$

Table 19: Cycle 149B, MCNP-Calculated HGRs, Neutron Flux, Depletion and Fission Density for AFIP-7, Plate Position 3, 53.6 EFPD (EOC) Average Center Lobe Power at 24.2 MW. ${ }^{2}$ 
Table 20: Cycle 149B, MCNP-Calculated HGRs and Neutron Flux for AFIP-7, Plate Position 4, 0 EFPD (BOC) Average Center Lobe Power at 24.2 MW. ${ }^{2}$

Table 21: Cycle 149B, MCNP-Calculated HGRs, Neutron Flux, Depletion and Fission Density for AFIP-7, Plate Position 4, 15 EFPD (MOC1) Average Center Lobe Power at 24.2 MW. ${ }^{2}$.

Table 22: Cycle 149B, MCNP-Calculated HGRs, Neutron Flux, Depletion and Fission Density for AFIP-7, Plate Position 4, 31 EFPD (MOC2) Average Center Lobe Power at 24.2 MW. ${ }^{2}$.

Table 23: Cycle 149B, MCNP-Calculated HGRs, Neutron Flux, Depletion and Fission Density for AFIP-7, Plate Position 4, 53.6 EFPD (EOC) Average Center Lobe Power at 24.2 MW. ${ }^{2}$.

Table 24: Cycle 150B, MCNP-Calculated HGRs and Neutron Flux for AFIP-7, Plate Position 1, 0 EFPD (BOC) Average Center Lobe Power at 24.2 MW. ${ }^{2}$

Table 25: Cycle 149B, MCNP-Calculated HGRs, Neutron Flux, Depletion and Fission Density for AFIP-7, Plate Position 1, 18 EFPD (MOC1) Average Center Lobe Power at 24.2 MW. ${ }^{2}$..

Table 26: Cycle 150B, MCNP-Calculated HGRs, Neutron Flux, Depletion and Fission Density for AFIP-7, Plate Position 1, 31 EFPD (MOC2) Average Center Lobe Power at 24.2 MW. ${ }^{2}$

Table 27: Cycle 150B, MCNP-Calculated HGRs, Neutron Flux, Depletion and Fission Density for AFIP-7, Plate Position 1, 42.0 EFPD (EOC) Average Center Lobe Power at 24.2 MW. ${ }^{2}$.

Table 28: Cycle 150B, MCNP-Calculated HGRs and Neutron Flux for AFIP-7, Plate Position 2, 0 EFPD (BOC) Average Center Lobe Power at 24.2 MW. ${ }^{2}$

Table 29: Cycle 150B, MCNP-Calculated HGRs, Neutron Flux, Depletion and Fission Density for AFIP-7, Plate Position 2, 18 EFPD (MOC1) Average Center Lobe Power at 24.2 MW. ${ }^{2}$

Table 30: Cycle 150B, MCNP-Calculated HGRs, Neutron Flux, Depletion and Fission Density for AFIP-7, Plate Position 2, 31 EFPD (MOC2) Average Center Lobe Power at 24.2 MW. ${ }^{2}$

Table 31: Cycle 150B, MCNP-Calculated HGRs, Neutron Flux, Depletion and Fission Density for AFIP-7, Plate Position 2, 42.0 EFPD (EOC) Average Center Lobe Power at 24.2 MW. ${ }^{2}$

Table 32: Cycle 150B, MCNP-Calculated HGRs and Neutron Flux for AFIP-7, Plate Position 3, 0 EFPD (BOC) Average Center Lobe Power at 24.2 MW. ${ }^{2}$

Table 33: Cycle 150B, MCNP-Calculated HGRs, Neutron Flux, Depletion and Fission Density for AFIP-7, Plate Position 3, 18 EFPD (MOC1) Average Center Lobe Power at 24.2 MW. ${ }^{2}$......

Table 34: Cycle 150B, MCNP-Calculated HGRs, Neutron Flux, Depletion and Fission Density for AFIP-7, Plate Position 3, 31 EFPD (MOC2) Average Center Lobe Power at 24.2 MW. ${ }^{2}$.

Table 35: Cycle 150B, MCNP-Calculated HGRs, Neutron Flux, Depletion and Fission Density for AFIP-7, Plate Position 3, 42.0 EFPD (EOC) Average Center Lobe Power at 24.2 MW. ${ }^{2}$. 
Table 36: Cycle 150B, MCNP-Calculated HGRs and Neutron Flux for AFIP-7, Plate Position 4, 0 EFPD (BOC) Average Center Lobe Power at 24.2 MW. ${ }^{2}$

Table 37: Cycle 150B, MCNP-Calculated HGRs, Neutron Flux, Depletion and Fission Density for AFIP-7, Plate Position 4, 18 EFPD (MOC1) Average Center Lobe Power at 24.2 MW. ${ }^{2}$.

Table 38: Cycle 150B, MCNP-Calculated HGRs, Neutron Flux, Depletion and Fission Density for AFIP-7, Plate Position 4, 31 EFPD (MOC2) Average Center Lobe Power at 24.2 MW. ${ }^{2}$.

Table 39: Cycle 150B, MCNP-Calculated HGRs, Neutron Flux, Depletion and Fission Density for AFIP-7, Plate Position 4, 42.0 EFPD (EOC) Average Center Lobe Power at 24.2 MW. ${ }^{2}$.

Table 40. Azimuthal L2AR for the AFIP-7 Plate Position 1 at BOC 149B............................................ 83

Table 41. Azimuthal L2AR for the AFIP-7 Plate 2 Position at BOC 149B. ............................................ 86

Table 42. Azimuthal L2AR for the AFIP-7 Plate 3 Position at BOC 149B. ............................................ 88

Table 43. Azimuthal L2AR for the AFIP-7 Plate 4 Position at BOC 149B. ............................................ 90

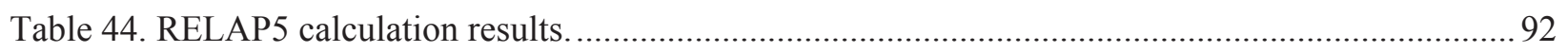

Table 45. Temperature $\left({ }^{\circ} \mathrm{C}\right)$ map of the north side of Plate Position 1 at EOC 149B............................. 99

Table 46. Temperature $\left({ }^{\circ} \mathrm{C}\right)$ map of the south side of Plate Position 1 at EOC 149B........................... 101

Table 47. Temperature $\left({ }^{\circ} \mathrm{C}\right)$ map of the north side of Plate Position 2 at EOC 149B........................... 103

Table 48. Temperature $\left({ }^{\circ} \mathrm{C}\right)$ map of the south side of Plate Position 2 at EOC 149B........................... 105

Table 49. Temperature $\left({ }^{\circ} \mathrm{C}\right)$ map of the north side of Plate Position 3 at EOC 149B............................ 107

Table 50. Temperature $\left({ }^{\circ} \mathrm{C}\right)$ map of the south side of Plate Position 3 at EOC 149B........................... 109

Table 51. Temperature $\left({ }^{\circ} \mathrm{C}\right)$ map of the north side of Plate Position 4 at EOC 149B............................ 111

Table 52. Temperature $\left({ }^{\circ} \mathrm{C}\right)$ map of the south side of Plate Position 4 at EOC 149B........................... 113

Table 53. Temperature $\left({ }^{\circ} \mathrm{C}\right)$ map of the north side of Plate Position 1 at EOC 150B............................ 115

Table 54. Temperature $\left({ }^{\circ} \mathrm{C}\right)$ map of the south side of Plate Position 1 at EOC 150B........................... 117

Table 55. Temperature $\left({ }^{\circ} \mathrm{C}\right)$ map of the north side of Plate Position 2 at EOC 150B............................ 119

Table 56. Temperature $\left({ }^{\circ} \mathrm{C}\right)$ map of the south side of Plate Position 2 at EOC 150B.......................... 121

Table 57. Temperature $\left({ }^{\circ} \mathrm{C}\right)$ map of the north side of Plate Position 3 at EOC 150B........................... 123

Table 58. Temperature $\left({ }^{\circ} \mathrm{C}\right)$ map of the south side of Plate Position 3 at EOC 150B......................... 125

Table 59. Temperature $\left({ }^{\circ} \mathrm{C}\right)$ map of the north side of Plate Position 4 at EOC 150B............................ 127

Table 60. Temperature $\left({ }^{\circ} \mathrm{C}\right)$ map of the south side of Plate Position 4 at EOC 150B........................... 129 


\section{ACRONYMS}

$\begin{array}{ll}\text { Al } & \text { Aluminum } \\ \text { ATR } & \text { Advanced Test Reactor } \\ \text { AFIP } & \text { ATR Full-size plate In center flux trap Position } \\ \text { CFT } & \text { Center Flux Trap } \\ \text { DAS } & \text { Data Acquisition System } \\ \text { EFPD } & \text { Effective Full Power Days } \\ \text { GTRI } & \text { Global Threat Reduction Initiative } \\ \text { FD } & \text { Fuel Development } \\ \text { HIP } & \text { Hot Isostatic Pressing } \\ \text { MCNP } & \text { Monte Carlo N-Particle } \\ \text { Mo } & \text { Molybdenum } \\ \text { RERTR } & \text { Reduced Enrichment Research and Test Reactor } \\ \text { U } & \text { Uranium } \\ \text { U-Mo } & \text { Uranium-Molybdenum } \\ \text { Zr } & \text { Zirconium }\end{array}$




\section{AFIP-7 Irradiation Summary Report \\ 1. EXPERIMENT GOALS}

In support of the Global Threat Reduction Initiative (GTRI) Fuel Development (FD) program (historically known as Reduced Enrichment for Research and Test Reactors [RERTR]), the Advanced Test Reactor (ATR) Full size plate In center flux trap Position (AFIP) experiment AFIP-7 was designed to evaluate the performance of monolithic uranium-molybdenum (U-Mo) fuels in geometries which are prototypic of US high power research reactor (HPRR) fuel plates ${ }^{1}$.

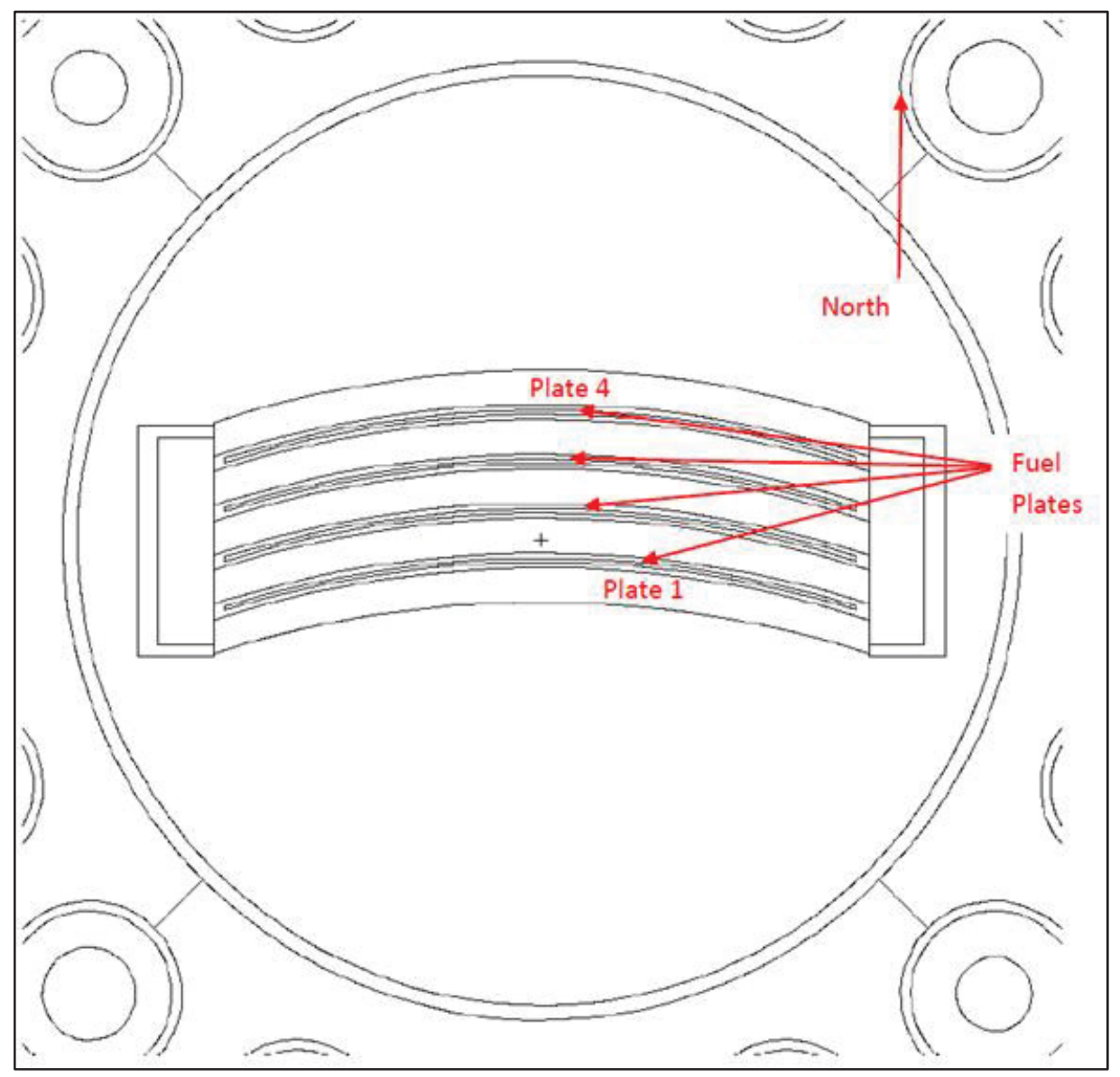

Figure 1: MCNP model X-Y view of the AFIP-7 test assembly ${ }^{2}$.

The AFIP-7 experiment was irradiated in the ATR Center Flux Trap (CFT) position. The test assembly holds 4 full length fuel plates. Each plate is approximately $40 \mathrm{in}$. in length, $2.473 \mathrm{in}$. in width and 0.050 in. in thickness with a curvature radius of $3.557 \mathrm{in}$. The fuel zone was nominally $38.5 \mathrm{in}$. in length, 2.163 in. in width and 0.013 in. in thickness. A diffusion barrier of $\sim 0.001$ in. thick zirconium was bonded to the top and bottom surface of the fuel foil. All four plates were hot isostatic pressed (HIP-ed). The experiment matrix is shown in Table 1 . Hafnium rods were inserted into four H-positions $(\mathrm{H}-1, \mathrm{H}-5, \mathrm{H}-9$ and H-13) surrounding the CFT. Each Hf rod is a solid rod with a diameter of 0.346 in. and contains approximately $984 \mathrm{~g}$ of $\mathrm{Hf}$. 
Table 1: Experiment matrix for AFIP-7 with as-built enrichments.

\begin{tabular}{|c|c|}
\hline \multicolumn{2}{|c|}{ AFIP-7 Experiment Matrix } \\
\hline \multirow{3}{*}{ Plate 1 } & U-10 Mo \\
& $19.56 \%$ enrich. \\
& $\mathbf{7 Z H - 1}$ \\
\hline & U-10 Mo \\
Plate 2 & $19.78 \%$ enrich. \\
& $\mathbf{7 Z H - 2}$ \\
\hline & U-10 Mo \\
Plate 3 & $19.88 \%$ enrich. \\
& $\mathbf{7 Z H - 3}$ \\
\hline & U-10 Mo \\
Plate 4 & $19.78 \%$ enrich. \\
& $\mathbf{7 Z H - 4}$ \\
\hline
\end{tabular}

\section{CONSTITUENT MASSES AND DENSITIES}

The constituent masses and densities for the 4 plates were obtained from the as-built data package ${ }^{3}$ plate summary sheets. Table 2 summarizes the constituent masses for the plates and Table 3 summarizes the constituent densities for the plates.

Table 2: AFIP-7 constituent masses ${ }^{3}$.

\begin{tabular}{|c|c|c|c|c|c|c|}
\hline $\begin{array}{c}\text { Fuel Plate } \\
\text { Location }\end{array}$ & $\begin{array}{c}\text { Fuel Plate } \\
\text { ID }\end{array}$ & $\begin{array}{c}\text { Total-U } \\
(\mathrm{g})\end{array}$ & $\begin{array}{c}\mathrm{U}-238 \\
(\mathrm{~g})\end{array}$ & $\begin{array}{c}\mathrm{U}-235 \\
(\mathrm{~g})\end{array}$ & $\begin{array}{c}\text { Mo } \\
(\mathrm{g})\end{array}$ & $\begin{array}{c}\mathrm{Zr} \\
(\mathrm{g})\end{array}$ \\
\hline 1 & $7 \mathrm{ZH}-1$ & 251.81 & 202.55 & 49.26 & 31.26 & 19.23 \\
\hline 2 & $7 \mathrm{ZH}-2$ & 283.71 & 227.58 & 56.13 & 30.32 & 14.11 \\
\hline 3 & $7 \mathrm{ZH}-3$ & 271.48 & 217.51 & 53.97 & 33.09 & 15.66 \\
\hline 4 & $7 \mathrm{ZH}-4$ & 283.64 & 227.53 & 56.11 & 30.31 & 14.10 \\
\hline
\end{tabular}

Table 3: AFIP-7 constituent densities.

\begin{tabular}{|c|c|c|c|c|c|c|c|c|}
\hline \multirow[b]{2}{*}{$\begin{array}{c}\text { Fuel Plate } \\
\text { Location }\end{array}$} & \multirow[b]{2}{*}{$\begin{array}{c}\text { Fuel } \\
\text { Plate ID }\end{array}$} & \multicolumn{5}{|c|}{ Fuel Foil } & \multicolumn{2}{|c|}{ Interlayer } \\
\hline & & $\begin{array}{l}\text { Volume } \\
\text { (cc) }\end{array}$ & $\begin{array}{c}\text { Total-U } \\
(\mathrm{g} / \mathrm{cc})\end{array}$ & $\begin{array}{l}\text { U-238 } \\
(\mathrm{g} / \mathrm{cc})\end{array}$ & $\begin{array}{l}\text { U-235 } \\
(\mathrm{g} / \mathrm{cc})\end{array}$ & $\begin{array}{l}\text { Mo } \\
\text { (g/cc) }\end{array}$ & $\begin{array}{c}\mathrm{Zr} \\
\text { Volume } \\
\text { (cc) }\end{array}$ & $\begin{array}{c}\mathrm{Zr} \\
(\mathrm{g} / \mathrm{cc})\end{array}$ \\
\hline 1 & $7 \mathrm{ZH}-1$ & 17.67 & 14.25 & 11.46 & 2.79 & 1.77 & 2.72 & 7.07 \\
\hline 2 & 7ZH-2 & 18.00 & 15.77 & 12.65 & 3.13 & 1.68 & 2.77 & 5.10 \\
\hline 3 & $7 \mathrm{ZH}-3$ & 17.87 & 15.19 & 12.17 & 3.02 & 1.85 & 2.75 & 5.70 \\
\hline 4 & $7 \mathrm{ZH}-4$ & 17.97 & 15.78 & 12.66 & 3.12 & 1.69 & 2.76 & 5.10 \\
\hline
\end{tabular}




\section{EXPERIMENT HARDWARE}

The experiment hardware list for AFIP-7 is shown in Table 4. The assembly retriever used for AFIP-1, -2 , $-3,-4$ and -6 was also used for AFIP-7 a new holder was designed to accommodate the new fuel plate design.

Table 4. AFIP-7 Irradiation Hardware Drawing List.

\begin{tabular}{c|c}
\hline Drawing Number & Drawing Title \\
\hline 602649 & ATR AFIP-7 Experimental Element Holder Assembly \\
\hline 602648 & ATR AFIP-7 Experimental Element Fuel Plate Assembly \\
\hline 602647 & ATR AFIP-7 Experimental Element Fuel Element Assembly and Details \\
\hline 602646 & ATR AFIP-7 Experimental Element Final Assembly \\
\hline 602650 & ATR AFIP-7 Experimental Element Holder Details \\
\hline 602687 & ATR AFIP-7 Hf Rod Basket Assembly \\
\hline 602688 & ATR AFIP-7 Hf Rod Assembly \\
\hline 602689 & ATR AFIP-7 Hf Rod Detail \\
\hline
\end{tabular}

The AFIP-7 test train assembly as shown in Figure 2 shows the main components of the test assembly, which includes the fuel plate assembly. Figure 3 shows the test train assembly with the retriever attached to the top. The retriever is used to get the test train assembly out of the reactor. Figure 4 has the specific fuel plate dimensions and nominal fuel foil dimensions. Figure 5 depicts the fuel element and Figure 6 is a radial cross section of the test train assembly and shows the locations of all the components. The Hf rods are intended to limit the increase in the center lobe source power due to the U-235 loading from the AFIP-7 experiment. 

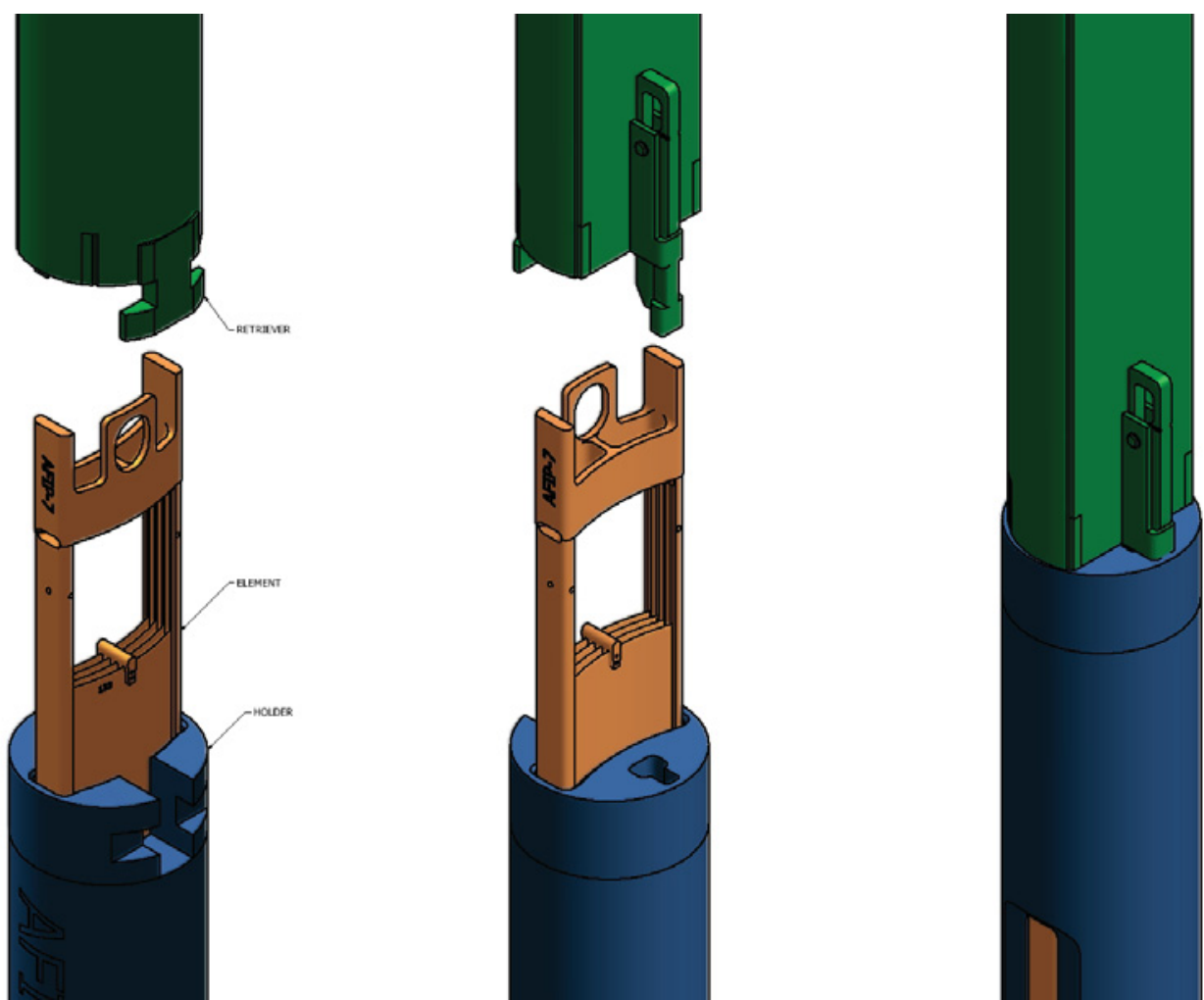

Figure 2: AFIP-7 Test Train Assembly Details. 


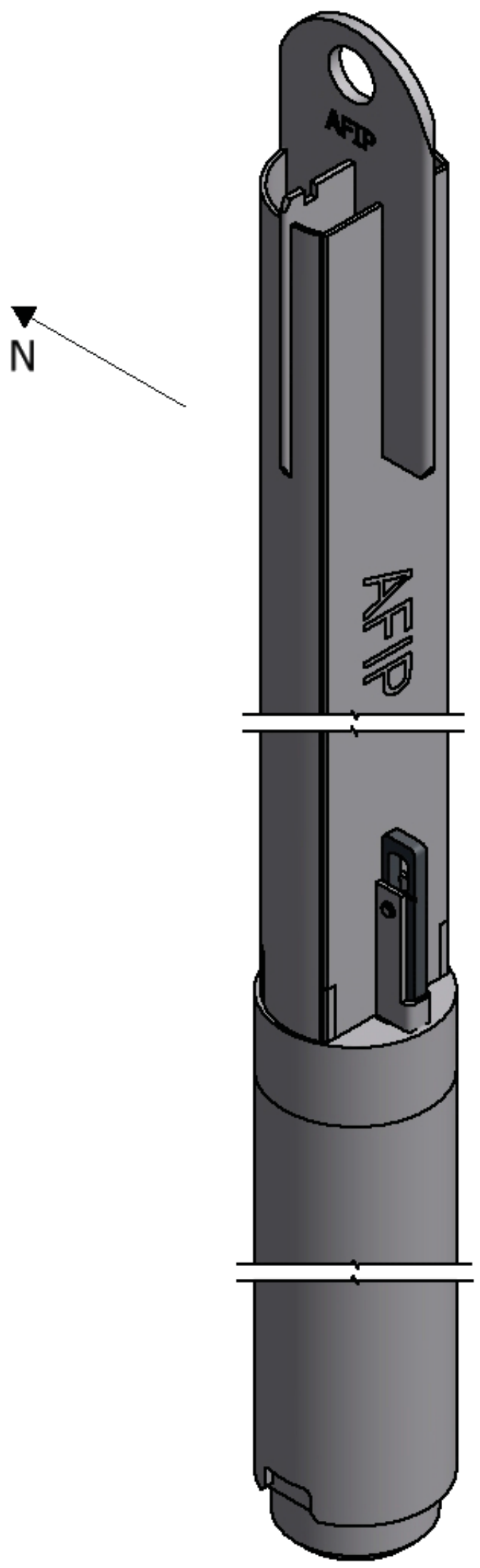

Figure 3: AFIP-7 Test Train Assembly. 


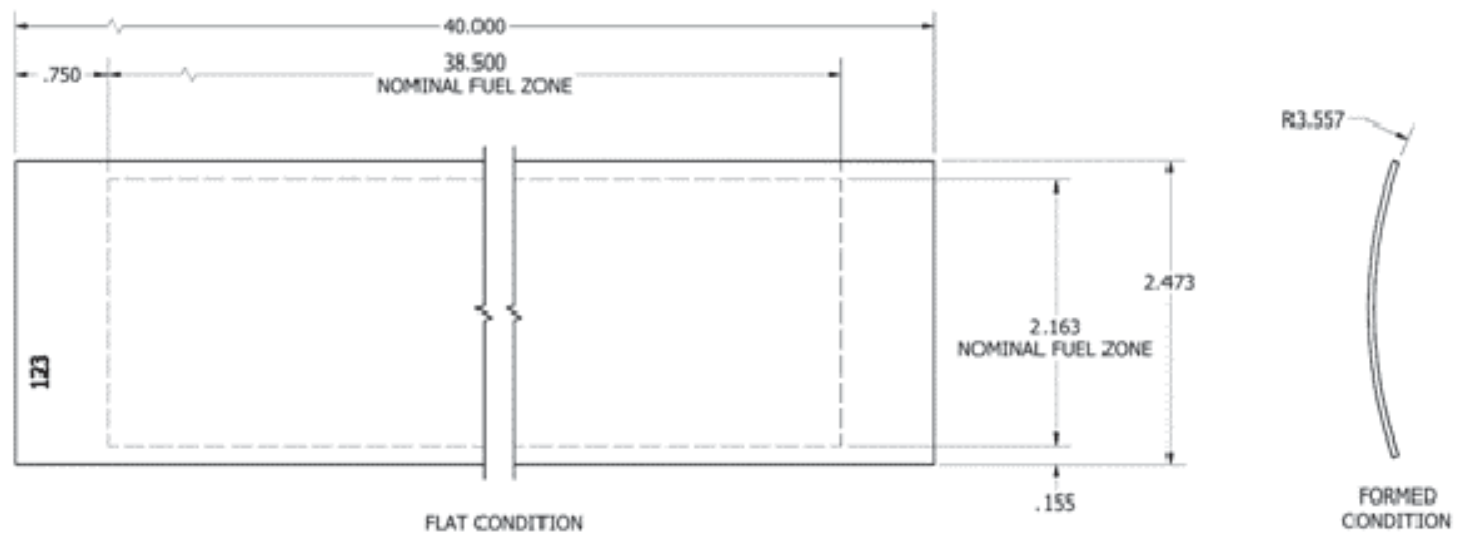

Figure 4: AFIP-7 Plate Dimensions.

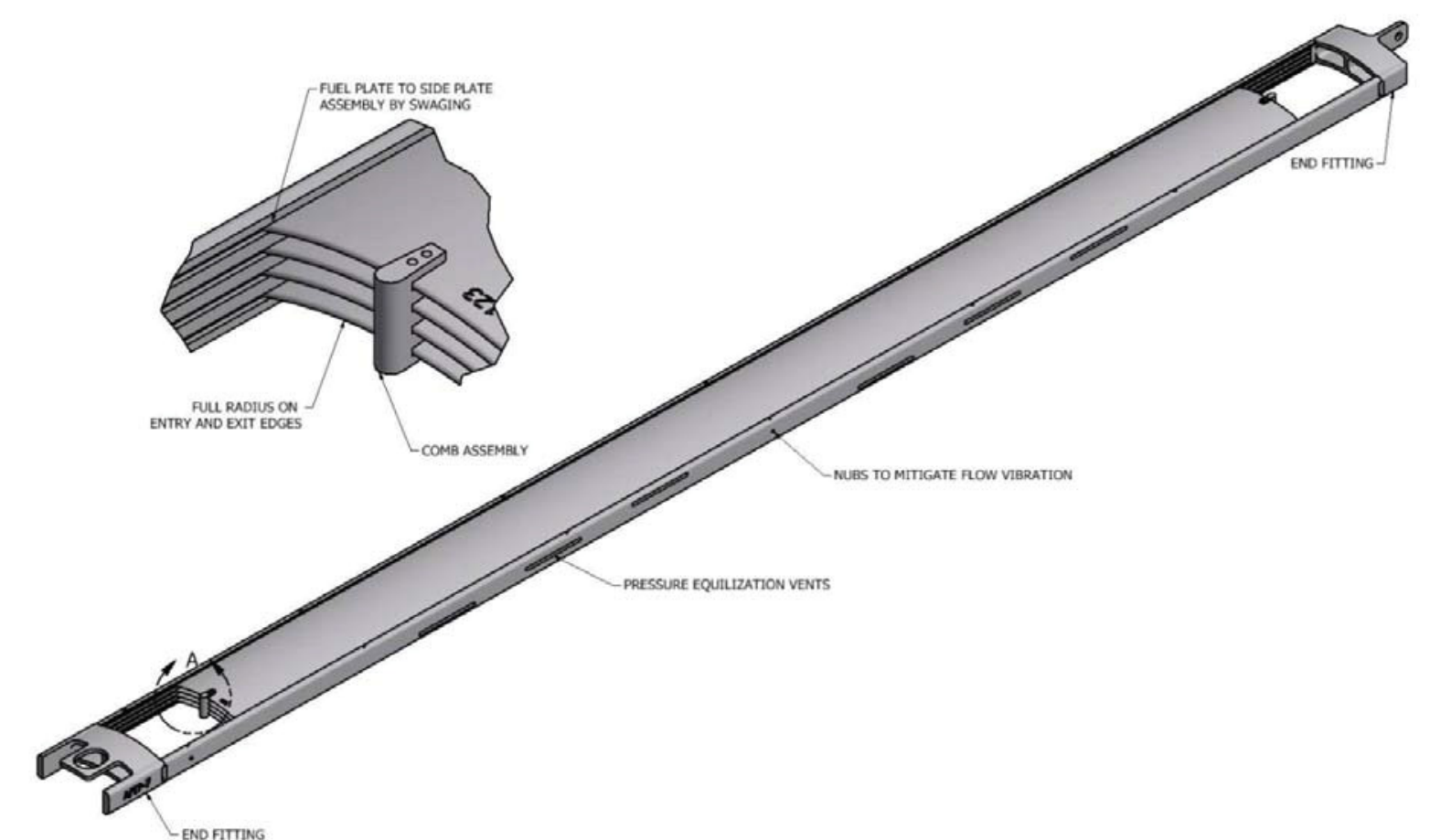

Figure 5: AFIP-7 Fuel Element. 

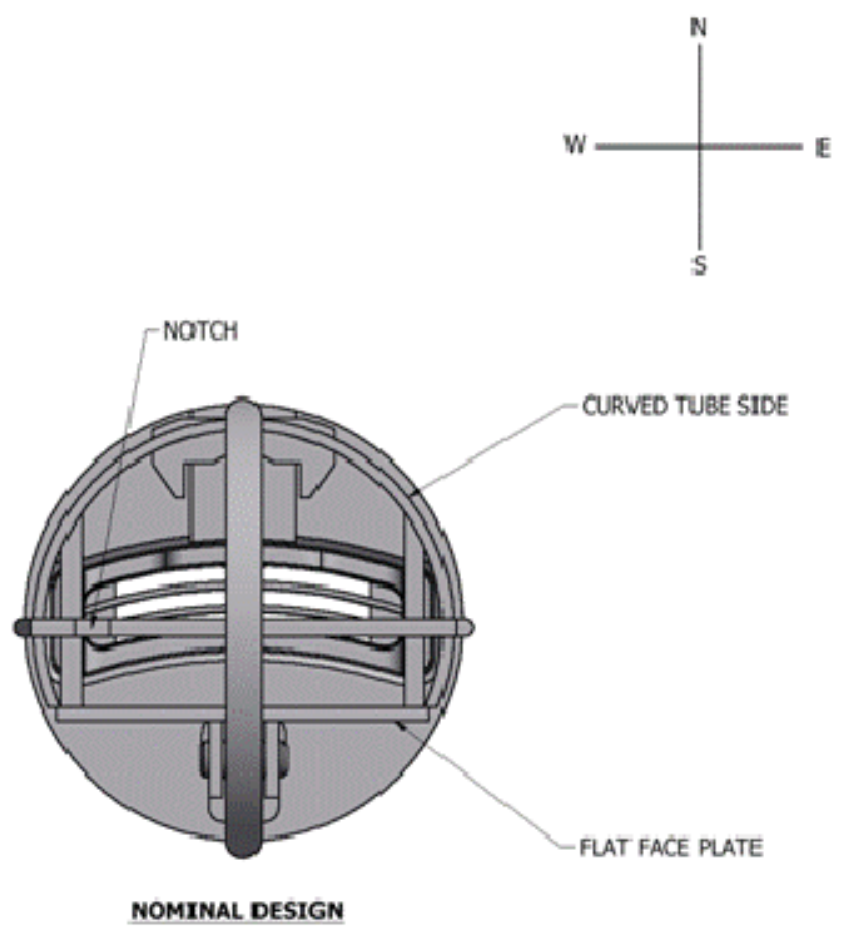

Figure 6: AFIP-7 radial cross-section view. 


\section{SAFETY ANALYSIS}

The safety analysis that was performed on the AFIP-7 experiment includes thermal/hydraulic analysis, physics analysis, and structural analysis. Table 5 summarizes the safety analyses performed on the AFIP-7 experiment.

Table 5: Summary table of the safety analyses done for the AFIP-7 experiment.

\begin{tabular}{|c|c|}
\hline ECAR Number & Description \\
\hline ECAR-1262 & $\begin{array}{l}\text { ATR Physics Analysis of the AFIP-7 Experiment in the Center } \\
\text { Flux Trap }\end{array}$ \\
\hline TEV-1253 & AFIP-7 Axial Power Distribution \\
\hline ECAR-1509 & $\begin{array}{l}\text { Analysis of the Effects of the AFIP-7 Four-Plate Test on the ATRC } \\
\text { Axial Flux Profile }\end{array}$ \\
\hline ECAR-1513 & Time to Oxide Spallation Expected During the AFIP-7 Experiment \\
\hline ECAR-1269 & AFIP-7 Thermal Evaluation \\
\hline ECAR-1326 & AFIP-7 Irradiation Assembly Structural Evaluation \\
\hline
\end{tabular}




\section{IRRADIATION HISTORY}

The AFIP-7 test assembly was irradiated during cycles 149B and 150B in the ATR Center Flux Trap (CFT). Cycle 149B ran for 53.6 effective full power days (EFPDs) with an average center lobe power of 24.2 MW (total core power of 107.2 MW). Cycle 150B ran for 42.0 EFPDs with an average center lobe power of 24.2 MW (total core power of 108.2 MW). This information is summarized in Table 6.

Table 6: Irradiation history for AFIP-7.

\begin{tabular}{|c|c|c|c|c|c|c|c|}
\hline ATR & AFIP & $\begin{array}{c}\text { Dates } \\
\text { Irradiated }\end{array}$ & $\begin{array}{c}\text { Cycle } \\
\text { EFPDs }\end{array}$ & $\begin{array}{c}\text { Mid-Cycle } \\
\text { Scram } \\
\text { Decay } \\
\text { Days }\end{array}$ & $\begin{array}{c}\text { Post-Cycle } \\
\text { Decay } \\
\text { Days }\end{array}$ & $\begin{array}{c}\text { Flux } \\
\text { Trap } \\
\text { Power } \\
\text { (MW) }\end{array}$ & $\begin{array}{c}\text { Total } \\
\text { Core } \\
\text { Power } \\
\text { (MW) }\end{array}$ \\
\hline 149B & AFIP-7 & $\begin{array}{c}06 / 06 / 2011- \\
07-30-2011\end{array}$ & 53.6 & 0 & 21 & 24.2 & 107.2 \\
\hline 150B & AFIP-7 & $\begin{array}{c}10 / 15 / 2011- \\
11 / 26 / 2011\end{array}$ & 42.0 & 0 & 17 & 24.2 & 108.2 \\
\hline
\end{tabular}

The power history for each cycle is obtained as an ATR Surveillance Report from the ATR Data Acquisition System (DAS). The plots of each lobe power on an hourly basis are shown in Figure 7 for Figure 8 for Cycle 149B and 150B, respectively.

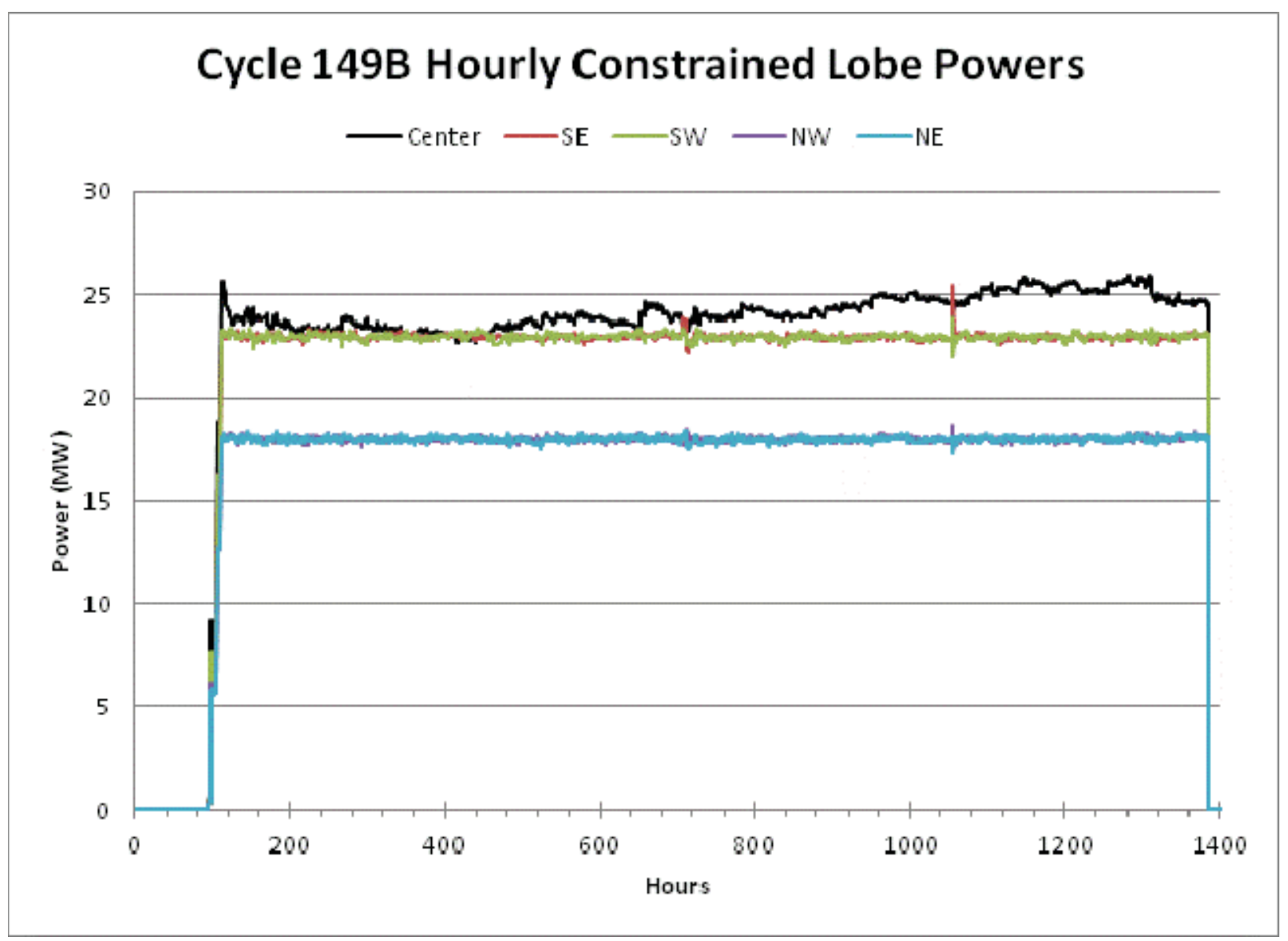

Figure 7: Hourly constrained lobe power history for Cycle 149B. 


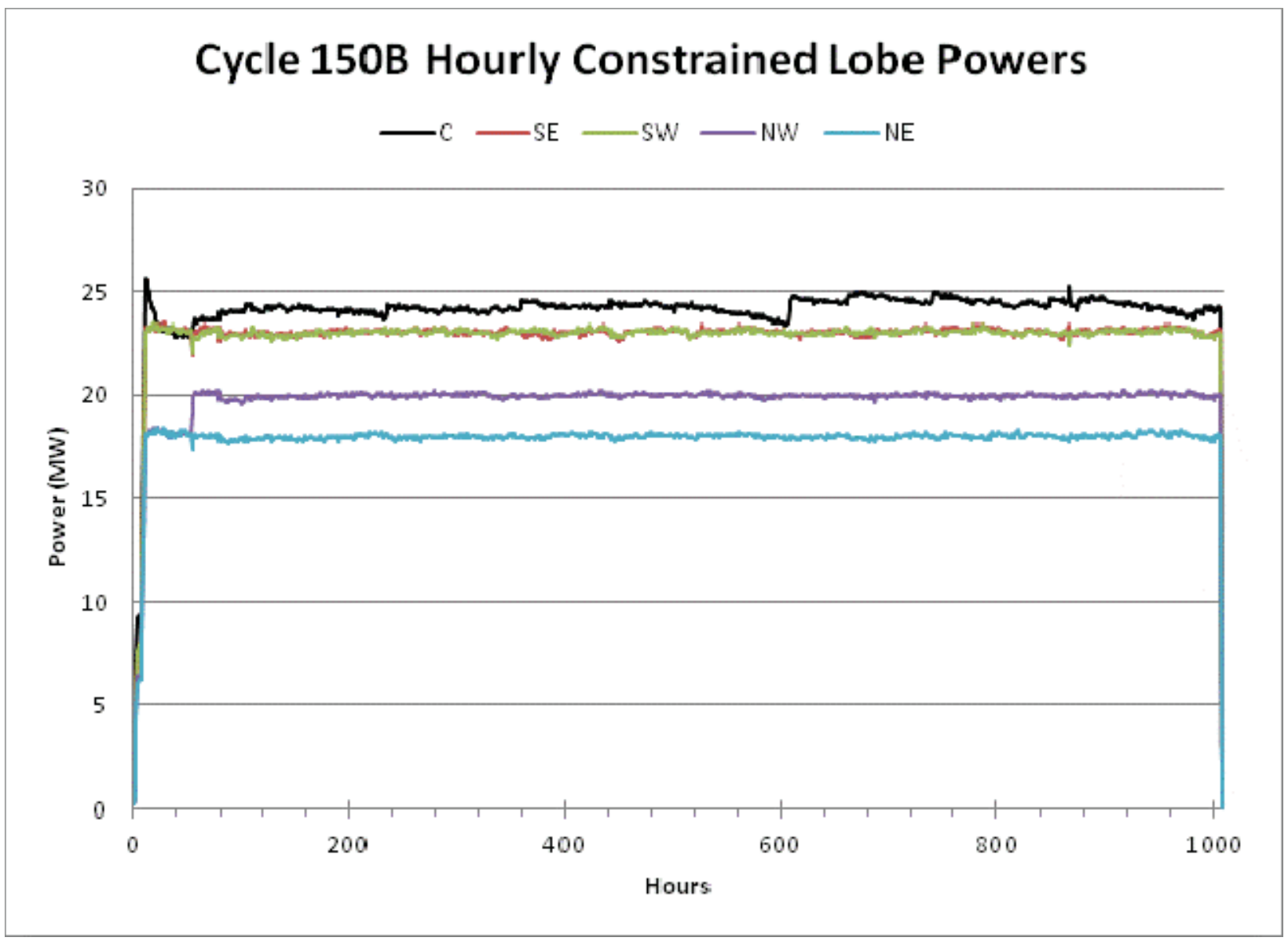

Figure 8: Hourly constrained lobe power history for Cycle 150B. 


\section{AS-RUN NUCLEAR ANALYSIS}

\subsection{Neutronics}

The as-run calculations were performed using the irradiation history in Table 6, the Monte Carlo N-Particle (MCNP) code, ORIGEN2.2 and $\mathrm{MCWO}^{2}$. The calculated as-run fission heat rates, fission densities, and as-run U-235 burnup results for the fueled plates reported have an uncertainty band $(1 \sigma)$ of $2.5 \%{ }^{2}$. The time intervals used to calculate the average plate power and burnup is shown in Table 7.

Table 7: Cycle breakdown.

\begin{tabular}{ccc}
\multicolumn{3}{c}{ Table 7: Cycle breakdown. } \\
$\begin{array}{c}\text { Time } \\
\text { Interval }\end{array}$ & $\begin{array}{c}\text { 149B } \\
\text { (days) }\end{array}$ & $\begin{array}{c}150 \mathrm{~B} \\
\text { (days) }\end{array}$ \\
\hline BOC & 0.0 & 0.0 \\
MOC 1 & 15.0 & 18.0 \\
MOC 2 & 16.0 & 13.0 \\
EOC & 22.6 & 11.0 \\
\hline Total EFPDs & 53.6 & 42.0 \\
\hline Cumulative & 53.6 & 95.6 \\
\hline
\end{tabular}

The MCNP-calculated plate power and burnup for the time intervals for each cycle are shown in Table 8 through Table 39. The plots of the fission power density and fission density as a function of the ATR Cycle time interval are in Appendix A. 
Table 8: Cycle 149B, MCNP-Calculated HGRs and Neutron Flux for AFIP-7, Plate Position 1, 0 EFPD (BOC) Average Center Lobe Power at 24.2 MW. ${ }^{2}$

\begin{tabular}{|c|c|c|c|c|}
\hline Node & $\begin{array}{l}\text { Distance from } \\
\text { Centerline (in.) }\end{array}$ & $\begin{array}{l}\text { Fission Power } \\
\text { Density (W/cc) }\end{array}$ & $\begin{array}{l}\text { Surface Heat } \\
\text { Flux }\left(W / \mathrm{cm}^{2}\right)\end{array}$ & $\begin{array}{c}\text { Neutron Flux } \\
\left(\mathbf{n} / \mathbf{c m}^{2} \mathbf{s e c}\right)\end{array}$ \\
\hline 1 & 19.25 & 6402.01 & 105.70 & $5.22 \mathrm{E}+14$ \\
\hline 2 & 18.75 & 6249.90 & 103.19 & $5.56 \mathrm{E}+14$ \\
\hline 3 & 18.25 & 6491.82 & 107.18 & $5.83 \mathrm{E}+14$ \\
\hline 4 & 17.75 & 6678.00 & 110.25 & $6.24 \mathrm{E}+14$ \\
\hline 5 & 17.25 & 7064.78 & 116.64 & $6.51 \mathrm{E}+14$ \\
\hline 6 & 16.75 & 7579.42 & 125.14 & $6.81 \mathrm{E}+14$ \\
\hline 7 & 16.25 & 7765.49 & 128.21 & $7.11 \mathrm{E}+14$ \\
\hline 8 & 15.75 & 7917.27 & 130.71 & $7.31 \mathrm{E}+14$ \\
\hline 9 & 15.25 & 8604.93 & 142.07 & $7.80 \mathrm{E}+14$ \\
\hline 10 & 14.75 & 8723.79 & 144.03 & $8.01 \mathrm{E}+14$ \\
\hline 11 & 14.25 & 9053.20 & 149.47 & $8.31 \mathrm{E}+14$ \\
\hline 13 & 13.75 & 9293.99 & 153.44 & $8.51 \mathrm{E}+14$ \\
\hline 14 & 13.25 & 9330.47 & 154.05 & $8.69 \mathrm{E}+14$ \\
\hline 15 & 12.75 & 9613.04 & 158.71 & $9.01 \mathrm{E}+14$ \\
\hline 16 & 12.25 & 10196.58 & 168.35 & $9.38 \mathrm{E}+14$ \\
\hline 17 & 11.75 & 10029.77 & 165.59 & $9.46 \mathrm{E}+14$ \\
\hline 18 & 11.25 & 10287.81 & 169.85 & $9.68 \mathrm{E}+14$ \\
\hline 19 & 10.75 & 10788.80 & 178.12 & $9.99 \mathrm{E}+14$ \\
\hline 20 & 10.25 & 11067.36 & 182.72 & $1.02 \mathrm{E}+15$ \\
\hline 21 & 9.75 & 11567.53 & 190.98 & $1.05 \mathrm{E}+15$ \\
\hline 22 & 9.25 & 11819.30 & 195.14 & $1.06 \mathrm{E}+15$ \\
\hline 23 & 8.75 & 12226.39 & 201.86 & $1.08 \mathrm{E}+15$ \\
\hline 24 & 8.25 & 12131.14 & 200.29 & $1.09 \mathrm{E}+15$ \\
\hline 25 & 7.75 & 12548.31 & 207.17 & $1.12 \mathrm{E}+15$ \\
\hline 26 & 7.25 & 12598.93 & 208.01 & $1.13 \mathrm{E}+15$ \\
\hline 27 & 6.75 & 12829.61 & 211.82 & $1.14 \mathrm{E}+15$ \\
\hline 28 & 6.25 & 12767.99 & 210.80 & $1.15 \mathrm{E}+15$ \\
\hline 29 & 5.75 & 13047.00 & 215.41 & $1.16 \mathrm{E}+15$ \\
\hline 30 & 5.25 & 13304.12 & 219.65 & $1.17 \mathrm{E}+15$ \\
\hline 31 & 4.75 & 13328.81 & 220.06 & $1.18 \mathrm{E}+15$ \\
\hline 32 & 4.25 & 13769.98 & 227.34 & $1.21 \mathrm{E}+15$ \\
\hline 33 & 3.75 & 13618.01 & 224.83 & $1.21 \mathrm{E}+15$ \\
\hline 34 & 3.25 & 13651.82 & 225.39 & $1.20 \mathrm{E}+15$ \\
\hline 35 & 2.75 & 13692.40 & 226.06 & $1.23 \mathrm{E}+15$ \\
\hline 36 & 2.25 & 13717.95 & 226.48 & $1.23 \mathrm{E}+15$ \\
\hline 37 & 1.75 & 13708.52 & 226.33 & $1.22 \mathrm{E}+15$ \\
\hline 38 & 1.25 & 13392.51 & 221.11 & $1.22 \mathrm{E}+15$ \\
\hline 39 & 0.75 & 13478.97 & 222.54 & $1.23 \mathrm{E}+15$ \\
\hline 40 & 0.25 & 13634.78 & 225.11 & $1.23 \mathrm{E}+15$ \\
\hline 41 & -0.25 & 13413.60 & 221.46 & $1.23 \mathrm{E}+15$ \\
\hline 42 & -0.75 & 13639.90 & 225.19 & $1.23 \mathrm{E}+15$ \\
\hline 43 & -1.25 & 13675.55 & 225.78 & $1.23 \mathrm{E}+15$ \\
\hline 44 & -1.75 & 14075.26 & 232.38 & $1.23 \mathrm{E}+15$ \\
\hline 45 & -2.25 & 14089.19 & 232.61 & $1.23 \mathrm{E}+15$ \\
\hline 46 & -2.75 & 13976.43 & 230.75 & $1.23 \mathrm{E}+15$ \\
\hline 47 & -3.25 & 13719.19 & 226.50 & $1.23 \mathrm{E}+15$ \\
\hline 48 & -3.75 & 13713.63 & 226.41 & $1.22 \mathrm{E}+15$ \\
\hline 49 & -4.25 & 13642.07 & 225.23 & $1.23 \mathrm{E}+15$ \\
\hline 50 & -4.75 & 13619.37 & 224.86 & $1.21 \mathrm{E}+15$ \\
\hline 51 & -5.25 & 13627.90 & 225.00 & $1.20 \mathrm{E}+15$ \\
\hline 52 & -5.75 & 13591.99 & 224.40 & $1.19 \mathrm{E}+15$ \\
\hline
\end{tabular}




\begin{tabular}{|l|l|l|l|l|}
\hline 53 & -6.25 & 13591.80 & 224.40 & $1.19 \mathrm{E}+15$ \\
\hline 54 & -6.75 & 13281.35 & 219.28 & $1.17 \mathrm{E}+15$ \\
\hline 55 & -7.25 & 13082.94 & 216.00 & $1.16 \mathrm{E}+15$ \\
\hline 56 & -7.75 & 12736.32 & 210.28 & $1.14 \mathrm{E}+15$ \\
\hline 57 & -8.25 & 12812.60 & 211.54 & $1.14 \mathrm{E}+15$ \\
\hline 58 & -8.75 & 12564.79 & 207.44 & $1.12 \mathrm{E}+15$ \\
\hline 59 & -9.25 & 12581.52 & 207.72 & $1.12 \mathrm{E}+15$ \\
\hline 60 & -9.75 & 12106.47 & 199.88 & $1.09 \mathrm{E}+15$ \\
\hline 61 & -10.25 & 11609.36 & 191.67 & $1.08 \mathrm{E}+15$ \\
\hline 62 & -10.75 & 11449.45 & 189.03 & $1.06 \mathrm{E}+15$ \\
\hline 63 & -11.25 & 11254.23 & 185.81 & $1.04 \mathrm{E}+15$ \\
\hline 64 & -11.75 & 10995.77 & 181.54 & $1.02 \mathrm{E}+15$ \\
\hline 65 & -12.25 & 10604.38 & 175.08 & $9.95 \mathrm{E}+14$ \\
\hline 66 & -12.75 & 10627.78 & 175.46 & $9.74 \mathrm{E}+14$ \\
\hline 67 & -13.25 & 10210.42 & 168.57 & $9.54 \mathrm{E}+14$ \\
\hline 68 & -13.75 & 10225.52 & 168.82 & $9.25 \mathrm{E}+14$ \\
\hline 69 & -14.25 & 10036.06 & 165.70 & $9.12 \mathrm{E}+14$ \\
\hline 70 & -14.75 & 9656.97 & 159.44 & $8.76 \mathrm{E}+14$ \\
\hline 71 & -15.25 & 9382.90 & 154.91 & $8.54 \mathrm{E}+14$ \\
\hline 72 & -15.75 & 8984.94 & 148.34 & $8.26 \mathrm{E}+14$ \\
\hline 73 & -16.25 & 8712.76 & 143.85 & $8.03 \mathrm{E}+14$ \\
\hline 74 & -16.75 & 8458.21 & 139.65 & $7.74 \mathrm{E}+14$ \\
\hline 75 & -17.25 & 8061.47 & 133.09 & $7.39 \mathrm{E}+14$ \\
\hline 76 & -17.75 & 7629.78 & 125.97 & $7.06 \mathrm{E}+14$ \\
\hline 77 & -18.25 & 7438.73 & 122.81 & $6.82 \mathrm{E}+14$ \\
\hline 78 & -18.75 & 7193.85 & 118.77 & $6.41 \mathrm{E}+14$ \\
\hline
\end{tabular}


Table 9: Cycle 149B, MCNP-Calculated HGRs, Neutron Flux, Depletion and Fission Density for AFIP-7, Plate Position 1, 15 EFPD (MOC1) Average Center Lobe Power at 24.2 MW. ${ }^{2}$

\begin{tabular}{|c|c|c|c|c|c|c|}
\hline Node & $\begin{array}{c}\text { Distance from } \\
\text { Centerline (in.) }\end{array}$ & $\begin{array}{l}\text { Fission Power } \\
\text { Density (W/cc) }\end{array}$ & $\begin{array}{l}\text { Surface Heat } \\
\text { Flux }\left(W / \mathrm{cm}^{2}\right)\end{array}$ & $\begin{array}{c}\text { Neutron Flux } \\
\left(\mathrm{n} / \mathrm{cm}^{2} \mathrm{sec}\right)\end{array}$ & $\begin{array}{l}\text { Fission Density } \\
\text { (fissions/cc) }\end{array}$ & $\begin{array}{c}\text { U-235 } \\
\text { Burnup (\%) }\end{array}$ \\
\hline 1 & 19.25 & 5341.07 & 88.18 & $4.97 \mathrm{E}+14$ & $2.50 \mathrm{E}+20$ & $4.13 \%$ \\
\hline 2 & 18.75 & 5082.17 & 83.91 & $5.28 \mathrm{E}+14$ & $2.44 \mathrm{E}+20$ & $4.05 \%$ \\
\hline 3 & 18.25 & 5363.80 & 88.56 & $5.49 \mathrm{E}+14$ & $2.54 \mathrm{E}+20$ & $4.21 \%$ \\
\hline 4 & 17.75 & 5650.29 & 93.29 & $5.79 \mathrm{E}+14$ & $2.61 \mathrm{E}+20$ & $4.30 \%$ \\
\hline 5 & 17.25 & 5948.91 & 98.22 & $6.10 \mathrm{E}+14$ & $2.76 \mathrm{E}+20$ & $4.55 \%$ \\
\hline 6 & 16.75 & 6069.28 & 100.20 & $6.36 \mathrm{E}+14$ & $2.96 \mathrm{E}+20$ & $4.89 \%$ \\
\hline 7 & 16.25 & 6333.43 & 104.56 & $6.55 \mathrm{E}+14$ & $3.03 \mathrm{E}+20$ & $4.97 \%$ \\
\hline 8 & 15.75 & 6460.28 & 106.66 & $6.79 \mathrm{E}+14$ & $3.09 \mathrm{E}+20$ & $5.05 \%$ \\
\hline 9 & 15.25 & 6771.92 & 111.80 & $7.11 \mathrm{E}+14$ & $3.36 \mathrm{E}+20$ & $5.56 \%$ \\
\hline 10 & 14.75 & 7000.50 & 115.58 & $7.23 \mathrm{E}+14$ & $3.41 \mathrm{E}+20$ & $5.56 \%$ \\
\hline 11 & 14.25 & 7073.46 & 116.78 & $7.47 \mathrm{E}+14$ & $3.54 \mathrm{E}+20$ & $5.81 \%$ \\
\hline 13 & 13.75 & 7216.39 & 119.14 & $7.75 \mathrm{E}+14$ & $3.63 \mathrm{E}+20$ & $5.98 \%$ \\
\hline 14 & 13.25 & 7178.04 & 118.51 & $7.87 \mathrm{E}+14$ & $3.65 \mathrm{E}+20$ & $5.98 \%$ \\
\hline 15 & 12.75 & 7321.55 & 120.88 & $8.04 \mathrm{E}+14$ & $3.76 \mathrm{E}+20$ & $6.15 \%$ \\
\hline 16 & 12.25 & 7468.09 & 123.30 & $8.23 \mathrm{E}+14$ & $3.98 \mathrm{E}+20$ & $6.48 \%$ \\
\hline 17 & 11.75 & 7597.20 & 125.43 & $8.36 \mathrm{E}+14$ & $3.92 \mathrm{E}+20$ & $6.40 \%$ \\
\hline 18 & 11.25 & 7734.51 & 127.70 & $8.48 \mathrm{E}+14$ & $4.02 \mathrm{E}+20$ & $6.57 \%$ \\
\hline 19 & 10.75 & 8017.17 & 132.36 & $8.61 \mathrm{E}+14$ & $4.21 \mathrm{E}+20$ & $6.92 \%$ \\
\hline 20 & 10.25 & 8070.86 & 133.25 & $8.75 \mathrm{E}+14$ & $4.32 \mathrm{E}+20$ & $7.08 \%$ \\
\hline 21 & 9.75 & 8442.94 & 139.39 & $8.98 \mathrm{E}+14$ & $4.52 \mathrm{E}+20$ & $7.34 \%$ \\
\hline 22 & 9.25 & 8383.53 & 138.41 & $9.02 \mathrm{E}+14$ & $4.62 \mathrm{E}+20$ & $7.50 \%$ \\
\hline 23 & 8.75 & 8547.13 & 141.11 & $9.18 \mathrm{E}+14$ & $4.78 \mathrm{E}+20$ & $7.76 \%$ \\
\hline 24 & 8.25 & 8773.76 & 144.85 & $9.28 \mathrm{E}+14$ & $4.74 \mathrm{E}+20$ & $7.67 \%$ \\
\hline 25 & 7.75 & 8638.51 & 142.62 & $9.29 \mathrm{E}+14$ & $4.90 \mathrm{E}+20$ & $7.92 \%$ \\
\hline 26 & 7.25 & 8775.00 & 144.88 & $9.48 \mathrm{E}+14$ & $4.92 \mathrm{E}+20$ & $8.01 \%$ \\
\hline 27 & 6.75 & 8868.93 & 146.43 & $9.51 \mathrm{E}+14$ & $5.01 \mathrm{E}+20$ & $8.09 \%$ \\
\hline 28 & 6.25 & 8946.79 & 147.71 & $9.59 \mathrm{E}+14$ & $4.99 \mathrm{E}+20$ & $8.09 \%$ \\
\hline 29 & 5.75 & 8948.41 & 147.74 & $9.64 \mathrm{E}+14$ & $5.10 \mathrm{E}+20$ & $8.26 \%$ \\
\hline 30 & 5.25 & 8948.06 & 147.73 & $9.69 \mathrm{E}+14$ & $5.20 \mathrm{E}+20$ & $8.43 \%$ \\
\hline 31 & 4.75 & 9129.56 & 150.73 & $9.82 \mathrm{E}+14$ & $5.21 \mathrm{E}+20$ & $8.43 \%$ \\
\hline 32 & 4.25 & 9106.10 & 150.34 & $9.89 \mathrm{E}+14$ & $5.38 \mathrm{E}+20$ & $8.68 \%$ \\
\hline 33 & 3.75 & 9212.24 & 152.09 & $9.84 \mathrm{E}+14$ & $5.32 \mathrm{E}+20$ & $8.60 \%$ \\
\hline 34 & 3.25 & 9230.94 & 152.40 & $9.87 \mathrm{E}+14$ & $5.33 \mathrm{E}+20$ & $8.60 \%$ \\
\hline 35 & 2.75 & 9090.18 & 150.08 & $9.92 \mathrm{E}+14$ & $5.35 \mathrm{E}+20$ & $8.60 \%$ \\
\hline 36 & 2.25 & 9225.54 & 152.31 & $9.96 \mathrm{E}+14$ & $5.36 \mathrm{E}+20$ & $8.68 \%$ \\
\hline 37 & 1.75 & 8926.39 & 147.37 & $9.95 \mathrm{E}+14$ & $5.36 \mathrm{E}+20$ & $8.68 \%$ \\
\hline 38 & 1.25 & 9147.04 & 151.02 & $9.99 \mathrm{E}+14$ & $5.23 \mathrm{E}+20$ & $8.43 \%$ \\
\hline 39 & 0.75 & 9087.94 & 150.04 & $9.98 \mathrm{E}+14$ & $5.27 \mathrm{E}+20$ & $8.51 \%$ \\
\hline 40 & 0.25 & 9052.57 & 149.46 & $1.00 \mathrm{E}+15$ & $5.33 \mathrm{E}+20$ & $8.60 \%$ \\
\hline 41 & -0.25 & 9226.17 & 152.32 & $1.00 \mathrm{E}+15$ & $5.24 \mathrm{E}+20$ & $8.51 \%$ \\
\hline 42 & -0.75 & 9124.29 & 150.64 & $1.00 \mathrm{E}+15$ & $5.33 \mathrm{E}+20$ & $8.60 \%$ \\
\hline 43 & -1.25 & 9263.32 & 152.94 & $1.01 \mathrm{E}+15$ & $5.34 \mathrm{E}+20$ & $8.60 \%$ \\
\hline 44 & -1.75 & 9031.13 & 149.10 & $9.97 \mathrm{E}+14$ & $5.50 \mathrm{E}+20$ & $8.85 \%$ \\
\hline 45 & -2.25 & 9365.85 & 154.63 & $9.98 \mathrm{E}+14$ & $5.50 \mathrm{E}+20$ & $8.85 \%$ \\
\hline 46 & -2.75 & 9255.04 & 152.80 & $9.98 \mathrm{E}+14$ & $5.46 \mathrm{E}+20$ & $8.85 \%$ \\
\hline 47 & -3.25 & 9171.18 & 151.42 & $9.97 \mathrm{E}+14$ & $5.36 \mathrm{E}+20$ & $8.68 \%$ \\
\hline 48 & -3.75 & 9342.59 & 154.25 & $9.92 \mathrm{E}+14$ & $5.36 \mathrm{E}+20$ & $8.68 \%$ \\
\hline 49 & -4.25 & 9239.06 & 152.54 & $1.00 \mathrm{E}+15$ & $5.33 \mathrm{E}+20$ & $8.60 \%$ \\
\hline 50 & -4.75 & 9199.97 & 151.89 & $9.90 \mathrm{E}+14$ & $5.32 \mathrm{E}+20$ & $8.60 \%$ \\
\hline 51 & -5.25 & 9294.68 & 153.46 & $9.86 \mathrm{E}+14$ & $5.32 \mathrm{E}+20$ & $8.60 \%$ \\
\hline 52 & -5.75 & 9163.55 & 151.29 & $9.81 \mathrm{E}+14$ & $5.31 \mathrm{E}+20$ & $8.60 \%$ \\
\hline
\end{tabular}




\begin{tabular}{|l|l|l|l|l|l|l|}
\hline 53 & -6.25 & 9060.58 & 149.59 & $9.76 \mathrm{E}+14$ & $5.31 \mathrm{E}+20$ & $8.60 \%$ \\
\hline 54 & -6.75 & 9017.55 & 148.88 & $9.74 \mathrm{E}+14$ & $5.19 \mathrm{E}+20$ & $8.35 \%$ \\
\hline 55 & -7.25 & 9057.11 & 149.53 & $9.66 \mathrm{E}+14$ & $5.11 \mathrm{E}+20$ & $8.26 \%$ \\
\hline 56 & -7.75 & 8869.18 & 146.43 & $9.63 \mathrm{E}+14$ & $4.98 \mathrm{E}+20$ & $8.09 \%$ \\
\hline 57 & -8.25 & 8953.13 & 147.82 & $9.58 \mathrm{E}+14$ & $5.01 \mathrm{E}+20$ & $8.09 \%$ \\
\hline 58 & -8.75 & 8771.80 & 144.82 & $9.49 \mathrm{E}+14$ & $4.91 \mathrm{E}+20$ & $7.92 \%$ \\
\hline 59 & -9.25 & 8750.25 & 144.47 & $9.34 \mathrm{E}+14$ & $4.92 \mathrm{E}+20$ & $7.92 \%$ \\
\hline 60 & -9.75 & 8392.52 & 138.56 & $9.26 \mathrm{E}+14$ & $4.73 \mathrm{E}+20$ & $7.67 \%$ \\
\hline 61 & -10.25 & 8412.82 & 138.90 & $9.20 \mathrm{E}+14$ & $4.54 \mathrm{E}+20$ & $7.42 \%$ \\
\hline 62 & -10.75 & 8249.45 & 136.20 & $9.06 \mathrm{E}+14$ & $4.47 \mathrm{E}+20$ & $7.25 \%$ \\
\hline 63 & -11.25 & 8168.26 & 134.86 & $8.90 \mathrm{E}+14$ & $4.40 \mathrm{E}+20$ & $7.17 \%$ \\
\hline 64 & -11.75 & 8133.22 & 134.28 & $8.81 \mathrm{E}+14$ & $4.30 \mathrm{E}+20$ & $7.00 \%$ \\
\hline 65 & -12.25 & 7940.52 & 131.10 & $8.70 \mathrm{E}+14$ & $4.14 \mathrm{E}+20$ & $6.82 \%$ \\
\hline 66 & -12.75 & 7987.76 & 131.88 & $8.61 \mathrm{E}+14$ & $4.15 \mathrm{E}+20$ & $6.73 \%$ \\
\hline 67 & -13.25 & 7957.01 & 131.37 & $8.42 \mathrm{E}+14$ & $3.99 \mathrm{E}+20$ & $6.48 \%$ \\
\hline 68 & -13.75 & 7822.66 & 129.15 & $8.29 \mathrm{E}+14$ & $3.99 \mathrm{E}+20$ & $6.48 \%$ \\
\hline 69 & -14.25 & 7729.07 & 127.61 & $8.12 \mathrm{E}+14$ & $3.92 \mathrm{E}+20$ & $6.40 \%$ \\
\hline 70 & -14.75 & 7585.46 & 125.24 & $7.99 \mathrm{E}+14$ & $3.77 \mathrm{E}+20$ & $6.15 \%$ \\
\hline 71 & -15.25 & 7253.68 & 119.76 & $7.70 \mathrm{E}+14$ & $3.67 \mathrm{E}+20$ & $5.98 \%$ \\
\hline 72 & -15.75 & 7144.72 & 117.96 & $7.54 \mathrm{E}+14$ & $3.51 \mathrm{E}+20$ & $5.73 \%$ \\
\hline 73 & -16.25 & 7044.86 & 116.31 & $7.36 \mathrm{E}+14$ & $3.40 \mathrm{E}+20$ & $5.56 \%$ \\
\hline 74 & -16.75 & 6899.96 & 113.92 & $7.17 \mathrm{E}+14$ & $3.30 \mathrm{E}+20$ & $5.39 \%$ \\
\hline 75 & -17.25 & 6668.69 & 110.10 & $6.97 \mathrm{E}+14$ & $3.15 \mathrm{E}+20$ & $5.22 \%$ \\
\hline 76 & -17.75 & 6380.85 & 105.35 & $6.66 \mathrm{E}+14$ & $2.98 \mathrm{E}+20$ & $4.89 \%$ \\
\hline 77 & -18.25 & 6120.72 & 101.05 & $6.35 \mathrm{E}+14$ & $2.91 \mathrm{E}+20$ & $4.80 \%$ \\
\hline 78 & -18.75 & 5980.26 & 98.73 & $6.18 \mathrm{E}+14$ & $2.81 \mathrm{E}+20$ & $4.63 \%$ \\
\hline
\end{tabular}


Table 10: Cycle 149B, MCNP-Calculated HGRs, Neutron Flux, Depletion and Fission Density for AFIP-7, Plate Position 1, 31 EFPD (MOC2) Average Center Lobe Power at 24.2 MW. ${ }^{2}$

\begin{tabular}{|c|c|c|c|c|c|c|}
\hline Node & $\begin{array}{c}\text { Distance from } \\
\text { Centerline (in.) }\end{array}$ & $\begin{array}{l}\text { Fission Power } \\
\text { Density (W/cc) }\end{array}$ & $\begin{array}{l}\text { Surface Heat } \\
\text { Flux }\left(W / \mathrm{cm}^{2}\right)\end{array}$ & $\begin{array}{c}\text { Neutron Flux } \\
\left(\mathrm{n} / \mathrm{cm}^{2} \mathrm{sec}\right)\end{array}$ & $\begin{array}{l}\text { Fission Density } \\
\text { (fissions/cc) }\end{array}$ & $\begin{array}{c}\text { U-235 } \\
\text { Burnup (\%) }\end{array}$ \\
\hline 1 & 19.25 & 5968.71 & 98.54 & $5.46 \mathrm{E}+14$ & $4.92 \mathrm{E}+20$ & $8.09 \%$ \\
\hline 2 & 18.75 & 5589.65 & 92.29 & $5.74 \mathrm{E}+14$ & $4.74 \mathrm{E}+20$ & $7.84 \%$ \\
\hline 3 & 18.25 & 5978.54 & 98.71 & $6.10 \mathrm{E}+14$ & $4.97 \mathrm{E}+20$ & $8.18 \%$ \\
\hline 4 & 17.75 & 6089.78 & 100.54 & $6.30 \mathrm{E}+14$ & $5.17 \mathrm{E}+20$ & $8.51 \%$ \\
\hline 5 & 17.25 & 6489.39 & 107.14 & $6.64 \mathrm{E}+14$ & $5.45 \mathrm{E}+20$ & $9.02 \%$ \\
\hline 6 & 16.75 & 6749.72 & 111.44 & $6.91 \mathrm{E}+14$ & $5.71 \mathrm{E}+20$ & $9.44 \%$ \\
\hline 7 & 16.25 & 6679.43 & 110.28 & $7.08 \mathrm{E}+14$ & $5.90 \mathrm{E}+20$ & $9.69 \%$ \\
\hline 8 & 15.75 & 7067.55 & 116.69 & $7.37 \mathrm{E}+14$ & $6.02 \mathrm{E}+20$ & $9.86 \%$ \\
\hline 9 & 15.25 & 7234.33 & 119.44 & $7.66 \mathrm{E}+14$ & $6.43 \mathrm{E}+20$ & $10.53 \%$ \\
\hline 10 & 14.75 & 7532.68 & 124.36 & $7.85 \mathrm{E}+14$ & $6.58 \mathrm{E}+20$ & $10.80 \%$ \\
\hline 11 & 14.25 & 7533.98 & 124.39 & $8.08 \mathrm{E}+14$ & $6.74 \mathrm{E}+20$ & $11.05 \%$ \\
\hline 13 & 13.75 & 7808.52 & 128.92 & $8.20 \mathrm{E}+14$ & $6.90 \mathrm{E}+20$ & $11.30 \%$ \\
\hline 14 & 13.25 & 7773.45 & 128.34 & $8.38 \mathrm{E}+14$ & $6.90 \mathrm{E}+20$ & $11.30 \%$ \\
\hline 15 & 12.75 & 8002.46 & 132.12 & $8.66 \mathrm{E}+14$ & $7.07 \mathrm{E}+20$ & $11.55 \%$ \\
\hline 16 & 12.25 & 8031.63 & 132.60 & $8.77 \mathrm{E}+14$ & $7.37 \mathrm{E}+20$ & $12.06 \%$ \\
\hline 17 & 11.75 & 8075.80 & 133.33 & $8.89 \mathrm{E}+14$ & $7.36 \mathrm{E}+20$ & $12.06 \%$ \\
\hline 18 & 11.25 & 8224.76 & 135.79 & $9.09 \mathrm{E}+14$ & $7.52 \mathrm{E}+20$ & $12.31 \%$ \\
\hline 19 & 10.75 & 8441.90 & 139.38 & $9.16 \mathrm{E}+14$ & $7.84 \mathrm{E}+20$ & $12.81 \%$ \\
\hline 20 & 10.25 & 8746.09 & 144.40 & $9.29 \mathrm{E}+14$ & $7.98 \mathrm{E}+20$ & $12.98 \%$ \\
\hline 21 & 9.75 & 8846.48 & 146.06 & $9.38 \mathrm{E}+14$ & $8.34 \mathrm{E}+20$ & $13.57 \%$ \\
\hline 22 & 9.25 & 8994.67 & 148.50 & $9.46 \mathrm{E}+14$ & $8.41 \mathrm{E}+20$ & $13.65 \%$ \\
\hline 23 & 8.75 & 9140.34 & 150.91 & $9.72 \mathrm{E}+14$ & $8.65 \mathrm{E}+20$ & $13.99 \%$ \\
\hline 24 & 8.25 & 9315.08 & 153.79 & $9.73 \mathrm{E}+14$ & $8.71 \mathrm{E}+20$ & $14.07 \%$ \\
\hline 25 & 7.75 & 9261.20 & 152.90 & $9.76 \mathrm{E}+14$ & $8.81 \mathrm{E}+20$ & $14.24 \%$ \\
\hline 26 & 7.25 & 9292.34 & 153.42 & $9.80 \mathrm{E}+14$ & $8.89 \mathrm{E}+20$ & $14.41 \%$ \\
\hline 27 & 6.75 & 9514.08 & 157.08 & $9.97 \mathrm{E}+14$ & $9.03 \mathrm{E}+20$ & $14.59 \%$ \\
\hline 28 & 6.25 & 9579.45 & 158.16 & $9.98 \mathrm{E}+14$ & $9.04 \mathrm{E}+20$ & $14.67 \%$ \\
\hline 29 & 5.75 & 9499.41 & 156.84 & $1.01 \mathrm{E}+15$ & $9.15 \mathrm{E}+20$ & $14.76 \%$ \\
\hline 30 & 5.25 & 9459.39 & 156.17 & $1.01 \mathrm{E}+15$ & $9.25 \mathrm{E}+20$ & $14.93 \%$ \\
\hline 31 & 4.75 & 9738.23 & 160.78 & $1.02 \mathrm{E}+15$ & $9.34 \mathrm{E}+20$ & $15.09 \%$ \\
\hline 32 & 4.25 & 9846.15 & 162.56 & $1.02 \mathrm{E}+15$ & $9.50 \mathrm{E}+20$ & $15.35 \%$ \\
\hline 33 & 3.75 & 9875.01 & 163.04 & $1.02 \mathrm{E}+15$ & $9.49 \mathrm{E}+20$ & $15.35 \%$ \\
\hline 34 & 3.25 & 9929.84 & 163.94 & $1.03 \mathrm{E}+15$ & $9.51 \mathrm{E}+20$ & $15.35 \%$ \\
\hline 35 & 2.75 & 9726.50 & 160.58 & $1.03 \mathrm{E}+15$ & $9.47 \mathrm{E}+20$ & $15.26 \%$ \\
\hline 36 & 2.25 & 9814.31 & 162.03 & $1.03 \mathrm{E}+15$ & $9.54 \mathrm{E}+20$ & $15.43 \%$ \\
\hline 37 & 1.75 & 9700.81 & 160.16 & $1.03 \mathrm{E}+15$ & $9.40 \mathrm{E}+20$ & $15.18 \%$ \\
\hline 38 & 1.25 & 9582.99 & 158.22 & $1.03 \mathrm{E}+15$ & $9.37 \mathrm{E}+20$ & $15.18 \%$ \\
\hline 39 & 0.75 & 9588.81 & 158.31 & $1.03 \mathrm{E}+15$ & $9.38 \mathrm{E}+20$ & $15.18 \%$ \\
\hline 40 & 0.25 & 9643.34 & 159.21 & $1.03 \mathrm{E}+15$ & $9.42 \mathrm{E}+20$ & $15.26 \%$ \\
\hline 41 & -0.25 & 9703.08 & 160.20 & $1.04 \mathrm{E}+15$ & $9.42 \mathrm{E}+20$ & $15.26 \%$ \\
\hline 42 & -0.75 & 9779.92 & 161.47 & $1.03 \mathrm{E}+15$ & $9.46 \mathrm{E}+20$ & $15.26 \%$ \\
\hline 43 & -1.25 & 9858.14 & 162.76 & $1.04 \mathrm{E}+15$ & $9.54 \mathrm{E}+20$ & $15.35 \%$ \\
\hline 44 & -1.75 & 9772.02 & 161.34 & $1.03 \mathrm{E}+15$ & $9.59 \mathrm{E}+20$ & $15.43 \%$ \\
\hline 45 & -2.25 & 9976.94 & 164.72 & $1.03 \mathrm{E}+15$ & $9.74 \mathrm{E}+20$ & $15.68 \%$ \\
\hline 46 & -2.75 & 9977.27 & 164.72 & $1.03 \mathrm{E}+15$ & $9.65 \mathrm{E}+20$ & $15.60 \%$ \\
\hline 47 & -3.25 & 9856.50 & 162.73 & $1.04 \mathrm{E}+15$ & $9.51 \mathrm{E}+20$ & $15.35 \%$ \\
\hline 48 & -3.75 & 9978.91 & 164.75 & $1.04 \mathrm{E}+15$ & $9.59 \mathrm{E}+20$ & $15.43 \%$ \\
\hline 49 & -4.25 & 9877.89 & 163.08 & $1.03 \mathrm{E}+15$ & $9.51 \mathrm{E}+20$ & $15.35 \%$ \\
\hline 50 & -4.75 & 9718.97 & 160.46 & $1.03 \mathrm{E}+15$ & $9.49 \mathrm{E}+20$ & $15.35 \%$ \\
\hline 51 & -5.25 & 9835.81 & 162.39 & $1.02 \mathrm{E}+15$ & $9.53 \mathrm{E}+20$ & $15.43 \%$ \\
\hline 52 & -5.75 & 9698.81 & 160.13 & $1.02 \mathrm{E}+15$ & $9.46 \mathrm{E}+20$ & $15.26 \%$ \\
\hline
\end{tabular}




\begin{tabular}{|l|l|l|l|l|l|l|}
\hline 53 & -6.25 & 9718.94 & 160.46 & $1.02 \mathrm{E}+15$ & $9.41 \mathrm{E}+20$ & $15.18 \%$ \\
\hline 54 & -6.75 & 9779.59 & 161.46 & $1.02 \mathrm{E}+15$ & $9.27 \mathrm{E}+20$ & $15.01 \%$ \\
\hline 55 & -7.25 & 9737.21 & 160.76 & $1.02 \mathrm{E}+15$ & $9.21 \mathrm{E}+20$ & $14.93 \%$ \\
\hline 56 & -7.75 & 9619.28 & 158.81 & $1.01 \mathrm{E}+15$ & $8.99 \mathrm{E}+20$ & $14.59 \%$ \\
\hline 57 & -8.25 & 9535.89 & 157.44 & $1.00 \mathrm{E}+15$ & $9.06 \mathrm{E}+20$ & $14.67 \%$ \\
\hline 58 & -8.75 & 9513.22 & 157.06 & $9.94 \mathrm{E}+14$ & $8.88 \mathrm{E}+20$ & $14.32 \%$ \\
\hline 59 & -9.25 & 9455.16 & 156.10 & $9.85 \mathrm{E}+14$ & $8.88 \mathrm{E}+20$ & $14.32 \%$ \\
\hline 60 & -9.75 & 9147.54 & 151.03 & $9.76 \mathrm{E}+14$ & $8.53 \mathrm{E}+20$ & $13.82 \%$ \\
\hline 61 & -10.25 & 9089.97 & 150.08 & $9.72 \mathrm{E}+14$ & $8.34 \mathrm{E}+20$ & $13.57 \%$ \\
\hline 62 & -10.75 & 8844.57 & 146.02 & $9.59 \mathrm{E}+14$ & $8.21 \mathrm{E}+20$ & $13.32 \%$ \\
\hline 63 & -11.25 & 8810.08 & 145.45 & $9.48 \mathrm{E}+14$ & $8.10 \mathrm{E}+20$ & $13.15 \%$ \\
\hline 64 & -11.75 & 8645.17 & 142.73 & $9.33 \mathrm{E}+14$ & $7.98 \mathrm{E}+20$ & $12.98 \%$ \\
\hline 65 & -12.25 & 8625.96 & 142.41 & $9.22 \mathrm{E}+14$ & $7.74 \mathrm{E}+20$ & $12.64 \%$ \\
\hline 66 & -12.75 & 8816.51 & 145.56 & $9.15 \mathrm{E}+14$ & $7.77 \mathrm{E}+20$ & $12.64 \%$ \\
\hline 67 & -13.25 & 8337.02 & 137.64 & $9.02 \mathrm{E}+14$ & $7.59 \mathrm{E}+20$ & $12.39 \%$ \\
\hline 68 & -13.75 & 8379.14 & 138.34 & $8.91 \mathrm{E}+14$ & $7.54 \mathrm{E}+20$ & $12.31 \%$ \\
\hline 69 & -14.25 & 8099.20 & 133.72 & $8.73 \mathrm{E}+14$ & $7.42 \mathrm{E}+20$ & $12.14 \%$ \\
\hline 70 & -14.75 & 8051.12 & 132.92 & $8.52 \mathrm{E}+14$ & $7.21 \mathrm{E}+20$ & $11.80 \%$ \\
\hline 71 & -15.25 & 7900.59 & 130.44 & $8.25 \mathrm{E}+14$ & $6.95 \mathrm{E}+20$ & $11.38 \%$ \\
\hline 72 & -15.75 & 7730.77 & 127.64 & $8.09 \mathrm{E}+14$ & $6.75 \mathrm{E}+20$ & $11.05 \%$ \\
\hline 73 & -16.25 & 7570.05 & 124.98 & $7.93 \mathrm{E}+14$ & $6.59 \mathrm{E}+20$ & $10.80 \%$ \\
\hline 74 & -16.75 & 7415.44 & 122.43 & $7.71 \mathrm{E}+14$ & $6.43 \mathrm{E}+20$ & $10.53 \%$ \\
\hline 75 & -17.25 & 7045.01 & 116.31 & $7.46 \mathrm{E}+14$ & $6.17 \mathrm{E}+20$ & $10.11 \%$ \\
\hline 76 & -17.75 & 6844.05 & 113.00 & $7.20 \mathrm{E}+14$ & $5.87 \mathrm{E}+20$ & $9.69 \%$ \\
\hline 77 & -18.25 & 6541.35 & 108.00 & $6.92 \mathrm{E}+14$ & $5.68 \mathrm{E}+20$ & $9.35 \%$ \\
\hline 78 & -18.75 & 6497.27 & 107.27 & $6.68 \mathrm{E}+14$ & $5.52 \mathrm{E}+20$ & $9.10 \%$ \\
\hline
\end{tabular}


Table 11: Cycle 149B, MCNP-Calculated HGRs, Neutron Flux, Depletion and Fission Density for AFIP-7, Plate Position 1, 53.6 EFPD (EOC) Average Center Lobe Power at 24.2 MW. ${ }^{2}$

\begin{tabular}{|c|c|c|c|c|c|c|}
\hline Node & $\begin{array}{c}\text { Distance from } \\
\text { Centerline (in.) }\end{array}$ & $\begin{array}{l}\text { Fission Power } \\
\text { Density (W/cc) }\end{array}$ & $\begin{array}{l}\text { Surface Heat } \\
\text { Flux }\left(W / \mathrm{cm}^{2}\right)\end{array}$ & $\begin{array}{c}\text { Neutron Flux } \\
\left(\mathrm{n} / \mathrm{cm}^{2} \mathrm{sec}\right)\end{array}$ & $\begin{array}{l}\text { Fission Density } \\
\text { (fissions/cc) }\end{array}$ & $\begin{array}{c}\text { U-235 } \\
\text { Burnup (\%) }\end{array}$ \\
\hline 1 & 19.25 & 5838.76 & 96.40 & $5.56 \mathrm{E}+14$ & $8.69 \mathrm{E}+20$ & $14.16 \%$ \\
\hline 2 & 18.75 & 5483.85 & 90.54 & $5.82 \mathrm{E}+14$ & $8.28 \mathrm{E}+20$ & $13.57 \%$ \\
\hline 3 & 18.25 & 5823.79 & 96.15 & $6.21 \mathrm{E}+14$ & $8.75 \mathrm{E}+20$ & $14.24 \%$ \\
\hline 4 & 17.75 & 5971.28 & 98.59 & $6.41 \mathrm{E}+14$ & $9.02 \mathrm{E}+20$ & $14.76 \%$ \\
\hline 5 & 17.25 & 6347.20 & 104.79 & $6.74 \mathrm{E}+14$ & $9.56 \mathrm{E}+20$ & $15.60 \%$ \\
\hline 6 & 16.75 & 6572.79 & 108.52 & $7.03 \mathrm{E}+14$ & $9.98 \mathrm{E}+20$ & $16.23 \%$ \\
\hline 7 & 16.25 & 6519.93 & 107.64 & $7.21 \mathrm{E}+14$ & $1.01 \mathrm{E}+21$ & $16.44 \%$ \\
\hline 8 & 15.75 & 6873.37 & 113.48 & $7.50 \mathrm{E}+14$ & $1.05 \mathrm{E}+21$ & $16.98 \%$ \\
\hline 9 & 15.25 & 7033.60 & 116.12 & $7.79 \mathrm{E}+14$ & $1.10 \mathrm{E}+21$ & $17.81 \%$ \\
\hline 10 & 14.75 & 7325.22 & 120.94 & $7.97 \mathrm{E}+14$ & $1.13 \mathrm{E}+21$ & $18.30 \%$ \\
\hline 11 & 14.25 & 7349.59 & 121.34 & $8.22 \mathrm{E}+14$ & $1.15 \mathrm{E}+21$ & $18.57 \%$ \\
\hline 13 & 13.75 & 7586.70 & 125.26 & $8.35 \mathrm{E}+14$ & $1.18 \mathrm{E}+21$ & $19.08 \%$ \\
\hline 14 & 13.25 & 7507.55 & 123.95 & $8.52 \mathrm{E}+14$ & $1.18 \mathrm{E}+21$ & $19.06 \%$ \\
\hline 15 & 12.75 & 7726.56 & 127.57 & $8.80 \mathrm{E}+14$ & $1.21 \mathrm{E}+21$ & $19.53 \%$ \\
\hline 16 & 12.25 & 7749.42 & 127.94 & $8.90 \mathrm{E}+14$ & $1.24 \mathrm{E}+21$ & $20.01 \%$ \\
\hline 17 & 11.75 & 7846.09 & 129.54 & $9.02 \mathrm{E}+14$ & $1.25 \mathrm{E}+21$ & $20.05 \%$ \\
\hline 18 & 11.25 & 7934.35 & 131.00 & $9.22 \mathrm{E}+14$ & $1.27 \mathrm{E}+21$ & $20.46 \%$ \\
\hline 19 & 10.75 & 8140.46 & 134.40 & $9.30 \mathrm{E}+14$ & $1.32 \mathrm{E}+21$ & $21.18 \%$ \\
\hline 20 & 10.25 & 8403.61 & 138.74 & $9.42 \mathrm{E}+14$ & $1.35 \mathrm{E}+21$ & $21.58 \%$ \\
\hline 21 & 9.75 & 8546.35 & 141.10 & $9.53 \mathrm{E}+14$ & $1.39 \mathrm{E}+21$ & $22.25 \%$ \\
\hline 22 & 9.25 & 8630.33 & 142.49 & $9.61 \mathrm{E}+14$ & $1.41 \mathrm{E}+21$ & $22.50 \%$ \\
\hline 23 & 8.75 & 8757.04 & 144.58 & $9.88 \mathrm{E}+14$ & $1.44 \mathrm{E}+21$ & $22.98 \%$ \\
\hline 24 & 8.25 & 8949.77 & 147.76 & $9.85 \mathrm{E}+14$ & $1.46 \mathrm{E}+21$ & $23.23 \%$ \\
\hline 25 & 7.75 & 8883.67 & 146.67 & $9.90 \mathrm{E}+14$ & $1.47 \mathrm{E}+21$ & $23.33 \%$ \\
\hline 26 & 7.25 & 8939.24 & 147.59 & $9.96 \mathrm{E}+14$ & $1.48 \mathrm{E}+21$ & $23.48 \%$ \\
\hline 27 & 6.75 & 9085.59 & 150.00 & $1.01 \mathrm{E}+15$ & $1.50 \mathrm{E}+21$ & $23.87 \%$ \\
\hline 28 & 6.25 & 9121.31 & 150.59 & $1.01 \mathrm{E}+15$ & $1.51 \mathrm{E}+21$ & $23.97 \%$ \\
\hline 29 & 5.75 & 9105.27 & 150.33 & $1.02 \mathrm{E}+15$ & $1.51 \mathrm{E}+21$ & $24.04 \%$ \\
\hline 30 & 5.25 & 9042.47 & 149.29 & $1.02 \mathrm{E}+15$ & $1.52 \mathrm{E}+21$ & $24.22 \%$ \\
\hline 31 & 4.75 & 9297.39 & 153.50 & $1.03 \mathrm{E}+15$ & $1.55 \mathrm{E}+21$ & $24.56 \%$ \\
\hline 32 & 4.25 & 9397.78 & 155.16 & $1.03 \mathrm{E}+15$ & $1.57 \mathrm{E}+21$ & $24.89 \%$ \\
\hline 33 & 3.75 & 9394.83 & 155.11 & $1.03 \mathrm{E}+15$ & $1.57 \mathrm{E}+21$ & $24.88 \%$ \\
\hline 34 & 3.25 & 9437.72 & 155.82 & $1.04 \mathrm{E}+15$ & $1.58 \mathrm{E}+21$ & $24.98 \%$ \\
\hline 35 & 2.75 & 9273.36 & 153.10 & $1.04 \mathrm{E}+15$ & $1.56 \mathrm{E}+21$ & $24.73 \%$ \\
\hline 36 & 2.25 & 9377.67 & 154.83 & $1.04 \mathrm{E}+15$ & $1.57 \mathrm{E}+21$ & $24.96 \%$ \\
\hline 37 & 1.75 & 9236.30 & 152.49 & $1.04 \mathrm{E}+15$ & $1.55 \mathrm{E}+21$ & $24.64 \%$ \\
\hline 38 & 1.25 & 9199.40 & 151.88 & $1.05 \mathrm{E}+15$ & $1.54 \mathrm{E}+21$ & $24.46 \%$ \\
\hline 39 & 0.75 & 9136.30 & 150.84 & $1.05 \mathrm{E}+15$ & $1.54 \mathrm{E}+21$ & $24.52 \%$ \\
\hline 40 & 0.25 & 9219.19 & 152.21 & $1.05 \mathrm{E}+15$ & $1.55 \mathrm{E}+21$ & $24.59 \%$ \\
\hline 41 & -0.25 & 9253.37 & 152.77 & $1.05 \mathrm{E}+15$ & $1.55 \mathrm{E}+21$ & $24.70 \%$ \\
\hline 42 & -0.75 & 9322.47 & 153.91 & $1.04 \mathrm{E}+15$ & $1.56 \mathrm{E}+21$ & $24.78 \%$ \\
\hline 43 & -1.25 & 9401.90 & 155.23 & $1.05 \mathrm{E}+15$ & $1.58 \mathrm{E}+21$ & $24.92 \%$ \\
\hline 44 & -1.75 & 9324.09 & 153.94 & $1.04 \mathrm{E}+15$ & $1.58 \mathrm{E}+21$ & $24.95 \%$ \\
\hline 45 & -2.25 & 9449.95 & 156.02 & $1.05 \mathrm{E}+15$ & $1.60 \mathrm{E}+21$ & $25.37 \%$ \\
\hline 46 & -2.75 & 9489.71 & 156.68 & $1.05 \mathrm{E}+15$ & $1.60 \mathrm{E}+21$ & $25.27 \%$ \\
\hline 47 & -3.25 & 9364.08 & 154.60 & $1.05 \mathrm{E}+15$ & $1.57 \mathrm{E}+21$ & $24.92 \%$ \\
\hline 48 & -3.75 & 9490.10 & 156.68 & $1.05 \mathrm{E}+15$ & $1.59 \mathrm{E}+21$ & $25.13 \%$ \\
\hline 49 & -4.25 & 9405.53 & 155.29 & $1.05 \mathrm{E}+15$ & $1.58 \mathrm{E}+21$ & $24.92 \%$ \\
\hline 50 & -4.75 & 9305.68 & 153.64 & $1.04 \mathrm{E}+15$ & $1.56 \mathrm{E}+21$ & $24.78 \%$ \\
\hline 51 & -5.25 & 9367.72 & 154.66 & $1.03 \mathrm{E}+15$ & $1.57 \mathrm{E}+21$ & $24.95 \%$ \\
\hline 52 & -5.75 & 9242.22 & 152.59 & $1.04 \mathrm{E}+15$ & $1.56 \mathrm{E}+21$ & $24.68 \%$ \\
\hline
\end{tabular}




\begin{tabular}{|l|l|l|l|l|l|l|}
\hline 53 & -6.25 & 9259.07 & 152.87 & $1.03 \mathrm{E}+15$ & $1.56 \mathrm{E}+21$ & $24.63 \%$ \\
\hline 54 & -6.75 & 9255.11 & 152.80 & $1.03 \mathrm{E}+15$ & $1.54 \mathrm{E}+21$ & $24.50 \%$ \\
\hline 55 & -7.25 & 9262.38 & 152.92 & $1.03 \mathrm{E}+15$ & $1.54 \mathrm{E}+21$ & $24.39 \%$ \\
\hline 56 & -7.75 & 9188.39 & 151.70 & $1.02 \mathrm{E}+15$ & $1.51 \mathrm{E}+21$ & $23.93 \%$ \\
\hline 57 & -8.25 & 9092.27 & 150.11 & $1.02 \mathrm{E}+15$ & $1.51 \mathrm{E}+21$ & $23.94 \%$ \\
\hline 58 & -8.75 & 9072.32 & 149.78 & $1.01 \mathrm{E}+15$ & $1.49 \mathrm{E}+21$ & $23.63 \%$ \\
\hline 59 & -9.25 & 8992.97 & 148.47 & $9.97 \mathrm{E}+14$ & $1.49 \mathrm{E}+21$ & $23.59 \%$ \\
\hline 60 & -9.75 & 8806.00 & 145.39 & $9.89 \mathrm{E}+14$ & $1.43 \mathrm{E}+21$ & $22.82 \%$ \\
\hline 61 & -10.25 & 8728.55 & 144.11 & $9.86 \mathrm{E}+14$ & $1.41 \mathrm{E}+21$ & $22.47 \%$ \\
\hline 62 & -10.75 & 8520.98 & 140.68 & $9.74 \mathrm{E}+14$ & $1.38 \mathrm{E}+21$ & $22.04 \%$ \\
\hline 63 & -11.25 & 8464.64 & 139.75 & $9.61 \mathrm{E}+14$ & $1.37 \mathrm{E}+21$ & $21.86 \%$ \\
\hline 64 & -11.75 & 8351.17 & 137.88 & $9.48 \mathrm{E}+14$ & $1.34 \mathrm{E}+21$ & $21.53 \%$ \\
\hline 65 & -12.25 & 8299.39 & 137.02 & $9.35 \mathrm{E}+14$ & $1.32 \mathrm{E}+21$ & $21.14 \%$ \\
\hline 66 & -12.75 & 8445.61 & 139.44 & $9.30 \mathrm{E}+14$ & $1.33 \mathrm{E}+21$ & $21.32 \%$ \\
\hline 67 & -13.25 & 8084.54 & 133.48 & $9.18 \mathrm{E}+14$ & $1.29 \mathrm{E}+21$ & $20.67 \%$ \\
\hline 68 & -13.75 & 8086.72 & 133.51 & $9.06 \mathrm{E}+14$ & $1.28 \mathrm{E}+21$ & $20.58 \%$ \\
\hline 69 & -14.25 & 7833.66 & 129.33 & $8.86 \mathrm{E}+14$ & $1.25 \mathrm{E}+21$ & $20.18 \%$ \\
\hline 70 & -14.75 & 7797.55 & 128.74 & $8.65 \mathrm{E}+14$ & $1.23 \mathrm{E}+21$ & $19.77 \%$ \\
\hline 71 & -15.25 & 7634.85 & 126.05 & $8.37 \mathrm{E}+14$ & $1.19 \mathrm{E}+21$ & $19.22 \%$ \\
\hline 72 & -15.75 & 7476.50 & 123.44 & $8.19 \mathrm{E}+14$ & $1.16 \mathrm{E}+21$ & $18.78 \%$ \\
\hline 73 & -16.25 & 7340.27 & 121.19 & $8.05 \mathrm{E}+14$ & $1.14 \mathrm{E}+21$ & $18.38 \%$ \\
\hline 74 & -16.75 & 7215.74 & 119.13 & $7.85 \mathrm{E}+14$ & $1.11 \mathrm{E}+21$ & $17.99 \%$ \\
\hline 75 & -17.25 & 6848.73 & 113.07 & $7.56 \mathrm{E}+14$ & $1.06 \mathrm{E}+21$ & $17.24 \%$ \\
\hline 76 & -17.75 & 6665.19 & 110.04 & $7.32 \mathrm{E}+14$ & $1.02 \mathrm{E}+21$ & $16.55 \%$ \\
\hline 77 & -18.25 & 6412.19 & 105.87 & $7.03 \mathrm{E}+14$ & $9.81 \mathrm{E}+20$ & $15.95 \%$ \\
\hline 78 & -18.75 & 6359.43 & 104.99 & $6.80 \mathrm{E}+14$ & $9.63 \mathrm{E}+20$ & $15.68 \%$ \\
\hline
\end{tabular}


Table 12: Cycle 149B, MCNP-Calculated HGRs and Neutron Flux for AFIP-7, Plate Position 2, 0 EFPD (BOC) Average Center Lobe Power at 24.2 MW. ${ }^{2}$

\begin{tabular}{|c|c|c|c|c|}
\hline Node & $\begin{array}{c}\text { Distance from } \\
\text { Centerline (in.) }\end{array}$ & $\begin{array}{l}\text { Fission Power } \\
\text { Density (W/cc) }\end{array}$ & $\begin{array}{l}\text { Surface Heat } \\
\text { Flux }\left(\mathrm{W} / \mathrm{cm}^{2}\right) \\
\end{array}$ & $\begin{array}{c}\text { Neutron Flux } \\
\left(\mathbf{n} / \mathrm{cm}^{2} \mathrm{sec}\right)\end{array}$ \\
\hline 1 & 19.25 & 6792.60 & 112.15 & $5.22 \mathrm{E}+14$ \\
\hline 2 & 18.75 & 6279.85 & 103.68 & $5.50 \mathrm{E}+14$ \\
\hline 3 & 18.25 & 6593.87 & 108.86 & $5.89 \mathrm{E}+14$ \\
\hline 4 & 17.75 & 7097.61 & 117.18 & $6.27 \mathrm{E}+14$ \\
\hline 5 & 17.25 & 7367.84 & 121.64 & $6.48 \mathrm{E}+14$ \\
\hline 6 & 16.75 & 7825.62 & 129.20 & $6.85 \mathrm{E}+14$ \\
\hline 7 & 16.25 & 8172.64 & 134.93 & $7.15 \mathrm{E}+14$ \\
\hline 8 & 15.75 & 8340.25 & 137.70 & $7.35 \mathrm{E}+14$ \\
\hline 9 & 15.25 & 8633.74 & 142.54 & $7.73 \mathrm{E}+14$ \\
\hline 10 & 14.75 & 9056.80 & 149.53 & $7.96 \mathrm{E}+14$ \\
\hline 11 & 14.25 & 9614.20 & 158.73 & $8.26 \mathrm{E}+14$ \\
\hline 13 & 13.75 & 9732.98 & 160.69 & $8.60 \mathrm{E}+14$ \\
\hline 14 & 13.25 & 9877.94 & 163.08 & $8.70 \mathrm{E}+14$ \\
\hline 15 & 12.75 & 9905.30 & 163.54 & $8.94 \mathrm{E}+14$ \\
\hline 16 & 12.25 & 10297.44 & 170.01 & $9.29 \mathrm{E}+14$ \\
\hline 17 & 11.75 & 10461.73 & 172.72 & $9.51 \mathrm{E}+14$ \\
\hline 18 & 11.25 & 10780.58 & 177.99 & $9.68 \mathrm{E}+14$ \\
\hline 19 & 10.75 & 11290.07 & 186.40 & $1.00 \mathrm{E}+15$ \\
\hline 20 & 10.25 & 11663.44 & 192.56 & $1.03 \mathrm{E}+15$ \\
\hline 21 & 9.75 & 11967.53 & 197.58 & $1.05 \mathrm{E}+15$ \\
\hline 22 & 9.25 & 12415.14 & 204.97 & $1.07 \mathrm{E}+15$ \\
\hline 23 & 8.75 & 12336.60 & 203.68 & $1.08 \mathrm{E}+15$ \\
\hline 24 & 8.25 & 12767.02 & 210.78 & $1.09 \mathrm{E}+15$ \\
\hline 25 & 7.75 & 12793.46 & 211.22 & $1.11 \mathrm{E}+15$ \\
\hline 26 & 7.25 & 12807.02 & 211.44 & $1.13 \mathrm{E}+15$ \\
\hline 27 & 6.75 & 13178.45 & 217.58 & $1.14 \mathrm{E}+15$ \\
\hline 28 & 6.25 & 13555.95 & 223.81 & $1.16 \mathrm{E}+15$ \\
\hline 29 & 5.75 & 13346.68 & 220.35 & $1.17 \mathrm{E}+15$ \\
\hline 30 & 5.25 & 13747.18 & 226.97 & $1.19 \mathrm{E}+15$ \\
\hline 31 & 4.75 & 14007.54 & 231.26 & $1.19 \mathrm{E}+15$ \\
\hline 32 & 4.25 & 13896.55 & 229.43 & $1.20 \mathrm{E}+15$ \\
\hline 33 & 3.75 & 14024.59 & 231.55 & $1.21 \mathrm{E}+15$ \\
\hline 34 & 3.25 & 14023.67 & 231.53 & $1.21 \mathrm{E}+15$ \\
\hline 35 & 2.75 & 14146.14 & 233.55 & $1.23 \mathrm{E}+15$ \\
\hline 36 & 2.25 & 14195.36 & 234.37 & $1.23 \mathrm{E}+15$ \\
\hline 37 & 1.75 & 14044.75 & 231.88 & $1.22 \mathrm{E}+15$ \\
\hline 38 & 1.25 & 13707.89 & 226.32 & $1.22 \mathrm{E}+15$ \\
\hline 39 & 0.75 & 13834.86 & 228.41 & $1.23 \mathrm{E}+15$ \\
\hline 40 & 0.25 & 13891.47 & 229.35 & $1.23 \mathrm{E}+15$ \\
\hline 41 & -0.25 & 14152.53 & 233.66 & $1.23 \mathrm{E}+15$ \\
\hline 42 & -0.75 & 14123.36 & 233.18 & $1.23 \mathrm{E}+15$ \\
\hline 43 & -1.25 & 14477.01 & 239.02 & $1.23 \mathrm{E}+15$ \\
\hline 44 & -1.75 & 14556.68 & 240.33 & $1.23 \mathrm{E}+15$ \\
\hline 45 & -2.25 & 14673.62 & 242.26 & $1.24 \mathrm{E}+15$ \\
\hline 46 & -2.75 & 14389.10 & 237.56 & $1.23 \mathrm{E}+15$ \\
\hline 47 & -3.25 & 14341.34 & 236.78 & $1.23 \mathrm{E}+15$ \\
\hline 48 & -3.75 & 14247.09 & 235.22 & $1.22 \mathrm{E}+15$ \\
\hline 49 & -4.25 & 14200.90 & 234.46 & $1.21 \mathrm{E}+15$ \\
\hline 50 & -4.75 & 14038.41 & 231.77 & $1.19 \mathrm{E}+15$ \\
\hline 51 & -5.25 & 14272.02 & 235.63 & $1.20 \mathrm{E}+15$ \\
\hline 52 & -5.75 & 13965.59 & 230.57 & $1.19 \mathrm{E}+15$ \\
\hline
\end{tabular}




\begin{tabular}{|l|l|l|l|l|}
\hline 53 & -6.25 & 13981.56 & 230.84 & $1.19 \mathrm{E}+15$ \\
\hline 54 & -6.75 & 13526.89 & 223.33 & $1.17 \mathrm{E}+15$ \\
\hline 55 & -7.25 & 13596.77 & 224.48 & $1.16 \mathrm{E}+15$ \\
\hline 56 & -7.75 & 13463.39 & 222.28 & $1.15 \mathrm{E}+15$ \\
\hline 57 & -8.25 & 13508.14 & 223.02 & $1.15 \mathrm{E}+15$ \\
\hline 58 & -8.75 & 13359.97 & 220.57 & $1.13 \mathrm{E}+15$ \\
\hline 59 & -9.25 & 12883.22 & 212.70 & $1.11 \mathrm{E}+15$ \\
\hline 60 & -9.75 & 12441.77 & 205.41 & $1.10 \mathrm{E}+15$ \\
\hline 61 & -10.25 & 11987.73 & 197.92 & $1.07 \mathrm{E}+15$ \\
\hline 62 & -10.75 & 11795.79 & 194.75 & $1.05 \mathrm{E}+15$ \\
\hline 63 & -11.25 & 11677.87 & 192.80 & $1.04 \mathrm{E}+15$ \\
\hline 64 & -11.75 & 11404.99 & 188.30 & $1.02 \mathrm{E}+15$ \\
\hline 65 & -12.25 & 11340.40 & 187.23 & $9.90 \mathrm{E}+14$ \\
\hline 66 & -12.75 & 11171.12 & 184.44 & $9.76 \mathrm{E}+14$ \\
\hline 67 & -13.25 & 11029.58 & 182.10 & $9.55 \mathrm{E}+14$ \\
\hline 68 & -13.75 & 10766.41 & 177.75 & $9.28 \mathrm{E}+14$ \\
\hline 69 & -14.25 & 10534.00 & 173.92 & $9.08 \mathrm{E}+14$ \\
\hline 70 & -14.75 & 10110.88 & 166.93 & $8.85 \mathrm{E}+14$ \\
\hline 71 & -15.25 & 9789.90 & 161.63 & $8.57 \mathrm{E}+14$ \\
\hline 72 & -15.75 & 9675.52 & 159.74 & $8.35 \mathrm{E}+14$ \\
\hline 73 & -16.25 & 9081.79 & 149.94 & $8.03 \mathrm{E}+14$ \\
\hline 74 & -16.75 & 8866.07 & 146.38 & $7.76 \mathrm{E}+14$ \\
\hline 75 & -17.25 & 8414.91 & 138.93 & $7.45 \mathrm{E}+14$ \\
\hline 76 & -17.75 & 7962.93 & 131.47 & $7.14 \mathrm{E}+14$ \\
\hline 77 & -18.25 & 7445.15 & 122.92 & $6.81 \mathrm{E}+14$ \\
\hline 78 & -18.75 & 7292.05 & 120.39 & $6.44 \mathrm{E}+14$ \\
\hline
\end{tabular}


Table 13: Cycle 149B, MCNP-Calculated HGRs, Neutron Flux, Depletion and Fission Density for AFIP-7, Plate Position 2, 15 EFPD (MOC1) Average Center Lobe Power at 24.2 MW. ${ }^{2}$

\begin{tabular}{|c|c|c|c|c|c|c|}
\hline Node & $\begin{array}{c}\text { Distance from } \\
\text { Centerline (in.) }\end{array}$ & $\begin{array}{l}\text { Fission Power } \\
\text { Density (W/cc) }\end{array}$ & $\begin{array}{l}\text { Surface Heat } \\
\text { Flux }\left(W / \mathrm{cm}^{2}\right)\end{array}$ & $\begin{array}{c}\text { Neutron Flux } \\
\left(\mathrm{n} / \mathrm{cm}^{2} \mathrm{sec}\right)\end{array}$ & $\begin{array}{l}\text { Fission Density } \\
\text { (fissions/cc) }\end{array}$ & $\begin{array}{c}\text { U-235 } \\
\text { Burnup (\%) }\end{array}$ \\
\hline 1 & 19.25 & 5631.41 & 92.97 & $4.97 \mathrm{E}+14$ & $2.65 \mathrm{E}+20$ & $3.92 \%$ \\
\hline 2 & 18.75 & 5239.45 & 86.50 & $5.22 \mathrm{E}+14$ & $2.45 \mathrm{E}+20$ & $3.62 \%$ \\
\hline 3 & 18.25 & 5328.73 & 87.98 & $5.47 \mathrm{E}+14$ & $2.58 \mathrm{E}+20$ & $3.84 \%$ \\
\hline 4 & 17.75 & 5771.04 & 95.28 & $5.80 \mathrm{E}+14$ & $2.77 \mathrm{E}+20$ & $4.14 \%$ \\
\hline 5 & 17.25 & 5905.47 & 97.50 & $6.06 \mathrm{E}+14$ & $2.88 \mathrm{E}+20$ & $4.29 \%$ \\
\hline 6 & 16.75 & 6244.15 & 103.09 & $6.37 \mathrm{E}+14$ & $3.06 \mathrm{E}+20$ & $4.52 \%$ \\
\hline 7 & 16.25 & 6325.46 & 104.43 & $6.56 \mathrm{E}+14$ & $3.19 \mathrm{E}+20$ & $4.67 \%$ \\
\hline 8 & 15.75 & 6679.04 & 110.27 & $6.78 \mathrm{E}+14$ & $3.26 \mathrm{E}+20$ & $4.82 \%$ \\
\hline 9 & 15.25 & 6628.73 & 109.44 & $7.06 \mathrm{E}+14$ & $3.37 \mathrm{E}+20$ & $4.97 \%$ \\
\hline 10 & 14.75 & 7124.54 & 117.63 & $7.25 \mathrm{E}+14$ & $3.54 \mathrm{E}+20$ & $5.19 \%$ \\
\hline 11 & 14.25 & 7105.74 & 117.32 & $7.39 \mathrm{E}+14$ & $3.76 \mathrm{E}+20$ & $5.49 \%$ \\
\hline 13 & 13.75 & 7249.03 & 119.68 & $7.62 \mathrm{E}+14$ & $3.80 \mathrm{E}+20$ & $5.57 \%$ \\
\hline 14 & 13.25 & 7224.88 & 119.28 & $7.83 \mathrm{E}+14$ & $3.86 \mathrm{E}+20$ & $5.64 \%$ \\
\hline 15 & 12.75 & 7312.53 & 120.73 & $8.02 \mathrm{E}+14$ & $3.87 \mathrm{E}+20$ & $5.72 \%$ \\
\hline 16 & 12.25 & 7707.91 & 127.26 & $8.19 \mathrm{E}+14$ & $4.02 \mathrm{E}+20$ & $5.87 \%$ \\
\hline 17 & 11.75 & 7793.11 & 128.66 & $8.37 \mathrm{E}+14$ & $4.09 \mathrm{E}+20$ & $6.02 \%$ \\
\hline 18 & 11.25 & 7933.94 & 130.99 & $8.50 \mathrm{E}+14$ & $4.21 \mathrm{E}+20$ & $6.17 \%$ \\
\hline 19 & 10.75 & 7906.06 & 130.53 & $8.56 \mathrm{E}+14$ & $4.41 \mathrm{E}+20$ & $6.47 \%$ \\
\hline 20 & 10.25 & 8162.42 & 134.76 & $8.74 \mathrm{E}+14$ & $4.56 \mathrm{E}+20$ & $6.63 \%$ \\
\hline 21 & 9.75 & 8487.52 & 140.13 & $8.90 \mathrm{E}+14$ & $4.68 \mathrm{E}+20$ & $6.86 \%$ \\
\hline 22 & 9.25 & 8376.34 & 138.29 & $9.04 \mathrm{E}+14$ & $4.85 \mathrm{E}+20$ & $7.08 \%$ \\
\hline 23 & 8.75 & 8637.17 & 142.60 & $9.05 \mathrm{E}+14$ & $4.82 \mathrm{E}+20$ & $7.01 \%$ \\
\hline 24 & 8.25 & 8642.07 & 142.68 & $9.14 \mathrm{E}+14$ & $4.99 \mathrm{E}+20$ & $7.23 \%$ \\
\hline 25 & 7.75 & 8703.04 & 143.69 & $9.17 \mathrm{E}+14$ & $5.00 \mathrm{E}+20$ & $7.31 \%$ \\
\hline 26 & 7.25 & 8610.35 & 142.16 & $9.37 \mathrm{E}+14$ & $5.00 \mathrm{E}+20$ & $7.31 \%$ \\
\hline 27 & 6.75 & 9016.98 & 148.87 & $9.55 \mathrm{E}+14$ & $5.15 \mathrm{E}+20$ & $7.46 \%$ \\
\hline 28 & 6.25 & 8791.87 & 145.15 & $9.50 \mathrm{E}+14$ & $5.30 \mathrm{E}+20$ & $7.68 \%$ \\
\hline 29 & 5.75 & 9008.00 & 148.72 & $9.52 \mathrm{E}+14$ & $5.21 \mathrm{E}+20$ & $7.53 \%$ \\
\hline 30 & 5.25 & 8925.83 & 147.37 & $9.60 \mathrm{E}+14$ & $5.37 \mathrm{E}+20$ & $7.76 \%$ \\
\hline 31 & 4.75 & 8931.10 & 147.45 & $9.68 \mathrm{E}+14$ & $5.47 \mathrm{E}+20$ & $7.91 \%$ \\
\hline 32 & 4.25 & 9049.19 & 149.40 & $9.76 \mathrm{E}+14$ & $5.43 \mathrm{E}+20$ & $7.91 \%$ \\
\hline 33 & 3.75 & 9026.94 & 149.03 & $9.80 \mathrm{E}+14$ & $5.48 \mathrm{E}+20$ & $7.98 \%$ \\
\hline 34 & 3.25 & 9157.62 & 151.19 & $9.84 \mathrm{E}+14$ & $5.48 \mathrm{E}+20$ & $7.98 \%$ \\
\hline 35 & 2.75 & 8999.75 & 148.59 & $9.83 \mathrm{E}+14$ & $5.53 \mathrm{E}+20$ & $7.98 \%$ \\
\hline 36 & 2.25 & 8962.45 & 147.97 & $9.75 \mathrm{E}+14$ & $5.55 \mathrm{E}+20$ & $8.06 \%$ \\
\hline 37 & 1.75 & 9058.54 & 149.56 & $9.82 \mathrm{E}+14$ & $5.49 \mathrm{E}+20$ & $7.98 \%$ \\
\hline 38 & 1.25 & 8890.69 & 146.79 & $9.86 \mathrm{E}+14$ & $5.36 \mathrm{E}+20$ & $7.76 \%$ \\
\hline 39 & 0.75 & 8929.74 & 147.43 & $9.85 \mathrm{E}+14$ & $5.40 \mathrm{E}+20$ & $7.83 \%$ \\
\hline 40 & 0.25 & 8867.54 & 146.40 & $9.90 \mathrm{E}+14$ & $5.43 \mathrm{E}+20$ & $7.91 \%$ \\
\hline 41 & -0.25 & 8977.40 & 148.22 & $9.91 \mathrm{E}+14$ & $5.53 \mathrm{E}+20$ & $8.06 \%$ \\
\hline 42 & -0.75 & 9069.92 & 149.74 & $9.98 \mathrm{E}+14$ & $5.52 \mathrm{E}+20$ & $7.98 \%$ \\
\hline 43 & -1.25 & 9081.16 & 149.93 & $9.90 \mathrm{E}+14$ & $5.66 \mathrm{E}+20$ & $8.21 \%$ \\
\hline 44 & -1.75 & 9053.43 & 149.47 & $9.82 \mathrm{E}+14$ & $5.69 \mathrm{E}+20$ & $8.21 \%$ \\
\hline 45 & -2.25 & 9100.79 & 150.25 & $9.78 \mathrm{E}+14$ & $5.73 \mathrm{E}+20$ & $8.28 \%$ \\
\hline 46 & -2.75 & 9139.49 & 150.89 & $9.88 \mathrm{E}+14$ & $5.62 \mathrm{E}+20$ & $8.13 \%$ \\
\hline 47 & -3.25 & 9041.33 & 149.27 & $9.84 \mathrm{E}+14$ & $5.60 \mathrm{E}+20$ & $8.13 \%$ \\
\hline 48 & -3.75 & 9230.15 & 152.39 & $9.80 \mathrm{E}+14$ & $5.57 \mathrm{E}+20$ & $8.06 \%$ \\
\hline 49 & -4.25 & 9010.72 & 148.77 & $9.89 \mathrm{E}+14$ & $5.55 \mathrm{E}+20$ & $8.06 \%$ \\
\hline 50 & -4.75 & 9170.76 & 151.41 & $9.79 \mathrm{E}+14$ & $5.48 \mathrm{E}+20$ & $7.98 \%$ \\
\hline 51 & -5.25 & 8998.16 & 148.56 & $9.73 \mathrm{E}+14$ & $5.58 \mathrm{E}+20$ & $8.06 \%$ \\
\hline 52 & -5.75 & 9016.63 & 148.86 & $9.64 \mathrm{E}+14$ & $5.46 \mathrm{E}+20$ & $7.91 \%$ \\
\hline
\end{tabular}




\begin{tabular}{|l|l|l|l|l|l|l|}
\hline 53 & -6.25 & 9045.30 & 149.34 & $9.64 \mathrm{E}+14$ & $5.46 \mathrm{E}+20$ & $7.91 \%$ \\
\hline 54 & -6.75 & 9015.03 & 148.84 & $9.67 \mathrm{E}+14$ & $5.28 \mathrm{E}+20$ & $7.68 \%$ \\
\hline 55 & -7.25 & 8810.90 & 145.47 & $9.59 \mathrm{E}+14$ & $5.31 \mathrm{E}+20$ & $7.68 \%$ \\
\hline 56 & -7.75 & 8867.35 & 146.40 & $9.56 \mathrm{E}+14$ & $5.26 \mathrm{E}+20$ & $7.61 \%$ \\
\hline 57 & -8.25 & 8799.50 & 145.28 & $9.39 \mathrm{E}+14$ & $5.28 \mathrm{E}+20$ & $7.68 \%$ \\
\hline 58 & -8.75 & 8641.14 & 142.67 & $9.33 \mathrm{E}+14$ & $5.22 \mathrm{E}+20$ & $7.61 \%$ \\
\hline 59 & -9.25 & 8755.96 & 144.56 & $9.28 \mathrm{E}+14$ & $5.03 \mathrm{E}+20$ & $7.31 \%$ \\
\hline 60 & -9.75 & 8559.36 & 141.32 & $9.23 \mathrm{E}+14$ & $4.86 \mathrm{E}+20$ & $7.08 \%$ \\
\hline 61 & -10.25 & 8245.58 & 136.13 & $9.10 \mathrm{E}+14$ & $4.68 \mathrm{E}+20$ & $6.86 \%$ \\
\hline 62 & -10.75 & 8310.89 & 137.21 & $9.02 \mathrm{E}+14$ & $4.61 \mathrm{E}+20$ & $6.78 \%$ \\
\hline 63 & -11.25 & 8157.52 & 134.68 & $8.90 \mathrm{E}+14$ & $4.56 \mathrm{E}+20$ & $6.71 \%$ \\
\hline 64 & -11.75 & 7986.27 & 131.85 & $8.74 \mathrm{E}+14$ & $4.46 \mathrm{E}+20$ & $6.47 \%$ \\
\hline 65 & -12.25 & 7915.63 & 130.69 & $8.63 \mathrm{E}+14$ & $4.43 \mathrm{E}+20$ & $6.47 \%$ \\
\hline 66 & -12.75 & 7976.58 & 131.69 & $8.41 \mathrm{E}+14$ & $4.36 \mathrm{E}+20$ & $6.39 \%$ \\
\hline 67 & -13.25 & 7967.08 & 131.54 & $8.37 \mathrm{E}+14$ & $4.31 \mathrm{E}+20$ & $6.32 \%$ \\
\hline 68 & -13.75 & 7824.45 & 129.18 & $8.24 \mathrm{E}+14$ & $4.21 \mathrm{E}+20$ & $6.17 \%$ \\
\hline 69 & -14.25 & 7829.81 & 129.27 & $8.06 \mathrm{E}+14$ & $4.12 \mathrm{E}+20$ & $6.02 \%$ \\
\hline 70 & -14.75 & 7600.85 & 125.49 & $7.94 \mathrm{E}+14$ & $3.95 \mathrm{E}+20$ & $5.79 \%$ \\
\hline 71 & -15.25 & 7368.07 & 121.65 & $7.77 \mathrm{E}+14$ & $3.82 \mathrm{E}+20$ & $5.64 \%$ \\
\hline 72 & -15.75 & 7207.42 & 118.99 & $7.50 \mathrm{E}+14$ & $3.78 \mathrm{E}+20$ & $5.57 \%$ \\
\hline 73 & -16.25 & 7011.09 & 115.75 & $7.30 \mathrm{E}+14$ & $3.55 \mathrm{E}+20$ & $5.19 \%$ \\
\hline 74 & -16.75 & 6937.12 & 114.53 & $7.11 \mathrm{E}+14$ & $3.46 \mathrm{E}+20$ & $5.12 \%$ \\
\hline 75 & -17.25 & 6780.04 & 111.94 & $6.89 \mathrm{E}+14$ & $3.29 \mathrm{E}+20$ & $4.82 \%$ \\
\hline 76 & -17.75 & 6430.76 & 106.17 & $6.63 \mathrm{E}+14$ & $3.11 \mathrm{E}+20$ & $4.59 \%$ \\
\hline 77 & -18.25 & 6208.14 & 102.50 & $6.39 \mathrm{E}+14$ & $2.91 \mathrm{E}+20$ & $4.29 \%$ \\
\hline 78 & -18.75 & 6016.35 & 99.33 & $6.10 \mathrm{E}+14$ & $2.85 \mathrm{E}+20$ & $4.22 \%$ \\
\hline
\end{tabular}


Table 14: Cycle 149B, MCNP-Calculated HGRs, Neutron Flux, Depletion and Fission Density for AFIP-7, Plate Position 2, 31 EFPD (MOC2) Average Center Lobe Power at 24.2 MW. ${ }^{2}$

\begin{tabular}{|c|c|c|c|c|c|c|}
\hline Node & $\begin{array}{c}\text { Distance from } \\
\text { Centerline (in.) }\end{array}$ & $\begin{array}{l}\text { Fission Power } \\
\text { Density (W/cc) }\end{array}$ & $\begin{array}{l}\text { Surface Heat } \\
\text { Flux }\left(W / \mathrm{cm}^{2}\right)\end{array}$ & $\begin{array}{c}\text { Neutron Flux } \\
\left(\mathrm{n} / \mathrm{cm}^{2} \mathrm{sec}\right)\end{array}$ & $\begin{array}{l}\text { Fission Density } \\
\text { (fissions/cc) }\end{array}$ & $\begin{array}{c}\text { U-235 } \\
\text { Burnup (\%) }\end{array}$ \\
\hline 1 & 19.25 & 6286.98 & 103.80 & $5.48 \mathrm{E}+14$ & $5.20 \mathrm{E}+20$ & $7.68 \%$ \\
\hline 2 & 18.75 & 5795.04 & 95.68 & $5.70 \mathrm{E}+14$ & $4.83 \mathrm{E}+20$ & $7.16 \%$ \\
\hline 3 & 18.25 & 6004.68 & 99.14 & $6.06 \mathrm{E}+14$ & $4.99 \mathrm{E}+20$ & $7.38 \%$ \\
\hline 4 & 17.75 & 6337.97 & 104.64 & $6.36 \mathrm{E}+14$ & $5.39 \mathrm{E}+20$ & $7.98 \%$ \\
\hline 5 & 17.25 & 6556.61 & 108.25 & $6.59 \mathrm{E}+14$ & $5.55 \mathrm{E}+20$ & $8.21 \%$ \\
\hline 6 & 16.75 & 6773.73 & 111.83 & $6.86 \mathrm{E}+14$ & $5.89 \mathrm{E}+20$ & $8.66 \%$ \\
\hline 7 & 16.25 & 6980.78 & 115.25 & $7.10 \mathrm{E}+14$ & $6.06 \mathrm{E}+20$ & $8.88 \%$ \\
\hline 8 & 15.75 & 7191.38 & 118.73 & $7.32 \mathrm{E}+14$ & $6.28 \mathrm{E}+20$ & $9.26 \%$ \\
\hline 9 & 15.25 & 7342.09 & 121.22 & $7.60 \mathrm{E}+14$ & $6.38 \mathrm{E}+20$ & $9.41 \%$ \\
\hline 10 & 14.75 & 7565.64 & 124.91 & $7.85 \mathrm{E}+14$ & $6.77 \mathrm{E}+20$ & $9.94 \%$ \\
\hline 11 & 14.25 & 7682.76 & 126.84 & $7.99 \mathrm{E}+14$ & $6.97 \mathrm{E}+20$ & $10.25 \%$ \\
\hline 13 & 13.75 & 7769.83 & 128.28 & $8.20 \mathrm{E}+14$ & $7.09 \mathrm{E}+20$ & $10.40 \%$ \\
\hline 14 & 13.25 & 7893.52 & 130.32 & $8.40 \mathrm{E}+14$ & $7.13 \mathrm{E}+20$ & $10.47 \%$ \\
\hline 15 & 12.75 & 8063.92 & 133.14 & $8.50 \mathrm{E}+14$ & $7.18 \mathrm{E}+20$ & $10.55 \%$ \\
\hline 16 & 12.25 & 8195.43 & 135.31 & $8.74 \mathrm{E}+14$ & $7.51 \mathrm{E}+20$ & $11.00 \%$ \\
\hline 17 & 11.75 & 8182.47 & 135.09 & $8.84 \mathrm{E}+14$ & $7.62 \mathrm{E}+20$ & $11.15 \%$ \\
\hline 18 & 11.25 & 8397.37 & 138.64 & $9.01 \mathrm{E}+14$ & $7.80 \mathrm{E}+20$ & $11.45 \%$ \\
\hline 19 & 10.75 & 8582.48 & 141.70 & $9.10 \mathrm{E}+14$ & $7.99 \mathrm{E}+20$ & $11.68 \%$ \\
\hline 20 & 10.25 & 8860.89 & 146.29 & $9.23 \mathrm{E}+14$ & $8.25 \mathrm{E}+20$ & $12.05 \%$ \\
\hline 21 & 9.75 & 9001.48 & 148.61 & $9.35 \mathrm{E}+14$ & $8.52 \mathrm{E}+20$ & $12.43 \%$ \\
\hline 22 & 9.25 & 9029.65 & 149.08 & $9.45 \mathrm{E}+14$ & $8.64 \mathrm{E}+20$ & $12.58 \%$ \\
\hline 23 & 8.75 & 9309.09 & 153.69 & $9.57 \mathrm{E}+14$ & $8.73 \mathrm{E}+20$ & $12.73 \%$ \\
\hline 24 & 8.25 & 9286.06 & 153.31 & $9.67 \mathrm{E}+14$ & $8.90 \mathrm{E}+20$ & $12.95 \%$ \\
\hline 25 & 7.75 & 9290.86 & 153.39 & $9.64 \mathrm{E}+14$ & $8.94 \mathrm{E}+20$ & $13.03 \%$ \\
\hline 26 & 7.25 & 9339.52 & 154.20 & $9.79 \mathrm{E}+14$ & $8.90 \mathrm{E}+20$ & $12.95 \%$ \\
\hline 27 & 6.75 & 9580.44 & 158.17 & $9.88 \mathrm{E}+14$ & $9.23 \mathrm{E}+20$ & $13.42 \%$ \\
\hline 28 & 6.25 & 9618.41 & 158.80 & $9.91 \mathrm{E}+14$ & $9.28 \mathrm{E}+20$ & $13.49 \%$ \\
\hline 29 & 5.75 & 9702.75 & 160.19 & $9.92 \mathrm{E}+14$ & $9.29 \mathrm{E}+20$ & $13.49 \%$ \\
\hline 30 & 5.25 & 9723.83 & 160.54 & $1.00 \mathrm{E}+15$ & $9.41 \mathrm{E}+20$ & $13.64 \%$ \\
\hline 31 & 4.75 & 9865.91 & 162.89 & $1.01 \mathrm{E}+15$ & $9.52 \mathrm{E}+20$ & $13.79 \%$ \\
\hline 32 & 4.25 & 9939.96 & 164.11 & $1.02 \mathrm{E}+15$ & $9.53 \mathrm{E}+20$ & $13.87 \%$ \\
\hline 33 & 3.75 & 9775.49 & 161.39 & $1.02 \mathrm{E}+15$ & $9.57 \mathrm{E}+20$ & $13.94 \%$ \\
\hline 34 & 3.25 & 9917.66 & 163.74 & $1.02 \mathrm{E}+15$ & $9.63 \mathrm{E}+20$ & $14.02 \%$ \\
\hline 35 & 2.75 & 9906.30 & 163.55 & $1.01 \mathrm{E}+15$ & $9.60 \mathrm{E}+20$ & $13.94 \%$ \\
\hline 36 & 2.25 & 9851.33 & 162.65 & $1.01 \mathrm{E}+15$ & $9.60 \mathrm{E}+20$ & $13.94 \%$ \\
\hline 37 & 1.75 & 9999.16 & 165.09 & $1.02 \mathrm{E}+15$ & $9.59 \mathrm{E}+20$ & $13.94 \%$ \\
\hline 38 & 1.25 & 9610.35 & 158.67 & $1.03 \mathrm{E}+15$ & $9.38 \mathrm{E}+20$ & $13.64 \%$ \\
\hline 39 & 0.75 & 9657.43 & 159.44 & $1.03 \mathrm{E}+15$ & $9.45 \mathrm{E}+20$ & $13.72 \%$ \\
\hline 40 & 0.25 & 9657.73 & 159.45 & $1.03 \mathrm{E}+15$ & $9.44 \mathrm{E}+20$ & $13.79 \%$ \\
\hline 41 & -0.25 & 9684.84 & 159.90 & $1.03 \mathrm{E}+15$ & $9.59 \mathrm{E}+20$ & $13.94 \%$ \\
\hline 42 & -0.75 & 9747.51 & 160.93 & $1.03 \mathrm{E}+15$ & $9.62 \mathrm{E}+20$ & $14.02 \%$ \\
\hline 43 & -1.25 & 9854.30 & 162.69 & $1.02 \mathrm{E}+15$ & $9.77 \mathrm{E}+20$ & $14.17 \%$ \\
\hline 44 & -1.75 & 9948.79 & 164.25 & $1.03 \mathrm{E}+15$ & $9.79 \mathrm{E}+20$ & $14.17 \%$ \\
\hline 45 & -2.25 & 9939.83 & 164.11 & $1.02 \mathrm{E}+15$ & $9.85 \mathrm{E}+20$ & $14.24 \%$ \\
\hline 46 & -2.75 & 9886.54 & 163.23 & $1.02 \mathrm{E}+15$ & $9.76 \mathrm{E}+20$ & $14.17 \%$ \\
\hline 47 & -3.25 & 9957.34 & 164.40 & $1.03 \mathrm{E}+15$ & $9.70 \mathrm{E}+20$ & $14.09 \%$ \\
\hline 48 & -3.75 & 10239.14 & 169.05 & $1.03 \mathrm{E}+15$ & $9.74 \mathrm{E}+20$ & $14.09 \%$ \\
\hline 49 & -4.25 & 9808.19 & 161.93 & $1.03 \mathrm{E}+15$ & $9.63 \mathrm{E}+20$ & $14.02 \%$ \\
\hline 50 & -4.75 & 9834.70 & 162.37 & $1.01 \mathrm{E}+15$ & $9.64 \mathrm{E}+20$ & $14.02 \%$ \\
\hline 51 & -5.25 & 9812.34 & 162.00 & $1.01 \mathrm{E}+15$ & $9.65 \mathrm{E}+20$ & $14.02 \%$ \\
\hline 52 & -5.75 & 9772.82 & 161.35 & $1.00 \mathrm{E}+15$ & $9.54 \mathrm{E}+20$ & $13.87 \%$ \\
\hline
\end{tabular}




\begin{tabular}{|l|l|l|l|l|l|l|}
\hline 53 & -6.25 & 9720.55 & 160.49 & $1.00 \mathrm{E}+15$ & $9.56 \mathrm{E}+20$ & $13.87 \%$ \\
\hline 54 & -6.75 & 9833.60 & 162.35 & $1.00 \mathrm{E}+15$ & $9.37 \mathrm{E}+20$ & $13.64 \%$ \\
\hline 55 & -7.25 & 9836.71 & 162.40 & $1.00 \mathrm{E}+15$ & $9.30 \mathrm{E}+20$ & $13.57 \%$ \\
\hline 56 & -7.75 & 9632.02 & 159.02 & $1.00 \mathrm{E}+15$ & $9.27 \mathrm{E}+20$ & $13.49 \%$ \\
\hline 57 & -8.25 & 9507.98 & 156.98 & $9.89 \mathrm{E}+14$ & $9.26 \mathrm{E}+20$ & $13.49 \%$ \\
\hline 58 & -8.75 & 9721.13 & 160.50 & $9.86 \mathrm{E}+14$ & $9.13 \mathrm{E}+20$ & $13.25 \%$ \\
\hline 59 & -9.25 & 9524.02 & 157.24 & $9.83 \mathrm{E}+14$ & $9.00 \mathrm{E}+20$ & $13.10 \%$ \\
\hline 60 & -9.75 & 9364.13 & 154.60 & $9.67 \mathrm{E}+14$ & $8.74 \mathrm{E}+20$ & $12.73 \%$ \\
\hline 61 & -10.25 & 9019.36 & 148.91 & $9.56 \mathrm{E}+14$ & $8.42 \mathrm{E}+20$ & $12.28 \%$ \\
\hline 62 & -10.75 & 8812.99 & 145.50 & $9.46 \mathrm{E}+14$ & $8.37 \mathrm{E}+20$ & $12.28 \%$ \\
\hline 63 & -11.25 & 8905.38 & 147.03 & $9.40 \mathrm{E}+14$ & $8.26 \mathrm{E}+20$ & $12.05 \%$ \\
\hline 64 & -11.75 & 8788.08 & 145.09 & $9.30 \mathrm{E}+14$ & $8.07 \mathrm{E}+20$ & $11.83 \%$ \\
\hline 65 & -12.25 & 8656.23 & 142.91 & $9.18 \mathrm{E}+14$ & $8.02 \mathrm{E}+20$ & $11.75 \%$ \\
\hline 66 & -12.75 & 8727.07 & 144.08 & $9.12 \mathrm{E}+14$ & $7.98 \mathrm{E}+20$ & $11.68 \%$ \\
\hline 67 & -13.25 & 8609.08 & 142.14 & $8.92 \mathrm{E}+14$ & $7.92 \mathrm{E}+20$ & $11.60 \%$ \\
\hline 68 & -13.75 & 8395.72 & 138.61 & $8.79 \mathrm{E}+14$ & $7.75 \mathrm{E}+20$ & $11.38 \%$ \\
\hline 69 & -14.25 & 8220.09 & 135.71 & $8.60 \mathrm{E}+14$ & $7.66 \mathrm{E}+20$ & $11.23 \%$ \\
\hline 70 & -14.75 & 8313.93 & 137.26 & $8.41 \mathrm{E}+14$ & $7.39 \mathrm{E}+20$ & $10.85 \%$ \\
\hline 71 & -15.25 & 7951.58 & 131.28 & $8.31 \mathrm{E}+14$ & $7.16 \mathrm{E}+20$ & $10.55 \%$ \\
\hline 72 & -15.75 & 7918.03 & 130.73 & $8.12 \mathrm{E}+14$ & $7.04 \mathrm{E}+20$ & $10.32 \%$ \\
\hline 73 & -16.25 & 7762.71 & 128.16 & $7.88 \mathrm{E}+14$ & $6.72 \mathrm{E}+20$ & $9.86 \%$ \\
\hline 74 & -16.75 & 7528.95 & 124.30 & $7.65 \mathrm{E}+14$ & $6.61 \mathrm{E}+20$ & $9.71 \%$ \\
\hline 75 & -17.25 & 7271.84 & 120.06 & $7.44 \mathrm{E}+14$ & $6.36 \mathrm{E}+20$ & $9.34 \%$ \\
\hline 76 & -17.75 & 6870.15 & 113.43 & $7.14 \mathrm{E}+14$ & $6.02 \mathrm{E}+20$ & $8.88 \%$ \\
\hline 77 & -18.25 & 6670.04 & 110.12 & $6.82 \mathrm{E}+14$ & $5.72 \mathrm{E}+20$ & $8.43 \%$ \\
\hline 78 & -18.75 & 6553.17 & 108.19 & $6.58 \mathrm{E}+14$ & $5.57 \mathrm{E}+20$ & $8.21 \%$ \\
\hline
\end{tabular}


Table 15: Cycle 149B, MCNP-Calculated HGRs, Neutron Flux, Depletion and Fission Density for AFIP-7, Plate Position 2, 53.6 EFPD (EOC) Average Center Lobe Power at 24.2 MW. ${ }^{2}$

\begin{tabular}{|c|c|c|c|c|c|c|}
\hline Node & $\begin{array}{c}\text { Distance from } \\
\text { Centerline (in.) }\end{array}$ & $\begin{array}{l}\text { Fission Power } \\
\text { Density (W/cc) }\end{array}$ & $\begin{array}{l}\text { Surface Heat } \\
\text { Flux }\left(W / \mathrm{cm}^{2}\right)\end{array}$ & $\begin{array}{c}\text { Neutron Flux } \\
\left(\mathrm{n} / \mathrm{cm}^{2} \mathrm{sec}\right)\end{array}$ & $\begin{array}{l}\text { Fission Density } \\
\text { (fissions/cc) }\end{array}$ & $\begin{array}{c}\text { U-235 } \\
\text { Burnup (\%) }\end{array}$ \\
\hline 1 & 19.25 & 6163.71 & 101.76 & $5.57 \mathrm{E}+14$ & $9.18 \mathrm{E}+20$ & $13.42 \%$ \\
\hline 2 & 18.75 & 5691.56 & 93.97 & $5.80 \mathrm{E}+14$ & $8.49 \mathrm{E}+20$ & $12.50 \%$ \\
\hline 3 & 18.25 & 5907.15 & 97.53 & $6.15 \mathrm{E}+14$ & $8.79 \mathrm{E}+20$ & $12.88 \%$ \\
\hline 4 & 17.75 & 6228.76 & 102.84 & $6.46 \mathrm{E}+14$ & $9.39 \mathrm{E}+20$ & $13.79 \%$ \\
\hline 5 & 17.25 & 6440.39 & 106.33 & $6.71 \mathrm{E}+14$ & $9.70 \mathrm{E}+20$ & $14.17 \%$ \\
\hline 6 & 16.75 & 6648.33 & 109.76 & $6.96 \mathrm{E}+14$ & $1.02 \mathrm{E}+21$ & $14.84 \%$ \\
\hline 7 & 16.25 & 6828.09 & 112.73 & $7.20 \mathrm{E}+14$ & $1.05 \mathrm{E}+21$ & $15.22 \%$ \\
\hline 8 & 15.75 & 7035.65 & 116.16 & $7.44 \mathrm{E}+14$ & $1.08 \mathrm{E}+21$ & $15.82 \%$ \\
\hline 9 & 15.25 & 7181.37 & 118.56 & $7.72 \mathrm{E}+14$ & $1.10 \mathrm{E}+21$ & $16.04 \%$ \\
\hline 10 & 14.75 & 7381.49 & 121.87 & $7.96 \mathrm{E}+14$ & $1.15 \mathrm{E}+21$ & $16.81 \%$ \\
\hline 11 & 14.25 & 7530.27 & 124.32 & $8.13 \mathrm{E}+14$ & $1.18 \mathrm{E}+21$ & $17.18 \%$ \\
\hline 13 & 13.75 & 7598.05 & 125.44 & $8.30 \mathrm{E}+14$ & $1.20 \mathrm{E}+21$ & $17.41 \%$ \\
\hline 14 & 13.25 & 7678.75 & 126.78 & $8.51 \mathrm{E}+14$ & $1.21 \mathrm{E}+21$ & $17.56 \%$ \\
\hline 15 & 12.75 & 7833.15 & 129.33 & $8.63 \mathrm{E}+14$ & $1.23 \mathrm{E}+21$ & $17.86 \%$ \\
\hline 16 & 12.25 & 7968.32 & 131.56 & $8.89 \mathrm{E}+14$ & $1.27 \mathrm{E}+21$ & $18.38 \%$ \\
\hline 17 & 11.75 & 7975.83 & 131.68 & $8.96 \mathrm{E}+14$ & $1.28 \mathrm{E}+21$ & $18.53 \%$ \\
\hline 18 & 11.25 & 8190.11 & 135.22 & $9.14 \mathrm{E}+14$ & $1.31 \mathrm{E}+21$ & $18.98 \%$ \\
\hline 19 & 10.75 & 8350.43 & 137.87 & $9.25 \mathrm{E}+14$ & $1.34 \mathrm{E}+21$ & $19.36 \%$ \\
\hline 20 & 10.25 & 8642.60 & 142.69 & $9.38 \mathrm{E}+14$ & $1.39 \mathrm{E}+21$ & $19.96 \%$ \\
\hline 21 & 9.75 & 8775.65 & 144.89 & $9.51 \mathrm{E}+14$ & $1.42 \mathrm{E}+21$ & $20.42 \%$ \\
\hline 22 & 9.25 & 8782.52 & 145.00 & $9.59 \mathrm{E}+14$ & $1.44 \mathrm{E}+21$ & $20.65 \%$ \\
\hline 23 & 8.75 & 9014.85 & 148.84 & $9.71 \mathrm{E}+14$ & $1.46 \mathrm{E}+21$ & $20.95 \%$ \\
\hline 24 & 8.25 & 8997.44 & 148.55 & $9.79 \mathrm{E}+14$ & $1.48 \mathrm{E}+21$ & $21.25 \%$ \\
\hline 25 & 7.75 & 9019.82 & 148.92 & $9.77 \mathrm{E}+14$ & $1.48 \mathrm{E}+21$ & $21.25 \%$ \\
\hline 26 & 7.25 & 9045.69 & 149.34 & $9.94 \mathrm{E}+14$ & $1.48 \mathrm{E}+21$ & $21.25 \%$ \\
\hline 27 & 6.75 & 9262.00 & 152.92 & $1.00 \mathrm{E}+15$ & $1.53 \mathrm{E}+21$ & $21.92 \%$ \\
\hline 28 & 6.25 & 9290.68 & 153.39 & $1.01 \mathrm{E}+15$ & $1.54 \mathrm{E}+21$ & $22.00 \%$ \\
\hline 29 & 5.75 & 9328.00 & 154.01 & $1.01 \mathrm{E}+15$ & $1.54 \mathrm{E}+21$ & $22.07 \%$ \\
\hline 30 & 5.25 & 9369.69 & 154.69 & $1.02 \mathrm{E}+15$ & $1.56 \mathrm{E}+21$ & $22.30 \%$ \\
\hline 31 & 4.75 & 9447.19 & 155.97 & $1.02 \mathrm{E}+15$ & $1.58 \mathrm{E}+21$ & $22.53 \%$ \\
\hline 32 & 4.25 & 9482.91 & 156.56 & $1.03 \mathrm{E}+15$ & $1.58 \mathrm{E}+21$ & $22.60 \%$ \\
\hline 33 & 3.75 & 9374.65 & 154.78 & $1.03 \mathrm{E}+15$ & $1.57 \mathrm{E}+21$ & $22.53 \%$ \\
\hline 34 & 3.25 & 9542.36 & 157.54 & $1.03 \mathrm{E}+15$ & $1.59 \mathrm{E}+21$ & $22.75 \%$ \\
\hline 35 & 2.75 & 9499.97 & 156.84 & $1.03 \mathrm{E}+15$ & $1.59 \mathrm{E}+21$ & $22.68 \%$ \\
\hline 36 & 2.25 & 9458.87 & 156.17 & $1.02 \mathrm{E}+15$ & $1.58 \mathrm{E}+21$ & $22.60 \%$ \\
\hline 37 & 1.75 & 9617.53 & 158.79 & $1.03 \mathrm{E}+15$ & $1.59 \mathrm{E}+21$ & $22.75 \%$ \\
\hline 38 & 1.25 & 9246.22 & 152.66 & $1.04 \mathrm{E}+15$ & $1.55 \mathrm{E}+21$ & $22.15 \%$ \\
\hline 39 & 0.75 & 9288.30 & 153.35 & $1.04 \mathrm{E}+15$ & $1.55 \mathrm{E}+21$ & $22.23 \%$ \\
\hline 40 & 0.25 & 9316.68 & 153.82 & $1.04 \mathrm{E}+15$ & $1.55 \mathrm{E}+21$ & $22.30 \%$ \\
\hline 41 & -0.25 & 9393.83 & 155.09 & $1.05 \mathrm{E}+15$ & $1.57 \mathrm{E}+21$ & $22.45 \%$ \\
\hline 42 & -0.75 & 9418.20 & 155.49 & $1.04 \mathrm{E}+15$ & $1.58 \mathrm{E}+21$ & $22.60 \%$ \\
\hline 43 & -1.25 & 9465.87 & 156.28 & $1.04 \mathrm{E}+15$ & $1.60 \mathrm{E}+21$ & $22.83 \%$ \\
\hline 44 & -1.75 & 9530.06 & 157.34 & $1.04 \mathrm{E}+15$ & $1.61 \mathrm{E}+21$ & $22.90 \%$ \\
\hline 45 & -2.25 & 9497.94 & 156.81 & $1.03 \mathrm{E}+15$ & $1.61 \mathrm{E}+21$ & $23.05 \%$ \\
\hline 46 & -2.75 & 9520.07 & 157.18 & $1.04 \mathrm{E}+15$ & $1.60 \mathrm{E}+21$ & $22.90 \%$ \\
\hline 47 & -3.25 & 9592.09 & 158.37 & $1.04 \mathrm{E}+15$ & $1.60 \mathrm{E}+21$ & $22.83 \%$ \\
\hline 48 & -3.75 & 9779.28 & 161.46 & $1.04 \mathrm{E}+15$ & $1.62 \mathrm{E}+21$ & $23.13 \%$ \\
\hline 49 & -4.25 & 9423.95 & 155.59 & $1.04 \mathrm{E}+15$ & $1.58 \mathrm{E}+21$ & $22.60 \%$ \\
\hline 50 & -4.75 & 9467.13 & 156.30 & $1.02 \mathrm{E}+15$ & $1.59 \mathrm{E}+21$ & $22.60 \%$ \\
\hline 51 & -5.25 & 9430.42 & 155.70 & $1.03 \mathrm{E}+15$ & $1.59 \mathrm{E}+21$ & $22.68 \%$ \\
\hline 52 & -5.75 & 9406.59 & 155.30 & $1.02 \mathrm{E}+15$ & $1.57 \mathrm{E}+21$ & $22.45 \%$ \\
\hline
\end{tabular}




\begin{tabular}{|l|l|l|l|l|l|l|}
\hline 53 & -6.25 & 9363.05 & 154.58 & $1.01 \mathrm{E}+15$ & $1.57 \mathrm{E}+21$ & $22.45 \%$ \\
\hline 54 & -6.75 & 9450.97 & 156.04 & $1.01 \mathrm{E}+15$ & $1.56 \mathrm{E}+21$ & $22.30 \%$ \\
\hline 55 & -7.25 & 9403.01 & 155.24 & $1.02 \mathrm{E}+15$ & $1.55 \mathrm{E}+21$ & $22.23 \%$ \\
\hline 56 & -7.75 & 9274.85 & 153.13 & $1.01 \mathrm{E}+15$ & $1.54 \mathrm{E}+21$ & $22.00 \%$ \\
\hline 57 & -8.25 & 9147.44 & 151.02 & $1.00 \mathrm{E}+15$ & $1.53 \mathrm{E}+21$ & $21.85 \%$ \\
\hline 58 & -8.75 & 9358.69 & 154.51 & $9.99 \mathrm{E}+14$ & $1.53 \mathrm{E}+21$ & $21.92 \%$ \\
\hline 59 & -9.25 & 9155.51 & 151.16 & $9.94 \mathrm{E}+14$ & $1.50 \mathrm{E}+21$ & $21.55 \%$ \\
\hline 60 & -9.75 & 9019.83 & 148.92 & $9.79 \mathrm{E}+14$ & $1.47 \mathrm{E}+21$ & $21.02 \%$ \\
\hline 61 & -10.25 & 8754.30 & 144.53 & $9.69 \mathrm{E}+14$ & $1.41 \mathrm{E}+21$ & $20.35 \%$ \\
\hline 62 & -10.75 & 8618.38 & 142.29 & $9.60 \mathrm{E}+14$ & $1.39 \mathrm{E}+21$ & $20.11 \%$ \\
\hline 63 & -11.25 & 8640.36 & 142.65 & $9.51 \mathrm{E}+14$ & $1.39 \mathrm{E}+21$ & $20.04 \%$ \\
\hline 64 & -11.75 & 8509.15 & 140.49 & $9.41 \mathrm{E}+14$ & $1.36 \mathrm{E}+21$ & $19.66 \%$ \\
\hline 65 & -12.25 & 8398.95 & 138.67 & $9.31 \mathrm{E}+14$ & $1.35 \mathrm{E}+21$ & $19.51 \%$ \\
\hline 66 & -12.75 & 8463.36 & 139.73 & $9.26 \mathrm{E}+14$ & $1.35 \mathrm{E}+21$ & $19.43 \%$ \\
\hline 67 & -13.25 & 8350.83 & 137.87 & $9.06 \mathrm{E}+14$ & $1.34 \mathrm{E}+21$ & $19.28 \%$ \\
\hline 68 & -13.75 & 8160.66 & 134.73 & $8.93 \mathrm{E}+14$ & $1.31 \mathrm{E}+21$ & $18.91 \%$ \\
\hline 69 & -14.25 & 8055.87 & 133.00 & $8.75 \mathrm{E}+14$ & $1.29 \mathrm{E}+21$ & $18.61 \%$ \\
\hline 70 & -14.75 & 8106.04 & 133.83 & $8.54 \mathrm{E}+14$ & $1.26 \mathrm{E}+21$ & $18.31 \%$ \\
\hline 71 & -15.25 & 7759.13 & 128.10 & $8.42 \mathrm{E}+14$ & $1.22 \mathrm{E}+21$ & $17.71 \%$ \\
\hline 72 & -15.75 & 7697.50 & 127.09 & $8.23 \mathrm{E}+14$ & $1.20 \mathrm{E}+21$ & $17.48 \%$ \\
\hline 73 & -16.25 & 7571.21 & 125.00 & $8.00 \mathrm{E}+14$ & $1.16 \mathrm{E}+21$ & $16.88 \%$ \\
\hline 74 & -16.75 & 7363.98 & 121.58 & $7.77 \mathrm{E}+14$ & $1.14 \mathrm{E}+21$ & $16.57 \%$ \\
\hline 75 & -17.25 & 7080.69 & 116.90 & $7.55 \mathrm{E}+14$ & $1.10 \mathrm{E}+21$ & $15.97 \%$ \\
\hline 76 & -17.75 & 6763.98 & 111.67 & $7.24 \mathrm{E}+14$ & $1.04 \mathrm{E}+21$ & $15.14 \%$ \\
\hline 77 & -18.25 & 6591.37 & 108.82 & $6.95 \mathrm{E}+14$ & $9.94 \mathrm{E}+20$ & $14.54 \%$ \\
\hline 78 & -18.75 & 6464.18 & 106.72 & $6.69 \mathrm{E}+14$ & $9.72 \mathrm{E}+20$ & $14.24 \%$ \\
\hline
\end{tabular}


Table 16: Cycle 149B, MCNP-Calculated HGRs and Neutron Flux for AFIP-7, Plate Position 3, 0 EFPD (BOC) Average Center Lobe Power at 24.2 MW. ${ }^{2}$

\begin{tabular}{|c|c|c|c|c|}
\hline Node & $\begin{array}{l}\text { Distance from } \\
\text { Centerline (in.) }\end{array}$ & $\begin{array}{l}\text { Fission Power } \\
\text { Density (W/cc) }\end{array}$ & $\begin{array}{l}\text { Surface Heat } \\
\text { Flux }\left(W / \mathrm{cm}^{2}\right)\end{array}$ & $\begin{array}{c}\text { Neutron Flux } \\
\left(\mathrm{n} / \mathrm{cm}^{2} \mathrm{sec}\right)\end{array}$ \\
\hline 1 & 19.25 & 6458.97 & 106.64 & $5.16 \mathrm{E}+14$ \\
\hline 2 & 18.75 & 6016.05 & 99.33 & $5.48 \mathrm{E}+14$ \\
\hline 3 & 18.25 & 6286.17 & 103.78 & $5.86 \mathrm{E}+14$ \\
\hline 4 & 17.75 & 6811.02 & 112.45 & $6.20 \mathrm{E}+14$ \\
\hline 5 & 17.25 & 7058.05 & 116.53 & $6.52 \mathrm{E}+14$ \\
\hline 6 & 16.75 & 7348.76 & 121.33 & $6.79 \mathrm{E}+14$ \\
\hline 7 & 16.25 & 7845.95 & 129.54 & $7.13 \mathrm{E}+14$ \\
\hline 8 & 15.75 & 8185.64 & 135.14 & $7.42 \mathrm{E}+14$ \\
\hline 9 & 15.25 & 8429.56 & 139.17 & $7.73 \mathrm{E}+14$ \\
\hline 10 & 14.75 & 8732.94 & 144.18 & $8.04 \mathrm{E}+14$ \\
\hline 11 & 14.25 & 9138.74 & 150.88 & $8.25 \mathrm{E}+14$ \\
\hline 13 & 13.75 & 9538.24 & 157.48 & $8.58 \mathrm{E}+14$ \\
\hline 14 & 13.25 & 9468.00 & 156.32 & $8.74 \mathrm{E}+14$ \\
\hline 15 & 12.75 & 9608.49 & 158.64 & $8.96 \mathrm{E}+14$ \\
\hline 16 & 12.25 & 9792.72 & 161.68 & $9.26 \mathrm{E}+14$ \\
\hline 17 & 11.75 & 10232.82 & 168.94 & $9.38 \mathrm{E}+14$ \\
\hline 18 & 11.25 & 10405.34 & 171.79 & $9.67 \mathrm{E}+14$ \\
\hline 19 & 10.75 & 10889.51 & 179.79 & $1.00 \mathrm{E}+15$ \\
\hline 20 & 10.25 & 11094.16 & 183.16 & $1.02 \mathrm{E}+15$ \\
\hline 21 & 9.75 & 11628.71 & 191.99 & $1.04 \mathrm{E}+15$ \\
\hline 22 & 9.25 & 11878.92 & 196.12 & $1.06 \mathrm{E}+15$ \\
\hline 23 & 8.75 & 12070.22 & 199.28 & $1.08 \mathrm{E}+15$ \\
\hline 24 & 8.25 & 12457.16 & 205.67 & $1.09 \mathrm{E}+15$ \\
\hline 25 & 7.75 & 12501.50 & 206.40 & $1.10 \mathrm{E}+15$ \\
\hline 26 & 7.25 & 12650.42 & 208.86 & $1.12 \mathrm{E}+15$ \\
\hline 27 & 6.75 & 13058.60 & 215.60 & $1.14 \mathrm{E}+15$ \\
\hline 28 & 6.25 & 13214.77 & 218.18 & $1.15 \mathrm{E}+15$ \\
\hline 29 & 5.75 & 13038.29 & 215.26 & $1.16 \mathrm{E}+15$ \\
\hline 30 & 5.25 & 13544.03 & 223.61 & $1.18 \mathrm{E}+15$ \\
\hline 31 & 4.75 & 13431.95 & 221.76 & $1.19 \mathrm{E}+15$ \\
\hline 32 & 4.25 & 13509.37 & 223.04 & $1.20 \mathrm{E}+15$ \\
\hline 33 & 3.75 & 13751.25 & 227.03 & $1.21 \mathrm{E}+15$ \\
\hline 34 & 3.25 & 13529.98 & 223.38 & $1.21 \mathrm{E}+15$ \\
\hline 35 & 2.75 & 13706.06 & 226.29 & $1.21 \mathrm{E}+15$ \\
\hline 36 & 2.25 & 13734.73 & 226.76 & $1.22 \mathrm{E}+15$ \\
\hline 37 & 1.75 & 13395.23 & 221.16 & $1.22 \mathrm{E}+15$ \\
\hline 38 & 1.25 & 13303.60 & 219.64 & $1.21 \mathrm{E}+15$ \\
\hline 39 & 0.75 & 13557.77 & 223.84 & $1.23 \mathrm{E}+15$ \\
\hline 40 & 0.25 & 13520.13 & 223.22 & $1.23 \mathrm{E}+15$ \\
\hline 41 & -0.25 & 13590.35 & 224.38 & $1.23 \mathrm{E}+15$ \\
\hline 42 & -0.75 & 13505.08 & 222.97 & $1.23 \mathrm{E}+15$ \\
\hline 43 & -1.25 & 13970.17 & 230.65 & $1.23 \mathrm{E}+15$ \\
\hline 44 & -1.75 & 13792.33 & 227.71 & $1.23 \mathrm{E}+15$ \\
\hline 45 & -2.25 & 14025.31 & 231.56 & $1.23 \mathrm{E}+15$ \\
\hline 46 & -2.75 & 14050.74 & 231.98 & $1.23 \mathrm{E}+15$ \\
\hline 47 & -3.25 & 13775.08 & 227.43 & $1.22 \mathrm{E}+15$ \\
\hline 48 & -3.75 & 13733.60 & 226.74 & $1.20 \mathrm{E}+15$ \\
\hline 49 & -4.25 & 13890.11 & 229.33 & $1.20 \mathrm{E}+15$ \\
\hline 50 & -4.75 & 13611.14 & 224.72 & $1.20 \mathrm{E}+15$ \\
\hline 51 & -5.25 & 13698.14 & 226.16 & $1.20 \mathrm{E}+15$ \\
\hline 52 & -5.75 & 13665.48 & 225.62 & $1.19 \mathrm{E}+15$ \\
\hline
\end{tabular}




\begin{tabular}{|l|l|l|l|l|}
\hline 53 & -6.25 & 13439.22 & 221.88 & $1.17 \mathrm{E}+15$ \\
\hline 54 & -6.75 & 13143.24 & 216.99 & $1.17 \mathrm{E}+15$ \\
\hline 55 & -7.25 & 13182.79 & 217.65 & $1.16 \mathrm{E}+15$ \\
\hline 56 & -7.75 & 13016.12 & 214.90 & $1.15 \mathrm{E}+15$ \\
\hline 57 & -8.25 & 12829.38 & 211.81 & $1.13 \mathrm{E}+15$ \\
\hline 58 & -8.75 & 12759.66 & 210.66 & $1.12 \mathrm{E}+15$ \\
\hline 59 & -9.25 & 12342.98 & 203.78 & $1.10 \mathrm{E}+15$ \\
\hline 60 & -9.75 & 12183.88 & 201.16 & $1.10 \mathrm{E}+15$ \\
\hline 61 & -10.25 & 11465.85 & 189.30 & $1.07 \mathrm{E}+15$ \\
\hline 62 & -10.75 & 11436.48 & 188.82 & $1.06 \mathrm{E}+15$ \\
\hline 63 & -11.25 & 11122.59 & 183.63 & $1.03 \mathrm{E}+15$ \\
\hline 64 & -11.75 & 10864.23 & 179.37 & $1.01 \mathrm{E}+15$ \\
\hline 65 & -12.25 & 10875.17 & 179.55 & $9.91 \mathrm{E}+14$ \\
\hline 66 & -12.75 & 10874.19 & 179.53 & $9.74 \mathrm{E}+14$ \\
\hline 67 & -13.25 & 10544.43 & 174.09 & $9.49 \mathrm{E}+14$ \\
\hline 68 & -13.75 & 10132.33 & 167.28 & $9.26 \mathrm{E}+14$ \\
\hline 69 & -14.25 & 10108.63 & 166.89 & $9.04 \mathrm{E}+14$ \\
\hline 70 & -14.75 & 9948.83 & 164.26 & $8.79 \mathrm{E}+14$ \\
\hline 71 & -15.25 & 9329.06 & 154.02 & $8.49 \mathrm{E}+14$ \\
\hline 72 & -15.75 & 9200.31 & 151.90 & $8.32 \mathrm{E}+14$ \\
\hline 73 & -16.25 & 8991.93 & 148.46 & $8.06 \mathrm{E}+14$ \\
\hline 74 & -16.75 & 8532.18 & 140.87 & $7.73 \mathrm{E}+14$ \\
\hline 75 & -17.25 & 8045.70 & 132.83 & $7.38 \mathrm{E}+14$ \\
\hline 76 & -17.75 & 7595.14 & 125.40 & $7.10 \mathrm{E}+14$ \\
\hline 77 & -18.25 & 7509.49 & 123.98 & $6.82 \mathrm{E}+14$ \\
\hline 78 & -18.75 & 6931.18 & 114.43 & $6.37 \mathrm{E}+14$ \\
\hline
\end{tabular}


Table 17: Cycle 149B, MCNP-Calculated HGRs, Neutron Flux, Depletion and Fission Density for AFIP-7, Plate Position 3, 15 EFPD (MOC1) Average Center Lobe Power at 24.2 MW. ${ }^{2}$

\begin{tabular}{|c|c|c|c|c|c|c|}
\hline Node & $\begin{array}{c}\text { Distance from } \\
\text { Centerline (in.) }\end{array}$ & $\begin{array}{l}\text { Fission Power } \\
\text { Density (W/cc) }\end{array}$ & $\begin{array}{l}\text { Surface Heat } \\
\text { Flux }\left(W / \mathrm{cm}^{2}\right)\end{array}$ & $\begin{array}{c}\text { Neutron Flux } \\
\left(\mathrm{n} / \mathrm{cm}^{2} \mathrm{sec}\right)\end{array}$ & $\begin{array}{l}\text { Fission Density } \\
\text { (fissions/cc) }\end{array}$ & $\begin{array}{c}\text { U-235 } \\
\text { Burnup (\%) }\end{array}$ \\
\hline 1 & 19.25 & 5544.03 & 91.53 & $4.96 \mathrm{E}+14$ & $2.52 \mathrm{E}+20$ & $3.81 \%$ \\
\hline 2 & 18.75 & 4997.85 & 82.51 & $5.17 \mathrm{E}+14$ & $2.35 \mathrm{E}+20$ & $3.58 \%$ \\
\hline 3 & 18.25 & 5298.74 & 87.48 & $5.51 \mathrm{E}+14$ & $2.46 \mathrm{E}+20$ & $3.73 \%$ \\
\hline 4 & 17.75 & 5454.65 & 90.06 & $5.75 \mathrm{E}+14$ & $2.66 \mathrm{E}+20$ & $4.04 \%$ \\
\hline 5 & 17.25 & 5884.73 & 97.16 & $6.09 \mathrm{E}+14$ & $2.76 \mathrm{E}+20$ & $4.20 \%$ \\
\hline 6 & 16.75 & 5914.54 & 97.65 & $6.27 \mathrm{E}+14$ & $2.87 \mathrm{E}+20$ & $4.36 \%$ \\
\hline 7 & 16.25 & 6195.40 & 102.29 & $6.53 \mathrm{E}+14$ & $3.07 \mathrm{E}+20$ & $4.67 \%$ \\
\hline 8 & 15.75 & 6278.79 & 103.66 & $6.78 \mathrm{E}+14$ & $3.20 \mathrm{E}+20$ & $4.82 \%$ \\
\hline 9 & 15.25 & 6685.13 & 110.37 & $7.03 \mathrm{E}+14$ & $3.29 \mathrm{E}+20$ & $4.98 \%$ \\
\hline 10 & 14.75 & 6718.08 & 110.92 & $7.30 \mathrm{E}+14$ & $3.41 \mathrm{E}+20$ & $5.21 \%$ \\
\hline 11 & 14.25 & 6823.43 & 112.65 & $7.38 \mathrm{E}+14$ & $3.57 \mathrm{E}+20$ & $5.44 \%$ \\
\hline 13 & 13.75 & 7004.33 & 115.64 & $7.61 \mathrm{E}+14$ & $3.73 \mathrm{E}+20$ & $5.67 \%$ \\
\hline 14 & 13.25 & 7023.62 & 115.96 & $7.80 \mathrm{E}+14$ & $3.70 \mathrm{E}+20$ & $5.60 \%$ \\
\hline 15 & 12.75 & 7181.48 & 118.57 & $8.02 \mathrm{E}+14$ & $3.75 \mathrm{E}+20$ & $5.67 \%$ \\
\hline 16 & 12.25 & 7237.29 & 119.49 & $8.12 \mathrm{E}+14$ & $3.83 \mathrm{E}+20$ & $5.83 \%$ \\
\hline 17 & 11.75 & 7547.07 & 124.60 & $8.35 \mathrm{E}+14$ & $4.00 \mathrm{E}+20$ & $6.06 \%$ \\
\hline 18 & 11.25 & 7465.29 & 123.25 & $8.36 \mathrm{E}+14$ & $4.06 \mathrm{E}+20$ & $6.14 \%$ \\
\hline 19 & 10.75 & 7821.04 & 129.13 & $8.58 \mathrm{E}+14$ & $4.25 \mathrm{E}+20$ & $6.37 \%$ \\
\hline 20 & 10.25 & 7814.37 & 129.02 & $8.72 \mathrm{E}+14$ & $4.33 \mathrm{E}+20$ & $6.53 \%$ \\
\hline 21 & 9.75 & 8249.22 & 136.19 & $8.88 \mathrm{E}+14$ & $4.54 \mathrm{E}+20$ & $6.84 \%$ \\
\hline 22 & 9.25 & 8156.15 & 134.66 & $9.01 \mathrm{E}+14$ & $4.64 \mathrm{E}+20$ & $6.91 \%$ \\
\hline 23 & 8.75 & 8258.95 & 136.36 & $8.98 \mathrm{E}+14$ & $4.72 \mathrm{E}+20$ & $7.08 \%$ \\
\hline 24 & 8.25 & 8314.47 & 137.27 & $9.23 \mathrm{E}+14$ & $4.87 \mathrm{E}+20$ & $7.31 \%$ \\
\hline 25 & 7.75 & 8349.59 & 137.85 & $9.16 \mathrm{E}+14$ & $4.88 \mathrm{E}+20$ & $7.31 \%$ \\
\hline 26 & 7.25 & 8640.32 & 142.65 & $9.32 \mathrm{E}+14$ & $4.94 \mathrm{E}+20$ & $7.39 \%$ \\
\hline 27 & 6.75 & 8609.29 & 142.14 & $9.39 \mathrm{E}+14$ & $5.10 \mathrm{E}+20$ & $7.62 \%$ \\
\hline 28 & 6.25 & 8561.66 & 141.35 & $9.42 \mathrm{E}+14$ & $5.16 \mathrm{E}+20$ & $7.70 \%$ \\
\hline 29 & 5.75 & 8707.12 & 143.75 & $9.58 \mathrm{E}+14$ & $5.09 \mathrm{E}+20$ & $7.62 \%$ \\
\hline 30 & 5.25 & 8686.14 & 143.41 & $9.59 \mathrm{E}+14$ & $5.29 \mathrm{E}+20$ & $7.93 \%$ \\
\hline 31 & 4.75 & 8676.67 & 143.25 & $9.67 \mathrm{E}+14$ & $5.25 \mathrm{E}+20$ & $7.86 \%$ \\
\hline 32 & 4.25 & 8803.81 & 145.35 & $9.73 \mathrm{E}+14$ & $5.28 \mathrm{E}+20$ & $7.86 \%$ \\
\hline 33 & 3.75 & 8658.36 & 142.95 & $9.73 \mathrm{E}+14$ & $5.37 \mathrm{E}+20$ & $8.01 \%$ \\
\hline 34 & 3.25 & 8847.59 & 146.07 & $9.69 \mathrm{E}+14$ & $5.29 \mathrm{E}+20$ & $7.86 \%$ \\
\hline 35 & 2.75 & 8936.62 & 147.54 & $9.78 \mathrm{E}+14$ & $5.35 \mathrm{E}+20$ & $8.01 \%$ \\
\hline 36 & 2.25 & 8618.72 & 142.30 & $9.71 \mathrm{E}+14$ & $5.37 \mathrm{E}+20$ & $8.01 \%$ \\
\hline 37 & 1.75 & 8828.08 & 145.75 & $9.85 \mathrm{E}+14$ & $5.23 \mathrm{E}+20$ & $7.86 \%$ \\
\hline 38 & 1.25 & 8605.77 & 142.08 & $9.79 \mathrm{E}+14$ & $5.20 \mathrm{E}+20$ & $7.78 \%$ \\
\hline 39 & 0.75 & 8527.59 & 140.79 & $9.85 \mathrm{E}+14$ & $5.30 \mathrm{E}+20$ & $7.93 \%$ \\
\hline 40 & 0.25 & 8683.21 & 143.36 & $9.87 \mathrm{E}+14$ & $5.28 \mathrm{E}+20$ & $7.93 \%$ \\
\hline 41 & -0.25 & 8626.89 & 142.43 & $9.81 \mathrm{E}+14$ & $5.31 \mathrm{E}+20$ & $7.93 \%$ \\
\hline 42 & -0.75 & 8808.70 & 145.43 & $9.88 \mathrm{E}+14$ & $5.28 \mathrm{E}+20$ & $7.86 \%$ \\
\hline 43 & -1.25 & 8863.52 & 146.34 & $9.87 \mathrm{E}+14$ & $5.46 \mathrm{E}+20$ & $8.17 \%$ \\
\hline 44 & -1.75 & 8775.60 & 144.89 & $9.83 \mathrm{E}+14$ & $5.39 \mathrm{E}+20$ & $8.01 \%$ \\
\hline 45 & -2.25 & 8825.40 & 145.71 & $9.82 \mathrm{E}+14$ & $5.48 \mathrm{E}+20$ & $8.17 \%$ \\
\hline 46 & -2.75 & 8726.27 & 144.07 & $9.77 \mathrm{E}+14$ & $5.49 \mathrm{E}+20$ & $8.17 \%$ \\
\hline 47 & -3.25 & 8778.81 & 144.94 & $9.78 \mathrm{E}+14$ & $5.38 \mathrm{E}+20$ & $8.01 \%$ \\
\hline 48 & -3.75 & 8850.72 & 146.13 & $9.76 \mathrm{E}+14$ & $5.37 \mathrm{E}+20$ & $8.01 \%$ \\
\hline 49 & -4.25 & 8858.72 & 146.26 & $9.81 \mathrm{E}+14$ & $5.43 \mathrm{E}+20$ & $8.09 \%$ \\
\hline 50 & -4.75 & 8835.22 & 145.87 & $9.77 \mathrm{E}+14$ & $5.32 \mathrm{E}+20$ & $7.93 \%$ \\
\hline 51 & -5.25 & 8800.52 & 145.30 & $9.71 \mathrm{E}+14$ & $5.35 \mathrm{E}+20$ & $8.01 \%$ \\
\hline 52 & -5.75 & 8671.37 & 143.16 & $9.67 \mathrm{E}+14$ & $5.34 \mathrm{E}+20$ & $8.01 \%$ \\
\hline
\end{tabular}




\begin{tabular}{|l|l|l|l|l|l|l|}
\hline 53 & -6.25 & 8688.79 & 143.45 & $9.64 \mathrm{E}+14$ & $5.25 \mathrm{E}+20$ & $7.86 \%$ \\
\hline 54 & -6.75 & 8795.66 & 145.22 & $9.63 \mathrm{E}+14$ & $5.13 \mathrm{E}+20$ & $7.70 \%$ \\
\hline 55 & -7.25 & 8577.51 & 141.61 & $9.53 \mathrm{E}+14$ & $5.15 \mathrm{E}+20$ & $7.70 \%$ \\
\hline 56 & -7.75 & 8654.03 & 142.88 & $9.50 \mathrm{E}+14$ & $5.08 \mathrm{E}+20$ & $7.62 \%$ \\
\hline 57 & -8.25 & 8553.92 & 141.23 & $9.35 \mathrm{E}+14$ & $5.01 \mathrm{E}+20$ & $7.47 \%$ \\
\hline 58 & -8.75 & 8468.67 & 139.82 & $9.27 \mathrm{E}+14$ & $4.98 \mathrm{E}+20$ & $7.47 \%$ \\
\hline 59 & -9.25 & 8395.60 & 138.61 & $9.27 \mathrm{E}+14$ & $4.82 \mathrm{E}+20$ & $7.24 \%$ \\
\hline 60 & -9.75 & 8197.19 & 135.34 & $9.18 \mathrm{E}+14$ & $4.76 \mathrm{E}+20$ & $7.16 \%$ \\
\hline 61 & -10.25 & 8109.17 & 133.88 & $9.11 \mathrm{E}+14$ & $4.48 \mathrm{E}+20$ & $6.76 \%$ \\
\hline 62 & -10.75 & 7897.08 & 130.38 & $8.96 \mathrm{E}+14$ & $4.47 \mathrm{E}+20$ & $6.76 \%$ \\
\hline 63 & -11.25 & 7911.05 & 130.61 & $8.89 \mathrm{E}+14$ & $4.35 \mathrm{E}+20$ & $6.53 \%$ \\
\hline 64 & -11.75 & 7727.75 & 127.59 & $8.74 \mathrm{E}+14$ & $4.24 \mathrm{E}+20$ & $6.37 \%$ \\
\hline 65 & -12.25 & 7709.40 & 127.28 & $8.56 \mathrm{E}+14$ & $4.25 \mathrm{E}+20$ & $6.37 \%$ \\
\hline 66 & -12.75 & 7768.77 & 128.26 & $8.43 \mathrm{E}+14$ & $4.25 \mathrm{E}+20$ & $6.37 \%$ \\
\hline 67 & -13.25 & 7761.94 & 128.15 & $8.35 \mathrm{E}+14$ & $4.12 \mathrm{E}+20$ & $6.22 \%$ \\
\hline 68 & -13.75 & 7550.92 & 124.67 & $8.22 \mathrm{E}+14$ & $3.96 \mathrm{E}+20$ & $5.98 \%$ \\
\hline 69 & -14.25 & 7559.20 & 124.80 & $8.05 \mathrm{E}+14$ & $3.95 \mathrm{E}+20$ & $5.98 \%$ \\
\hline 70 & -14.75 & 7219.28 & 119.19 & $7.89 \mathrm{E}+14$ & $3.89 \mathrm{E}+20$ & $5.91 \%$ \\
\hline 71 & -15.25 & 7152.04 & 118.08 & $7.68 \mathrm{E}+14$ & $3.64 \mathrm{E}+20$ & $5.52 \%$ \\
\hline 72 & -15.75 & 7007.02 & 115.69 & $7.47 \mathrm{E}+14$ & $3.59 \mathrm{E}+20$ & $5.44 \%$ \\
\hline 73 & -16.25 & 6876.63 & 113.53 & $7.29 \mathrm{E}+14$ & $3.51 \mathrm{E}+20$ & $5.29 \%$ \\
\hline 74 & -16.75 & 6756.22 & 111.55 & $7.10 \mathrm{E}+14$ & $3.33 \mathrm{E}+20$ & $5.05 \%$ \\
\hline 75 & -17.25 & 6402.69 & 105.71 & $6.85 \mathrm{E}+14$ & $3.14 \mathrm{E}+20$ & $4.74 \%$ \\
\hline 76 & -17.75 & 6185.43 & 102.12 & $6.60 \mathrm{E}+14$ & $2.97 \mathrm{E}+20$ & $4.51 \%$ \\
\hline 77 & -18.25 & 5876.14 & 97.02 & $6.35 \mathrm{E}+14$ & $2.93 \mathrm{E}+20$ & $4.43 \%$ \\
\hline 78 & -18.75 & 5814.33 & 95.99 & $6.07 \mathrm{E}+14$ & $2.71 \mathrm{E}+20$ & $4.12 \%$ \\
\hline
\end{tabular}


Table 18: Cycle 149B, MCNP-Calculated HGRs, Neutron Flux, Depletion and Fission Density for AFIP-7, Plate Position 3, 31 EFPD (MOC2) Average Center Lobe Power at 24.2 MW. ${ }^{2}$

\begin{tabular}{|c|c|c|c|c|c|c|}
\hline Node & $\begin{array}{c}\text { Distance from } \\
\text { Centerline (in.) }\end{array}$ & $\begin{array}{l}\text { Fission Power } \\
\text { Density (W/cc) }\end{array}$ & $\begin{array}{l}\text { Surface Heat } \\
\text { Flux }\left(W / \mathrm{cm}^{2}\right)\end{array}$ & $\begin{array}{c}\text { Neutron Flux } \\
\left(\mathrm{n} / \mathrm{cm}^{2} \mathrm{sec}\right)\end{array}$ & $\begin{array}{l}\text { Fission Density } \\
\text { (fissions/cc) }\end{array}$ & $\begin{array}{c}\text { U-235 } \\
\text { Burnup (\%) }\end{array}$ \\
\hline 1 & 19.25 & 6158.41 & 101.68 & $5.44 \mathrm{E}+14$ & $5.03 \mathrm{E}+20$ & $7.62 \%$ \\
\hline 2 & 18.75 & 5627.32 & 92.91 & $5.72 \mathrm{E}+14$ & $4.61 \mathrm{E}+20$ & $7.08 \%$ \\
\hline 3 & 18.25 & 5730.53 & 94.61 & $6.00 \mathrm{E}+14$ & $4.86 \mathrm{E}+20$ & $7.39 \%$ \\
\hline 4 & 17.75 & 5900.68 & 97.42 & $6.22 \mathrm{E}+14$ & $5.13 \mathrm{E}+20$ & $7.86 \%$ \\
\hline 5 & 17.25 & 6280.34 & 103.69 & $6.57 \mathrm{E}+14$ & $5.42 \mathrm{E}+20$ & $8.25 \%$ \\
\hline 6 & 16.75 & 6561.97 & 108.34 & $6.87 \mathrm{E}+14$ & $5.55 \mathrm{E}+20$ & $8.48 \%$ \\
\hline 7 & 16.25 & 6820.80 & 112.61 & $7.09 \mathrm{E}+14$ & $5.87 \mathrm{E}+20$ & $8.94 \%$ \\
\hline 8 & 15.75 & 6960.15 & 114.91 & $7.32 \mathrm{E}+14$ & $6.04 \mathrm{E}+20$ & $9.18 \%$ \\
\hline 9 & 15.25 & 7149.09 & 118.03 & $7.47 \mathrm{E}+14$ & $6.32 \mathrm{E}+20$ & $9.56 \%$ \\
\hline 10 & 14.75 & 7281.81 & 120.22 & $7.75 \mathrm{E}+14$ & $6.45 \mathrm{E}+20$ & $9.80 \%$ \\
\hline 11 & 14.25 & 7494.07 & 123.73 & $7.89 \mathrm{E}+14$ & $6.66 \mathrm{E}+20$ & $10.11 \%$ \\
\hline 13 & 13.75 & 7517.16 & 124.11 & $8.16 \mathrm{E}+14$ & $6.90 \mathrm{E}+20$ & $10.49 \%$ \\
\hline 14 & 13.25 & 7750.84 & 127.97 & $8.31 \mathrm{E}+14$ & $6.88 \mathrm{E}+20$ & $10.42 \%$ \\
\hline 15 & 12.75 & 7989.04 & 131.90 & $8.54 \mathrm{E}+14$ & $7.01 \mathrm{E}+20$ & $10.66 \%$ \\
\hline 16 & 12.25 & 7934.69 & 131.00 & $8.76 \mathrm{E}+14$ & $7.10 \mathrm{E}+20$ & $10.74 \%$ \\
\hline 17 & 11.75 & 8056.80 & 133.02 & $8.87 \mathrm{E}+14$ & $7.42 \mathrm{E}+20$ & $11.20 \%$ \\
\hline 18 & 11.25 & 8168.94 & 134.87 & $8.97 \mathrm{E}+14$ & $7.45 \mathrm{E}+20$ & $11.28 \%$ \\
\hline 19 & 10.75 & 8291.60 & 136.89 & $9.07 \mathrm{E}+14$ & $7.80 \mathrm{E}+20$ & $11.75 \%$ \\
\hline 20 & 10.25 & 8471.00 & 139.86 & $9.23 \mathrm{E}+14$ & $7.87 \mathrm{E}+20$ & $11.90 \%$ \\
\hline 21 & 9.75 & 8646.85 & 142.76 & $9.29 \mathrm{E}+14$ & $8.28 \mathrm{E}+20$ & $12.45 \%$ \\
\hline 22 & 9.25 & 8843.20 & 146.00 & $9.46 \mathrm{E}+14$ & $8.33 \mathrm{E}+20$ & $12.52 \%$ \\
\hline 23 & 8.75 & 8877.04 & 146.56 & $9.55 \mathrm{E}+14$ & $8.46 \mathrm{E}+20$ & $12.68 \%$ \\
\hline 24 & 8.25 & 8854.68 & 146.19 & $9.61 \mathrm{E}+14$ & $8.63 \mathrm{E}+20$ & $12.99 \%$ \\
\hline 25 & 7.75 & 9056.92 & 149.53 & $9.66 \mathrm{E}+14$ & $8.66 \mathrm{E}+20$ & $12.99 \%$ \\
\hline 26 & 7.25 & 9273.72 & 153.11 & $9.77 \mathrm{E}+14$ & $8.85 \mathrm{E}+20$ & $13.30 \%$ \\
\hline 27 & 6.75 & 9241.31 & 152.57 & $9.77 \mathrm{E}+14$ & $9.00 \mathrm{E}+20$ & $13.45 \%$ \\
\hline 28 & 6.25 & 9193.16 & 151.78 & $9.79 \mathrm{E}+14$ & $9.04 \mathrm{E}+20$ & $13.53 \%$ \\
\hline 29 & 5.75 & 9279.63 & 153.21 & $9.93 \mathrm{E}+14$ & $9.04 \mathrm{E}+20$ & $13.53 \%$ \\
\hline 30 & 5.25 & 9398.36 & 155.17 & $1.00 \mathrm{E}+15$ & $9.22 \mathrm{E}+20$ & $13.84 \%$ \\
\hline 31 & 4.75 & 9430.45 & 155.70 & $1.00 \mathrm{E}+15$ & $9.18 \mathrm{E}+20$ & $13.76 \%$ \\
\hline 32 & 4.25 & 9490.83 & 156.69 & $1.00 \mathrm{E}+15$ & $9.26 \mathrm{E}+20$ & $13.84 \%$ \\
\hline 33 & 3.75 & 9495.43 & 156.77 & $1.01 \mathrm{E}+15$ & $9.29 \mathrm{E}+20$ & $13.92 \%$ \\
\hline 34 & 3.25 & 9539.24 & 157.49 & $1.01 \mathrm{E}+15$ & $9.29 \mathrm{E}+20$ & $13.92 \%$ \\
\hline 35 & 2.75 & 9490.02 & 156.68 & $1.01 \mathrm{E}+15$ & $9.40 \mathrm{E}+20$ & $14.09 \%$ \\
\hline 36 & 2.25 & 9406.25 & 155.30 & $1.01 \mathrm{E}+15$ & $9.27 \mathrm{E}+20$ & $13.84 \%$ \\
\hline 37 & 1.75 & 9559.48 & 157.83 & $1.02 \mathrm{E}+15$ & $9.23 \mathrm{E}+20$ & $13.84 \%$ \\
\hline 38 & 1.25 & 9219.59 & 152.22 & $1.02 \mathrm{E}+15$ & $9.09 \mathrm{E}+20$ & $13.61 \%$ \\
\hline 39 & 0.75 & 9378.12 & 154.83 & $1.03 \mathrm{E}+15$ & $9.16 \mathrm{E}+20$ & $13.76 \%$ \\
\hline 40 & 0.25 & 9349.92 & 154.37 & $1.03 \mathrm{E}+15$ & $9.21 \mathrm{E}+20$ & $13.84 \%$ \\
\hline 41 & -0.25 & 9281.20 & 153.23 & $1.01 \mathrm{E}+15$ & $9.22 \mathrm{E}+20$ & $13.84 \%$ \\
\hline 42 & -0.75 & 9470.61 & 156.36 & $1.03 \mathrm{E}+15$ & $9.26 \mathrm{E}+20$ & $13.84 \%$ \\
\hline 43 & -1.25 & 9640.86 & 159.17 & $1.03 \mathrm{E}+15$ & $9.47 \mathrm{E}+20$ & $14.16 \%$ \\
\hline 44 & -1.75 & 9513.63 & 157.07 & $1.02 \mathrm{E}+15$ & $9.36 \mathrm{E}+20$ & $14.00 \%$ \\
\hline 45 & -2.25 & 9480.48 & 156.52 & $1.02 \mathrm{E}+15$ & $9.48 \mathrm{E}+20$ & $14.16 \%$ \\
\hline 46 & -2.75 & 9521.76 & 157.20 & $1.02 \mathrm{E}+15$ & $9.44 \mathrm{E}+20$ & $14.16 \%$ \\
\hline 47 & -3.25 & 9570.19 & 158.00 & $1.02 \mathrm{E}+15$ & $9.36 \mathrm{E}+20$ & $14.00 \%$ \\
\hline 48 & -3.75 & 9715.91 & 160.41 & $1.02 \mathrm{E}+15$ & $9.37 \mathrm{E}+20$ & $14.00 \%$ \\
\hline 49 & -4.25 & 9589.38 & 158.32 & $1.01 \mathrm{E}+15$ & $9.44 \mathrm{E}+20$ & $14.16 \%$ \\
\hline 50 & -4.75 & 9531.86 & 157.37 & $1.01 \mathrm{E}+15$ & $9.32 \mathrm{E}+20$ & $13.92 \%$ \\
\hline 51 & -5.25 & 9645.32 & 159.24 & $1.02 \mathrm{E}+15$ & $9.34 \mathrm{E}+20$ & $14.00 \%$ \\
\hline 52 & -5.75 & 9407.04 & 155.31 & $1.00 \mathrm{E}+15$ & $9.26 \mathrm{E}+20$ & $13.92 \%$ \\
\hline
\end{tabular}




\begin{tabular}{|l|l|l|l|l|l|l|}
\hline 53 & -6.25 & 9437.10 & 155.81 & $9.98 \mathrm{E}+14$ & $9.18 \mathrm{E}+20$ & $13.76 \%$ \\
\hline 54 & -6.75 & 9578.32 & 158.14 & $1.01 \mathrm{E}+15$ & $9.12 \mathrm{E}+20$ & $13.69 \%$ \\
\hline 55 & -7.25 & 9376.35 & 154.80 & $9.94 \mathrm{E}+14$ & $9.03 \mathrm{E}+20$ & $13.53 \%$ \\
\hline 56 & -7.75 & 9335.65 & 154.13 & $9.97 \mathrm{E}+14$ & $9.00 \mathrm{E}+20$ & $13.53 \%$ \\
\hline 57 & -8.25 & 9309.62 & 153.70 & $9.84 \mathrm{E}+14$ & $8.88 \mathrm{E}+20$ & $13.30 \%$ \\
\hline 58 & -8.75 & 9135.14 & 150.82 & $9.80 \mathrm{E}+14$ & $8.82 \mathrm{E}+20$ & $13.22 \%$ \\
\hline 59 & -9.25 & 9088.08 & 150.04 & $9.75 \mathrm{E}+14$ & $8.62 \mathrm{E}+20$ & $12.99 \%$ \\
\hline 60 & -9.75 & 8985.86 & 148.36 & $9.63 \mathrm{E}+14$ & $8.47 \mathrm{E}+20$ & $12.76 \%$ \\
\hline 61 & -10.25 & 8843.40 & 146.00 & $9.54 \mathrm{E}+14$ & $8.15 \mathrm{E}+20$ & $12.29 \%$ \\
\hline 62 & -10.75 & 8664.01 & 143.04 & $9.47 \mathrm{E}+14$ & $8.04 \mathrm{E}+20$ & $12.13 \%$ \\
\hline 63 & -11.25 & 8658.83 & 142.96 & $9.39 \mathrm{E}+14$ & $7.93 \mathrm{E}+20$ & $11.98 \%$ \\
\hline 64 & -11.75 & 8515.65 & 140.59 & $9.25 \mathrm{E}+14$ & $7.74 \mathrm{E}+20$ & $11.67 \%$ \\
\hline 65 & -12.25 & 8469.24 & 139.83 & $9.14 \mathrm{E}+14$ & $7.74 \mathrm{E}+20$ & $11.67 \%$ \\
\hline 66 & -12.75 & 8386.24 & 138.46 & $8.98 \mathrm{E}+14$ & $7.77 \mathrm{E}+20$ & $11.67 \%$ \\
\hline 67 & -13.25 & 8274.10 & 136.61 & $8.90 \mathrm{E}+14$ & $7.63 \mathrm{E}+20$ & $11.51 \%$ \\
\hline 68 & -13.75 & 8124.72 & 134.14 & $8.73 \mathrm{E}+14$ & $7.38 \mathrm{E}+20$ & $11.13 \%$ \\
\hline 69 & -14.25 & 8271.32 & 136.56 & $8.61 \mathrm{E}+14$ & $7.37 \mathrm{E}+20$ & $11.13 \%$ \\
\hline 70 & -14.75 & 7860.97 & 129.78 & $8.43 \mathrm{E}+14$ & $7.16 \mathrm{E}+20$ & $10.82 \%$ \\
\hline 71 & -15.25 & 7790.10 & 128.61 & $8.25 \mathrm{E}+14$ & $6.88 \mathrm{E}+20$ & $10.42 \%$ \\
\hline 72 & -15.75 & 7627.26 & 125.93 & $8.04 \mathrm{E}+14$ & $6.77 \mathrm{E}+20$ & $10.26 \%$ \\
\hline 73 & -16.25 & 7375.18 & 121.76 & $7.82 \mathrm{E}+14$ & $6.63 \mathrm{E}+20$ & $10.03 \%$ \\
\hline 74 & -16.75 & 7271.42 & 120.05 & $7.63 \mathrm{E}+14$ & $6.39 \mathrm{E}+20$ & $9.72 \%$ \\
\hline 75 & -17.25 & 7000.90 & 115.58 & $7.39 \mathrm{E}+14$ & $6.04 \mathrm{E}+20$ & $9.18 \%$ \\
\hline 76 & -17.75 & 6541.81 & 108.01 & $7.11 \mathrm{E}+14$ & $5.77 \mathrm{E}+20$ & $8.79 \%$ \\
\hline 77 & -18.25 & 6562.94 & 108.35 & $6.96 \mathrm{E}+14$ & $5.59 \mathrm{E}+20$ & $8.48 \%$ \\
\hline 78 & -18.75 & 6292.60 & 103.89 & $6.52 \mathrm{E}+14$ & $5.34 \mathrm{E}+20$ & $8.17 \%$ \\
\hline
\end{tabular}


Table 19: Cycle 149B, MCNP-Calculated HGRs, Neutron Flux, Depletion and Fission Density for AFIP-7, Plate Position 3, 53.6 EFPD (EOC) Average Center Lobe Power at 24.2 MW. ${ }^{2}$

\begin{tabular}{|c|c|c|c|c|c|c|}
\hline Node & $\begin{array}{c}\text { Distance from } \\
\text { Centerline (in.) }\end{array}$ & $\begin{array}{l}\text { Fission Power } \\
\text { Density (W/cc) }\end{array}$ & $\begin{array}{l}\text { Surface Heat } \\
\text { Flux }\left(W / \mathrm{cm}^{2}\right)\end{array}$ & $\begin{array}{c}\text { Neutron Flux } \\
\left(\mathrm{n} / \mathrm{cm}^{2} \mathrm{sec}\right)\end{array}$ & $\begin{array}{l}\text { Fission Density } \\
\text { (fissions/cc) }\end{array}$ & $\begin{array}{c}\text { U-235 } \\
\text { Burnup (\%) }\end{array}$ \\
\hline 1 & 19.25 & 6010.43 & 99.23 & $5.53 \mathrm{E}+14$ & $8.93 \mathrm{E}+20$ & $13.45 \%$ \\
\hline 2 & 18.75 & 5534.61 & 91.38 & $5.80 \mathrm{E}+14$ & $8.17 \mathrm{E}+20$ & $12.37 \%$ \\
\hline 3 & 18.25 & 5635.28 & 93.04 & $6.11 \mathrm{E}+14$ & $8.48 \mathrm{E}+20$ & $12.83 \%$ \\
\hline 4 & 17.75 & 5797.34 & 95.71 & $6.33 \mathrm{E}+14$ & $8.86 \mathrm{E}+20$ & $13.38 \%$ \\
\hline 5 & 17.25 & 6179.30 & 102.02 & $6.68 \mathrm{E}+14$ & $9.39 \mathrm{E}+20$ & $14.16 \%$ \\
\hline 6 & 16.75 & 6445.54 & 106.42 & $6.97 \mathrm{E}+14$ & $9.70 \mathrm{E}+20$ & $14.63 \%$ \\
\hline 7 & 16.25 & 6669.61 & 110.12 & $7.21 \mathrm{E}+14$ & $1.02 \mathrm{E}+21$ & $15.33 \%$ \\
\hline 8 & 15.75 & 6798.93 & 112.25 & $7.42 \mathrm{E}+14$ & $1.04 \mathrm{E}+21$ & $15.71 \%$ \\
\hline 9 & 15.25 & 6964.31 & 114.98 & $7.58 \mathrm{E}+14$ & $1.08 \mathrm{E}+21$ & $16.26 \%$ \\
\hline 10 & 14.75 & 7138.34 & 117.85 & $7.86 \mathrm{E}+14$ & $1.11 \mathrm{E}+21$ & $16.65 \%$ \\
\hline 11 & 14.25 & 7317.31 & 120.81 & $7.99 \mathrm{E}+14$ & $1.14 \mathrm{E}+21$ & $17.11 \%$ \\
\hline 13 & 13.75 & 7321.24 & 120.87 & $8.27 \mathrm{E}+14$ & $1.17 \mathrm{E}+21$ & $17.50 \%$ \\
\hline 14 & 13.25 & 7547.07 & 124.60 & $8.43 \mathrm{E}+14$ & $1.18 \mathrm{E}+21$ & $17.67 \%$ \\
\hline 15 & 12.75 & 7759.05 & 128.10 & $8.66 \mathrm{E}+14$ & $1.21 \mathrm{E}+21$ & $18.05 \%$ \\
\hline 16 & 12.25 & 7723.53 & 127.52 & $8.90 \mathrm{E}+14$ & $1.21 \mathrm{E}+21$ & $18.13 \%$ \\
\hline 17 & 11.75 & 7861.26 & 129.79 & $9.01 \mathrm{E}+14$ & $1.25 \mathrm{E}+21$ & $18.67 \%$ \\
\hline 18 & 11.25 & 7964.07 & 131.49 & $9.10 \mathrm{E}+14$ & $1.26 \mathrm{E}+21$ & $18.83 \%$ \\
\hline 19 & 10.75 & 8105.54 & 133.82 & $9.19 \mathrm{E}+14$ & $1.30 \mathrm{E}+21$ & $19.37 \%$ \\
\hline 20 & 10.25 & 8242.37 & 136.08 & $9.38 \mathrm{E}+14$ & $1.32 \mathrm{E}+21$ & $19.68 \%$ \\
\hline 21 & 9.75 & 8366.54 & 138.13 & $9.44 \mathrm{E}+14$ & $1.37 \mathrm{E}+21$ & $20.38 \%$ \\
\hline 22 & 9.25 & 8577.54 & 141.62 & $9.58 \mathrm{E}+14$ & $1.39 \mathrm{E}+21$ & $20.61 \%$ \\
\hline 23 & 8.75 & 8584.87 & 141.74 & $9.70 \mathrm{E}+14$ & $1.41 \mathrm{E}+21$ & $20.85 \%$ \\
\hline 24 & 8.25 & 8601.90 & 142.02 & $9.74 \mathrm{E}+14$ & $1.42 \mathrm{E}+21$ & $21.08 \%$ \\
\hline 25 & 7.75 & 8774.12 & 144.86 & $9.79 \mathrm{E}+14$ & $1.44 \mathrm{E}+21$ & $21.32 \%$ \\
\hline 26 & 7.25 & 8906.51 & 147.05 & $9.89 \mathrm{E}+14$ & $1.47 \mathrm{E}+21$ & $21.79 \%$ \\
\hline 27 & 6.75 & 8874.87 & 146.52 & $9.91 \mathrm{E}+14$ & $1.48 \mathrm{E}+21$ & $21.94 \%$ \\
\hline 28 & 6.25 & 8891.28 & 146.79 & $9.96 \mathrm{E}+14$ & $1.48 \mathrm{E}+21$ & $21.94 \%$ \\
\hline 29 & 5.75 & 8942.75 & 147.64 & $1.01 \mathrm{E}+15$ & $1.49 \mathrm{E}+21$ & $22.02 \%$ \\
\hline 30 & 5.25 & 9055.15 & 149.50 & $1.01 \mathrm{E}+15$ & $1.52 \mathrm{E}+21$ & $22.42 \%$ \\
\hline 31 & 4.75 & 9091.26 & 150.10 & $1.02 \mathrm{E}+15$ & $1.51 \mathrm{E}+21$ & $22.33 \%$ \\
\hline 32 & 4.25 & 9137.45 & 150.86 & $1.02 \mathrm{E}+15$ & $1.53 \mathrm{E}+21$ & $22.50 \%$ \\
\hline 33 & 3.75 & 9130.49 & 150.74 & $1.02 \mathrm{E}+15$ & $1.53 \mathrm{E}+21$ & $22.58 \%$ \\
\hline 34 & 3.25 & 9211.01 & 152.07 & $1.03 \mathrm{E}+15$ & $1.53 \mathrm{E}+21$ & $22.60 \%$ \\
\hline 35 & 2.75 & 9119.85 & 150.57 & $1.02 \mathrm{E}+15$ & $1.54 \mathrm{E}+21$ & $22.72 \%$ \\
\hline 36 & 2.25 & 9117.12 & 150.52 & $1.02 \mathrm{E}+15$ & $1.52 \mathrm{E}+21$ & $22.45 \%$ \\
\hline 37 & 1.75 & 9165.92 & 151.33 & $1.03 \mathrm{E}+15$ & $1.53 \mathrm{E}+21$ & $22.55 \%$ \\
\hline 38 & 1.25 & 8933.29 & 147.49 & $1.03 \mathrm{E}+15$ & $1.49 \mathrm{E}+21$ & $22.02 \%$ \\
\hline 39 & 0.75 & 9021.88 & 148.95 & $1.04 \mathrm{E}+15$ & $1.51 \mathrm{E}+21$ & $22.28 \%$ \\
\hline 40 & 0.25 & 8987.66 & 148.39 & $1.04 \mathrm{E}+15$ & $1.51 \mathrm{E}+21$ & $22.37 \%$ \\
\hline 41 & -0.25 & 8962.53 & 147.97 & $1.03 \mathrm{E}+15$ & $1.51 \mathrm{E}+21$ & $22.29 \%$ \\
\hline 42 & -0.75 & 9117.93 & 150.54 & $1.04 \mathrm{E}+15$ & $1.52 \mathrm{E}+21$ & $22.50 \%$ \\
\hline 43 & -1.25 & 9258.14 & 152.85 & $1.04 \mathrm{E}+15$ & $1.56 \mathrm{E}+21$ & $22.93 \%$ \\
\hline 44 & -1.75 & 9115.89 & 150.50 & $1.03 \mathrm{E}+15$ & $1.54 \mathrm{E}+21$ & $22.65 \%$ \\
\hline 45 & -2.25 & 9146.46 & 151.01 & $1.04 \mathrm{E}+15$ & $1.55 \mathrm{E}+21$ & $22.81 \%$ \\
\hline 46 & -2.75 & 9177.38 & 151.52 & $1.03 \mathrm{E}+15$ & $1.55 \mathrm{E}+21$ & $22.81 \%$ \\
\hline 47 & -3.25 & 9189.12 & 151.71 & $1.03 \mathrm{E}+15$ & $1.54 \mathrm{E}+21$ & $22.74 \%$ \\
\hline 48 & -3.75 & 9318.23 & 153.84 & $1.03 \mathrm{E}+15$ & $1.55 \mathrm{E}+21$ & $22.86 \%$ \\
\hline 49 & -4.25 & 9258.10 & 152.85 & $1.03 \mathrm{E}+15$ & $1.55 \mathrm{E}+21$ & $22.86 \%$ \\
\hline 50 & -4.75 & 9134.96 & 150.82 & $1.02 \mathrm{E}+15$ & $1.53 \mathrm{E}+21$ & $22.65 \%$ \\
\hline 51 & -5.25 & 9292.28 & 153.42 & $1.03 \mathrm{E}+15$ & $1.54 \mathrm{E}+21$ & $22.78 \%$ \\
\hline 52 & -5.75 & 9103.21 & 150.29 & $1.02 \mathrm{E}+15$ & $1.52 \mathrm{E}+21$ & $22.50 \%$ \\
\hline
\end{tabular}




\begin{tabular}{|l|l|l|l|l|l|l|}
\hline 53 & -6.25 & 9050.79 & 149.43 & $1.01 \mathrm{E}+15$ & $1.51 \mathrm{E}+21$ & $22.38 \%$ \\
\hline 54 & -6.75 & 9159.72 & 151.23 & $1.02 \mathrm{E}+15$ & $1.52 \mathrm{E}+21$ & $22.38 \%$ \\
\hline 55 & -7.25 & 8998.84 & 148.57 & $1.01 \mathrm{E}+15$ & $1.50 \mathrm{E}+21$ & $22.10 \%$ \\
\hline 56 & -7.75 & 8966.23 & 148.03 & $1.01 \mathrm{E}+15$ & $1.49 \mathrm{E}+21$ & $22.02 \%$ \\
\hline 57 & -8.25 & 8928.15 & 147.40 & $9.94 \mathrm{E}+14$ & $1.48 \mathrm{E}+21$ & $21.87 \%$ \\
\hline 58 & -8.75 & 8843.03 & 146.00 & $9.94 \mathrm{E}+14$ & $1.46 \mathrm{E}+21$ & $21.56 \%$ \\
\hline 59 & -9.25 & 8813.11 & 145.50 & $9.87 \mathrm{E}+14$ & $1.44 \mathrm{E}+21$ & $21.32 \%$ \\
\hline 60 & -9.75 & 8698.65 & 143.61 & $9.75 \mathrm{E}+14$ & $1.42 \mathrm{E}+21$ & $21.00 \%$ \\
\hline 61 & -10.25 & 8532.42 & 140.87 & $9.67 \mathrm{E}+14$ & $1.37 \mathrm{E}+21$ & $20.46 \%$ \\
\hline 62 & -10.75 & 8395.21 & 138.60 & $9.60 \mathrm{E}+14$ & $1.35 \mathrm{E}+21$ & $20.07 \%$ \\
\hline 63 & -11.25 & 8411.02 & 138.87 & $9.50 \mathrm{E}+14$ & $1.34 \mathrm{E}+21$ & $19.91 \%$ \\
\hline 64 & -11.75 & 8280.96 & 136.72 & $9.37 \mathrm{E}+14$ & $1.31 \mathrm{E}+21$ & $19.53 \%$ \\
\hline 65 & -12.25 & 8195.51 & 135.31 & $9.26 \mathrm{E}+14$ & $1.31 \mathrm{E}+21$ & $19.53 \%$ \\
\hline 66 & -12.75 & 8137.28 & 134.35 & $9.11 \mathrm{E}+14$ & $1.31 \mathrm{E}+21$ & $19.45 \%$ \\
\hline 67 & -13.25 & 8031.07 & 132.59 & $9.03 \mathrm{E}+14$ & $1.29 \mathrm{E}+21$ & $19.22 \%$ \\
\hline 68 & -13.75 & 7869.44 & 129.92 & $8.86 \mathrm{E}+14$ & $1.25 \mathrm{E}+21$ & $18.67 \%$ \\
\hline 69 & -14.25 & 8044.65 & 132.82 & $8.74 \mathrm{E}+14$ & $1.26 \mathrm{E}+21$ & $18.83 \%$ \\
\hline 70 & -14.75 & 7685.43 & 126.89 & $8.56 \mathrm{E}+14$ & $1.21 \mathrm{E}+21$ & $18.13 \%$ \\
\hline 71 & -15.25 & 7600.17 & 125.48 & $8.38 \mathrm{E}+14$ & $1.18 \mathrm{E}+21$ & $17.67 \%$ \\
\hline 72 & -15.75 & 7441.80 & 122.86 & $8.15 \mathrm{E}+14$ & $1.16 \mathrm{E}+21$ & $17.34 \%$ \\
\hline 73 & -16.25 & 7210.71 & 119.05 & $7.92 \mathrm{E}+14$ & $1.13 \mathrm{E}+21$ & $16.96 \%$ \\
\hline 74 & -16.75 & 7134.20 & 117.79 & $7.76 \mathrm{E}+14$ & $1.10 \mathrm{E}+21$ & $16.49 \%$ \\
\hline 75 & -17.25 & 6794.69 & 112.18 & $7.49 \mathrm{E}+14$ & $1.05 \mathrm{E}+21$ & $15.71 \%$ \\
\hline 76 & -17.75 & 6420.80 & 106.01 & $7.22 \mathrm{E}+14$ & $9.90 \mathrm{E}+20$ & $14.86 \%$ \\
\hline 77 & -18.25 & 6465.36 & 106.74 & $7.08 \mathrm{E}+14$ & $9.74 \mathrm{E}+20$ & $14.71 \%$ \\
\hline 78 & -18.75 & 6203.67 & 102.42 & $6.65 \mathrm{E}+14$ & $9.32 \mathrm{E}+20$ & $14.09 \%$ \\
\hline
\end{tabular}


Table 20: Cycle 149B, MCNP-Calculated HGRs and Neutron Flux for AFIP-7, Plate Position 4, 0 EFPD (BOC) Average Center Lobe Power at 24.2 MW. ${ }^{2}$

\begin{tabular}{|c|c|c|c|c|}
\hline Node & $\begin{array}{l}\text { Distance from } \\
\text { Centerline (in.) }\end{array}$ & $\begin{array}{l}\text { Fission Power } \\
\text { Density (W/cc) }\end{array}$ & $\begin{array}{l}\text { Surface Heat } \\
\text { Flux }\left(\mathrm{W} / \mathrm{cm}^{2}\right)\end{array}$ & $\begin{array}{c}\text { Neutron Flux } \\
\left(\mathrm{n} / \mathrm{cm}^{2} \mathrm{sec}\right)\end{array}$ \\
\hline 1 & 19.25 & 6835.61 & 112.86 & $5.24 \mathrm{E}+14$ \\
\hline 2 & 18.75 & 6491.81 & 107.18 & $5.49 \mathrm{E}+14$ \\
\hline 3 & 18.25 & 6777.68 & 111.90 & $5.85 \mathrm{E}+14$ \\
\hline 4 & 17.75 & 7447.87 & 122.96 & $6.17 \mathrm{E}+14$ \\
\hline 5 & 17.25 & 7579.06 & 125.13 & $6.40 \mathrm{E}+14$ \\
\hline 6 & 16.75 & 8102.90 & 133.78 & $6.83 \mathrm{E}+14$ \\
\hline 7 & 16.25 & 8413.99 & 138.92 & $7.12 \mathrm{E}+14$ \\
\hline 8 & 15.75 & 8806.84 & 145.40 & $7.37 \mathrm{E}+14$ \\
\hline 9 & 15.25 & 9066.94 & 149.70 & $7.57 \mathrm{E}+14$ \\
\hline 10 & 14.75 & 9530.98 & 157.36 & $7.95 \mathrm{E}+14$ \\
\hline 11 & 14.25 & 9642.55 & 159.20 & $8.21 \mathrm{E}+14$ \\
\hline 13 & 13.75 & 9804.42 & 161.87 & $8.48 \mathrm{E}+14$ \\
\hline 14 & 13.25 & 10165.42 & 167.83 & $8.69 \mathrm{E}+14$ \\
\hline 15 & 12.75 & 10272.18 & 169.59 & $8.91 \mathrm{E}+14$ \\
\hline 16 & 12.25 & 10710.70 & 176.83 & $9.12 \mathrm{E}+14$ \\
\hline 17 & 11.75 & 10896.14 & 179.90 & $9.43 \mathrm{E}+14$ \\
\hline 18 & 11.25 & 11090.78 & 183.11 & $9.63 \mathrm{E}+14$ \\
\hline 19 & 10.75 & 11602.65 & 191.56 & $9.90 \mathrm{E}+14$ \\
\hline 20 & 10.25 & 12031.19 & 198.63 & $1.01 \mathrm{E}+15$ \\
\hline 21 & 9.75 & 12579.74 & 207.69 & $1.03 \mathrm{E}+15$ \\
\hline 22 & 9.25 & 12595.21 & 207.95 & $1.05 \mathrm{E}+15$ \\
\hline 23 & 8.75 & 12747.16 & 210.46 & $1.07 \mathrm{E}+15$ \\
\hline 24 & 8.25 & 13266.02 & 219.02 & $1.09 \mathrm{E}+15$ \\
\hline 25 & 7.75 & 13494.20 & 222.79 & $1.10 \mathrm{E}+15$ \\
\hline 26 & 7.25 & 13668.20 & 225.66 & $1.11 \mathrm{E}+15$ \\
\hline 27 & 6.75 & 13826.52 & 228.28 & $1.12 \mathrm{E}+15$ \\
\hline 28 & 6.25 & 14183.59 & 234.17 & $1.14 \mathrm{E}+15$ \\
\hline 29 & 5.75 & 14109.46 & 232.95 & $1.15 \mathrm{E}+15$ \\
\hline 30 & 5.25 & 14375.73 & 237.34 & $1.17 \mathrm{E}+15$ \\
\hline 31 & 4.75 & 14474.32 & 238.97 & $1.18 \mathrm{E}+15$ \\
\hline 32 & 4.25 & 14572.32 & 240.59 & $1.19 \mathrm{E}+15$ \\
\hline 33 & 3.75 & 14583.55 & 240.77 & $1.19 \mathrm{E}+15$ \\
\hline 34 & 3.25 & 14516.01 & 239.66 & $1.20 \mathrm{E}+15$ \\
\hline 35 & 2.75 & 14750.25 & 243.53 & $1.22 \mathrm{E}+15$ \\
\hline 36 & 2.25 & 14707.00 & 242.81 & $1.21 \mathrm{E}+15$ \\
\hline 37 & 1.75 & 14708.46 & 242.84 & $1.22 \mathrm{E}+15$ \\
\hline 38 & 1.25 & 14529.22 & 239.88 & $1.23 \mathrm{E}+15$ \\
\hline 39 & 0.75 & 14571.99 & 240.58 & $1.22 \mathrm{E}+15$ \\
\hline 40 & 0.25 & 14246.94 & 235.22 & $1.22 \mathrm{E}+15$ \\
\hline 41 & -0.25 & 14633.39 & 241.60 & $1.22 \mathrm{E}+15$ \\
\hline 42 & -0.75 & 14823.35 & 244.73 & $1.22 \mathrm{E}+15$ \\
\hline 43 & -1.25 & 14953.40 & 246.88 & $1.23 \mathrm{E}+15$ \\
\hline 44 & -1.75 & 15092.31 & 249.17 & $1.24 \mathrm{E}+15$ \\
\hline 45 & -2.25 & 15234.24 & 251.52 & $1.23 \mathrm{E}+15$ \\
\hline 46 & -2.75 & 14843.60 & 245.07 & $1.22 \mathrm{E}+15$ \\
\hline 47 & -3.25 & 14887.78 & 245.80 & $1.21 \mathrm{E}+15$ \\
\hline 48 & -3.75 & 14985.11 & 247.40 & $1.21 \mathrm{E}+15$ \\
\hline 49 & -4.25 & 15116.81 & 249.58 & $1.20 \mathrm{E}+15$ \\
\hline 50 & -4.75 & 15018.89 & 247.96 & $1.20 \mathrm{E}+15$ \\
\hline 51 & -5.25 & 14844.17 & 245.08 & $1.20 \mathrm{E}+15$ \\
\hline 52 & -5.75 & 14627.53 & 241.50 & $1.18 \mathrm{E}+15$ \\
\hline
\end{tabular}




\begin{tabular}{|l|l|l|l|l|}
\hline 53 & -6.25 & 14418.94 & 238.06 & $1.17 \mathrm{E}+15$ \\
\hline 54 & -6.75 & 14360.85 & 237.10 & $1.16 \mathrm{E}+15$ \\
\hline 55 & -7.25 & 14090.96 & 232.64 & $1.16 \mathrm{E}+15$ \\
\hline 56 & -7.75 & 13886.43 & 229.26 & $1.14 \mathrm{E}+15$ \\
\hline 57 & -8.25 & 14039.27 & 231.79 & $1.13 \mathrm{E}+15$ \\
\hline 58 & -8.75 & 13633.68 & 225.09 & $1.12 \mathrm{E}+15$ \\
\hline 59 & -9.25 & 13547.84 & 223.67 & $1.10 \mathrm{E}+15$ \\
\hline 60 & -9.75 & 13080.94 & 215.97 & $1.09 \mathrm{E}+15$ \\
\hline 61 & -10.25 & 12689.43 & 209.50 & $1.07 \mathrm{E}+15$ \\
\hline 62 & -10.75 & 12420.49 & 205.06 & $1.05 \mathrm{E}+15$ \\
\hline 63 & -11.25 & 12066.40 & 199.22 & $1.03 \mathrm{E}+15$ \\
\hline 64 & -11.75 & 11966.89 & 197.57 & $1.00 \mathrm{E}+15$ \\
\hline 65 & -12.25 & 11704.84 & 193.25 & $9.82 \mathrm{E}+14$ \\
\hline 66 & -12.75 & 11559.72 & 190.85 & $9.66 \mathrm{E}+14$ \\
\hline 67 & -13.25 & 11268.53 & 186.04 & $9.48 \mathrm{E}+14$ \\
\hline 68 & -13.75 & 10927.37 & 180.41 & $9.24 \mathrm{E}+14$ \\
\hline 69 & -14.25 & 10818.49 & 178.61 & $9.01 \mathrm{E}+14$ \\
\hline 70 & -14.75 & 10606.38 & 175.11 & $8.92 \mathrm{E}+14$ \\
\hline 71 & -15.25 & 10381.52 & 171.40 & $8.62 \mathrm{E}+14$ \\
\hline 72 & -15.75 & 9899.41 & 163.44 & $8.28 \mathrm{E}+14$ \\
\hline 73 & -16.25 & 9592.41 & 158.37 & $8.07 \mathrm{E}+14$ \\
\hline 74 & -16.75 & 9097.34 & 150.20 & $7.75 \mathrm{E}+14$ \\
\hline 75 & -17.25 & 8790.26 & 145.13 & $7.41 \mathrm{E}+14$ \\
\hline 76 & -17.75 & 8349.95 & 137.86 & $7.07 \mathrm{E}+14$ \\
\hline 77 & -18.25 & 8017.63 & 132.37 & $6.74 \mathrm{E}+14$ \\
\hline 78 & -18.75 & 7741.26 & 127.81 & $6.50 \mathrm{E}+14$ \\
\hline
\end{tabular}


Table 21: Cycle 149B, MCNP-Calculated HGRs, Neutron Flux, Depletion and Fission Density for AFIP-7, Plate Position 4, 15 EFPD (MOC1) Average Center Lobe Power at 24.2 MW. ${ }^{2}$

\begin{tabular}{|c|c|c|c|c|c|c|}
\hline Node & $\begin{array}{c}\text { Distance from } \\
\text { Centerline (in.) }\end{array}$ & $\begin{array}{l}\text { Fission Power } \\
\text { Density (W/cc) }\end{array}$ & $\begin{array}{l}\text { Surface Heat } \\
\text { Flux }\left(W / \mathrm{cm}^{2}\right)\end{array}$ & $\begin{array}{c}\text { Neutron Flux } \\
\left(\mathrm{n} / \mathrm{cm}^{2} \mathrm{sec}\right)\end{array}$ & $\begin{array}{l}\text { Fission Density } \\
\text { (fissions/cc) }\end{array}$ & $\begin{array}{c}\text { U-235 } \\
\text { Burnup (\%) }\end{array}$ \\
\hline 1 & 19.25 & 6127.93 & 101.17 & $5.01 \mathrm{E}+14$ & $2.67 \mathrm{E}+20$ & $3.91 \%$ \\
\hline 2 & 18.75 & 5749.70 & 94.93 & $5.20 \mathrm{E}+14$ & $2.54 \mathrm{E}+20$ & $3.69 \%$ \\
\hline 3 & 18.25 & 5973.31 & 98.62 & $5.45 \mathrm{E}+14$ & $2.65 \mathrm{E}+20$ & $3.91 \%$ \\
\hline 4 & 17.75 & 6123.66 & 101.10 & $5.80 \mathrm{E}+14$ & $2.91 \mathrm{E}+20$ & $4.29 \%$ \\
\hline 5 & 17.25 & 6402.60 & 105.71 & $6.07 \mathrm{E}+14$ & $2.96 \mathrm{E}+20$ & $4.36 \%$ \\
\hline 6 & 16.75 & 6579.27 & 108.62 & $6.29 \mathrm{E}+14$ & $3.17 \mathrm{E}+20$ & $4.66 \%$ \\
\hline 7 & 16.25 & 6921.69 & 114.28 & $6.56 \mathrm{E}+14$ & $3.29 \mathrm{E}+20$ & $4.81 \%$ \\
\hline 8 & 15.75 & 7089.73 & 117.05 & $6.76 \mathrm{E}+14$ & $3.44 \mathrm{E}+20$ & $5.04 \%$ \\
\hline 9 & 15.25 & 7416.91 & 122.45 & $7.10 \mathrm{E}+14$ & $3.54 \mathrm{E}+20$ & $5.19 \%$ \\
\hline 10 & 14.75 & 7614.82 & 125.72 & $7.24 \mathrm{E}+14$ & $3.72 \mathrm{E}+20$ & $5.41 \%$ \\
\hline 11 & 14.25 & 7681.98 & 126.83 & $7.43 \mathrm{E}+14$ & $3.77 \mathrm{E}+20$ & $5.49 \%$ \\
\hline 13 & 13.75 & 7917.05 & 130.71 & $7.71 \mathrm{E}+14$ & $3.83 \mathrm{E}+20$ & $5.56 \%$ \\
\hline 14 & 13.25 & 7913.26 & 130.65 & $7.84 \mathrm{E}+14$ & $3.97 \mathrm{E}+20$ & $5.79 \%$ \\
\hline 15 & 12.75 & 8056.50 & 133.01 & $8.06 \mathrm{E}+14$ & $4.01 \mathrm{E}+20$ & $5.86 \%$ \\
\hline 16 & 12.25 & 8292.66 & 136.91 & $8.22 \mathrm{E}+14$ & $4.18 \mathrm{E}+20$ & $6.09 \%$ \\
\hline 17 & 11.75 & 8414.41 & 138.92 & $8.32 \mathrm{E}+14$ & $4.26 \mathrm{E}+20$ & $6.16 \%$ \\
\hline 18 & 11.25 & 8647.27 & 142.77 & $8.48 \mathrm{E}+14$ & $4.33 \mathrm{E}+20$ & $6.31 \%$ \\
\hline 19 & 10.75 & 8770.61 & 144.80 & $8.67 \mathrm{E}+14$ & $4.53 \mathrm{E}+20$ & $6.63 \%$ \\
\hline 20 & 10.25 & 8883.08 & 146.66 & $8.70 \mathrm{E}+14$ & $4.70 \mathrm{E}+20$ & $6.85 \%$ \\
\hline 21 & 9.75 & 9199.55 & 151.88 & $8.98 \mathrm{E}+14$ & $4.91 \mathrm{E}+20$ & $7.08 \%$ \\
\hline 22 & 9.25 & 9339.00 & 154.19 & $9.05 \mathrm{E}+14$ & $4.92 \mathrm{E}+20$ & $7.15 \%$ \\
\hline 23 & 8.75 & 9409.52 & 155.35 & $9.09 \mathrm{E}+14$ & $4.98 \mathrm{E}+20$ & $7.23 \%$ \\
\hline 24 & 8.25 & 9469.45 & 156.34 & $9.14 \mathrm{E}+14$ & $5.18 \mathrm{E}+20$ & $7.53 \%$ \\
\hline 25 & 7.75 & 9665.65 & 159.58 & $9.27 \mathrm{E}+14$ & $5.27 \mathrm{E}+20$ & $7.60 \%$ \\
\hline 26 & 7.25 & 9682.72 & 159.86 & $9.33 \mathrm{E}+14$ & $5.34 \mathrm{E}+20$ & $7.75 \%$ \\
\hline 27 & 6.75 & 9764.85 & 161.22 & $9.44 \mathrm{E}+14$ & $5.40 \mathrm{E}+20$ & $7.75 \%$ \\
\hline 28 & 6.25 & 9673.93 & 159.72 & $9.46 \mathrm{E}+14$ & $5.54 \mathrm{E}+20$ & $7.98 \%$ \\
\hline 29 & 5.75 & 9644.84 & 159.24 & $9.68 \mathrm{E}+14$ & $5.51 \mathrm{E}+20$ & $7.98 \%$ \\
\hline 30 & 5.25 & 9823.04 & 162.18 & $9.65 \mathrm{E}+14$ & $5.62 \mathrm{E}+20$ & $8.13 \%$ \\
\hline 31 & 4.75 & 9974.64 & 164.68 & $9.73 \mathrm{E}+14$ & $5.65 \mathrm{E}+20$ & $8.13 \%$ \\
\hline 32 & 4.25 & 9931.87 & 163.98 & $9.75 \mathrm{E}+14$ & $5.69 \mathrm{E}+20$ & $8.20 \%$ \\
\hline 33 & 3.75 & 9879.64 & 163.11 & $9.74 \mathrm{E}+14$ & $5.70 \mathrm{E}+20$ & $8.20 \%$ \\
\hline 34 & 3.25 & 10093.79 & 166.65 & $9.88 \mathrm{E}+14$ & $5.67 \mathrm{E}+20$ & $8.20 \%$ \\
\hline 35 & 2.75 & 10165.99 & 167.84 & $9.94 \mathrm{E}+14$ & $5.76 \mathrm{E}+20$ & $8.28 \%$ \\
\hline 36 & 2.25 & 9847.80 & 162.59 & $9.88 \mathrm{E}+14$ & $5.75 \mathrm{E}+20$ & $8.28 \%$ \\
\hline 37 & 1.75 & 10086.43 & 166.53 & $9.99 \mathrm{E}+14$ & $5.75 \mathrm{E}+20$ & $8.28 \%$ \\
\hline 38 & 1.25 & 9865.74 & 162.88 & $9.94 \mathrm{E}+14$ & $5.68 \mathrm{E}+20$ & $8.20 \%$ \\
\hline 39 & 0.75 & 9821.49 & 162.15 & $9.96 \mathrm{E}+14$ & $5.69 \mathrm{E}+20$ & $8.20 \%$ \\
\hline 40 & 0.25 & 9977.75 & 164.73 & $9.99 \mathrm{E}+14$ & $5.57 \mathrm{E}+20$ & $8.05 \%$ \\
\hline 41 & -0.25 & 9898.82 & 163.43 & $9.93 \mathrm{E}+14$ & $5.72 \mathrm{E}+20$ & $8.20 \%$ \\
\hline 42 & -0.75 & 9910.87 & 163.63 & $9.93 \mathrm{E}+14$ & $5.79 \mathrm{E}+20$ & $8.35 \%$ \\
\hline 43 & -1.25 & 10006.51 & 165.21 & $9.97 \mathrm{E}+14$ & $5.84 \mathrm{E}+20$ & $8.43 \%$ \\
\hline 44 & -1.75 & 10060.05 & 166.09 & $9.92 \mathrm{E}+14$ & $5.90 \mathrm{E}+20$ & $8.50 \%$ \\
\hline 45 & -2.25 & 10118.69 & 167.06 & $9.92 \mathrm{E}+14$ & $5.95 \mathrm{E}+20$ & $8.58 \%$ \\
\hline 46 & -2.75 & 10120.59 & 167.09 & $9.88 \mathrm{E}+14$ & $5.80 \mathrm{E}+20$ & $8.35 \%$ \\
\hline 47 & -3.25 & 10056.58 & 166.03 & $9.96 \mathrm{E}+14$ & $5.82 \mathrm{E}+20$ & $8.35 \%$ \\
\hline 48 & -3.75 & 9912.53 & 163.66 & $9.83 \mathrm{E}+14$ & $5.85 \mathrm{E}+20$ & $8.43 \%$ \\
\hline 49 & -4.25 & 10085.10 & 166.50 & $9.88 \mathrm{E}+14$ & $5.91 \mathrm{E}+20$ & $8.50 \%$ \\
\hline 50 & -4.75 & 9926.79 & 163.89 & $9.79 \mathrm{E}+14$ & $5.87 \mathrm{E}+20$ & $8.43 \%$ \\
\hline 51 & -5.25 & 10029.26 & 165.58 & $9.79 \mathrm{E}+14$ & $5.80 \mathrm{E}+20$ & $8.35 \%$ \\
\hline 52 & -5.75 & 9945.50 & 164.20 & $9.68 \mathrm{E}+14$ & $5.71 \mathrm{E}+20$ & $8.20 \%$ \\
\hline
\end{tabular}




\begin{tabular}{|l|l|l|l|l|l|l|}
\hline 53 & -6.25 & 9994.93 & 165.02 & $9.66 \mathrm{E}+14$ & $5.63 \mathrm{E}+20$ & $8.13 \%$ \\
\hline 54 & -6.75 & 9836.37 & 162.40 & $9.73 \mathrm{E}+14$ & $5.61 \mathrm{E}+20$ & $8.05 \%$ \\
\hline 55 & -7.25 & 9790.22 & 161.64 & $9.54 \mathrm{E}+14$ & $5.50 \mathrm{E}+20$ & $7.90 \%$ \\
\hline 56 & -7.75 & 9681.08 & 159.83 & $9.52 \mathrm{E}+14$ & $5.42 \mathrm{E}+20$ & $7.83 \%$ \\
\hline 57 & -8.25 & 9709.76 & 160.31 & $9.46 \mathrm{E}+14$ & $5.48 \mathrm{E}+20$ & $7.90 \%$ \\
\hline 58 & -8.75 & 9679.68 & 159.81 & $9.39 \mathrm{E}+14$ & $5.33 \mathrm{E}+20$ & $7.68 \%$ \\
\hline 59 & -9.25 & 9527.72 & 157.30 & $9.39 \mathrm{E}+14$ & $5.29 \mathrm{E}+20$ & $7.60 \%$ \\
\hline 60 & -9.75 & 9351.67 & 154.40 & $9.22 \mathrm{E}+14$ & $5.11 \mathrm{E}+20$ & $7.38 \%$ \\
\hline 61 & -10.25 & 9244.21 & 152.62 & $9.22 \mathrm{E}+14$ & $4.96 \mathrm{E}+20$ & $7.15 \%$ \\
\hline 62 & -10.75 & 9050.89 & 149.43 & $9.08 \mathrm{E}+14$ & $4.85 \mathrm{E}+20$ & $7.08 \%$ \\
\hline 63 & -11.25 & 9075.25 & 149.83 & $8.96 \mathrm{E}+14$ & $4.71 \mathrm{E}+20$ & $6.85 \%$ \\
\hline 64 & -11.75 & 8774.21 & 144.86 & $8.77 \mathrm{E}+14$ & $4.68 \mathrm{E}+20$ & $6.78 \%$ \\
\hline 65 & -12.25 & 8516.11 & 140.60 & $8.55 \mathrm{E}+14$ & $4.57 \mathrm{E}+20$ & $6.63 \%$ \\
\hline 66 & -12.75 & 8688.55 & 143.45 & $8.48 \mathrm{E}+14$ & $4.52 \mathrm{E}+20$ & $6.54 \%$ \\
\hline 67 & -13.25 & 8584.08 & 141.72 & $8.37 \mathrm{E}+14$ & $4.40 \mathrm{E}+20$ & $6.39 \%$ \\
\hline 68 & -13.75 & 8533.09 & 140.88 & $8.25 \mathrm{E}+14$ & $4.27 \mathrm{E}+20$ & $6.24 \%$ \\
\hline 69 & -14.25 & 8448.61 & 139.49 & $8.15 \mathrm{E}+14$ & $4.23 \mathrm{E}+20$ & $6.16 \%$ \\
\hline 70 & -14.75 & 8222.27 & 135.75 & $7.90 \mathrm{E}+14$ & $4.14 \mathrm{E}+20$ & $6.01 \%$ \\
\hline 71 & -15.25 & 8079.46 & 133.39 & $7.79 \mathrm{E}+14$ & $4.06 \mathrm{E}+20$ & $5.94 \%$ \\
\hline 72 & -15.75 & 7797.67 & 128.74 & $7.51 \mathrm{E}+14$ & $3.87 \mathrm{E}+20$ & $5.64 \%$ \\
\hline 73 & -16.25 & 7619.78 & 125.80 & $7.25 \mathrm{E}+14$ & $3.75 \mathrm{E}+20$ & $5.49 \%$ \\
\hline 74 & -16.75 & 7396.15 & 122.11 & $7.07 \mathrm{E}+14$ & $3.55 \mathrm{E}+20$ & $5.19 \%$ \\
\hline 75 & -17.25 & 7298.49 & 120.50 & $6.91 \mathrm{E}+14$ & $3.43 \mathrm{E}+20$ & $5.04 \%$ \\
\hline 76 & -17.75 & 6846.43 & 113.03 & $6.60 \mathrm{E}+14$ & $3.26 \mathrm{E}+20$ & $4.74 \%$ \\
\hline 77 & -18.25 & 6772.99 & 111.82 & $6.36 \mathrm{E}+14$ & $3.13 \mathrm{E}+20$ & $4.59 \%$ \\
\hline 78 & -18.75 & 6457.48 & 106.61 & $6.04 \mathrm{E}+14$ & $3.02 \mathrm{E}+20$ & $4.44 \%$ \\
\hline
\end{tabular}


Table 22: Cycle 149B, MCNP-Calculated HGRs, Neutron Flux, Depletion and Fission Density for AFIP-7, Plate Position 4, 31 EFPD (MOC2) Average Center Lobe Power at 24.2 MW. ${ }^{2}$

\begin{tabular}{|c|c|c|c|c|c|c|}
\hline Node & $\begin{array}{c}\text { Distance from } \\
\text { Centerline (in.) }\end{array}$ & $\begin{array}{l}\text { Fission Power } \\
\text { Density (W/cc) }\end{array}$ & $\begin{array}{l}\text { Surface Heat } \\
\text { Flux }\left(W / \mathrm{cm}^{2}\right)\end{array}$ & $\begin{array}{c}\text { Neutron Flux } \\
\left(\mathrm{n} / \mathrm{cm}^{2} \mathrm{sec}\right)\end{array}$ & $\begin{array}{l}\text { Fission Density } \\
\text { (fissions/cc) }\end{array}$ & $\begin{array}{c}\text { U-235 } \\
\text { Burnup (\%) }\end{array}$ \\
\hline 1 & 19.25 & 6541.11 & 107.99 & $5.42 \mathrm{E}+14$ & $5.45 \mathrm{E}+20$ & $7.98 \%$ \\
\hline 2 & 18.75 & 6143.94 & 101.44 & $5.75 \mathrm{E}+14$ & $5.14 \mathrm{E}+20$ & $7.53 \%$ \\
\hline 3 & 18.25 & 6297.53 & 103.97 & $6.02 \mathrm{E}+14$ & $5.35 \mathrm{E}+20$ & $7.90 \%$ \\
\hline 4 & 17.75 & 6612.74 & 109.18 & $6.26 \mathrm{E}+14$ & $5.68 \mathrm{E}+20$ & $8.35 \%$ \\
\hline 5 & 17.25 & 7006.62 & 115.68 & $6.61 \mathrm{E}+14$ & $5.86 \mathrm{E}+20$ & $8.65 \%$ \\
\hline 6 & 16.75 & 7291.44 & 120.38 & $6.79 E+14$ & $6.15 \mathrm{E}+20$ & $9.03 \%$ \\
\hline 7 & 16.25 & 7505.64 & 123.92 & $7.08 \mathrm{E}+14$ & $6.42 \mathrm{E}+20$ & $9.40 \%$ \\
\hline 8 & 15.75 & 7781.90 & 128.48 & $7.29 \mathrm{E}+14$ & $6.65 \mathrm{E}+20$ & $9.78 \%$ \\
\hline 9 & 15.25 & 7947.63 & 131.22 & $7.57 \mathrm{E}+14$ & $6.90 \mathrm{E}+20$ & $10.09 \%$ \\
\hline 10 & 14.75 & 8190.32 & 135.22 & $7.78 \mathrm{E}+14$ & $7.17 \mathrm{E}+20$ & $10.47 \%$ \\
\hline 11 & 14.25 & 8266.63 & 136.48 & $7.96 \mathrm{E}+14$ & $7.25 \mathrm{E}+20$ & $10.62 \%$ \\
\hline 13 & 13.75 & 8467.87 & 139.80 & $8.14 \mathrm{E}+14$ & $7.42 \mathrm{E}+20$ & $10.84 \%$ \\
\hline 14 & 13.25 & 8477.36 & 139.96 & $8.33 \mathrm{E}+14$ & $7.56 \mathrm{E}+20$ & $11.07 \%$ \\
\hline 15 & 12.75 & 8464.75 & 139.75 & $8.58 \mathrm{E}+14$ & $7.66 \mathrm{E}+20$ & $11.22 \%$ \\
\hline 16 & 12.25 & 9002.75 & 148.64 & $8.75 \mathrm{E}+14$ & $7.94 \mathrm{E}+20$ & $11.59 \%$ \\
\hline 17 & 11.75 & 8778.47 & 144.93 & $8.84 \mathrm{E}+14$ & $8.07 \mathrm{E}+20$ & $11.74 \%$ \\
\hline 18 & 11.25 & 9231.76 & 152.42 & $9.02 \mathrm{E}+14$ & $8.25 \mathrm{E}+20$ & $12.04 \%$ \\
\hline 19 & 10.75 & 9173.04 & 151.45 & $9.16 \mathrm{E}+14$ & $8.50 \mathrm{E}+20$ & $12.34 \%$ \\
\hline 20 & 10.25 & 9459.43 & 156.18 & $9.20 \mathrm{E}+14$ & $8.72 \mathrm{E}+20$ & $12.64 \%$ \\
\hline 21 & 9.75 & 9785.44 & 161.56 & $9.44 \mathrm{E}+14$ & $9.08 \mathrm{E}+20$ & $13.17 \%$ \\
\hline 22 & 9.25 & 9898.40 & 163.42 & $9.39 \mathrm{E}+14$ & $9.15 \mathrm{E}+20$ & $13.24 \%$ \\
\hline 23 & 8.75 & 10025.02 & 165.51 & $9.59 \mathrm{E}+14$ & $9.24 \mathrm{E}+20$ & $13.39 \%$ \\
\hline 24 & 8.25 & 10132.97 & 167.30 & $9.64 \mathrm{E}+14$ & $9.47 \mathrm{E}+20$ & $13.71 \%$ \\
\hline 25 & 7.75 & 10042.79 & 165.81 & $9.66 \mathrm{E}+14$ & $9.65 \mathrm{E}+20$ & $13.93 \%$ \\
\hline 26 & 7.25 & 10283.61 & 169.78 & $9.75 \mathrm{E}+14$ & $9.72 \mathrm{E}+20$ & $14.08 \%$ \\
\hline 27 & 6.75 & 10302.68 & 170.10 & $9.76 \mathrm{E}+14$ & $9.82 \mathrm{E}+20$ & $14.16 \%$ \\
\hline 28 & 6.25 & 10296.53 & 170.00 & $9.83 \mathrm{E}+14$ & $9.92 \mathrm{E}+20$ & $14.31 \%$ \\
\hline 29 & 5.75 & 10338.15 & 170.68 & $1.01 \mathrm{E}+15$ & $9.88 \mathrm{E}+20$ & $14.23 \%$ \\
\hline 30 & 5.25 & 10553.17 & 174.23 & $1.01 \mathrm{E}+15$ & $1.01 \mathrm{E}+21$ & $14.53 \%$ \\
\hline 31 & 4.75 & 10498.54 & 173.33 & $1.00 \mathrm{E}+15$ & $1.02 \mathrm{E}+21$ & $14.68 \%$ \\
\hline 32 & 4.25 & 10601.35 & 175.03 & $1.00 \mathrm{E}+15$ & $1.02 \mathrm{E}+21$ & $14.68 \%$ \\
\hline 33 & 3.75 & 10526.91 & 173.80 & $1.00 \mathrm{E}+15$ & $1.02 \mathrm{E}+21$ & $14.68 \%$ \\
\hline 34 & 3.25 & 10690.47 & 176.50 & $1.03 \mathrm{E}+15$ & $1.02 \mathrm{E}+21$ & $14.76 \%$ \\
\hline 35 & 2.75 & 10713.11 & 176.87 & $1.03 \mathrm{E}+15$ & $1.04 \mathrm{E}+21$ & $14.91 \%$ \\
\hline 36 & 2.25 & 10484.43 & 173.10 & $1.02 \mathrm{E}+15$ & $1.02 \mathrm{E}+21$ & $14.68 \%$ \\
\hline 37 & 1.75 & 10505.22 & 173.44 & $1.03 \mathrm{E}+15$ & $1.03 \mathrm{E}+21$ & $14.91 \%$ \\
\hline 38 & 1.25 & 10378.59 & 171.35 & $1.03 \mathrm{E}+15$ & $1.01 \mathrm{E}+21$ & $14.61 \%$ \\
\hline 39 & 0.75 & 10471.67 & 172.89 & $1.03 \mathrm{E}+15$ & $1.01 \mathrm{E}+21$ & $14.61 \%$ \\
\hline 40 & 0.25 & 10597.87 & 174.97 & $1.04 \mathrm{E}+15$ & $1.01 \mathrm{E}+21$ & $14.53 \%$ \\
\hline 41 & -0.25 & 10506.88 & 173.47 & $1.03 \mathrm{E}+15$ & $1.02 \mathrm{E}+21$ & $14.68 \%$ \\
\hline 42 & -0.75 & 10761.30 & 177.67 & $1.04 \mathrm{E}+15$ & $1.03 \mathrm{E}+21$ & $14.83 \%$ \\
\hline 43 & -1.25 & 10666.88 & 176.11 & $1.04 \mathrm{E}+15$ & $1.04 \mathrm{E}+21$ & $14.98 \%$ \\
\hline 44 & -1.75 & 10720.88 & 177.00 & $1.03 \mathrm{E}+15$ & $1.05 \mathrm{E}+21$ & $15.06 \%$ \\
\hline 45 & -2.25 & 10597.89 & 174.97 & $1.03 \mathrm{E}+15$ & $1.05 \mathrm{E}+21$ & $15.13 \%$ \\
\hline 46 & -2.75 & 10767.81 & 177.78 & $1.03 \mathrm{E}+15$ & $1.04 \mathrm{E}+21$ & $14.91 \%$ \\
\hline 47 & -3.25 & 10709.56 & 176.81 & $1.03 \mathrm{E}+15$ & $1.04 \mathrm{E}+21$ & $14.91 \%$ \\
\hline 48 & -3.75 & 10503.41 & 173.41 & $1.02 \mathrm{E}+15$ & $1.03 \mathrm{E}+21$ & $14.91 \%$ \\
\hline 49 & -4.25 & 10730.28 & 177.16 & $1.02 \mathrm{E}+15$ & $1.05 \mathrm{E}+21$ & $15.06 \%$ \\
\hline 50 & -4.75 & 10640.09 & 175.67 & $1.02 \mathrm{E}+15$ & $1.04 \mathrm{E}+21$ & $14.91 \%$ \\
\hline 51 & -5.25 & 10584.01 & 174.74 & $1.02 \mathrm{E}+15$ & $1.03 \mathrm{E}+21$ & $14.91 \%$ \\
\hline 52 & -5.75 & 10488.61 & 173.17 & $1.00 \mathrm{E}+15$ & $1.02 \mathrm{E}+21$ & $14.76 \%$ \\
\hline
\end{tabular}




\begin{tabular}{|l|l|l|l|l|l|l|}
\hline 53 & -6.25 & 10542.15 & 174.05 & $1.01 \mathrm{E}+15$ & $1.02 \mathrm{E}+21$ & $14.61 \%$ \\
\hline 54 & -6.75 & 10582.88 & 174.72 & $1.01 \mathrm{E}+15$ & $1.01 \mathrm{E}+21$ & $14.53 \%$ \\
\hline 55 & -7.25 & 10426.06 & 172.13 & $9.90 \mathrm{E}+14$ & $9.94 \mathrm{E}+20$ & $14.31 \%$ \\
\hline 56 & -7.75 & 10191.04 & 168.25 & $9.84 \mathrm{E}+14$ & $9.81 \mathrm{E}+20$ & $14.16 \%$ \\
\hline 57 & -8.25 & 10273.28 & 169.61 & $9.84 \mathrm{E}+14$ & $9.88 \mathrm{E}+20$ & $14.23 \%$ \\
\hline 58 & -8.75 & 10320.84 & 170.40 & $9.84 \mathrm{E}+14$ & $9.71 \mathrm{E}+20$ & $14.01 \%$ \\
\hline 59 & -9.25 & 10207.60 & 168.53 & $9.82 \mathrm{E}+14$ & $9.61 \mathrm{E}+20$ & $13.86 \%$ \\
\hline 60 & -9.75 & 9852.61 & 162.67 & $9.68 \mathrm{E}+14$ & $9.35 \mathrm{E}+20$ & $13.56 \%$ \\
\hline 61 & -10.25 & 9903.36 & 163.50 & $9.63 \mathrm{E}+14$ & $9.14 \mathrm{E}+20$ & $13.24 \%$ \\
\hline 62 & -10.75 & 9705.95 & 160.25 & $9.48 \mathrm{E}+14$ & $8.95 \mathrm{E}+20$ & $13.02 \%$ \\
\hline 63 & -11.25 & 9614.25 & 158.73 & $9.44 \mathrm{E}+14$ & $8.82 \mathrm{E}+20$ & $12.79 \%$ \\
\hline 64 & -11.75 & 9350.38 & 154.37 & $9.29 \mathrm{E}+14$ & $8.65 \mathrm{E}+20$ & $12.57 \%$ \\
\hline 65 & -12.25 & 9220.77 & 152.23 & $9.10 \mathrm{E}+14$ & $8.43 \mathrm{E}+20$ & $12.27 \%$ \\
\hline 66 & -12.75 & 9183.77 & 151.62 & $9.05 \mathrm{E}+14$ & $8.45 \mathrm{E}+20$ & $12.27 \%$ \\
\hline 67 & -13.25 & 9184.37 & 151.63 & $8.96 \mathrm{E}+14$ & $8.29 \mathrm{E}+20$ & $12.04 \%$ \\
\hline 68 & -13.75 & 9159.11 & 151.22 & $8.73 \mathrm{E}+14$ & $8.13 \mathrm{E}+20$ & $11.82 \%$ \\
\hline 69 & -14.25 & 8883.25 & 146.66 & $8.62 \mathrm{E}+14$ & $8.05 \mathrm{E}+20$ & $11.74 \%$ \\
\hline 70 & -14.75 & 8733.10 & 144.18 & $8.49 \mathrm{E}+14$ & $7.87 \mathrm{E}+20$ & $11.44 \%$ \\
\hline 71 & -15.25 & 8755.35 & 144.55 & $8.31 \mathrm{E}+14$ & $7.71 \mathrm{E}+20$ & $11.22 \%$ \\
\hline 72 & -15.75 & 8582.08 & 141.69 & $8.08 \mathrm{E}+14$ & $7.40 \mathrm{E}+20$ & $10.77 \%$ \\
\hline 73 & -16.25 & 8148.02 & 134.52 & $7.78 \mathrm{E}+14$ & $7.20 \mathrm{E}+20$ & $10.54 \%$ \\
\hline 74 & -16.75 & 7851.67 & 129.63 & $7.60 \mathrm{E}+14$ & $6.90 \mathrm{E}+20$ & $10.09 \%$ \\
\hline 75 & -17.25 & 7731.03 & 127.64 & $7.43 \mathrm{E}+14$ & $6.74 \mathrm{E}+20$ & $9.85 \%$ \\
\hline 76 & -17.75 & 7328.65 & 121.00 & $7.13 \mathrm{E}+14$ & $6.36 \mathrm{E}+20$ & $9.33 \%$ \\
\hline 77 & -18.25 & 7308.75 & 120.67 & $6.87 \mathrm{E}+14$ & $6.20 \mathrm{E}+20$ & $9.10 \%$ \\
\hline 78 & -18.75 & 6949.30 & 114.73 & $6.55 \mathrm{E}+14$ & $5.95 \mathrm{E}+20$ & $8.73 \%$ \\
\hline
\end{tabular}


Table 23: Cycle 149B, MCNP-Calculated HGRs, Neutron Flux, Depletion and Fission Density for AFIP-7, Plate Position 4, 53.6 EFPD (EOC) Average Center Lobe Power at 24.2 MW. ${ }^{2}$

\begin{tabular}{|c|c|c|c|c|c|c|}
\hline Node & $\begin{array}{c}\text { Distance from } \\
\text { Centerline (in.) }\end{array}$ & $\begin{array}{l}\text { Fission Power } \\
\text { Density (W/cc) }\end{array}$ & $\begin{array}{l}\text { Surface Heat } \\
\text { Flux }\left(W / \mathrm{cm}^{2}\right)\end{array}$ & $\begin{array}{c}\text { Neutron Flux } \\
\left(\mathrm{n} / \mathrm{cm}^{2} \mathrm{sec}\right)\end{array}$ & $\begin{array}{l}\text { Fission Density } \\
\text { (fissions/cc) }\end{array}$ & $\begin{array}{c}\text { U-235 } \\
\text { Burnup (\%) }\end{array}$ \\
\hline 1 & 19.25 & 6420.54 & 106.00 & $5.53 \mathrm{E}+14$ & $9.58 \mathrm{E}+20$ & $13.93 \%$ \\
\hline 2 & 18.75 & 6074.95 & 100.30 & $5.85 \mathrm{E}+14$ & $9.03 \mathrm{E}+20$ & $13.17 \%$ \\
\hline 3 & 18.25 & 6195.86 & 102.29 & $6.12 \mathrm{E}+14$ & $9.34 \mathrm{E}+20$ & $13.63 \%$ \\
\hline 4 & 17.75 & 6492.12 & 107.18 & $6.37 \mathrm{E}+14$ & $9.86 \mathrm{E}+20$ & $14.38 \%$ \\
\hline 5 & 17.25 & 6826.39 & 112.70 & $6.71 \mathrm{E}+14$ & $1.03 \mathrm{E}+21$ & $14.98 \%$ \\
\hline 6 & 16.75 & 7102.76 & 117.27 & $6.90 \mathrm{E}+14$ & $1.08 \mathrm{E}+21$ & $15.66 \%$ \\
\hline 7 & 16.25 & 7321.24 & 120.87 & $7.18 \mathrm{E}+14$ & $1.12 \mathrm{E}+21$ & $16.18 \%$ \\
\hline 8 & 15.75 & 7566.23 & 124.92 & $7.39 \mathrm{E}+14$ & $1.16 \mathrm{E}+21$ & $16.78 \%$ \\
\hline 9 & 15.25 & 7698.40 & 127.10 & $7.68 \mathrm{E}+14$ & $1.19 \mathrm{E}+21$ & $17.24 \%$ \\
\hline 10 & 14.75 & 7946.93 & 131.20 & $7.91 \mathrm{E}+14$ & $1.23 \mathrm{E}+21$ & $17.84 \%$ \\
\hline 11 & 14.25 & 8047.89 & 132.87 & $8.09 \mathrm{E}+14$ & $1.25 \mathrm{E}+21$ & $17.99 \%$ \\
\hline 13 & 13.75 & 8220.02 & 135.71 & $8.26 \mathrm{E}+14$ & $1.28 \mathrm{E}+21$ & $18.37 \%$ \\
\hline 14 & 13.25 & 8226.31 & 135.82 & $8.45 \mathrm{E}+14$ & $1.29 \mathrm{E}+21$ & $18.59 \%$ \\
\hline 15 & 12.75 & 8214.28 & 135.62 & $8.71 \mathrm{E}+14$ & $1.30 \mathrm{E}+21$ & $18.74 \%$ \\
\hline 16 & 12.25 & 8650.69 & 142.82 & $8.86 \mathrm{E}+14$ & $1.36 \mathrm{E}+21$ & $19.57 \%$ \\
\hline 17 & 11.75 & 8512.65 & 140.54 & $8.96 \mathrm{E}+14$ & $1.36 \mathrm{E}+21$ & $19.57 \%$ \\
\hline 18 & 11.25 & 8907.56 & 147.06 & $9.14 \mathrm{E}+14$ & $1.41 \mathrm{E}+21$ & $20.26 \%$ \\
\hline 19 & 10.75 & 8895.12 & 146.86 & $9.29 \mathrm{E}+14$ & $1.43 \mathrm{E}+21$ & $20.48 \%$ \\
\hline 20 & 10.25 & 9119.54 & 150.56 & $9.34 \mathrm{E}+14$ & $1.47 \mathrm{E}+21$ & $21.08 \%$ \\
\hline 21 & 9.75 & 9378.35 & 154.84 & $9.58 \mathrm{E}+14$ & $1.53 \mathrm{E}+21$ & $21.76 \%$ \\
\hline 22 & 9.25 & 9507.61 & 156.97 & $9.54 \mathrm{E}+14$ & $1.54 \mathrm{E}+21$ & $21.98 \%$ \\
\hline 23 & 8.75 & 9666.83 & 159.60 & $9.70 \mathrm{E}+14$ & $1.56 \mathrm{E}+21$ & $22.21 \%$ \\
\hline 24 & 8.25 & 9712.16 & 160.35 & $9.78 \mathrm{E}+14$ & $1.59 \mathrm{E}+21$ & $22.58 \%$ \\
\hline 25 & 7.75 & 9666.55 & 159.59 & $9.79 \mathrm{E}+14$ & $1.60 \mathrm{E}+21$ & $22.73 \%$ \\
\hline 26 & 7.25 & 9866.32 & 162.89 & $9.90 \mathrm{E}+14$ & $1.62 \mathrm{E}+21$ & $23.11 \%$ \\
\hline 27 & 6.75 & 9893.96 & 163.35 & $9.91 \mathrm{E}+14$ & $1.63 \mathrm{E}+21$ & $23.18 \%$ \\
\hline 28 & 6.25 & 9853.95 & 162.69 & $9.97 \mathrm{E}+14$ & $1.64 \mathrm{E}+21$ & $23.33 \%$ \\
\hline 29 & 5.75 & 9900.28 & 163.45 & $1.02 \mathrm{E}+15$ & $1.64 \mathrm{E}+21$ & $23.33 \%$ \\
\hline 30 & 5.25 & 10113.53 & 166.97 & $1.02 \mathrm{E}+15$ & $1.67 \mathrm{E}+21$ & $23.80 \%$ \\
\hline 31 & 4.75 & 10065.92 & 166.19 & $1.02 \mathrm{E}+15$ & $1.68 \mathrm{E}+21$ & $23.80 \%$ \\
\hline 32 & 4.25 & 10115.61 & 167.01 & $1.01 \mathrm{E}+15$ & $1.69 \mathrm{E}+21$ & $23.95 \%$ \\
\hline 33 & 3.75 & 10070.35 & 166.26 & $1.02 \mathrm{E}+15$ & $1.68 \mathrm{E}+21$ & $23.87 \%$ \\
\hline 34 & 3.25 & 10206.31 & 168.51 & $1.04 \mathrm{E}+15$ & $1.70 \mathrm{E}+21$ & $24.10 \%$ \\
\hline 35 & 2.75 & 10259.03 & 169.38 & $1.04 \mathrm{E}+15$ & $1.71 \mathrm{E}+21$ & $24.25 \%$ \\
\hline 36 & 2.25 & 9986.52 & 164.88 & $1.04 \mathrm{E}+15$ & $1.68 \mathrm{E}+21$ & $23.87 \%$ \\
\hline 37 & 1.75 & 10038.77 & 165.74 & $1.04 \mathrm{E}+15$ & $1.69 \mathrm{E}+21$ & $24.10 \%$ \\
\hline 38 & 1.25 & 9989.53 & 164.93 & $1.04 \mathrm{E}+15$ & $1.67 \mathrm{E}+21$ & $23.72 \%$ \\
\hline 39 & 0.75 & 10001.90 & 165.13 & $1.04 \mathrm{E}+15$ & $1.68 \mathrm{E}+21$ & $23.80 \%$ \\
\hline 40 & 0.25 & 10112.53 & 166.96 & $1.05 \mathrm{E}+15$ & $1.68 \mathrm{E}+21$ & $23.80 \%$ \\
\hline 41 & -0.25 & 10060.51 & 166.10 & $1.04 \mathrm{E}+15$ & $1.68 \mathrm{E}+21$ & $23.87 \%$ \\
\hline 42 & -0.75 & 10219.38 & 168.72 & $1.05 \mathrm{E}+15$ & $1.71 \mathrm{E}+21$ & $24.17 \%$ \\
\hline 43 & -1.25 & 10198.69 & 168.38 & $1.05 \mathrm{E}+15$ & $1.71 \mathrm{E}+21$ & $24.25 \%$ \\
\hline 44 & -1.75 & 10245.08 & 169.15 & $1.05 \mathrm{E}+15$ & $1.72 \mathrm{E}+21$ & $24.40 \%$ \\
\hline 45 & -2.25 & 10139.71 & 167.41 & $1.04 \mathrm{E}+15$ & $1.72 \mathrm{E}+21$ & $24.40 \%$ \\
\hline 46 & -2.75 & 10268.35 & 169.53 & $1.04 \mathrm{E}+15$ & $1.72 \mathrm{E}+21$ & $24.32 \%$ \\
\hline 47 & -3.25 & 10252.44 & 169.27 & $1.04 \mathrm{E}+15$ & $1.71 \mathrm{E}+21$ & $24.25 \%$ \\
\hline 48 & -3.75 & 10036.56 & 165.70 & $1.03 \mathrm{E}+15$ & $1.70 \mathrm{E}+21$ & $24.10 \%$ \\
\hline 49 & -4.25 & 10236.57 & 169.01 & $1.03 \mathrm{E}+15$ & $1.73 \mathrm{E}+21$ & $24.40 \%$ \\
\hline 50 & -4.75 & 10161.93 & 167.77 & $1.03 \mathrm{E}+15$ & $1.71 \mathrm{E}+21$ & $24.17 \%$ \\
\hline 51 & -5.25 & 10128.53 & 167.22 & $1.03 \mathrm{E}+15$ & $1.70 \mathrm{E}+21$ & $24.17 \%$ \\
\hline 52 & -5.75 & 9998.55 & 165.08 & $1.01 \mathrm{E}+15$ & $1.68 \mathrm{E}+21$ & $23.95 \%$ \\
\hline
\end{tabular}




\begin{tabular}{|l|l|l|l|l|l|l|}
\hline 53 & -6.25 & 10095.38 & 166.67 & $1.02 \mathrm{E}+15$ & $1.68 \mathrm{E}+21$ & $23.87 \%$ \\
\hline 54 & -6.75 & 10096.70 & 166.70 & $1.02 \mathrm{E}+15$ & $1.68 \mathrm{E}+21$ & $23.80 \%$ \\
\hline 55 & -7.25 & 9949.89 & 164.27 & $1.00 \mathrm{E}+15$ & $1.65 \mathrm{E}+21$ & $23.41 \%$ \\
\hline 56 & -7.75 & 9777.20 & 161.42 & $9.99 \mathrm{E}+14$ & $1.62 \mathrm{E}+21$ & $23.11 \%$ \\
\hline 57 & -8.25 & 9868.22 & 162.92 & $9.98 \mathrm{E}+14$ & $1.64 \mathrm{E}+21$ & $23.26 \%$ \\
\hline 58 & -8.75 & 9913.62 & 163.67 & $9.99 \mathrm{E}+14$ & $1.62 \mathrm{E}+21$ & $23.03 \%$ \\
\hline 59 & -9.25 & 9749.94 & 160.97 & $9.95 \mathrm{E}+14$ & $1.61 \mathrm{E}+21$ & $22.81 \%$ \\
\hline 60 & -9.75 & 9519.65 & 157.17 & $9.82 \mathrm{E}+14$ & $1.56 \mathrm{E}+21$ & $22.21 \%$ \\
\hline 61 & -10.25 & 9494.13 & 156.75 & $9.79 \mathrm{E}+14$ & $1.54 \mathrm{E}+21$ & $21.98 \%$ \\
\hline 62 & -10.75 & 9339.15 & 154.19 & $9.64 \mathrm{E}+14$ & $1.51 \mathrm{E}+21$ & $21.53 \%$ \\
\hline 63 & -11.25 & 9251.94 & 152.75 & $9.57 \mathrm{E}+14$ & $1.49 \mathrm{E}+21$ & $21.31 \%$ \\
\hline 64 & -11.75 & 9011.37 & 148.78 & $9.40 \mathrm{E}+14$ & $1.46 \mathrm{E}+21$ & $20.86 \%$ \\
\hline 65 & -12.25 & 8890.76 & 146.79 & $9.23 \mathrm{E}+14$ & $1.43 \mathrm{E}+21$ & $20.41 \%$ \\
\hline 66 & -12.75 & 8873.53 & 146.50 & $9.19 \mathrm{E}+14$ & $1.43 \mathrm{E}+21$ & $20.41 \%$ \\
\hline 67 & -13.25 & 8823.37 & 145.67 & $9.09 \mathrm{E}+14$ & $1.41 \mathrm{E}+21$ & $20.26 \%$ \\
\hline 68 & -13.75 & 8868.26 & 146.41 & $8.86 \mathrm{E}+14$ & $1.39 \mathrm{E}+21$ & $19.94 \%$ \\
\hline 69 & -14.25 & 8606.51 & 142.09 & $8.78 \mathrm{E}+14$ & $1.37 \mathrm{E}+21$ & $19.64 \%$ \\
\hline 70 & -14.75 & 8456.66 & 139.62 & $8.62 \mathrm{E}+14$ & $1.34 \mathrm{E}+21$ & $19.27 \%$ \\
\hline 71 & -15.25 & 8432.92 & 139.23 & $8.44 \mathrm{E}+14$ & $1.32 \mathrm{E}+21$ & $19.04 \%$ \\
\hline 72 & -15.75 & 8295.41 & 136.96 & $8.19 \mathrm{E}+14$ & $1.28 \mathrm{E}+21$ & $18.44 \%$ \\
\hline 73 & -16.25 & 7925.03 & 130.84 & $7.89 \mathrm{E}+14$ & $1.23 \mathrm{E}+21$ & $17.77 \%$ \\
\hline 74 & -16.75 & 7671.66 & 126.66 & $7.72 \mathrm{E}+14$ & $1.19 \mathrm{E}+21$ & $17.17 \%$ \\
\hline 75 & -17.25 & 7520.87 & 124.17 & $7.54 \mathrm{E}+14$ & $1.16 \mathrm{E}+21$ & $16.87 \%$ \\
\hline 76 & -17.75 & 7180.85 & 118.56 & $7.25 \mathrm{E}+14$ & $1.10 \mathrm{E}+21$ & $15.88 \%$ \\
\hline 77 & -18.25 & 7120.59 & 117.56 & $7.00 \mathrm{E}+14$ & $1.08 \mathrm{E}+21$ & $15.73 \%$ \\
\hline 78 & -18.75 & 6818.88 & 112.58 & $6.66 \mathrm{E}+14$ & $1.03 \mathrm{E}+21$ & $15.06 \%$ \\
\hline
\end{tabular}


Table 24: Cycle 150B, MCNP-Calculated HGRs and Neutron Flux for AFIP-7, Plate Position 1, 0 EFPD (BOC) Average Center Lobe Power at 24.2 MW. ${ }^{2}$

\begin{tabular}{|c|c|c|c|c|}
\hline Node & $\begin{array}{c}\text { Distance from } \\
\text { Centerline (in.) }\end{array}$ & $\begin{array}{l}\text { Fission Power } \\
\text { Density (W/cc) }\end{array}$ & $\begin{array}{l}\text { Surface Heat } \\
\text { Flux }\left(\mathrm{W} / \mathrm{cm}^{2}\right) \\
\end{array}$ & $\begin{array}{c}\text { Neutron Flux } \\
\left(\mathbf{n} / \mathrm{cm}^{2} \mathrm{sec}\right)\end{array}$ \\
\hline 1 & 19.25 & 5638.37 & 93.09 & $5.39 \mathrm{E}+14$ \\
\hline 2 & 18.75 & 5325.13 & 87.92 & $5.61 \mathrm{E}+14$ \\
\hline 3 & 18.25 & 5558.76 & 91.78 & $5.92 \mathrm{E}+14$ \\
\hline 4 & 17.75 & 5805.12 & 95.84 & $6.31 \mathrm{E}+14$ \\
\hline 5 & 17.25 & 6093.68 & 100.61 & $6.64 \mathrm{E}+14$ \\
\hline 6 & 16.75 & 6398.43 & 105.64 & $6.88 \mathrm{E}+14$ \\
\hline 7 & 16.25 & 6595.89 & 108.90 & $7.17 \mathrm{E}+14$ \\
\hline 8 & 15.75 & 6733.31 & 111.17 & $7.47 \mathrm{E}+14$ \\
\hline 9 & 15.25 & 6879.35 & 113.58 & $7.70 \mathrm{E}+14$ \\
\hline 10 & 14.75 & 6937.34 & 114.54 & $7.88 \mathrm{E}+14$ \\
\hline 11 & 14.25 & 7280.18 & 120.20 & $8.19 \mathrm{E}+14$ \\
\hline 13 & 13.75 & 7359.31 & 121.50 & $8.45 \mathrm{E}+14$ \\
\hline 14 & 13.25 & 7326.17 & 120.96 & $8.58 \mathrm{E}+14$ \\
\hline 15 & 12.75 & 7588.06 & 125.28 & $8.75 \mathrm{E}+14$ \\
\hline 16 & 12.25 & 7695.60 & 127.05 & $8.95 \mathrm{E}+14$ \\
\hline 17 & 11.75 & 7880.71 & 130.11 & $9.15 \mathrm{E}+14$ \\
\hline 18 & 11.25 & 8136.06 & 134.33 & $9.45 \mathrm{E}+14$ \\
\hline 19 & 10.75 & 8279.01 & 136.69 & $9.53 \mathrm{E}+14$ \\
\hline 20 & 10.25 & 8485.85 & 140.10 & $9.68 \mathrm{E}+14$ \\
\hline 21 & 9.75 & 8649.86 & 142.81 & $9.85 \mathrm{E}+14$ \\
\hline 22 & 9.25 & 8734.13 & 144.20 & $1.00 \mathrm{E}+15$ \\
\hline 23 & 8.75 & 8887.67 & 146.74 & $1.01 \mathrm{E}+15$ \\
\hline 24 & 8.25 & 9146.40 & 151.01 & $1.03 \mathrm{E}+15$ \\
\hline 25 & 7.75 & 9176.21 & 151.50 & $1.04 \mathrm{E}+15$ \\
\hline 26 & 7.25 & 9257.25 & 152.84 & $1.04 \mathrm{E}+15$ \\
\hline 27 & 6.75 & 9236.15 & 152.49 & $1.05 \mathrm{E}+15$ \\
\hline 28 & 6.25 & 9392.73 & 155.07 & $1.07 \mathrm{E}+15$ \\
\hline 29 & 5.75 & 9708.55 & 160.29 & $1.09 \mathrm{E}+15$ \\
\hline 30 & 5.25 & 9631.44 & 159.02 & $1.09 \mathrm{E}+15$ \\
\hline 31 & 4.75 & 9650.78 & 159.33 & $1.09 \mathrm{E}+15$ \\
\hline 32 & 4.25 & 9613.54 & 158.72 & $1.11 \mathrm{E}+15$ \\
\hline 33 & 3.75 & 9628.78 & 158.97 & $1.11 \mathrm{E}+15$ \\
\hline 34 & 3.25 & 9433.34 & 155.74 & $1.10 \mathrm{E}+15$ \\
\hline 35 & 2.75 & 9836.59 & 162.40 & $1.11 \mathrm{E}+15$ \\
\hline 36 & 2.25 & 9766.60 & 161.25 & $1.11 \mathrm{E}+15$ \\
\hline 37 & 1.75 & 9850.28 & 162.63 & $1.12 \mathrm{E}+15$ \\
\hline 38 & 1.25 & 9649.82 & 159.32 & $1.12 \mathrm{E}+15$ \\
\hline 39 & 0.75 & 9759.78 & 161.13 & $1.13 \mathrm{E}+15$ \\
\hline 40 & 0.25 & 9515.15 & 157.10 & $1.12 \mathrm{E}+15$ \\
\hline 41 & -0.25 & 9617.18 & 158.78 & $1.13 \mathrm{E}+15$ \\
\hline 42 & -0.75 & 9723.37 & 160.53 & $1.13 \mathrm{E}+15$ \\
\hline 43 & -1.25 & 9748.07 & 160.94 & $1.13 \mathrm{E}+15$ \\
\hline 44 & -1.75 & 9772.42 & 161.34 & $1.12 \mathrm{E}+15$ \\
\hline 45 & -2.25 & 9954.74 & 164.35 & $1.13 \mathrm{E}+15$ \\
\hline 46 & -2.75 & 9907.00 & 163.56 & $1.13 \mathrm{E}+15$ \\
\hline 47 & -3.25 & 9949.61 & 164.27 & $1.13 \mathrm{E}+15$ \\
\hline 48 & -3.75 & 9821.51 & 162.15 & $1.11 \mathrm{E}+15$ \\
\hline 49 & -4.25 & 9830.22 & 162.30 & $1.12 \mathrm{E}+15$ \\
\hline 50 & -4.75 & 9804.86 & 161.88 & $1.11 \mathrm{E}+15$ \\
\hline 51 & -5.25 & 9598.33 & 158.47 & $1.10 \mathrm{E}+15$ \\
\hline 52 & -5.75 & 9612.03 & 158.69 & $1.10 \mathrm{E}+15$ \\
\hline
\end{tabular}




\begin{tabular}{|l|l|l|l|l|}
\hline 53 & -6.25 & 9704.15 & 160.22 & $1.10 \mathrm{E}+15$ \\
\hline 54 & -6.75 & 9639.34 & 159.15 & $1.09 \mathrm{E}+15$ \\
\hline 55 & -7.25 & 9598.43 & 158.47 & $1.09 \mathrm{E}+15$ \\
\hline 56 & -7.75 & 9605.31 & 158.58 & $1.07 \mathrm{E}+15$ \\
\hline 57 & -8.25 & 9224.77 & 152.30 & $1.06 \mathrm{E}+15$ \\
\hline 58 & -8.75 & 9099.01 & 150.22 & $1.05 \mathrm{E}+15$ \\
\hline 59 & -9.25 & 8982.49 & 148.30 & $1.04 \mathrm{E}+15$ \\
\hline 60 & -9.75 & 9062.98 & 149.63 & $1.04 \mathrm{E}+15$ \\
\hline 61 & -10.25 & 8870.68 & 146.45 & $1.03 \mathrm{E}+15$ \\
\hline 62 & -10.75 & 8688.54 & 143.45 & $1.00 \mathrm{E}+15$ \\
\hline 63 & -11.25 & 8593.51 & 141.88 & $9.92 \mathrm{E}+14$ \\
\hline 64 & -11.75 & 8509.69 & 140.50 & $9.83 \mathrm{E}+14$ \\
\hline 65 & -12.25 & 8325.12 & 137.45 & $9.60 \mathrm{E}+14$ \\
\hline 66 & -12.75 & 8193.72 & 135.28 & $9.50 \mathrm{E}+14$ \\
\hline 67 & -13.25 & 8175.52 & 134.98 & $9.28 \mathrm{E}+14$ \\
\hline 68 & -13.75 & 7997.13 & 132.03 & $9.10 \mathrm{E}+14$ \\
\hline 69 & -14.25 & 7922.78 & 130.81 & $8.94 \mathrm{E}+14$ \\
\hline 70 & -14.75 & 7547.79 & 124.61 & $8.69 \mathrm{E}+14$ \\
\hline 71 & -15.25 & 7632.12 & 126.01 & $8.49 \mathrm{E}+14$ \\
\hline 72 & -15.75 & 7419.15 & 122.49 & $8.25 \mathrm{E}+14$ \\
\hline 73 & -16.25 & 7311.64 & 120.72 & $8.05 \mathrm{E}+14$ \\
\hline 74 & -16.75 & 6822.00 & 112.63 & $7.76 \mathrm{E}+14$ \\
\hline 75 & -17.25 & 6830.30 & 112.77 & $7.48 \mathrm{E}+14$ \\
\hline 76 & -17.75 & 6543.43 & 108.03 & $7.18 \mathrm{E}+14$ \\
\hline 77 & -18.25 & 6297.74 & 103.98 & $6.97 \mathrm{E}+14$ \\
\hline 78 & -18.75 & 6306.42 & 104.12 & $6.69 \mathrm{E}+14$ \\
\hline
\end{tabular}


Table 25: Cycle 149B, MCNP-Calculated HGRs, Neutron Flux, Depletion and Fission Density for AFIP-7, Plate Position 1, 18 EFPD (MOC1) Average Center Lobe Power at 24.2 MW. ${ }^{2}$

\begin{tabular}{|c|c|c|c|c|c|c|}
\hline Node & $\begin{array}{c}\text { Distance from } \\
\text { Centerline (in.) }\end{array}$ & $\begin{array}{l}\text { Fission Power } \\
\text { Density (W/cc) }\end{array}$ & $\begin{array}{l}\text { Surface Heat } \\
\text { Flux }\left(W / \mathrm{cm}^{2}\right)\end{array}$ & $\begin{array}{c}\text { Neutron Flux } \\
\left(\mathrm{n} / \mathrm{cm}^{2} \mathrm{sec}\right)\end{array}$ & $\begin{array}{l}\text { Fission Density } \\
\text { (fissions/cc) }\end{array}$ & $\begin{array}{c}\text { U-235 } \\
\text { Burnup (\%) }\end{array}$ \\
\hline 1 & 19.25 & 5550.50 & 91.64 & $5.26 \mathrm{E}+14$ & $1.13 \mathrm{E}+21$ & $18.43 \%$ \\
\hline 2 & 18.75 & 5348.01 & 88.30 & $5.54 \mathrm{E}+14$ & $1.08 \mathrm{E}+21$ & $17.57 \%$ \\
\hline 3 & 18.25 & 5436.03 & 89.75 & $5.85 \mathrm{E}+14$ & $1.14 \mathrm{E}+21$ & $18.45 \%$ \\
\hline 4 & 17.75 & 5790.28 & 95.60 & $6.14 \mathrm{E}+14$ & $1.17 \mathrm{E}+21$ & $19.08 \%$ \\
\hline 5 & 17.25 & 5977.31 & 98.69 & $6.46 \mathrm{E}+14$ & $1.24 \mathrm{E}+21$ & $20.09 \%$ \\
\hline 6 & 16.75 & 6066.70 & 100.16 & $6.64 \mathrm{E}+14$ & $1.30 \mathrm{E}+21$ & $20.99 \%$ \\
\hline 7 & 16.25 & 6303.74 & 104.07 & $6.89 \mathrm{E}+14$ & $1.32 \mathrm{E}+21$ & $21.32 \%$ \\
\hline 8 & 15.75 & 6364.85 & 105.08 & $7.14 \mathrm{E}+14$ & $1.37 \mathrm{E}+21$ & $21.97 \%$ \\
\hline 9 & 15.25 & 6694.71 & 110.53 & $7.46 \mathrm{E}+14$ & $1.42 \mathrm{E}+21$ & $22.89 \%$ \\
\hline 10 & 14.75 & 6806.45 & 112.37 & $7.64 \mathrm{E}+14$ & $1.46 \mathrm{E}+21$ & $23.41 \%$ \\
\hline 11 & 14.25 & 6977.95 & 115.21 & $7.83 \mathrm{E}+14$ & $1.49 \mathrm{E}+21$ & $23.91 \%$ \\
\hline 13 & 13.75 & 7041.73 & 116.26 & $8.07 \mathrm{E}+14$ & $1.53 \mathrm{E}+21$ & $24.49 \%$ \\
\hline 14 & 13.25 & 7016.05 & 115.83 & $8.19 \mathrm{E}+14$ & $1.53 \mathrm{E}+21$ & $24.45 \%$ \\
\hline 15 & 12.75 & 7264.98 & 119.94 & $8.29 \mathrm{E}+14$ & $1.57 \mathrm{E}+21$ & $25.06 \%$ \\
\hline 16 & 12.25 & 7437.36 & 122.79 & $8.51 \mathrm{E}+14$ & $1.61 \mathrm{E}+21$ & $25.61 \%$ \\
\hline 17 & 11.75 & 7466.72 & 123.28 & $8.68 \mathrm{E}+14$ & $1.62 \mathrm{E}+21$ & $25.81 \%$ \\
\hline 18 & 11.25 & 7764.88 & 128.20 & $8.97 \mathrm{E}+14$ & $1.65 \mathrm{E}+21$ & $26.37 \%$ \\
\hline 19 & 10.75 & 7813.04 & 128.99 & $9.05 \mathrm{E}+14$ & $1.71 \mathrm{E}+21$ & $27.18 \%$ \\
\hline 20 & 10.25 & 7946.31 & 131.19 & $9.12 \mathrm{E}+14$ & $1.75 \mathrm{E}+21$ & $27.71 \%$ \\
\hline 21 & 9.75 & 8168.13 & 134.86 & $9.32 \mathrm{E}+14$ & $1.80 \mathrm{E}+21$ & $28.48 \%$ \\
\hline 22 & 9.25 & 8245.30 & 136.13 & $9.36 \mathrm{E}+14$ & $1.82 \mathrm{E}+21$ & $28.80 \%$ \\
\hline 23 & 8.75 & 8297.33 & 136.99 & $9.50 \mathrm{E}+14$ & $1.86 \mathrm{E}+21$ & $29.33 \%$ \\
\hline 24 & 8.25 & 8484.15 & 140.07 & $9.58 \mathrm{E}+14$ & $1.89 \mathrm{E}+21$ & $29.78 \%$ \\
\hline 25 & 7.75 & 8495.37 & 140.26 & $9.72 \mathrm{E}+14$ & $1.90 \mathrm{E}+21$ & $29.89 \%$ \\
\hline 26 & 7.25 & 8488.88 & 140.15 & $9.74 \mathrm{E}+14$ & $1.91 \mathrm{E}+21$ & $30.10 \%$ \\
\hline 27 & 6.75 & 8667.26 & 143.10 & $9.84 \mathrm{E}+14$ & $1.94 \mathrm{E}+21$ & $30.48 \%$ \\
\hline 28 & 6.25 & 8777.76 & 144.92 & $9.97 \mathrm{E}+14$ & $1.95 \mathrm{E}+21$ & $30.69 \%$ \\
\hline 29 & 5.75 & 8881.80 & 146.64 & $1.00 \mathrm{E}+15$ & $1.97 \mathrm{E}+21$ & $30.94 \%$ \\
\hline 30 & 5.25 & 8704.91 & 143.72 & $1.01 \mathrm{E}+15$ & $1.98 \mathrm{E}+21$ & $31.08 \%$ \\
\hline 31 & 4.75 & 8855.77 & 146.21 & $1.02 \mathrm{E}+15$ & $2.00 \mathrm{E}+21$ & $31.45 \%$ \\
\hline 32 & 4.25 & 8951.32 & 147.79 & $1.02 \mathrm{E}+15$ & $2.02 \mathrm{E}+21$ & $31.71 \%$ \\
\hline 33 & 3.75 & 8885.23 & 146.70 & $1.02 \mathrm{E}+15$ & $2.03 \mathrm{E}+21$ & $31.70 \%$ \\
\hline 34 & 3.25 & 8841.02 & 145.97 & $1.02 \mathrm{E}+15$ & $2.02 \mathrm{E}+21$ & $31.69 \%$ \\
\hline 35 & 2.75 & 8980.57 & 148.27 & $1.02 \mathrm{E}+15$ & $2.02 \mathrm{E}+21$ & $31.71 \%$ \\
\hline 36 & 2.25 & 8911.31 & 147.13 & $1.03 \mathrm{E}+15$ & $2.03 \mathrm{E}+21$ & $31.90 \%$ \\
\hline 37 & 1.75 & 8937.50 & 147.56 & $1.03 \mathrm{E}+15$ & $2.02 \mathrm{E}+21$ & $31.63 \%$ \\
\hline 38 & 1.25 & 8865.27 & 146.37 & $1.04 \mathrm{E}+15$ & $2.00 \mathrm{E}+21$ & $31.28 \%$ \\
\hline 39 & 0.75 & 8979.48 & 148.25 & $1.04 \mathrm{E}+15$ & $2.00 \mathrm{E}+21$ & $31.43 \%$ \\
\hline 40 & 0.25 & 8860.06 & 146.28 & $1.04 \mathrm{E}+15$ & $2.00 \mathrm{E}+21$ & $31.32 \%$ \\
\hline 41 & -0.25 & 9008.98 & 148.74 & $1.04 \mathrm{E}+15$ & $2.01 \mathrm{E}+21$ & $31.55 \%$ \\
\hline 42 & -0.75 & 9047.14 & 149.37 & $1.05 \mathrm{E}+15$ & $2.02 \mathrm{E}+21$ & $31.67 \%$ \\
\hline 43 & -1.25 & 8945.92 & 147.70 & $1.04 \mathrm{E}+15$ & $2.03 \mathrm{E}+21$ & $31.84 \%$ \\
\hline 44 & -1.75 & 8998.22 & 148.56 & $1.03 \mathrm{E}+15$ & $2.04 \mathrm{E}+21$ & $31.88 \%$ \\
\hline 45 & -2.25 & 8929.72 & 147.43 & $1.04 \mathrm{E}+15$ & $2.07 \mathrm{E}+21$ & $32.41 \%$ \\
\hline 46 & -2.75 & 9170.94 & 151.41 & $1.05 \mathrm{E}+15$ & $2.06 \mathrm{E}+21$ & $32.29 \%$ \\
\hline 47 & -3.25 & 9075.89 & 149.84 & $1.04 \mathrm{E}+15$ & $2.04 \mathrm{E}+21$ & $31.98 \%$ \\
\hline 48 & -3.75 & 8986.33 & 148.36 & $1.03 \mathrm{E}+15$ & $2.05 \mathrm{E}+21$ & $32.11 \%$ \\
\hline 49 & -4.25 & 8974.26 & 148.17 & $1.04 \mathrm{E}+15$ & $2.04 \mathrm{E}+21$ & $31.88 \%$ \\
\hline 50 & -4.75 & 8977.56 & 148.22 & $1.03 \mathrm{E}+15$ & $2.02 \mathrm{E}+21$ & $31.76 \%$ \\
\hline 51 & -5.25 & 8853.22 & 146.17 & $1.03 \mathrm{E}+15$ & $2.03 \mathrm{E}+21$ & $31.77 \%$ \\
\hline 52 & -5.75 & 8939.89 & 147.60 & $1.03 \mathrm{E}+15$ & $2.01 \mathrm{E}+21$ & $31.52 \%$ \\
\hline
\end{tabular}




\begin{tabular}{|l|l|l|l|l|l|l|}
\hline 53 & -6.25 & 8927.35 & 147.39 & $1.03 \mathrm{E}+15$ & $2.01 \mathrm{E}+21$ & $31.52 \%$ \\
\hline 54 & -6.75 & 8801.77 & 145.32 & $1.02 \mathrm{E}+15$ & $2.00 \mathrm{E}+21$ & $31.35 \%$ \\
\hline 55 & -7.25 & 8854.97 & 146.20 & $1.01 \mathrm{E}+15$ & $1.99 \mathrm{E}+21$ & $31.24 \%$ \\
\hline 56 & -7.75 & 8871.45 & 146.47 & $1.00 \mathrm{E}+15$ & $1.96 \mathrm{E}+21$ & $30.73 \%$ \\
\hline 57 & -8.25 & 8710.54 & 143.81 & $9.93 \mathrm{E}+14$ & $1.94 \mathrm{E}+21$ & $30.54 \%$ \\
\hline 58 & -8.75 & 8656.39 & 142.92 & $9.93 \mathrm{E}+14$ & $1.92 \mathrm{E}+21$ & $30.15 \%$ \\
\hline 59 & -9.25 & 8450.90 & 139.52 & $9.72 \mathrm{E}+14$ & $1.91 \mathrm{E}+21$ & $30.05 \%$ \\
\hline 60 & -9.75 & 8511.26 & 140.52 & $9.67 \mathrm{E}+14$ & $1.86 \mathrm{E}+21$ & $29.32 \%$ \\
\hline 61 & -10.25 & 8132.32 & 134.26 & $9.63 \mathrm{E}+14$ & $1.83 \mathrm{E}+21$ & $28.83 \%$ \\
\hline 62 & -10.75 & 8106.48 & 133.84 & $9.46 \mathrm{E}+14$ & $1.79 \mathrm{E}+21$ & $28.30 \%$ \\
\hline 63 & -11.25 & 8011.71 & 132.27 & $9.34 \mathrm{E}+14$ & $1.77 \mathrm{E}+21$ & $28.05 \%$ \\
\hline 64 & -11.75 & 8008.78 & 132.22 & $9.32 \mathrm{E}+14$ & $1.74 \mathrm{E}+21$ & $27.68 \%$ \\
\hline 65 & -12.25 & 7945.07 & 131.17 & $9.05 \mathrm{E}+14$ & $1.71 \mathrm{E}+21$ & $27.18 \%$ \\
\hline 66 & -12.75 & 7765.26 & 128.20 & $8.96 \mathrm{E}+14$ & $1.72 \mathrm{E}+21$ & $27.28 \%$ \\
\hline 67 & -13.25 & 7729.58 & 127.62 & $8.83 \mathrm{E}+14$ & $1.67 \mathrm{E}+21$ & $26.60 \%$ \\
\hline 68 & -13.75 & 7655.53 & 126.39 & $8.66 \mathrm{E}+14$ & $1.66 \mathrm{E}+21$ & $26.38 \%$ \\
\hline 69 & -14.25 & 7477.39 & 123.45 & $8.51 \mathrm{E}+14$ & $1.63 \mathrm{E}+21$ & $25.95 \%$ \\
\hline 70 & -14.75 & 7301.00 & 120.54 & $8.30 \mathrm{E}+14$ & $1.58 \mathrm{E}+21$ & $25.29 \%$ \\
\hline 71 & -15.25 & 7256.39 & 119.80 & $8.19 \mathrm{E}+14$ & $1.55 \mathrm{E}+21$ & $24.80 \%$ \\
\hline 72 & -15.75 & 7225.74 & 119.30 & $7.98 \mathrm{E}+14$ & $1.51 \mathrm{E}+21$ & $24.21 \%$ \\
\hline 73 & -16.25 & 6977.39 & 115.20 & $7.76 \mathrm{E}+14$ & $1.48 \mathrm{E}+21$ & $23.75 \%$ \\
\hline 74 & -16.75 & 6777.08 & 111.89 & $7.53 \mathrm{E}+14$ & $1.43 \mathrm{E}+21$ & $23.05 \%$ \\
\hline 75 & -17.25 & 6643.36 & 109.68 & $7.24 \mathrm{E}+14$ & $1.38 \mathrm{E}+21$ & $22.30 \%$ \\
\hline 76 & -17.75 & 6406.64 & 105.77 & $7.08 \mathrm{E}+14$ & $1.33 \mathrm{E}+21$ & $21.41 \%$ \\
\hline 77 & -18.25 & 6191.60 & 102.22 & $6.83 \mathrm{E}+14$ & $1.28 \mathrm{E}+21$ & $20.64 \%$ \\
\hline 78 & -18.75 & 6070.15 & 100.22 & $6.50 \mathrm{E}+14$ & $1.26 \mathrm{E}+21$ & $20.37 \%$ \\
\hline
\end{tabular}


Table 26: Cycle 150B, MCNP-Calculated HGRs, Neutron Flux, Depletion and Fission Density for AFIP-7, Plate Position 1, 31 EFPD (MOC2) Average Center Lobe Power at 24.2 MW. ${ }^{2}$

\begin{tabular}{|c|c|c|c|c|c|c|}
\hline Node & $\begin{array}{c}\text { Distance from } \\
\text { Centerline (in.) }\end{array}$ & $\begin{array}{l}\text { Fission Power } \\
\text { Density (W/cc) }\end{array}$ & $\begin{array}{l}\text { Surface Heat } \\
\text { Flux }\left(W / \mathrm{cm}^{2}\right)\end{array}$ & $\begin{array}{c}\text { Neutron Flux } \\
\left(\mathrm{n} / \mathrm{cm}^{2} \mathrm{sec}\right)\end{array}$ & $\begin{array}{l}\text { Fission Density } \\
\text { (fissions/cc) }\end{array}$ & $\begin{array}{c}\text { U-235 } \\
\text { Burnup (\%) }\end{array}$ \\
\hline 1 & 19.25 & 5682.51 & 93.82 & $5.40 \mathrm{E}+14$ & $1.34 \mathrm{E}+21$ & $21.65 \%$ \\
\hline 2 & 18.75 & 5299.85 & 87.50 & $5.62 \mathrm{E}+14$ & $1.27 \mathrm{E}+21$ & $20.69 \%$ \\
\hline 3 & 18.25 & 5628.59 & 92.93 & $6.06 \mathrm{E}+14$ & $1.34 \mathrm{E}+21$ & $21.62 \%$ \\
\hline 4 & 17.75 & 5848.32 & 96.56 & $6.31 \mathrm{E}+14$ & $1.39 \mathrm{E}+21$ & $22.44 \%$ \\
\hline 5 & 17.25 & 6088.96 & 100.53 & $6.63 \mathrm{E}+14$ & $1.46 \mathrm{E}+21$ & $23.52 \%$ \\
\hline 6 & 16.75 & 6292.11 & 103.88 & $6.82 \mathrm{E}+14$ & $1.52 \mathrm{E}+21$ & $24.49 \%$ \\
\hline 7 & 16.25 & 6395.97 & 105.60 & $7.02 \mathrm{E}+14$ & $1.55 \mathrm{E}+21$ & $24.95 \%$ \\
\hline 8 & 15.75 & 6634.54 & 109.54 & $7.38 \mathrm{E}+14$ & $1.60 \mathrm{E}+21$ & $25.60 \%$ \\
\hline 9 & 15.25 & 6747.49 & 111.40 & $7.57 \mathrm{E}+14$ & $1.67 \mathrm{E}+21$ & $26.72 \%$ \\
\hline 10 & 14.75 & 6962.51 & 114.95 & $7.77 \mathrm{E}+14$ & $1.71 \mathrm{E}+21$ & $27.26 \%$ \\
\hline 11 & 14.25 & 7139.94 & 117.88 & $8.01 \mathrm{E}+14$ & $1.75 \mathrm{E}+21$ & $27.86 \%$ \\
\hline 13 & 13.75 & 7234.11 & 119.44 & $8.22 \mathrm{E}+14$ & $1.79 \mathrm{E}+21$ & $28.45 \%$ \\
\hline 14 & 13.25 & 7204.80 & 118.95 & $8.29 \mathrm{E}+14$ & $1.78 \mathrm{E}+21$ & $28.40 \%$ \\
\hline 15 & 12.75 & 7490.51 & 123.67 & $8.63 \mathrm{E}+14$ & $1.84 \mathrm{E}+21$ & $29.15 \%$ \\
\hline 16 & 12.25 & 7470.86 & 123.34 & $8.66 \mathrm{E}+14$ & $1.88 \mathrm{E}+21$ & $29.78 \%$ \\
\hline 17 & 11.75 & 7684.13 & 126.86 & $8.83 \mathrm{E}+14$ & $1.89 \mathrm{E}+21$ & $29.99 \%$ \\
\hline 18 & 11.25 & 7837.27 & 129.39 & $9.07 \mathrm{E}+14$ & $1.94 \mathrm{E}+21$ & $30.71 \%$ \\
\hline 19 & 10.75 & 8006.53 & 132.19 & $9.23 \mathrm{E}+14$ & $1.99 \mathrm{E}+21$ & $31.53 \%$ \\
\hline 20 & 10.25 & 8146.52 & 134.50 & $9.29 \mathrm{E}+14$ & $2.04 \mathrm{E}+21$ & $32.12 \%$ \\
\hline 21 & 9.75 & 8282.97 & 136.75 & $9.47 \mathrm{E}+14$ & $2.10 \mathrm{E}+21$ & $33.00 \%$ \\
\hline 22 & 9.25 & 8241.65 & 136.07 & $9.48 \mathrm{E}+14$ & $2.12 \mathrm{E}+21$ & $33.39 \%$ \\
\hline 23 & 8.75 & 8465.48 & 139.77 & $9.71 \mathrm{E}+14$ & $2.16 \mathrm{E}+21$ & $33.91 \%$ \\
\hline 24 & 8.25 & 8496.41 & 140.28 & $9.75 \mathrm{E}+14$ & $2.20 \mathrm{E}+21$ & $34.46 \%$ \\
\hline 25 & 7.75 & 8579.62 & 141.65 & $9.88 \mathrm{E}+14$ & $2.21 \mathrm{E}+21$ & $34.58 \%$ \\
\hline 26 & 7.25 & 8711.23 & 143.82 & $9.95 \mathrm{E}+14$ & $2.22 \mathrm{E}+21$ & $34.78 \%$ \\
\hline 27 & 6.75 & 8860.39 & 146.29 & $1.00 \mathrm{E}+15$ & $2.26 \mathrm{E}+21$ & $35.21 \%$ \\
\hline 28 & 6.25 & 8745.58 & 144.39 & $1.01 \mathrm{E}+15$ & $2.27 \mathrm{E}+21$ & $35.51 \%$ \\
\hline 29 & 5.75 & 8742.12 & 144.33 & $1.01 \mathrm{E}+15$ & $2.30 \mathrm{E}+21$ & $35.80 \%$ \\
\hline 30 & 5.25 & 8758.93 & 144.61 & $1.02 \mathrm{E}+15$ & $2.29 \mathrm{E}+21$ & $35.84 \%$ \\
\hline 31 & 4.75 & 8851.93 & 146.15 & $1.03 \mathrm{E}+15$ & $2.33 \mathrm{E}+21$ & $36.31 \%$ \\
\hline 32 & 4.25 & 8888.63 & 146.75 & $1.03 \mathrm{E}+15$ & $2.35 \mathrm{E}+21$ & $36.57 \%$ \\
\hline 33 & 3.75 & 8893.20 & 146.83 & $1.03 \mathrm{E}+15$ & $2.35 \mathrm{E}+21$ & $36.54 \%$ \\
\hline 34 & 3.25 & 8803.35 & 145.34 & $1.03 \mathrm{E}+15$ & $2.35 \mathrm{E}+21$ & $36.53 \%$ \\
\hline 35 & 2.75 & 9034.59 & 149.16 & $1.04 \mathrm{E}+15$ & $2.35 \mathrm{E}+21$ & $36.61 \%$ \\
\hline 36 & 2.25 & 8905.77 & 147.03 & $1.04 \mathrm{E}+15$ & $2.36 \mathrm{E}+21$ & $36.78 \%$ \\
\hline 37 & 1.75 & 9058.80 & 149.56 & $1.05 \mathrm{E}+15$ & $2.34 \mathrm{E}+21$ & $36.50 \%$ \\
\hline 38 & 1.25 & 8906.42 & 147.04 & $1.05 \mathrm{E}+15$ & $2.32 \mathrm{E}+21$ & $36.10 \%$ \\
\hline 39 & 0.75 & 9000.54 & 148.60 & $1.06 \mathrm{E}+15$ & $2.33 \mathrm{E}+21$ & $36.33 \%$ \\
\hline 40 & 0.25 & 8828.61 & 145.76 & $1.06 \mathrm{E}+15$ & $2.32 \mathrm{E}+21$ & $36.14 \%$ \\
\hline 41 & -0.25 & 8844.76 & 146.03 & $1.05 \mathrm{E}+15$ & $2.34 \mathrm{E}+21$ & $36.46 \%$ \\
\hline 42 & -0.75 & 9031.77 & 149.11 & $1.05 \mathrm{E}+15$ & $2.35 \mathrm{E}+21$ & $36.59 \%$ \\
\hline 43 & -1.25 & 8899.72 & 146.93 & $1.05 \mathrm{E}+15$ & $2.36 \mathrm{E}+21$ & $36.71 \%$ \\
\hline 44 & -1.75 & 9140.95 & 150.92 & $1.05 \mathrm{E}+15$ & $2.36 \mathrm{E}+21$ & $36.78 \%$ \\
\hline 45 & -2.25 & 9004.56 & 148.67 & $1.05 \mathrm{E}+15$ & $2.40 \mathrm{E}+21$ & $37.26 \%$ \\
\hline 46 & -2.75 & 9015.78 & 148.85 & $1.05 \mathrm{E}+15$ & $2.40 \mathrm{E}+21$ & $37.27 \%$ \\
\hline 47 & -3.25 & 9012.58 & 148.80 & $1.05 \mathrm{E}+15$ & $2.37 \mathrm{E}+21$ & $36.94 \%$ \\
\hline 48 & -3.75 & 9085.87 & 150.01 & $1.05 \mathrm{E}+15$ & $2.38 \mathrm{E}+21$ & $37.01 \%$ \\
\hline 49 & -4.25 & 8962.06 & 147.96 & $1.05 \mathrm{E}+15$ & $2.37 \mathrm{E}+21$ & $36.77 \%$ \\
\hline 50 & -4.75 & 9006.64 & 148.70 & $1.03 \mathrm{E}+15$ & $2.35 \mathrm{E}+21$ & $36.66 \%$ \\
\hline 51 & -5.25 & 9052.50 & 149.46 & $1.04 \mathrm{E}+15$ & $2.35 \mathrm{E}+21$ & $36.59 \%$ \\
\hline 52 & -5.75 & 8875.57 & 146.54 & $1.04 \mathrm{E}+15$ & $2.34 \mathrm{E}+21$ & $36.40 \%$ \\
\hline
\end{tabular}




\begin{tabular}{|l|l|l|l|l|l|l|}
\hline 53 & -6.25 & 8887.81 & 146.74 & $1.03 \mathrm{E}+15$ & $2.34 \mathrm{E}+21$ & $36.40 \%$ \\
\hline 54 & -6.75 & 9038.59 & 149.23 & $1.03 \mathrm{E}+15$ & $2.32 \mathrm{E}+21$ & $36.14 \%$ \\
\hline 55 & -7.25 & 8783.21 & 145.01 & $1.03 \mathrm{E}+15$ & $2.31 \mathrm{E}+21$ & $36.10 \%$ \\
\hline 56 & -7.75 & 8954.72 & 147.84 & $1.02 \mathrm{E}+15$ & $2.28 \mathrm{E}+21$ & $35.56 \%$ \\
\hline 57 & -8.25 & 8679.45 & 143.30 & $1.01 \mathrm{E}+15$ & $2.26 \mathrm{E}+21$ & $35.31 \%$ \\
\hline 58 & -8.75 & 8706.50 & 143.74 & $1.00 \mathrm{E}+15$ & $2.23 \mathrm{E}+21$ & $34.91 \%$ \\
\hline 59 & -9.25 & 8395.54 & 138.61 & $9.84 \mathrm{E}+14$ & $2.22 \mathrm{E}+21$ & $34.72 \%$ \\
\hline 60 & -9.75 & 8548.29 & 141.13 & $9.87 \mathrm{E}+14$ & $2.17 \mathrm{E}+21$ & $34.01 \%$ \\
\hline 61 & -10.25 & 8397.83 & 138.65 & $9.73 \mathrm{E}+14$ & $2.12 \mathrm{E}+21$ & $33.34 \%$ \\
\hline 62 & -10.75 & 8182.86 & 135.10 & $9.66 \mathrm{E}+14$ & $2.08 \mathrm{E}+21$ & $32.79 \%$ \\
\hline 63 & -11.25 & 8233.05 & 135.93 & $9.57 \mathrm{E}+14$ & $2.06 \mathrm{E}+21$ & $32.50 \%$ \\
\hline 64 & -11.75 & 8194.24 & 135.29 & $9.47 \mathrm{E}+14$ & $2.04 \mathrm{E}+21$ & $32.13 \%$ \\
\hline 65 & -12.25 & 7840.56 & 129.45 & $9.29 \mathrm{E}+14$ & $2.00 \mathrm{E}+21$ & $31.60 \%$ \\
\hline 66 & -12.75 & 7995.53 & 132.01 & $9.16 \mathrm{E}+14$ & $2.00 \mathrm{E}+21$ & $31.60 \%$ \\
\hline 67 & -13.25 & 7854.45 & 129.68 & $8.99 \mathrm{E}+14$ & $1.95 \mathrm{E}+21$ & $30.93 \%$ \\
\hline 68 & -13.75 & 7763.71 & 128.18 & $8.80 \mathrm{E}+14$ & $1.94 \mathrm{E}+21$ & $30.66 \%$ \\
\hline 69 & -14.25 & 7643.34 & 126.19 & $8.72 \mathrm{E}+14$ & $1.90 \mathrm{E}+21$ & $30.13 \%$ \\
\hline 70 & -14.75 & 7428.24 & 122.64 & $8.50 \mathrm{E}+14$ & $1.85 \mathrm{E}+21$ & $29.39 \%$ \\
\hline 71 & -15.25 & 7440.49 & 122.84 & $8.32 \mathrm{E}+14$ & $1.82 \mathrm{E}+21$ & $28.89 \%$ \\
\hline 72 & -15.75 & 7170.56 & 118.39 & $8.07 \mathrm{E}+14$ & $1.78 \mathrm{E}+21$ & $28.30 \%$ \\
\hline 73 & -16.25 & 7092.44 & 117.10 & $7.89 \mathrm{E}+14$ & $1.74 \mathrm{E}+21$ & $27.70 \%$ \\
\hline 74 & -16.75 & 6852.05 & 113.13 & $7.61 \mathrm{E}+14$ & $1.68 \mathrm{E}+21$ & $26.90 \%$ \\
\hline 75 & -17.25 & 6491.11 & 107.17 & $7.34 \mathrm{E}+14$ & $1.63 \mathrm{E}+21$ & $26.11 \%$ \\
\hline 76 & -17.75 & 6417.68 & 105.96 & $7.11 \mathrm{E}+14$ & $1.56 \mathrm{E}+21$ & $25.08 \%$ \\
\hline 77 & -18.25 & 6216.46 & 102.63 & $6.91 \mathrm{E}+14$ & $1.50 \mathrm{E}+21$ & $24.19 \%$ \\
\hline 78 & -18.75 & 6174.22 & 101.94 & $6.63 \mathrm{E}+14$ & $1.48 \mathrm{E}+21$ & $23.87 \%$ \\
\hline
\end{tabular}


Table 27: Cycle 150B, MCNP-Calculated HGRs, Neutron Flux, Depletion and Fission Density for AFIP-7, Plate Position 1, 42.0 EFPD (EOC) Average Center Lobe Power at 24.2 MW. ${ }^{2}$

\begin{tabular}{|c|c|c|c|c|c|c|}
\hline Node & $\begin{array}{c}\text { Distance from } \\
\text { Centerline (in.) }\end{array}$ & $\begin{array}{l}\text { Fission Power } \\
\text { Density (W/cc) }\end{array}$ & $\begin{array}{l}\text { Surface Heat } \\
\text { Flux }\left(W / \mathrm{cm}^{2}\right)\end{array}$ & $\begin{array}{c}\text { Neutron Flux } \\
\left(\mathrm{n} / \mathrm{cm}^{2} \mathrm{sec}\right)\end{array}$ & $\begin{array}{l}\text { Fission Density } \\
\text { (fissions/cc) }\end{array}$ & $\begin{array}{c}\text { U-235 } \\
\text { Burnup (\%) }\end{array}$ \\
\hline 1 & 19.25 & 5625.61 & 92.88 & $5.46 \mathrm{E}+14$ & $1.51 \mathrm{E}+21$ & $24.40 \%$ \\
\hline 2 & 18.75 & 5301.43 & 87.53 & $5.70 \mathrm{E}+14$ & $1.44 \mathrm{E}+21$ & $23.27 \%$ \\
\hline 3 & 18.25 & 5596.04 & 92.39 & $6.12 \mathrm{E}+14$ & $1.51 \mathrm{E}+21$ & $24.36 \%$ \\
\hline 4 & 17.75 & 5828.25 & 96.22 & $6.38 \mathrm{E}+14$ & $1.57 \mathrm{E}+21$ & $25.27 \%$ \\
\hline 5 & 17.25 & 6061.31 & 100.07 & $6.68 \mathrm{E}+14$ & $1.65 \mathrm{E}+21$ & $26.44 \%$ \\
\hline 6 & 16.75 & 6228.72 & 102.84 & $6.88 \mathrm{E}+14$ & $1.72 \mathrm{E}+21$ & $27.50 \%$ \\
\hline 7 & 16.25 & 6350.37 & 104.84 & $7.09 \mathrm{E}+14$ & $1.75 \mathrm{E}+21$ & $28.00 \%$ \\
\hline 8 & 15.75 & 6561.02 & 108.32 & $7.44 \mathrm{E}+14$ & $1.80 \mathrm{E}+21$ & $28.75 \%$ \\
\hline 9 & 15.25 & 6692.50 & 110.49 & $7.62 \mathrm{E}+14$ & $1.88 \mathrm{E}+21$ & $29.91 \%$ \\
\hline 10 & 14.75 & 6887.59 & 113.71 & $7.84 \mathrm{E}+14$ & $1.92 \mathrm{E}+21$ & $30.54 \%$ \\
\hline 11 & 14.25 & 7066.66 & 116.67 & $8.07 \mathrm{E}+14$ & $1.97 \mathrm{E}+21$ & $31.22 \%$ \\
\hline 13 & 13.75 & 7163.22 & 118.26 & $8.28 \mathrm{E}+14$ & $2.01 \mathrm{E}+21$ & $31.84 \%$ \\
\hline 14 & 13.25 & 7165.84 & 118.31 & $8.36 \mathrm{E}+14$ & $2.00 \mathrm{E}+21$ & $31.77 \%$ \\
\hline 15 & 12.75 & 7375.31 & 121.77 & $8.68 \mathrm{E}+14$ & $2.07 \mathrm{E}+21$ & $32.64 \%$ \\
\hline 16 & 12.25 & 7405.67 & 122.27 & $8.74 \mathrm{E}+14$ & $2.11 \mathrm{E}+21$ & $33.25 \%$ \\
\hline 17 & 11.75 & 7603.37 & 125.53 & $8.92 \mathrm{E}+14$ & $2.13 \mathrm{E}+21$ & $33.58 \%$ \\
\hline 18 & 11.25 & 7755.54 & 128.04 & $9.17 \mathrm{E}+14$ & $2.18 \mathrm{E}+21$ & $34.35 \%$ \\
\hline 19 & 10.75 & 7841.61 & 129.46 & $9.30 \mathrm{E}+14$ & $2.24 \mathrm{E}+21$ & $35.24 \%$ \\
\hline 20 & 10.25 & 8028.57 & 132.55 & $9.36 \mathrm{E}+14$ & $2.29 \mathrm{E}+21$ & $35.87 \%$ \\
\hline 21 & 9.75 & 8165.58 & 134.81 & $9.53 \mathrm{E}+14$ & $2.35 \mathrm{E}+21$ & $36.82 \%$ \\
\hline 22 & 9.25 & 8143.36 & 134.45 & $9.57 \mathrm{E}+14$ & $2.38 \mathrm{E}+21$ & $37.20 \%$ \\
\hline 23 & 8.75 & 8317.50 & 137.32 & $9.78 \mathrm{E}+14$ & $2.42 \mathrm{E}+21$ & $37.78 \%$ \\
\hline 24 & 8.25 & 8361.17 & 138.04 & $9.84 \mathrm{E}+14$ & $2.46 \mathrm{E}+21$ & $38.34 \%$ \\
\hline 25 & 7.75 & 8407.57 & 138.81 & $9.95 \mathrm{E}+14$ & $2.47 \mathrm{E}+21$ & $38.52 \%$ \\
\hline 26 & 7.25 & 8541.34 & 141.02 & $1.00 \mathrm{E}+15$ & $2.49 \mathrm{E}+21$ & $38.76 \%$ \\
\hline 27 & 6.75 & 8661.61 & 143.00 & $1.01 \mathrm{E}+15$ & $2.53 \mathrm{E}+21$ & $39.23 \%$ \\
\hline 28 & 6.25 & 8600.30 & 141.99 & $1.02 \mathrm{E}+15$ & $2.54 \mathrm{E}+21$ & $39.47 \%$ \\
\hline 29 & 5.75 & 8590.17 & 141.82 & $1.02 \mathrm{E}+15$ & $2.57 \mathrm{E}+21$ & $39.76 \%$ \\
\hline 30 & 5.25 & 8594.32 & 141.89 & $1.03 \mathrm{E}+15$ & $2.56 \mathrm{E}+21$ & $39.82 \%$ \\
\hline 31 & 4.75 & 8692.02 & 143.51 & $1.04 \mathrm{E}+15$ & $2.60 \mathrm{E}+21$ & $40.32 \%$ \\
\hline 32 & 4.25 & 8715.89 & 143.90 & $1.04 \mathrm{E}+15$ & $2.63 \mathrm{E}+21$ & $40.59 \%$ \\
\hline 33 & 3.75 & 8726.58 & 144.08 & $1.04 \mathrm{E}+15$ & $2.62 \mathrm{E}+21$ & $40.55 \%$ \\
\hline 34 & 3.25 & 8646.53 & 142.75 & $1.04 \mathrm{E}+15$ & $2.62 \mathrm{E}+21$ & $40.52 \%$ \\
\hline 35 & 2.75 & 8902.64 & 146.98 & $1.05 \mathrm{E}+15$ & $2.63 \mathrm{E}+21$ & $40.69 \%$ \\
\hline 36 & 2.25 & 8757.53 & 144.59 & $1.05 \mathrm{E}+15$ & $2.63 \mathrm{E}+21$ & $40.80 \%$ \\
\hline 37 & 1.75 & 8869.39 & 146.43 & $1.06 \mathrm{E}+15$ & $2.62 \mathrm{E}+21$ & $40.58 \%$ \\
\hline 38 & 1.25 & 8714.13 & 143.87 & $1.05 \mathrm{E}+15$ & $2.59 \mathrm{E}+21$ & $40.13 \%$ \\
\hline 39 & 0.75 & 8815.00 & 145.54 & $1.07 \mathrm{E}+15$ & $2.61 \mathrm{E}+21$ & $40.41 \%$ \\
\hline 40 & 0.25 & 8684.76 & 143.39 & $1.07 \mathrm{E}+15$ & $2.59 \mathrm{E}+21$ & $40.11 \%$ \\
\hline 41 & -0.25 & 8742.36 & 144.34 & $1.06 \mathrm{E}+15$ & $2.61 \mathrm{E}+21$ & $40.46 \%$ \\
\hline 42 & -0.75 & 8855.51 & 146.20 & $1.06 \mathrm{E}+15$ & $2.63 \mathrm{E}+21$ & $40.66 \%$ \\
\hline 43 & -1.25 & 8734.92 & 144.21 & $1.05 \mathrm{E}+15$ & $2.64 \mathrm{E}+21$ & $40.73 \%$ \\
\hline 44 & -1.75 & 8964.29 & 148.00 & $1.06 \mathrm{E}+15$ & $2.65 \mathrm{E}+21$ & $40.91 \%$ \\
\hline 45 & -2.25 & 8854.95 & 146.20 & $1.06 \mathrm{E}+15$ & $2.68 \mathrm{E}+21$ & $41.32 \%$ \\
\hline 46 & -2.75 & 8832.31 & 145.82 & $1.06 \mathrm{E}+15$ & $2.67 \mathrm{E}+21$ & $41.35 \%$ \\
\hline 47 & -3.25 & 8867.66 & 146.41 & $1.06 \mathrm{E}+15$ & $2.65 \mathrm{E}+21$ & $41.01 \%$ \\
\hline 48 & -3.75 & 8869.50 & 146.44 & $1.06 \mathrm{E}+15$ & $2.66 \mathrm{E}+21$ & $41.12 \%$ \\
\hline 49 & -4.25 & 8793.01 & 145.17 & $1.06 \mathrm{E}+15$ & $2.64 \mathrm{E}+21$ & $40.81 \%$ \\
\hline 50 & -4.75 & 8857.28 & 146.23 & $1.04 \mathrm{E}+15$ & $2.63 \mathrm{E}+21$ & $40.73 \%$ \\
\hline 51 & -5.25 & 8854.76 & 146.19 & $1.05 \mathrm{E}+15$ & $2.63 \mathrm{E}+21$ & $40.67 \%$ \\
\hline 52 & -5.75 & 8711.01 & 143.82 & $1.05 \mathrm{E}+15$ & $2.61 \mathrm{E}+21$ & $40.42 \%$ \\
\hline
\end{tabular}




\begin{tabular}{|l|l|l|l|l|l|l|}
\hline 53 & -6.25 & 8716.63 & 143.91 & $1.04 \mathrm{E}+15$ & $2.61 \mathrm{E}+21$ & $40.42 \%$ \\
\hline 54 & -6.75 & 8831.41 & 145.81 & $1.04 \mathrm{E}+15$ & $2.60 \mathrm{E}+21$ & $40.23 \%$ \\
\hline 55 & -7.25 & 8617.55 & 142.28 & $1.04 \mathrm{E}+15$ & $2.58 \mathrm{E}+21$ & $40.11 \%$ \\
\hline 56 & -7.75 & 8792.78 & 145.17 & $1.03 \mathrm{E}+15$ & $2.56 \mathrm{E}+21$ & $39.62 \%$ \\
\hline 57 & -8.25 & 8528.87 & 140.81 & $1.02 \mathrm{E}+15$ & $2.53 \mathrm{E}+21$ & $39.27 \%$ \\
\hline 58 & -8.75 & 8554.64 & 141.24 & $1.01 \mathrm{E}+15$ & $2.50 \mathrm{E}+21$ & $38.90 \%$ \\
\hline 59 & -9.25 & 8276.25 & 136.64 & $9.94 \mathrm{E}+14$ & $2.48 \mathrm{E}+21$ & $38.57 \%$ \\
\hline 60 & -9.75 & 8421.08 & 139.03 & $9.96 \mathrm{E}+14$ & $2.43 \mathrm{E}+21$ & $37.92 \%$ \\
\hline 61 & -10.25 & 8236.81 & 135.99 & $9.82 \mathrm{E}+14$ & $2.38 \mathrm{E}+21$ & $37.16 \%$ \\
\hline 62 & -10.75 & 8042.64 & 132.78 & $9.74 \mathrm{E}+14$ & $2.34 \mathrm{E}+21$ & $36.56 \%$ \\
\hline 63 & -11.25 & 8061.24 & 133.09 & $9.64 \mathrm{E}+14$ & $2.32 \mathrm{E}+21$ & $36.29 \%$ \\
\hline 64 & -11.75 & 8053.22 & 132.96 & $9.56 \mathrm{E}+14$ & $2.29 \mathrm{E}+21$ & $35.91 \%$ \\
\hline 65 & -12.25 & 7785.13 & 128.53 & $9.38 \mathrm{E}+14$ & $2.24 \mathrm{E}+21$ & $35.23 \%$ \\
\hline 66 & -12.75 & 7859.39 & 129.76 & $9.23 \mathrm{E}+14$ & $2.25 \mathrm{E}+21$ & $35.30 \%$ \\
\hline 67 & -13.25 & 7715.91 & 127.39 & $9.06 \mathrm{E}+14$ & $2.20 \mathrm{E}+21$ & $34.58 \%$ \\
\hline 68 & -13.75 & 7654.31 & 126.37 & $8.88 \mathrm{E}+14$ & $2.18 \mathrm{E}+21$ & $34.28 \%$ \\
\hline 69 & -14.25 & 7535.95 & 124.42 & $8.80 \mathrm{E}+14$ & $2.14 \mathrm{E}+21$ & $33.69 \%$ \\
\hline 70 & -14.75 & 7367.52 & 121.64 & $8.55 \mathrm{E}+14$ & $2.08 \mathrm{E}+21$ & $32.88 \%$ \\
\hline 71 & -15.25 & 7354.86 & 121.43 & $8.38 \mathrm{E}+14$ & $2.05 \mathrm{E}+21$ & $32.39 \%$ \\
\hline 72 & -15.75 & 7139.73 & 117.88 & $8.14 \mathrm{E}+14$ & $2.00 \mathrm{E}+21$ & $31.66 \%$ \\
\hline 73 & -16.25 & 7012.79 & 115.78 & $7.95 \mathrm{E}+14$ & $1.96 \mathrm{E}+21$ & $31.04 \%$ \\
\hline 74 & -16.75 & 6799.11 & 112.25 & $7.67 \mathrm{E}+14$ & $1.89 \mathrm{E}+21$ & $30.15 \%$ \\
\hline 75 & -17.25 & 6452.24 & 106.53 & $7.40 \mathrm{E}+14$ & $1.83 \mathrm{E}+21$ & $29.19 \%$ \\
\hline 76 & -17.75 & 6371.24 & 105.19 & $7.16 \mathrm{E}+14$ & $1.76 \mathrm{E}+21$ & $28.13 \%$ \\
\hline 77 & -18.25 & 6197.72 & 102.32 & $6.97 \mathrm{E}+14$ & $1.70 \mathrm{E}+21$ & $27.18 \%$ \\
\hline 78 & -18.75 & 6118.09 & 101.01 & $6.70 \mathrm{E}+14$ & $1.67 \mathrm{E}+21$ & $26.84 \%$ \\
\hline
\end{tabular}


Table 28: Cycle 150B, MCNP-Calculated HGRs and Neutron Flux for AFIP-7, Plate Position 2, 0 EFPD (BOC) Average Center Lobe Power at 24.2 MW. ${ }^{2}$

\begin{tabular}{|c|c|c|c|c|}
\hline Node & $\begin{array}{c}\text { Distance from } \\
\text { Centerline (in.) }\end{array}$ & $\begin{array}{l}\text { Fission Power } \\
\text { Density (W/cc) }\end{array}$ & $\begin{array}{l}\text { Surface Heat } \\
\text { Flux }\left(\mathrm{W} / \mathrm{cm}^{2}\right) \\
\end{array}$ & $\begin{array}{c}\text { Neutron Flux } \\
\left(\mathbf{n} / \mathrm{cm}^{2} \mathrm{sec}\right)\end{array}$ \\
\hline 1 & 19.25 & 5881.33 & 97.10 & $5.34 \mathrm{E}+14$ \\
\hline 2 & 18.75 & 5527.50 & 91.26 & $5.62 \mathrm{E}+14$ \\
\hline 3 & 18.25 & 5636.16 & 93.05 & $5.98 \mathrm{E}+14$ \\
\hline 4 & 17.75 & 5848.91 & 96.57 & $6.24 \mathrm{E}+14$ \\
\hline 5 & 17.25 & 6348.06 & 104.81 & $6.56 \mathrm{E}+14$ \\
\hline 6 & 16.75 & 6549.51 & 108.13 & $6.82 \mathrm{E}+14$ \\
\hline 7 & 16.25 & 6712.66 & 110.83 & $7.11 \mathrm{E}+14$ \\
\hline 8 & 15.75 & 6802.60 & 112.31 & $7.33 \mathrm{E}+14$ \\
\hline 9 & 15.25 & 7170.27 & 118.38 & $7.63 \mathrm{E}+14$ \\
\hline 10 & 14.75 & 7435.76 & 122.76 & $7.91 \mathrm{E}+14$ \\
\hline 11 & 14.25 & 7400.29 & 122.18 & $8.05 \mathrm{E}+14$ \\
\hline 13 & 13.75 & 7442.60 & 122.88 & $8.31 \mathrm{E}+14$ \\
\hline 14 & 13.25 & 7659.20 & 126.45 & $8.46 \mathrm{E}+14$ \\
\hline 15 & 12.75 & 7909.22 & 130.58 & $8.76 \mathrm{E}+14$ \\
\hline 16 & 12.25 & 7907.16 & 130.55 & $8.88 \mathrm{E}+14$ \\
\hline 17 & 11.75 & 8246.99 & 136.16 & $9.15 \mathrm{E}+14$ \\
\hline 18 & 11.25 & 8257.69 & 136.33 & $9.34 \mathrm{E}+14$ \\
\hline 19 & 10.75 & 8398.93 & 138.67 & $9.50 \mathrm{E}+14$ \\
\hline 20 & 10.25 & 8637.94 & 142.61 & $9.65 \mathrm{E}+14$ \\
\hline 21 & 9.75 & 8732.26 & 144.17 & $9.80 \mathrm{E}+14$ \\
\hline 22 & 9.25 & 9015.63 & 148.85 & $9.92 \mathrm{E}+14$ \\
\hline 23 & 8.75 & 9118.66 & 150.55 & $1.01 \mathrm{E}+15$ \\
\hline 24 & 8.25 & 9400.11 & 155.20 & $1.02 \mathrm{E}+15$ \\
\hline 25 & 7.75 & 9335.56 & 154.13 & $1.03 \mathrm{E}+15$ \\
\hline 26 & 7.25 & 9520.55 & 157.18 & $1.05 \mathrm{E}+15$ \\
\hline 27 & 6.75 & 9497.96 & 156.81 & $1.05 \mathrm{E}+15$ \\
\hline 28 & 6.25 & 9616.27 & 158.76 & $1.07 \mathrm{E}+15$ \\
\hline 29 & 5.75 & 9826.22 & 162.23 & $1.08 \mathrm{E}+15$ \\
\hline 30 & 5.25 & 9785.40 & 161.56 & $1.09 \mathrm{E}+15$ \\
\hline 31 & 4.75 & 9782.88 & 161.52 & $1.09 \mathrm{E}+15$ \\
\hline 32 & 4.25 & 9786.83 & 161.58 & $1.10 \mathrm{E}+15$ \\
\hline 33 & 3.75 & 9968.89 & 164.59 & $1.10 \mathrm{E}+15$ \\
\hline 34 & 3.25 & 9717.84 & 160.44 & $1.10 \mathrm{E}+15$ \\
\hline 35 & 2.75 & 10016.12 & 165.37 & $1.10 \mathrm{E}+15$ \\
\hline 36 & 2.25 & 10103.89 & 166.82 & $1.12 \mathrm{E}+15$ \\
\hline 37 & 1.75 & 9809.10 & 161.95 & $1.12 \mathrm{E}+15$ \\
\hline 38 & 1.25 & 9812.96 & 162.01 & $1.12 \mathrm{E}+15$ \\
\hline 39 & 0.75 & 9794.18 & 161.70 & $1.12 \mathrm{E}+15$ \\
\hline 40 & 0.25 & 9807.85 & 161.93 & $1.12 \mathrm{E}+15$ \\
\hline 41 & -0.25 & 9859.06 & 162.77 & $1.12 \mathrm{E}+15$ \\
\hline 42 & -0.75 & 9886.03 & 163.22 & $1.12 \mathrm{E}+15$ \\
\hline 43 & -1.25 & 9818.03 & 162.10 & $1.12 \mathrm{E}+15$ \\
\hline 44 & -1.75 & 10006.52 & 165.21 & $1.12 \mathrm{E}+15$ \\
\hline 45 & -2.25 & 10294.07 & 169.96 & $1.13 \mathrm{E}+15$ \\
\hline 46 & -2.75 & 10157.59 & 167.70 & $1.12 \mathrm{E}+15$ \\
\hline 47 & -3.25 & 10009.42 & 165.26 & $1.12 \mathrm{E}+15$ \\
\hline 48 & -3.75 & 10011.90 & 165.30 & $1.11 \mathrm{E}+15$ \\
\hline 49 & -4.25 & 9739.41 & 160.80 & $1.10 \mathrm{E}+15$ \\
\hline 50 & -4.75 & 10017.94 & 165.40 & $1.10 \mathrm{E}+15$ \\
\hline 51 & -5.25 & 10122.79 & 167.13 & $1.10 \mathrm{E}+15$ \\
\hline 52 & -5.75 & 9828.57 & 162.27 & $1.10 \mathrm{E}+15$ \\
\hline
\end{tabular}




\begin{tabular}{|l|l|l|l|l|}
\hline 53 & -6.25 & 9874.10 & 163.02 & $1.09 \mathrm{E}+15$ \\
\hline 54 & -6.75 & 9811.60 & 161.99 & $1.08 \mathrm{E}+15$ \\
\hline 55 & -7.25 & 9790.60 & 161.64 & $1.08 \mathrm{E}+15$ \\
\hline 56 & -7.75 & 9732.60 & 160.69 & $1.07 \mathrm{E}+15$ \\
\hline 57 & -8.25 & 9570.90 & 158.02 & $1.06 \mathrm{E}+15$ \\
\hline 58 & -8.75 & 9398.82 & 155.17 & $1.04 \mathrm{E}+15$ \\
\hline 59 & -9.25 & 9293.46 & 153.44 & $1.04 \mathrm{E}+15$ \\
\hline 60 & -9.75 & 9221.77 & 152.25 & $1.03 \mathrm{E}+15$ \\
\hline 61 & -10.25 & 8952.81 & 147.81 & $1.02 \mathrm{E}+15$ \\
\hline 62 & -10.75 & 8791.10 & 145.14 & $1.00 \mathrm{E}+15$ \\
\hline 63 & -11.25 & 8782.22 & 144.99 & $9.92 \mathrm{E}+14$ \\
\hline 64 & -11.75 & 8519.08 & 140.65 & $9.72 \mathrm{E}+14$ \\
\hline 65 & -12.25 & 8639.02 & 142.63 & $9.57 \mathrm{E}+14$ \\
\hline 66 & -12.75 & 8471.42 & 139.86 & $9.37 \mathrm{E}+14$ \\
\hline 67 & -13.25 & 8309.03 & 137.18 & $9.14 \mathrm{E}+14$ \\
\hline 68 & -13.75 & 8184.04 & 135.12 & $8.93 \mathrm{E}+14$ \\
\hline 69 & -14.25 & 8120.75 & 134.07 & $8.82 \mathrm{E}+14$ \\
\hline 70 & -14.75 & 7899.48 & 130.42 & $8.63 \mathrm{E}+14$ \\
\hline 71 & -15.25 & 7688.43 & 126.94 & $8.49 \mathrm{E}+14$ \\
\hline 72 & -15.75 & 7572.51 & 125.02 & $8.25 \mathrm{E}+14$ \\
\hline 73 & -16.25 & 7409.64 & 122.33 & $7.93 \mathrm{E}+14$ \\
\hline 74 & -16.75 & 7157.65 & 118.17 & $7.75 \mathrm{E}+14$ \\
\hline 75 & -17.25 & 6764.50 & 111.68 & $7.40 \mathrm{E}+14$ \\
\hline 76 & -17.75 & 6728.25 & 111.08 & $7.19 \mathrm{E}+14$ \\
\hline 77 & -18.25 & 6393.94 & 105.56 & $6.90 \mathrm{E}+14$ \\
\hline 78 & -18.75 & 6285.99 & 103.78 & $6.61 \mathrm{E}+14$ \\
\hline
\end{tabular}


Table 29: Cycle 150B, MCNP-Calculated HGRs, Neutron Flux, Depletion and Fission Density for AFIP-7, Plate Position 2, 18 EFPD (MOC1) Average Center Lobe Power at 24.2 MW. ${ }^{2}$

\begin{tabular}{|c|c|c|c|c|c|c|}
\hline Node & $\begin{array}{c}\text { Distance from } \\
\text { Centerline (in.) }\end{array}$ & $\begin{array}{l}\text { Fission Power } \\
\text { Density (W/cc) }\end{array}$ & $\begin{array}{l}\text { Surface Heat } \\
\text { Flux }\left(W / \mathrm{cm}^{2}\right)\end{array}$ & $\begin{array}{c}\text { Neutron Flux } \\
\left(\mathrm{n} / \mathrm{cm}^{2} \mathrm{sec}\right)\end{array}$ & $\begin{array}{l}\text { Fission Density } \\
\text { (fissions/cc) }\end{array}$ & $\begin{array}{c}\text { U-235 } \\
\text { Burnup (\%) }\end{array}$ \\
\hline 1 & 19.25 & 5925.96 & 97.84 & $5.31 \mathrm{E}+14$ & $1.19 \mathrm{E}+21$ & $17.41 \%$ \\
\hline 2 & 18.75 & 5377.36 & 88.78 & $5.48 \mathrm{E}+14$ & $1.11 \mathrm{E}+21$ & $16.27 \%$ \\
\hline 3 & 18.25 & 5483.53 & 90.53 & $5.80 \mathrm{E}+14$ & $1.14 \mathrm{E}+21$ & $16.72 \%$ \\
\hline 4 & 17.75 & 5975.98 & 98.66 & $6.09 \mathrm{E}+14$ & $1.21 \mathrm{E}+21$ & $17.78 \%$ \\
\hline 5 & 17.25 & 6284.65 & 103.76 & $6.39 \mathrm{E}+14$ & $1.27 \mathrm{E}+21$ & $18.46 \%$ \\
\hline 6 & 16.75 & 6421.00 & 106.01 & $6.63 \mathrm{E}+14$ & $1.32 \mathrm{E}+21$ & $19.28 \%$ \\
\hline 7 & 16.25 & 6533.71 & 107.87 & $6.85 \mathrm{E}+14$ & $1.36 \mathrm{E}+21$ & $19.73 \%$ \\
\hline 8 & 15.75 & 6806.18 & 112.37 & $7.14 \mathrm{E}+14$ & $1.40 \mathrm{E}+21$ & $20.42 \%$ \\
\hline 9 & 15.25 & 7044.01 & 116.30 & $7.36 \mathrm{E}+14$ & $1.44 \mathrm{E}+21$ & $20.87 \%$ \\
\hline 10 & 14.75 & 7120.67 & 117.56 & $7.62 \mathrm{E}+14$ & $1.50 \mathrm{E}+21$ & $21.77 \%$ \\
\hline 11 & 14.25 & 7186.20 & 118.64 & $7.74 \mathrm{E}+14$ & $1.53 \mathrm{E}+21$ & $22.15 \%$ \\
\hline 13 & 13.75 & 7159.18 & 118.20 & $7.96 \mathrm{E}+14$ & $1.55 \mathrm{E}+21$ & $22.38 \%$ \\
\hline 14 & 13.25 & 7377.52 & 121.80 & $8.11 \mathrm{E}+14$ & $1.57 \mathrm{E}+21$ & $22.68 \%$ \\
\hline 15 & 12.75 & 7558.76 & 124.80 & $8.35 \mathrm{E}+14$ & $1.60 \mathrm{E}+21$ & $23.05 \%$ \\
\hline 16 & 12.25 & 7577.58 & 125.11 & $8.47 \mathrm{E}+14$ & $1.64 \mathrm{E}+21$ & $23.58 \%$ \\
\hline 17 & 11.75 & 7713.24 & 127.35 & $8.69 \mathrm{E}+14$ & $1.67 \mathrm{E}+21$ & $23.96 \%$ \\
\hline 18 & 11.25 & 7812.03 & 128.98 & $8.81 \mathrm{E}+14$ & $1.70 \mathrm{E}+21$ & $24.41 \%$ \\
\hline 19 & 10.75 & 7940.92 & 131.10 & $8.95 \mathrm{E}+14$ & $1.74 \mathrm{E}+21$ & $24.88 \%$ \\
\hline 20 & 10.25 & 8173.17 & 134.94 & $9.11 \mathrm{E}+14$ & $1.79 \mathrm{E}+21$ & $25.63 \%$ \\
\hline 21 & 9.75 & 8330.78 & 137.54 & $9.22 \mathrm{E}+14$ & $1.83 \mathrm{E}+21$ & $26.15 \%$ \\
\hline 22 & 9.25 & 8553.65 & 141.22 & $9.34 \mathrm{E}+14$ & $1.86 \mathrm{E}+21$ & $26.54 \%$ \\
\hline 23 & 8.75 & 8628.04 & 142.45 & $9.43 \mathrm{E}+14$ & $1.89 \mathrm{E}+21$ & $26.92 \%$ \\
\hline 24 & 8.25 & 8785.46 & 145.05 & $9.56 \mathrm{E}+14$ & $1.92 \mathrm{E}+21$ & $27.32 \%$ \\
\hline 25 & 7.75 & 8574.50 & 141.56 & $9.64 \mathrm{E}+14$ & $1.92 \mathrm{E}+21$ & $27.33 \%$ \\
\hline 26 & 7.25 & 8804.24 & 145.36 & $9.65 \mathrm{E}+14$ & $1.93 \mathrm{E}+21$ & $27.43 \%$ \\
\hline 27 & 6.75 & 8929.44 & 147.43 & $9.78 \mathrm{E}+14$ & $1.97 \mathrm{E}+21$ & $28.09 \%$ \\
\hline 28 & 6.25 & 8977.79 & 148.22 & $9.85 \mathrm{E}+14$ & $1.99 \mathrm{E}+21$ & $28.24 \%$ \\
\hline 29 & 5.75 & 9217.65 & 152.18 & $9.99 \mathrm{E}+14$ & $2.00 \mathrm{E}+21$ & $28.44 \%$ \\
\hline 30 & 5.25 & 8955.22 & 147.85 & $1.01 \mathrm{E}+15$ & $2.02 \mathrm{E}+21$ & $28.61 \%$ \\
\hline 31 & 4.75 & 9147.68 & 151.03 & $1.00 \mathrm{E}+15$ & $2.03 \mathrm{E}+21$ & $28.83 \%$ \\
\hline 32 & 4.25 & 9163.32 & 151.29 & $1.02 \mathrm{E}+15$ & $2.04 \mathrm{E}+21$ & $28.93 \%$ \\
\hline 33 & 3.75 & 9225.77 & 152.32 & $1.02 \mathrm{E}+15$ & $2.04 \mathrm{E}+21$ & $28.97 \%$ \\
\hline 34 & 3.25 & 9106.41 & 150.35 & $1.01 \mathrm{E}+15$ & $2.05 \mathrm{E}+21$ & $29.03 \%$ \\
\hline 35 & 2.75 & 9217.77 & 152.19 & $1.02 \mathrm{E}+15$ & $2.06 \mathrm{E}+21$ & $29.17 \%$ \\
\hline 36 & 2.25 & 9069.16 & 149.73 & $1.02 \mathrm{E}+15$ & $2.06 \mathrm{E}+21$ & $29.17 \%$ \\
\hline 37 & 1.75 & 9035.16 & 149.17 & $1.02 \mathrm{E}+15$ & $2.05 \mathrm{E}+21$ & $29.10 \%$ \\
\hline 38 & 1.25 & 9014.29 & 148.83 & $1.03 \mathrm{E}+15$ & $2.01 \mathrm{E}+21$ & $28.51 \%$ \\
\hline 39 & 0.75 & 9170.92 & 151.41 & $1.03 \mathrm{E}+15$ & $2.02 \mathrm{E}+21$ & $28.58 \%$ \\
\hline 40 & 0.25 & 9068.41 & 149.72 & $1.03 \mathrm{E}+15$ & $2.02 \mathrm{E}+21$ & $28.68 \%$ \\
\hline 41 & -0.25 & 9136.39 & 150.84 & $1.03 \mathrm{E}+15$ & $2.03 \mathrm{E}+21$ & $28.83 \%$ \\
\hline 42 & -0.75 & 9134.03 & 150.80 & $1.03 \mathrm{E}+15$ & $2.04 \mathrm{E}+21$ & $28.98 \%$ \\
\hline 43 & -1.25 & 9105.83 & 150.34 & $1.04 \mathrm{E}+15$ & $2.06 \mathrm{E}+21$ & $29.16 \%$ \\
\hline 44 & -1.75 & 9313.13 & 153.76 & $1.03 \mathrm{E}+15$ & $2.08 \mathrm{E}+21$ & $29.38 \%$ \\
\hline 45 & -2.25 & 9309.36 & 153.70 & $1.03 \mathrm{E}+15$ & $2.10 \mathrm{E}+21$ & $29.66 \%$ \\
\hline 46 & -2.75 & 9302.47 & 153.58 & $1.04 \mathrm{E}+15$ & $2.08 \mathrm{E}+21$ & $29.46 \%$ \\
\hline 47 & -3.25 & 9270.18 & 153.05 & $1.03 \mathrm{E}+15$ & $2.07 \mathrm{E}+21$ & $29.28 \%$ \\
\hline 48 & -3.75 & 9272.53 & 153.09 & $1.03 \mathrm{E}+15$ & $2.09 \mathrm{E}+21$ & $29.57 \%$ \\
\hline 49 & -4.25 & 9051.41 & 149.44 & $1.02 \mathrm{E}+15$ & $2.04 \mathrm{E}+21$ & $28.92 \%$ \\
\hline 50 & -4.75 & 9215.81 & 152.15 & $1.02 \mathrm{E}+15$ & $2.06 \mathrm{E}+21$ & $29.10 \%$ \\
\hline 51 & -5.25 & 9086.49 & 150.02 & $1.01 \mathrm{E}+15$ & $2.06 \mathrm{E}+21$ & $29.21 \%$ \\
\hline 52 & -5.75 & 9106.61 & 150.35 & $1.02 \mathrm{E}+15$ & $2.03 \mathrm{E}+21$ & $28.79 \%$ \\
\hline
\end{tabular}




\begin{tabular}{|l|l|l|l|l|l|l|}
\hline 53 & -6.25 & 9164.28 & 151.30 & $1.02 \mathrm{E}+15$ & $2.03 \mathrm{E}+21$ & $28.86 \%$ \\
\hline 54 & -6.75 & 9079.50 & 149.90 & $1.01 \mathrm{E}+15$ & $2.02 \mathrm{E}+21$ & $28.68 \%$ \\
\hline 55 & -7.25 & 9130.14 & 150.74 & $1.01 \mathrm{E}+15$ & $2.01 \mathrm{E}+21$ & $28.61 \%$ \\
\hline 56 & -7.75 & 8999.65 & 148.58 & $1.00 \mathrm{E}+15$ & $1.99 \mathrm{E}+21$ & $28.28 \%$ \\
\hline 57 & -8.25 & 8965.08 & 148.01 & $9.92 \mathrm{E}+14$ & $1.98 \mathrm{E}+21$ & $28.08 \%$ \\
\hline 58 & -8.75 & 8801.30 & 145.31 & $9.78 \mathrm{E}+14$ & $1.97 \mathrm{E}+21$ & $28.02 \%$ \\
\hline 59 & -9.25 & 8856.06 & 146.21 & $9.75 \mathrm{E}+14$ & $1.94 \mathrm{E}+21$ & $27.59 \%$ \\
\hline 60 & -9.75 & 8622.74 & 142.36 & $9.64 \mathrm{E}+14$ & $1.90 \mathrm{E}+21$ & $27.03 \%$ \\
\hline 61 & -10.25 & 8436.29 & 139.28 & $9.52 \mathrm{E}+14$ & $1.83 \mathrm{E}+21$ & $26.15 \%$ \\
\hline 62 & -10.75 & 8292.42 & 136.91 & $9.46 \mathrm{E}+14$ & $1.81 \mathrm{E}+21$ & $25.84 \%$ \\
\hline 63 & -11.25 & 8269.09 & 136.52 & $9.31 \mathrm{E}+14$ & $1.80 \mathrm{E}+21$ & $25.75 \%$ \\
\hline 64 & -11.75 & 8166.78 & 134.83 & $9.17 \mathrm{E}+14$ & $1.76 \mathrm{E}+21$ & $25.27 \%$ \\
\hline 65 & -12.25 & 8169.03 & 134.87 & $9.06 \mathrm{E}+14$ & $1.75 \mathrm{E}+21$ & $25.17 \%$ \\
\hline 66 & -12.75 & 8007.94 & 132.21 & $8.94 \mathrm{E}+14$ & $1.75 \mathrm{E}+21$ & $25.03 \%$ \\
\hline 67 & -13.25 & 7838.31 & 129.41 & $8.68 \mathrm{E}+14$ & $1.73 \mathrm{E}+21$ & $24.77 \%$ \\
\hline 68 & -13.75 & 7759.56 & 128.11 & $8.53 \mathrm{E}+14$ & $1.69 \mathrm{E}+21$ & $24.26 \%$ \\
\hline 69 & -14.25 & 7829.52 & 129.27 & $8.44 \mathrm{E}+14$ & $1.67 \mathrm{E}+21$ & $23.96 \%$ \\
\hline 70 & -14.75 & 7628.48 & 125.95 & $8.29 \mathrm{E}+14$ & $1.64 \mathrm{E}+21$ & $23.50 \%$ \\
\hline 71 & -15.25 & 7556.84 & 124.76 & $8.09 \mathrm{E}+14$ & $1.58 \mathrm{E}+21$ & $22.75 \%$ \\
\hline 72 & -15.75 & 7452.86 & 123.05 & $7.92 \mathrm{E}+14$ & $1.56 \mathrm{E}+21$ & $22.53 \%$ \\
\hline 73 & -16.25 & 7165.86 & 118.31 & $7.73 \mathrm{E}+14$ & $1.51 \mathrm{E}+21$ & $21.85 \%$ \\
\hline 74 & -16.75 & 7021.99 & 115.93 & $7.42 \mathrm{E}+14$ & $1.47 \mathrm{E}+21$ & $21.32 \%$ \\
\hline 75 & -17.25 & 6768.77 & 111.75 & $7.21 \mathrm{E}+14$ & $1.41 \mathrm{E}+21$ & $20.50 \%$ \\
\hline 76 & -17.75 & 6604.02 & 109.03 & $6.95 \mathrm{E}+14$ & $1.35 \mathrm{E}+21$ & $19.66 \%$ \\
\hline 77 & -18.25 & 6350.46 & 104.85 & $6.79 \mathrm{E}+14$ & $1.29 \mathrm{E}+21$ & $18.83 \%$ \\
\hline 78 & -18.75 & 6192.63 & 102.24 & $6.40 \mathrm{E}+14$ & $1.27 \mathrm{E}+21$ & $18.46 \%$ \\
\hline
\end{tabular}


Table 30: Cycle 150B, MCNP-Calculated HGRs, Neutron Flux, Depletion and Fission Density for AFIP-7, Plate Position 2, 31 EFPD (MOC2) Average Center Lobe Power at 24.2 MW. ${ }^{2}$

\begin{tabular}{|c|c|c|c|c|c|c|}
\hline Node & $\begin{array}{c}\text { Distance from } \\
\text { Centerline (in.) }\end{array}$ & $\begin{array}{l}\text { Fission Power } \\
\text { Density (W/cc) }\end{array}$ & $\begin{array}{l}\text { Surface Heat } \\
\text { Flux }\left(W / \mathrm{cm}^{2}\right)\end{array}$ & $\begin{array}{c}\text { Neutron Flux } \\
\left(\mathrm{n} / \mathrm{cm}^{2} \mathrm{sec}\right)\end{array}$ & $\begin{array}{l}\text { Fission Density } \\
\text { (fissions/cc) }\end{array}$ & $\begin{array}{c}\text { U-235 } \\
\text { Burnup (\%) }\end{array}$ \\
\hline 1 & 19.25 & 5934.41 & 97.98 & $5.39 \mathrm{E}+14$ & $1.41 \mathrm{E}+21$ & $20.50 \%$ \\
\hline 2 & 18.75 & 5549.89 & 91.63 & $5.63 \mathrm{E}+14$ & $1.31 \mathrm{E}+21$ & $19.06 \%$ \\
\hline 3 & 18.25 & 5806.62 & 95.87 & $5.99 \mathrm{E}+14$ & $1.35 \mathrm{E}+21$ & $19.58 \%$ \\
\hline 4 & 17.75 & 5903.43 & 97.47 & $6.18 \mathrm{E}+14$ & $1.43 \mathrm{E}+21$ & $20.87 \%$ \\
\hline 5 & 17.25 & 6200.16 & 102.36 & $6.54 \mathrm{E}+14$ & $1.50 \mathrm{E}+21$ & $21.70 \%$ \\
\hline 6 & 16.75 & 6581.57 & 108.66 & $6.87 \mathrm{E}+14$ & $1.56 \mathrm{E}+21$ & $22.60 \%$ \\
\hline 7 & 16.25 & 6579.22 & 108.62 & $7.09 \mathrm{E}+14$ & $1.60 \mathrm{E}+21$ & $23.13 \%$ \\
\hline 8 & 15.75 & 6951.52 & 114.77 & $7.32 \mathrm{E}+14$ & $1.65 \mathrm{E}+21$ & $23.89 \%$ \\
\hline 9 & 15.25 & 6981.90 & 115.27 & $7.57 \mathrm{E}+14$ & $1.70 \mathrm{E}+21$ & $24.49 \%$ \\
\hline 10 & 14.75 & 7208.83 & 119.02 & $7.72 \mathrm{E}+14$ & $1.77 \mathrm{E}+21$ & $25.40 \%$ \\
\hline 11 & 14.25 & 7119.52 & 117.54 & $7.84 \mathrm{E}+14$ & $1.79 \mathrm{E}+21$ & $25.83 \%$ \\
\hline 13 & 13.75 & 7291.27 & 120.38 & $8.09 \mathrm{E}+14$ & $1.81 \mathrm{E}+21$ & $26.02 \%$ \\
\hline 14 & 13.25 & 7512.66 & 124.03 & $8.30 \mathrm{E}+14$ & $1.84 \mathrm{E}+21$ & $26.47 \%$ \\
\hline 15 & 12.75 & 7621.23 & 125.83 & $8.51 \mathrm{E}+14$ & $1.88 \mathrm{E}+21$ & $26.89 \%$ \\
\hline 16 & 12.25 & 7795.44 & 128.70 & $8.64 \mathrm{E}+14$ & $1.92 \mathrm{E}+21$ & $27.48 \%$ \\
\hline 17 & 11.75 & 7837.04 & 129.39 & $8.95 \mathrm{E}+14$ & $1.95 \mathrm{E}+21$ & $27.92 \%$ \\
\hline 18 & 11.25 & 8012.75 & 132.29 & $9.02 \mathrm{E}+14$ & $1.99 \mathrm{E}+21$ & $28.42 \%$ \\
\hline 19 & 10.75 & 8179.99 & 135.05 & $9.06 \mathrm{E}+14$ & $2.03 \mathrm{E}+21$ & $28.91 \%$ \\
\hline 20 & 10.25 & 8081.02 & 133.42 & $9.21 \mathrm{E}+14$ & $2.09 \mathrm{E}+21$ & $29.76 \%$ \\
\hline 21 & 9.75 & 8504.61 & 140.41 & $9.41 \mathrm{E}+14$ & $2.14 \mathrm{E}+21$ & $30.36 \%$ \\
\hline 22 & 9.25 & 8713.57 & 143.86 & $9.57 \mathrm{E}+14$ & $2.17 \mathrm{E}+21$ & $30.85 \%$ \\
\hline 23 & 8.75 & 8722.83 & 144.01 & $9.60 \mathrm{E}+14$ & $2.21 \mathrm{E}+21$ & $31.24 \%$ \\
\hline 24 & 8.25 & 8735.31 & 144.22 & $9.68 \mathrm{E}+14$ & $2.24 \mathrm{E}+21$ & $31.72 \%$ \\
\hline 25 & 7.75 & 8776.93 & 144.91 & $9.73 \mathrm{E}+14$ & $2.23 \mathrm{E}+21$ & $31.66 \%$ \\
\hline 26 & 7.25 & 8848.50 & 146.09 & $9.83 \mathrm{E}+14$ & $2.25 \mathrm{E}+21$ & $31.86 \%$ \\
\hline 27 & 6.75 & 8942.14 & 147.63 & $9.91 \mathrm{E}+14$ & $2.30 \mathrm{E}+21$ & $32.55 \%$ \\
\hline 28 & 6.25 & 8956.84 & 147.88 & $1.00 \mathrm{E}+15$ & $2.32 \mathrm{E}+21$ & $32.74 \%$ \\
\hline 29 & 5.75 & 9038.34 & 149.22 & $1.01 \mathrm{E}+15$ & $2.34 \mathrm{E}+21$ & $33.05 \%$ \\
\hline 30 & 5.25 & 8979.36 & 148.25 & $1.01 \mathrm{E}+15$ & $2.34 \mathrm{E}+21$ & $33.09 \%$ \\
\hline 31 & 4.75 & 9082.32 & 149.95 & $1.02 \mathrm{E}+15$ & $2.37 \mathrm{E}+21$ & $33.40 \%$ \\
\hline 32 & 4.25 & 9130.33 & 150.74 & $1.03 \mathrm{E}+15$ & $2.38 \mathrm{E}+21$ & $33.51 \%$ \\
\hline 33 & 3.75 & 9006.00 & 148.69 & $1.02 \mathrm{E}+15$ & $2.38 \mathrm{E}+21$ & $33.56 \%$ \\
\hline 34 & 3.25 & 9221.99 & 152.26 & $1.03 \mathrm{E}+15$ & $2.38 \mathrm{E}+21$ & $33.58 \%$ \\
\hline 35 & 2.75 & 9216.92 & 152.17 & $1.03 \mathrm{E}+15$ & $2.39 \mathrm{E}+21$ & $33.78 \%$ \\
\hline 36 & 2.25 & 9118.26 & 150.54 & $1.04 \mathrm{E}+15$ & $2.39 \mathrm{E}+21$ & $33.69 \%$ \\
\hline 37 & 1.75 & 9060.76 & 149.59 & $1.04 \mathrm{E}+15$ & $2.38 \mathrm{E}+21$ & $33.59 \%$ \\
\hline 38 & 1.25 & 9190.55 & 151.74 & $1.05 \mathrm{E}+15$ & $2.34 \mathrm{E}+21$ & $33.01 \%$ \\
\hline 39 & 0.75 & 9126.72 & 150.68 & $1.05 \mathrm{E}+15$ & $2.35 \mathrm{E}+21$ & $33.16 \%$ \\
\hline 40 & 0.25 & 9046.22 & 149.35 & $1.05 \mathrm{E}+15$ & $2.35 \mathrm{E}+21$ & $33.22 \%$ \\
\hline 41 & -0.25 & 9228.21 & 152.36 & $1.04 \mathrm{E}+15$ & $2.37 \mathrm{E}+21$ & $33.38 \%$ \\
\hline 42 & -0.75 & 9239.26 & 152.54 & $1.05 \mathrm{E}+15$ & $2.38 \mathrm{E}+21$ & $33.51 \%$ \\
\hline 43 & -1.25 & 9143.62 & 150.96 & $1.05 \mathrm{E}+15$ & $2.39 \mathrm{E}+21$ & $33.68 \%$ \\
\hline 44 & -1.75 & 9335.42 & 154.13 & $1.05 \mathrm{E}+15$ & $2.42 \mathrm{E}+21$ & $34.00 \%$ \\
\hline 45 & -2.25 & 9354.20 & 154.44 & $1.05 \mathrm{E}+15$ & $2.44 \mathrm{E}+21$ & $34.29 \%$ \\
\hline 46 & -2.75 & 9262.22 & 152.92 & $1.04 \mathrm{E}+15$ & $2.42 \mathrm{E}+21$ & $34.09 \%$ \\
\hline 47 & -3.25 & 9291.72 & 153.41 & $1.05 \mathrm{E}+15$ & $2.41 \mathrm{E}+21$ & $33.90 \%$ \\
\hline 48 & -3.75 & 9256.24 & 152.82 & $1.04 \mathrm{E}+15$ & $2.43 \mathrm{E}+21$ & $34.19 \%$ \\
\hline 49 & -4.25 & 9339.25 & 154.19 & $1.04 \mathrm{E}+15$ & $2.37 \mathrm{E}+21$ & $33.41 \%$ \\
\hline 50 & -4.75 & 9250.28 & 152.72 & $1.03 \mathrm{E}+15$ & $2.39 \mathrm{E}+21$ & $33.70 \%$ \\
\hline 51 & -5.25 & 9148.29 & 151.04 & $1.04 \mathrm{E}+15$ & $2.39 \mathrm{E}+21$ & $33.74 \%$ \\
\hline 52 & -5.75 & 9053.25 & 149.47 & $1.03 \mathrm{E}+15$ & $2.37 \mathrm{E}+21$ & $33.32 \%$ \\
\hline
\end{tabular}




\begin{tabular}{|l|l|l|l|l|l|l|}
\hline 53 & -6.25 & 9047.08 & 149.37 & $1.02 \mathrm{E}+15$ & $2.37 \mathrm{E}+21$ & $33.41 \%$ \\
\hline 54 & -6.75 & 9146.07 & 151.00 & $1.02 \mathrm{E}+15$ & $2.35 \mathrm{E}+21$ & $33.21 \%$ \\
\hline 55 & -7.25 & 9040.77 & 149.26 & $1.01 \mathrm{E}+15$ & $2.35 \mathrm{E}+21$ & $33.17 \%$ \\
\hline 56 & -7.75 & 8895.61 & 146.87 & $1.01 \mathrm{E}+15$ & $2.32 \mathrm{E}+21$ & $32.79 \%$ \\
\hline 57 & -8.25 & 9041.78 & 149.28 & $1.01 \mathrm{E}+15$ & $2.31 \mathrm{E}+21$ & $32.57 \%$ \\
\hline 58 & -8.75 & 8819.85 & 145.62 & $9.95 \mathrm{E}+14$ & $2.29 \mathrm{E}+21$ & $32.44 \%$ \\
\hline 59 & -9.25 & 8830.24 & 145.79 & $9.88 \mathrm{E}+14$ & $2.26 \mathrm{E}+21$ & $32.04 \%$ \\
\hline 60 & -9.75 & 8655.55 & 142.90 & $9.75 \mathrm{E}+14$ & $2.22 \mathrm{E}+21$ & $31.35 \%$ \\
\hline 61 & -10.25 & 8397.22 & 138.64 & $9.68 \mathrm{E}+14$ & $2.14 \mathrm{E}+21$ & $30.40 \%$ \\
\hline 62 & -10.75 & 8356.46 & 137.97 & $9.54 \mathrm{E}+14$ & $2.11 \mathrm{E}+21$ & $30.03 \%$ \\
\hline 63 & -11.25 & 8384.93 & 138.44 & $9.44 \mathrm{E}+14$ & $2.10 \mathrm{E}+21$ & $29.93 \%$ \\
\hline 64 & -11.75 & 8274.28 & 136.61 & $9.35 \mathrm{E}+14$ & $2.06 \mathrm{E}+21$ & $29.40 \%$ \\
\hline 65 & -12.25 & 8278.03 & 136.67 & $9.28 \mathrm{E}+14$ & $2.05 \mathrm{E}+21$ & $29.32 \%$ \\
\hline 66 & -12.75 & 8202.66 & 135.43 & $9.03 \mathrm{E}+14$ & $2.04 \mathrm{E}+21$ & $29.10 \%$ \\
\hline 67 & -13.25 & 8173.03 & 134.94 & $8.89 \mathrm{E}+14$ & $2.01 \mathrm{E}+21$ & $28.76 \%$ \\
\hline 68 & -13.75 & 8039.17 & 132.73 & $8.88 \mathrm{E}+14$ & $1.98 \mathrm{E}+21$ & $28.24 \%$ \\
\hline 69 & -14.25 & 7908.17 & 130.56 & $8.59 \mathrm{E}+14$ & $1.95 \mathrm{E}+21$ & $27.96 \%$ \\
\hline 70 & -14.75 & 7662.97 & 126.52 & $8.50 \mathrm{E}+14$ & $1.92 \mathrm{E}+21$ & $27.43 \%$ \\
\hline 71 & -15.25 & 7579.35 & 125.14 & $8.24 \mathrm{E}+14$ & $1.86 \mathrm{E}+21$ & $26.63 \%$ \\
\hline 72 & -15.75 & 7404.79 & 122.25 & $8.05 \mathrm{E}+14$ & $1.83 \mathrm{E}+21$ & $26.35 \%$ \\
\hline 73 & -16.25 & 7237.17 & 119.49 & $7.88 \mathrm{E}+14$ & $1.77 \mathrm{E}+21$ & $25.52 \%$ \\
\hline 74 & -16.75 & 7055.27 & 116.48 & $7.58 \mathrm{E}+14$ & $1.73 \mathrm{E}+21$ & $24.90 \%$ \\
\hline 75 & -17.25 & 6825.05 & 112.68 & $7.33 \mathrm{E}+14$ & $1.66 \mathrm{E}+21$ & $23.96 \%$ \\
\hline 76 & -17.75 & 6508.09 & 107.45 & $6.97 \mathrm{E}+14$ & $1.60 \mathrm{E}+21$ & $23.05 \%$ \\
\hline 77 & -18.25 & 6329.56 & 104.50 & $6.83 \mathrm{E}+14$ & $1.53 \mathrm{E}+21$ & $22.15 \%$ \\
\hline 78 & -18.75 & 6263.02 & 103.40 & $6.58 \mathrm{E}+14$ & $1.49 \mathrm{E}+21$ & $21.70 \%$ \\
\hline
\end{tabular}


Table 31: Cycle 150B, MCNP-Calculated HGRs, Neutron Flux, Depletion and Fission Density for AFIP-7, Plate Position 2, 42.0 EFPD (EOC) Average Center Lobe Power at 24.2 MW. ${ }^{2}$

\begin{tabular}{|c|c|c|c|c|c|c|}
\hline Node & $\begin{array}{c}\text { Distance from } \\
\text { Centerline (in.) }\end{array}$ & $\begin{array}{l}\text { Fission Power } \\
\text { Density (W/cc) }\end{array}$ & $\begin{array}{l}\text { Surface Heat } \\
\text { Flux }\left(W / \mathrm{cm}^{2}\right)\end{array}$ & $\begin{array}{c}\text { Neutron Flux } \\
\left(\mathrm{n} / \mathrm{cm}^{2} \mathrm{sec}\right)\end{array}$ & $\begin{array}{l}\text { Fission Density } \\
\text { (fissions/cc) }\end{array}$ & $\begin{array}{c}\text { U-235 } \\
\text { Burnup (\%) }\end{array}$ \\
\hline 1 & 19.25 & 5939.32 & 98.06 & $5.45 \mathrm{E}+14$ & $1.60 \mathrm{E}+21$ & $23.05 \%$ \\
\hline 2 & 18.75 & 5504.95 & 90.89 & $5.68 \mathrm{E}+14$ & $1.48 \mathrm{E}+21$ & $21.55 \%$ \\
\hline 3 & 18.25 & 5789.08 & 95.58 & $6.06 \mathrm{E}+14$ & $1.52 \mathrm{E}+21$ & $22.15 \%$ \\
\hline 4 & 17.75 & 5924.35 & 97.81 & $6.25 \mathrm{E}+14$ & $1.62 \mathrm{E}+21$ & $23.43 \%$ \\
\hline 5 & 17.25 & 6184.46 & 102.11 & $6.61 \mathrm{E}+14$ & $1.69 \mathrm{E}+21$ & $24.41 \%$ \\
\hline 6 & 16.75 & 6517.83 & 107.61 & $6.94 \mathrm{E}+14$ & $1.76 \mathrm{E}+21$ & $25.47 \%$ \\
\hline 7 & 16.25 & 6549.34 & 108.13 & $7.15 \mathrm{E}+14$ & $1.81 \mathrm{E}+21$ & $26.00 \%$ \\
\hline 8 & 15.75 & 6922.94 & 114.30 & $7.40 \mathrm{E}+14$ & $1.87 \mathrm{E}+21$ & $26.93 \%$ \\
\hline 9 & 15.25 & 6939.57 & 114.57 & $7.64 \mathrm{E}+14$ & $1.91 \mathrm{E}+21$ & $27.49 \%$ \\
\hline 10 & 14.75 & 7147.99 & 118.01 & $7.79 \mathrm{E}+14$ & $1.99 \mathrm{E}+21$ & $28.51 \%$ \\
\hline 11 & 14.25 & 7094.13 & 117.12 & $7.90 \mathrm{E}+14$ & $2.01 \mathrm{E}+21$ & $28.88 \%$ \\
\hline 13 & 13.75 & 7269.01 & 120.01 & $8.17 \mathrm{E}+14$ & $2.04 \mathrm{E}+21$ & $29.13 \%$ \\
\hline 14 & 13.25 & 7459.78 & 123.16 & $8.38 \mathrm{E}+14$ & $2.07 \mathrm{E}+21$ & $29.67 \%$ \\
\hline 15 & 12.75 & 7580.58 & 125.16 & $8.60 \mathrm{E}+14$ & $2.11 \mathrm{E}+21$ & $30.15 \%$ \\
\hline 16 & 12.25 & 7702.60 & 127.17 & $8.71 \mathrm{E}+14$ & $2.16 \mathrm{E}+21$ & $30.80 \%$ \\
\hline 17 & 11.75 & 7787.02 & 128.56 & $9.03 \mathrm{E}+14$ & $2.19 \mathrm{E}+21$ & $31.24 \%$ \\
\hline 18 & 11.25 & 7942.73 & 131.13 & $9.08 \mathrm{E}+14$ & $2.23 \mathrm{E}+21$ & $31.80 \%$ \\
\hline 19 & 10.75 & 8093.19 & 133.62 & $9.13 \mathrm{E}+14$ & $2.28 \mathrm{E}+21$ & $32.35 \%$ \\
\hline 20 & 10.25 & 8027.77 & 132.54 & $9.28 \mathrm{E}+14$ & $2.34 \mathrm{E}+21$ & $33.16 \%$ \\
\hline 21 & 9.75 & 8425.72 & 139.11 & $9.49 \mathrm{E}+14$ & $2.40 \mathrm{E}+21$ & $33.93 \%$ \\
\hline 22 & 9.25 & 8630.48 & 142.49 & $9.63 \mathrm{E}+14$ & $2.44 \mathrm{E}+21$ & $34.50 \%$ \\
\hline 23 & 8.75 & 8620.60 & 142.33 & $9.68 \mathrm{E}+14$ & $2.47 \mathrm{E}+21$ & $34.88 \%$ \\
\hline 24 & 8.25 & 8607.12 & 142.10 & $9.77 \mathrm{E}+14$ & $2.51 \mathrm{E}+21$ & $35.39 \%$ \\
\hline 25 & 7.75 & 8676.99 & 143.26 & $9.81 \mathrm{E}+14$ & $2.50 \mathrm{E}+21$ & $35.33 \%$ \\
\hline 26 & 7.25 & 8726.89 & 144.08 & $9.91 \mathrm{E}+14$ & $2.52 \mathrm{E}+21$ & $35.54 \%$ \\
\hline 27 & 6.75 & 8797.61 & 145.25 & $1.00 \mathrm{E}+15$ & $2.58 \mathrm{E}+21$ & $36.25 \%$ \\
\hline 28 & 6.25 & 8870.87 & 146.46 & $1.01 \mathrm{E}+15$ & $2.59 \mathrm{E}+21$ & $36.45 \%$ \\
\hline 29 & 5.75 & 8951.77 & 147.79 & $1.02 \mathrm{E}+15$ & $2.62 \mathrm{E}+21$ & $36.82 \%$ \\
\hline 30 & 5.25 & 8852.72 & 146.16 & $1.02 \mathrm{E}+15$ & $2.62 \mathrm{E}+21$ & $36.83 \%$ \\
\hline 31 & 4.75 & 9003.21 & 148.64 & $1.03 \mathrm{E}+15$ & $2.65 \mathrm{E}+21$ & $37.15 \%$ \\
\hline 32 & 4.25 & 9008.64 & 148.73 & $1.04 \mathrm{E}+15$ & $2.66 \mathrm{E}+21$ & $37.30 \%$ \\
\hline 33 & 3.75 & 8939.57 & 147.59 & $1.03 \mathrm{E}+15$ & $2.66 \mathrm{E}+21$ & $37.30 \%$ \\
\hline 34 & 3.25 & 9116.32 & 150.51 & $1.04 \mathrm{E}+15$ & $2.66 \mathrm{E}+21$ & $37.38 \%$ \\
\hline 35 & 2.75 & 9108.38 & 150.38 & $1.04 \mathrm{E}+15$ & $2.68 \mathrm{E}+21$ & $37.62 \%$ \\
\hline 36 & 2.25 & 9021.20 & 148.94 & $1.04 \mathrm{E}+15$ & $2.67 \mathrm{E}+21$ & $37.45 \%$ \\
\hline 37 & 1.75 & 8965.39 & 148.02 & $1.05 \mathrm{E}+15$ & $2.66 \mathrm{E}+21$ & $37.34 \%$ \\
\hline 38 & 1.25 & 9052.71 & 149.46 & $1.06 \mathrm{E}+15$ & $2.62 \mathrm{E}+21$ & $36.83 \%$ \\
\hline 39 & 0.75 & 9000.66 & 148.60 & $1.06 \mathrm{E}+15$ & $2.63 \mathrm{E}+21$ & $36.94 \%$ \\
\hline 40 & 0.25 & 8934.62 & 147.51 & $1.06 \mathrm{E}+15$ & $2.63 \mathrm{E}+21$ & $36.98 \%$ \\
\hline 41 & -0.25 & 9094.45 & 150.15 & $1.05 \mathrm{E}+15$ & $2.65 \mathrm{E}+21$ & $37.19 \%$ \\
\hline 42 & -0.75 & 9093.03 & 150.13 & $1.05 \mathrm{E}+15$ & $2.66 \mathrm{E}+21$ & $37.33 \%$ \\
\hline 43 & -1.25 & 9026.73 & 149.03 & $1.05 \mathrm{E}+15$ & $2.68 \mathrm{E}+21$ & $37.44 \%$ \\
\hline 44 & -1.75 & 9204.23 & 151.96 & $1.06 \mathrm{E}+15$ & $2.71 \mathrm{E}+21$ & $37.84 \%$ \\
\hline 45 & -2.25 & 9219.07 & 152.21 & $1.06 \mathrm{E}+15$ & $2.73 \mathrm{E}+21$ & $38.16 \%$ \\
\hline 46 & -2.75 & 9160.76 & 151.24 & $1.05 \mathrm{E}+15$ & $2.70 \mathrm{E}+21$ & $37.92 \%$ \\
\hline 47 & -3.25 & 9194.92 & 151.81 & $1.06 \mathrm{E}+15$ & $2.70 \mathrm{E}+21$ & $37.74 \%$ \\
\hline 48 & -3.75 & 9105.85 & 150.34 & $1.05 \mathrm{E}+15$ & $2.72 \mathrm{E}+21$ & $38.02 \%$ \\
\hline 49 & -4.25 & 9188.46 & 151.70 & $1.05 \mathrm{E}+15$ & $2.66 \mathrm{E}+21$ & $37.27 \%$ \\
\hline 50 & -4.75 & 9135.41 & 150.83 & $1.04 \mathrm{E}+15$ & $2.68 \mathrm{E}+21$ & $37.53 \%$ \\
\hline 51 & -5.25 & 8979.53 & 148.25 & $1.04 \mathrm{E}+15$ & $2.68 \mathrm{E}+21$ & $37.53 \%$ \\
\hline 52 & -5.75 & 8933.02 & 147.48 & $1.04 \mathrm{E}+15$ & $2.65 \mathrm{E}+21$ & $37.09 \%$ \\
\hline
\end{tabular}




\begin{tabular}{|l|l|l|l|l|l|l|}
\hline 53 & -6.25 & 8913.61 & 147.16 & $1.03 \mathrm{E}+15$ & $2.65 \mathrm{E}+21$ & $37.15 \%$ \\
\hline 54 & -6.75 & 9000.73 & 148.60 & $1.03 \mathrm{E}+15$ & $2.63 \mathrm{E}+21$ & $37.02 \%$ \\
\hline 55 & -7.25 & 8944.92 & 147.68 & $1.02 \mathrm{E}+15$ & $2.63 \mathrm{E}+21$ & $36.93 \%$ \\
\hline 56 & -7.75 & 8831.69 & 145.81 & $1.02 \mathrm{E}+15$ & $2.60 \mathrm{E}+21$ & $36.49 \%$ \\
\hline 57 & -8.25 & 8912.09 & 147.14 & $1.02 \mathrm{E}+15$ & $2.58 \mathrm{E}+21$ & $36.33 \%$ \\
\hline 58 & -8.75 & 8708.58 & 143.78 & $1.00 \mathrm{E}+15$ & $2.56 \mathrm{E}+21$ & $36.12 \%$ \\
\hline 59 & -9.25 & 8765.15 & 144.71 & $9.98 \mathrm{E}+14$ & $2.54 \mathrm{E}+21$ & $35.72 \%$ \\
\hline 60 & -9.75 & 8547.56 & 141.12 & $9.83 \mathrm{E}+14$ & $2.48 \mathrm{E}+21$ & $34.96 \%$ \\
\hline 61 & -10.25 & 8306.69 & 137.14 & $9.75 \mathrm{E}+14$ & $2.40 \mathrm{E}+21$ & $33.90 \%$ \\
\hline 62 & -10.75 & 8277.37 & 136.66 & $9.62 \mathrm{E}+14$ & $2.37 \mathrm{E}+21$ & $33.55 \%$ \\
\hline 63 & -11.25 & 8266.82 & 136.49 & $9.53 \mathrm{E}+14$ & $2.36 \mathrm{E}+21$ & $33.45 \%$ \\
\hline 64 & -11.75 & 8188.72 & 135.20 & $9.44 \mathrm{E}+14$ & $2.32 \mathrm{E}+21$ & $32.89 \%$ \\
\hline 65 & -12.25 & 8195.39 & 135.31 & $9.36 \mathrm{E}+14$ & $2.31 \mathrm{E}+21$ & $32.81 \%$ \\
\hline 66 & -12.75 & 8083.47 & 133.46 & $9.11 \mathrm{E}+14$ & $2.29 \mathrm{E}+21$ & $32.56 \%$ \\
\hline 67 & -13.25 & 8035.18 & 132.66 & $8.95 \mathrm{E}+14$ & $2.27 \mathrm{E}+21$ & $32.20 \%$ \\
\hline 68 & -13.75 & 7984.93 & 131.83 & $8.95 \mathrm{E}+14$ & $2.22 \mathrm{E}+21$ & $31.67 \%$ \\
\hline 69 & -14.25 & 7831.31 & 129.29 & $8.67 \mathrm{E}+14$ & $2.20 \mathrm{E}+21$ & $31.31 \%$ \\
\hline 70 & -14.75 & 7625.50 & 125.90 & $8.59 \mathrm{E}+14$ & $2.15 \mathrm{E}+21$ & $30.68 \%$ \\
\hline 71 & -15.25 & 7543.49 & 124.54 & $8.32 \mathrm{E}+14$ & $2.09 \mathrm{E}+21$ & $29.87 \%$ \\
\hline 72 & -15.75 & 7380.00 & 121.84 & $8.13 \mathrm{E}+14$ & $2.06 \mathrm{E}+21$ & $29.51 \%$ \\
\hline 73 & -16.25 & 7223.08 & 119.25 & $7.95 \mathrm{E}+14$ & $2.00 \mathrm{E}+21$ & $28.62 \%$ \\
\hline 74 & -16.75 & 7038.86 & 116.21 & $7.63 \mathrm{E}+14$ & $1.95 \mathrm{E}+21$ & $27.94 \%$ \\
\hline 75 & -17.25 & 6788.61 & 112.08 & $7.40 \mathrm{E}+14$ & $1.87 \mathrm{E}+21$ & $26.93 \%$ \\
\hline 76 & -17.75 & 6484.62 & 107.06 & $7.05 \mathrm{E}+14$ & $1.80 \mathrm{E}+21$ & $25.89 \%$ \\
\hline 77 & -18.25 & 6344.65 & 104.75 & $6.89 \mathrm{E}+14$ & $1.72 \mathrm{E}+21$ & $24.87 \%$ \\
\hline 78 & -18.75 & 6251.38 & 103.21 & $6.63 \mathrm{E}+14$ & $1.69 \mathrm{E}+21$ & $24.41 \%$ \\
\hline
\end{tabular}


Table 32: Cycle 150B, MCNP-Calculated HGRs and Neutron Flux for AFIP-7, Plate Position 3, 0 EFPD (BOC) Average Center Lobe Power at 24.2 MW. ${ }^{2}$

\begin{tabular}{|c|c|c|c|c|}
\hline Node & $\begin{array}{c}\text { Distance from } \\
\text { Centerline (in.) }\end{array}$ & $\begin{array}{l}\text { Fission Power } \\
\text { Density (W/cc) }\end{array}$ & $\begin{array}{l}\text { Surface Heat } \\
\text { Flux }\left(\mathrm{W} / \mathrm{cm}^{2}\right) \\
\end{array}$ & $\begin{array}{c}\text { Neutron Flux } \\
\left(\mathbf{n} / \mathrm{cm}^{2} \mathrm{sec}\right)\end{array}$ \\
\hline 1 & 19.25 & 5661.29 & 93.47 & $5.36 \mathrm{E}+14$ \\
\hline 2 & 18.75 & 5463.61 & 90.20 & $5.61 \mathrm{E}+14$ \\
\hline 3 & 18.25 & 5463.79 & 90.21 & $5.92 \mathrm{E}+14$ \\
\hline 4 & 17.75 & 5871.56 & 96.94 & $6.30 \mathrm{E}+14$ \\
\hline 5 & 17.25 & 6066.63 & 100.16 & $6.57 \mathrm{E}+14$ \\
\hline 6 & 16.75 & 6300.56 & 104.02 & $6.81 \mathrm{E}+14$ \\
\hline 7 & 16.25 & 6503.17 & 107.37 & $7.13 \mathrm{E}+14$ \\
\hline 8 & 15.75 & 6736.71 & 111.22 & $7.33 \mathrm{E}+14$ \\
\hline 9 & 15.25 & 6927.79 & 114.38 & $7.60 \mathrm{E}+14$ \\
\hline 10 & 14.75 & 6999.95 & 115.57 & $7.87 \mathrm{E}+14$ \\
\hline 11 & 14.25 & 7264.50 & 119.94 & $8.09 \mathrm{E}+14$ \\
\hline 13 & 13.75 & 7129.59 & 117.71 & $8.27 \mathrm{E}+14$ \\
\hline 14 & 13.25 & 7504.39 & 123.90 & $8.48 \mathrm{E}+14$ \\
\hline 15 & 12.75 & 7479.89 & 123.49 & $8.66 \mathrm{E}+14$ \\
\hline 16 & 12.25 & 7766.75 & 128.23 & $8.88 \mathrm{E}+14$ \\
\hline 17 & 11.75 & 7750.86 & 127.97 & $9.14 \mathrm{E}+14$ \\
\hline 18 & 11.25 & 7934.06 & 130.99 & $9.23 \mathrm{E}+14$ \\
\hline 19 & 10.75 & 8146.23 & 134.49 & $9.45 \mathrm{E}+14$ \\
\hline 20 & 10.25 & 8325.41 & 137.45 & $9.61 \mathrm{E}+14$ \\
\hline 21 & 9.75 & 8544.15 & 141.06 & $9.81 \mathrm{E}+14$ \\
\hline 22 & 9.25 & 8781.42 & 144.98 & $9.98 \mathrm{E}+14$ \\
\hline 23 & 8.75 & 8731.91 & 144.16 & $1.01 \mathrm{E}+15$ \\
\hline 24 & 8.25 & 9002.98 & 148.64 & $1.02 \mathrm{E}+15$ \\
\hline 25 & 7.75 & 9282.67 & 153.26 & $1.03 \mathrm{E}+15$ \\
\hline 26 & 7.25 & 9164.45 & 151.31 & $1.04 \mathrm{E}+15$ \\
\hline 27 & 6.75 & 9155.68 & 151.16 & $1.05 \mathrm{E}+15$ \\
\hline 28 & 6.25 & 9357.50 & 154.49 & $1.06 \mathrm{E}+15$ \\
\hline 29 & 5.75 & 9389.38 & 155.02 & $1.07 \mathrm{E}+15$ \\
\hline 30 & 5.25 & 9386.47 & 154.97 & $1.07 \mathrm{E}+15$ \\
\hline 31 & 4.75 & 9421.84 & 155.55 & $1.08 \mathrm{E}+15$ \\
\hline 32 & 4.25 & 9527.66 & 157.30 & $1.09 \mathrm{E}+15$ \\
\hline 33 & 3.75 & 9687.66 & 159.94 & $1.10 \mathrm{E}+15$ \\
\hline 34 & 3.25 & 9713.75 & 160.37 & $1.09 \mathrm{E}+15$ \\
\hline 35 & 2.75 & 9572.00 & 158.03 & $1.10 \mathrm{E}+15$ \\
\hline 36 & 2.25 & 9706.57 & 160.26 & $1.12 \mathrm{E}+15$ \\
\hline 37 & 1.75 & 9444.46 & 155.93 & $1.11 \mathrm{E}+15$ \\
\hline 38 & 1.25 & 9616.27 & 158.76 & $1.11 \mathrm{E}+15$ \\
\hline 39 & 0.75 & 9515.93 & 157.11 & $1.11 \mathrm{E}+15$ \\
\hline 40 & 0.25 & 9476.14 & 156.45 & $1.11 \mathrm{E}+15$ \\
\hline 41 & -0.25 & 9627.36 & 158.95 & $1.12 \mathrm{E}+15$ \\
\hline 42 & -0.75 & 9611.10 & 158.68 & $1.12 \mathrm{E}+15$ \\
\hline 43 & -1.25 & 9433.66 & 155.75 & $1.11 \mathrm{E}+15$ \\
\hline 44 & -1.75 & 9771.74 & 161.33 & $1.12 \mathrm{E}+15$ \\
\hline 45 & -2.25 & 9764.72 & 161.22 & $1.11 \mathrm{E}+15$ \\
\hline 46 & -2.75 & 9741.60 & 160.83 & $1.11 \mathrm{E}+15$ \\
\hline 47 & -3.25 & 9634.94 & 159.07 & $1.11 \mathrm{E}+15$ \\
\hline 48 & -3.75 & 9593.90 & 158.40 & $1.10 \mathrm{E}+15$ \\
\hline 49 & -4.25 & 9714.79 & 160.39 & $1.10 \mathrm{E}+15$ \\
\hline 50 & -4.75 & 9541.39 & 157.53 & $1.10 \mathrm{E}+15$ \\
\hline 51 & -5.25 & 9571.09 & 158.02 & $1.09 \mathrm{E}+15$ \\
\hline 52 & -5.75 & 9472.58 & 156.39 & $1.09 \mathrm{E}+15$ \\
\hline
\end{tabular}




\begin{tabular}{|l|l|l|l|l|}
\hline 53 & -6.25 & 9511.64 & 157.04 & $1.09 \mathrm{E}+15$ \\
\hline 54 & -6.75 & 9351.87 & 154.40 & $1.08 \mathrm{E}+15$ \\
\hline 55 & -7.25 & 9397.00 & 155.14 & $1.06 \mathrm{E}+15$ \\
\hline 56 & -7.75 & 9188.14 & 151.70 & $1.05 \mathrm{E}+15$ \\
\hline 57 & -8.25 & 9270.34 & 153.05 & $1.06 \mathrm{E}+15$ \\
\hline 58 & -8.75 & 9054.12 & 149.48 & $1.03 \mathrm{E}+15$ \\
\hline 59 & -9.25 & 9018.09 & 148.89 & $1.03 \mathrm{E}+15$ \\
\hline 60 & -9.75 & 8863.14 & 146.33 & $1.02 \mathrm{E}+15$ \\
\hline 61 & -10.25 & 8747.93 & 144.43 & $1.01 \mathrm{E}+15$ \\
\hline 62 & -10.75 & 8628.09 & 142.45 & $9.99 \mathrm{E}+14$ \\
\hline 63 & -11.25 & 8515.61 & 140.59 & $9.84 \mathrm{E}+14$ \\
\hline 64 & -11.75 & 8340.75 & 137.71 & $9.67 \mathrm{E}+14$ \\
\hline 65 & -12.25 & 8129.18 & 134.21 & $9.56 \mathrm{E}+14$ \\
\hline 66 & -12.75 & 8221.09 & 135.73 & $9.39 \mathrm{E}+14$ \\
\hline 67 & -13.25 & 8042.79 & 132.79 & $9.11 \mathrm{E}+14$ \\
\hline 68 & -13.75 & 7953.32 & 131.31 & $8.93 \mathrm{E}+14$ \\
\hline 69 & -14.25 & 7677.82 & 126.76 & $8.82 \mathrm{E}+14$ \\
\hline 70 & -14.75 & 7582.16 & 125.18 & $8.64 \mathrm{E}+14$ \\
\hline 71 & -15.25 & 7408.32 & 122.31 & $8.41 \mathrm{E}+14$ \\
\hline 72 & -15.75 & 7311.02 & 120.71 & $8.21 \mathrm{E}+14$ \\
\hline 73 & -16.25 & 6955.12 & 114.83 & $7.90 \mathrm{E}+14$ \\
\hline 74 & -16.75 & 6826.80 & 112.71 & $7.64 \mathrm{E}+14$ \\
\hline 75 & -17.25 & 6600.11 & 108.97 & $7.43 \mathrm{E}+14$ \\
\hline 76 & -17.75 & 6365.79 & 105.10 & $7.13 \mathrm{E}+14$ \\
\hline 77 & -18.25 & 6155.47 & 101.63 & $6.81 \mathrm{E}+14$ \\
\hline 78 & -18.75 & 6220.88 & 102.71 & $6.60 \mathrm{E}+14$ \\
\hline
\end{tabular}


Table 33: Cycle 150B, MCNP-Calculated HGRs, Neutron Flux, Depletion and Fission Density for AFIP-7, Plate Position 3, 18 EFPD (MOC1) Average Center Lobe Power at 24.2 MW. ${ }^{2}$

\begin{tabular}{|c|c|c|c|c|c|c|}
\hline Node & $\begin{array}{c}\text { Distance from } \\
\text { Centerline (in.) }\end{array}$ & $\begin{array}{l}\text { Fission Power } \\
\text { Density (W/cc) }\end{array}$ & $\begin{array}{l}\text { Surface Heat } \\
\text { Flux }\left(W / \mathrm{cm}^{2}\right)\end{array}$ & $\begin{array}{c}\text { Neutron Flux } \\
\left(\mathrm{n} / \mathrm{cm}^{2} \mathrm{sec}\right)\end{array}$ & $\begin{array}{l}\text { Fission Density } \\
\text { (fissions/cc) }\end{array}$ & $\begin{array}{c}\text { U-235 } \\
\text { Burnup (\%) }\end{array}$ \\
\hline 1 & 19.25 & 5497.61 & 90.77 & $5.21 \mathrm{E}+14$ & $1.16 \mathrm{E}+21$ & $17.42 \%$ \\
\hline 2 & 18.75 & 5223.71 & 86.24 & $5.48 \mathrm{E}+14$ & $1.07 \mathrm{E}+21$ & $16.26 \%$ \\
\hline 3 & 18.25 & 5444.03 & 89.88 & $5.83 \mathrm{E}+14$ & $1.11 \mathrm{E}+21$ & $16.65 \%$ \\
\hline 4 & 17.75 & 5798.73 & 95.74 & $6.13 \mathrm{E}+14$ & $1.16 \mathrm{E}+21$ & $17.50 \%$ \\
\hline 5 & 17.25 & 5968.92 & 98.55 & $6.31 \mathrm{E}+14$ & $1.22 \mathrm{E}+21$ & $18.44 \%$ \\
\hline 6 & 16.75 & 6171.21 & 101.89 & $6.57 \mathrm{E}+14$ & $1.27 \mathrm{E}+21$ & $18.98 \%$ \\
\hline 7 & 16.25 & 6383.18 & 105.39 & $6.89 \mathrm{E}+14$ & $1.32 \mathrm{E}+21$ & $19.84 \%$ \\
\hline 8 & 15.75 & 6657.66 & 109.92 & $7.12 \mathrm{E}+14$ & $1.36 \mathrm{E}+21$ & $20.38 \%$ \\
\hline 9 & 15.25 & 6737.38 & 111.23 & $7.34 \mathrm{E}+14$ & $1.41 \mathrm{E}+21$ & $21.00 \%$ \\
\hline 10 & 14.75 & 6953.58 & 114.80 & $7.58 \mathrm{E}+14$ & $1.43 \mathrm{E}+21$ & $21.40 \%$ \\
\hline 11 & 14.25 & 7068.23 & 116.70 & $7.72 \mathrm{E}+14$ & $1.48 \mathrm{E}+21$ & $22.10 \%$ \\
\hline 13 & 13.75 & 6970.86 & 115.09 & $7.93 \mathrm{E}+14$ & $1.50 \mathrm{E}+21$ & $22.37 \%$ \\
\hline 14 & 13.25 & 7102.07 & 117.26 & $8.06 \mathrm{E}+14$ & $1.53 \mathrm{E}+21$ & $22.77 \%$ \\
\hline 15 & 12.75 & 7273.09 & 120.08 & $8.32 \mathrm{E}+14$ & $1.56 \mathrm{E}+21$ & $23.16 \%$ \\
\hline 16 & 12.25 & 7418.83 & 122.48 & $8.48 \mathrm{E}+14$ & $1.58 \mathrm{E}+21$ & $23.43 \%$ \\
\hline 17 & 11.75 & 7463.05 & 123.21 & $8.66 \mathrm{E}+14$ & $1.62 \mathrm{E}+21$ & $23.97 \%$ \\
\hline 18 & 11.25 & 7672.27 & 126.67 & $8.76 \mathrm{E}+14$ & $1.63 \mathrm{E}+21$ & $24.24 \%$ \\
\hline 19 & 10.75 & 7730.06 & 127.62 & $8.89 \mathrm{E}+14$ & $1.69 \mathrm{E}+21$ & $24.89 \%$ \\
\hline 20 & 10.25 & 7955.69 & 131.35 & $9.11 \mathrm{E}+14$ & $1.71 \mathrm{E}+21$ & $25.29 \%$ \\
\hline 21 & 9.75 & 8178.99 & 135.04 & $9.20 \mathrm{E}+14$ & $1.78 \mathrm{E}+21$ & $26.21 \%$ \\
\hline 22 & 9.25 & 8250.40 & 136.21 & $9.35 \mathrm{E}+14$ & $1.81 \mathrm{E}+21$ & $26.56 \%$ \\
\hline 23 & 8.75 & 8389.18 & 138.51 & $9.46 \mathrm{E}+14$ & $1.82 \mathrm{E}+21$ & $26.75 \%$ \\
\hline 24 & 8.25 & 8344.94 & 137.77 & $9.49 \mathrm{E}+14$ & $1.85 \mathrm{E}+21$ & $27.15 \%$ \\
\hline 25 & 7.75 & 8537.20 & 140.95 & $9.57 \mathrm{E}+14$ & $1.88 \mathrm{E}+21$ & $27.54 \%$ \\
\hline 26 & 7.25 & 8649.05 & 142.80 & $9.68 \mathrm{E}+14$ & $1.90 \mathrm{E}+21$ & $27.91 \%$ \\
\hline 27 & 6.75 & 8639.08 & 142.63 & $9.75 \mathrm{E}+14$ & $1.91 \mathrm{E}+21$ & $28.06 \%$ \\
\hline 28 & 6.25 & 8696.77 & 143.58 & $9.82 \mathrm{E}+14$ & $1.92 \mathrm{E}+21$ & $28.24 \%$ \\
\hline 29 & 5.75 & 8755.56 & 144.55 & $9.92 \mathrm{E}+14$ & $1.93 \mathrm{E}+21$ & $28.30 \%$ \\
\hline 30 & 5.25 & 8786.99 & 145.07 & $9.93 \mathrm{E}+14$ & $1.96 \mathrm{E}+21$ & $28.72 \%$ \\
\hline 31 & 4.75 & 8792.48 & 145.16 & $1.00 \mathrm{E}+15$ & $1.96 \mathrm{E}+21$ & $28.61 \%$ \\
\hline 32 & 4.25 & 8818.44 & 145.59 & $1.01 \mathrm{E}+15$ & $1.97 \mathrm{E}+21$ & $28.86 \%$ \\
\hline 33 & 3.75 & 8902.56 & 146.98 & $1.01 \mathrm{E}+15$ & $1.98 \mathrm{E}+21$ & $29.05 \%$ \\
\hline 34 & 3.25 & 8925.71 & 147.36 & $1.01 \mathrm{E}+15$ & $1.99 \mathrm{E}+21$ & $29.06 \%$ \\
\hline 35 & 2.75 & 8828.01 & 145.75 & $1.02 \mathrm{E}+15$ & $1.99 \mathrm{E}+21$ & $29.13 \%$ \\
\hline 36 & 2.25 & 8914.35 & 147.18 & $1.03 \mathrm{E}+15$ & $1.98 \mathrm{E}+21$ & $28.92 \%$ \\
\hline 37 & 1.75 & 8716.71 & 143.91 & $1.02 \mathrm{E}+15$ & $1.97 \mathrm{E}+21$ & $28.86 \%$ \\
\hline 38 & 1.25 & 8778.04 & 144.93 & $1.02 \mathrm{E}+15$ & $1.94 \mathrm{E}+21$ & $28.46 \%$ \\
\hline 39 & 0.75 & 8752.69 & 144.51 & $1.03 \mathrm{E}+15$ & $1.96 \mathrm{E}+21$ & $28.64 \%$ \\
\hline 40 & 0.25 & 8770.06 & 144.79 & $1.03 \mathrm{E}+15$ & $1.96 \mathrm{E}+21$ & $28.74 \%$ \\
\hline 41 & -0.25 & 8867.75 & 146.41 & $1.02 \mathrm{E}+15$ & $1.96 \mathrm{E}+21$ & $28.72 \%$ \\
\hline 42 & -0.75 & 8893.83 & 146.84 & $1.03 \mathrm{E}+15$ & $1.98 \mathrm{E}+21$ & $28.91 \%$ \\
\hline 43 & -1.25 & 8773.69 & 144.85 & $1.02 \mathrm{E}+15$ & $2.00 \mathrm{E}+21$ & $29.23 \%$ \\
\hline 44 & -1.75 & 9069.15 & 149.73 & $1.03 \mathrm{E}+15$ & $2.00 \mathrm{E}+21$ & $29.15 \%$ \\
\hline 45 & -2.25 & 9060.13 & 149.58 & $1.03 \mathrm{E}+15$ & $2.01 \mathrm{E}+21$ & $29.31 \%$ \\
\hline 46 & -2.75 & 9000.93 & 148.61 & $1.02 \mathrm{E}+15$ & $2.00 \mathrm{E}+21$ & $29.31 \%$ \\
\hline 47 & -3.25 & 8854.45 & 146.19 & $1.03 \mathrm{E}+15$ & $1.99 \mathrm{E}+21$ & $29.19 \%$ \\
\hline 48 & -3.75 & 8844.34 & 146.02 & $1.02 \mathrm{E}+15$ & $2.00 \mathrm{E}+21$ & $29.26 \%$ \\
\hline 49 & -4.25 & 8975.61 & 148.19 & $1.02 \mathrm{E}+15$ & $2.01 \mathrm{E}+21$ & $29.35 \%$ \\
\hline 50 & -4.75 & 8910.01 & 147.10 & $1.02 \mathrm{E}+15$ & $1.98 \mathrm{E}+21$ & $29.03 \%$ \\
\hline 51 & -5.25 & 8827.64 & 145.74 & $1.00 \mathrm{E}+15$ & $1.99 \mathrm{E}+21$ & $29.19 \%$ \\
\hline 52 & -5.75 & 8955.04 & 147.85 & $1.02 \mathrm{E}+15$ & $1.97 \mathrm{E}+21$ & $28.81 \%$ \\
\hline
\end{tabular}




\begin{tabular}{|l|l|l|l|l|l|l|}
\hline 53 & -6.25 & 8832.83 & 145.83 & $1.01 \mathrm{E}+15$ & $1.96 \mathrm{E}+21$ & $28.75 \%$ \\
\hline 54 & -6.75 & 8690.14 & 143.47 & $1.01 \mathrm{E}+15$ & $1.96 \mathrm{E}+21$ & $28.64 \%$ \\
\hline 55 & -7.25 & 8854.27 & 146.18 & $9.98 \mathrm{E}+14$ & $1.94 \mathrm{E}+21$ & $28.38 \%$ \\
\hline 56 & -7.75 & 8683.21 & 143.36 & $9.90 \mathrm{E}+14$ & $1.92 \mathrm{E}+21$ & $28.17 \%$ \\
\hline 57 & -8.25 & 8568.20 & 141.46 & $9.79 \mathrm{E}+14$ & $1.91 \mathrm{E}+21$ & $28.04 \%$ \\
\hline 58 & -8.75 & 8591.54 & 141.85 & $9.74 \mathrm{E}+14$ & $1.88 \mathrm{E}+21$ & $27.68 \%$ \\
\hline 59 & -9.25 & 8563.73 & 141.39 & $9.68 \mathrm{E}+14$ & $1.86 \mathrm{E}+21$ & $27.37 \%$ \\
\hline 60 & -9.75 & 8294.30 & 136.94 & $9.51 \mathrm{E}+14$ & $1.83 \mathrm{E}+21$ & $26.96 \%$ \\
\hline 61 & -10.25 & 8186.58 & 135.16 & $9.45 \mathrm{E}+14$ & $1.79 \mathrm{E}+21$ & $26.35 \%$ \\
\hline 62 & -10.75 & 8086.09 & 133.50 & $9.40 \mathrm{E}+14$ & $1.76 \mathrm{E}+21$ & $25.91 \%$ \\
\hline 63 & -11.25 & 7982.98 & 131.80 & $9.24 \mathrm{E}+14$ & $1.74 \mathrm{E}+21$ & $25.70 \%$ \\
\hline 64 & -11.75 & 7821.71 & 129.14 & $9.17 \mathrm{E}+14$ & $1.70 \mathrm{E}+21$ & $25.20 \%$ \\
\hline 65 & -12.25 & 7876.24 & 130.04 & $9.03 \mathrm{E}+14$ & $1.69 \mathrm{E}+21$ & $25.02 \%$ \\
\hline 66 & -12.75 & 7764.96 & 128.20 & $8.90 \mathrm{E}+14$ & $1.69 \mathrm{E}+21$ & $25.02 \%$ \\
\hline 67 & -13.25 & 7682.01 & 126.83 & $8.60 \mathrm{E}+14$ & $1.66 \mathrm{E}+21$ & $24.70 \%$ \\
\hline 68 & -13.75 & 7542.32 & 124.52 & $8.54 \mathrm{E}+14$ & $1.63 \mathrm{E}+21$ & $24.10 \%$ \\
\hline 69 & -14.25 & 7475.68 & 123.42 & $8.38 \mathrm{E}+14$ & $1.62 \mathrm{E}+21$ & $24.01 \%$ \\
\hline 70 & -14.75 & 7351.32 & 121.37 & $8.20 \mathrm{E}+14$ & $1.57 \mathrm{E}+21$ & $23.30 \%$ \\
\hline 71 & -15.25 & 7283.76 & 120.25 & $8.06 \mathrm{E}+14$ & $1.53 \mathrm{E}+21$ & $22.76 \%$ \\
\hline 72 & -15.75 & 7190.13 & 118.71 & $7.91 \mathrm{E}+14$ & $1.50 \mathrm{E}+21$ & $22.32 \%$ \\
\hline 73 & -16.25 & 6957.34 & 114.87 & $7.68 \mathrm{E}+14$ & $1.46 \mathrm{E}+21$ & $21.71 \%$ \\
\hline 74 & -16.75 & 6787.00 & 112.05 & $7.44 \mathrm{E}+14$ & $1.42 \mathrm{E}+21$ & $21.17 \%$ \\
\hline 75 & -17.25 & 6529.60 & 107.80 & $7.22 \mathrm{E}+14$ & $1.36 \mathrm{E}+21$ & $20.30 \%$ \\
\hline 76 & -17.75 & 6288.22 & 103.82 & $6.94 \mathrm{E}+14$ & $1.29 \mathrm{E}+21$ & $19.29 \%$ \\
\hline 77 & -18.25 & 6071.36 & 100.24 & $6.69 \mathrm{E}+14$ & $1.26 \mathrm{E}+21$ & $18.98 \%$ \\
\hline 78 & -18.75 & 6092.57 & 100.59 & $6.33 \mathrm{E}+14$ & $1.22 \mathrm{E}+21$ & $18.36 \%$ \\
\hline
\end{tabular}


Table 34: Cycle 150B, MCNP-Calculated HGRs, Neutron Flux, Depletion and Fission Density for AFIP-7, Plate Position 3, 31 EFPD (MOC2) Average Center Lobe Power at 24.2 MW. ${ }^{2}$

\begin{tabular}{|c|c|c|c|c|c|c|}
\hline Node & $\begin{array}{c}\text { Distance from } \\
\text { Centerline (in.) }\end{array}$ & $\begin{array}{l}\text { Fission Power } \\
\text { Density (W/cc) }\end{array}$ & $\begin{array}{l}\text { Surface Heat } \\
\text { Flux }\left(W / \mathrm{cm}^{2}\right)\end{array}$ & $\begin{array}{c}\text { Neutron Flux } \\
\left(\mathrm{n} / \mathrm{cm}^{2} \mathrm{sec}\right)\end{array}$ & $\begin{array}{l}\text { Fission Density } \\
\text { (fissions/cc) }\end{array}$ & $\begin{array}{c}\text { U-235 } \\
\text { Burnup (\%) }\end{array}$ \\
\hline 1 & 19.25 & 5644.60 & 93.19 & $5.36 \mathrm{E}+14$ & $1.36 \mathrm{E}+21$ & $20.38 \%$ \\
\hline 2 & 18.75 & 5323.94 & 87.90 & $5.66 \mathrm{E}+14$ & $1.27 \mathrm{E}+21$ & $19.06 \%$ \\
\hline 3 & 18.25 & 5611.49 & 92.65 & $5.94 \mathrm{E}+14$ & $1.30 \mathrm{E}+21$ & $19.60 \%$ \\
\hline 4 & 17.75 & 5863.75 & 96.81 & $6.21 \mathrm{E}+14$ & $1.38 \mathrm{E}+21$ & $20.61 \%$ \\
\hline 5 & 17.25 & 6053.37 & 99.94 & $6.47 \mathrm{E}+14$ & $1.44 \mathrm{E}+21$ & $21.63 \%$ \\
\hline 6 & 16.75 & 6269.10 & 103.50 & $6.79 E+14$ & $1.49 \mathrm{E}+21$ & $22.29 \%$ \\
\hline 7 & 16.25 & 6478.12 & 106.95 & $7.06 \mathrm{E}+14$ & $1.56 \mathrm{E}+21$ & $23.26 \%$ \\
\hline 8 & 15.75 & 6571.01 & 108.49 & $7.31 \mathrm{E}+14$ & $1.61 \mathrm{E}+21$ & $23.95 \%$ \\
\hline 9 & 15.25 & 6796.35 & 112.21 & $7.52 \mathrm{E}+14$ & $1.66 \mathrm{E}+21$ & $24.62 \%$ \\
\hline 10 & 14.75 & 6868.17 & 113.39 & $7.76 \mathrm{E}+14$ & $1.69 \mathrm{E}+21$ & $25.11 \%$ \\
\hline 11 & 14.25 & 7070.11 & 116.73 & $7.92 \mathrm{E}+14$ & $1.74 \mathrm{E}+21$ & $25.82 \%$ \\
\hline 13 & 13.75 & 7051.51 & 116.42 & $8.01 \mathrm{E}+14$ & $1.76 \mathrm{E}+21$ & $26.07 \%$ \\
\hline 14 & 13.25 & 7305.04 & 120.61 & $8.33 \mathrm{E}+14$ & $1.79 \mathrm{E}+21$ & $26.53 \%$ \\
\hline 15 & 12.75 & 7397.20 & 122.13 & $8.51 \mathrm{E}+14$ & $1.82 \mathrm{E}+21$ & $27.01 \%$ \\
\hline 16 & 12.25 & 7493.82 & 123.72 & $8.63 \mathrm{E}+14$ & $1.85 \mathrm{E}+21$ & $27.33 \%$ \\
\hline 17 & 11.75 & 7684.95 & 126.88 & $8.80 \mathrm{E}+14$ & $1.89 \mathrm{E}+21$ & $27.89 \%$ \\
\hline 18 & 11.25 & 7783.43 & 128.50 & $8.94 \mathrm{E}+14$ & $1.92 \mathrm{E}+21$ & $28.28 \%$ \\
\hline 19 & 10.75 & 7908.03 & 130.56 & $9.06 \mathrm{E}+14$ & $1.97 \mathrm{E}+21$ & $28.94 \%$ \\
\hline 20 & 10.25 & 7944.50 & 131.16 & $9.22 \mathrm{E}+14$ & $2.01 \mathrm{E}+21$ & $29.44 \%$ \\
\hline 21 & 9.75 & 8131.79 & 134.26 & $9.32 \mathrm{E}+14$ & $2.08 \mathrm{E}+21$ & $30.46 \%$ \\
\hline 22 & 9.25 & 8467.23 & 139.79 & $9.49 \mathrm{E}+14$ & $2.11 \mathrm{E}+21$ & $30.85 \%$ \\
\hline 23 & 8.75 & 8446.38 & 139.45 & $9.53 \mathrm{E}+14$ & $2.12 \mathrm{E}+21$ & $31.11 \%$ \\
\hline 24 & 8.25 & 8538.07 & 140.96 & $9.64 \mathrm{E}+14$ & $2.15 \mathrm{E}+21$ & $31.49 \%$ \\
\hline 25 & 7.75 & 8457.58 & 139.63 & $9.67 \mathrm{E}+14$ & $2.19 \mathrm{E}+21$ & $31.96 \%$ \\
\hline 26 & 7.25 & 8482.90 & 140.05 & $9.75 \mathrm{E}+14$ & $2.22 \mathrm{E}+21$ & $32.40 \%$ \\
\hline 27 & 6.75 & 8670.70 & 143.15 & $9.92 \mathrm{E}+14$ & $2.23 \mathrm{E}+21$ & $32.53 \%$ \\
\hline 28 & 6.25 & 8657.44 & 142.93 & $9.96 \mathrm{E}+14$ & $2.24 \mathrm{E}+21$ & $32.73 \%$ \\
\hline 29 & 5.75 & 8650.54 & 142.82 & $9.96 \mathrm{E}+14$ & $2.25 \mathrm{E}+21$ & $32.83 \%$ \\
\hline 30 & 5.25 & 8680.79 & 143.32 & $1.01 \mathrm{E}+15$ & $2.28 \mathrm{E}+21$ & $33.25 \%$ \\
\hline 31 & 4.75 & 8801.36 & 145.31 & $1.02 \mathrm{E}+15$ & $2.28 \mathrm{E}+21$ & $33.14 \%$ \\
\hline 32 & 4.25 & 8904.46 & 147.01 & $1.03 \mathrm{E}+15$ & $2.30 \mathrm{E}+21$ & $33.39 \%$ \\
\hline 33 & 3.75 & 8791.13 & 145.14 & $1.02 \mathrm{E}+15$ & $2.31 \mathrm{E}+21$ & $33.64 \%$ \\
\hline 34 & 3.25 & 8933.28 & 147.49 & $1.03 \mathrm{E}+15$ & $2.32 \mathrm{E}+21$ & $33.67 \%$ \\
\hline 35 & 2.75 & 8860.15 & 146.28 & $1.03 \mathrm{E}+15$ & $2.31 \mathrm{E}+21$ & $33.68 \%$ \\
\hline 36 & 2.25 & 9039.00 & 149.23 & $1.04 \mathrm{E}+15$ & $2.30 \mathrm{E}+21$ & $33.51 \%$ \\
\hline 37 & 1.75 & 8861.89 & 146.31 & $1.04 \mathrm{E}+15$ & $2.29 \mathrm{E}+21$ & $33.35 \%$ \\
\hline 38 & 1.25 & 8910.39 & 147.11 & $1.04 \mathrm{E}+15$ & $2.27 \mathrm{E}+21$ & $32.97 \%$ \\
\hline 39 & 0.75 & 8911.65 & 147.13 & $1.05 \mathrm{E}+15$ & $2.28 \mathrm{E}+21$ & $33.14 \%$ \\
\hline 40 & 0.25 & 8751.71 & 144.49 & $1.04 \mathrm{E}+15$ & $2.28 \mathrm{E}+21$ & $33.28 \%$ \\
\hline 41 & -0.25 & 8863.74 & 146.34 & $1.03 \mathrm{E}+15$ & $2.29 \mathrm{E}+21$ & $33.28 \%$ \\
\hline 42 & -0.75 & 8924.61 & 147.35 & $1.05 \mathrm{E}+15$ & $2.30 \mathrm{E}+21$ & $33.47 \%$ \\
\hline 43 & -1.25 & 8717.20 & 143.92 & $1.03 \mathrm{E}+15$ & $2.32 \mathrm{E}+21$ & $33.72 \%$ \\
\hline 44 & -1.75 & 8867.86 & 146.41 & $1.05 \mathrm{E}+15$ & $2.33 \mathrm{E}+21$ & $33.81 \%$ \\
\hline 45 & -2.25 & 9001.96 & 148.62 & $1.04 \mathrm{E}+15$ & $2.34 \mathrm{E}+21$ & $33.96 \%$ \\
\hline 46 & -2.75 & 9008.39 & 148.73 & $1.04 \mathrm{E}+15$ & $2.33 \mathrm{E}+21$ & $33.94 \%$ \\
\hline 47 & -3.25 & 9079.87 & 149.91 & $1.03 \mathrm{E}+15$ & $2.32 \mathrm{E}+21$ & $33.76 \%$ \\
\hline 48 & -3.75 & 8908.05 & 147.07 & $1.04 \mathrm{E}+15$ & $2.33 \mathrm{E}+21$ & $33.81 \%$ \\
\hline 49 & -4.25 & 8943.77 & 147.66 & $1.03 \mathrm{E}+15$ & $2.34 \mathrm{E}+21$ & $33.96 \%$ \\
\hline 50 & -4.75 & 8951.42 & 147.79 & $1.03 \mathrm{E}+15$ & $2.31 \mathrm{E}+21$ & $33.64 \%$ \\
\hline 51 & -5.25 & 8943.05 & 147.65 & $1.01 \mathrm{E}+15$ & $2.32 \mathrm{E}+21$ & $33.76 \%$ \\
\hline 52 & -5.75 & 8721.18 & 143.99 & $1.02 \mathrm{E}+15$ & $2.29 \mathrm{E}+21$ & $33.42 \%$ \\
\hline
\end{tabular}




\begin{tabular}{|l|l|l|l|l|l|l|}
\hline 53 & -6.25 & 8784.89 & 145.04 & $1.02 \mathrm{E}+15$ & $2.29 \mathrm{E}+21$ & $33.29 \%$ \\
\hline 54 & -6.75 & 8789.90 & 145.12 & $1.02 \mathrm{E}+15$ & $2.28 \mathrm{E}+21$ & $33.11 \%$ \\
\hline 55 & -7.25 & 8703.65 & 143.70 & $1.00 \mathrm{E}+15$ & $2.26 \mathrm{E}+21$ & $32.93 \%$ \\
\hline 56 & -7.75 & 8655.99 & 142.91 & $1.00 \mathrm{E}+15$ & $2.24 \mathrm{E}+21$ & $32.67 \%$ \\
\hline 57 & -8.25 & 8641.77 & 142.68 & $9.95 \mathrm{E}+14$ & $2.23 \mathrm{E}+21$ & $32.46 \%$ \\
\hline 58 & -8.75 & 8393.24 & 138.57 & $9.86 \mathrm{E}+14$ & $2.20 \mathrm{E}+21$ & $32.14 \%$ \\
\hline 59 & -9.25 & 8481.09 & 140.02 & $9.83 \mathrm{E}+14$ & $2.17 \mathrm{E}+21$ & $31.83 \%$ \\
\hline 60 & -9.75 & 8438.32 & 139.32 & $9.70 \mathrm{E}+14$ & $2.14 \mathrm{E}+21$ & $31.27 \%$ \\
\hline 61 & -10.25 & 8253.12 & 136.26 & $9.63 \mathrm{E}+14$ & $2.09 \mathrm{E}+21$ & $30.62 \%$ \\
\hline 62 & -10.75 & 8261.36 & 136.40 & $9.52 \mathrm{E}+14$ & $2.05 \mathrm{E}+21$ & $30.12 \%$ \\
\hline 63 & -11.25 & 8128.34 & 134.20 & $9.48 \mathrm{E}+14$ & $2.03 \mathrm{E}+21$ & $29.89 \%$ \\
\hline 64 & -11.75 & 8028.24 & 132.55 & $9.39 \mathrm{E}+14$ & $1.99 \mathrm{E}+21$ & $29.30 \%$ \\
\hline 65 & -12.25 & 8044.86 & 132.82 & $9.24 \mathrm{E}+14$ & $1.98 \mathrm{E}+21$ & $29.14 \%$ \\
\hline 66 & -12.75 & 8029.75 & 132.57 & $9.06 \mathrm{E}+14$ & $1.98 \mathrm{E}+21$ & $29.09 \%$ \\
\hline 67 & -13.25 & 7793.28 & 128.67 & $8.97 \mathrm{E}+14$ & $1.95 \mathrm{E}+21$ & $28.73 \%$ \\
\hline 68 & -13.75 & 7684.15 & 126.87 & $8.75 \mathrm{E}+14$ & $1.90 \mathrm{E}+21$ & $28.07 \%$ \\
\hline 69 & -14.25 & 7601.32 & 125.50 & $8.67 \mathrm{E}+14$ & $1.90 \mathrm{E}+21$ & $27.94 \%$ \\
\hline 70 & -14.75 & 7405.14 & 122.26 & $8.35 \mathrm{E}+14$ & $1.84 \mathrm{E}+21$ & $27.18 \%$ \\
\hline 71 & -15.25 & 7327.68 & 120.98 & $8.23 \mathrm{E}+14$ & $1.80 \mathrm{E}+21$ & $26.61 \%$ \\
\hline 72 & -15.75 & 7294.90 & 120.44 & $8.12 \mathrm{E}+14$ & $1.77 \mathrm{E}+21$ & $26.12 \%$ \\
\hline 73 & -16.25 & 7036.76 & 116.18 & $7.86 \mathrm{E}+14$ & $1.71 \mathrm{E}+21$ & $25.39 \%$ \\
\hline 74 & -16.75 & 6760.96 & 111.62 & $7.62 \mathrm{E}+14$ & $1.67 \mathrm{E}+21$ & $24.80 \%$ \\
\hline 75 & -17.25 & 6738.19 & 111.25 & $7.42 \mathrm{E}+14$ & $1.60 \mathrm{E}+21$ & $23.80 \%$ \\
\hline 76 & -17.75 & 6314.51 & 104.25 & $7.03 \mathrm{E}+14$ & $1.52 \mathrm{E}+21$ & $22.67 \%$ \\
\hline 77 & -18.25 & 6232.38 & 102.90 & $6.85 \mathrm{E}+14$ & $1.49 \mathrm{E}+21$ & $22.23 \%$ \\
\hline 78 & -18.75 & 6186.01 & 102.13 & $6.53 \mathrm{E}+14$ & $1.45 \mathrm{E}+21$ & $21.63 \%$ \\
\hline
\end{tabular}


Table 35: Cycle 150B, MCNP-Calculated HGRs, Neutron Flux, Depletion and Fission Density for AFIP-7, Plate Position 3, 42.0 EFPD (EOC) Average Center Lobe Power at 24.2 MW. ${ }^{2}$

\begin{tabular}{|c|c|c|c|c|c|c|}
\hline Node & $\begin{array}{c}\text { Distance from } \\
\text { Centerline (in.) }\end{array}$ & $\begin{array}{l}\text { Fission Power } \\
\text { Density (W/cc) }\end{array}$ & $\begin{array}{l}\text { Surface Heat } \\
\text { Flux }\left(W / \mathrm{cm}^{2}\right)\end{array}$ & $\begin{array}{c}\text { Neutron Flux } \\
\left(\mathrm{n} / \mathrm{cm}^{2} \mathrm{sec}\right)\end{array}$ & $\begin{array}{l}\text { Fission Density } \\
\text { (fissions/cc) }\end{array}$ & $\begin{array}{c}\text { U-235 } \\
\text { Burnup (\%) }\end{array}$ \\
\hline 1 & 19.25 & 5645.18 & 93.20 & $5.41 \mathrm{E}+14$ & $1.54 \mathrm{E}+21$ & $22.95 \%$ \\
\hline 2 & 18.75 & 5350.74 & 88.34 & $5.71 \mathrm{E}+14$ & $1.43 \mathrm{E}+21$ & $21.48 \%$ \\
\hline 3 & 18.25 & 5624.71 & 92.86 & $6.00 \mathrm{E}+14$ & $1.48 \mathrm{E}+21$ & $22.18 \%$ \\
\hline 4 & 17.75 & 5830.84 & 96.27 & $6.27 \mathrm{E}+14$ & $1.56 \mathrm{E}+21$ & $23.27 \%$ \\
\hline 5 & 17.25 & 6061.23 & 100.07 & $6.54 \mathrm{E}+14$ & $1.63 \mathrm{E}+21$ & $24.33 \%$ \\
\hline 6 & 16.75 & 6245.06 & 103.11 & $6.84 \mathrm{E}+14$ & $1.69 \mathrm{E}+21$ & $25.10 \%$ \\
\hline 7 & 16.25 & 6463.87 & 106.72 & $7.11 \mathrm{E}+14$ & $1.76 \mathrm{E}+21$ & $26.14 \%$ \\
\hline 8 & 15.75 & 6567.63 & 108.43 & $7.38 \mathrm{E}+14$ & $1.81 \mathrm{E}+21$ & $26.85 \%$ \\
\hline 9 & 15.25 & 6745.96 & 111.38 & $7.58 \mathrm{E}+14$ & $1.87 \mathrm{E}+21$ & $27.64 \%$ \\
\hline 10 & 14.75 & 6842.75 & 112.97 & $7.81 \mathrm{E}+14$ & $1.90 \mathrm{E}+21$ & $28.15 \%$ \\
\hline 11 & 14.25 & 7022.85 & 115.95 & $7.98 \mathrm{E}+14$ & $1.96 \mathrm{E}+21$ & $28.95 \%$ \\
\hline 13 & 13.75 & 7029.62 & 116.06 & $8.09 \mathrm{E}+14$ & $1.97 \mathrm{E}+21$ & $29.18 \%$ \\
\hline 14 & 13.25 & 7262.17 & 119.90 & $8.41 \mathrm{E}+14$ & $2.02 \mathrm{E}+21$ & $29.75 \%$ \\
\hline 15 & 12.75 & 7341.48 & 121.21 & $8.61 \mathrm{E}+14$ & $2.05 \mathrm{E}+21$ & $30.27 \%$ \\
\hline 16 & 12.25 & 7446.02 & 122.93 & $8.72 \mathrm{E}+14$ & $2.08 \mathrm{E}+21$ & $30.63 \%$ \\
\hline 17 & 11.75 & 7632.38 & 126.01 & $8.88 \mathrm{E}+14$ & $2.13 \mathrm{E}+21$ & $31.25 \%$ \\
\hline 18 & 11.25 & 7730.61 & 127.63 & $9.01 \mathrm{E}+14$ & $2.16 \mathrm{E}+21$ & $31.67 \%$ \\
\hline 19 & 10.75 & 7827.67 & 129.23 & $9.14 \mathrm{E}+14$ & $2.21 \mathrm{E}+21$ & $32.37 \%$ \\
\hline 20 & 10.25 & 7896.05 & 130.36 & $9.29 \mathrm{E}+14$ & $2.25 \mathrm{E}+21$ & $32.89 \%$ \\
\hline 21 & 9.75 & 8098.62 & 133.71 & $9.41 \mathrm{E}+14$ & $2.33 \mathrm{E}+21$ & $33.99 \%$ \\
\hline 22 & 9.25 & 8353.24 & 137.91 & $9.56 \mathrm{E}+14$ & $2.37 \mathrm{E}+21$ & $34.52 \%$ \\
\hline 23 & 8.75 & 8345.35 & 137.78 & $9.60 \mathrm{E}+14$ & $2.38 \mathrm{E}+21$ & $34.76 \%$ \\
\hline 24 & 8.25 & 8446.53 & 139.45 & $9.70 \mathrm{E}+14$ & $2.42 \mathrm{E}+21$ & $35.18 \%$ \\
\hline 25 & 7.75 & 8386.03 & 138.45 & $9.77 \mathrm{E}+14$ & $2.45 \mathrm{E}+21$ & $35.63 \%$ \\
\hline 26 & 7.25 & 8400.62 & 138.69 & $9.82 \mathrm{E}+14$ & $2.48 \mathrm{E}+21$ & $36.06 \%$ \\
\hline 27 & 6.75 & 8579.70 & 141.65 & $1.00 \mathrm{E}+15$ & $2.50 \mathrm{E}+21$ & $36.25 \%$ \\
\hline 28 & 6.25 & 8568.99 & 141.47 & $1.00 \mathrm{E}+15$ & $2.51 \mathrm{E}+21$ & $36.44 \%$ \\
\hline 29 & 5.75 & 8606.23 & 142.09 & $1.01 \mathrm{E}+15$ & $2.52 \mathrm{E}+21$ & $36.55 \%$ \\
\hline 30 & 5.25 & 8560.25 & 141.33 & $1.02 \mathrm{E}+15$ & $2.55 \mathrm{E}+21$ & $36.99 \%$ \\
\hline 31 & 4.75 & 8712.35 & 143.84 & $1.03 \mathrm{E}+15$ & $2.55 \mathrm{E}+21$ & $36.90 \%$ \\
\hline 32 & 4.25 & 8769.66 & 144.79 & $1.03 \mathrm{E}+15$ & $2.57 \mathrm{E}+21$ & $37.21 \%$ \\
\hline 33 & 3.75 & 8700.65 & 143.65 & $1.03 \mathrm{E}+15$ & $2.58 \mathrm{E}+21$ & $37.43 \%$ \\
\hline 34 & 3.25 & 8817.98 & 145.58 & $1.03 \mathrm{E}+15$ & $2.59 \mathrm{E}+21$ & $37.50 \%$ \\
\hline 35 & 2.75 & 8779.48 & 144.95 & $1.04 \mathrm{E}+15$ & $2.59 \mathrm{E}+21$ & $37.48 \%$ \\
\hline 36 & 2.25 & 8905.00 & 147.02 & $1.05 \mathrm{E}+15$ & $2.58 \mathrm{E}+21$ & $37.36 \%$ \\
\hline 37 & 1.75 & 8720.31 & 143.97 & $1.04 \mathrm{E}+15$ & $2.56 \mathrm{E}+21$ & $37.14 \%$ \\
\hline 38 & 1.25 & 8794.35 & 145.19 & $1.05 \mathrm{E}+15$ & $2.54 \mathrm{E}+21$ & $36.78 \%$ \\
\hline 39 & 0.75 & 8822.28 & 145.66 & $1.06 \mathrm{E}+15$ & $2.55 \mathrm{E}+21$ & $36.93 \%$ \\
\hline 40 & 0.25 & 8623.29 & 142.37 & $1.05 \mathrm{E}+15$ & $2.55 \mathrm{E}+21$ & $37.05 \%$ \\
\hline 41 & -0.25 & 8790.99 & 145.14 & $1.04 \mathrm{E}+15$ & $2.56 \mathrm{E}+21$ & $37.06 \%$ \\
\hline 42 & -0.75 & 8801.11 & 145.31 & $1.05 \mathrm{E}+15$ & $2.58 \mathrm{E}+21$ & $37.27 \%$ \\
\hline 43 & -1.25 & 8611.67 & 142.18 & $1.04 \mathrm{E}+15$ & $2.59 \mathrm{E}+21$ & $37.44 \%$ \\
\hline 44 & -1.75 & 8784.32 & 145.03 & $1.06 \mathrm{E}+15$ & $2.60 \mathrm{E}+21$ & $37.58 \%$ \\
\hline 45 & -2.25 & 8904.89 & 147.02 & $1.05 \mathrm{E}+15$ & $2.61 \mathrm{E}+21$ & $37.80 \%$ \\
\hline 46 & -2.75 & 8899.67 & 146.93 & $1.04 \mathrm{E}+15$ & $2.61 \mathrm{E}+21$ & $37.79 \%$ \\
\hline 47 & -3.25 & 8951.68 & 147.79 & $1.05 \mathrm{E}+15$ & $2.60 \mathrm{E}+21$ & $37.65 \%$ \\
\hline 48 & -3.75 & 8807.88 & 145.42 & $1.05 \mathrm{E}+15$ & $2.60 \mathrm{E}+21$ & $37.62 \%$ \\
\hline 49 & -4.25 & 8798.97 & 145.27 & $1.04 \mathrm{E}+15$ & $2.61 \mathrm{E}+21$ & $37.79 \%$ \\
\hline 50 & -4.75 & 8830.63 & 145.79 & $1.03 \mathrm{E}+15$ & $2.59 \mathrm{E}+21$ & $37.48 \%$ \\
\hline 51 & -5.25 & 8806.17 & 145.39 & $1.02 \mathrm{E}+15$ & $2.59 \mathrm{E}+21$ & $37.59 \%$ \\
\hline 52 & -5.75 & 8598.75 & 141.97 & $1.03 \mathrm{E}+15$ & $2.56 \mathrm{E}+21$ & $37.14 \%$ \\
\hline
\end{tabular}




\begin{tabular}{|l|l|l|l|l|l|l|}
\hline 53 & -6.25 & 8693.18 & 143.52 & $1.03 \mathrm{E}+15$ & $2.56 \mathrm{E}+21$ & $37.06 \%$ \\
\hline 54 & -6.75 & 8674.59 & 143.22 & $1.03 \mathrm{E}+15$ & $2.55 \mathrm{E}+21$ & $36.87 \%$ \\
\hline 55 & -7.25 & 8650.37 & 142.82 & $1.01 \mathrm{E}+15$ & $2.53 \mathrm{E}+21$ & $36.66 \%$ \\
\hline 56 & -7.75 & 8550.25 & 141.16 & $1.01 \mathrm{E}+15$ & $2.51 \mathrm{E}+21$ & $36.39 \%$ \\
\hline 57 & -8.25 & 8571.07 & 141.51 & $1.00 \mathrm{E}+15$ & $2.49 \mathrm{E}+21$ & $36.17 \%$ \\
\hline 58 & -8.75 & 8301.65 & 137.06 & $9.94 \mathrm{E}+14$ & $2.46 \mathrm{E}+21$ & $35.76 \%$ \\
\hline 59 & -9.25 & 8402.19 & 138.72 & $9.91 \mathrm{E}+14$ & $2.44 \mathrm{E}+21$ & $35.49 \%$ \\
\hline 60 & -9.75 & 8336.43 & 137.63 & $9.78 \mathrm{E}+14$ & $2.40 \mathrm{E}+21$ & $34.92 \%$ \\
\hline 61 & -10.25 & 8133.43 & 134.28 & $9.72 \mathrm{E}+14$ & $2.34 \mathrm{E}+21$ & $34.18 \%$ \\
\hline 62 & -10.75 & 8167.65 & 134.85 & $9.60 \mathrm{E}+14$ & $2.31 \mathrm{E}+21$ & $33.69 \%$ \\
\hline 63 & -11.25 & 8029.90 & 132.57 & $9.57 \mathrm{E}+14$ & $2.28 \mathrm{E}+21$ & $33.43 \%$ \\
\hline 64 & -11.75 & 7947.24 & 131.21 & $9.46 \mathrm{E}+14$ & $2.24 \mathrm{E}+21$ & $32.77 \%$ \\
\hline 65 & -12.25 & 7980.56 & 131.76 & $9.32 \mathrm{E}+14$ & $2.23 \mathrm{E}+21$ & $32.63 \%$ \\
\hline 66 & -12.75 & 7955.67 & 131.35 & $9.14 \mathrm{E}+14$ & $2.23 \mathrm{E}+21$ & $32.58 \%$ \\
\hline 67 & -13.25 & 7728.91 & 127.60 & $9.04 \mathrm{E}+14$ & $2.19 \mathrm{E}+21$ & $32.14 \%$ \\
\hline 68 & -13.75 & 7655.59 & 126.39 & $8.84 \mathrm{E}+14$ & $2.14 \mathrm{E}+21$ & $31.44 \%$ \\
\hline 69 & -14.25 & 7556.41 & 124.76 & $8.75 \mathrm{E}+14$ & $2.13 \mathrm{E}+21$ & $31.27 \%$ \\
\hline 70 & -14.75 & 7402.12 & 122.21 & $8.43 \mathrm{E}+14$ & $2.07 \mathrm{E}+21$ & $30.43 \%$ \\
\hline 71 & -15.25 & 7277.33 & 120.15 & $8.32 \mathrm{E}+14$ & $2.02 \mathrm{E}+21$ & $29.85 \%$ \\
\hline 72 & -15.75 & 7238.14 & 119.50 & $8.18 \mathrm{E}+14$ & $1.99 \mathrm{E}+21$ & $29.32 \%$ \\
\hline 73 & -16.25 & 7012.05 & 115.77 & $7.91 \mathrm{E}+14$ & $1.93 \mathrm{E}+21$ & $28.51 \%$ \\
\hline 74 & -16.75 & 6723.95 & 111.01 & $7.68 \mathrm{E}+14$ & $1.88 \mathrm{E}+21$ & $27.78 \%$ \\
\hline 75 & -17.25 & 6695.45 & 110.54 & $7.49 \mathrm{E}+14$ & $1.80 \mathrm{E}+21$ & $26.82 \%$ \\
\hline 76 & -17.75 & 6302.42 & 104.05 & $7.10 \mathrm{E}+14$ & $1.72 \mathrm{E}+21$ & $25.50 \%$ \\
\hline 77 & -18.25 & 6226.03 & 102.79 & $6.90 \mathrm{E}+14$ & $1.68 \mathrm{E}+21$ & $25.02 \%$ \\
\hline 78 & -18.75 & 6170.61 & 101.88 & $6.59 \mathrm{E}+14$ & $1.64 \mathrm{E}+21$ & $24.42 \%$ \\
\hline
\end{tabular}


Table 36: Cycle 150B, MCNP-Calculated HGRs and Neutron Flux for AFIP-7, Plate Position 4, 0 EFPD (BOC) Average Center Lobe Power at 24.2 MW. ${ }^{2}$

\begin{tabular}{|c|c|c|c|c|}
\hline Node & $\begin{array}{l}\text { Distance from } \\
\text { Centerline (in.) }\end{array}$ & $\begin{array}{l}\text { Fission Power } \\
\text { Density (W/cc) }\end{array}$ & $\begin{array}{l}\text { Surface Heat } \\
\text { Flux }\left(W / \mathrm{cm}^{2}\right)\end{array}$ & $\begin{array}{c}\text { Neutron Flux } \\
\left(\mathrm{n} / \mathrm{cm}^{2} \mathrm{sec}\right)\end{array}$ \\
\hline 1 & 19.25 & 6119.39 & 101.03 & $5.33 \mathrm{E}+14$ \\
\hline 2 & 18.75 & 6002.07 & 99.09 & $5.68 \mathrm{E}+14$ \\
\hline 3 & 18.25 & 6021.70 & 99.42 & $5.95 \mathrm{E}+14$ \\
\hline 4 & 17.75 & 6198.98 & 102.35 & $6.25 \mathrm{E}+14$ \\
\hline 5 & 17.25 & 6579.66 & 108.63 & $6.53 \mathrm{E}+14$ \\
\hline 6 & 16.75 & 6706.09 & 110.72 & $6.77 \mathrm{E}+14$ \\
\hline 7 & 16.25 & 7194.10 & 118.77 & $7.13 \mathrm{E}+14$ \\
\hline 8 & 15.75 & 7315.95 & 120.79 & $7.36 \mathrm{E}+14$ \\
\hline 9 & 15.25 & 7427.37 & 122.63 & $7.57 \mathrm{E}+14$ \\
\hline 10 & 14.75 & 7710.64 & 127.30 & $7.85 \mathrm{E}+14$ \\
\hline 11 & 14.25 & 7898.14 & 130.40 & $8.09 \mathrm{E}+14$ \\
\hline 13 & 13.75 & 8014.18 & 132.31 & $8.26 \mathrm{E}+14$ \\
\hline 14 & 13.25 & 8189.53 & 135.21 & $8.50 \mathrm{E}+14$ \\
\hline 15 & 12.75 & 8135.83 & 134.32 & $8.64 \mathrm{E}+14$ \\
\hline 16 & 12.25 & 8439.03 & 139.33 & $8.87 \mathrm{E}+14$ \\
\hline 17 & 11.75 & 8862.76 & 146.32 & $9.09 \mathrm{E}+14$ \\
\hline 18 & 11.25 & 8766.23 & 144.73 & $9.32 \mathrm{E}+14$ \\
\hline 19 & 10.75 & 8969.19 & 148.08 & $9.50 \mathrm{E}+14$ \\
\hline 20 & 10.25 & 9287.20 & 153.33 & $9.67 \mathrm{E}+14$ \\
\hline 21 & 9.75 & 9509.57 & 157.00 & $9.87 \mathrm{E}+14$ \\
\hline 22 & 9.25 & 9586.39 & 158.27 & $9.96 \mathrm{E}+14$ \\
\hline 23 & 8.75 & 9697.19 & 160.10 & $1.01 \mathrm{E}+15$ \\
\hline 24 & 8.25 & 9847.54 & 162.58 & $1.02 \mathrm{E}+15$ \\
\hline 25 & 7.75 & 10076.21 & 166.36 & $1.04 \mathrm{E}+15$ \\
\hline 26 & 7.25 & 10008.52 & 165.24 & $1.04 \mathrm{E}+15$ \\
\hline 27 & 6.75 & 10190.34 & 168.24 & $1.05 \mathrm{E}+15$ \\
\hline 28 & 6.25 & 10114.27 & 166.99 & $1.06 \mathrm{E}+15$ \\
\hline 29 & 5.75 & 10405.96 & 171.80 & $1.07 \mathrm{E}+15$ \\
\hline 30 & 5.25 & 10378.69 & 171.35 & $1.08 \mathrm{E}+15$ \\
\hline 31 & 4.75 & 10488.08 & 173.16 & $1.09 \mathrm{E}+15$ \\
\hline 32 & 4.25 & 10280.62 & 169.73 & $1.09 \mathrm{E}+15$ \\
\hline 33 & 3.75 & 10571.17 & 174.53 & $1.09 \mathrm{E}+15$ \\
\hline 34 & 3.25 & 10683.32 & 176.38 & $1.10 \mathrm{E}+15$ \\
\hline 35 & 2.75 & 10759.20 & 177.63 & $1.11 \mathrm{E}+15$ \\
\hline 36 & 2.25 & 10803.36 & 178.36 & $1.12 \mathrm{E}+15$ \\
\hline 37 & 1.75 & 10536.01 & 173.95 & $1.11 \mathrm{E}+15$ \\
\hline 38 & 1.25 & 10500.67 & 173.37 & $1.12 \mathrm{E}+15$ \\
\hline 39 & 0.75 & 10411.07 & 171.89 & $1.12 \mathrm{E}+15$ \\
\hline 40 & 0.25 & 10710.40 & 176.83 & $1.12 \mathrm{E}+15$ \\
\hline 41 & -0.25 & 10517.17 & 173.64 & $1.12 \mathrm{E}+15$ \\
\hline 42 & -0.75 & 10558.64 & 174.32 & $1.12 \mathrm{E}+15$ \\
\hline 43 & -1.25 & 10640.89 & 175.68 & $1.13 \mathrm{E}+15$ \\
\hline 44 & -1.75 & 10828.60 & 178.78 & $1.13 \mathrm{E}+15$ \\
\hline 45 & -2.25 & 10841.65 & 179.00 & $1.12 \mathrm{E}+15$ \\
\hline 46 & -2.75 & 10731.42 & 177.18 & $1.11 \mathrm{E}+15$ \\
\hline 47 & -3.25 & 10595.77 & 174.94 & $1.11 \mathrm{E}+15$ \\
\hline 48 & -3.75 & 10682.52 & 176.37 & $1.11 \mathrm{E}+15$ \\
\hline 49 & -4.25 & 10556.63 & 174.29 & $1.10 \mathrm{E}+15$ \\
\hline 50 & -4.75 & 10614.87 & 175.25 & $1.10 \mathrm{E}+15$ \\
\hline 51 & -5.25 & 10473.76 & 172.92 & $1.10 \mathrm{E}+15$ \\
\hline 52 & -5.75 & 10390.38 & 171.55 & $1.09 \mathrm{E}+15$ \\
\hline
\end{tabular}




\begin{tabular}{|l|l|l|l|l|}
\hline 53 & -6.25 & 10475.44 & 172.95 & $1.09 \mathrm{E}+15$ \\
\hline 54 & -6.75 & 10295.62 & 169.98 & $1.08 \mathrm{E}+15$ \\
\hline 55 & -7.25 & 10359.33 & 171.03 & $1.07 \mathrm{E}+15$ \\
\hline 56 & -7.75 & 10241.10 & 169.08 & $1.06 \mathrm{E}+15$ \\
\hline 57 & -8.25 & 10111.83 & 166.95 & $1.05 \mathrm{E}+15$ \\
\hline 58 & -8.75 & 10113.24 & 166.97 & $1.04 \mathrm{E}+15$ \\
\hline 59 & -9.25 & 9942.50 & 164.15 & $1.04 \mathrm{E}+15$ \\
\hline 60 & -9.75 & 9903.05 & 163.50 & $1.02 \mathrm{E}+15$ \\
\hline 61 & -10.25 & 9500.80 & 156.86 & $1.02 \mathrm{E}+15$ \\
\hline 62 & -10.75 & 9379.79 & 154.86 & $1.00 \mathrm{E}+15$ \\
\hline 63 & -11.25 & 9328.24 & 154.01 & $9.91 \mathrm{E}+14$ \\
\hline 64 & -11.75 & 9131.05 & 150.75 & $9.70 \mathrm{E}+14$ \\
\hline 65 & -12.25 & 8911.51 & 147.13 & $9.56 \mathrm{E}+14$ \\
\hline 66 & -12.75 & 9043.95 & 149.32 & $9.43 \mathrm{E}+14$ \\
\hline 67 & -13.25 & 8778.92 & 144.94 & $9.17 \mathrm{E}+14$ \\
\hline 68 & -13.75 & 8685.22 & 143.39 & $9.06 \mathrm{E}+14$ \\
\hline 69 & -14.25 & 8681.14 & 143.33 & $8.87 \mathrm{E}+14$ \\
\hline 70 & -14.75 & 8356.08 & 137.96 & $8.67 \mathrm{E}+14$ \\
\hline 71 & -15.25 & 8096.23 & 133.67 & $8.39 \mathrm{E}+14$ \\
\hline 72 & -15.75 & 8020.43 & 132.42 & $8.18 \mathrm{E}+14$ \\
\hline 73 & -16.25 & 7718.09 & 127.43 & $8.01 \mathrm{E}+14$ \\
\hline 74 & -16.75 & 7514.54 & 124.07 & $7.68 \mathrm{E}+14$ \\
\hline 75 & -17.25 & 7273.08 & 120.08 & $7.46 \mathrm{E}+14$ \\
\hline 76 & -17.75 & 7027.03 & 116.02 & $7.12 \mathrm{E}+14$ \\
\hline 77 & -18.25 & 6795.68 & 112.20 & $6.85 \mathrm{E}+14$ \\
\hline 78 & -18.75 & 6791.28 & 112.12 & $6.66 \mathrm{E}+14$ \\
\hline
\end{tabular}


Table 37: Cycle 150B, MCNP-Calculated HGRs, Neutron Flux, Depletion and Fission Density for AFIP-7, Plate Position 4, 18 EFPD (MOC1) Average Center Lobe Power at 24.2 MW. ${ }^{2}$

\begin{tabular}{|c|c|c|c|c|c|c|}
\hline Node & $\begin{array}{c}\text { Distance from } \\
\text { Centerline (in.) }\end{array}$ & $\begin{array}{l}\text { Fission Power } \\
\text { Density (W/cc) }\end{array}$ & $\begin{array}{l}\text { Surface Heat } \\
\text { Flux }\left(W / \mathrm{cm}^{2}\right)\end{array}$ & $\begin{array}{c}\text { Neutron Flux } \\
\left(\mathrm{n} / \mathrm{cm}^{2} \mathrm{sec}\right)\end{array}$ & $\begin{array}{l}\text { Fission Density } \\
\text { (fissions/cc) }\end{array}$ & $\begin{array}{c}\text { U-235 } \\
\text { Burnup (\%) }\end{array}$ \\
\hline 1 & 19.25 & 6089.83 & 100.54 & $5.29 \mathrm{E}+14$ & $1.25 \mathrm{E}+21$ & $18.07 \%$ \\
\hline 2 & 18.75 & 5868.41 & 96.89 & $5.48 \mathrm{E}+14$ & $1.18 \mathrm{E}+21$ & $17.17 \%$ \\
\hline 3 & 18.25 & 5981.08 & 98.75 & $5.79 \mathrm{E}+14$ & $1.22 \mathrm{E}+21$ & $17.69 \%$ \\
\hline 4 & 17.75 & 6296.53 & 103.96 & $6.09 \mathrm{E}+14$ & $1.28 \mathrm{E}+21$ & $18.52 \%$ \\
\hline 5 & 17.25 & 6497.36 & 107.27 & $6.34 \mathrm{E}+14$ & $1.34 \mathrm{E}+21$ & $19.34 \%$ \\
\hline 6 & 16.75 & 6690.78 & 110.46 & $6.62 \mathrm{E}+14$ & $1.39 \mathrm{E}+21$ & $20.10 \%$ \\
\hline 7 & 16.25 & 7080.46 & 116.90 & $6.90 \mathrm{E}+14$ & $1.46 \mathrm{E}+21$ & $21.01 \%$ \\
\hline 8 & 15.75 & 7249.66 & 119.69 & $7.18 \mathrm{E}+14$ & $1.50 \mathrm{E}+21$ & $21.61 \%$ \\
\hline 9 & 15.25 & 7378.88 & 121.83 & $7.28 \mathrm{E}+14$ & $1.54 \mathrm{E}+21$ & $22.21 \%$ \\
\hline 10 & 14.75 & 7449.37 & 122.99 & $7.57 \mathrm{E}+14$ & $1.60 \mathrm{E}+21$ & $22.88 \%$ \\
\hline 11 & 14.25 & 7757.34 & 128.07 & $7.74 \mathrm{E}+14$ & $1.62 \mathrm{E}+21$ & $23.18 \%$ \\
\hline 13 & 13.75 & 7839.22 & 129.43 & $8.00 \mathrm{E}+14$ & $1.65 \mathrm{E}+21$ & $23.63 \%$ \\
\hline 14 & 13.25 & 7913.66 & 130.65 & $8.14 \mathrm{E}+14$ & $1.68 \mathrm{E}+21$ & $24.02 \%$ \\
\hline 15 & 12.75 & 7950.13 & 131.26 & $8.31 \mathrm{E}+14$ & $1.68 \mathrm{E}+21$ & $24.10 \%$ \\
\hline 16 & 12.25 & 8115.80 & 133.99 & $8.46 \mathrm{E}+14$ & $1.76 \mathrm{E}+21$ & $25.12 \%$ \\
\hline 17 & 11.75 & 8239.60 & 136.04 & $8.66 \mathrm{E}+14$ & $1.78 \mathrm{E}+21$ & $25.33 \%$ \\
\hline 18 & 11.25 & 8444.89 & 139.43 & $8.84 \mathrm{E}+14$ & $1.82 \mathrm{E}+21$ & $25.91 \%$ \\
\hline 19 & 10.75 & 8575.20 & 141.58 & $9.03 \mathrm{E}+14$ & $1.85 \mathrm{E}+21$ & $26.35 \%$ \\
\hline 20 & 10.25 & 8883.45 & 146.67 & $9.10 \mathrm{E}+14$ & $1.91 \mathrm{E}+21$ & $27.09 \%$ \\
\hline 21 & 9.75 & 9050.50 & 149.42 & $9.23 \mathrm{E}+14$ & $1.97 \mathrm{E}+21$ & $27.92 \%$ \\
\hline 22 & 9.25 & 9059.34 & 149.57 & $9.32 \mathrm{E}+14$ & $1.99 \mathrm{E}+21$ & $28.16 \%$ \\
\hline 23 & 8.75 & 9266.21 & 152.99 & $9.48 \mathrm{E}+14$ & $2.01 \mathrm{E}+21$ & $28.51 \%$ \\
\hline 24 & 8.25 & 9165.10 & 151.32 & $9.52 \mathrm{E}+14$ & $2.05 \mathrm{E}+21$ & $28.92 \%$ \\
\hline 25 & 7.75 & 9205.20 & 151.98 & $9.63 \mathrm{E}+14$ & $2.07 \mathrm{E}+21$ & $29.22 \%$ \\
\hline 26 & 7.25 & 9453.80 & 156.08 & $9.75 \mathrm{E}+14$ & $2.09 \mathrm{E}+21$ & $29.52 \%$ \\
\hline 27 & 6.75 & 9560.18 & 157.84 & $9.80 \mathrm{E}+14$ & $2.11 \mathrm{E}+21$ & $29.76 \%$ \\
\hline 28 & 6.25 & 9611.92 & 158.69 & $9.89 \mathrm{E}+14$ & $2.12 \mathrm{E}+21$ & $29.84 \%$ \\
\hline 29 & 5.75 & 9721.31 & 160.50 & $1.01 \mathrm{E}+15$ & $2.13 \mathrm{E}+21$ & $29.96 \%$ \\
\hline 30 & 5.25 & 9693.89 & 160.05 & $1.01 \mathrm{E}+15$ & $2.16 \mathrm{E}+21$ & $30.39 \%$ \\
\hline 31 & 4.75 & 9691.72 & 160.01 & $1.01 \mathrm{E}+15$ & $2.17 \mathrm{E}+21$ & $30.50 \%$ \\
\hline 32 & 4.25 & 9669.33 & 159.64 & $1.01 \mathrm{E}+15$ & $2.17 \mathrm{E}+21$ & $30.55 \%$ \\
\hline 33 & 3.75 & 9619.31 & 158.81 & $1.01 \mathrm{E}+15$ & $2.18 \mathrm{E}+21$ & $30.60 \%$ \\
\hline 34 & 3.25 & 9967.74 & 164.57 & $1.02 \mathrm{E}+15$ & $2.20 \mathrm{E}+21$ & $30.86 \%$ \\
\hline 35 & 2.75 & 9804.10 & 161.87 & $1.03 \mathrm{E}+15$ & $2.22 \mathrm{E}+21$ & $31.11 \%$ \\
\hline 36 & 2.25 & 9767.98 & 161.27 & $1.03 \mathrm{E}+15$ & $2.19 \mathrm{E}+21$ & $30.76 \%$ \\
\hline 37 & 1.75 & 9702.42 & 160.19 & $1.03 \mathrm{E}+15$ & $2.19 \mathrm{E}+21$ & $30.77 \%$ \\
\hline 38 & 1.25 & 9554.47 & 157.74 & $1.03 \mathrm{E}+15$ & $2.16 \mathrm{E}+21$ & $30.45 \%$ \\
\hline 39 & 0.75 & 9707.01 & 160.26 & $1.03 \mathrm{E}+15$ & $2.16 \mathrm{E}+21$ & $30.41 \%$ \\
\hline 40 & 0.25 & 9814.30 & 162.03 & $1.03 \mathrm{E}+15$ & $2.18 \mathrm{E}+21$ & $30.64 \%$ \\
\hline 41 & -0.25 & 9693.41 & 160.04 & $1.03 \mathrm{E}+15$ & $2.18 \mathrm{E}+21$ & $30.57 \%$ \\
\hline 42 & -0.75 & 9891.12 & 163.30 & $1.03 \mathrm{E}+15$ & $2.20 \mathrm{E}+21$ & $30.87 \%$ \\
\hline 43 & -1.25 & 10012.28 & 165.30 & $1.04 \mathrm{E}+15$ & $2.21 \mathrm{E}+21$ & $31.04 \%$ \\
\hline 44 & -1.75 & 9921.95 & 163.81 & $1.03 \mathrm{E}+15$ & $2.23 \mathrm{E}+21$ & $31.27 \%$ \\
\hline 45 & -2.25 & 9985.10 & 164.85 & $1.04 \mathrm{E}+15$ & $2.23 \mathrm{E}+21$ & $31.31 \%$ \\
\hline 46 & -2.75 & 9915.71 & 163.71 & $1.03 \mathrm{E}+15$ & $2.22 \mathrm{E}+21$ & $31.16 \%$ \\
\hline 47 & -3.25 & 9827.22 & 162.25 & $1.03 \mathrm{E}+15$ & $2.21 \mathrm{E}+21$ & $31.02 \%$ \\
\hline 48 & -3.75 & 9861.75 & 162.82 & $1.03 \mathrm{E}+15$ & $2.20 \mathrm{E}+21$ & $30.90 \%$ \\
\hline 49 & -4.25 & 9832.74 & 162.34 & $1.02 \mathrm{E}+15$ & $2.22 \mathrm{E}+21$ & $31.12 \%$ \\
\hline 50 & -4.75 & 9824.72 & 162.21 & $1.01 \mathrm{E}+15$ & $2.21 \mathrm{E}+21$ & $30.96 \%$ \\
\hline 51 & -5.25 & 9760.96 & 161.15 & $1.02 \mathrm{E}+15$ & $2.19 \mathrm{E}+21$ & $30.87 \%$ \\
\hline 52 & -5.75 & 9727.60 & 160.60 & $1.02 \mathrm{E}+15$ & $2.17 \mathrm{E}+21$ & $30.55 \%$ \\
\hline
\end{tabular}




\begin{tabular}{|l|l|l|l|l|l|l|}
\hline 53 & -6.25 & 9810.43 & 161.97 & $1.02 \mathrm{E}+15$ & $2.17 \mathrm{E}+21$ & $30.54 \%$ \\
\hline 54 & -6.75 & 9775.47 & 161.39 & $1.00 \mathrm{E}+15$ & $2.16 \mathrm{E}+21$ & $30.35 \%$ \\
\hline 55 & -7.25 & 9757.65 & 161.10 & $1.00 \mathrm{E}+15$ & $2.14 \mathrm{E}+21$ & $30.07 \%$ \\
\hline 56 & -7.75 & 9499.42 & 156.84 & $9.91 \mathrm{E}+14$ & $2.11 \mathrm{E}+21$ & $29.67 \%$ \\
\hline 57 & -8.25 & 9609.12 & 158.65 & $9.90 \mathrm{E}+14$ & $2.11 \mathrm{E}+21$ & $29.70 \%$ \\
\hline 58 & -8.75 & 9362.22 & 154.57 & $9.78 \mathrm{E}+14$ & $2.10 \mathrm{E}+21$ & $29.57 \%$ \\
\hline 59 & -9.25 & 9301.54 & 153.57 & $9.72 \mathrm{E}+14$ & $2.07 \mathrm{E}+21$ & $29.24 \%$ \\
\hline 60 & -9.75 & 9147.69 & 151.03 & $9.61 \mathrm{E}+14$ & $2.02 \mathrm{E}+21$ & $28.59 \%$ \\
\hline 61 & -10.25 & 9127.73 & 150.70 & $9.52 \mathrm{E}+14$ & $1.99 \mathrm{E}+21$ & $28.10 \%$ \\
\hline 62 & -10.75 & 8777.83 & 144.92 & $9.44 \mathrm{E}+14$ & $1.95 \mathrm{E}+21$ & $27.66 \%$ \\
\hline 63 & -11.25 & 8699.70 & 143.63 & $9.31 \mathrm{E}+14$ & $1.93 \mathrm{E}+21$ & $27.34 \%$ \\
\hline 64 & -11.75 & 8797.74 & 145.25 & $9.19 \mathrm{E}+14$ & $1.88 \mathrm{E}+21$ & $26.76 \%$ \\
\hline 65 & -12.25 & 8602.11 & 142.02 & $9.03 \mathrm{E}+14$ & $1.84 \mathrm{E}+21$ & $26.26 \%$ \\
\hline 66 & -12.75 & 8559.80 & 141.32 & $8.91 \mathrm{E}+14$ & $1.85 \mathrm{E}+21$ & $26.31 \%$ \\
\hline 67 & -13.25 & 8587.54 & 141.78 & $8.78 \mathrm{E}+14$ & $1.82 \mathrm{E}+21$ & $25.95 \%$ \\
\hline 68 & -13.75 & 8256.69 & 136.32 & $8.58 \mathrm{E}+14$ & $1.80 \mathrm{E}+21$ & $25.65 \%$ \\
\hline 69 & -14.25 & 8291.86 & 136.90 & $8.38 \mathrm{E}+14$ & $1.77 \mathrm{E}+21$ & $25.33 \%$ \\
\hline 70 & -14.75 & 8088.30 & 133.54 & $8.25 \mathrm{E}+14$ & $1.73 \mathrm{E}+21$ & $24.75 \%$ \\
\hline 71 & -15.25 & 7938.67 & 131.07 & $8.11 \mathrm{E}+14$ & $1.71 \mathrm{E}+21$ & $24.40 \%$ \\
\hline 72 & -15.75 & 7873.10 & 129.98 & $7.87 \mathrm{E}+14$ & $1.66 \mathrm{E}+21$ & $23.72 \%$ \\
\hline 73 & -16.25 & 7545.42 & 124.57 & $7.71 \mathrm{E}+14$ & $1.60 \mathrm{E}+21$ & $22.88 \%$ \\
\hline 74 & -16.75 & 7485.47 & 123.59 & $7.45 \mathrm{E}+14$ & $1.54 \mathrm{E}+21$ & $22.13 \%$ \\
\hline 75 & -17.25 & 7233.78 & 119.43 & $7.31 \mathrm{E}+14$ & $1.50 \mathrm{E}+21$ & $21.68 \%$ \\
\hline 76 & -17.75 & 6965.03 & 114.99 & $6.95 \mathrm{E}+14$ & $1.43 \mathrm{E}+21$ & $20.56 \%$ \\
\hline 77 & -18.25 & 6742.34 & 111.32 & $6.71 \mathrm{E}+14$ & $1.40 \mathrm{E}+21$ & $20.26 \%$ \\
\hline 78 & -18.75 & 6500.51 & 107.32 & $6.42 \mathrm{E}+14$ & $1.35 \mathrm{E}+21$ & $19.57 \%$ \\
\hline
\end{tabular}


Table 38: Cycle 150B, MCNP-Calculated HGRs, Neutron Flux, Depletion and Fission Density for AFIP-7, Plate Position 4, 31 EFPD (MOC2) Average Center Lobe Power at 24.2 MW. ${ }^{2}$

\begin{tabular}{|c|c|c|c|c|c|c|}
\hline Node & $\begin{array}{c}\text { Distance from } \\
\text { Centerline (in.) }\end{array}$ & $\begin{array}{l}\text { Fission Power } \\
\text { Density (W/cc) }\end{array}$ & $\begin{array}{l}\text { Surface Heat } \\
\text { Flux }\left(W / \mathrm{cm}^{2}\right)\end{array}$ & $\begin{array}{c}\text { Neutron Flux } \\
\left(\mathrm{n} / \mathrm{cm}^{2} \mathrm{sec}\right)\end{array}$ & $\begin{array}{l}\text { Fission Density } \\
\text { (fissions/cc) }\end{array}$ & $\begin{array}{c}\text { U-235 } \\
\text { Burnup (\%) }\end{array}$ \\
\hline 1 & 19.25 & 6165.66 & 101.80 & $5.35 \mathrm{E}+14$ & $1.47 \mathrm{E}+21$ & $21.23 \%$ \\
\hline 2 & 18.75 & 5945.82 & 98.17 & $5.67 \mathrm{E}+14$ & $1.40 \mathrm{E}+21$ & $20.26 \%$ \\
\hline 3 & 18.25 & 6035.74 & 99.65 & $5.94 \mathrm{E}+14$ & $1.44 \mathrm{E}+21$ & $20.78 \%$ \\
\hline 4 & 17.75 & 6391.14 & 105.52 & $6.27 \mathrm{E}+14$ & $1.51 \mathrm{E}+21$ & $21.83 \%$ \\
\hline 5 & 17.25 & 6567.37 & 108.43 & $6.50 \mathrm{E}+14$ & $1.58 \mathrm{E}+21$ & $22.73 \%$ \\
\hline 6 & 16.75 & 6875.90 & 113.52 & $6.81 \mathrm{E}+14$ & $1.64 \mathrm{E}+21$ & $23.56 \%$ \\
\hline 7 & 16.25 & 7112.16 & 117.42 & $7.09 \mathrm{E}+14$ & $1.71 \mathrm{E}+21$ & $24.62 \%$ \\
\hline 8 & 15.75 & 7143.35 & 117.94 & $7.28 \mathrm{E}+14$ & $1.77 \mathrm{E}+21$ & $25.35 \%$ \\
\hline 9 & 15.25 & 7293.03 & 120.41 & $7.49 \mathrm{E}+14$ & $1.81 \mathrm{E}+21$ & $25.95 \%$ \\
\hline 10 & 14.75 & 7546.61 & 124.59 & $7.73 \mathrm{E}+14$ & $1.87 \mathrm{E}+21$ & $26.72 \%$ \\
\hline 11 & 14.25 & 7803.50 & 128.84 & $7.89 \mathrm{E}+14$ & $1.90 \mathrm{E}+21$ & $27.14 \%$ \\
\hline 13 & 13.75 & 7876.75 & 130.05 & $8.13 \mathrm{E}+14$ & $1.94 \mathrm{E}+21$ & $27.64 \%$ \\
\hline 14 & 13.25 & 8055.37 & 132.99 & $8.40 \mathrm{E}+14$ & $1.97 \mathrm{E}+21$ & $28.04 \%$ \\
\hline 15 & 12.75 & 8020.03 & 132.41 & $8.45 \mathrm{E}+14$ & $1.98 \mathrm{E}+21$ & $28.09 \%$ \\
\hline 16 & 12.25 & 8218.58 & 135.69 & $8.67 \mathrm{E}+14$ & $2.06 \mathrm{E}+21$ & $29.21 \%$ \\
\hline 17 & 11.75 & 8330.07 & 137.53 & $8.81 \mathrm{E}+14$ & $2.08 \mathrm{E}+21$ & $29.47 \%$ \\
\hline 18 & 11.25 & 8491.37 & 140.19 & $8.91 \mathrm{E}+14$ & $2.13 \mathrm{E}+21$ & $30.15 \%$ \\
\hline 19 & 10.75 & 8589.06 & 141.81 & $9.10 \mathrm{E}+14$ & $2.17 \mathrm{E}+21$ & $30.64 \%$ \\
\hline 20 & 10.25 & 8664.74 & 143.05 & $9.25 \mathrm{E}+14$ & $2.23 \mathrm{E}+21$ & $31.54 \%$ \\
\hline 21 & 9.75 & 8886.97 & 146.72 & $9.40 \mathrm{E}+14$ & $2.31 \mathrm{E}+21$ & $32.42 \%$ \\
\hline 22 & 9.25 & 9149.23 & 151.05 & $9.53 \mathrm{E}+14$ & $2.32 \mathrm{E}+21$ & $32.69 \%$ \\
\hline 23 & 8.75 & 9150.19 & 151.07 & $9.69 \mathrm{E}+14$ & $2.35 \mathrm{E}+21$ & $33.12 \%$ \\
\hline 24 & 8.25 & 9116.43 & 150.51 & $9.67 \mathrm{E}+14$ & $2.39 \mathrm{E}+21$ & $33.46 \%$ \\
\hline 25 & 7.75 & 9196.74 & 151.84 & $9.84 \mathrm{E}+14$ & $2.41 \mathrm{E}+21$ & $33.79 \%$ \\
\hline 26 & 7.25 & 9355.72 & 154.46 & $9.90 \mathrm{E}+14$ & $2.44 \mathrm{E}+21$ & $34.19 \%$ \\
\hline 27 & 6.75 & 9453.40 & 156.08 & $9.88 \mathrm{E}+14$ & $2.46 \mathrm{E}+21$ & $34.50 \%$ \\
\hline 28 & 6.25 & 9660.89 & 159.50 & $1.01 \mathrm{E}+15$ & $2.47 \mathrm{E}+21$ & $34.58 \%$ \\
\hline 29 & 5.75 & 9644.50 & 159.23 & $1.02 \mathrm{E}+15$ & $2.49 \mathrm{E}+21$ & $34.75 \%$ \\
\hline 30 & 5.25 & 9485.59 & 156.61 & $1.03 \mathrm{E}+15$ & $2.52 \mathrm{E}+21$ & $35.15 \%$ \\
\hline 31 & 4.75 & 9751.82 & 161.00 & $1.02 \mathrm{E}+15$ & $2.53 \mathrm{E}+21$ & $35.24 \%$ \\
\hline 32 & 4.25 & 9693.23 & 160.04 & $1.02 \mathrm{E}+15$ & $2.53 \mathrm{E}+21$ & $35.31 \%$ \\
\hline 33 & 3.75 & 9828.60 & 162.27 & $1.03 \mathrm{E}+15$ & $2.53 \mathrm{E}+21$ & $35.34 \%$ \\
\hline 34 & 3.25 & 9660.16 & 159.49 & $1.03 \mathrm{E}+15$ & $2.57 \mathrm{E}+21$ & $35.75 \%$ \\
\hline 35 & 2.75 & 9737.43 & 160.76 & $1.04 \mathrm{E}+15$ & $2.58 \mathrm{E}+21$ & $35.91 \%$ \\
\hline 36 & 2.25 & 9827.47 & 162.25 & $1.05 \mathrm{E}+15$ & $2.55 \mathrm{E}+21$ & $35.56 \%$ \\
\hline 37 & 1.75 & 9809.01 & 161.95 & $1.04 \mathrm{E}+15$ & $2.55 \mathrm{E}+21$ & $35.54 \%$ \\
\hline 38 & 1.25 & 9653.13 & 159.37 & $1.04 \mathrm{E}+15$ & $2.51 \mathrm{E}+21$ & $35.15 \%$ \\
\hline 39 & 0.75 & 9794.43 & 161.71 & $1.05 \mathrm{E}+15$ & $2.52 \mathrm{E}+21$ & $35.18 \%$ \\
\hline 40 & 0.25 & 9804.57 & 161.87 & $1.06 \mathrm{E}+15$ & $2.54 \mathrm{E}+21$ & $35.45 \%$ \\
\hline 41 & -0.25 & 9746.31 & 160.91 & $1.05 \mathrm{E}+15$ & $2.53 \mathrm{E}+21$ & $35.34 \%$ \\
\hline 42 & -0.75 & 9697.70 & 160.11 & $1.05 \mathrm{E}+15$ & $2.57 \mathrm{E}+21$ & $35.71 \%$ \\
\hline 43 & -1.25 & 9759.92 & 161.14 & $1.05 \mathrm{E}+15$ & $2.58 \mathrm{E}+21$ & $35.93 \%$ \\
\hline 44 & -1.75 & 9761.84 & 161.17 & $1.04 \mathrm{E}+15$ & $2.59 \mathrm{E}+21$ & $36.14 \%$ \\
\hline 45 & -2.25 & 9957.13 & 164.39 & $1.06 \mathrm{E}+15$ & $2.60 \mathrm{E}+21$ & $36.20 \%$ \\
\hline 46 & -2.75 & 9975.17 & 164.69 & $1.05 \mathrm{E}+15$ & $2.59 \mathrm{E}+21$ & $36.03 \%$ \\
\hline 47 & -3.25 & 9744.76 & 160.89 & $1.05 \mathrm{E}+15$ & $2.57 \mathrm{E}+21$ & $35.85 \%$ \\
\hline 48 & -3.75 & 9739.50 & 160.80 & $1.04 \mathrm{E}+15$ & $2.56 \mathrm{E}+21$ & $35.74 \%$ \\
\hline 49 & -4.25 & 9799.31 & 161.79 & $1.04 \mathrm{E}+15$ & $2.58 \mathrm{E}+21$ & $35.95 \%$ \\
\hline 50 & -4.75 & 9708.98 & 160.30 & $1.04 \mathrm{E}+15$ & $2.57 \mathrm{E}+21$ & $35.79 \%$ \\
\hline 51 & -5.25 & 9734.29 & 160.71 & $1.03 \mathrm{E}+15$ & $2.55 \mathrm{E}+21$ & $35.70 \%$ \\
\hline 52 & -5.75 & 9620.70 & 158.84 & $1.03 \mathrm{E}+15$ & $2.53 \mathrm{E}+21$ & $35.33 \%$ \\
\hline
\end{tabular}




\begin{tabular}{|l|l|l|l|l|l|l|}
\hline 53 & -6.25 & 9761.42 & 161.16 & $1.03 \mathrm{E}+15$ & $2.53 \mathrm{E}+21$ & $35.36 \%$ \\
\hline 54 & -6.75 & 9726.43 & 160.58 & $1.02 \mathrm{E}+15$ & $2.52 \mathrm{E}+21$ & $35.16 \%$ \\
\hline 55 & -7.25 & 9577.08 & 158.12 & $1.02 \mathrm{E}+15$ & $2.50 \mathrm{E}+21$ & $34.86 \%$ \\
\hline 56 & -7.75 & 9553.39 & 157.73 & $1.00 \mathrm{E}+15$ & $2.45 \mathrm{E}+21$ & $34.36 \%$ \\
\hline 57 & -8.25 & 9644.16 & 159.23 & $1.01 \mathrm{E}+15$ & $2.46 \mathrm{E}+21$ & $34.41 \%$ \\
\hline 58 & -8.75 & 9506.66 & 156.95 & $1.00 \mathrm{E}+15$ & $2.44 \mathrm{E}+21$ & $34.21 \%$ \\
\hline 59 & -9.25 & 9240.99 & 152.57 & $9.90 \mathrm{E}+14$ & $2.41 \mathrm{E}+21$ & $33.86 \%$ \\
\hline 60 & -9.75 & 9385.51 & 154.95 & $9.83 \mathrm{E}+14$ & $2.36 \mathrm{E}+21$ & $33.12 \%$ \\
\hline 61 & -10.25 & 9282.93 & 153.26 & $9.73 \mathrm{E}+14$ & $2.32 \mathrm{E}+21$ & $32.64 \%$ \\
\hline 62 & -10.75 & 8979.92 & 148.26 & $9.61 \mathrm{E}+14$ & $2.27 \mathrm{E}+21$ & $32.05 \%$ \\
\hline 63 & -11.25 & 8914.33 & 147.18 & $9.51 \mathrm{E}+14$ & $2.25 \mathrm{E}+21$ & $31.69 \%$ \\
\hline 64 & -11.75 & 8733.28 & 144.19 & $9.38 \mathrm{E}+14$ & $2.21 \mathrm{E}+21$ & $31.14 \%$ \\
\hline 65 & -12.25 & 8737.76 & 144.26 & $9.30 \mathrm{E}+14$ & $2.16 \mathrm{E}+21$ & $30.59 \%$ \\
\hline 66 & -12.75 & 8659.91 & 142.98 & $9.04 \mathrm{E}+14$ & $2.16 \mathrm{E}+21$ & $30.60 \%$ \\
\hline 67 & -13.25 & 8490.97 & 140.19 & $8.90 \mathrm{E}+14$ & $2.14 \mathrm{E}+21$ & $30.26 \%$ \\
\hline 68 & -13.75 & 8523.51 & 140.72 & $8.74 \mathrm{E}+14$ & $2.10 \mathrm{E}+21$ & $29.81 \%$ \\
\hline 69 & -14.25 & 8428.52 & 139.15 & $8.61 \mathrm{E}+14$ & $2.08 \mathrm{E}+21$ & $29.51 \%$ \\
\hline 70 & -14.75 & 8141.06 & 134.41 & $8.48 \mathrm{E}+14$ & $2.03 \mathrm{E}+21$ & $28.84 \%$ \\
\hline 71 & -15.25 & 8017.43 & 132.37 & $8.31 \mathrm{E}+14$ & $2.00 \mathrm{E}+21$ & $28.40 \%$ \\
\hline 72 & -15.75 & 8007.26 & 132.20 & $8.07 \mathrm{E}+14$ & $1.95 \mathrm{E}+21$ & $27.71 \%$ \\
\hline 73 & -16.25 & 7728.57 & 127.60 & $7.89 \mathrm{E}+14$ & $1.87 \mathrm{E}+21$ & $26.72 \%$ \\
\hline 74 & -16.75 & 7501.47 & 123.85 & $7.60 \mathrm{E}+14$ & $1.81 \mathrm{E}+21$ & $26.01 \%$ \\
\hline 75 & -17.25 & 7110.37 & 117.39 & $7.41 \mathrm{E}+14$ & $1.77 \mathrm{E}+21$ & $25.36 \%$ \\
\hline 76 & -17.75 & 6893.69 & 113.81 & $7.08 \mathrm{E}+14$ & $1.69 \mathrm{E}+21$ & $24.17 \%$ \\
\hline 77 & -18.25 & 6914.68 & 114.16 & $6.90 \mathrm{E}+14$ & $1.65 \mathrm{E}+21$ & $23.72 \%$ \\
\hline 78 & -18.75 & 6640.64 & 109.64 & $6.58 \mathrm{E}+14$ & $1.59 \mathrm{E}+21$ & $22.88 \%$ \\
\hline
\end{tabular}


Table 39: Cycle 150B, MCNP-Calculated HGRs, Neutron Flux, Depletion and Fission Density for AFIP-7, Plate Position 4, 42.0 EFPD (EOC) Average Center Lobe Power at 24.2 MW. ${ }^{2}$

\begin{tabular}{|c|c|c|c|c|c|c|}
\hline Node & $\begin{array}{c}\text { Distance from } \\
\text { Centerline (in.) }\end{array}$ & $\begin{array}{l}\text { Fission Power } \\
\text { Density (W/cc) }\end{array}$ & $\begin{array}{l}\text { Surface Heat } \\
\text { Flux }\left(W / \mathrm{cm}^{2}\right)\end{array}$ & $\begin{array}{c}\text { Neutron Flux } \\
\left(\mathrm{n} / \mathrm{cm}^{2} \mathrm{sec}\right)\end{array}$ & $\begin{array}{l}\text { Fission Density } \\
\text { (fissions/cc) }\end{array}$ & $\begin{array}{c}\text { U-235 } \\
\text { Burnup (\%) }\end{array}$ \\
\hline 1 & 19.25 & 6185.52 & 102.12 & $5.41 \mathrm{E}+14$ & $1.66 \mathrm{E}+21$ & $23.87 \%$ \\
\hline 2 & 18.75 & 5941.36 & 98.09 & $5.72 \mathrm{E}+14$ & $1.58 \mathrm{E}+21$ & $22.81 \%$ \\
\hline 3 & 18.25 & 6079.68 & 100.38 & $6.00 \mathrm{E}+14$ & $1.62 \mathrm{E}+21$ & $23.41 \%$ \\
\hline 4 & 17.75 & 6394.39 & 105.57 & $6.34 \mathrm{E}+14$ & $1.71 \mathrm{E}+21$ & $24.55 \%$ \\
\hline 5 & 17.25 & 6537.81 & 107.94 & $6.56 \mathrm{E}+14$ & $1.78 \mathrm{E}+21$ & $25.57 \%$ \\
\hline 6 & 16.75 & 6826.14 & 112.70 & $6.87 \mathrm{E}+14$ & $1.85 \mathrm{E}+21$ & $26.52 \%$ \\
\hline 7 & 16.25 & 7090.43 & 117.06 & $7.16 \mathrm{E}+14$ & $1.93 \mathrm{E}+21$ & $27.67 \%$ \\
\hline 8 & 15.75 & 7122.76 & 117.60 & $7.35 \mathrm{E}+14$ & $1.99 \mathrm{E}+21$ & $28.41 \%$ \\
\hline 9 & 15.25 & 7256.65 & 119.81 & $7.55 \mathrm{E}+14$ & $2.04 \mathrm{E}+21$ & $29.06 \%$ \\
\hline 10 & 14.75 & 7501.15 & 123.84 & $7.81 \mathrm{E}+14$ & $2.10 \mathrm{E}+21$ & $29.92 \%$ \\
\hline 11 & 14.25 & 7742.50 & 127.83 & $7.97 \mathrm{E}+14$ & $2.14 \mathrm{E}+21$ & $30.44 \%$ \\
\hline 13 & 13.75 & 7845.70 & 129.53 & $8.22 \mathrm{E}+14$ & $2.18 \mathrm{E}+21$ & $30.97 \%$ \\
\hline 14 & 13.25 & 7977.93 & 131.72 & $8.47 \mathrm{E}+14$ & $2.21 \mathrm{E}+21$ & $31.44 \%$ \\
\hline 15 & 12.75 & 7977.04 & 131.70 & $8.52 \mathrm{E}+14$ & $2.22 \mathrm{E}+21$ & $31.46 \%$ \\
\hline 16 & 12.25 & 8170.86 & 134.90 & $8.78 \mathrm{E}+14$ & $2.31 \mathrm{E}+21$ & $32.66 \%$ \\
\hline 17 & 11.75 & 8274.30 & 136.61 & $8.90 \mathrm{E}+14$ & $2.34 \mathrm{E}+21$ & $32.95 \%$ \\
\hline 18 & 11.25 & 8411.22 & 138.87 & $9.00 \mathrm{E}+14$ & $2.39 \mathrm{E}+21$ & $33.69 \%$ \\
\hline 19 & 10.75 & 8519.52 & 140.66 & $9.18 \mathrm{E}+14$ & $2.43 \mathrm{E}+21$ & $34.23 \%$ \\
\hline 20 & 10.25 & 8626.71 & 142.43 & $9.34 \mathrm{E}+14$ & $2.50 \mathrm{E}+21$ & $35.15 \%$ \\
\hline 21 & 9.75 & 8829.43 & 145.77 & $9.49 \mathrm{E}+14$ & $2.58 \mathrm{E}+21$ & $36.10 \%$ \\
\hline 22 & 9.25 & 9048.62 & 149.39 & $9.60 \mathrm{E}+14$ & $2.60 \mathrm{E}+21$ & $36.46 \%$ \\
\hline 23 & 8.75 & 9044.49 & 149.32 & $9.78 \mathrm{E}+14$ & $2.63 \mathrm{E}+21$ & $36.90 \%$ \\
\hline 24 & 8.25 & 9046.64 & 149.36 & $9.74 \mathrm{E}+14$ & $2.67 \mathrm{E}+21$ & $37.23 \%$ \\
\hline 25 & 7.75 & 9120.78 & 150.58 & $9.91 \mathrm{E}+14$ & $2.69 \mathrm{E}+21$ & $37.58 \%$ \\
\hline 26 & 7.25 & 9215.87 & 152.15 & $9.97 \mathrm{E}+14$ & $2.73 \mathrm{E}+21$ & $38.04 \%$ \\
\hline 27 & 6.75 & 9320.14 & 153.88 & $9.99 \mathrm{E}+14$ & $2.75 \mathrm{E}+21$ & $38.39 \%$ \\
\hline 28 & 6.25 & 9515.72 & 157.10 & $1.01 \mathrm{E}+15$ & $2.77 \mathrm{E}+21$ & $38.53 \%$ \\
\hline 29 & 5.75 & 9510.89 & 157.02 & $1.03 \mathrm{E}+15$ & $2.78 \mathrm{E}+21$ & $38.69 \%$ \\
\hline 30 & 5.25 & 9406.47 & 155.30 & $1.03 \mathrm{E}+15$ & $2.81 \mathrm{E}+21$ & $39.04 \%$ \\
\hline 31 & 4.75 & 9650.87 & 159.34 & $1.03 \mathrm{E}+15$ & $2.83 \mathrm{E}+21$ & $39.20 \%$ \\
\hline 32 & 4.25 & 9550.09 & 157.67 & $1.03 \mathrm{E}+15$ & $2.82 \mathrm{E}+21$ & $39.28 \%$ \\
\hline 33 & 3.75 & 9658.71 & 159.47 & $1.04 \mathrm{E}+15$ & $2.83 \mathrm{E}+21$ & $39.34 \%$ \\
\hline 34 & 3.25 & 9566.30 & 157.94 & $1.04 \mathrm{E}+15$ & $2.86 \mathrm{E}+21$ & $39.68 \%$ \\
\hline 35 & 2.75 & 9609.88 & 158.66 & $1.05 \mathrm{E}+15$ & $2.88 \mathrm{E}+21$ & $39.88 \%$ \\
\hline 36 & 2.25 & 9648.41 & 159.30 & $1.06 \mathrm{E}+15$ & $2.85 \mathrm{E}+21$ & $39.56 \%$ \\
\hline 37 & 1.75 & 9666.75 & 159.60 & $1.05 \mathrm{E}+15$ & $2.85 \mathrm{E}+21$ & $39.54 \%$ \\
\hline 38 & 1.25 & 9544.88 & 157.59 & $1.05 \mathrm{E}+15$ & $2.81 \mathrm{E}+21$ & $39.08 \%$ \\
\hline 39 & 0.75 & 9651.50 & 159.35 & $1.06 \mathrm{E}+15$ & $2.82 \mathrm{E}+21$ & $39.16 \%$ \\
\hline 40 & 0.25 & 9654.78 & 159.40 & $1.07 \mathrm{E}+15$ & $2.84 \mathrm{E}+21$ & $39.45 \%$ \\
\hline 41 & -0.25 & 9623.32 & 158.88 & $1.06 \mathrm{E}+15$ & $2.83 \mathrm{E}+21$ & $39.30 \%$ \\
\hline 42 & -0.75 & 9584.95 & 158.25 & $1.06 \mathrm{E}+15$ & $2.86 \mathrm{E}+21$ & $39.64 \%$ \\
\hline 43 & -1.25 & 9653.08 & 159.37 & $1.06 \mathrm{E}+15$ & $2.88 \mathrm{E}+21$ & $39.90 \%$ \\
\hline 44 & -1.75 & 9620.07 & 158.83 & $1.05 \mathrm{E}+15$ & $2.90 \mathrm{E}+21$ & $40.12 \%$ \\
\hline 45 & -2.25 & 9814.22 & 162.03 & $1.07 \mathrm{E}+15$ & $2.90 \mathrm{E}+21$ & $40.24 \%$ \\
\hline 46 & -2.75 & 9768.68 & 161.28 & $1.06 \mathrm{E}+15$ & $2.89 \mathrm{E}+21$ & $40.09 \%$ \\
\hline 47 & -3.25 & 9615.73 & 158.76 & $1.06 \mathrm{E}+15$ & $2.87 \mathrm{E}+21$ & $39.83 \%$ \\
\hline 48 & -3.75 & 9647.07 & 159.27 & $1.05 \mathrm{E}+15$ & $2.86 \mathrm{E}+21$ & $39.71 \%$ \\
\hline 49 & -4.25 & 9649.30 & 159.31 & $1.05 \mathrm{E}+15$ & $2.88 \mathrm{E}+21$ & $39.94 \%$ \\
\hline 50 & -4.75 & 9567.10 & 157.95 & $1.04 \mathrm{E}+15$ & $2.87 \mathrm{E}+21$ & $39.75 \%$ \\
\hline 51 & -5.25 & 9552.03 & 157.70 & $1.04 \mathrm{E}+15$ & $2.85 \mathrm{E}+21$ & $39.69 \%$ \\
\hline 52 & -5.75 & 9464.20 & 156.25 & $1.04 \mathrm{E}+15$ & $2.83 \mathrm{E}+21$ & $39.25 \%$ \\
\hline
\end{tabular}




\begin{tabular}{|l|l|l|l|l|l|l|}
\hline 53 & -6.25 & 9578.03 & 158.13 & $1.03 \mathrm{E}+15$ & $2.83 \mathrm{E}+21$ & $39.35 \%$ \\
\hline 54 & -6.75 & 9583.24 & 158.22 & $1.03 \mathrm{E}+15$ & $2.82 \mathrm{E}+21$ & $39.14 \%$ \\
\hline 55 & -7.25 & 9496.09 & 156.78 & $1.03 \mathrm{E}+15$ & $2.79 \mathrm{E}+21$ & $38.79 \%$ \\
\hline 56 & -7.75 & 9431.00 & 155.71 & $1.01 \mathrm{E}+15$ & $2.75 \mathrm{E}+21$ & $38.28 \%$ \\
\hline 57 & -8.25 & 9497.04 & 156.80 & $1.02 \mathrm{E}+15$ & $2.76 \mathrm{E}+21$ & $38.35 \%$ \\
\hline 58 & -8.75 & 9306.97 & 153.66 & $1.01 \mathrm{E}+15$ & $2.73 \mathrm{E}+21$ & $38.09 \%$ \\
\hline 59 & -9.25 & 9162.67 & 151.28 & $9.98 \mathrm{E}+14$ & $2.70 \mathrm{E}+21$ & $37.68 \%$ \\
\hline 60 & -9.75 & 9266.43 & 152.99 & $9.91 \mathrm{E}+14$ & $2.65 \mathrm{E}+21$ & $37.00 \%$ \\
\hline 61 & -10.25 & 9123.34 & 150.63 & $9.82 \mathrm{E}+14$ & $2.61 \mathrm{E}+21$ & $36.46 \%$ \\
\hline 62 & -10.75 & 8821.42 & 145.64 & $9.69 \mathrm{E}+14$ & $2.55 \mathrm{E}+21$ & $35.78 \%$ \\
\hline 63 & -11.25 & 8789.63 & 145.12 & $9.60 \mathrm{E}+14$ & $2.52 \mathrm{E}+21$ & $35.40 \%$ \\
\hline 64 & -11.75 & 8678.69 & 143.29 & $9.47 \mathrm{E}+14$ & $2.48 \mathrm{E}+21$ & $34.76 \%$ \\
\hline 65 & -12.25 & 8634.00 & 142.55 & $9.37 \mathrm{E}+14$ & $2.43 \mathrm{E}+21$ & $34.24 \%$ \\
\hline 66 & -12.75 & 8577.74 & 141.62 & $9.12 \mathrm{E}+14$ & $2.43 \mathrm{E}+21$ & $34.23 \%$ \\
\hline 67 & -13.25 & 8445.00 & 139.43 & $9.01 \mathrm{E}+14$ & $2.40 \mathrm{E}+21$ & $33.81 \%$ \\
\hline 68 & -13.75 & 8441.09 & 139.36 & $8.82 \mathrm{E}+14$ & $2.37 \mathrm{E}+21$ & $33.39 \%$ \\
\hline 69 & -14.25 & 8371.03 & 138.21 & $8.69 \mathrm{E}+14$ & $2.34 \mathrm{E}+21$ & $33.02 \%$ \\
\hline 70 & -14.75 & 8091.67 & 133.59 & $8.56 \mathrm{E}+14$ & $2.28 \mathrm{E}+21$ & $32.27 \%$ \\
\hline 71 & -15.25 & 7955.94 & 131.35 & $8.40 \mathrm{E}+14$ & $2.24 \mathrm{E}+21$ & $31.79 \%$ \\
\hline 72 & -15.75 & 8002.67 & 132.12 & $8.13 \mathrm{E}+14$ & $2.19 \mathrm{E}+21$ & $31.07 \%$ \\
\hline 73 & -16.25 & 7652.36 & 126.34 & $7.95 \mathrm{E}+14$ & $2.11 \mathrm{E}+21$ & $29.99 \%$ \\
\hline 74 & -16.75 & 7475.44 & 123.42 & $7.69 \mathrm{E}+14$ & $2.05 \mathrm{E}+21$ & $29.21 \%$ \\
\hline 75 & -17.25 & 7083.99 & 116.96 & $7.48 \mathrm{E}+14$ & $1.99 \mathrm{E}+21$ & $28.40 \%$ \\
\hline 76 & -17.75 & 6891.16 & 113.77 & $7.15 \mathrm{E}+14$ & $1.90 \mathrm{E}+21$ & $27.12 \%$ \\
\hline 77 & -18.25 & 6874.94 & 113.51 & $6.95 \mathrm{E}+14$ & $1.86 \mathrm{E}+21$ & $26.72 \%$ \\
\hline 78 & -18.75 & 6649.00 & 109.77 & $6.64 \mathrm{E}+14$ & $1.80 \mathrm{E}+21$ & $25.77 \%$ \\
\hline
\end{tabular}



iी

눙

त

$\stackrel{1}{\circ}$

तี

으을

뉸

. $\frac{\pi}{0} \frac{0}{\pi}$

证

o. .

$\exists$

으응

च

䒕

के

क्षे

$0 \frac{\pi}{0}$

흔

पै

.릉

吾

䜦

ए

它

$\exists$ 唃

을 멈

च छ

클

즘

$\theta \pi$

$\exists 0$

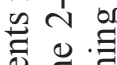

电

के

다욜

응 릴

인 몽

(1)

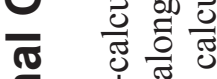

득흔

ह

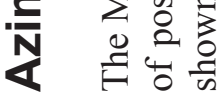

บ

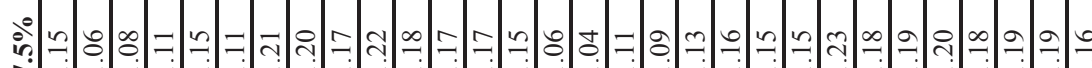

认े

î̉.

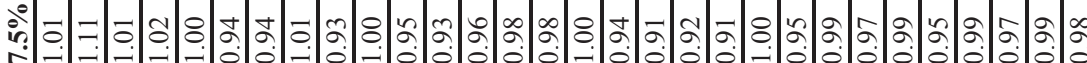

îं

2

eำ

î̉

in:

紊

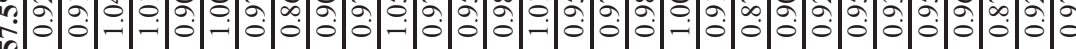

$\stackrel{\frac{5}{5}}{\frac{2}{0}}$

iी

赵赵

\%

छ)

î.

○

îำ

彳్లో

îำ

+

îํ:

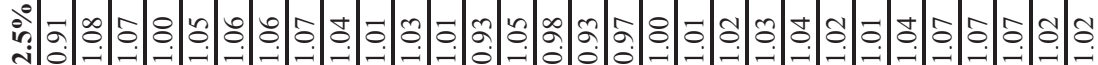

inํ:

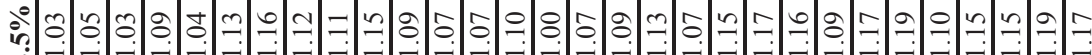
के

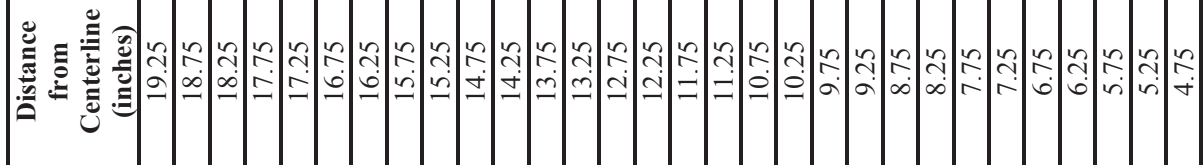




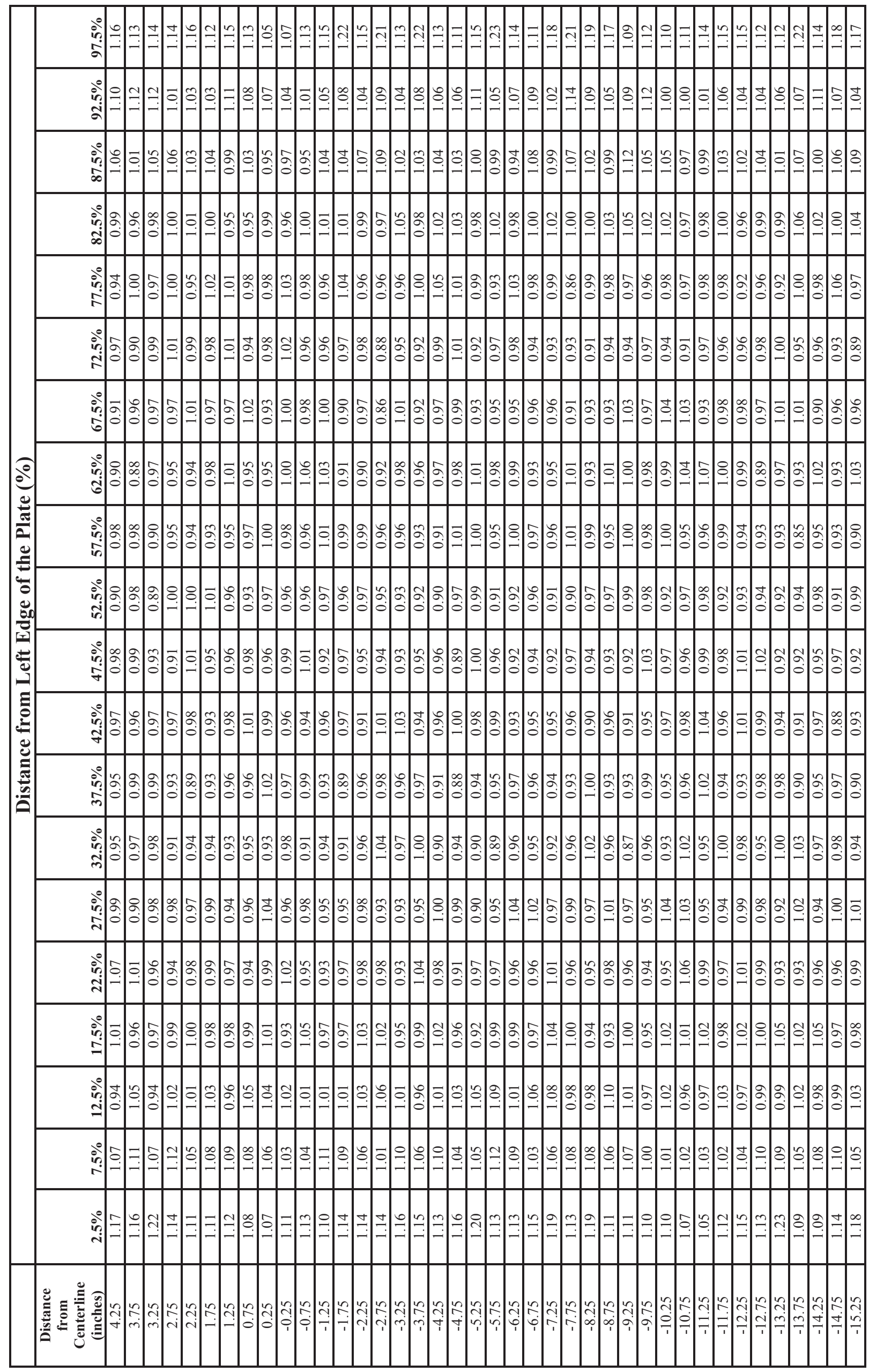




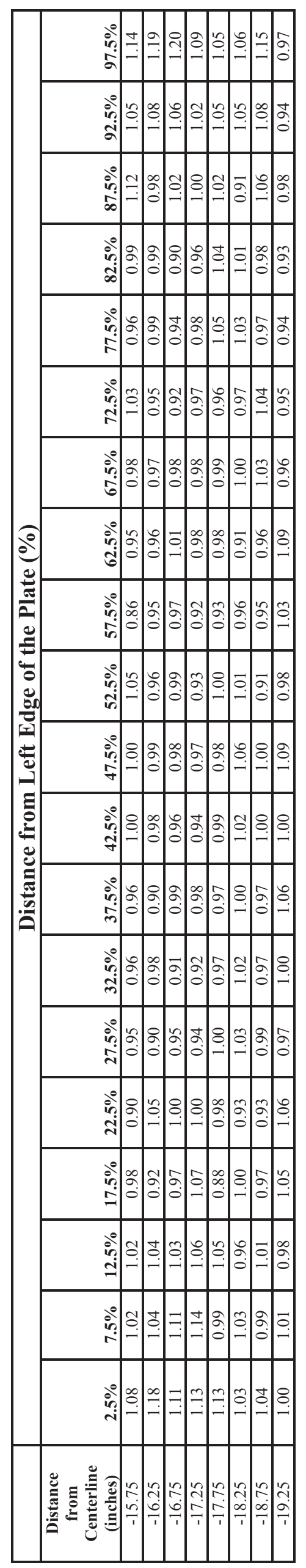




\begin{tabular}{|c|c|c|c|c|c|c|c|c|c|c|c|c|c|c|c|c|c|c|c|c|c|c|c|c|c|c|c|c|}
\hline & $\begin{array}{c}0 \\
\text { in: } \\
5\end{array}$ & \begin{tabular}{l|l|l|}
$\circ$ & $\bullet$ \\
\hdashline & -
\end{tabular} & $=$ & $\underset{-}{-}$ & & & $\vec{T}$ & & & $\Rightarrow$ & & & & & $\exists$ & $\stackrel{\nexists}{-}$ & $\stackrel{\infty}{-\infty}$ & & $\vec{\sim}$ & & $=\infty$ & & $\stackrel{\overbrace{}}{\overbrace =}$ & & & & & $\stackrel{\circ}{=}$ \\
\hline & ปू. & & & 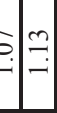 & & & ণิ) & & $\begin{array}{lll} \\
\end{array}$ & & & & $\stackrel{8}{-}$ & & & & & & & & $\Rightarrow$ & & & & & & & $\stackrel{8}{\circ}$ \\
\hline & i⿱宀⿻心㇒. & 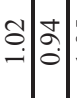 & $\approx$ & $\because$ & & & 8 & $\stackrel{\overbrace{}}{=}$ & $\because$ & $\stackrel{0}{-}$ & 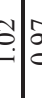 & & & & \pm & 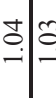 & & $\stackrel{\square}{\square}$ & $\stackrel{-}{-}$ & $\stackrel{-}{-} \dot{0}$ & 2 & 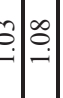 & & & & & & $\stackrel{\infty}{0}$ \\
\hline & $\begin{array}{l}i_{i} \\
i \\
\infty\end{array}$ & $\therefore:$ & $\overrightarrow{-}$ & $=0$ & & & $\mid \begin{array}{l}0 \\
2 \\
0\end{array}$ & & s. & $\overrightarrow{0}$ & 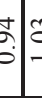 & $\hat{\delta}$ & & & $\overrightarrow{\overrightarrow{-}}$ & 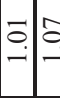 & $\stackrel{8}{-1}$ & बे. & 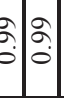 & $\stackrel{\theta}{-} \delta$ & 2 & & $:$ & & $\overrightarrow{-}$ & & & $\stackrel{\Omega}{-}$ \\
\hline & : & & $=\overrightarrow{0}$ & $\stackrel{t}{\rightarrow}$ & & & t. & & 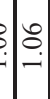 & $\stackrel{0}{\circ}$ & 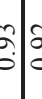 & हे & & & $\begin{array}{l}\infty \\
0 \\
0 \\
0\end{array}$ & \begin{tabular}{l|l}
$\infty$ & $\infty$ \\
0 & 0 \\
0 & 0
\end{tabular} & 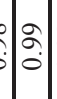 & $\begin{array}{l}\infty \\
\stackrel{0}{0} \\
0\end{array}$ & $\stackrel{c}{\infty}: \infty$ & & $\overrightarrow{-}$ & & & & & & & बे \\
\hline & i⿱宀 & \begin{tabular}{l|l|l|}
$\because$ & \\
\hdashline & 0 \\
$\vdots$
\end{tabular} & 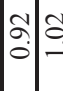 & 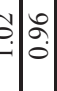 & & & : & \begin{tabular}{l|l}
0 \\
\hdashline \\
\hdashline
\end{tabular} & & $\overrightarrow{-}$ & 8 & & & 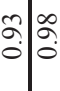 & 2 & $\overrightarrow{\dot{\sigma}} \overrightarrow{0}$ & $\stackrel{8}{8}$ & $\stackrel{\overbrace{}}{-}$ & 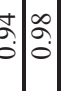 & (t) & $\therefore$ & 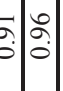 & $\begin{array}{lll}0 & 0 \\
0 & 0 & 0\end{array}$ & & 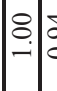 & & & $\stackrel{\infty}{2}$ \\
\hline & : & & $\mid$\begin{tabular}{c}
0 \\
\hdashline \\
\hdashline
\end{tabular} & : & & & $\hat{a}$ & $\hat{\circ}$ & 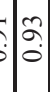 & $\because$ & 6 & $\stackrel{0}{\circ}$ & & & $\hat{0}$ & 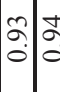 & $\overrightarrow{-}$ & ڤ.. & o. & & $\left(\begin{array}{l}\infty \\
0 \\
0\end{array}\right.$ & & & $\begin{array}{ll}0 \\
0 \\
0\end{array}$ & $\hat{\circ}$ & & & $\bar{\sigma}$ \\
\hline eे & : & & 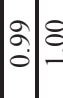 & $\vec{b}$ & & & 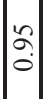 & & & & $=$ & $\stackrel{8}{2}$ & & & 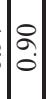 & 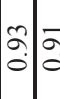 & 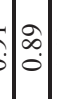 & $\stackrel{8}{2}$ & 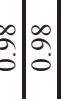 & & $\left(\begin{array}{lll}\infty & 8 \\
0 & 0\end{array}\right.$ & & 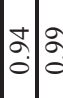 & & $\hat{\varrho}$ & & & $\stackrel{\infty}{\circ}$ \\
\hline 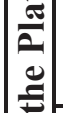 & in & $\vec{\circ}=$ & 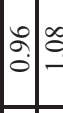 & 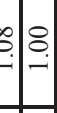 & & & $\delta$ & $\hat{a}$ & & $\left|\begin{array}{c}\hat{0} \\
0 \\
0\end{array}\right|$ & 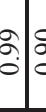 & $\stackrel{2}{\circ}$ & & \begin{tabular}{l|l}
$:$ & 0 \\
\hdashline & 0 \\
\hdashline
\end{tabular} & : & $\begin{array}{l}\circ \\
\vdots \\
\vdots\end{array}$ & \begin{tabular}{c|c}
$\vdots$ \\
$\vdots$ \\
\hdashline
\end{tabular} & $\stackrel{8}{8}$ & $\begin{array}{lll}0 \\
0\end{array}$ & 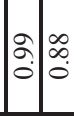 & $\tilde{\sigma}$ & & $\mid \begin{array}{l}0 \\
0 \\
0 \\
0\end{array}$ & $\delta$ & {$\left[\begin{array}{l}2 \\
\hat{0}\end{array}\right.$} & b. & & 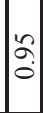 \\
\hline $\mid \begin{array}{l}0 \\
0 \\
0 \\
0\end{array}$ & in & & 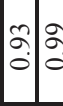 & : & & & 2 & & & $\tilde{\delta}$ & 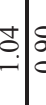 & d. & & & $\begin{array}{l}0 \\
0 \\
0\end{array}$ & \begin{tabular}{l|l|l}
$\circ$ & 2 \\
$\vdots$ & 0
\end{tabular} & 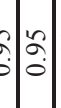 & $\delta$ & $\hat{0}$ & & $\overline{\hat{0}}$ & & & & $\because$ & & & $\stackrel{\overbrace{}}{-}$ \\
\hline $\mid$ & †े & \begin{tabular}{lll}
0 \\
$\vdots$ \\
\hdashline
\end{tabular} & $\stackrel{2}{\circ}$ & 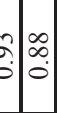 & & & $\delta$ & & & a. & a & S. & & & $\begin{array}{l}1 \\
2 \\
0 \\
0\end{array}$ & \begin{tabular}{|l|l}
$\vec{a}$ & $\infty$ \\
$\dot{0}$ & $\infty$ \\
0
\end{tabular} & & ฉి. & 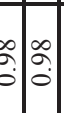 & & \begin{tabular}{l|l}
$\vdots$ & 0 \\
$\vdots$ & 0
\end{tabular} & 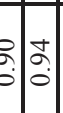 & & مी & $\hat{0}$ & & & $\hat{\partial}$ \\
\hline$\stackrel{0}{0}$ & îj. & & & $\dot{b} \vec{\circ}$ & & & वे & & & 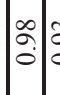 & & & & & $\stackrel{l}{\infty}$ & 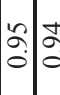 & & \begin{tabular}{l|l}
$\infty$ & $\infty$ \\
$\vdots$ & $\vdots$
\end{tabular} & 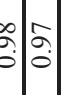 & & $\therefore$ & & & & 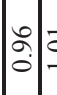 & & & $\stackrel{l}{2}$ \\
\hline$\stackrel{n}{a}$ & 角 & \begin{tabular}{l|l|l|}
$\infty$ & $\overrightarrow{0}$ \\
\hdashline & -
\end{tabular} & \begin{tabular}{|l|l}
$\infty$ & 2 \\
$\infty$ & $\vdots$ \\
0 & 0
\end{tabular} & ç: & & & ô & 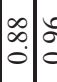 & $\stackrel{\circ}{\circ}$ & $\grave{\circ}$ & \begin{tabular}{lll}
$\infty$ & 0 \\
\hdashline & 0 & 0
\end{tabular} & & $\overrightarrow{-}$ & & $\hat{0}$ & \begin{tabular}{l|l}
2 & 2 \\
0 & 0
\end{tabular} & $\begin{array}{l} \pm \\
0 \\
0\end{array}$ & $\dot{a}$ & $\mid$ & & $\overline{0}$ & $\stackrel{2}{2}$ & & $\dot{0}$ & & & & $\stackrel{8}{-}$ \\
\hline & 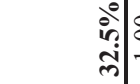 & & & $\begin{array}{lll}\circ & \circ \\
: & 0 \\
\end{array}$ & & & $\vec{\delta}$ & \begin{tabular}{l|l}
2 \\
$\vdots$ \\
0
\end{tabular} & 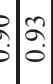 & 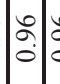 & 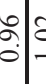 & aे & $\stackrel{8}{-}$ & & $\stackrel{0}{0}$ & 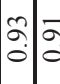 & $\hat{\circ}$ & $\begin{array}{l}0 \\
\vdots \\
\vdots\end{array}$ & :ُ. & & $\stackrel{8}{-}$ & $\stackrel{0}{0}$ & & 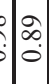 & & & & $\begin{array}{l}\infty \\
0 \\
0\end{array}$ \\
\hline & in: & & & 02 & & & $\hat{\alpha}$ & 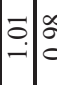 & & $\widehat{\sigma}$ & 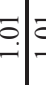 & aे & & $\stackrel{8}{-}$ & 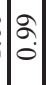 & $\hat{a} \mid \hat{a}$ & $\hat{\circ}$ & a. & $\stackrel{-}{-}$ & & 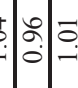 & $\vec{c}: 0$. & & & $\delta$ & 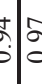 & & 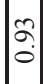 \\
\hline & $\begin{array}{l}\text { î̀ं } \\
\text { ते }\end{array}$ & 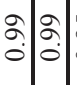 & 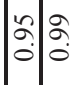 & 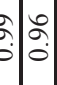 & & & 2 & & 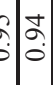 & 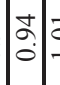 & 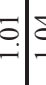 & $\stackrel{\infty}{0}$ & & & $\stackrel{\vec{\sigma}}{-}$ & $\mid$\begin{tabular}{l|l}
$\circ$ \\
0 \\
0 & 0 \\
0
\end{tabular} & 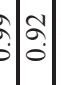 & 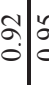 & $\hat{0}: \hat{\circ}$ & & 2. & $\stackrel{0}{0}$ & के. & & $\left(\begin{array}{l}0 \\
0 \\
0\end{array}\right.$ & & & $\stackrel{\overrightarrow{.}}{-}$ \\
\hline & : & 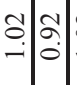 & & $\stackrel{8}{0}$ & & & $\widehat{\alpha}$ & & & $\bar{\sigma} \mid$ & \begin{tabular}{lll}
$\infty$ & 0 \\
\hdashline & 0 & 0
\end{tabular} & & & & : & & & $\stackrel{\Omega}{\circ}$ & $\stackrel{t}{*}$ & & 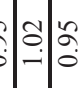 & $\stackrel{0}{0}$ & & & & $:$ & & $\hat{o}$ \\
\hline & 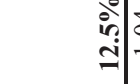 & $\stackrel{0}{-}$ & 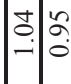 & $\widehat{c}$ & & & $\stackrel{\infty}{\stackrel{\infty}{-}}$ & \begin{tabular}{l|r}
$\infty$ & $=$ \\
0 & $=$
\end{tabular} & & $\stackrel{0}{-}$ & 8 & & $\stackrel{+}{0}$ & $\stackrel{0}{-}$ & : & 0 & : & ठे. & 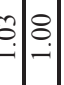 & 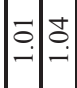 & 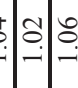 & : & & & 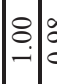 & ó & & $\stackrel{\overrightarrow{-}}{-}$ \\
\hline & : & & 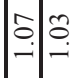 & 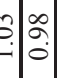 & & & 8 & 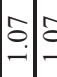 & $-\infty$ & 0 & $\Phi$ & & : & $\mid$\begin{tabular}{l}
$\infty$ \\
\hdashline \\
\hdashline
\end{tabular} & 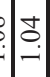 & 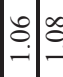 & 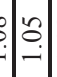 & $\stackrel{\infty}{\infty}$ & $=$ & 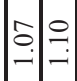 & 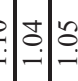 & : & & & $\mid$ & $\approx$ & & : \\
\hline & iृ: & & & & & & 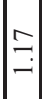 & & & & & & & & & & & & $=$ & & 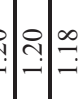 & & & & & & & $\stackrel{9}{=}$ \\
\hline & 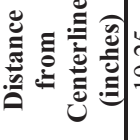 & $=$ & 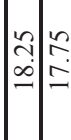 & $=2$ & & & & $\mid \begin{array}{l}n \\
\dot{ \pm} \\
\pm\end{array}$ & & 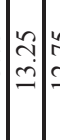 & $\approx=$ & & & 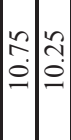 & 10 & & $\left.\begin{array}{c}c \\
0 \\
0 \\
\infty \\
\infty\end{array}\right]$ & & 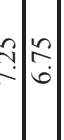 & (ָુ) & 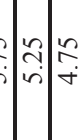 & 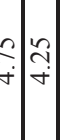 & 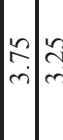 & & ì & & & กิ \\
\hline
\end{tabular}




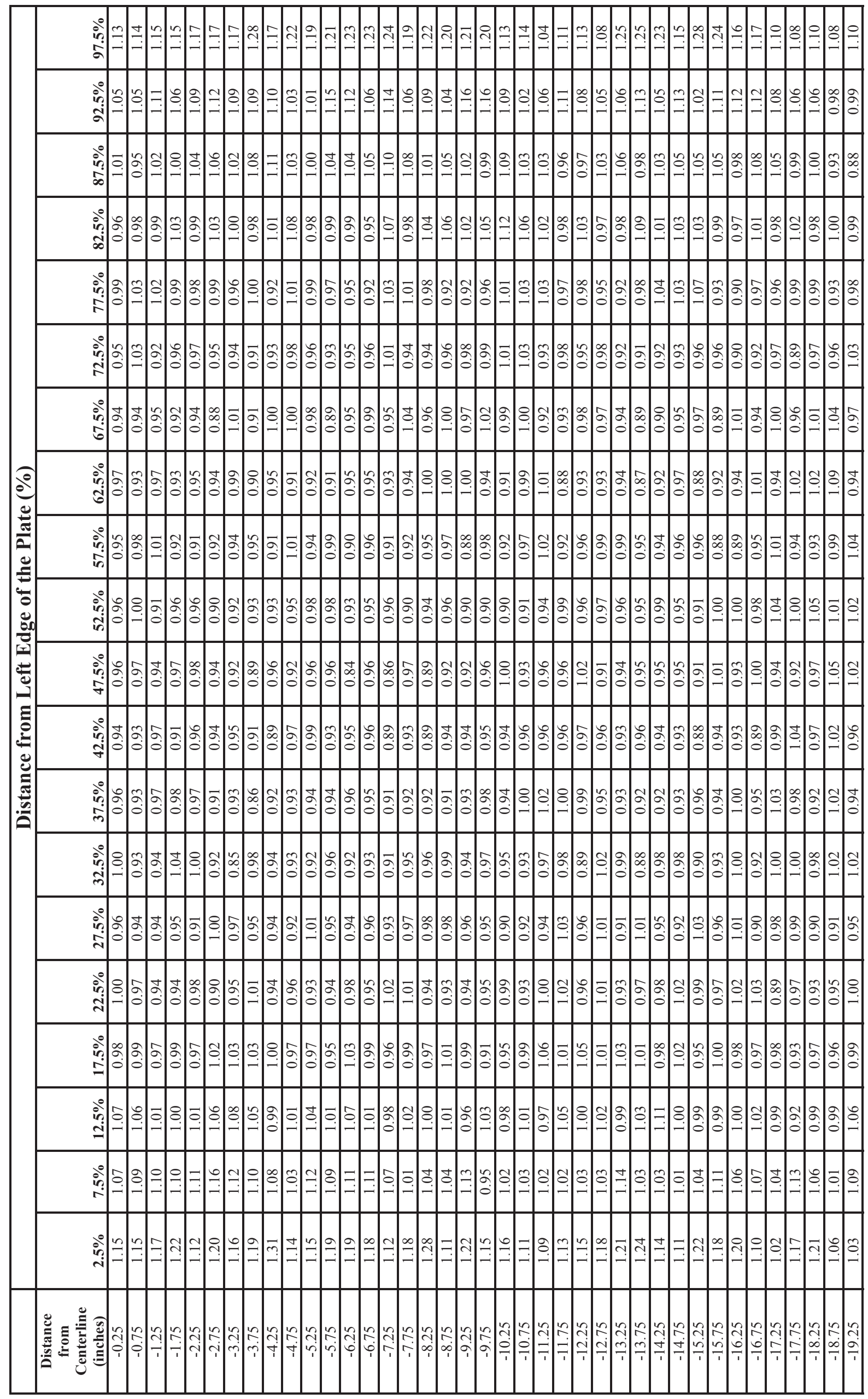




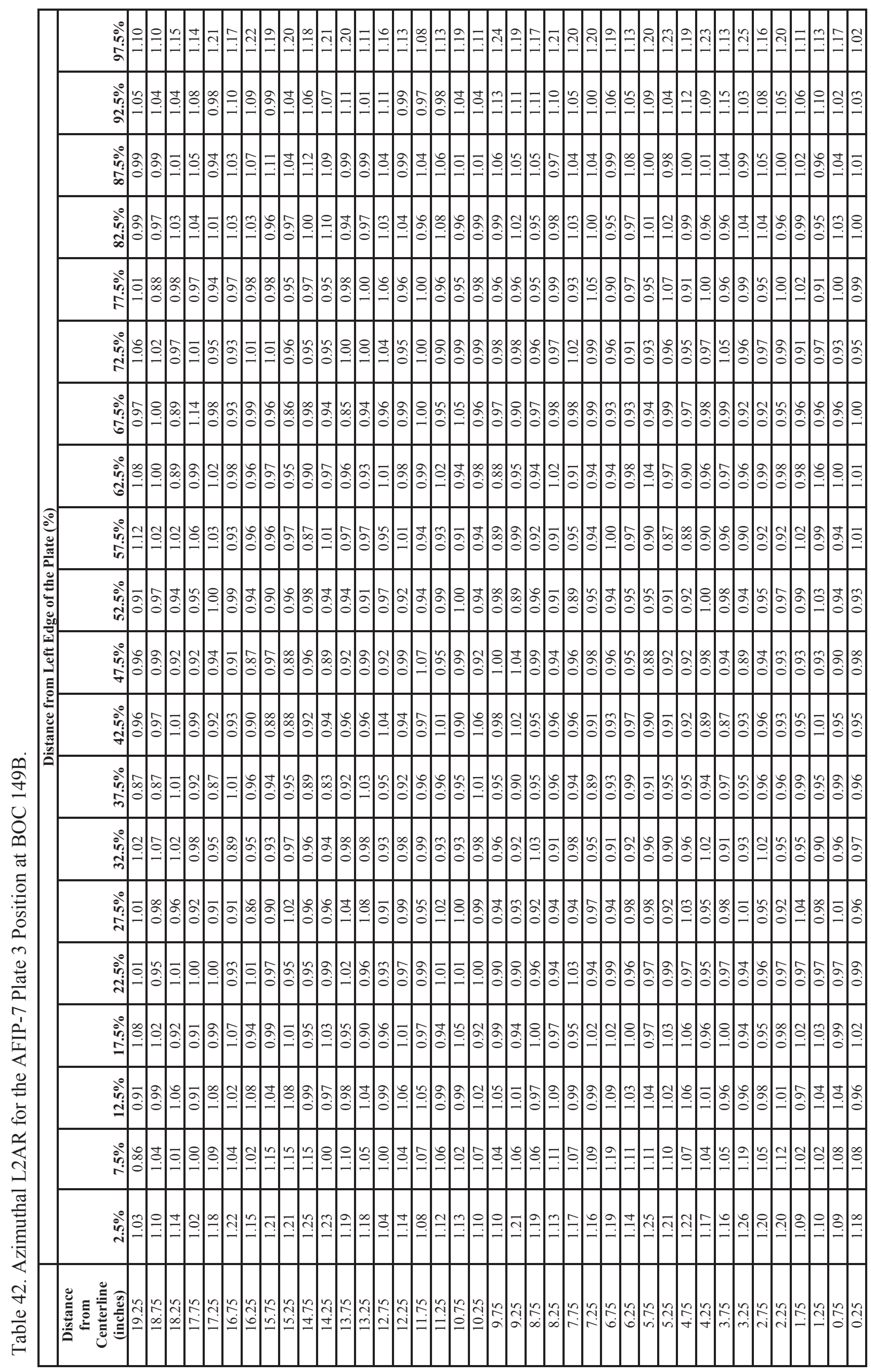




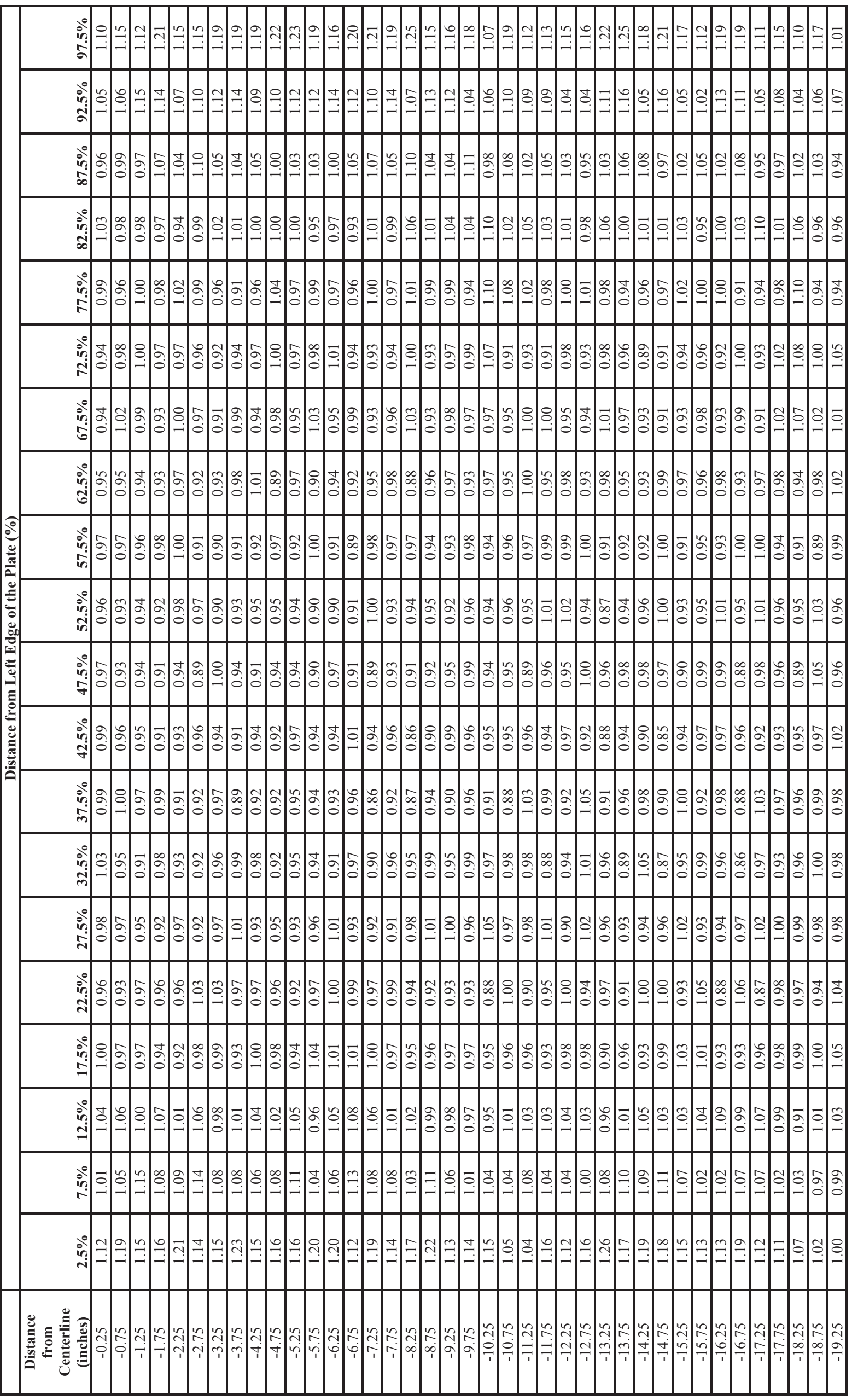




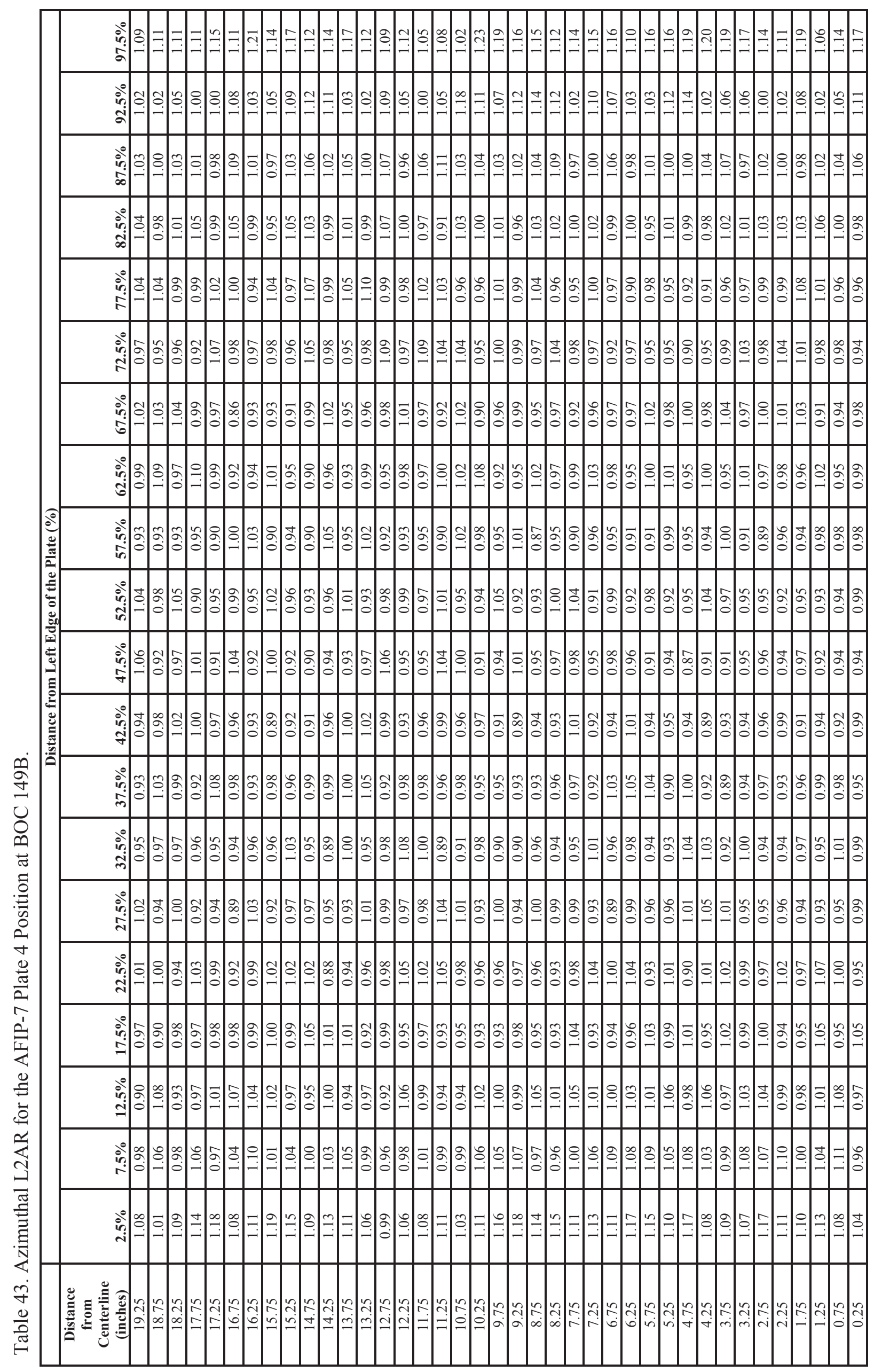




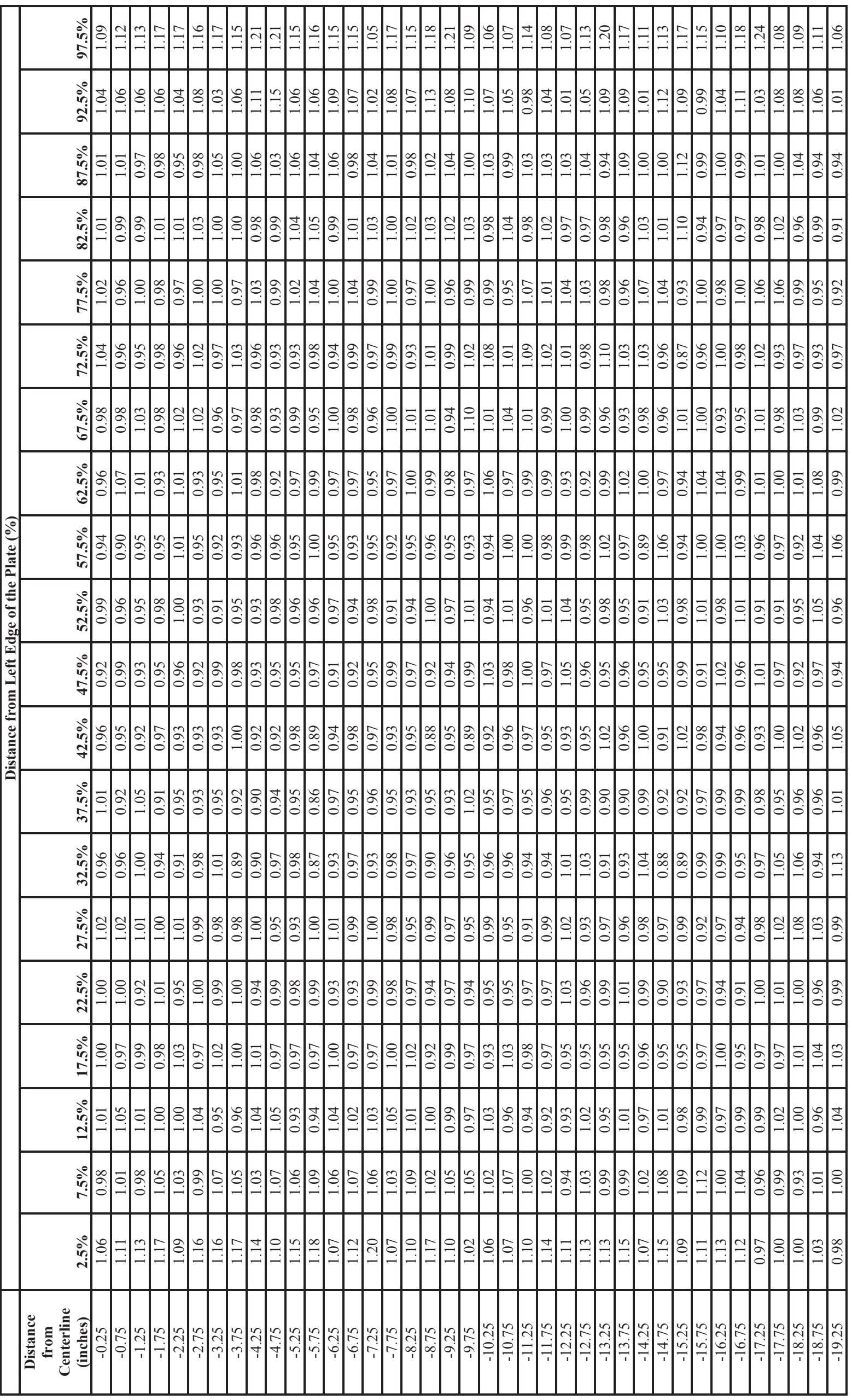




\section{HYDRAULIC TESTING}

Flow tests were not performed on the AFIP-7 test vehicle. To determine the flow rates through the experiment, the AFIP-7 experiment was modeled and analyzed using the RELAP5 code $^{4}$. A summary of the RELAP5 results are shown in Table 44.

Table 44. RELAP5 calculation results.

\begin{tabular}{cc}
\hline & $\begin{array}{c}\text { Flow Rate } \\
\text { (gpm) }\end{array}$ \\
\hline \hline Inner channel & 31.971 \\
Outer channel & 29.774 \\
\hline \hline Total & 155.461 \\
\hline
\end{tabular}




\section{AS-RUN THERMAL ANALYSIS}

The thermal as-run analysis was performed using the as-built geometry, MCNP-calculated surface heat flux $\left(\mathrm{W} / \mathrm{cm}^{2}\right)$ and nominal coolant channel flow rate. ABAQUS ${ }^{5}$ was used to calculate the coolant channel temperatures and plate surface temperatures.

The heat transfer correlation used to calculate these temperatures was calculated from the Colburn equation (equation 5-50c from Reference 6):

$$
N u=\frac{h D}{k}=0.023 R e^{0.8} \operatorname{Pr}^{0.3}
$$

Where $\mathrm{Nu}$ is the Nusselt number, $\mathrm{h}$ is the heat transfer coefficient, $\mathrm{D}$ is the hydraulic diameter, $\mathrm{k}$ is the thermal conductivity, Re is the Reynolds number and Pr is the Prandlt number.

\subsection{Coolant Channel Temperature}

The coolant temperature was analyzed at the five flow channels in the test assembly. The top coolant channel (channel 5) is north of plate position 4, the bottom coolant channel (channel 1) is south of plate position 1 (refer to Figure 9). For each cycle interval, the coolant temperature was plotted as a function of location along the test assembly with $0.0 \mathrm{in}$. being at the top of the assembly. These plots are show in Figure 10 through Figure 17.

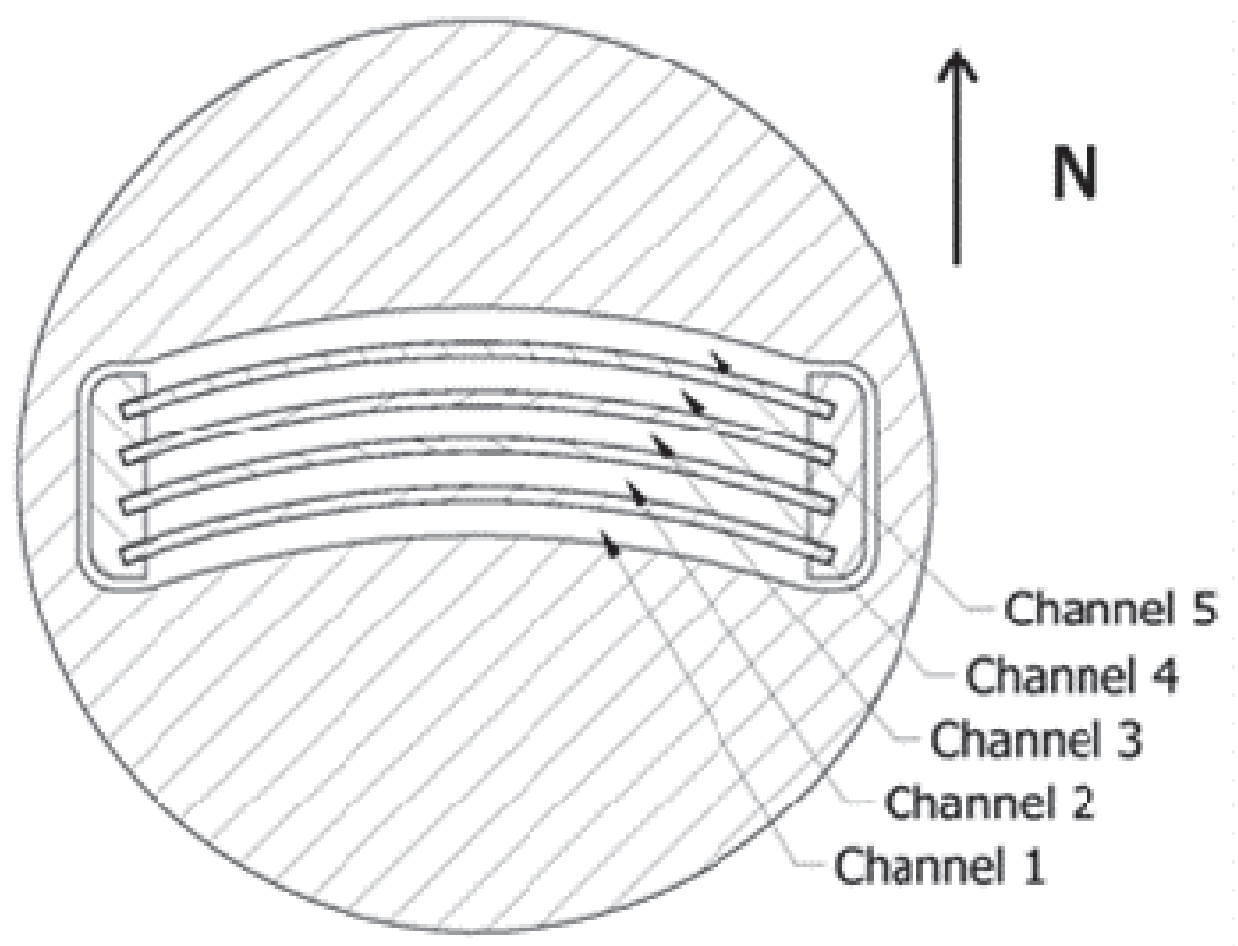

Figure 9. Coolant channel diagram. 


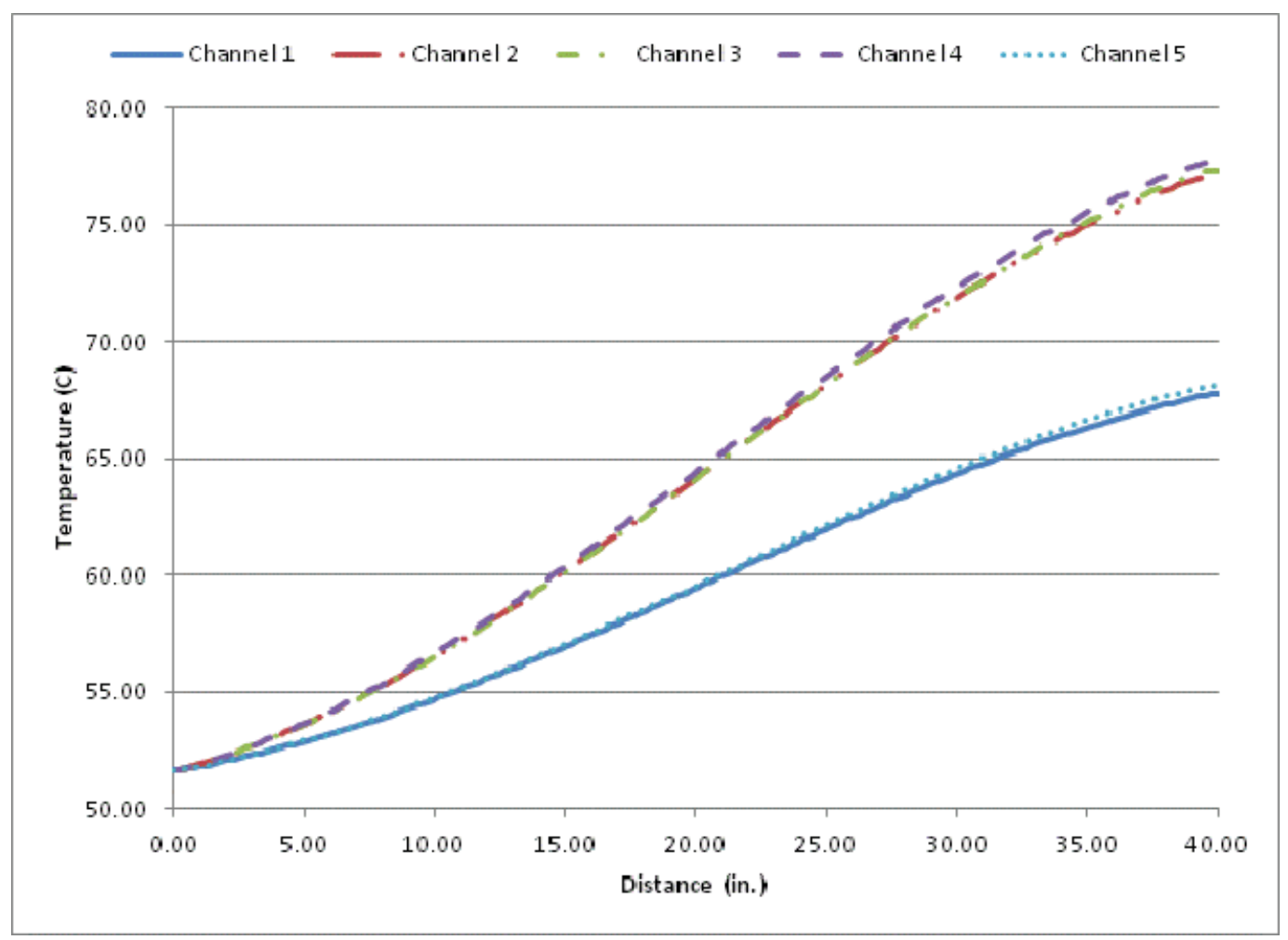

Figure 10: Coolant channel temperatures as a function of location along the AFIP-7 test assembly at BOC 149B (0.0 EFPD).

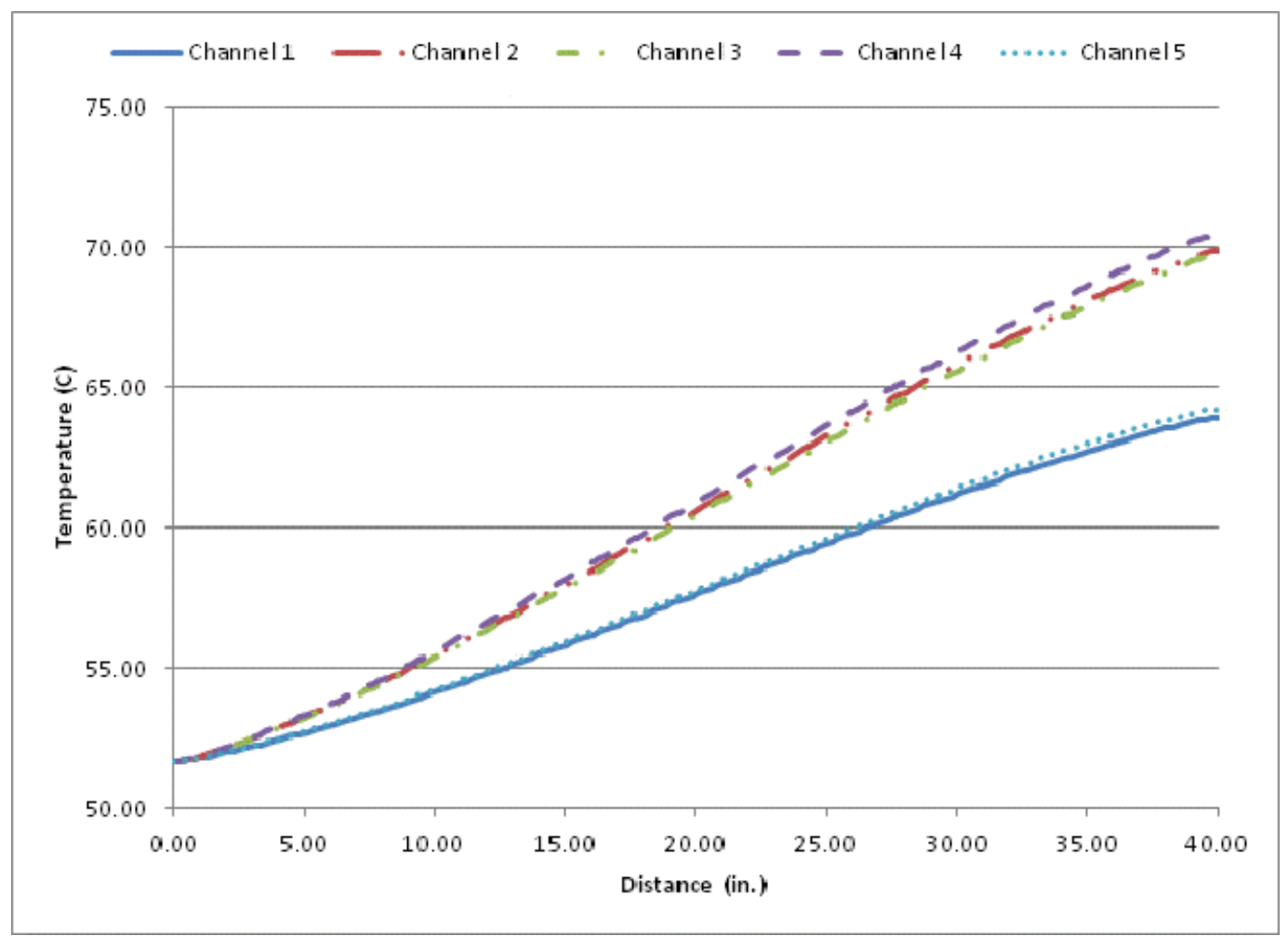

Figure 11: Coolant channel temperatures as a function of location along the AFIP-7 test assembly at MOC1 149B (15.0 EFPD). 


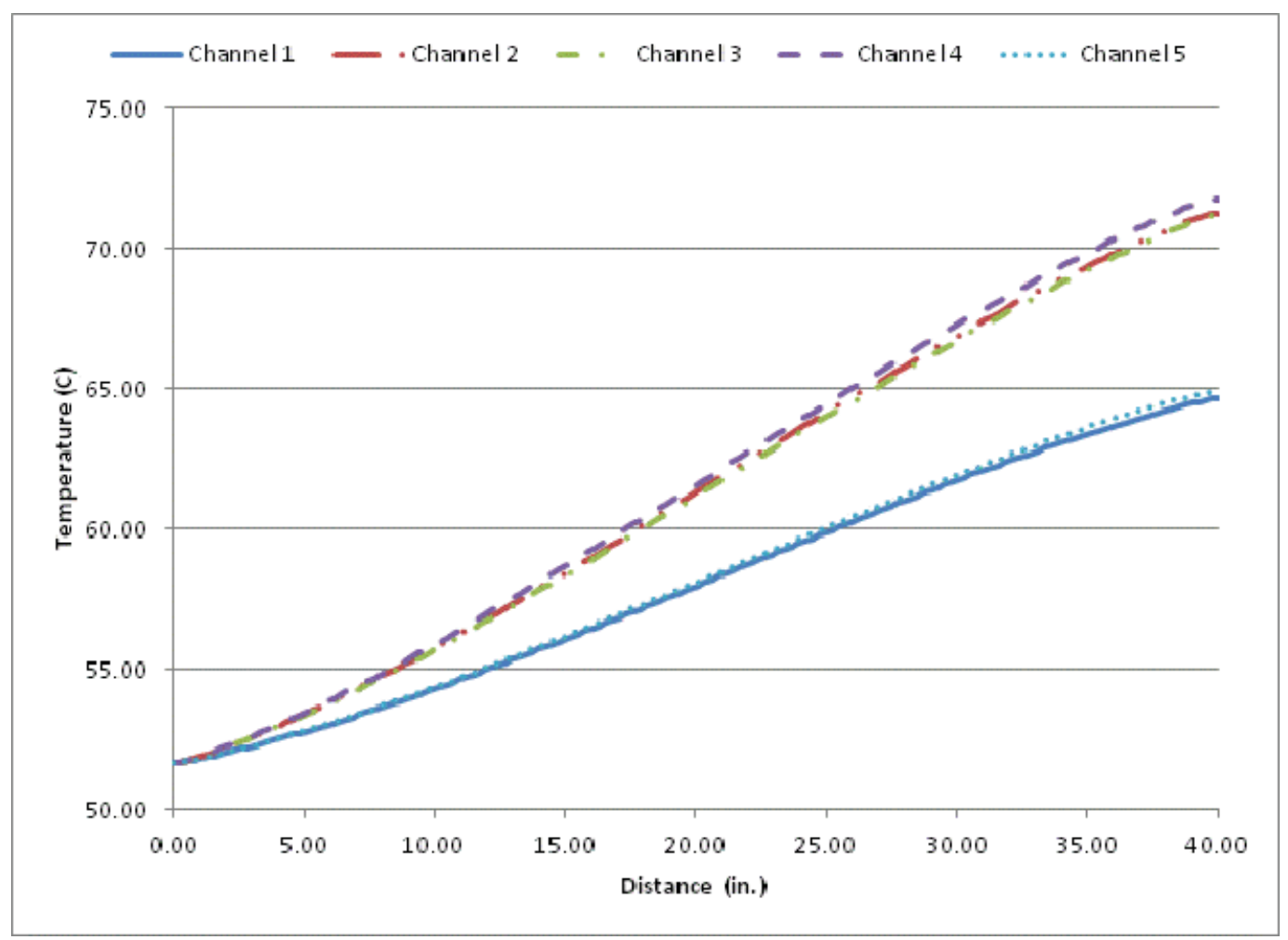

Figure 12: Coolant channel temperatures as a function of location along the AFIP-7 test assembly at MOC2 149B (31.0 EFPD).

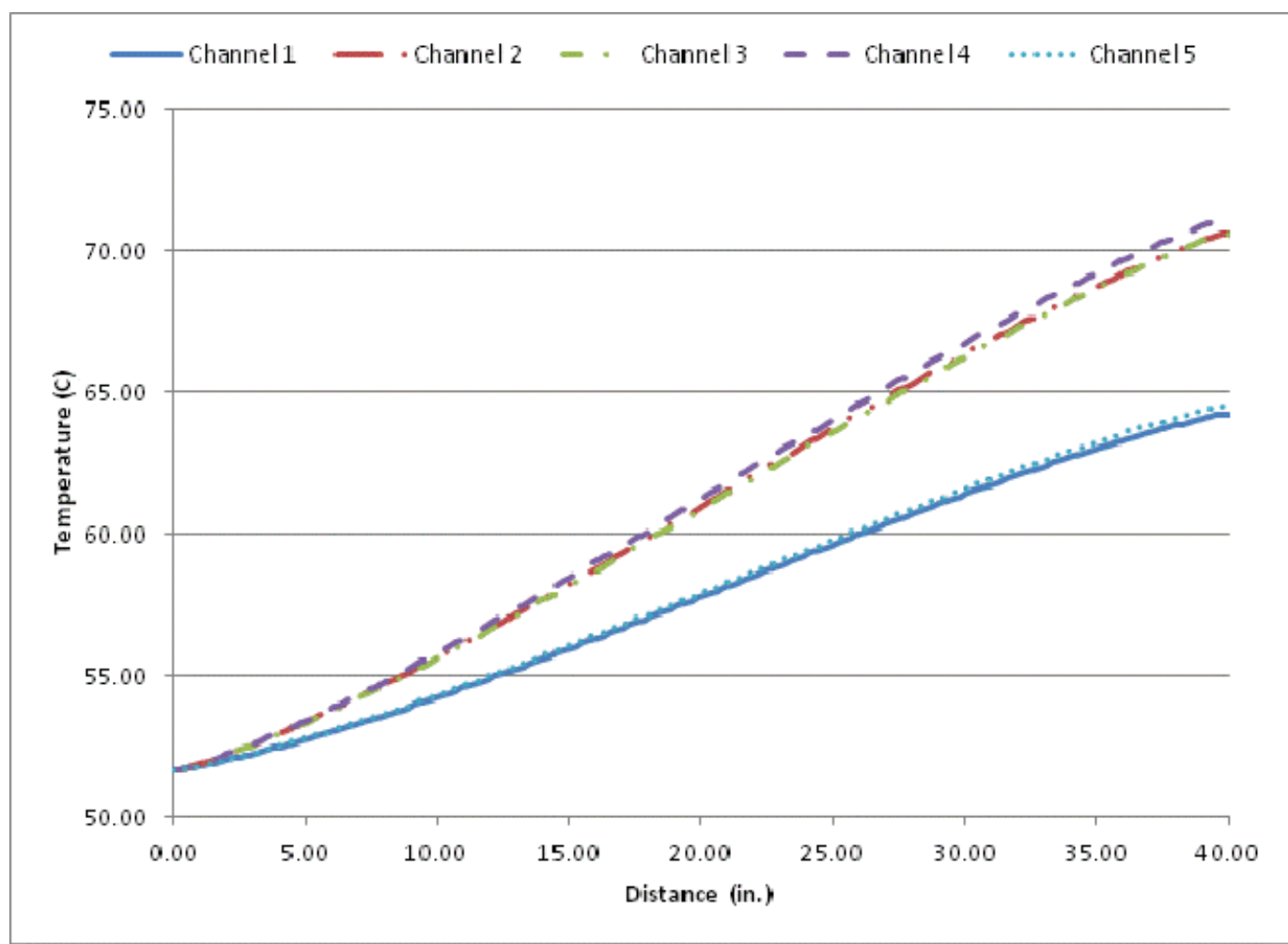

Figure 13: Coolant channel temperatures as a function of location along the AFIP-7 test assembly at EOC 149B (53.6 EFPD). 


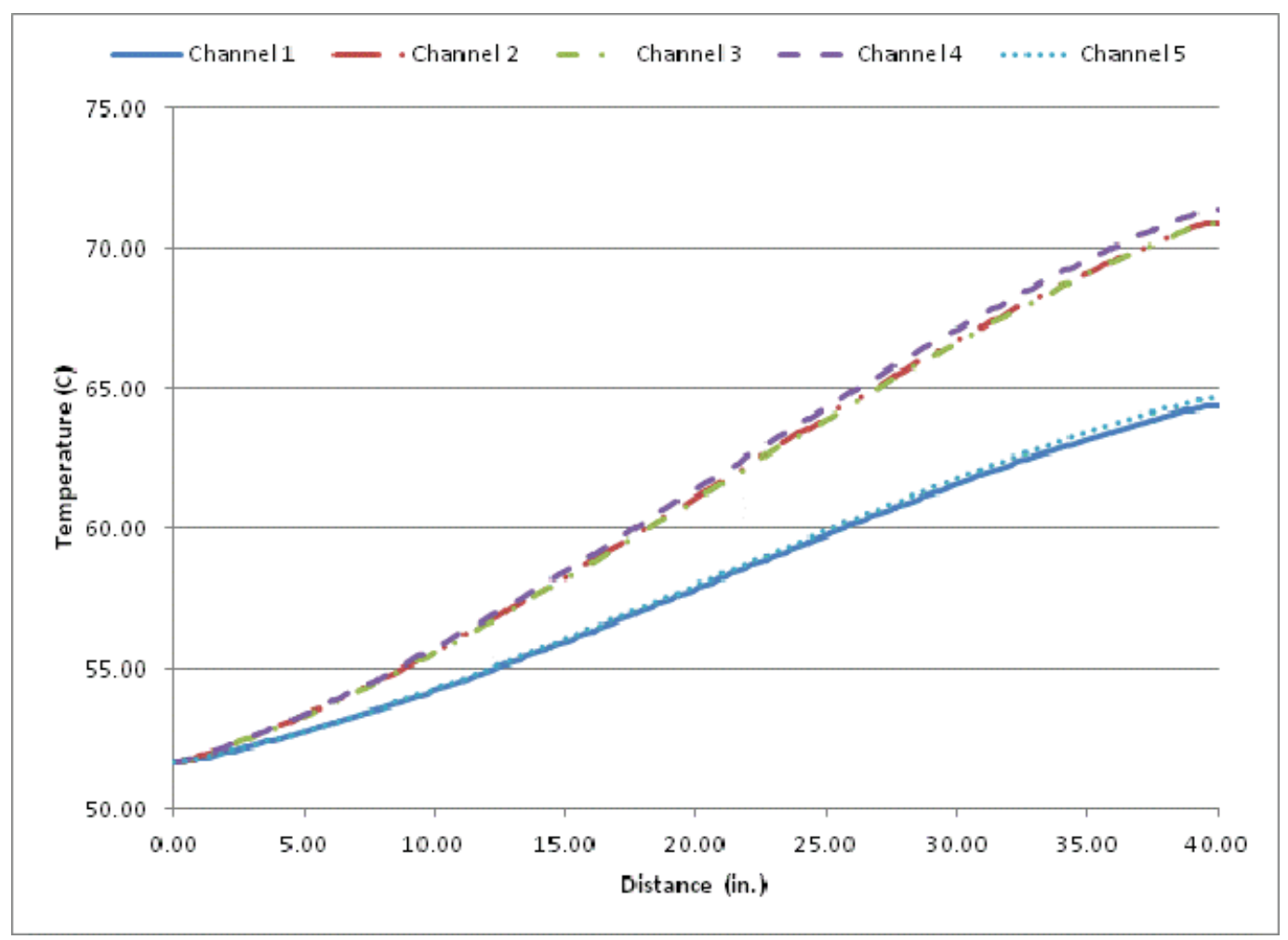

Figure 14: Coolant channel temperatures as a function of location along the AFIP-7 test assembly at BOC 150B (0 EFPD, cumulative 53.6 EFPD).

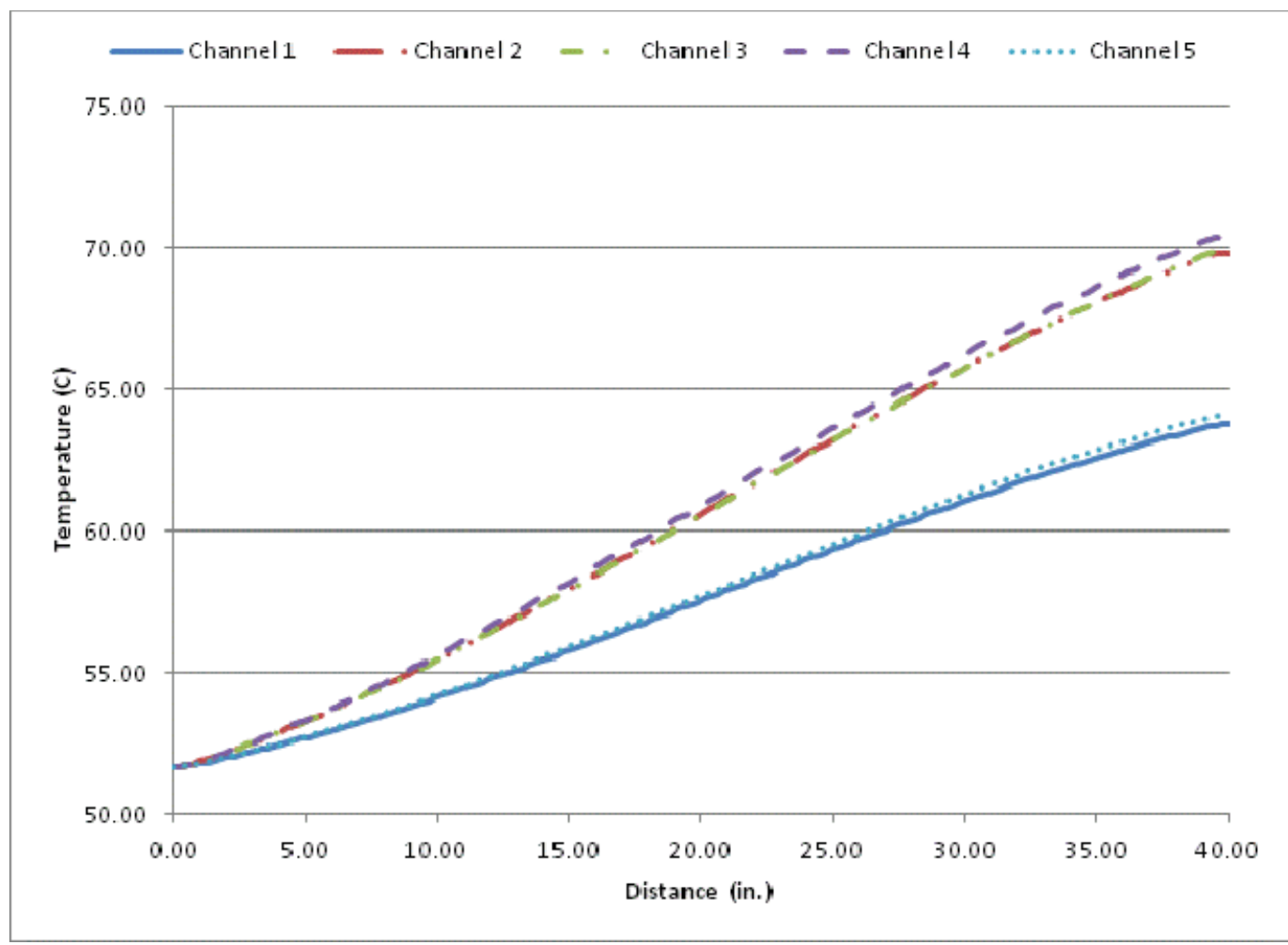

Figure 15: Coolant channel temperatures as a function of location along the AFIP-7 test assembly at MOC1 150B (18 EFPD, cumulative 71.6 EFPD). 


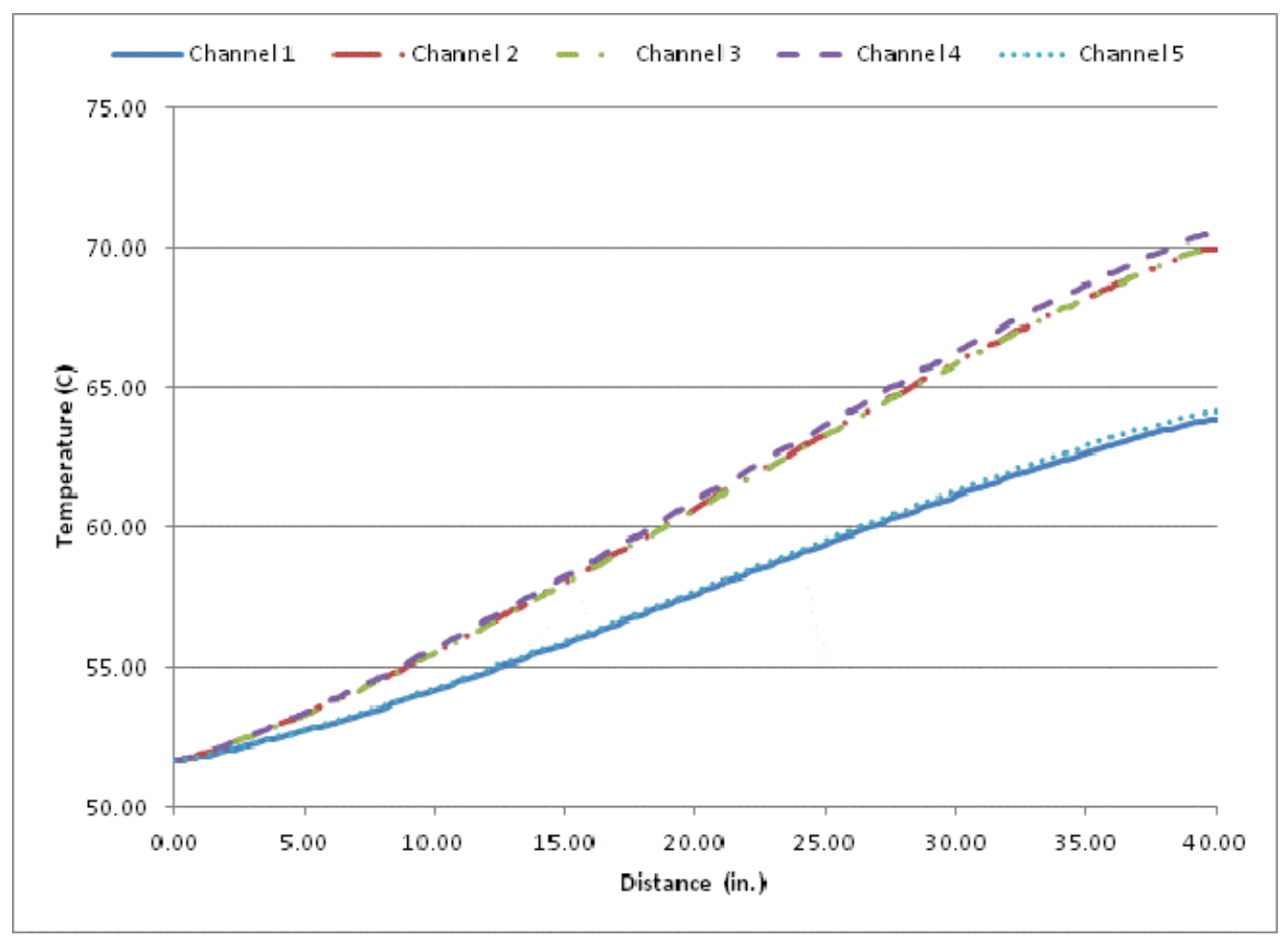

Figure 16: Coolant channel temperatures as a function of location along the AFIP-7 test assembly at MOC2 150B (31 EFPD, cumulative 84.6 EFPD).

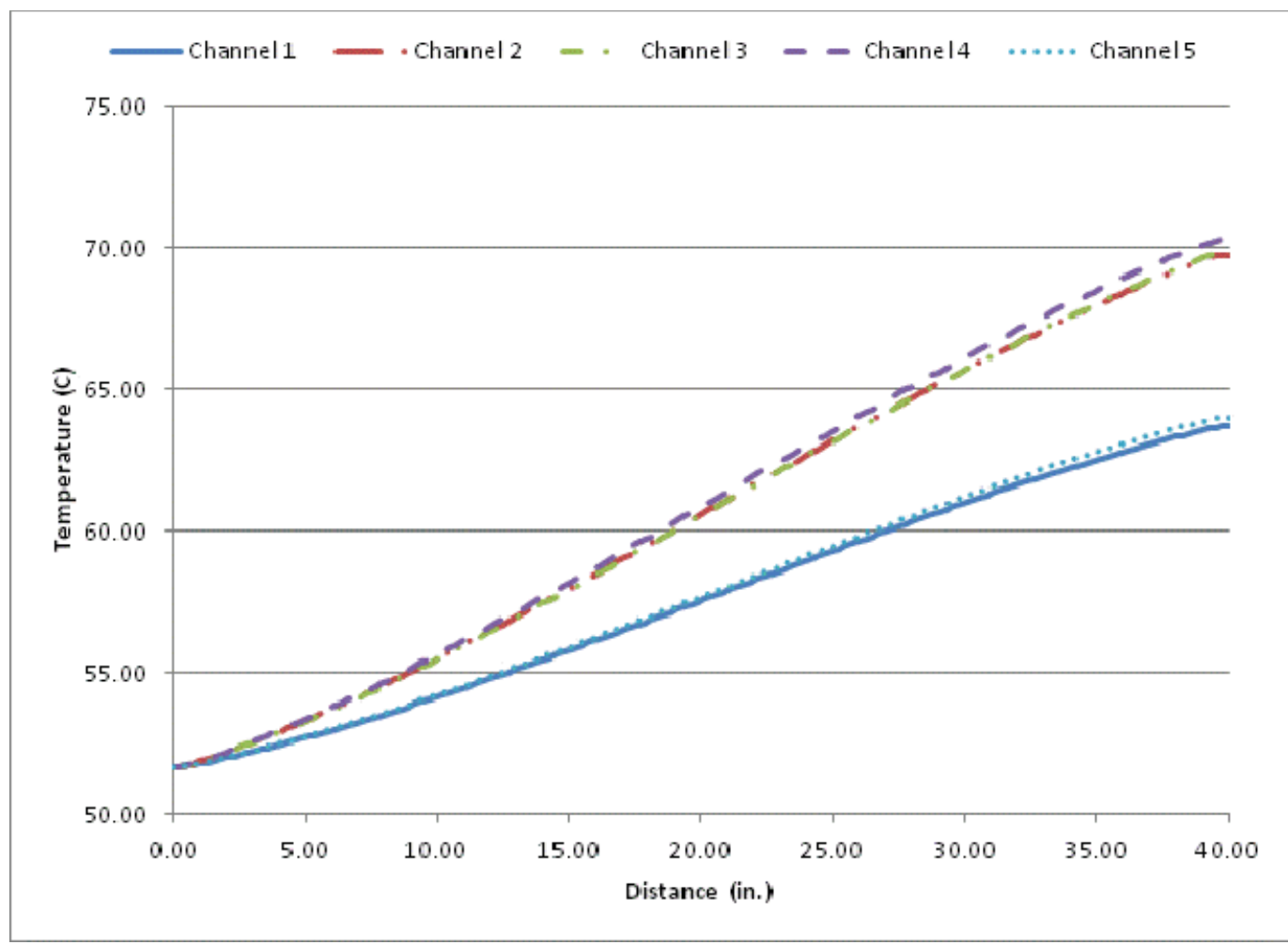

Figure 17: Coolant channel temperatures as a function of location along the AFIP-7 test assembly at EOC 150B (42.0 EFPD, cumulative 95.6 EFPD). 


\subsection{Plate Surface Temperature}

The plate surface temperatures were analyzed at each time step for each side of the plate, with the top side of the plate facing north. Table 45 through Table 52 tabulate the 2D map of the temperatures for each side of the plate at EOC for cycle 149B. Table 53 through Table 60 tabulate the 2D map of the temperatures for each side of plate at EOC for cycle 150B. The plate surface temperatures for each plate at every time step are tabulated in Appendix B. 
च

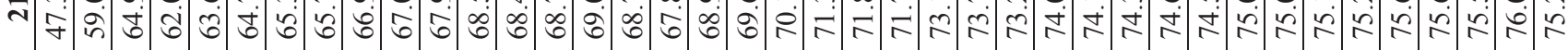

그

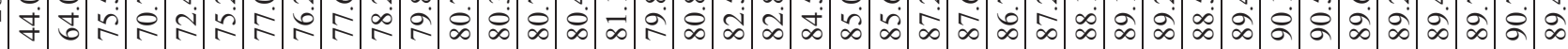

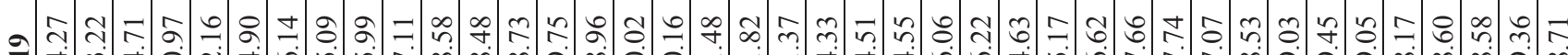

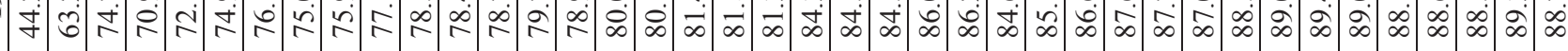

$\infty$ กิ J

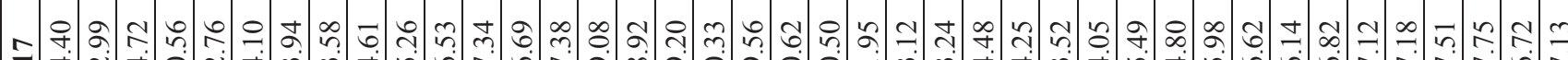

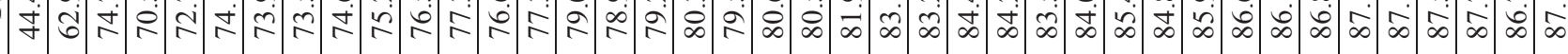

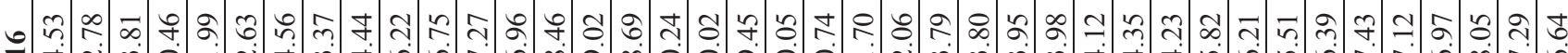

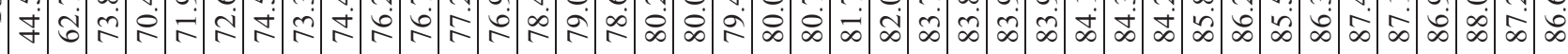

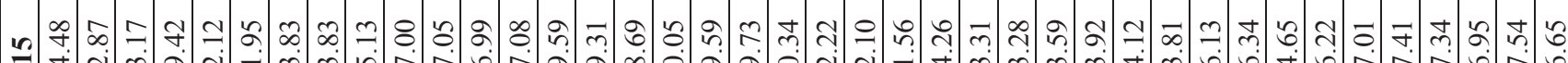
舟

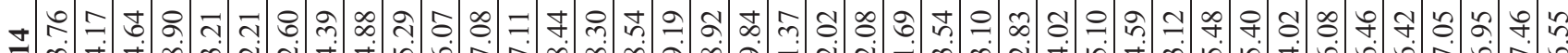

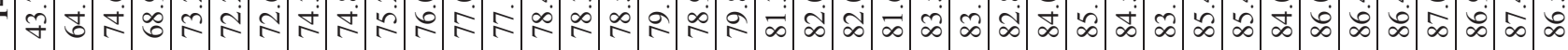

の $=$ 守

ง กี กิ ㅈ. 过

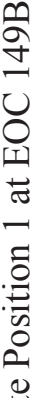

$=$ † f. 舟

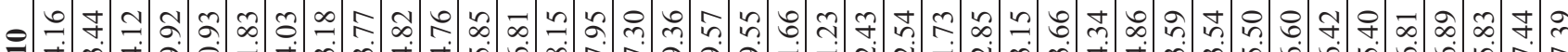

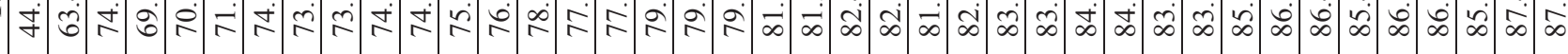

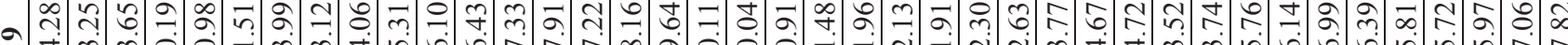

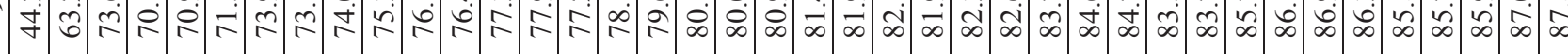
ஸी J

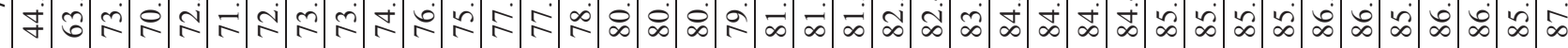

๑

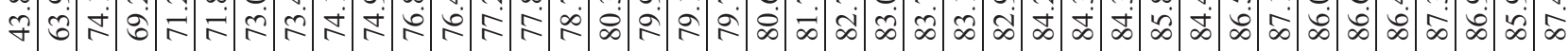

\&

世 $=$ ตி

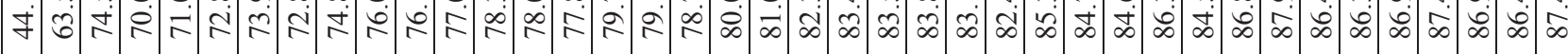

猋

- ำ

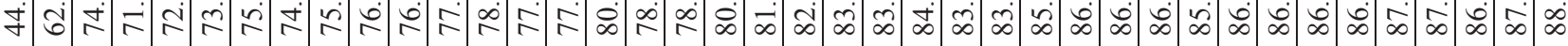

లి ఢุ

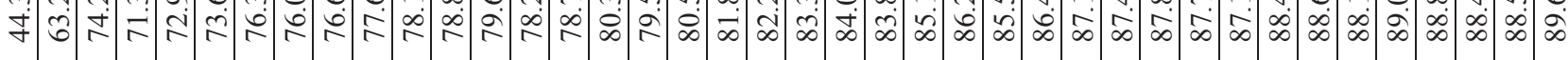

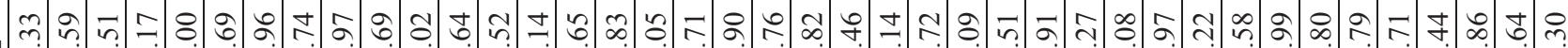

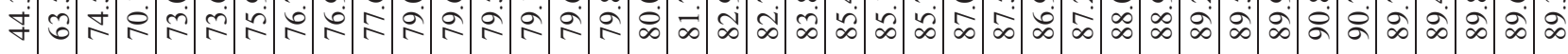

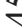

$\frac{\frac{\pi}{2}}{\frac{\pi}{\sigma}}$

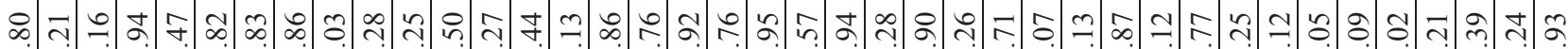

字的 㝵

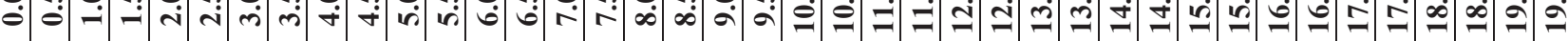


a ๆ

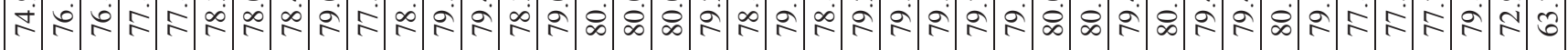

ๆ

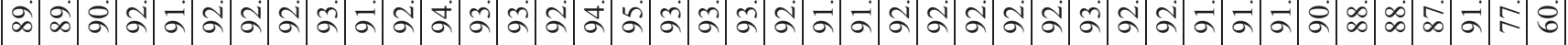
ภे

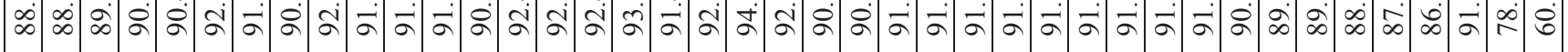

กㄴ.

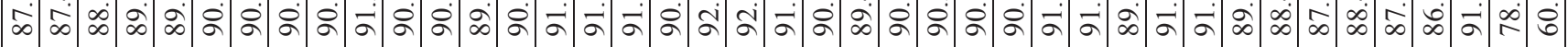

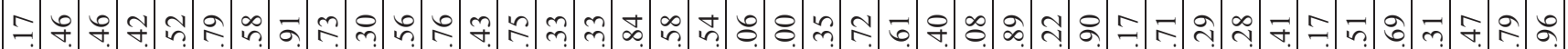

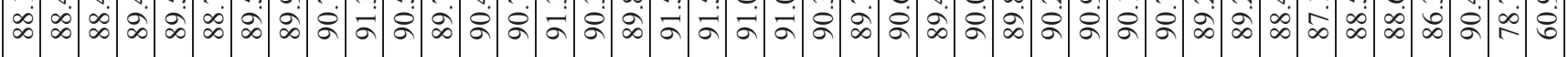

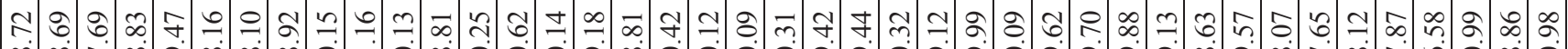

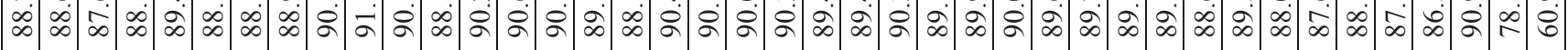

๙

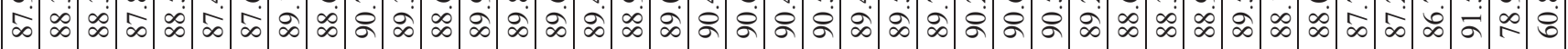

ஸุ

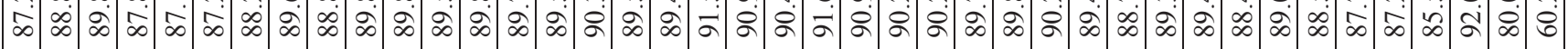

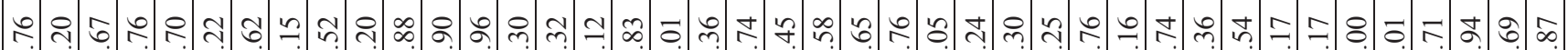

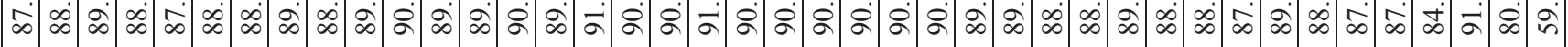

구

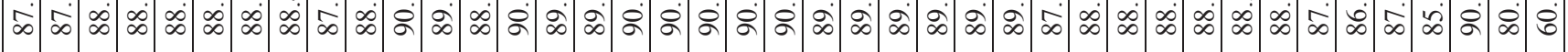

లి

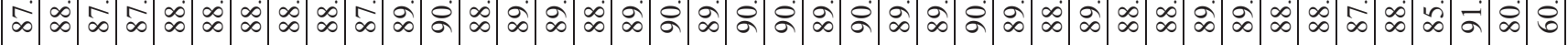

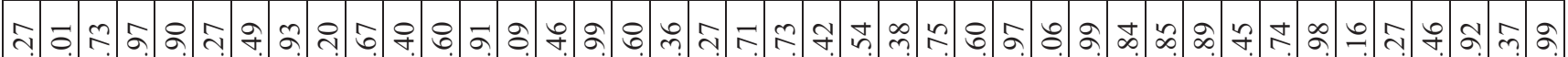

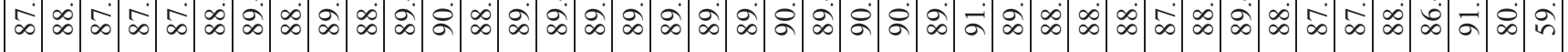

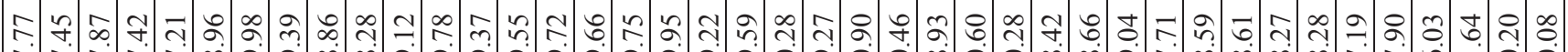

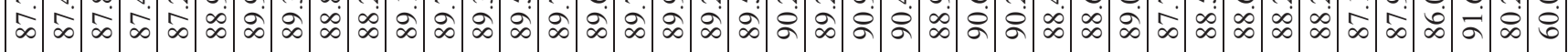

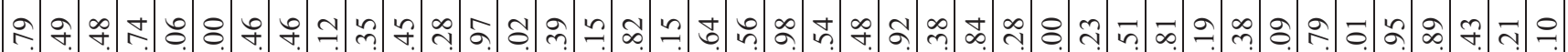

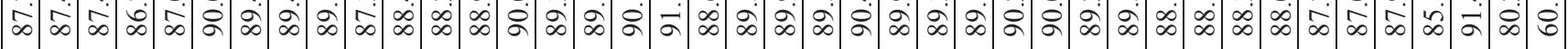

ล.

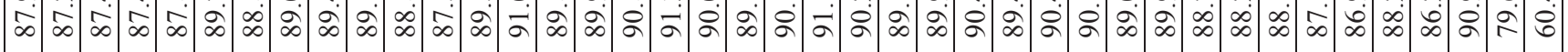

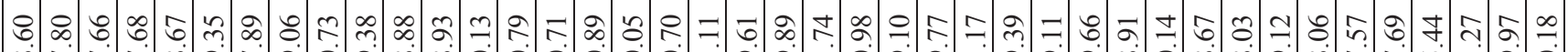

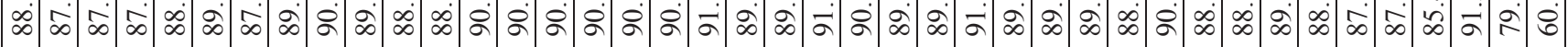

๘ तิ

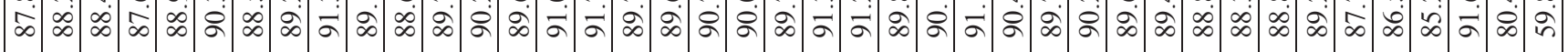

\& f.

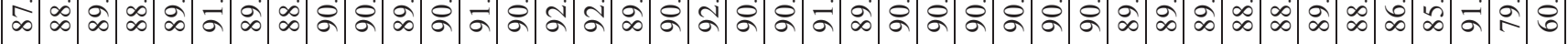

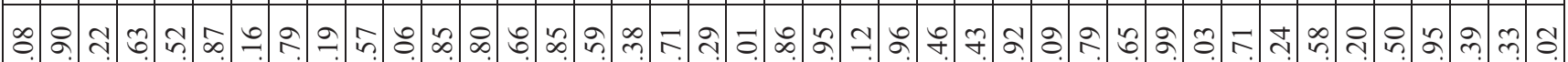

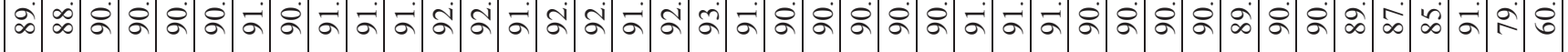

子.

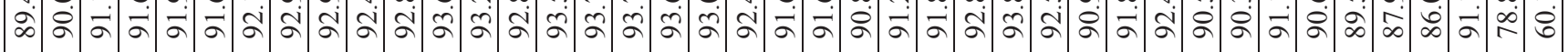

守

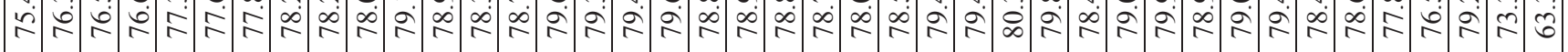

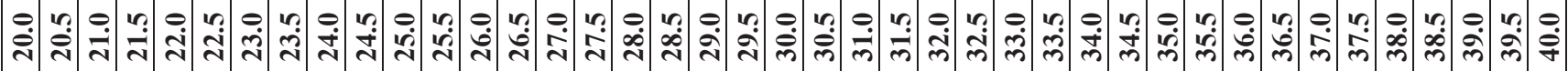


- సิำ

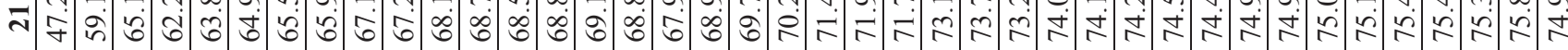

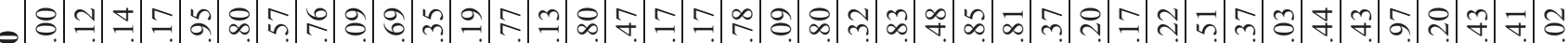

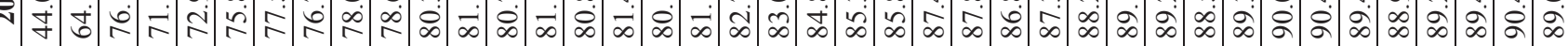

- సี

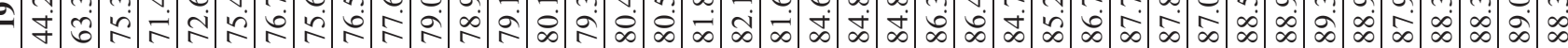

丮 ప t

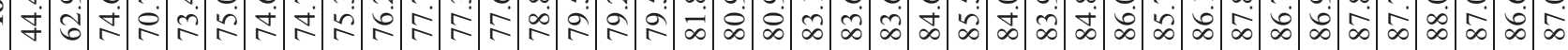

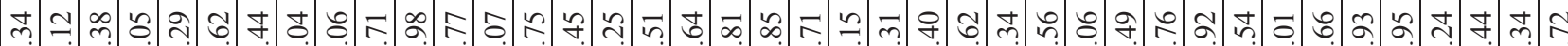
$=$ च

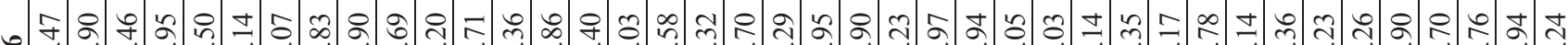

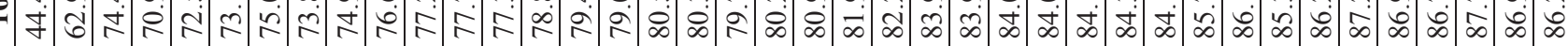

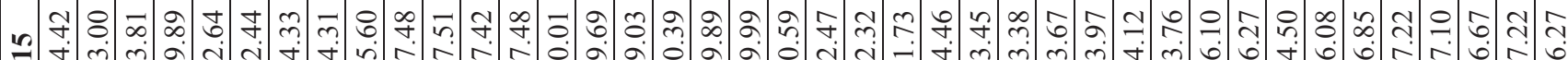
舟

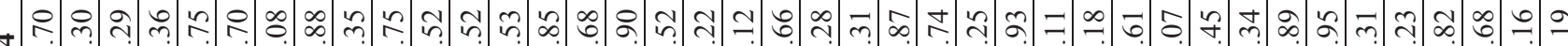

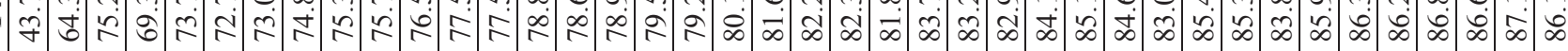

m ₹.

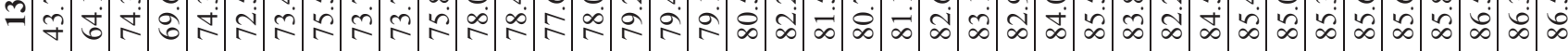

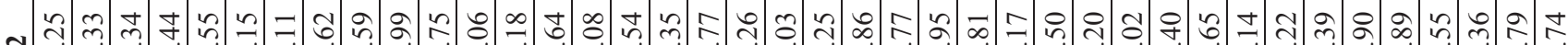

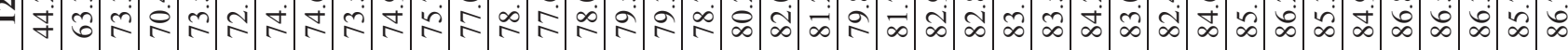

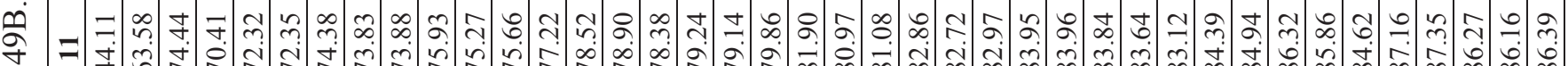
I $\quad$ b

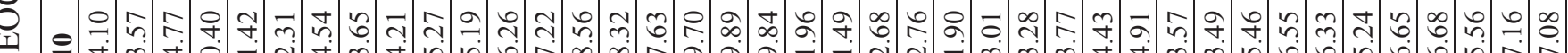

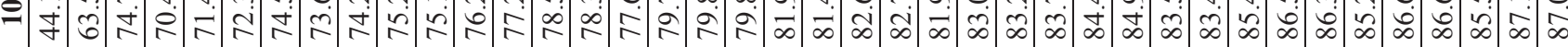

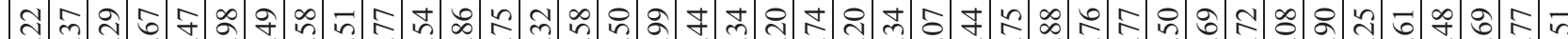
舟 Ұ 怙 กฺ่

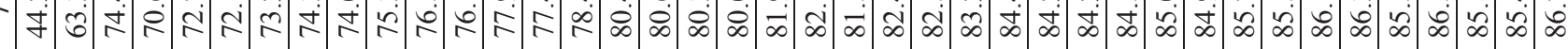

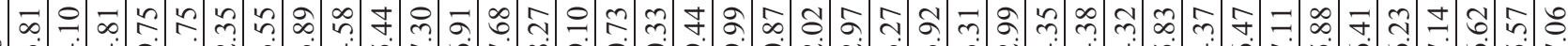

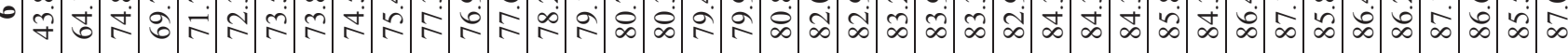
\&

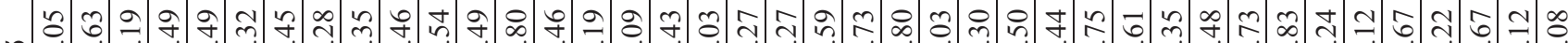

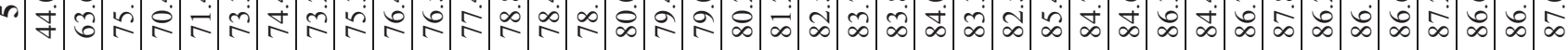

(2) กำ

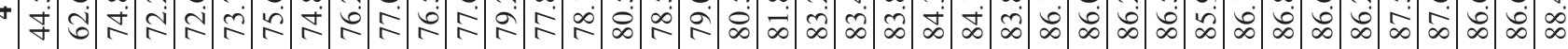

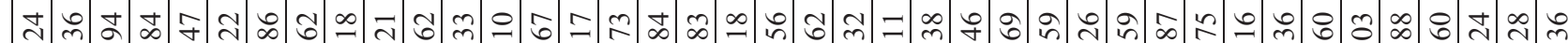

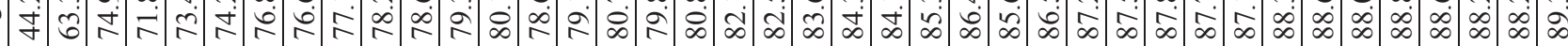

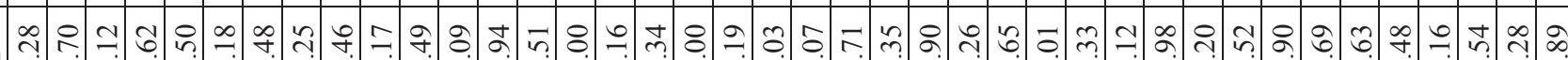
过

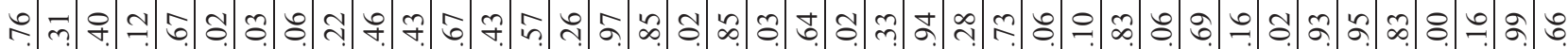
守

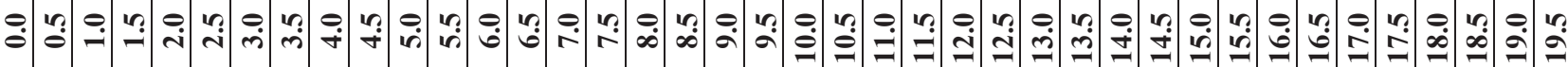




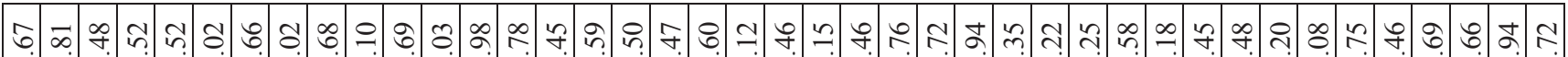

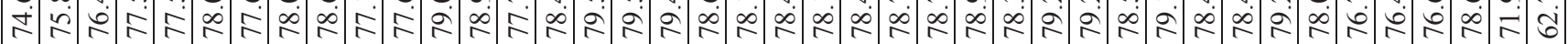

t

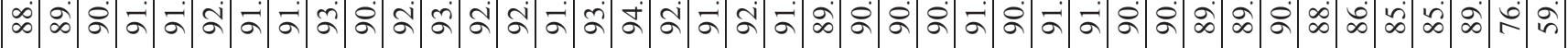

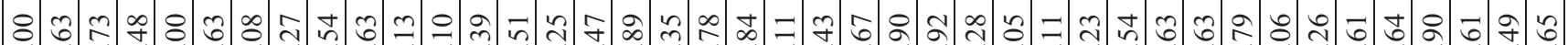

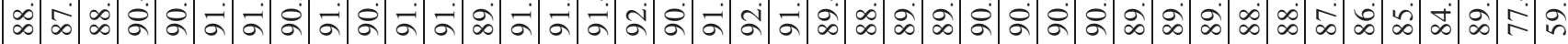

œ

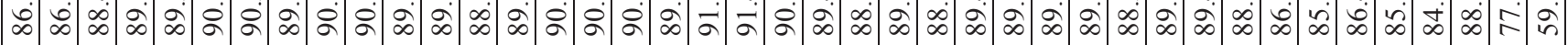

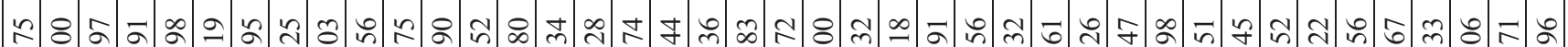

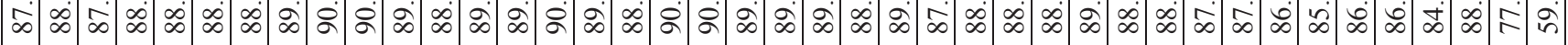

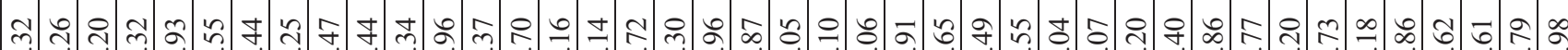

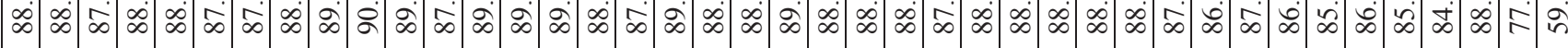

そิ

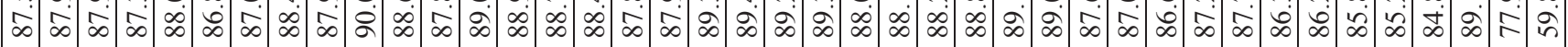

万எ f.

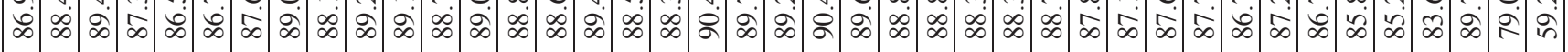

F.

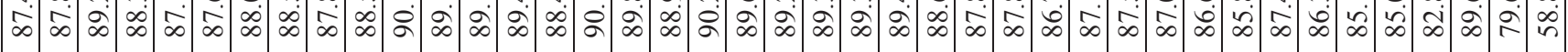

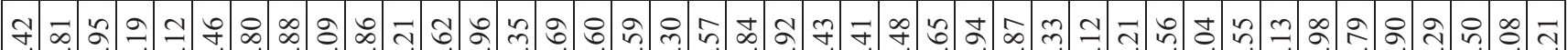

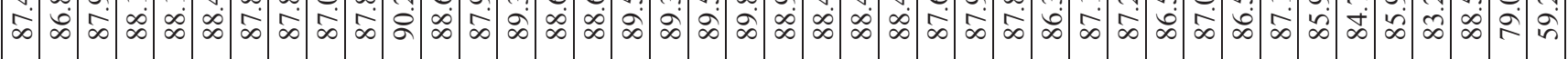

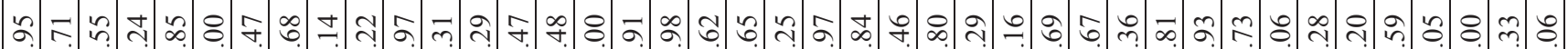

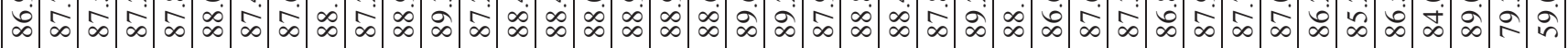

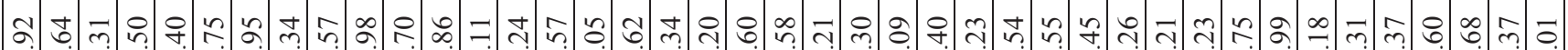

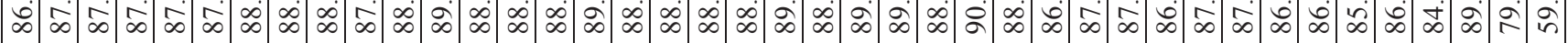

fำ

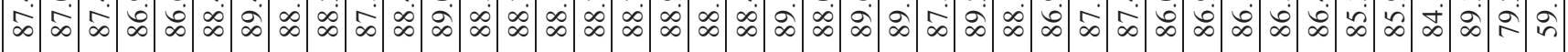

भ.

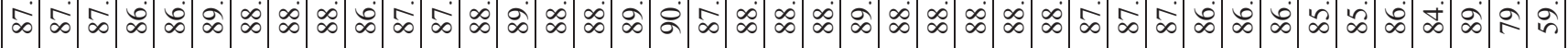

ठำ

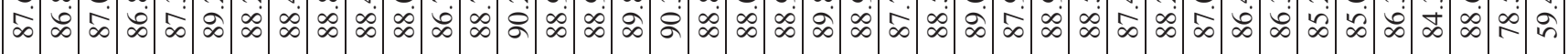

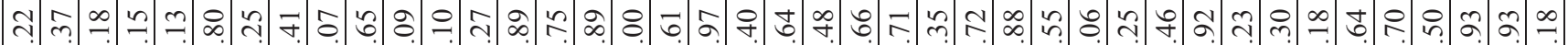

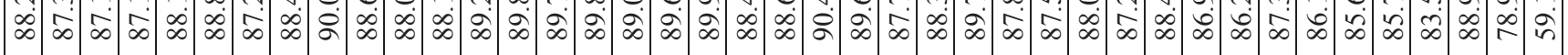

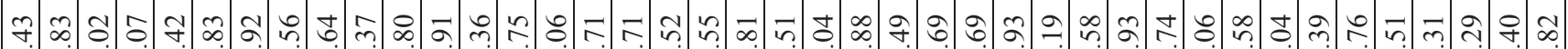

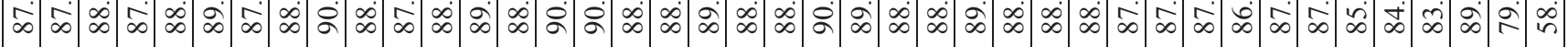

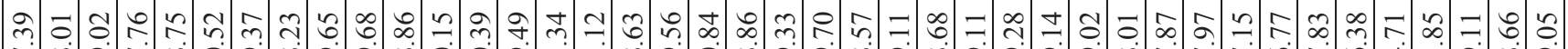

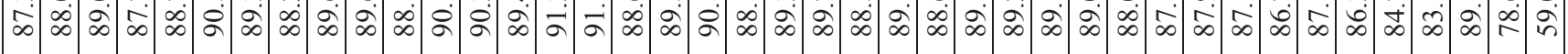

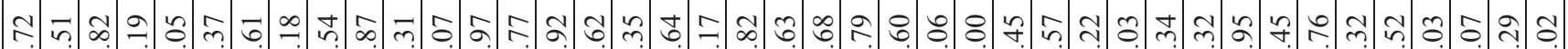

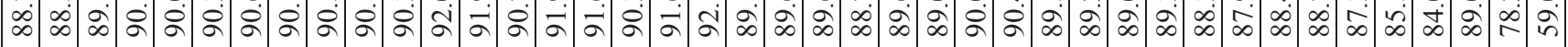

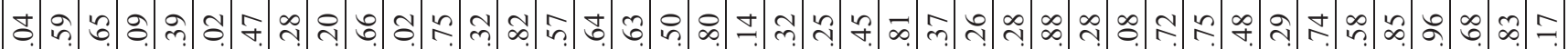

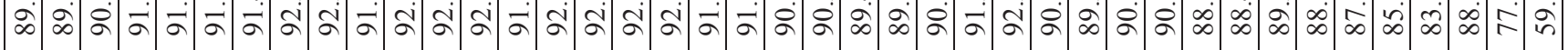

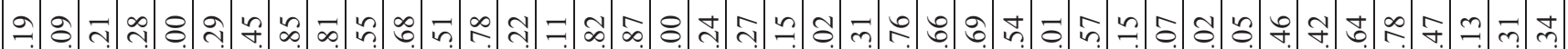

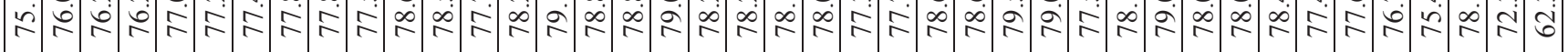

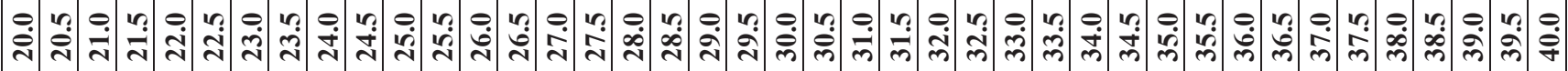




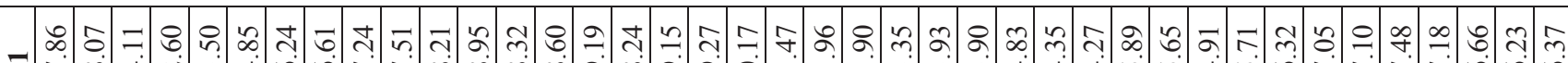
ন

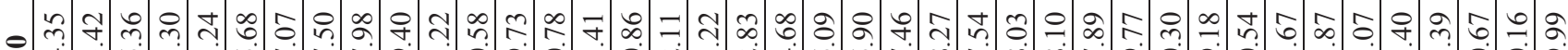

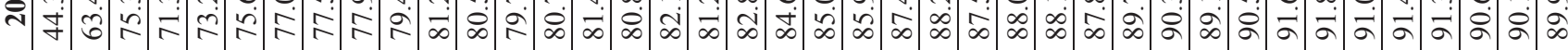

ค

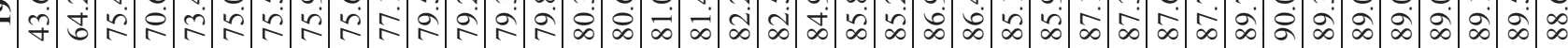

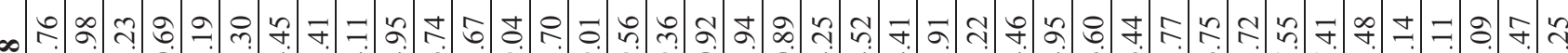

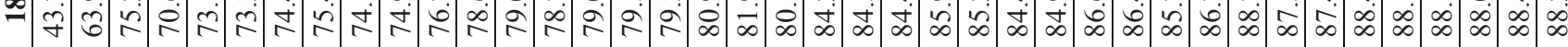

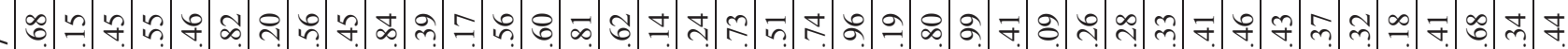

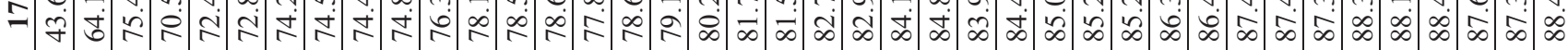

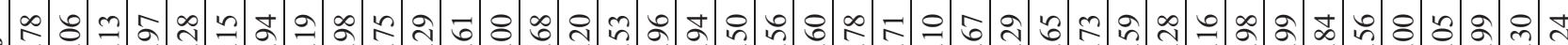

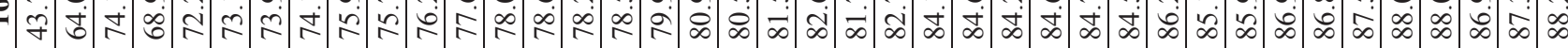

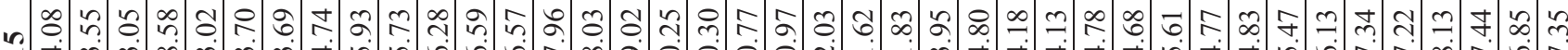

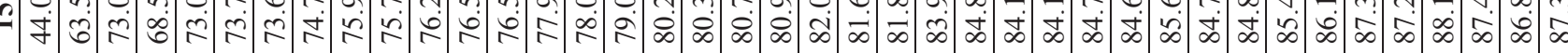

- సิ

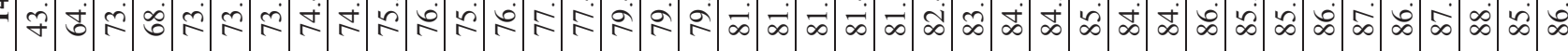

m

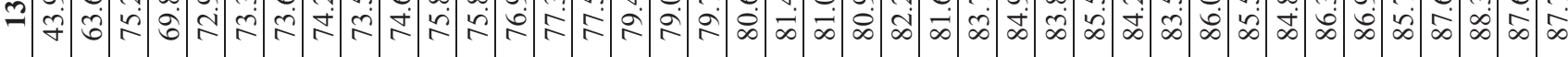

ง

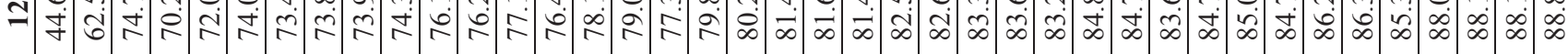

号

-

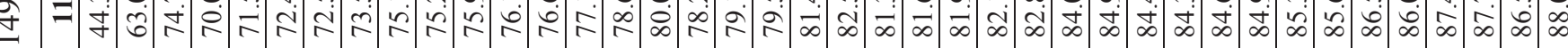
•

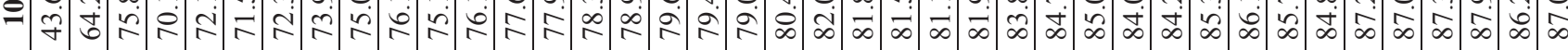
m -

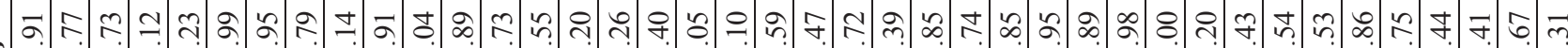

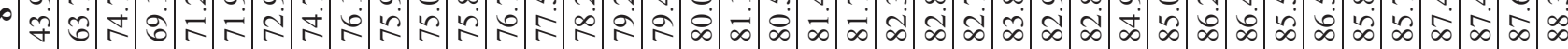

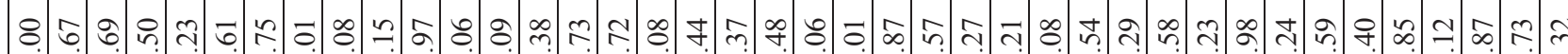
过

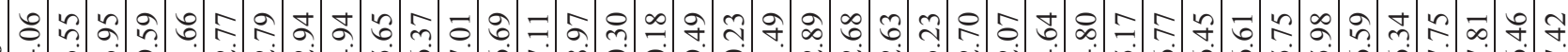

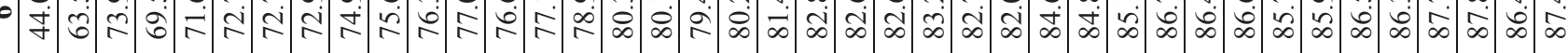

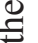

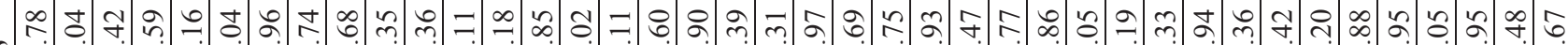

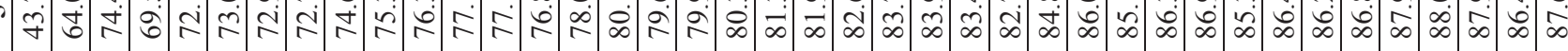

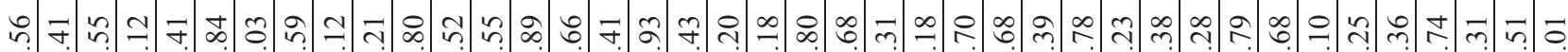
f)

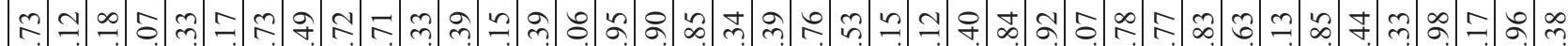

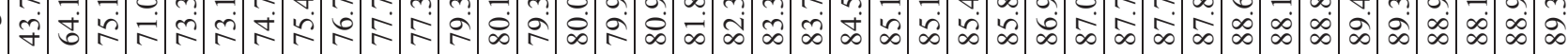

$\checkmark$ f $=$ m. Jं

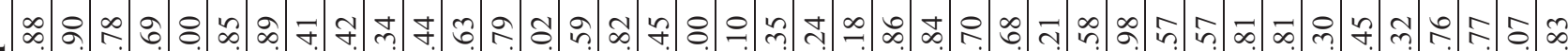

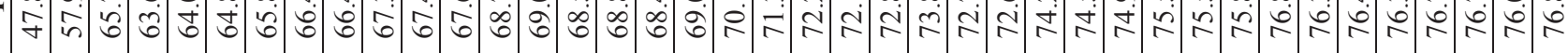
車 $\dot{0}-\dot{0}-\dot{\mathrm{i}}$ ن 


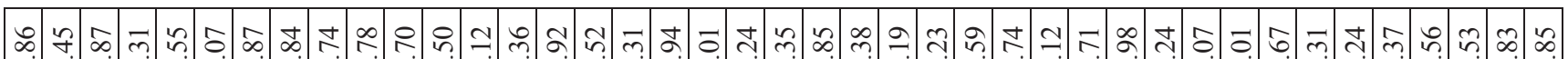

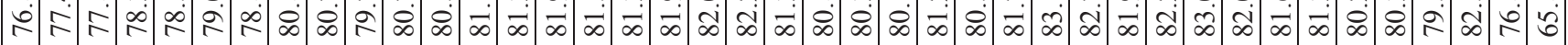

क

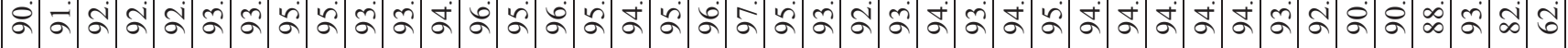

유유 유

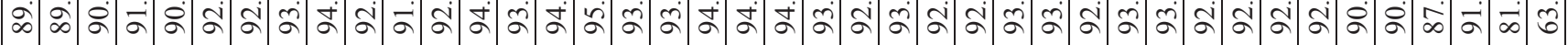

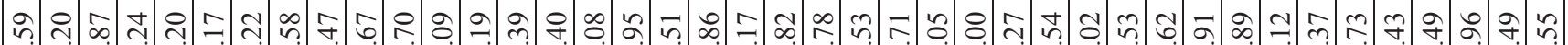

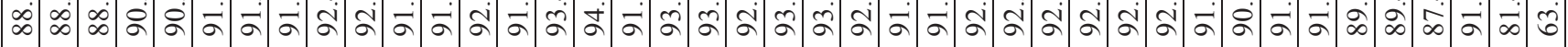

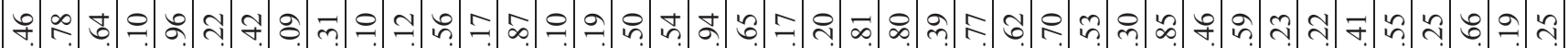

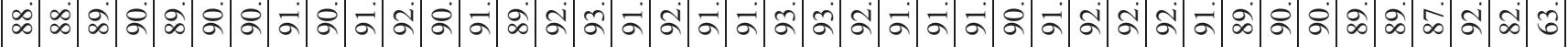

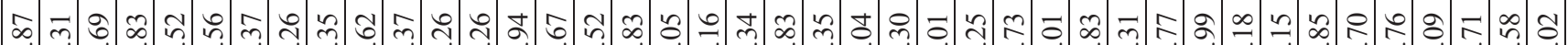

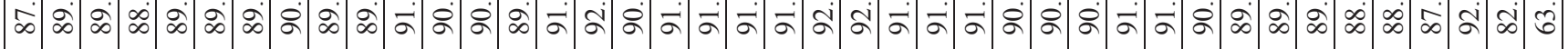

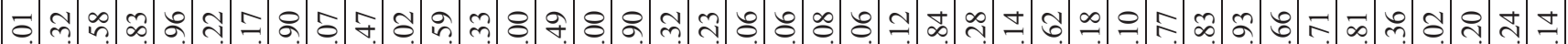

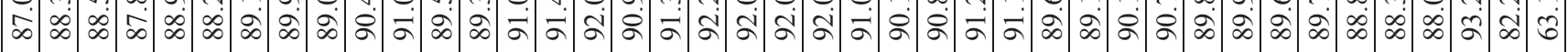

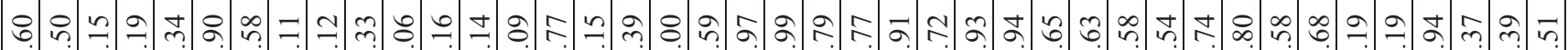

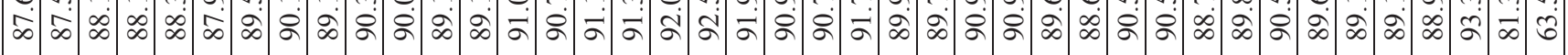

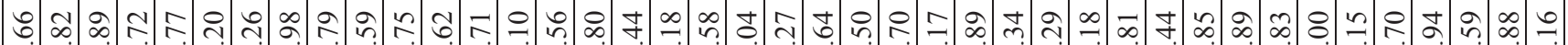

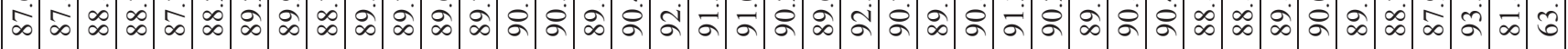

นิ

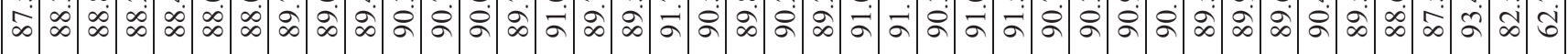

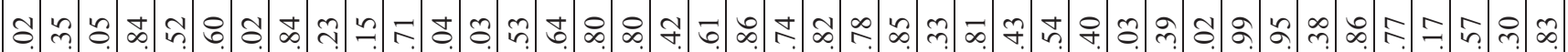

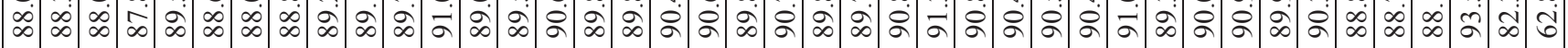

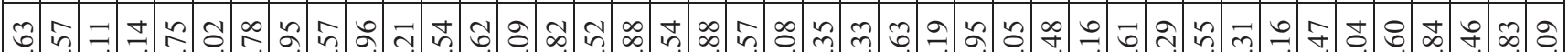

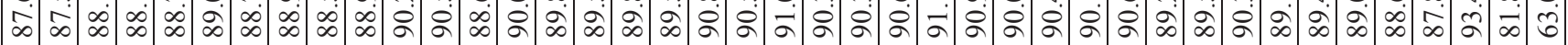

๓

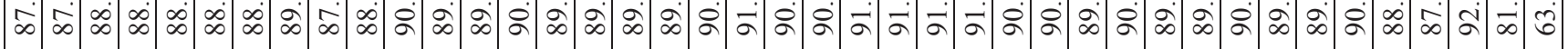

বㄷำ

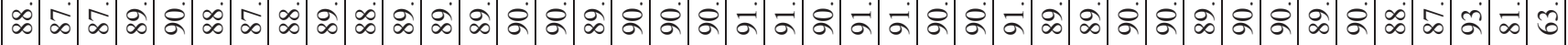
$\circ=$ ㄱำ

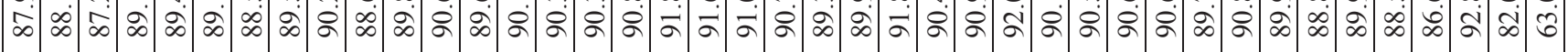

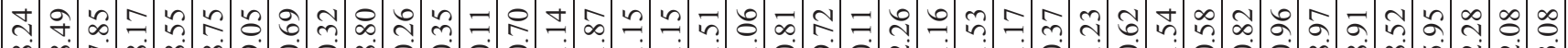

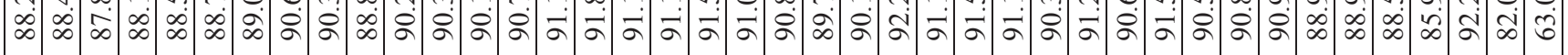

ช.

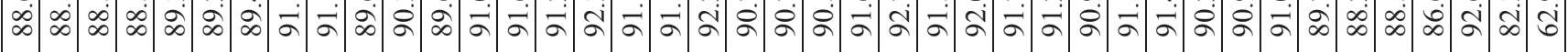

ఐ

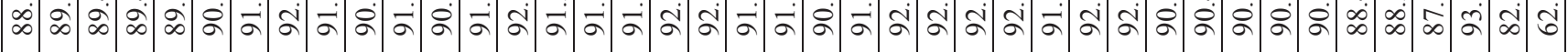

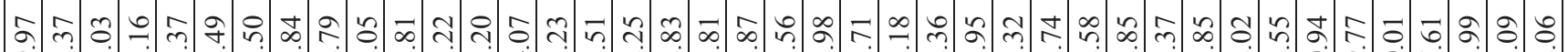

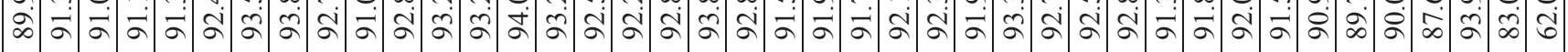

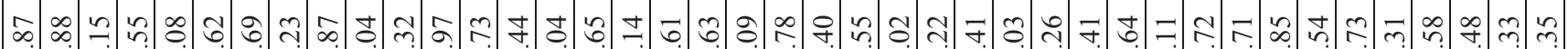

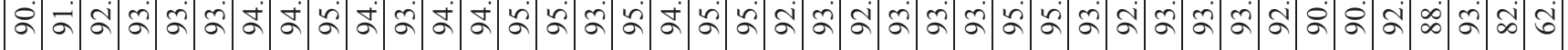
ప

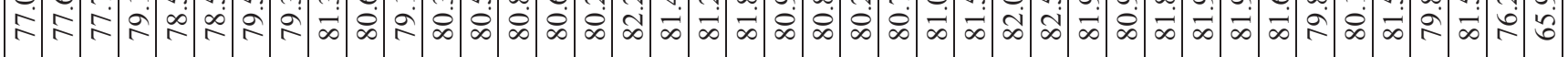

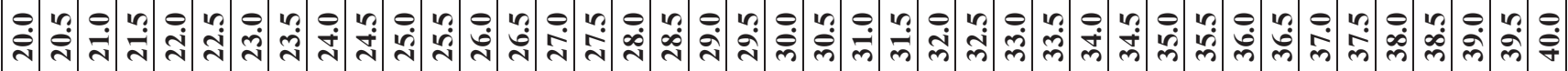




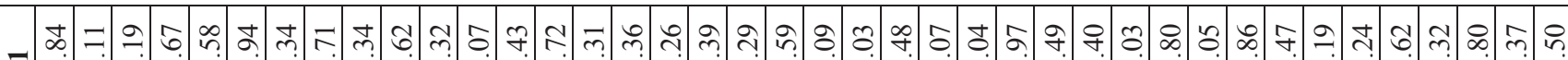

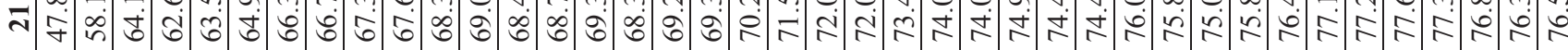

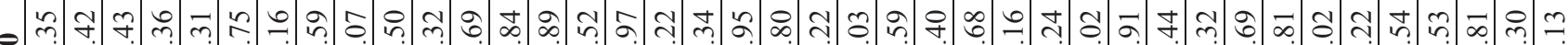

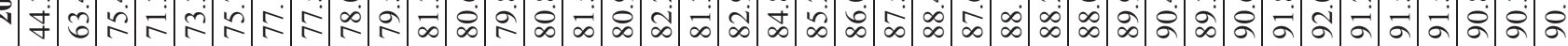

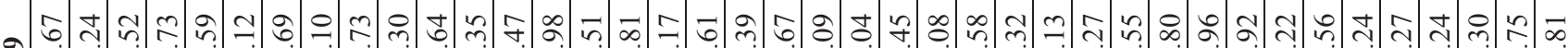
$=$ 子ं

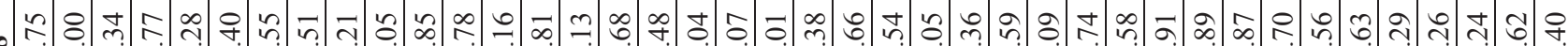

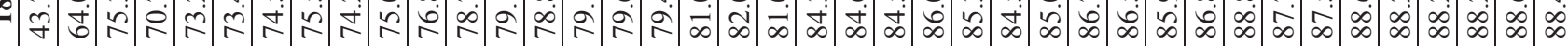

ก โิ

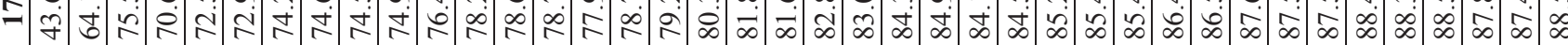

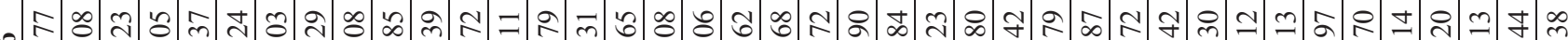

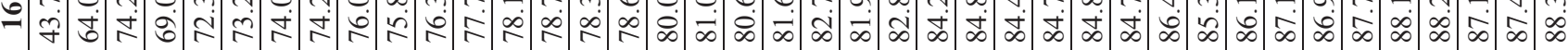

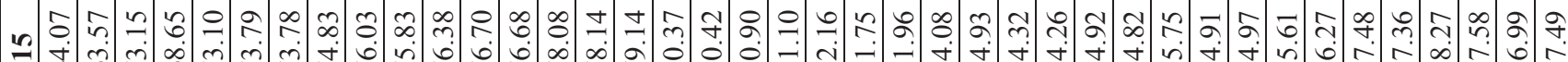

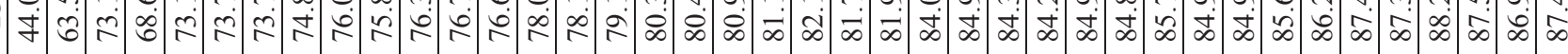

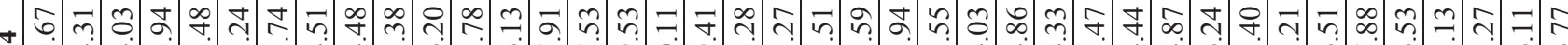

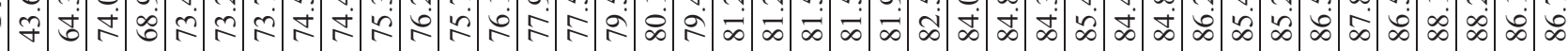

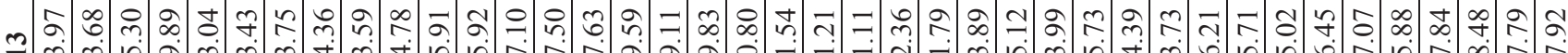

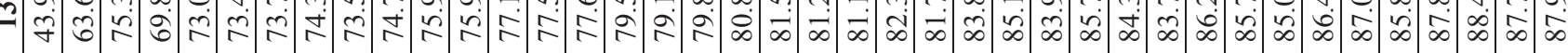

ㄱำ $=$ I

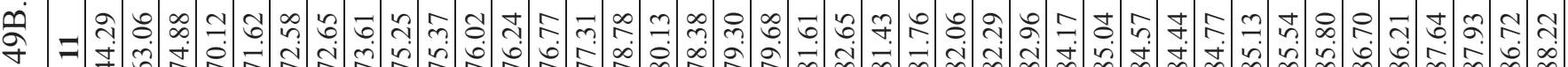

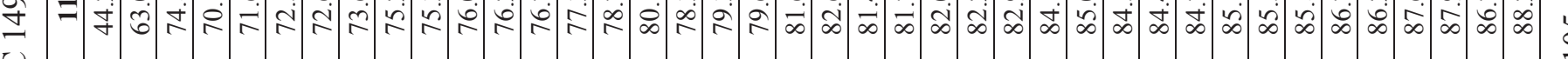
○ ๑

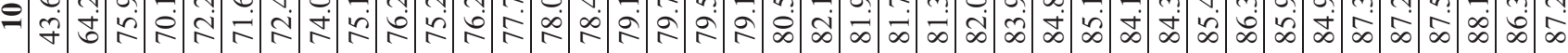
กิ ๓ૈ -

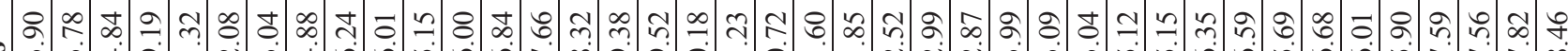

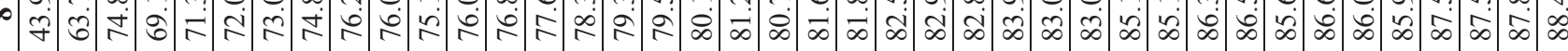
\&

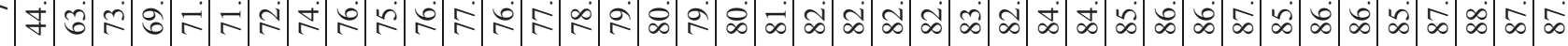
そ กิ

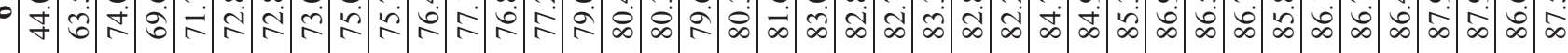
\&

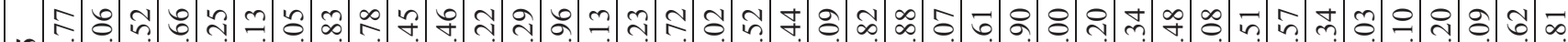

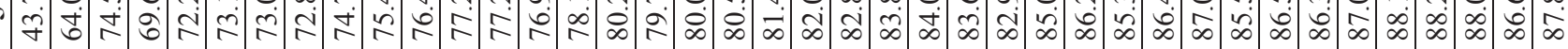

छี

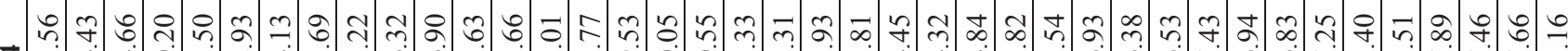

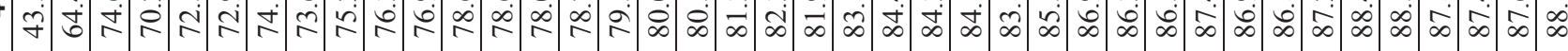

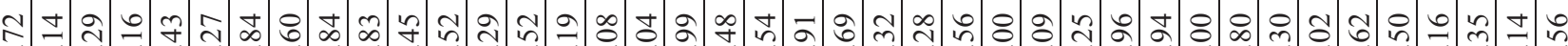

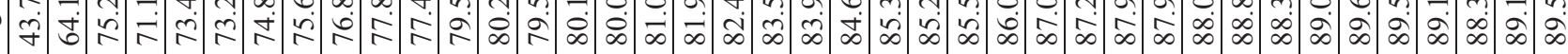
:

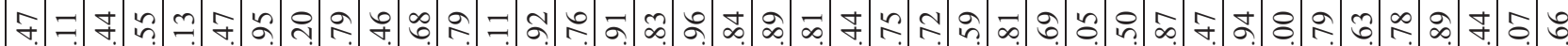

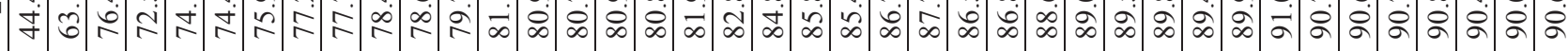

$\underset{+}{+}$

$\frac{0}{\frac{\pi}{\pi}}$

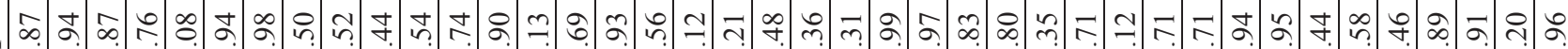
守 约 e. $\dot{0}-\dot{\theta}-\dot{i}$ i 
ร की

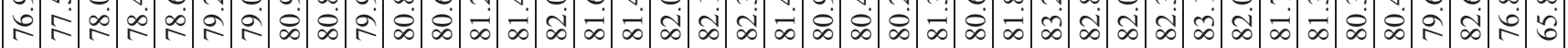

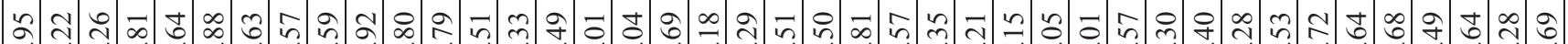

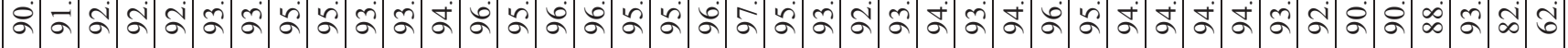

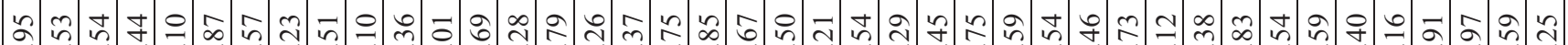

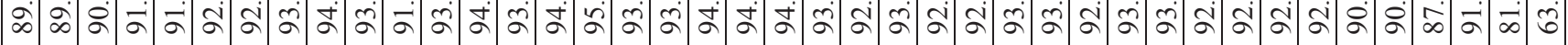

구

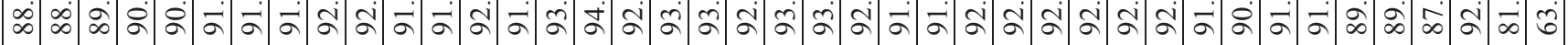

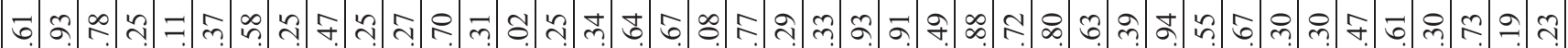

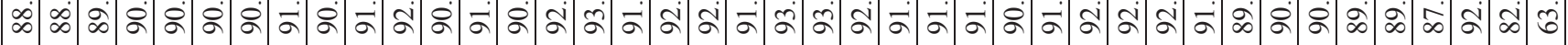

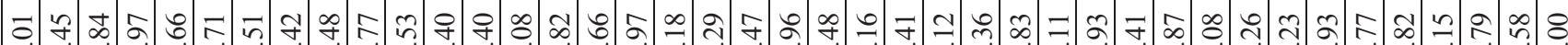

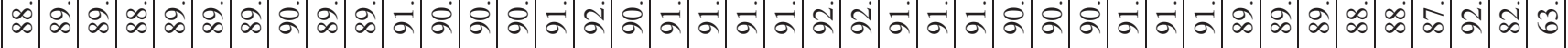

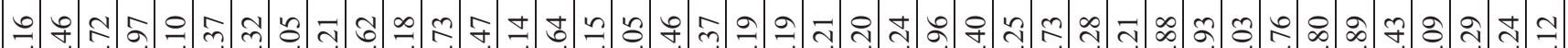

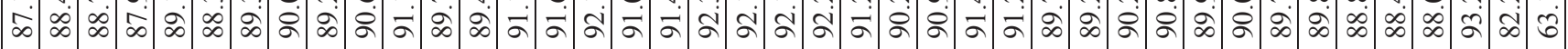

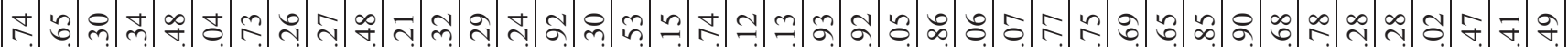

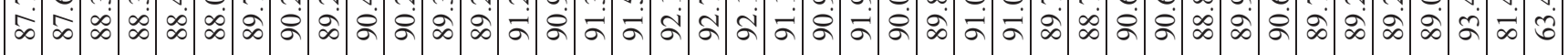

\&

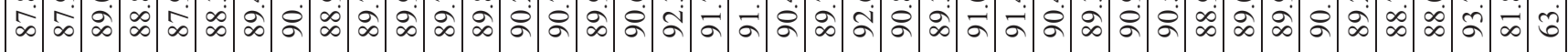

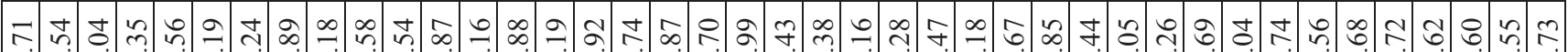

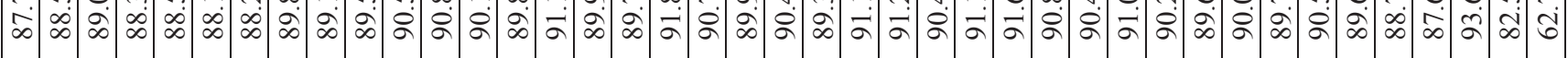

ㄱำ

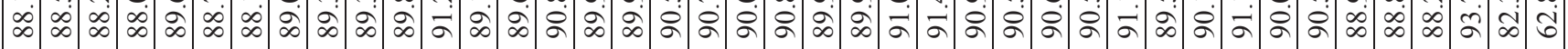

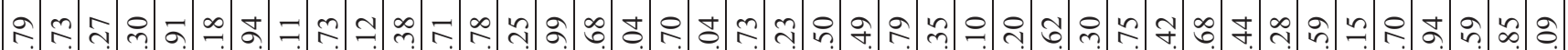

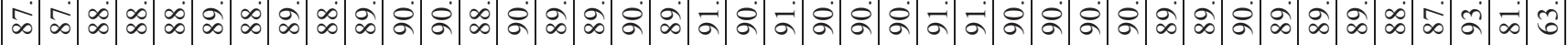

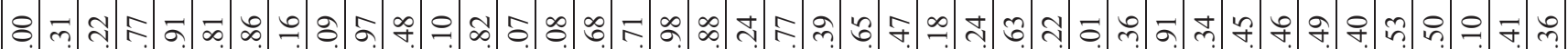

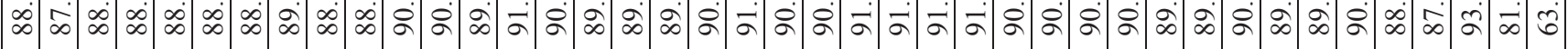

舟兄

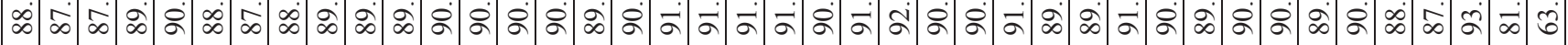
= $\tilde{m}$ กิ

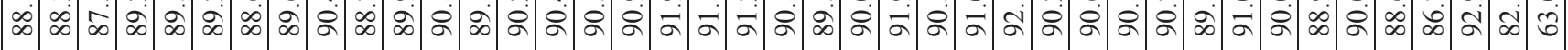

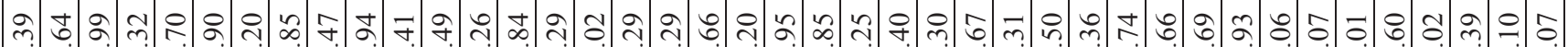

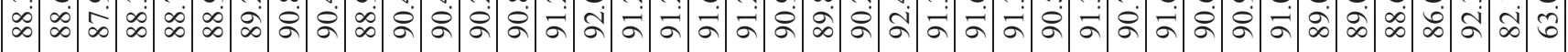

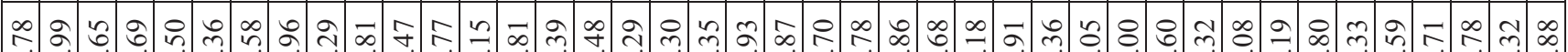

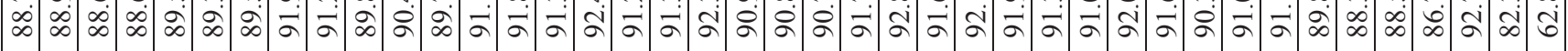

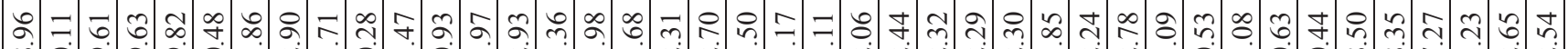

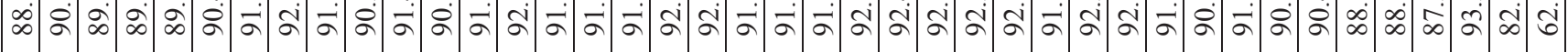

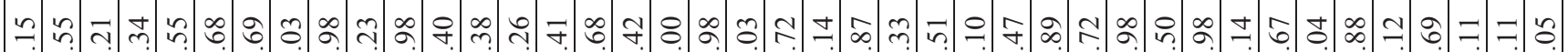

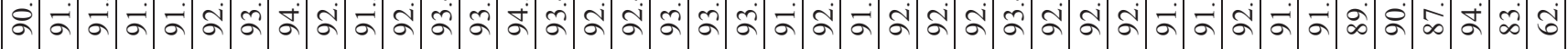

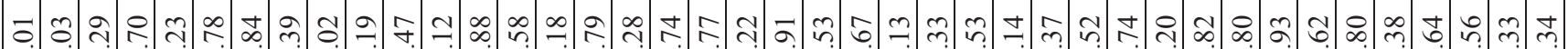

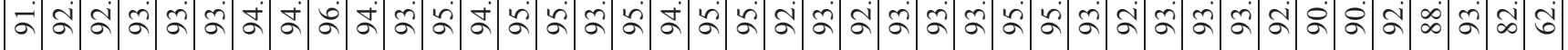

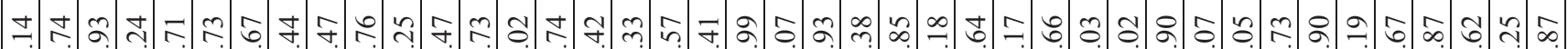

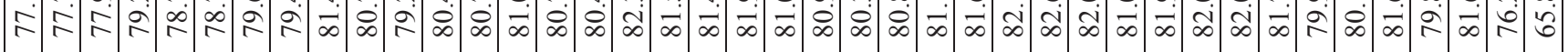

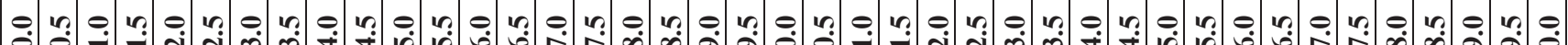

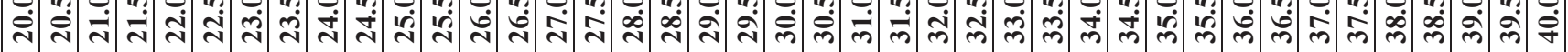


ন

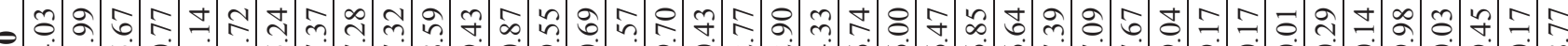

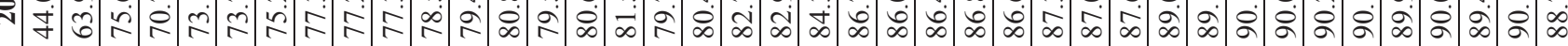

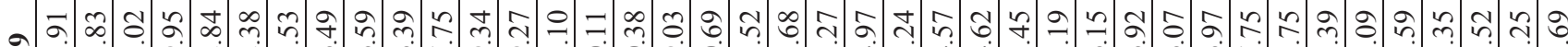

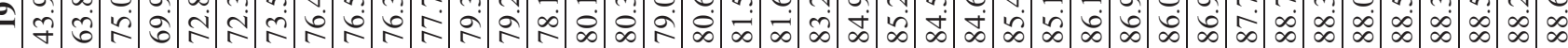

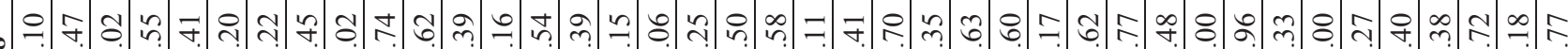

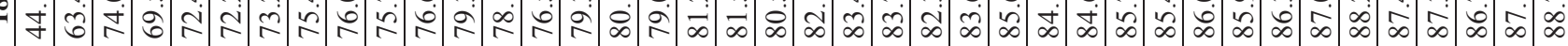
t

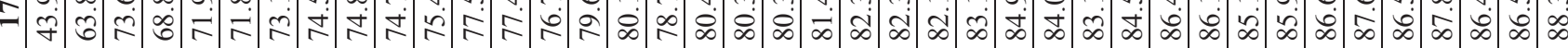

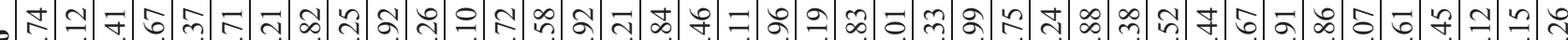

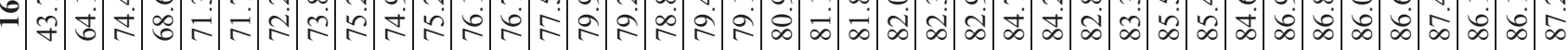

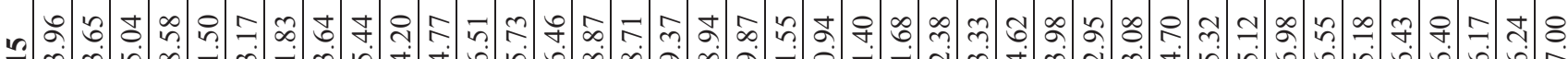

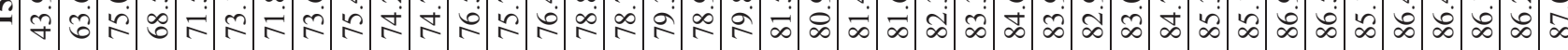

๑

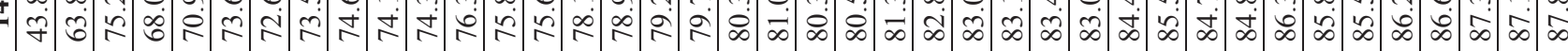

m

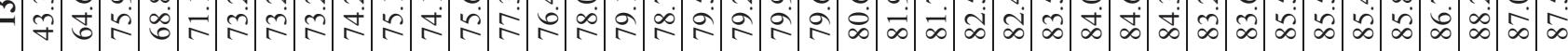

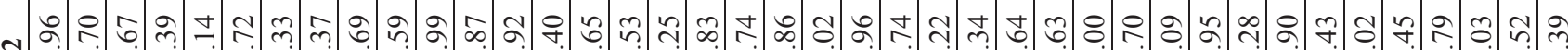

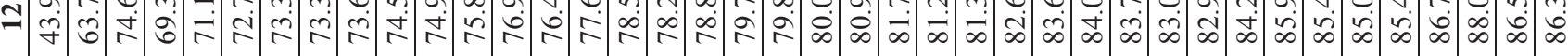

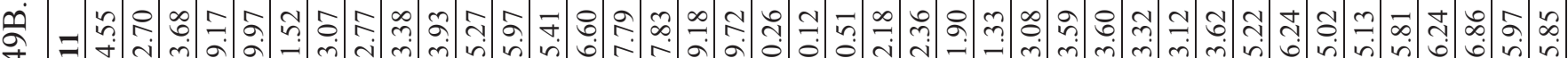

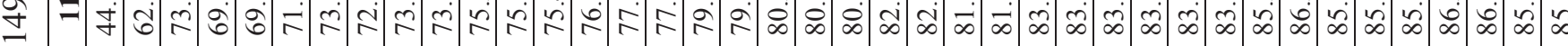
○ f $=$ 过

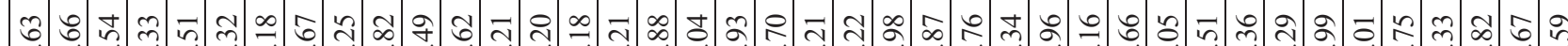

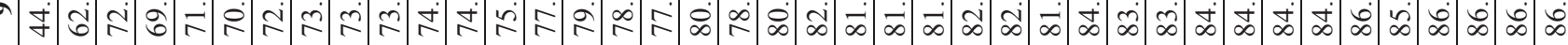

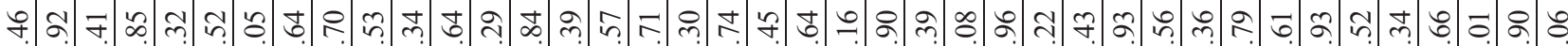

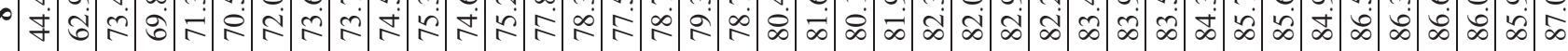
б

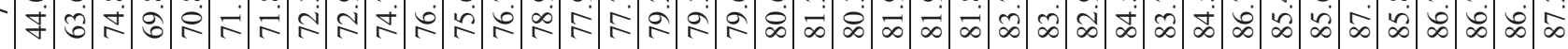

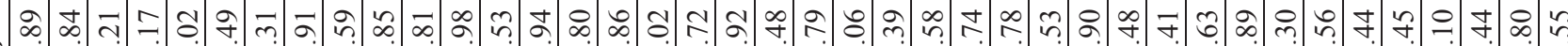

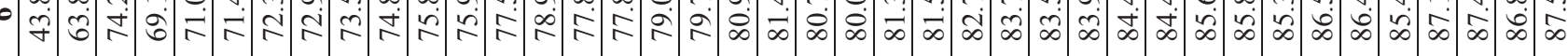

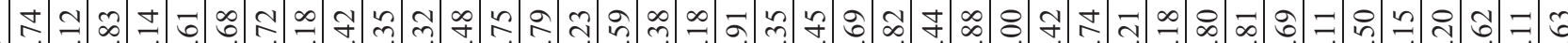

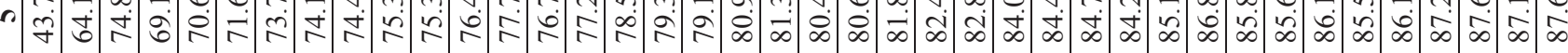

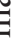

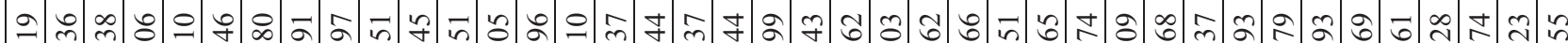

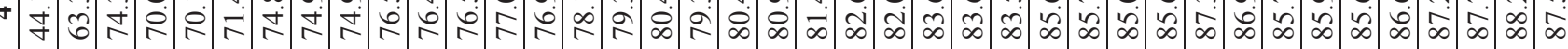

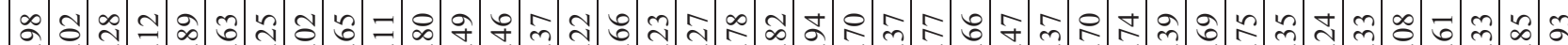

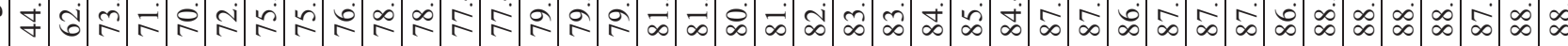

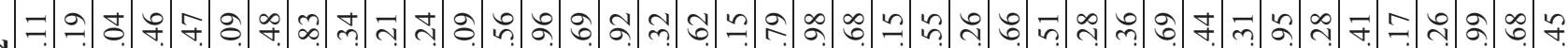
守 g

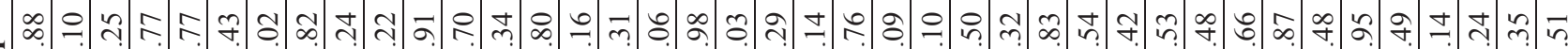

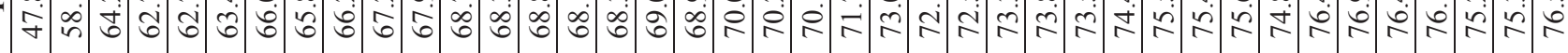
e.

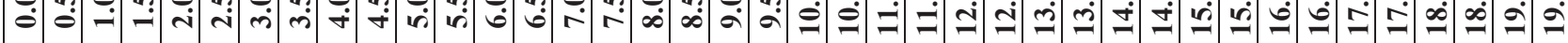


ๆา ต

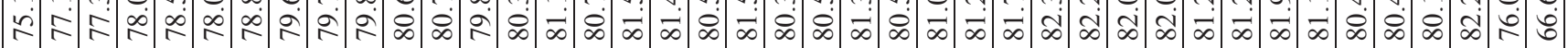

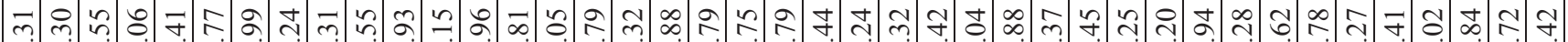

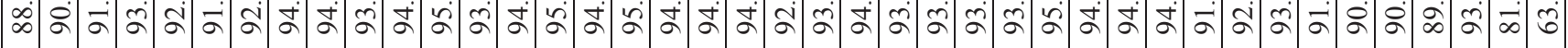

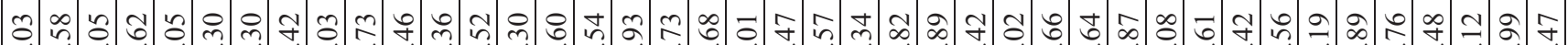

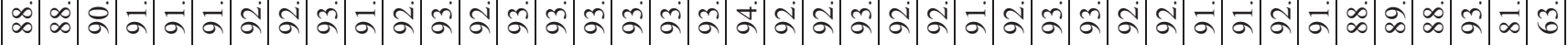

nิ่

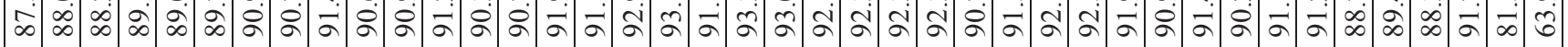

ప.

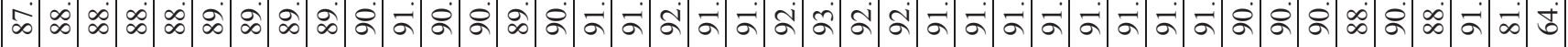

m

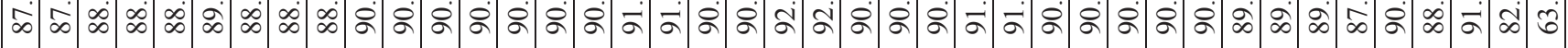

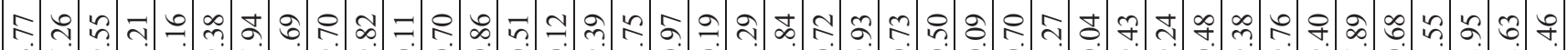

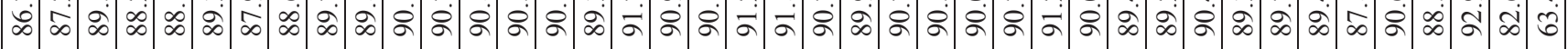

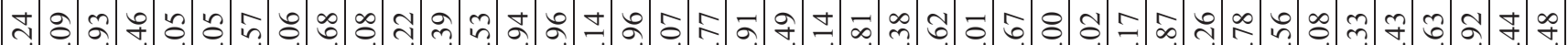

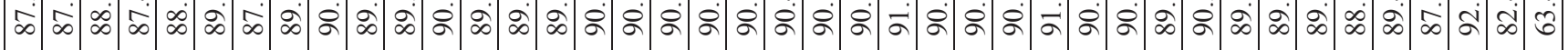

m

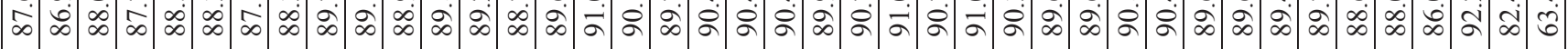

สุ่ ลำ

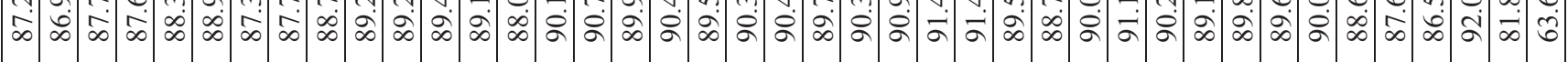

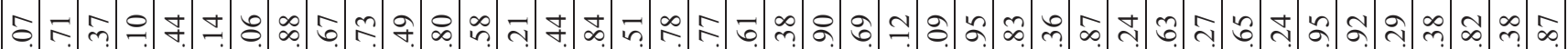

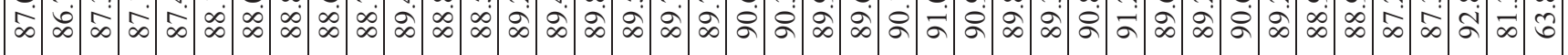
ᄀ

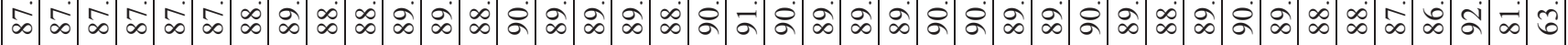

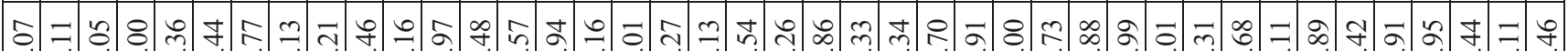

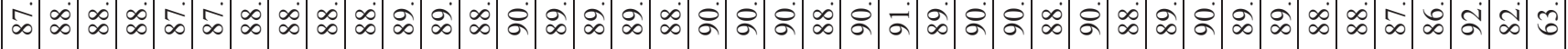

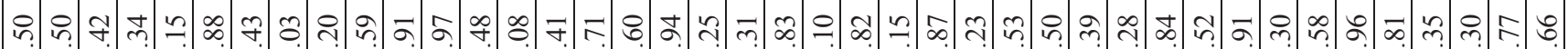

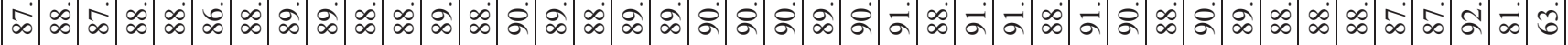
苟 = m

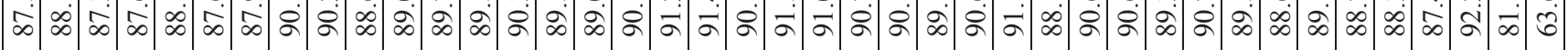

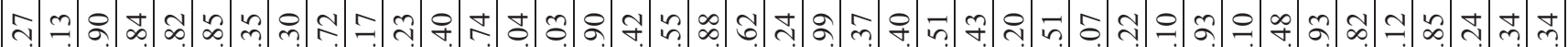

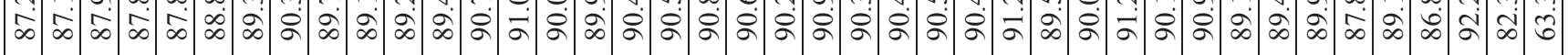

ป

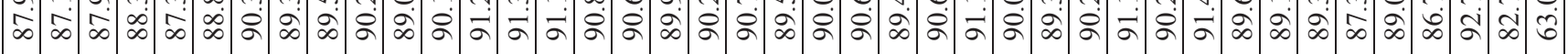

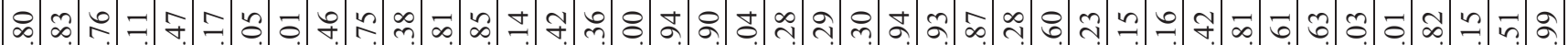

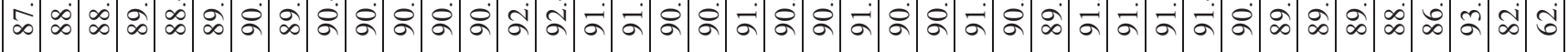

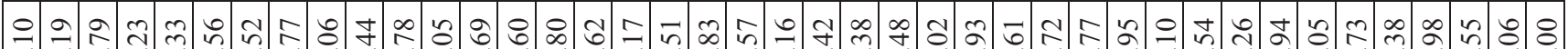

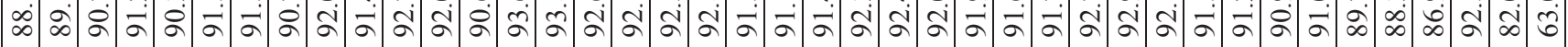

‡.

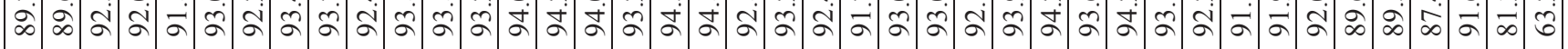

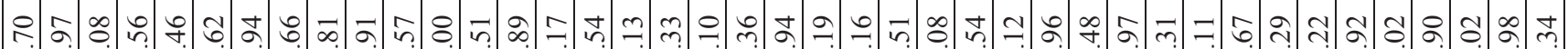

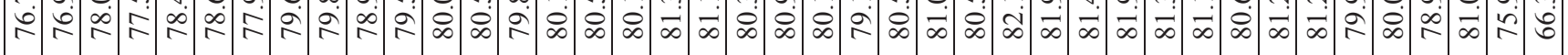
우 ปิ่ 


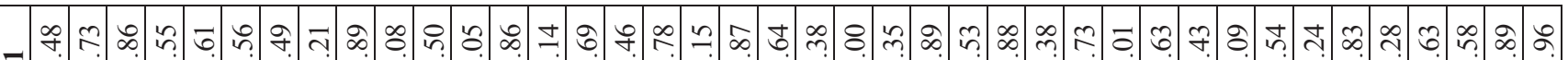

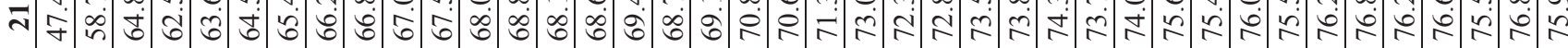

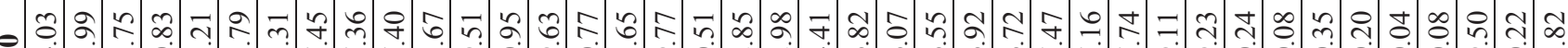

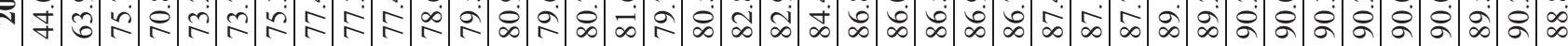

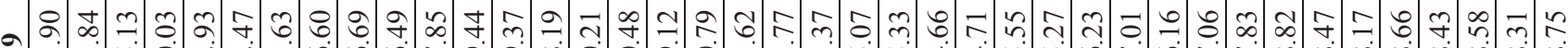

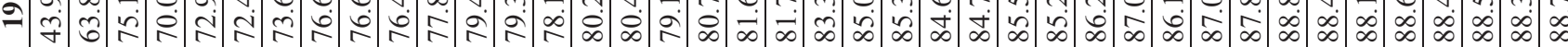

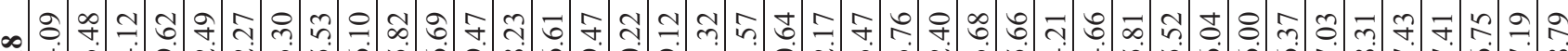
过

๙

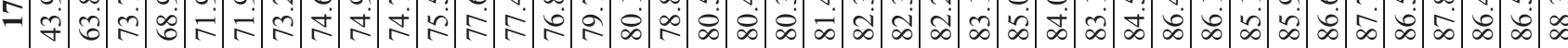

古

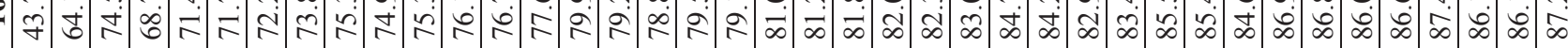

๓ $\curvearrowleft$ $\because$ ص

Љ

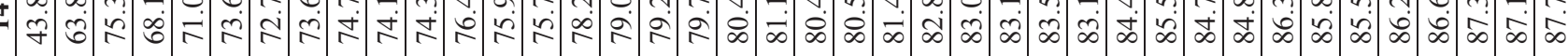

m

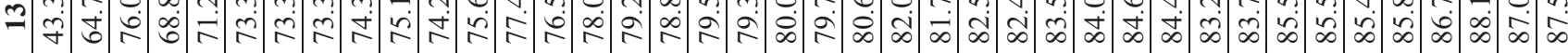

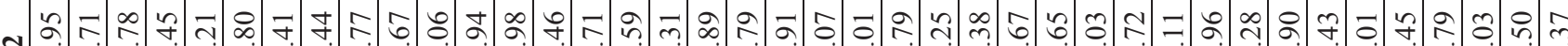

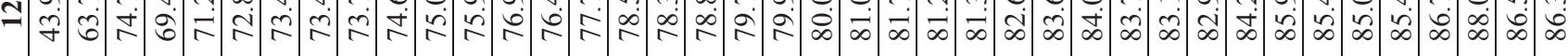

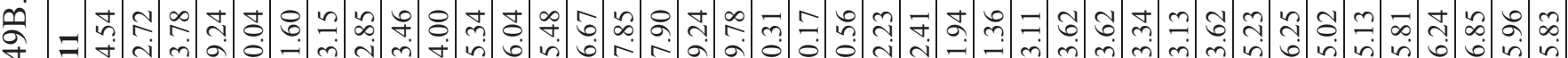

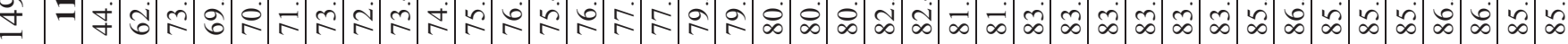

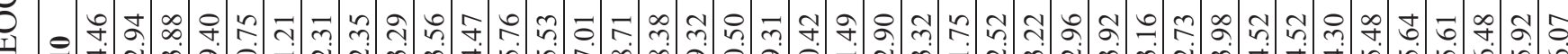

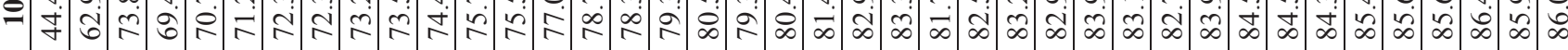

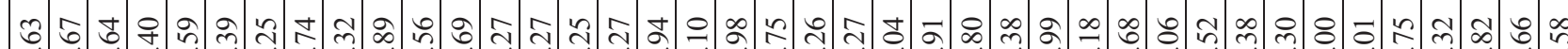

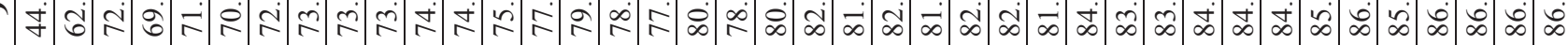
ๆ

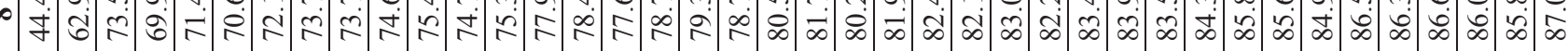
-

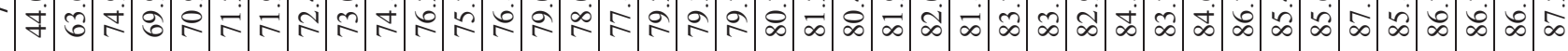

- $\infty$ $\infty$ m 守 $\stackrel{乛}{\Xi}$

華

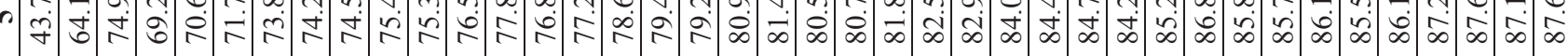

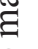

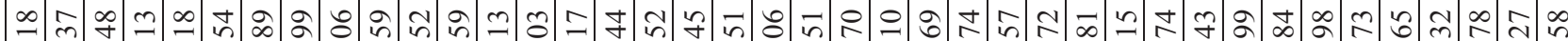

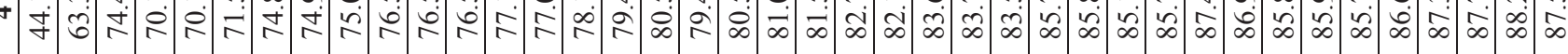

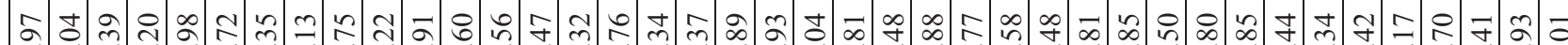

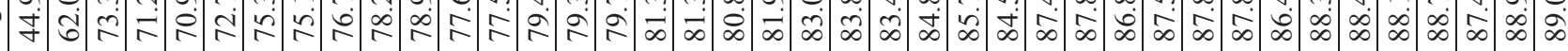

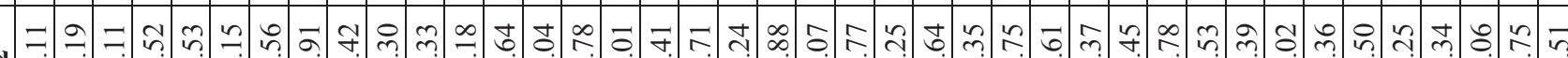
守

in

$\frac{0}{\frac{\pi}{a}}$

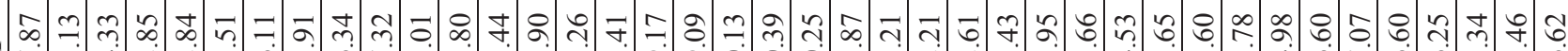

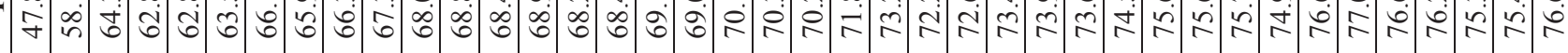
-

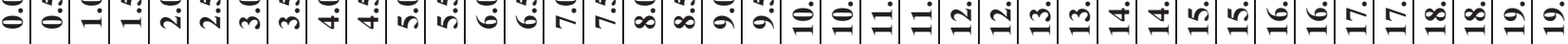


งิ สุ

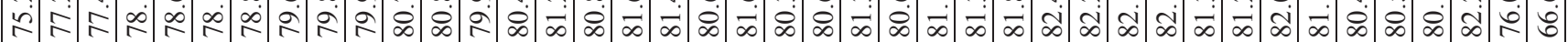

m

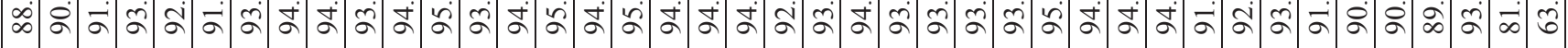

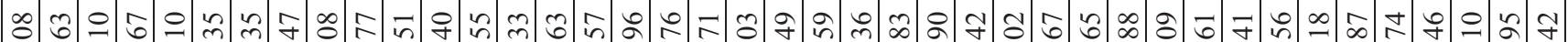

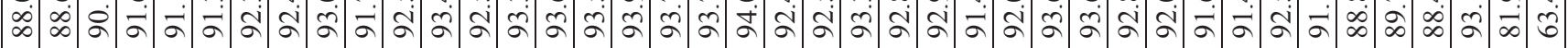

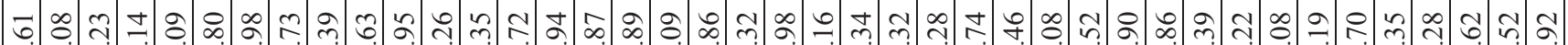

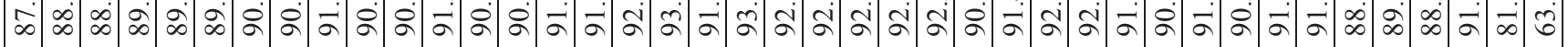

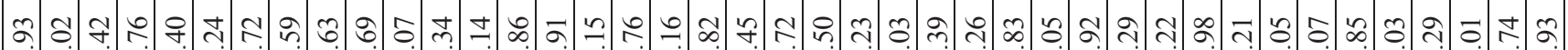

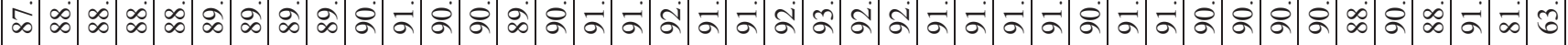

윌

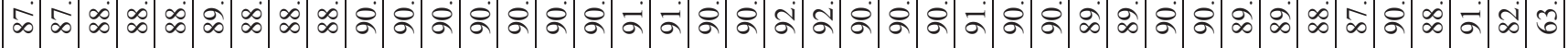

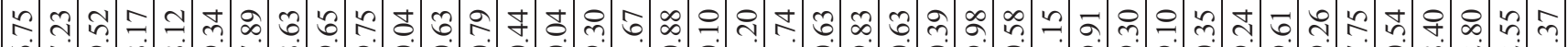

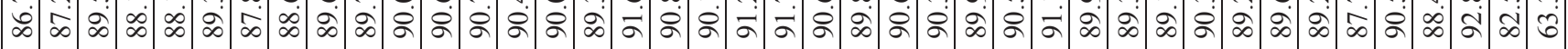

สิ

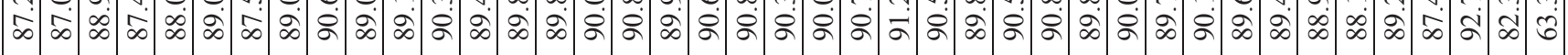

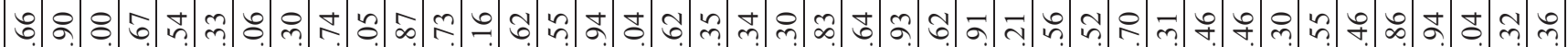

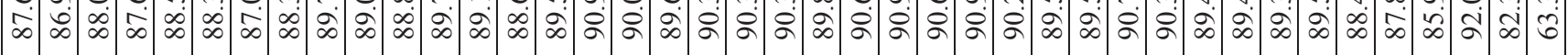

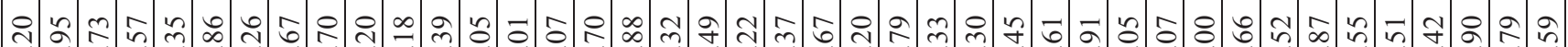

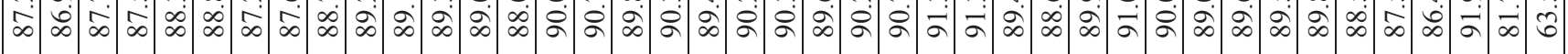

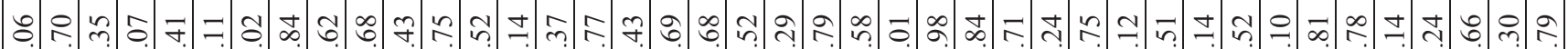

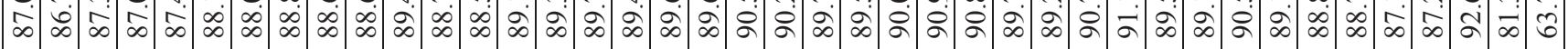

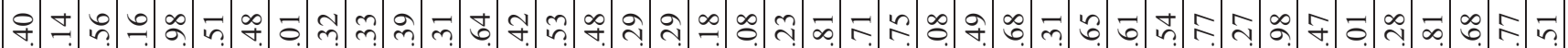

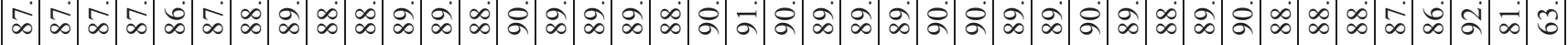

\&의

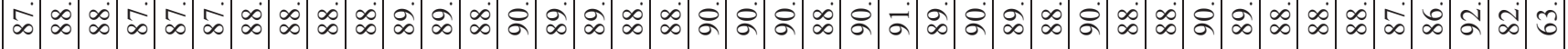

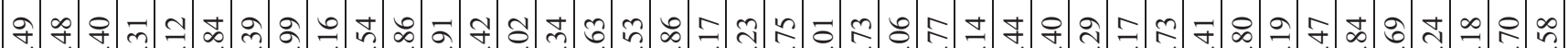

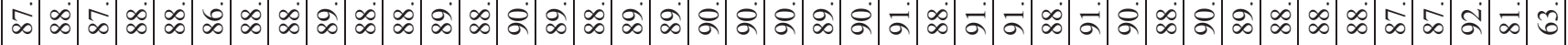

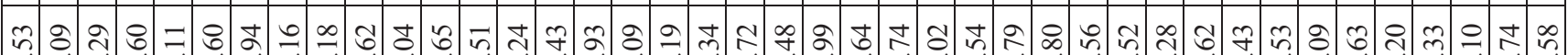

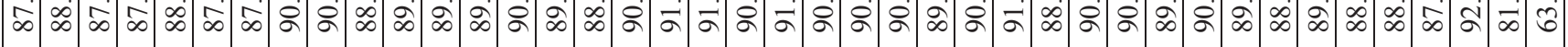

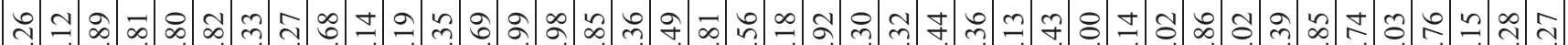

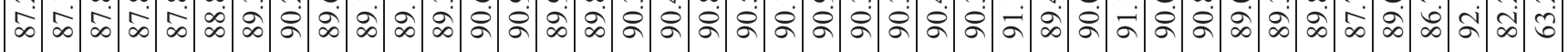

ปప

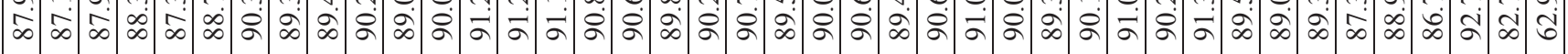

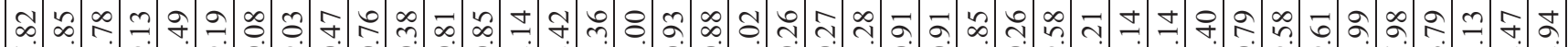

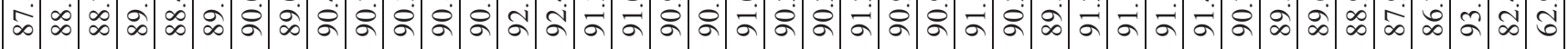

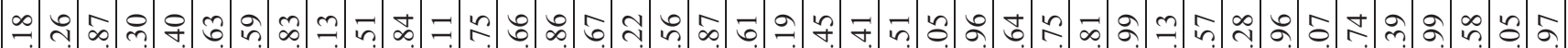

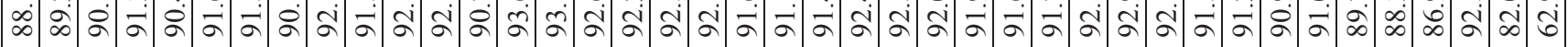

ప.

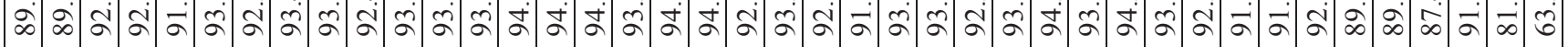

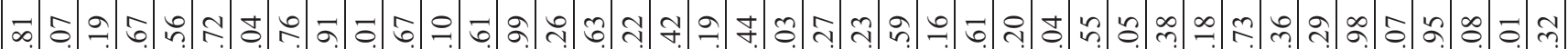

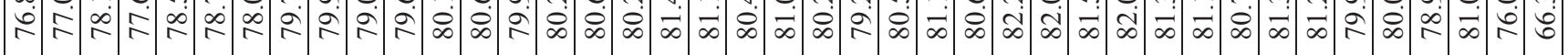
我 ปิ่ 


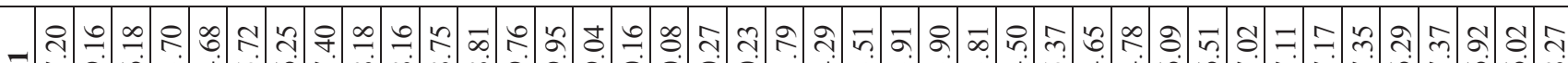

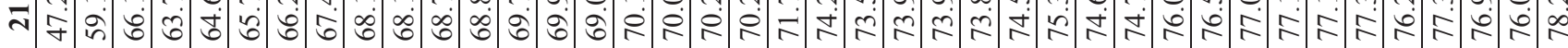

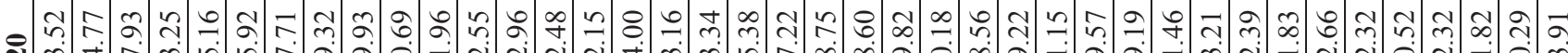

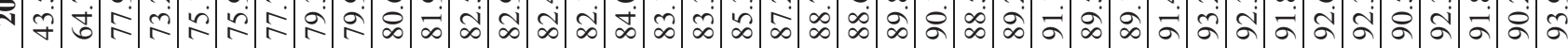

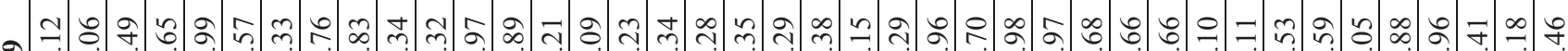

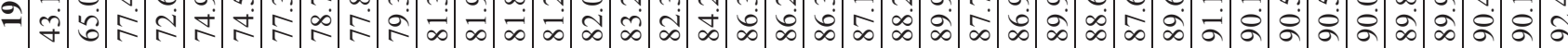

$\infty$ రำ ำ

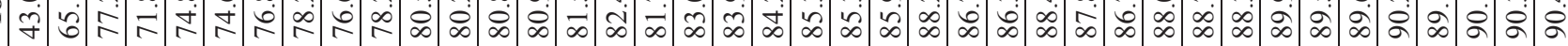

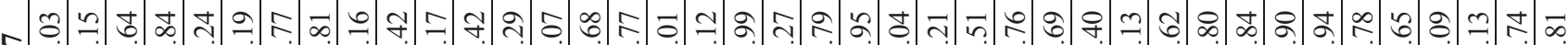

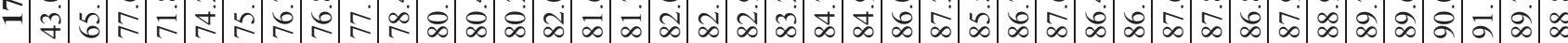

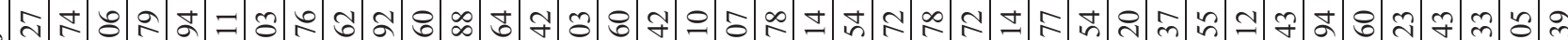

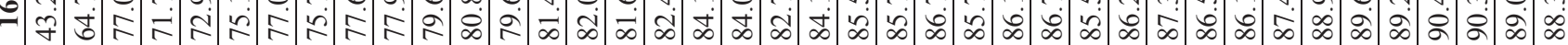

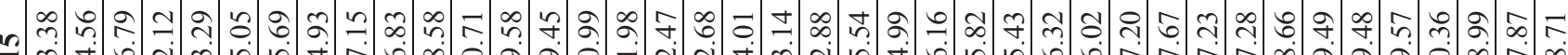

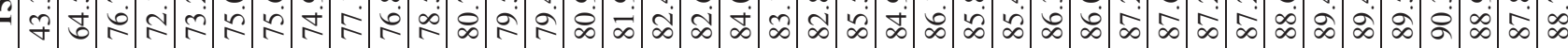

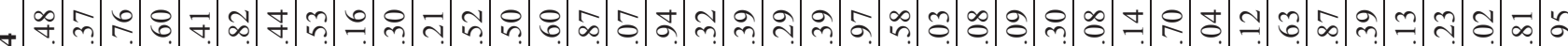

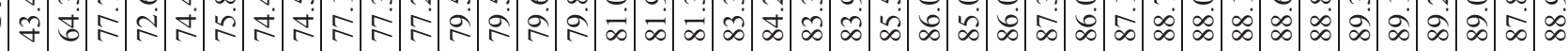

๓ ㅊ.่.

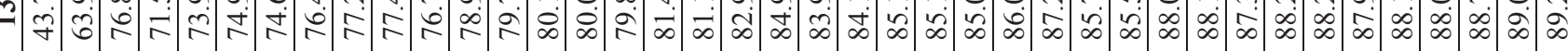

ภे

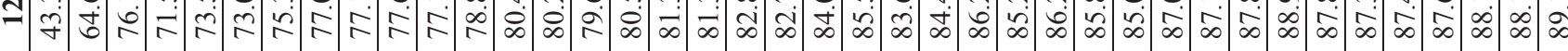

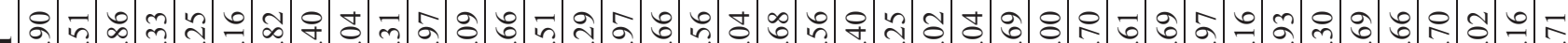

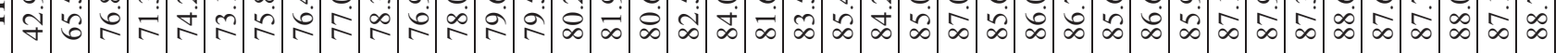

穴

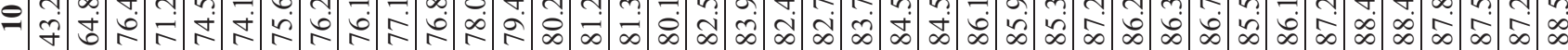

न

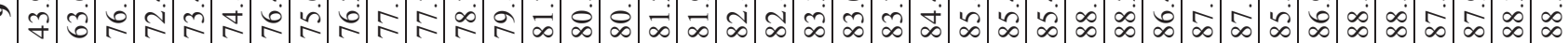

ลำ

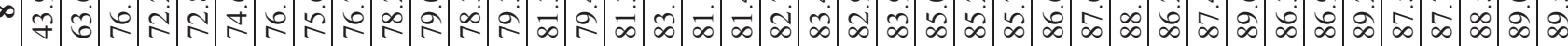

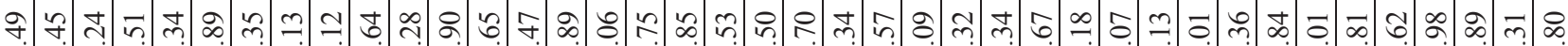

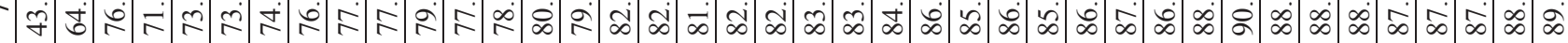

กิ

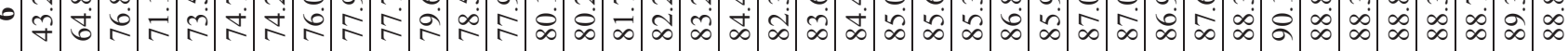

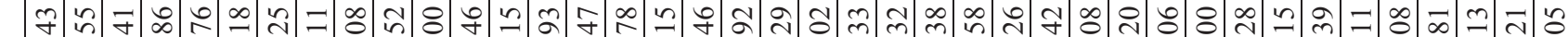

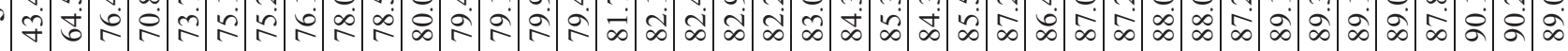

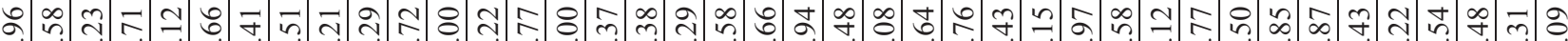

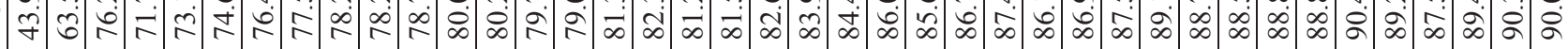

ஜ ᄀ

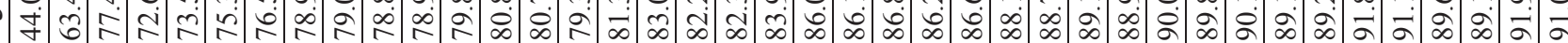
₹

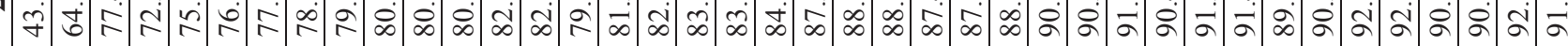

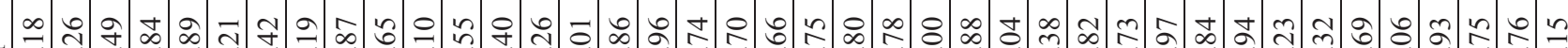
守 @̈ 
d:4

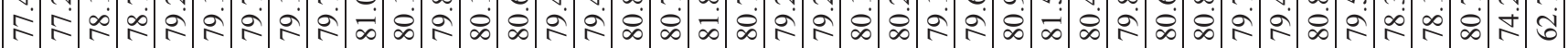

ळ t)

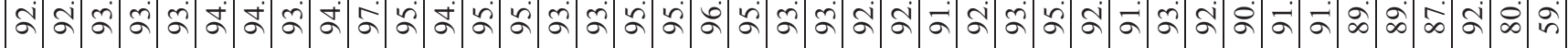

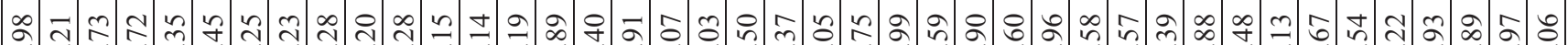

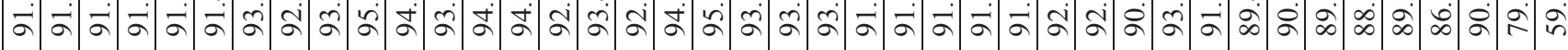

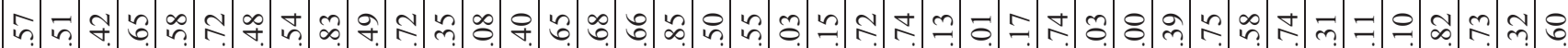

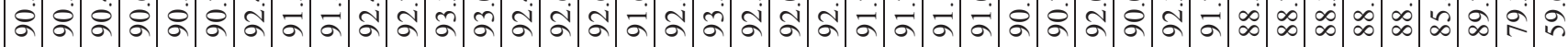

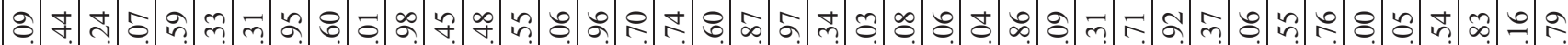

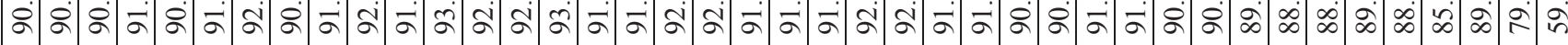

의 의

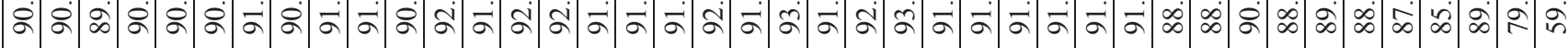

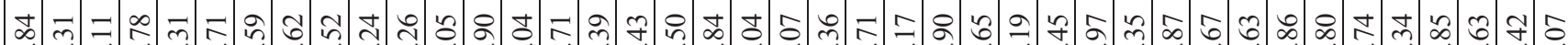

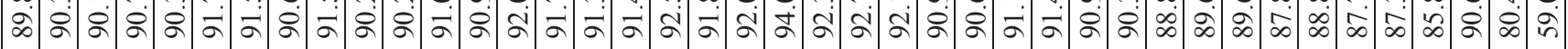

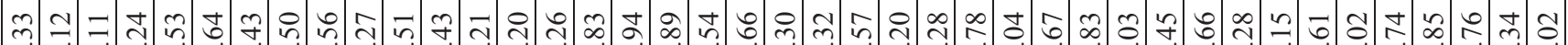
ळे àं

กิ่

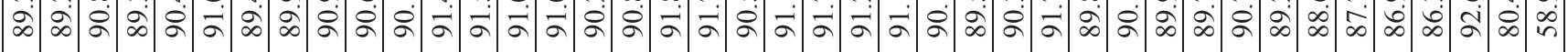

ㄷำ

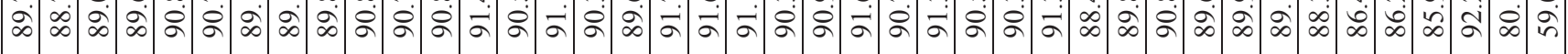

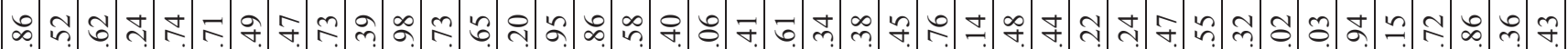

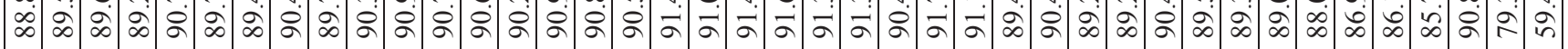
๘)

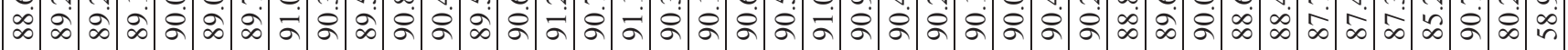

\&

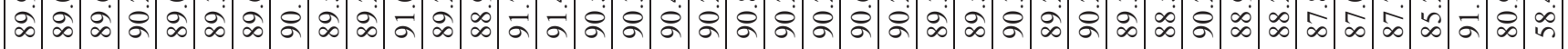

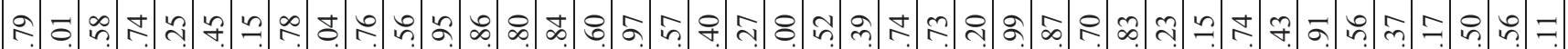

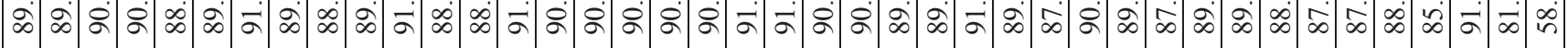

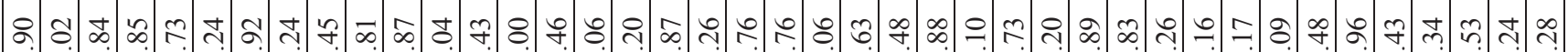

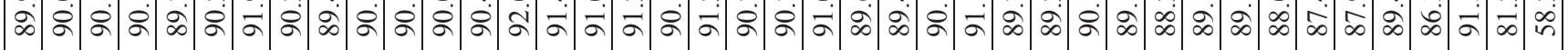

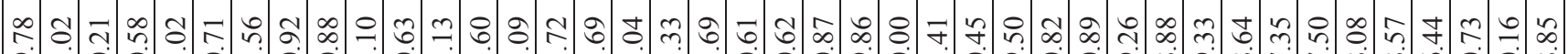

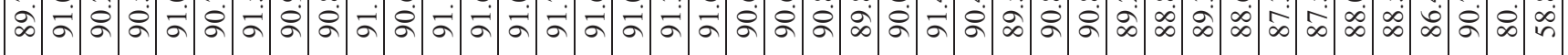

సิ츠의

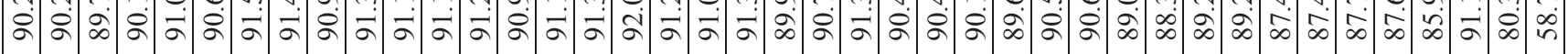

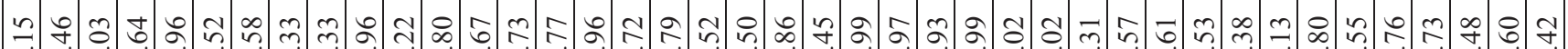

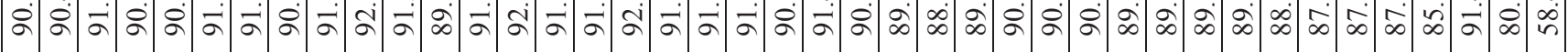

๙

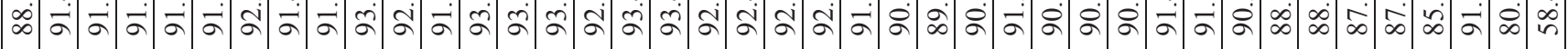

$f$ ㄱำ

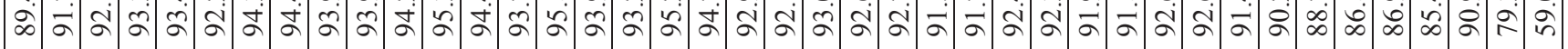
๑)

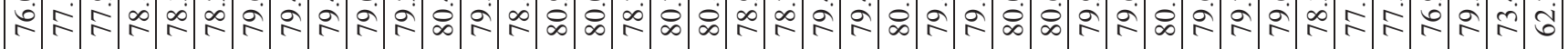




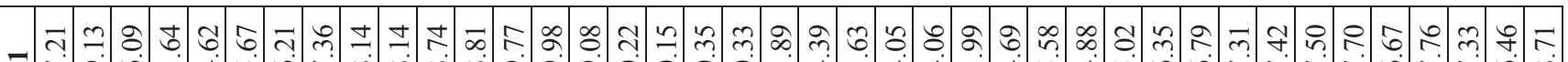

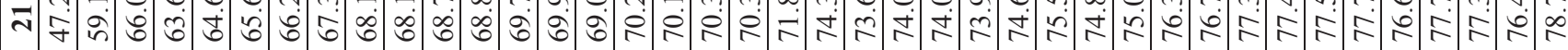

-

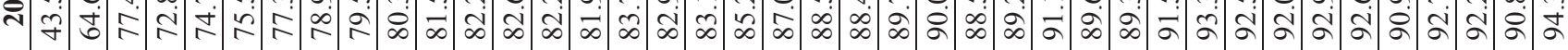
- ニ ฉ

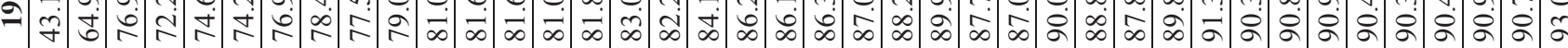

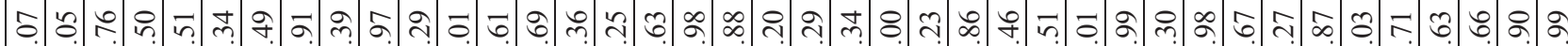

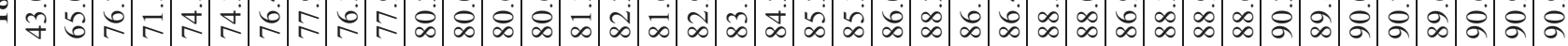
ठ․

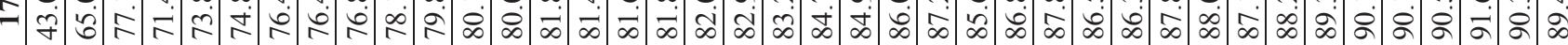

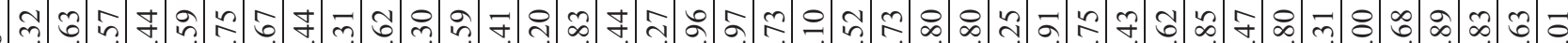

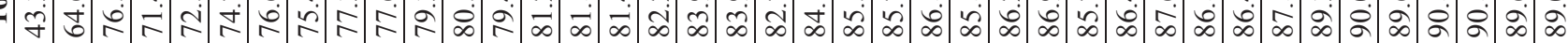

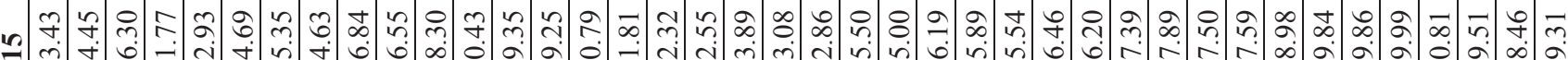

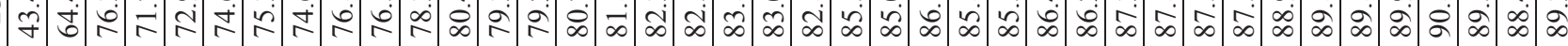

กิ

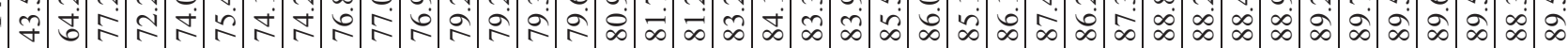

m ஜ $=$ भ

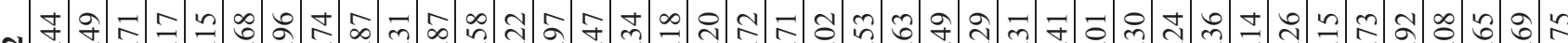

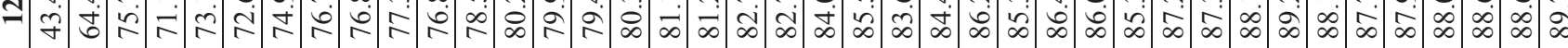



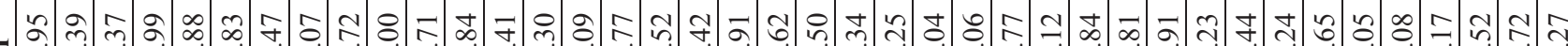

$=$ पे

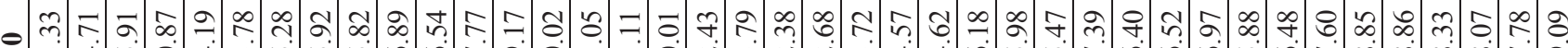

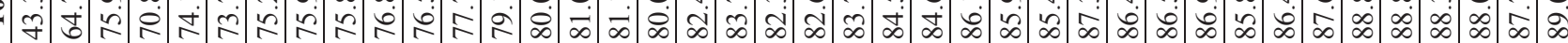

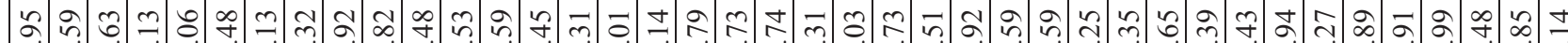

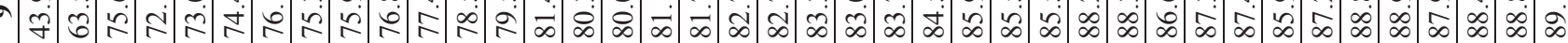
ลุ. 守 芒声

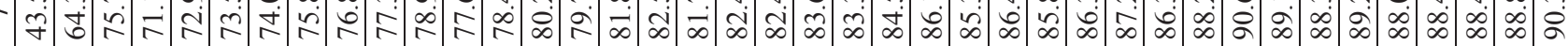

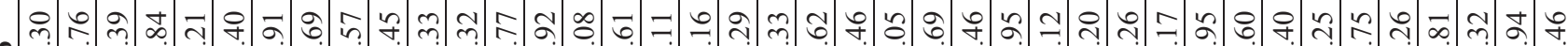

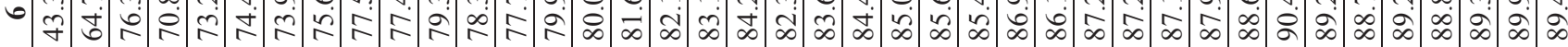

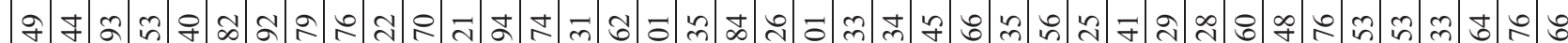

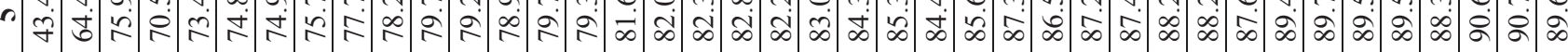

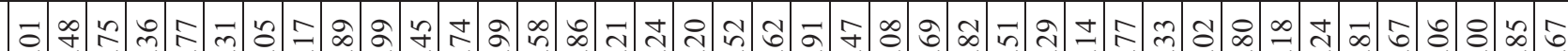

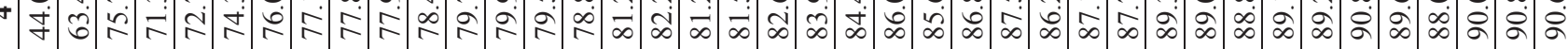

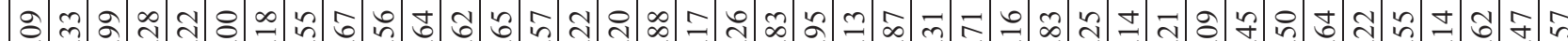
我

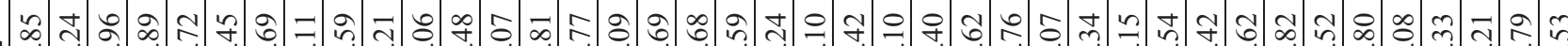
fं

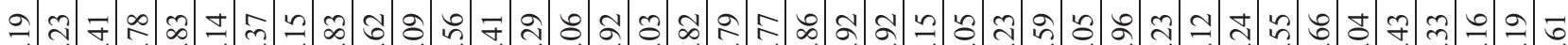
守的它 ச̊ 
ป⿻

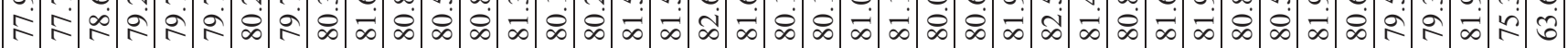

mై

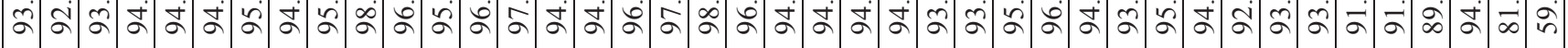

施

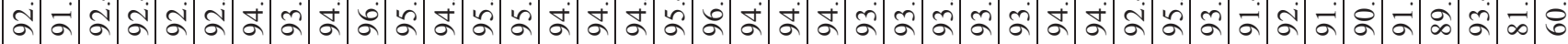

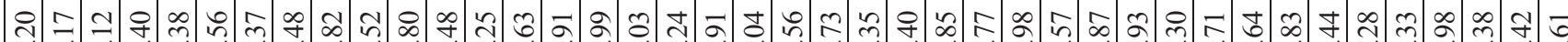

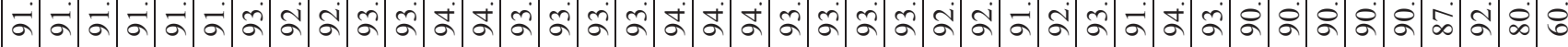

$\Re=\backsim$ m

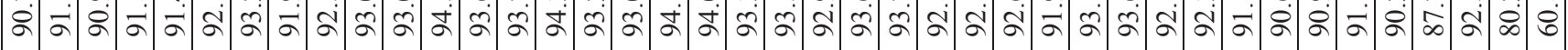

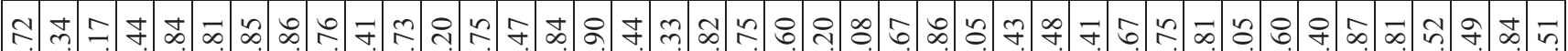

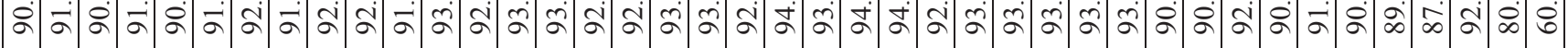

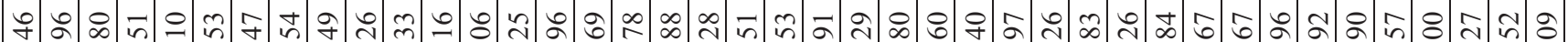

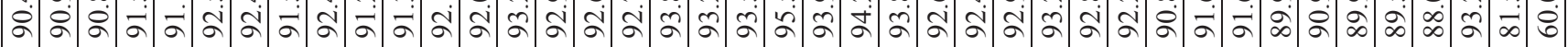

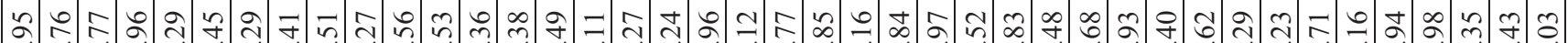

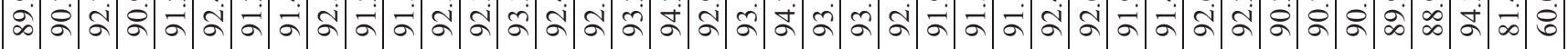

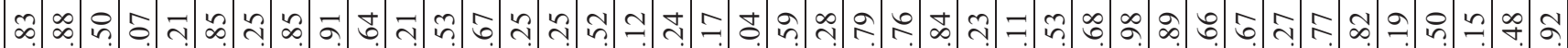

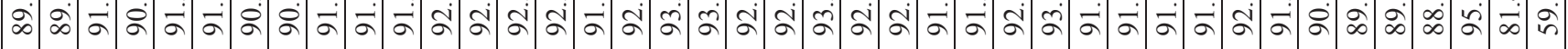

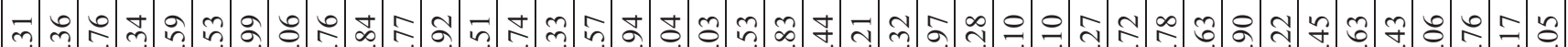

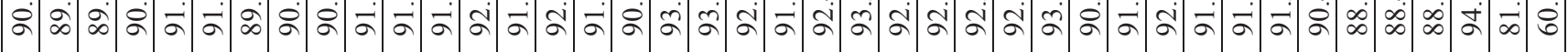

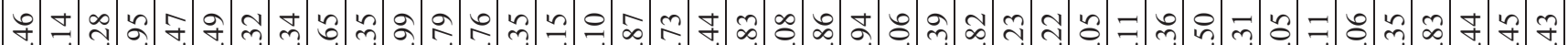

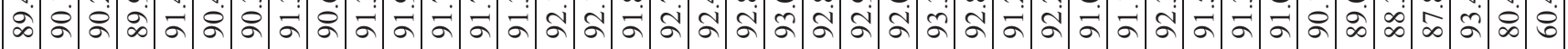

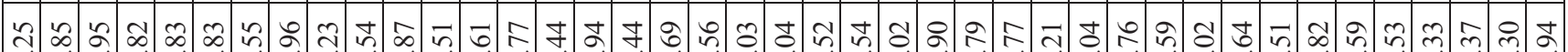

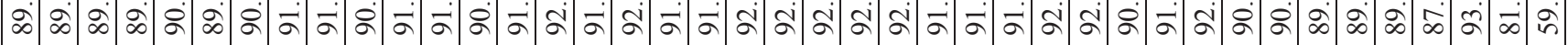

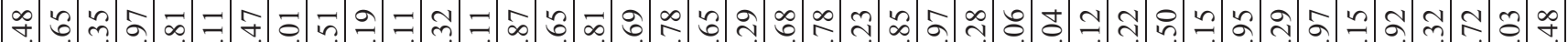

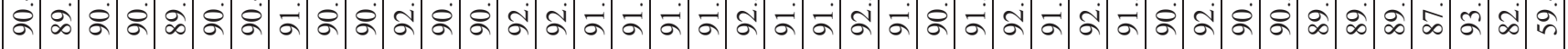

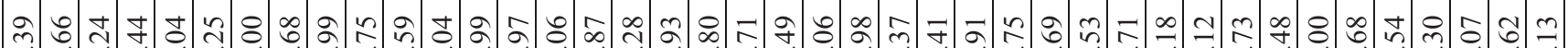

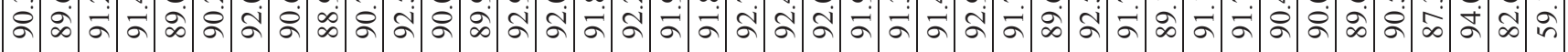

जี

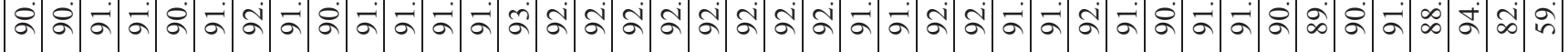

우유.

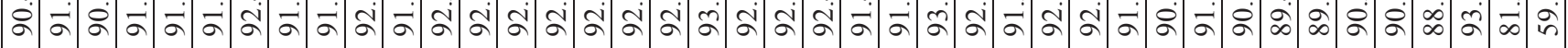

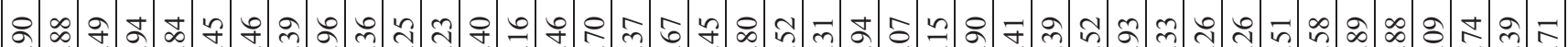

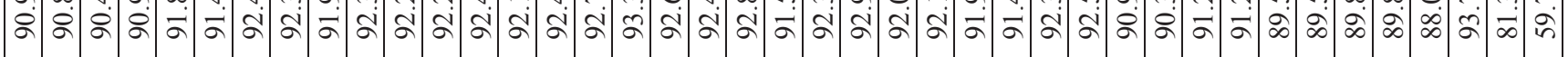

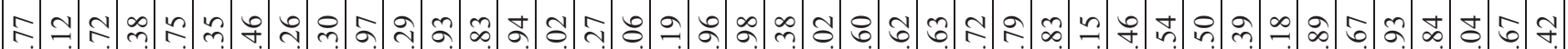

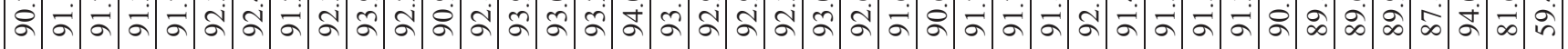

ๆ ช

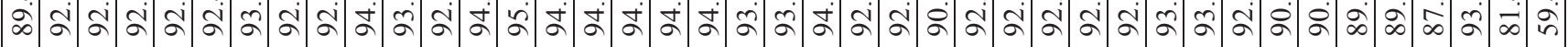

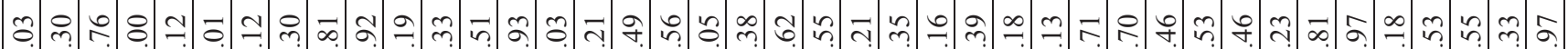

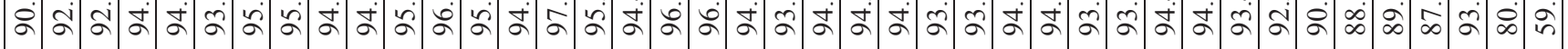

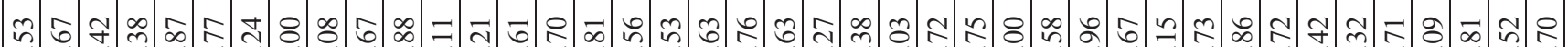

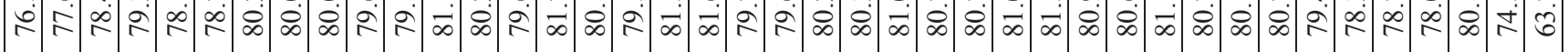


ה

-

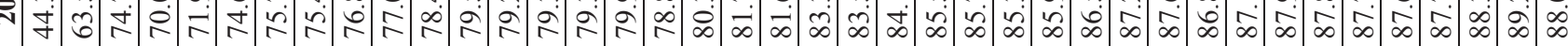

$=$ 势

$\stackrel{\infty}{=}$

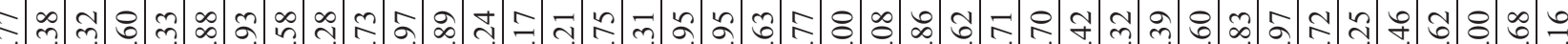
Ұं

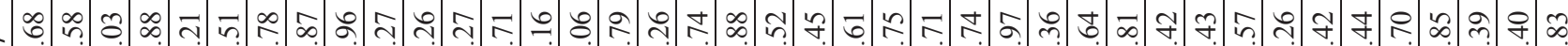
$=$ 过

\& 过

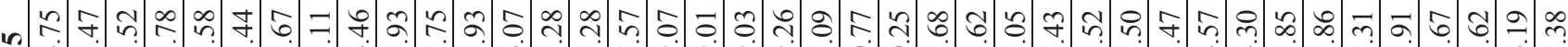
$\because$ a

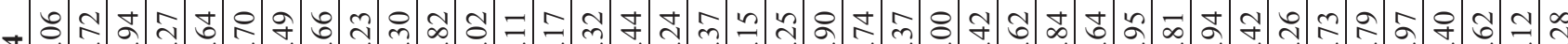

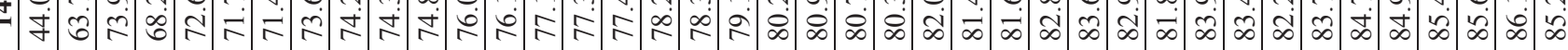

m น $=$ 我

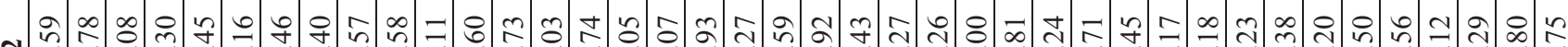

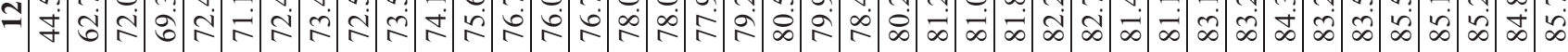

n

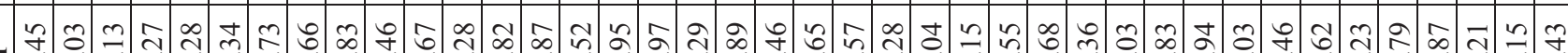

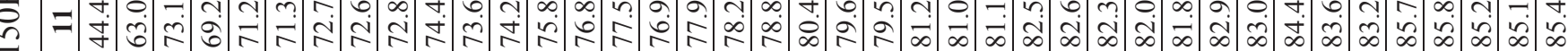

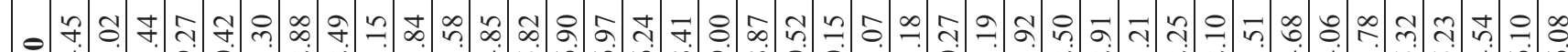
$=$ 过

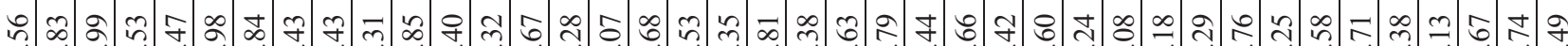

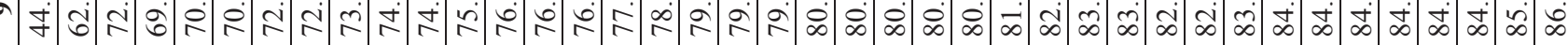

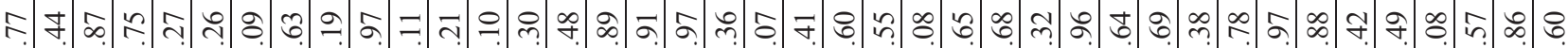

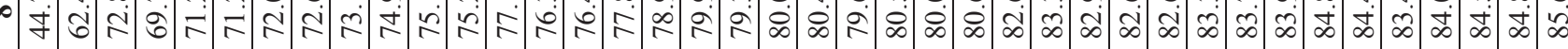

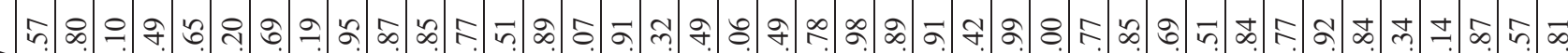

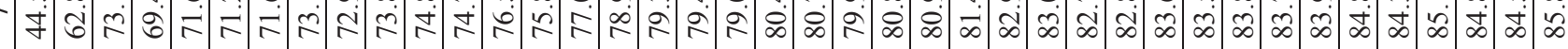

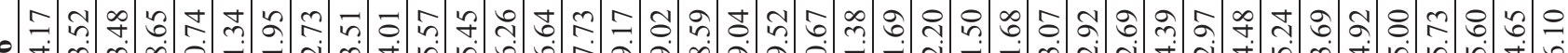

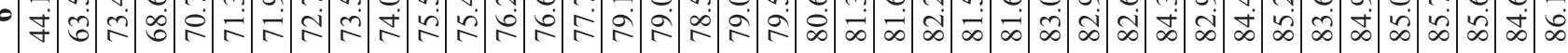

\&

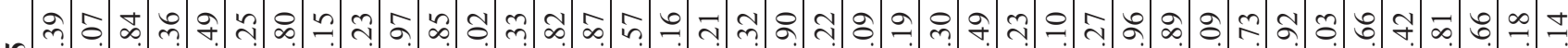

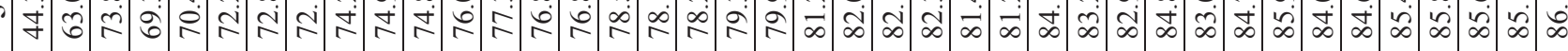
$\Xi$

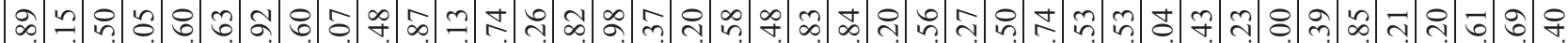

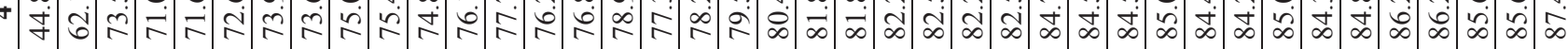

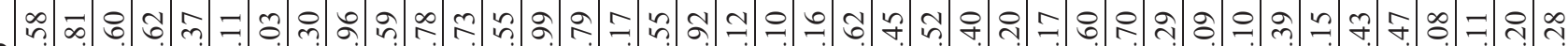

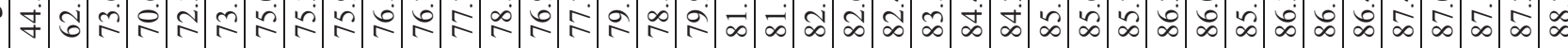

অ

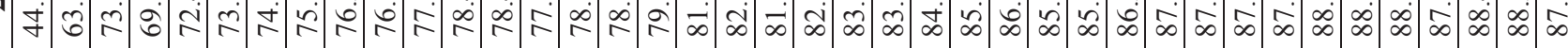

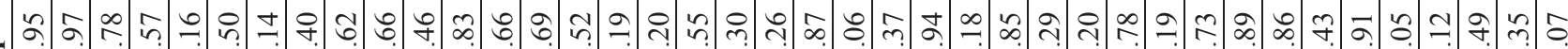
卉 的 車

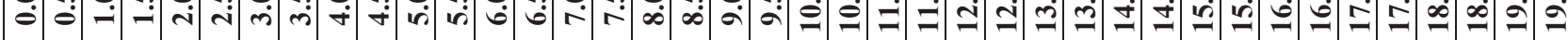


$\infty$ :

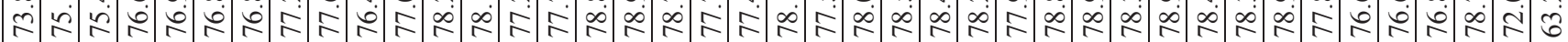

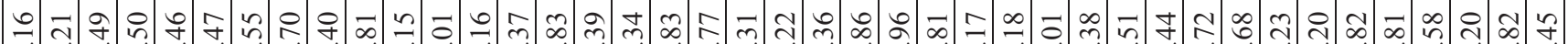

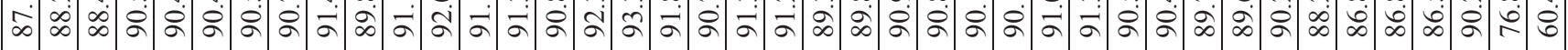

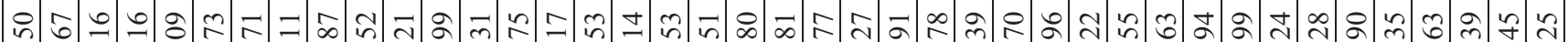

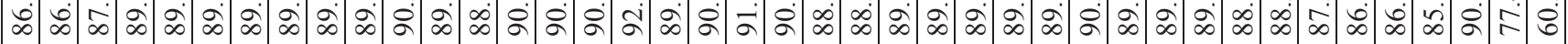

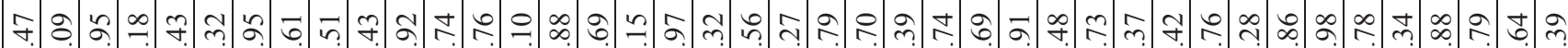

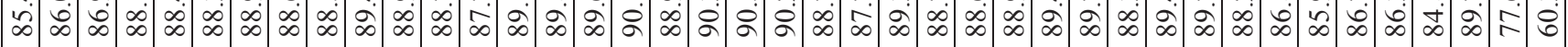

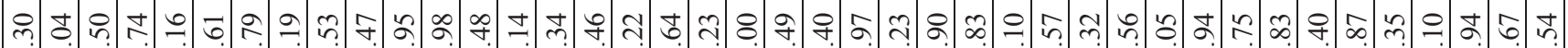

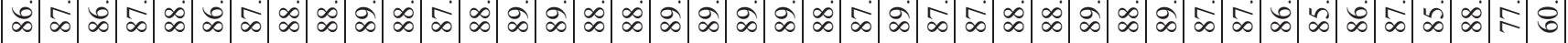

๙

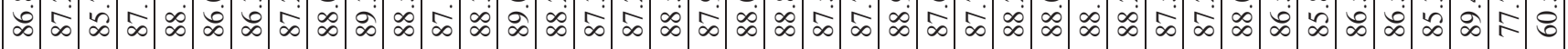

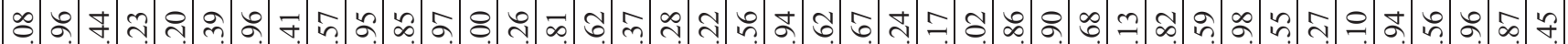

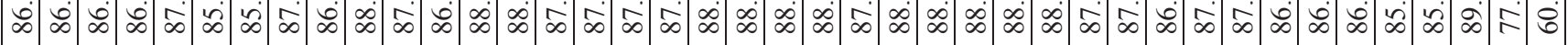

ๆ

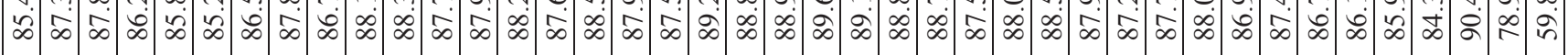

б. ํ.

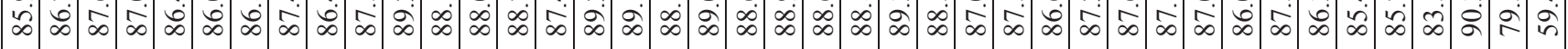

ภ.

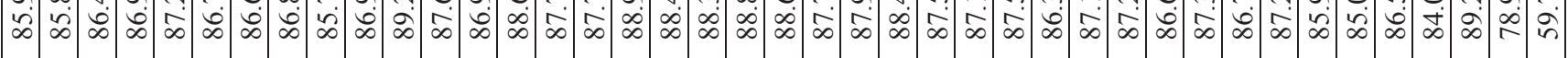

ศ โิ

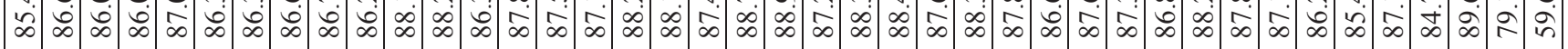

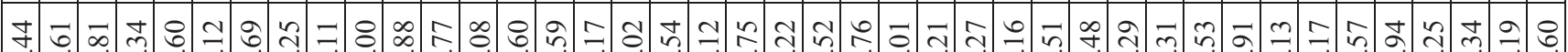

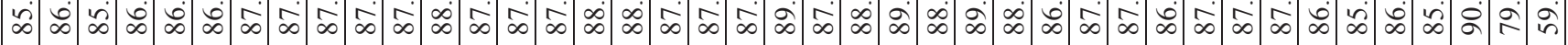

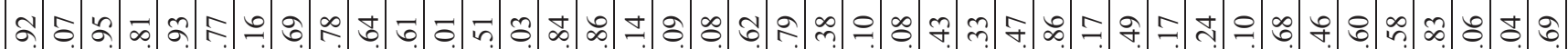

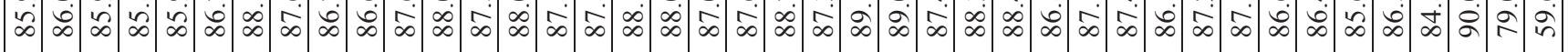

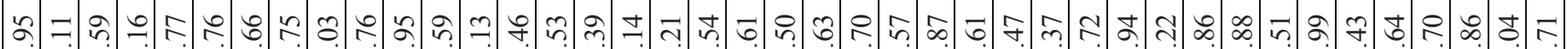

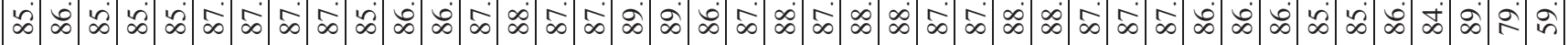

‡:

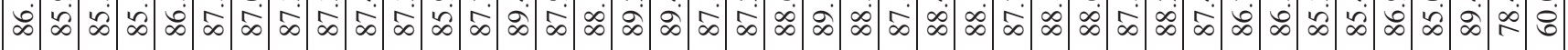

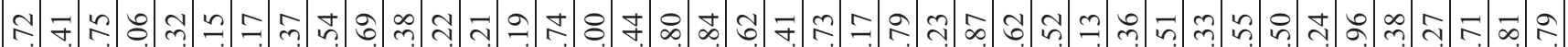

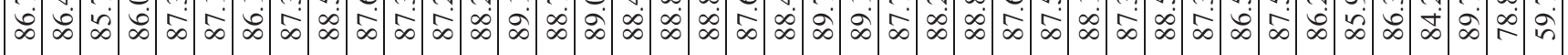

ฉ๘

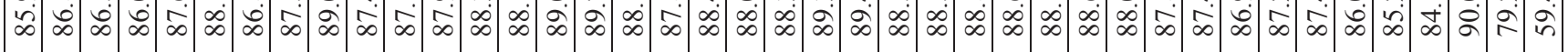

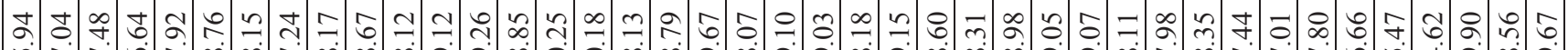

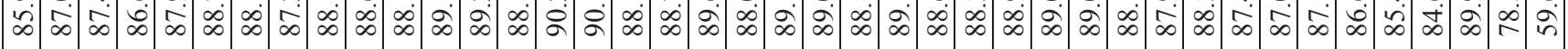

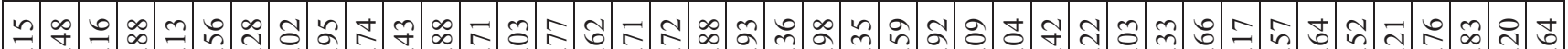

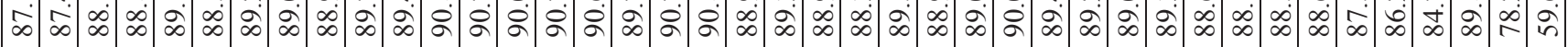

ที

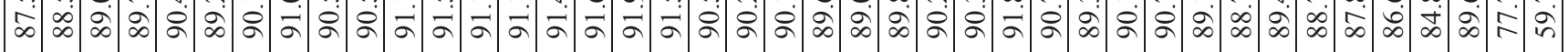

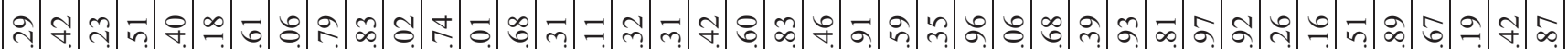

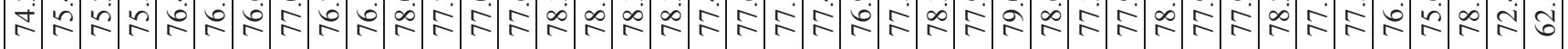
节 
- f ה

ते ๕

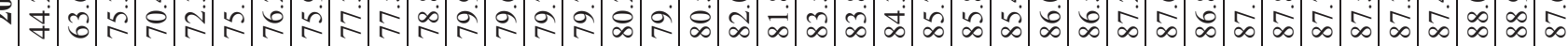

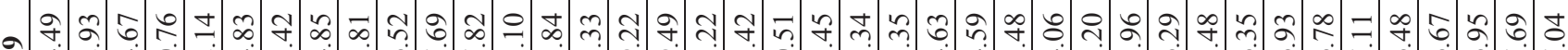

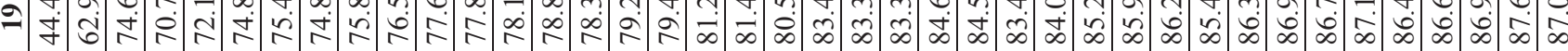

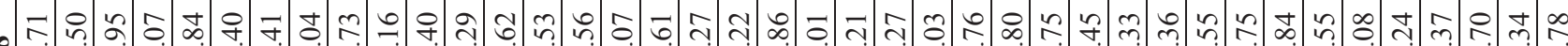

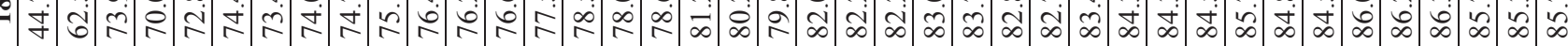

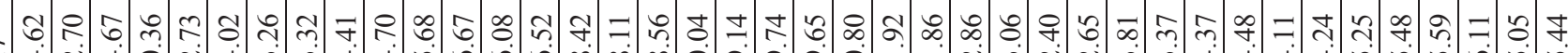

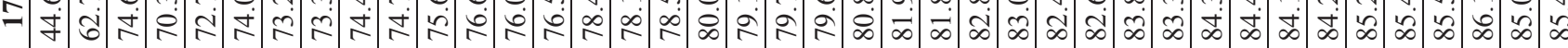

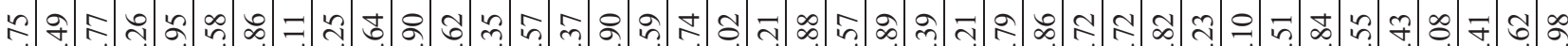

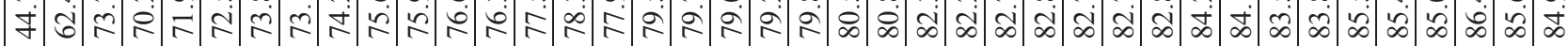

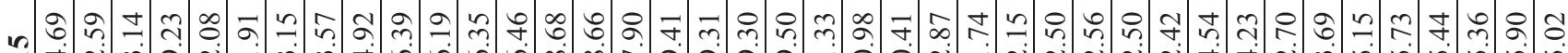

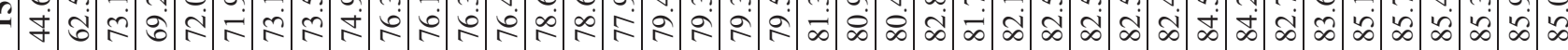

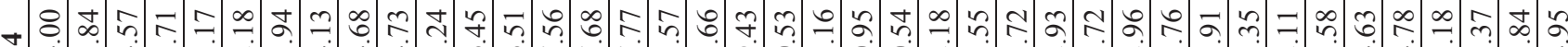

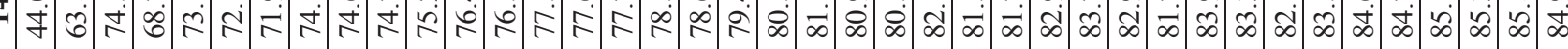

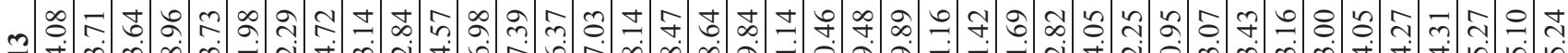

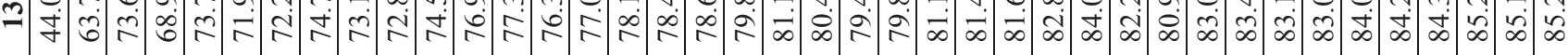

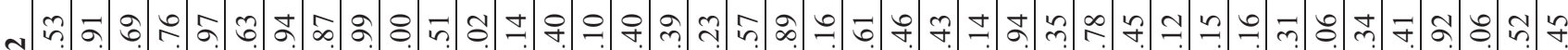

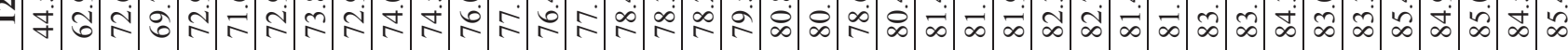

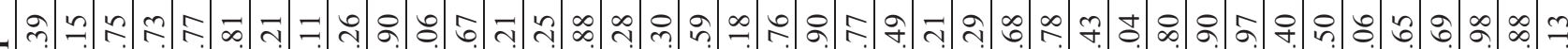

$=$ 过

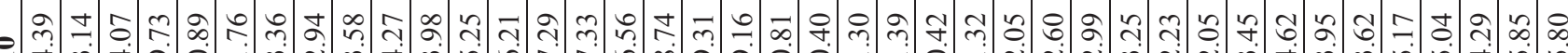

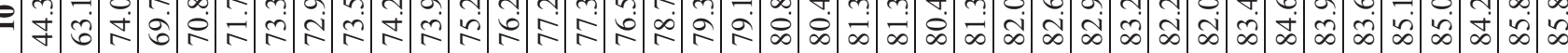

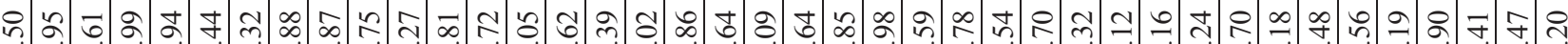

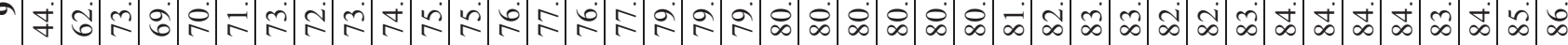

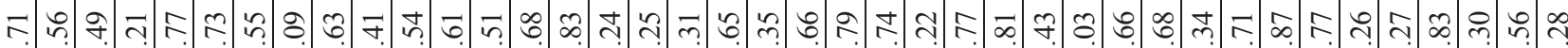

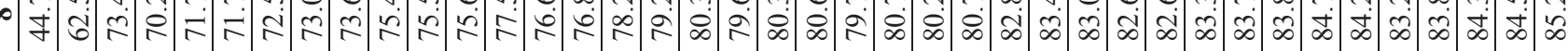

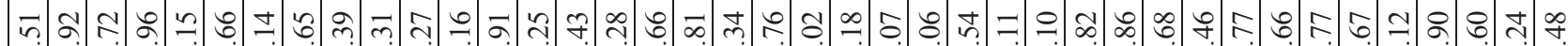
f

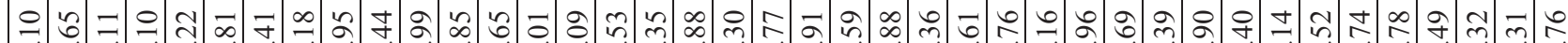

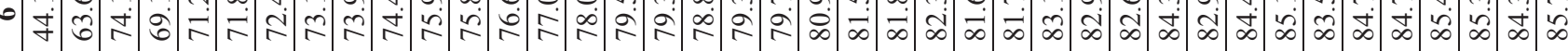

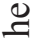

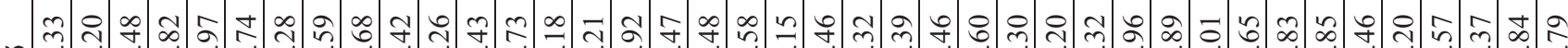

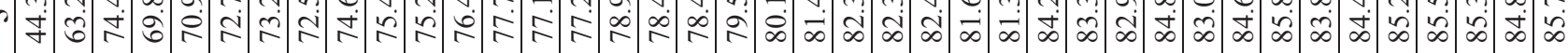

छี

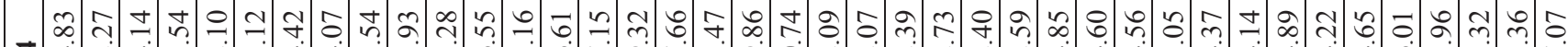

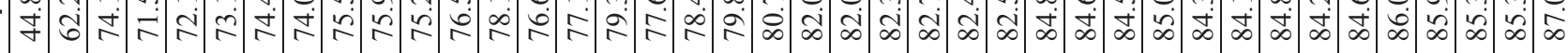

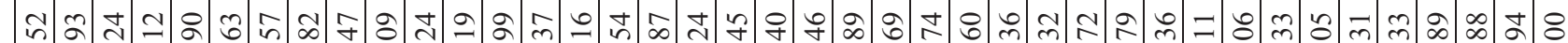
过

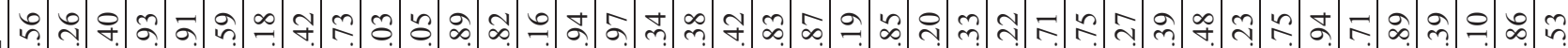

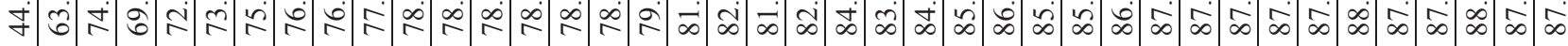

in

- $\delta$ 의

守 向

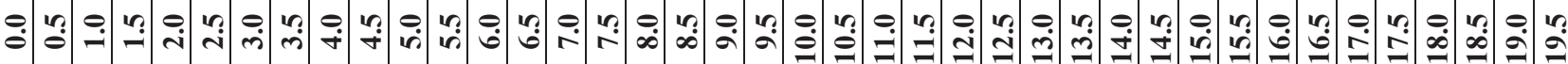




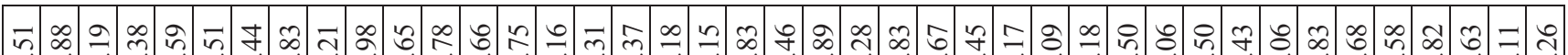

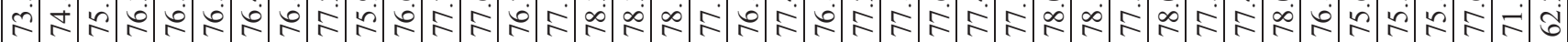

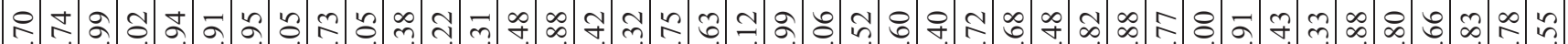

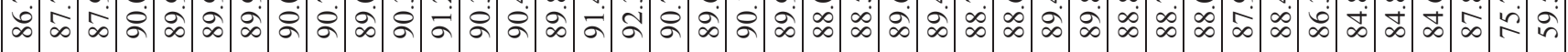

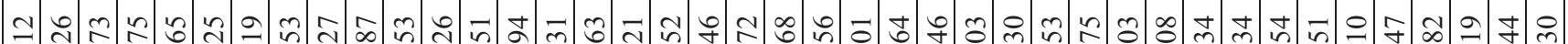

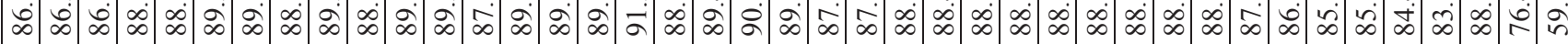

ذt古

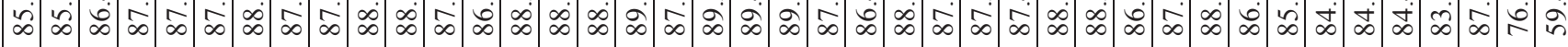

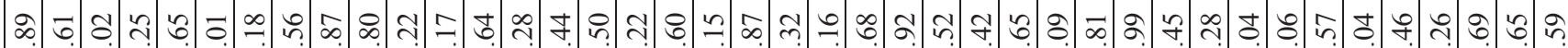

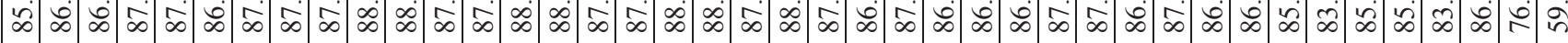

Ұ

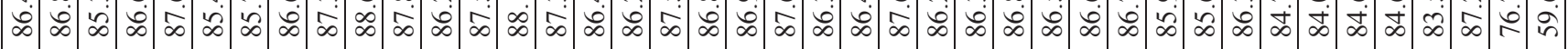

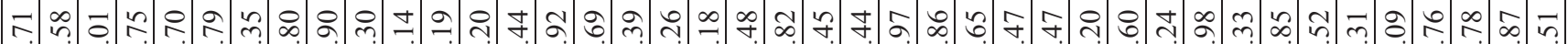

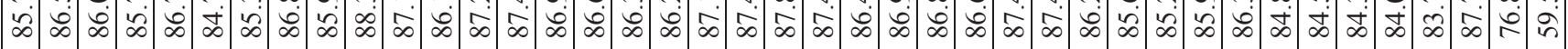

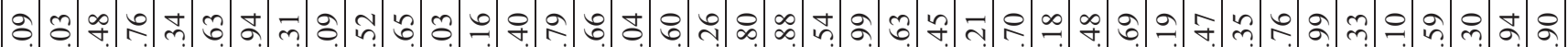

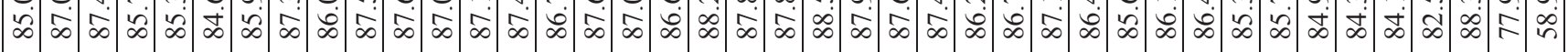

กิ f

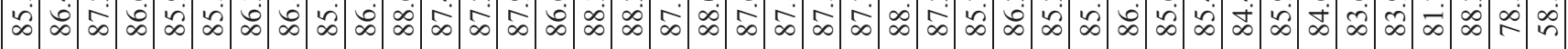

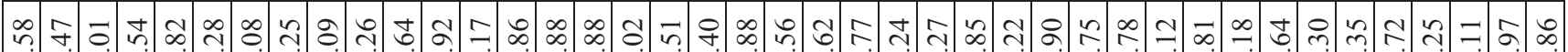

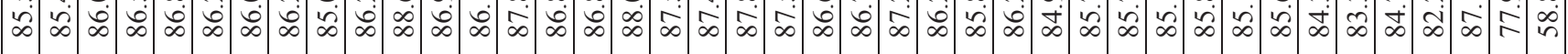

$\exists$ กิ

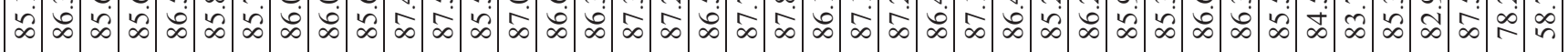

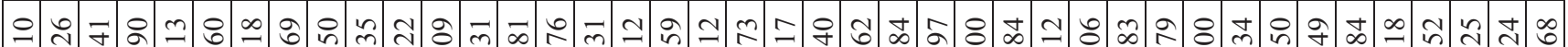

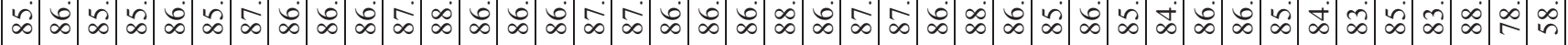

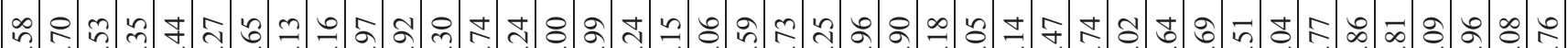

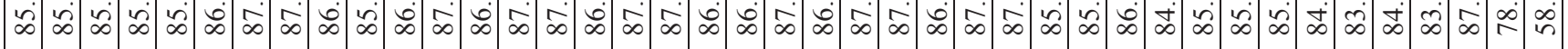

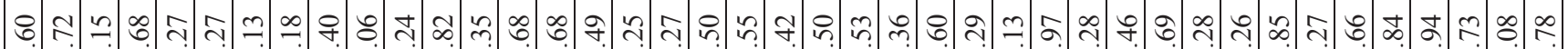

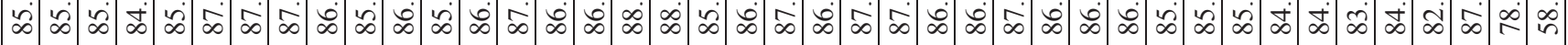

ㅇำ m

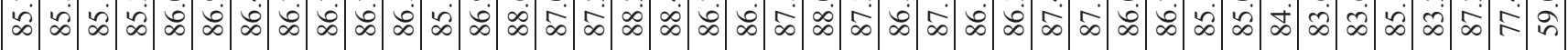

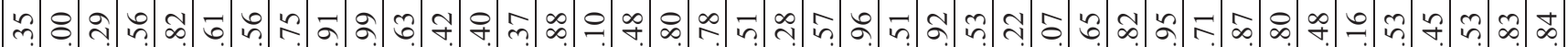

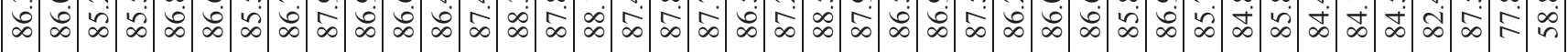

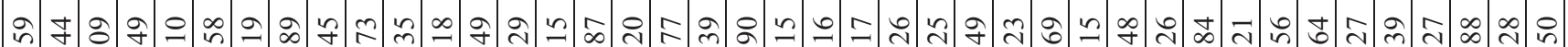

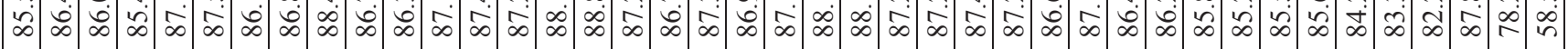

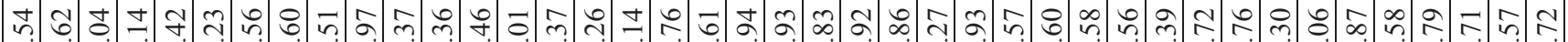

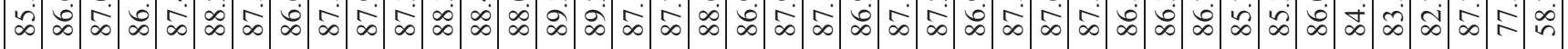

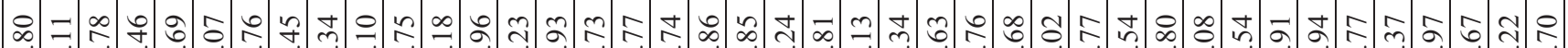

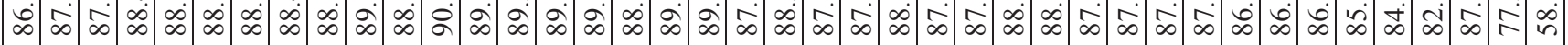

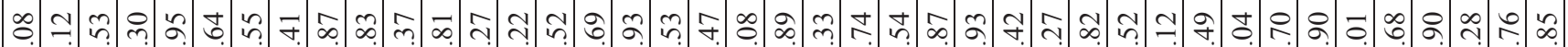

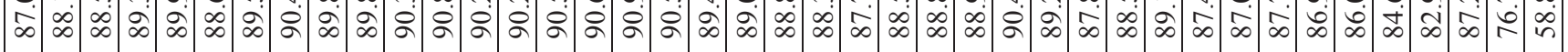
б ص

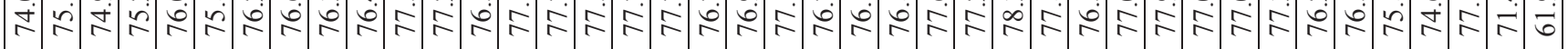
ㄴ. ปิ่ 
- ت

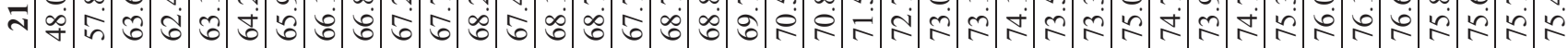

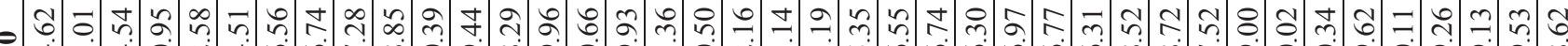

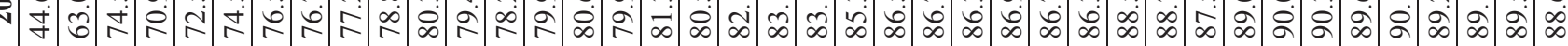

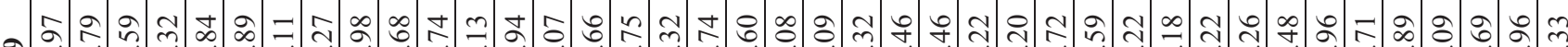

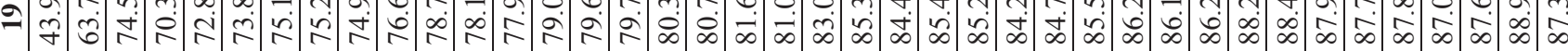

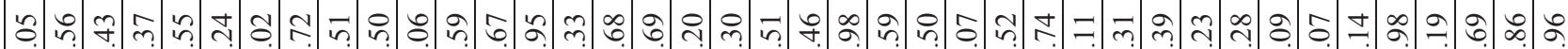

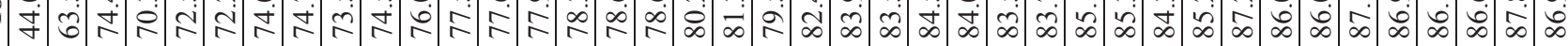

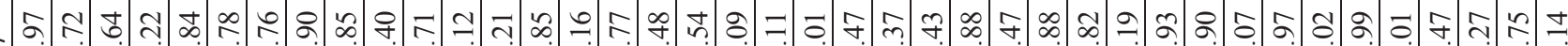

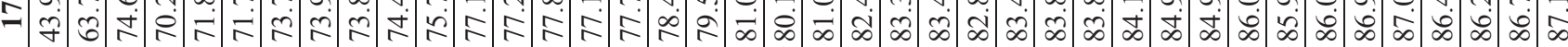

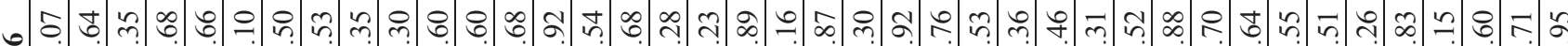

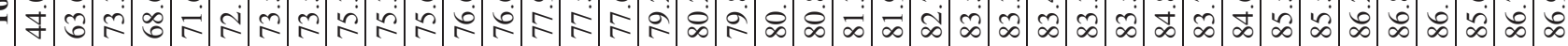

$\because$ กุ

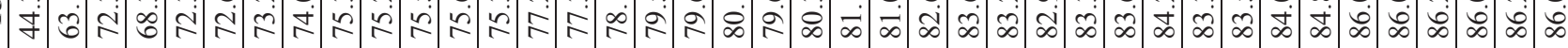

-

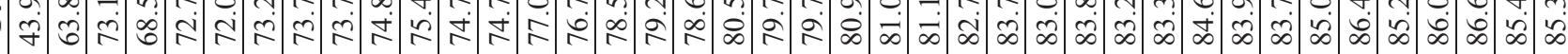

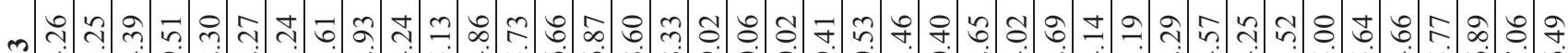

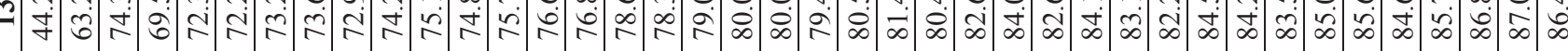

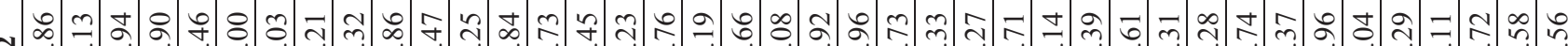

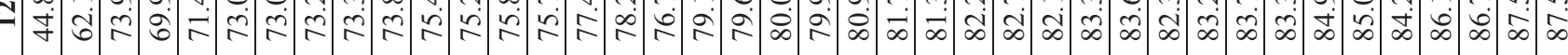

ก ก

$=$ च

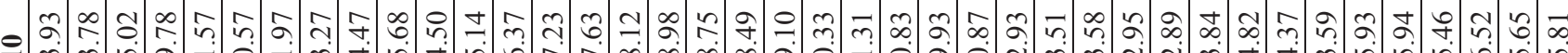
字家

๙ิ

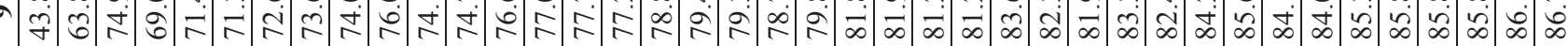

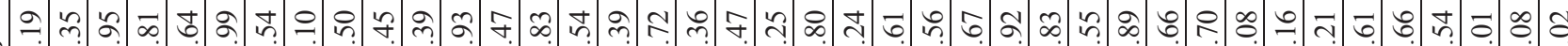

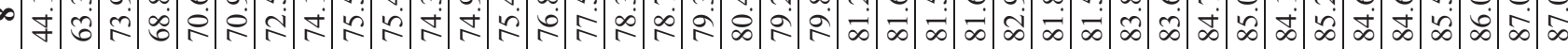

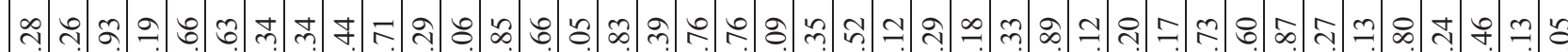

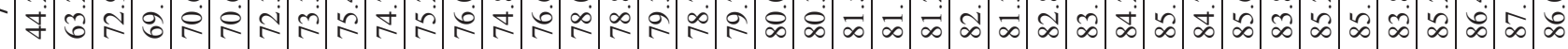

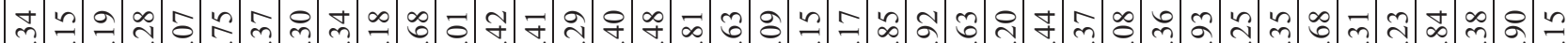

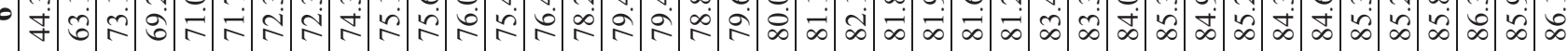
E

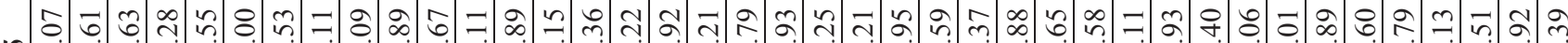
过

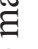

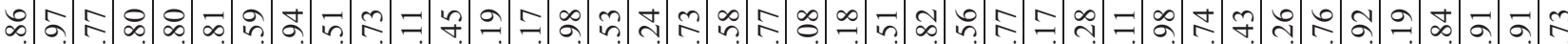

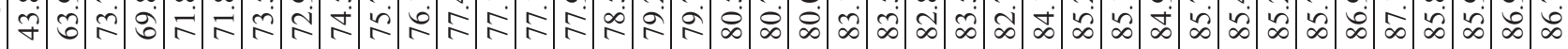

ชర

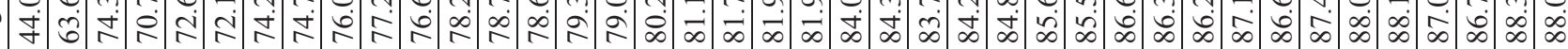

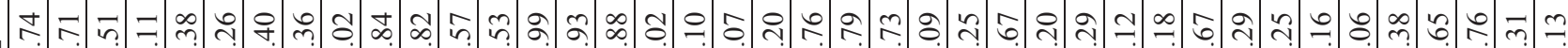

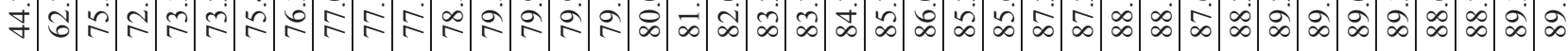
$\ddot{n}$

$\frac{1}{0}$ ช 守 的

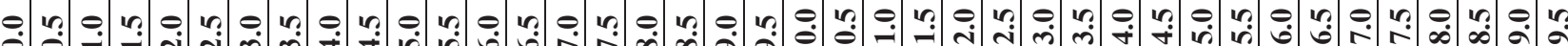

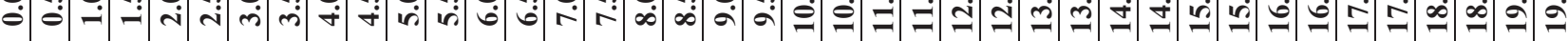


ฮร) ร.

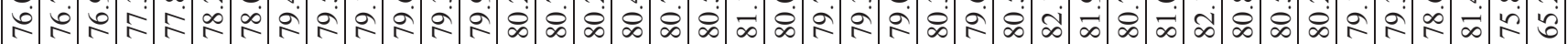

กิ

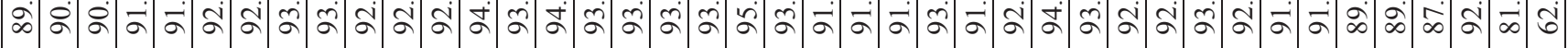

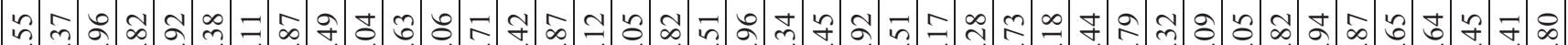

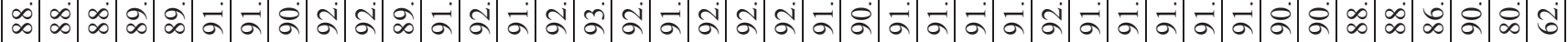

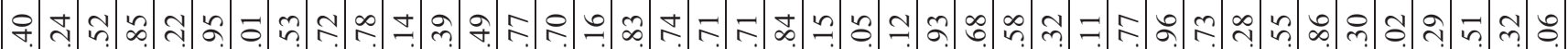

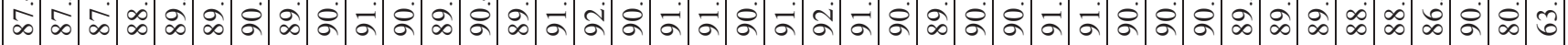

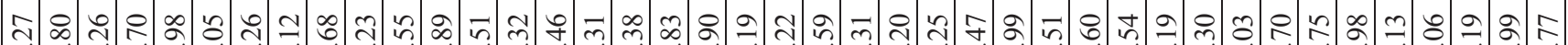

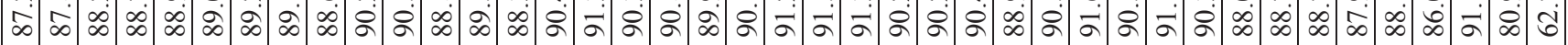

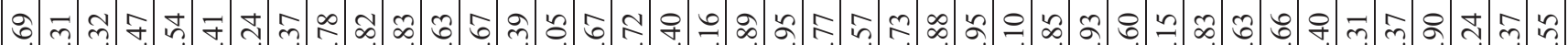

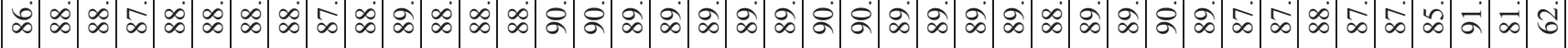

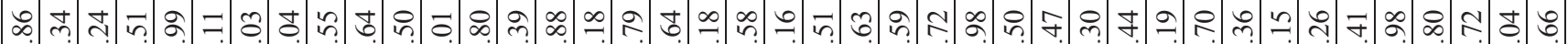

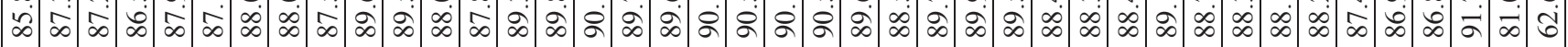

ว

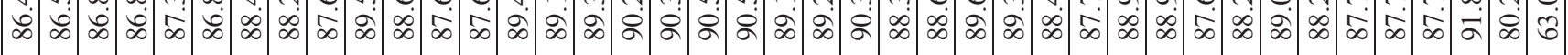

子

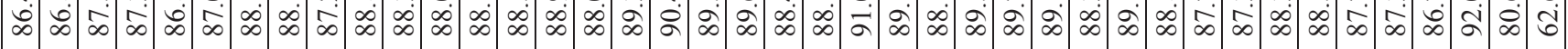

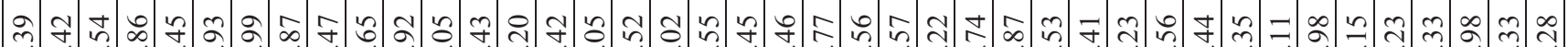

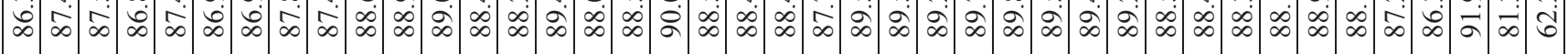

ఫ

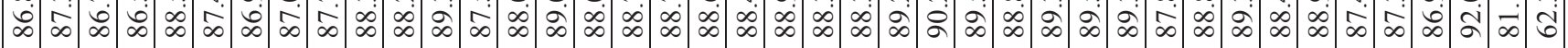

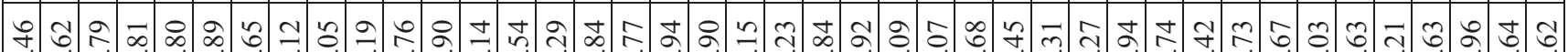

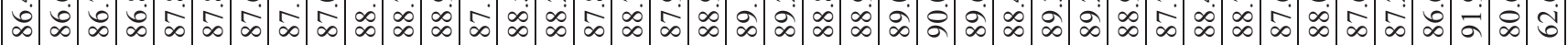

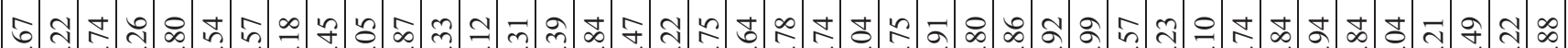

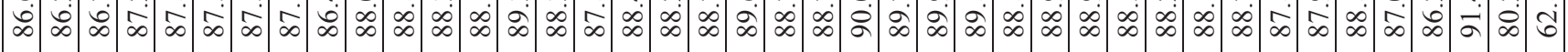

तุ

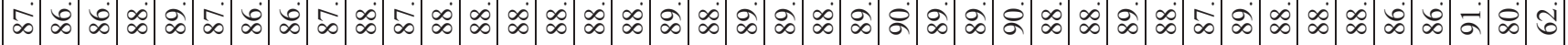
我

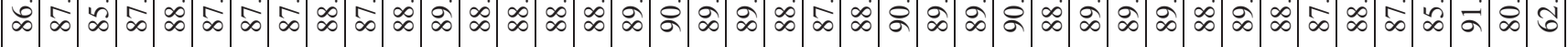

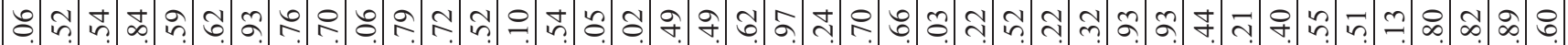

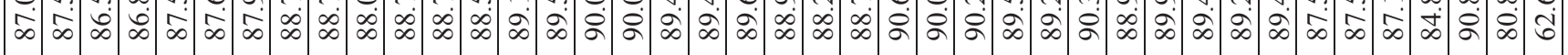

qٔ

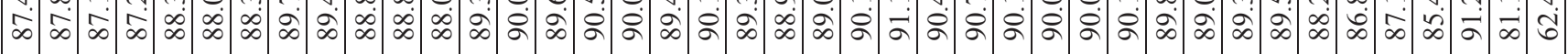

ธุ

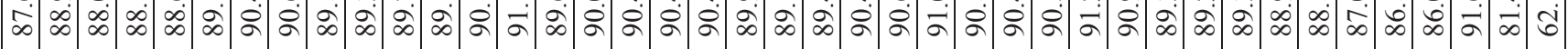

ㄱำ

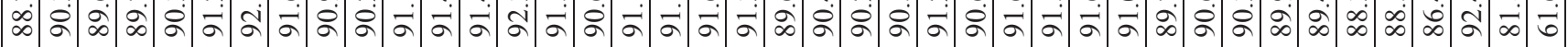

ทิ่

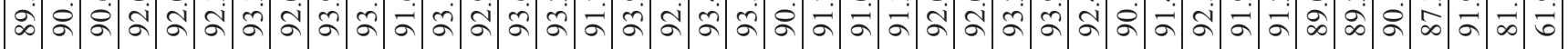

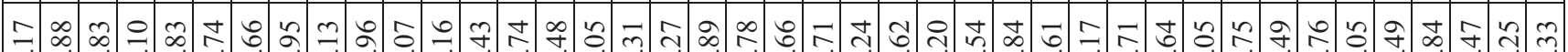

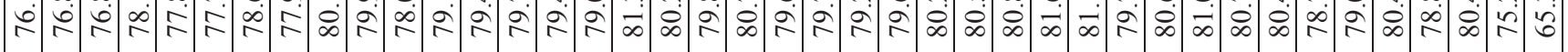
ثิ山 
- Б. ন

고

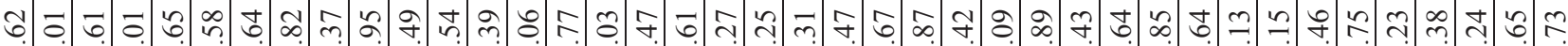

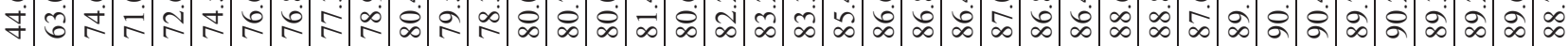

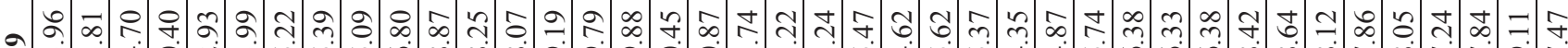

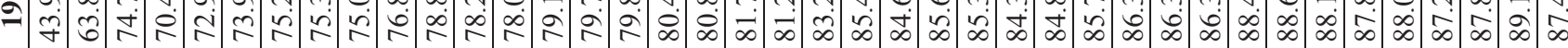

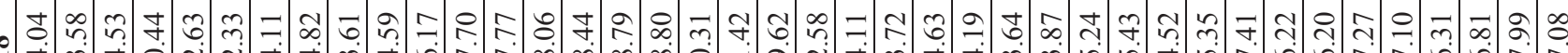

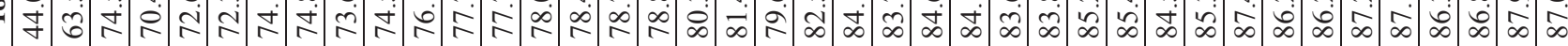

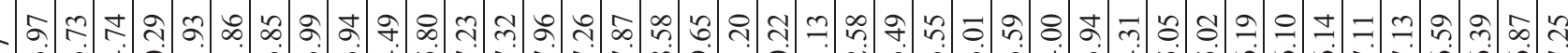

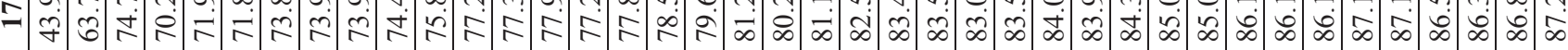

-

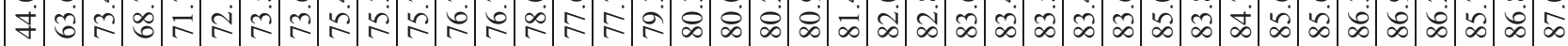

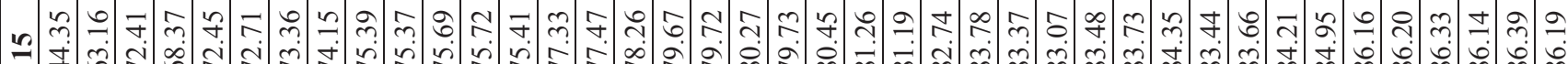

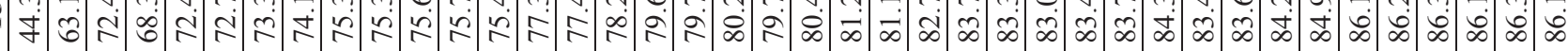

+

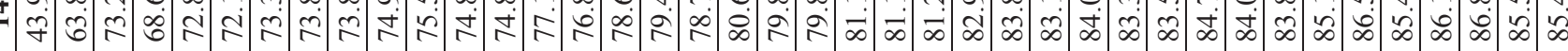

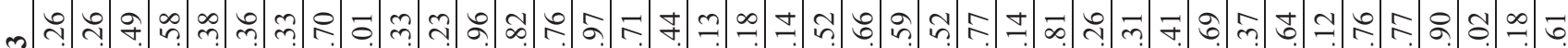

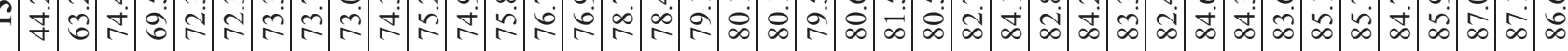

ح $\exists$ 舟

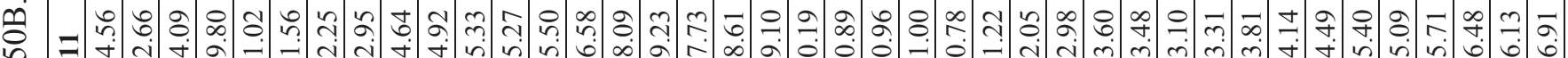

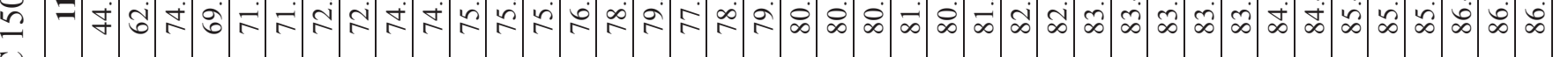
○

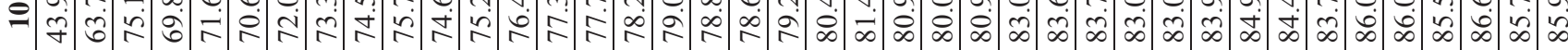

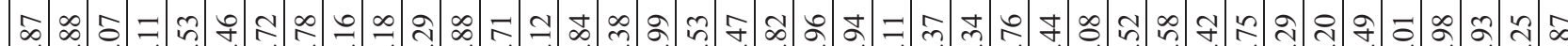

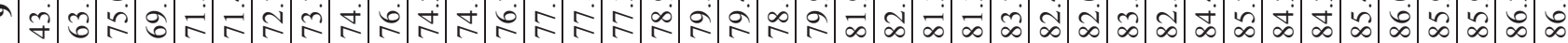
$\infty$ ก 莳

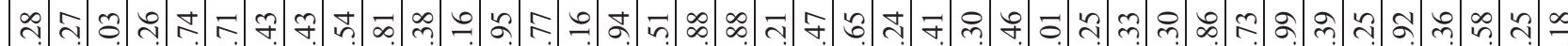
-

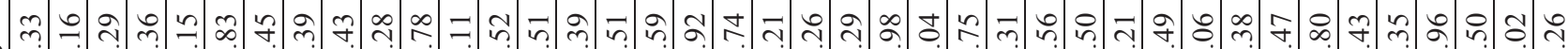

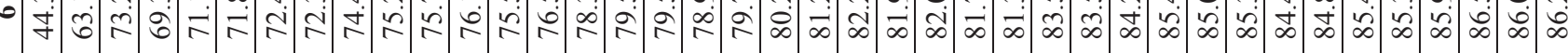
\&

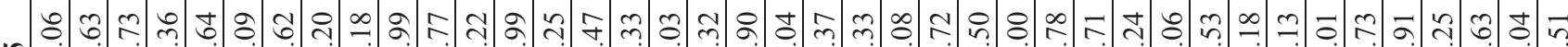

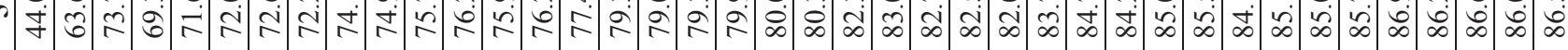

छี

- $\infty$. 守

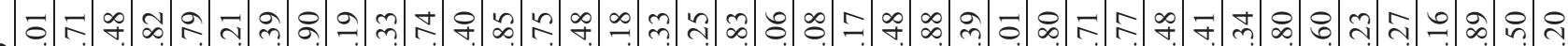

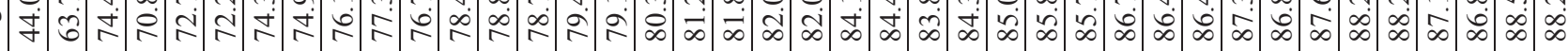

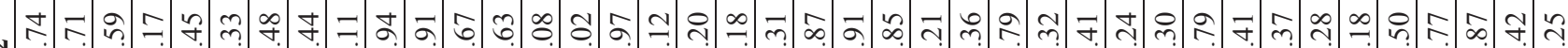

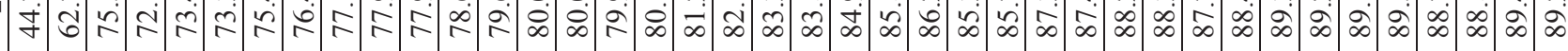

ڤั 
จ

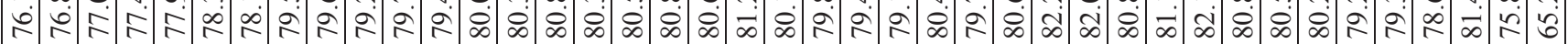

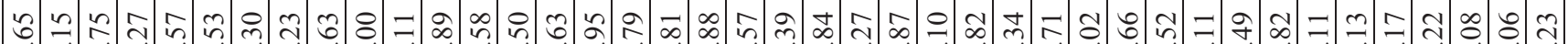

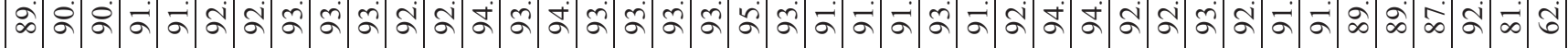

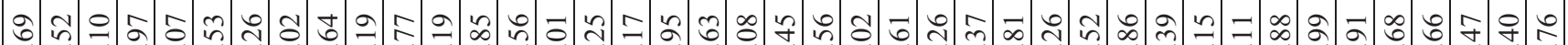

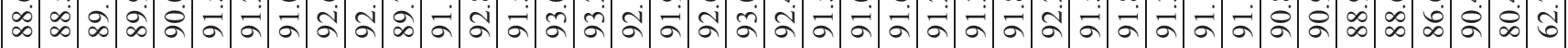

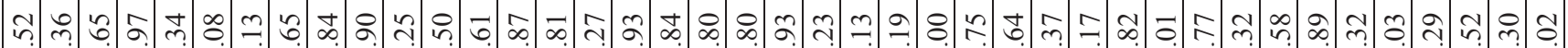
टे

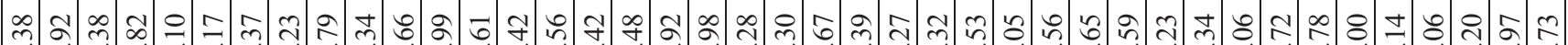

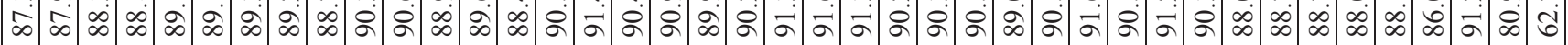

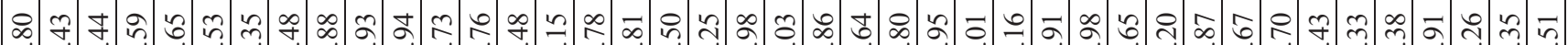

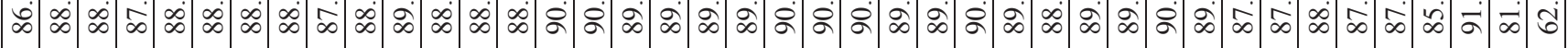

\& f

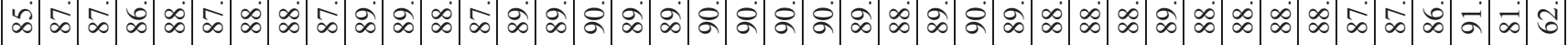

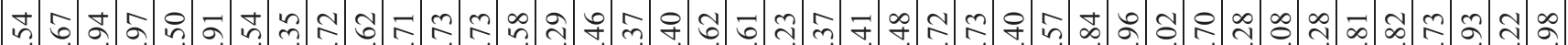

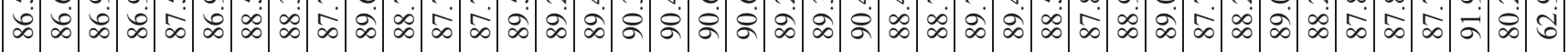

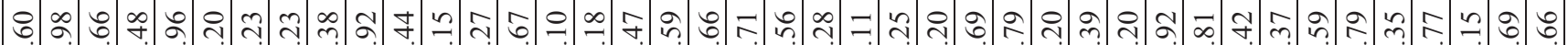

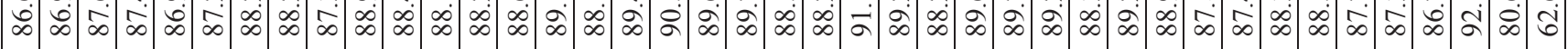

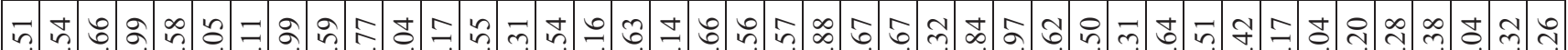

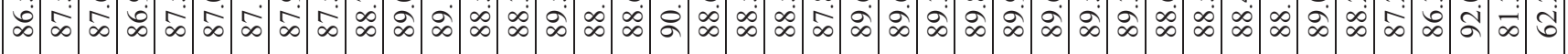

\&ำ

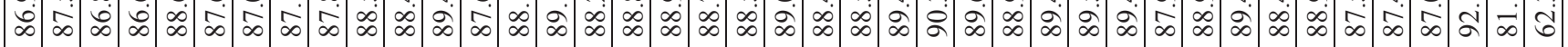

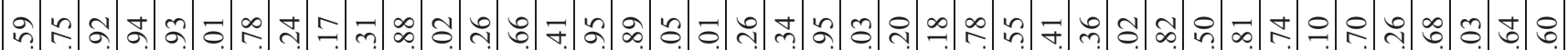

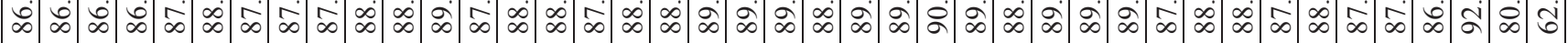

○

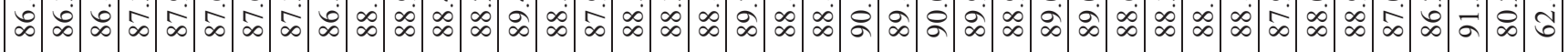

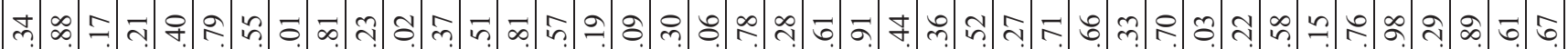

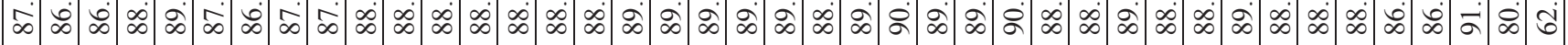
\&

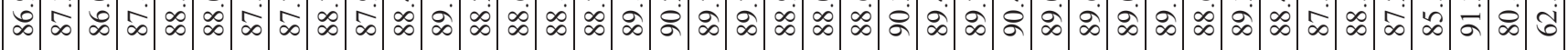

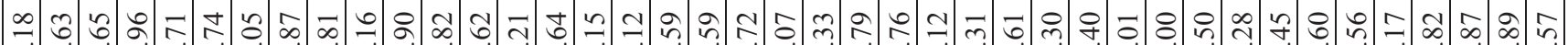

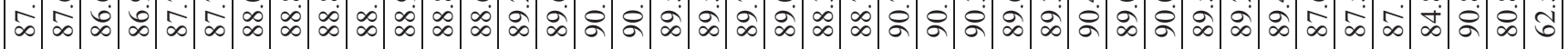

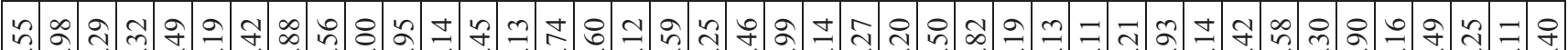

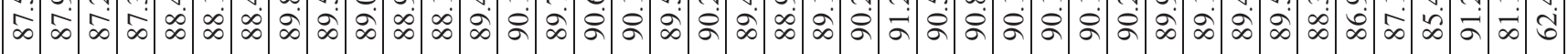

m $\infty$ สิ

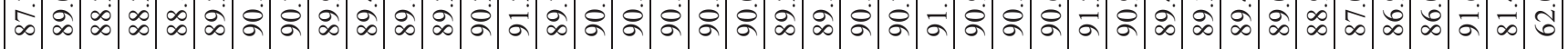

$\infty$ 乎

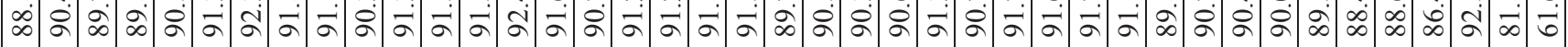

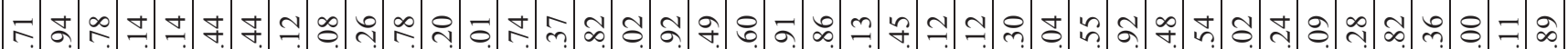

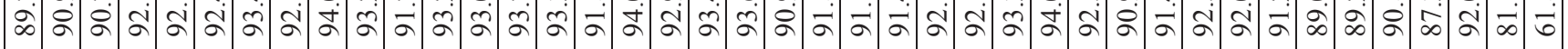

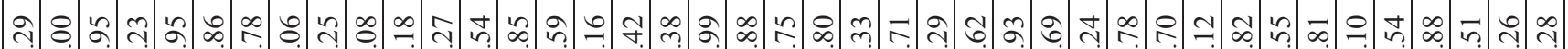

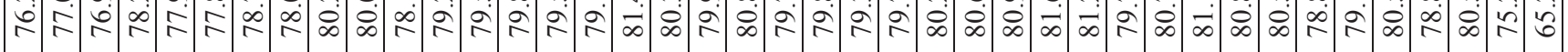

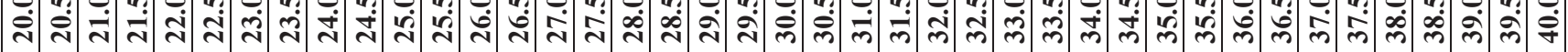




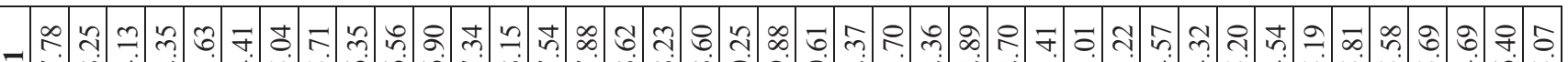
च

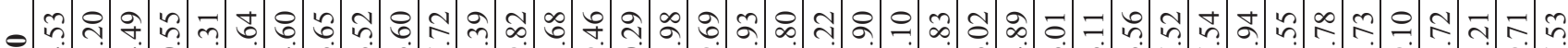

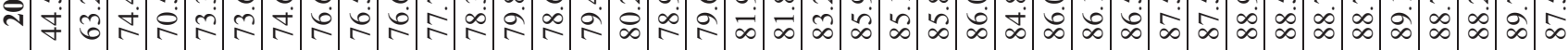

๑ ᄀน

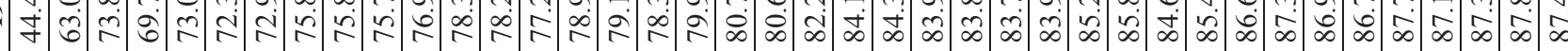

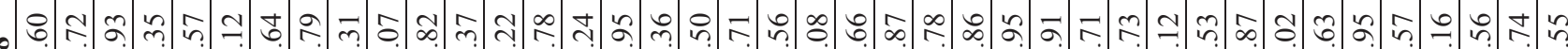

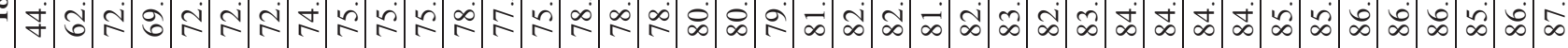
J t) ำ

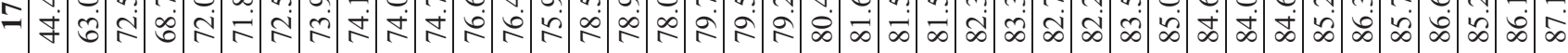

- †่

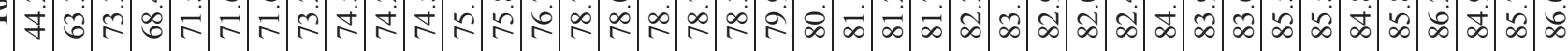

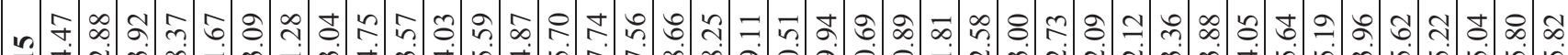

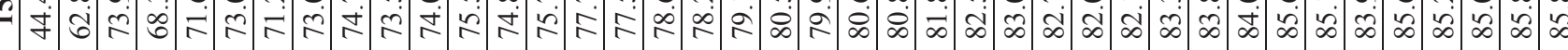

-

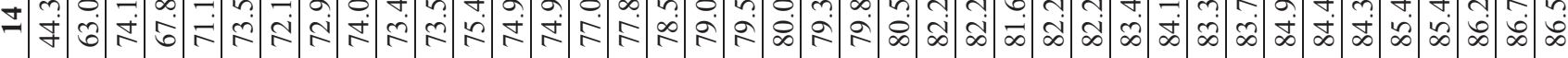

๑

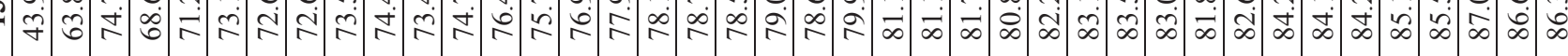

4

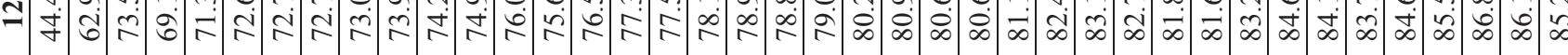

n

- రิ

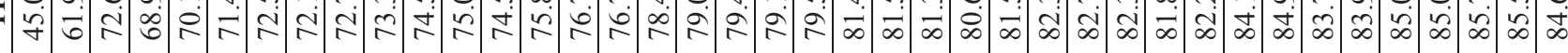
ప స 구

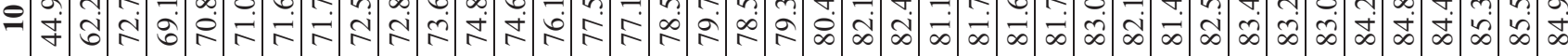

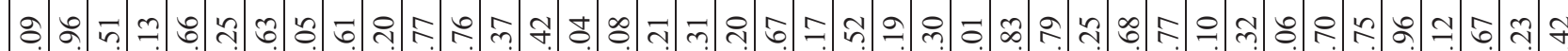

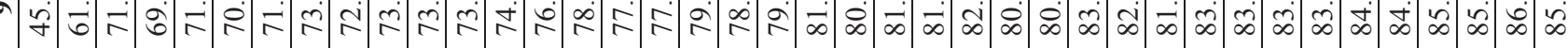

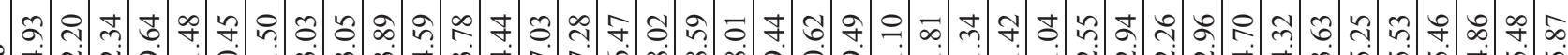

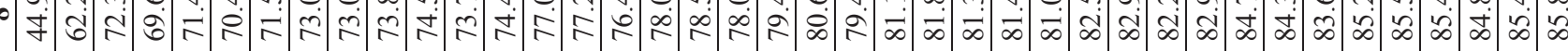

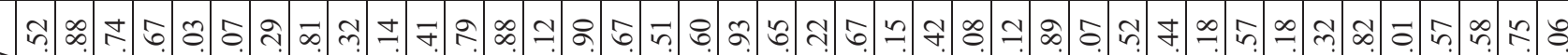

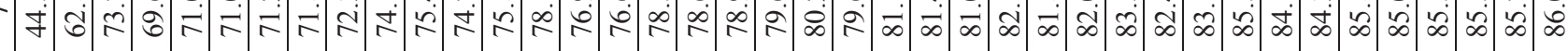

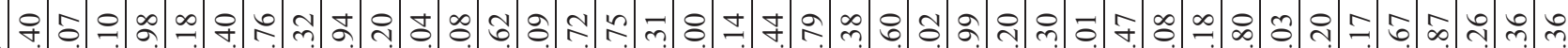

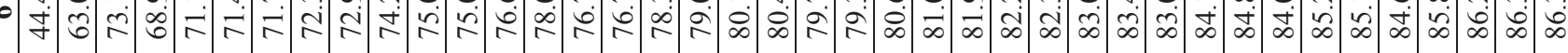

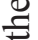

ำ

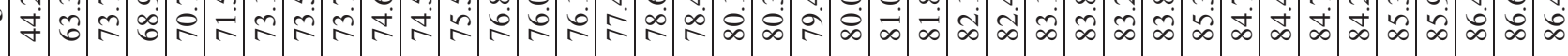

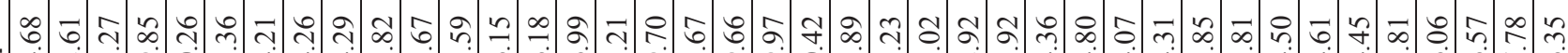

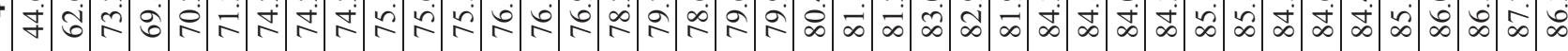

ๆ

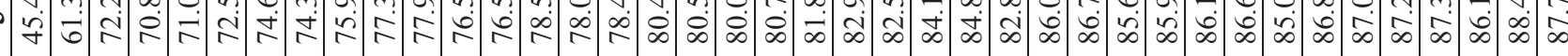
苞 দ̆

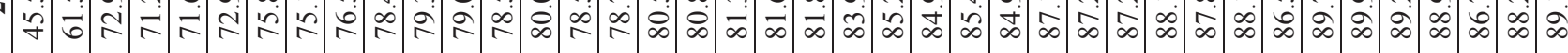
$-\exists$ ప

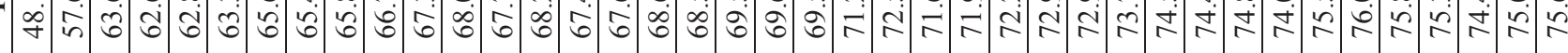

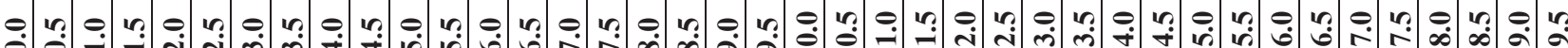

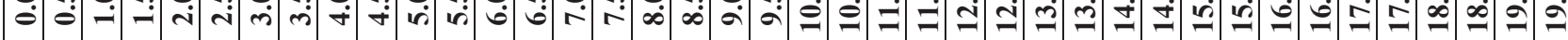




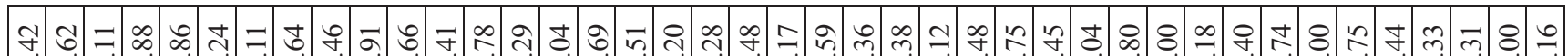

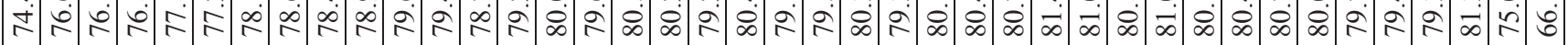

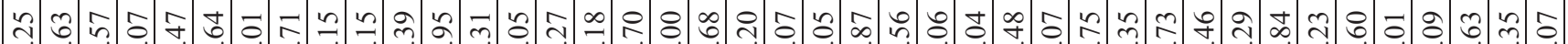

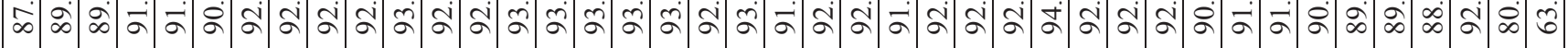

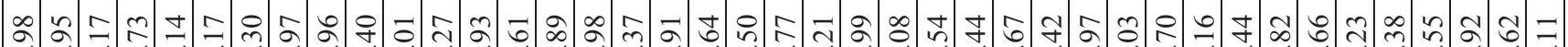
ळ

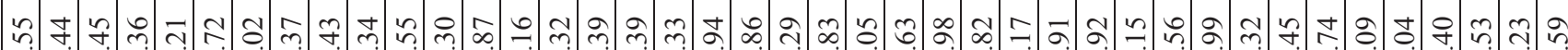

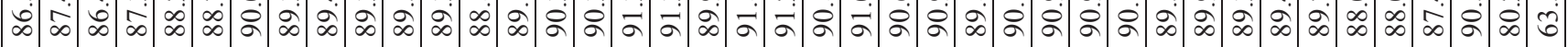

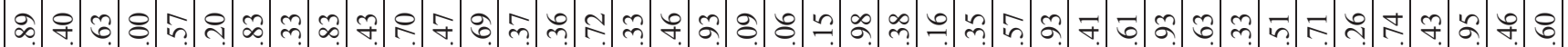

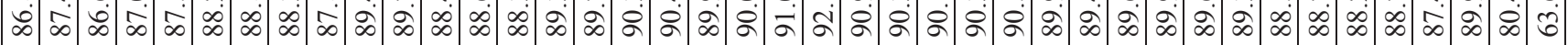

ஓे

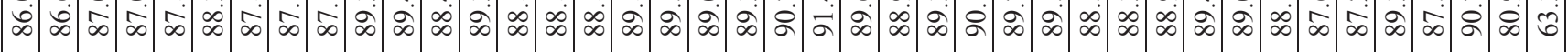

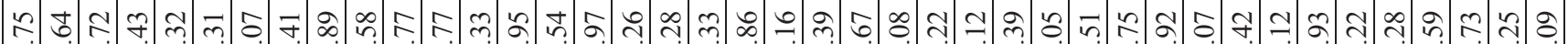

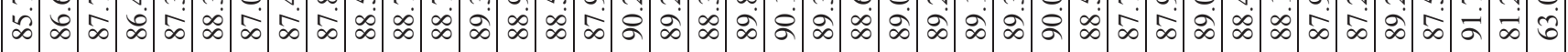

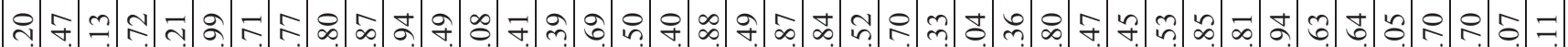

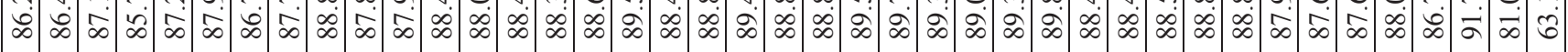

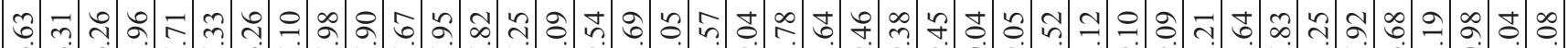

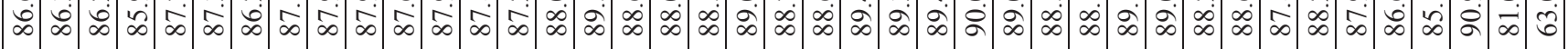

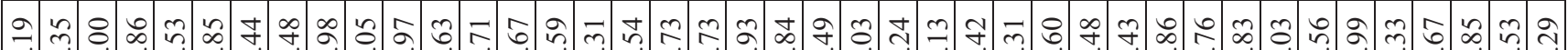

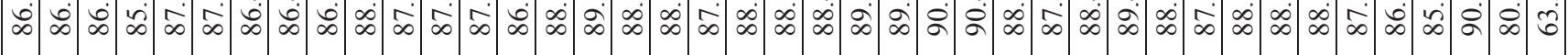

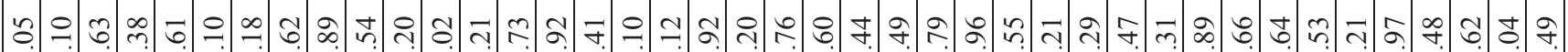

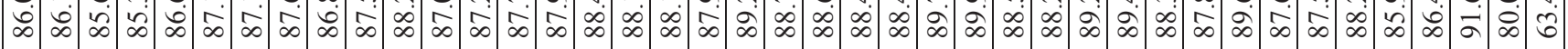

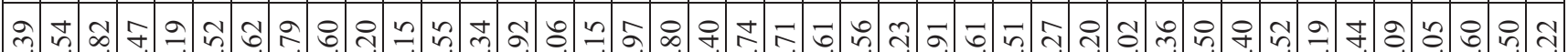

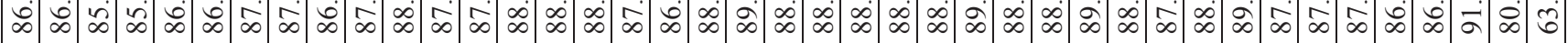

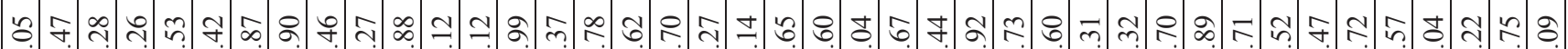

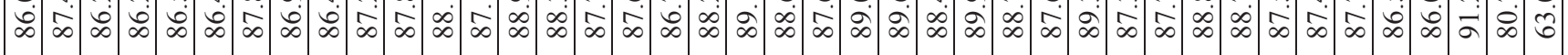

子น

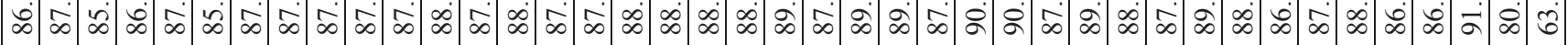

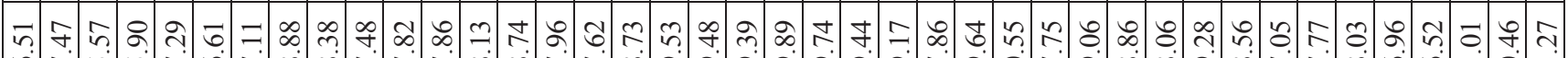

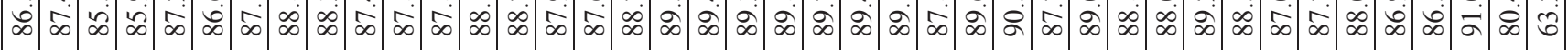

ปุ กิ

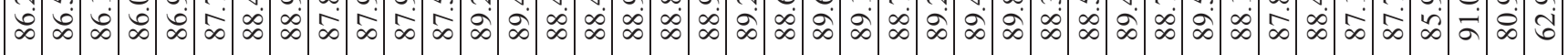

片

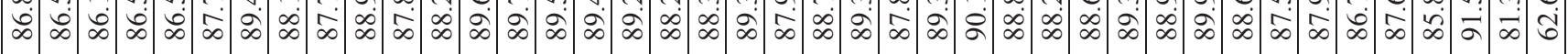

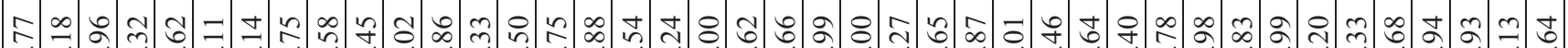

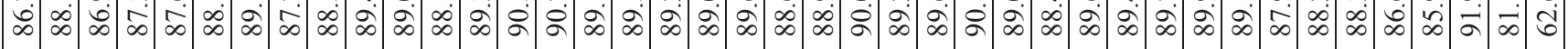

๖ น

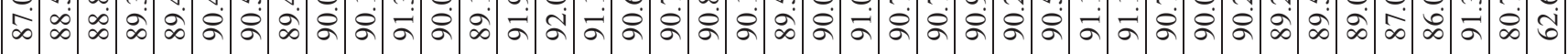

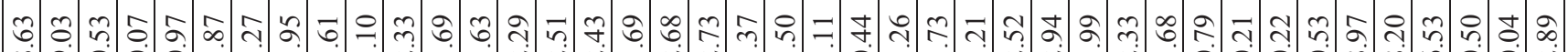

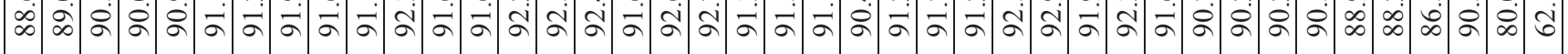

๑)

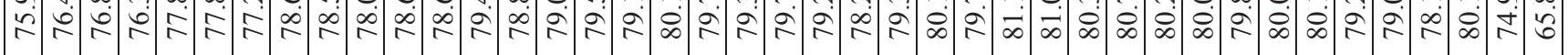




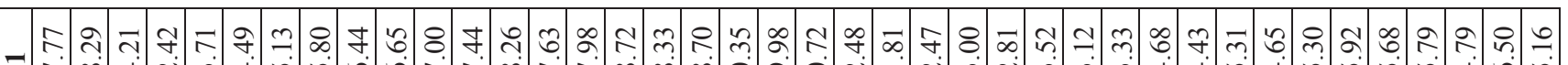
ন

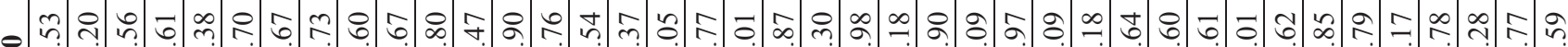

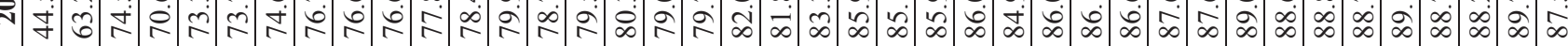

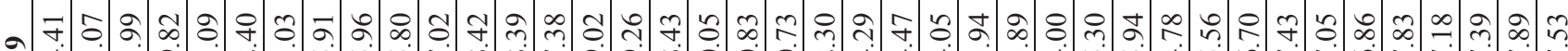

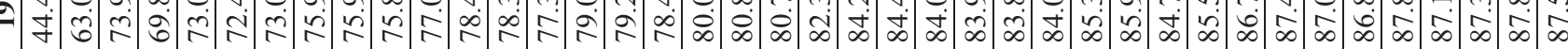

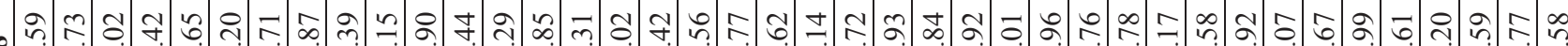

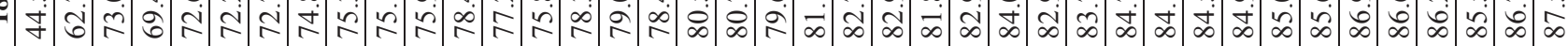

ㄱำ $=$ च

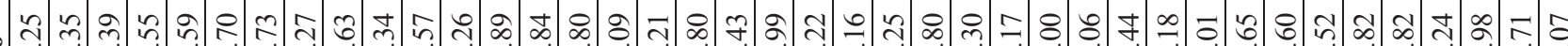

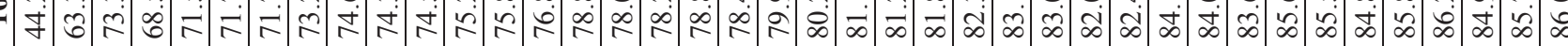

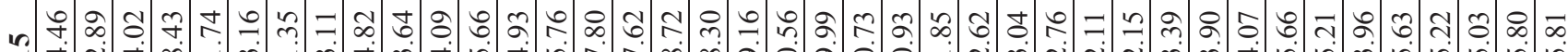

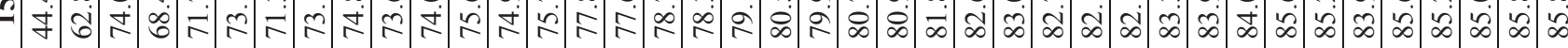

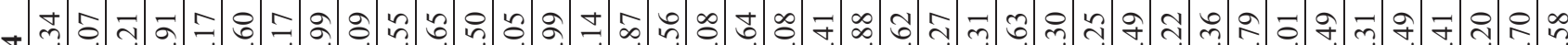

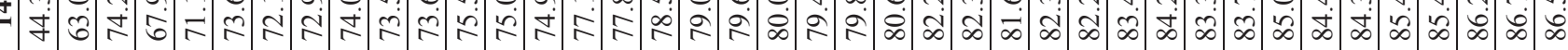

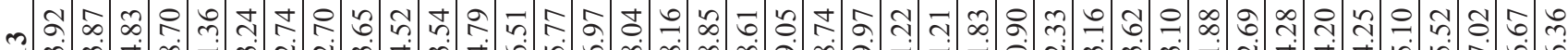

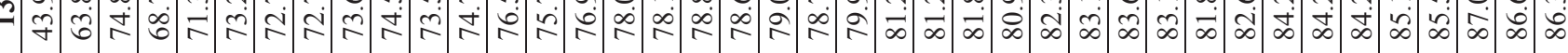

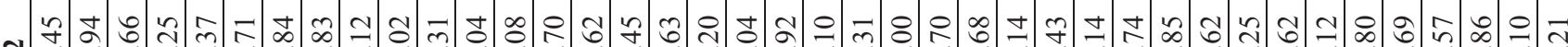

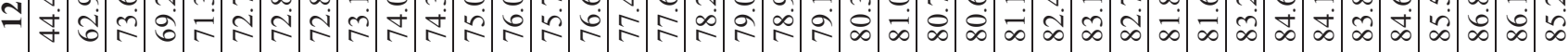

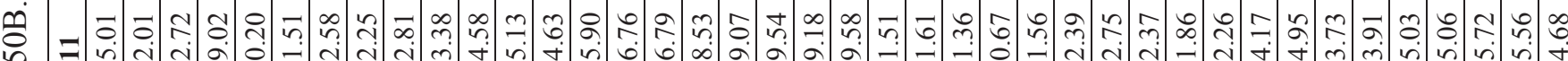
$=$ 守

-

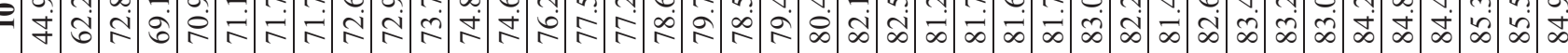

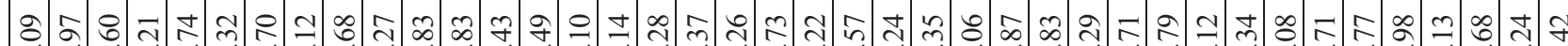

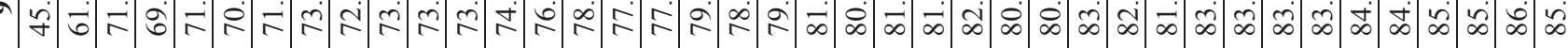

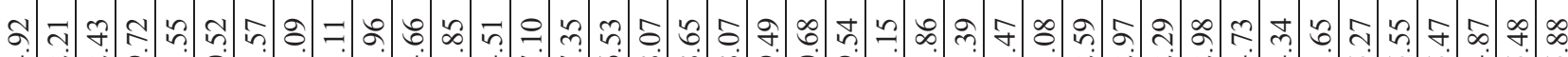

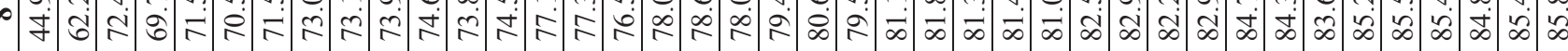
Бิ

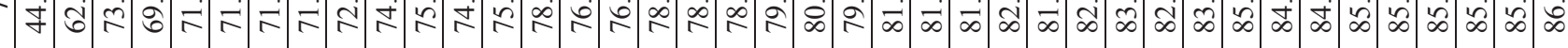

ભి

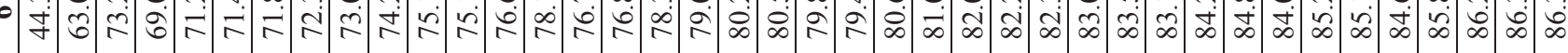
\&

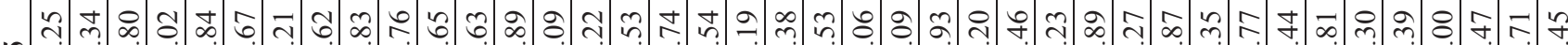

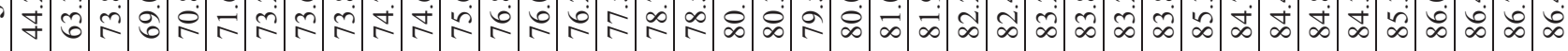
西

โธ่

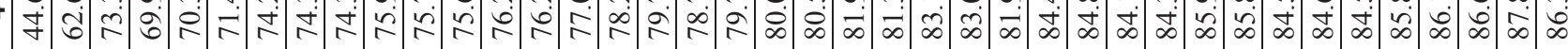

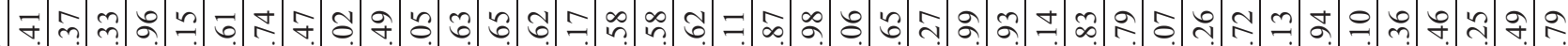

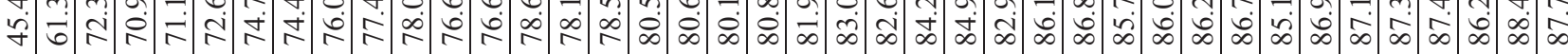

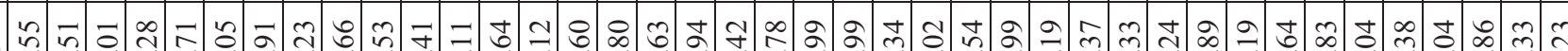

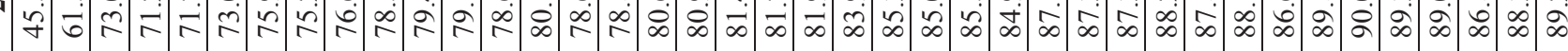

$\infty$

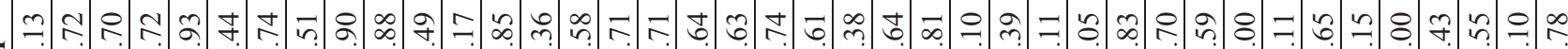
古 車

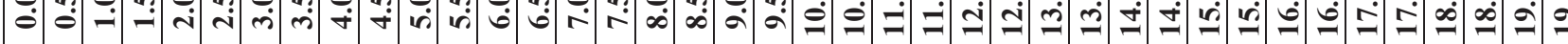




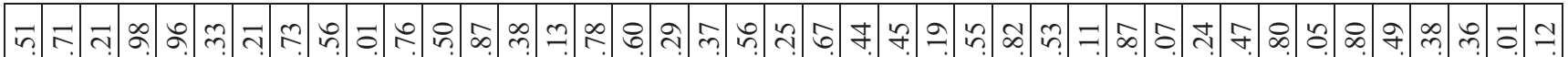

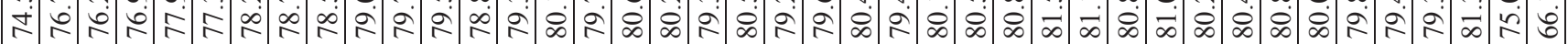

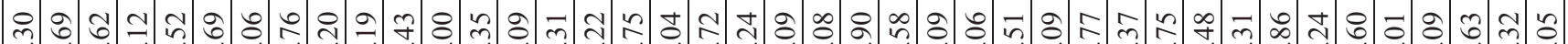

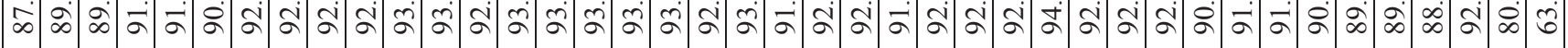

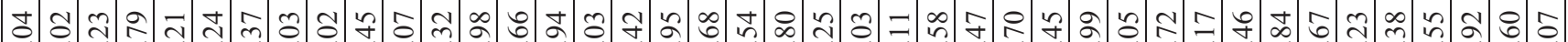

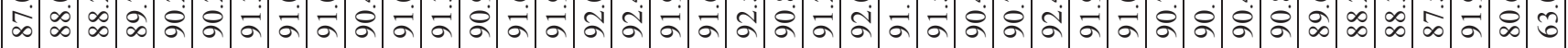

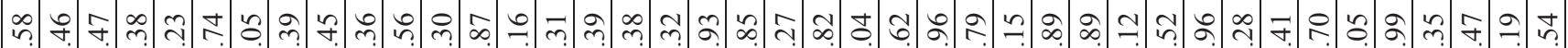

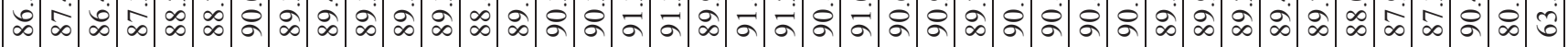

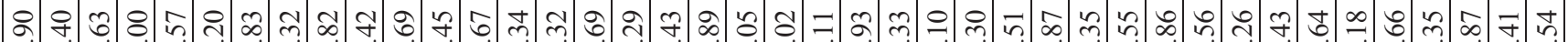

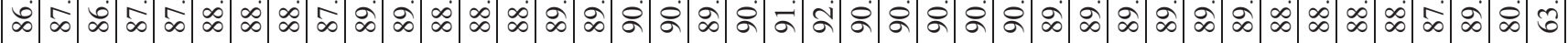

g)

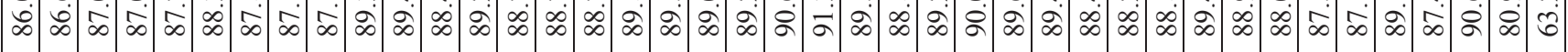

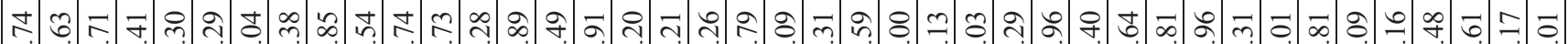

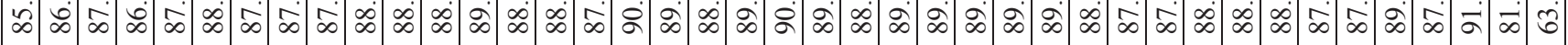

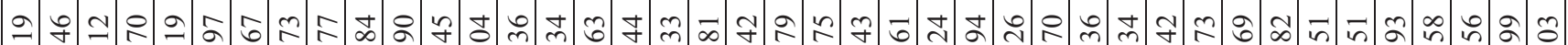

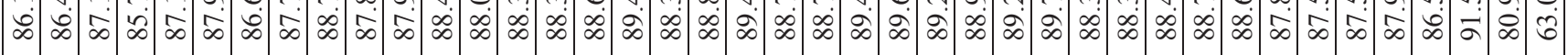

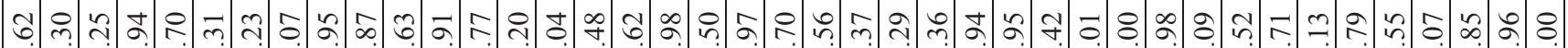

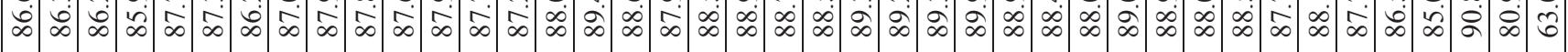

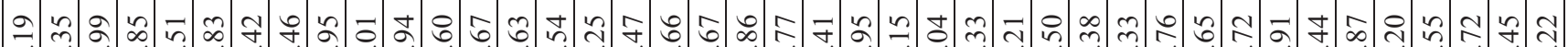

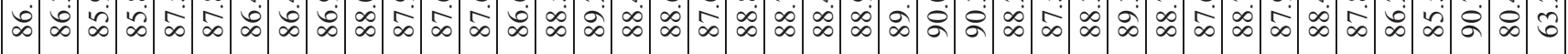

\&의

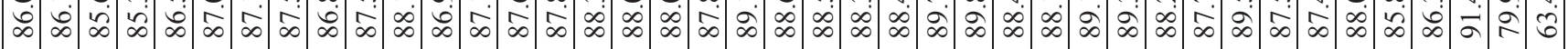

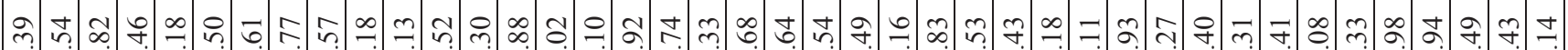

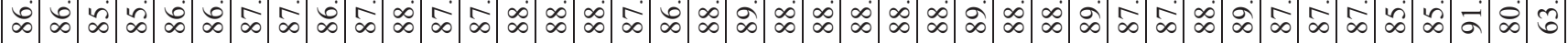

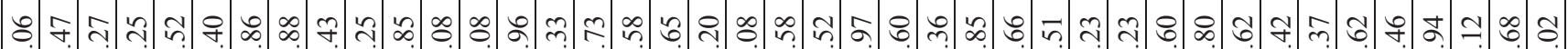

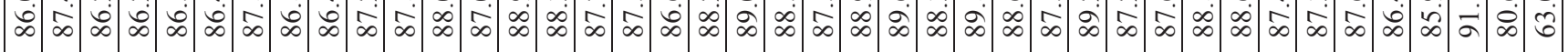

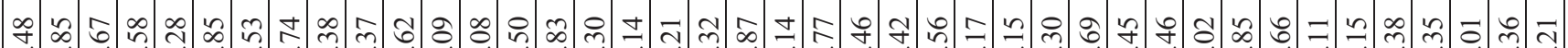

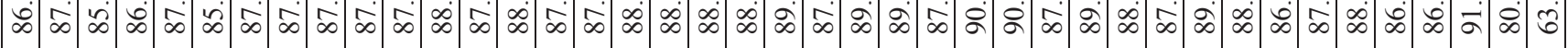
ที ร

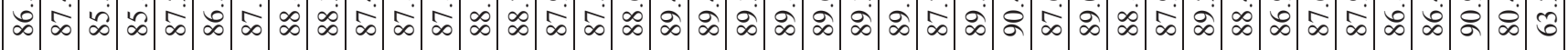

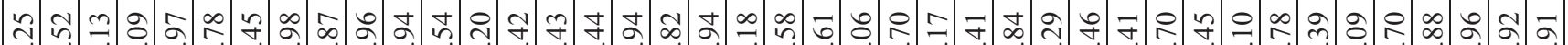

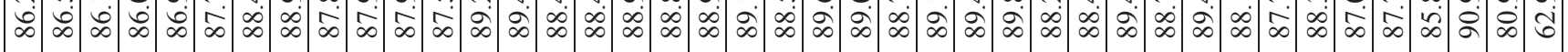

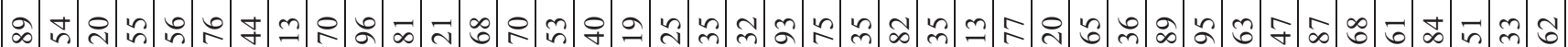

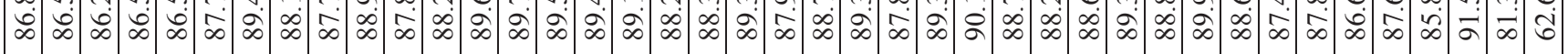

๑ สิ

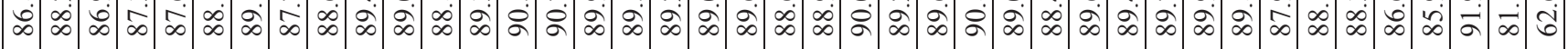

J t

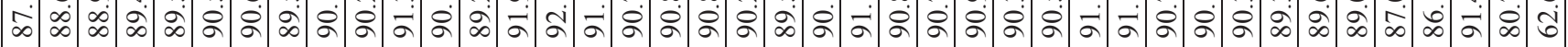

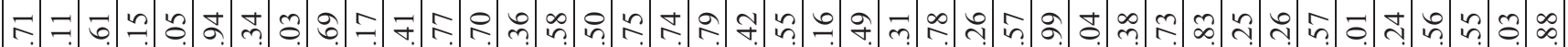

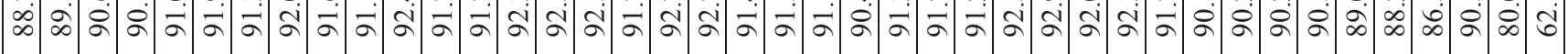

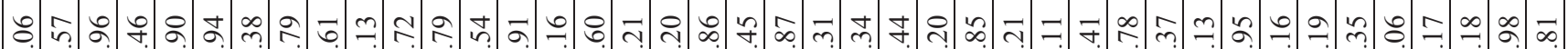

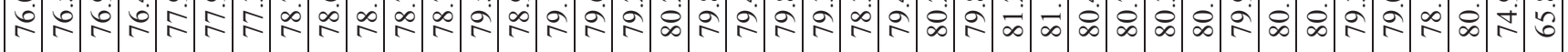

우

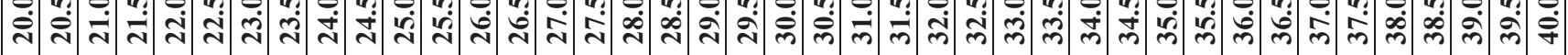




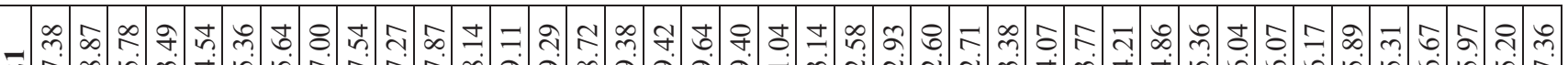

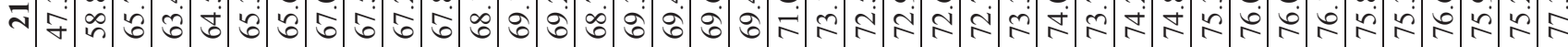

ఫ

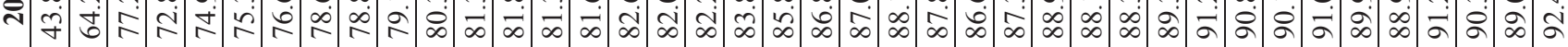

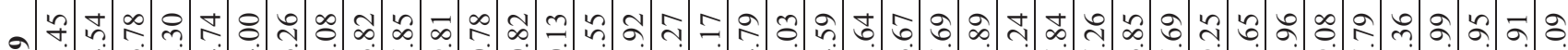

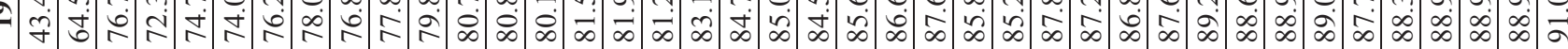

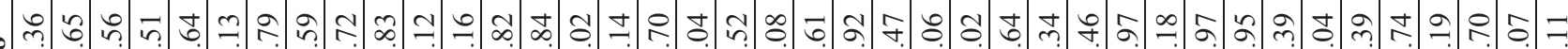

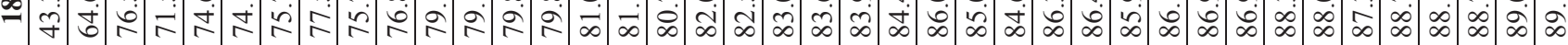
กิ่

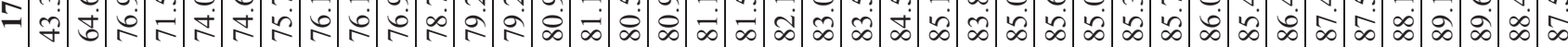

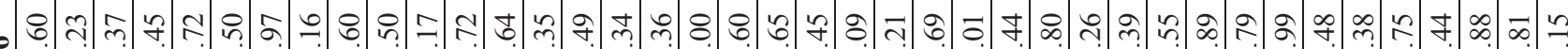

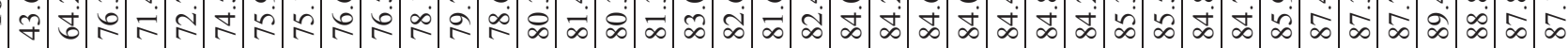

ㅈ.

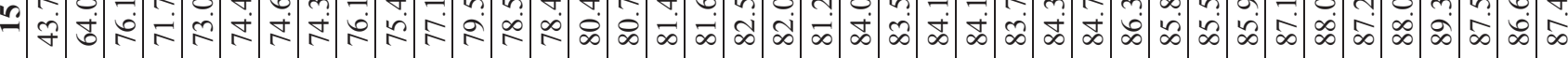

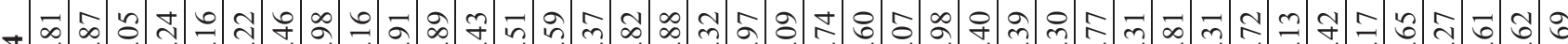
定

$m$ ○ी

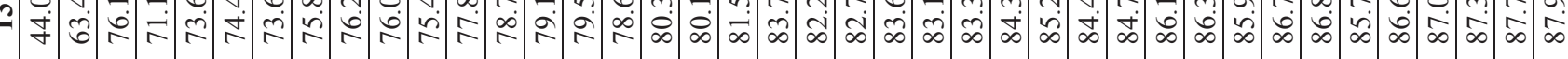

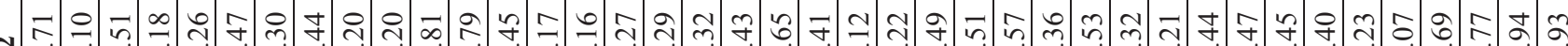

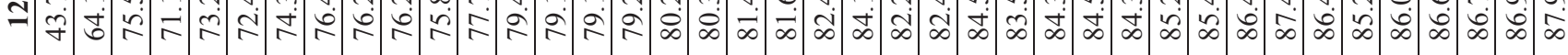

?

- ปุ

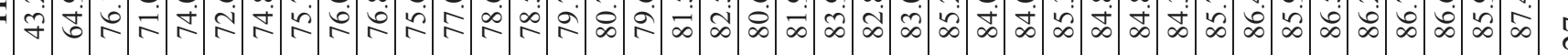

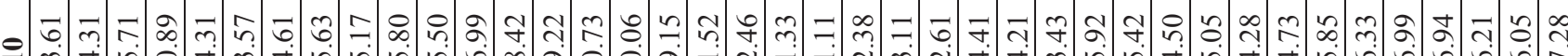

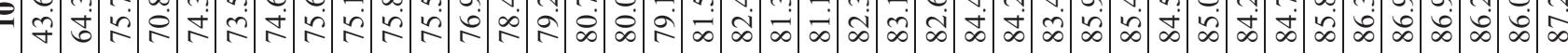

సี สี

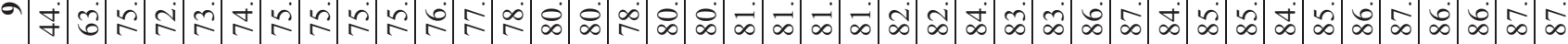

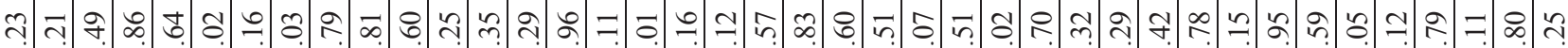

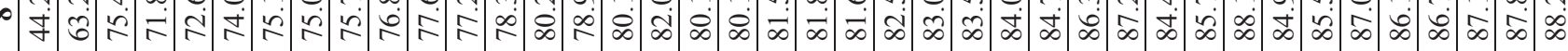

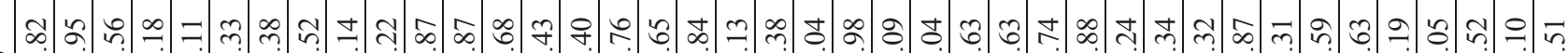
穴

மீ

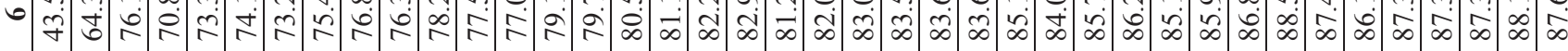

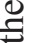

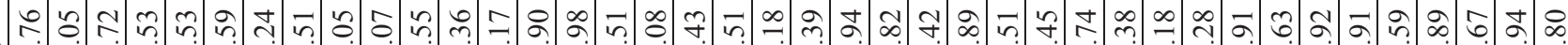

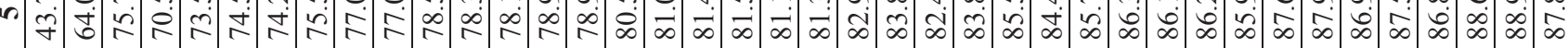

छี

సิ $=$ ம

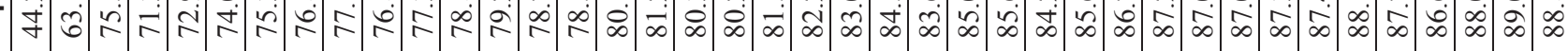

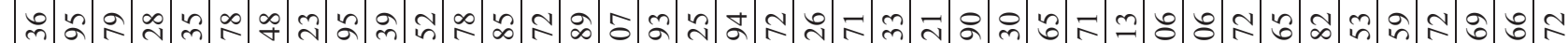
Jं

ح의

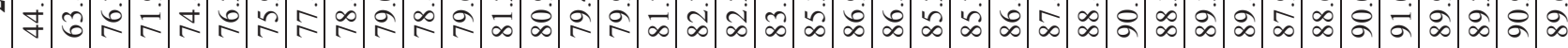

in

-

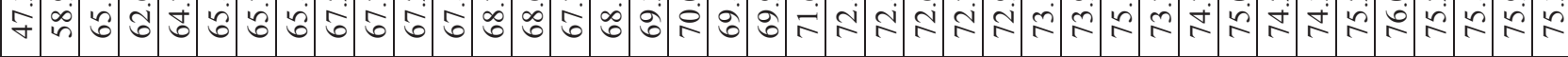
过象 


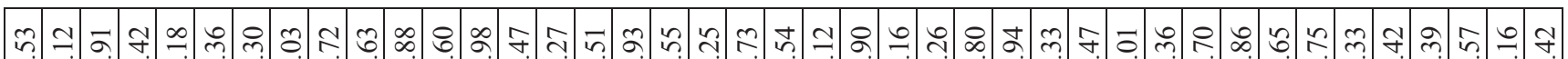

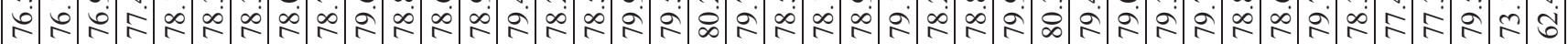

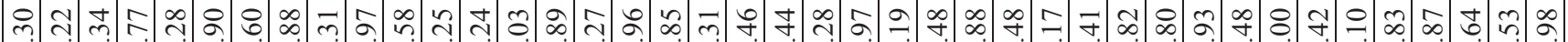

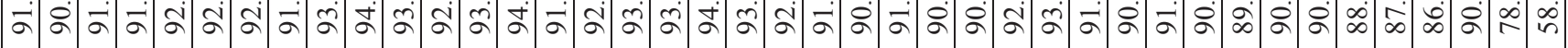

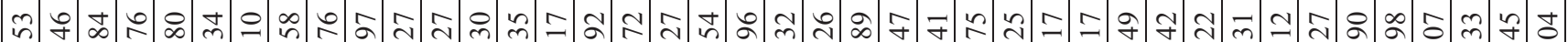

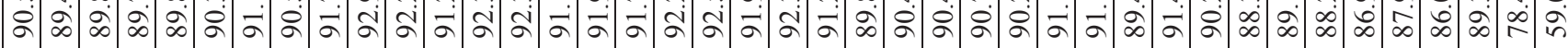

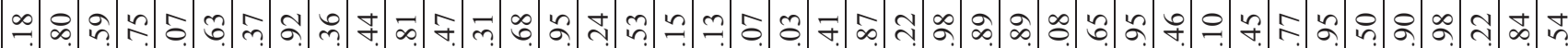

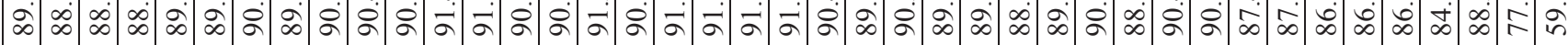

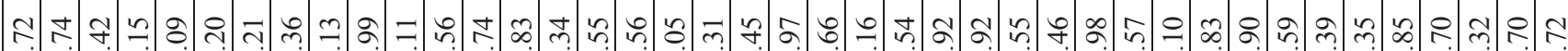

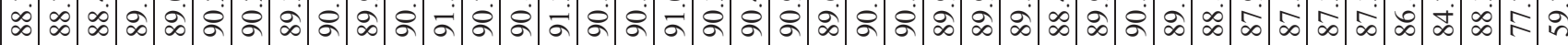

ㄱำ

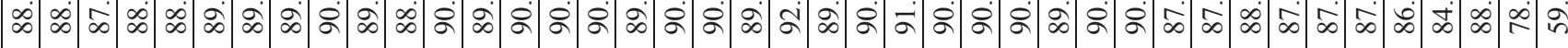

ศ

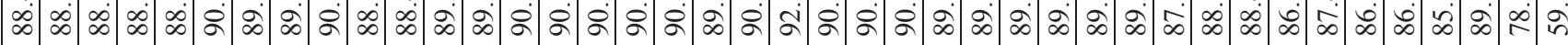

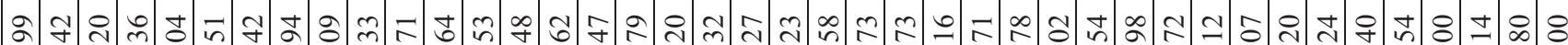

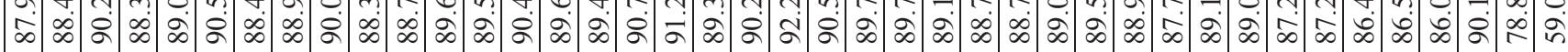

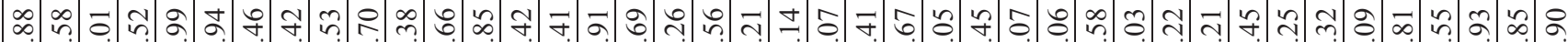

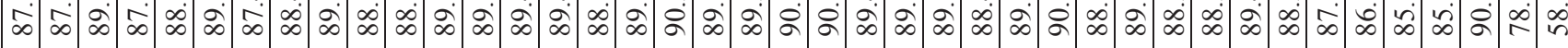

m

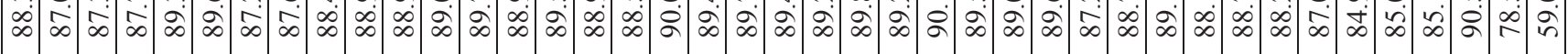

กิ

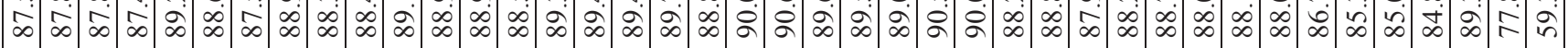

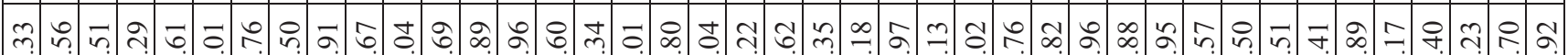

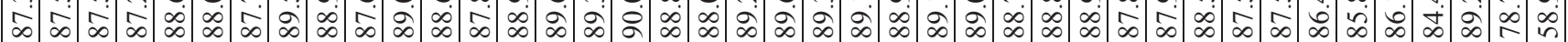

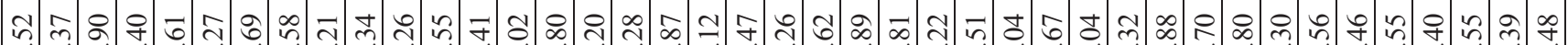

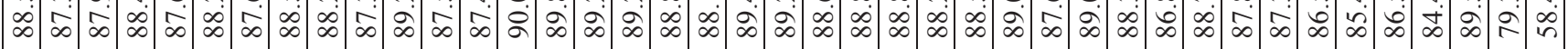

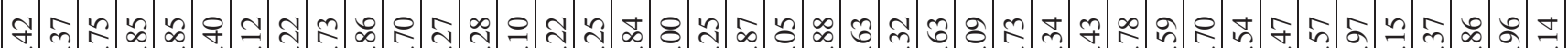

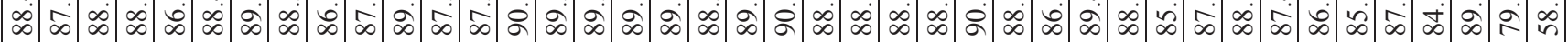

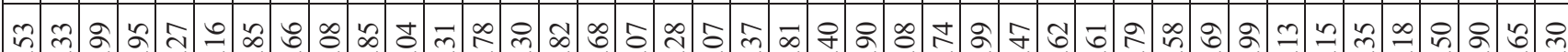

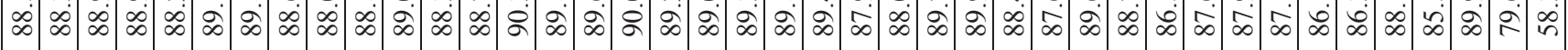

Fळి ભి

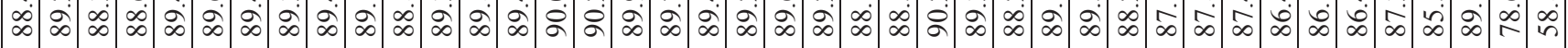

$\infty$ గి

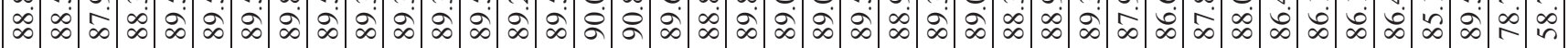

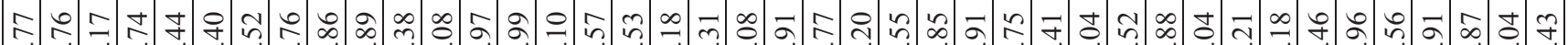

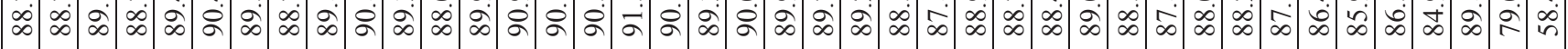

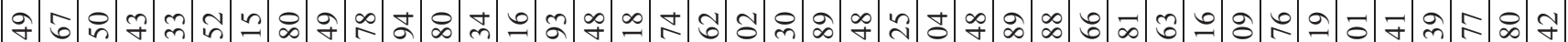

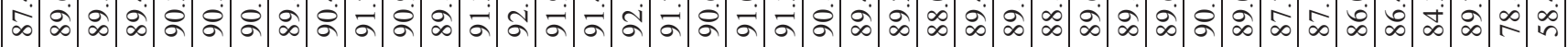

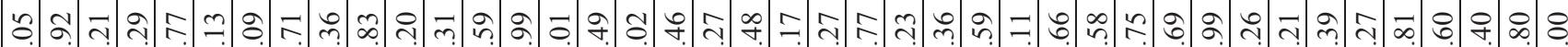

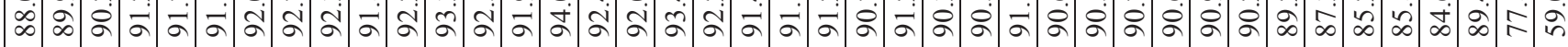

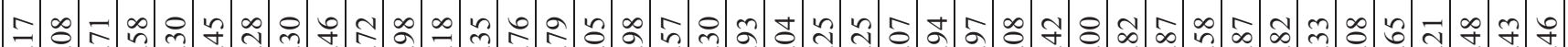

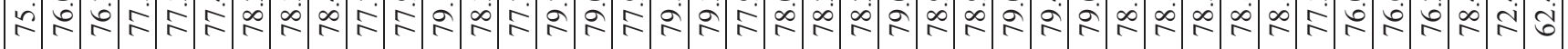

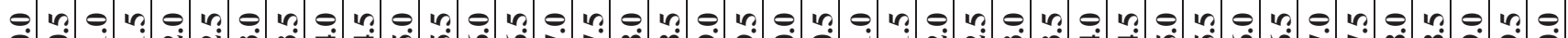

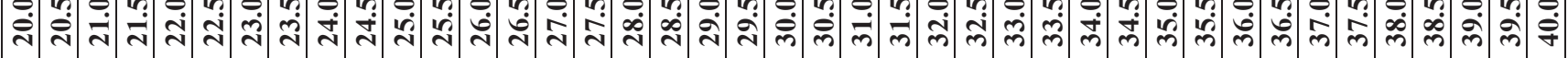


- ஸे ন

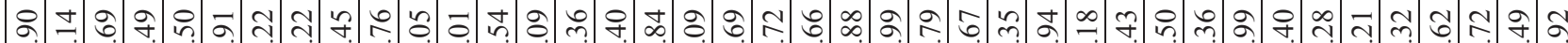

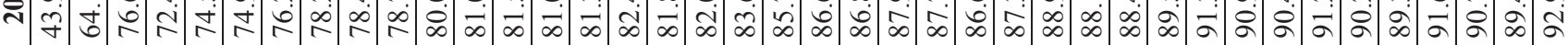

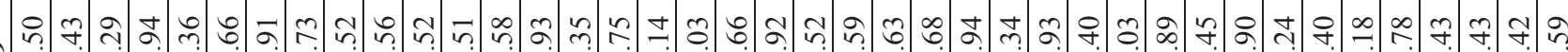

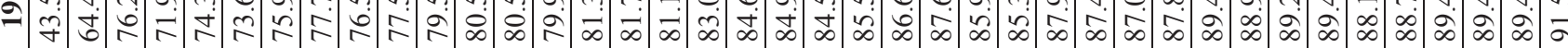

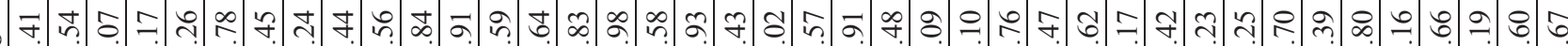

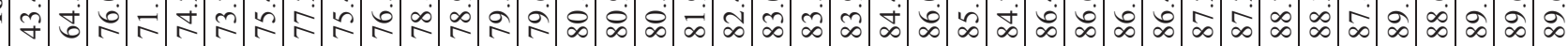

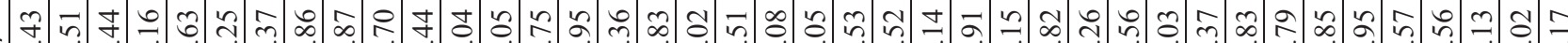

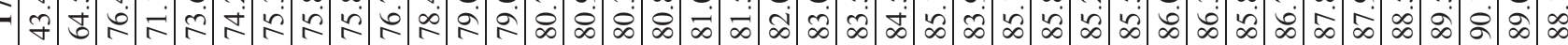

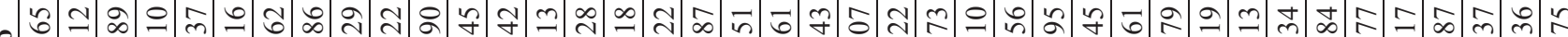

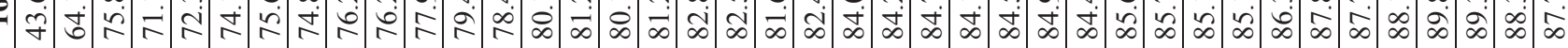

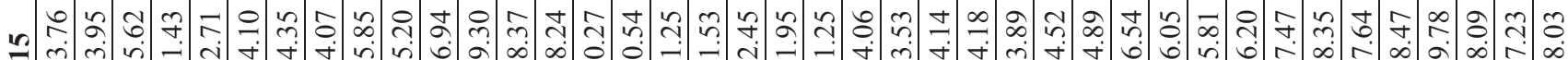

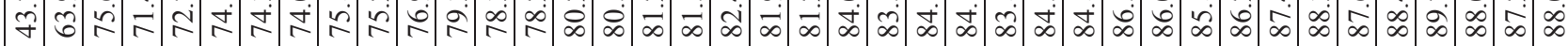

-

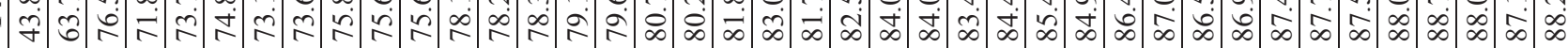

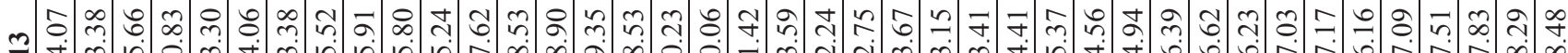

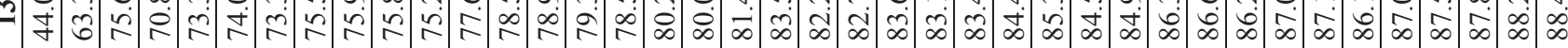

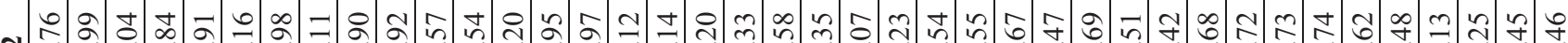
च

?

- సิ

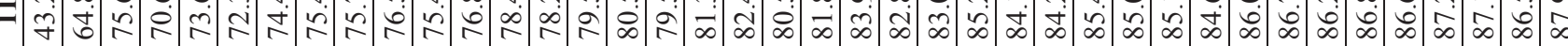

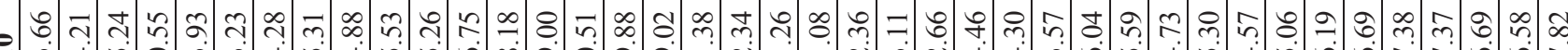

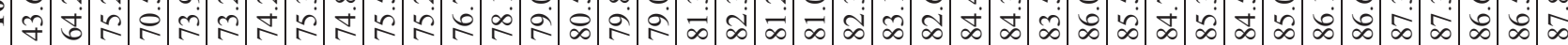

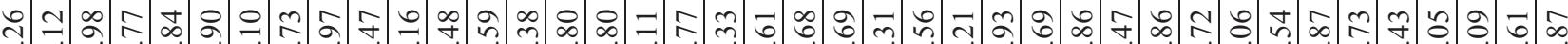

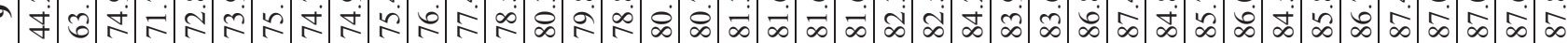

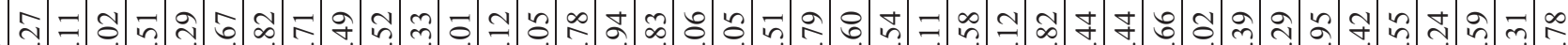

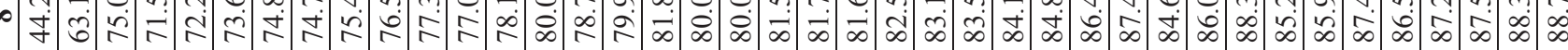

क人 守

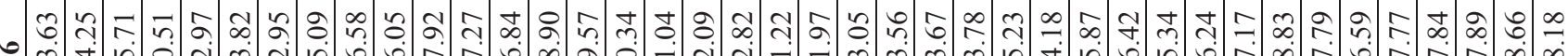

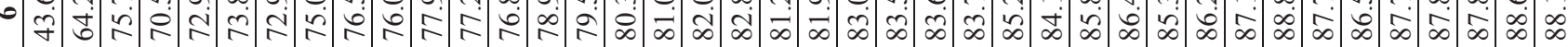

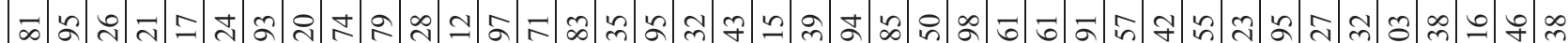

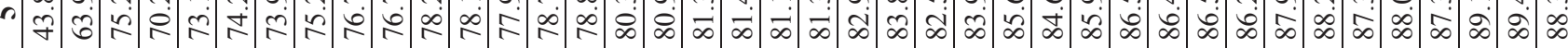

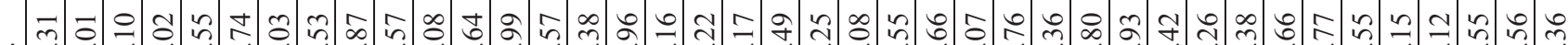

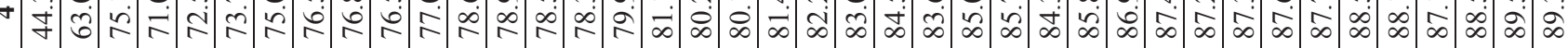
으 古

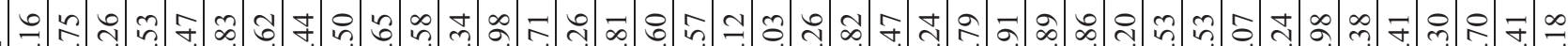
过

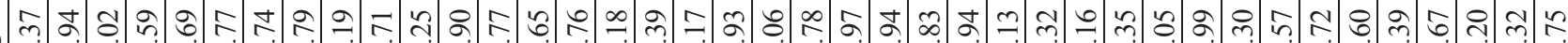

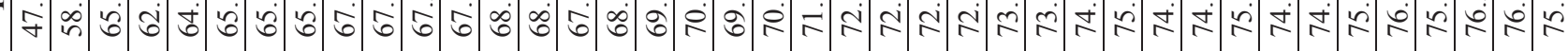

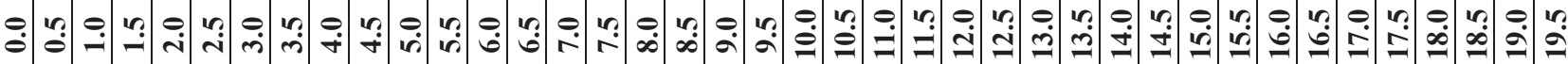




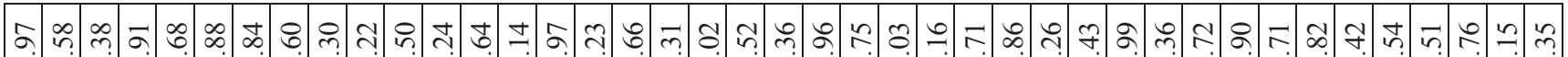

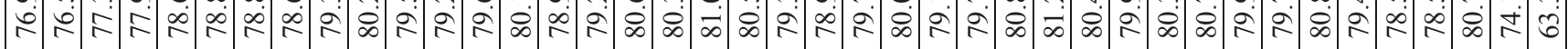

๘

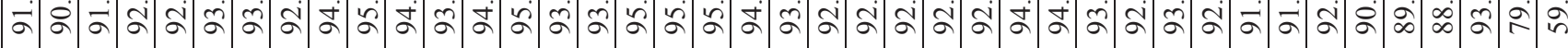

\&) f f

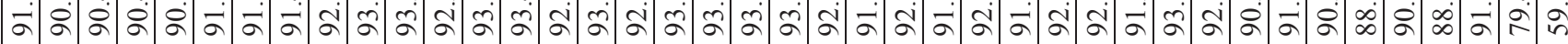

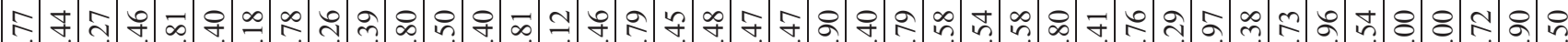

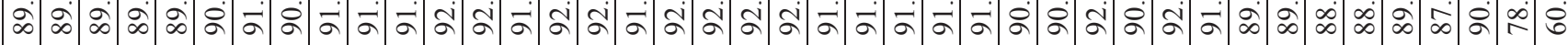

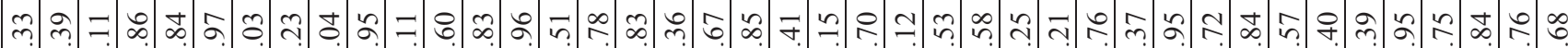

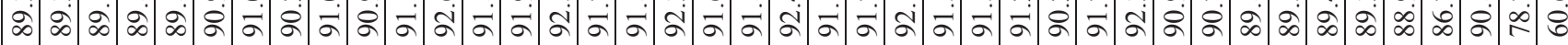

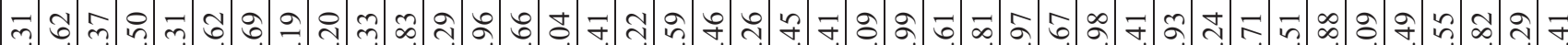

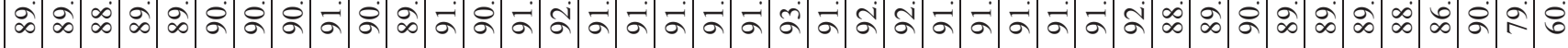

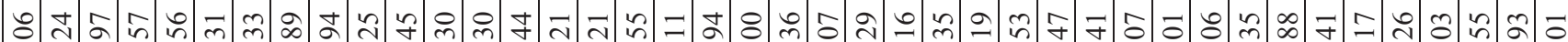

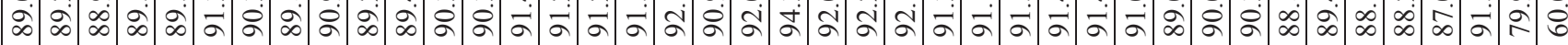

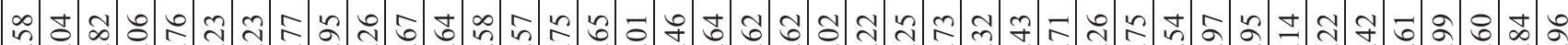

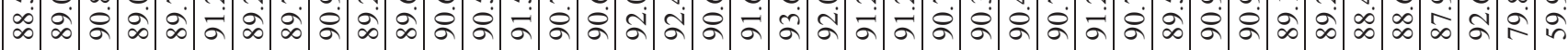

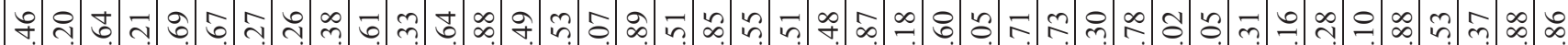

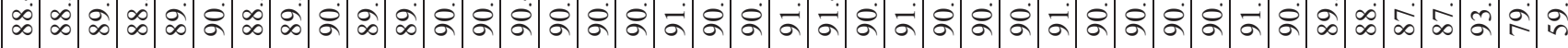

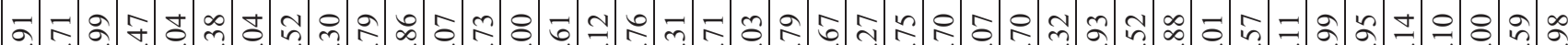

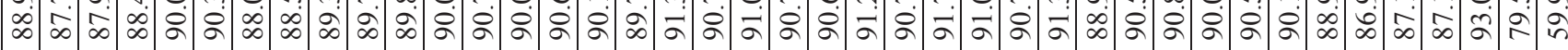

의 f f

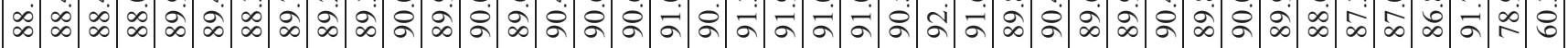

ฉ. = $=\infty$ m

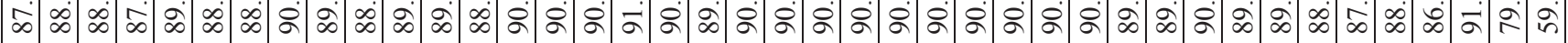

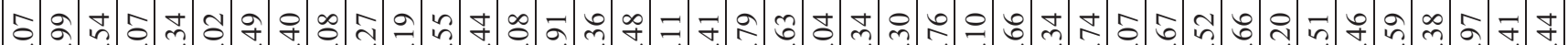

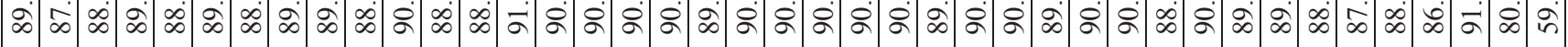

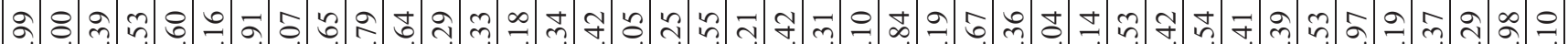

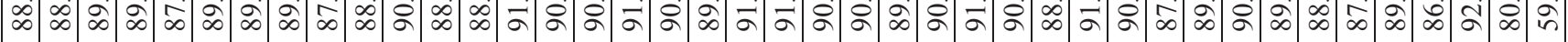

$=\varnothing$ tढ

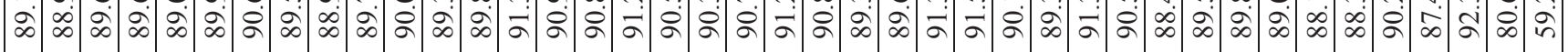

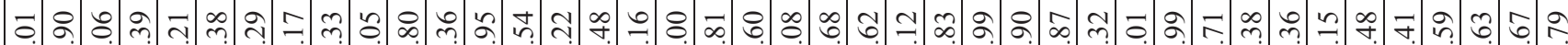

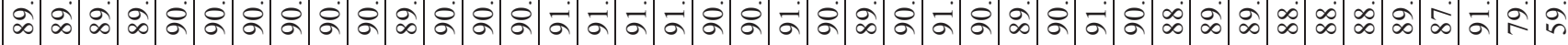

$\infty$ 긍 t

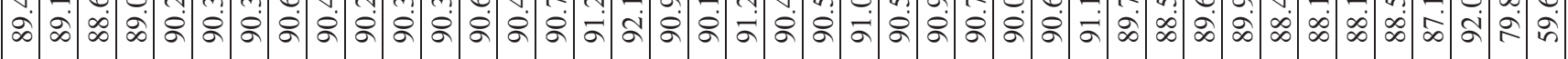

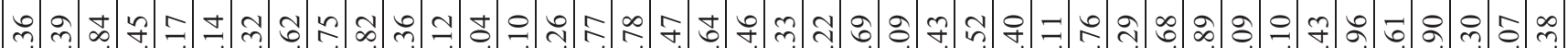

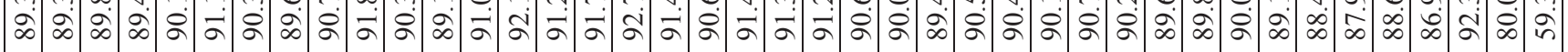

จ

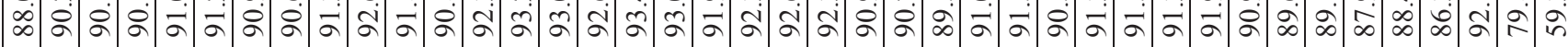

b

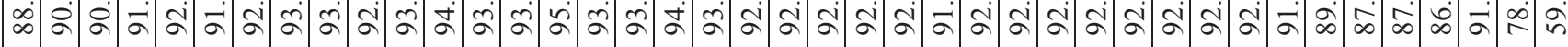

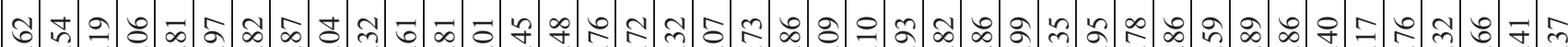

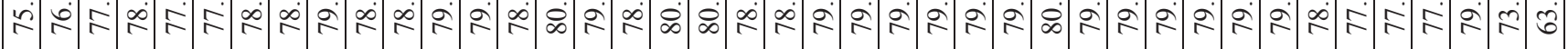




\section{REFERENCES}

1. "Experiment Control Plan for the AFIP-7 Fuel Irradiation in the ATR," PLN-3447, Revision 1, January, 2011.

2. J. W. Nielsen, G. S. Chang. "As-Run Neutronic Analysis for the AFIP-7 Experiment Irradiated in the Center Flux Trap of the ATR, Cycles 149B and 150B,”ECAR-1890, April 2012.

3. RERTR Project Personnel, "RERTR AFIP-7 Irradiation Experiment in the Advanced Test Reactor: As-Built Data Package,” AFIP-7 Job ID 3543571, May 2011.

4. G. A. Roth, “AFIP-7 Thermal Evaluation,” ECAR-1269. May 2011.

5. P. E. Murray, "Validation of ABAQUS Standard 6.7-3 Heat Transfer", ECAR-131, January 2008.

6. R. H. Perry, D. W. Green, "Perry's Chemical Engineer's Handbook," $7^{\text {th }}$ Edition, McGraw-Hill, 1997.

7. G. A. Roth, "As-Run Thermal Analysis for the AFIP-7 Experiment Irradiated in the Center Flux Trap of the ATR, Cycles 149B and 150B," ECAR-1894, June 2012. 


\section{Appendix A: Individual Plate Power and Fission Density Plots}



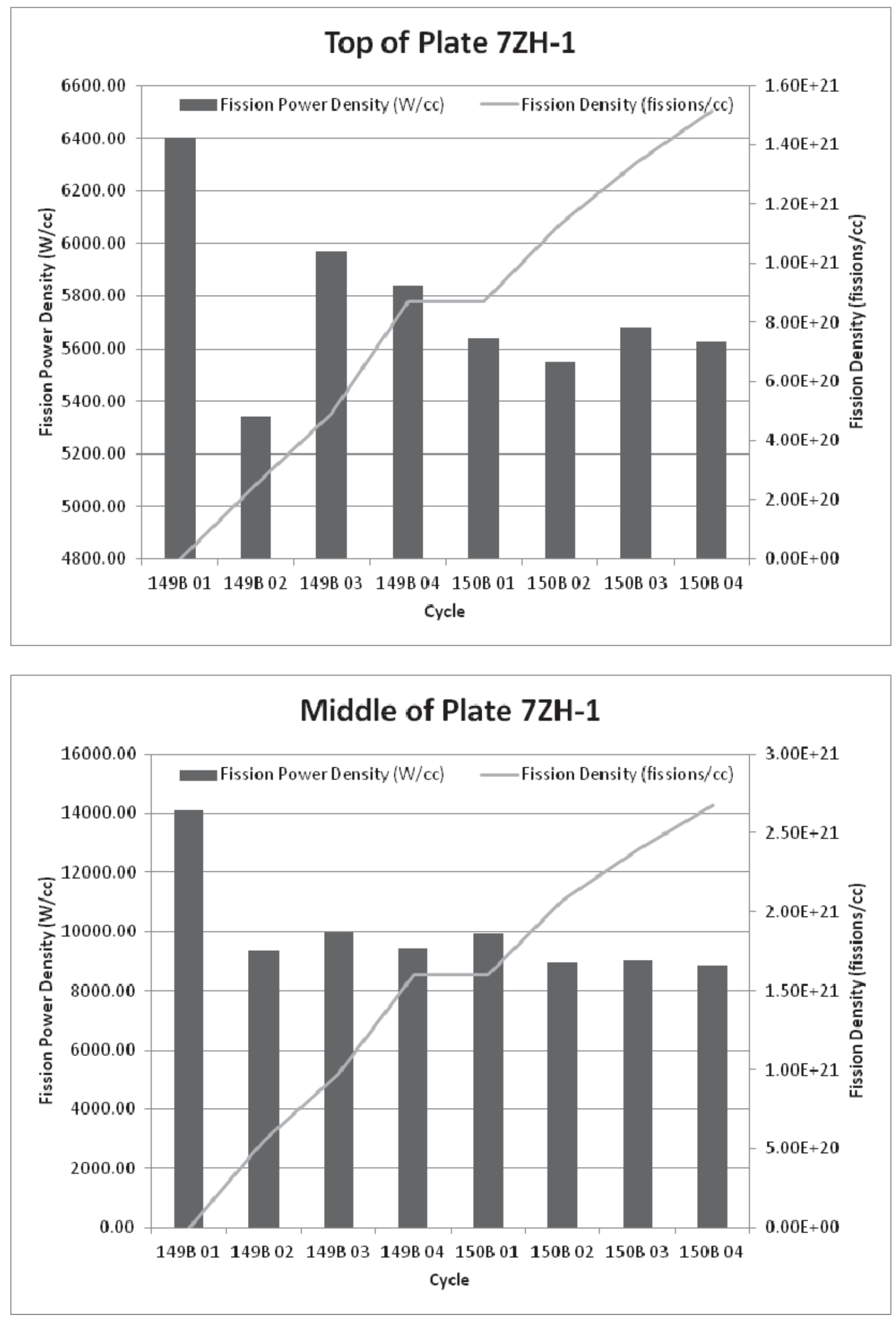

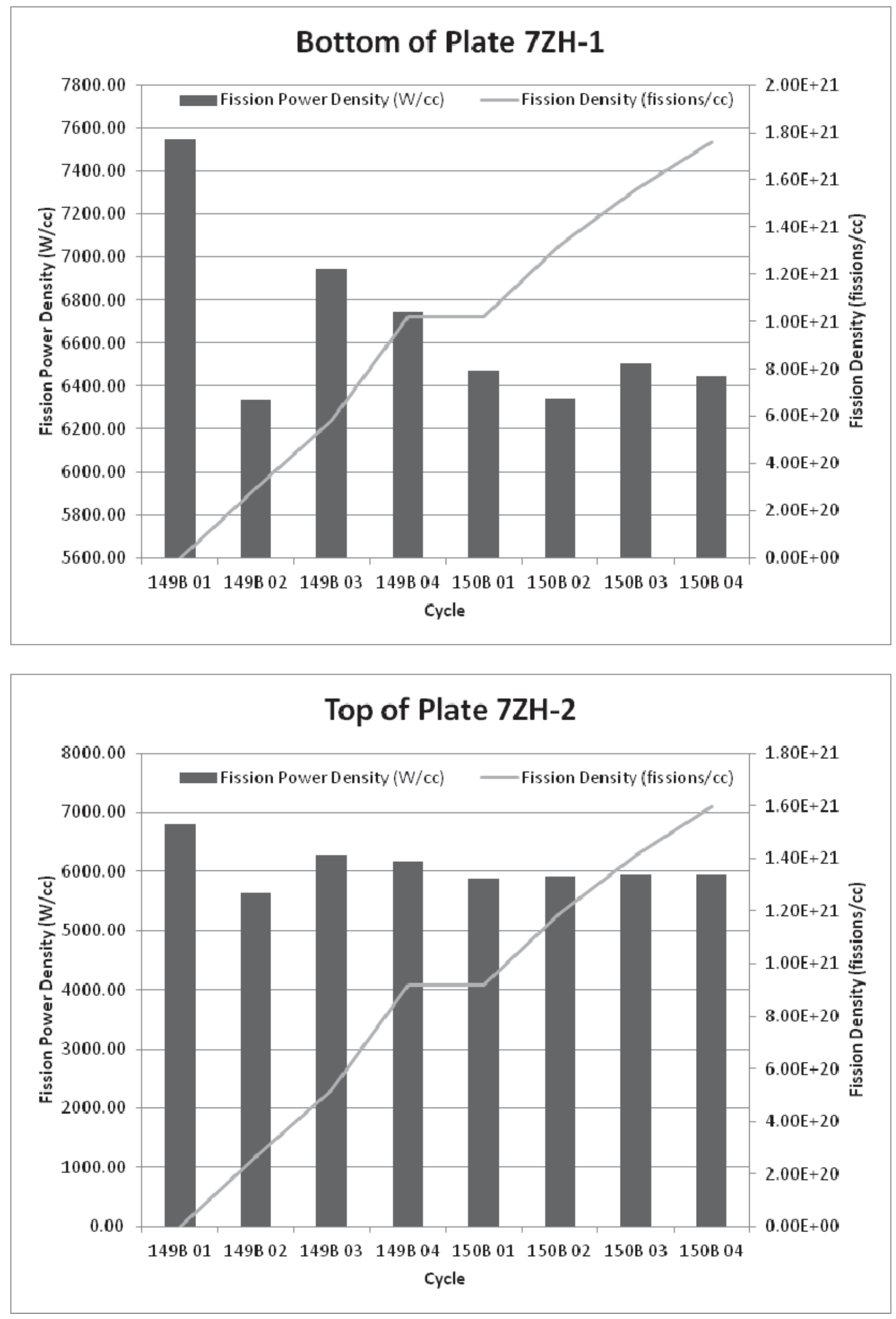

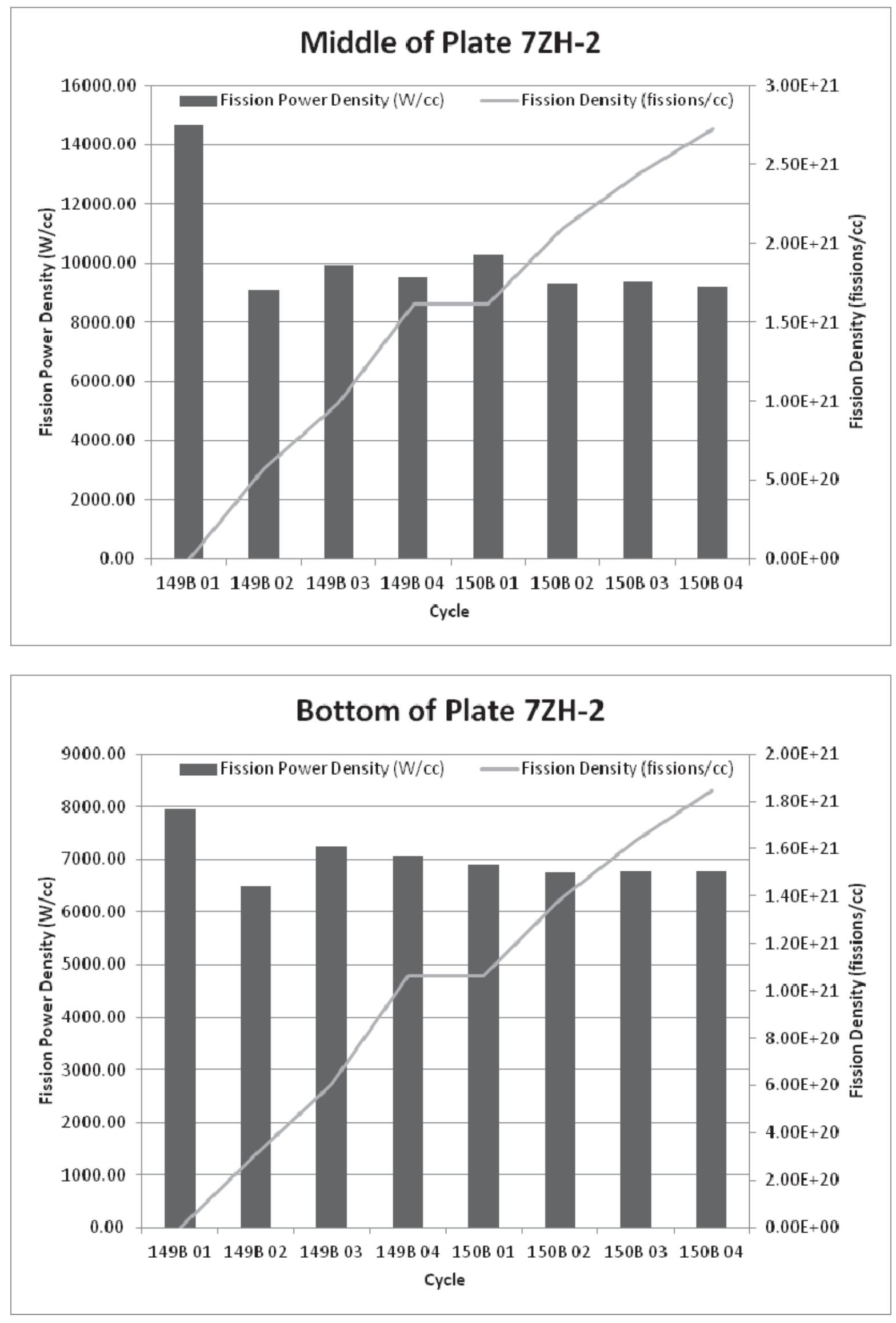

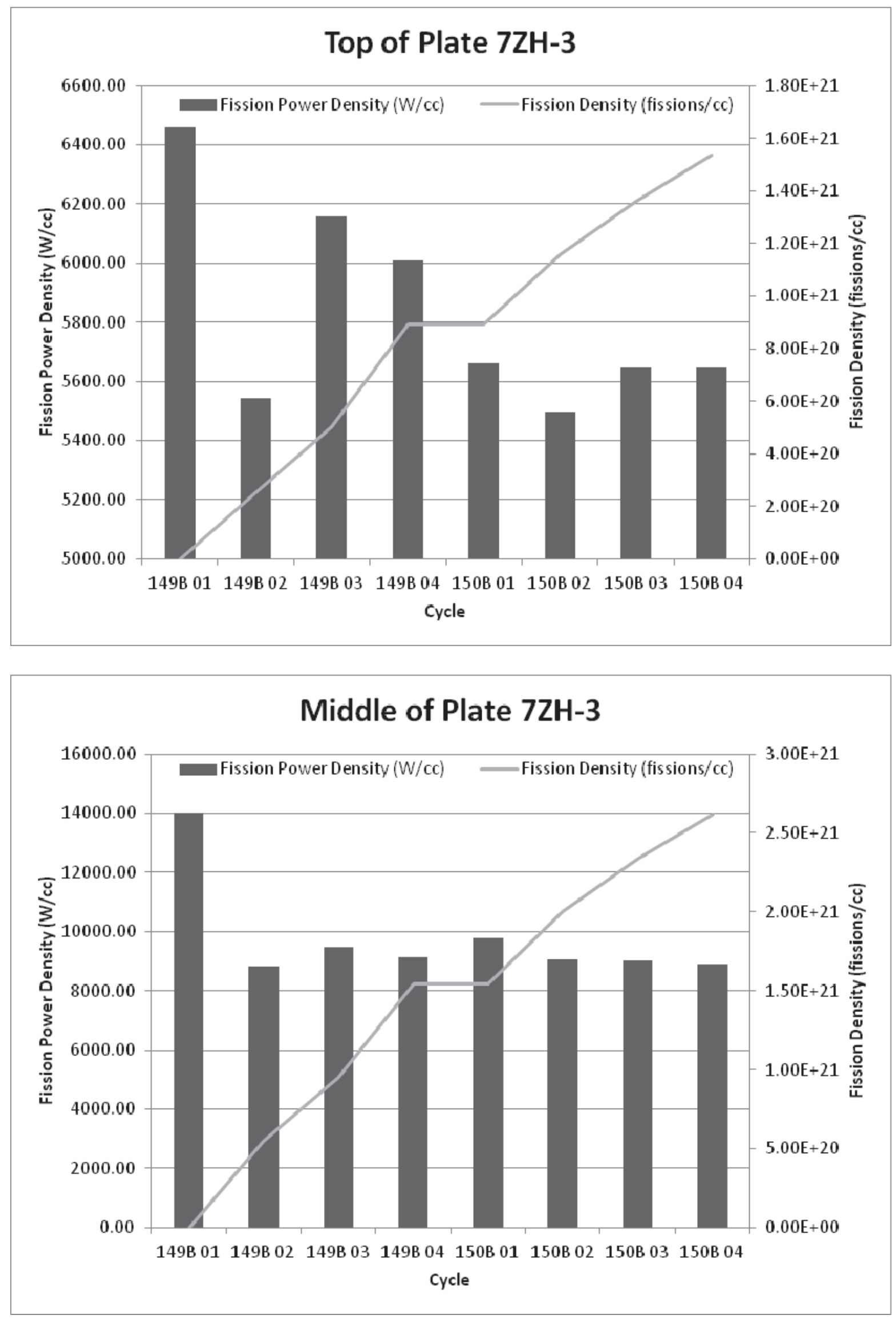

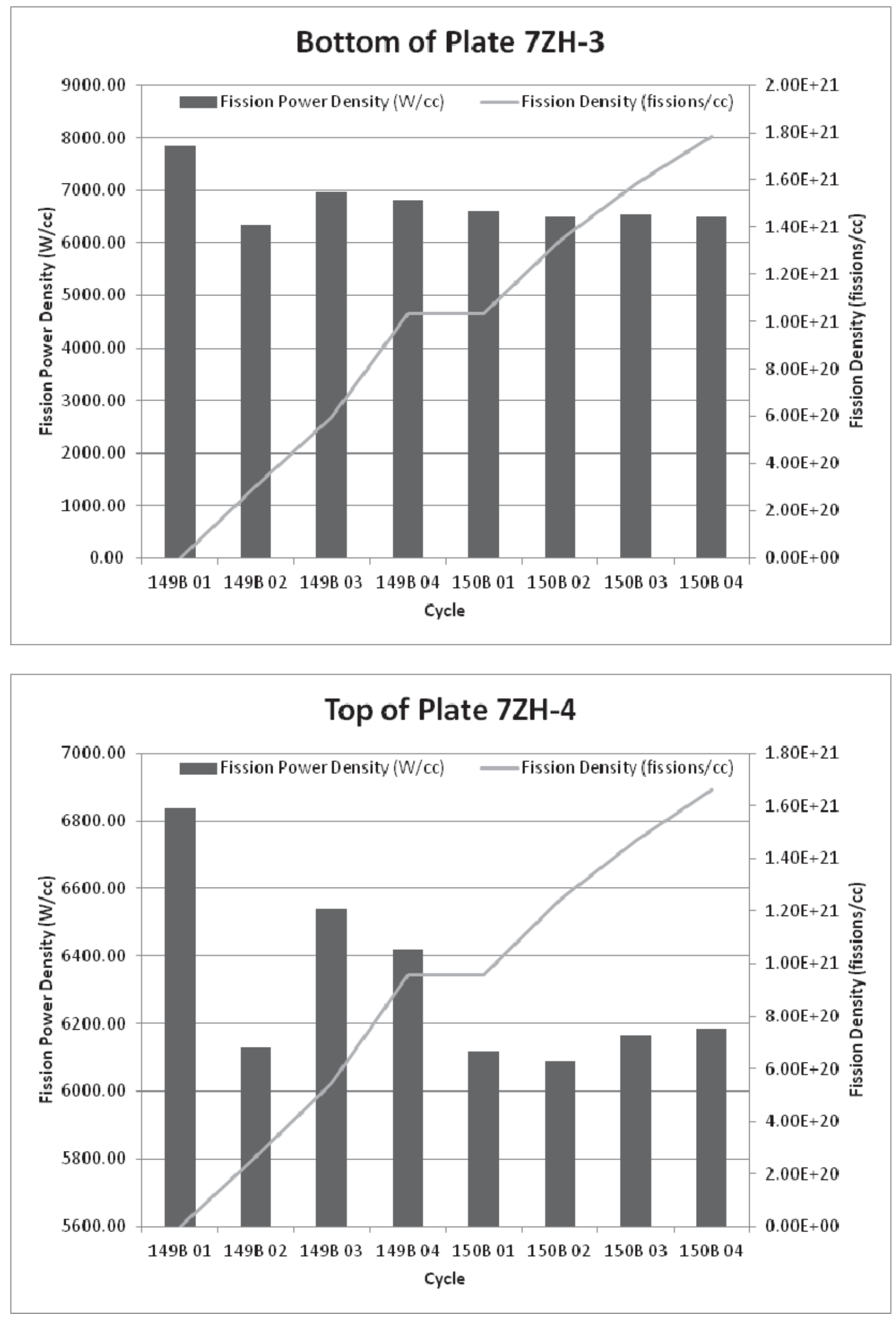

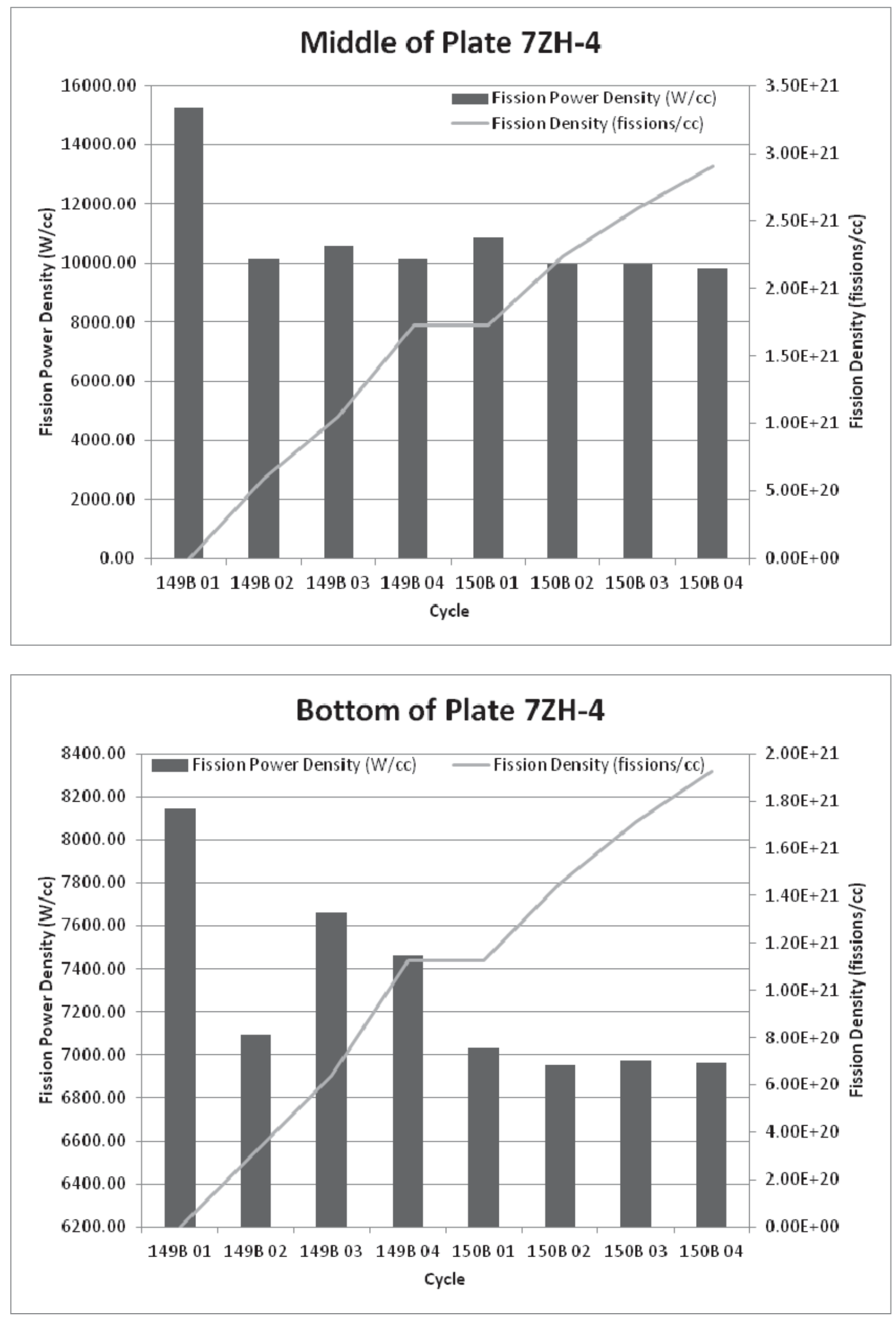
Appendix B: Plate Surface Temperatures 


\section{Appendix B}

\section{Index of Tables}

Table B - 1: Temperature $\left({ }^{\circ} \mathrm{C}\right)$ map of the north side of plate position 1 at BOC 149B (0 EFPD)......... 143

Table B - 2: Temperature $\left({ }^{\circ} \mathrm{C}\right)$ map of the south side of plate position 1 at BOC 149B (0 EFPD)......... 145

Table B - 3: Temperature $\left({ }^{\circ} \mathrm{C}\right)$ map of the north side of plate position 2 at BOC 149B (0 EFPD)......... 147

Table B - 4: Temperature $\left({ }^{\circ} \mathrm{C}\right)$ map of the south side of plate position 2 at BOC 149B (0 EFPD)......... 149

Table B - 5: Temperature $\left({ }^{\circ} \mathrm{C}\right)$ map of the north side of plate position 3 at BOC 149B (0 EFPD)......... 151

Table B - 6: Temperature $\left({ }^{\circ} \mathrm{C}\right)$ map of the south side of plate position 3 at BOC 149B (0 EFPD)......... 153

Table B - 7: Temperature $\left({ }^{\circ} \mathrm{C}\right)$ map of the north side of plate position 4 at BOC 149B (0 EFPD)......... 155

Table B - 8: Temperature $\left({ }^{\circ} \mathrm{C}\right)$ map of the south side of plate position 4 at BOC 149B (0 EFPD)......... 157

Table B - 9: Temperature $\left({ }^{\circ} \mathrm{C}\right)$ map of the north side of plate position 1 at MOC1 149B (15.0

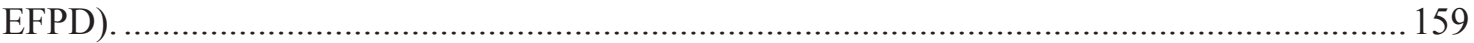

Table B - 10: Temperature $\left({ }^{\circ} \mathrm{C}\right)$ map of the south side of plate position 1 at MOC1 149B (15.0

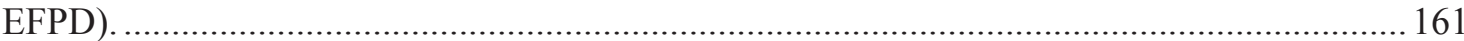

Table B - 11: Temperature $\left({ }^{\circ} \mathrm{C}\right)$ map of the north side of plate position 2 at MOC1 149B (15.0

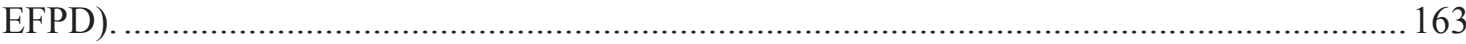

Table B - 12: Temperature $\left({ }^{\circ} \mathrm{C}\right)$ map of the south side of plate position 2 at MOC1 149B (15.0

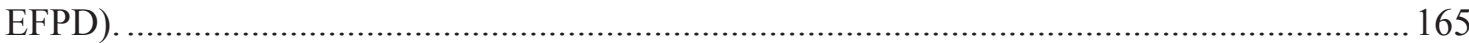

Table B - 13: Temperature $\left({ }^{\circ} \mathrm{C}\right)$ map of the north side of plate position 3 at MOC1 149B (15.0 EFPD).

Table B - 14: Temperature $\left({ }^{\circ} \mathrm{C}\right)$ map of the south side of plate position 3 at MOC1 149B (15.0 EFPD).

Table B - 15: Temperature $\left({ }^{\circ} \mathrm{C}\right)$ map of the north side of plate position 4 at MOC1 149B (15.0 EFPD).

Table B - 16: Temperature $\left({ }^{\circ} \mathrm{C}\right)$ map of the south side of plate position 4 at MOC1 149B (15.0 EFPD).

Table B - 17: Temperature $\left({ }^{\circ} \mathrm{C}\right)$ map of the north side of plate position 1 at MOC2 149B (31.0 EFPD).

Table B - 18: Temperature $\left({ }^{\circ} \mathrm{C}\right)$ map of the south side of plate position 1 at MOC2 149B (31.0 EFPD)

Table B - 19: Temperature $\left({ }^{\circ} \mathrm{C}\right)$ map of the north side of plate position 2 at MOC2 149B (31.0 EFPD).

Table B - 20: Temperature $\left({ }^{\circ} \mathrm{C}\right)$ map of the south side of plate position 2 at MOC2 149B (31.0 EFPD)

Table B - 21: Temperature $\left({ }^{\circ} \mathrm{C}\right)$ map of the north side of plate position 3 at MOC2 149B (31.0 EFPD).

Table B - 22: Temperature $\left({ }^{\circ} \mathrm{C}\right)$ map of the south side of plate position 3 at MOC2 149B (31.0 EFPD).

Table B - 23: Temperature $\left({ }^{\circ} \mathrm{C}\right)$ map of the north side of plate position 4 at MOC2 149B (31.0 EFPD).

Table B - 24: Temperature $\left({ }^{\circ} \mathrm{C}\right)$ map of the south side of position 4 at MOC2 149B (31.0 EFPD). 
Table B - 25: Temperature $\left({ }^{\circ} \mathrm{C}\right)$ map of the north side of plate position 1 at EOC 149A (53.6 EFPD).

Table B - 26: Temperature $\left({ }^{\circ} \mathrm{C}\right)$ map of the south side of position 1 at EOC 149B (53.6 EFPD)

Table B - 27: Temperature $\left({ }^{\circ} \mathrm{C}\right)$ map of the north side of plate position 2 at EOC 149B (53.6 EFPD).

Table B - 28: Temperature $\left({ }^{\circ} \mathrm{C}\right)$ map of the south side of plate position 2 at EOC 149B (53.6 EFPD).

Table B - 29: Temperature $\left({ }^{\circ} \mathrm{C}\right)$ map of the north side of plate position 3 at EOC 149B (53.6 EFPD).

Table B - 30: Temperature $\left({ }^{\circ} \mathrm{C}\right)$ map of the south side of plate position 3 at EOC 149B $(53.6$ EFPD).

Table B - 31: Temperature $\left({ }^{\circ} \mathrm{C}\right)$ map of the north side of plate position 4 at EOC 149B (53.6 EFPD).

Table B - 32: Temperature $\left({ }^{\circ} \mathrm{C}\right)$ map of the south side of plate position 4 at EOC 149B $(53.6$ EFPD).

Table B - 33: Temperature $\left({ }^{\circ} \mathrm{C}\right)$ map of the north side of plate position 1 at BOC 150B (total 53.6 EFPD).

Table B - 34: Temperature $\left({ }^{\circ} \mathrm{C}\right)$ map of the south side of plate position 1 at BOC 150B (total 53.6 EFPD).

Table B - 35: Temperature $\left({ }^{\circ} \mathrm{C}\right)$ map of the north side of plate position 2 at BOC 150B (total 53.6 EFPD).

Table B - 36: Temperature $\left({ }^{\circ} \mathrm{C}\right)$ map of the south side of plate position 2 at BOC 150B (total 53.6 EFPD).

Table B - 37: Temperature $\left({ }^{\circ} \mathrm{C}\right)$ map of the north side of plate position 3 at BOC 150B (total 53.6 EFPD).

Table B - 38: Temperature $\left({ }^{\circ} \mathrm{C}\right)$ map of the south side of plate position 3 at BOC 150B (total 53.6 EFPD).

Table B - 39: Temperature $\left({ }^{\circ} \mathrm{C}\right)$ map of the north side of plate position 4 at BOC 150B (total 53.6 EFPD).

Table B - 40: Temperature $\left({ }^{\circ} \mathrm{C}\right)$ map of the south side of plate position 4 at BOC 150B (total 53.6 EFPD).

Table B - 41: Temperature $\left({ }^{\circ} \mathrm{C}\right)$ map of the north side of plate position 1 at MOC1 150B (total 71.6 EFPD).

Table B - 42: Temperature $\left({ }^{\circ} \mathrm{C}\right)$ map of the south side of plate position 1 at MOC1 150B (total 71.6 EFPD).

Table B - 43: Temperature $\left({ }^{\circ} \mathrm{C}\right)$ map of the north side of plate position 2 at MOC1 150B (total 71.6 EFPD).

Table B - 44: Temperature $\left({ }^{\circ} \mathrm{C}\right)$ map of the south side of plate position 2 at MOC1 150B (total 71.6 EFPD).

Table B - 45: Temperature $\left({ }^{\circ} \mathrm{C}\right)$ map of the north side of plate position 3 at MOC1 150B (total 71.6 EFPD).

Table B - 46: Temperature $\left({ }^{\circ} \mathrm{C}\right)$ map of the south side of plate position 3 at MOC1 150B (total 71.6 EFPD). 
Table B - 47: Temperature $\left({ }^{\circ} \mathrm{C}\right)$ map of the north side of plate position 4 at MOC1 150B (total 71.6 EFPD).

Table B - 48: Temperature $\left({ }^{\circ} \mathrm{C}\right)$ map of the south side of plate position 4 at MOC1 150B (total 71.6 EFPD).

Table B - 49: Temperature $\left({ }^{\circ} \mathrm{C}\right)$ map of the north side of plate position 1 at MOC2 150B (total 84.6 EFPD).

Table B - 50: Temperature $\left({ }^{\circ} \mathrm{C}\right)$ map of the south side of plate position 1 at MOC2 150B (total 84.6 EFPD).

Table B - 51: Temperature $\left({ }^{\circ} \mathrm{C}\right)$ map of the north side of plate position 2 at MOC2 150B (total 84.6 EFPD).

Table B - 52: Temperature $\left({ }^{\circ} \mathrm{C}\right)$ map of the south side of plate position 2 at MOC2 150B (total 84.6 EFPD).

Table B - 53: Temperature $\left({ }^{\circ} \mathrm{C}\right)$ map of the north side of plate position 3 at MOC2 150B (total 84.6 EFPD).

Table B - 54: Temperature $\left({ }^{\circ} \mathrm{C}\right)$ map of the south side of plate position 3 at MOC2 150B (total 84.6 EFPD).

Table B - 55: Temperature $\left({ }^{\circ} \mathrm{C}\right)$ map of the north side of plate position 4 at MOC2 150B (total 84.6 EFPD).

Table B - 56: Temperature $\left({ }^{\circ} \mathrm{C}\right)$ map of the south side of plate position 4 at MOC2 150B (total 84.6 EFPD).

Table B - 57: Temperature $\left({ }^{\circ} \mathrm{C}\right)$ map of the north side of plate position 1 at EOC 150B (total 95.6 EFPD).

Table B - 58: Temperature $\left({ }^{\circ} \mathrm{C}\right)$ map of the south side of plate position 1 at EOC 150B (total 95.6 EFPD).

Table B - 59: Temperature $\left({ }^{\circ} \mathrm{C}\right)$ map of the north side of plate position 2 at EOC 150B (total 95.6 EFPD).

Table B - 60: Temperature $\left({ }^{\circ} \mathrm{C}\right)$ map of the south side of plate position 2 at EOC 150B (total 95.6 EFPD).

Table B - 61: Temperature $\left({ }^{\circ} \mathrm{C}\right)$ map of the north side of plate position 3 at EOC 150B (total 95.6 EFPD).

Table B - 62: Temperature $\left({ }^{\circ} \mathrm{C}\right)$ map of the south side of plate position 3 at EOC 150B (total 95.6 EFPD).

Table B - 63: Temperature $\left({ }^{\circ} \mathrm{C}\right)$ map of the north side of plate position 4 at EOC 150B (total 95.6 EFPD).

Table B - 64: Temperature $\left({ }^{\circ} \mathrm{C}\right)$ map of the south side of plate position 4 at EOC 150B (total 95.6 EFPD). 


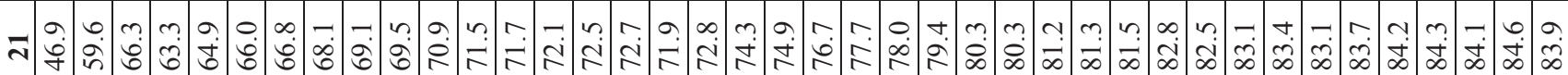

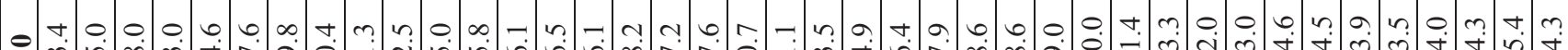

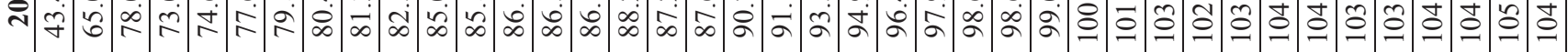

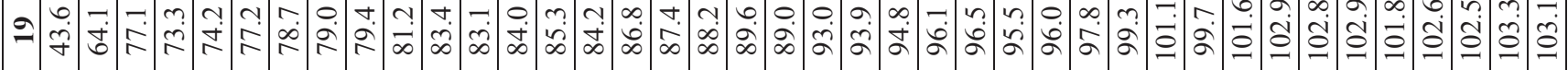

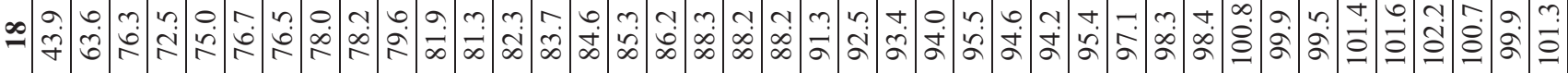

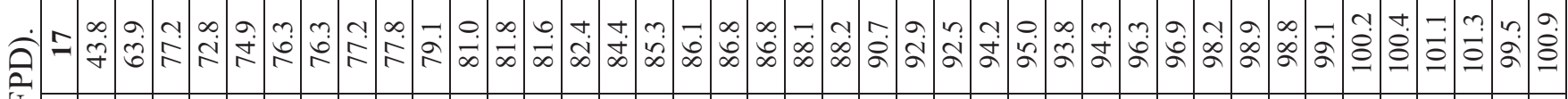

空

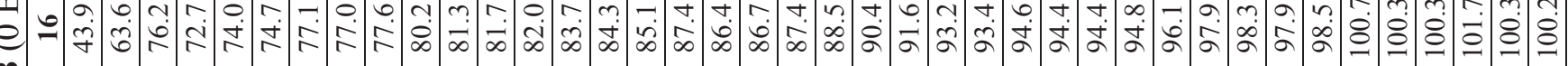

a

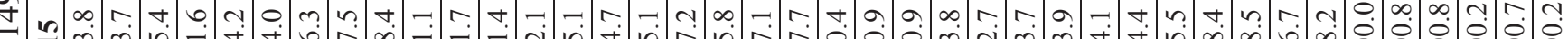

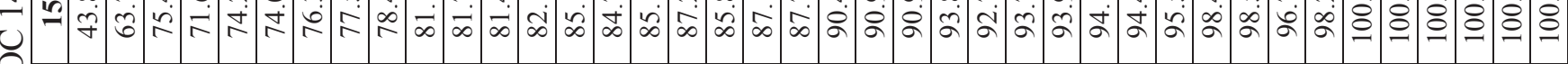
I

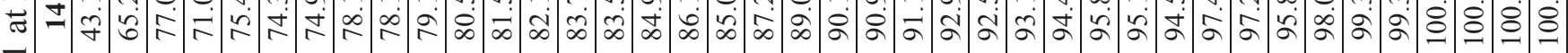

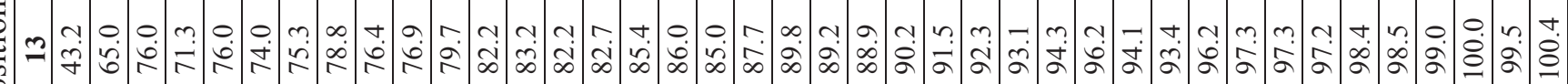

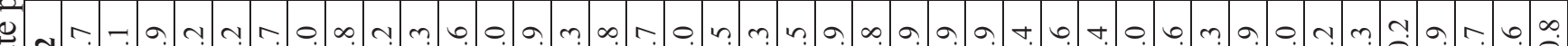

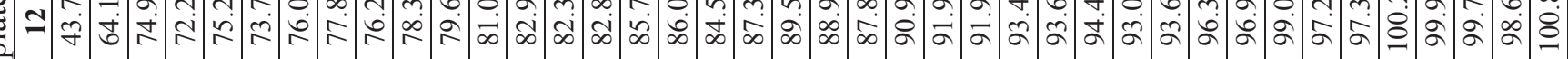

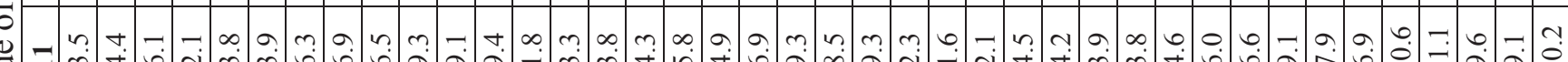

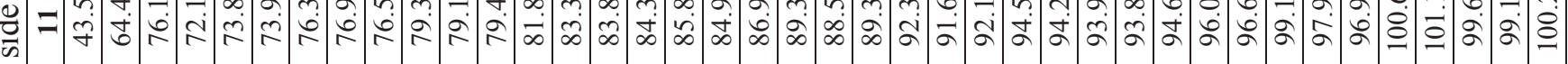

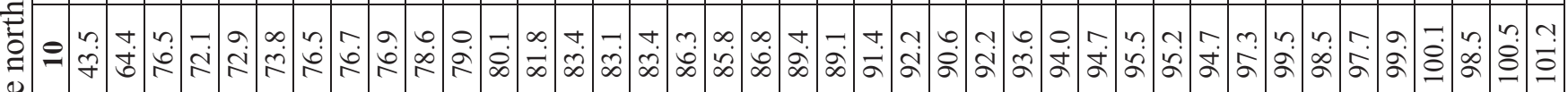

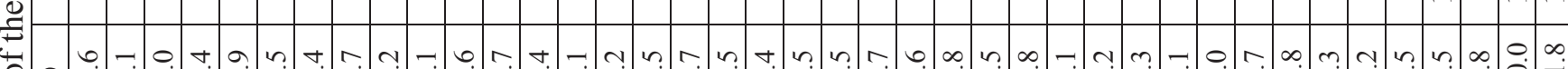

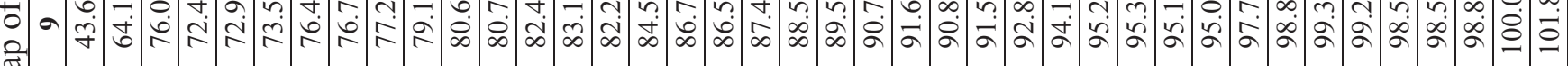

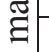

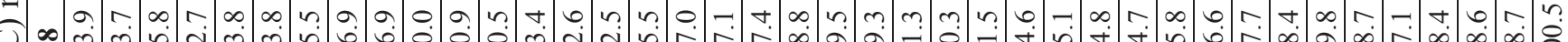

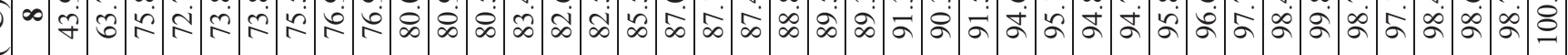

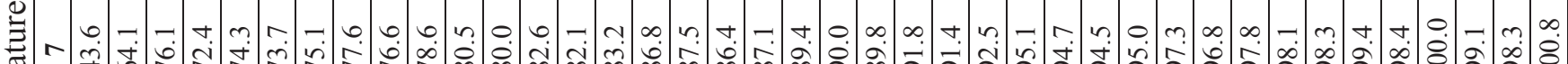
可 馬 $\ddot{-1}$

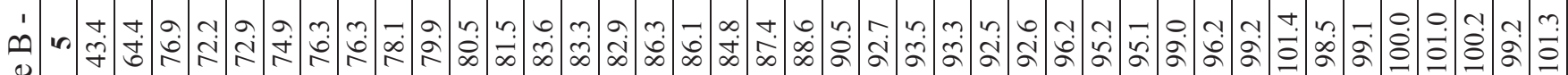

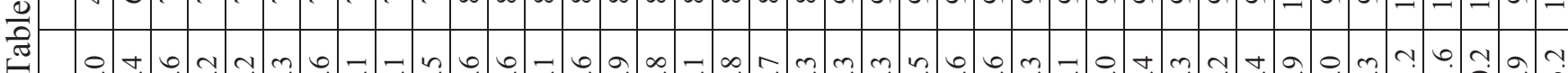

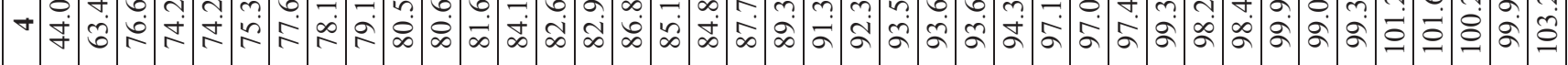

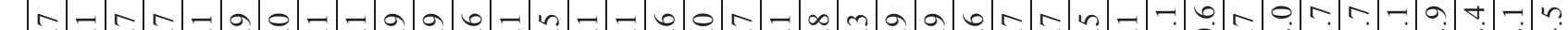

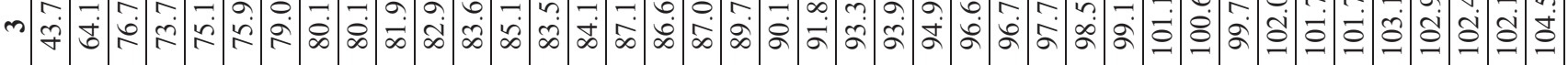
r. n

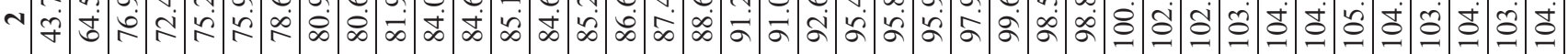

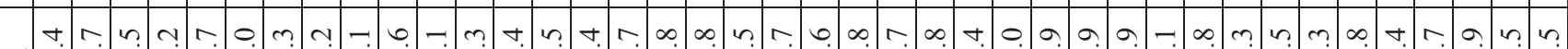

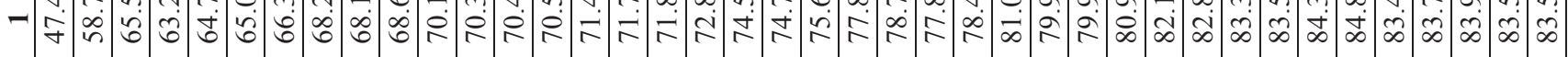
-

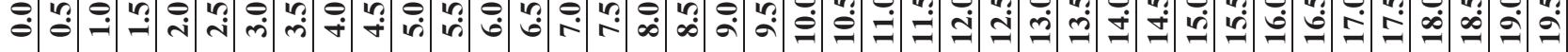




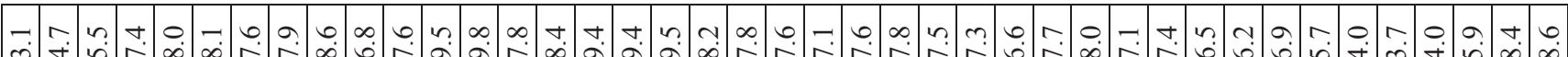

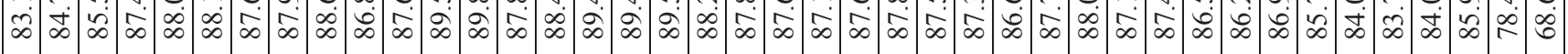
ᄀ央 仓ी

O t d

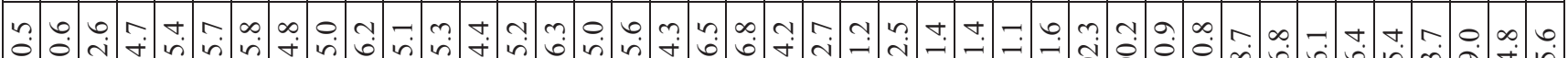
ఏ丶

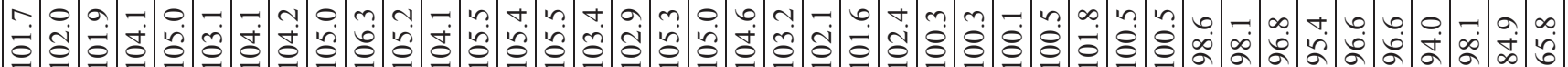

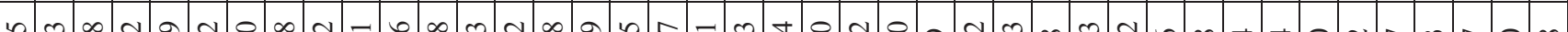

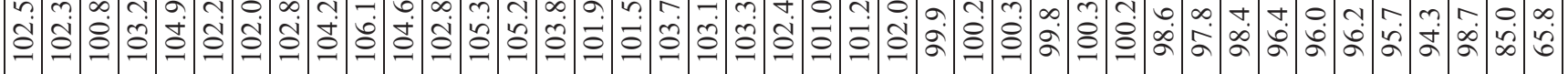

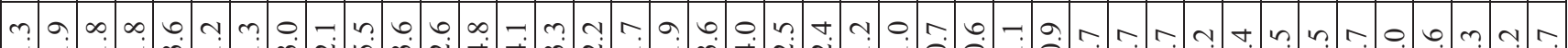

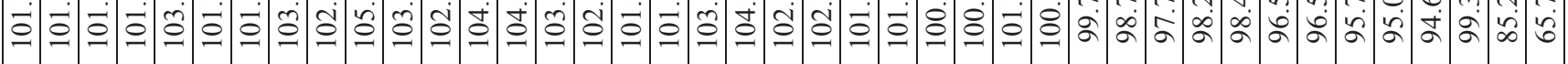

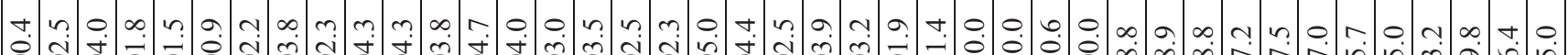
¿ी J:

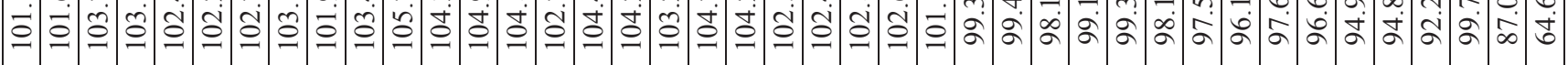

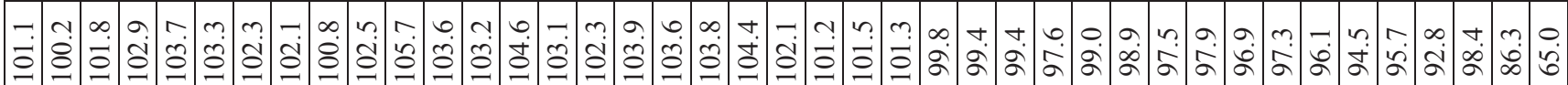
+

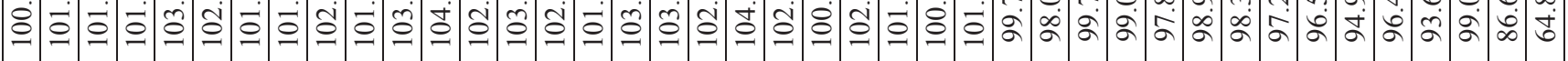

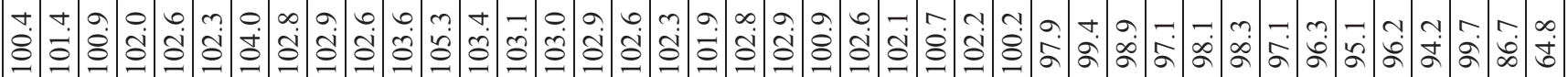

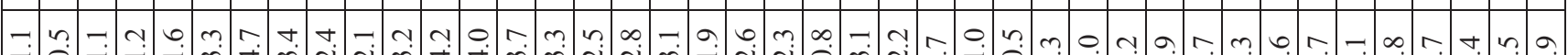
ఏ) c @) t. m 0 c c 0 . ఏ) m.

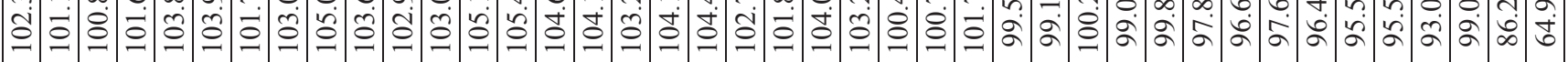

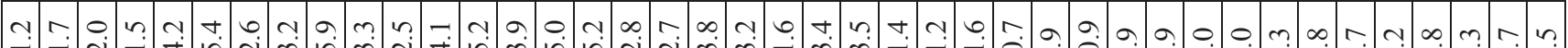
ఏ) c 0 t. t r m r r n

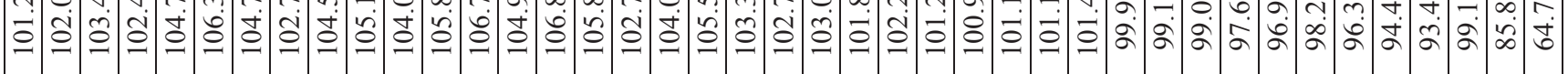

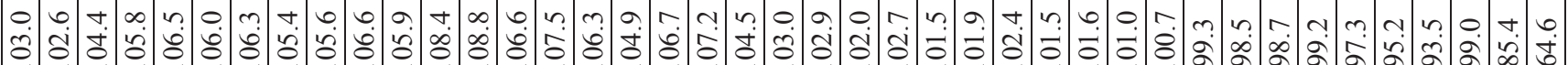

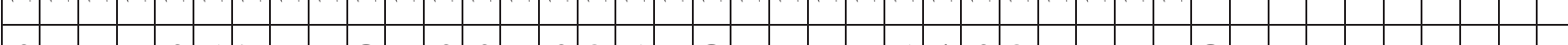

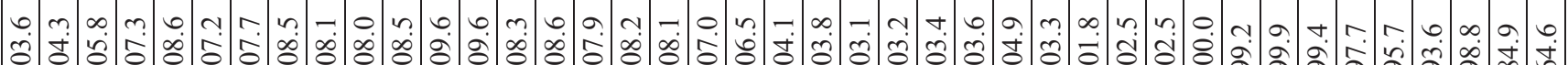
仓ิ)

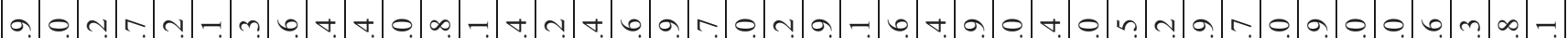

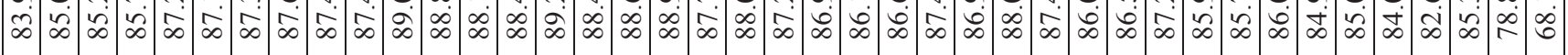

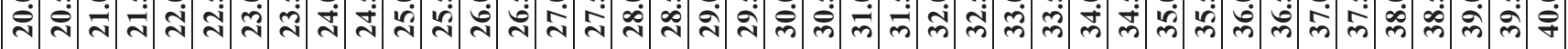


च $\infty+\infty$

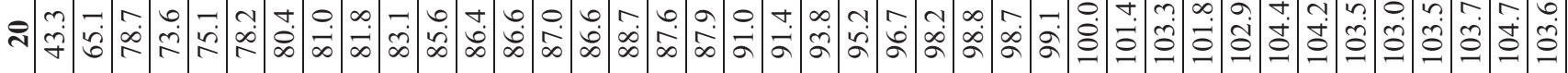

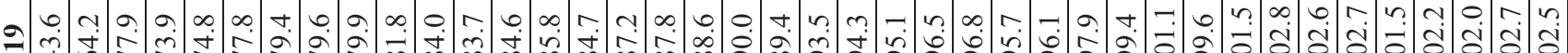

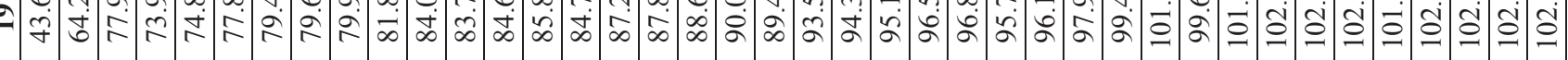

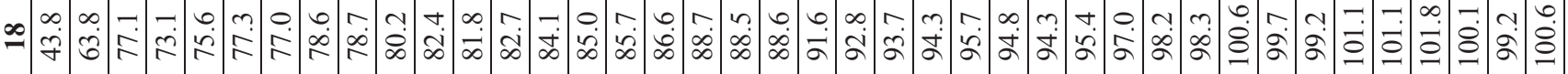

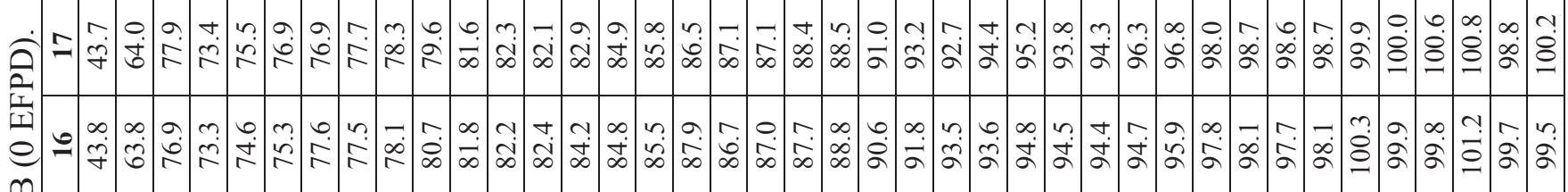
a

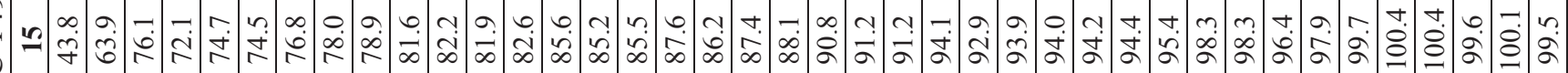
○)

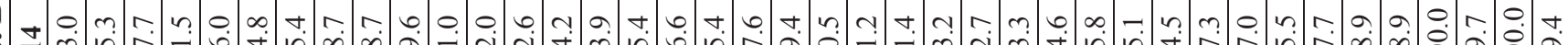

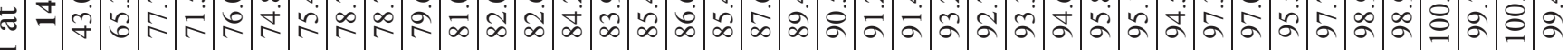
כ

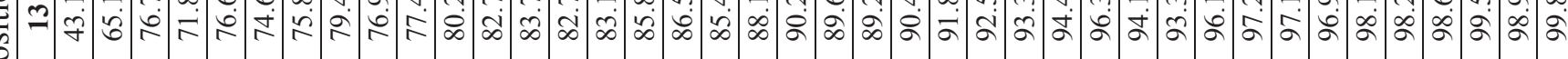

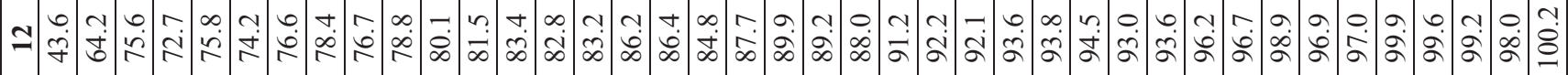

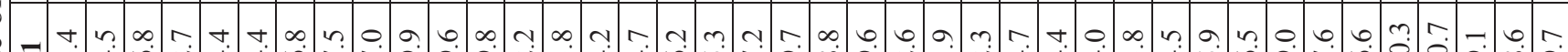

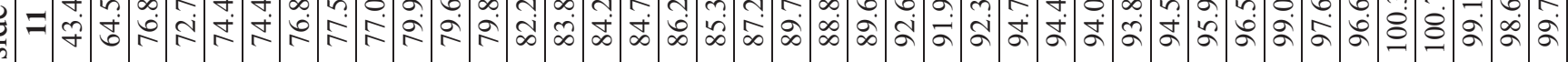

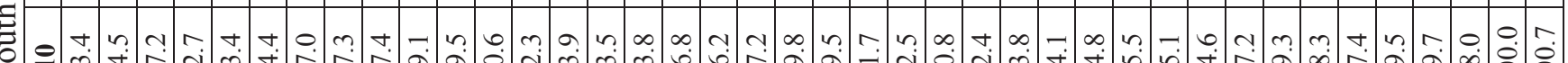

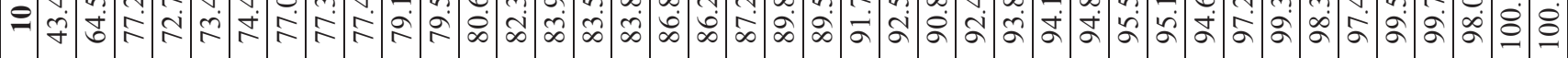
$\stackrel{0}{ \pm}$

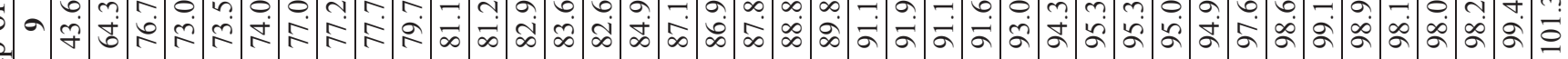
:

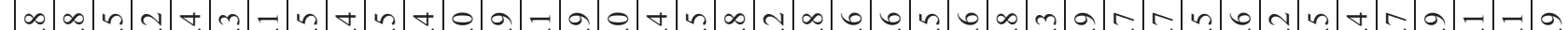
事

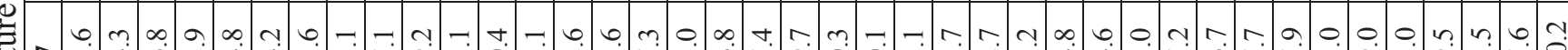

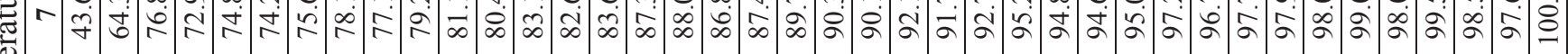

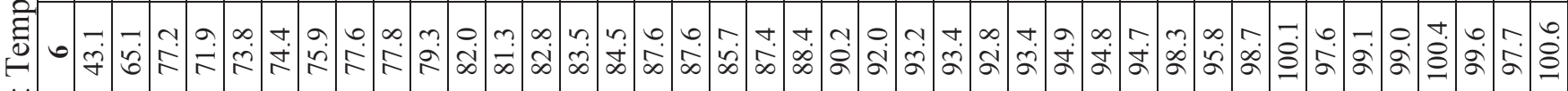

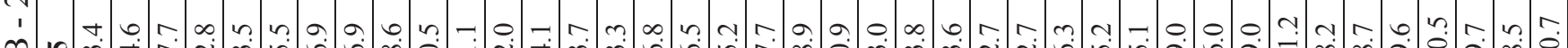

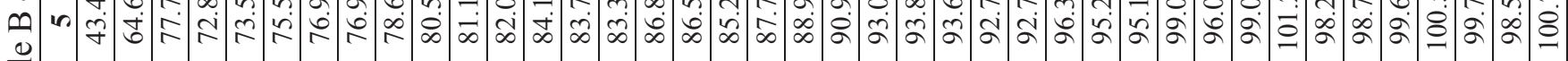

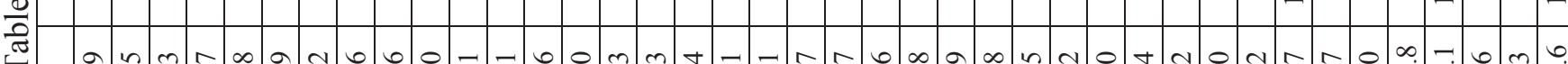

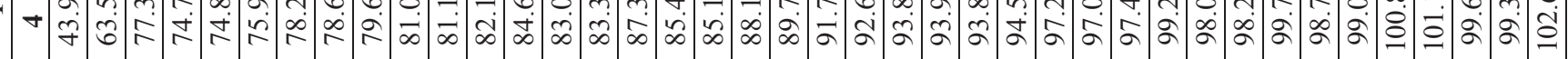

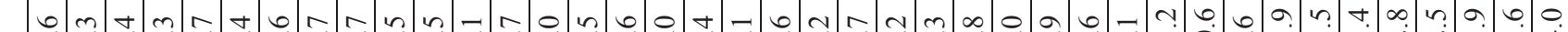

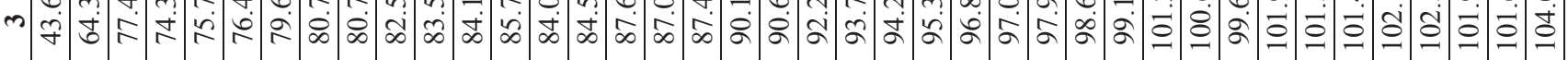

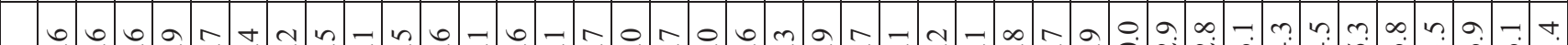

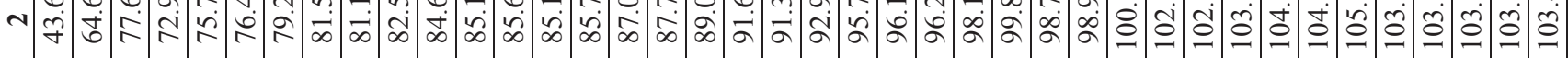

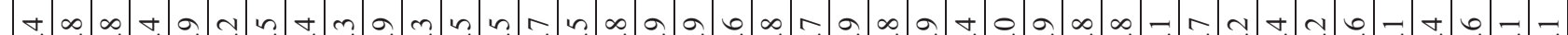

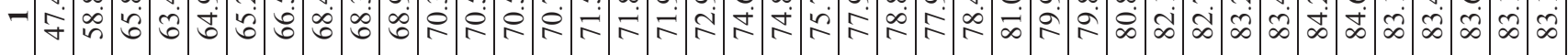
审 


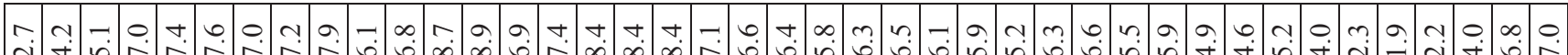

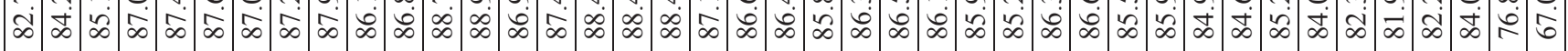

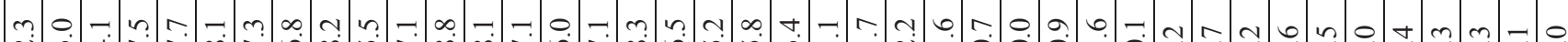
ปீ m б으 ठㅇ - n

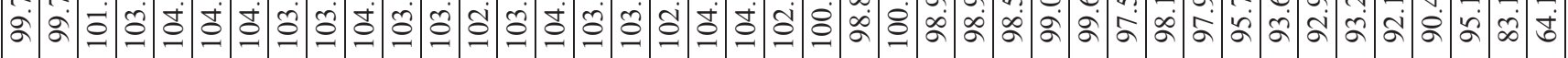

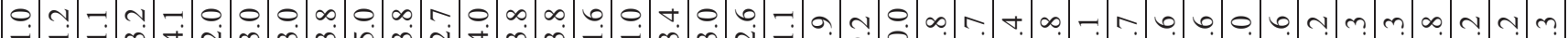

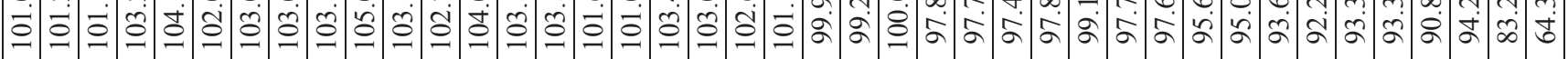

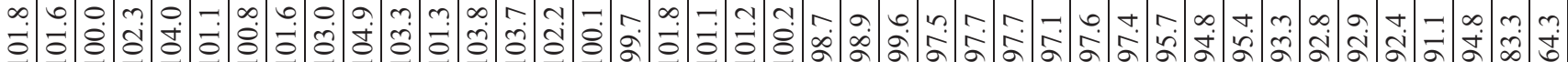

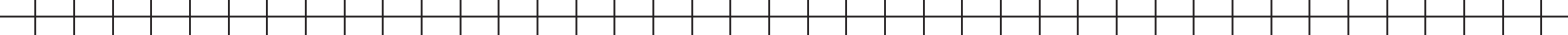

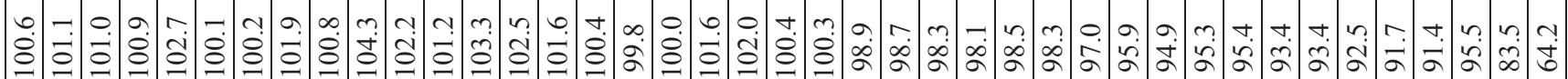
ᄀ.

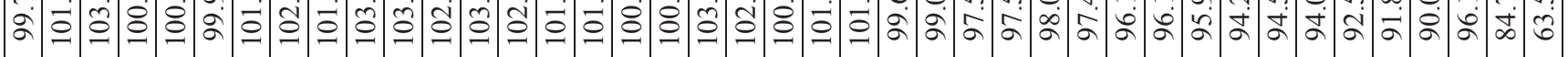

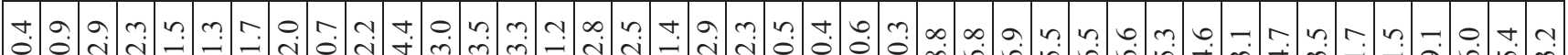
过

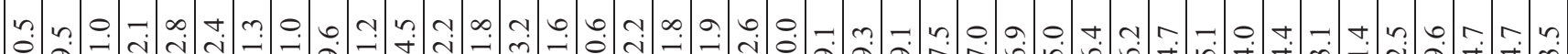

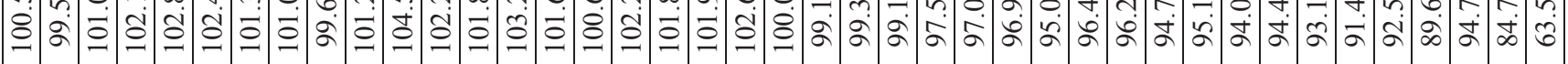

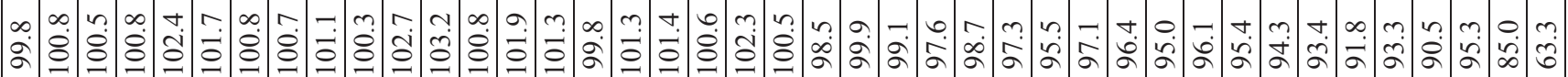

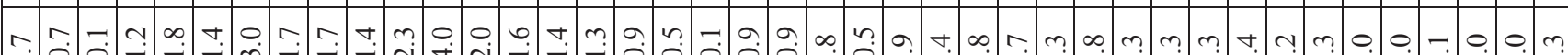

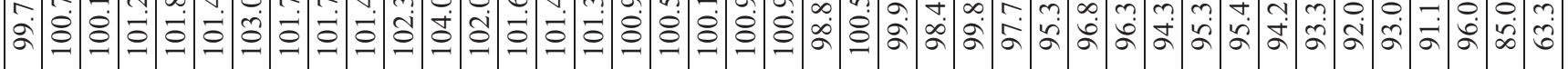

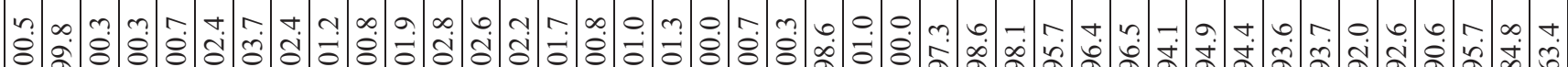

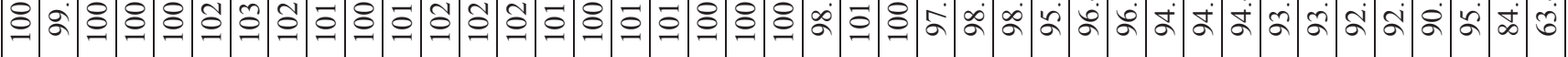

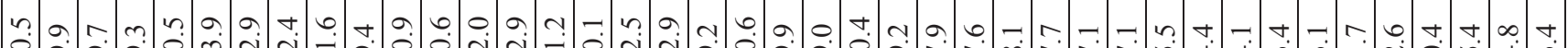

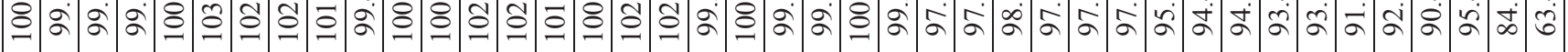

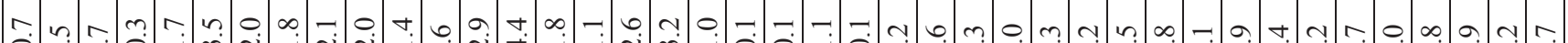
இें o. m.

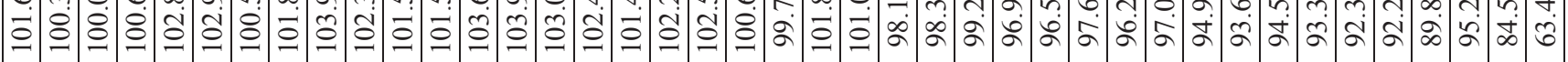

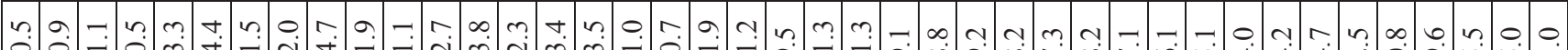

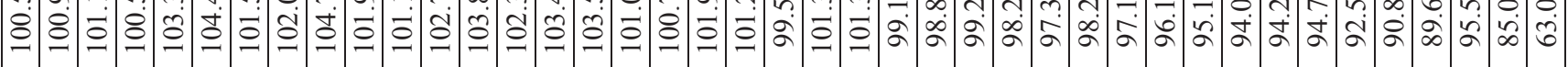

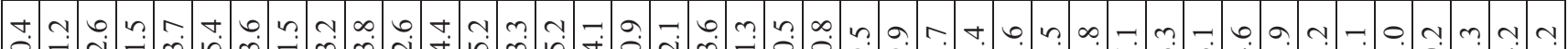
¿

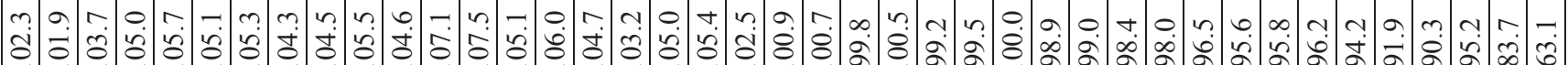

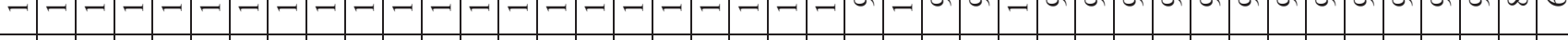

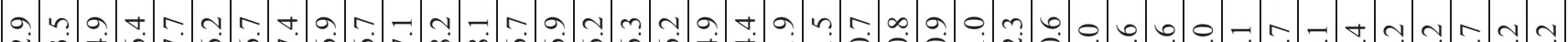
过

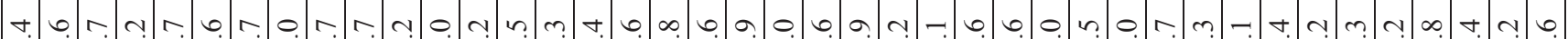

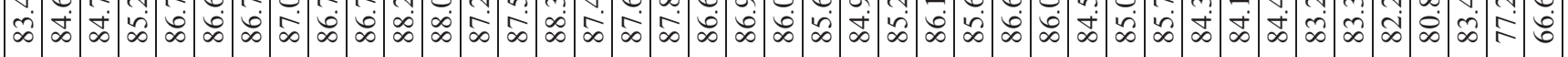

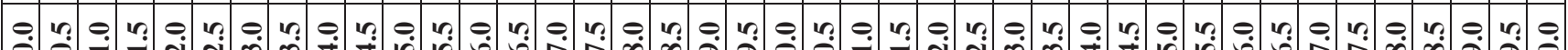

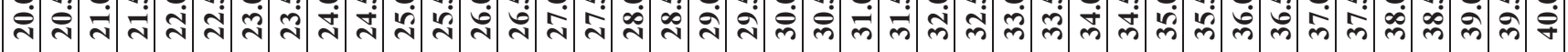




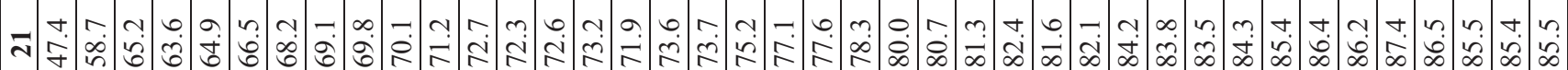

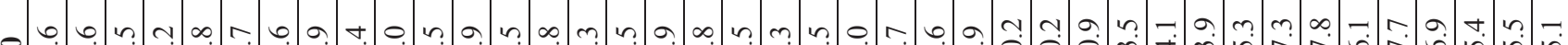

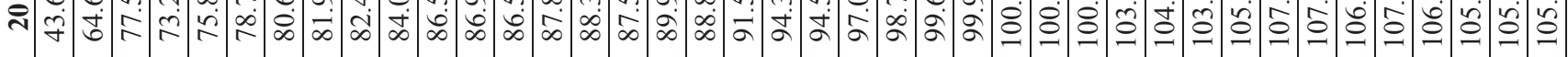

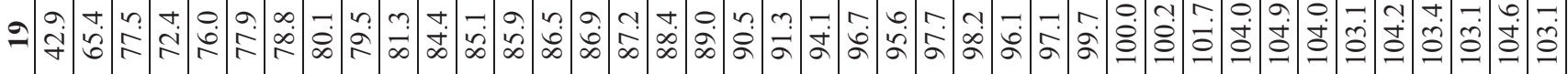

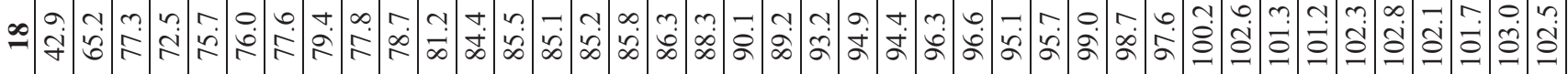

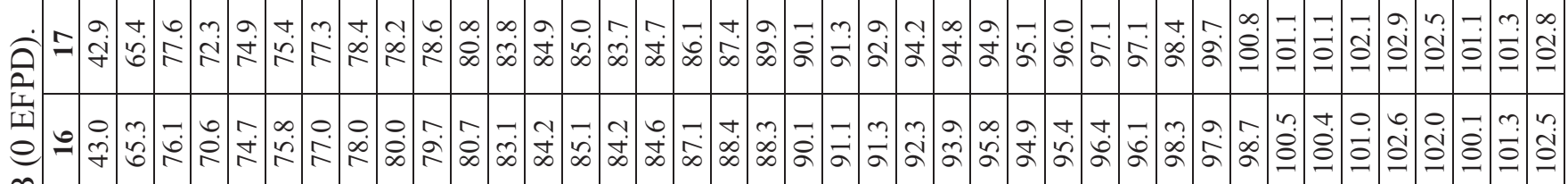
a

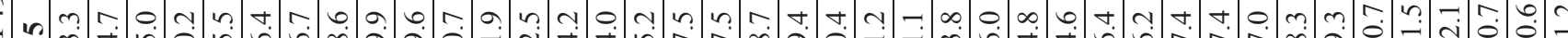

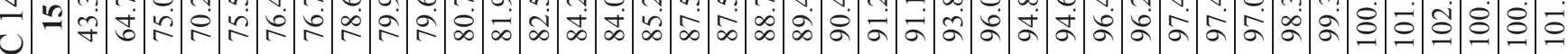

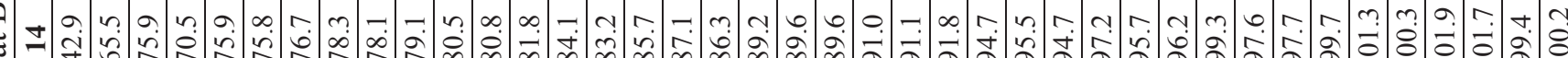

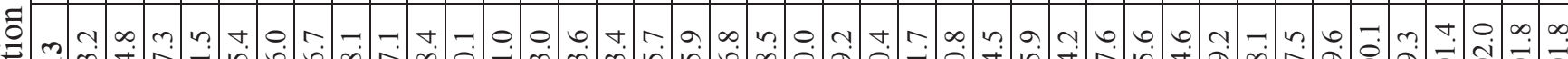

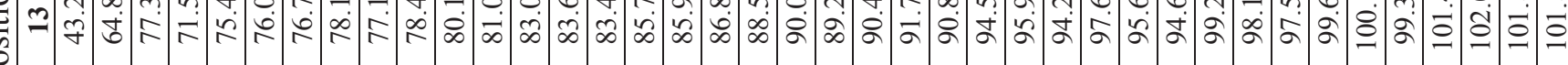

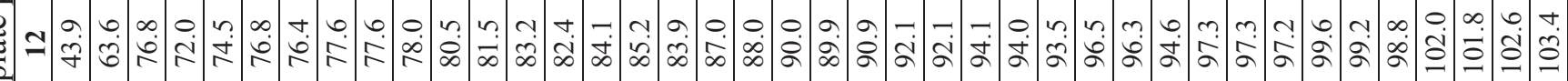

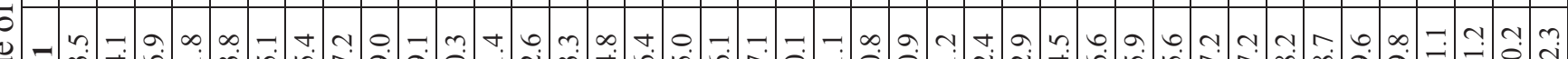

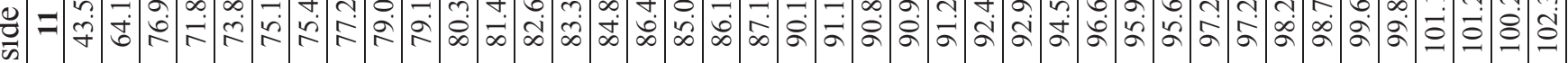

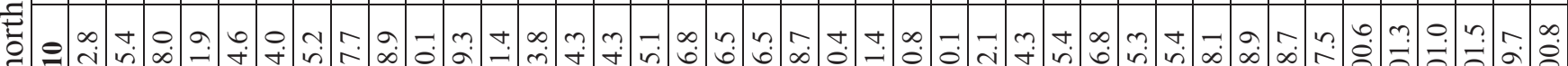
fं $\Xi$

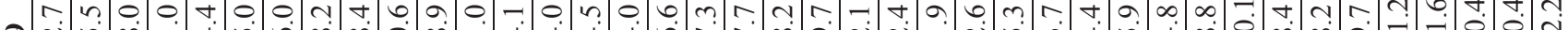

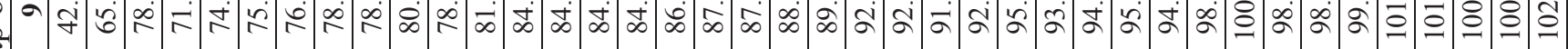

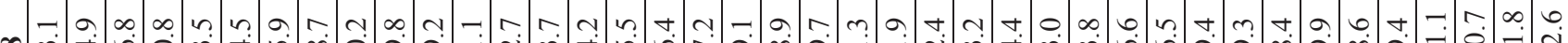
क fं

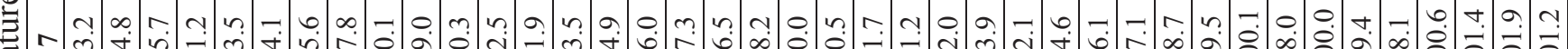

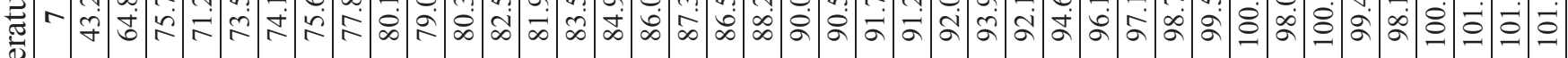
苟 m

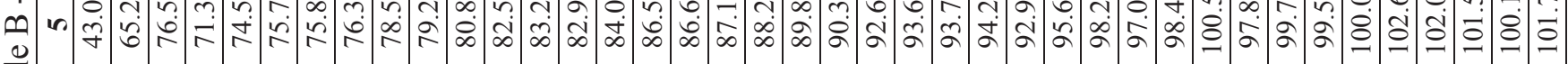

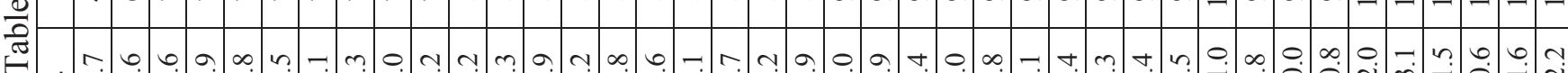

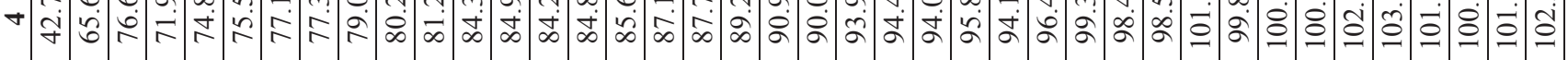

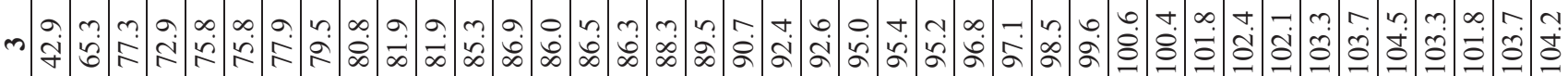

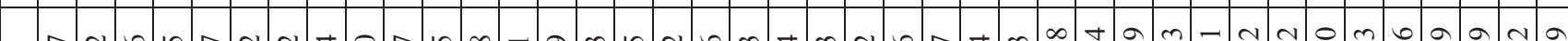

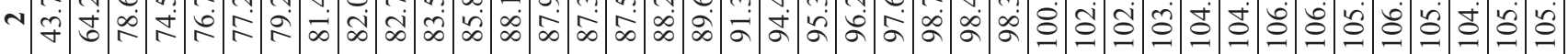

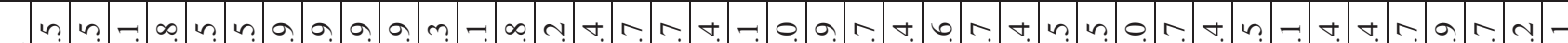

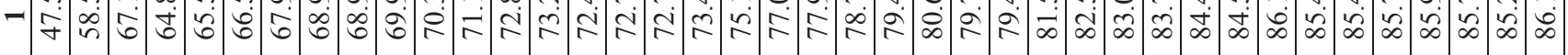
-

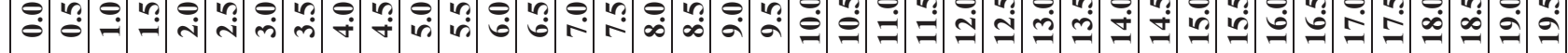




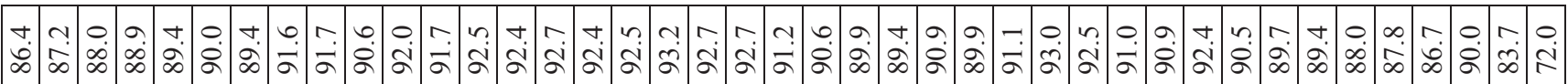

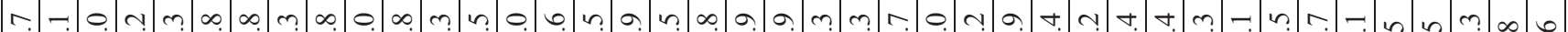

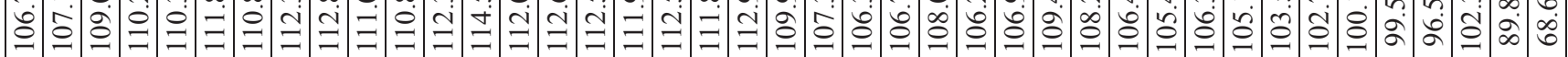
- 幺

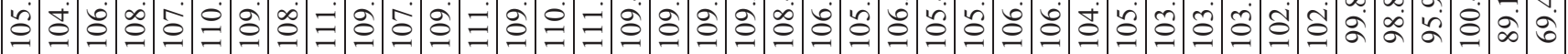

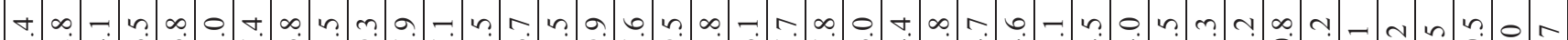
仓ी N.r.m. 舟全全

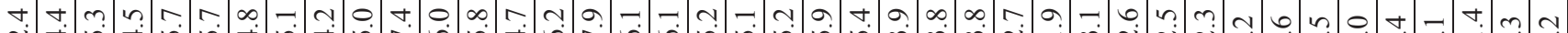
ḋ ت: 휘

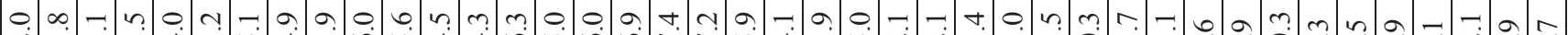

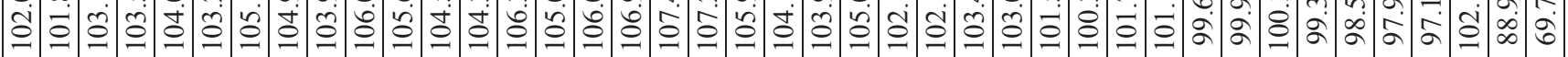

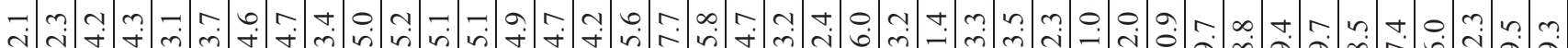

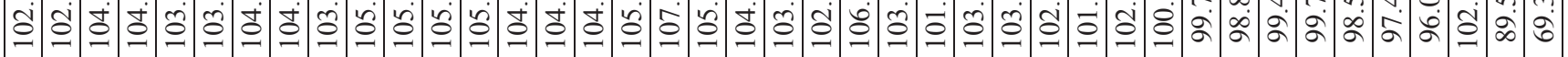

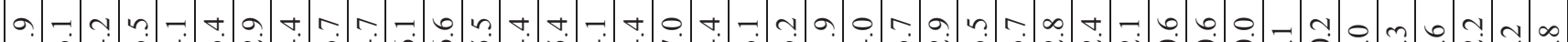
ఏ人 0 过

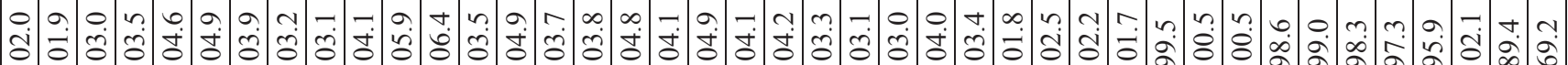

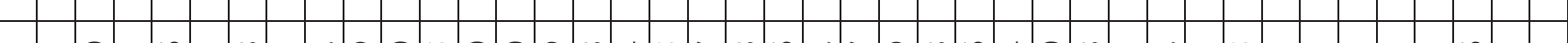

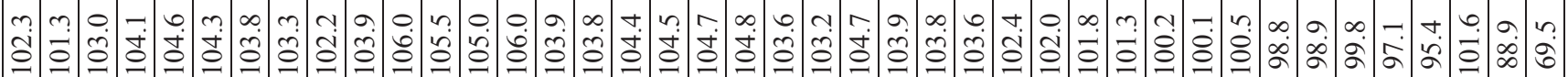

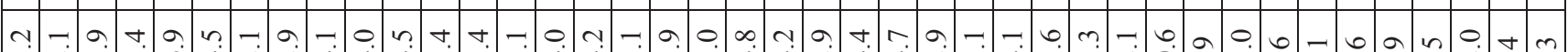

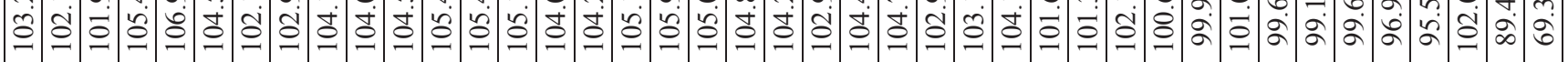

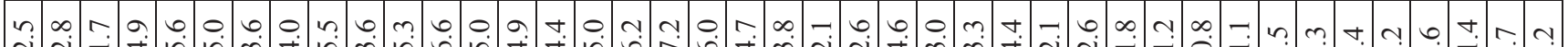
仓ิ

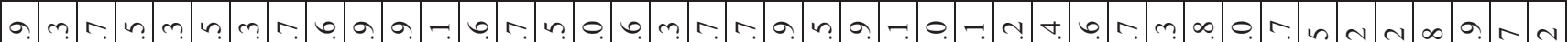
仓े

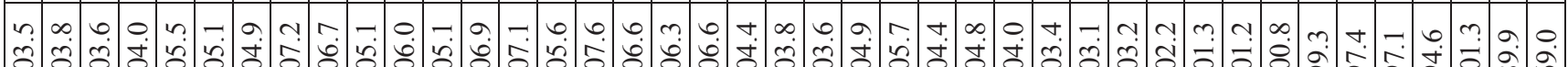
仓

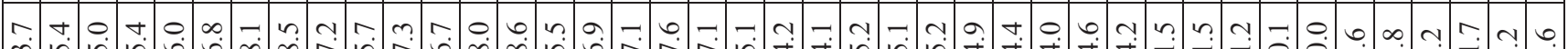

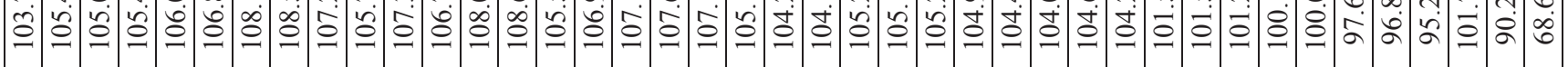

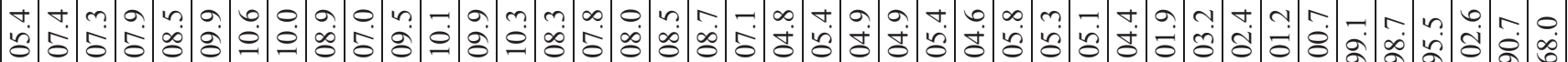

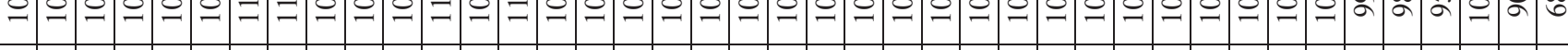
$\infty$ c c 0.0 . ఏ리

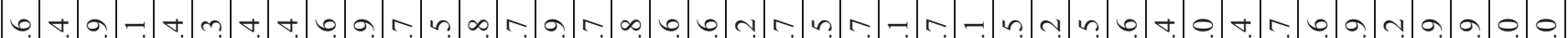

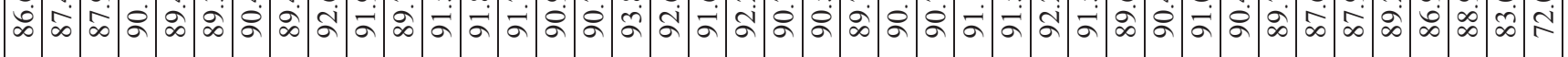




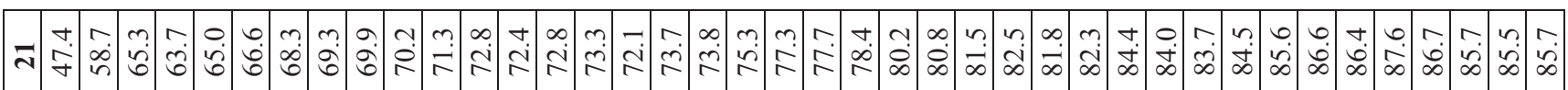

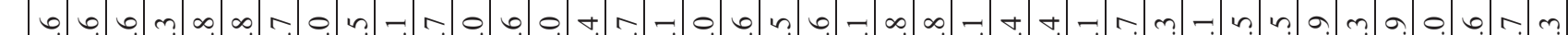

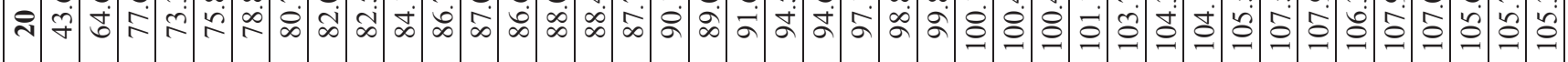

m n r n

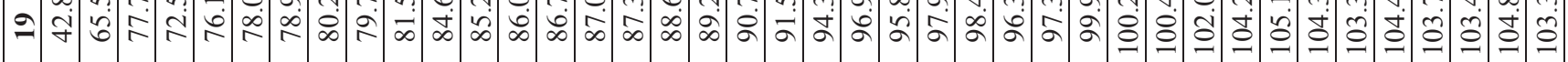

a c n

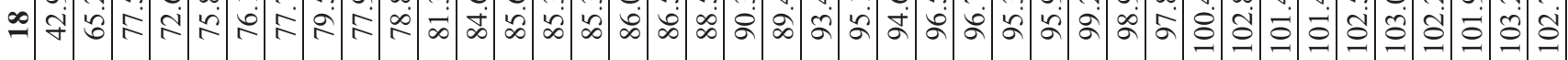

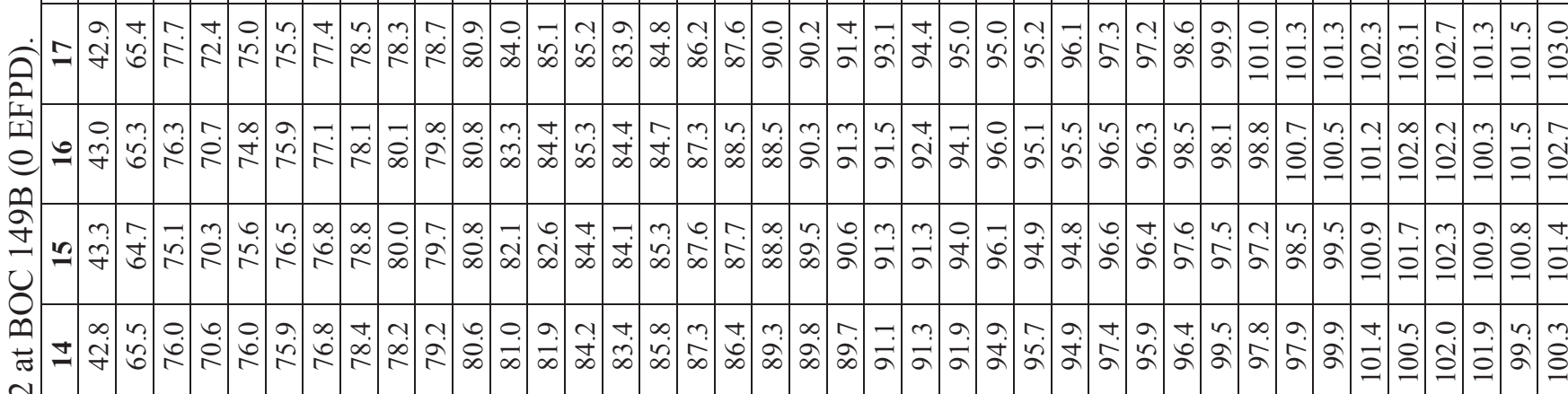

f

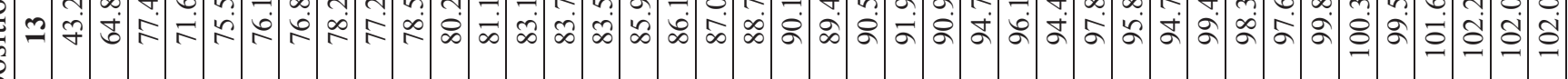

ง 光 =

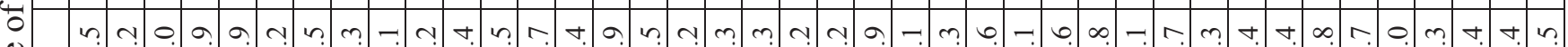

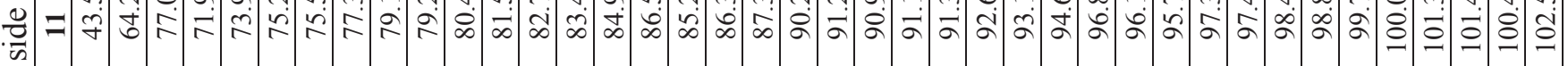

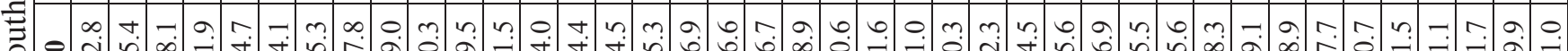

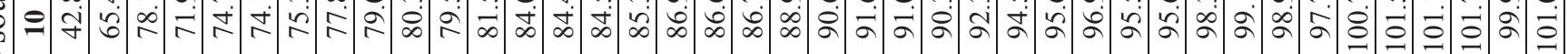
$\stackrel{8}{\ddagger}$

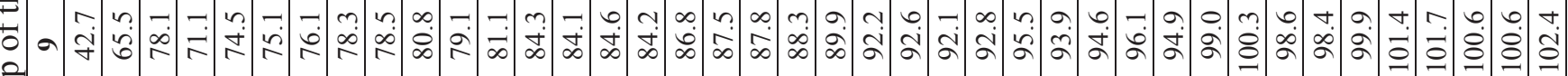
్ㅕㄹ

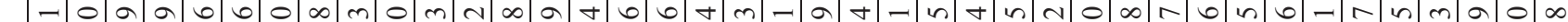
$\infty$ fं

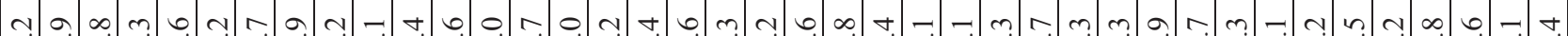

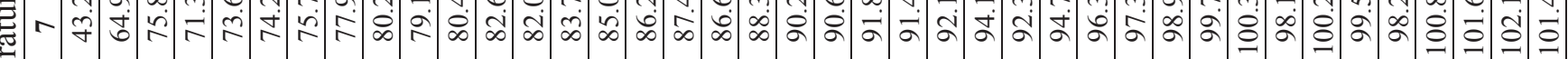
ڤ్)

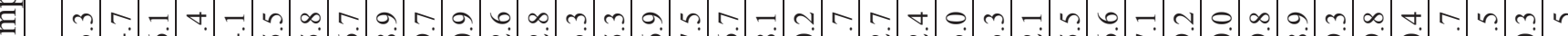

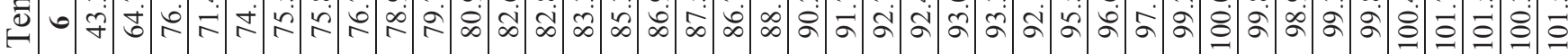
r

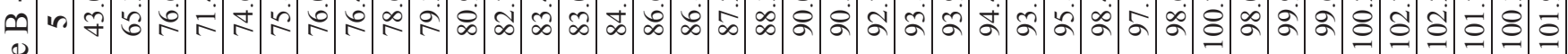

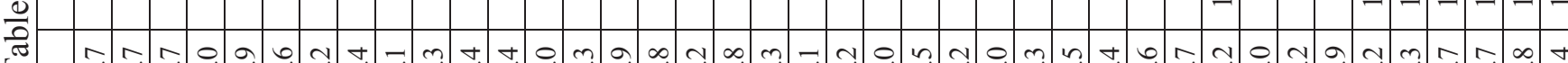

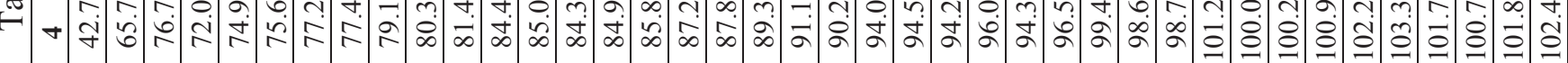

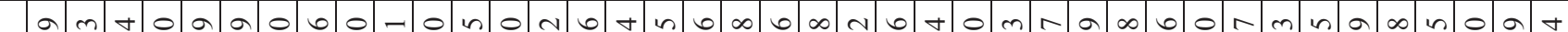
m jل

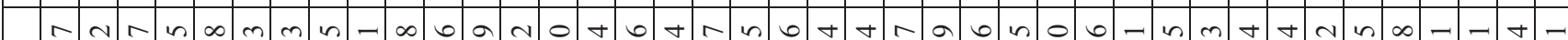

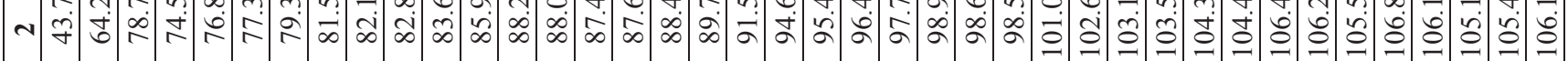
n n

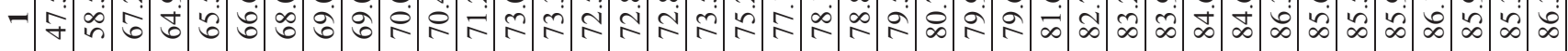
事 


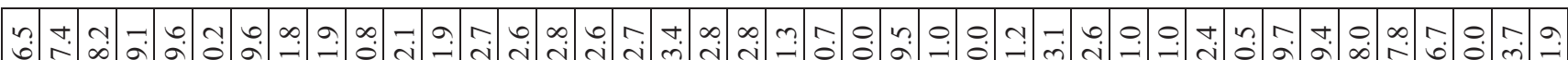

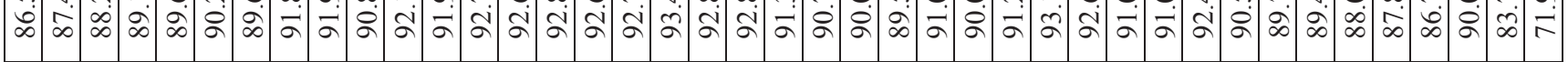
a c c c t n

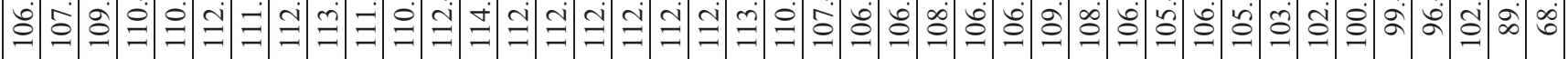

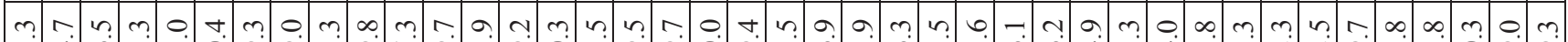
ఏ人 o. ف의

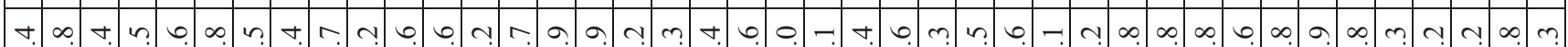
ف일

n ¿ी m c c

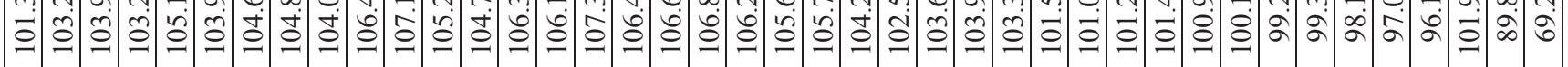

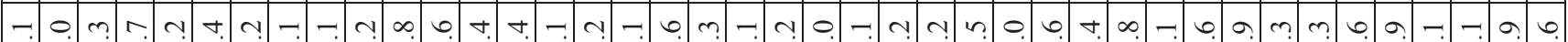
de

c t t ¿ి

ᄀ m m.

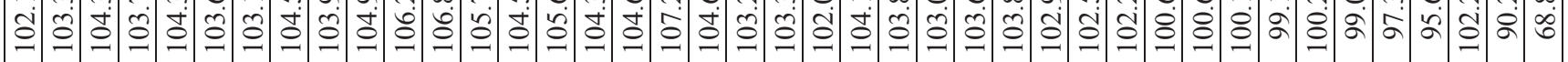

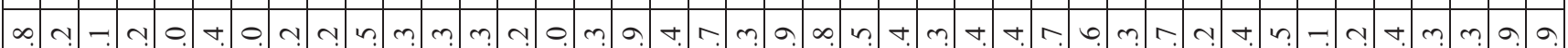
dீ

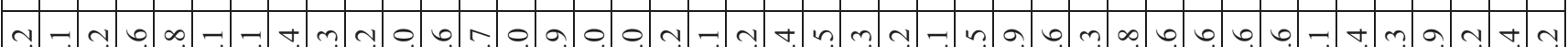
ḋ

n

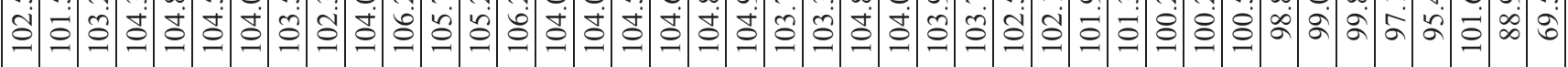
m m 仓ी

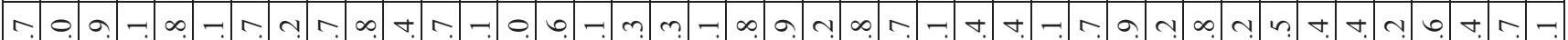
¿̇

- t $\infty$ n n 0 n $a$. 仓̊

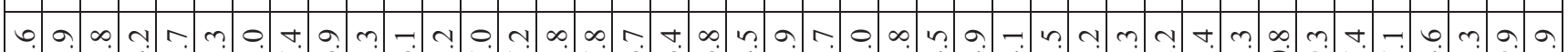
فे a. bे

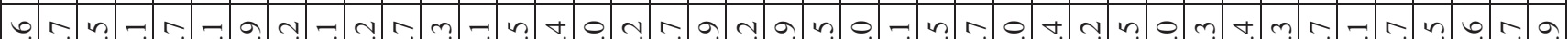

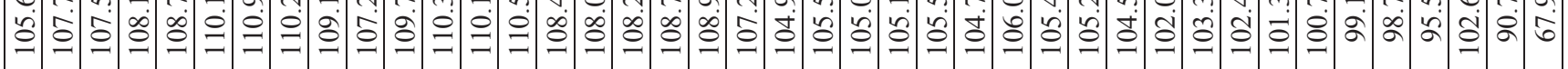

o.

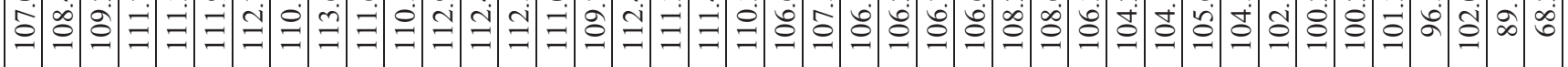

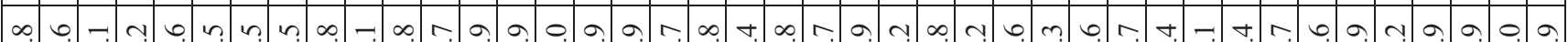

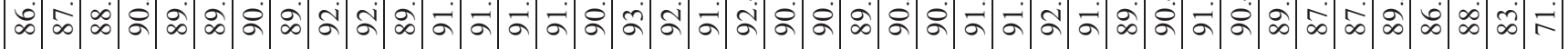




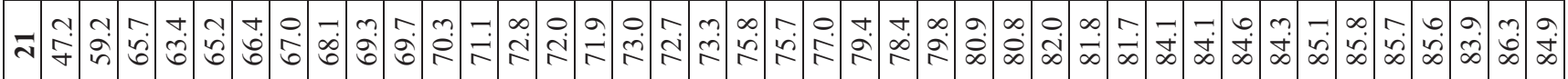

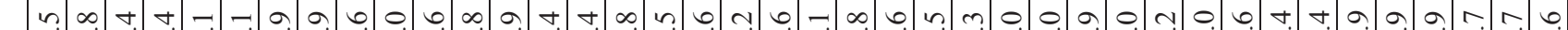

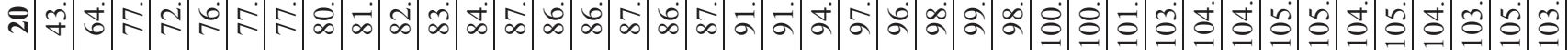

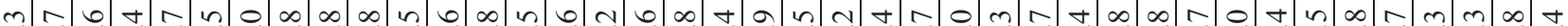

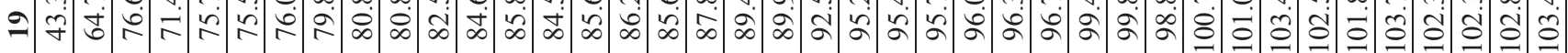

n m 0 o n m o o

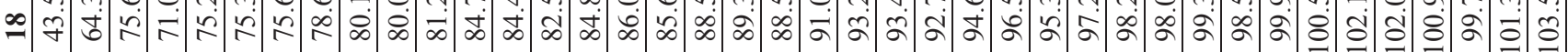

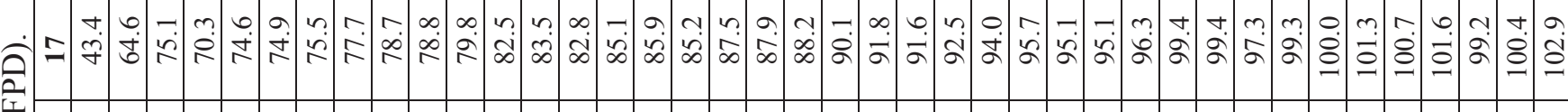
I e =

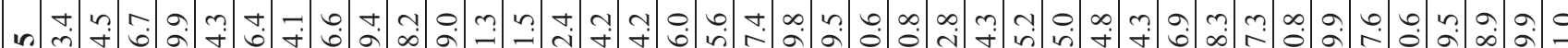
○ $\exists$ F

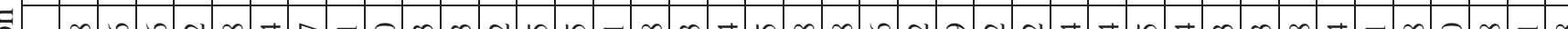

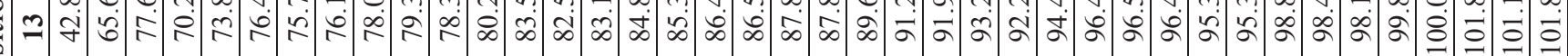

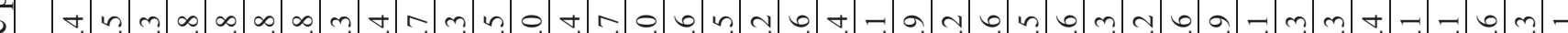

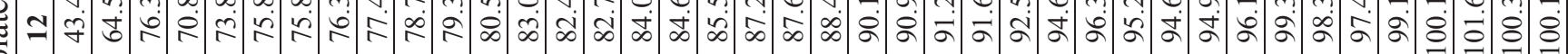

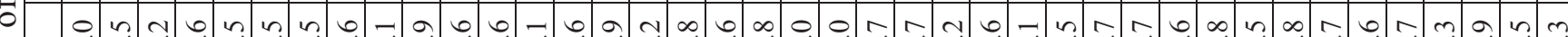

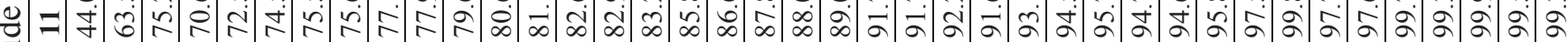

(7)

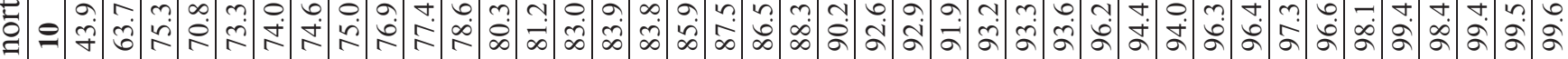
苞毛 :ี

- r to t m 0 + $\infty$ fं

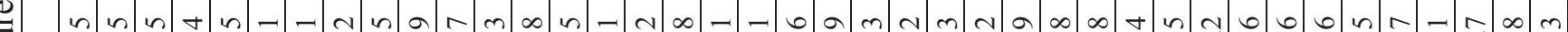
矛 :

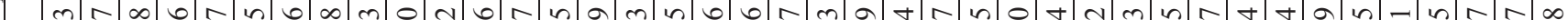

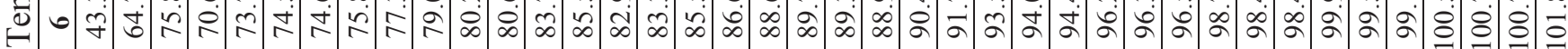

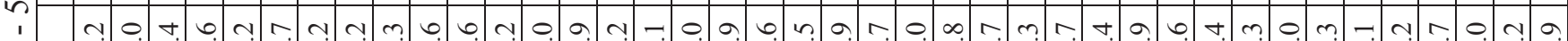

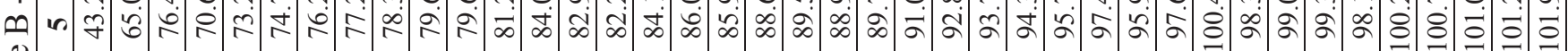
$\frac{\pi}{a}$
0

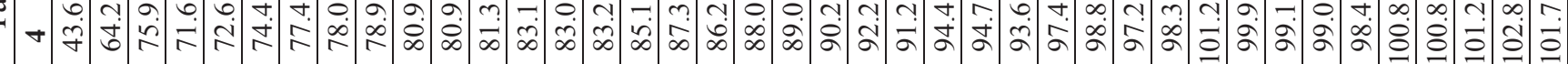

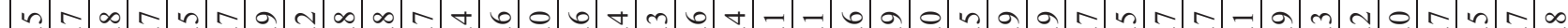

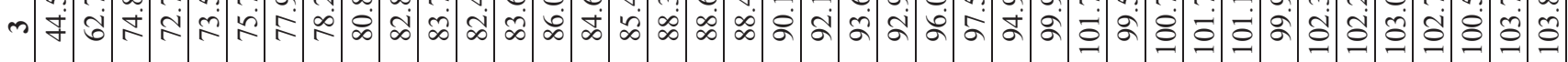

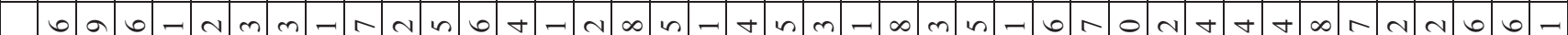

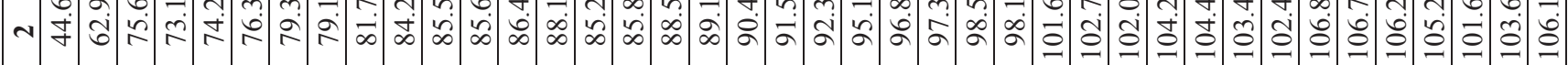

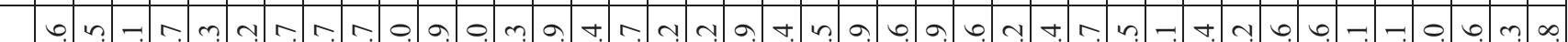

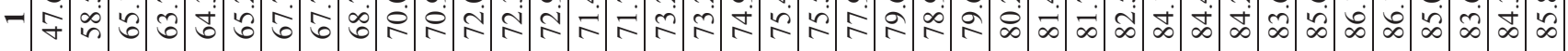

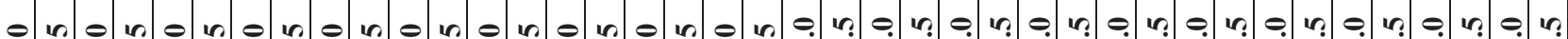

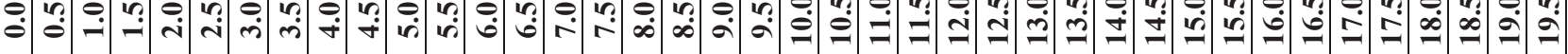




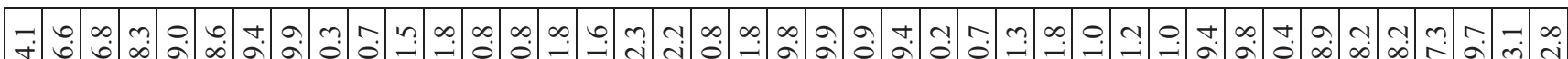

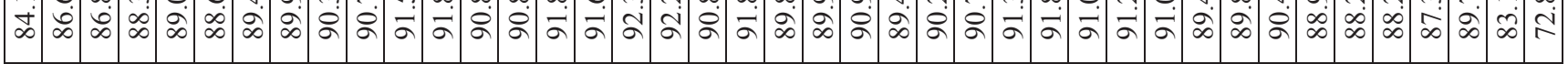

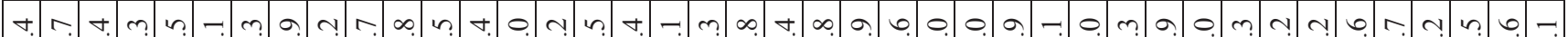

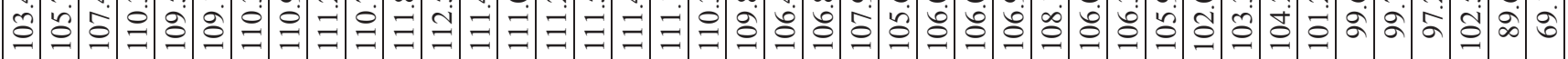

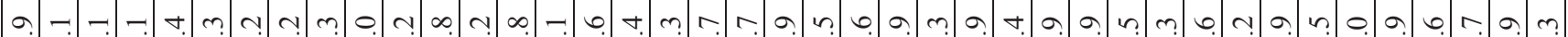
¿े 八 t 仓ิ)

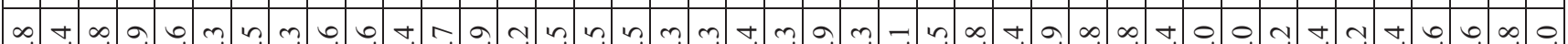
ḋ п m

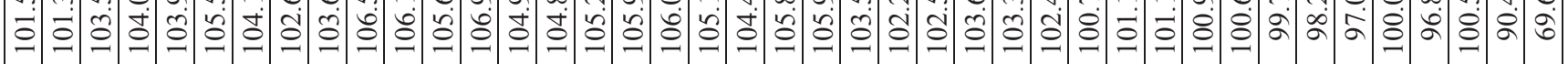

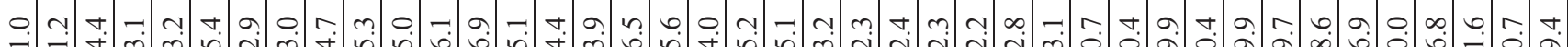
ఏ) †. 0.0 .

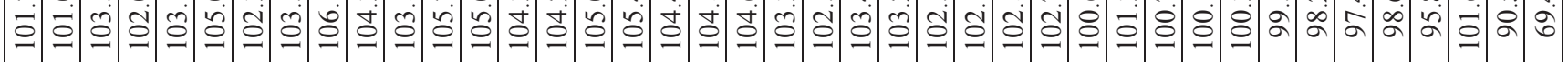

t $\infty$ c m m $\infty$ a r n

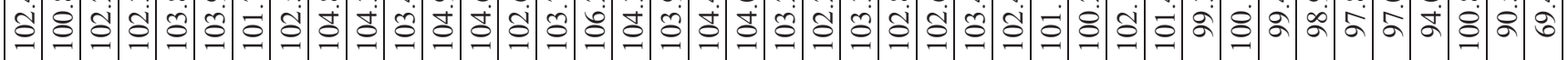

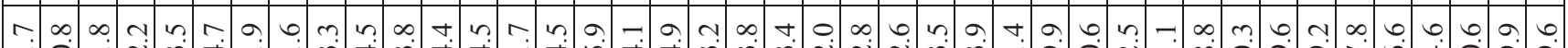

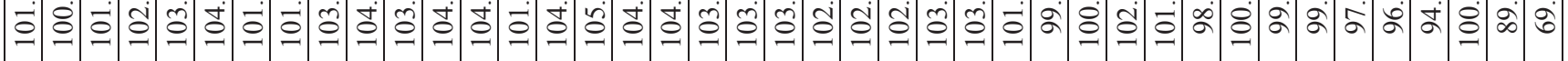
n

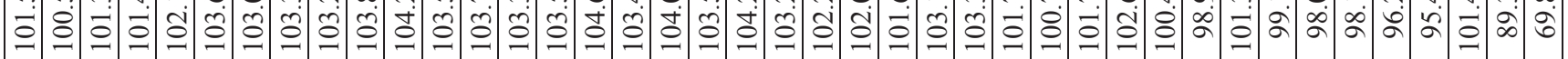
o r

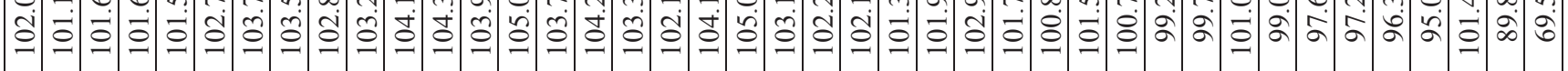

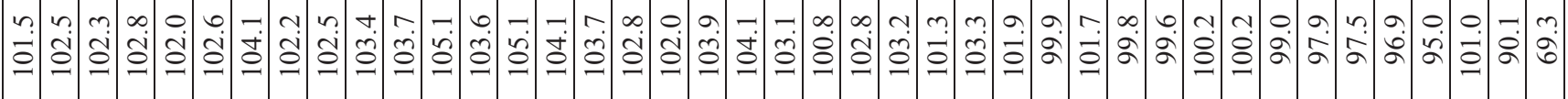

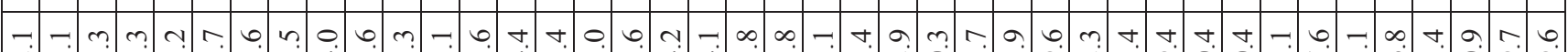
仓ิ

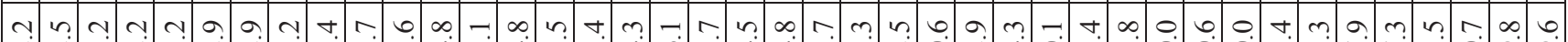

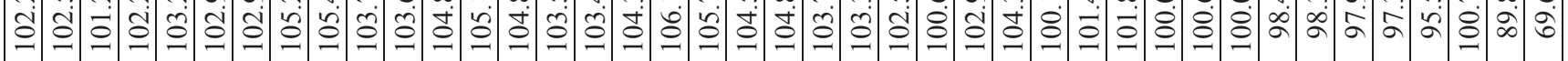

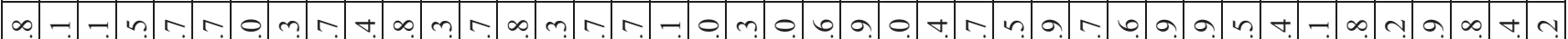
¿ी

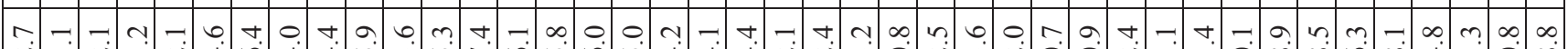

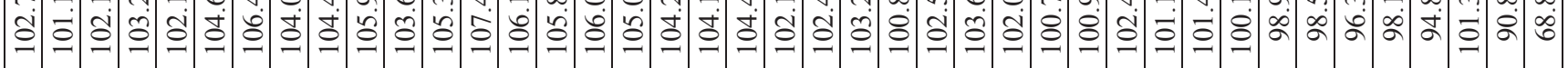
o. ḋ

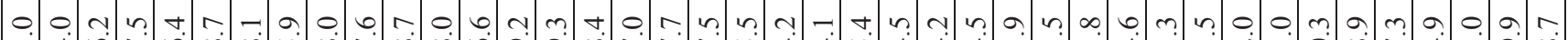
فำ

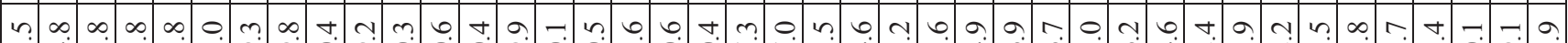

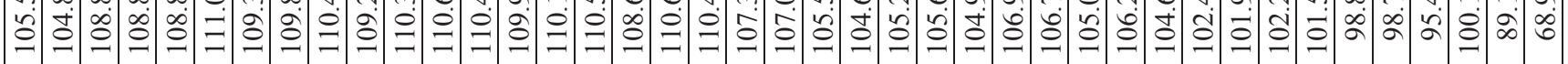

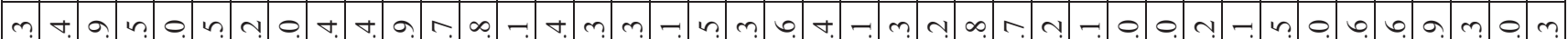

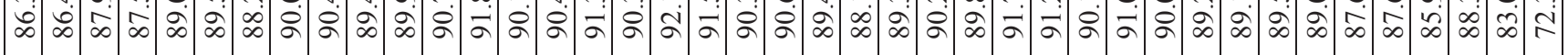




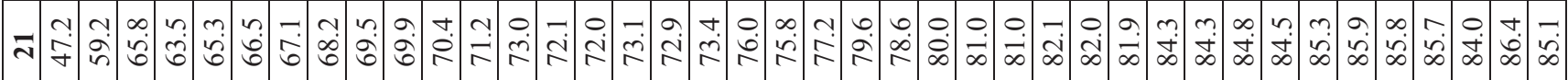
$\sin \infty+4-0$ or จิ f)

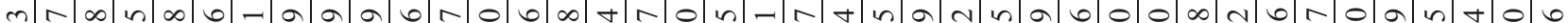

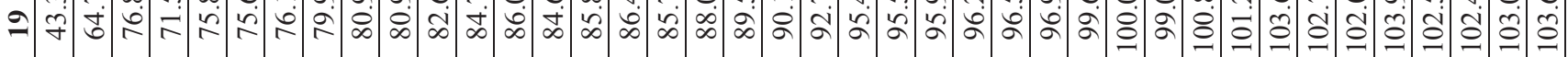

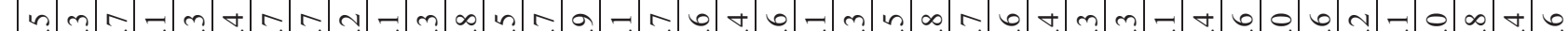

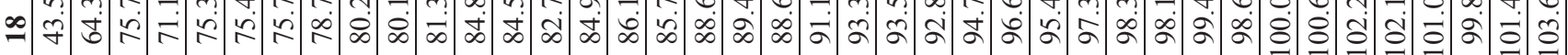

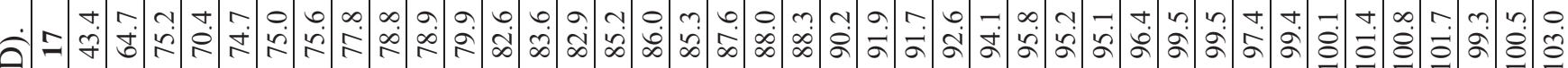
它

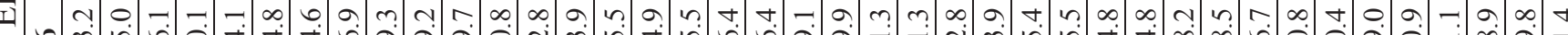

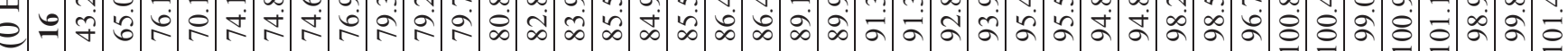
ติ

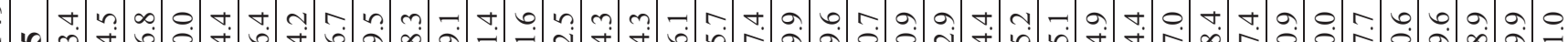
O ma otra

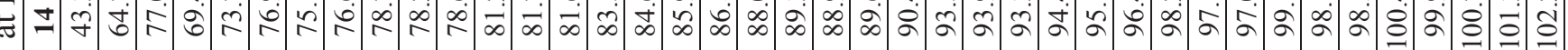
7

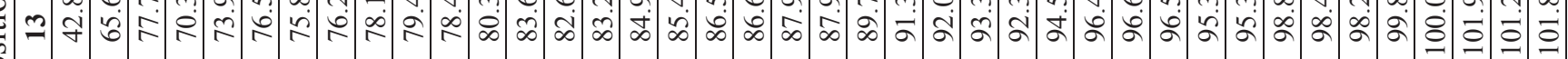

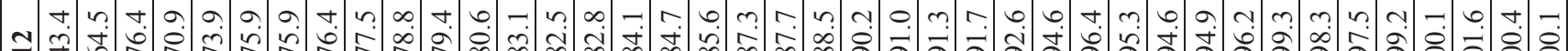

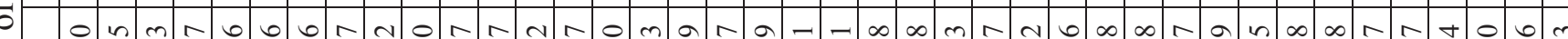

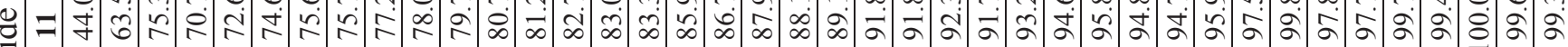

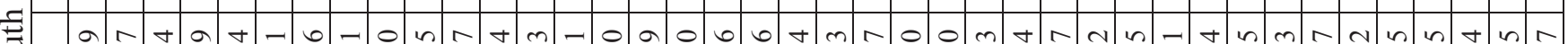

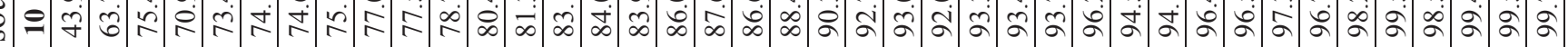
$\stackrel{0}{ \pm}$

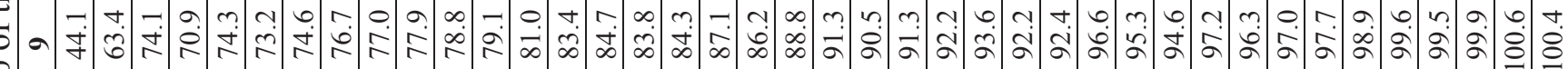
పี

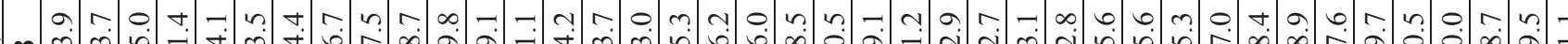

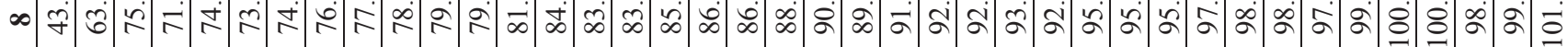

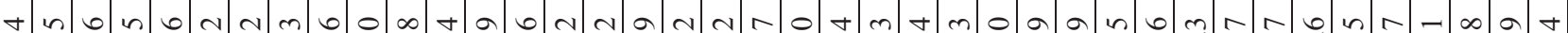

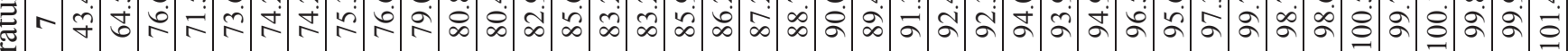
Фे.

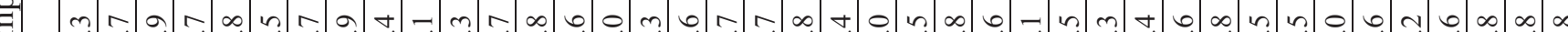

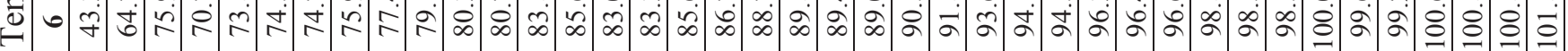

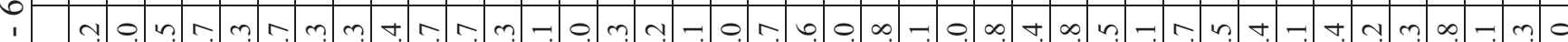
๑ n a

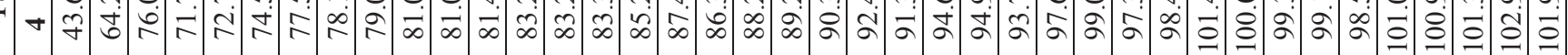

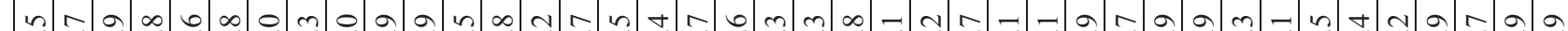

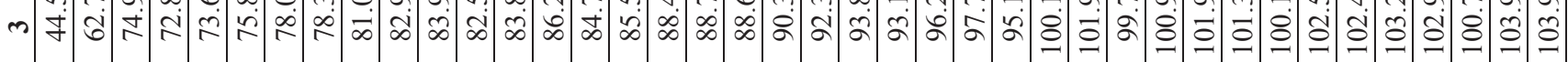
o a $a$ a m t t t a

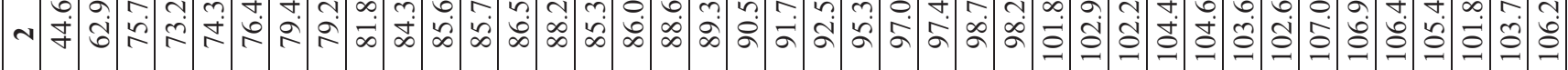

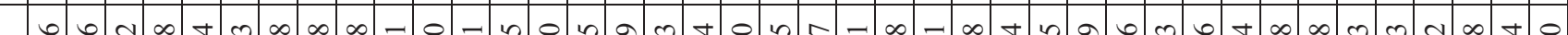

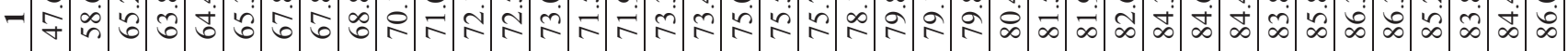

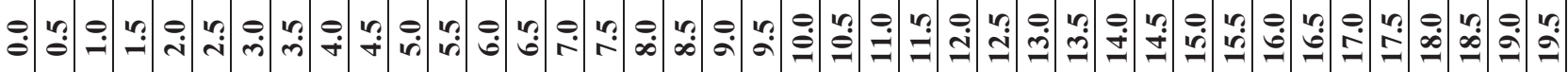


บ

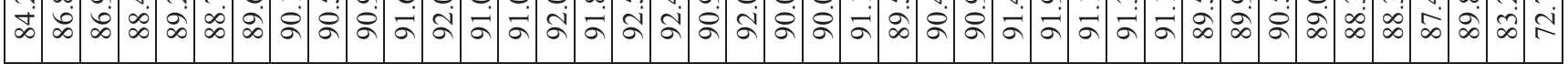
n 仓

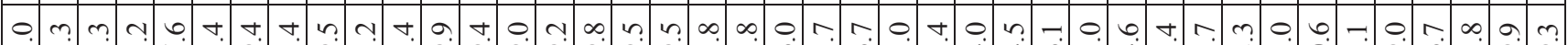
仓ी

m n ¿े

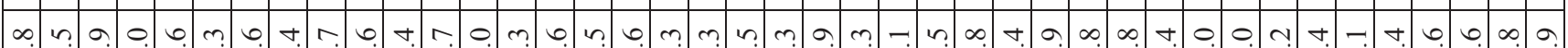
ḋ o t ఏ)

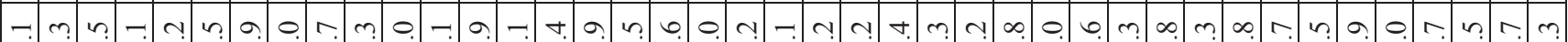
을

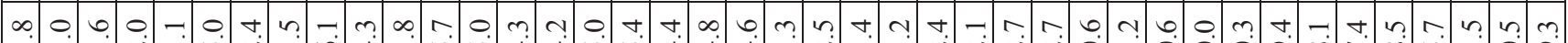
ఏ)

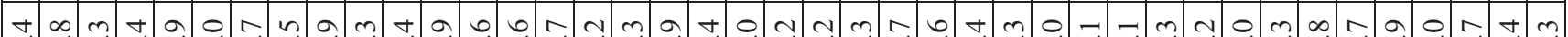

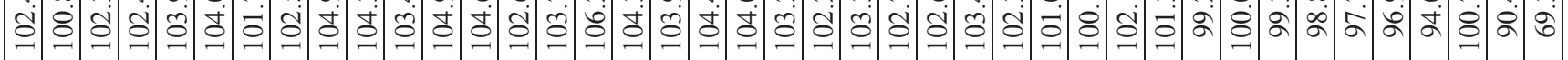

r.

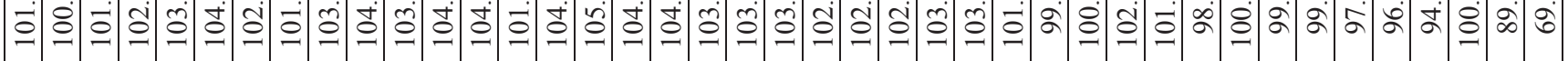
n

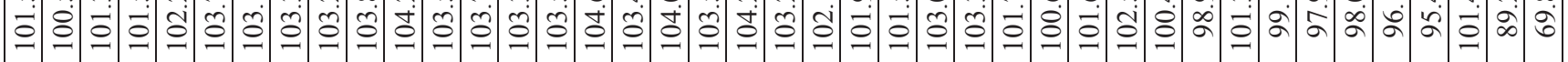

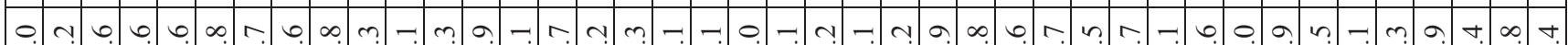
ḋ d

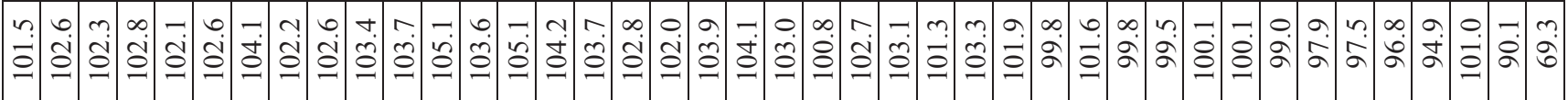

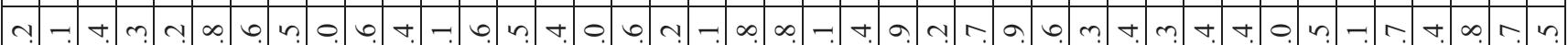
仓ิ 八

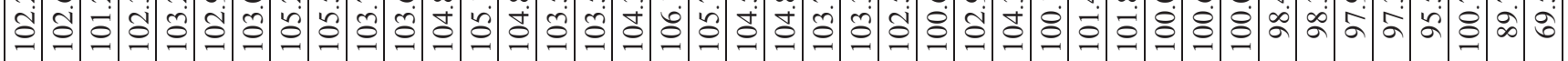

$\infty$ - J ¿ी

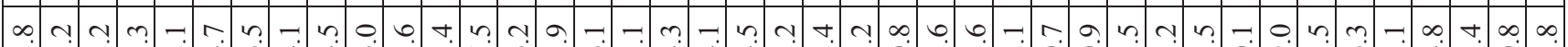
¿ी r. r. t n $\infty$ m m ḋ

c c t

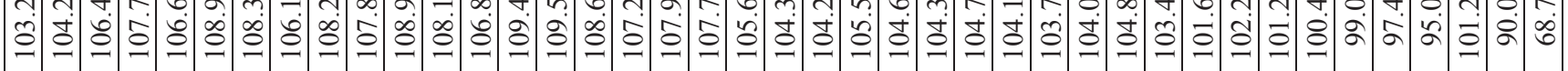

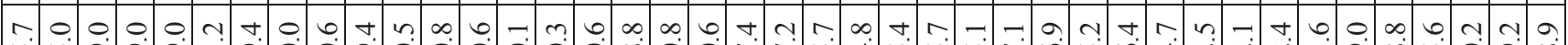
宅 n

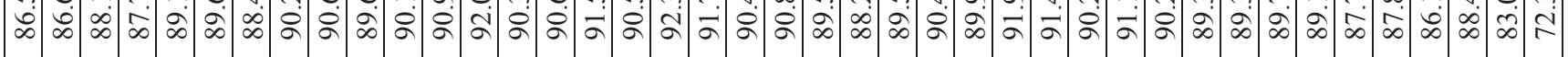
e $\varphi_{0}$.

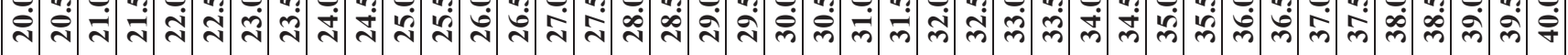




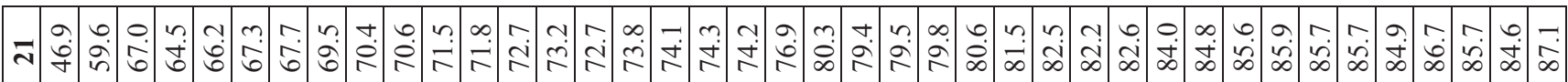
o o t o a r t a

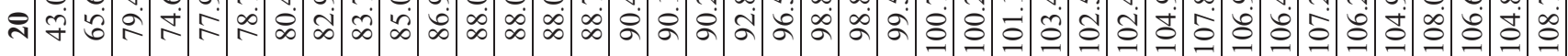

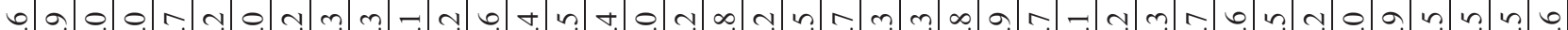
2 भं

non-

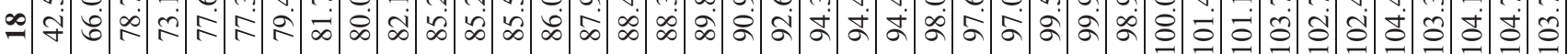

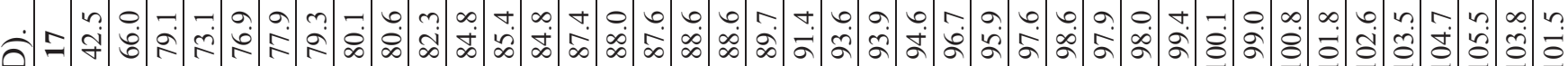
บ

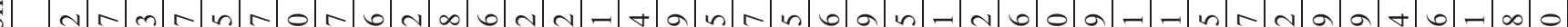

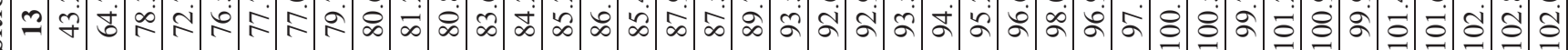

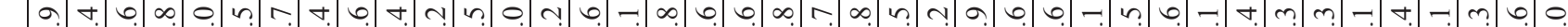

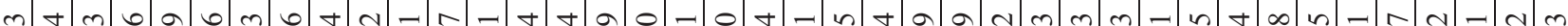

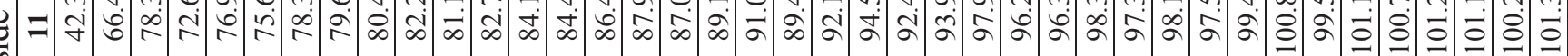

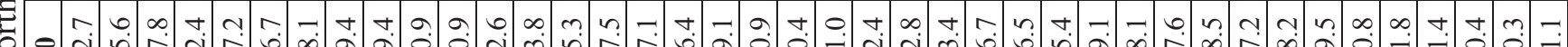

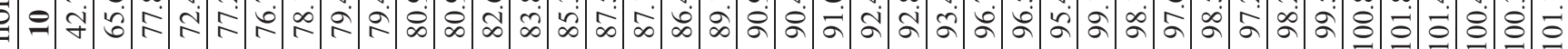
ฐ

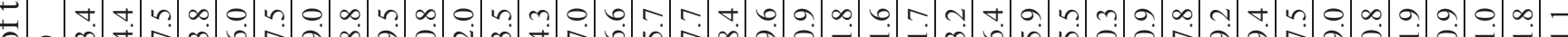
త్ $\infty$ fं

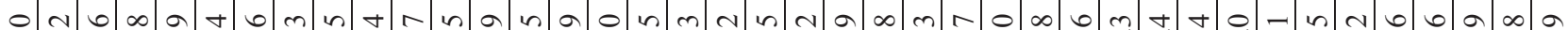

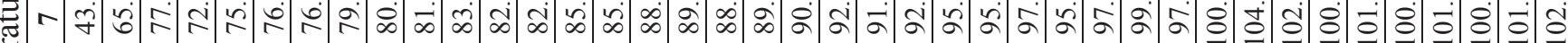

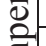

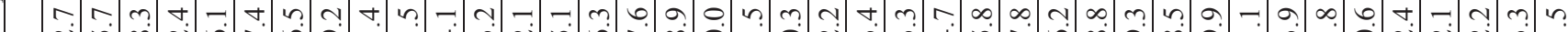
i a m $\infty$ o t a

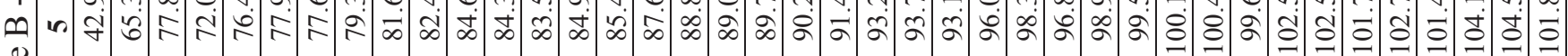
疍

n m

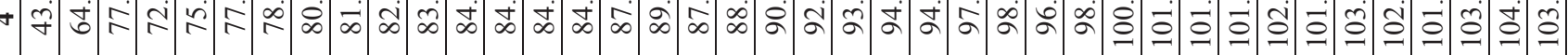

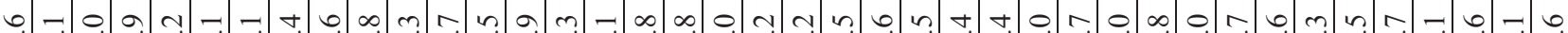

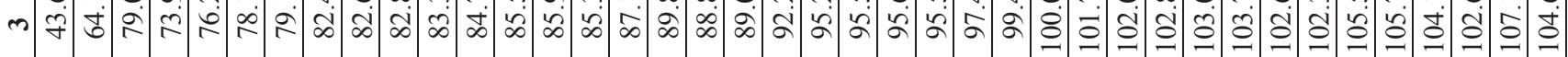

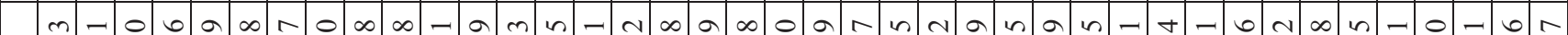

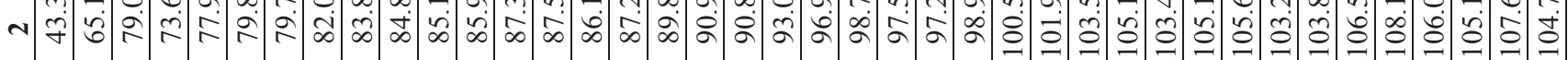

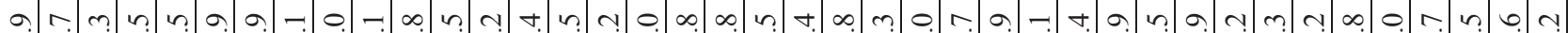
- 守 की

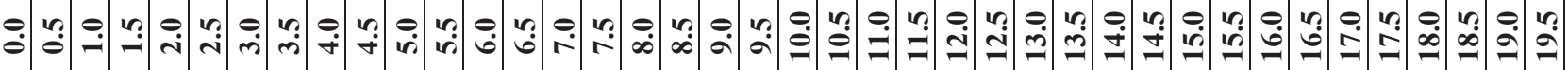




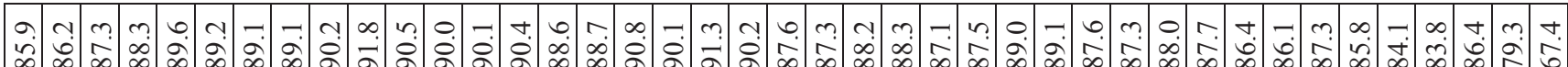

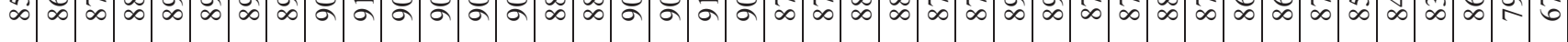
- - $\infty$ o o 0 c a -

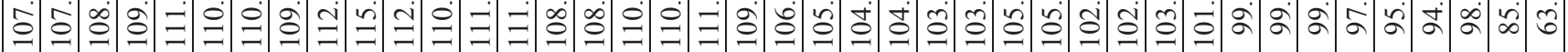

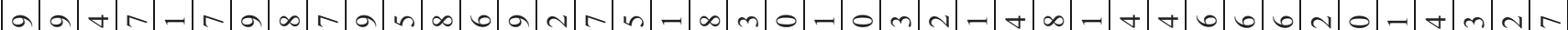
๖े

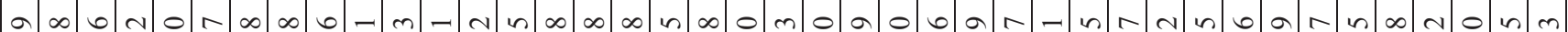
仓ी

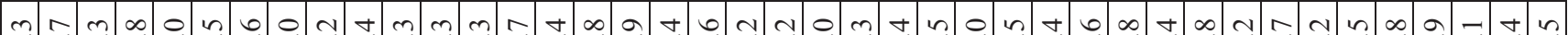
仓ी

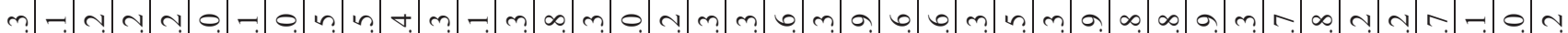
仓ी

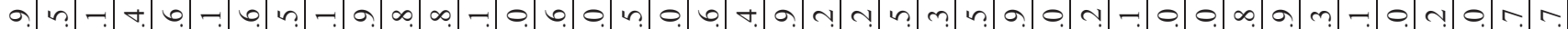
ḋ

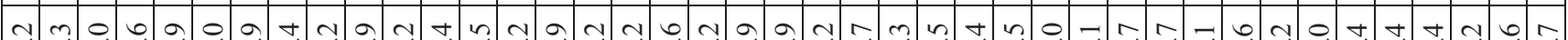

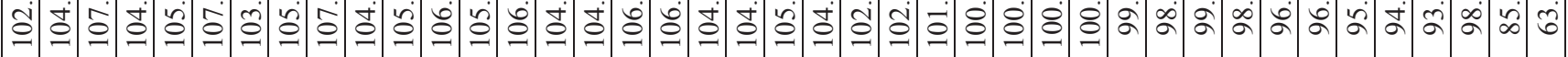

- 0 m $m$ a n

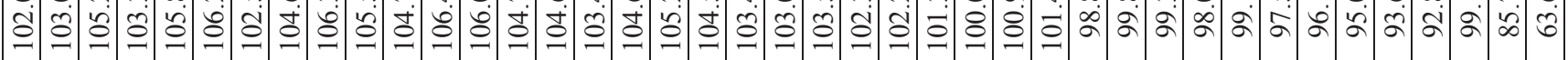

rmamn-tr ḋ $n+t-4 \pi$ t

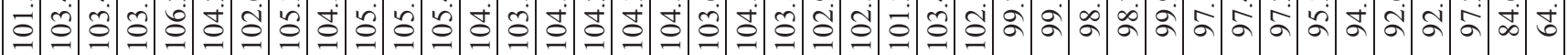

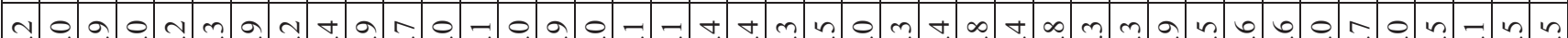

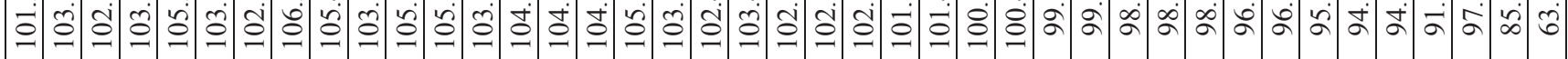

o n 仓்

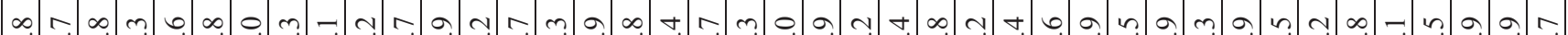
อิ o-4n一 @ீ)

m 仓்

n t r. n 仓ी

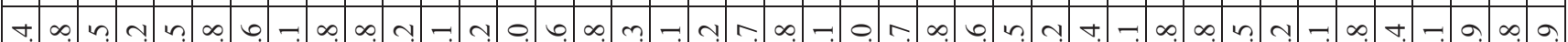
อి

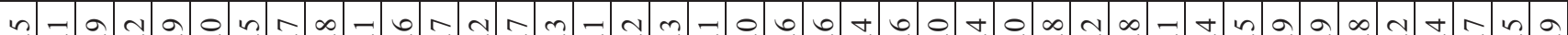

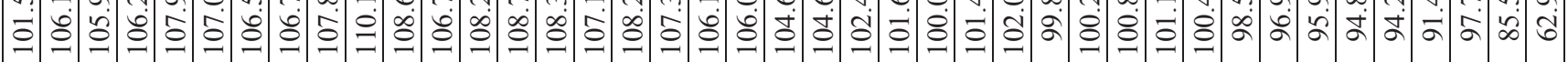

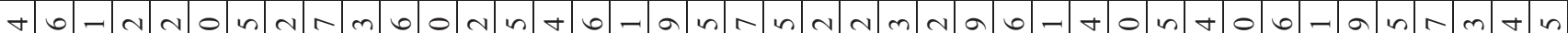

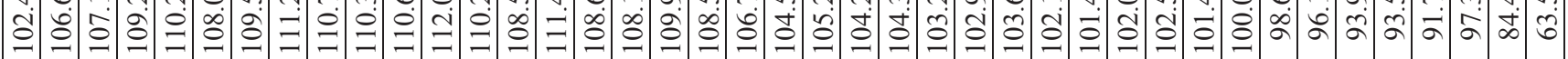
- - -

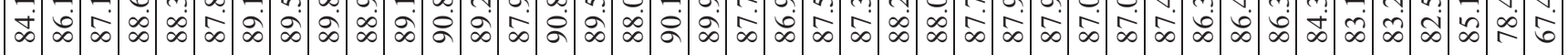

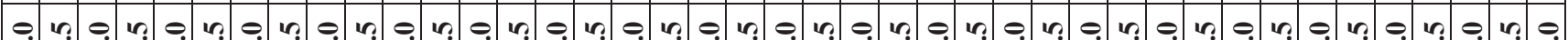
ปิ) 


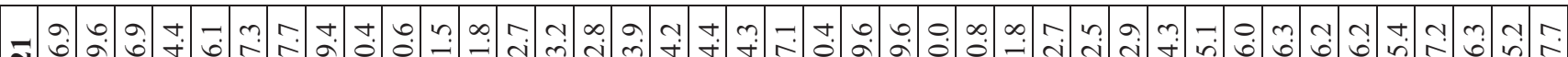
$\bar{N} \dot{\dagger}$ 它

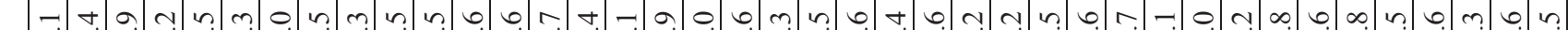

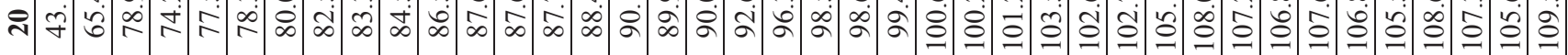

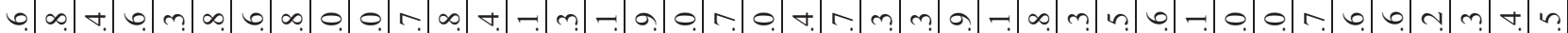
$=$ ᄀं

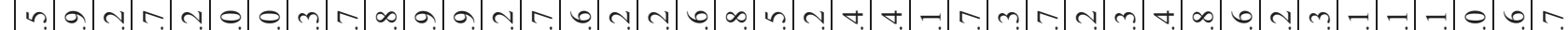
๑ fं

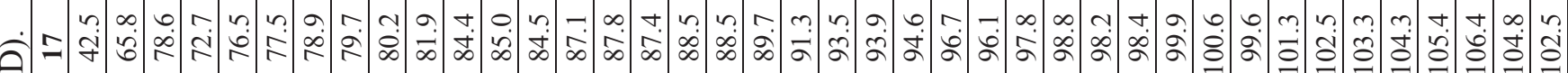
II

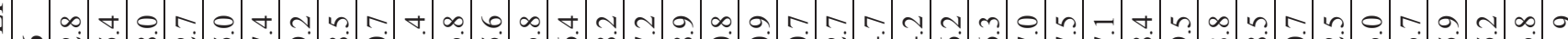

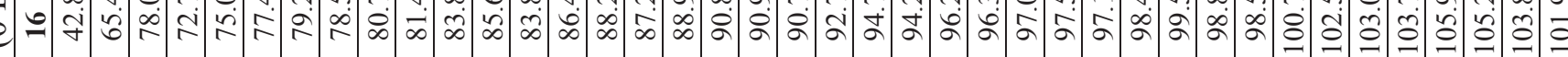

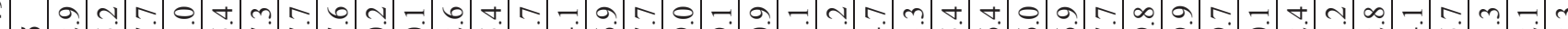
थ (1)

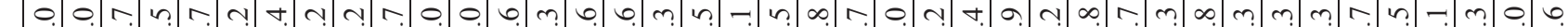

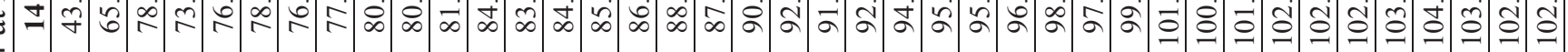
z

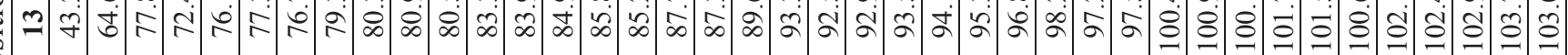

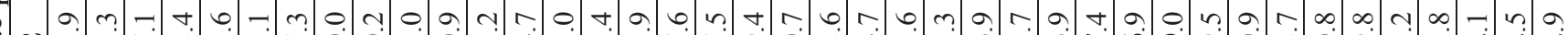

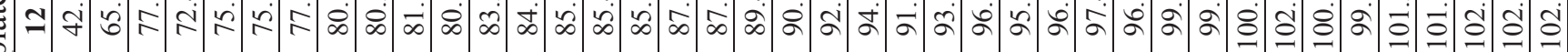

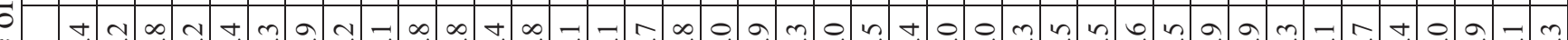

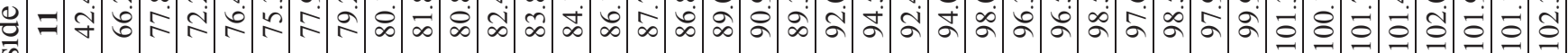

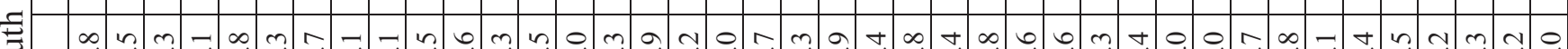

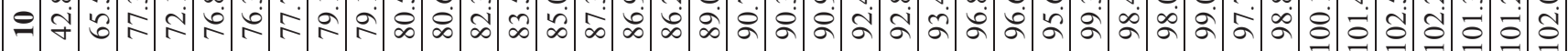
$\stackrel{8}{\Xi}$

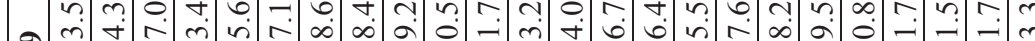
켤 m-

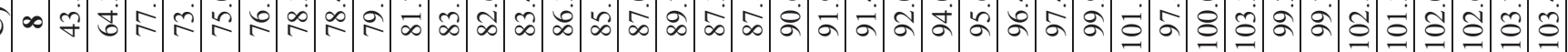
D - t t

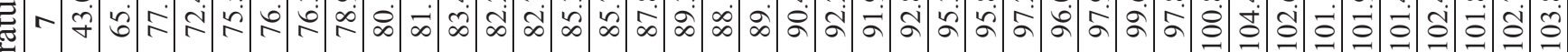
$\bar{\Phi}$

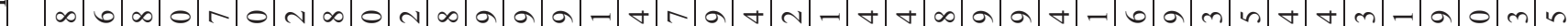

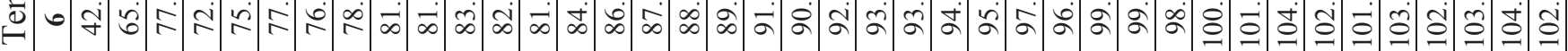

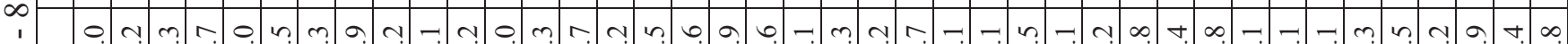

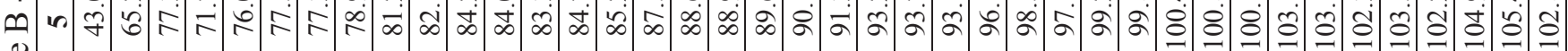
造

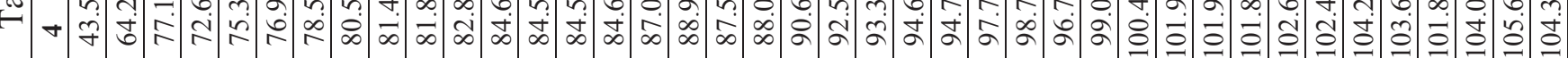

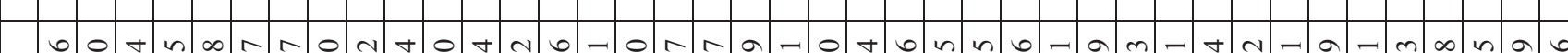

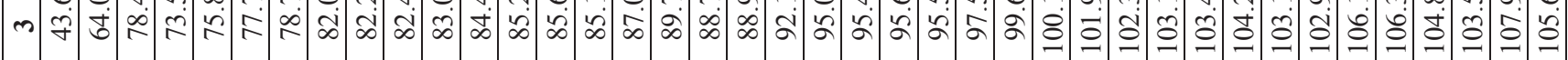

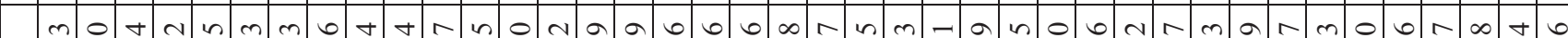

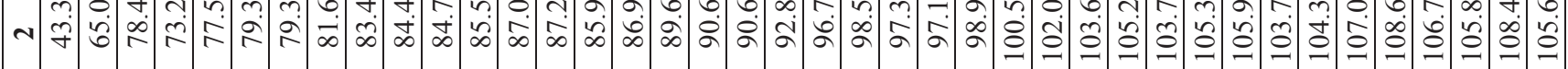

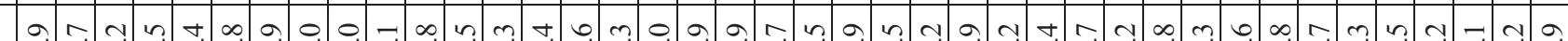
- 它 - 


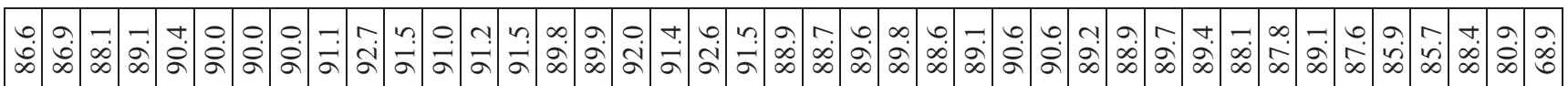

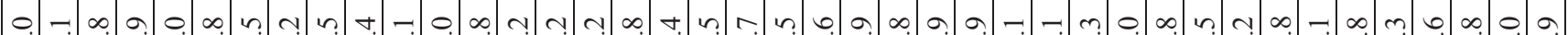

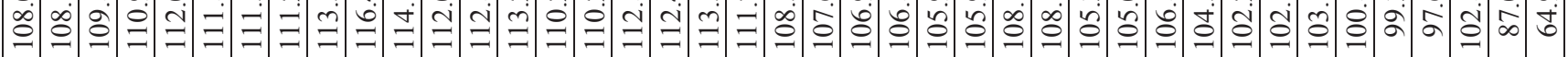

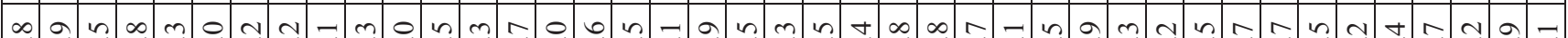

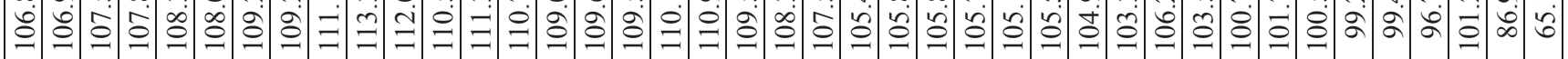
o. ఏి + $\infty$ n 过家

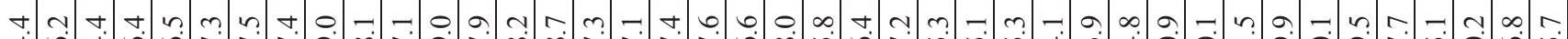

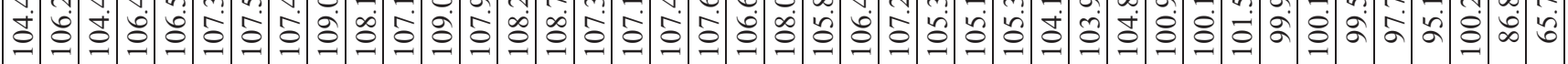
o. 过

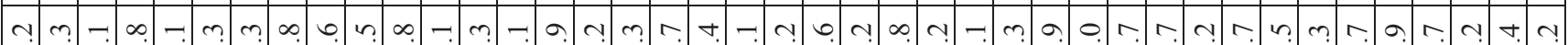
¿ी

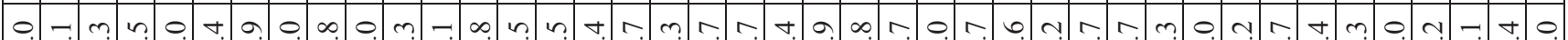
ف의

r. m.

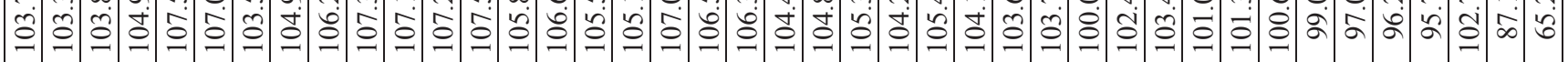

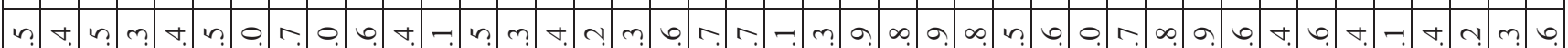
ḋ

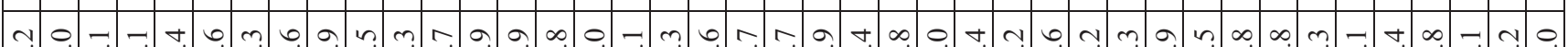

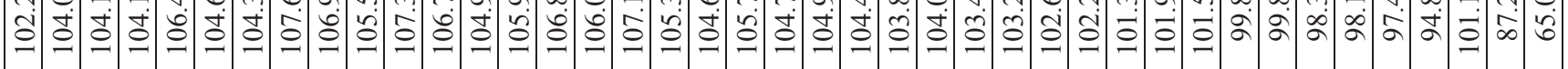

O ^. 过全 a. $\infty$ a

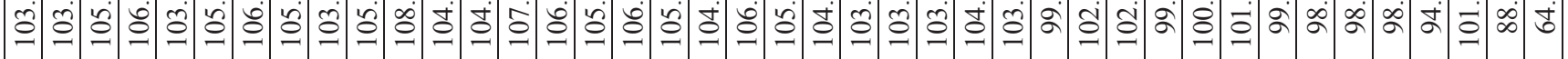
o c m o o m t t r. m m m m 过

a

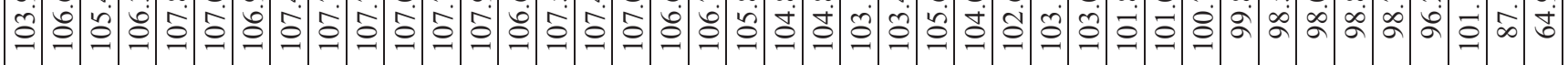

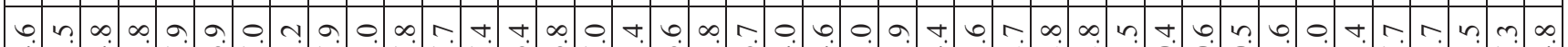
过全

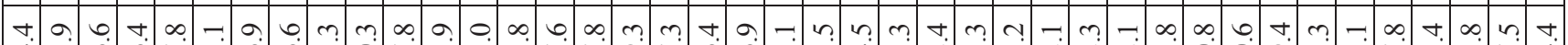

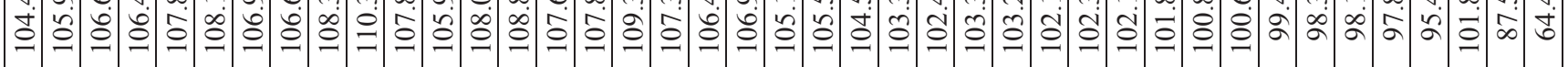
n -

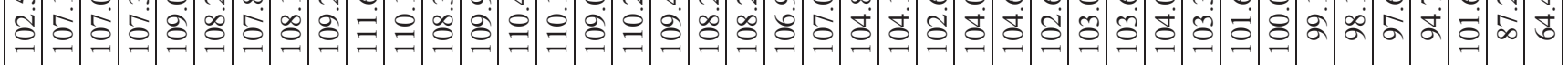

ナ. 仓̊)

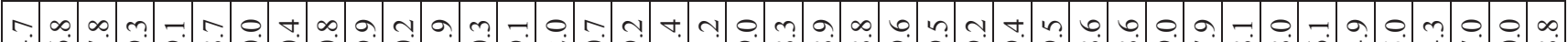

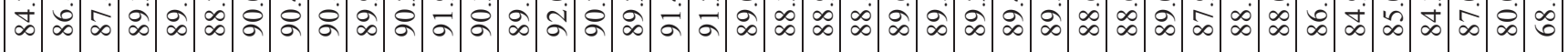
e $\varphi_{0}$.

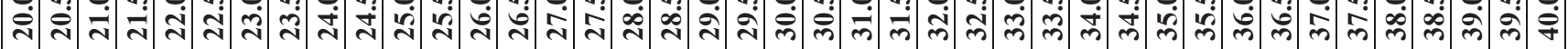




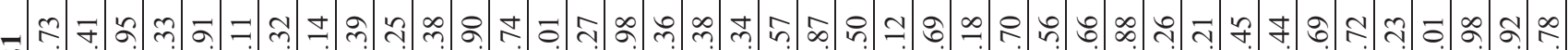

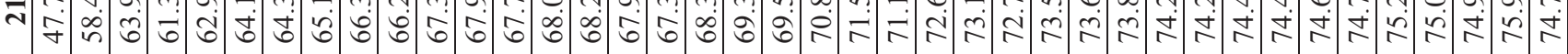

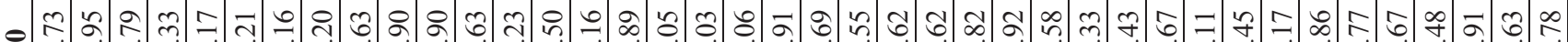

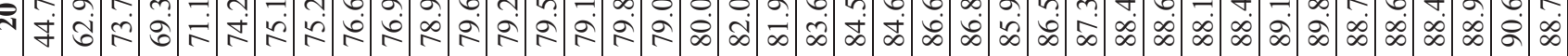

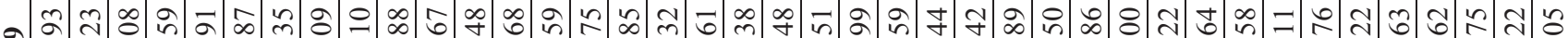

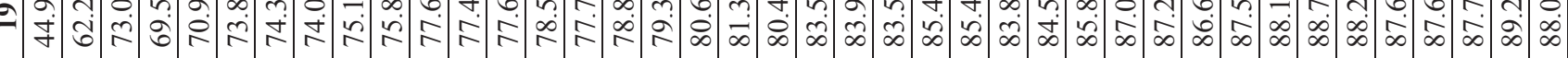

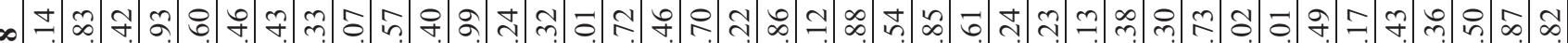

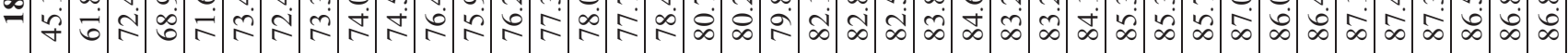

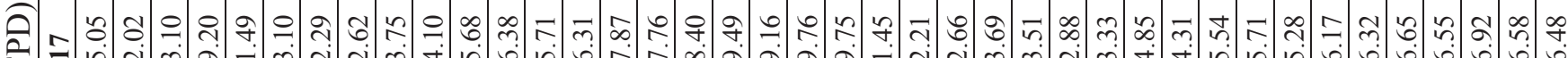

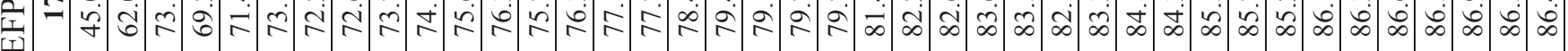
ம.

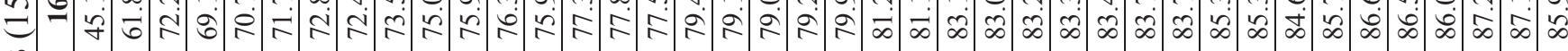

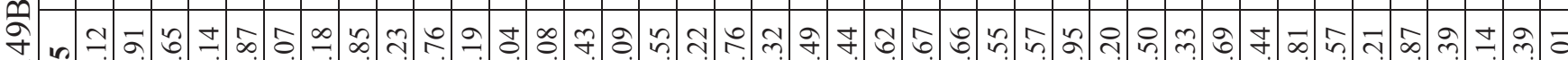

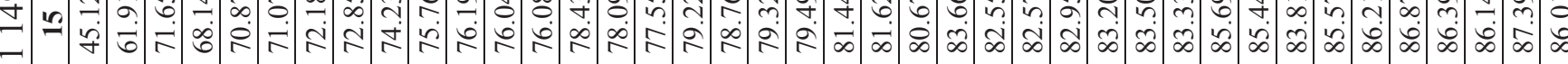
อ ษ

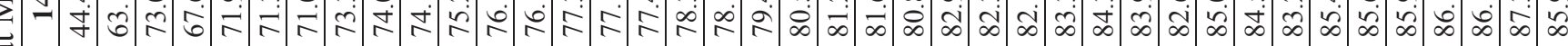

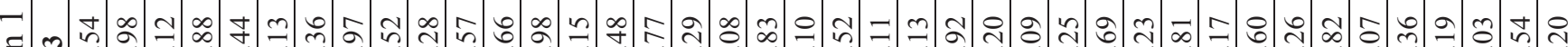

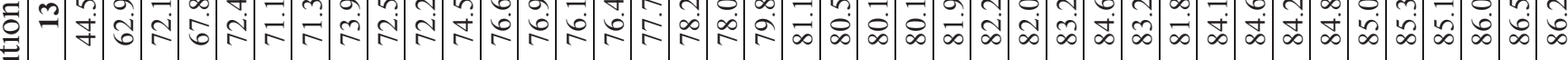
ด ล

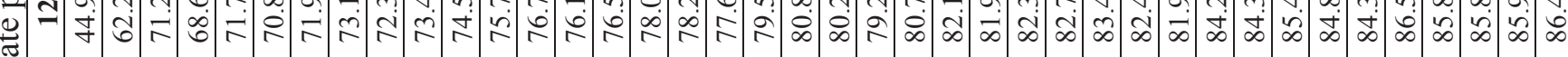
政 †ิ

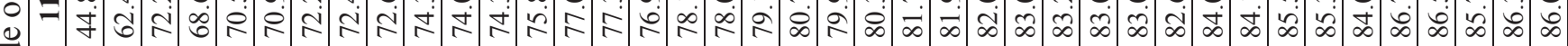

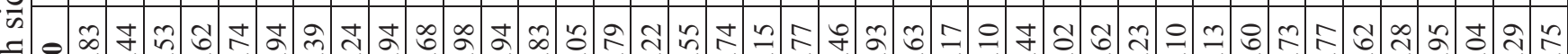

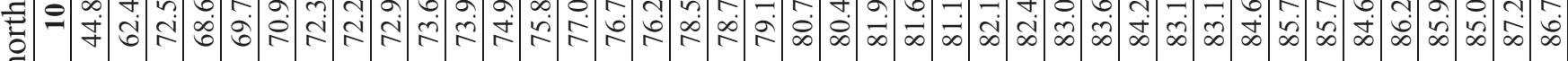

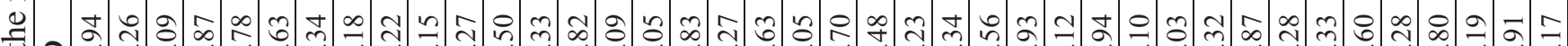

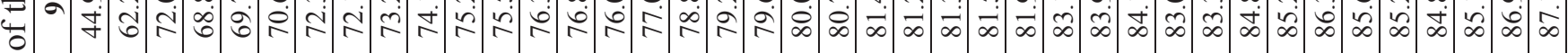

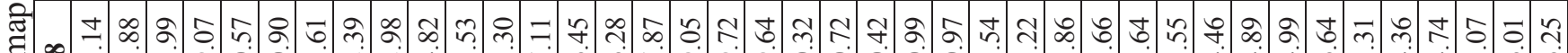

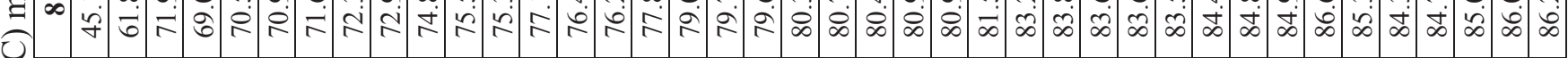
舟

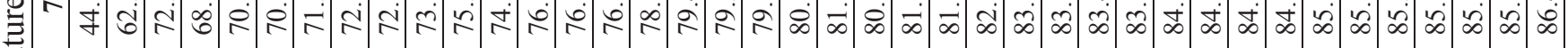
矛 氙

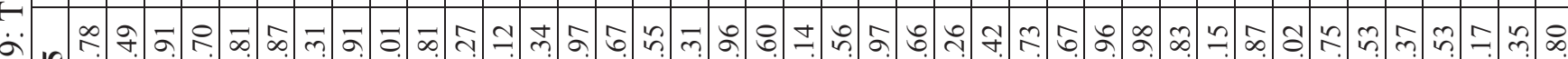

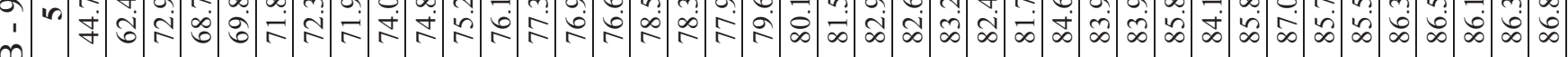

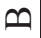

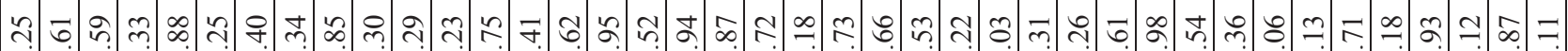

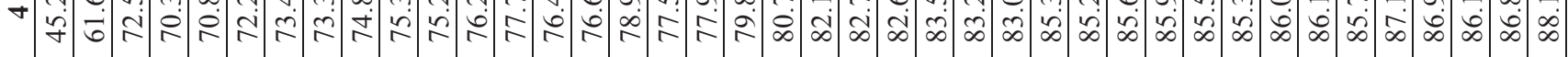

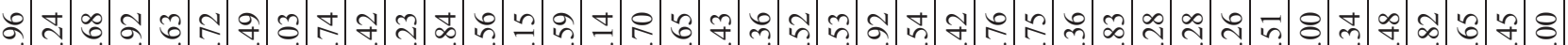

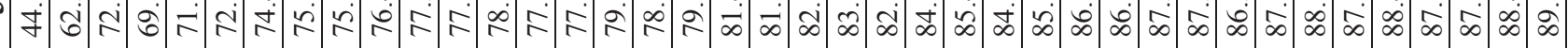
$\infty$ 两

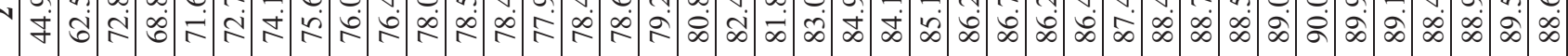

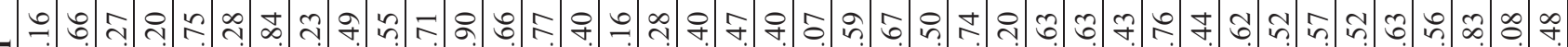

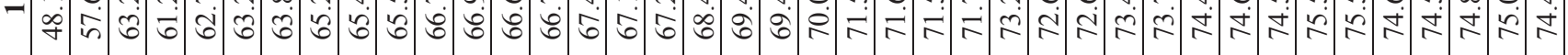
官 


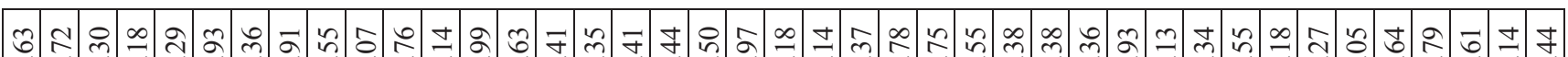

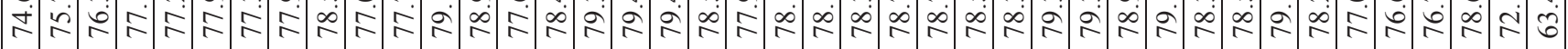
: $\infty$ œ ఫ.

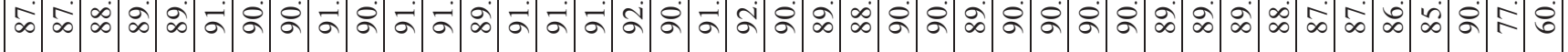
œ

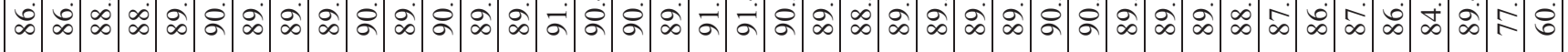

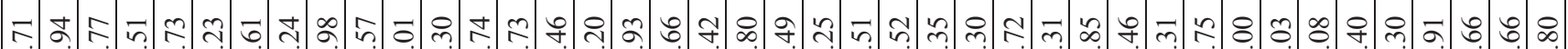

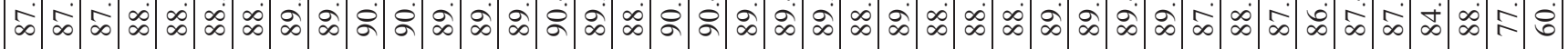

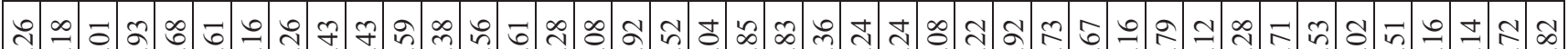

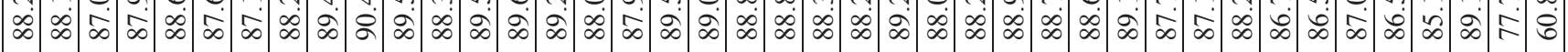

官 $\infty$ b

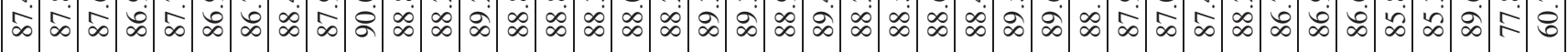

ఫ

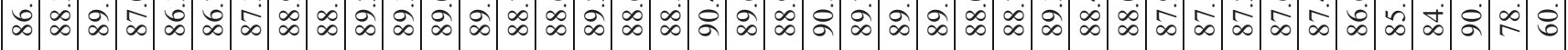

m. $\infty$ :

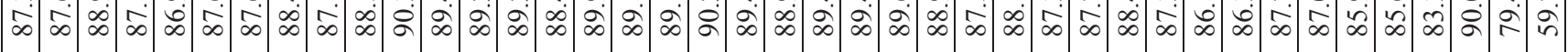
m.

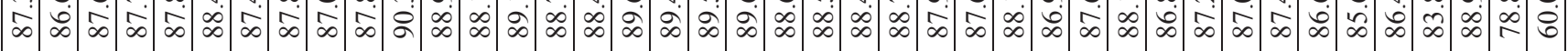
虽

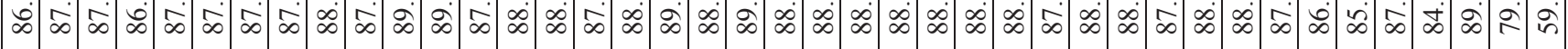

๙

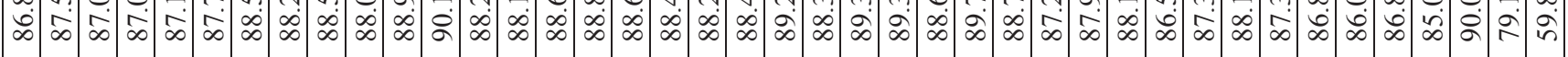

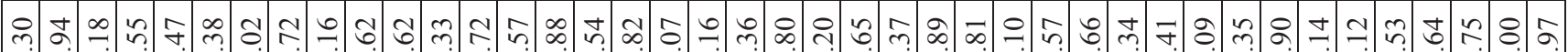

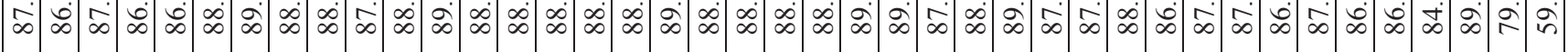

m.

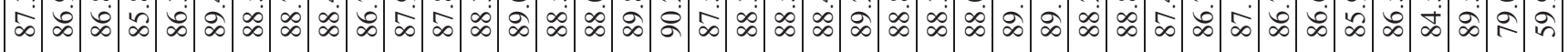

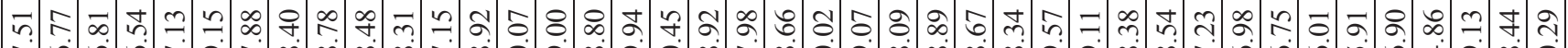

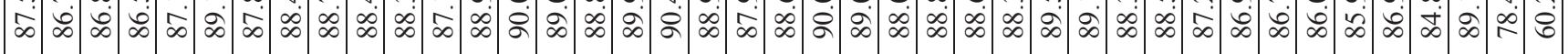

$\exists$ तิ

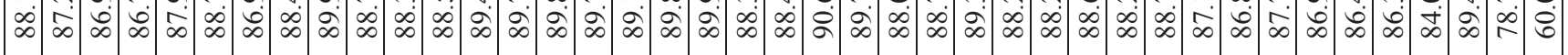
m: ㄴ. $\infty$ ก

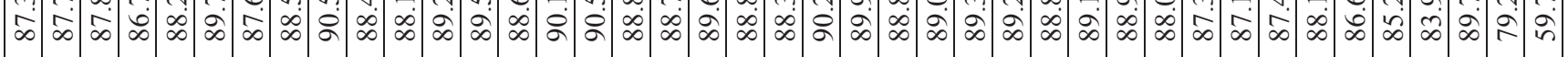
m.

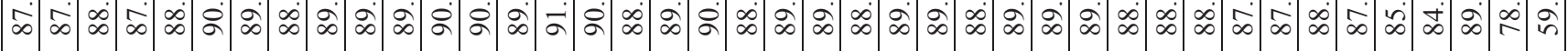

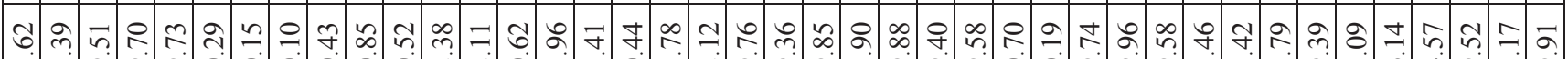

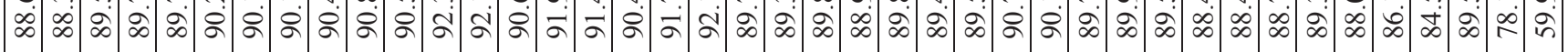

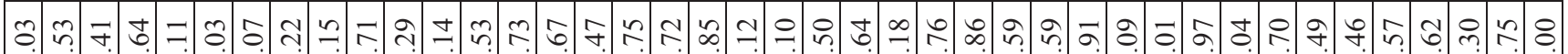

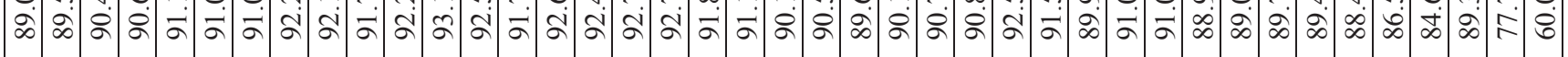

ఫ

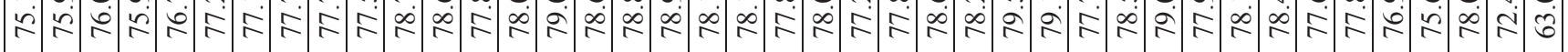


ส

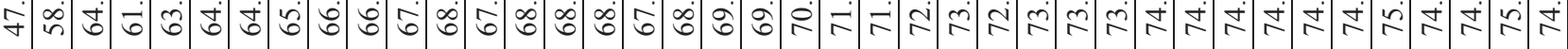
-

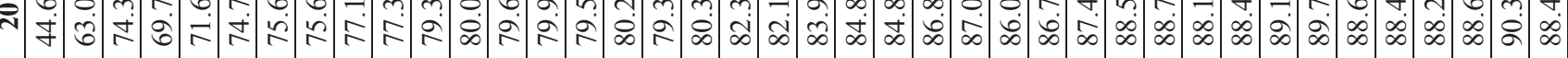

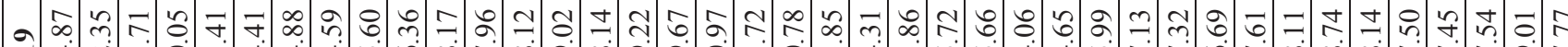

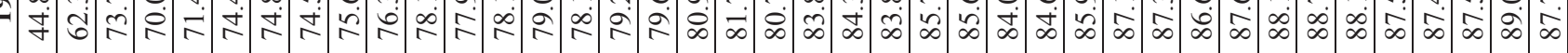

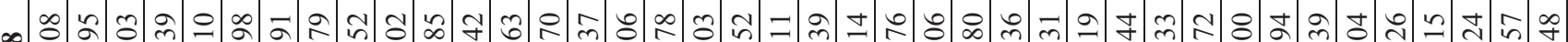

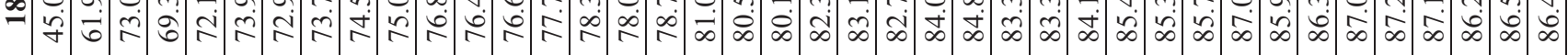

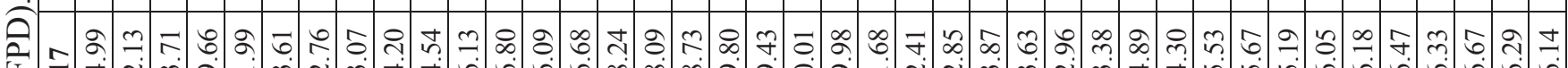
在=

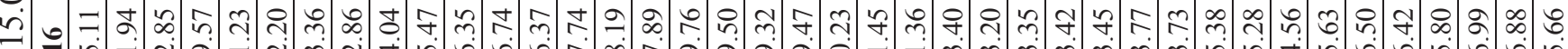
ت a 守

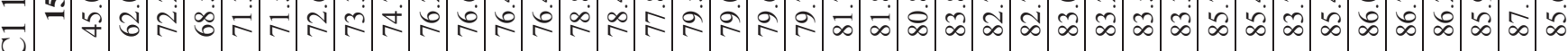

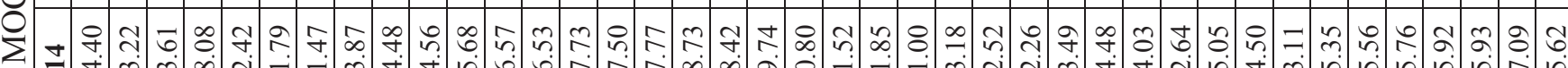

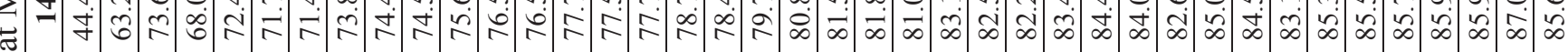
च

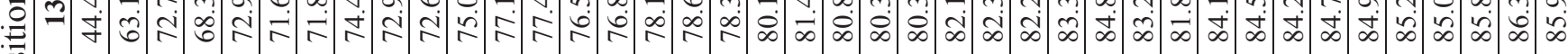

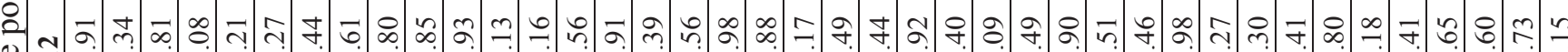

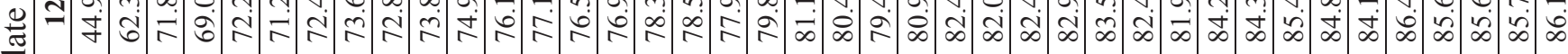

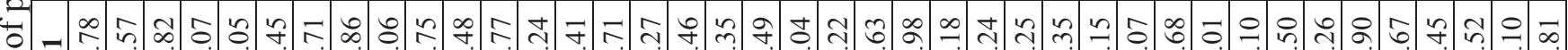

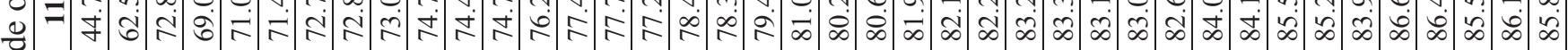

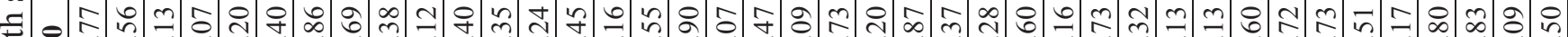

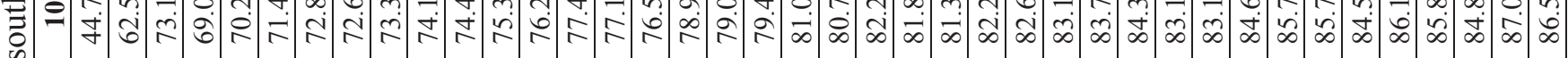

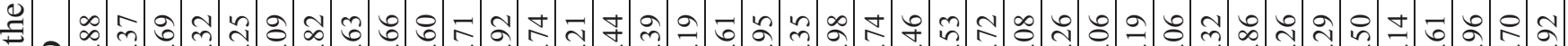
फ्ठ శิ

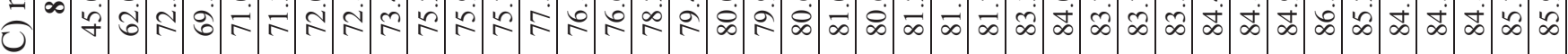
O

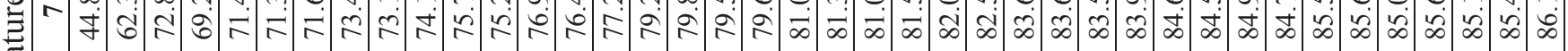

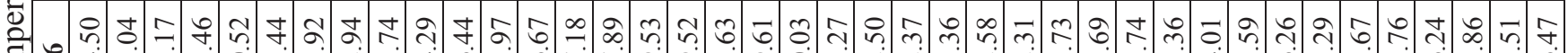
昰 ¿

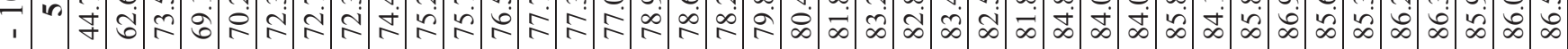
๑

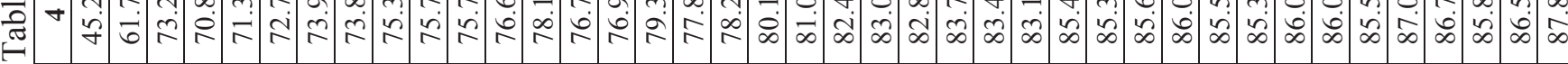

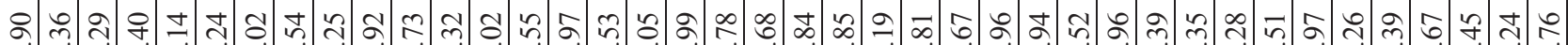

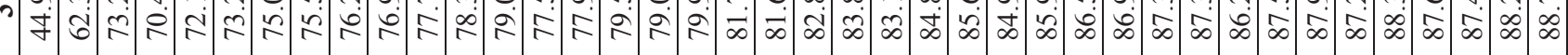

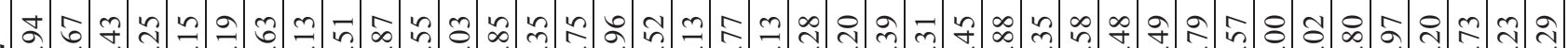

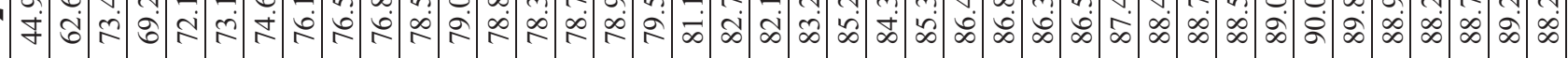

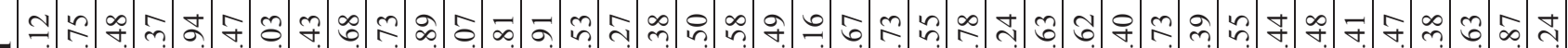

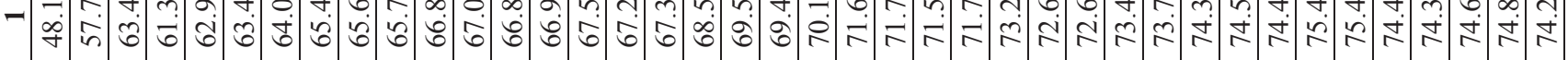

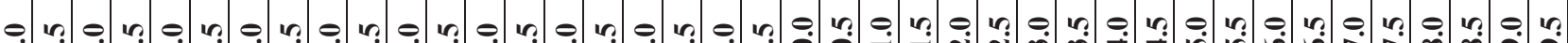

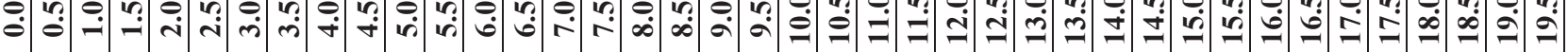


ก.

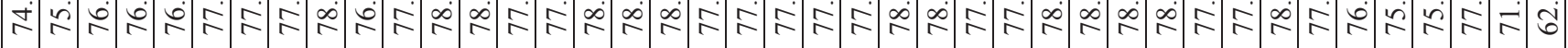

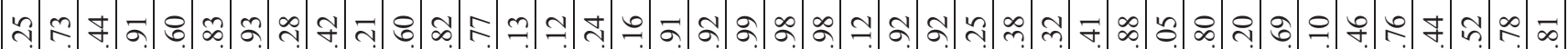

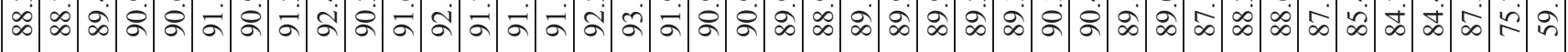

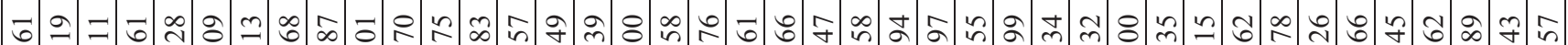

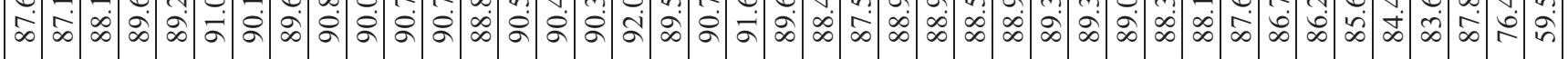

我

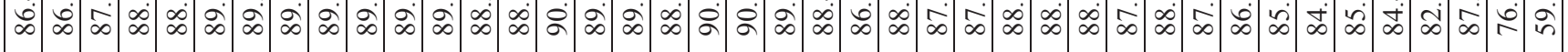

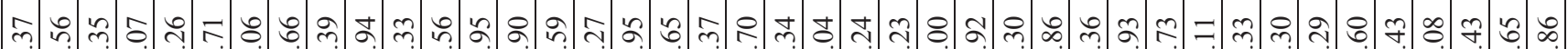

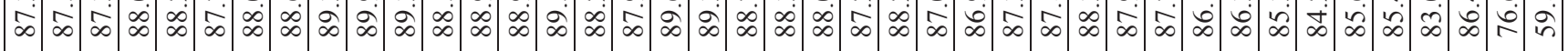

t.

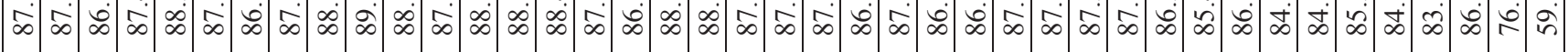

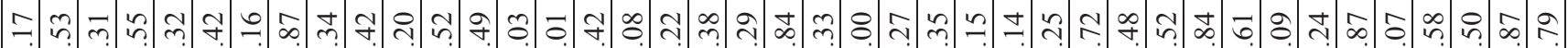

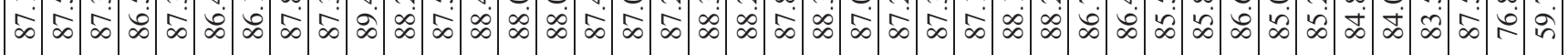

苗

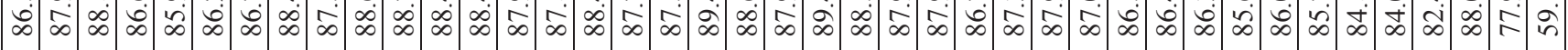

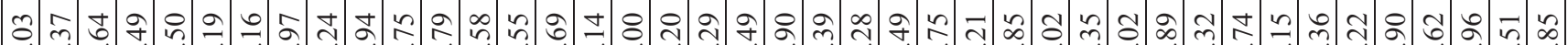

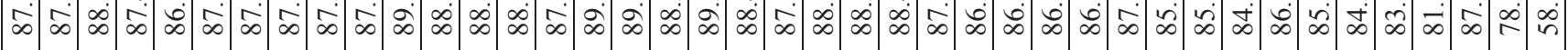

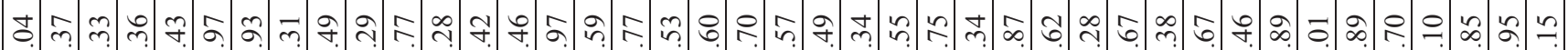

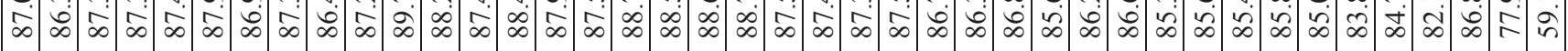

กิ กำ ๆ

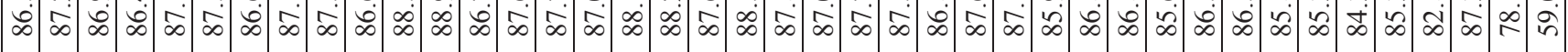

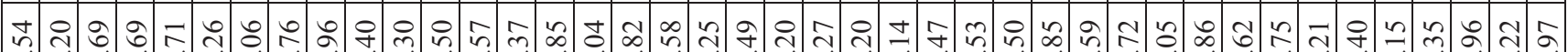

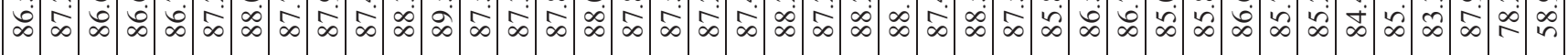

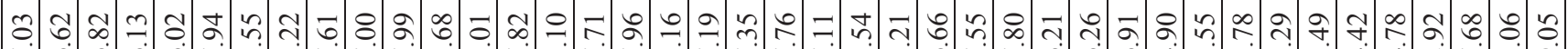

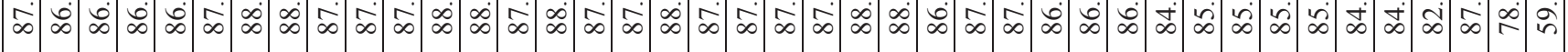

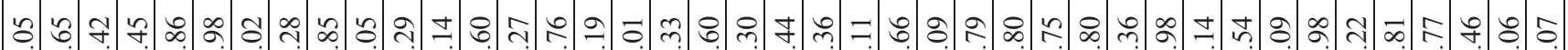

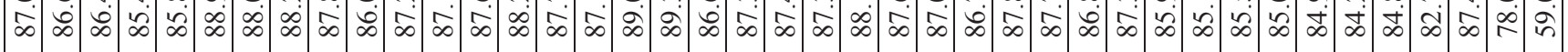

స় ᄀ ᄀ

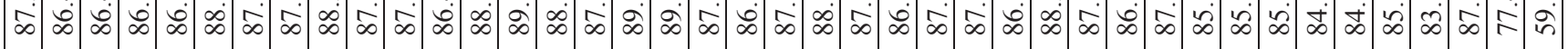

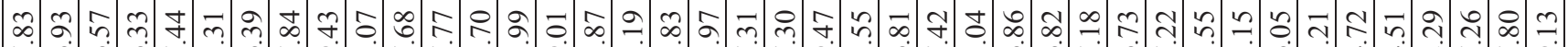

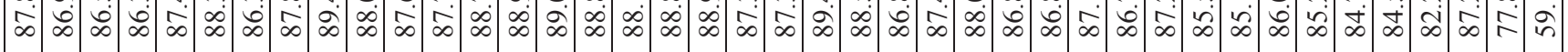

$\because$ ○

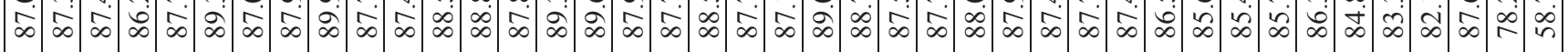
\&

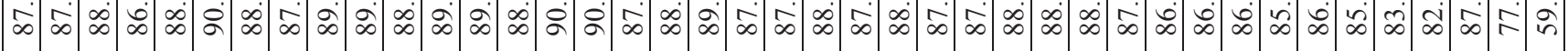

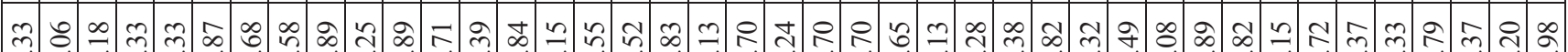

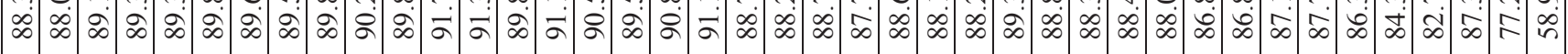

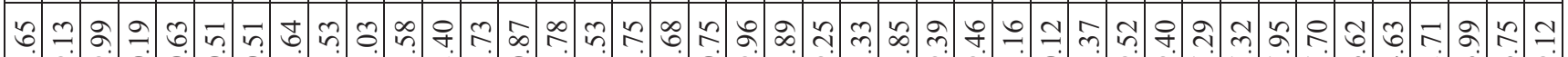

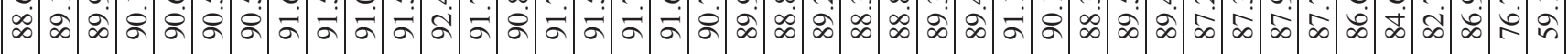

क)

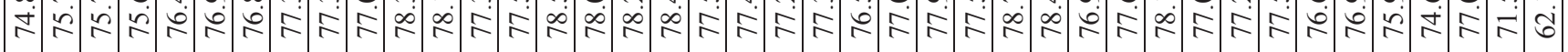
我

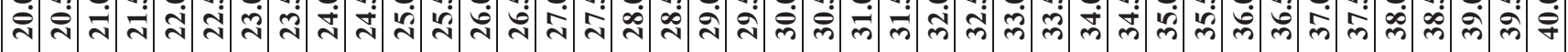




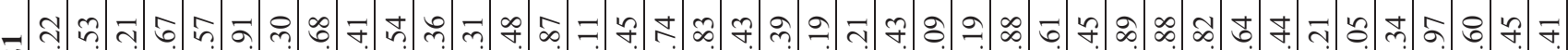

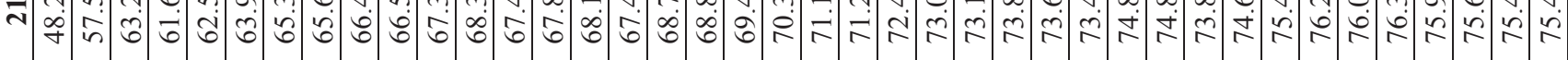

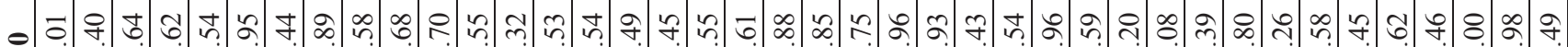
守

- लेప

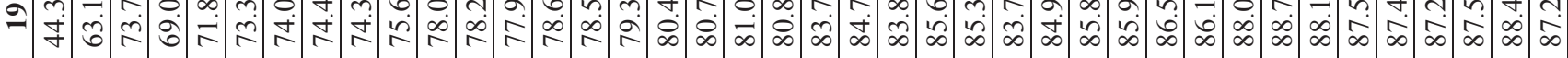

手

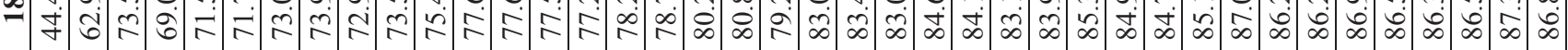

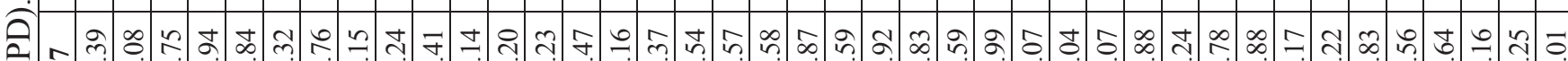

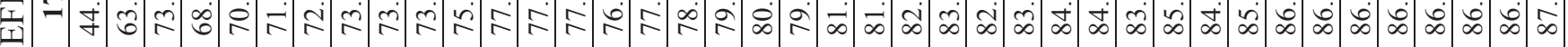

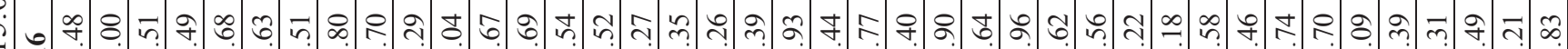

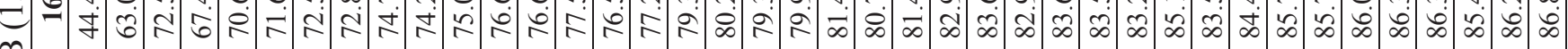

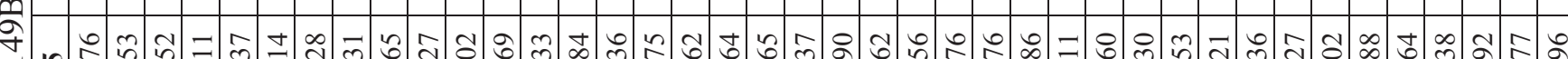
In乞ํ.

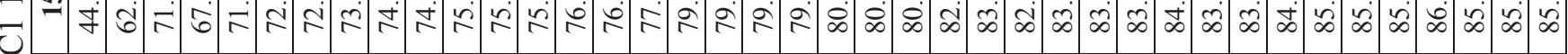

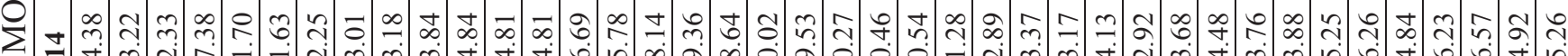

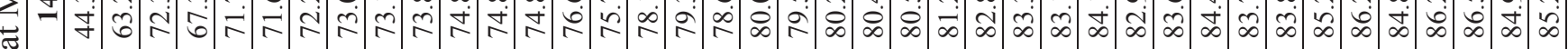
ปૈ

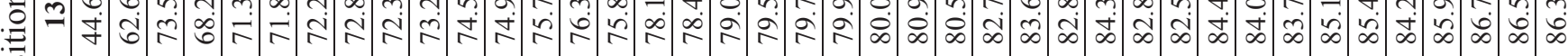

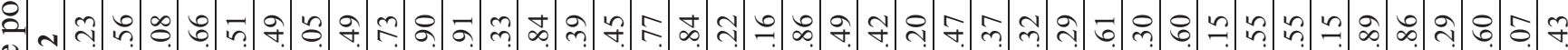

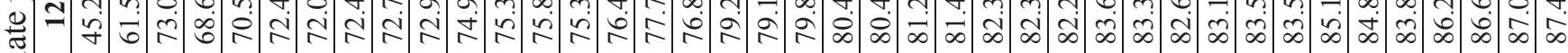

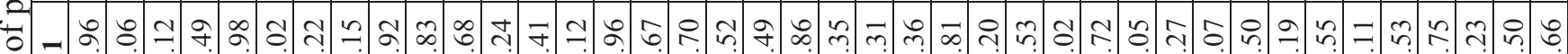

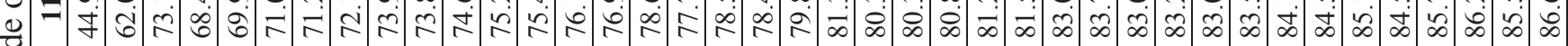
" n

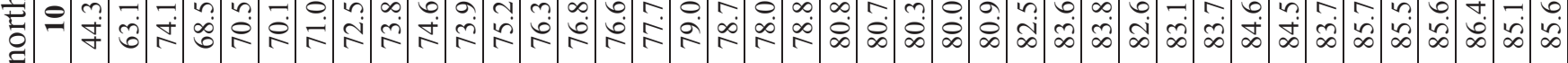
ક फ्ठ

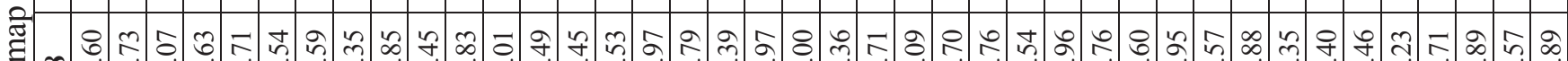

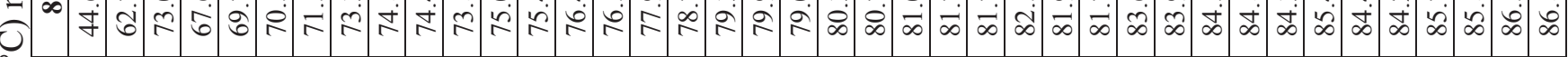
0 $\infty$ t

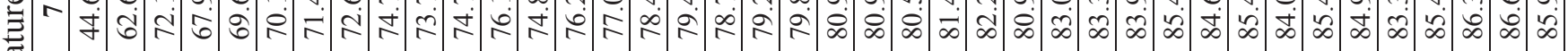

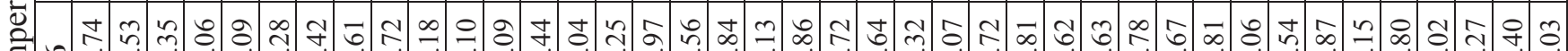
包

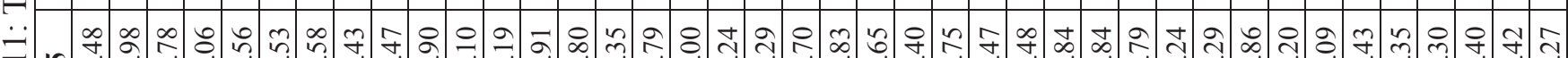

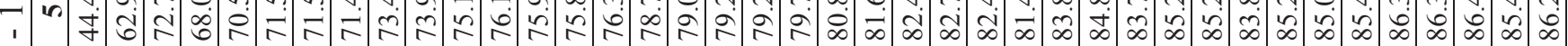
๑

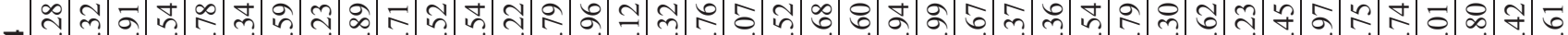

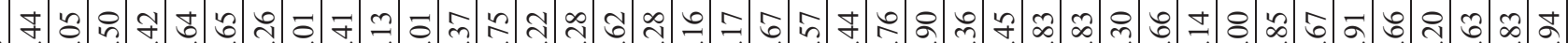

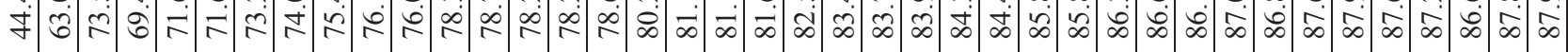

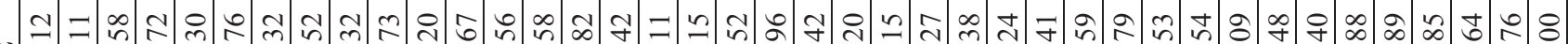

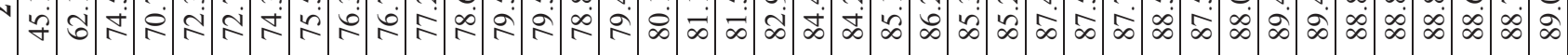

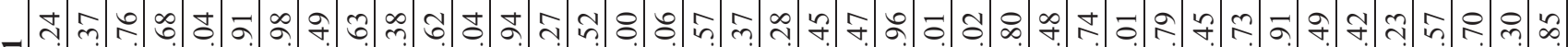

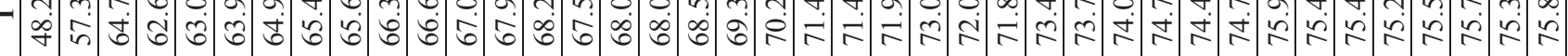
- n

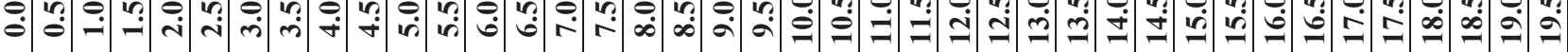




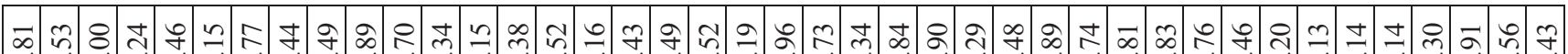

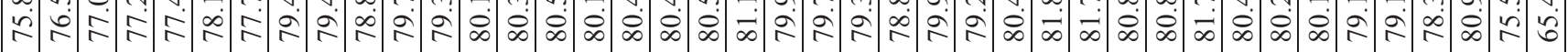

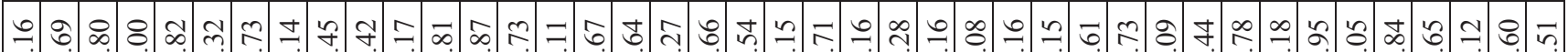

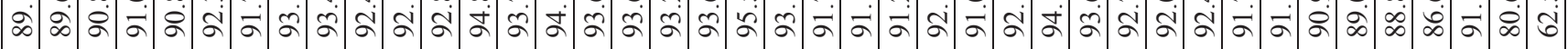
तิ

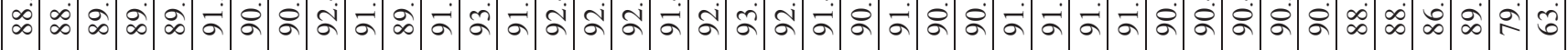

\&

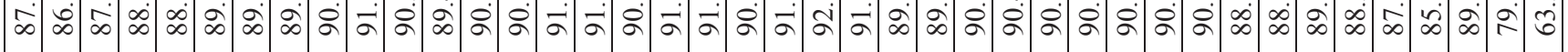

๙

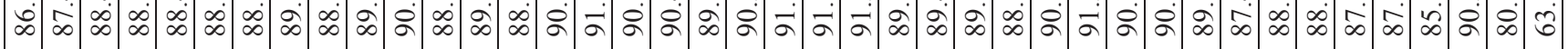

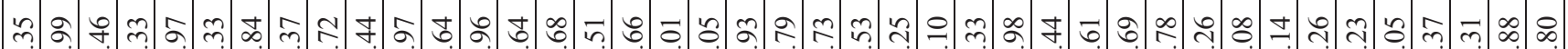

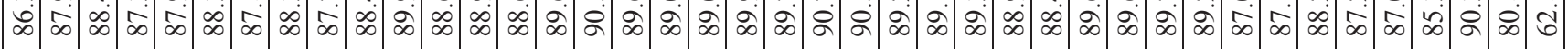

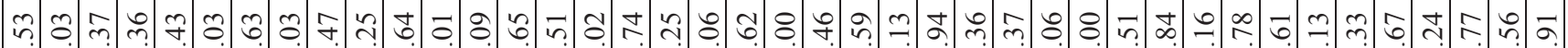

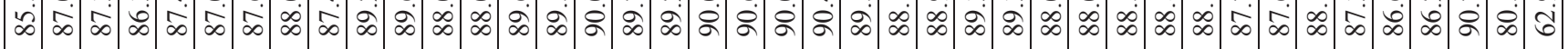

의

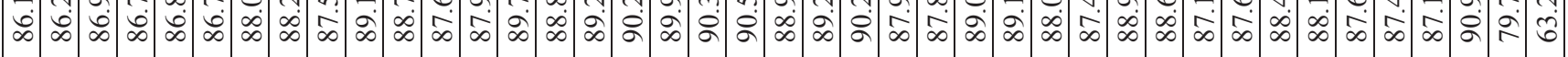

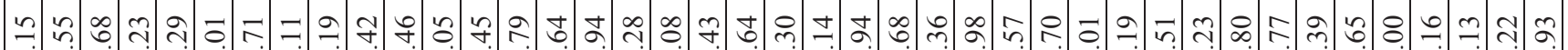

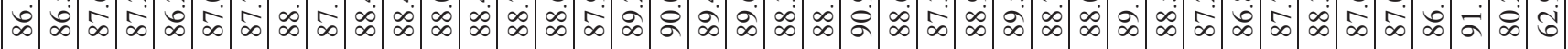

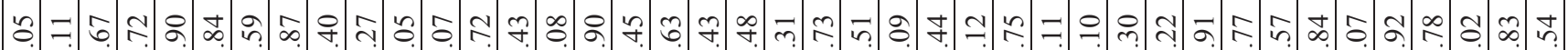

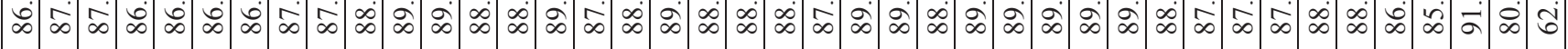

Иุ

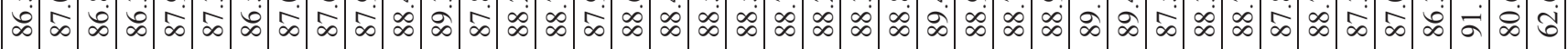

m

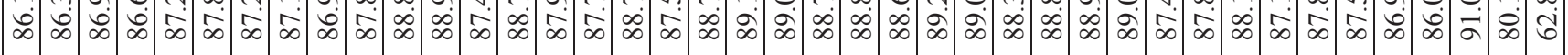

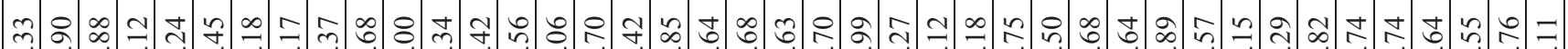

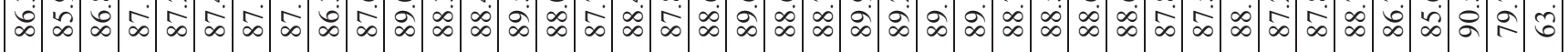

$\infty$ t

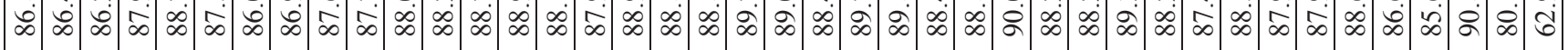

भ

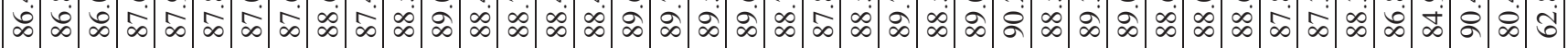

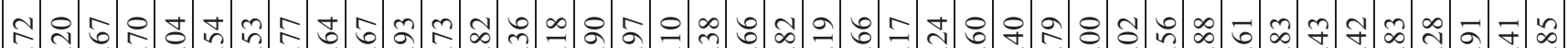

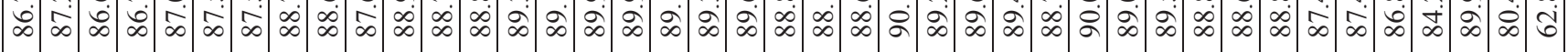

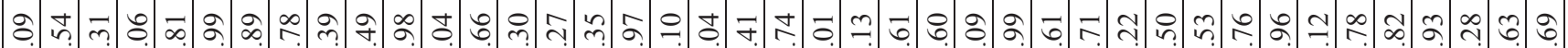

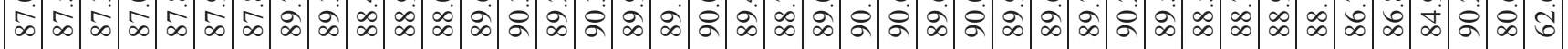
กิ

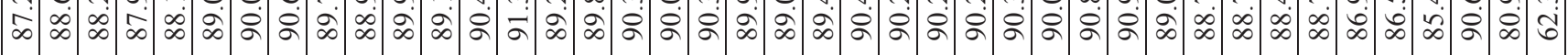

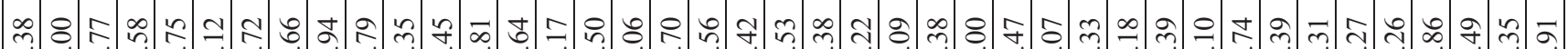

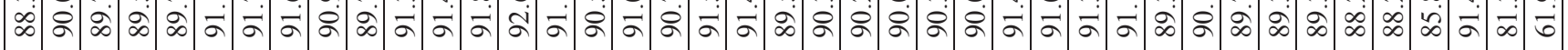

สุ f

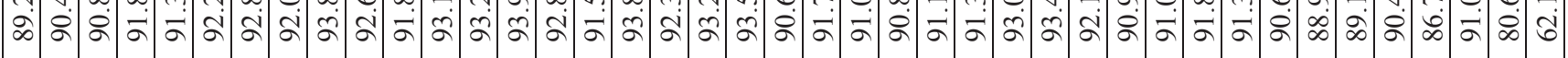

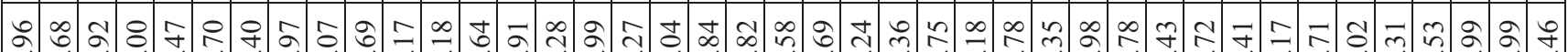

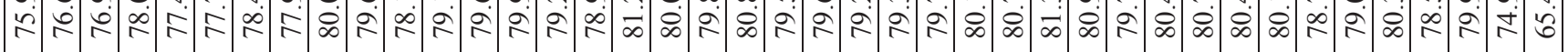




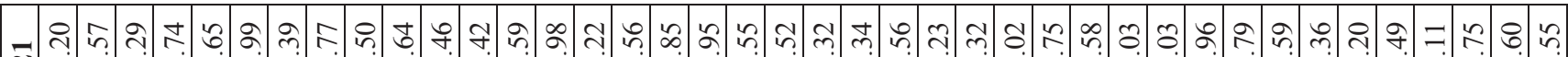

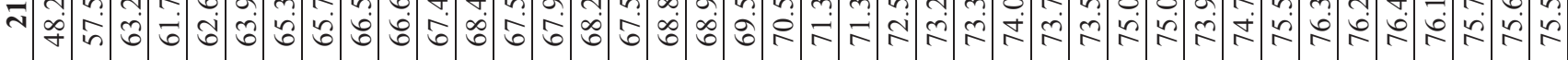

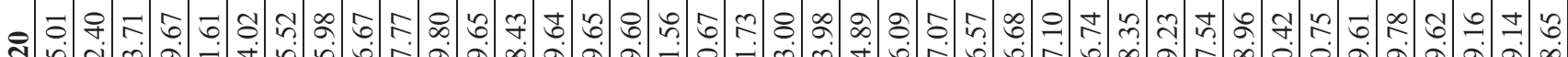

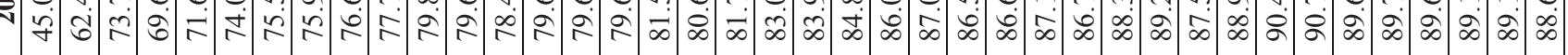

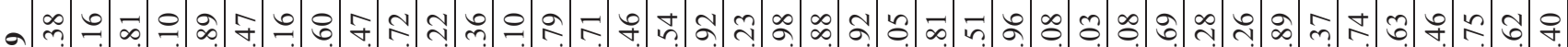

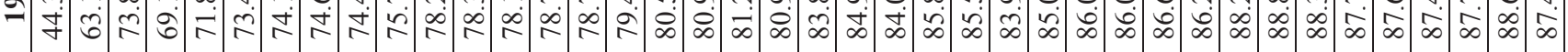

$\infty$ 守直

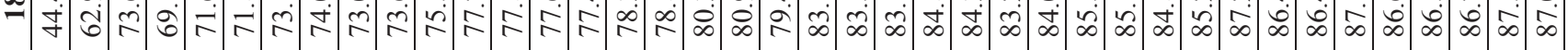
فी

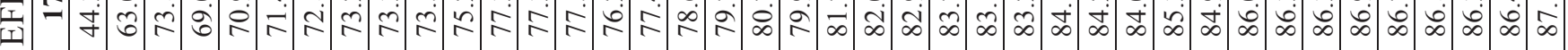
ப் f

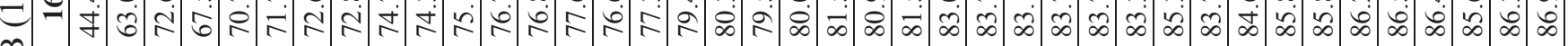
向

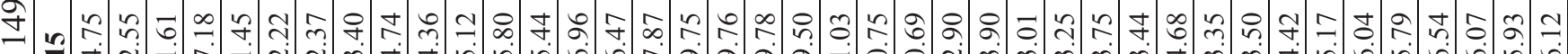

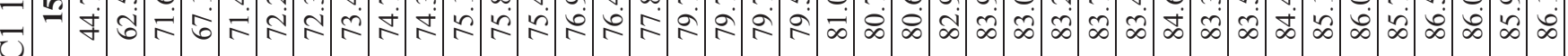

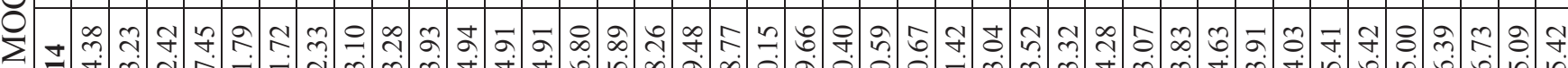

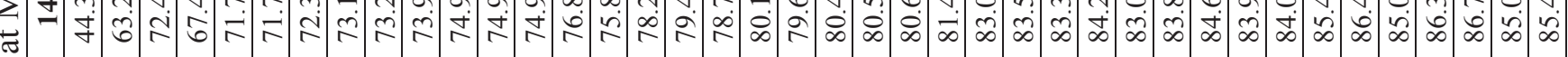
ปี

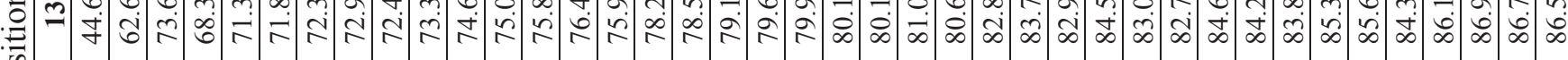

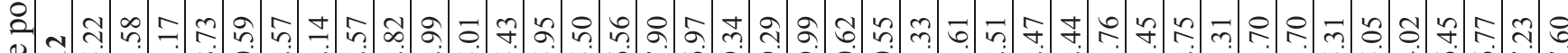

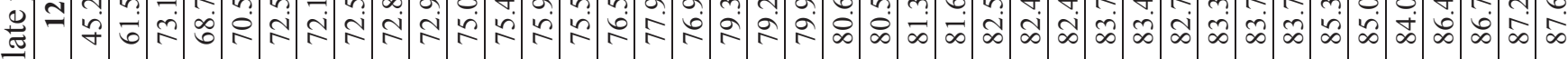

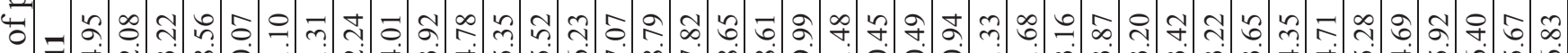

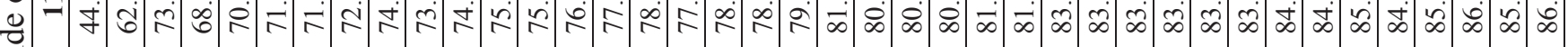

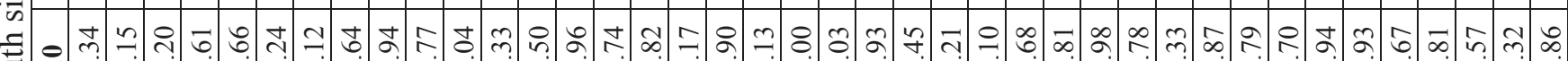

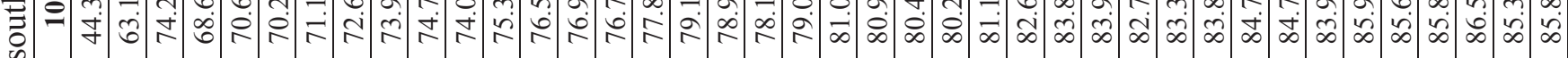

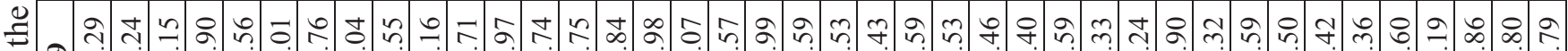

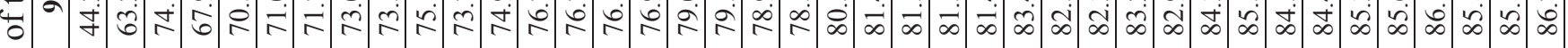

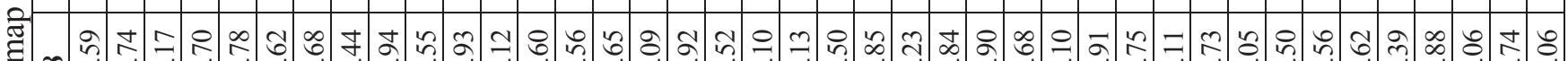

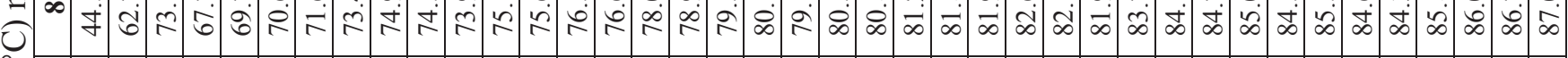
0

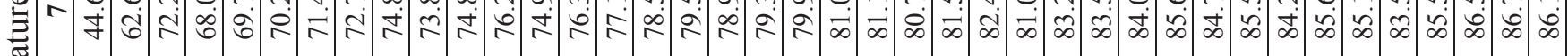

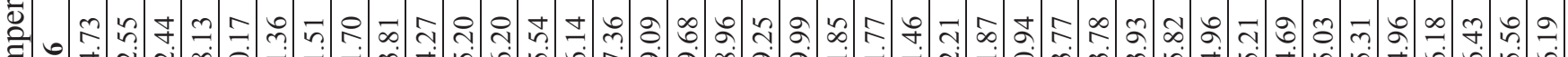

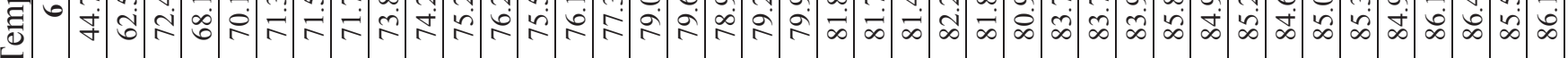

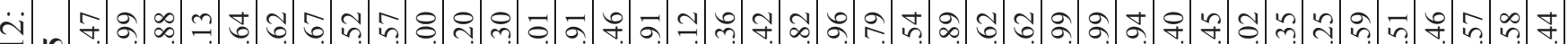

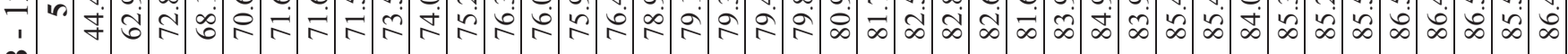

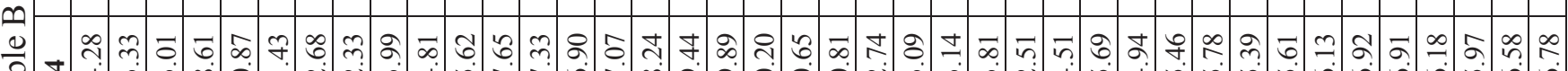
考 - f

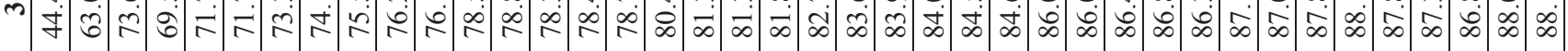

ง =

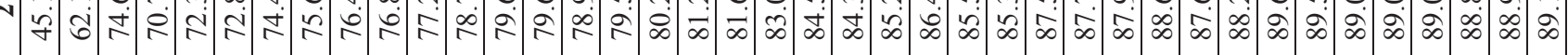
- 定 $\because$ 急 


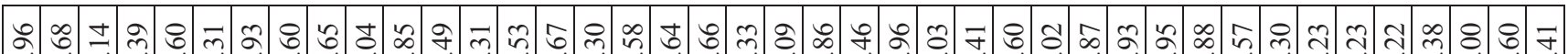

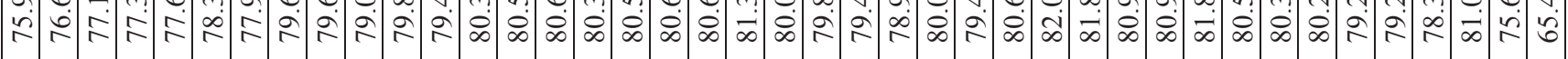
m ๘

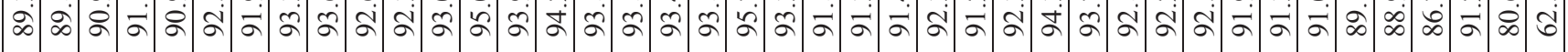

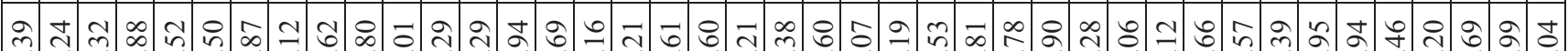

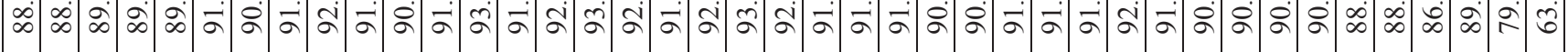

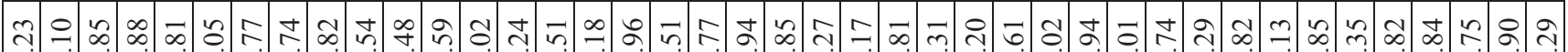

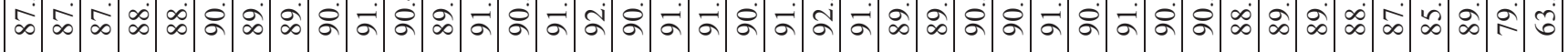

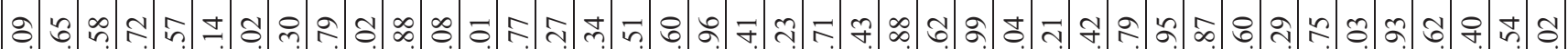

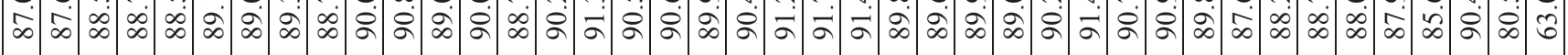

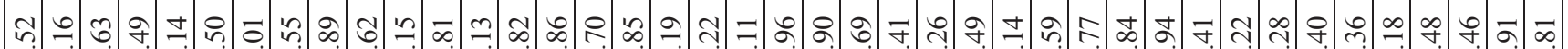

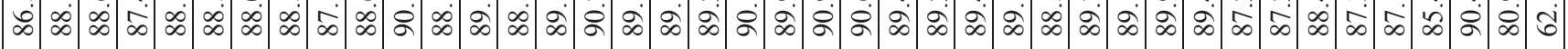

은

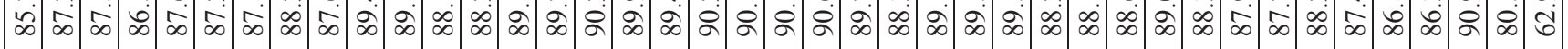

办

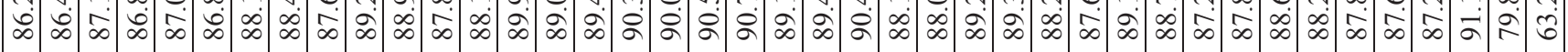

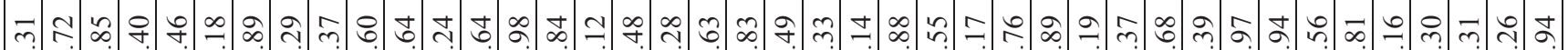

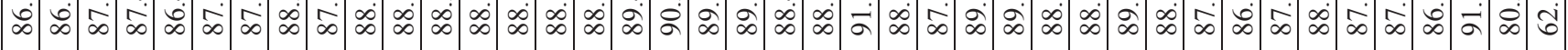
สิ

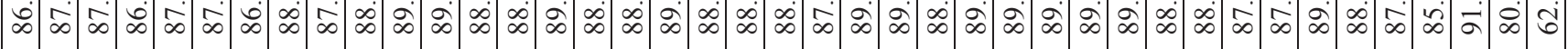

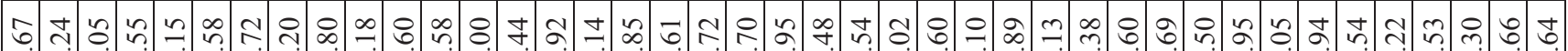

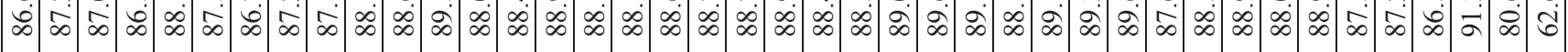

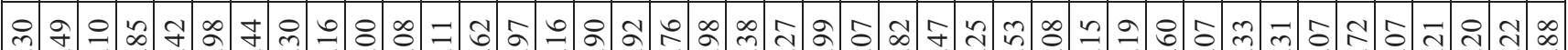

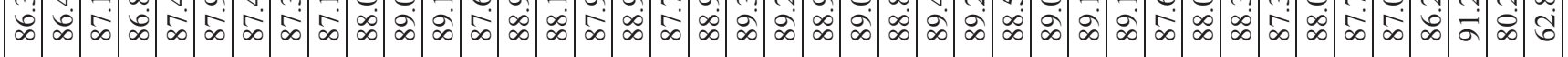

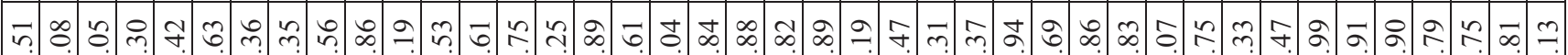

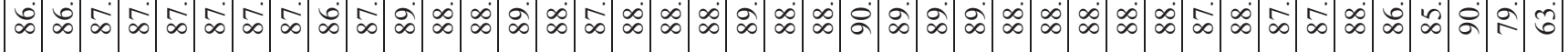

$\curvearrowleft$ 再

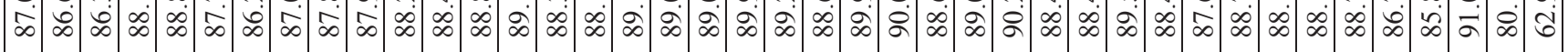

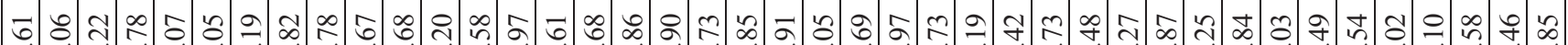

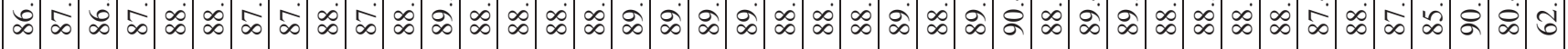

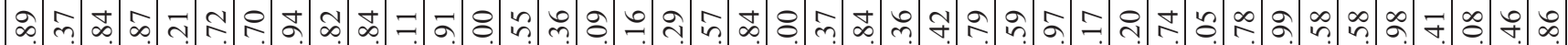

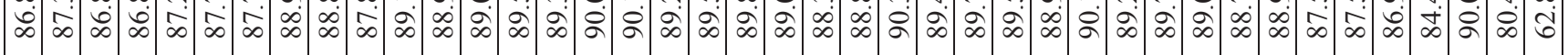

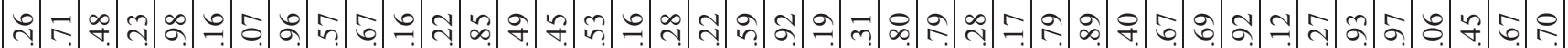

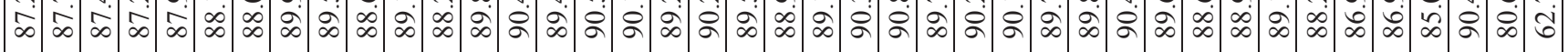
7.

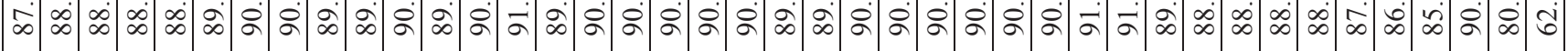

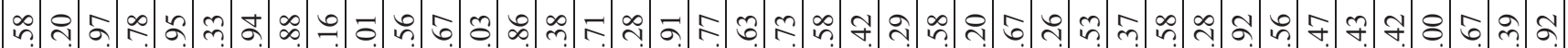

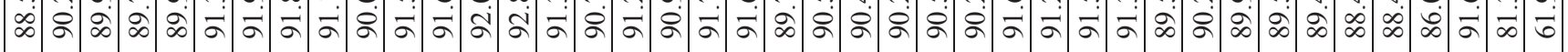

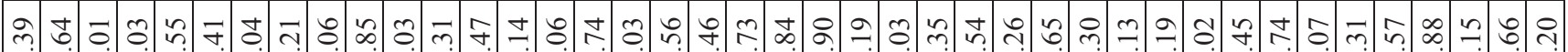

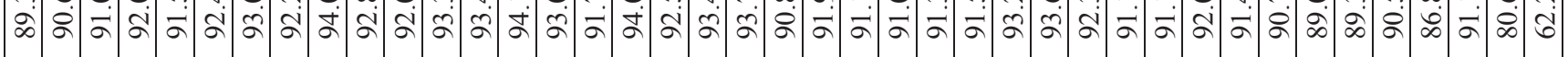

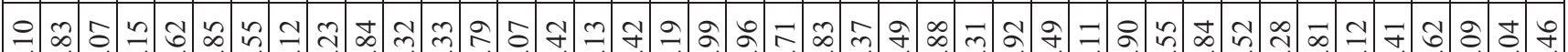

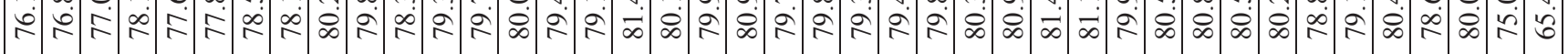

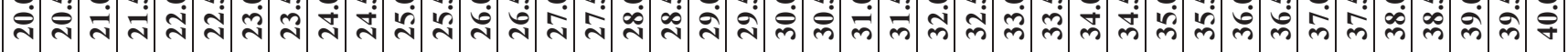




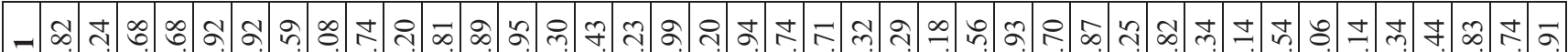
卉至

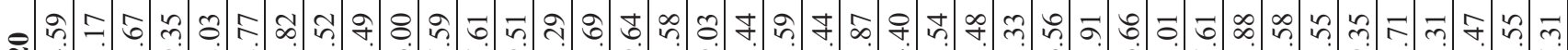

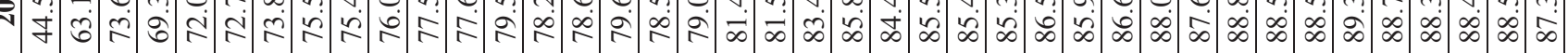

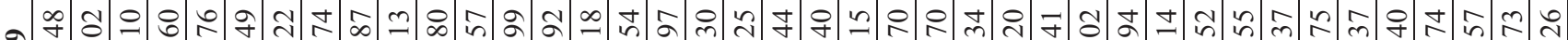

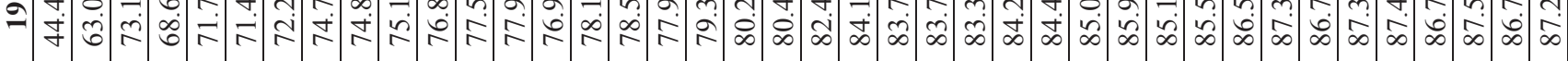

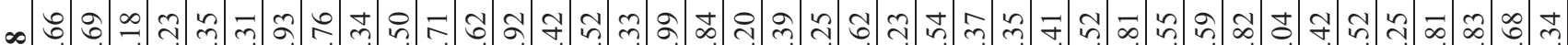

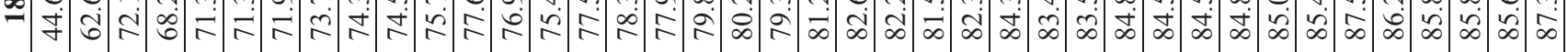
ఏి

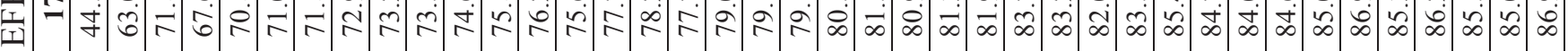
•

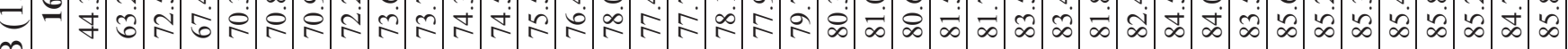

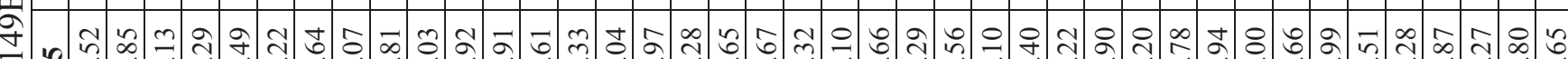

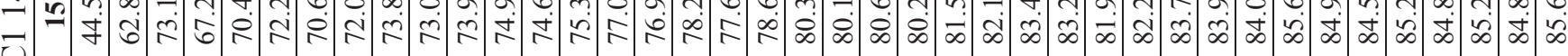

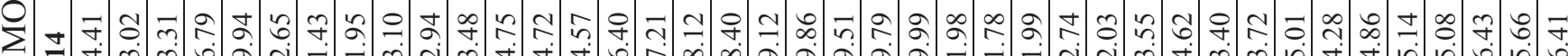

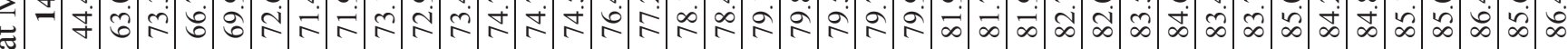

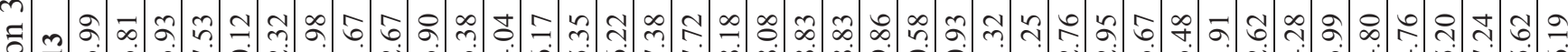

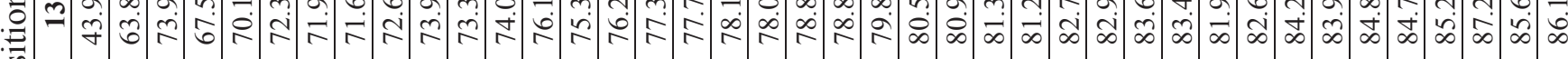
ㄱ ก

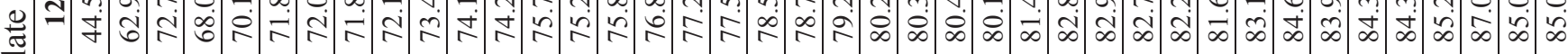
प्ठ = ప.

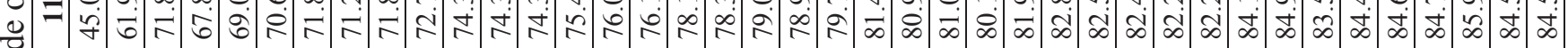

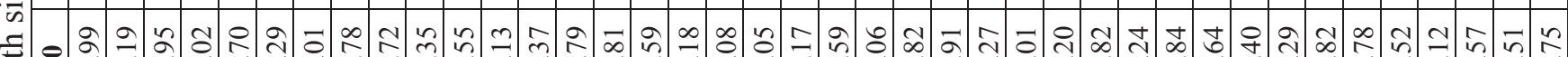

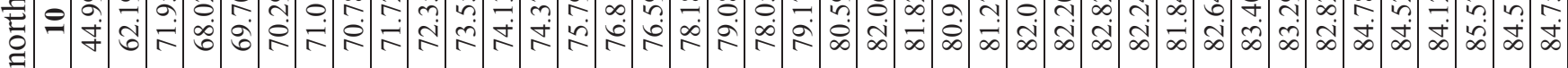

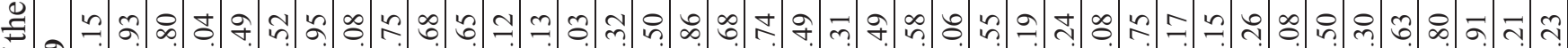
फ़

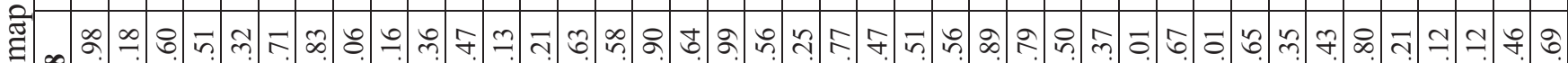

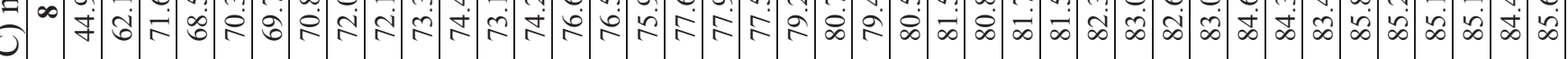

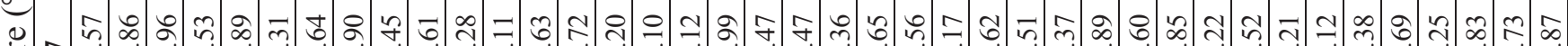

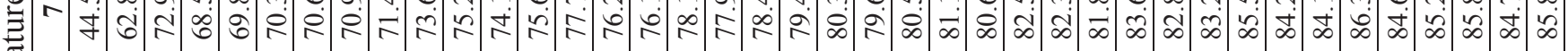
节

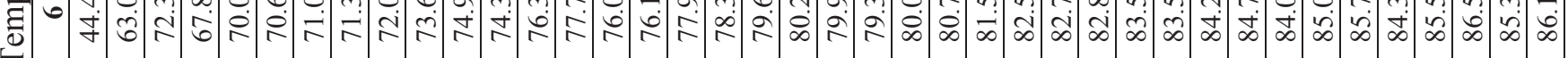

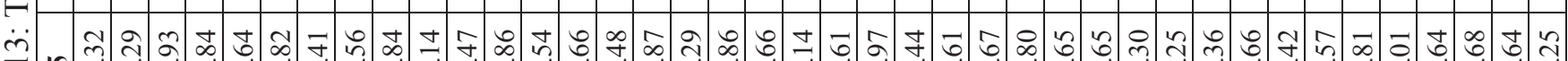

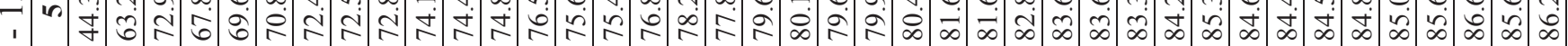
d m 豞

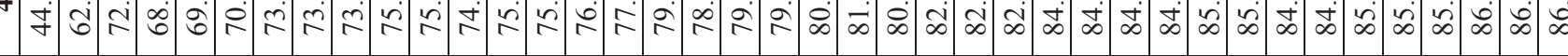

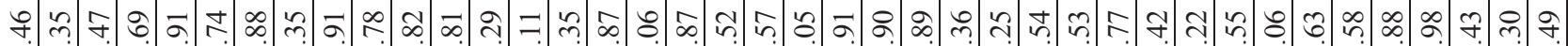

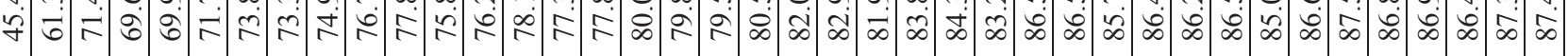
兄

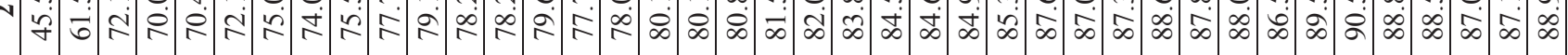
- Є

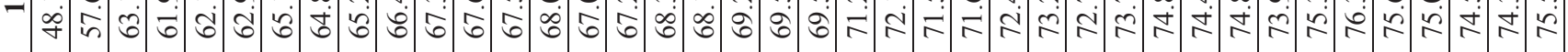
$\because$ 急 


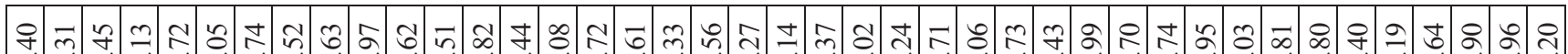

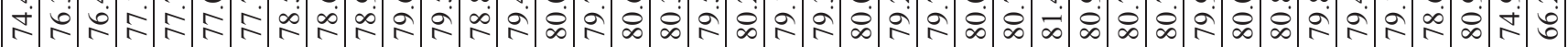

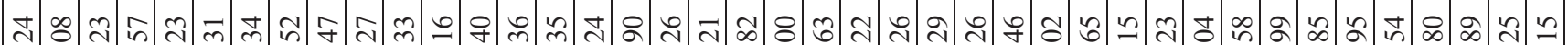

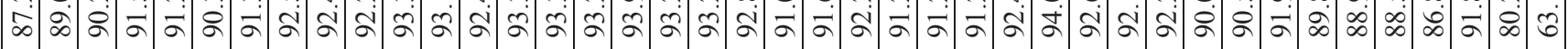

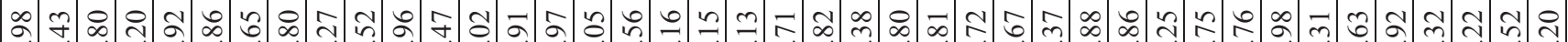

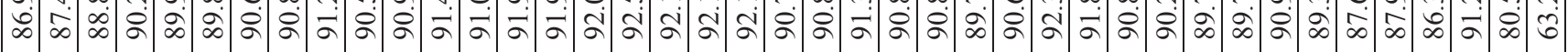

ที่

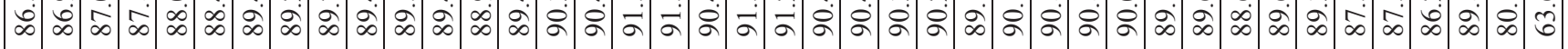

के

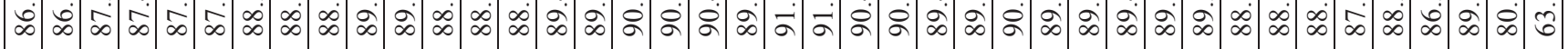

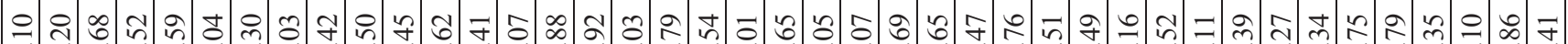

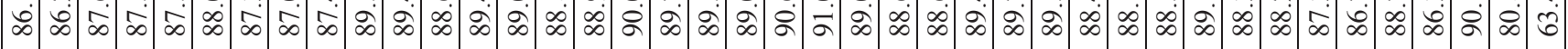

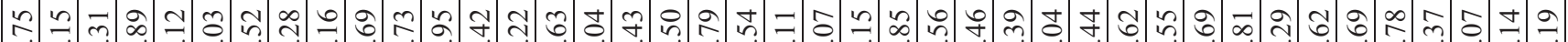

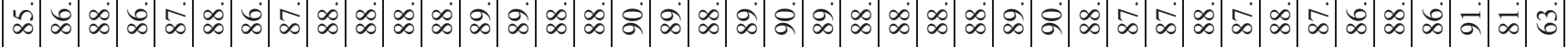

స़

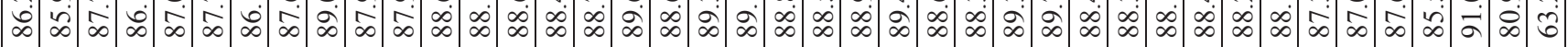

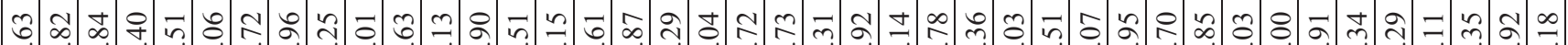

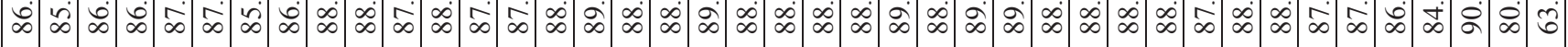

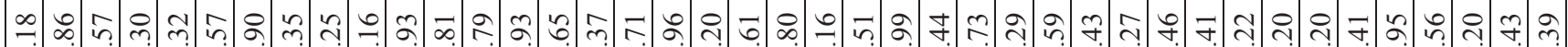

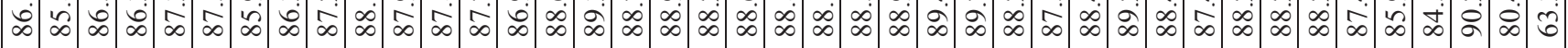

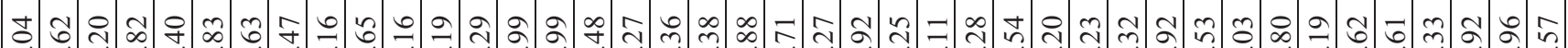

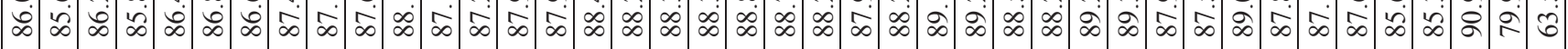

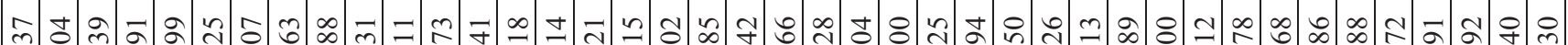

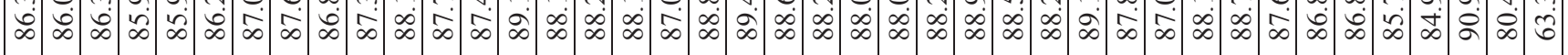

t) $\infty$ 의

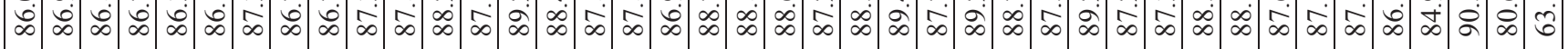

ๆ

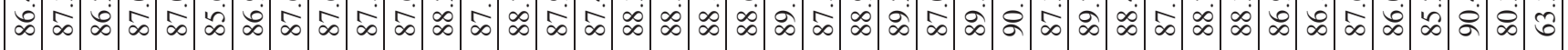

只

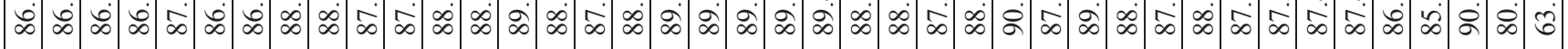

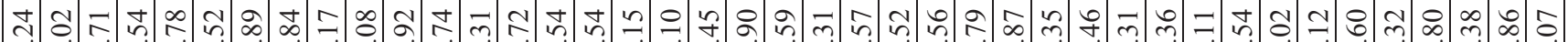

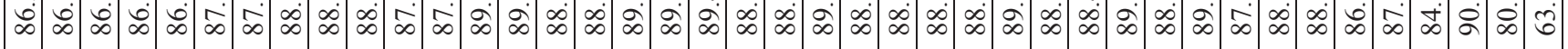

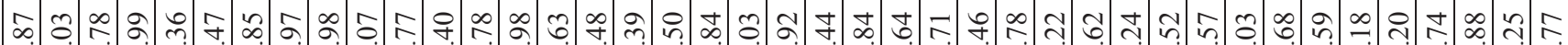

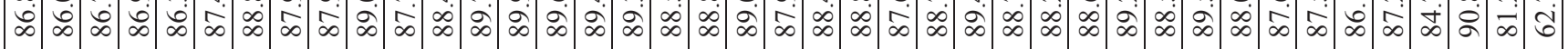

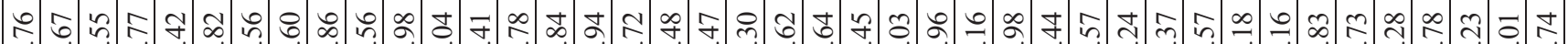

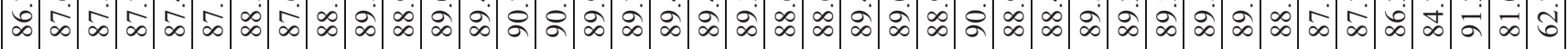
\&)

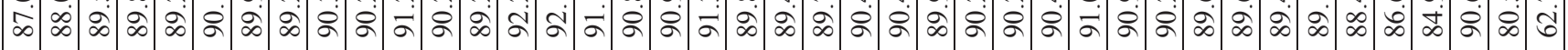

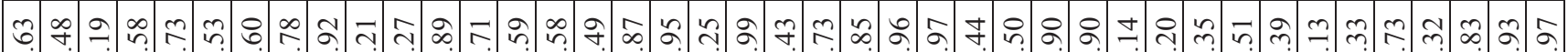

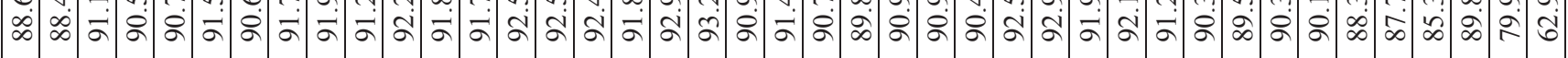

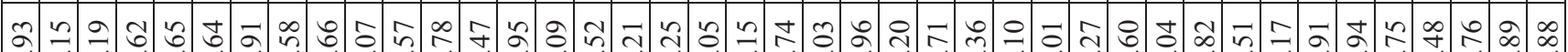

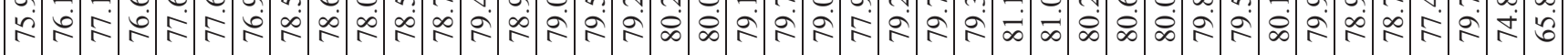


- $\infty$ సุ

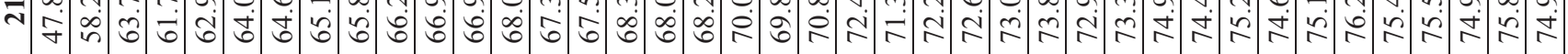

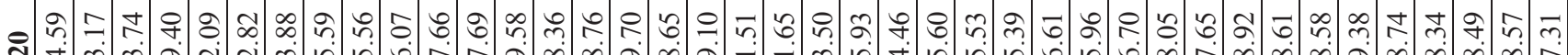

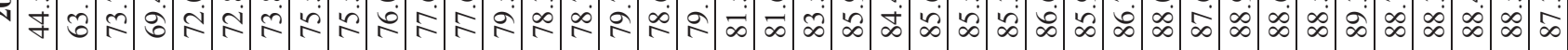

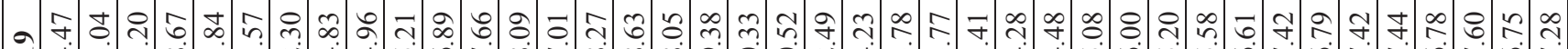

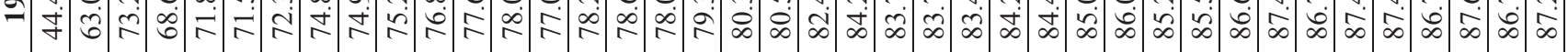

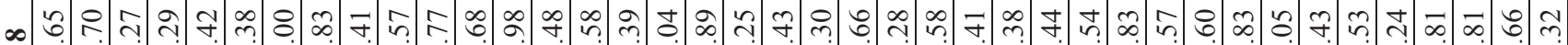

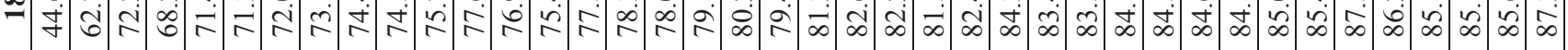

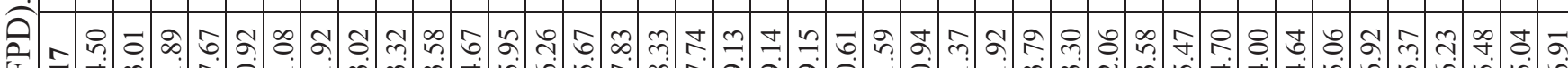
盾=

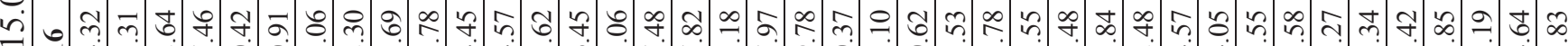

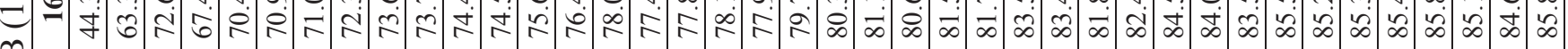
a

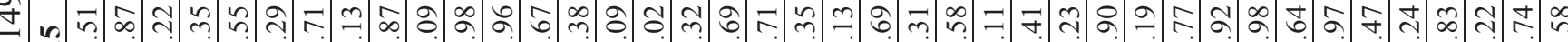

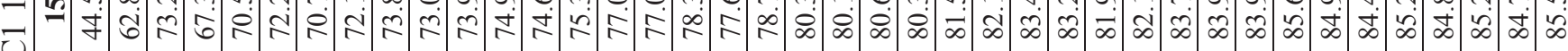

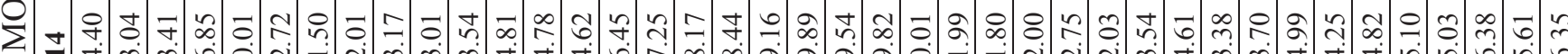

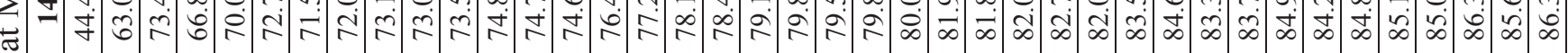
m

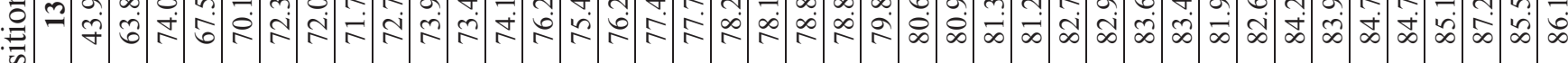

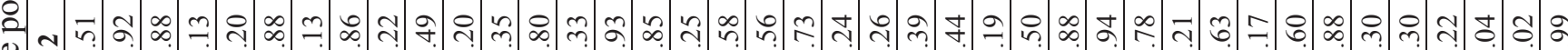

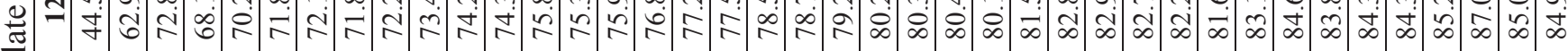
幽 =

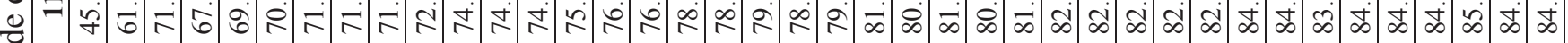

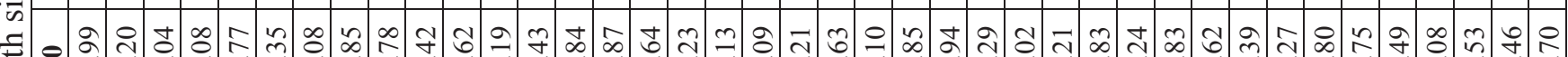

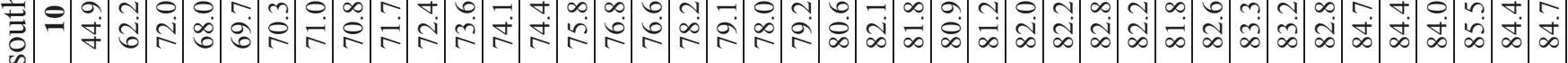

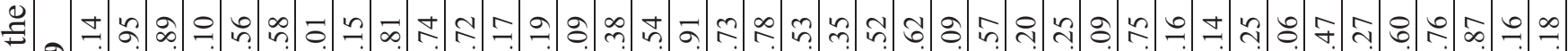
फ्ठ

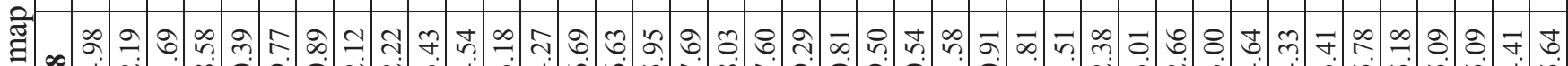
U.

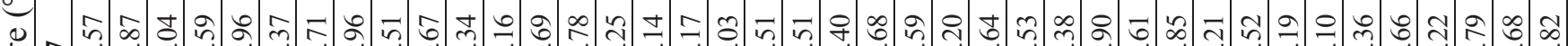

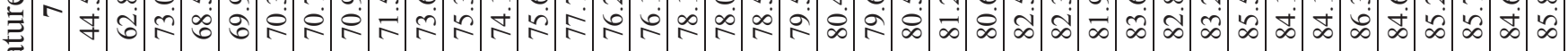

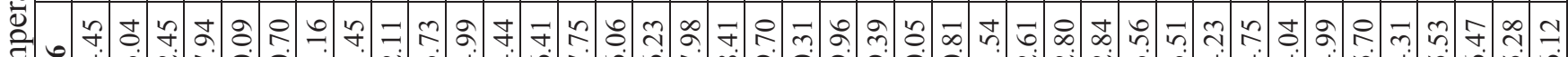

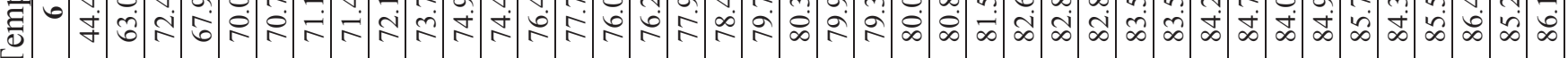

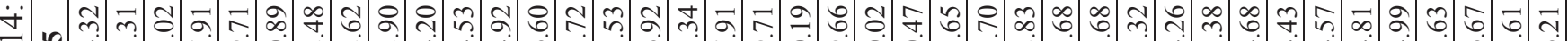
1 ๑

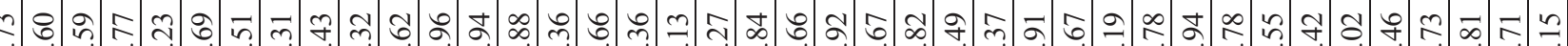
$\checkmark$ 莳

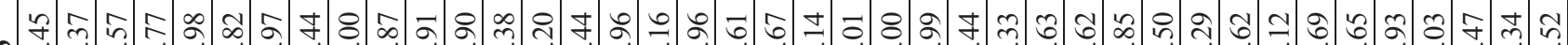

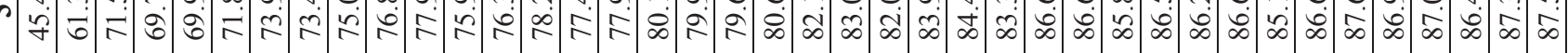

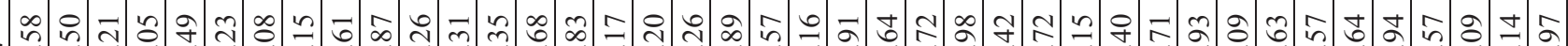

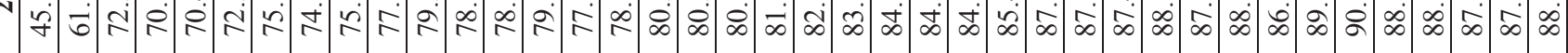

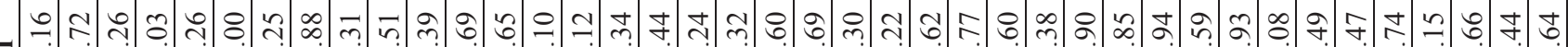

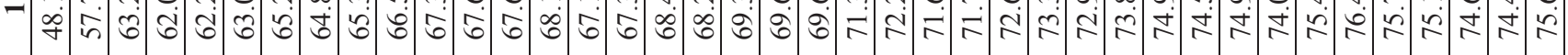

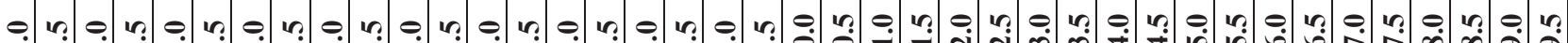

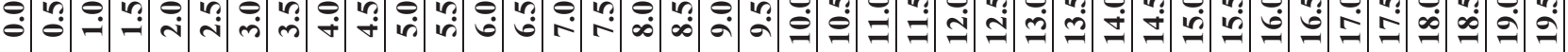




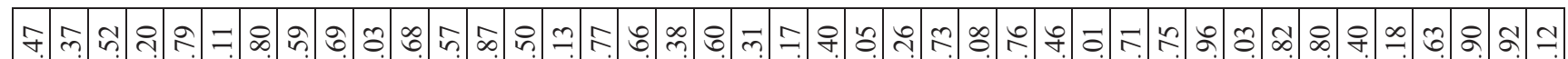

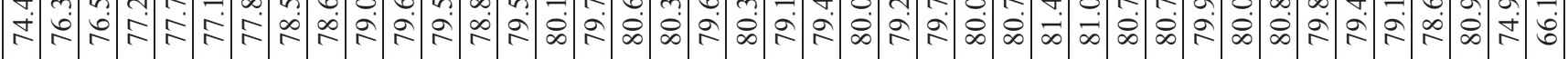
ปุ

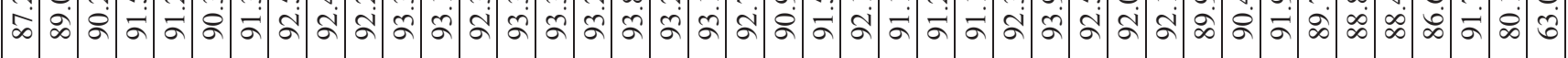
ฉ.

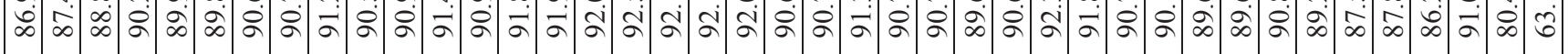
กุ. 8 .

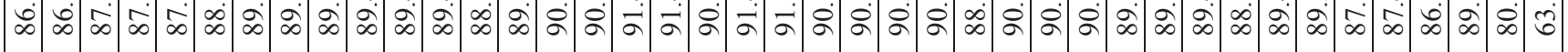

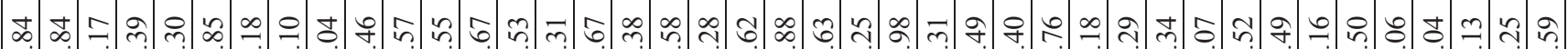

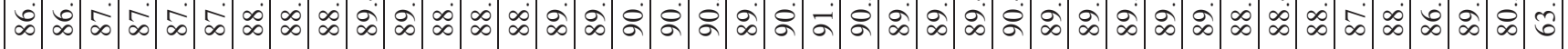

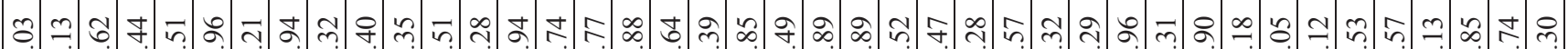

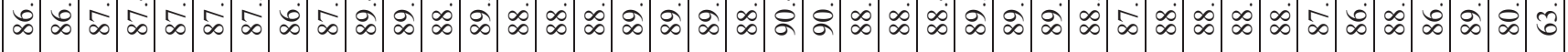
b.

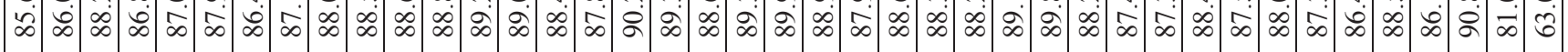

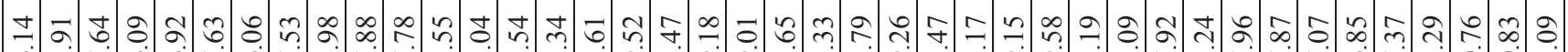

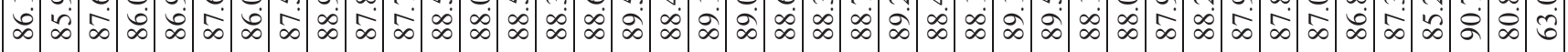

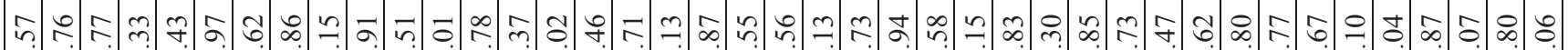

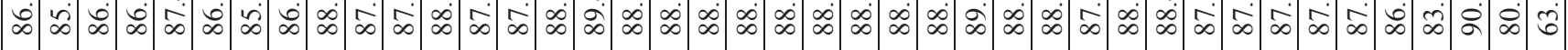

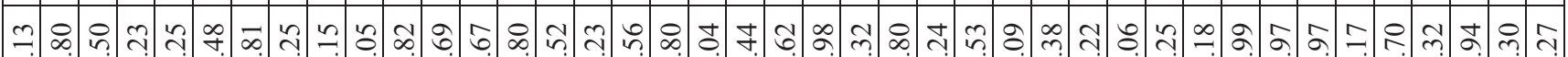

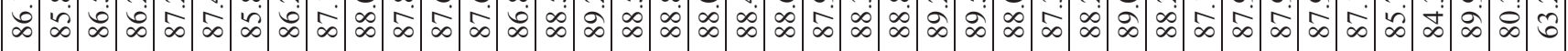

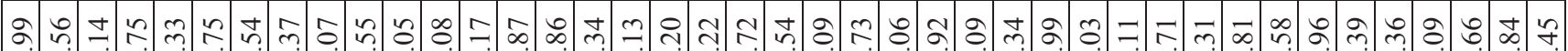

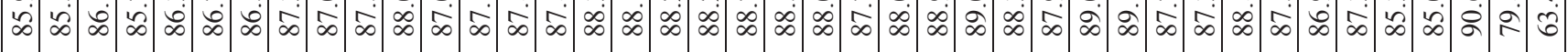

๙ె

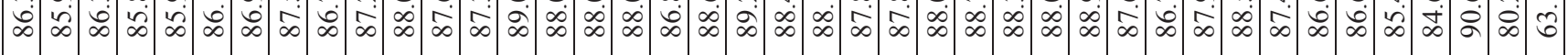

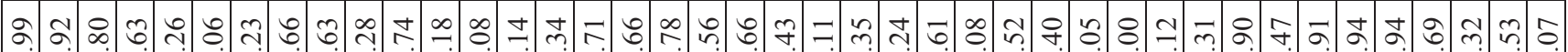

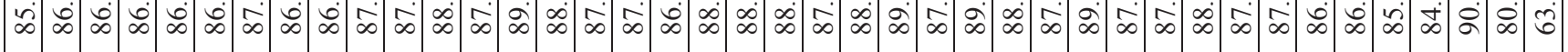

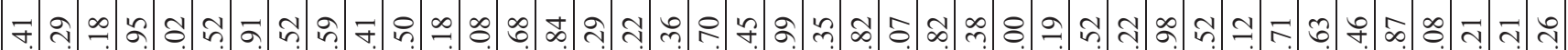

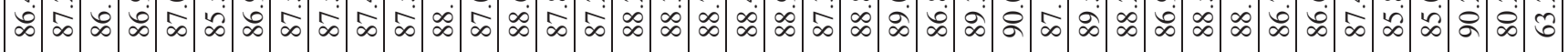

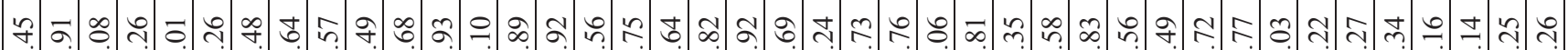

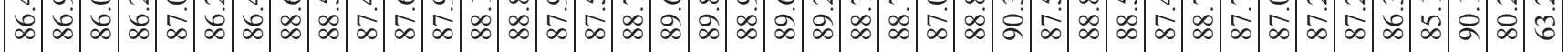

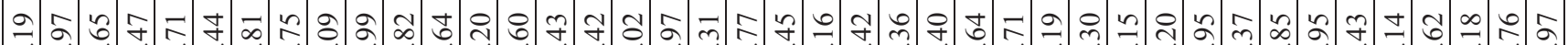

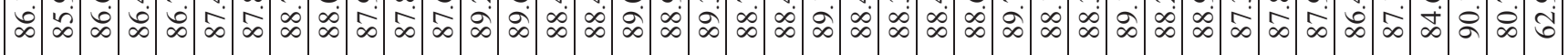
ळ.

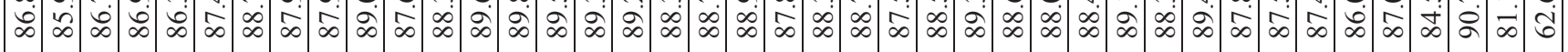

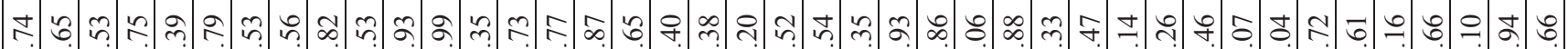

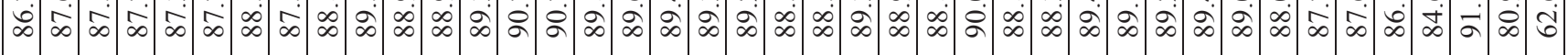
จำ

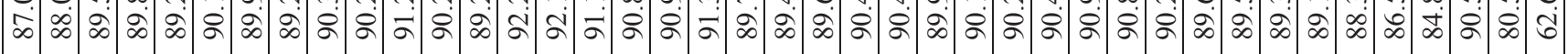

๘

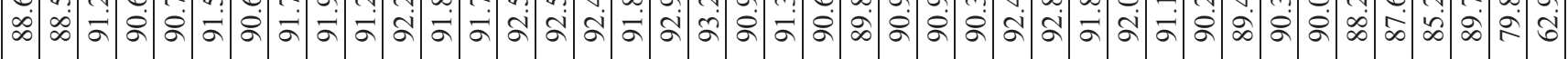

ชุ

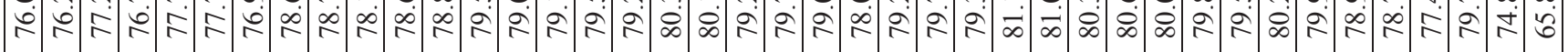
过

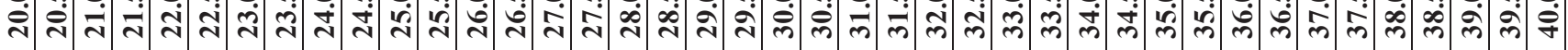


- ㅇำ

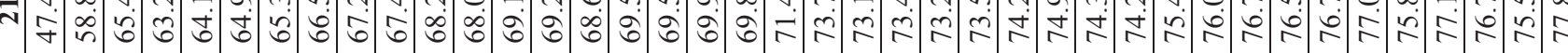
-

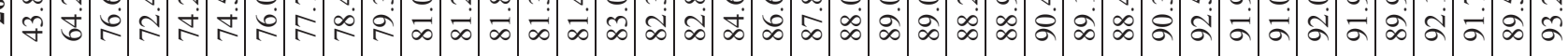

- 守

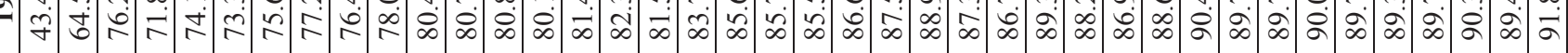

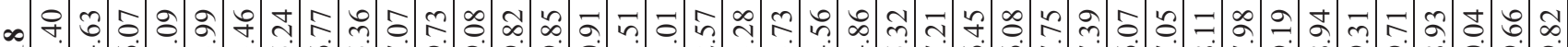
$=$ f

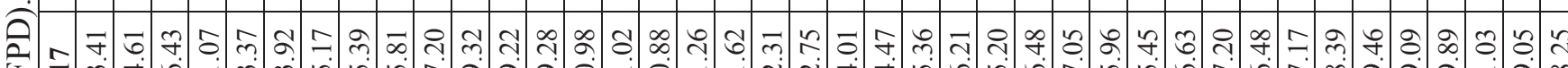

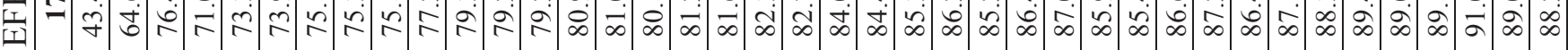
•

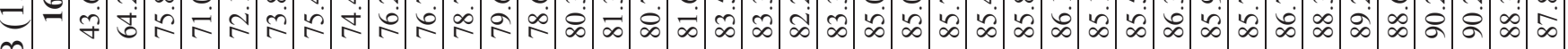
手 म

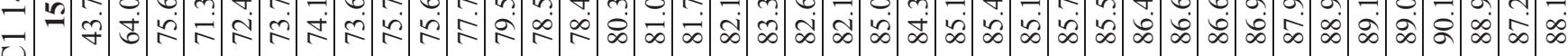

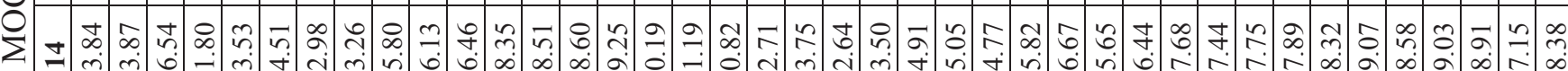

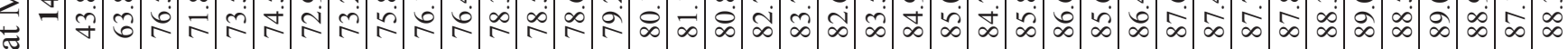
ナ๐

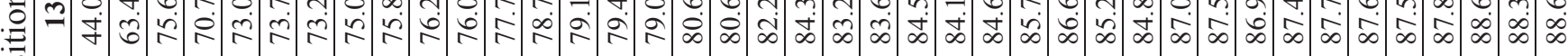

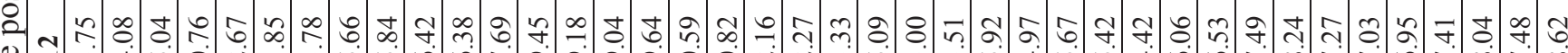

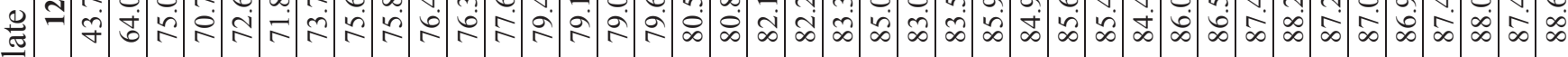
Ч్ర =

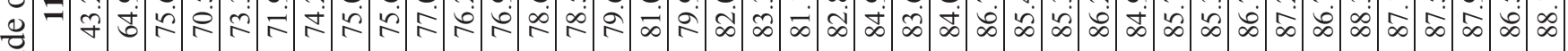
๘

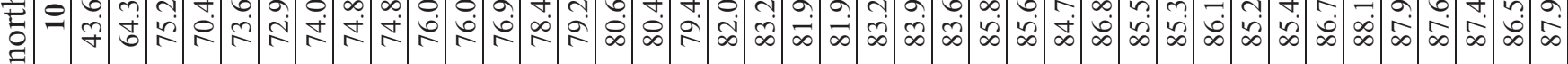

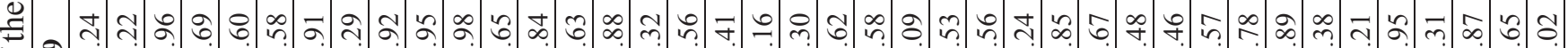
㫕

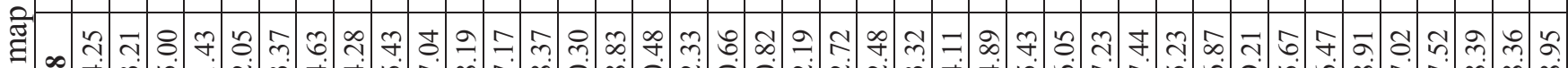

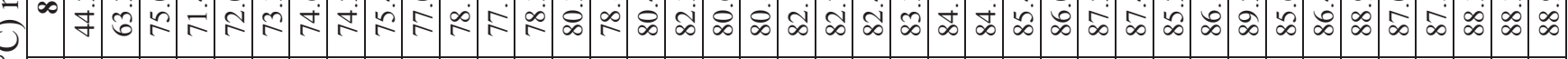

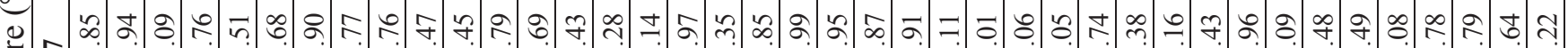

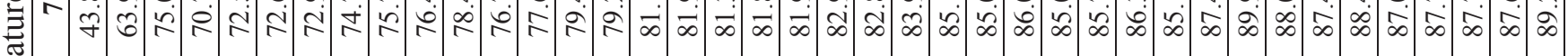
节

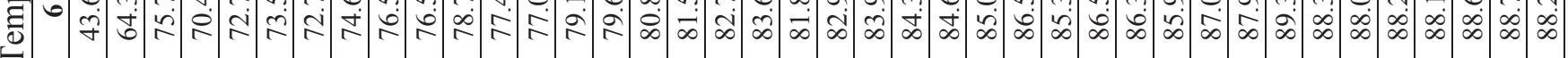

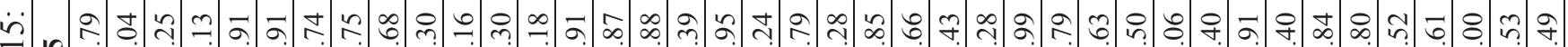

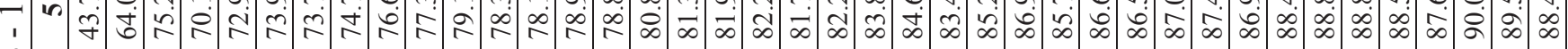
๑)

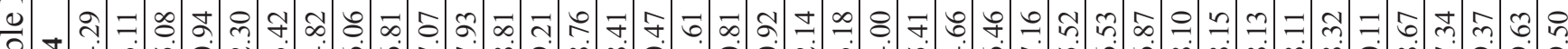

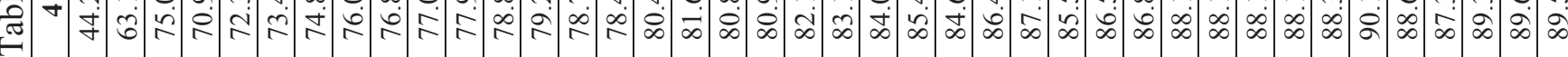

m

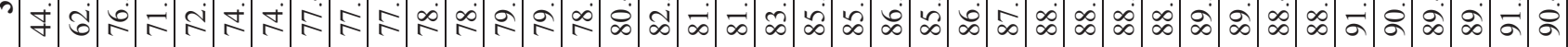

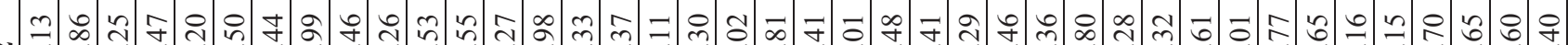

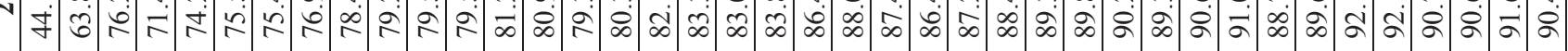
-

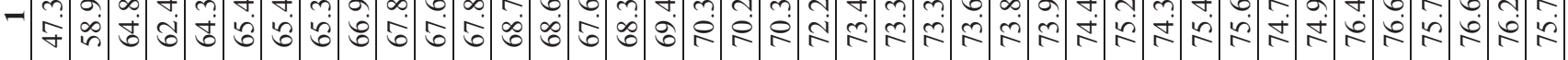
- n 官 


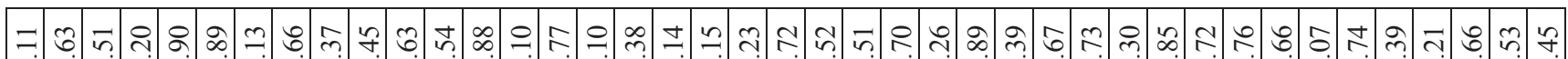

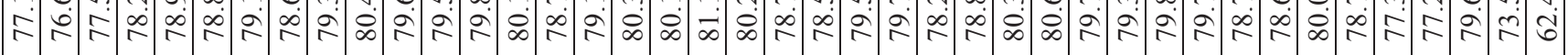

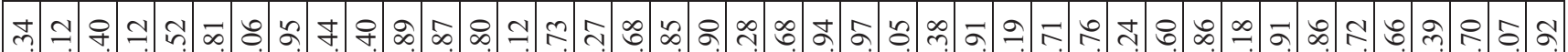

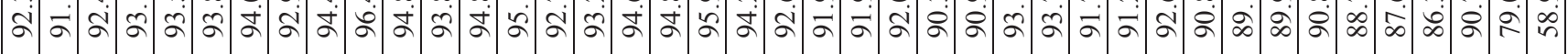

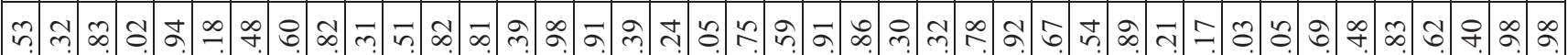

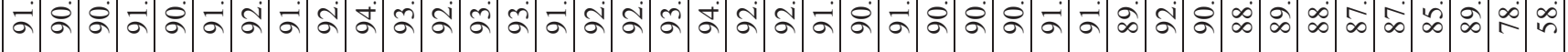

ఫ t

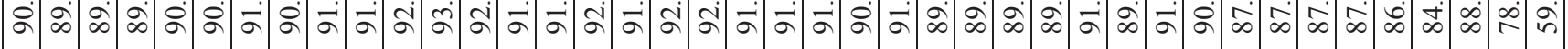

:

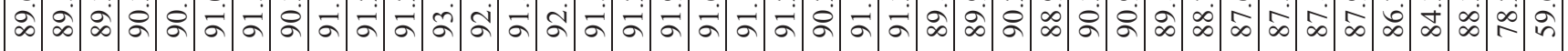

: ஊ

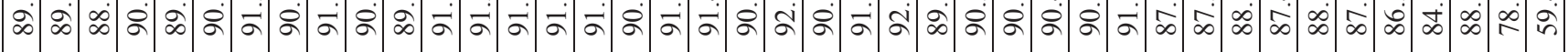

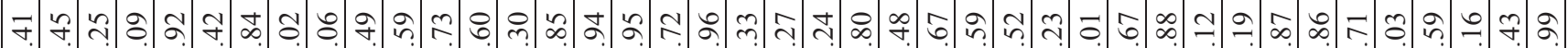

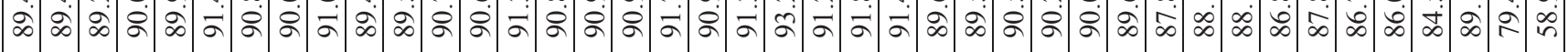

б

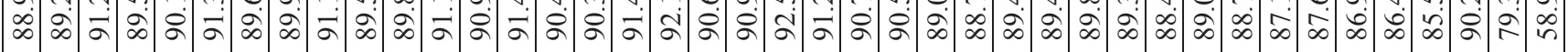

\&

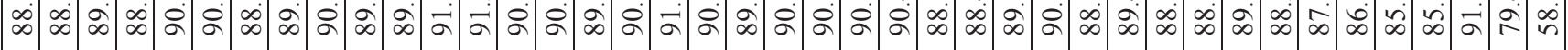
సิे

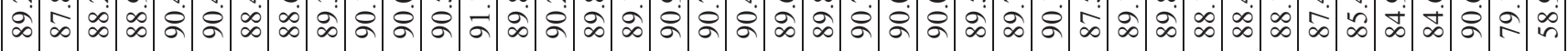

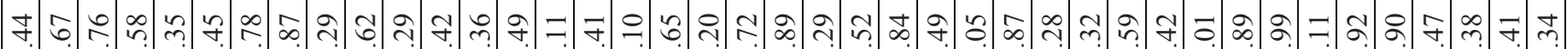

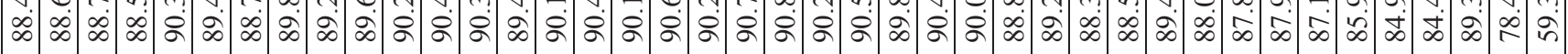

ป

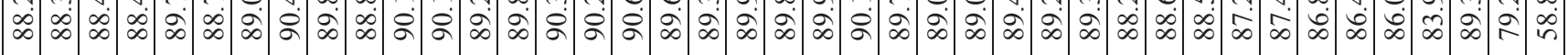

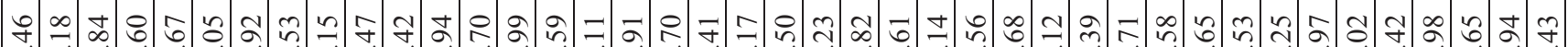

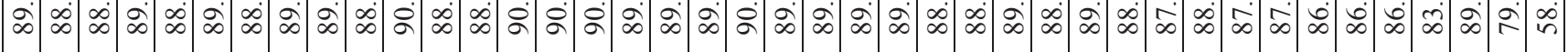

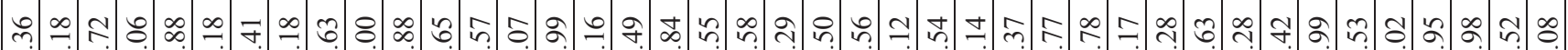

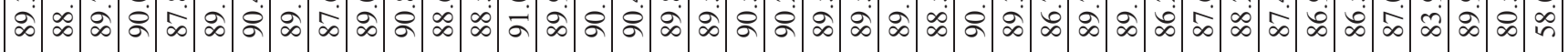

f

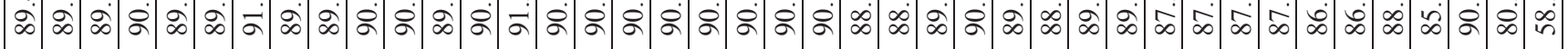

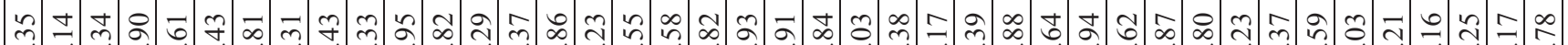

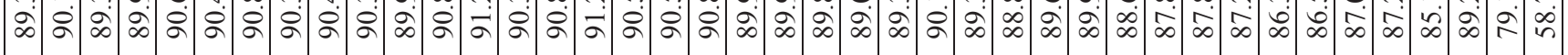

œ

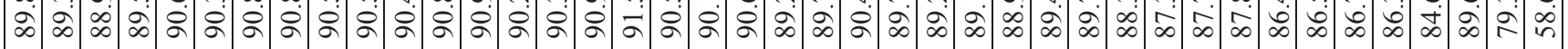
군

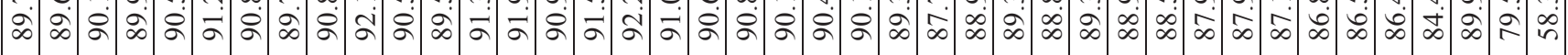
Fn

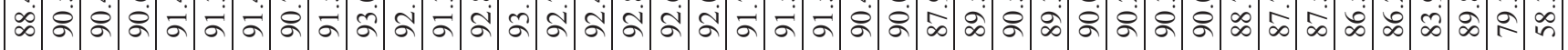

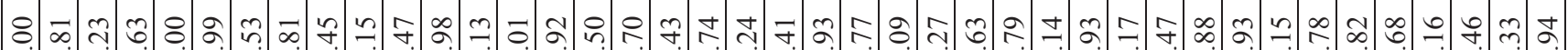

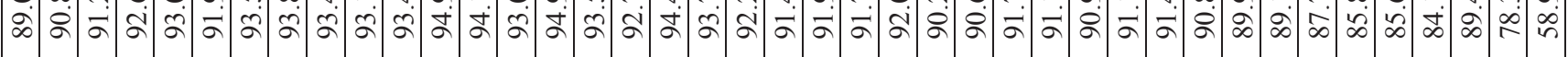

글 m

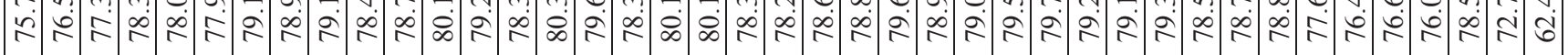


- Ұ.

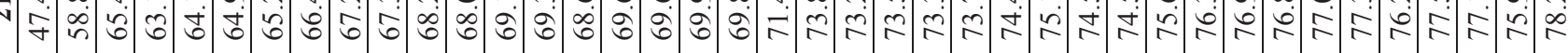

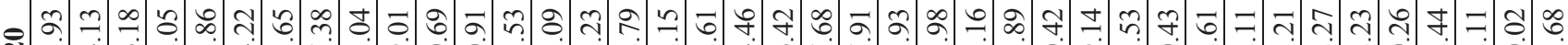

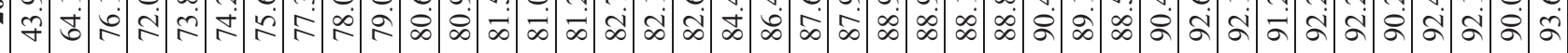

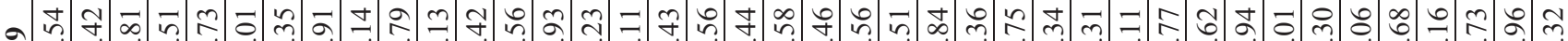

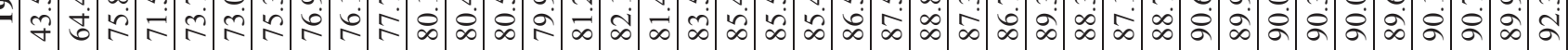

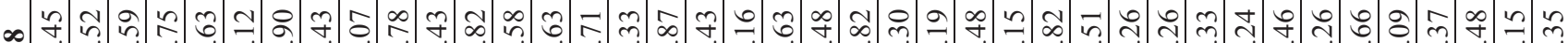

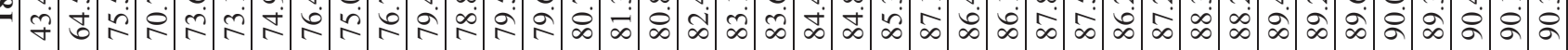
全 在= ㅁำ

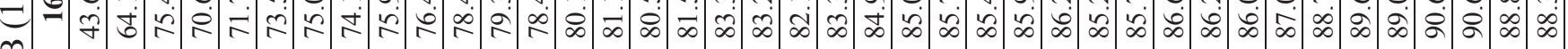
เด 寸

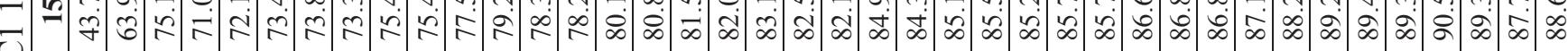

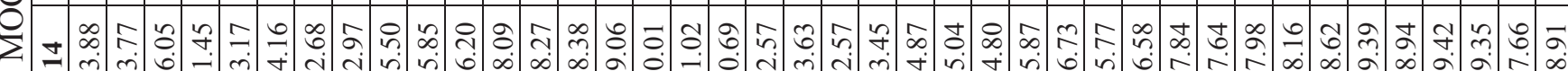
$\vec{z}$
$\gamma$ ป

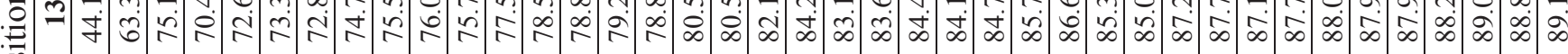

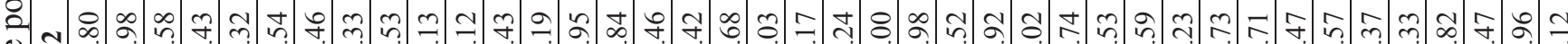
考二

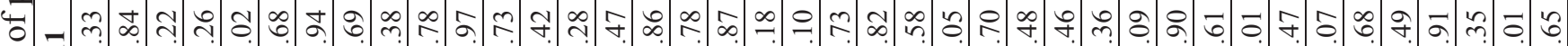

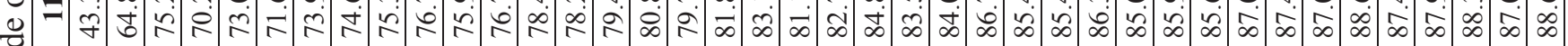
"

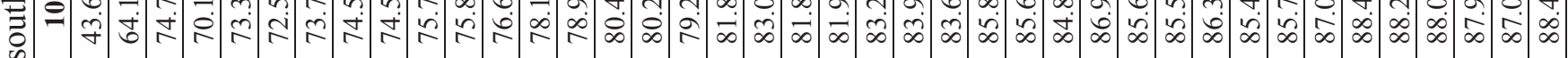

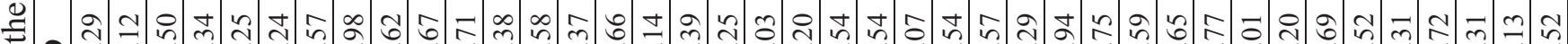
फ्ठ

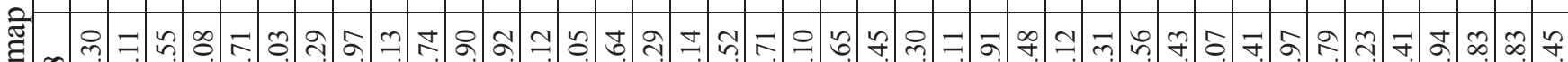

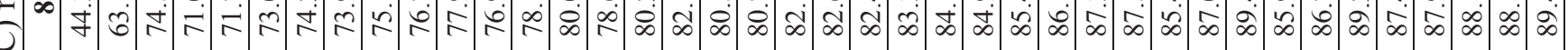

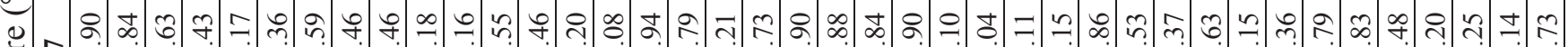

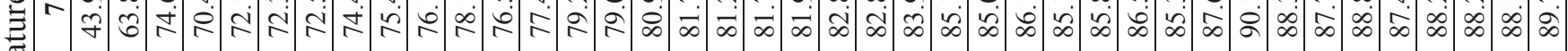

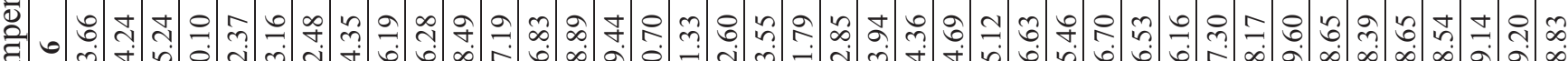

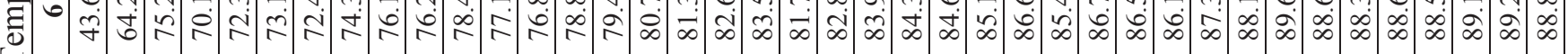
ㅂ.

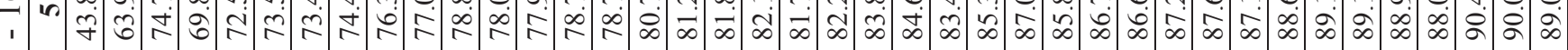
( t)

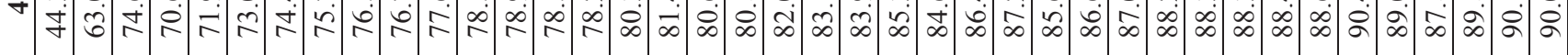

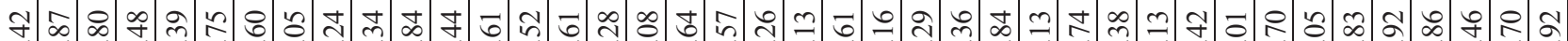
古

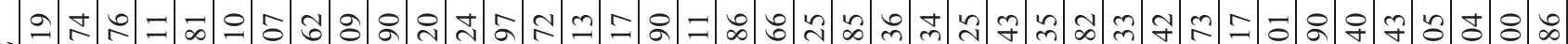

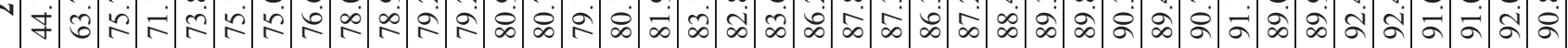
- లి

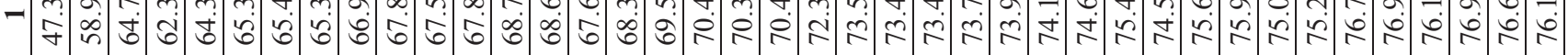
- n

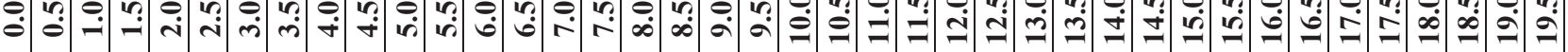


$4 \infty$

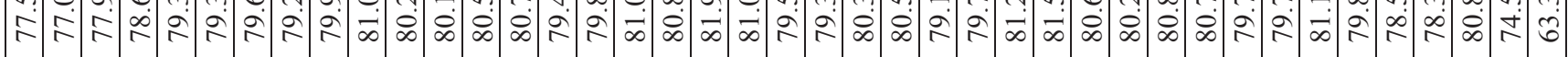
$\infty$ t

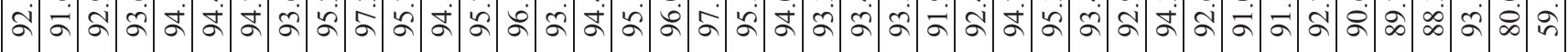

ఫ $\partial$. f.

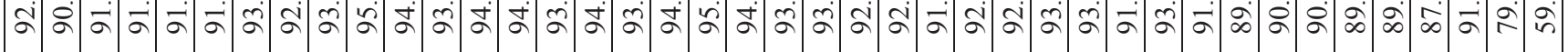

b

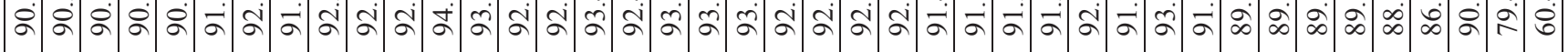

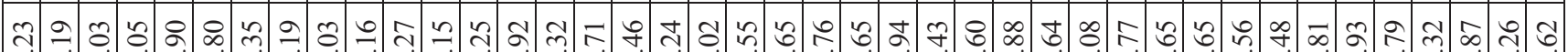

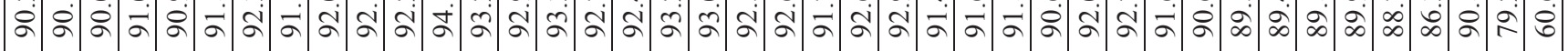

สิน

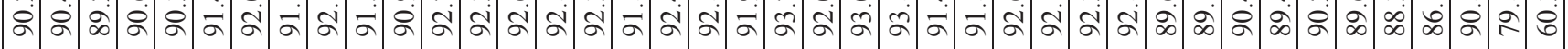

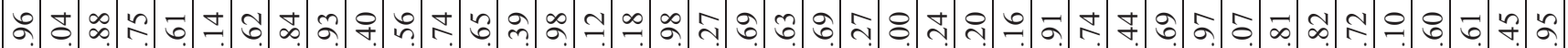

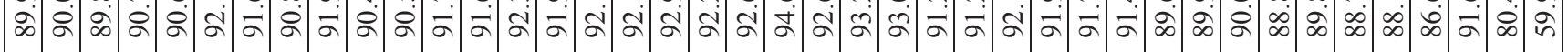

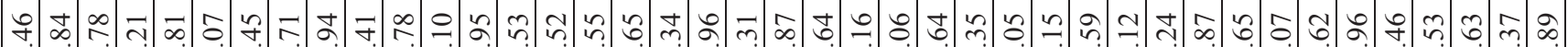

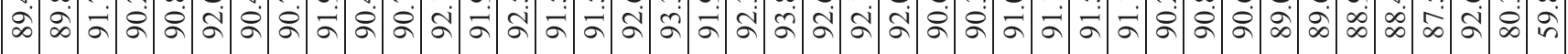

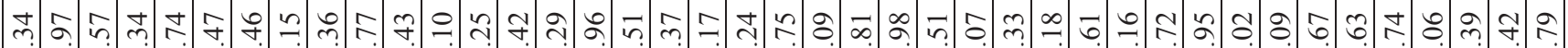

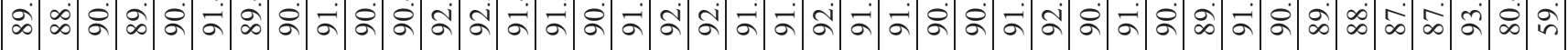

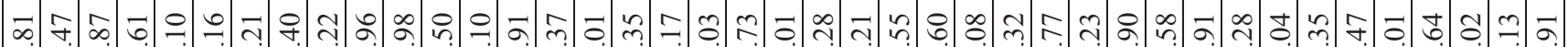

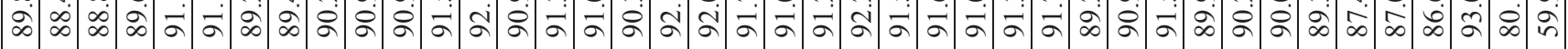
ล. กิ กิ สิ

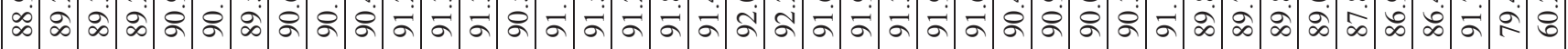

‥

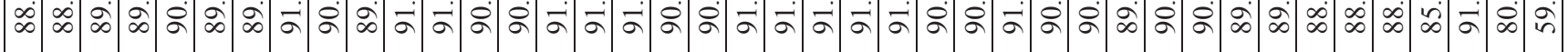

$\approx$ ?

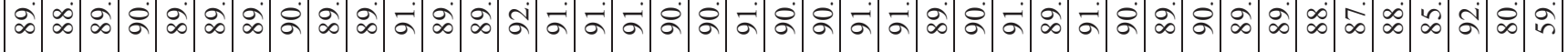

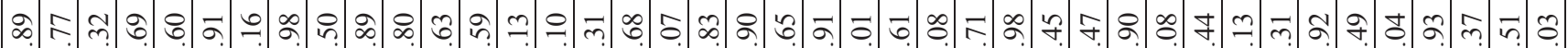

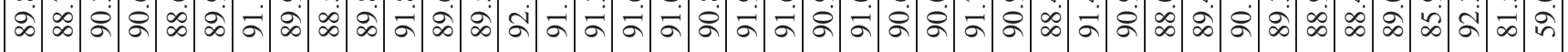

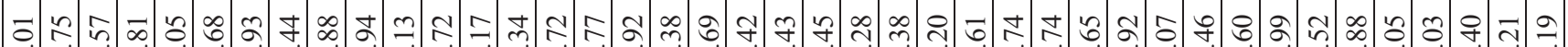

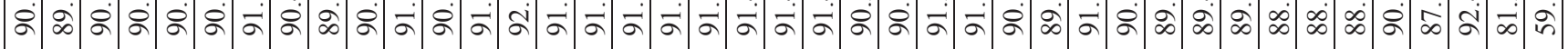

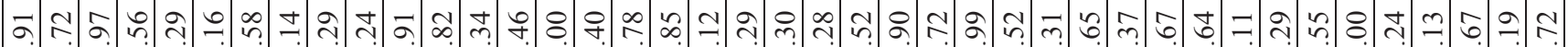

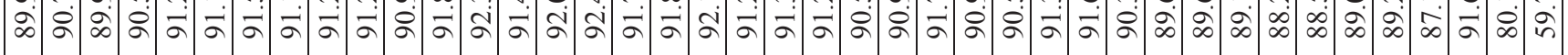

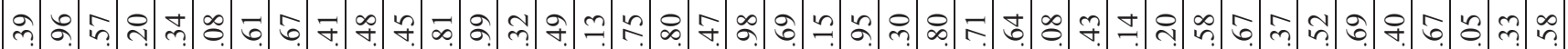

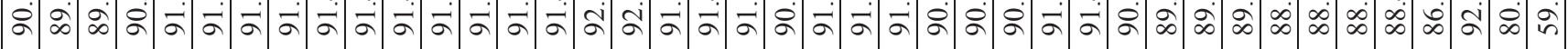

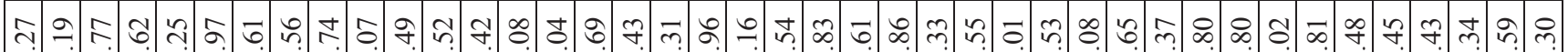

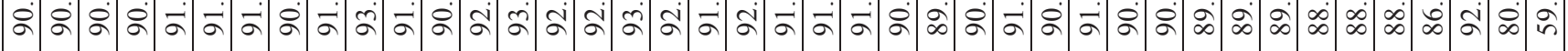
人.

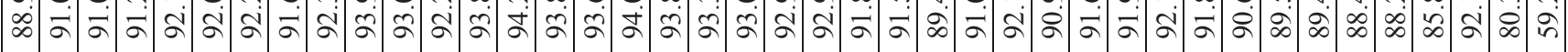

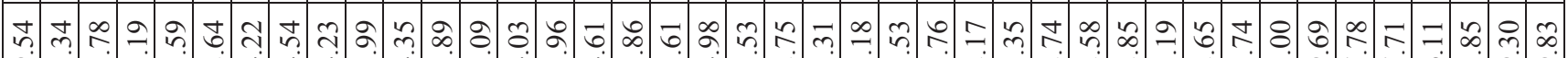

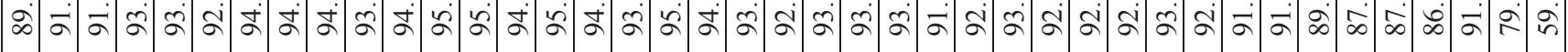

$\because$ t)

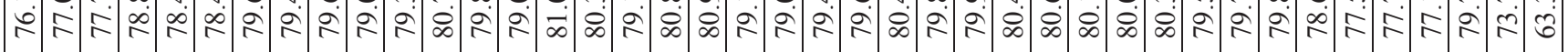

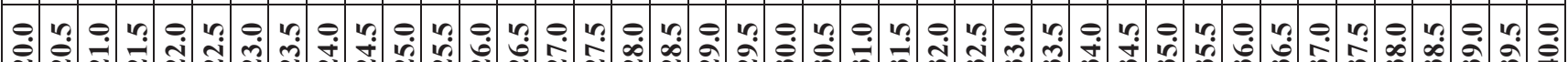

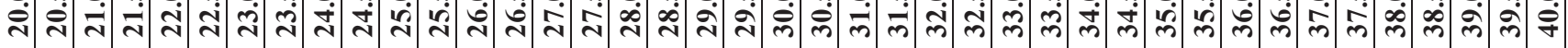


- ภิด

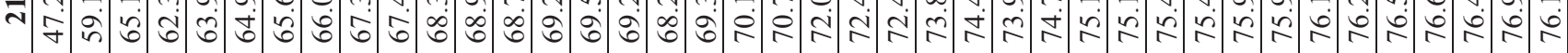

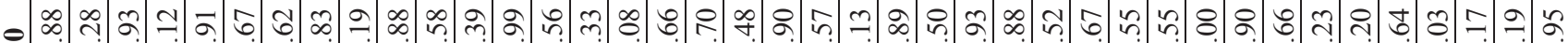

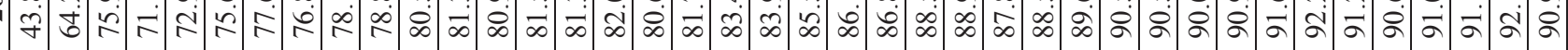

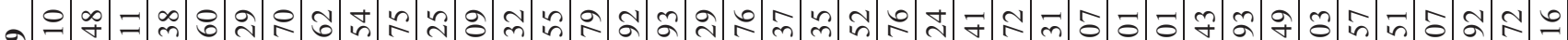

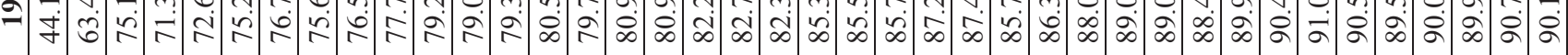

$\infty$ లి

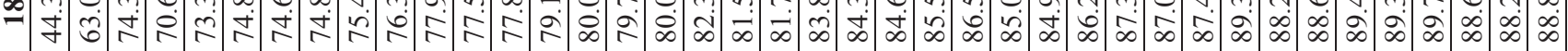

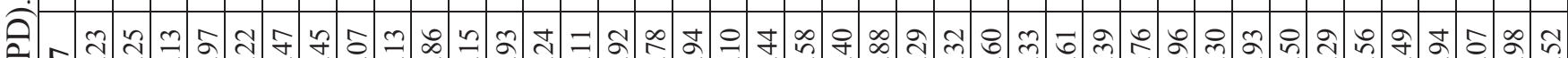

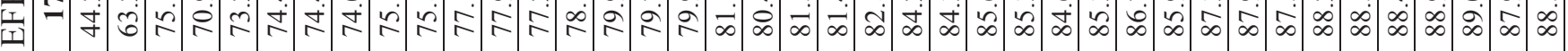
๑

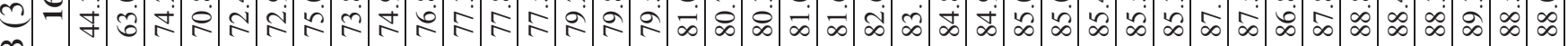

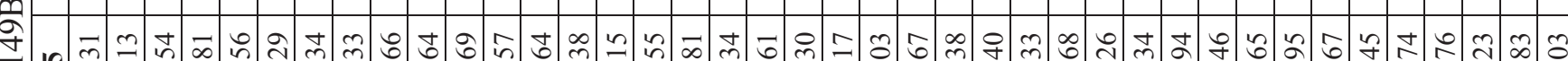

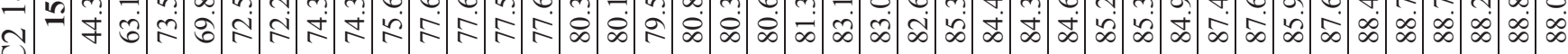

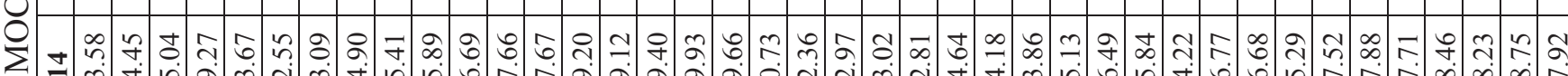

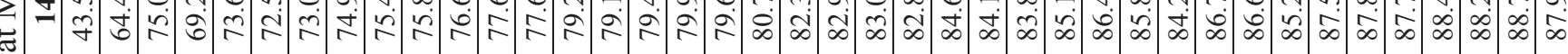
चี

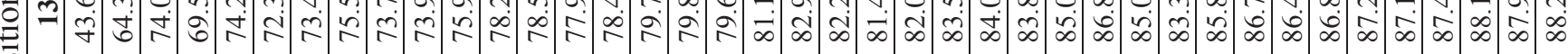
ป

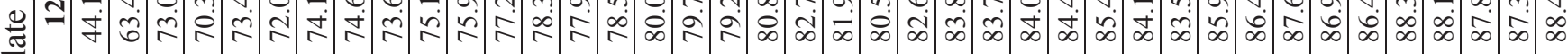
苟

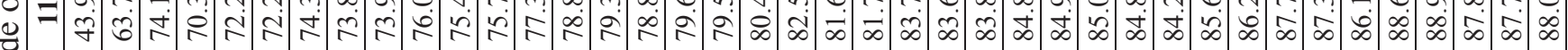

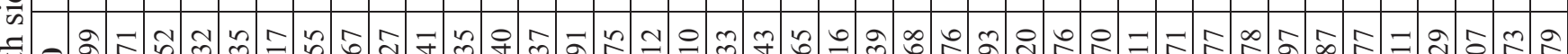

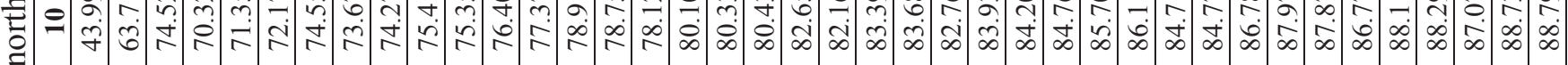
ક $=$ ก

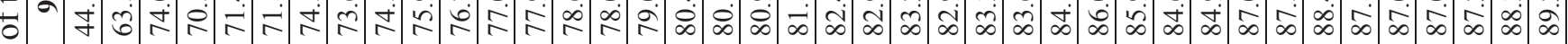

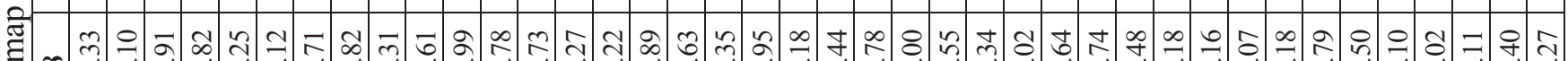

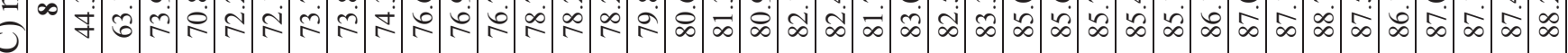

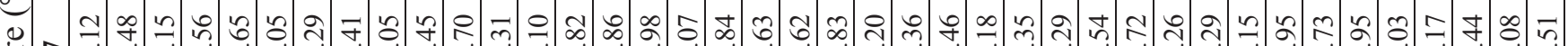

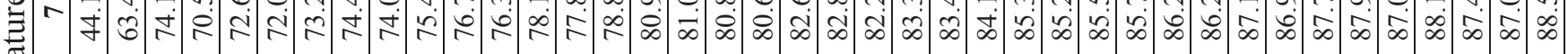
节

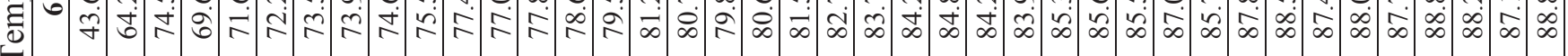

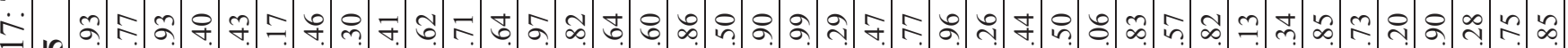

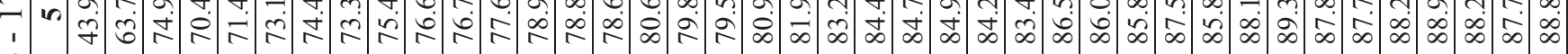
๑)

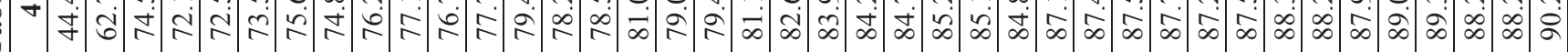

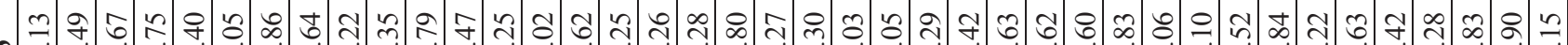

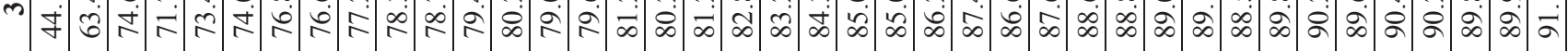

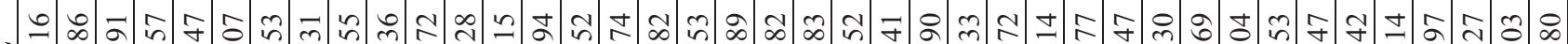

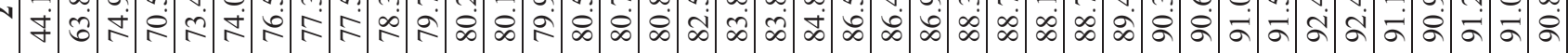

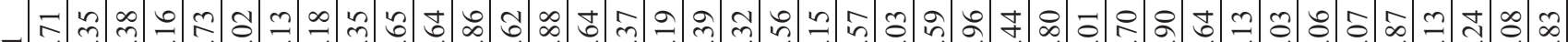

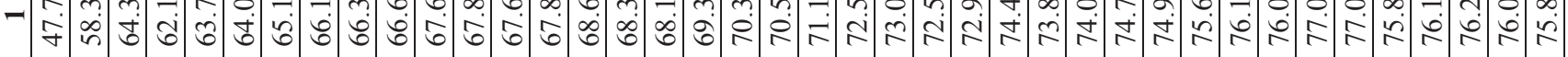

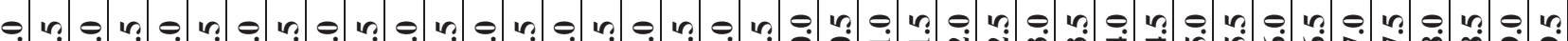
官 
Q

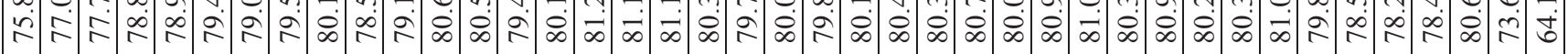

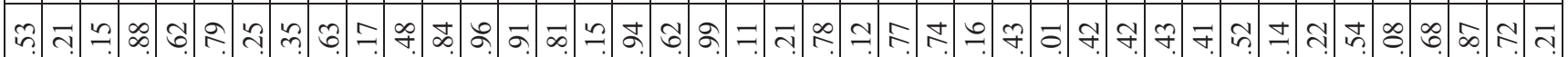

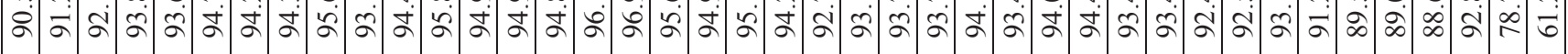

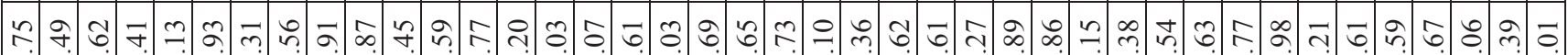

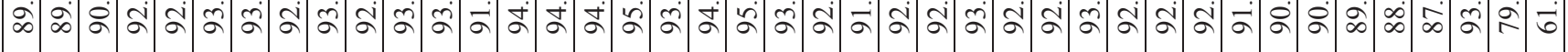

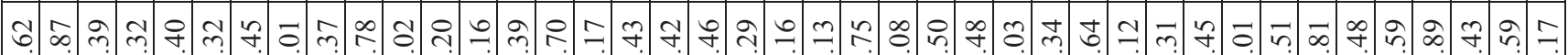

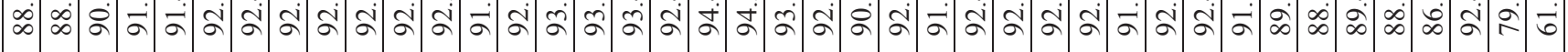

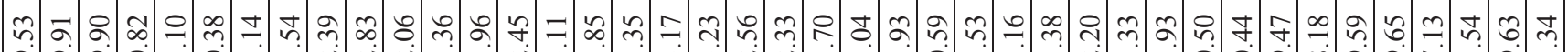

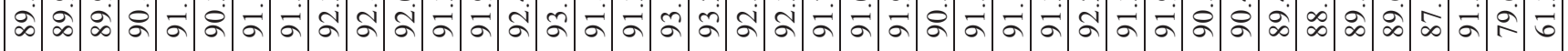

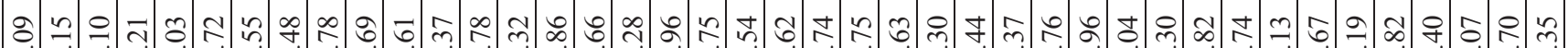

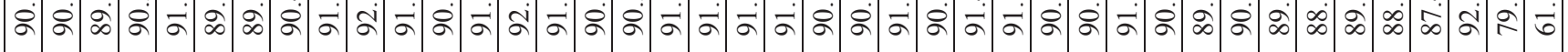
ণิ

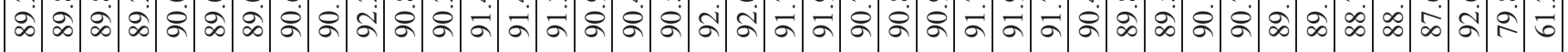

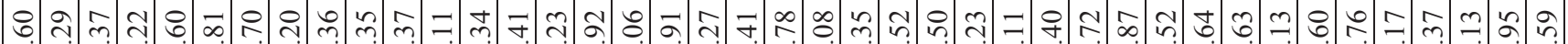

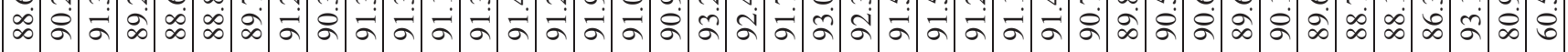

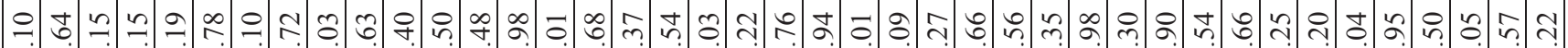

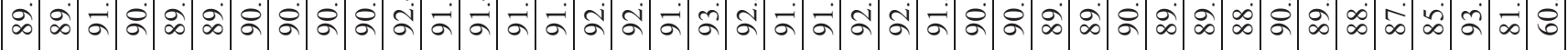

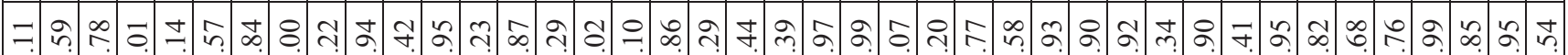

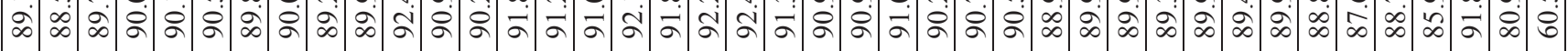

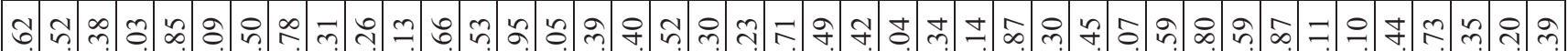

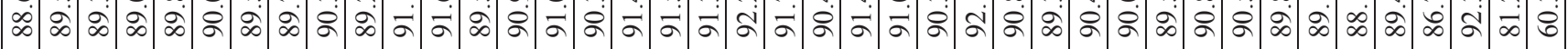
ทำ

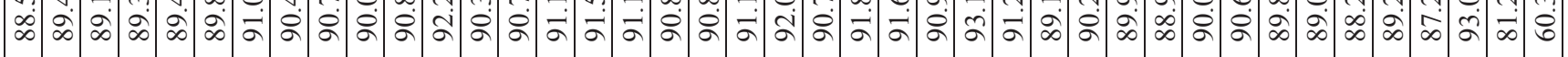

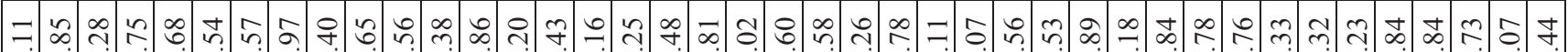

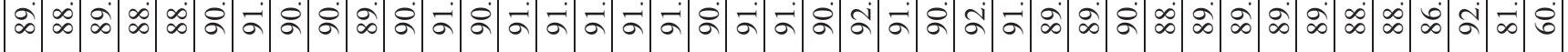

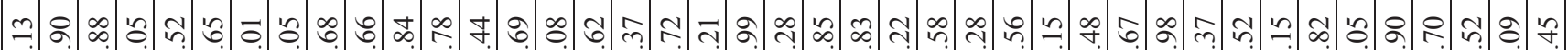

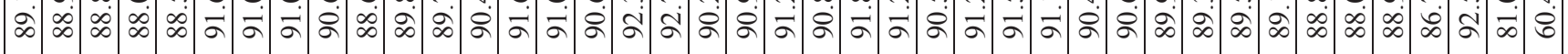

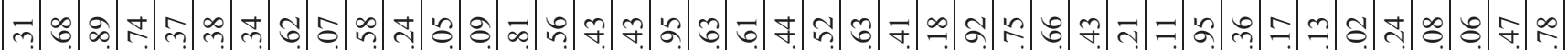

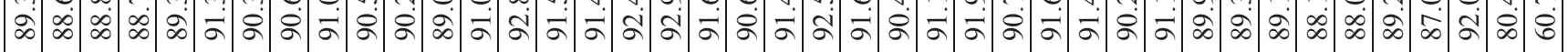
$\approx$ กิ

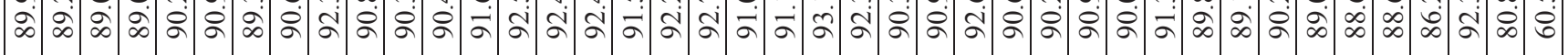

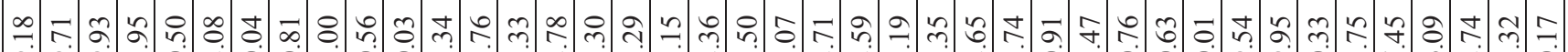

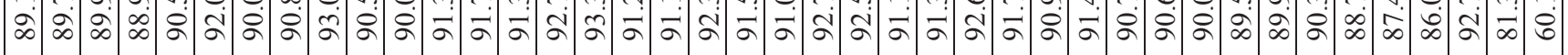

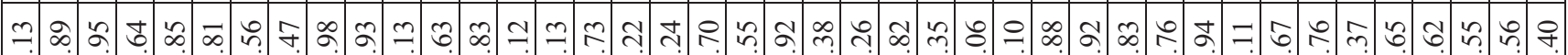

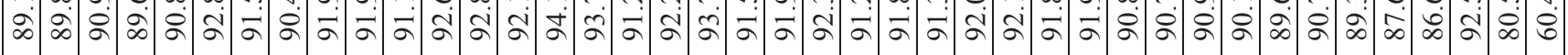
守

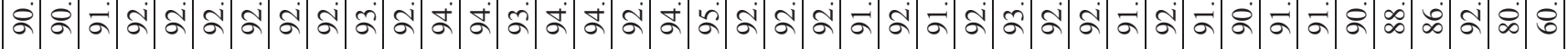
б.

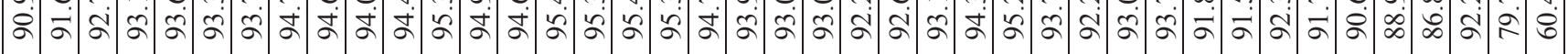

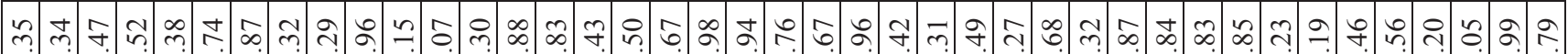

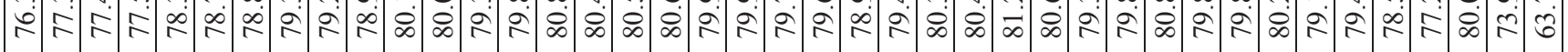




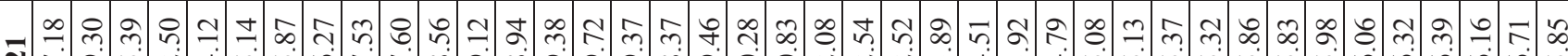

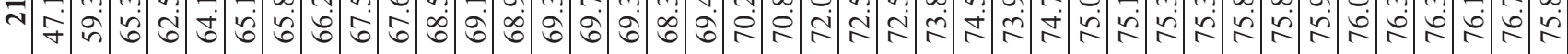

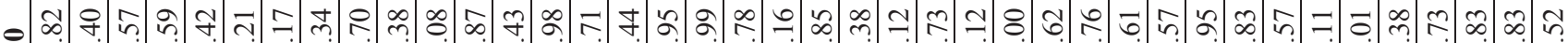

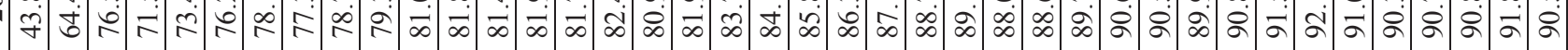

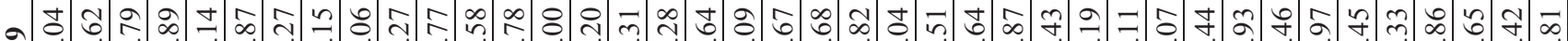

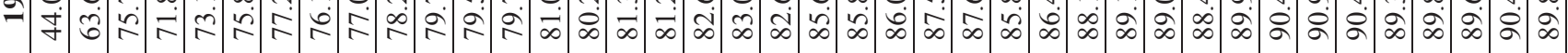

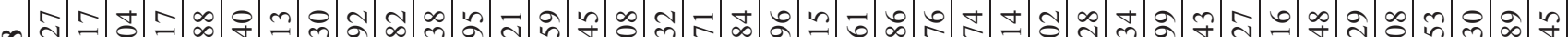

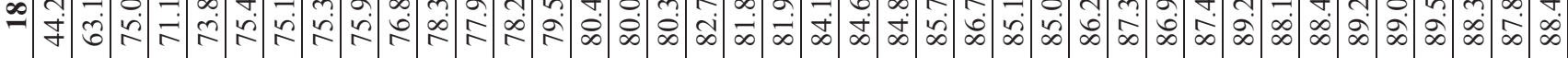

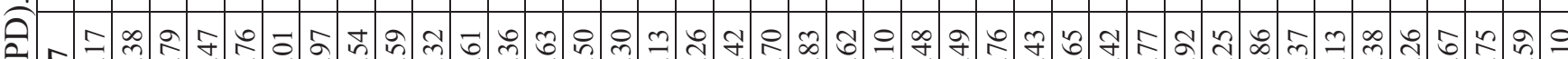

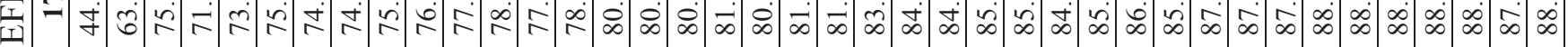
-

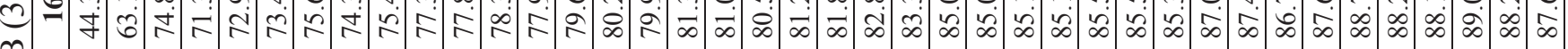

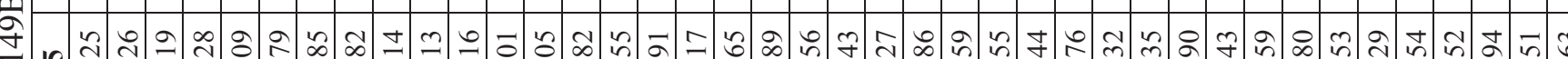

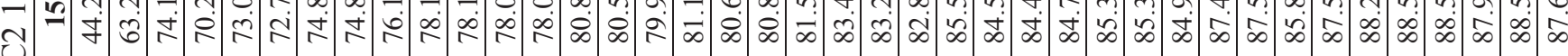

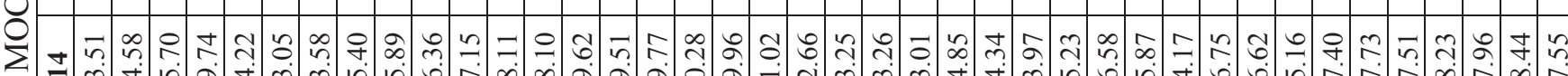

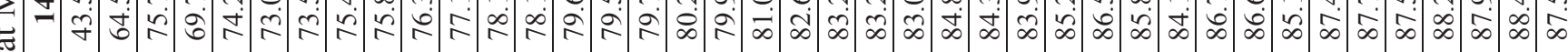

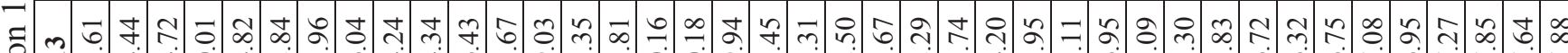

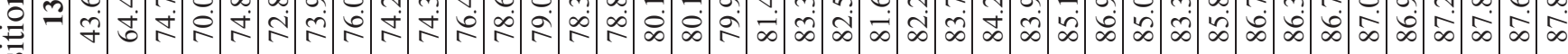

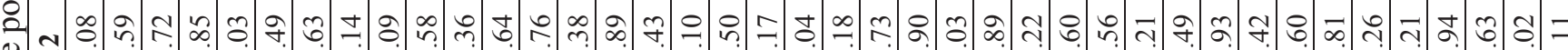
馬 =

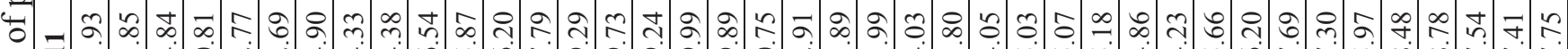

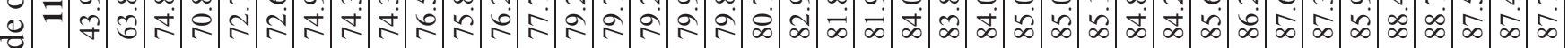

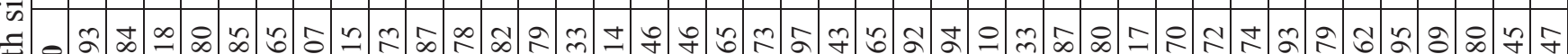

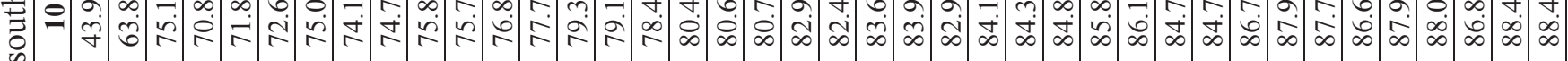
ક फै

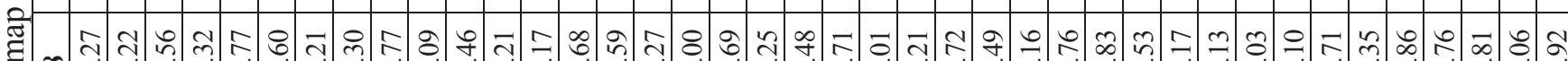

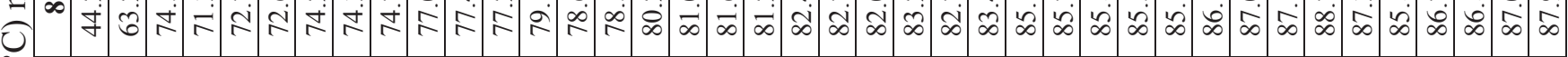
0

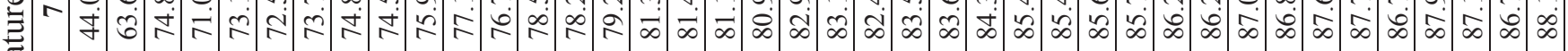
节

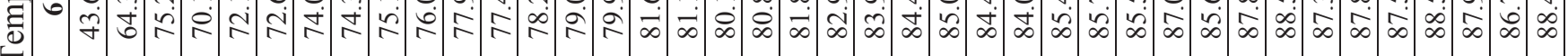

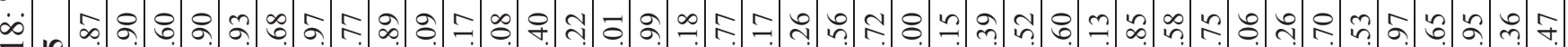

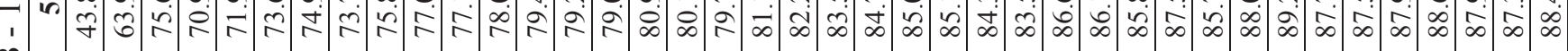
๑

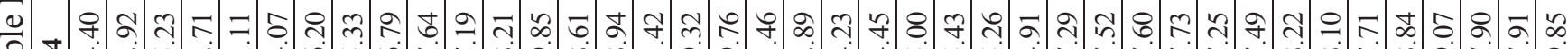

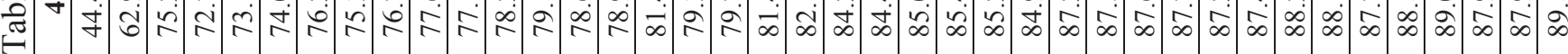

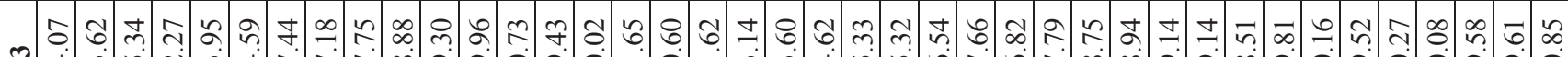

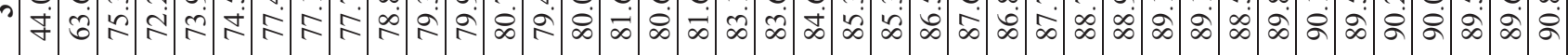
N =

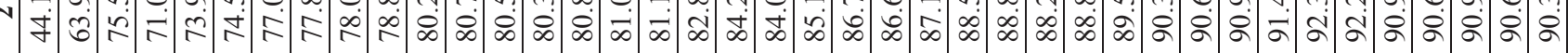

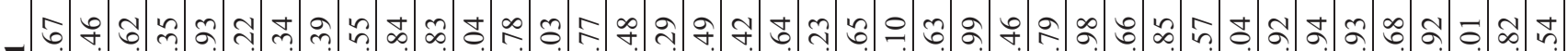

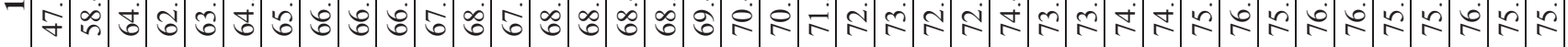




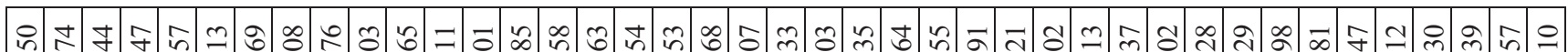

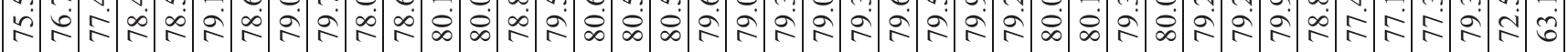
t잉

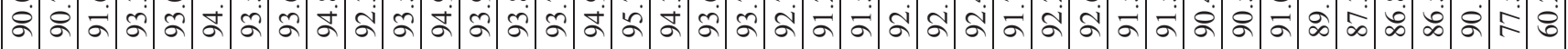

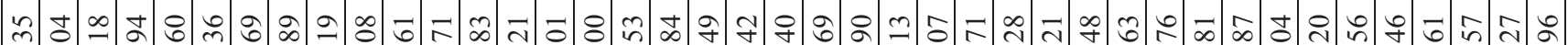

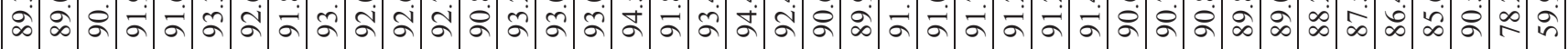
ก

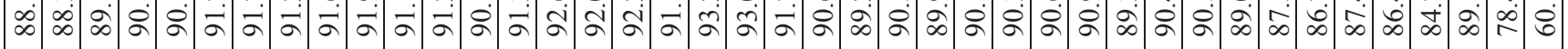

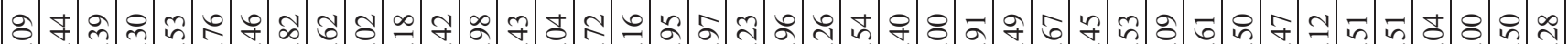

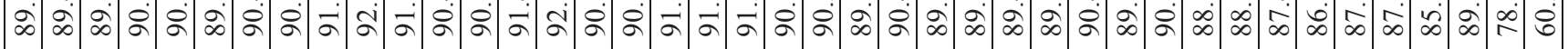

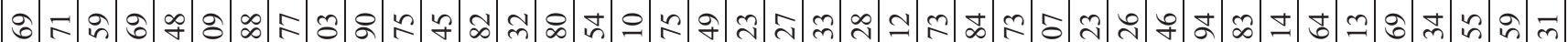

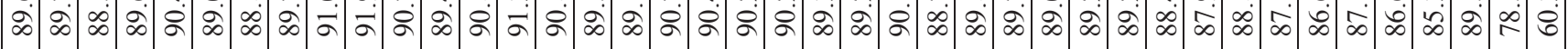

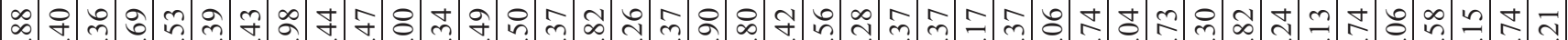

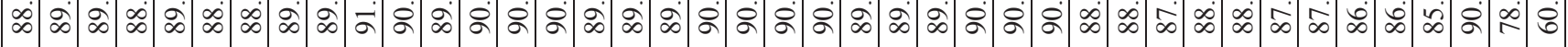

तิ

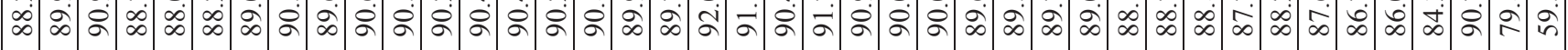

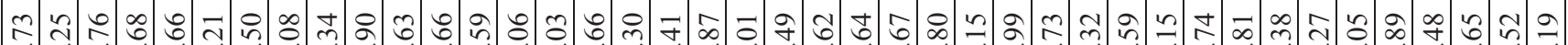

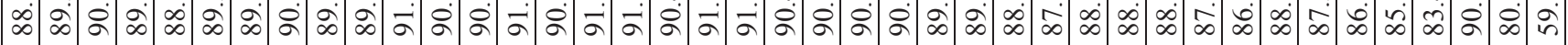
근

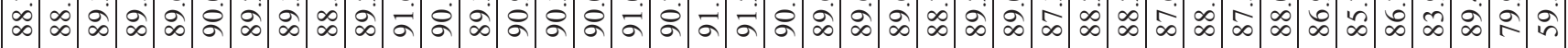

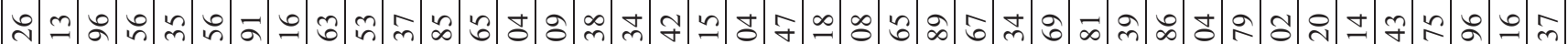

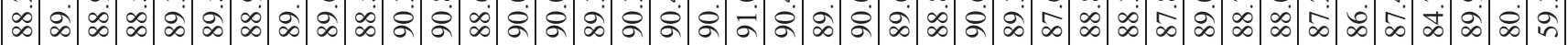

तి 유

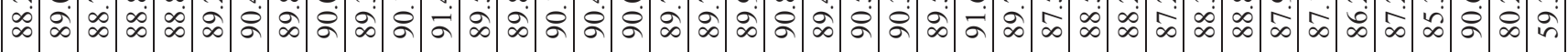

ㄱำ

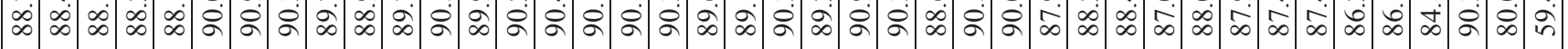

욱ำ

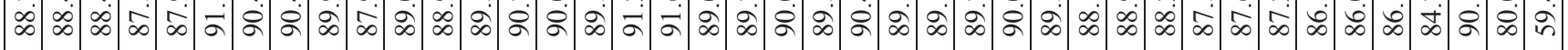

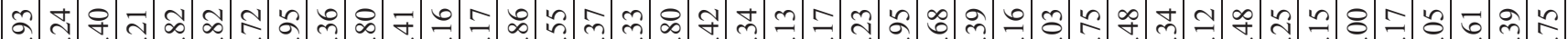

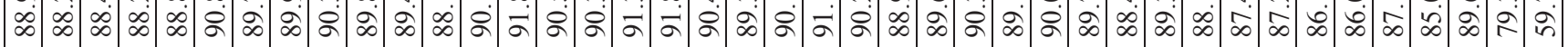

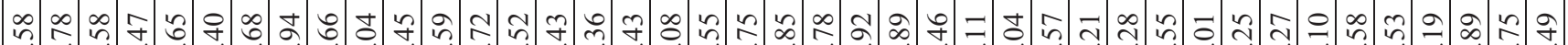

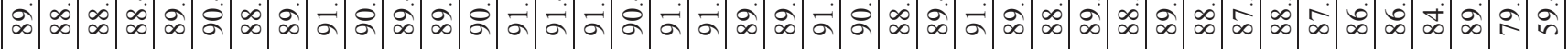

윅

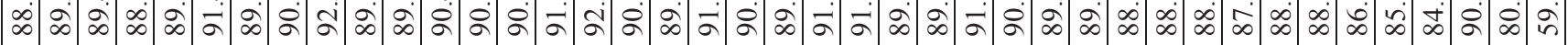

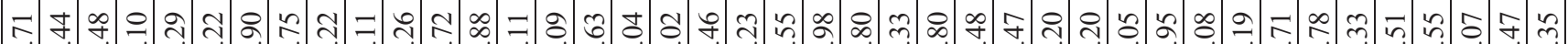

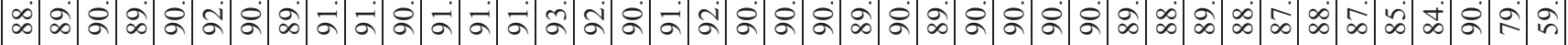
의

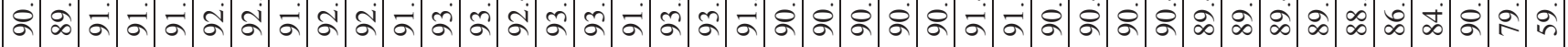

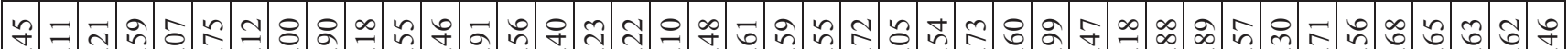

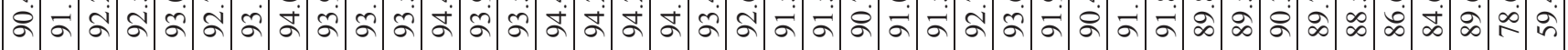

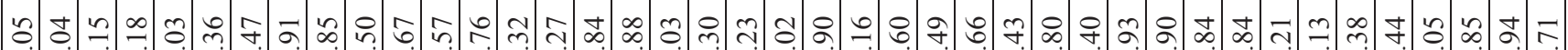

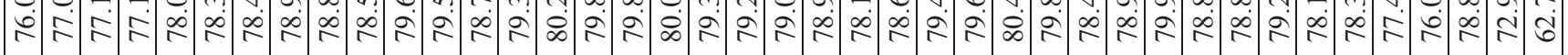


-

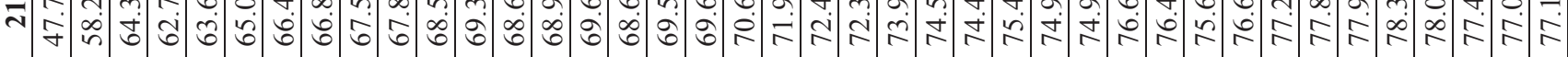
๑ กิน

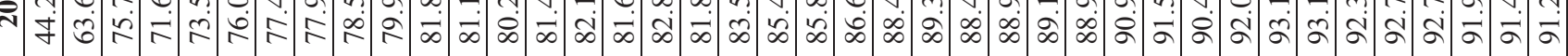

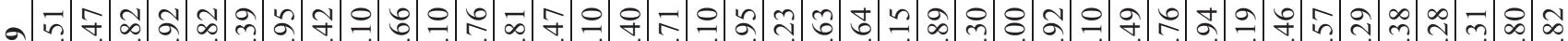

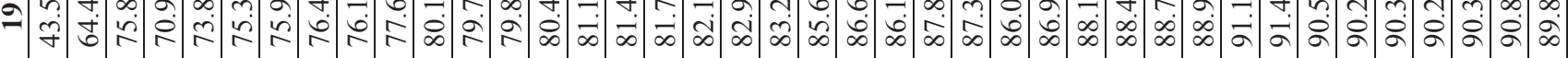

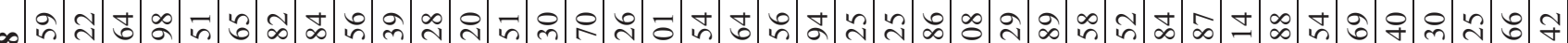

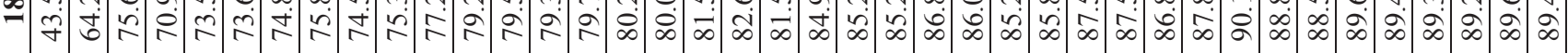

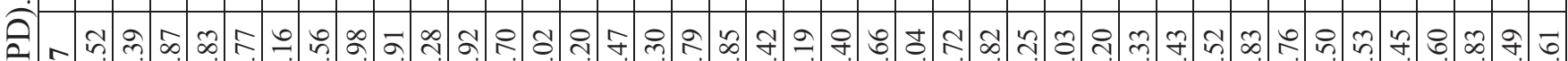

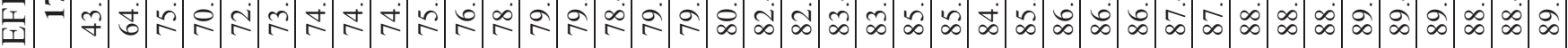

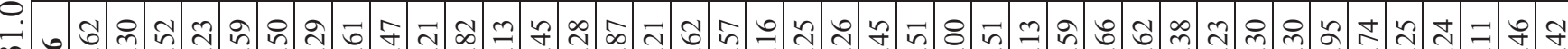
(2)

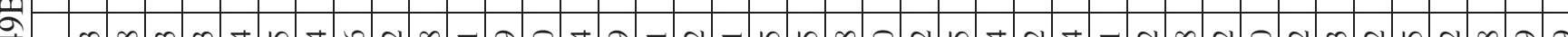

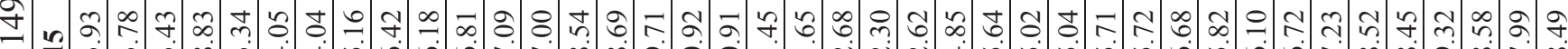

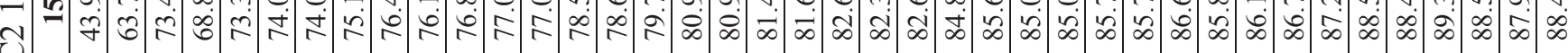

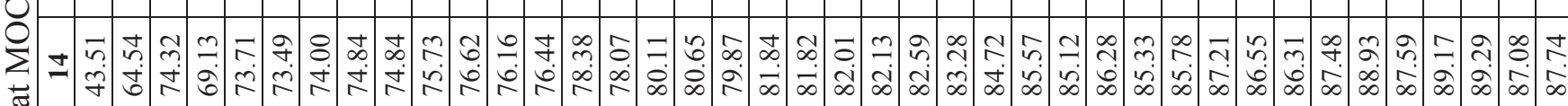
ปี

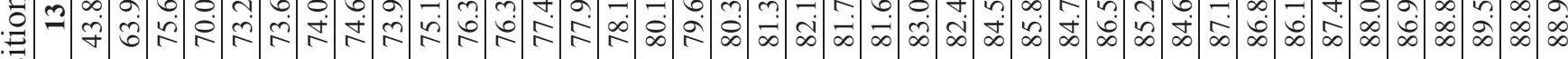

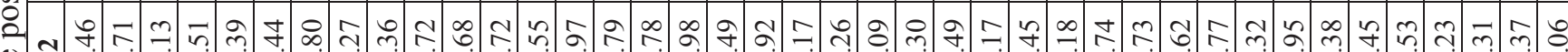

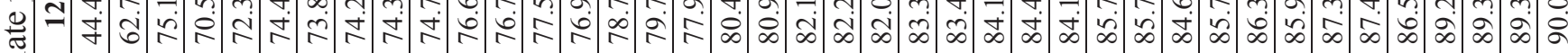

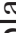

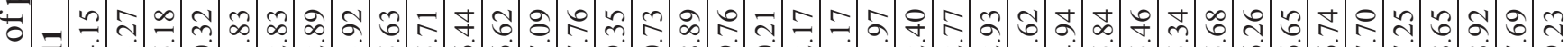

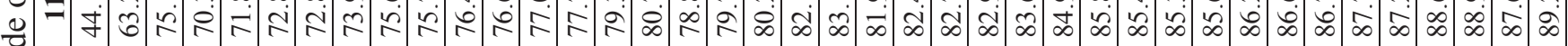

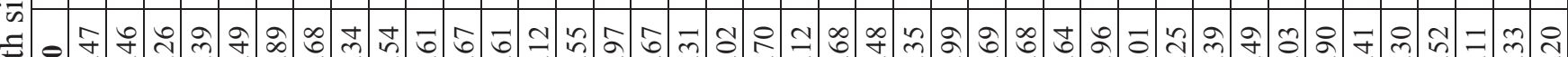

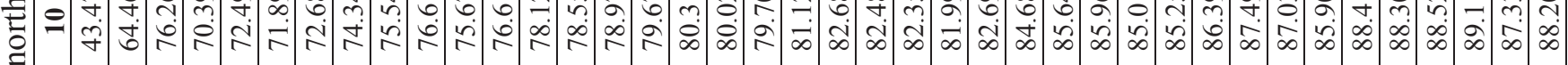
ఫ)

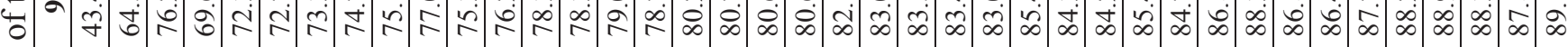

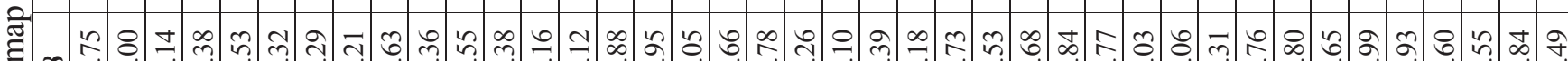

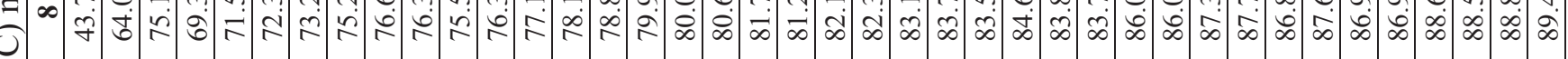
0 $\curvearrowleft$ б

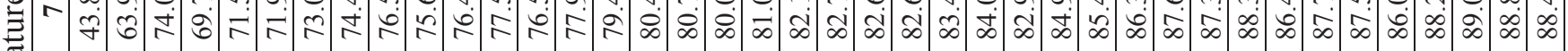

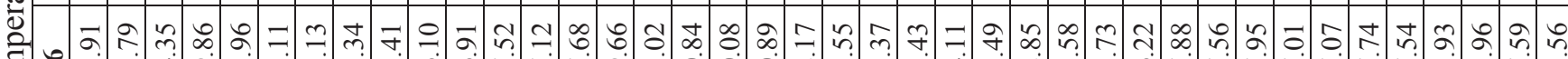

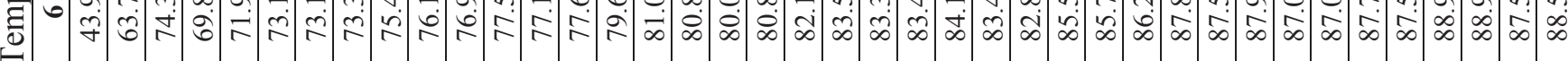

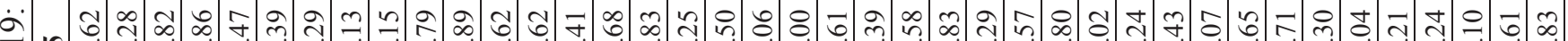

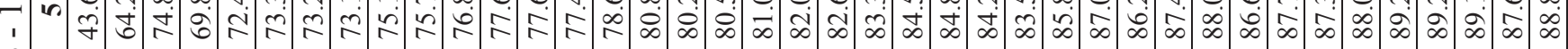
๑

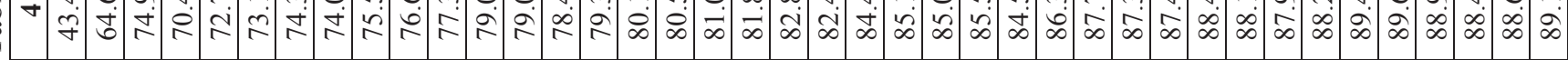
กิ

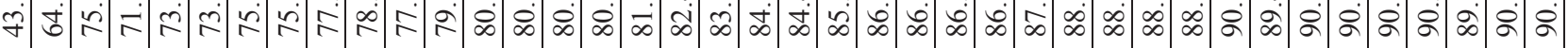

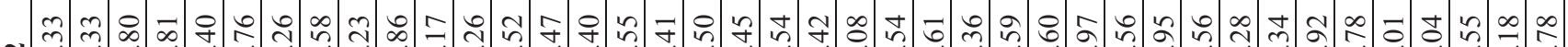

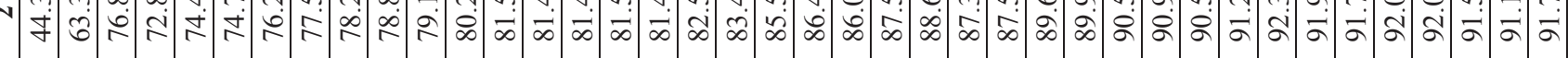

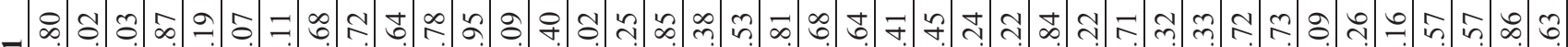

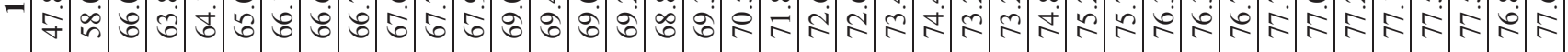
苞 


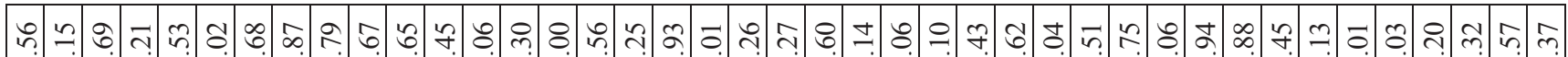

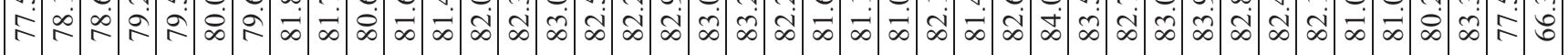

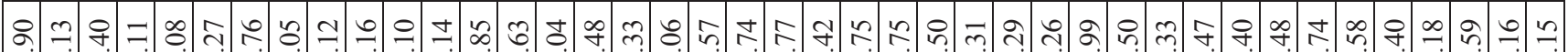

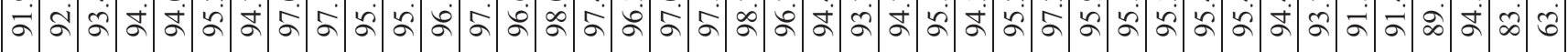

๙

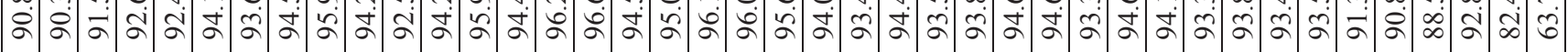

б

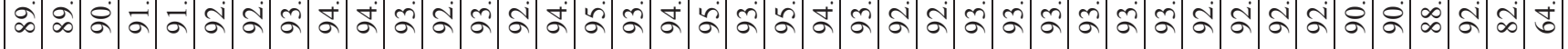

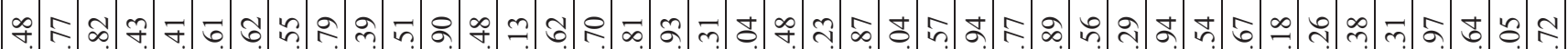

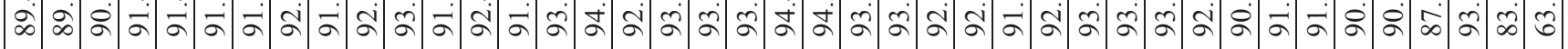

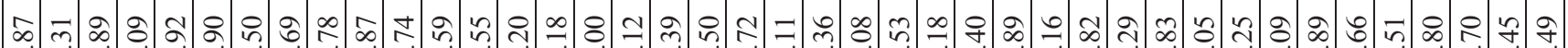

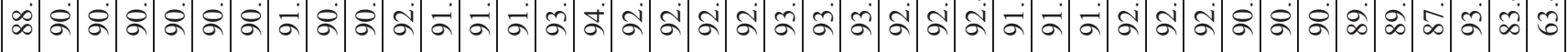

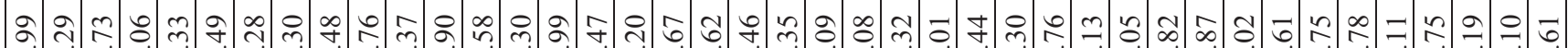

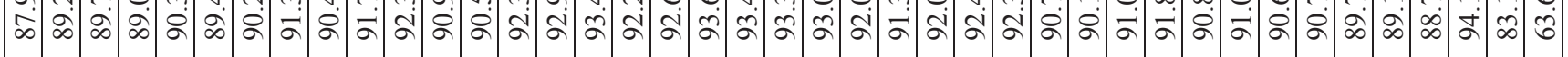

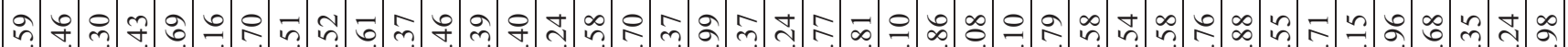

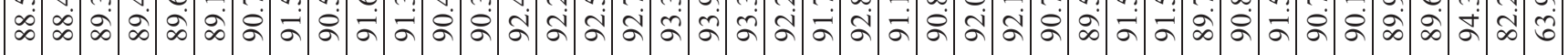

๘)

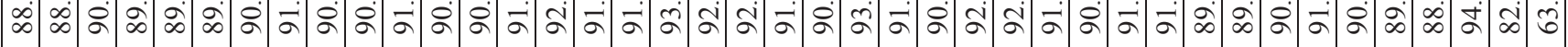

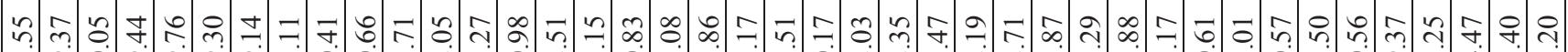

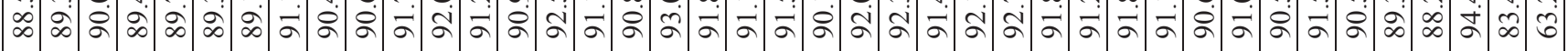

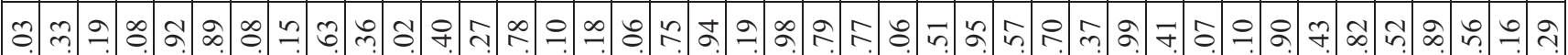

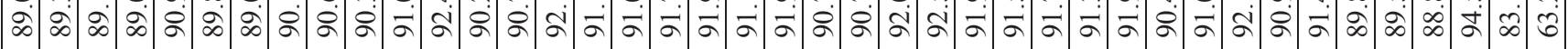

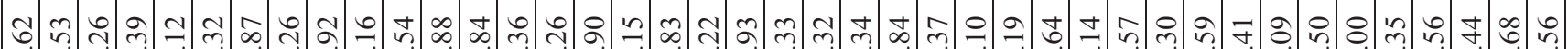

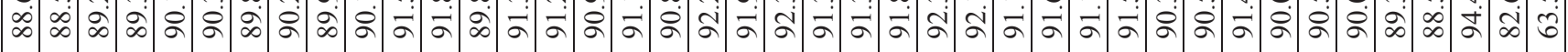

œ

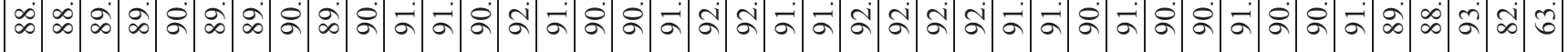

₹

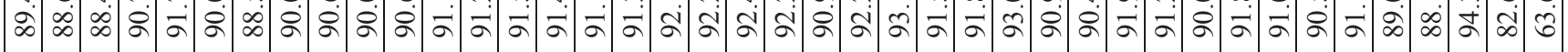

\&

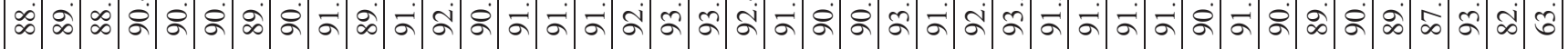

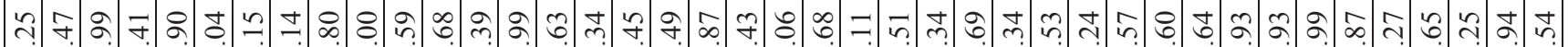

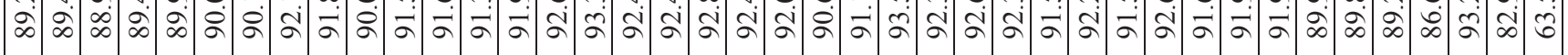
๒ ๘

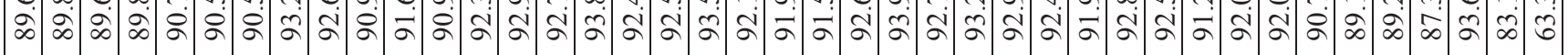

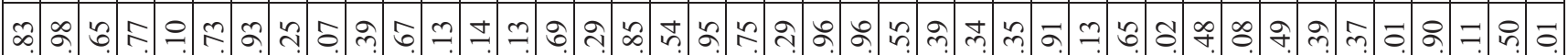

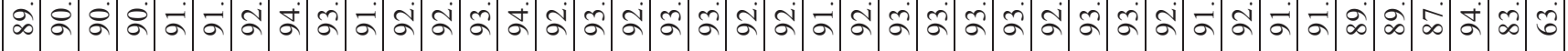

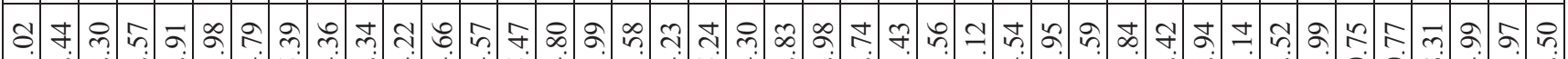

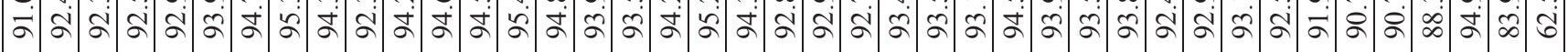

ஃ。 f ने

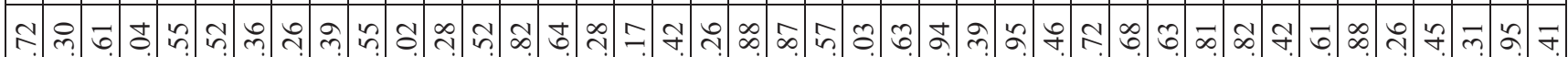

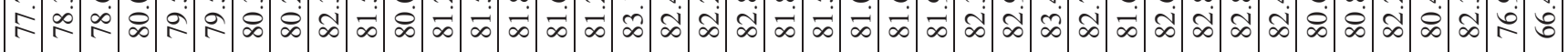
我

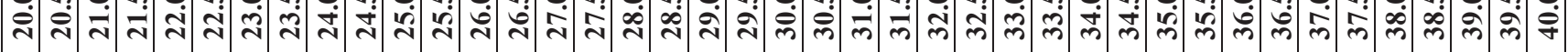


- 둔

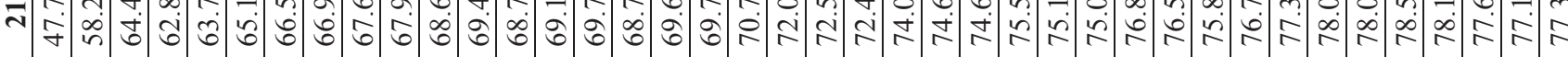
- \ิ ț

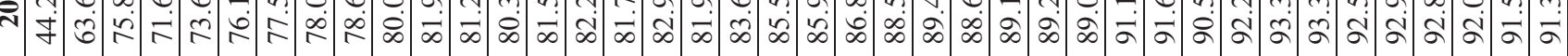

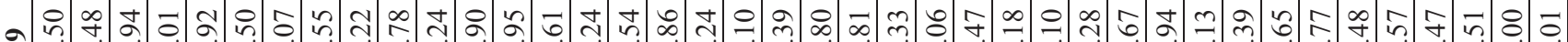

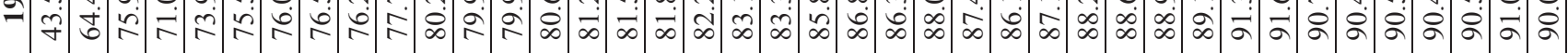

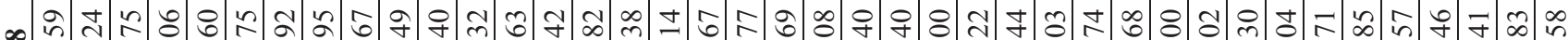

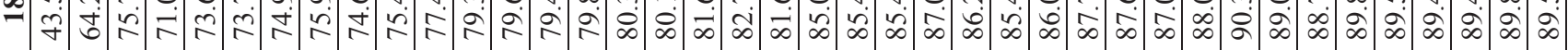

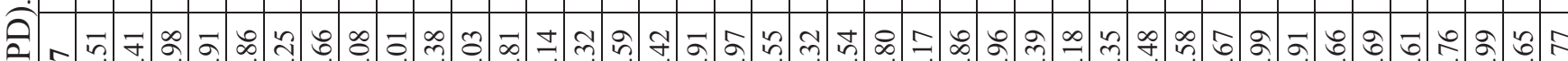

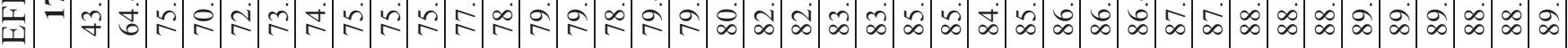

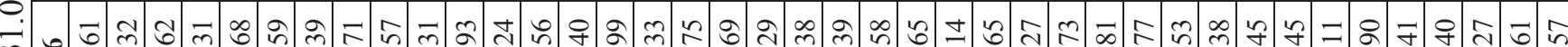

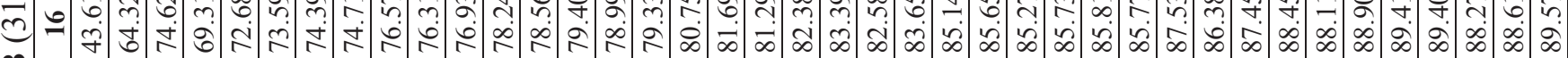

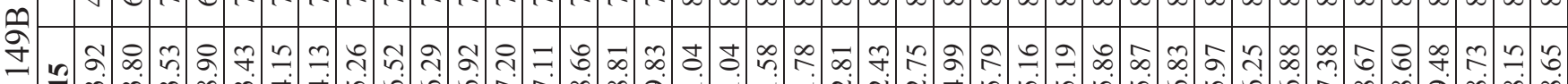

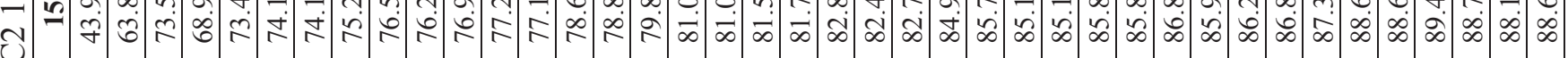

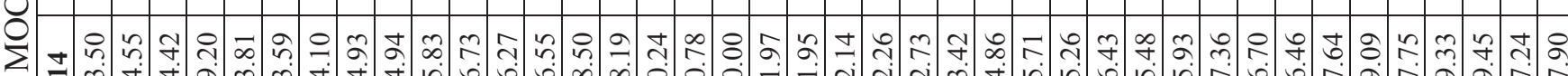

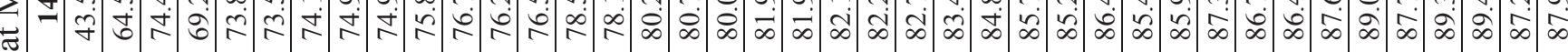

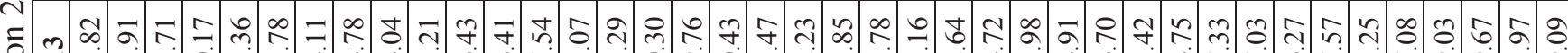

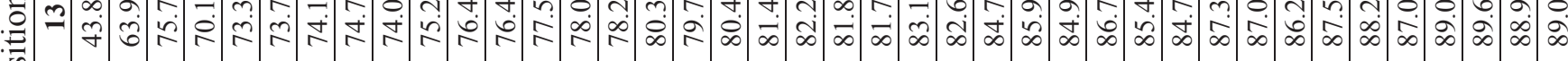
¿

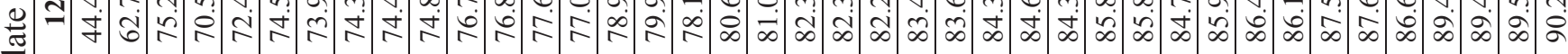

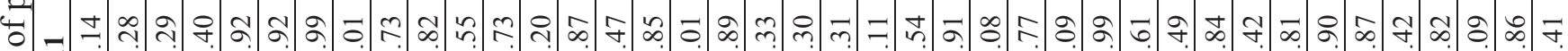

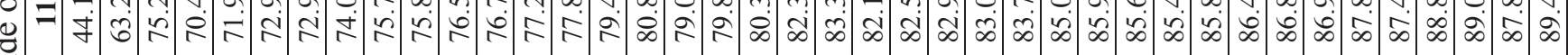

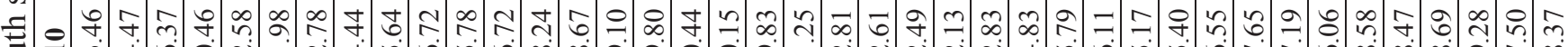

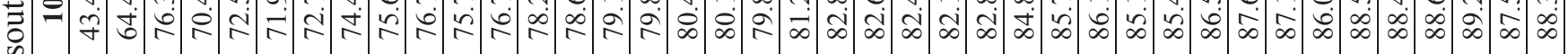

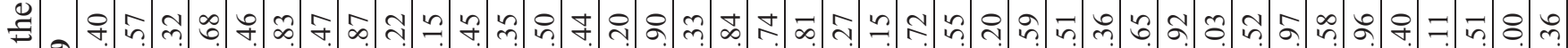
फ्ठ 羟

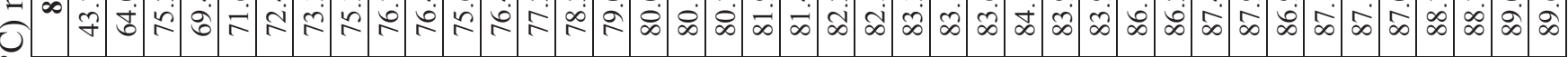

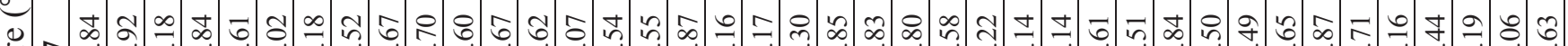

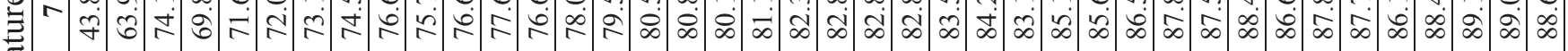

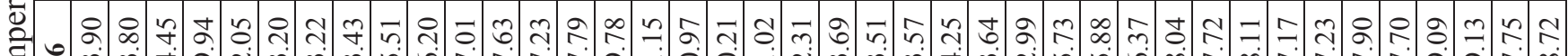

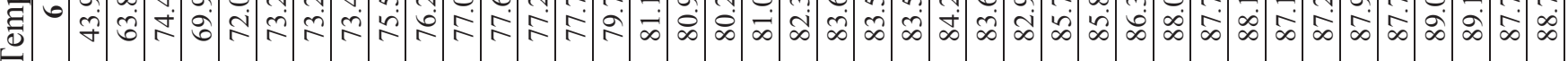

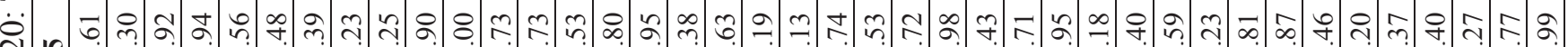

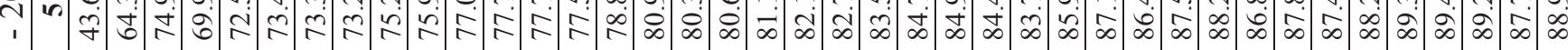
m

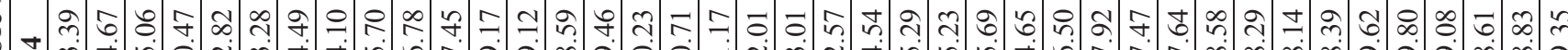

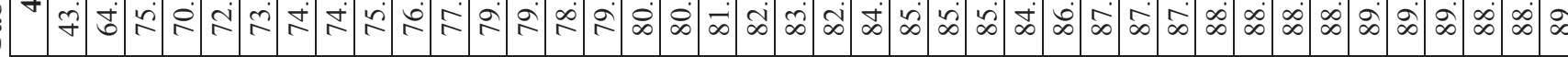

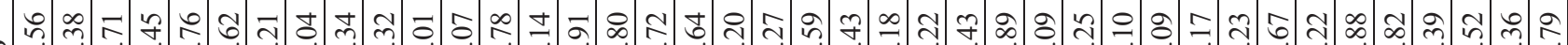

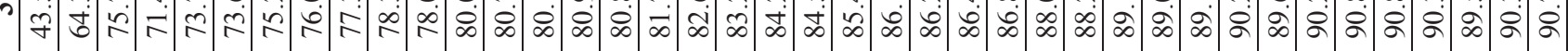

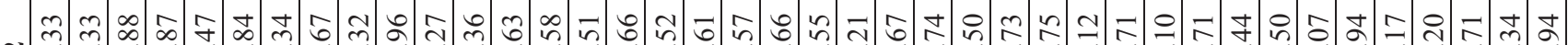

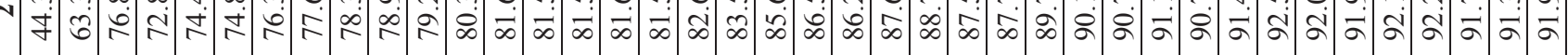

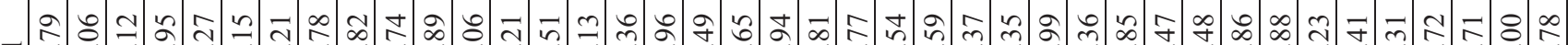

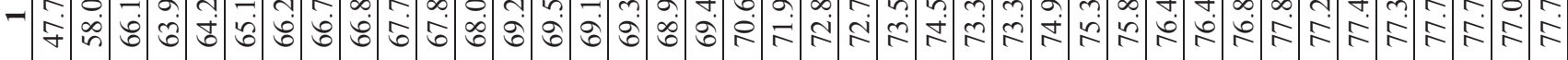
- n 官 
त

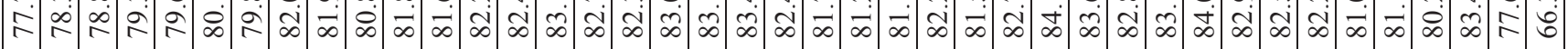

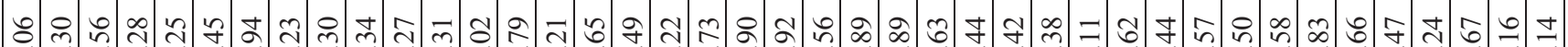

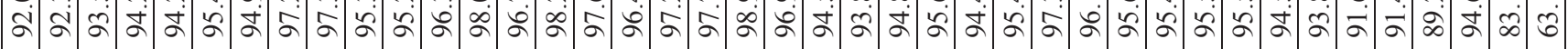

d

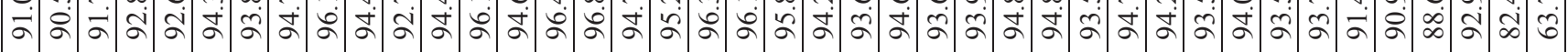

$\therefore$ m సี

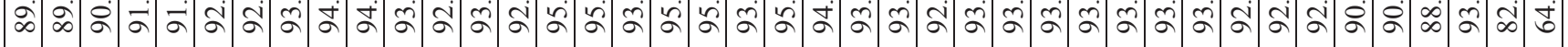

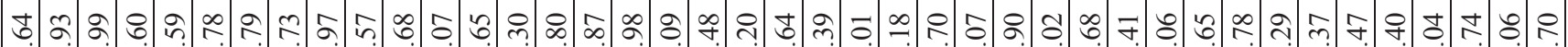

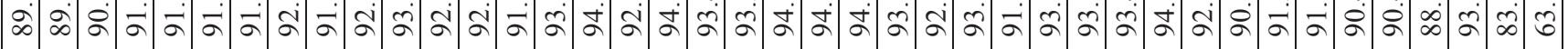

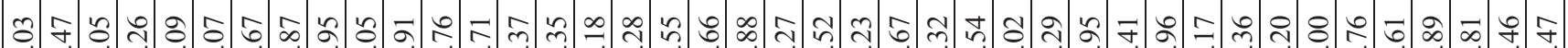

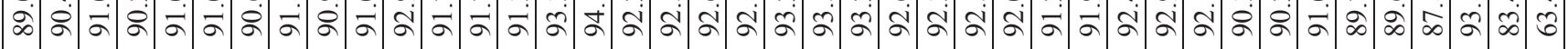

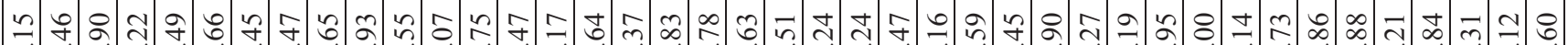

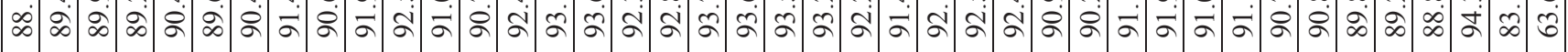

$\approx$ 눙

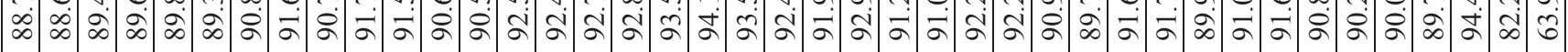

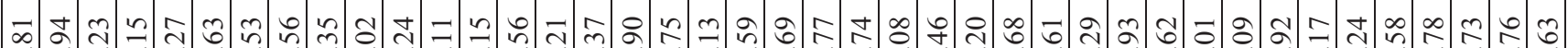

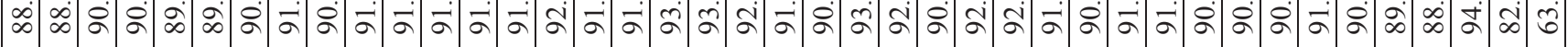

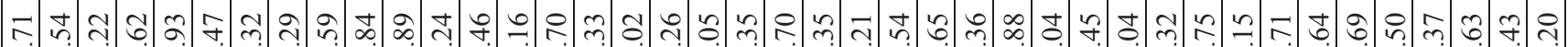

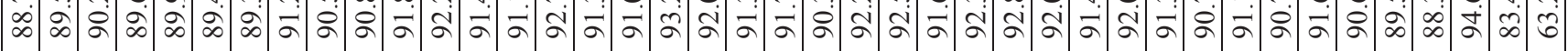

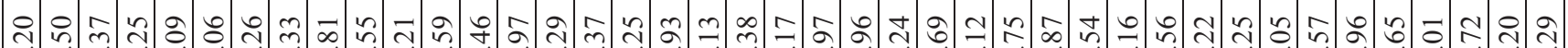
ळ

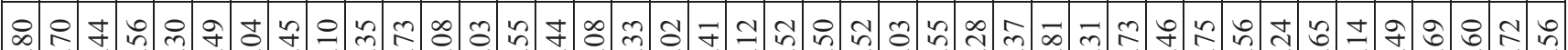

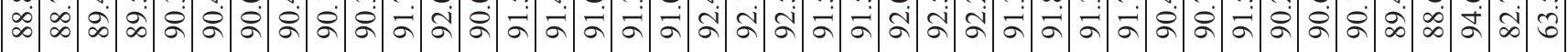

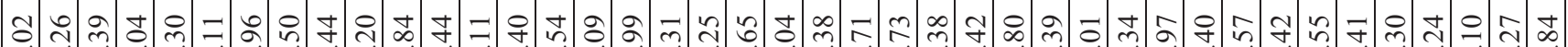

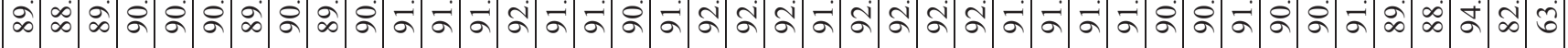

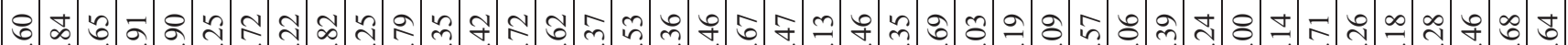

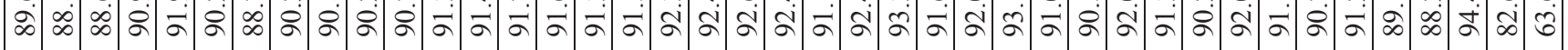

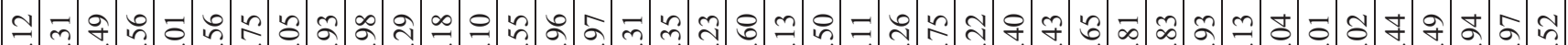

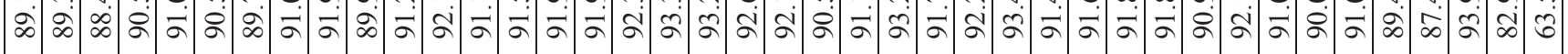

ᄀ tน

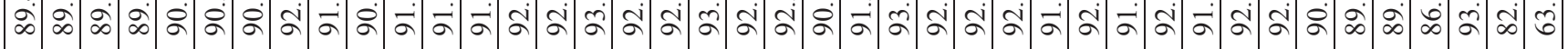

ळ.

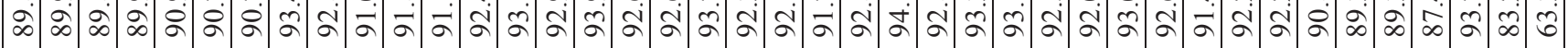

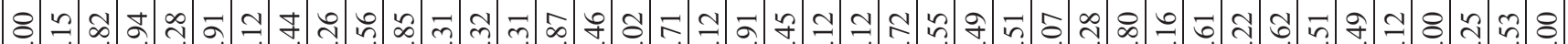

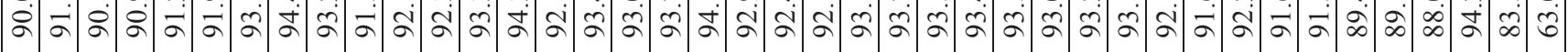
ᄀี

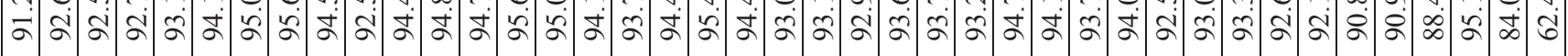

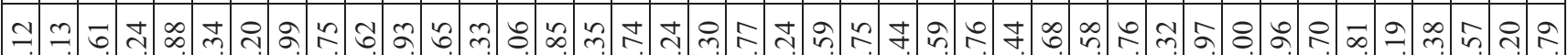
হं

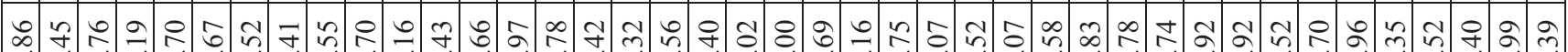

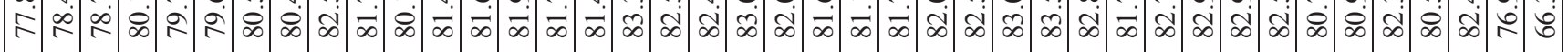




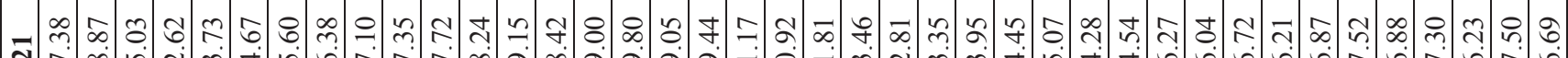

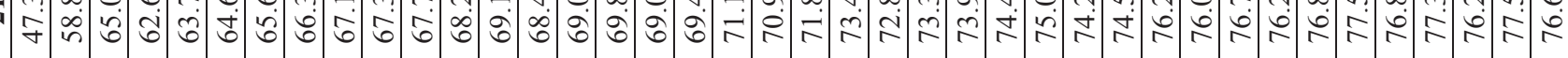
- ळ

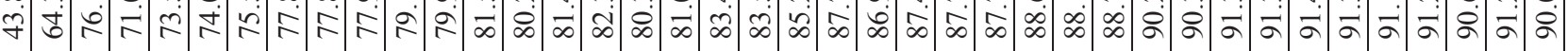

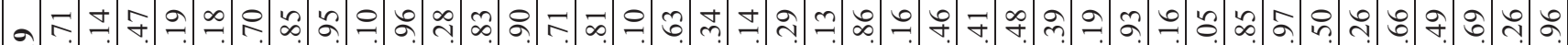

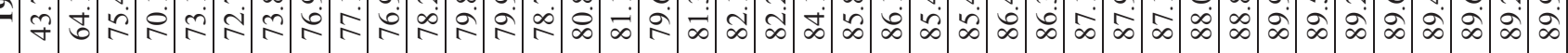

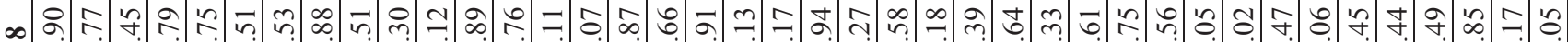

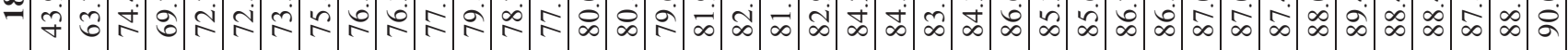
全

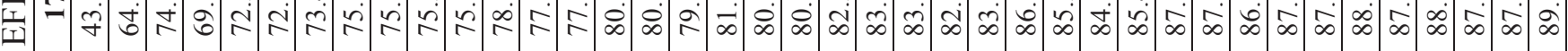

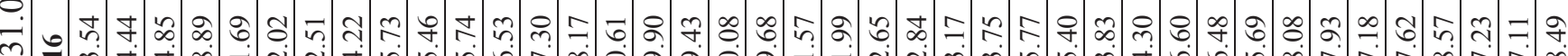

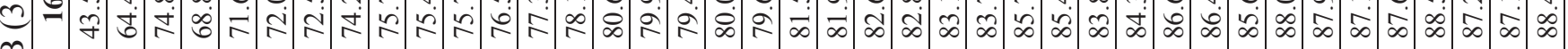
की ปn

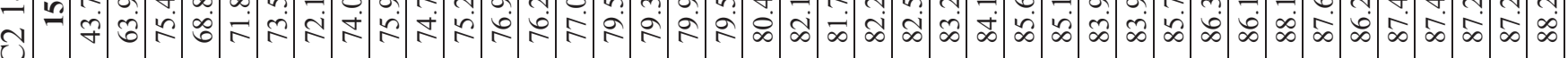

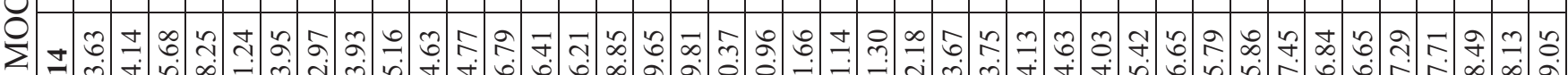

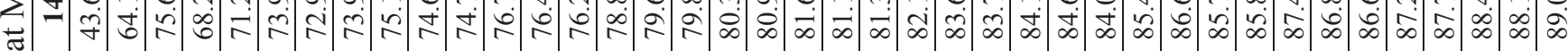
m

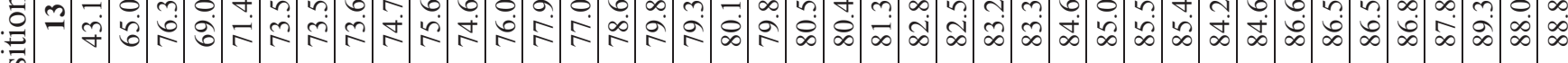
ఏ ₹

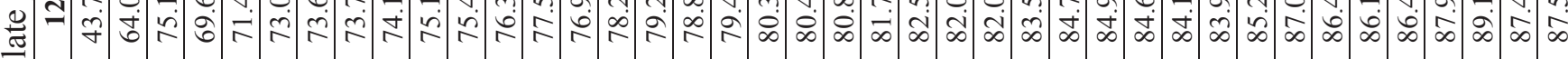
苛= 导 =

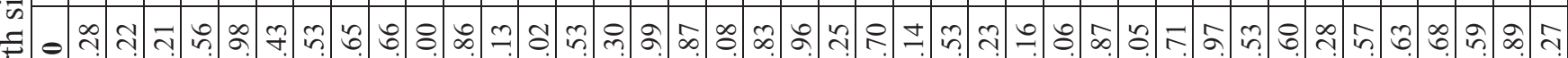

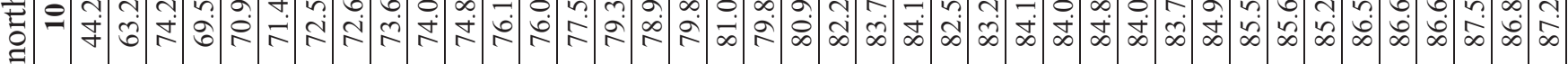

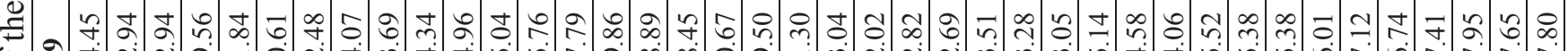
फ्ठ

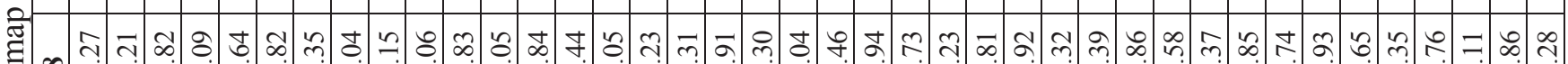
- 0 d

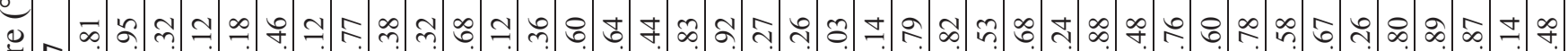

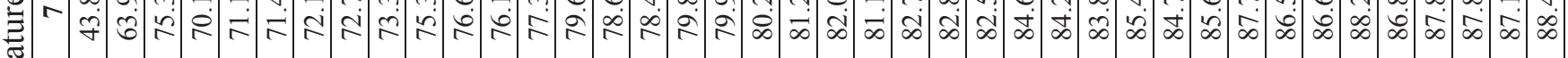
节

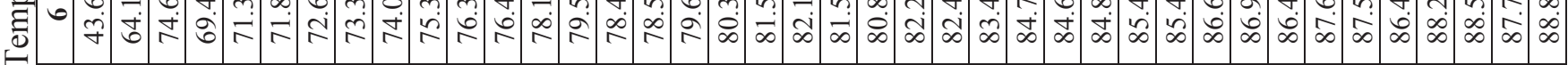

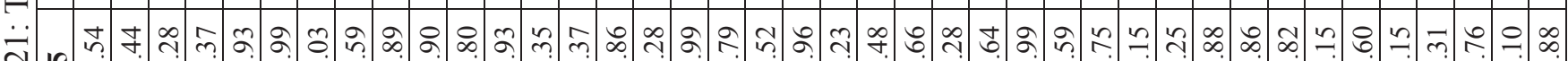

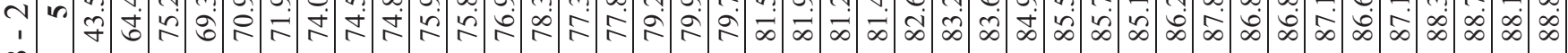
光

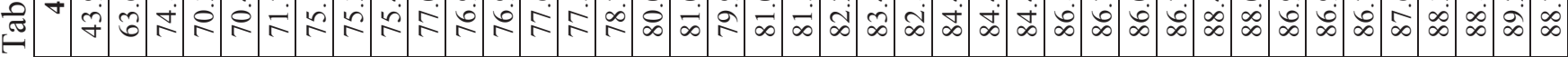

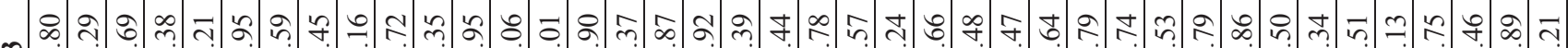

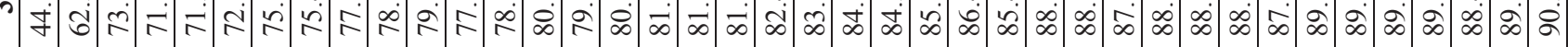

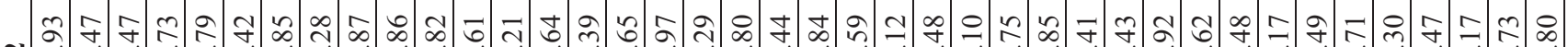
$N$ J

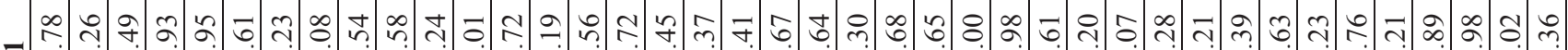

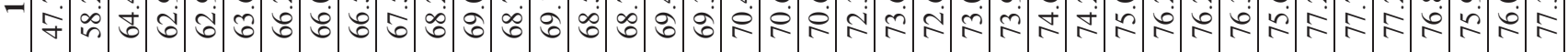
$\because$ 急 
穴

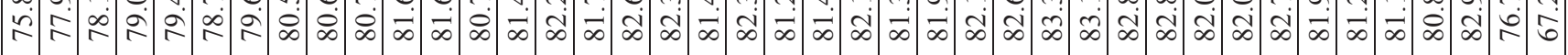

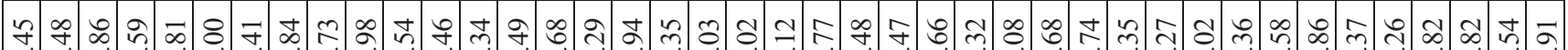

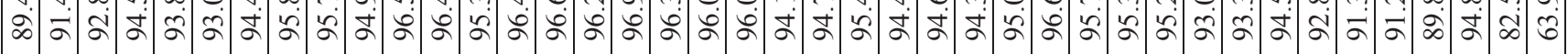

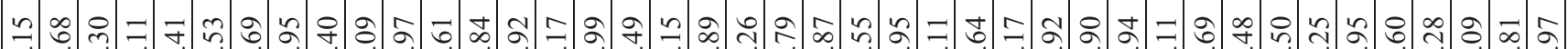

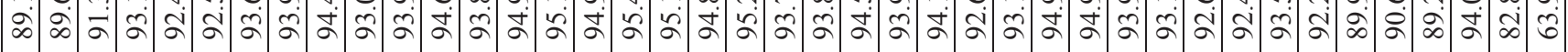

ㄱㄱ Ұㅇำ

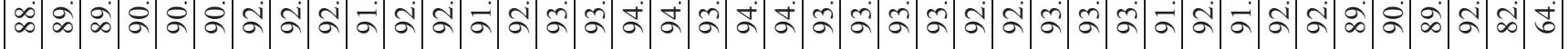

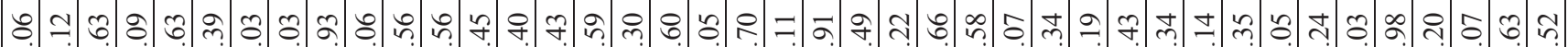

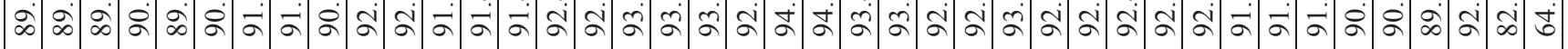

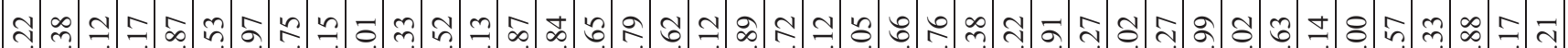

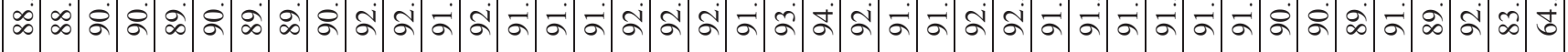

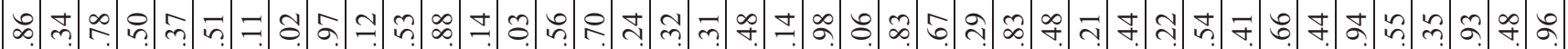

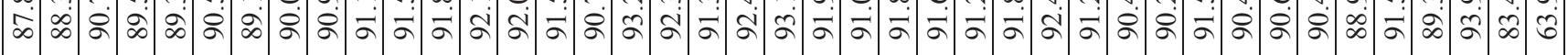

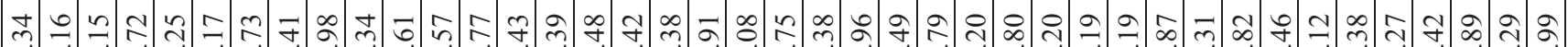

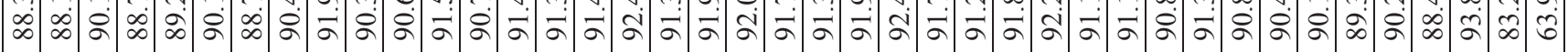

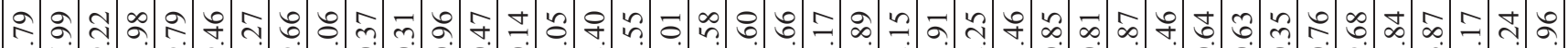

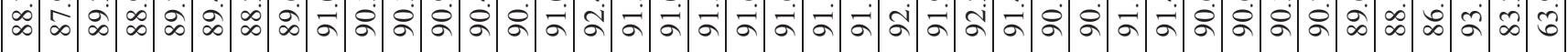

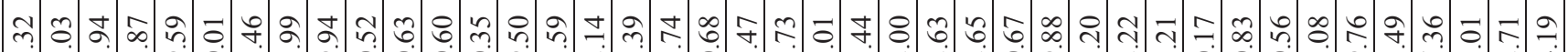

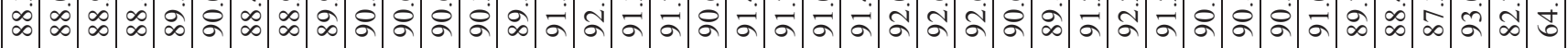
ㄷำ

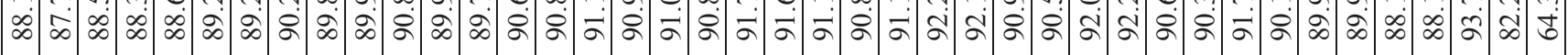
ก

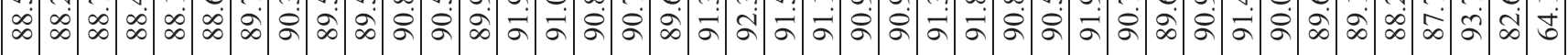

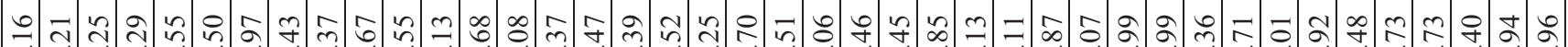

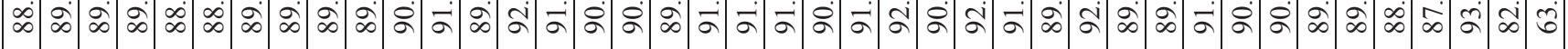

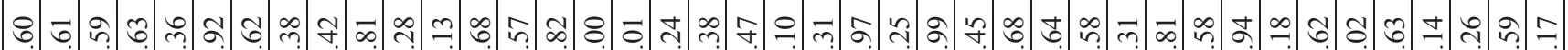

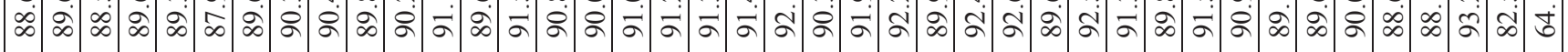

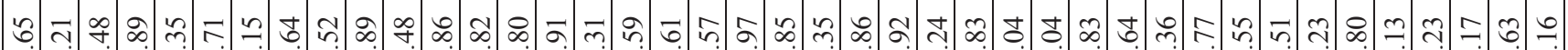

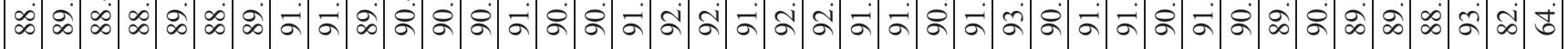

๓

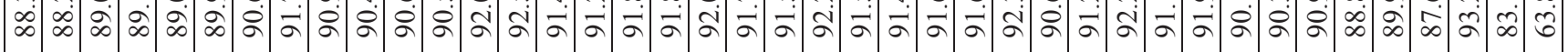

ذ

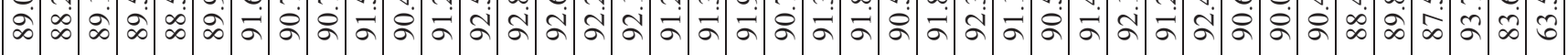

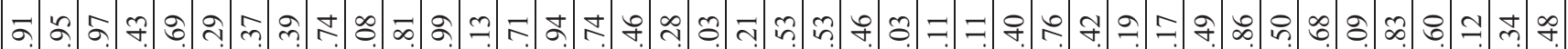

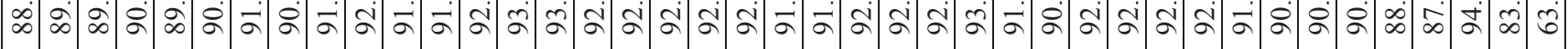

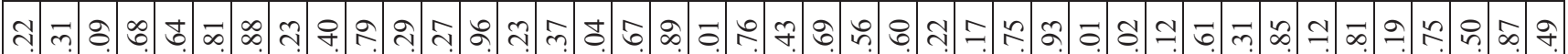

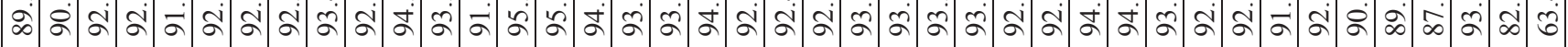

ఫ.

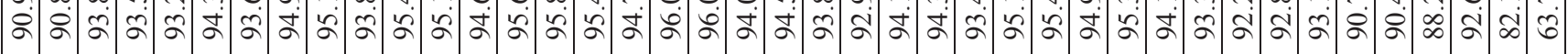

子

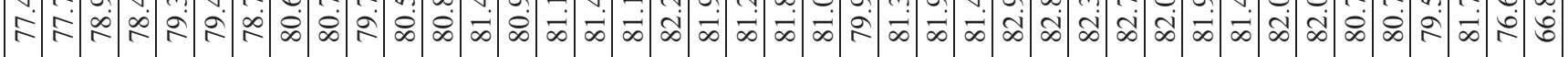
竎 
-

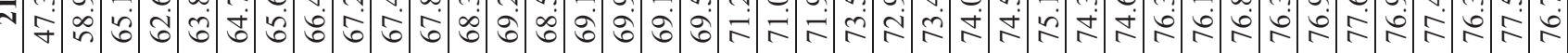
न

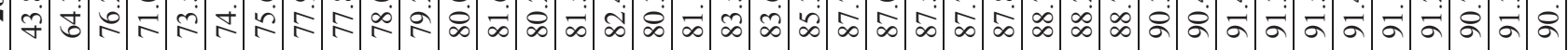

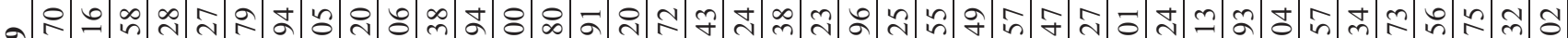

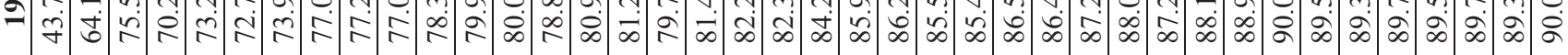

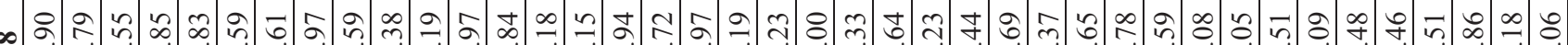

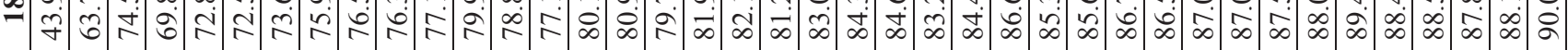

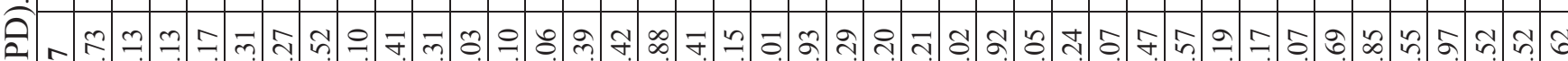
盾 = అ)

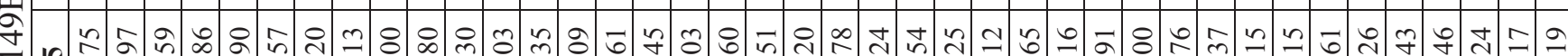

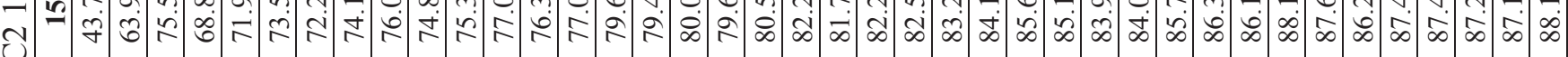

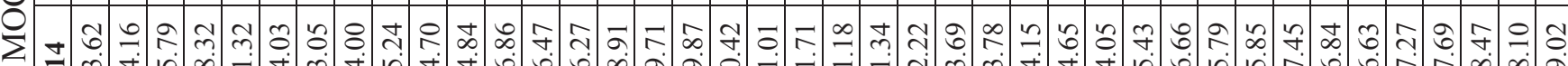

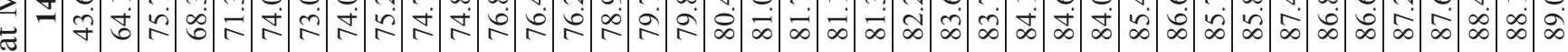
m

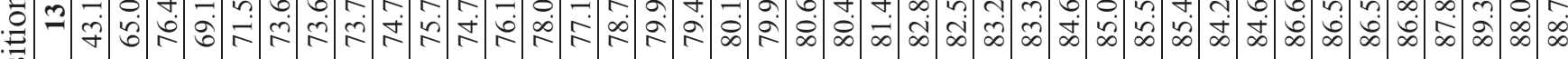

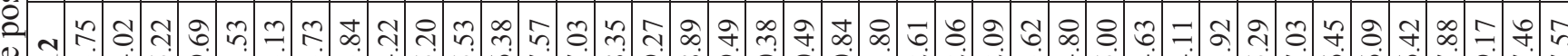

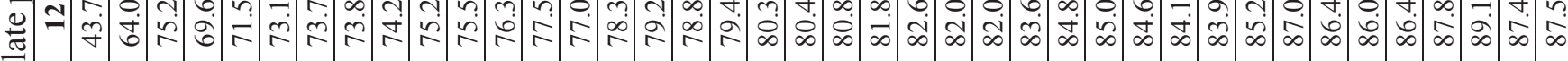

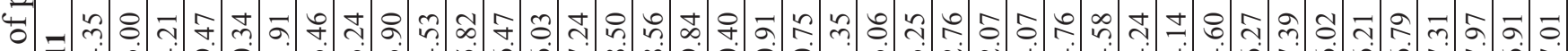

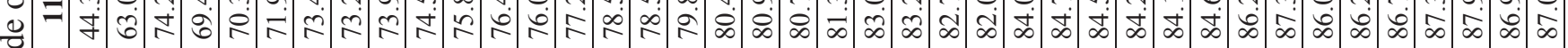

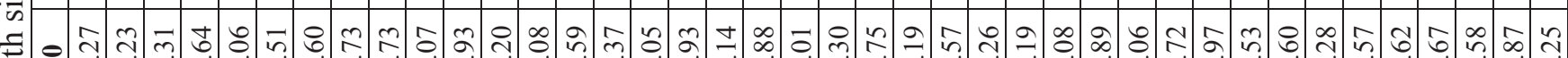

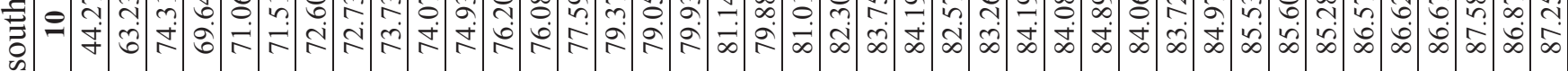

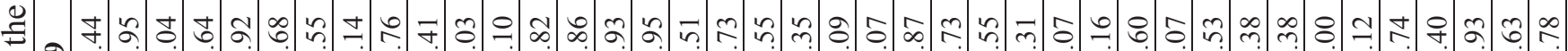
फ्ठ สิ

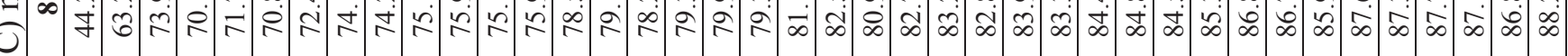

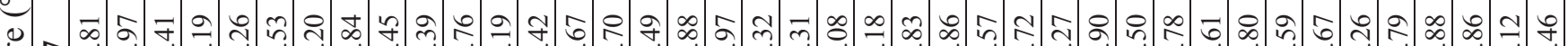

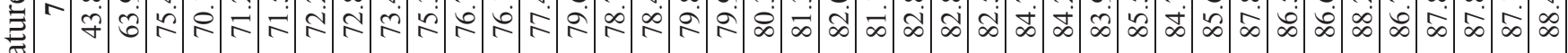

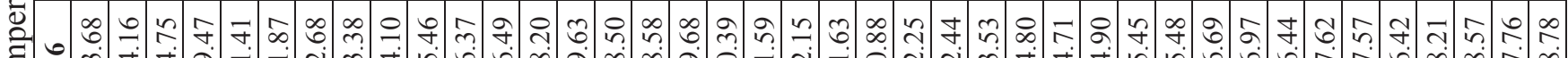
包 त่ तै س ๑

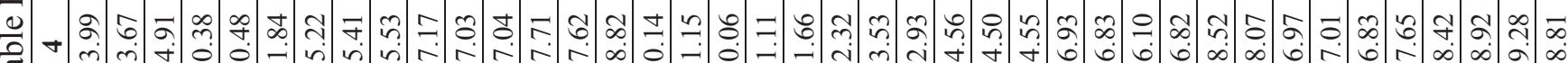

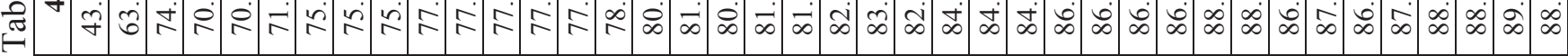

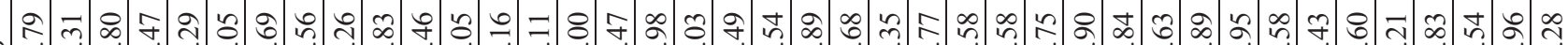

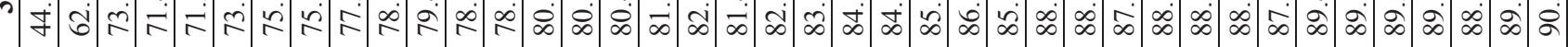

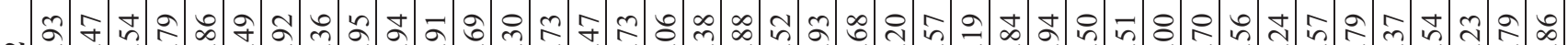
N $\dot{f}$ Uं - 유

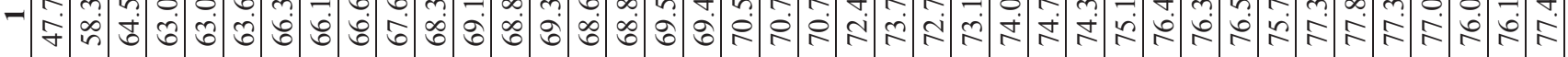

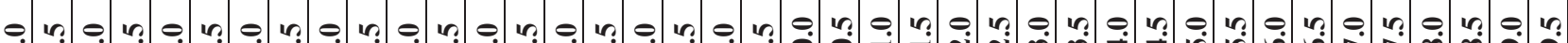

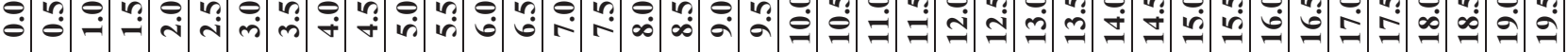


๙2

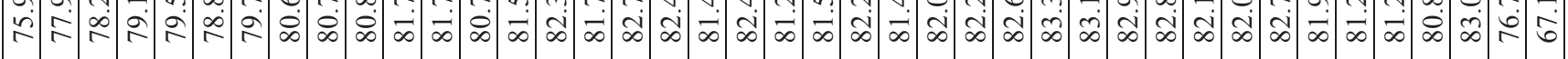
舟

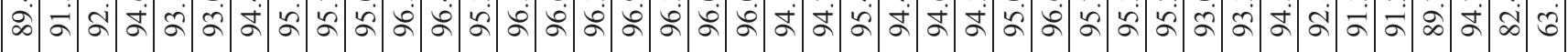

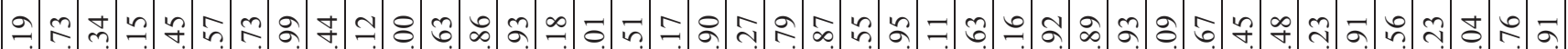

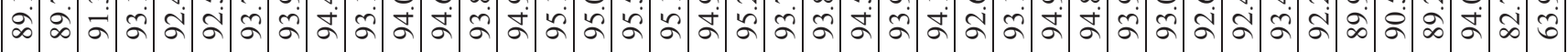

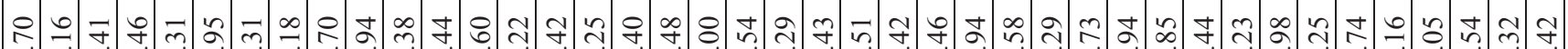

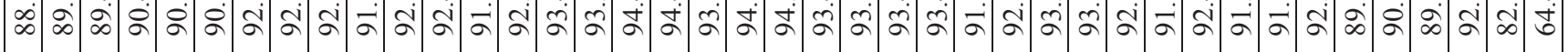

t)

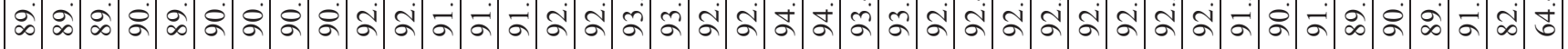

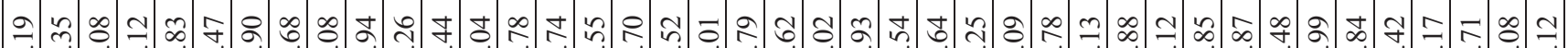

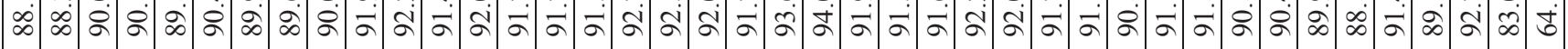

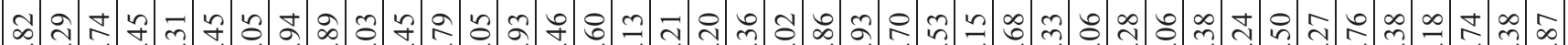

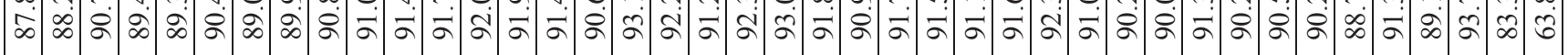

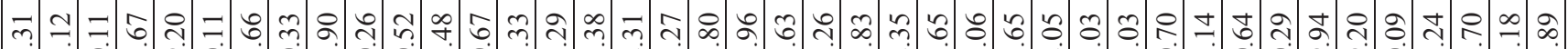

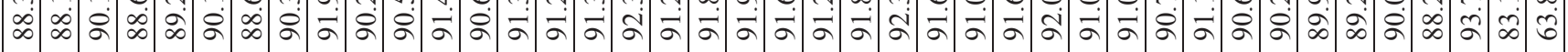

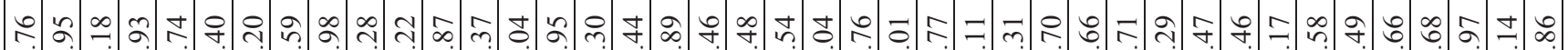

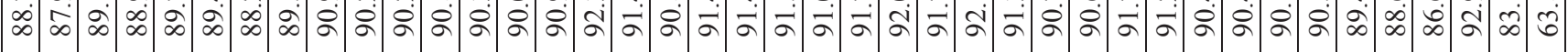
तิ \&:

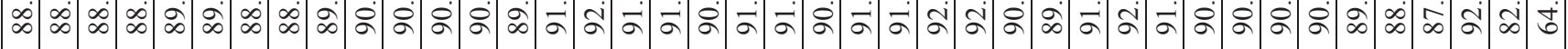

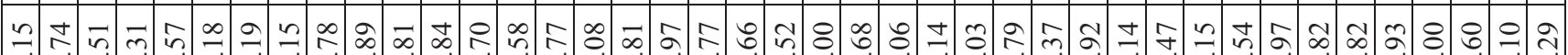

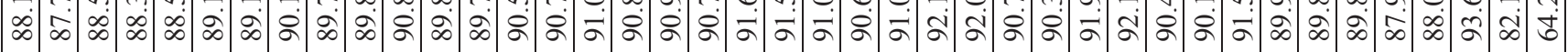

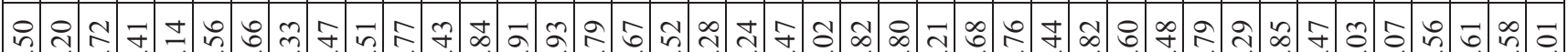

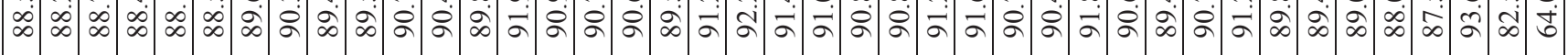

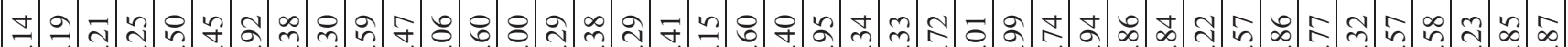

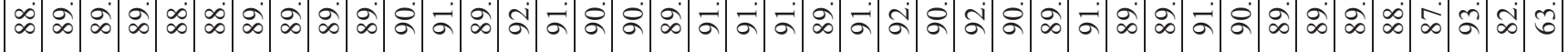

$\infty$ n

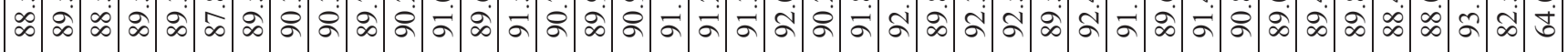

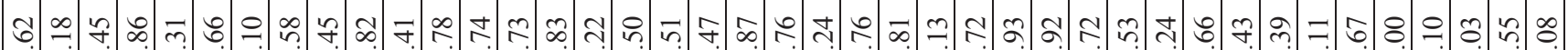

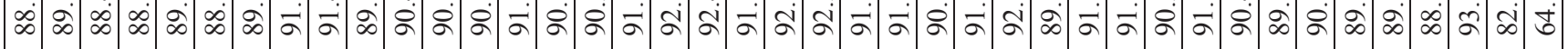

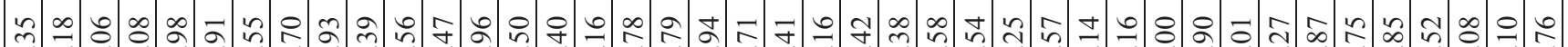

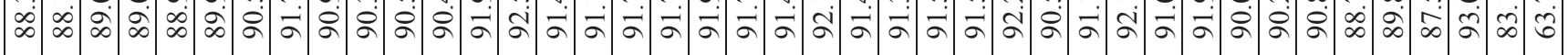

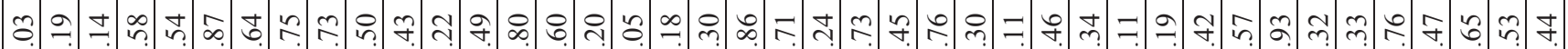

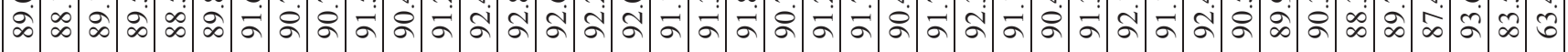
๙

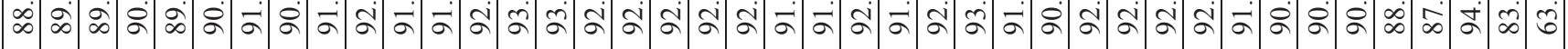
ণิ

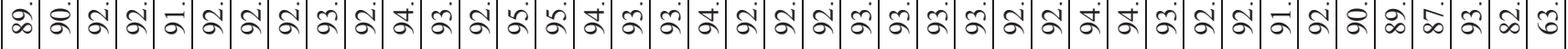

à $\infty$ n

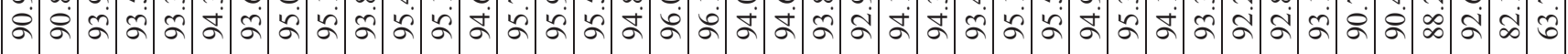
药

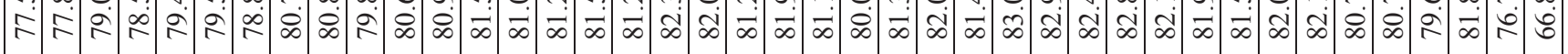
我

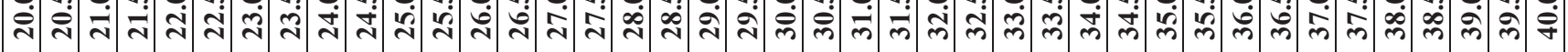




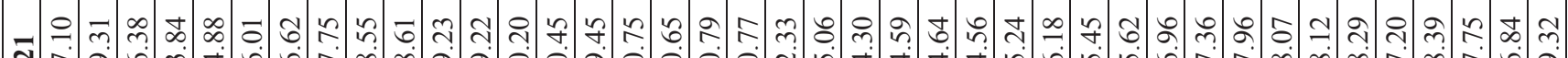

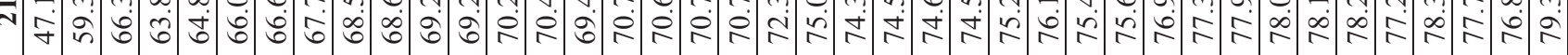
-

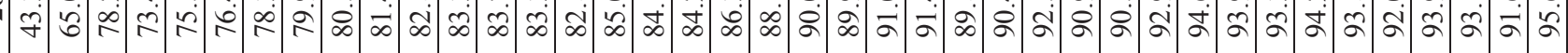

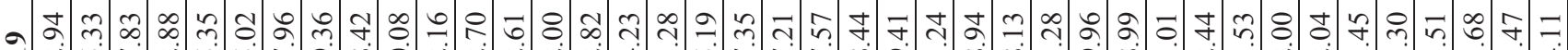

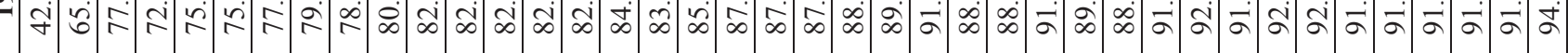

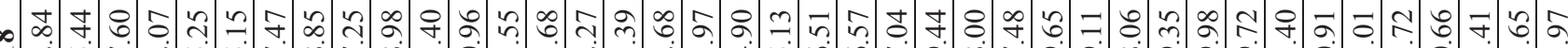

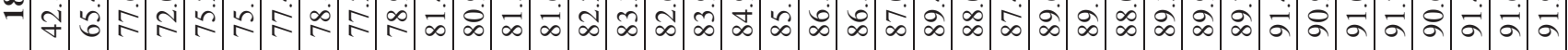
Әิ)

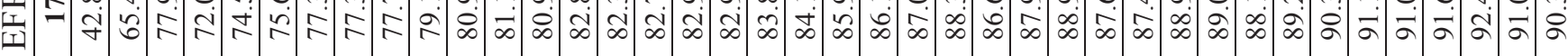

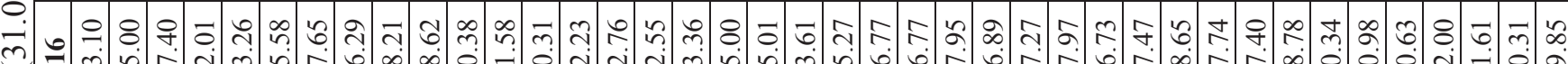

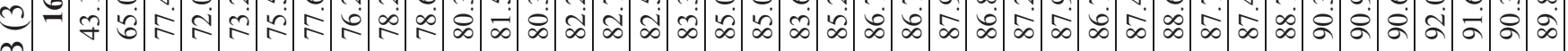
क

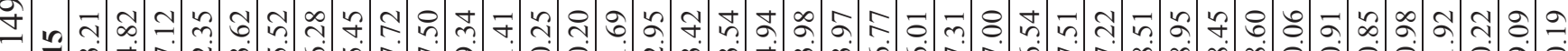

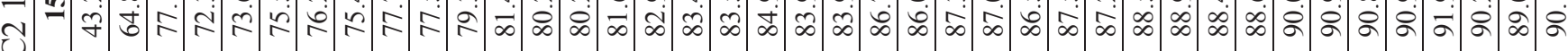

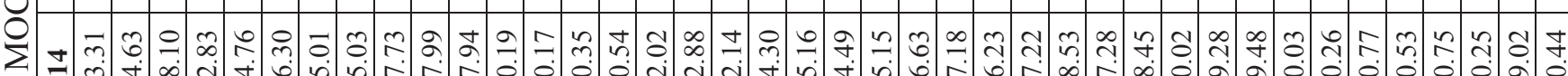

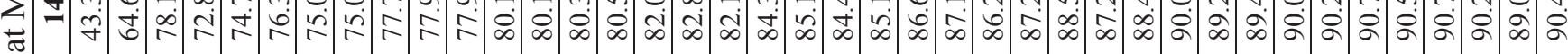

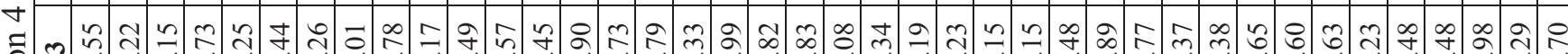

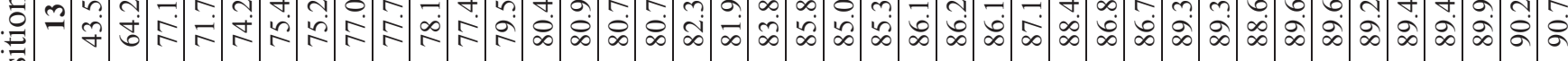
ด ส

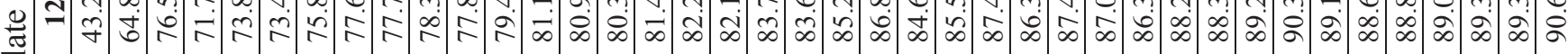

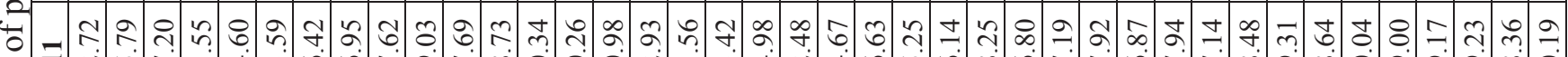

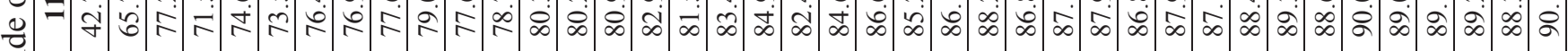

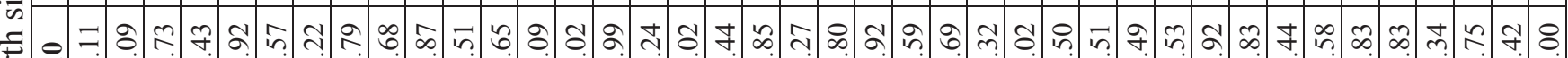

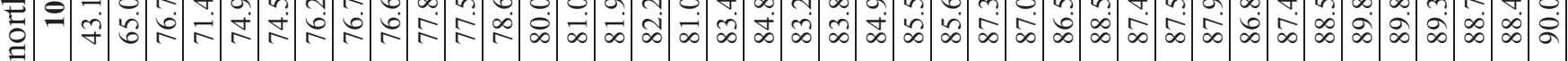
Е

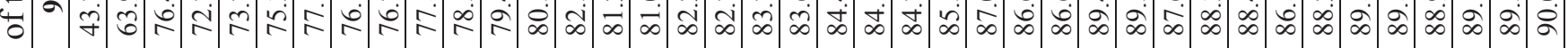

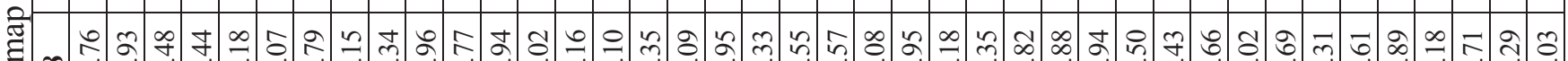

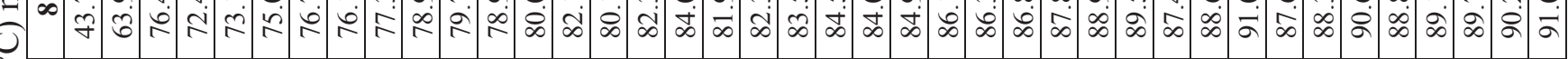

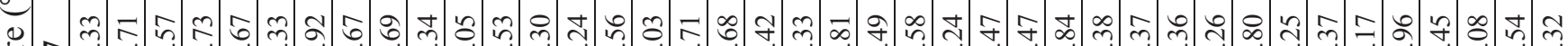

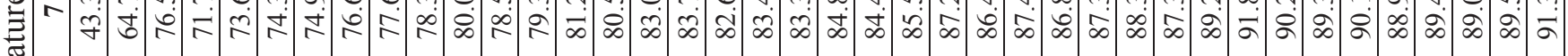
艺

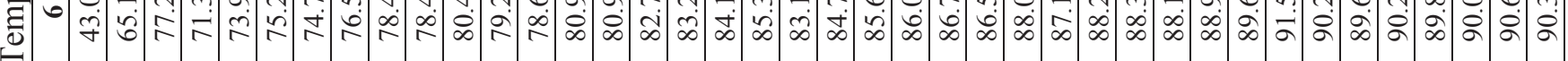

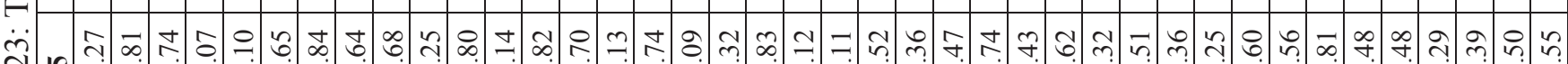

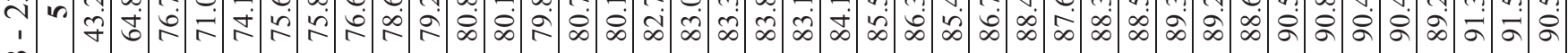

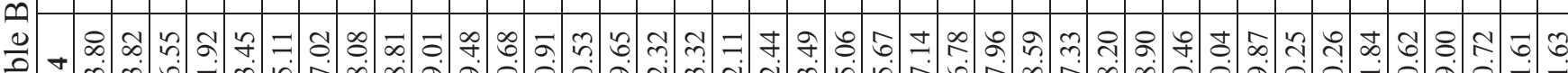
䒕

के

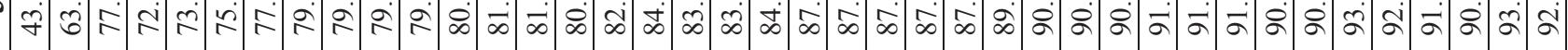

ง

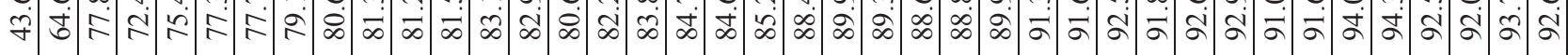
- 字的定

舟 


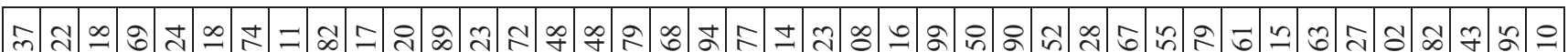
定 m

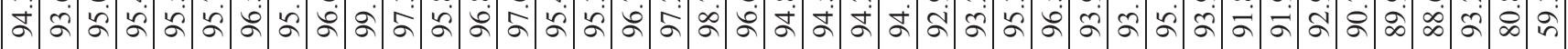

\%

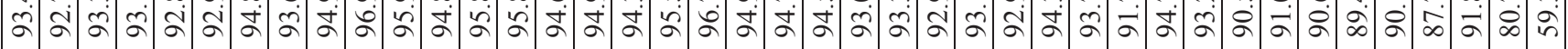

ล́

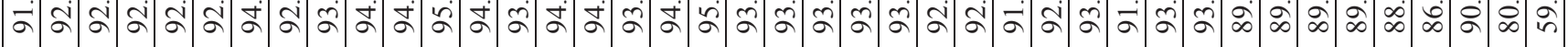

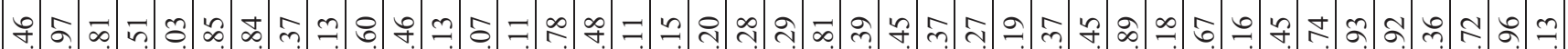

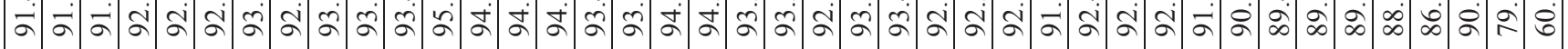

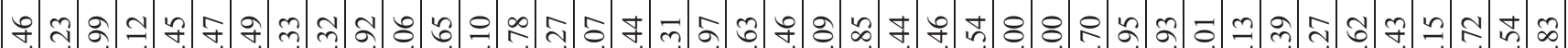

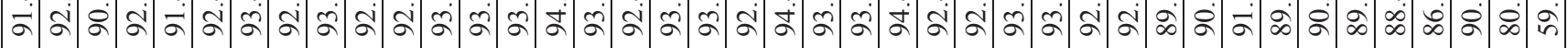

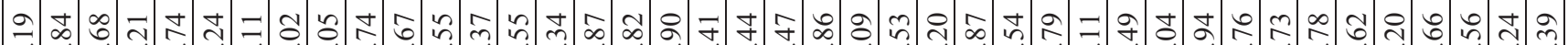

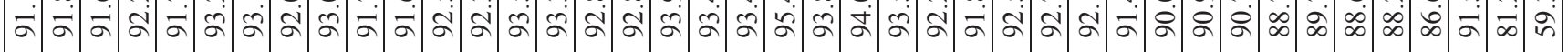

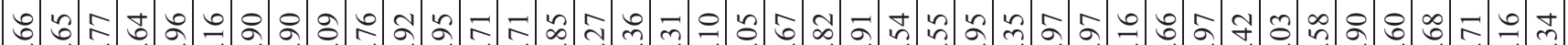

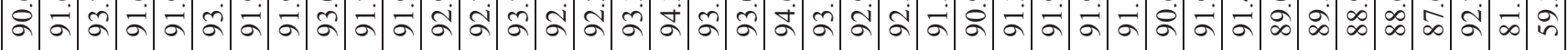

经毒

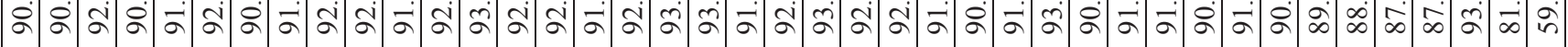

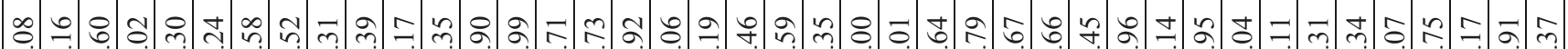

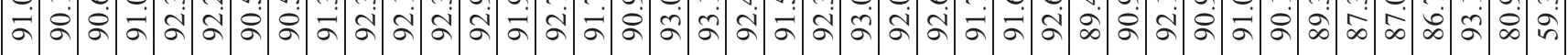
$\infty$ \&

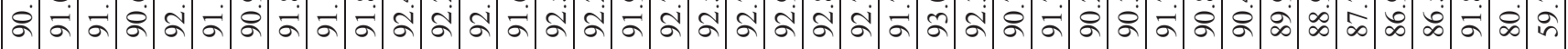

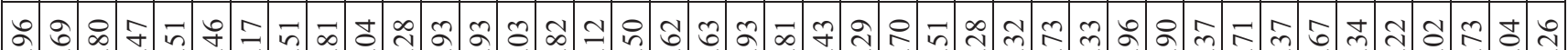

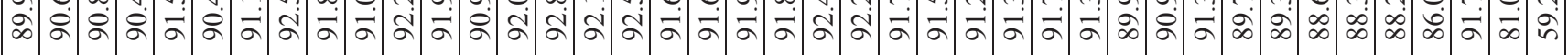

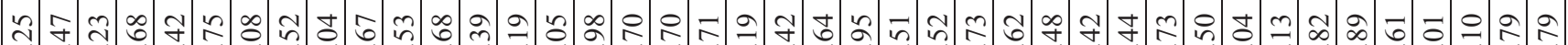

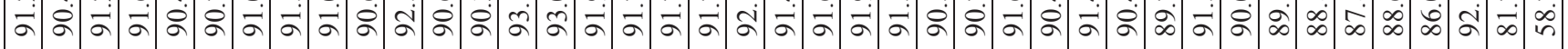

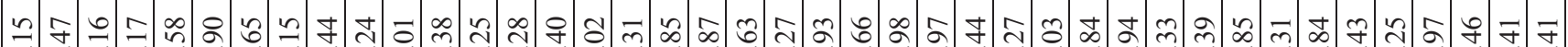

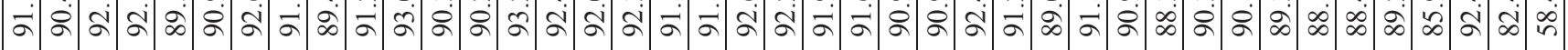

సి

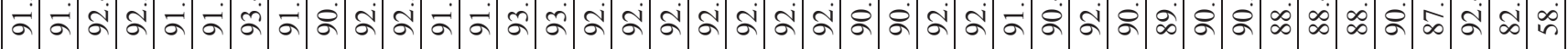

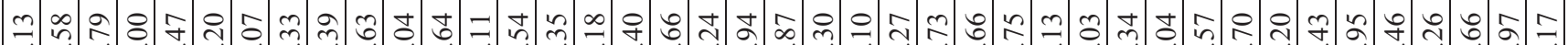

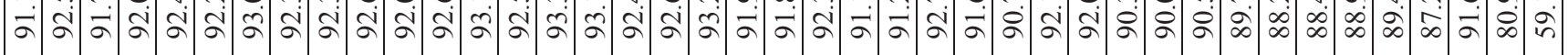

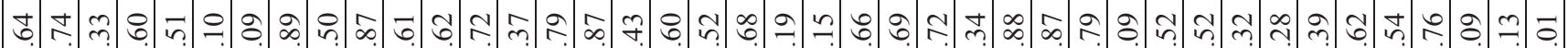
ने बे बं ก

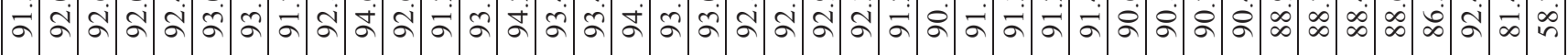

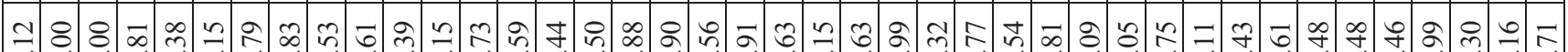

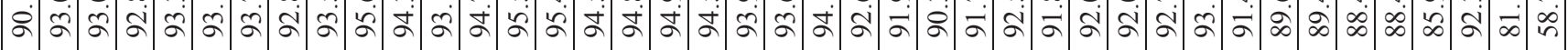

হ. m.

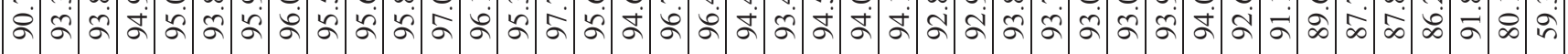

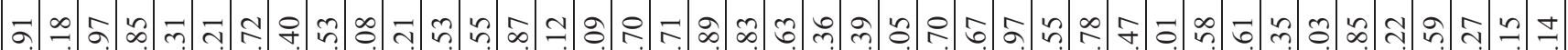

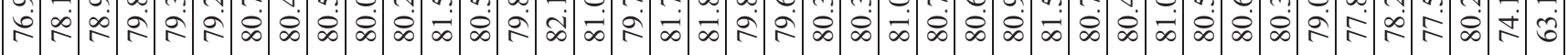




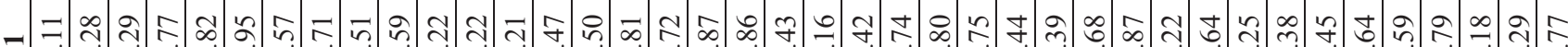

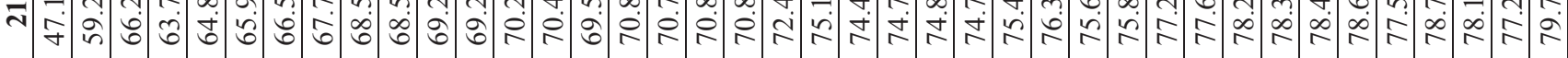

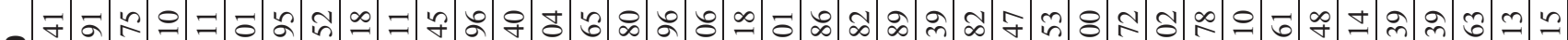

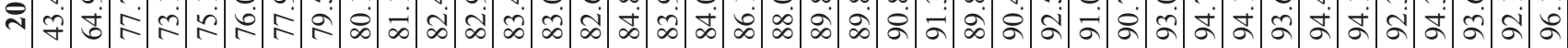

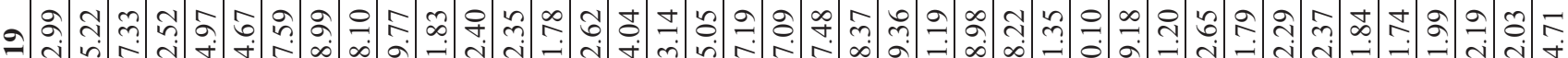
f

$\infty$ \&

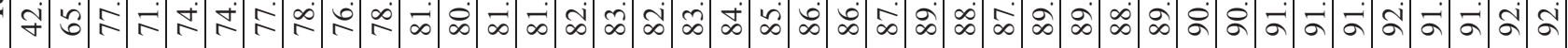

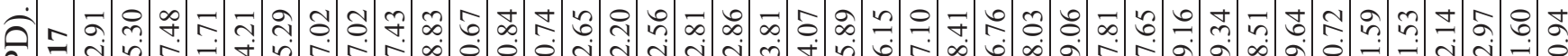

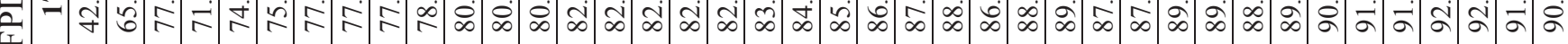
○. ๑

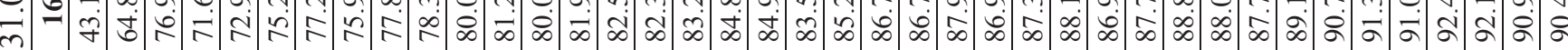

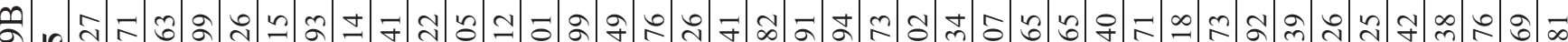

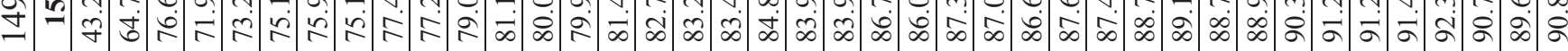

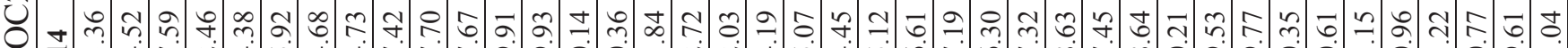

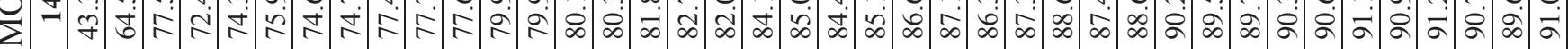
t)

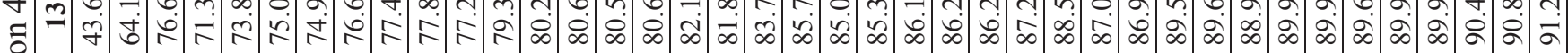
:

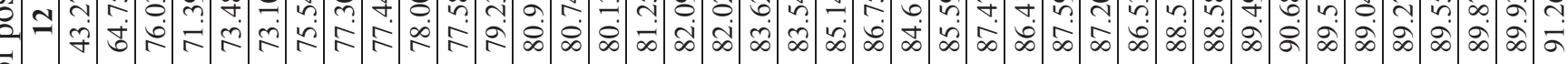

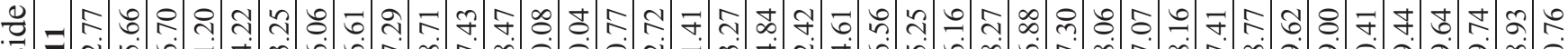

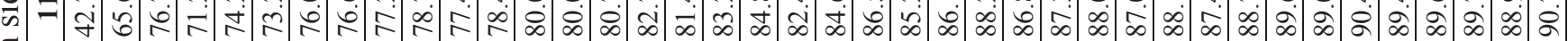

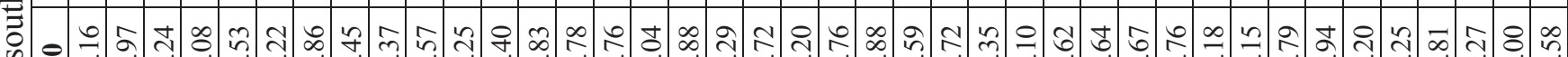

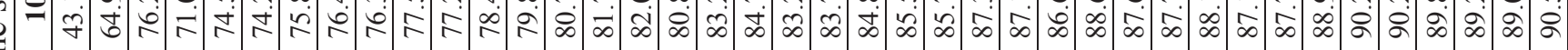
要

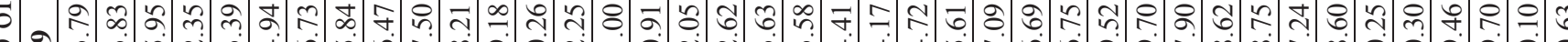

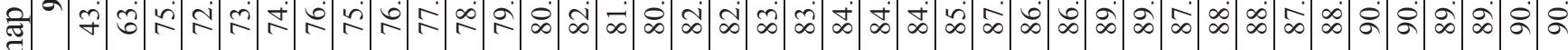

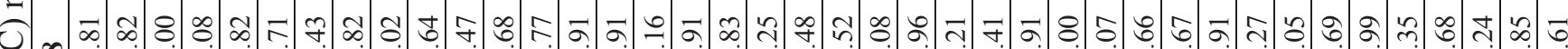

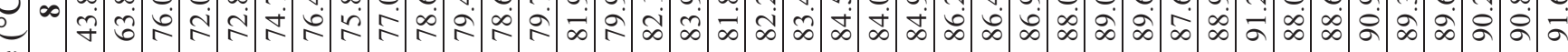

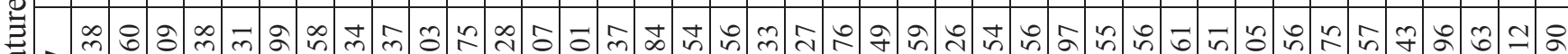

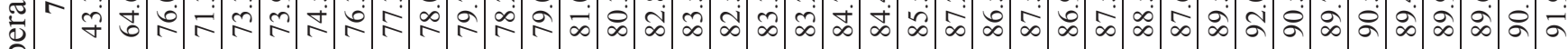

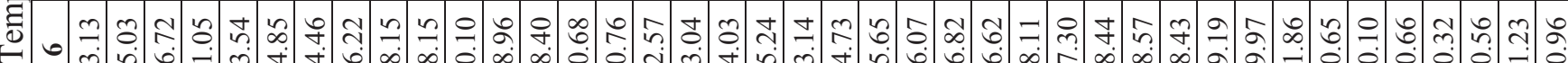

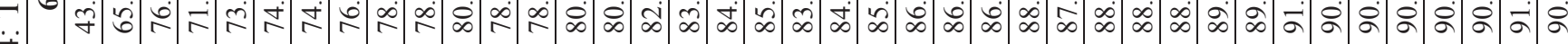

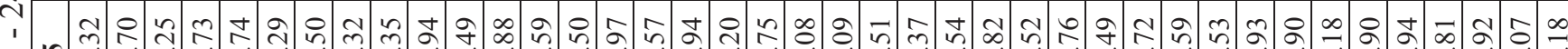
ص س 考 + भु) t

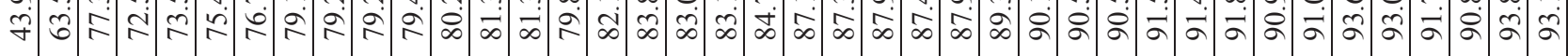

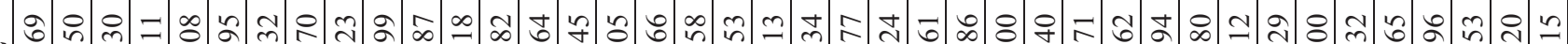

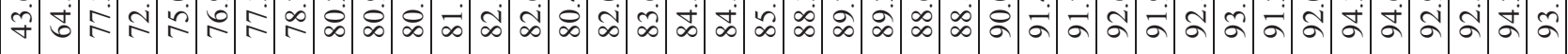
- ఫ)

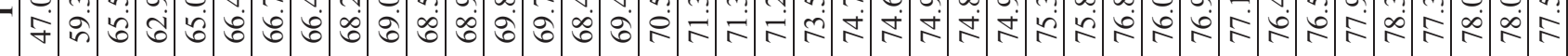




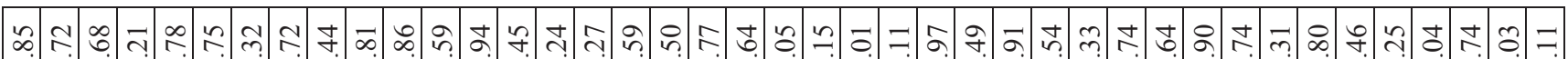

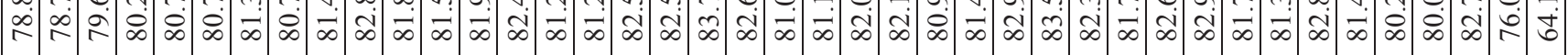

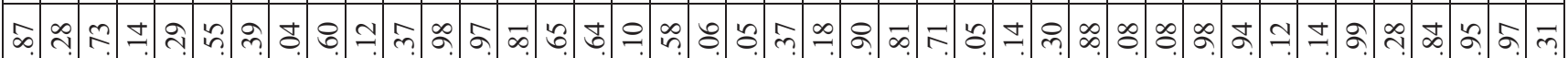

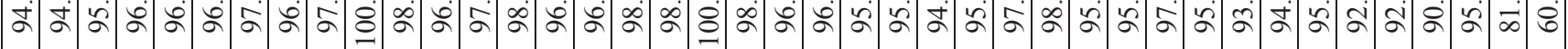

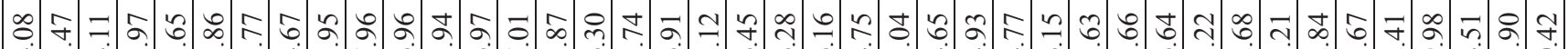

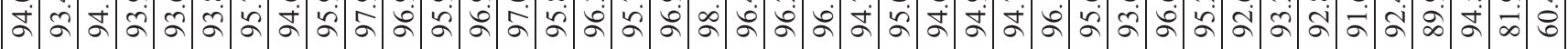

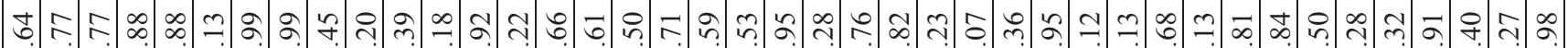

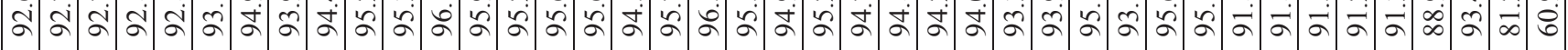

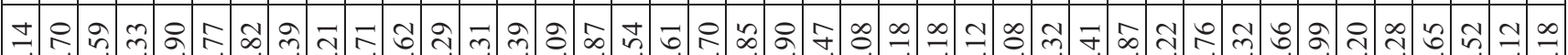
হं রi হ̇

ๆ

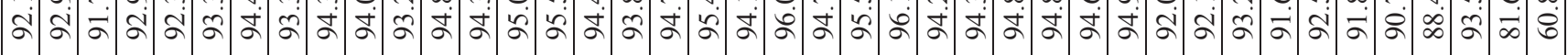

舟

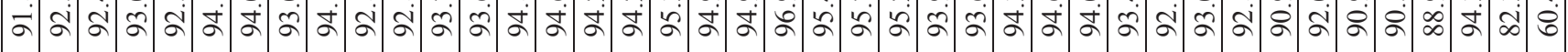

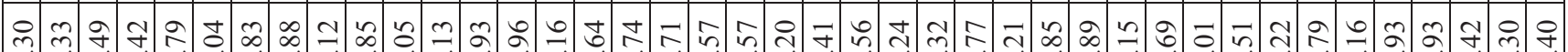

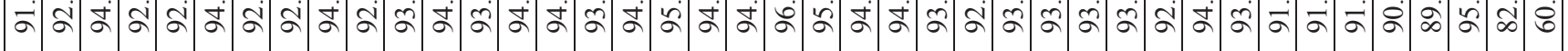

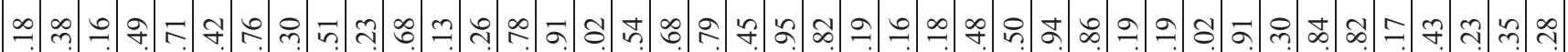

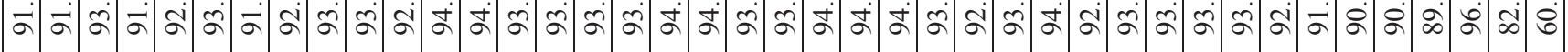

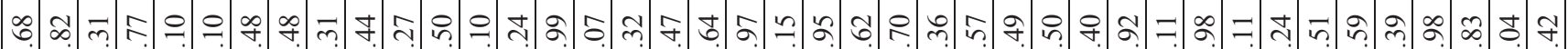

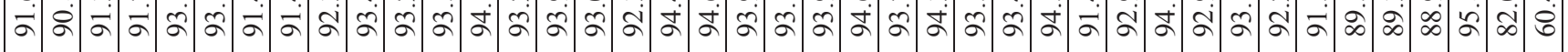

ఇ

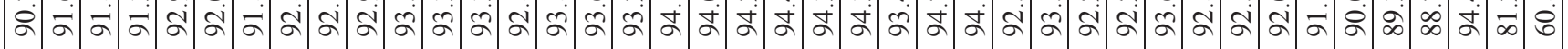
ஜ

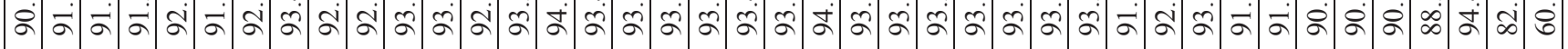

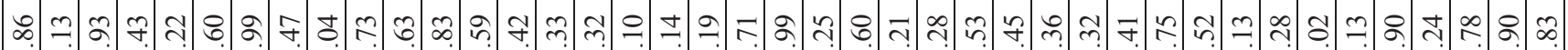

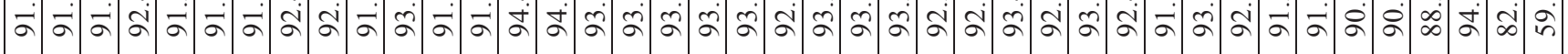

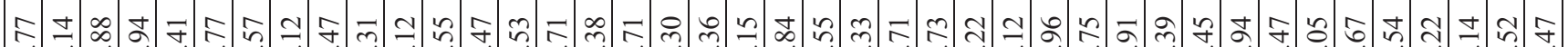

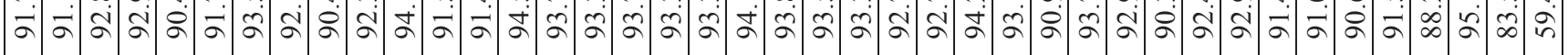

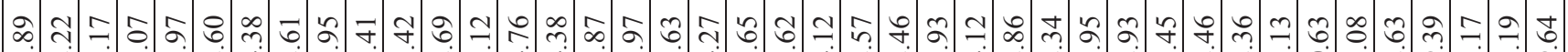

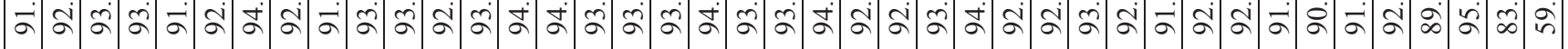

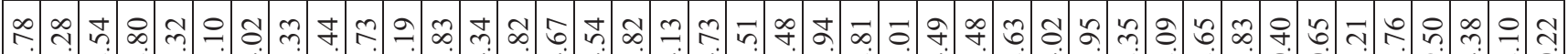

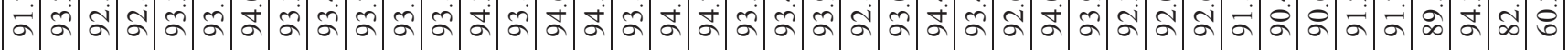

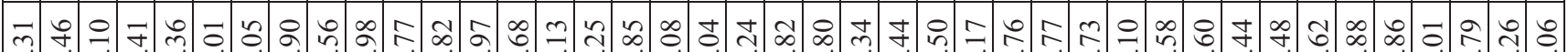

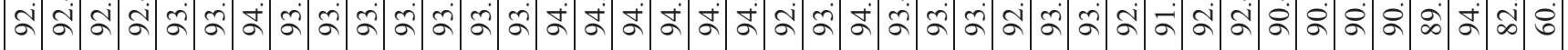
구 구

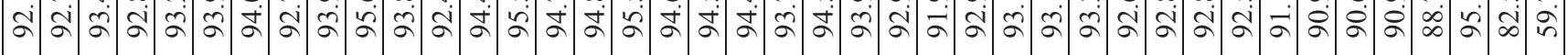
ก

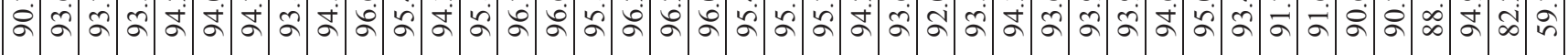

q) ூ.

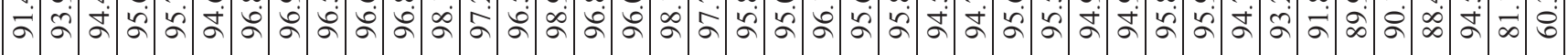
q.

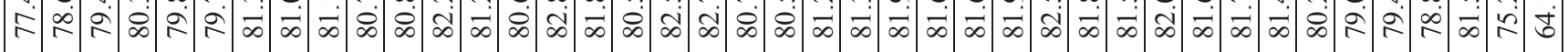




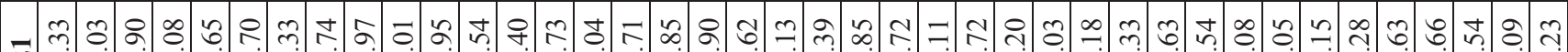

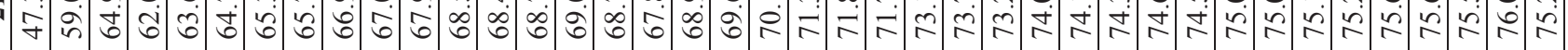

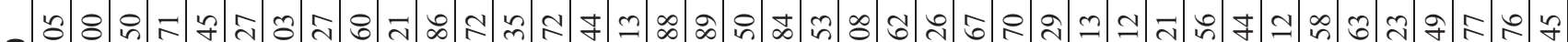
๙

$\Rightarrow$ ㄱำ

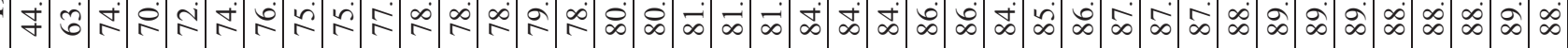

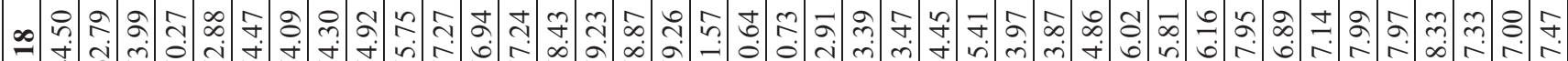
(2) 워 在 = ஸ் ש̂.

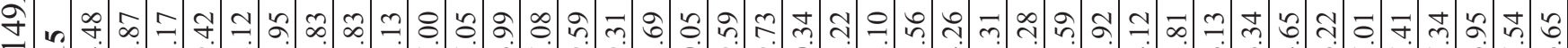

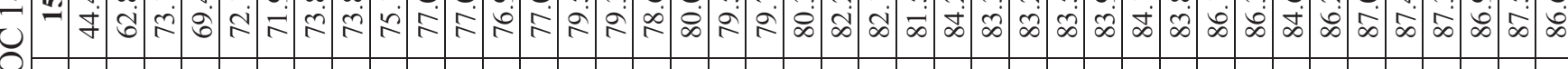

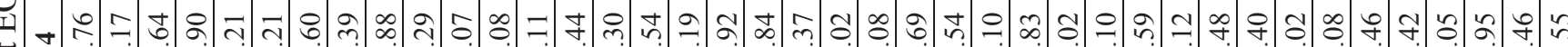

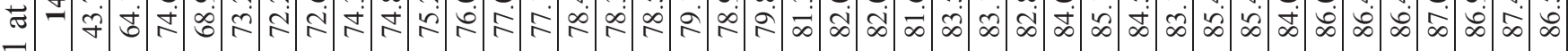
こ

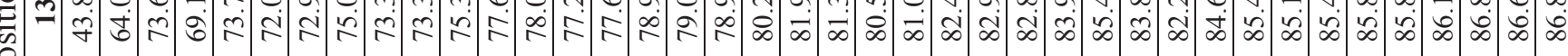

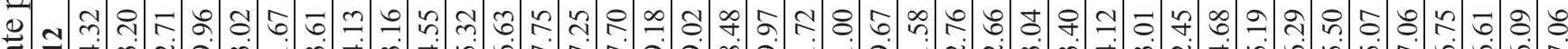

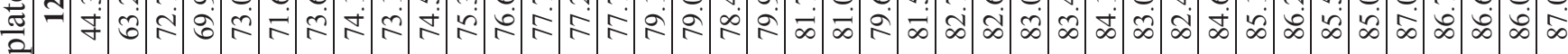
- 궁

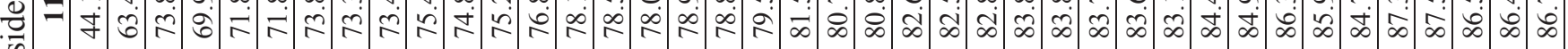
馬

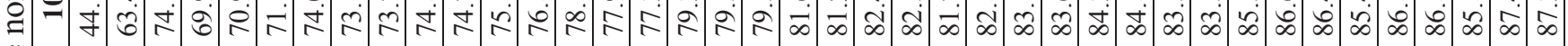
艺

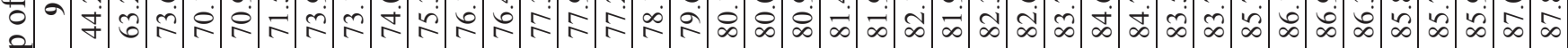

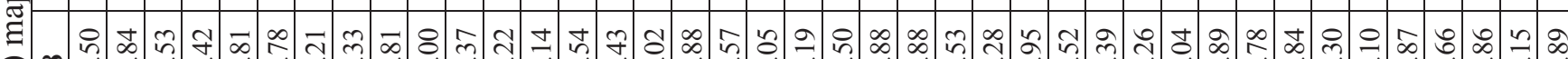

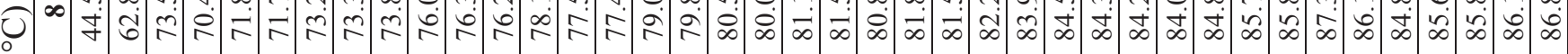

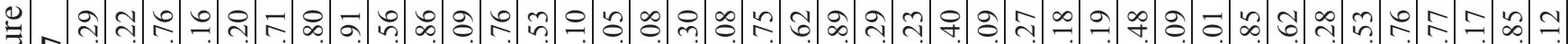

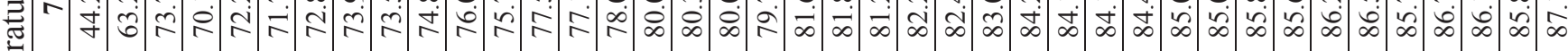
音 勿 乞 மூ

$\frac{0}{2}+\sigma$ ก E

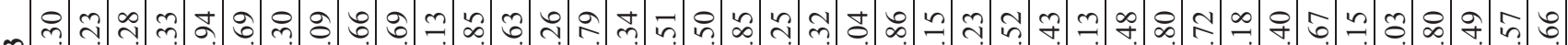

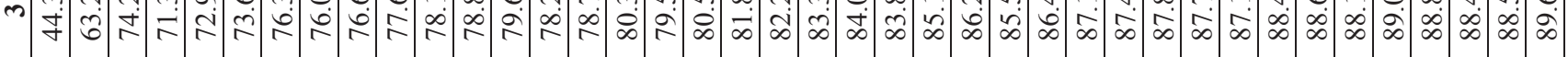
m N

-

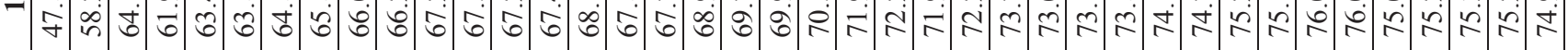
○ 


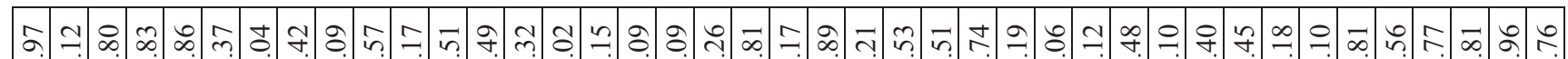

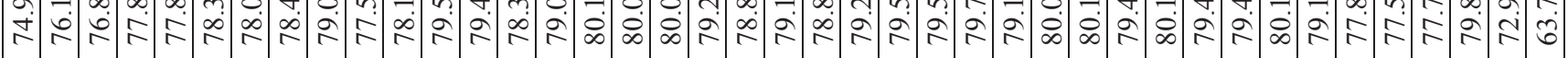
ๆ

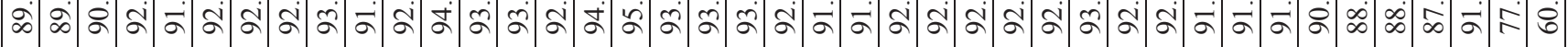

กิ

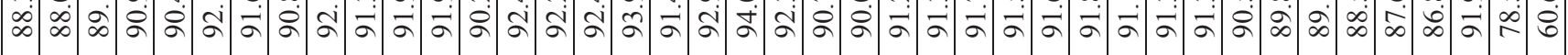

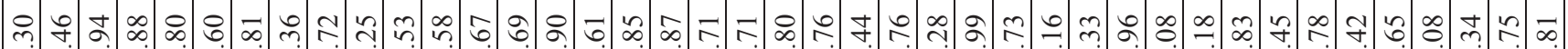

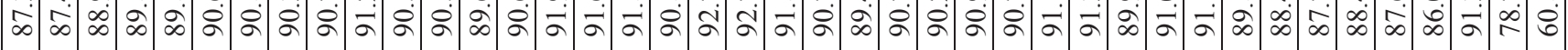

ㄱำ

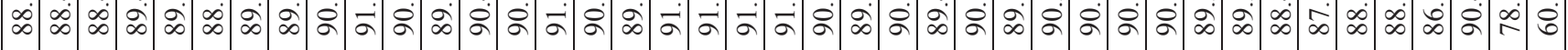

ㄱํ.

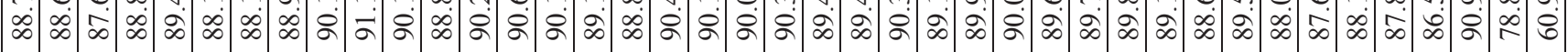

๙

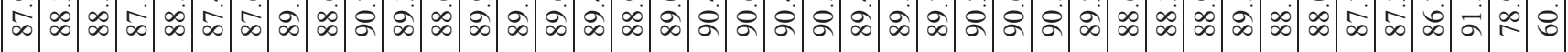

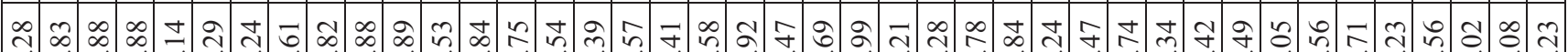

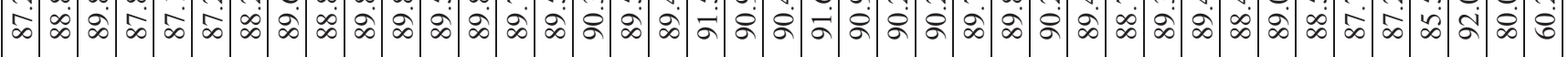

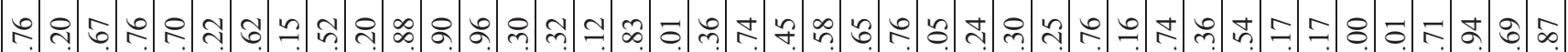

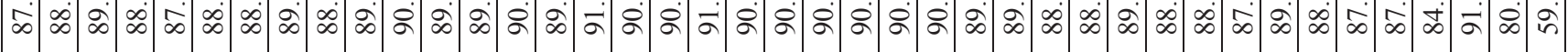

ㄱ.

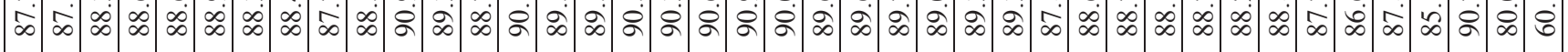

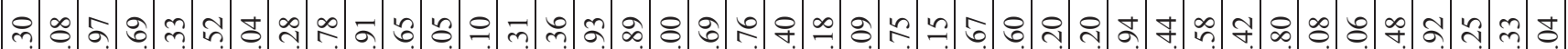

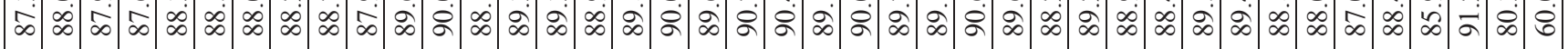

กิ

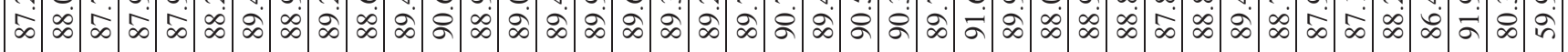

ลาน

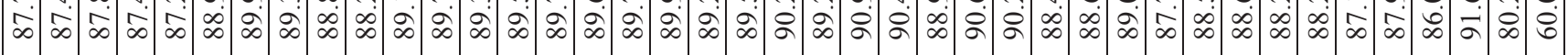

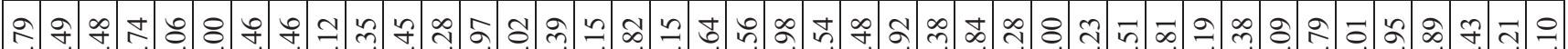

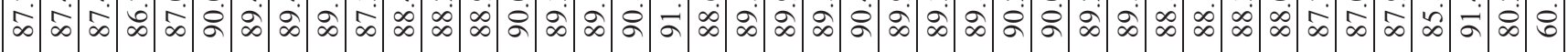
ઈ.

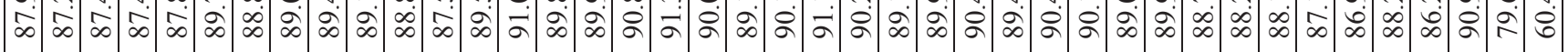
๑ $\infty$ :

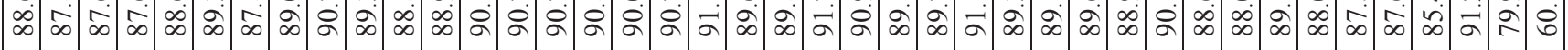

๘ กิ

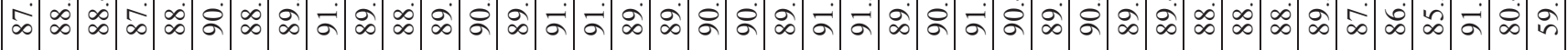

\&.

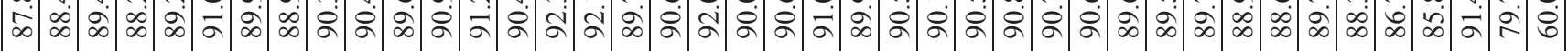

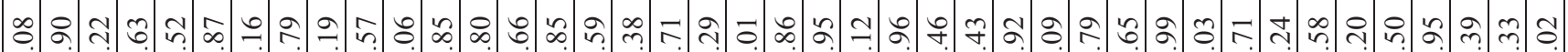

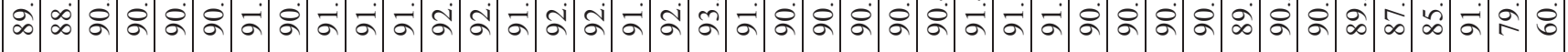

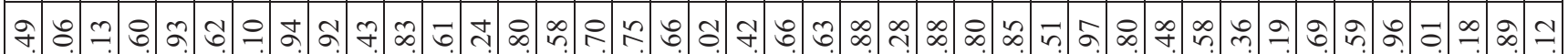

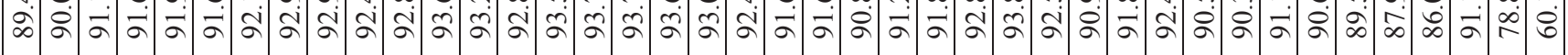

舟

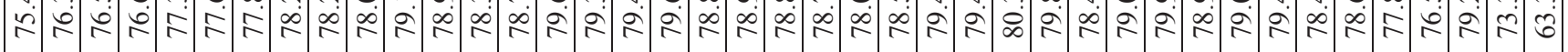
过

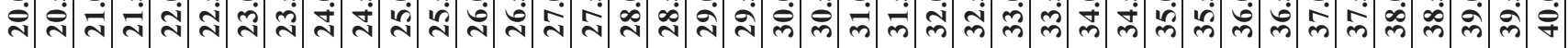




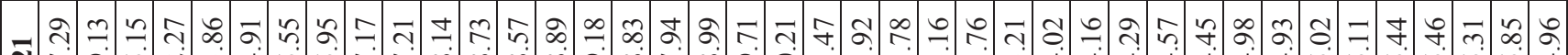

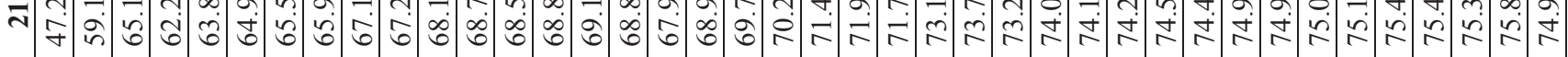

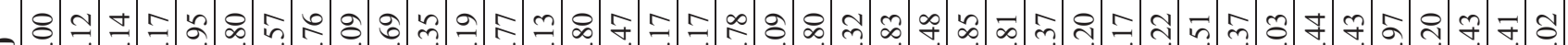

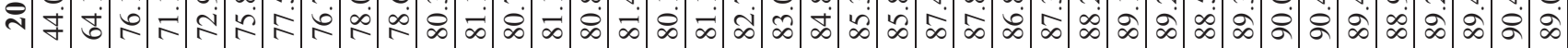

$=$ ᄀ

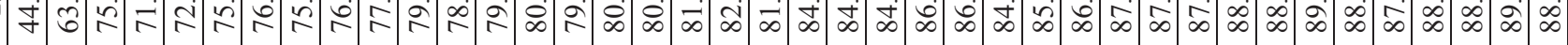

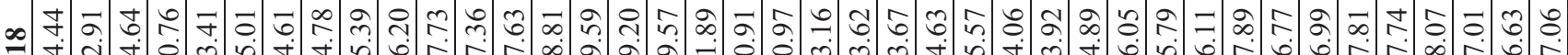
古

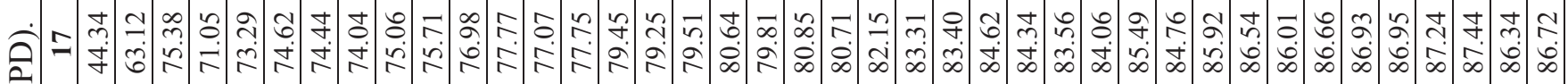

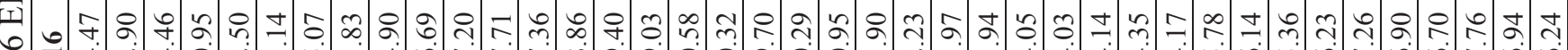

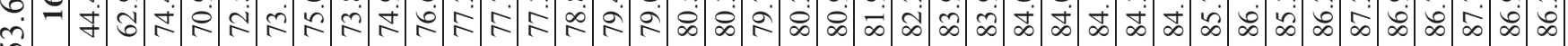

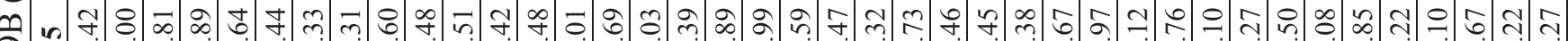

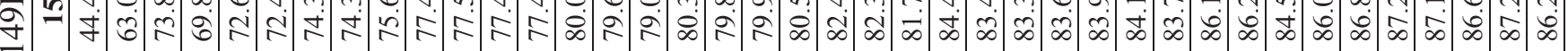

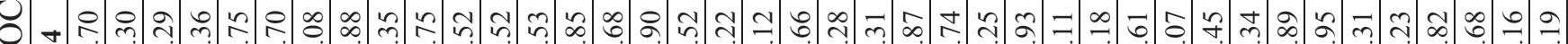

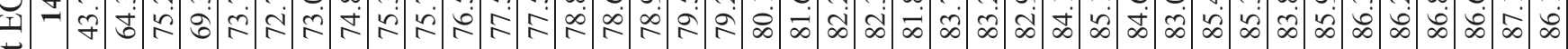
-

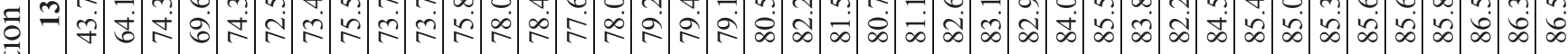
๙ क्षे

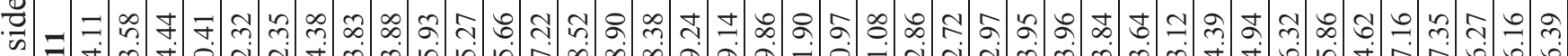

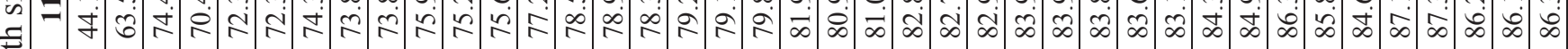
亏ำ

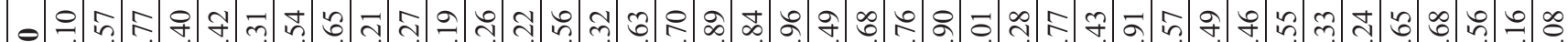

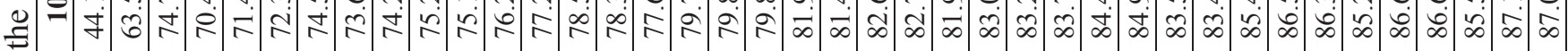

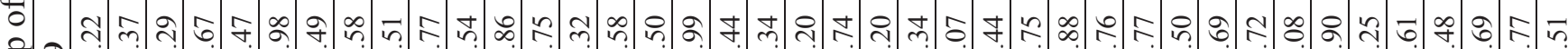
寻 (ব)

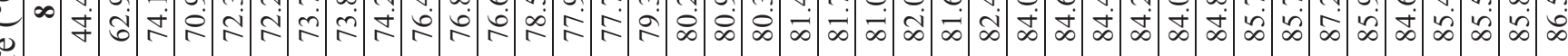
言 ๙ ठ

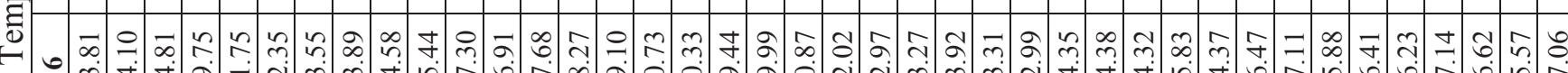

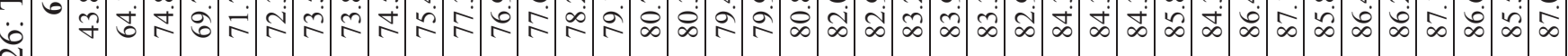

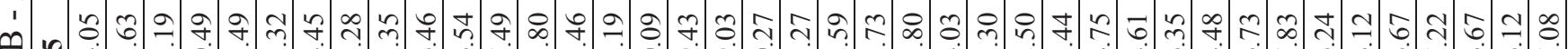
光 䒕

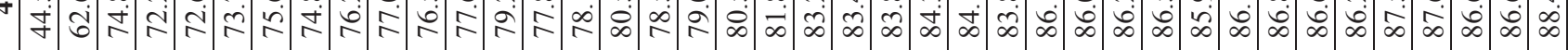

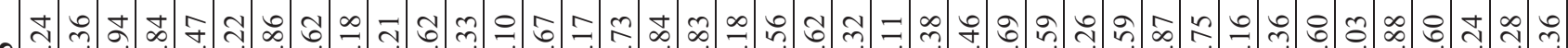

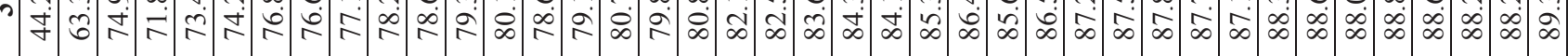

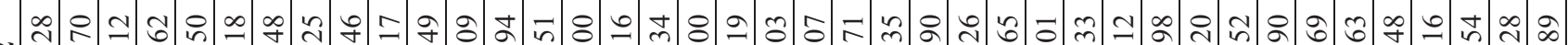

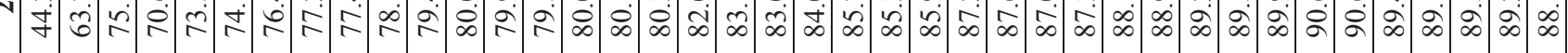

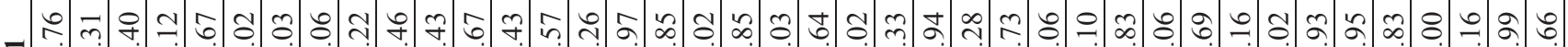

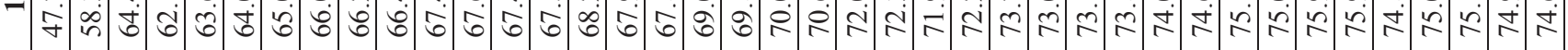
- 


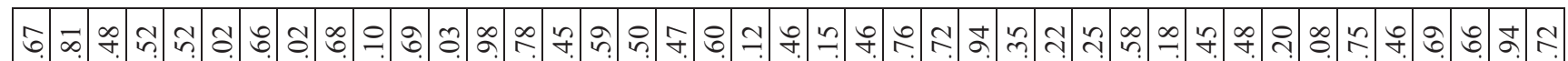

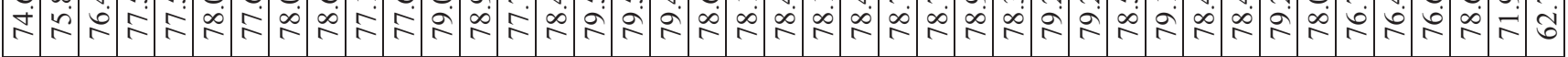

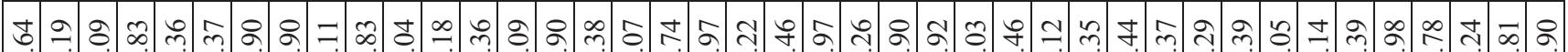

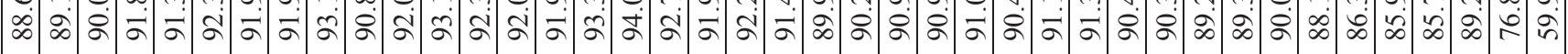

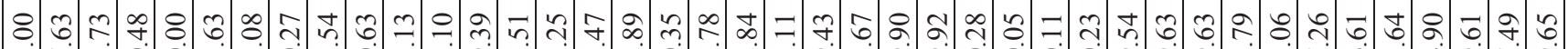

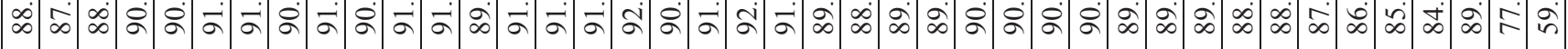

œ

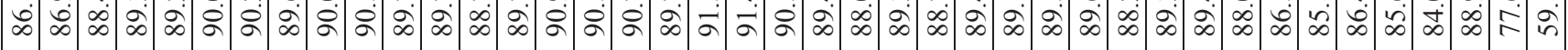

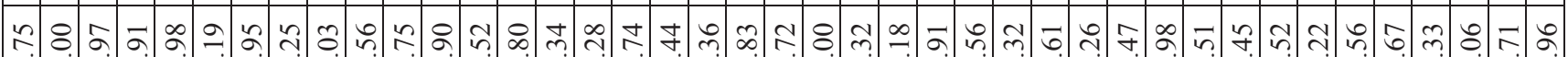

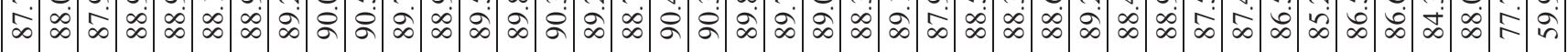

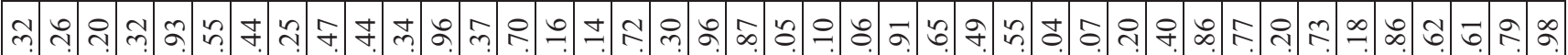

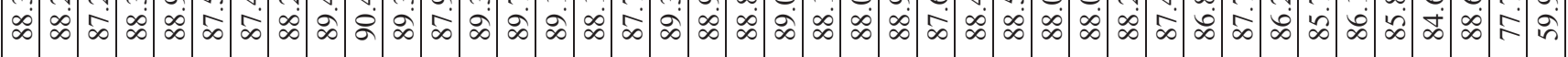

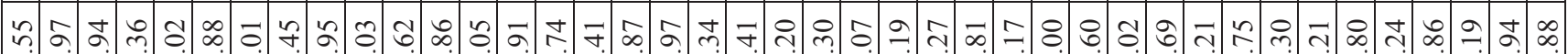

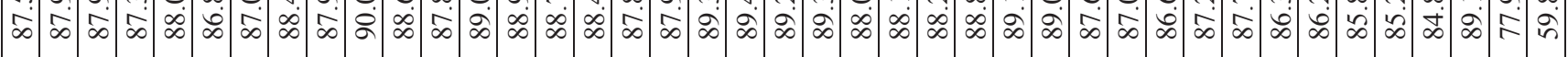

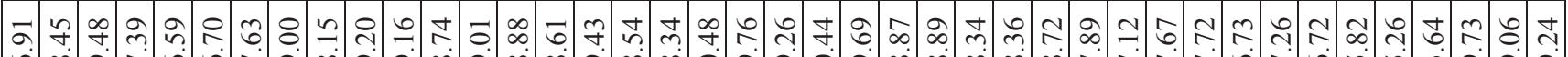

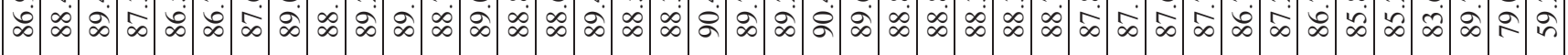

ఫ.

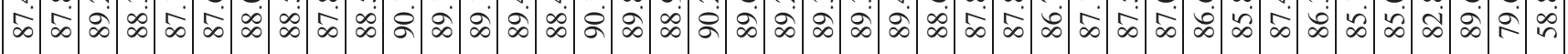

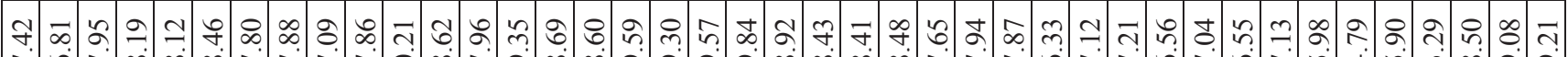

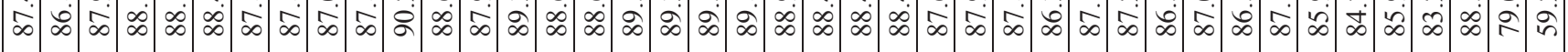

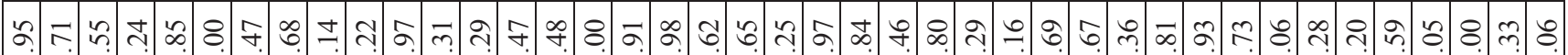

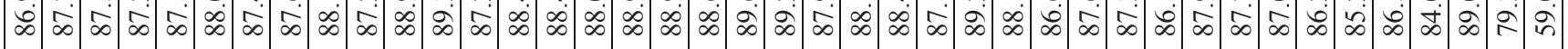

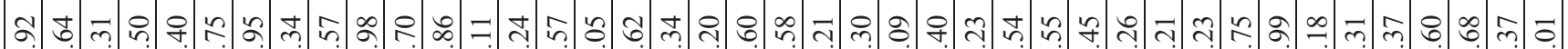

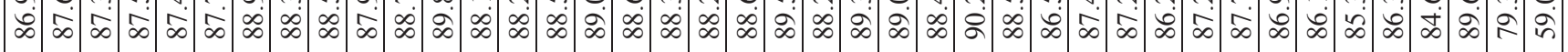

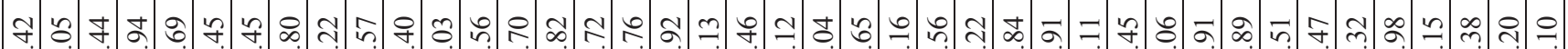

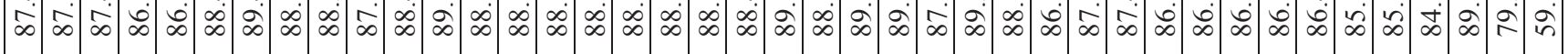

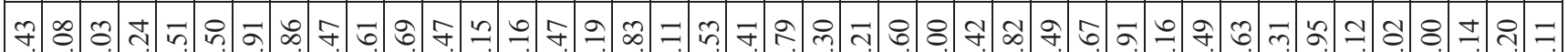

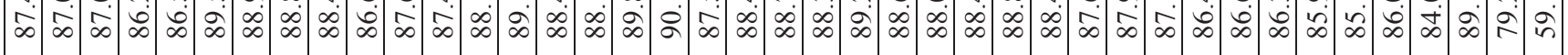
๑

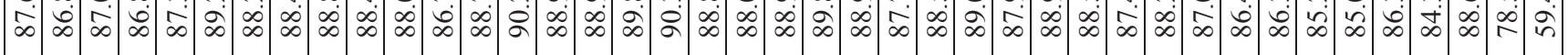

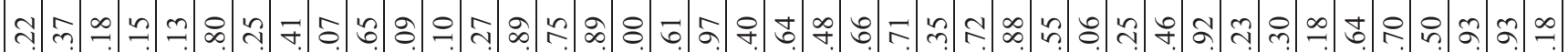

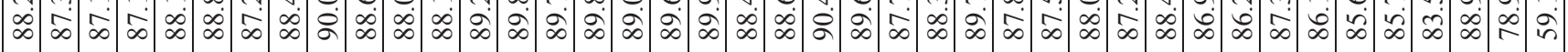

ๆ.

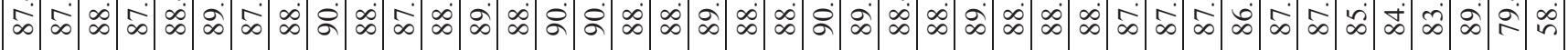

mิ

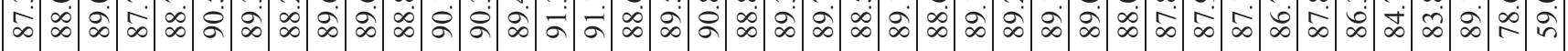

ล

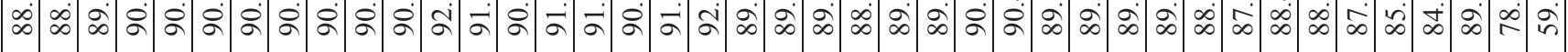

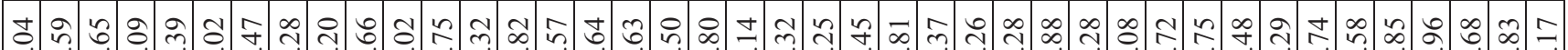

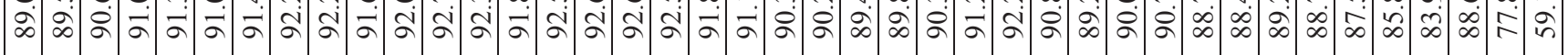

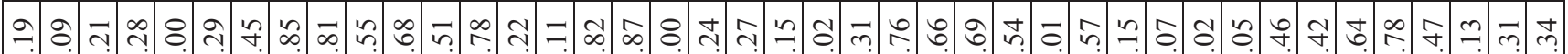

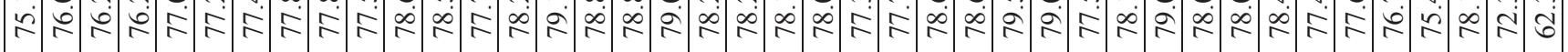
我

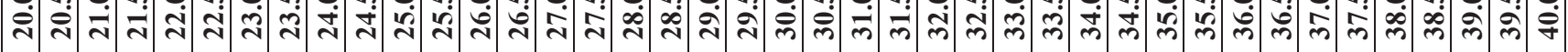




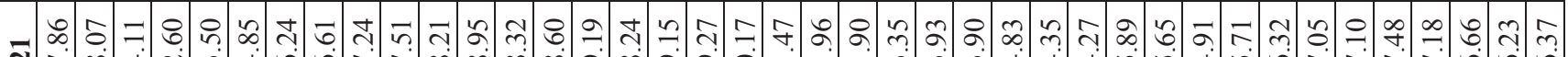

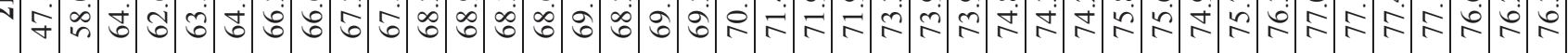

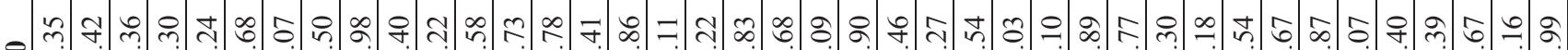

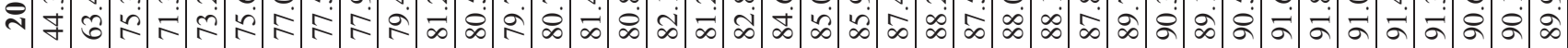

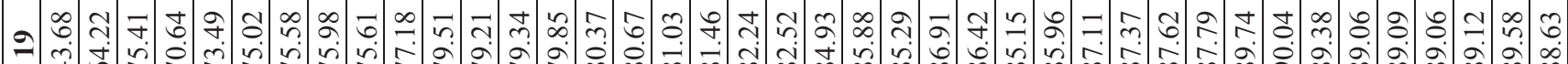

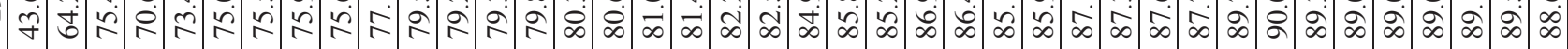

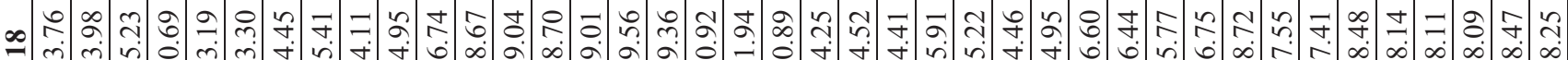
fं

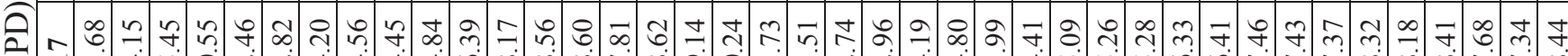

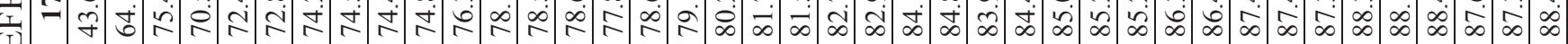

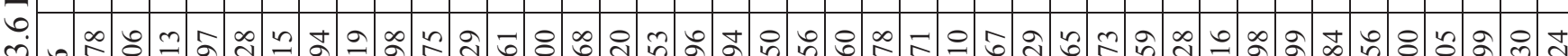

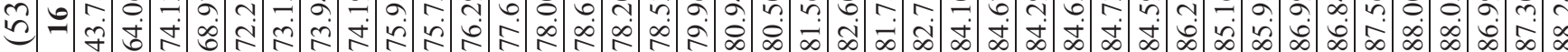

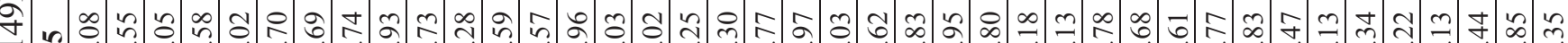

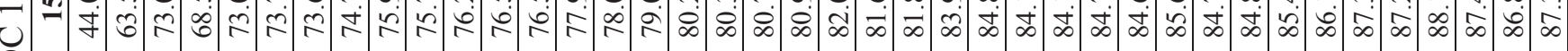

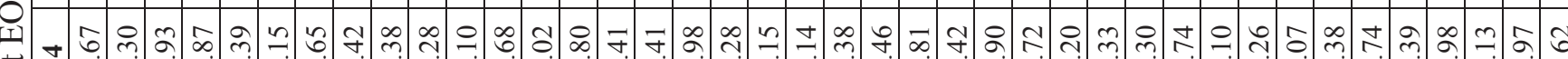

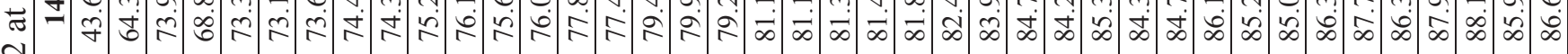
శే

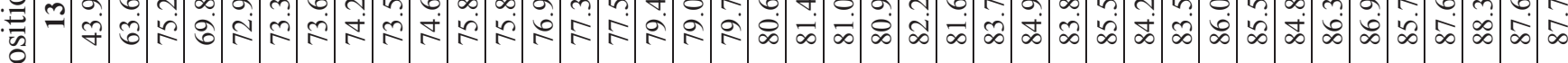
จ

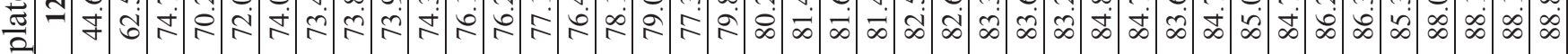
0 -

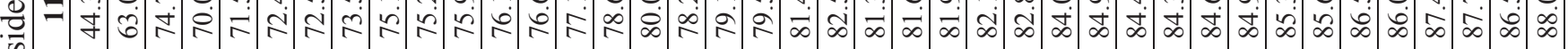

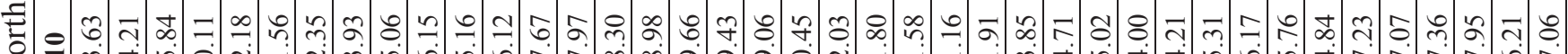

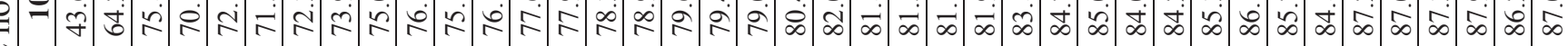
至 $\infty$ m

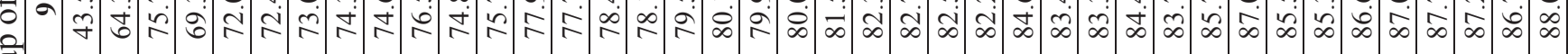

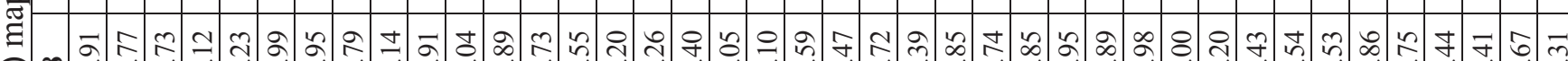

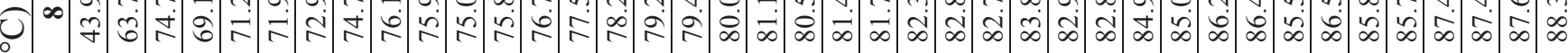

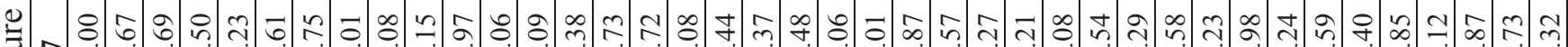

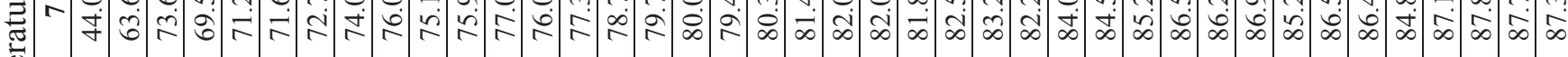

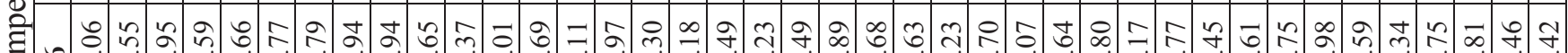
包 خิ

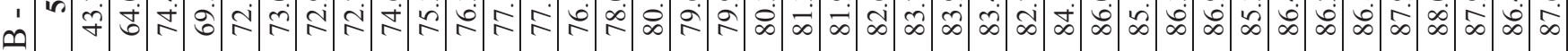

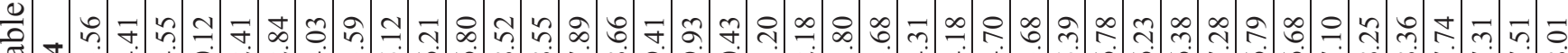

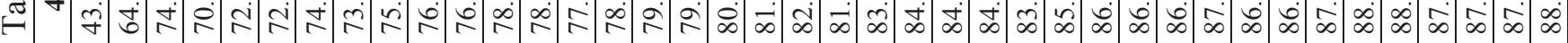

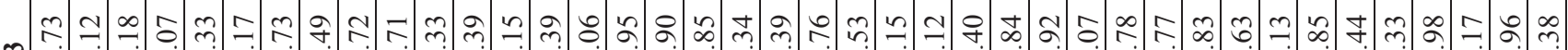

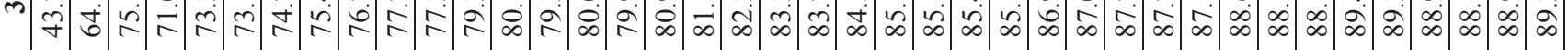

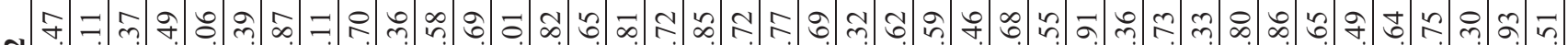

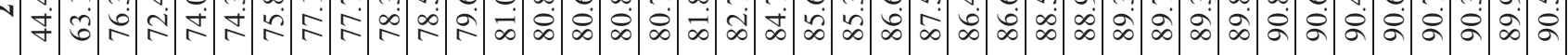

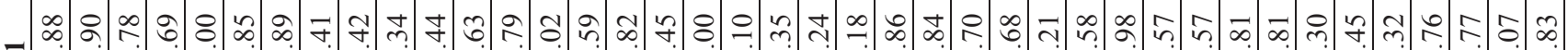

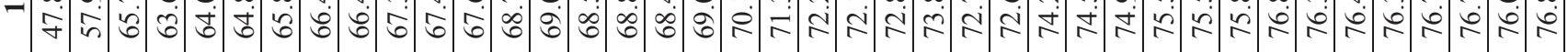
웅 官 


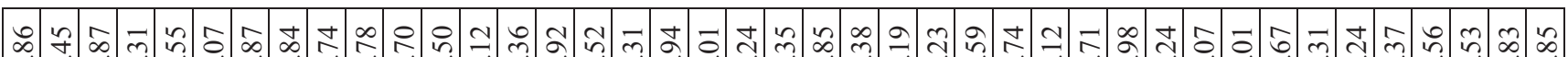

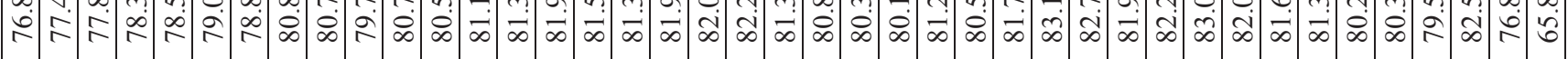

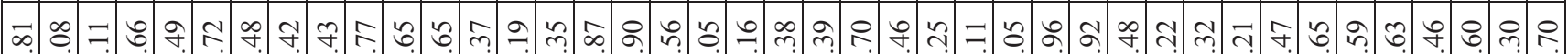

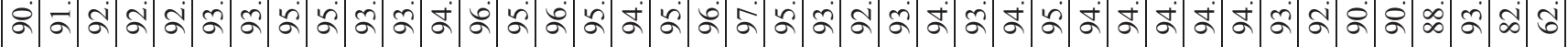

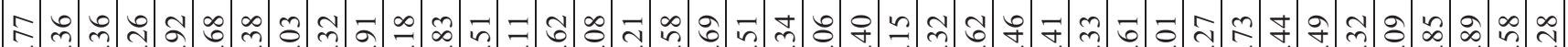

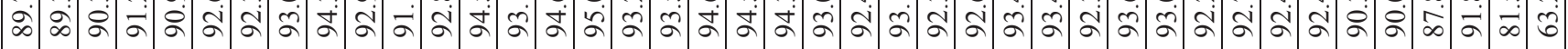
ทิ กิ

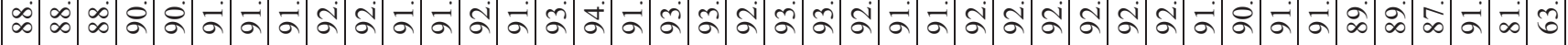

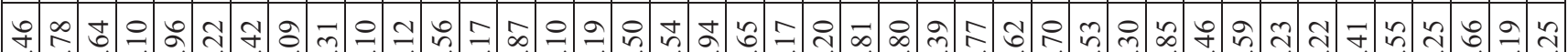

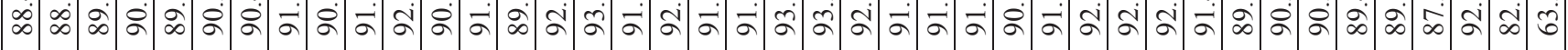

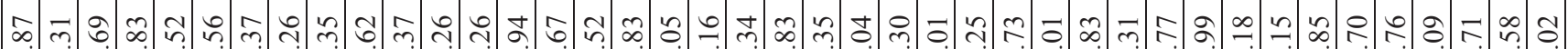

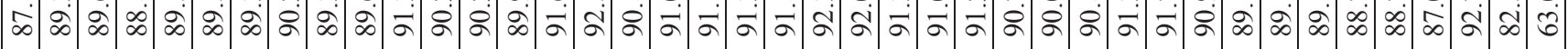

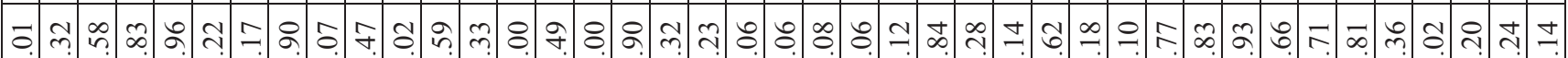

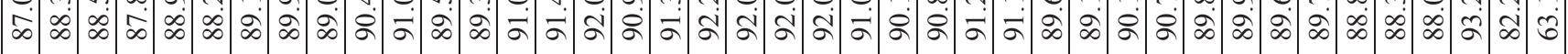
ㅇำ

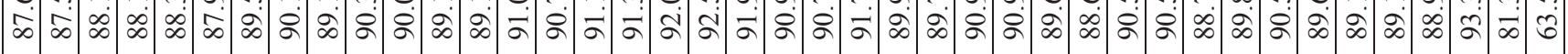

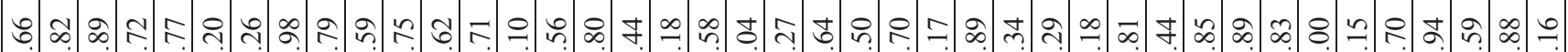

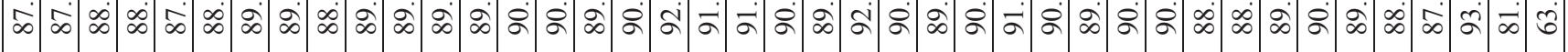

กิ

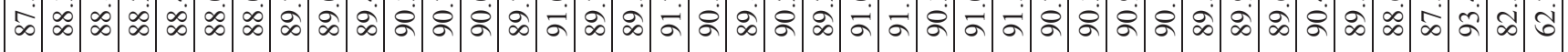

ชิ

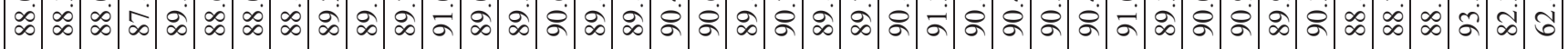

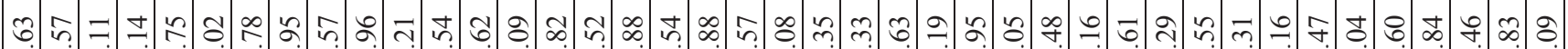

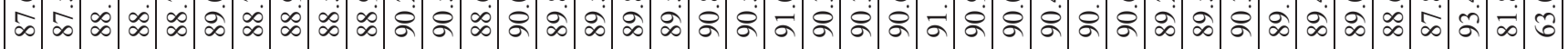

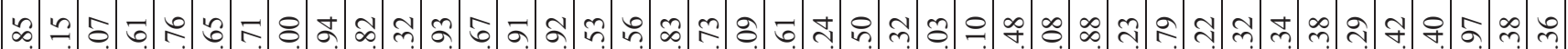

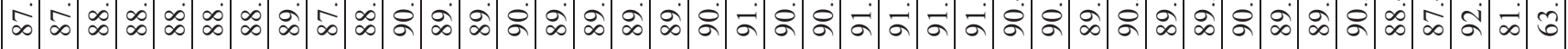

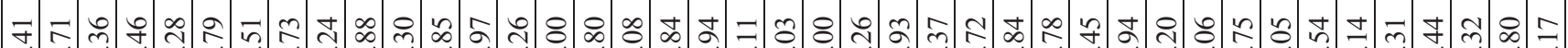

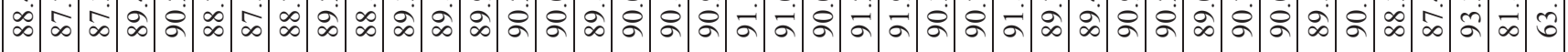

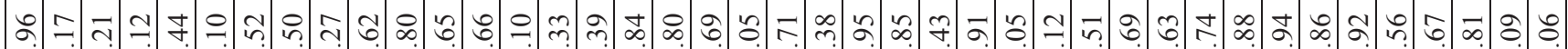

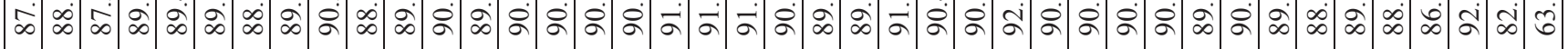

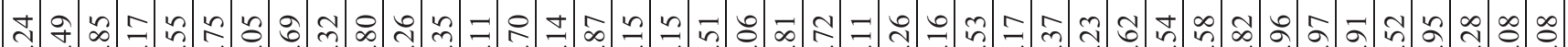

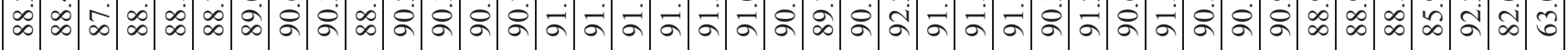
ช.

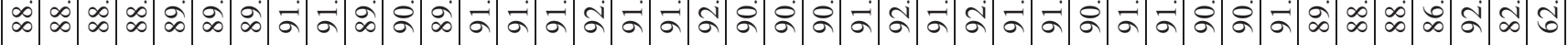
চ

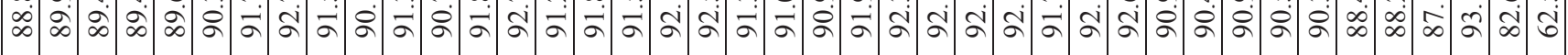

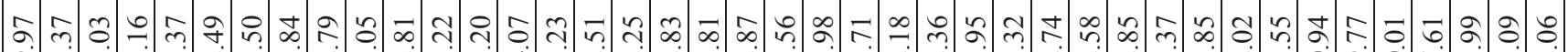

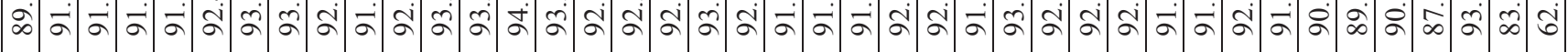

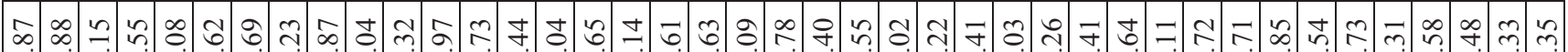

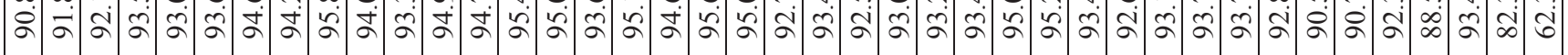
-

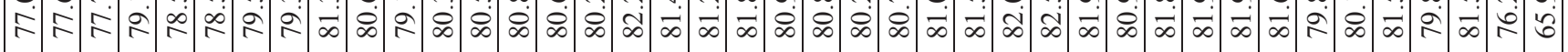




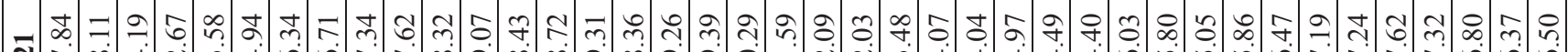

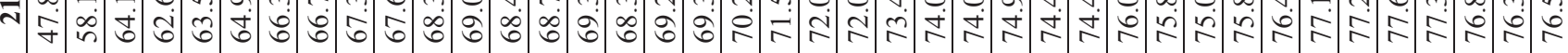

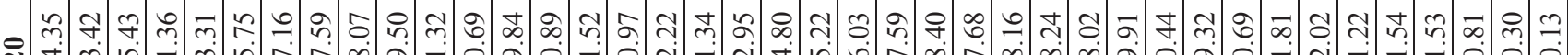

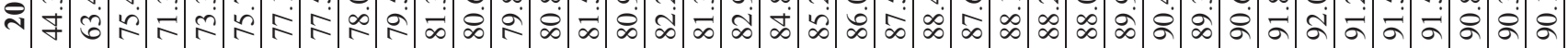

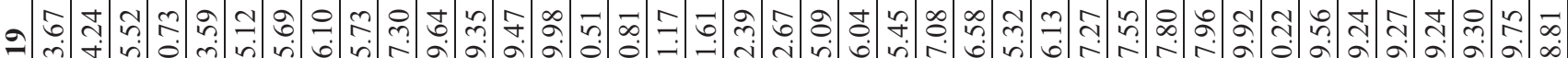

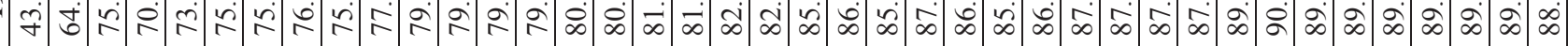

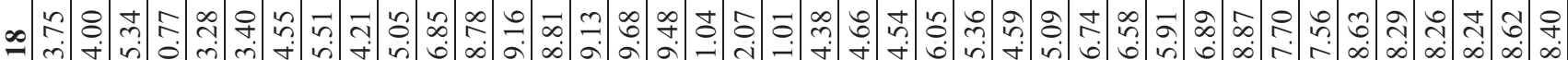
f

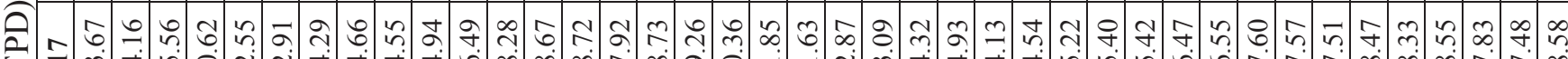

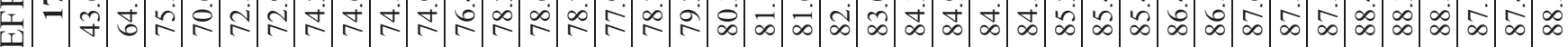

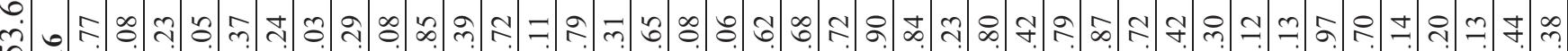

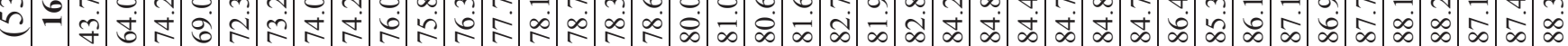

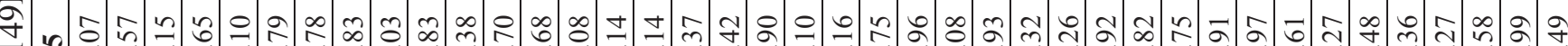

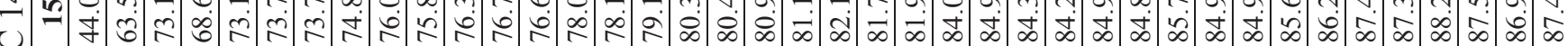
○一

士

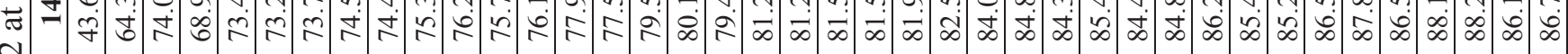

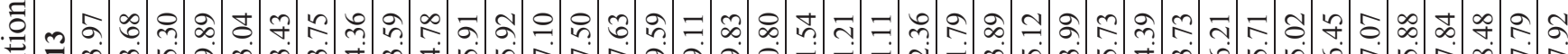

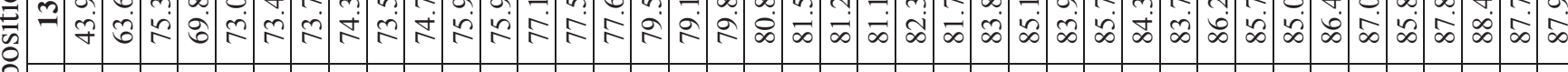

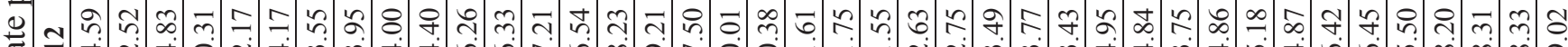

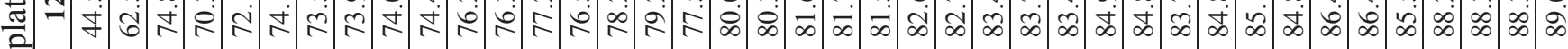
ป

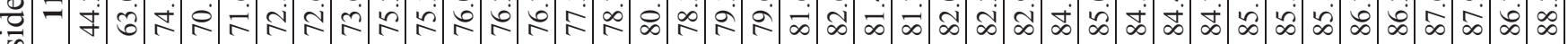

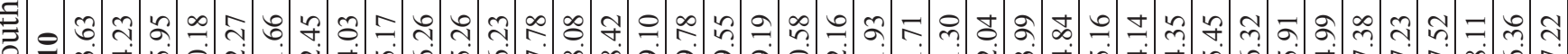

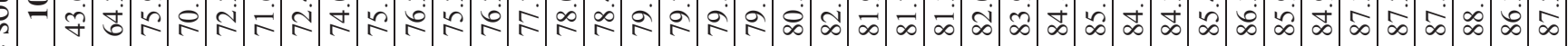

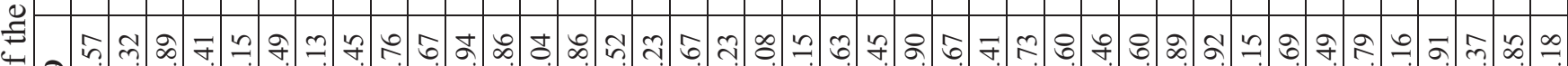

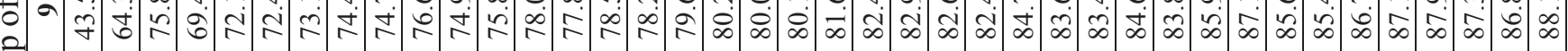
ఫ్

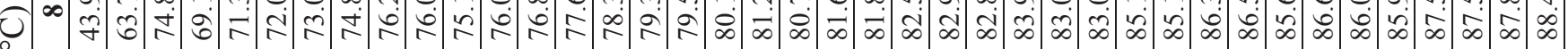

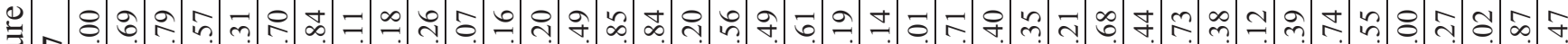

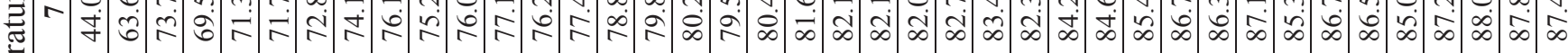

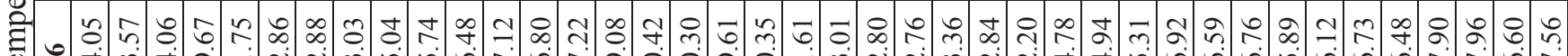
年

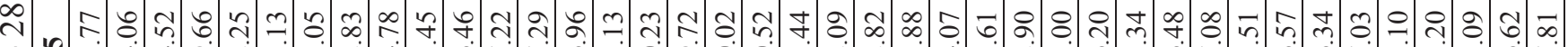

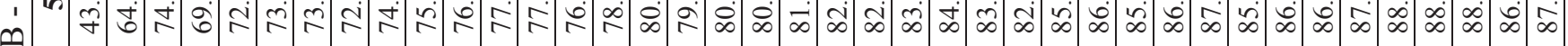

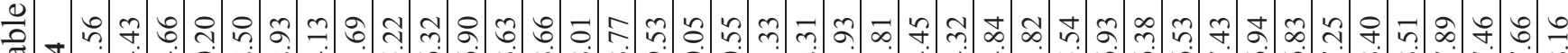

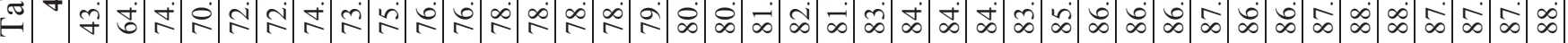

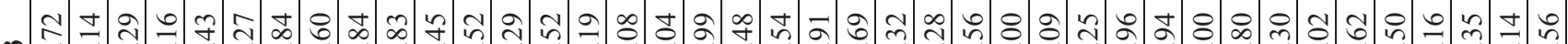

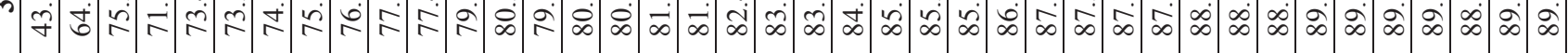

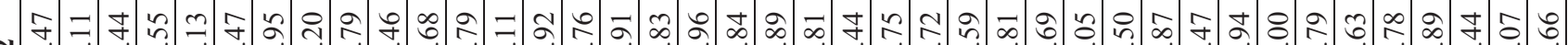

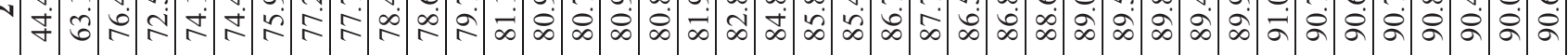
- $\begin{aligned} & f \\ & \text { in }\end{aligned}$ - n

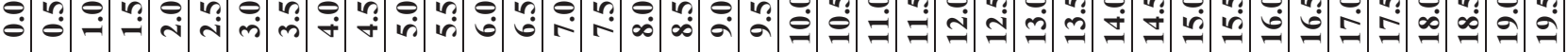


ฉ

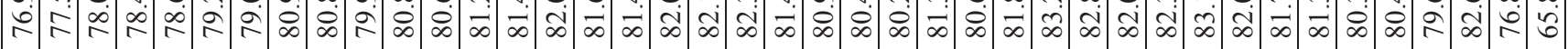
๙

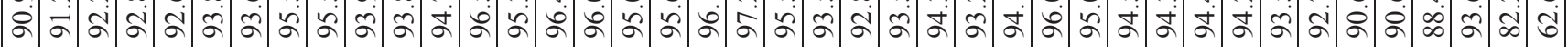

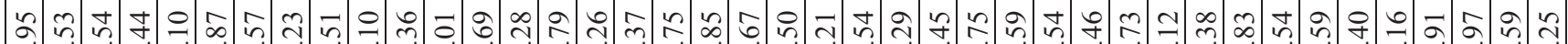

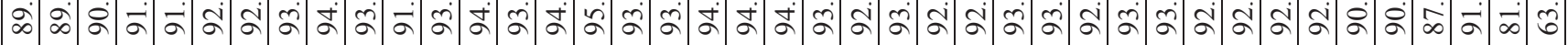

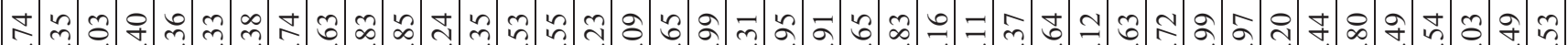
ळ

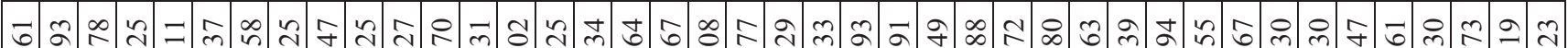

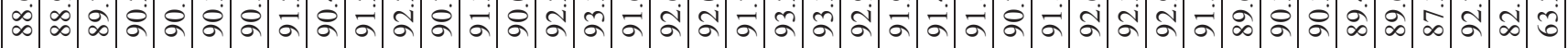

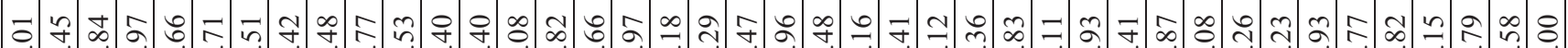

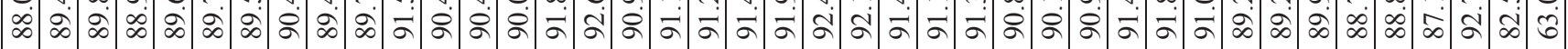

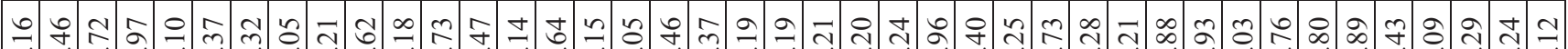

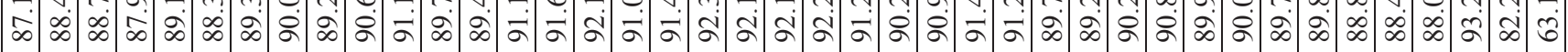

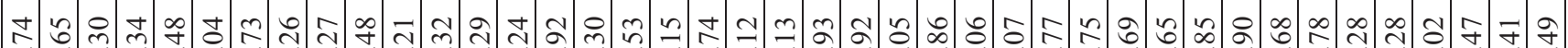

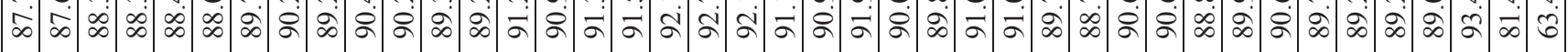

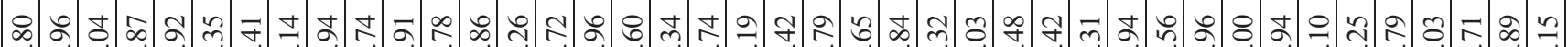

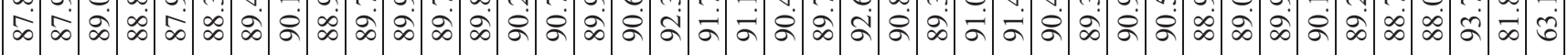

대유.

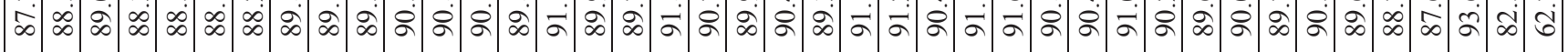

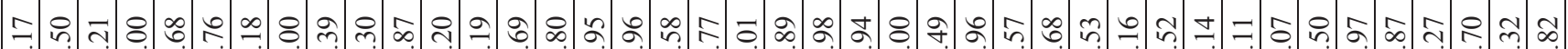

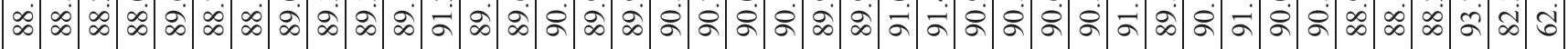

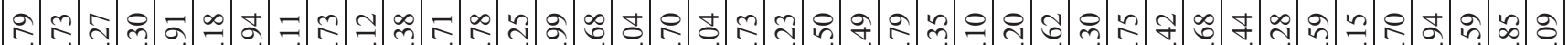

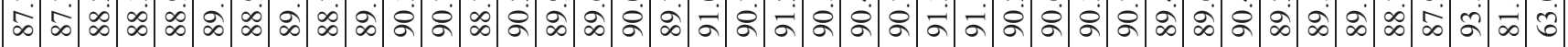

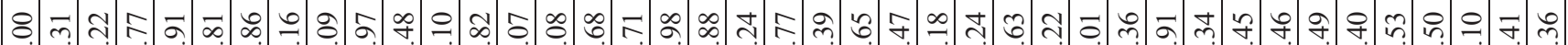

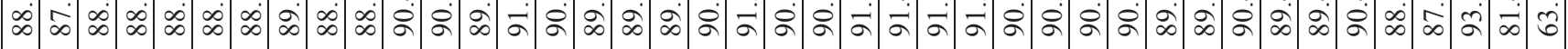

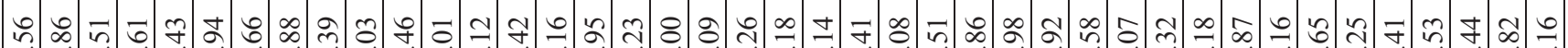

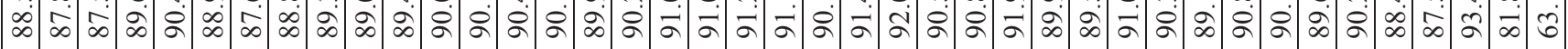

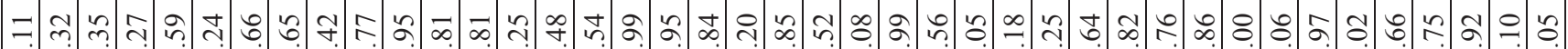

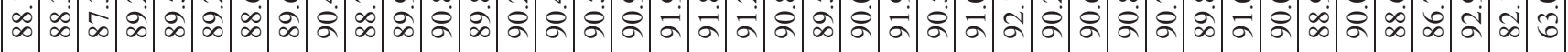

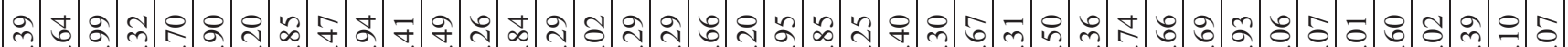

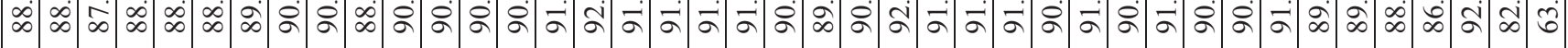

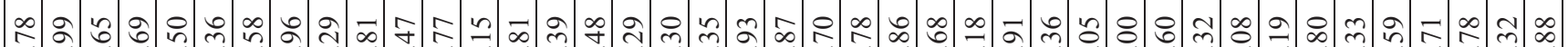

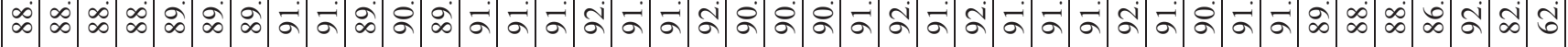

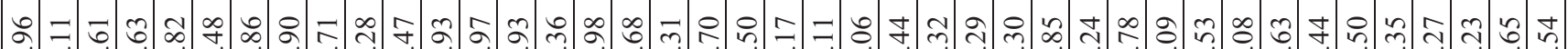

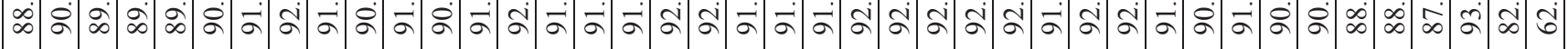

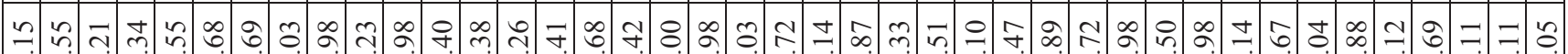

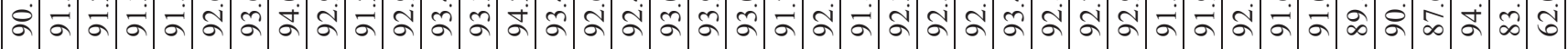

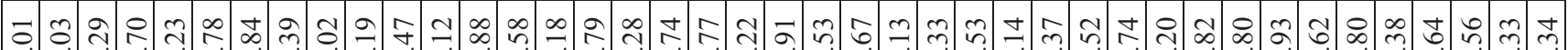

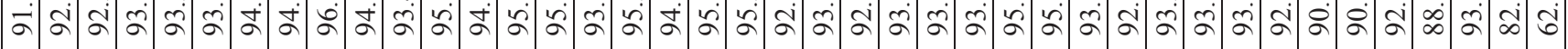

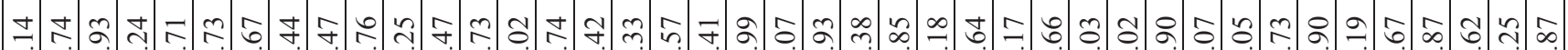

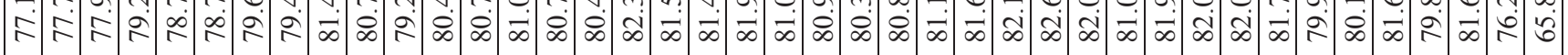

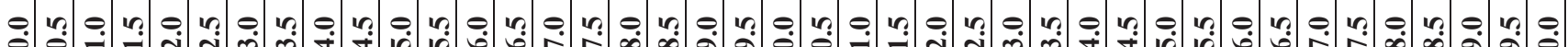
जें 
-

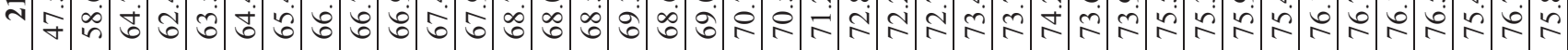

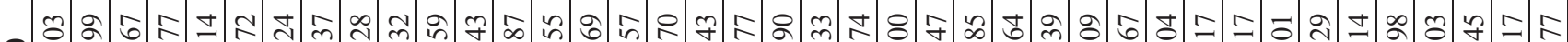

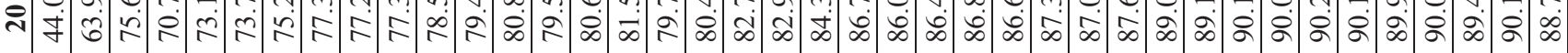

$=$ б.

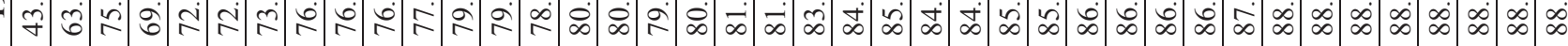

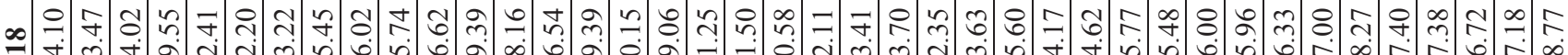

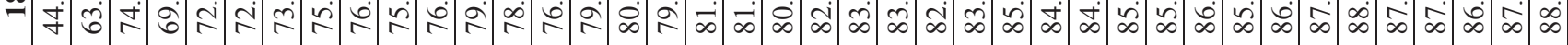
दी

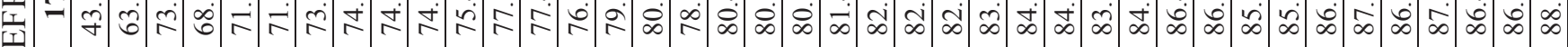

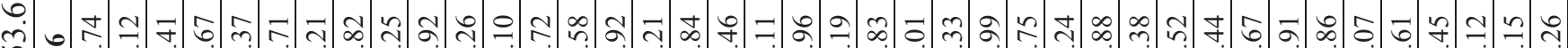

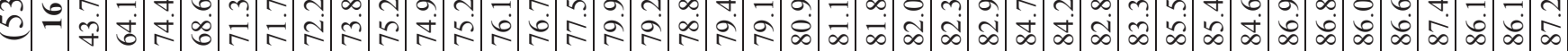
守n

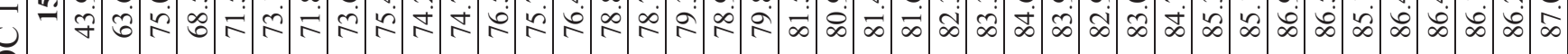
- ఫ.

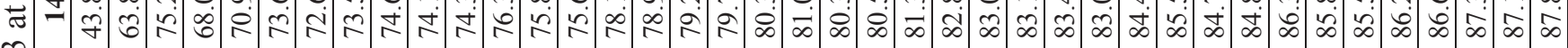

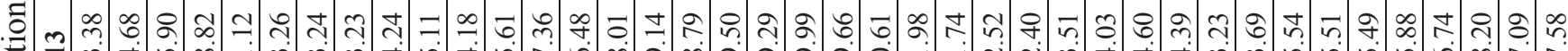

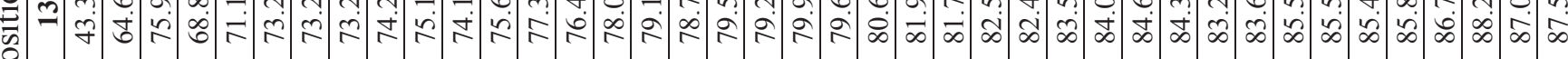
๑

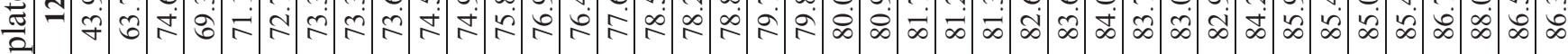
-

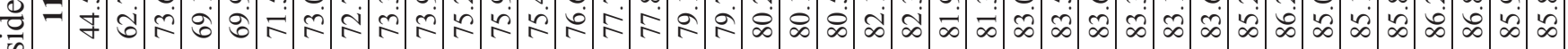
馬

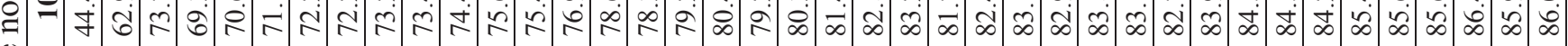
苞

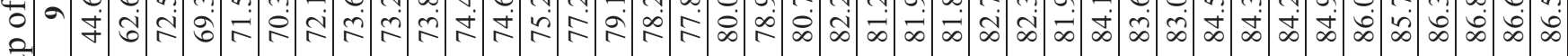

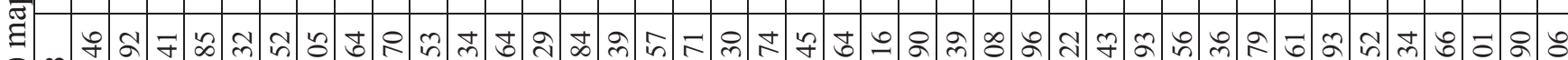

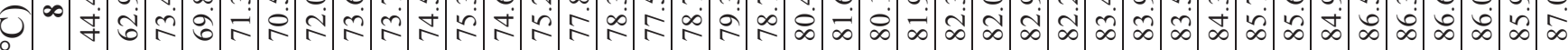

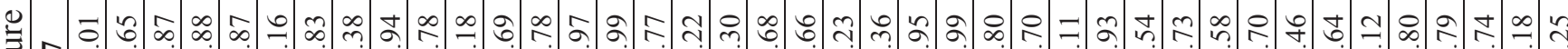

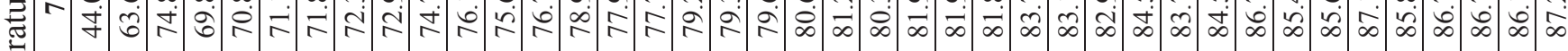

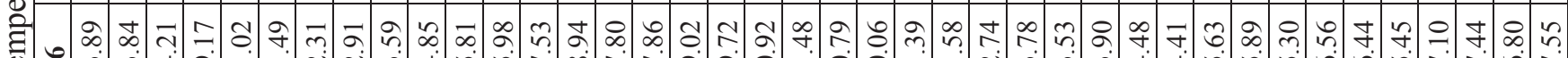

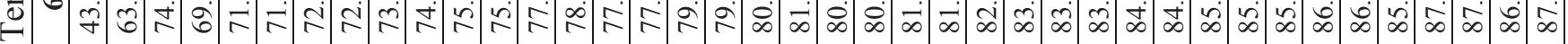
ปิ n'

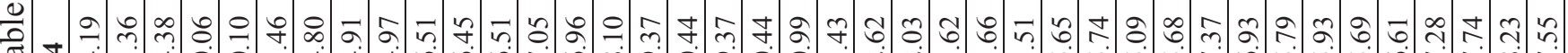

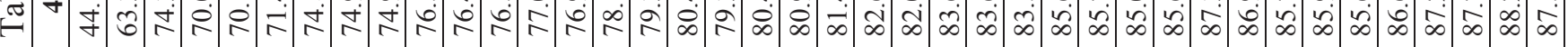

m $\infty$ రి

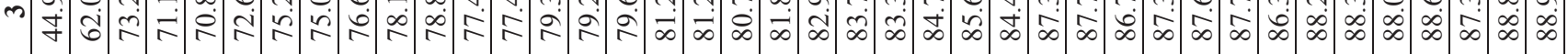

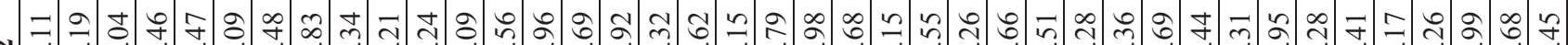

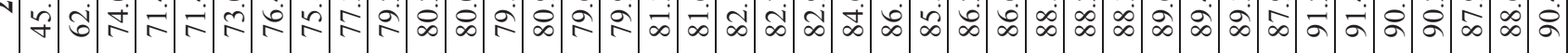
$-\infty$ -

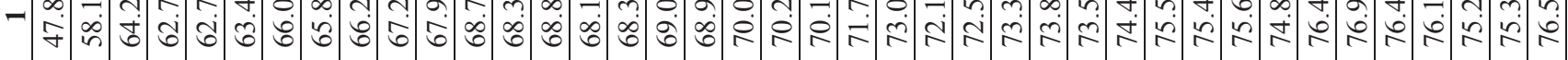
- n 官 
Im m

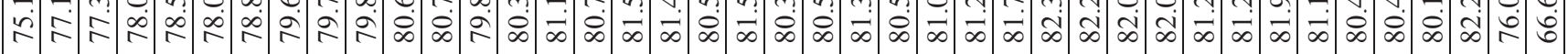

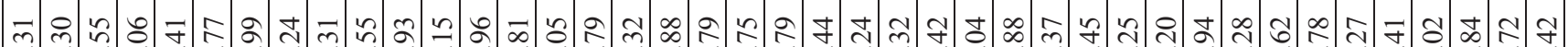

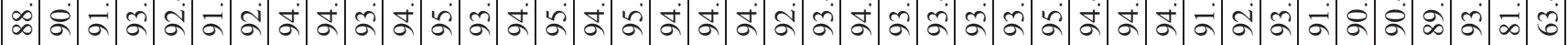

ชำ

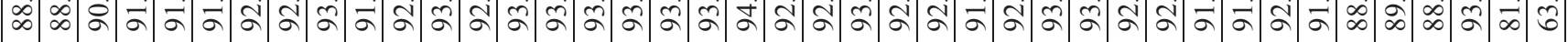

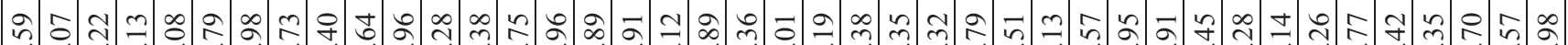

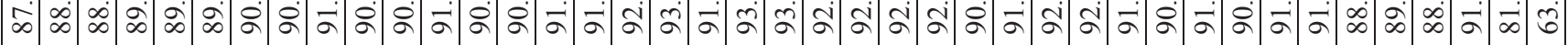

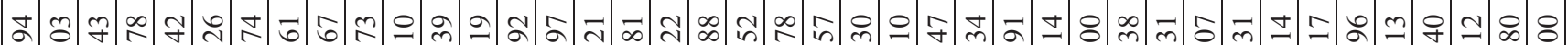

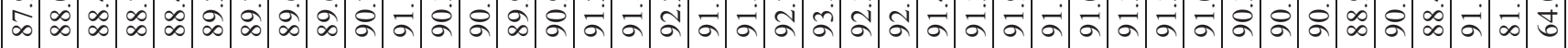

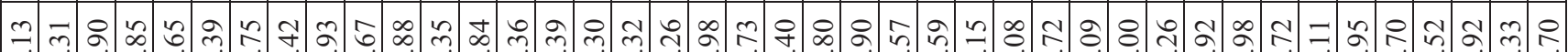

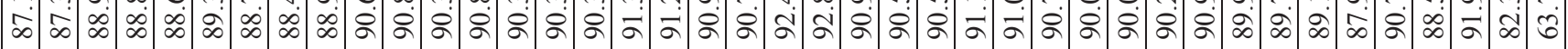

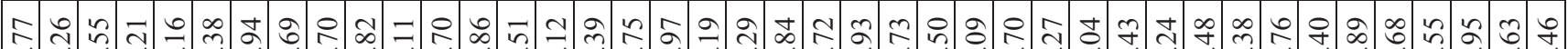

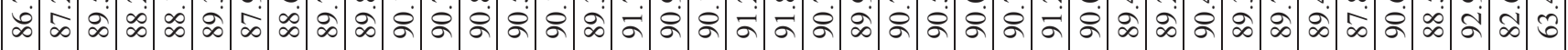

ป

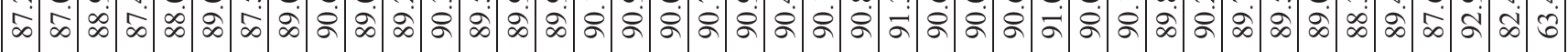

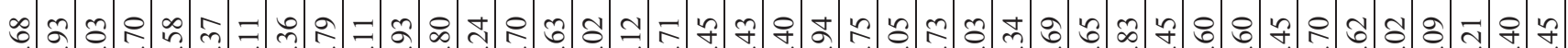

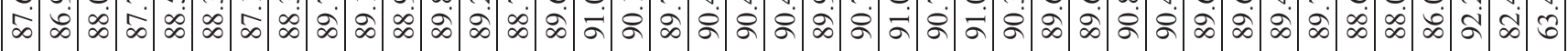

สิ่人

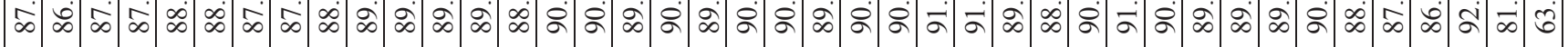

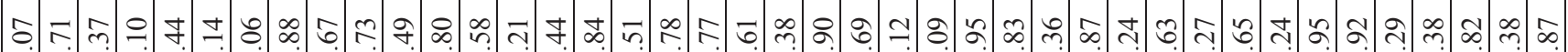

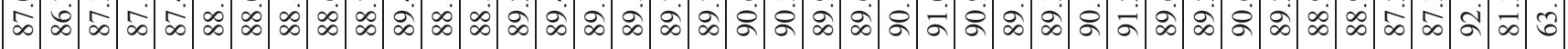

ᄀ

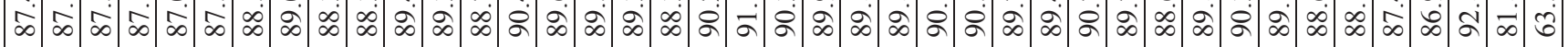

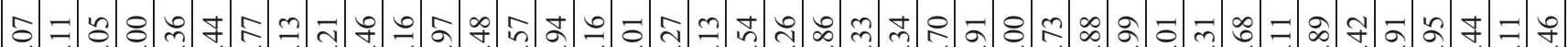

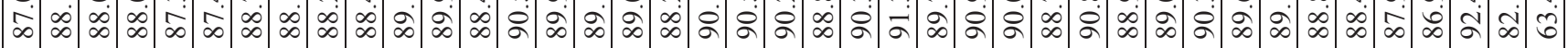

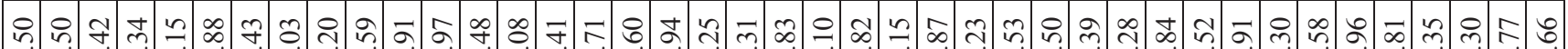

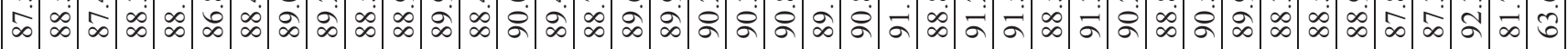

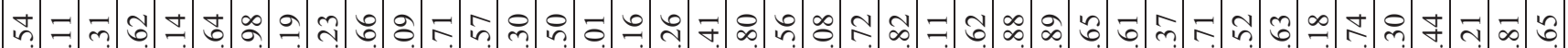

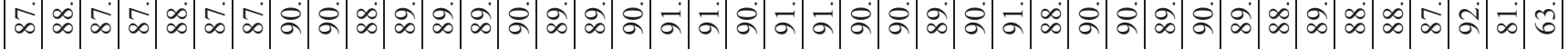

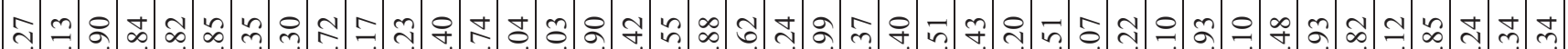

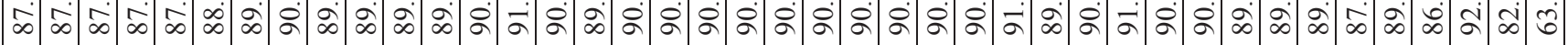

ป

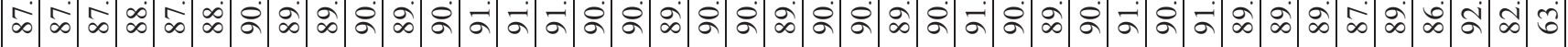

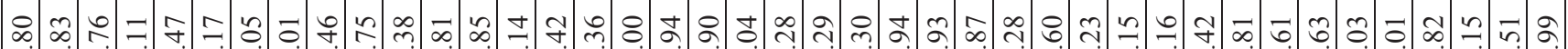

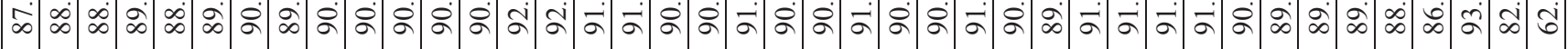

읯ำ

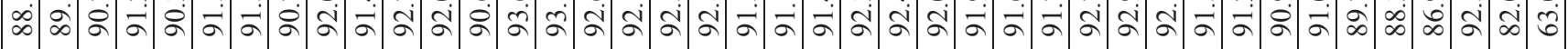

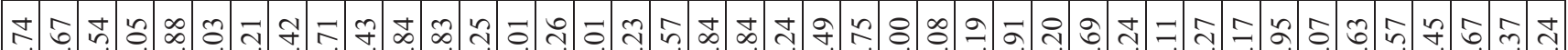

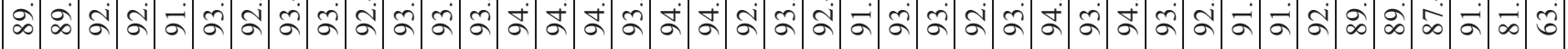

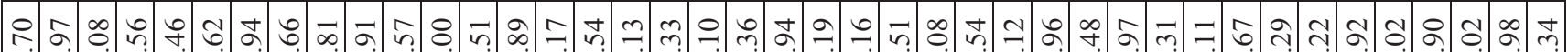

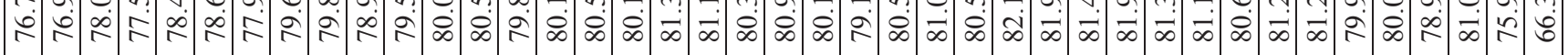




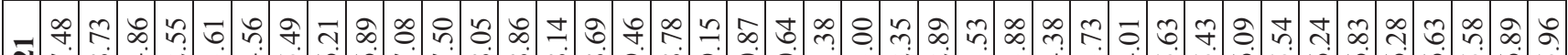

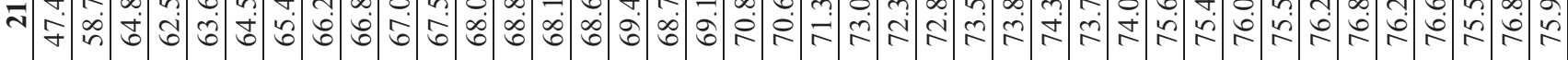

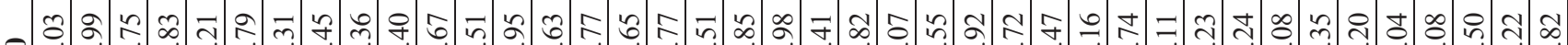

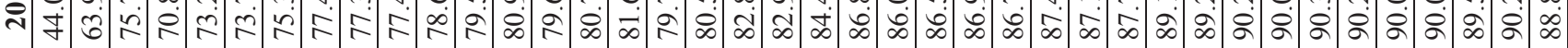

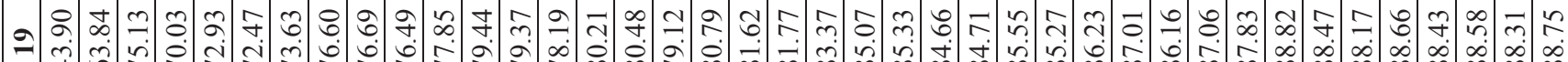
r)

$\infty$ ○े

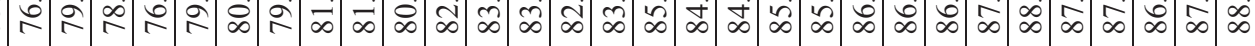

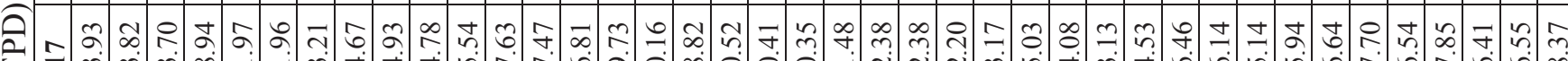

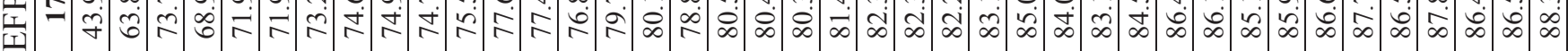

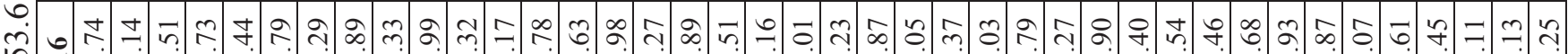

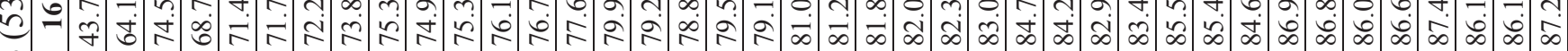

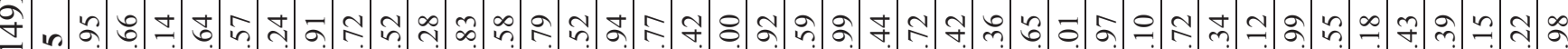
U

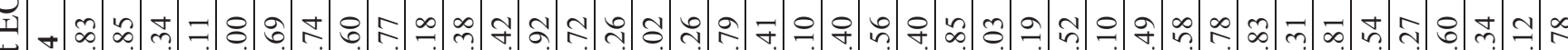

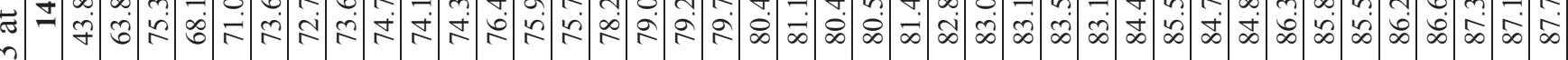
.

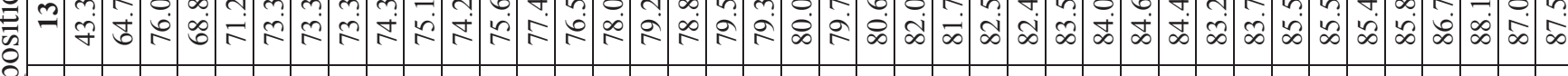

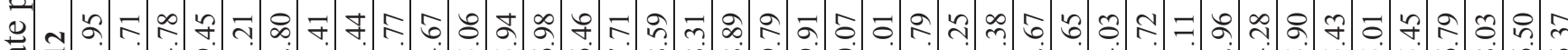

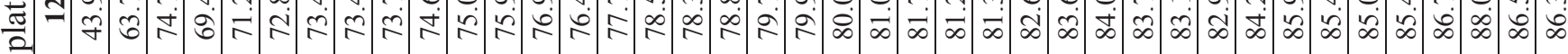

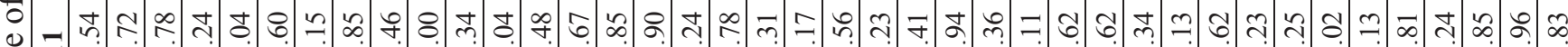

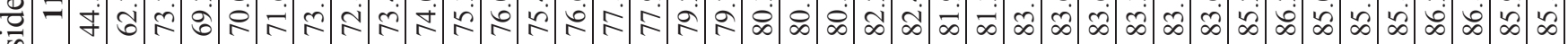

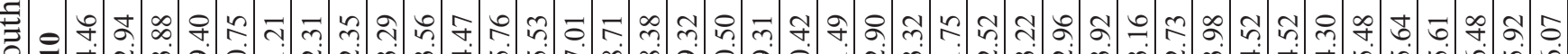

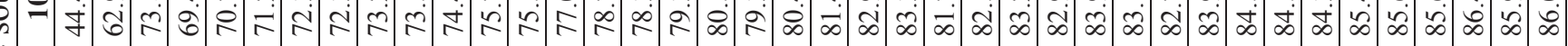

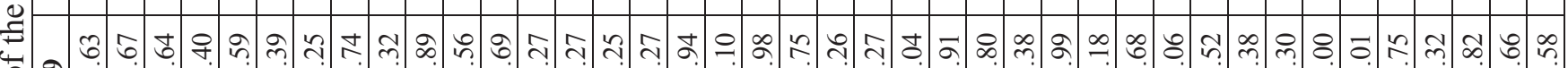

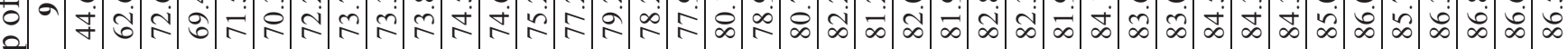

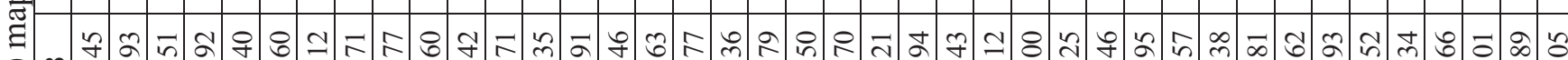

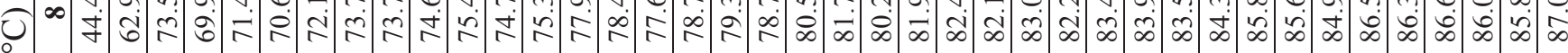

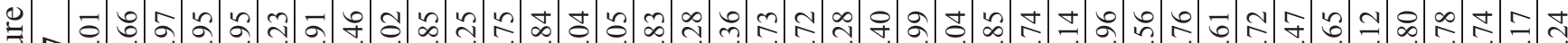

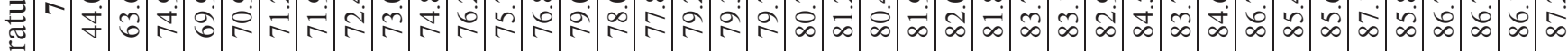
节 光 ๑)

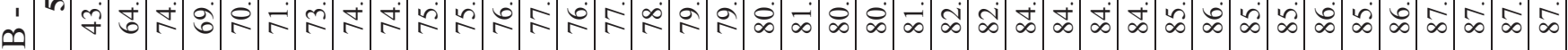

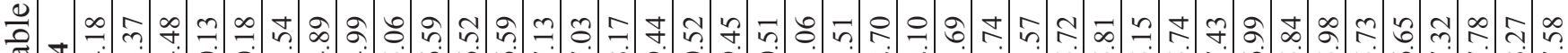

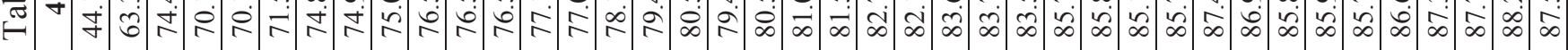

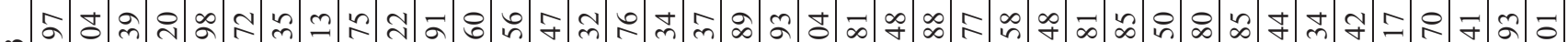

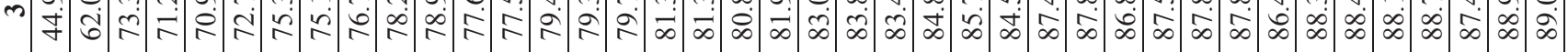

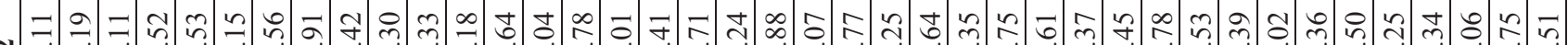

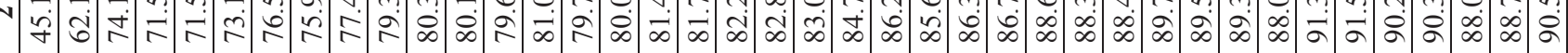
-

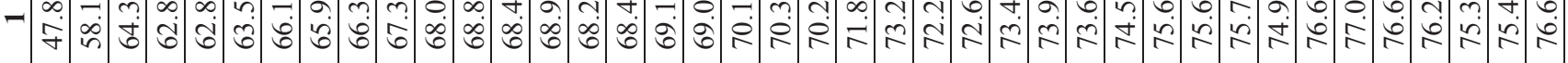

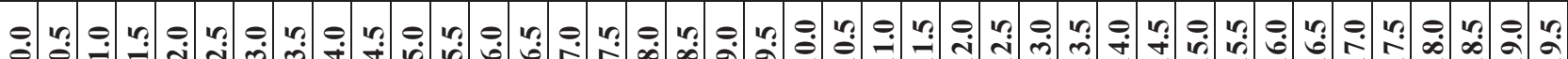


สิ สิ

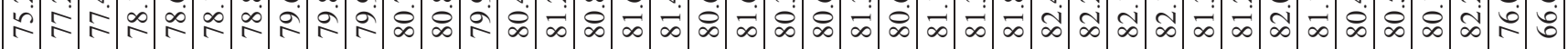
m

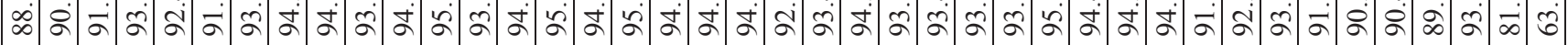

ஜ 6 응의

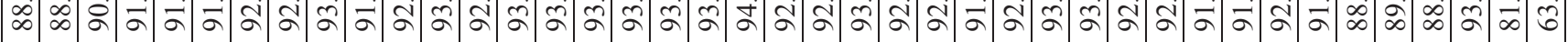

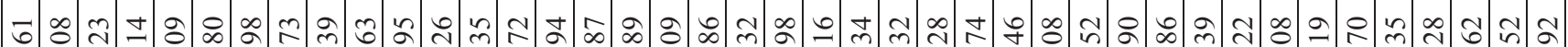

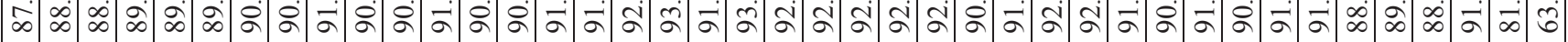

๙

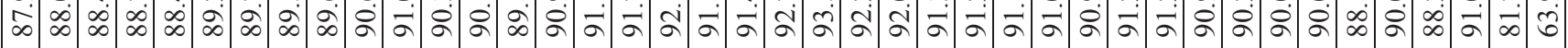

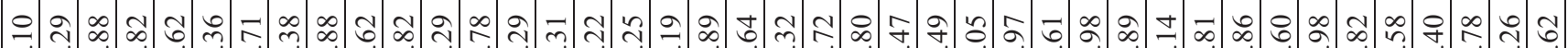

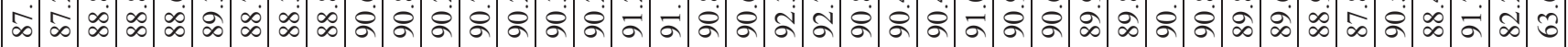

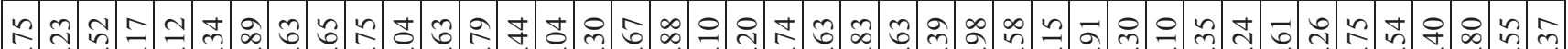

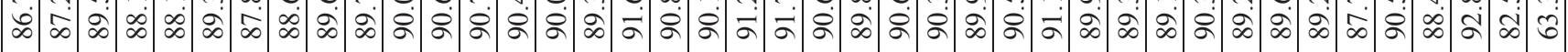

สิ

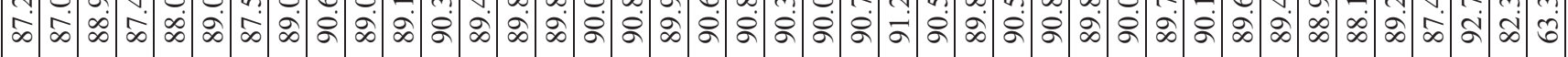

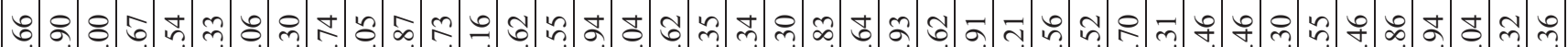

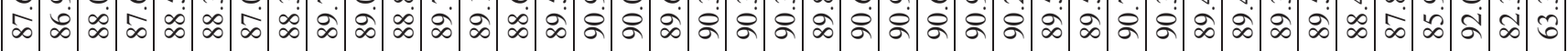

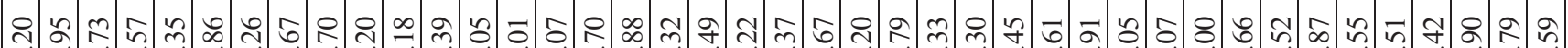

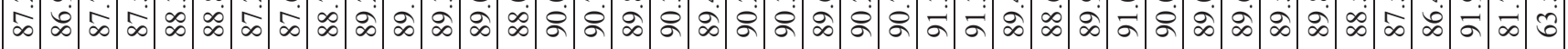

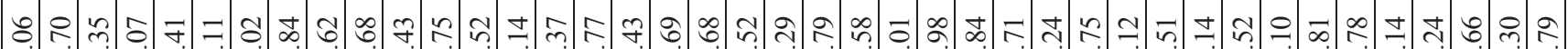

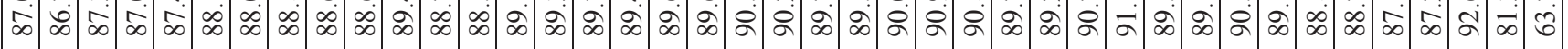

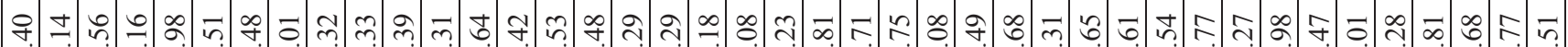

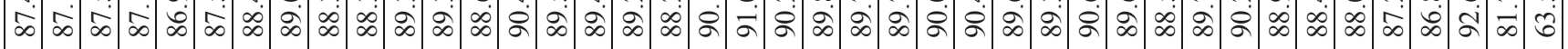

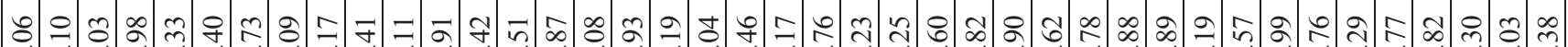

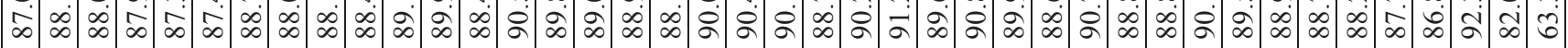
子小

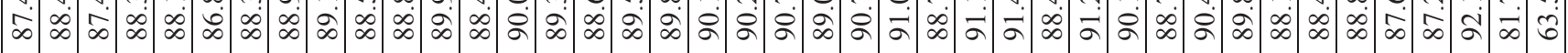
กิ

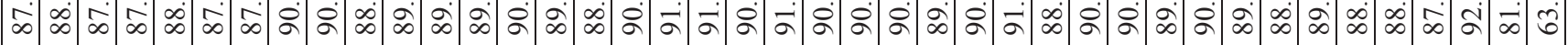
స్ ᄀ

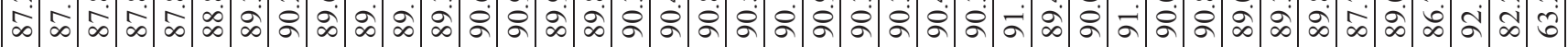
ป

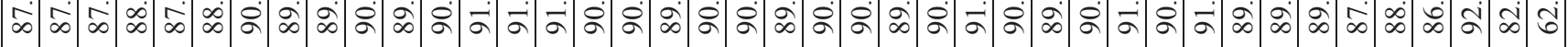

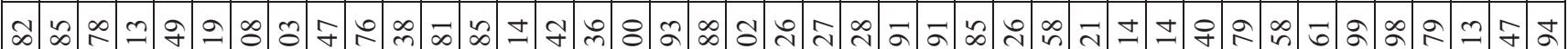

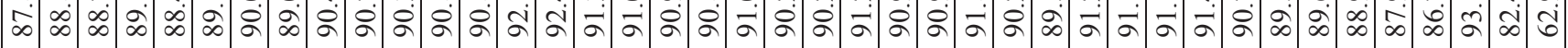

$\infty$ 눙

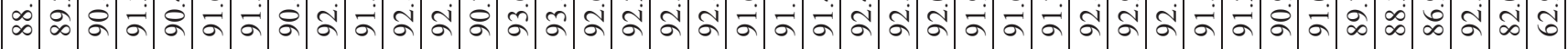

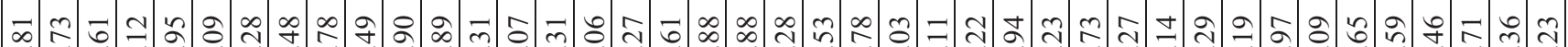

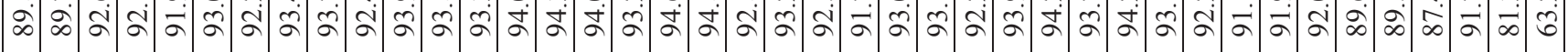

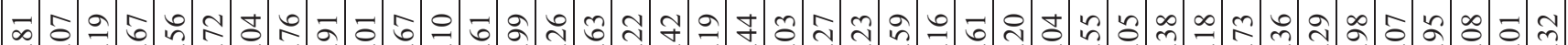

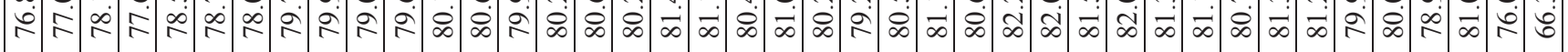




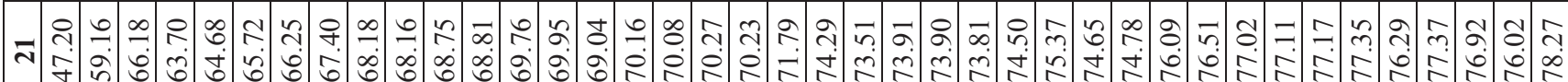

กิ

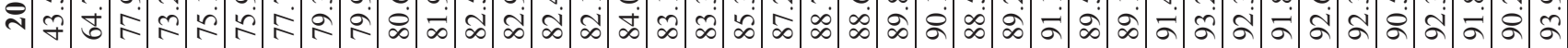

$=$ -

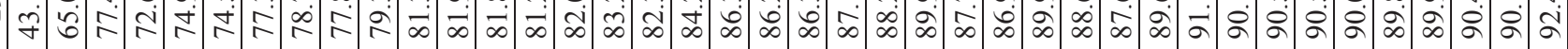

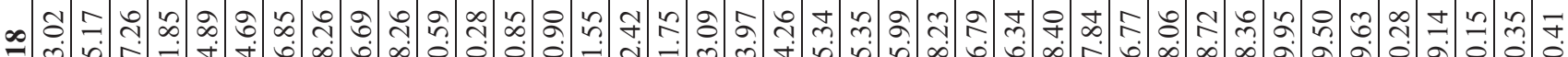
fु

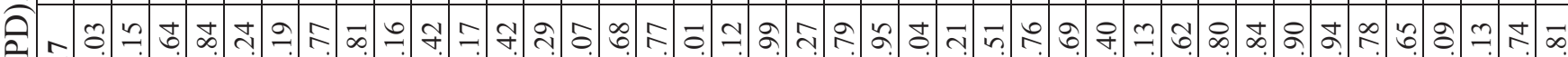

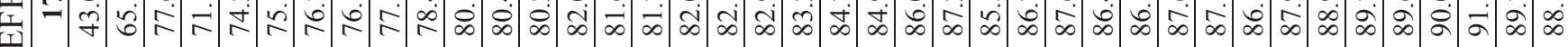

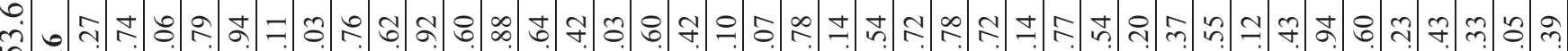

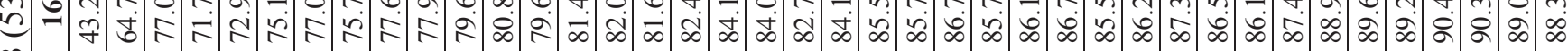

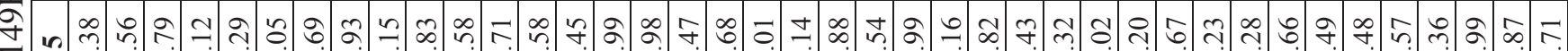

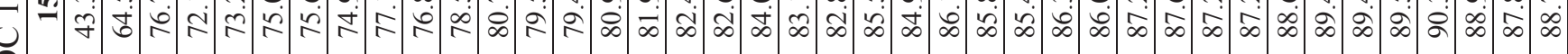

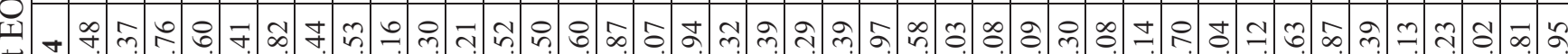

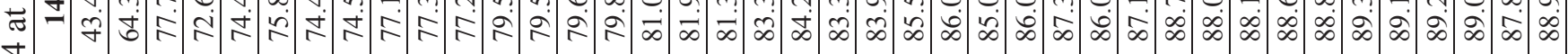

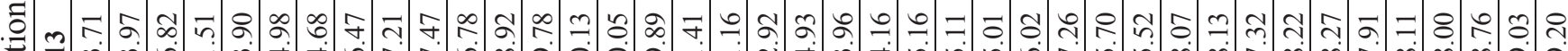

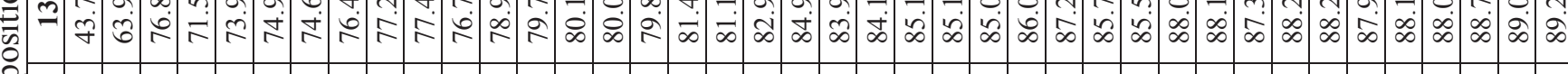

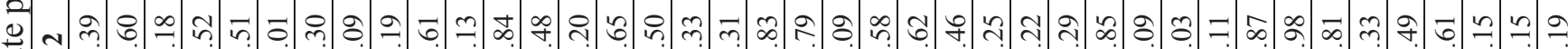

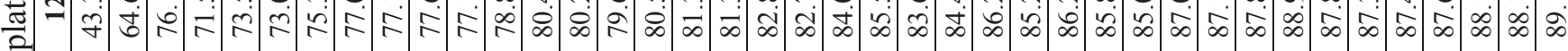

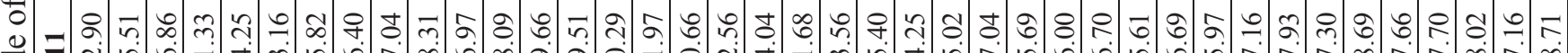

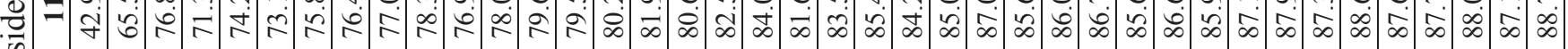
馬

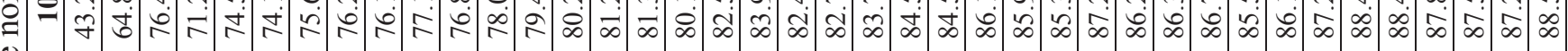

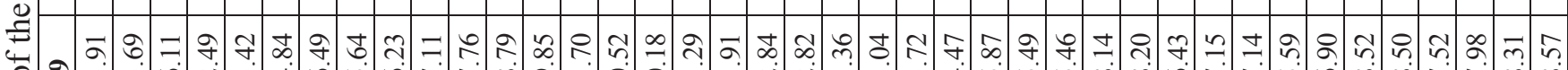

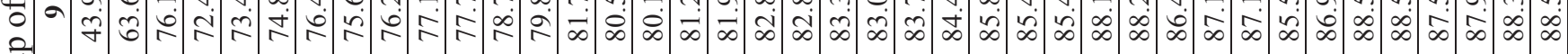
‡

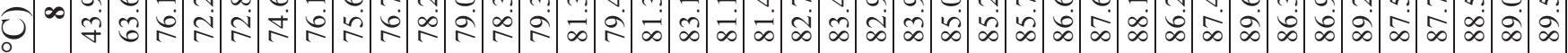

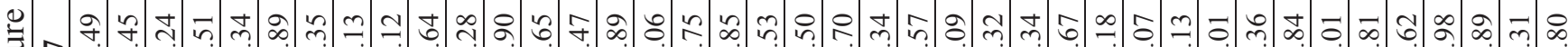

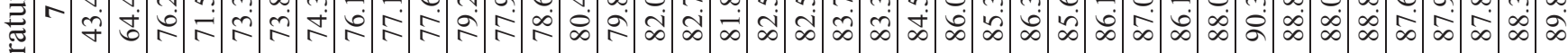
๑

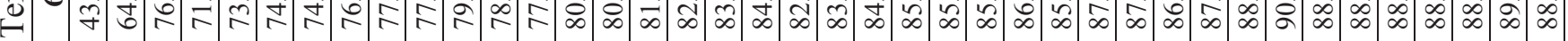
m ๆ

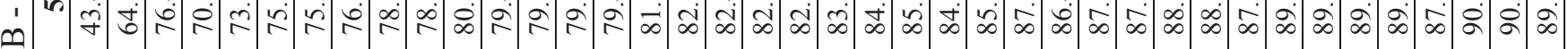
光-

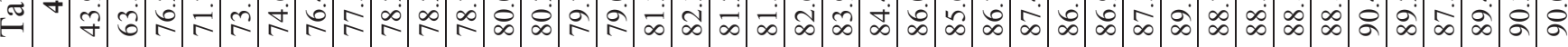

m ₹ f

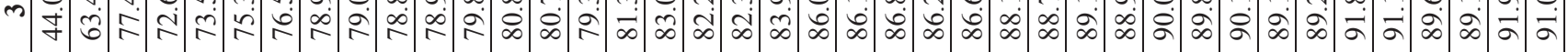
ᄀ

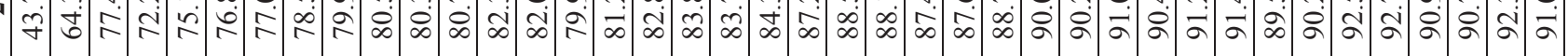

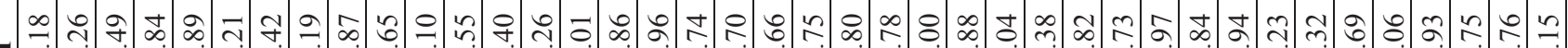

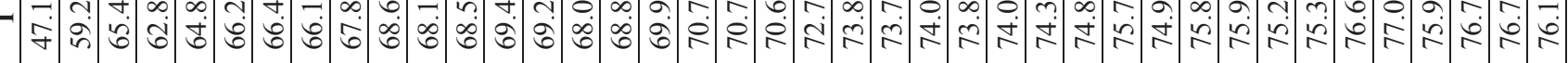
- n

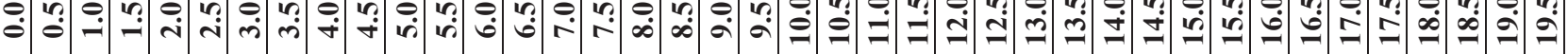


군

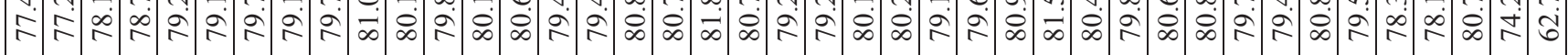
๑ t)

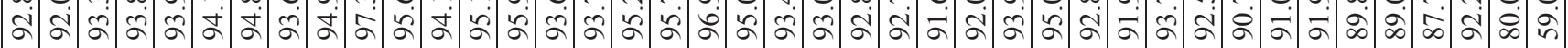

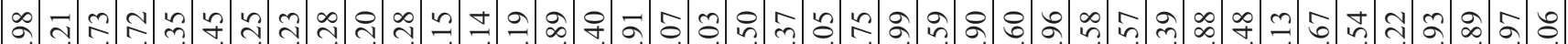

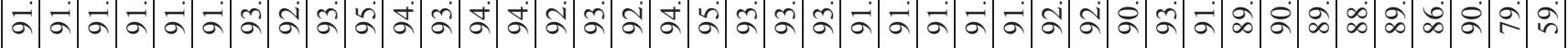

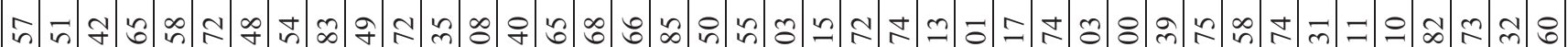

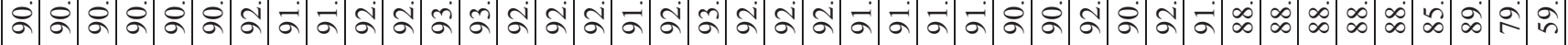

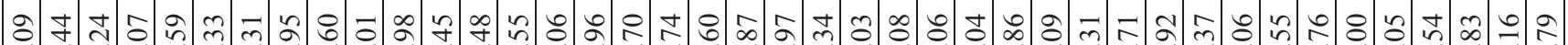

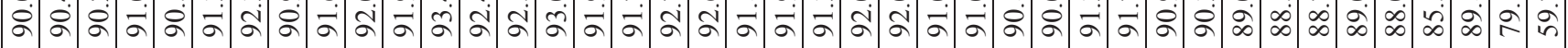

읭 b to

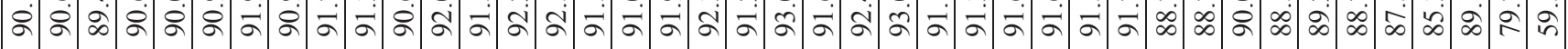

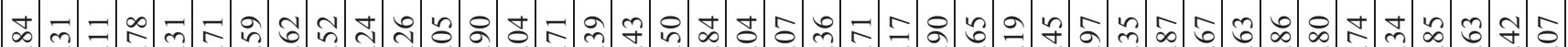

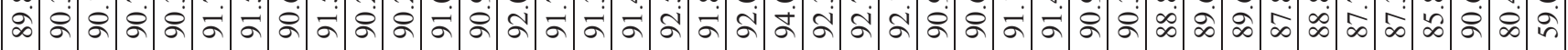

m) $=$ ปี

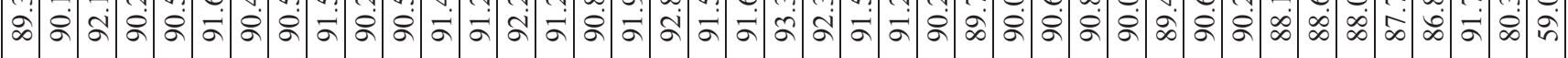

กุ่

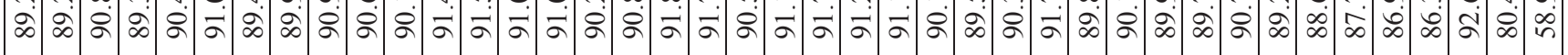

ㅊำ

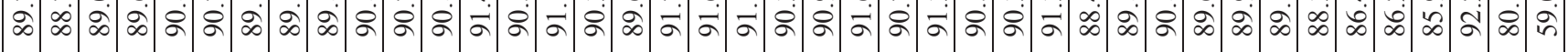

$\infty$ ก

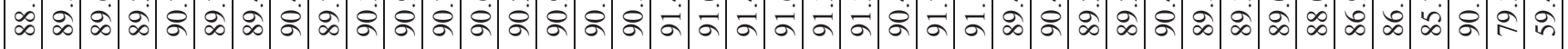

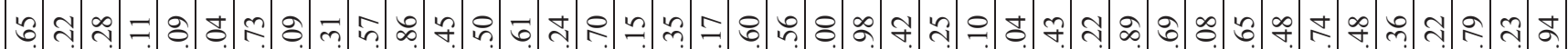

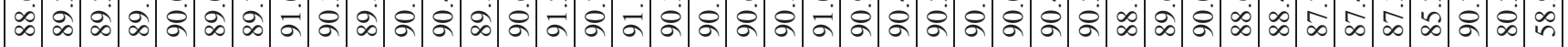

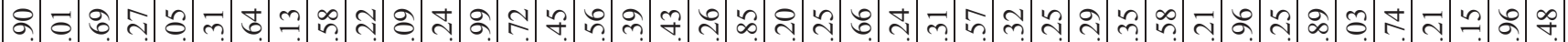

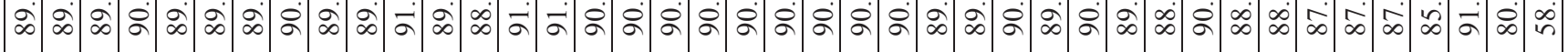

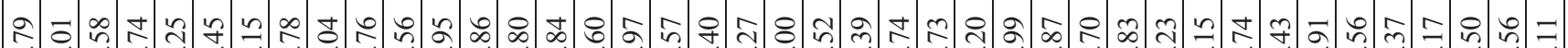

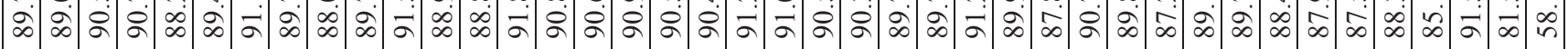

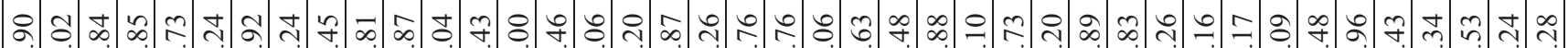

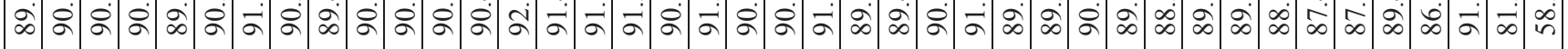

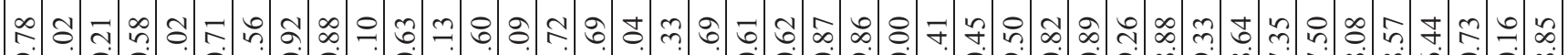

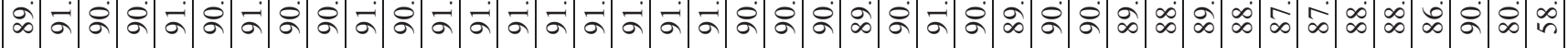
तิ

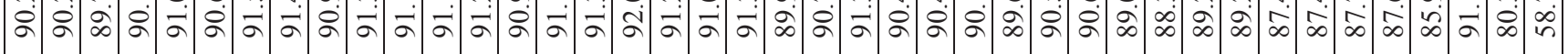

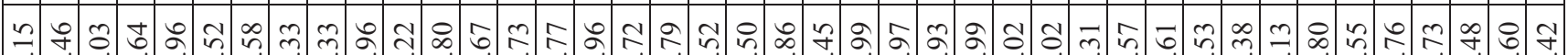

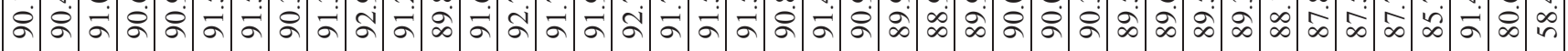

๙

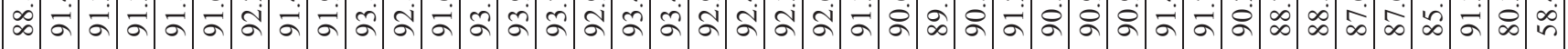

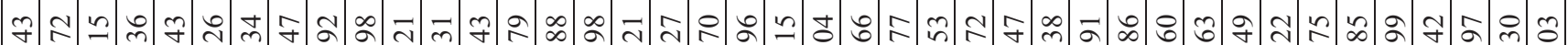

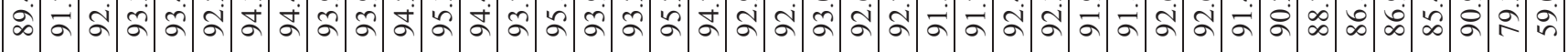

๘)

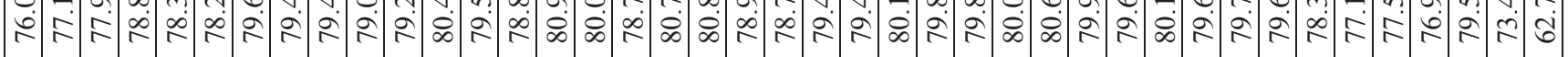




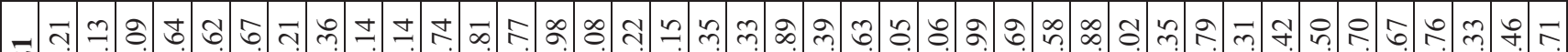

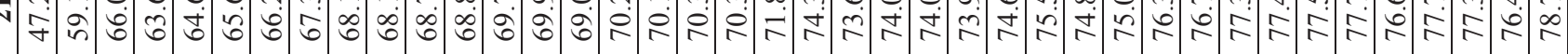

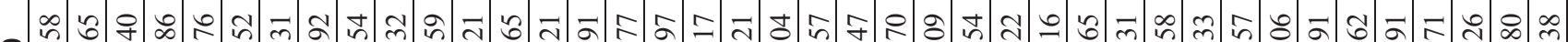
సิ

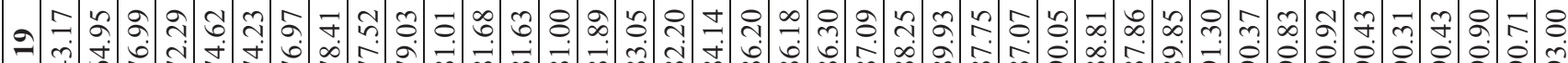

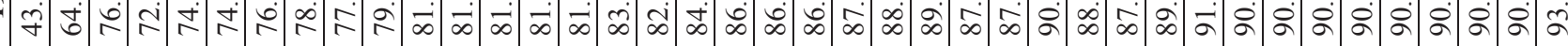

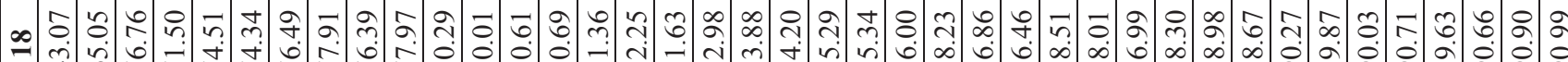
守

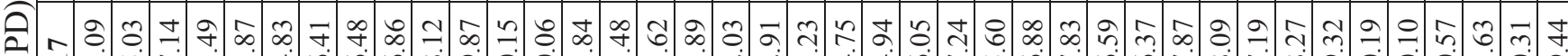

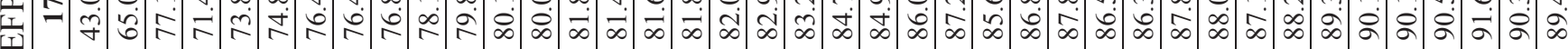

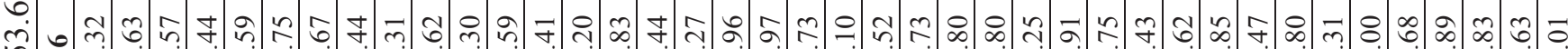

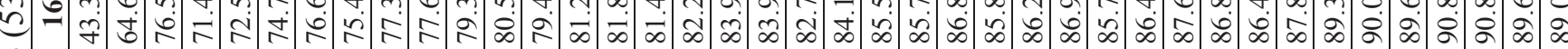
守

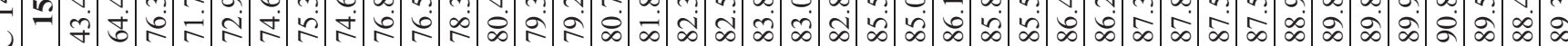
I

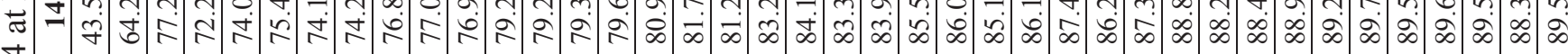
苟 일

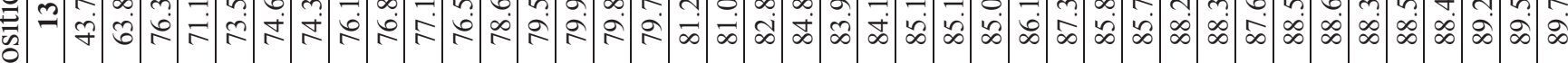

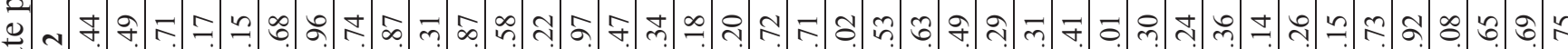

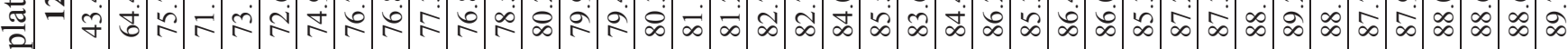
0 ஊ

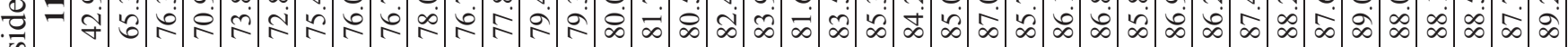

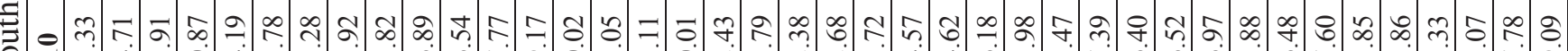

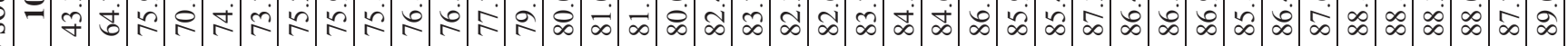
Е

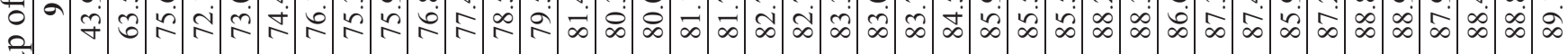

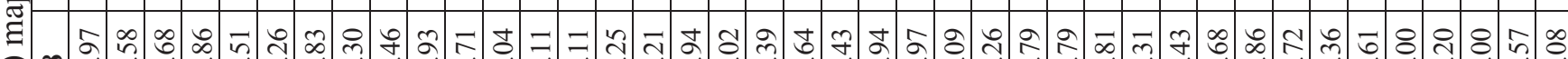

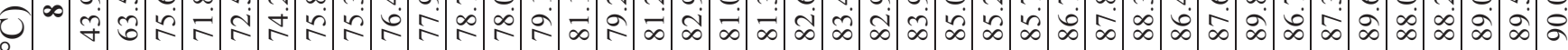
ઇ

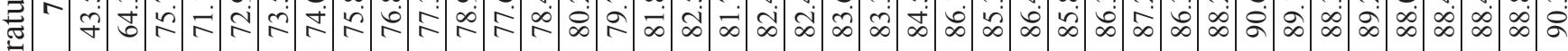

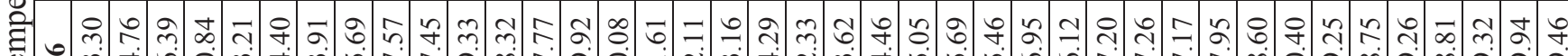
包

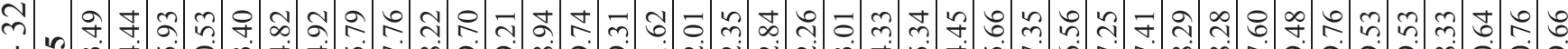

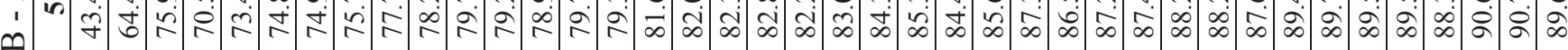

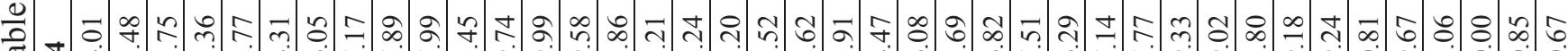

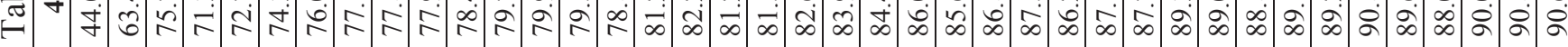

-

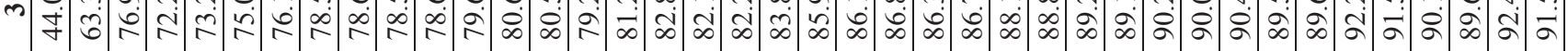

œे ț

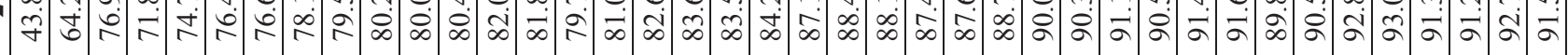

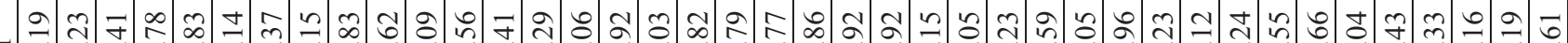

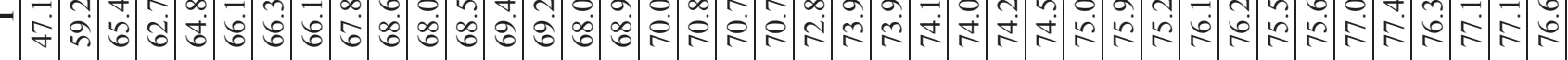
- n

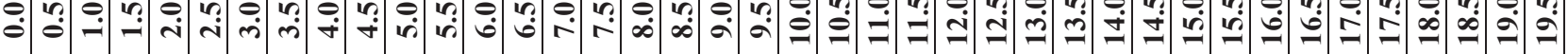




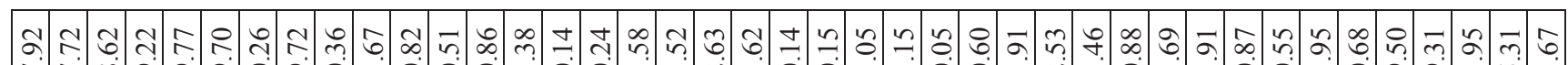

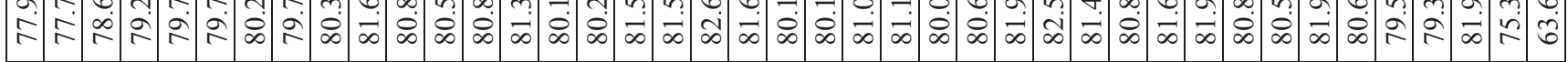
m.

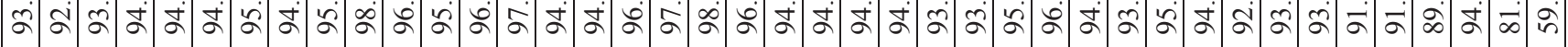

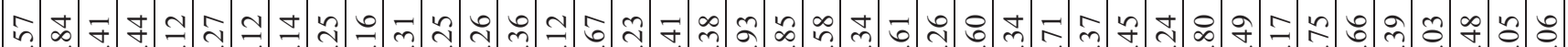

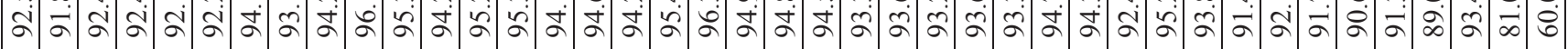

กิ = ㄱำ

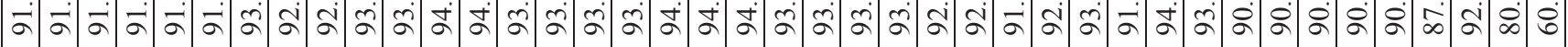

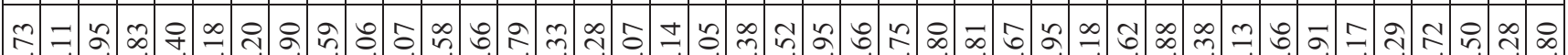

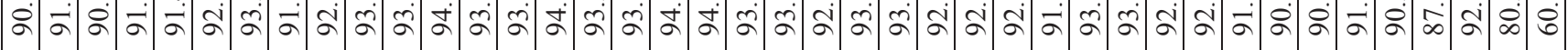

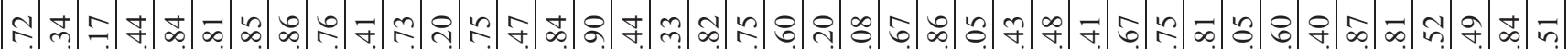

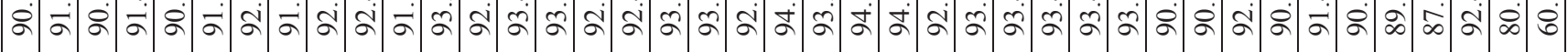

f.

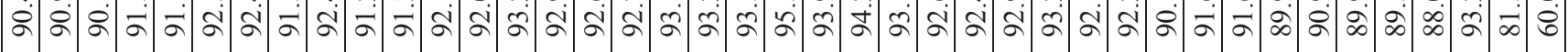

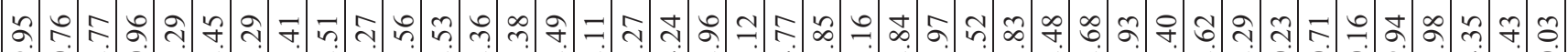

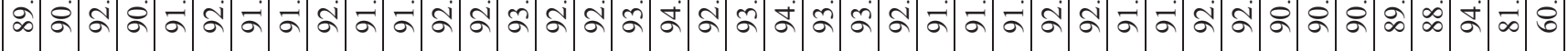

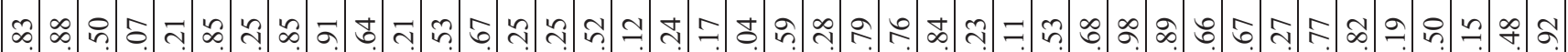

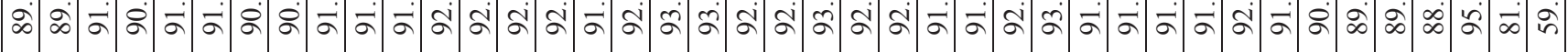

m

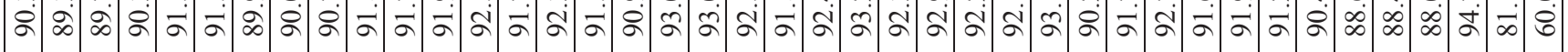

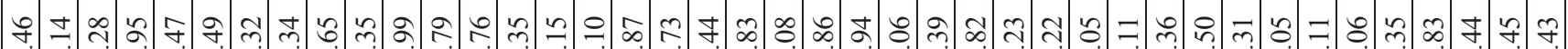

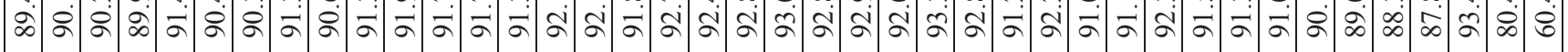

กุ

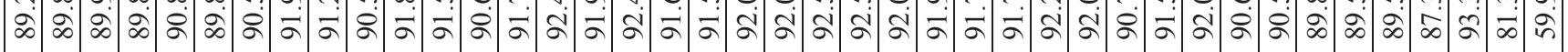

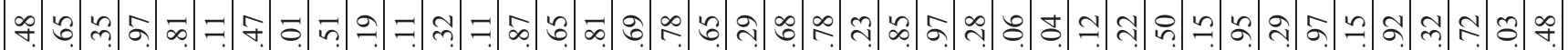

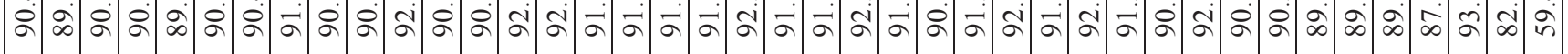

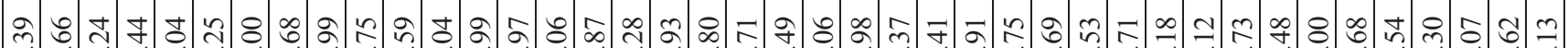

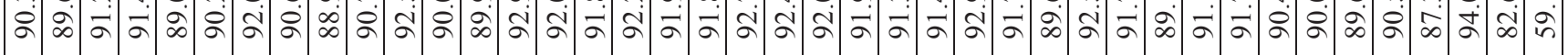
пี

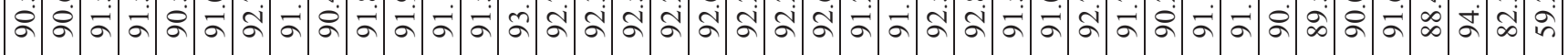
ใน

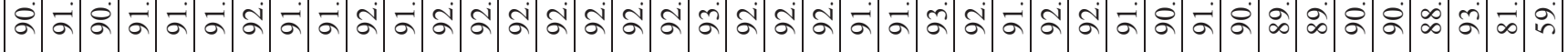

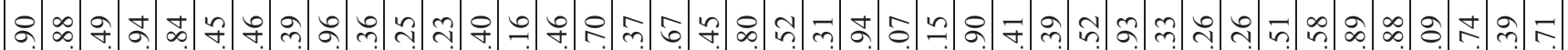

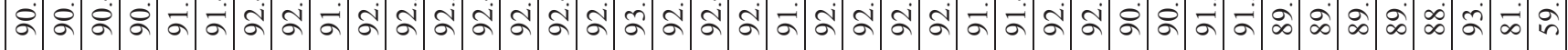

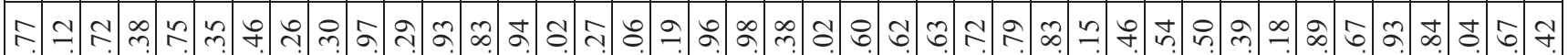

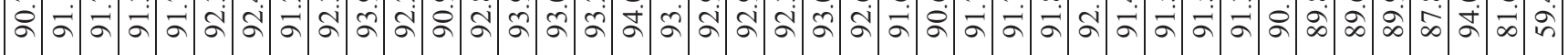
ศ. ช. ఫ

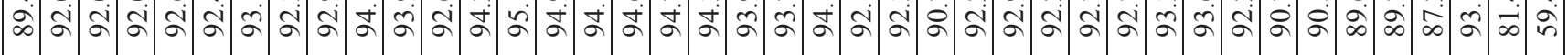

ชุ ฉ nీ

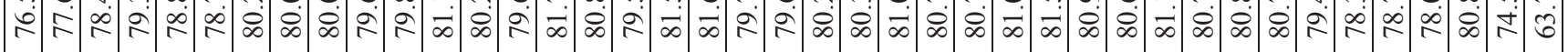
竎 


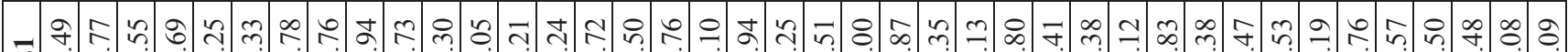

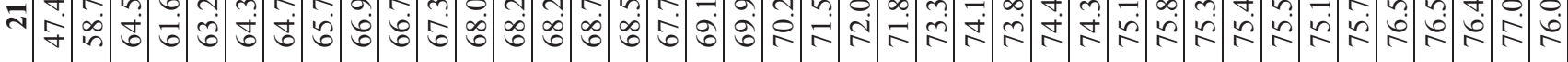
ஸी

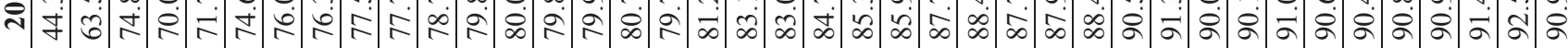

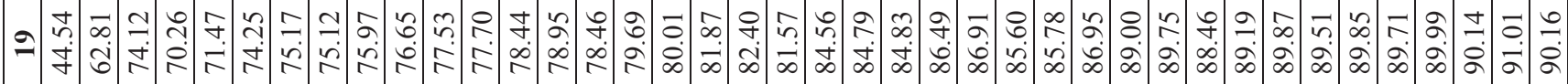

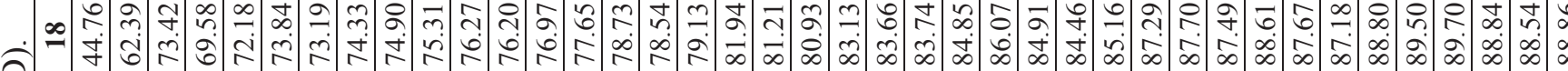

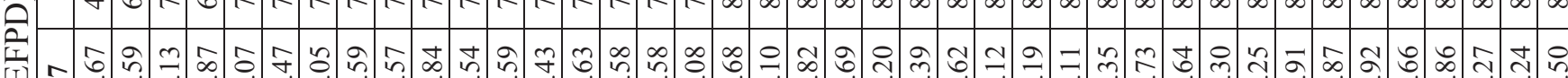

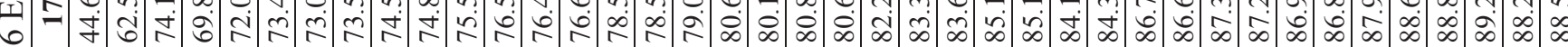

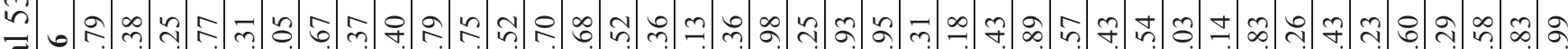
吾

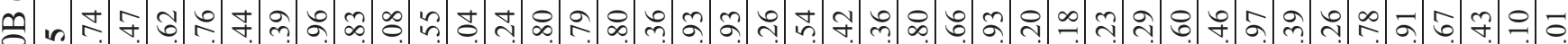

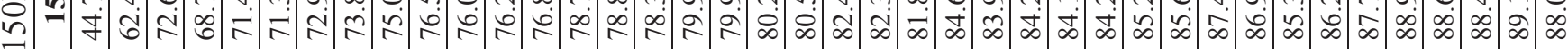

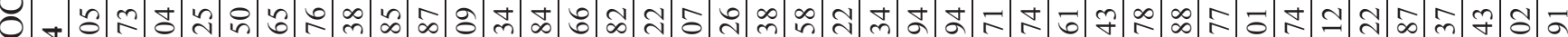

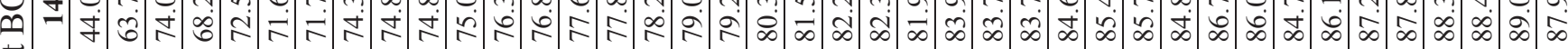
- m

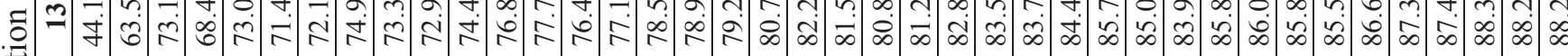
ح

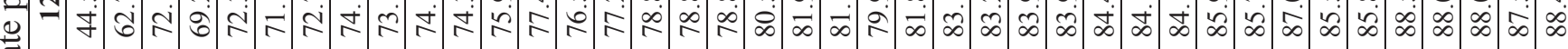

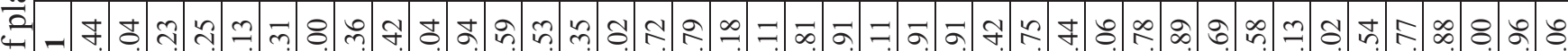

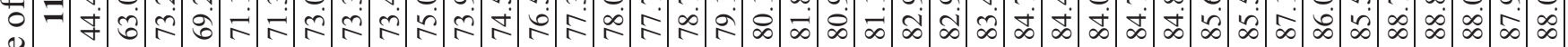

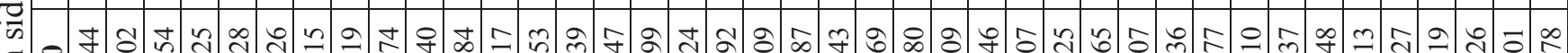

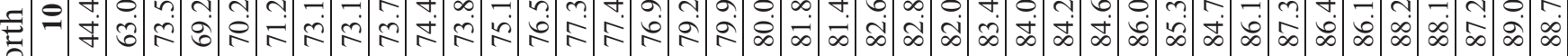
ฮ) 艺

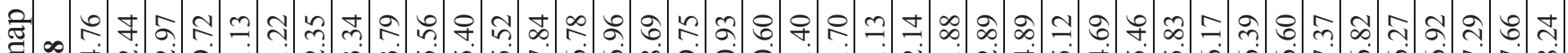

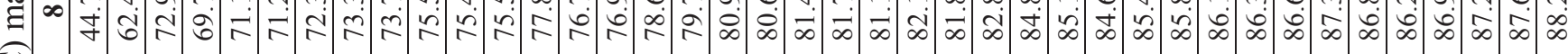

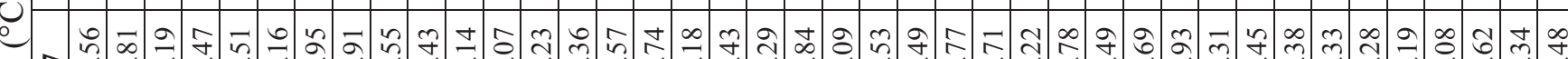

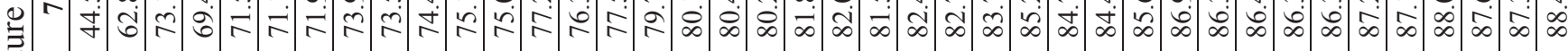
矛

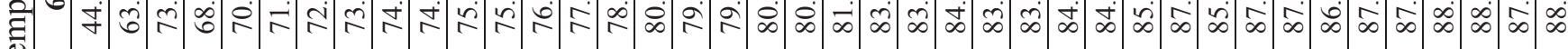

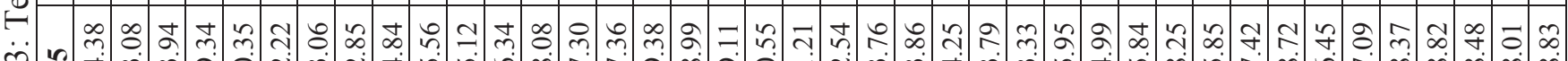

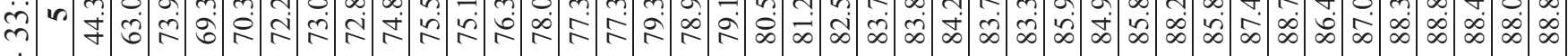

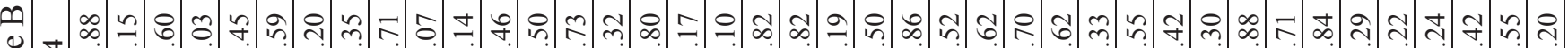

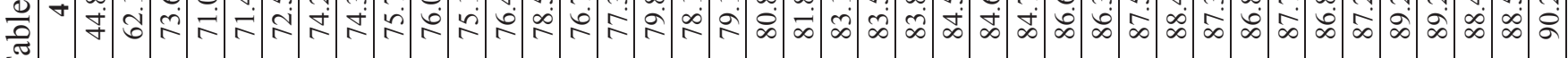

F m $\quad$ t -

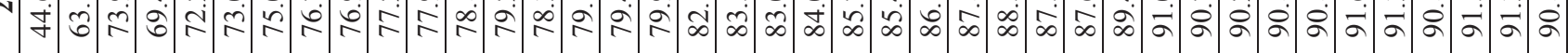
- 守 in $\because$ 急 


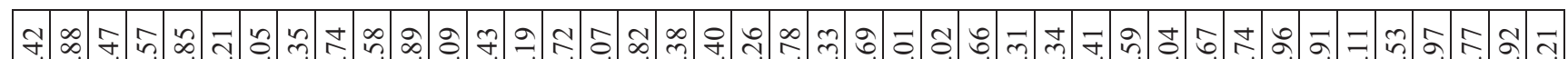

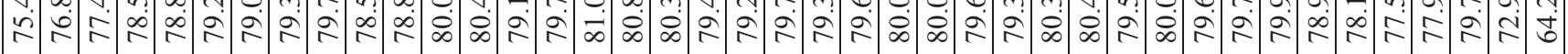

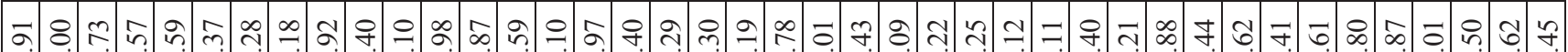

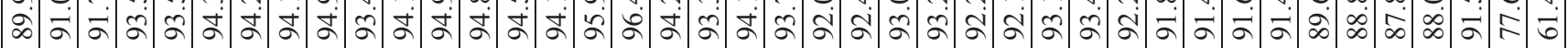

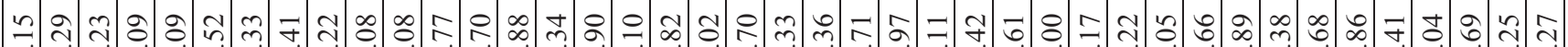

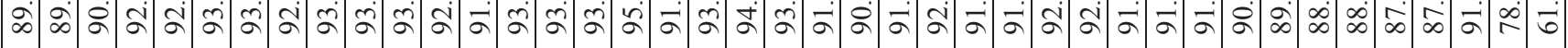
ఫ車

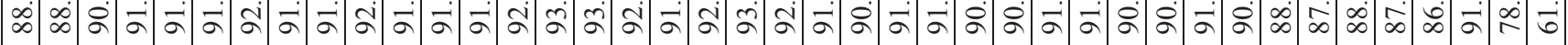

t

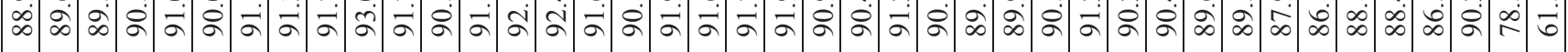

只

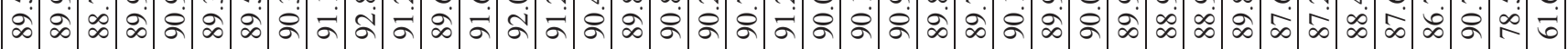

ดี

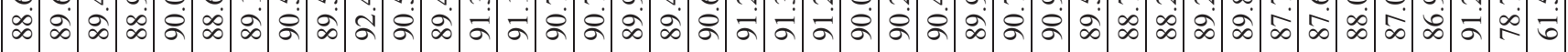

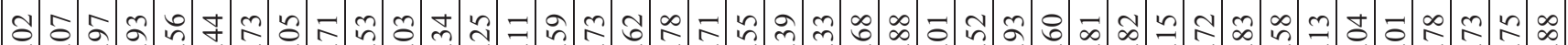

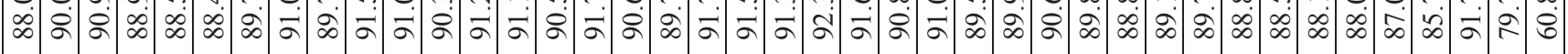

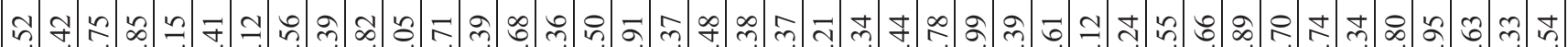

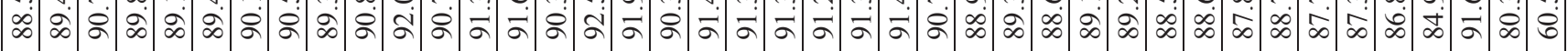

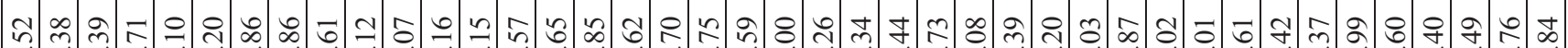

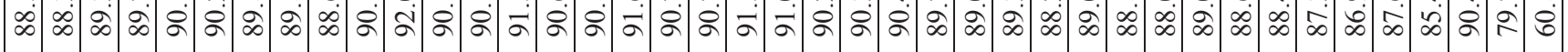

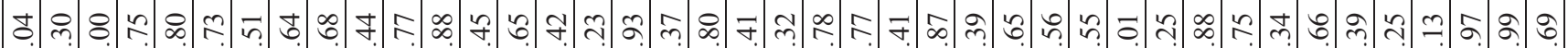

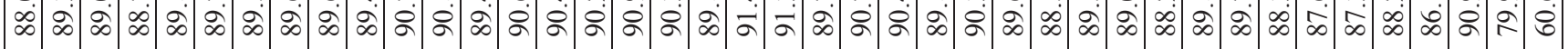

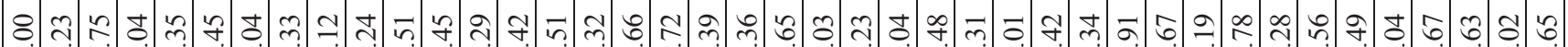

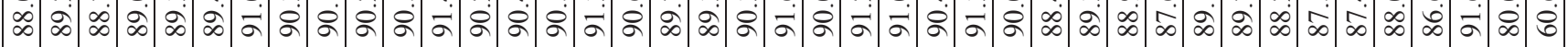

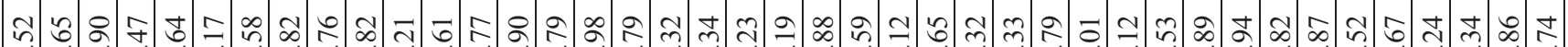

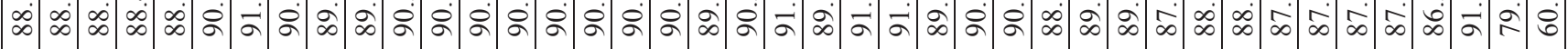
n

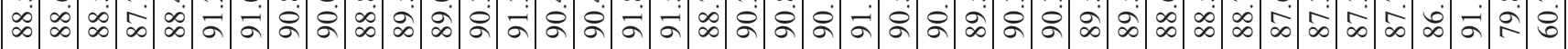

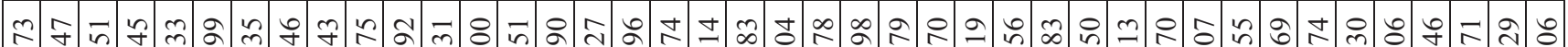

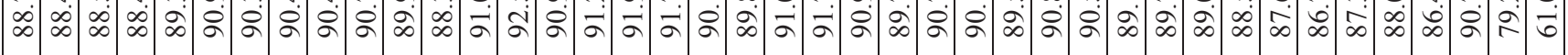

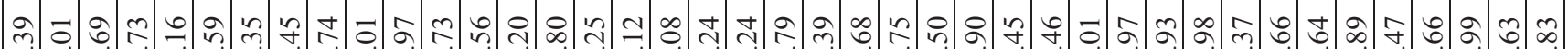

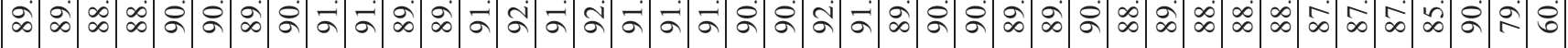

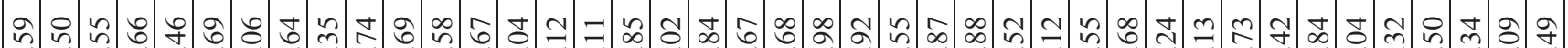

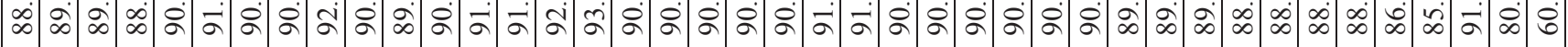
芛

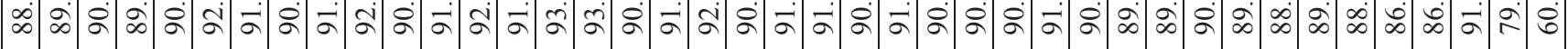

œ

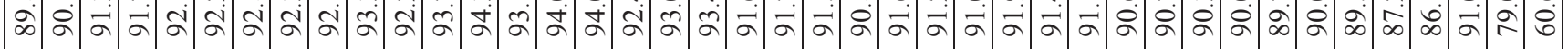

ণิ

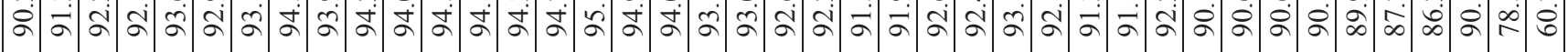
๙

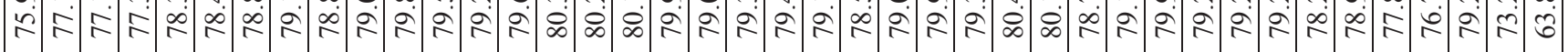




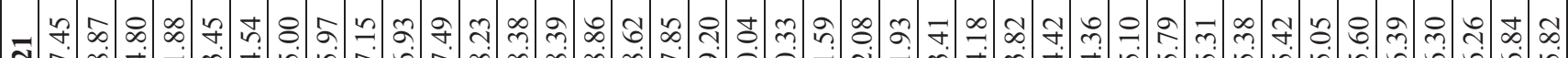

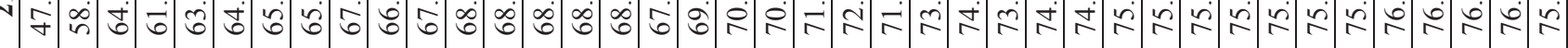

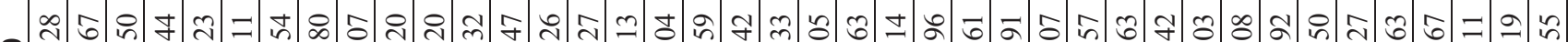

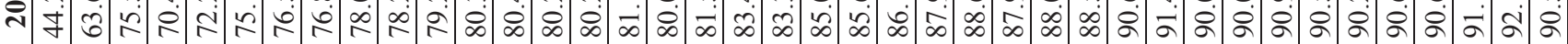

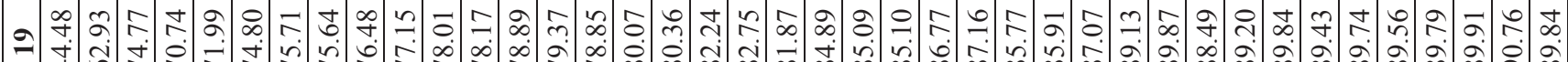

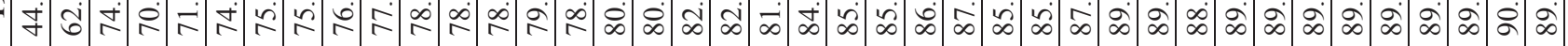

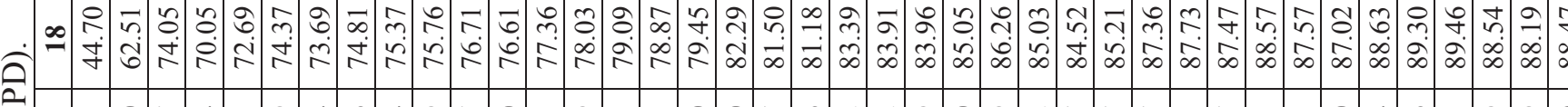

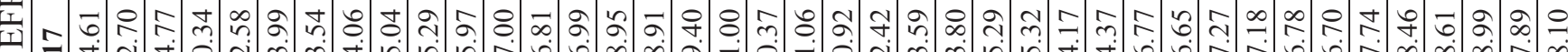

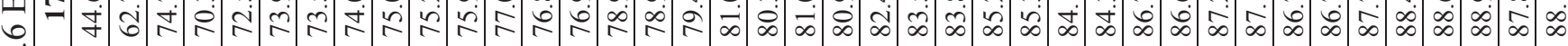

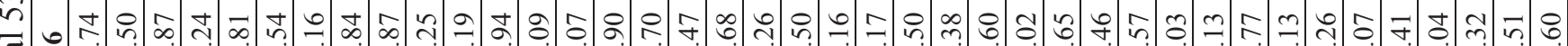

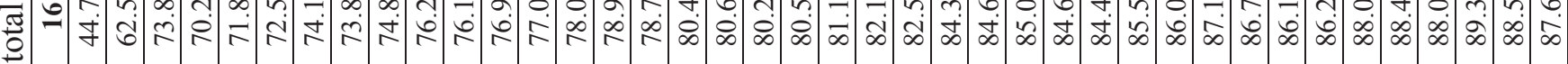
ஜ

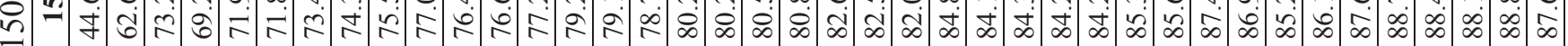
○ จ

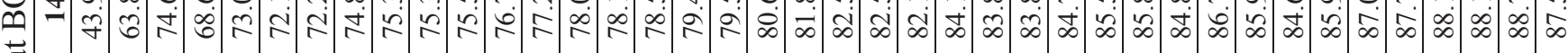

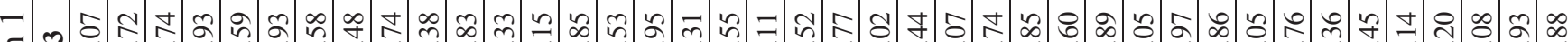

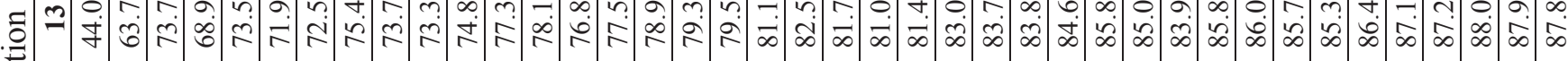

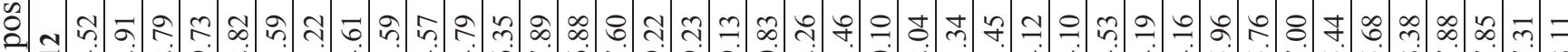
D.

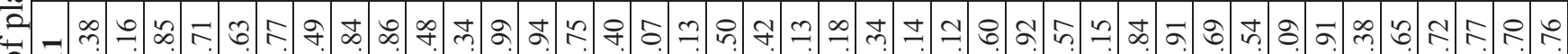

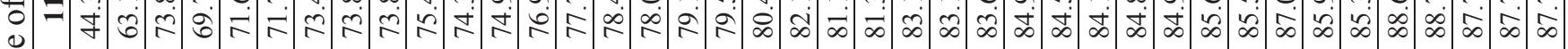

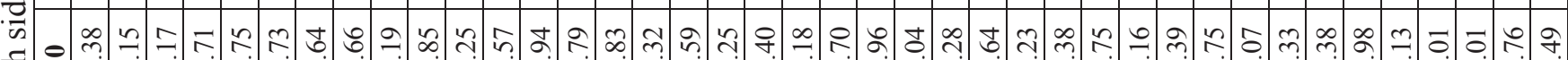

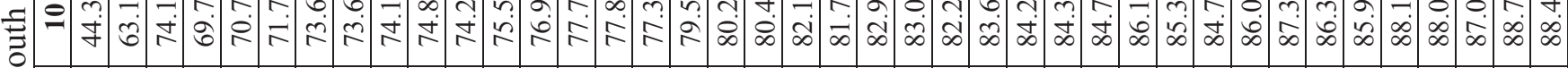
g f

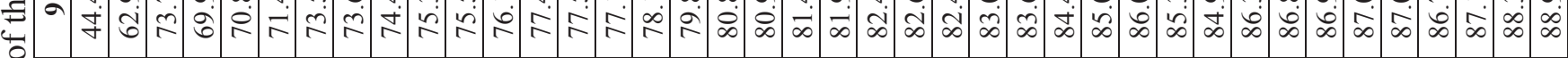
สิ $\infty$ ำ

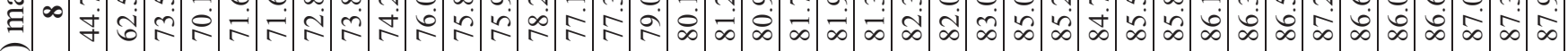

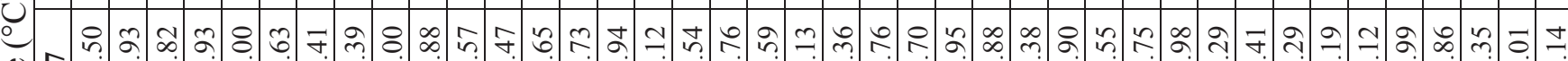
可 J

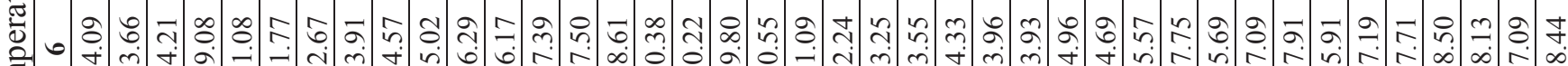

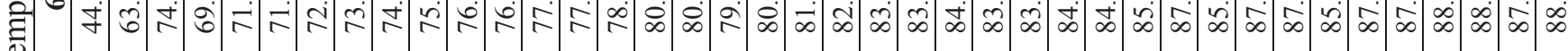

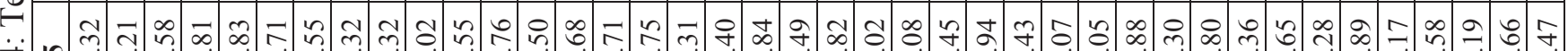

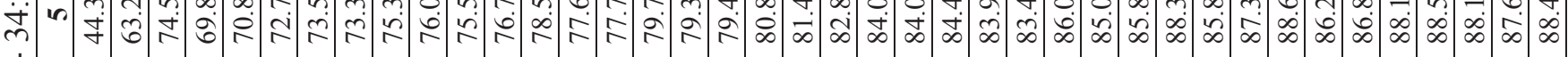

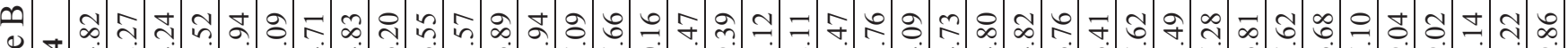

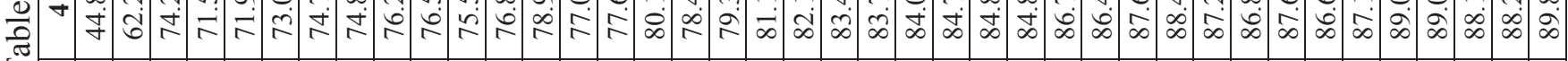

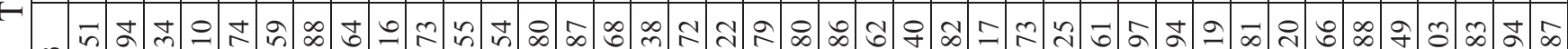

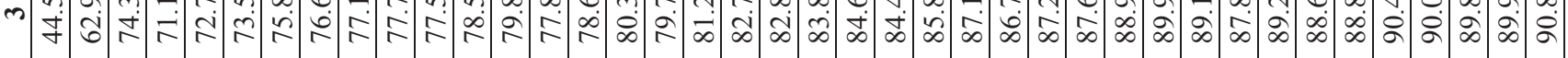

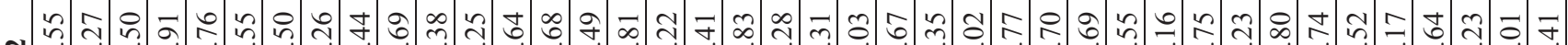
N - ప

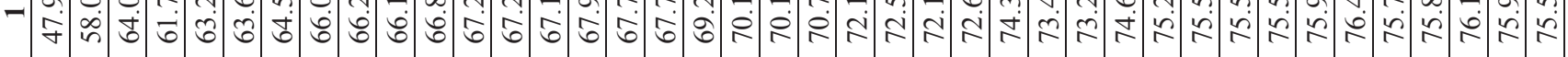

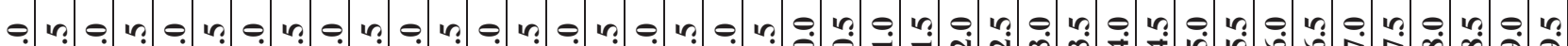

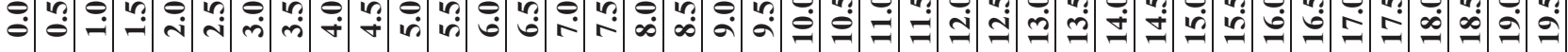




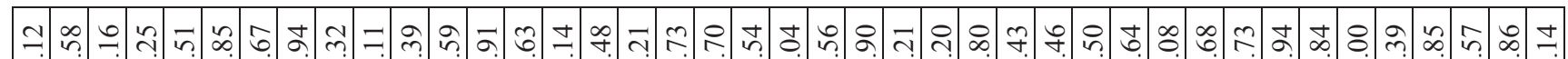

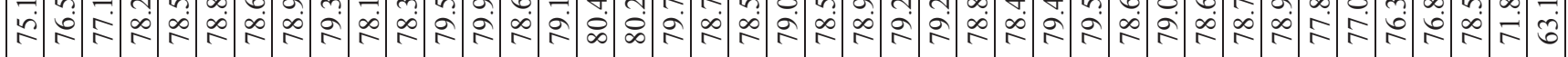

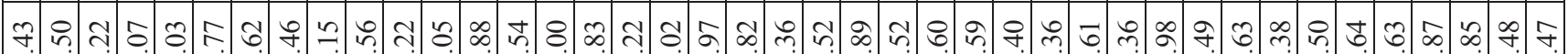

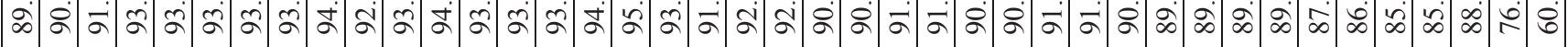

そ ฉ

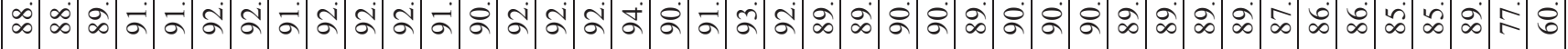

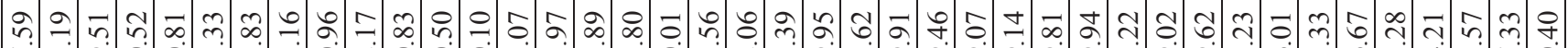

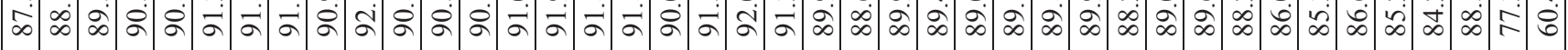

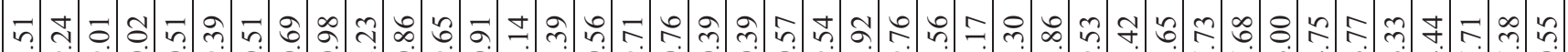

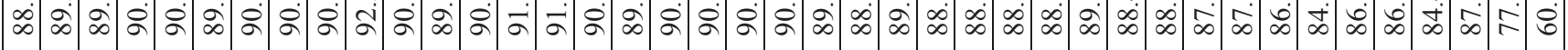

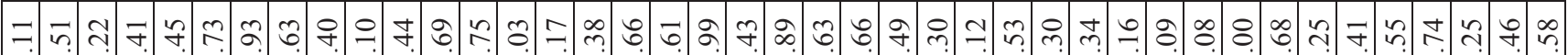

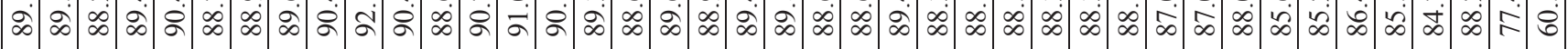
ల)

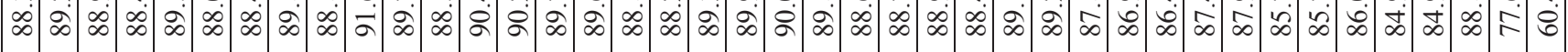

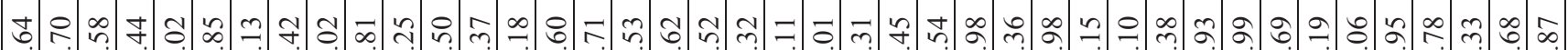

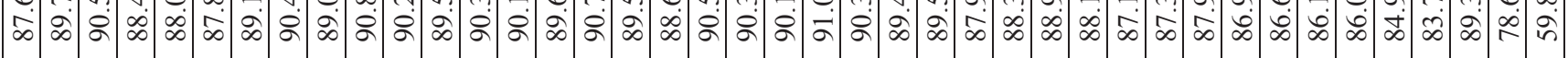

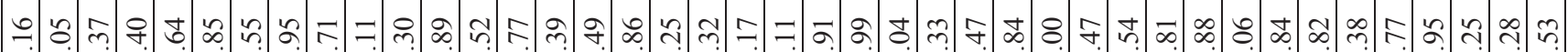

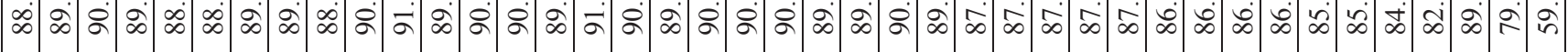

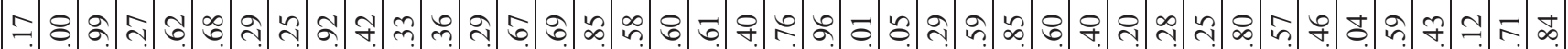

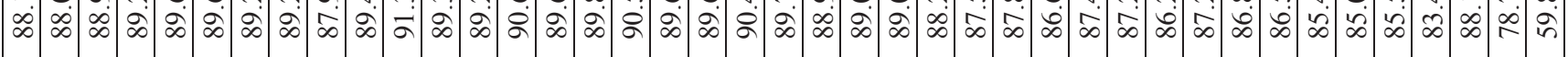

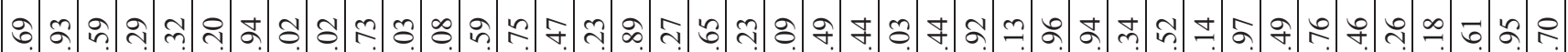

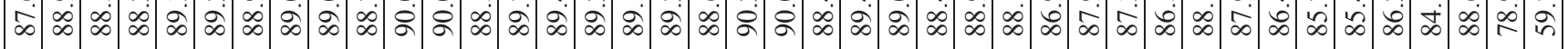

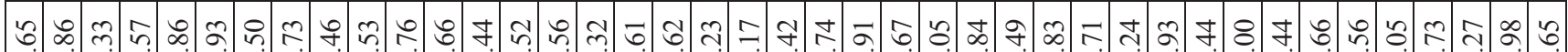

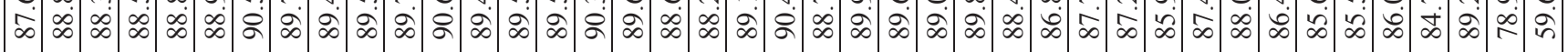

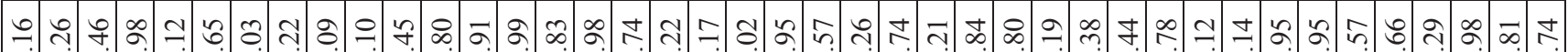

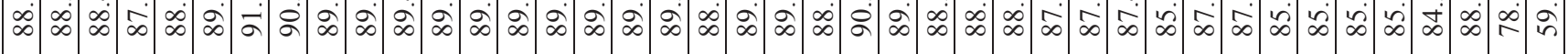
ㄱำ

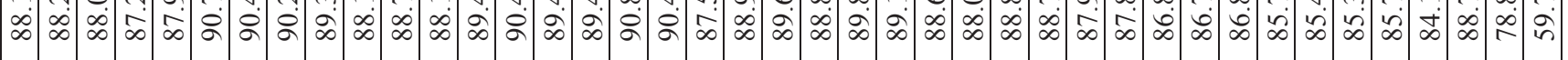

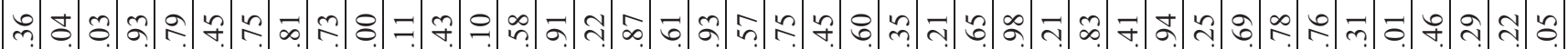

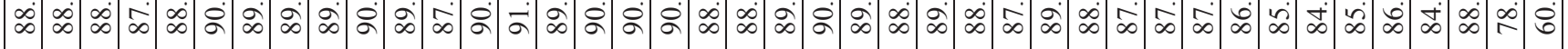
\&!

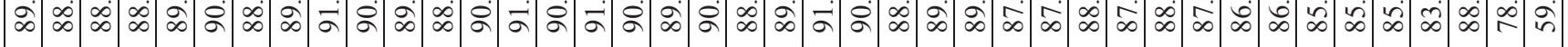

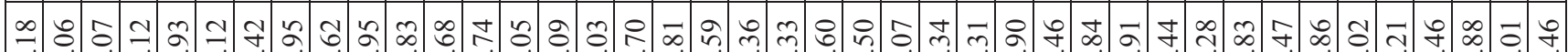

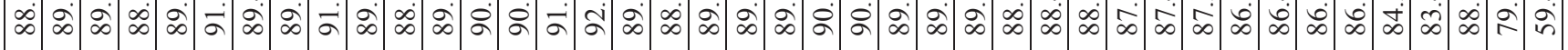

ๆ กำ

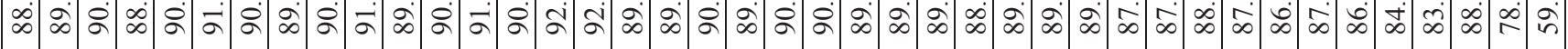

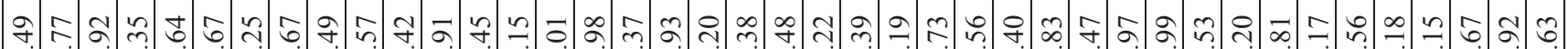

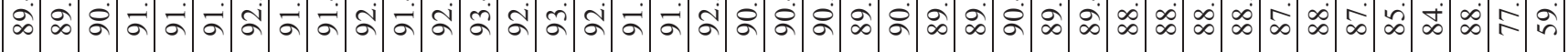

๘

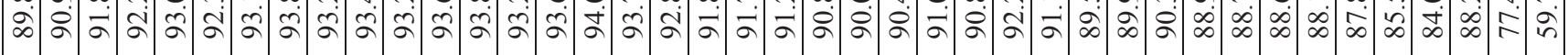

:

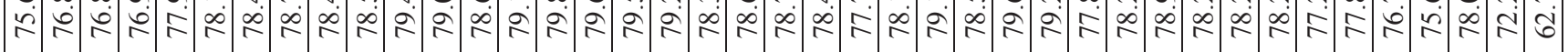
过

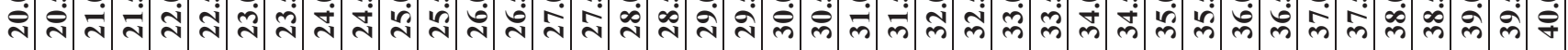


- \& 응 ส

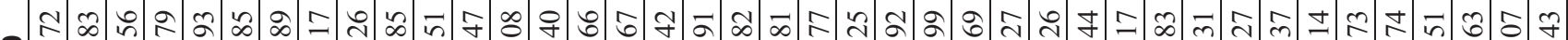
๙ 过

웅

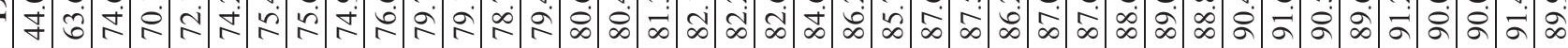
Q

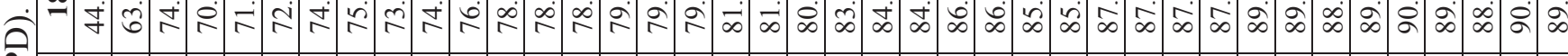

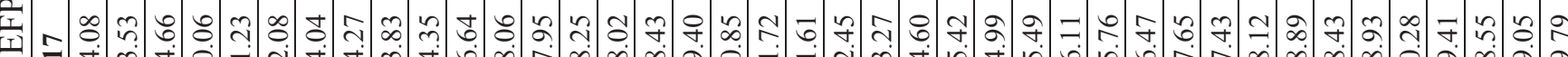

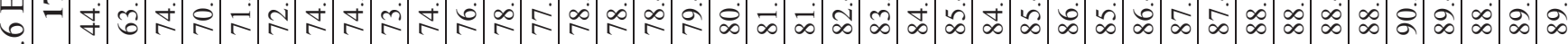

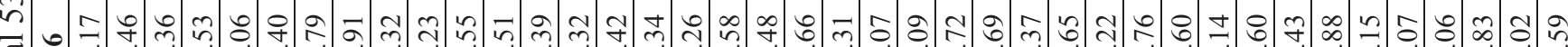

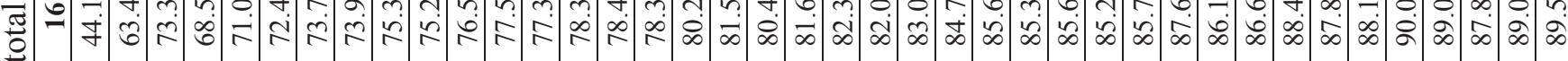

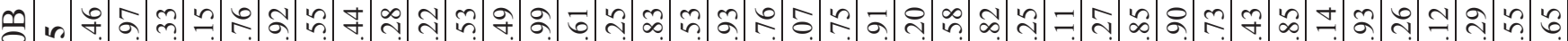

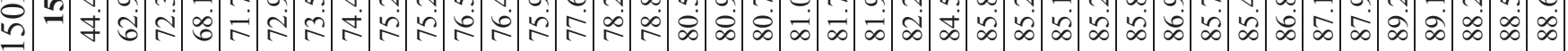
- ㅎㅇㅇㅢ

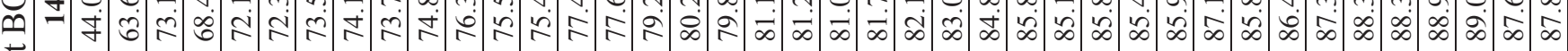
ง

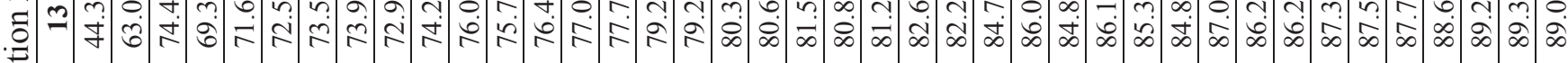
ง

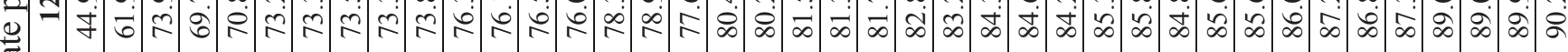

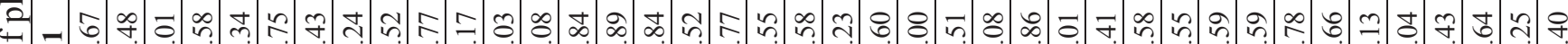

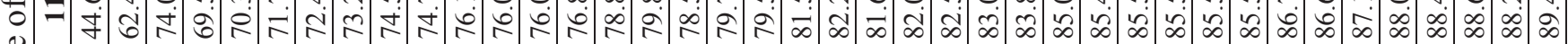

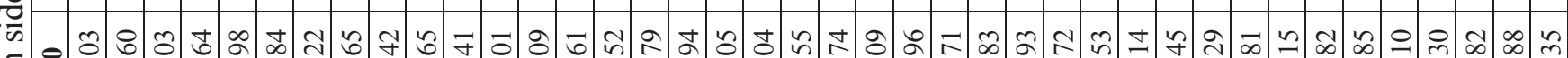

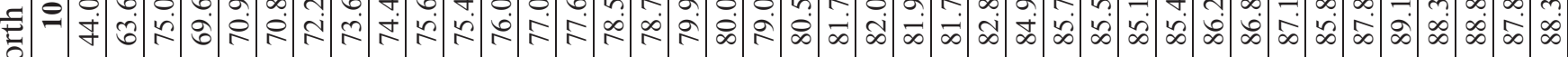

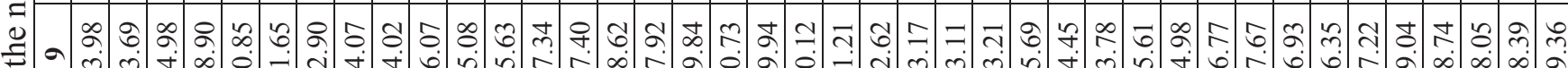

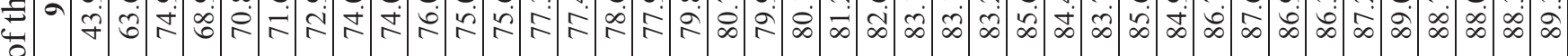
สิ

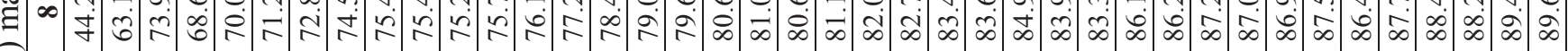
。 m 看

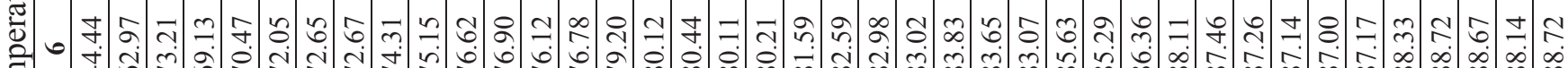

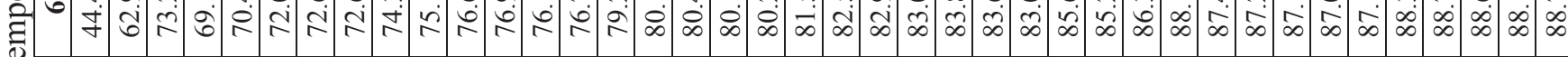
$\because$ 구의 mे n

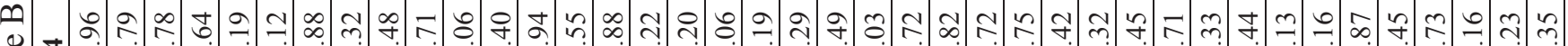

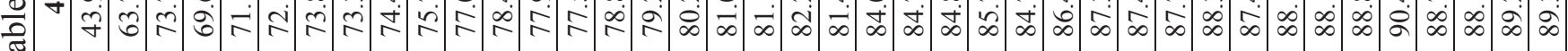

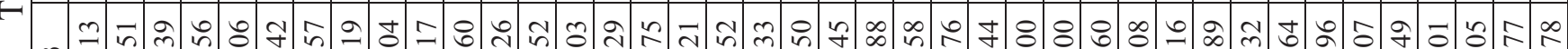

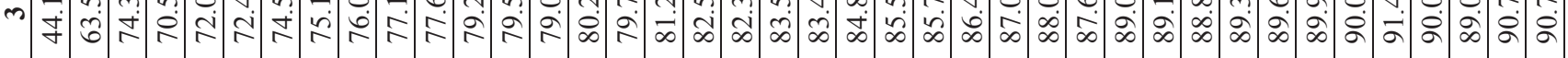

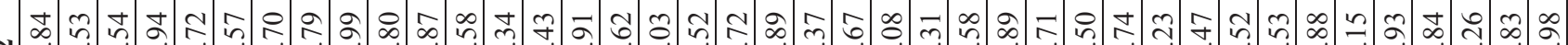
过

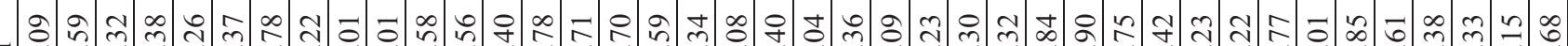

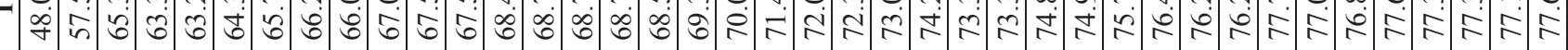
en

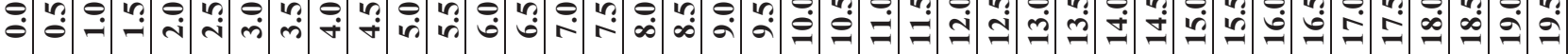




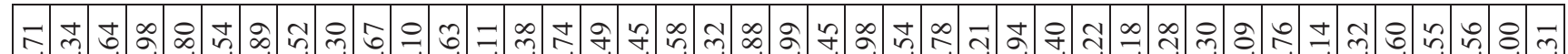

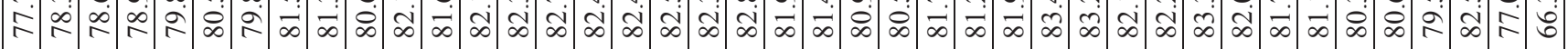

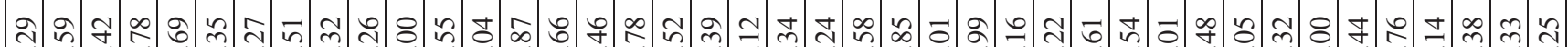

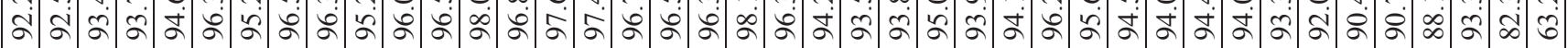

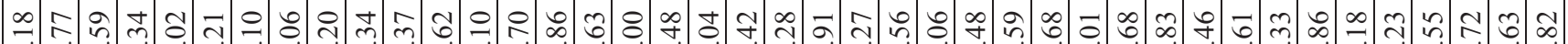

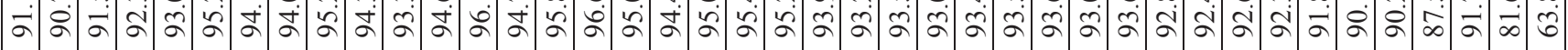

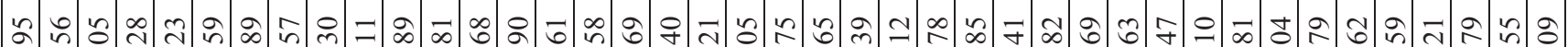

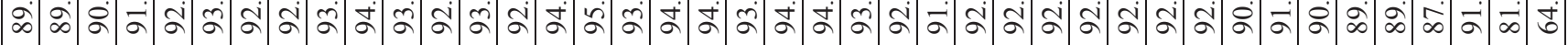

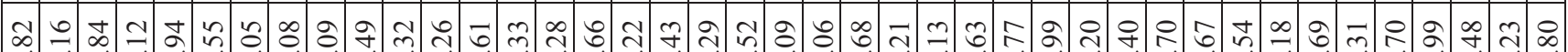

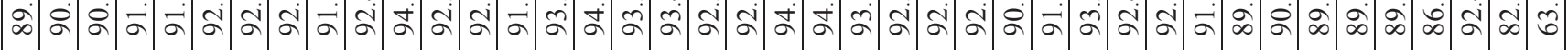
ริ त)

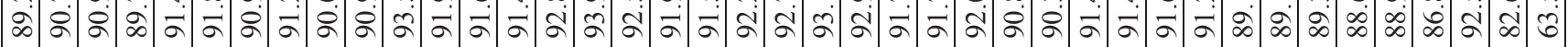

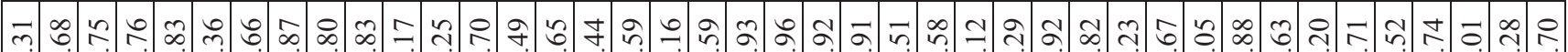

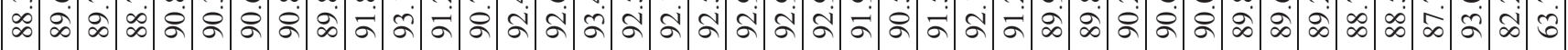

万৷

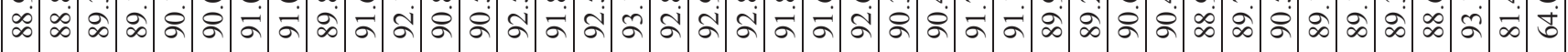

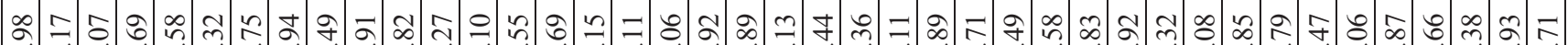

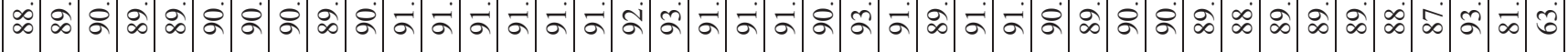

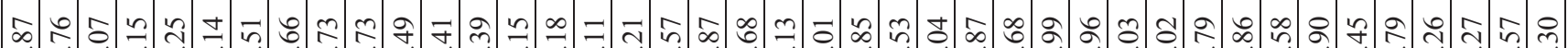

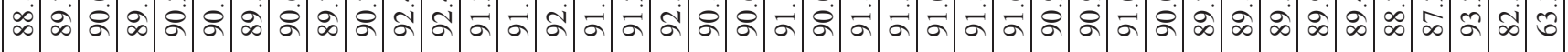

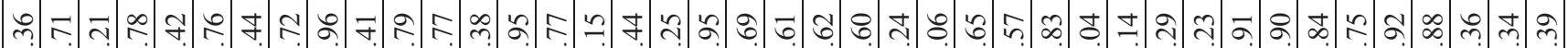

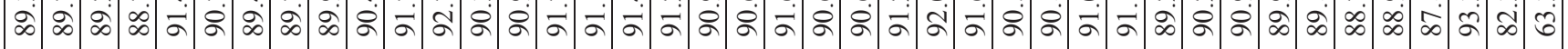

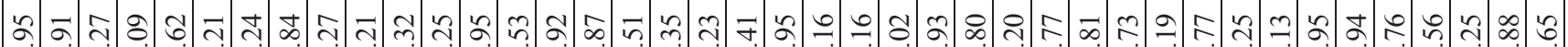

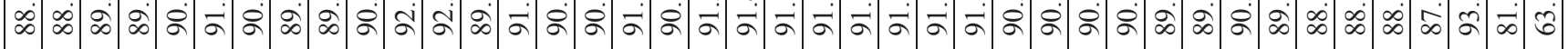

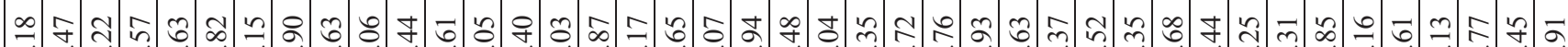

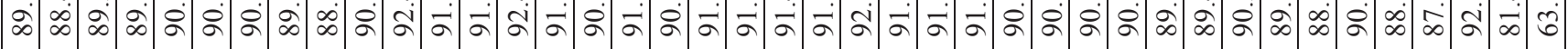

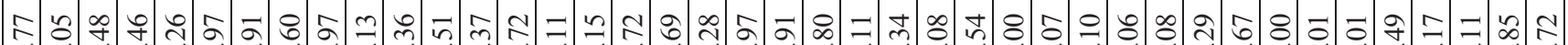

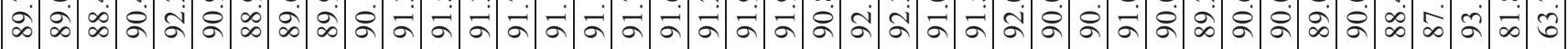

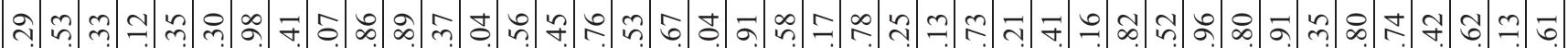

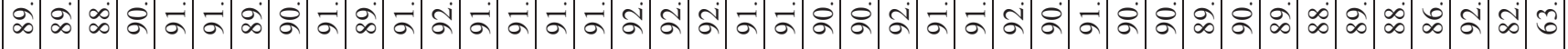
กิ

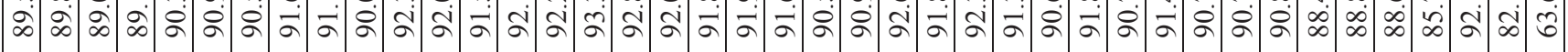
ฉส

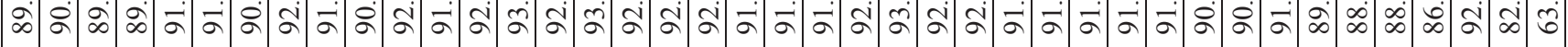

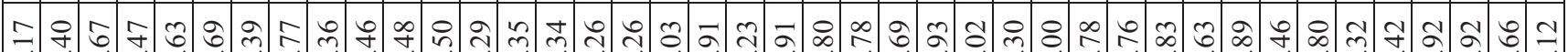

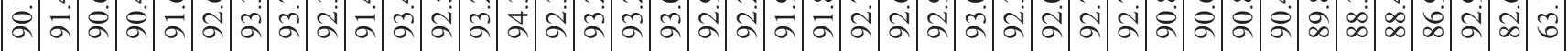

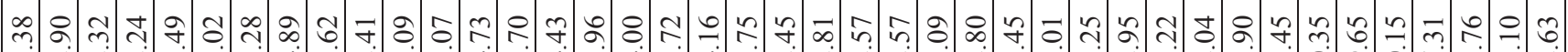

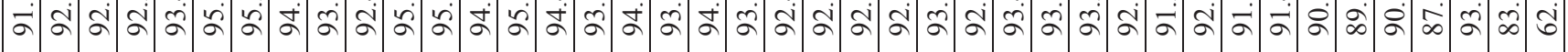

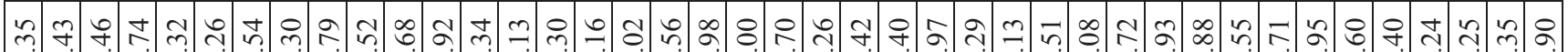

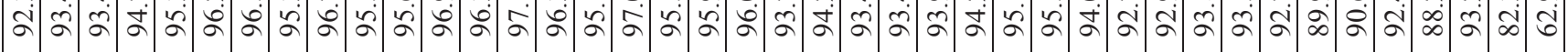

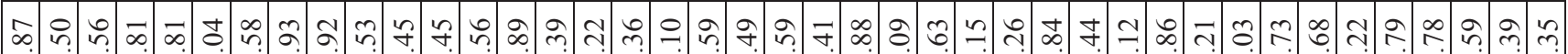

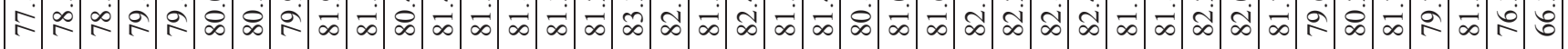
-

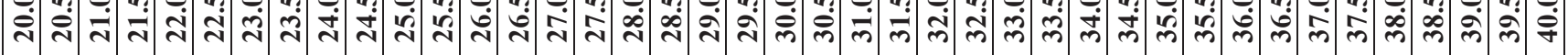




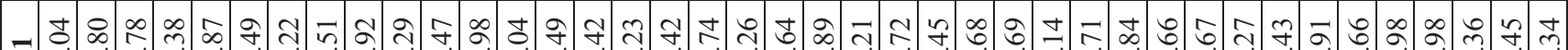

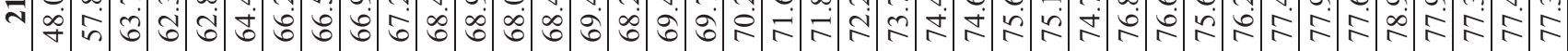

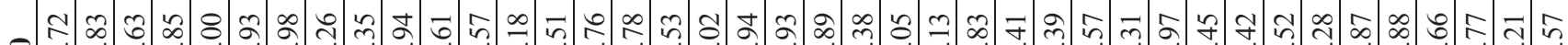

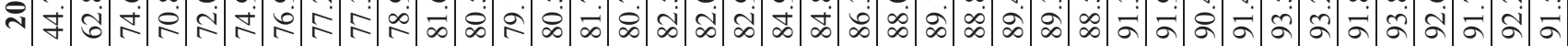

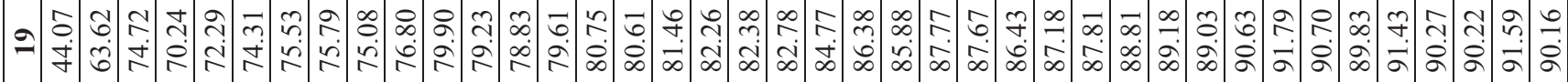

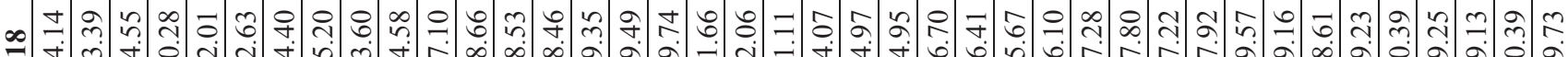
(-) 牙

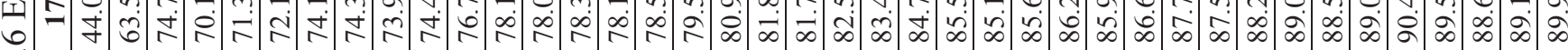
n. 至

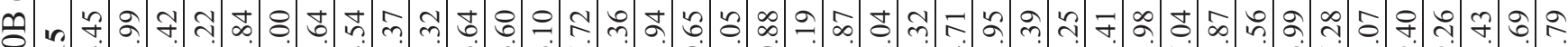

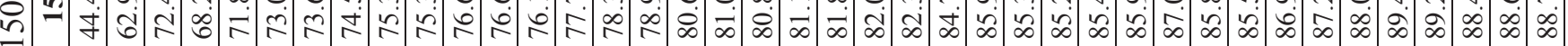
○

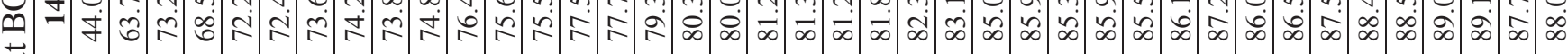
ᄀ

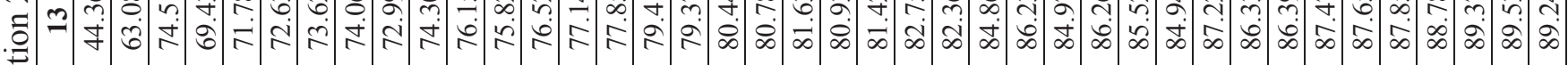
ఏ

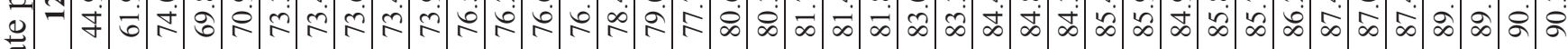

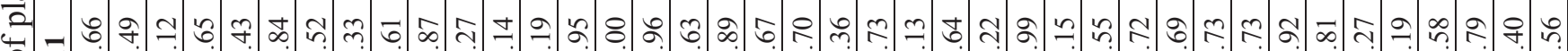

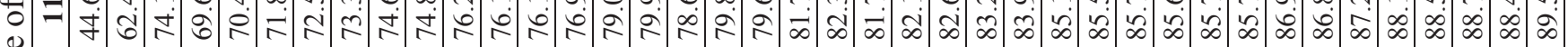

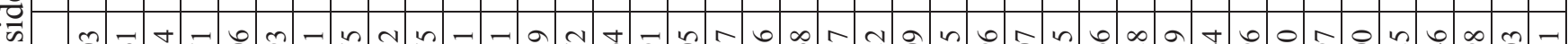
플

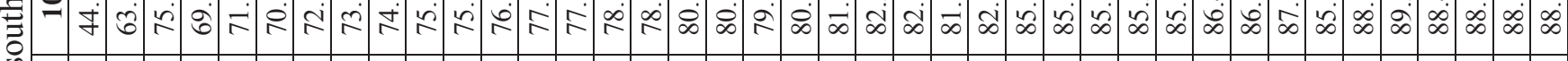
Ð a

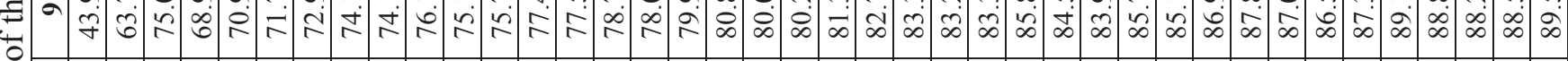

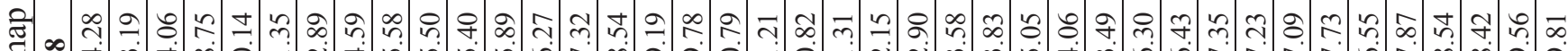

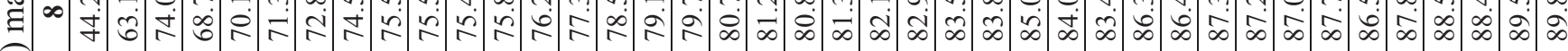

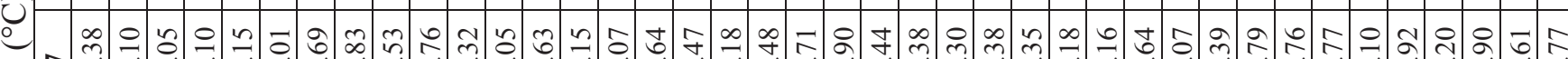

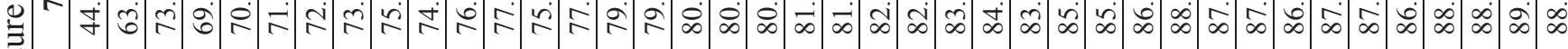

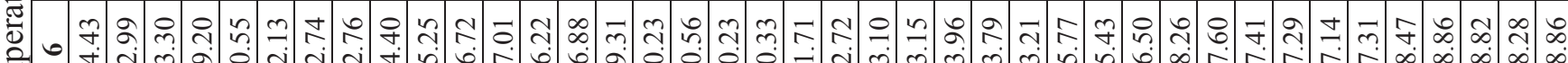
च $\quad$ J

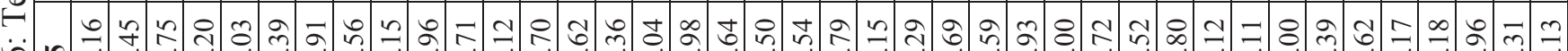

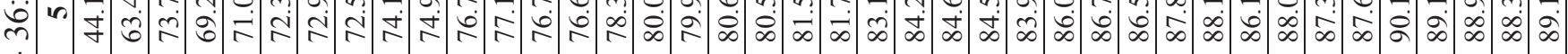
๑ -

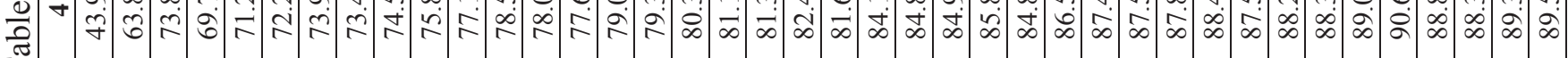

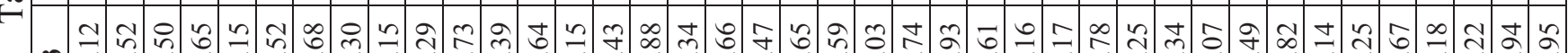

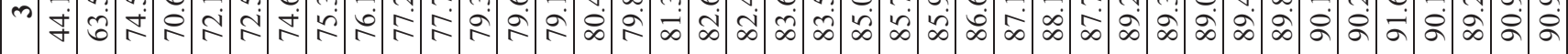

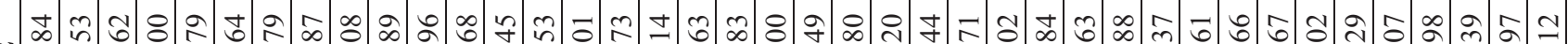
f

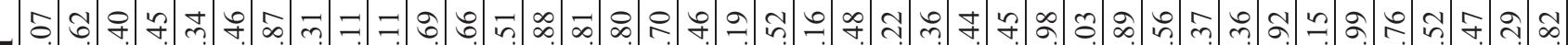
- 定 舟 


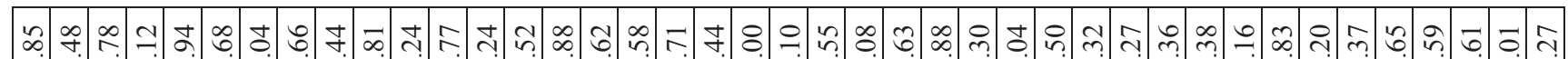

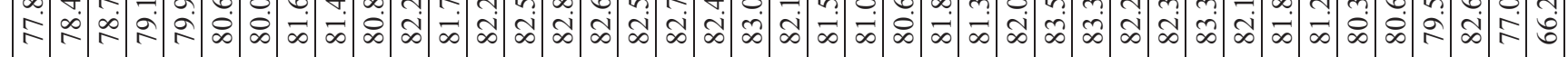

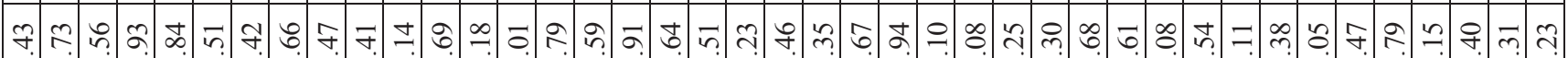

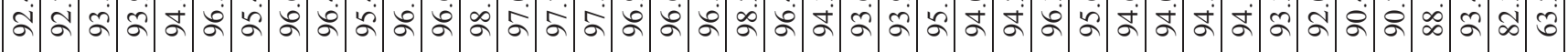

m.

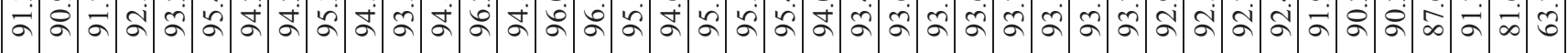

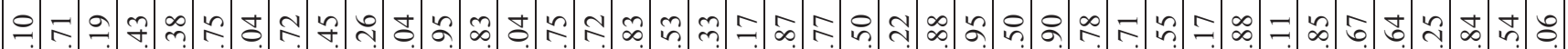

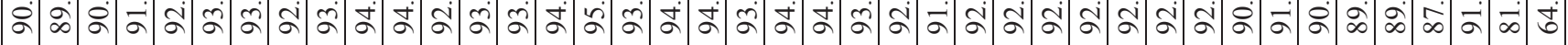
ஃ

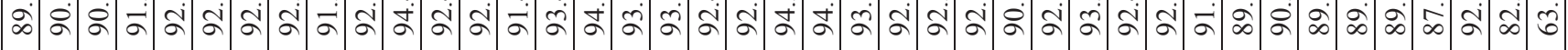
m

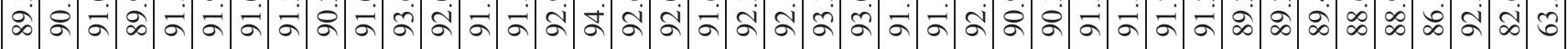

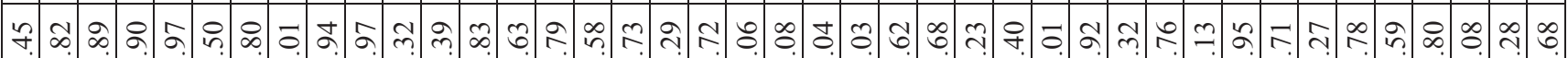

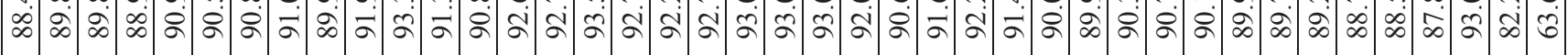

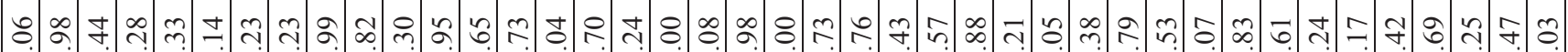

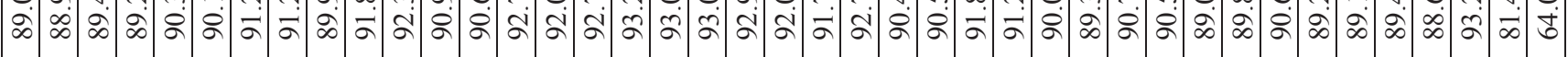

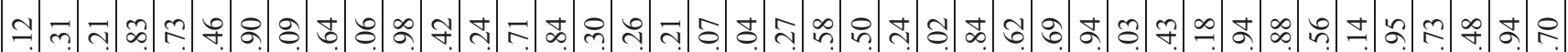

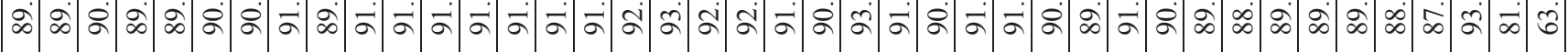

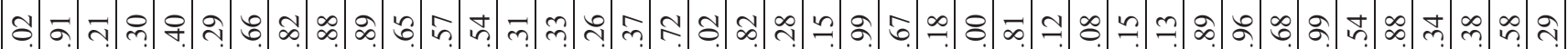

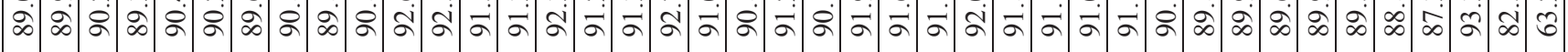
చี

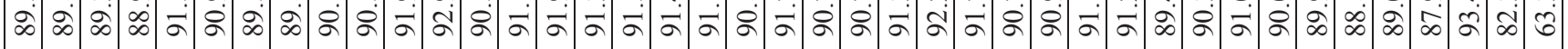
=

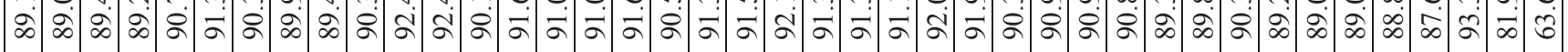
m

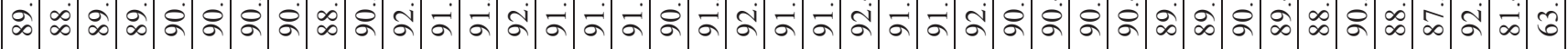

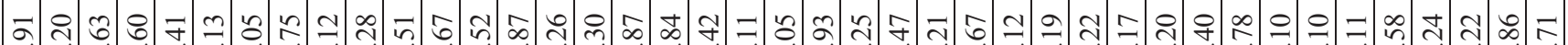

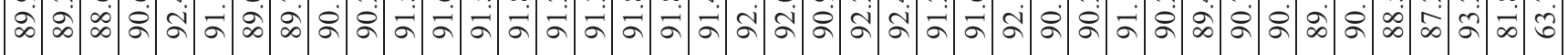
Ұ

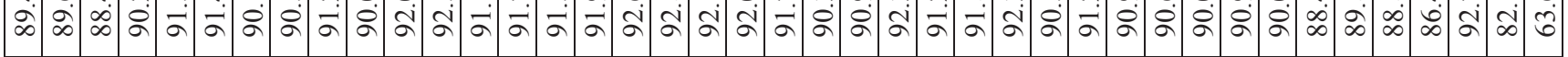

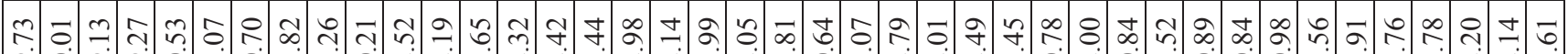

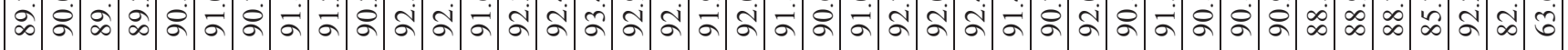

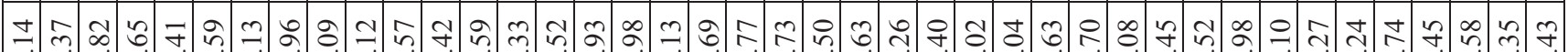

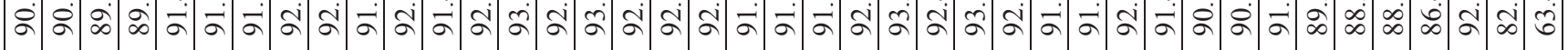

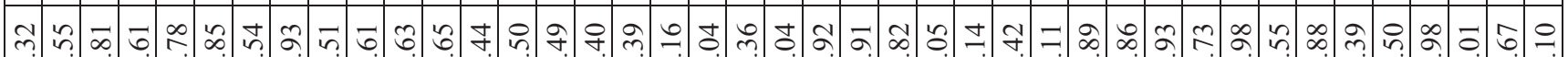

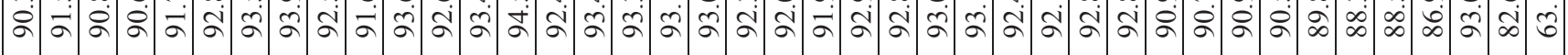
只

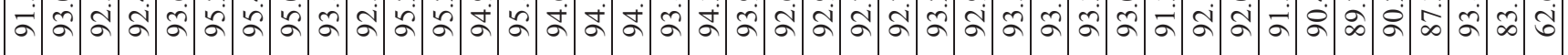

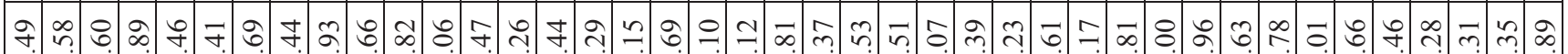

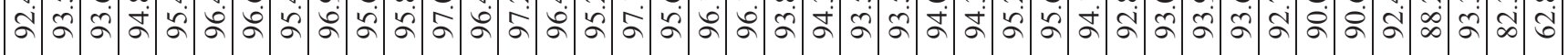

ஏ t.

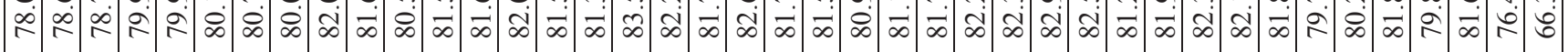
过

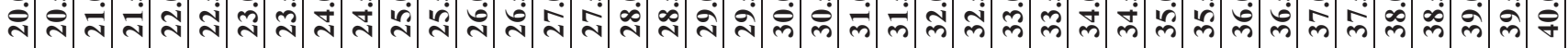




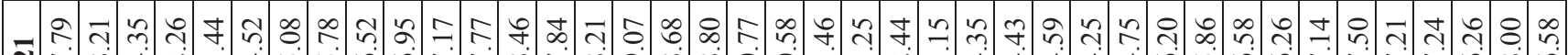

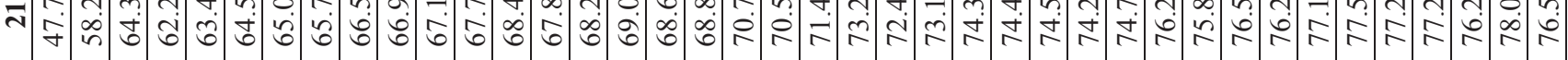

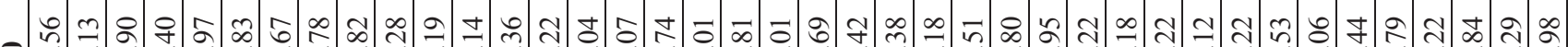

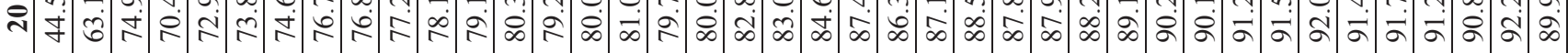

- 孝 f

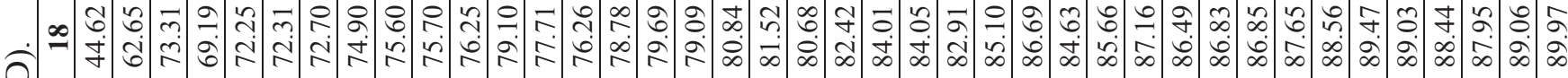
牙 =

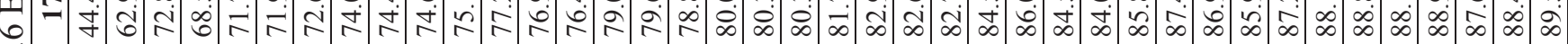
n๑ 至 ఇ

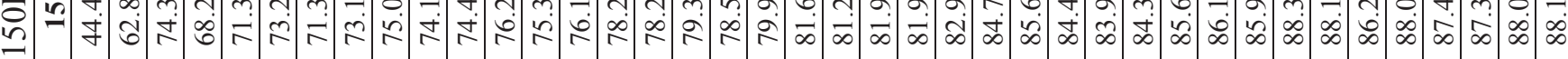

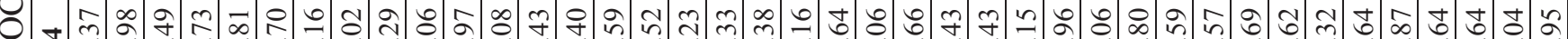

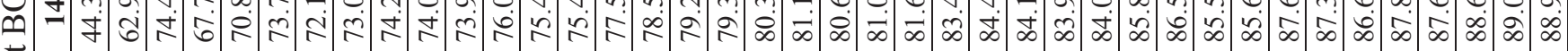

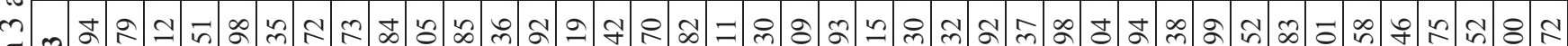

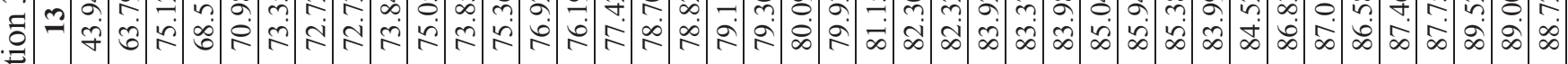

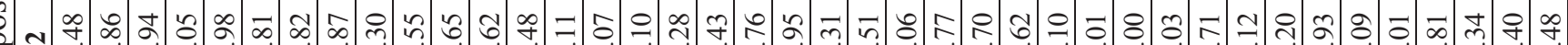

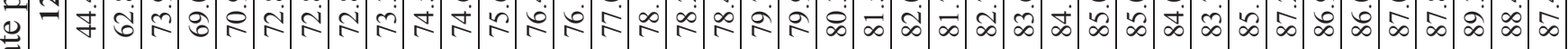

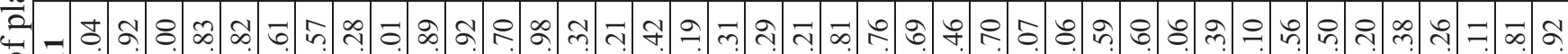

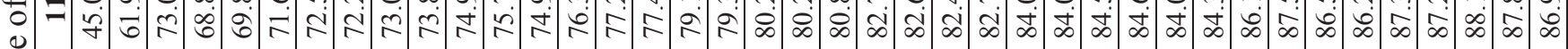

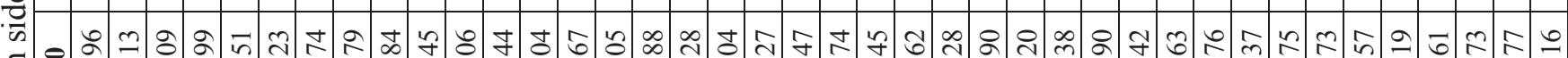

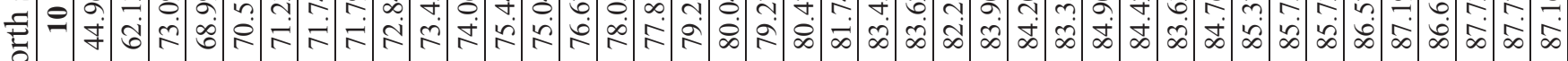

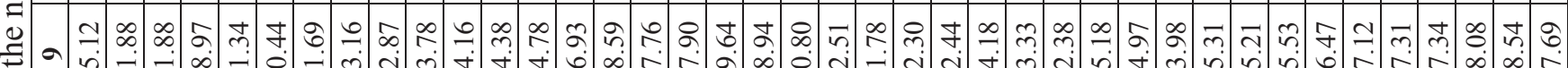

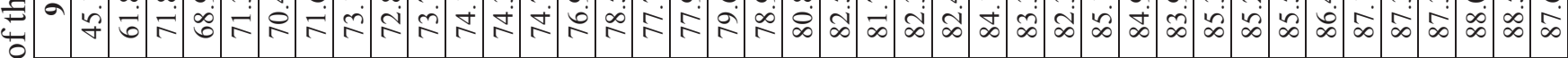
สิ

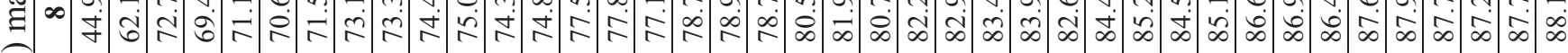

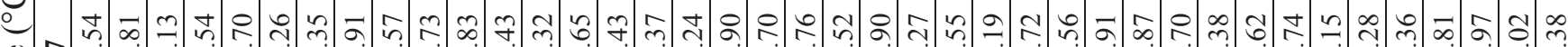

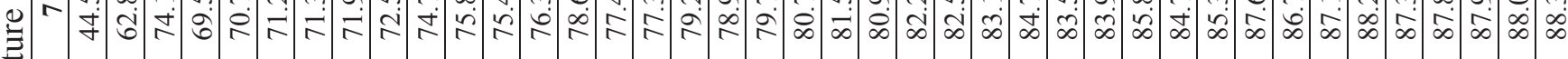

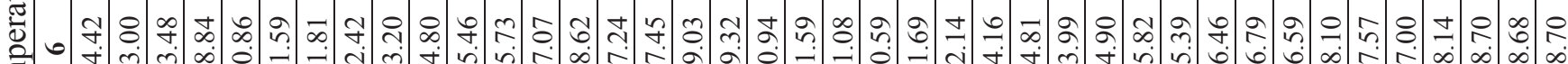

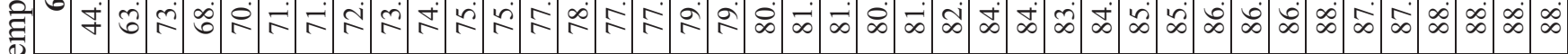

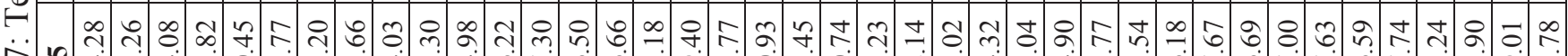

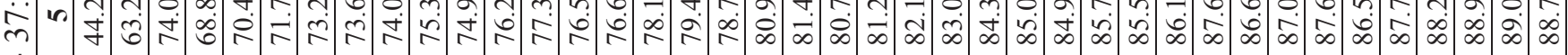

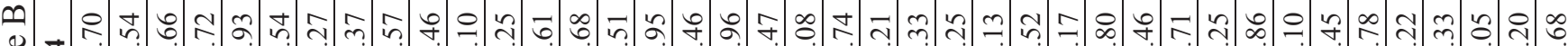

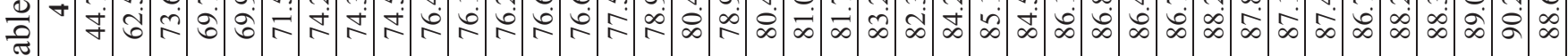

ᄀ ริ

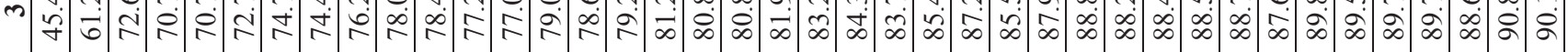

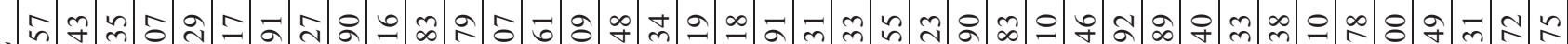

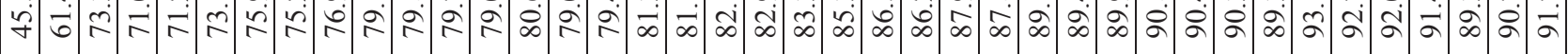

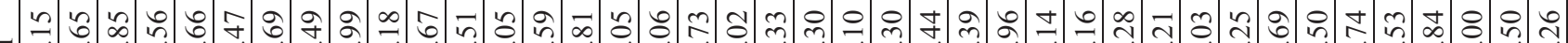

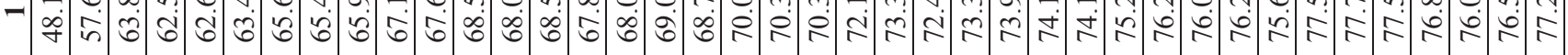

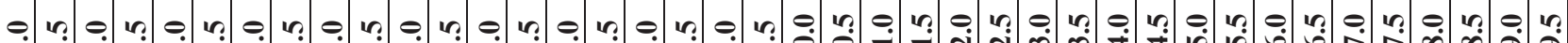

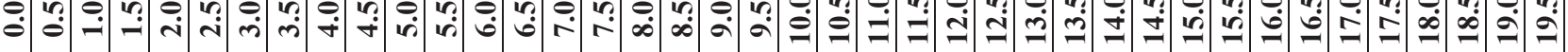




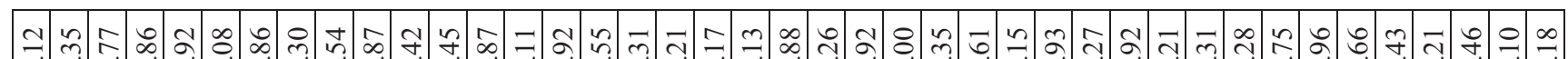

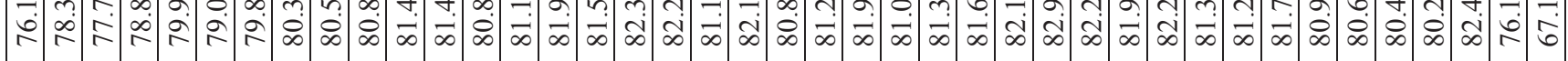

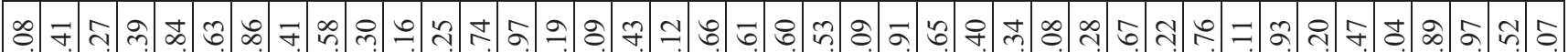

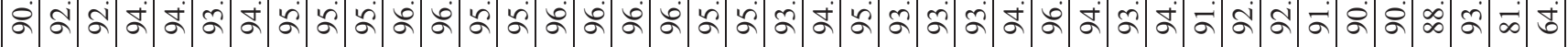

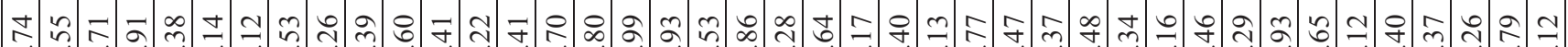

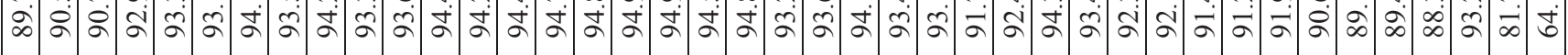

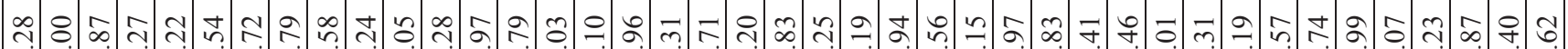

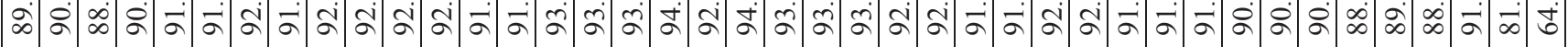

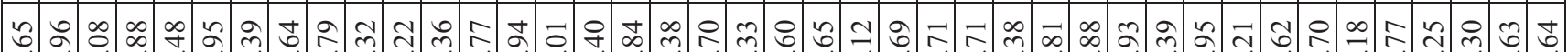

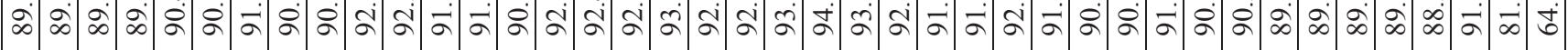

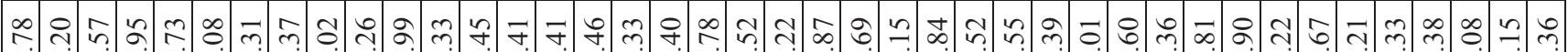

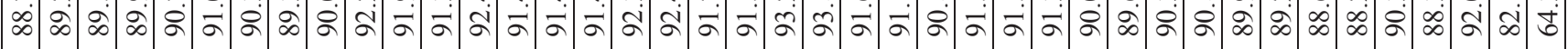

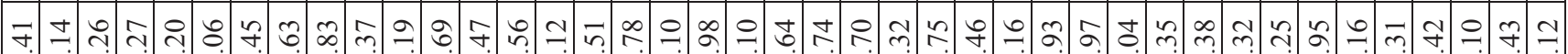

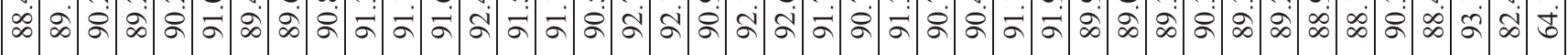
\&

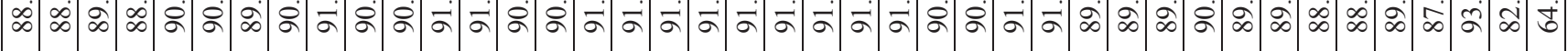

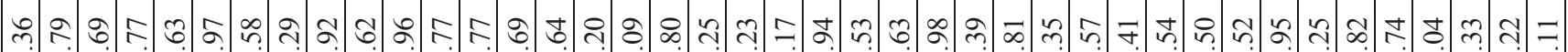

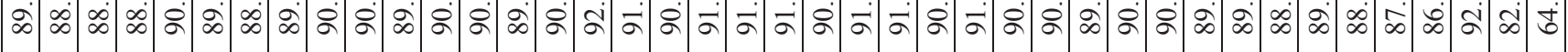

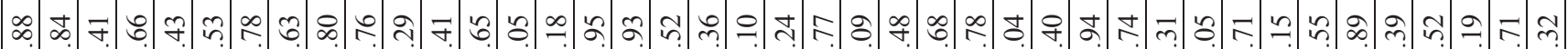

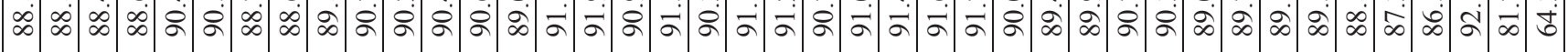

t苗

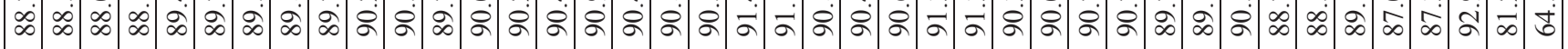

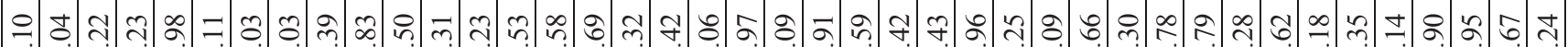

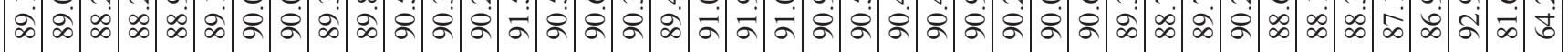

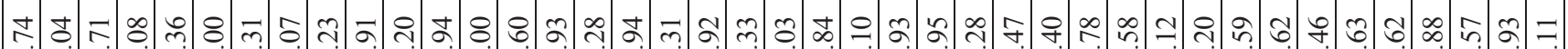

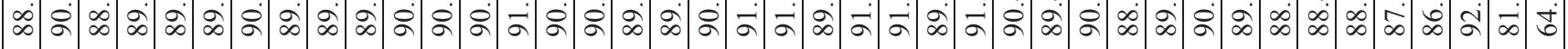
누

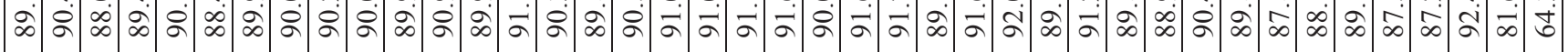
กฺ

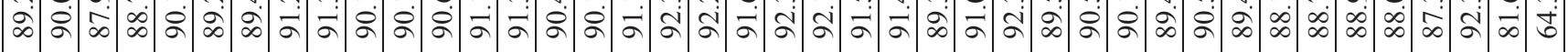
t)

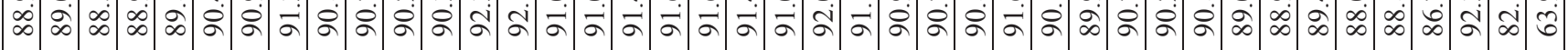

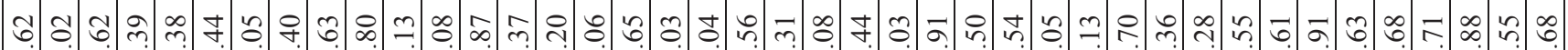

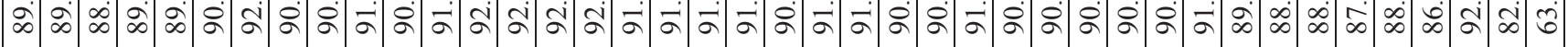
দี $\bar{\infty}$ f

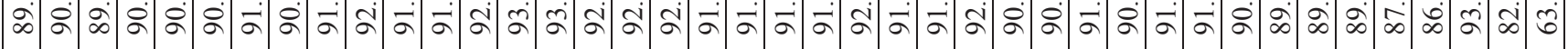

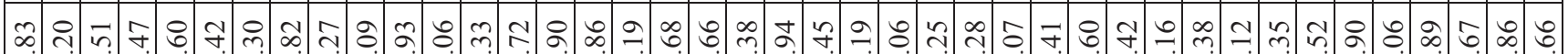

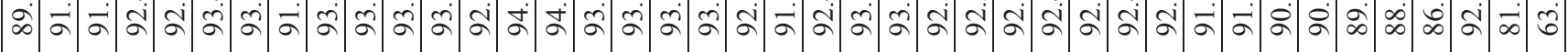

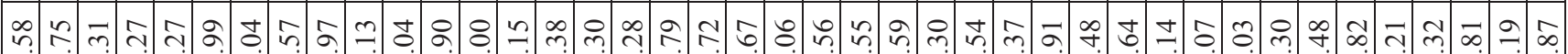

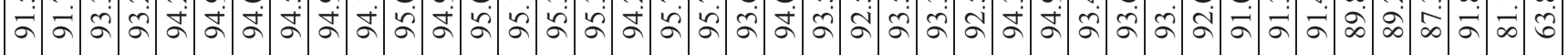

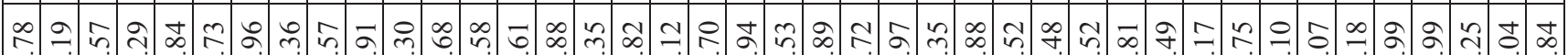

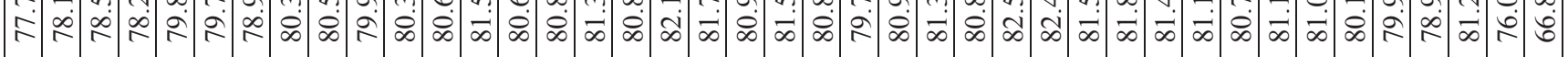
竎 


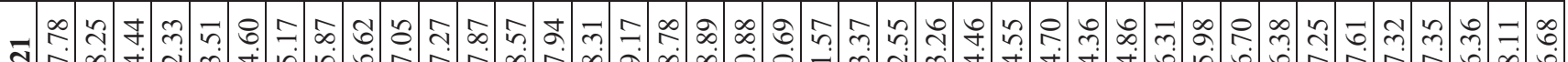

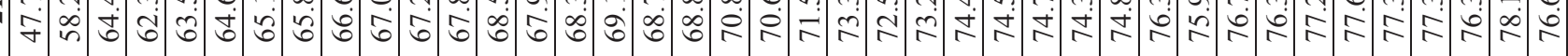

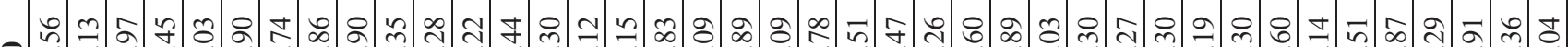
حิ 过

$=\underset{f}{f}$, f

$\infty$ б

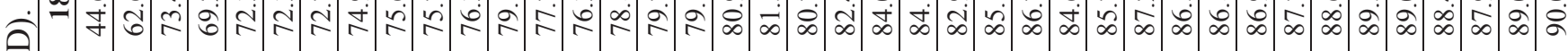

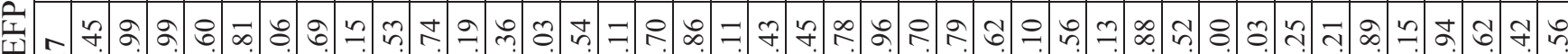

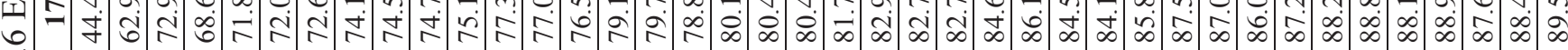

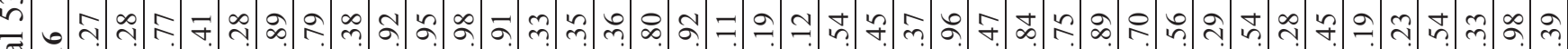

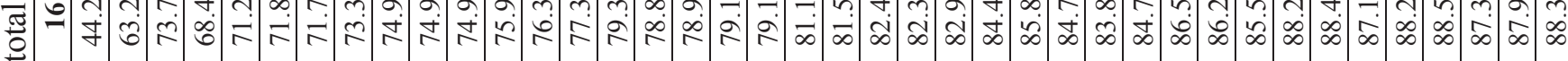

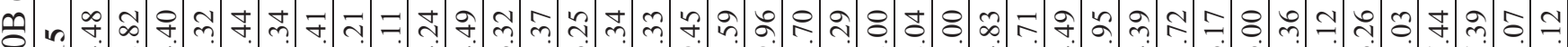

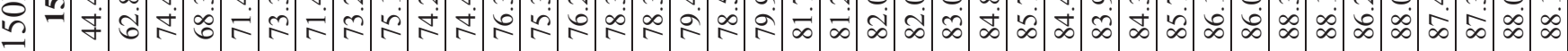
- +

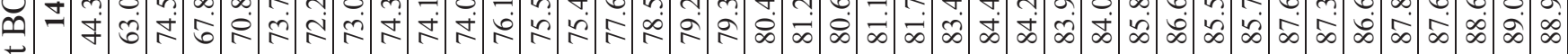
m

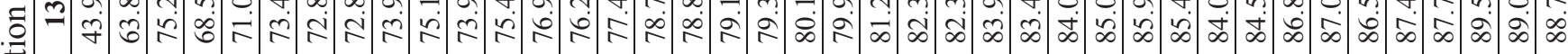

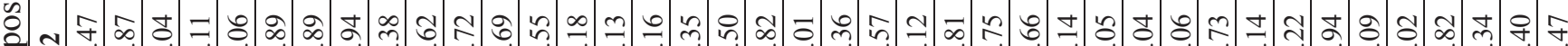

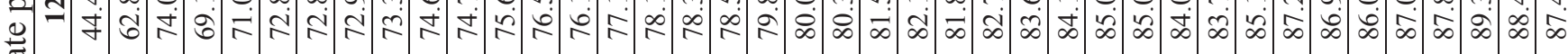

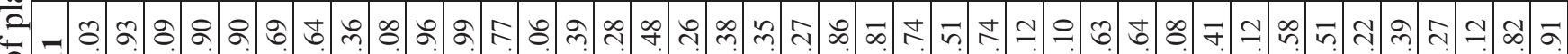

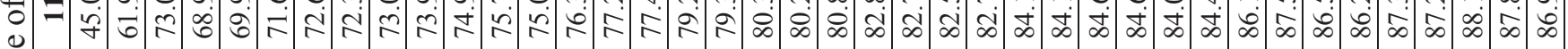

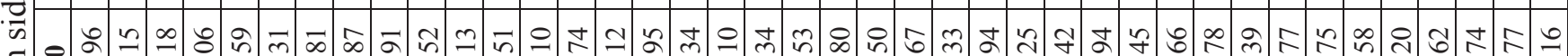

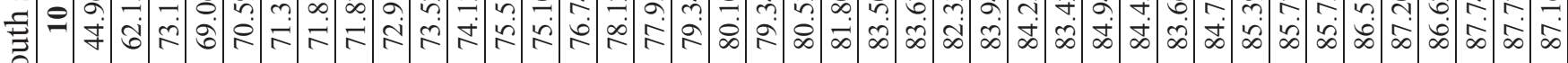
b =

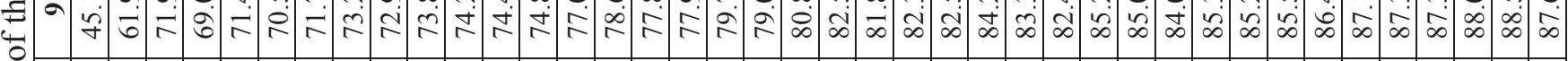

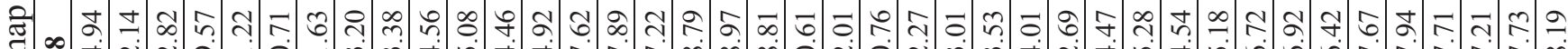

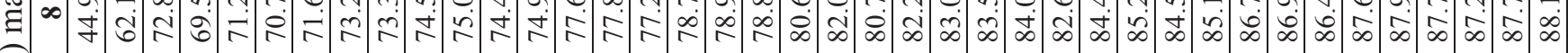

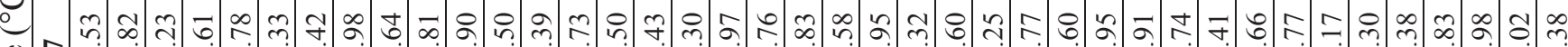

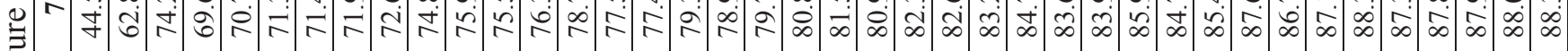

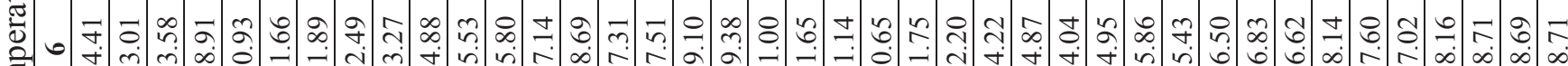

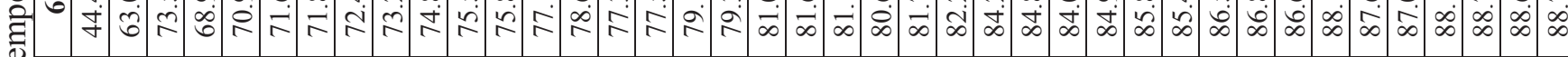

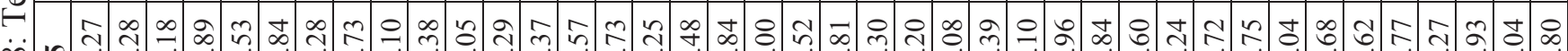

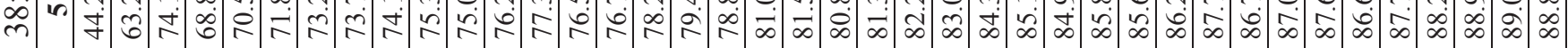

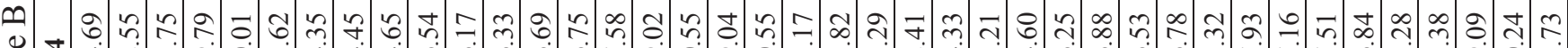

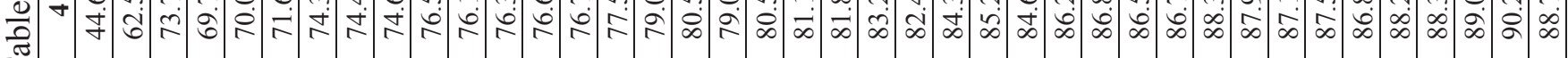
- Ұ

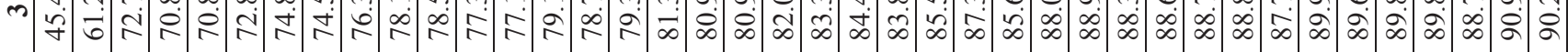

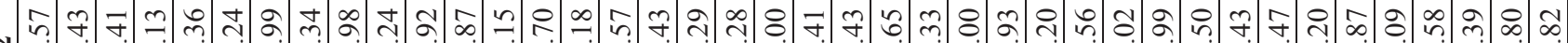

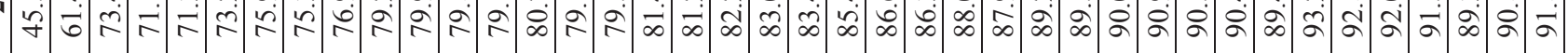

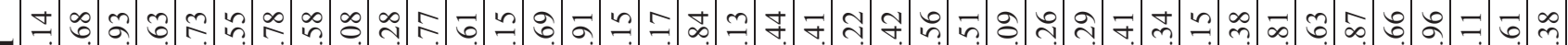

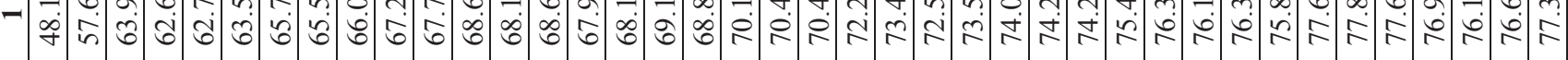

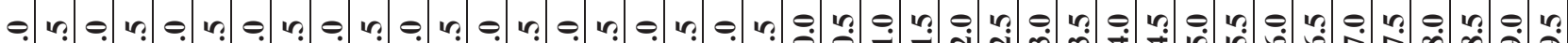

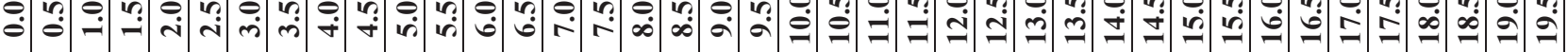




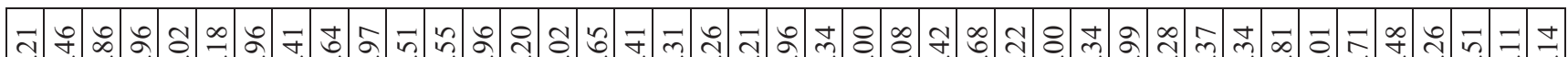

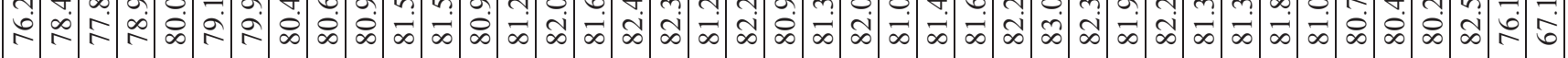

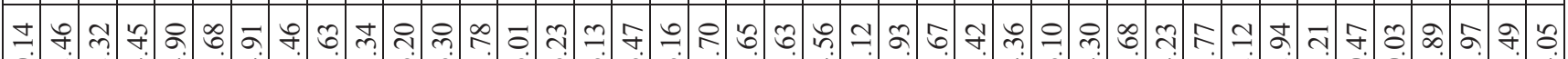

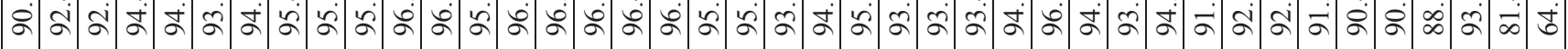

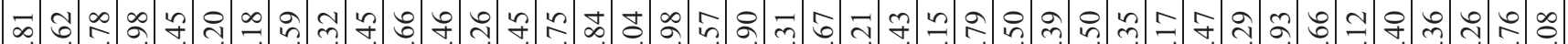

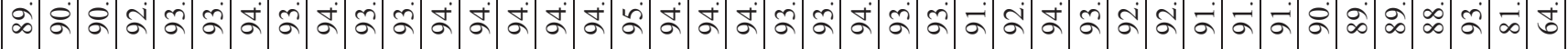

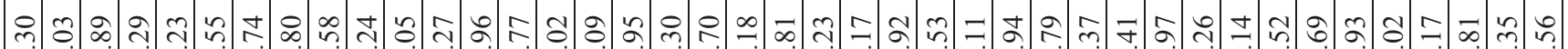

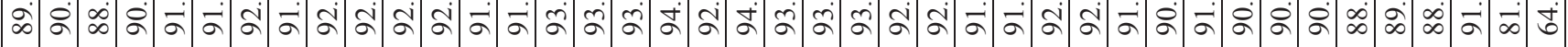
๘)

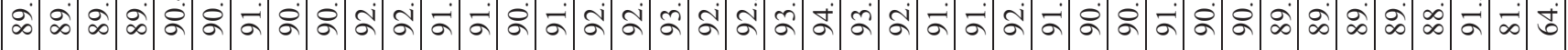

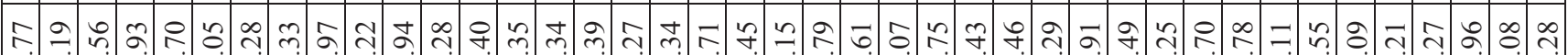

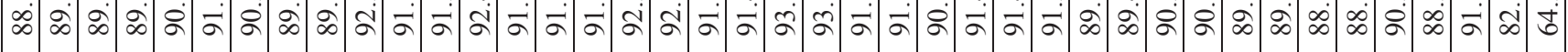

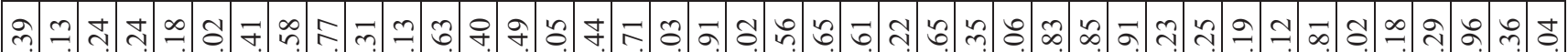

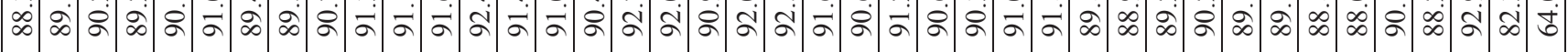

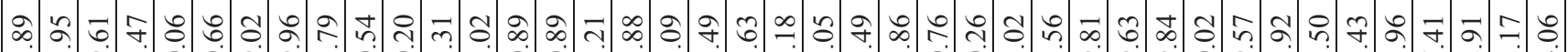

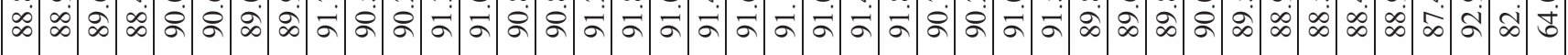

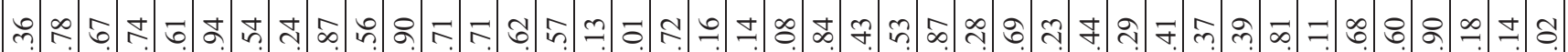

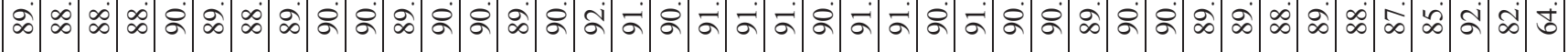

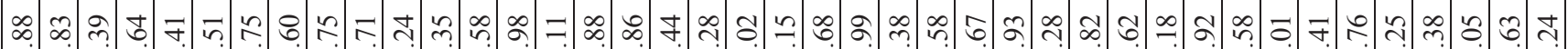

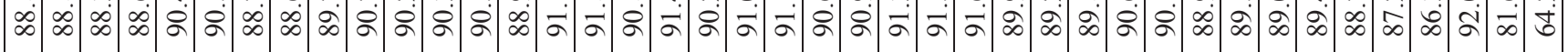

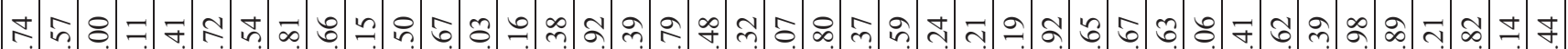

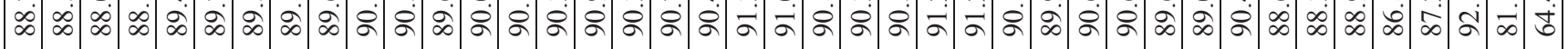

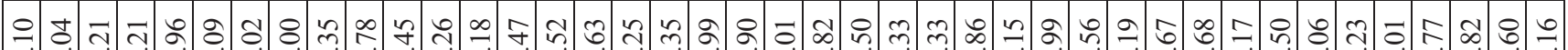

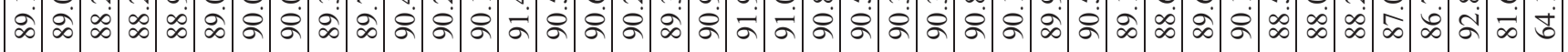

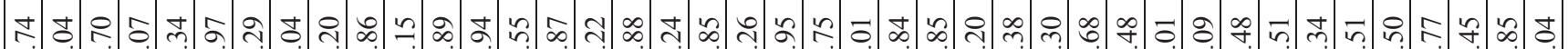

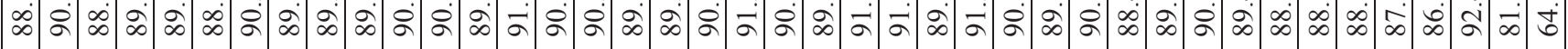
2) 守

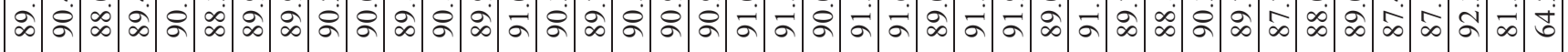
กุ t

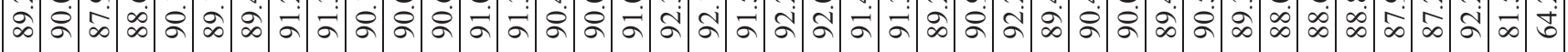

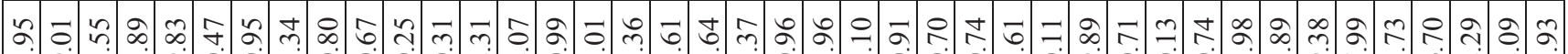

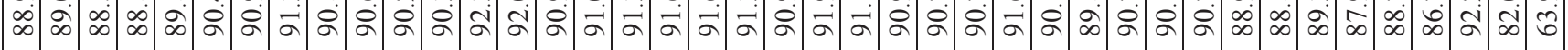

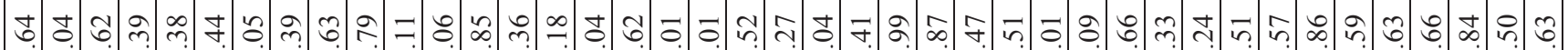

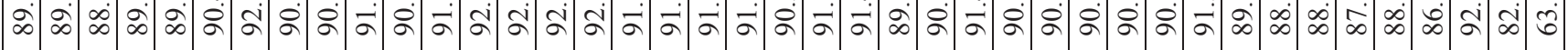
nิ

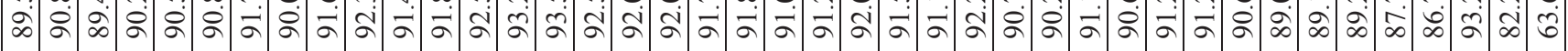

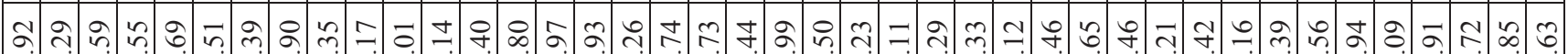

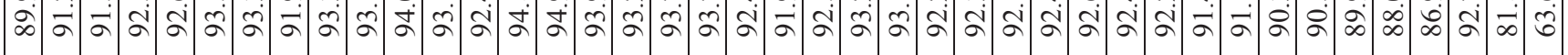

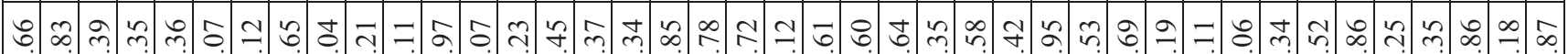

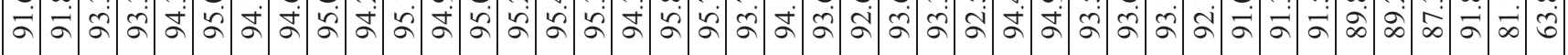
ळ.

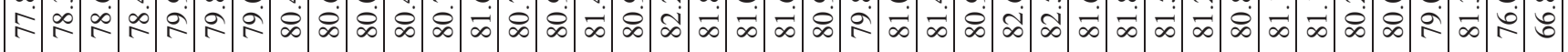




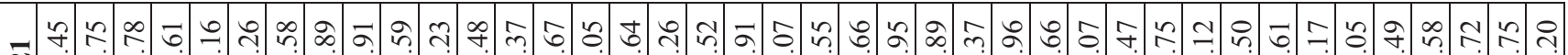

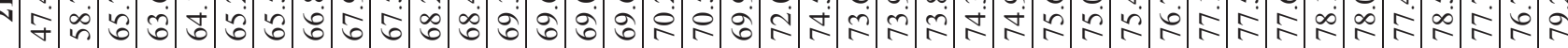

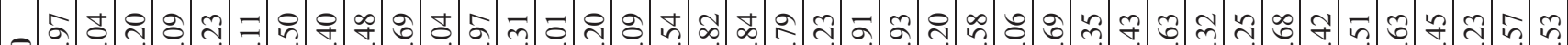

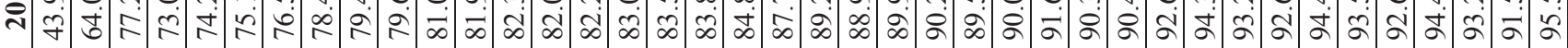

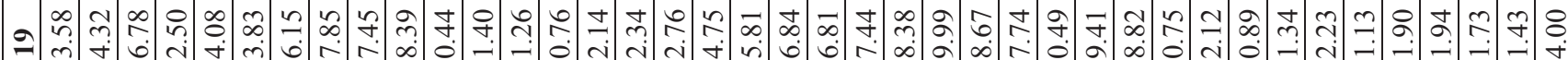

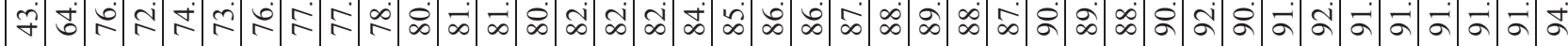

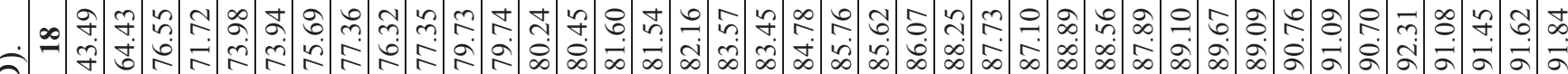

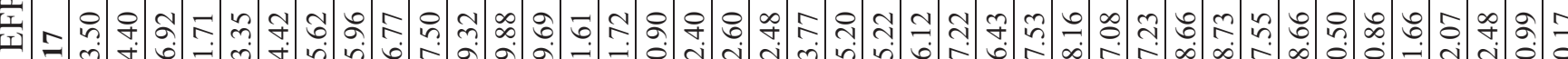
b-

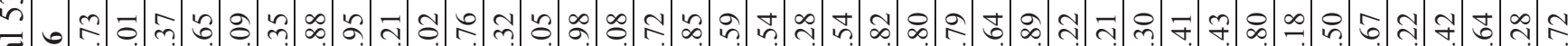

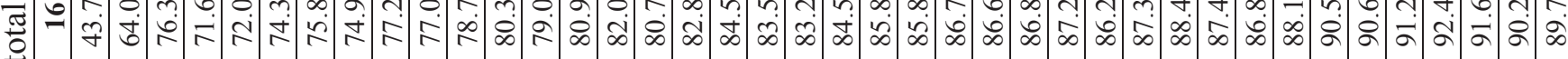

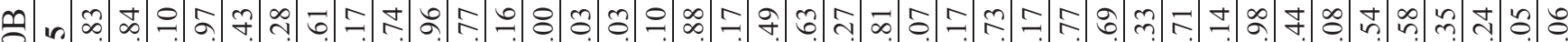

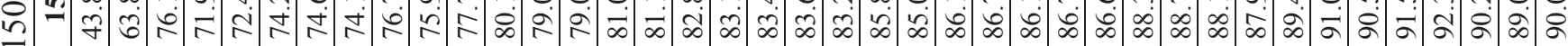

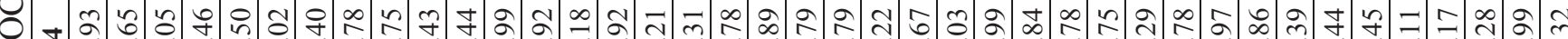
$\oplus$ I

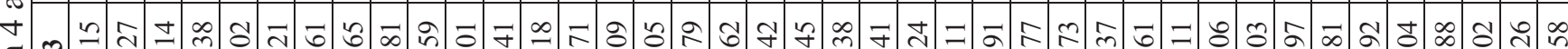

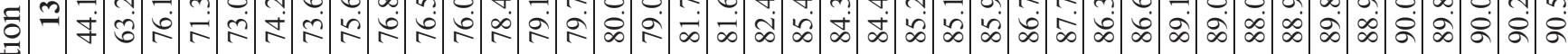

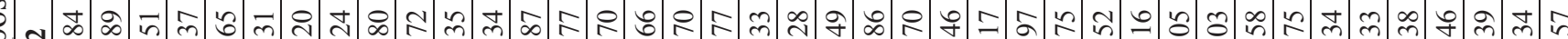
ब न

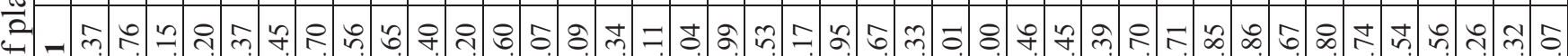

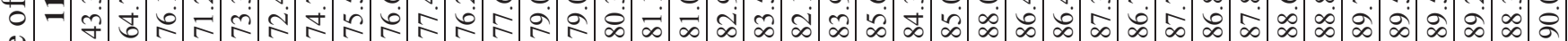
.

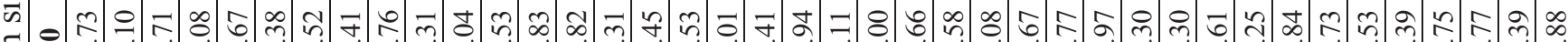

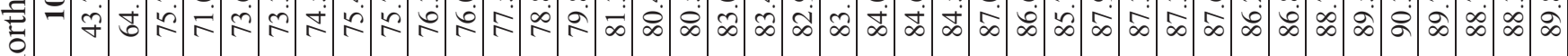

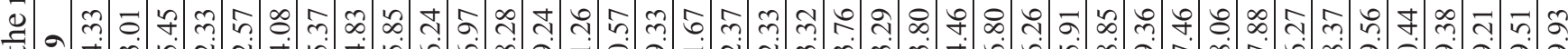
毛 चิ

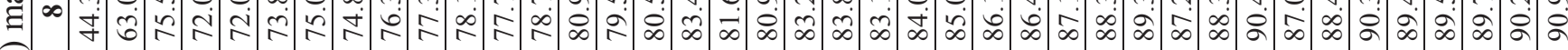

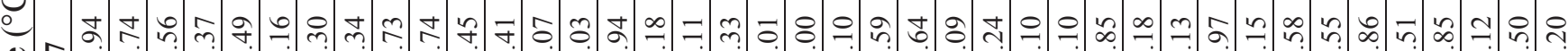

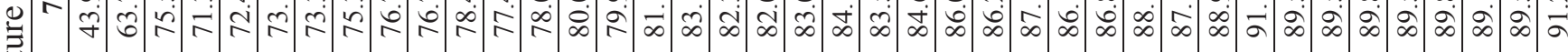

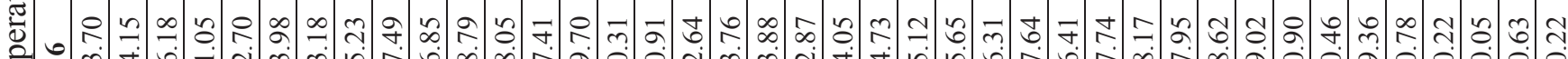

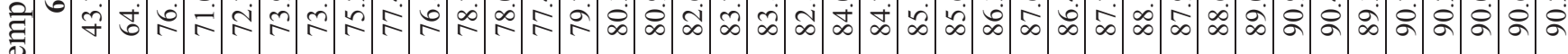

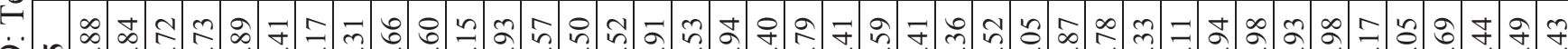

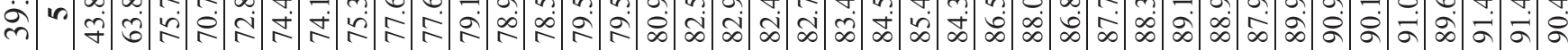

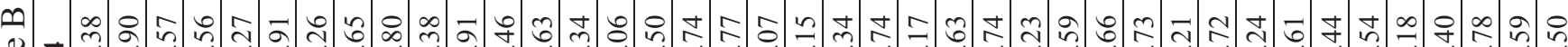
类

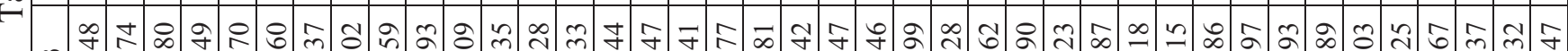

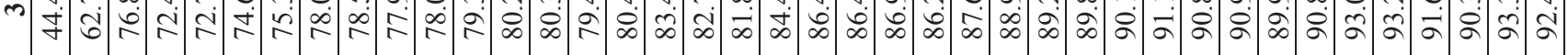

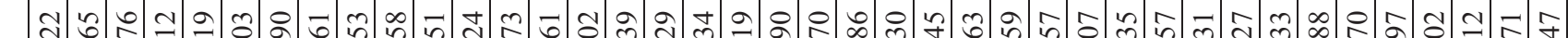

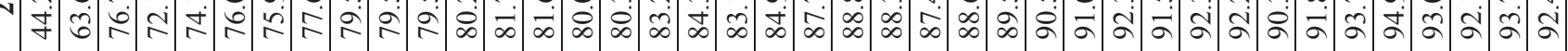

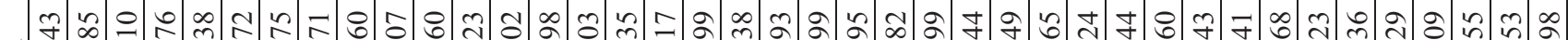

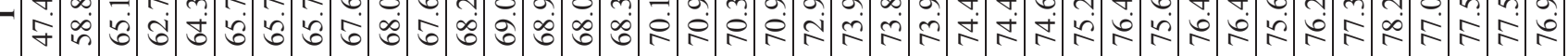

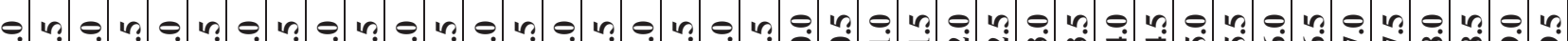

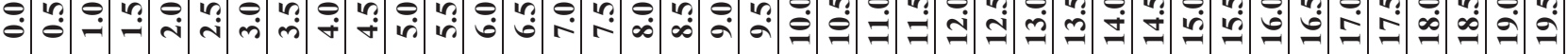




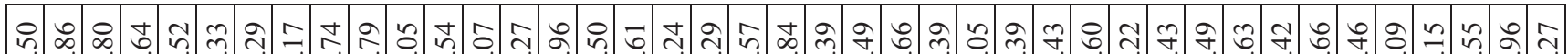

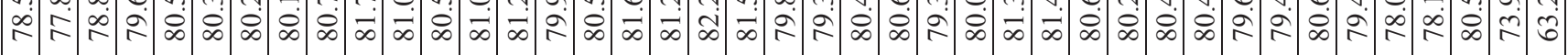

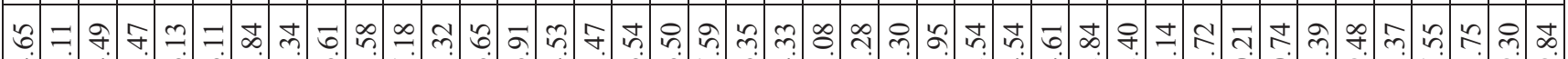

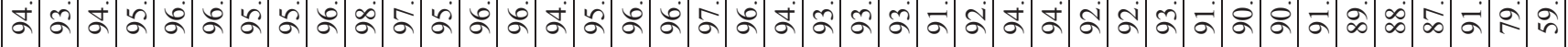

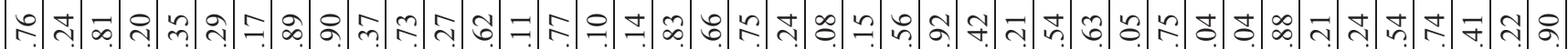

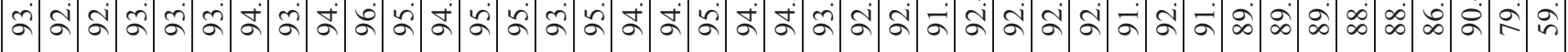

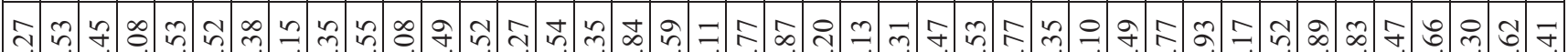

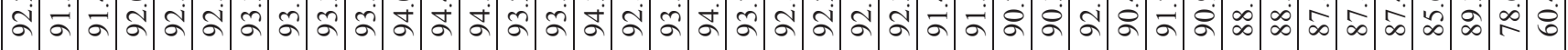

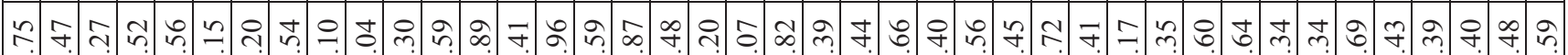

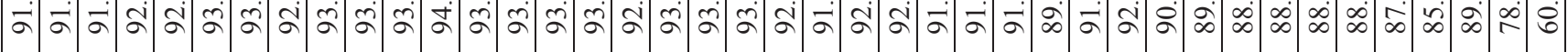

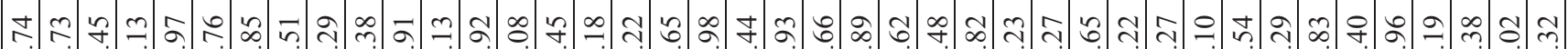

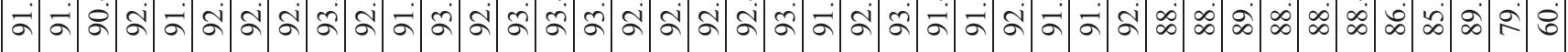

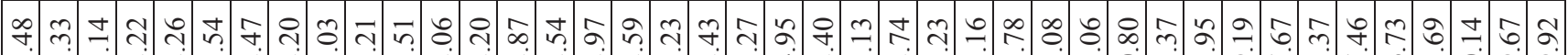

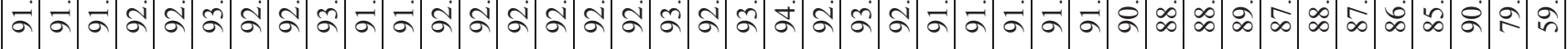

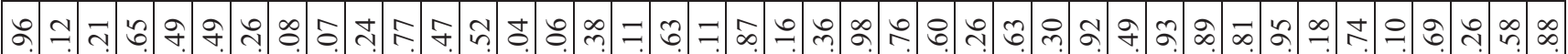

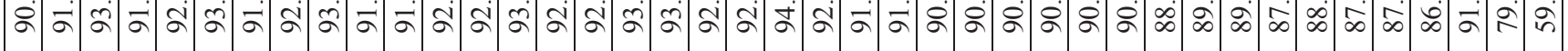

œ

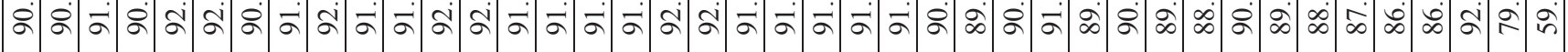

กำ

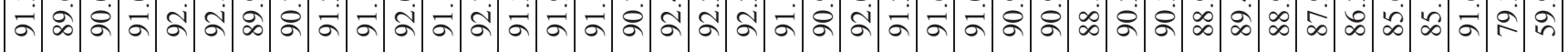
f 운

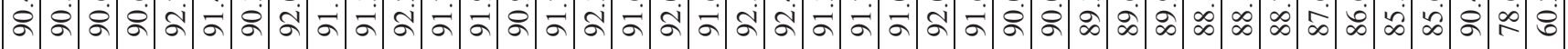
กิ ฉ்

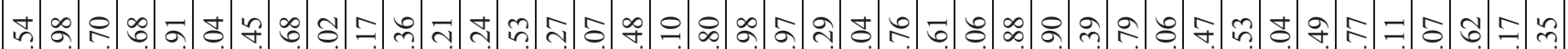

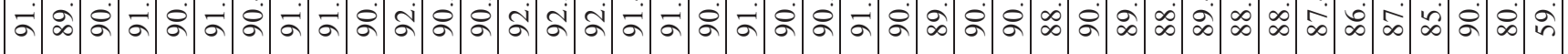
‡)

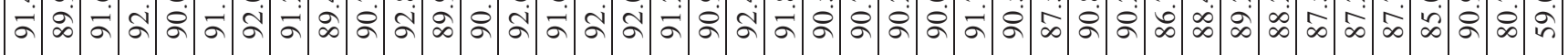
นก

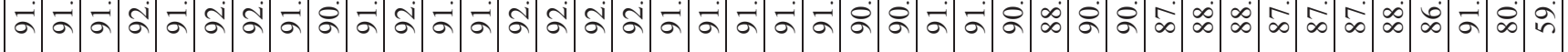

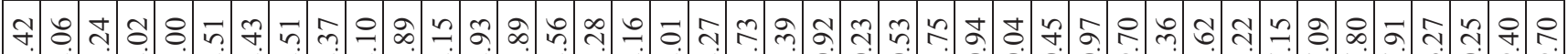

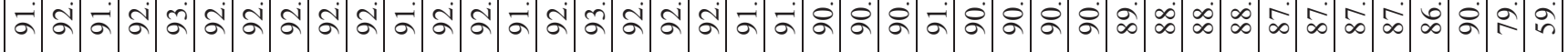

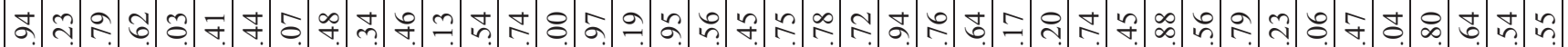

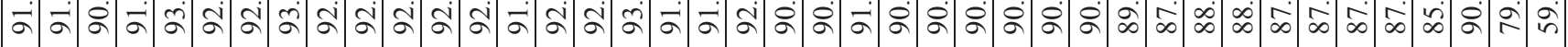

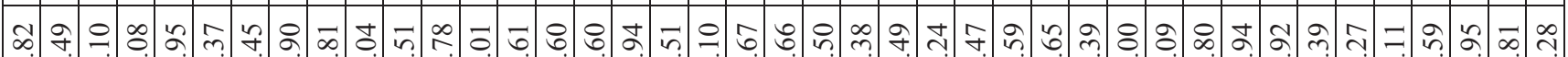

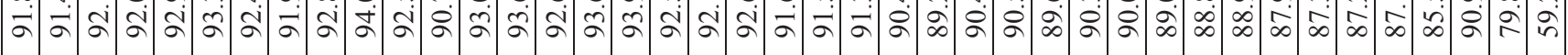

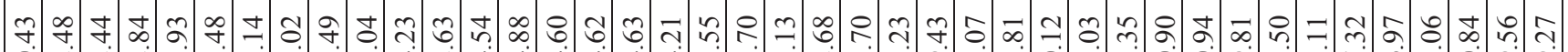

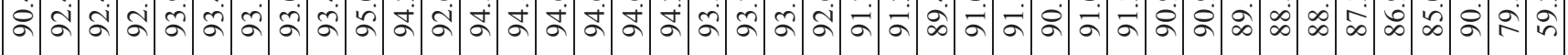

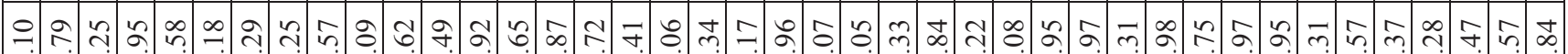

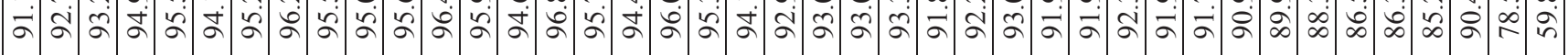

ชุ

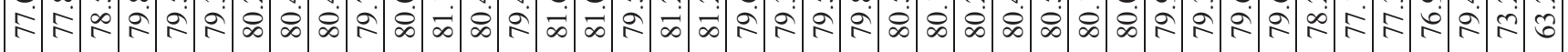




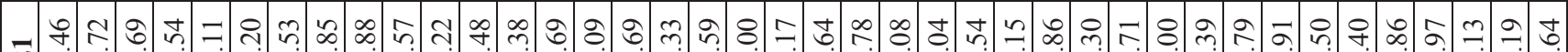

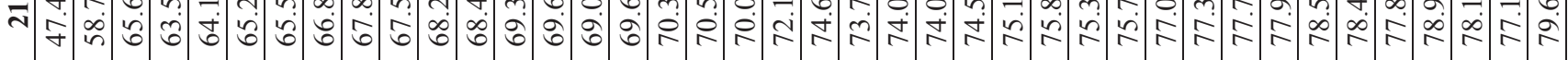

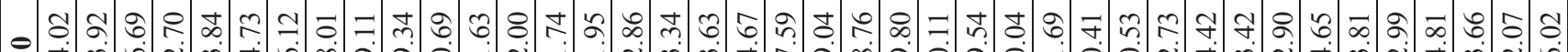

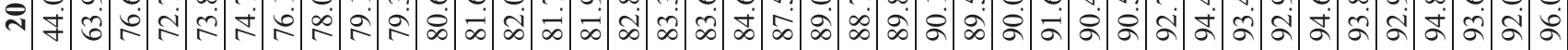

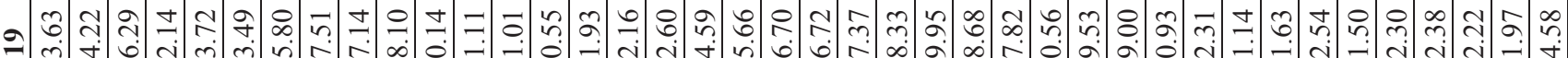

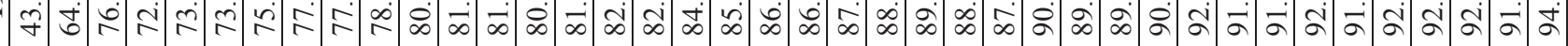

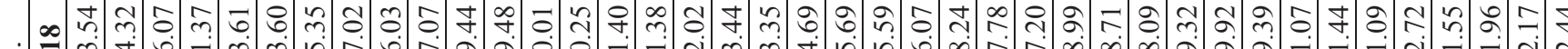

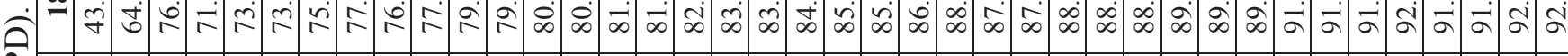

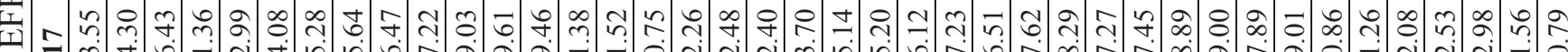

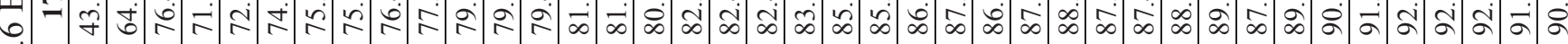

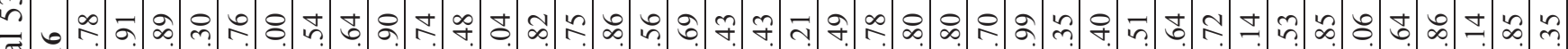
哥

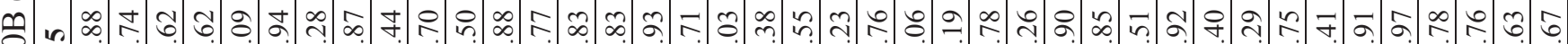

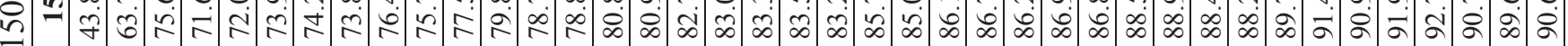
○

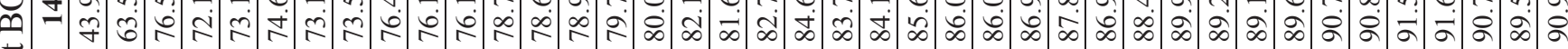
寸

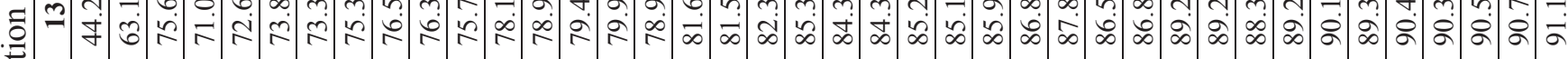

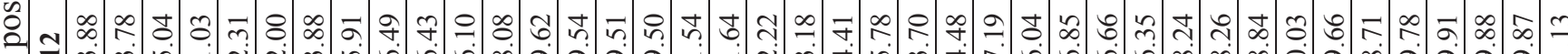

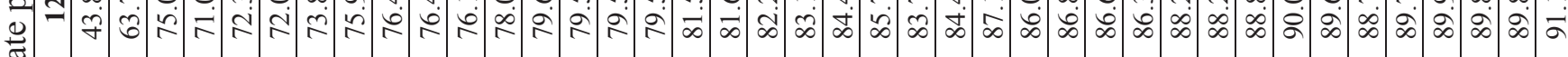

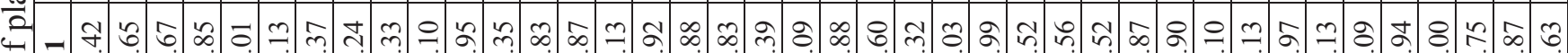

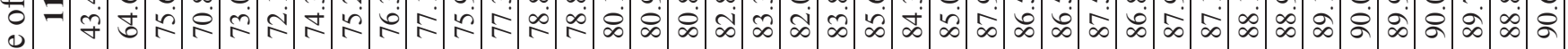

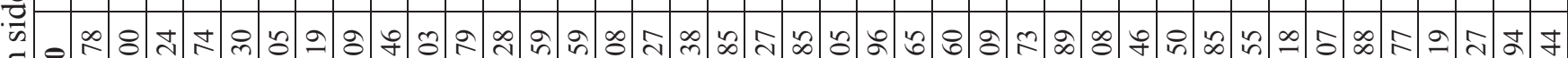
声=

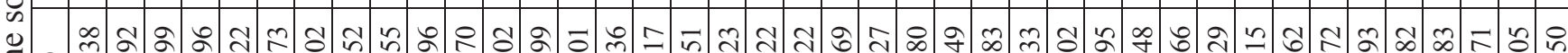

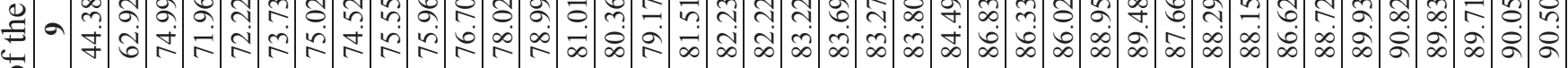

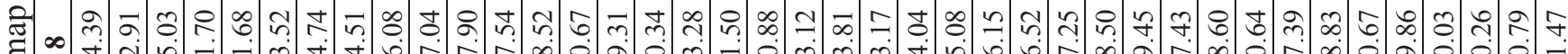

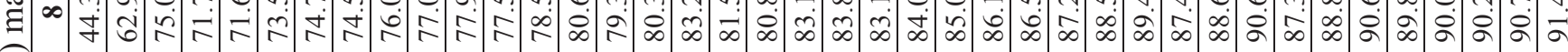
-

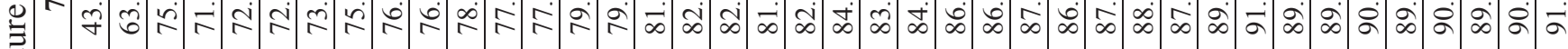

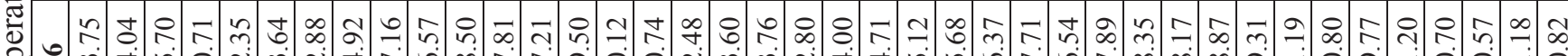

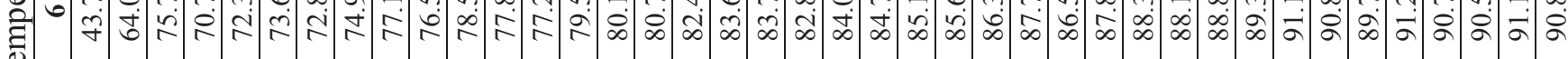

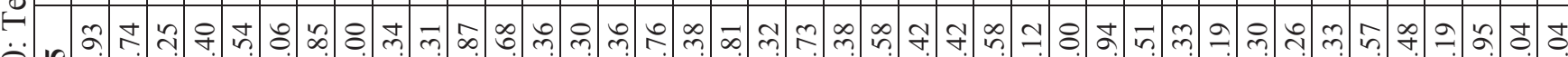

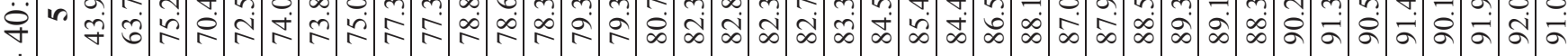

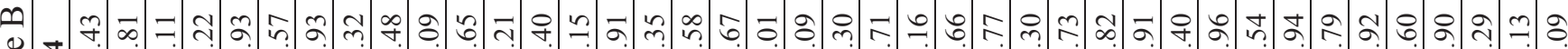
光

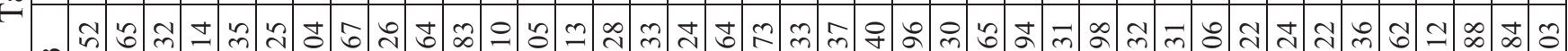

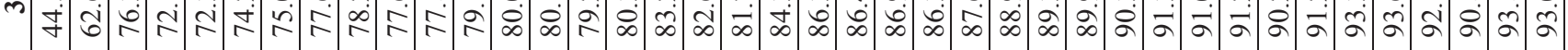

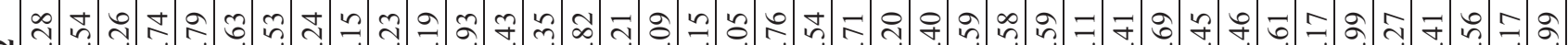
N - J - 守 它 -

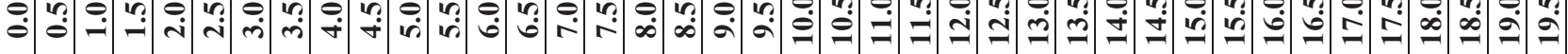




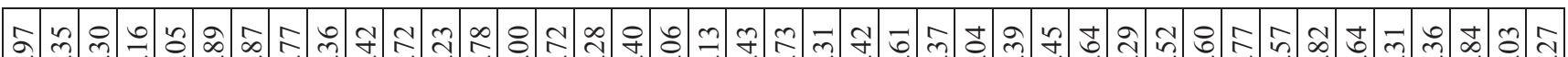

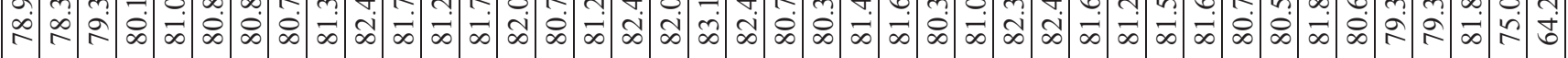
귝ำ

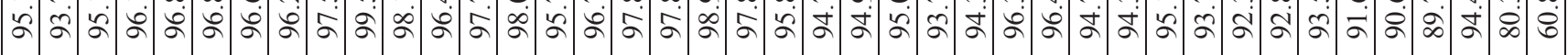

m

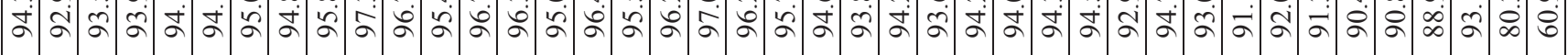

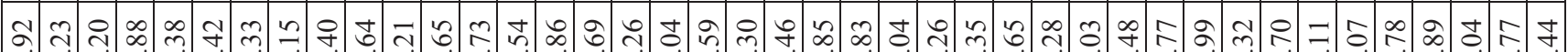

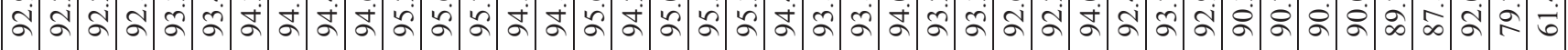

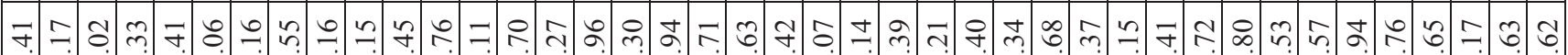

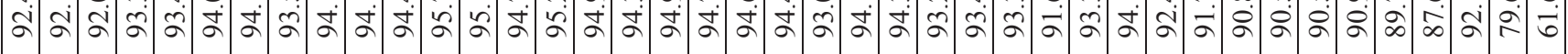

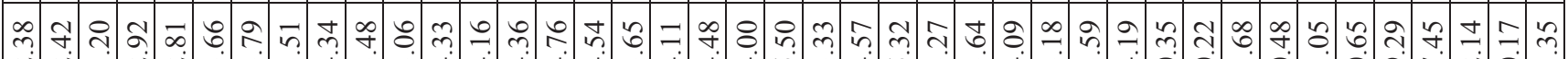

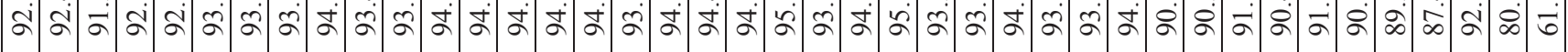
두

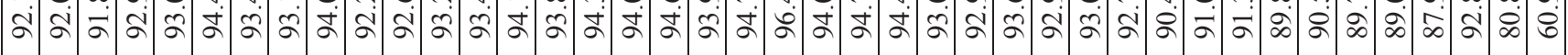

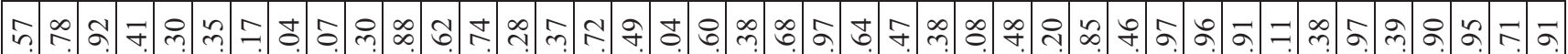

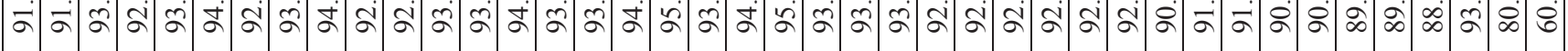
น.

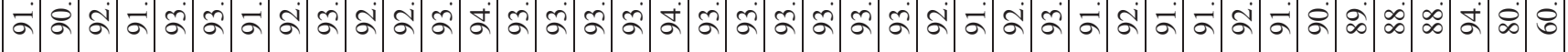

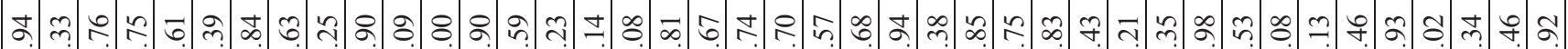

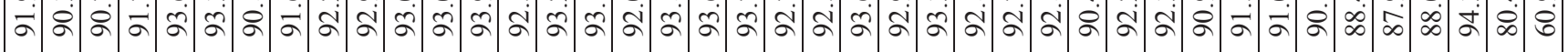
๑) ๆ

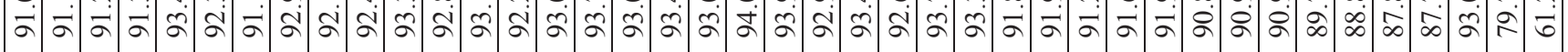

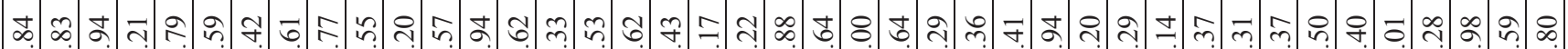

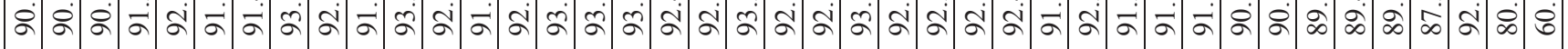

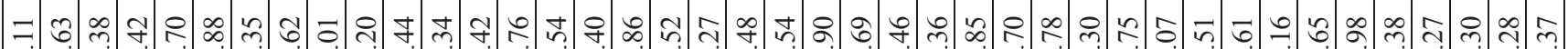

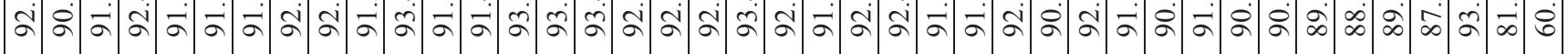

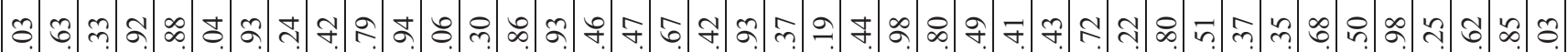

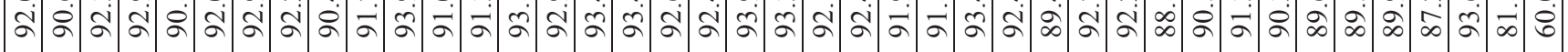
은 হं ন

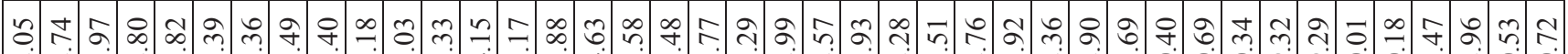

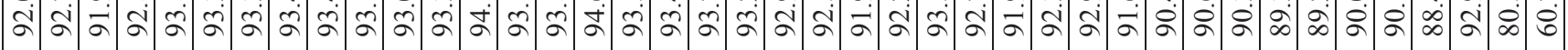

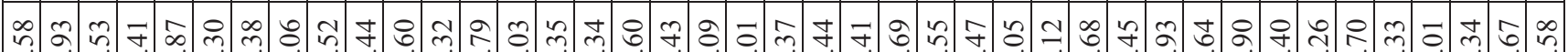

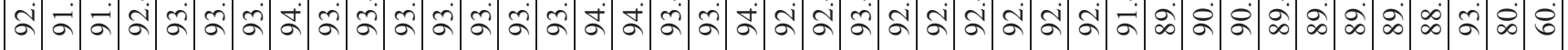

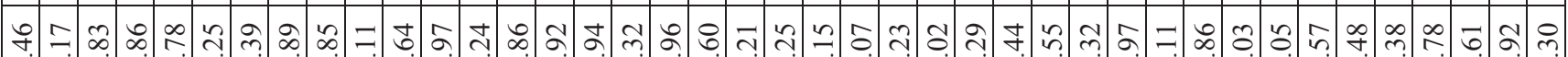

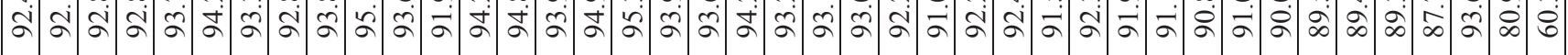

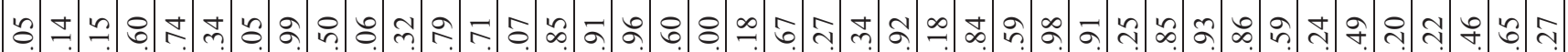

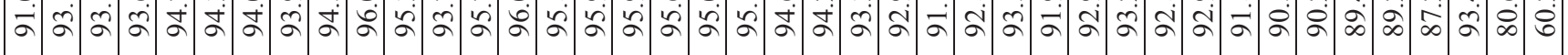
₹ ले

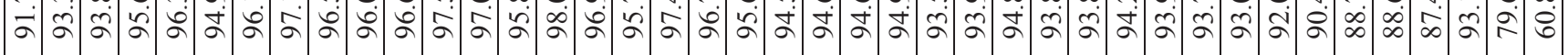
กิ

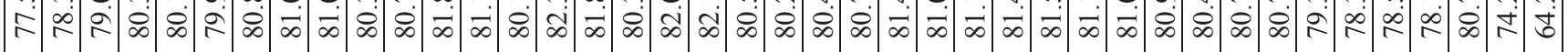




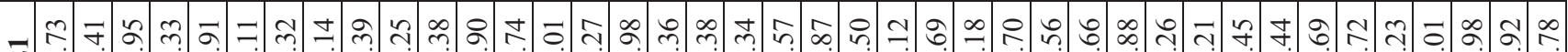

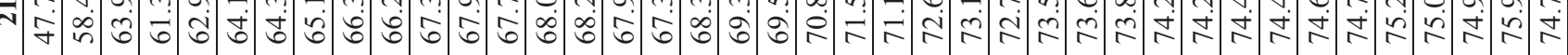

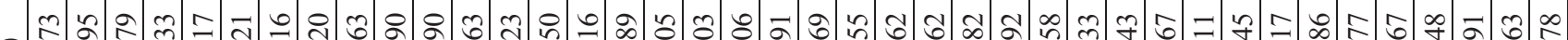

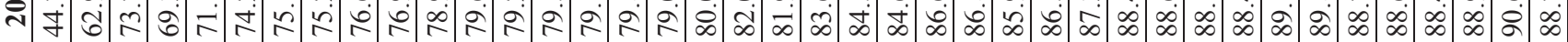

-

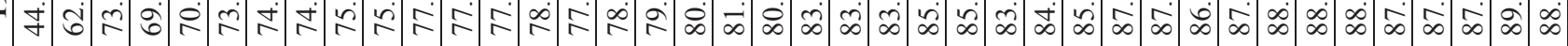

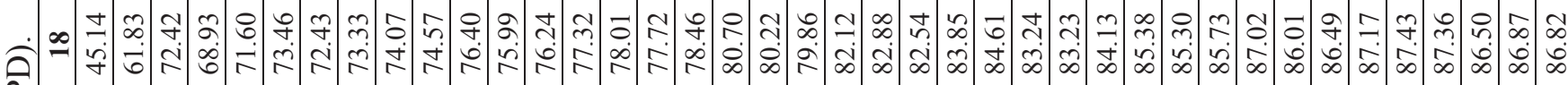

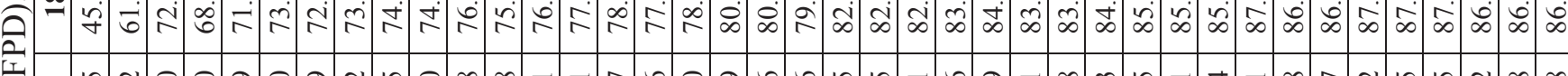

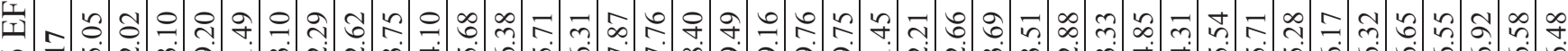
0 -

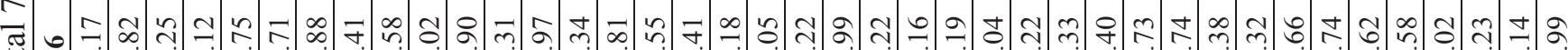

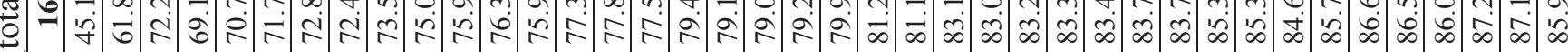

ஜ

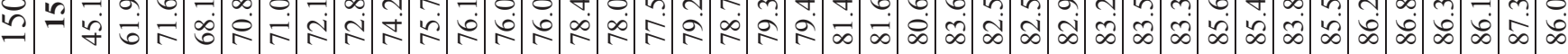
రำㅇำ

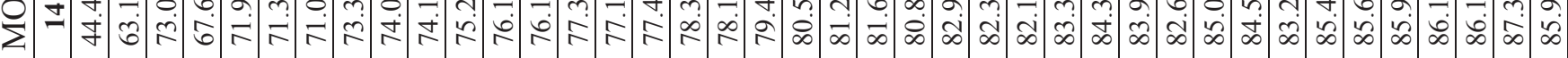
牙

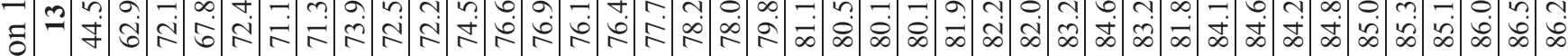
:

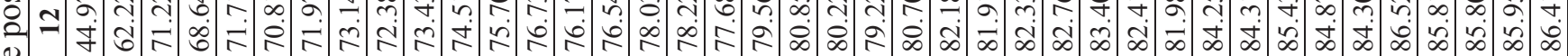
党 ㄱ Љ

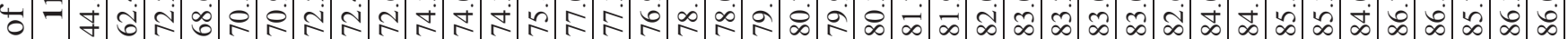
: क m

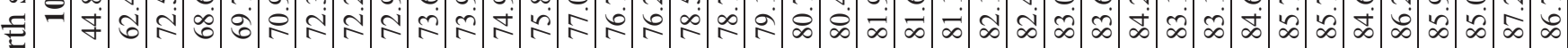
పี

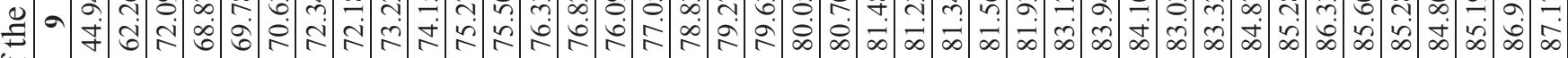

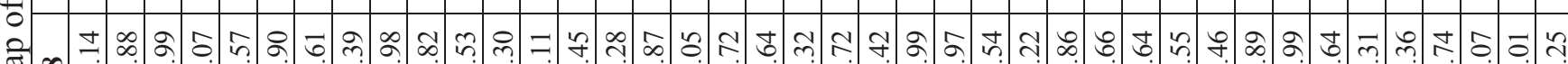
吾 $\infty$ 字

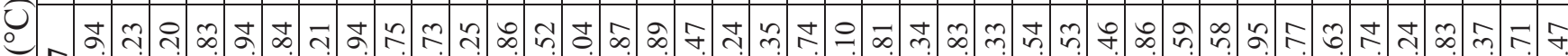

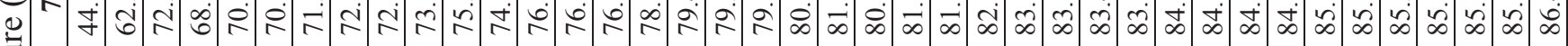

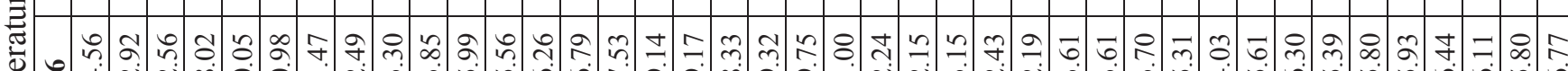

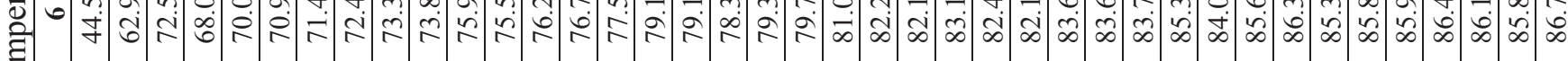

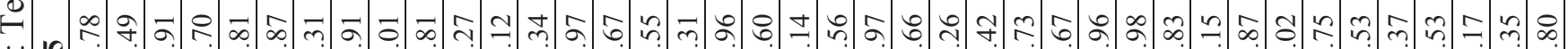

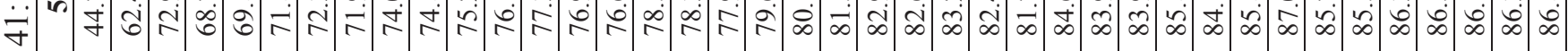

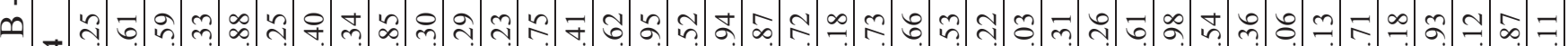

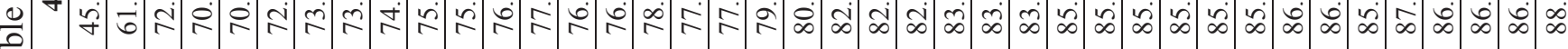
。ำ

- 웡

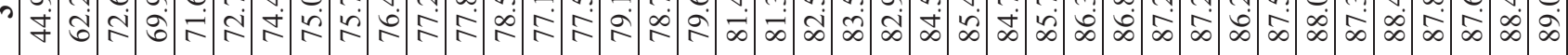
$\approx$ 约

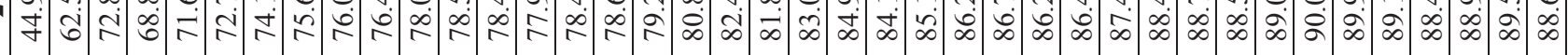

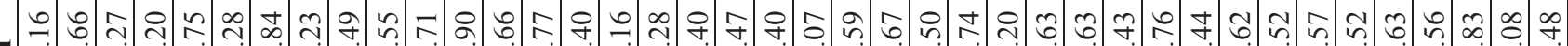

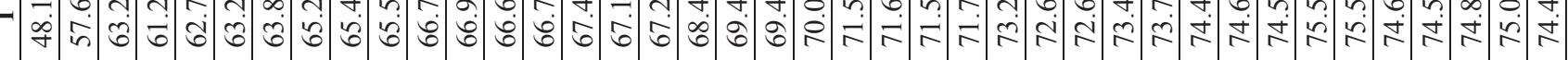

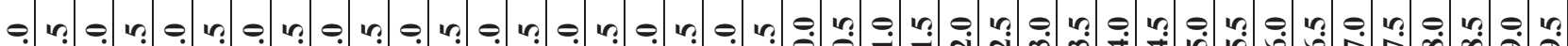

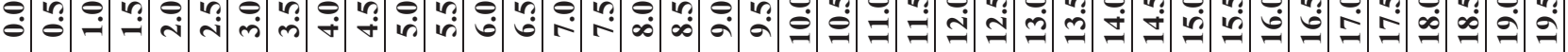




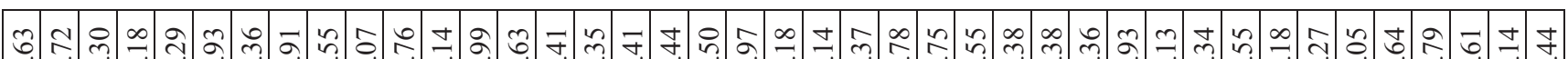

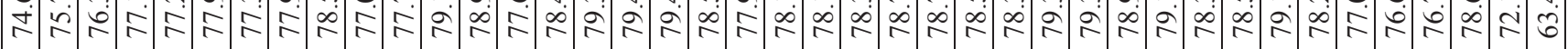
:

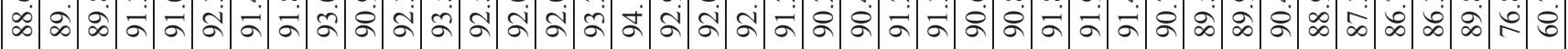

ప. के

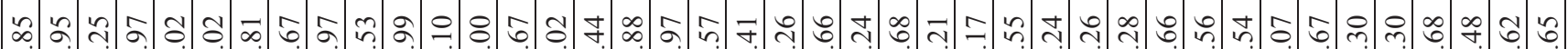

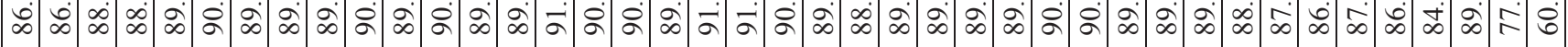

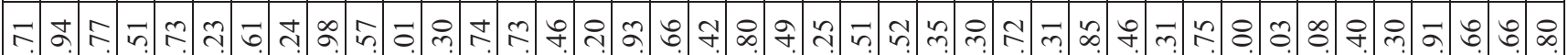

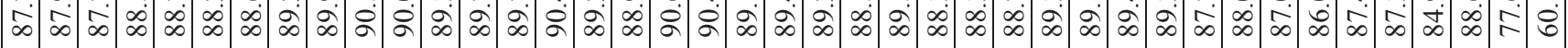

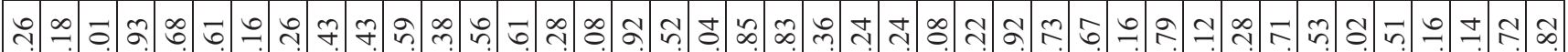

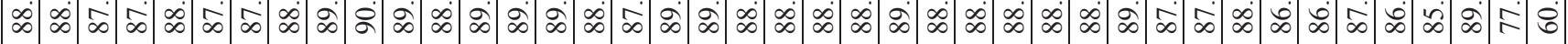

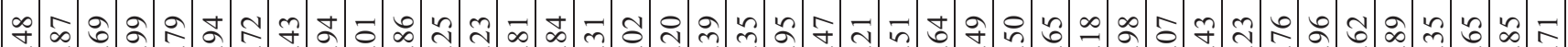

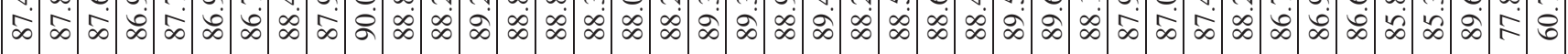

夰

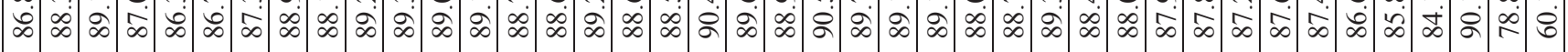

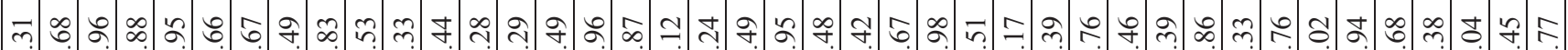

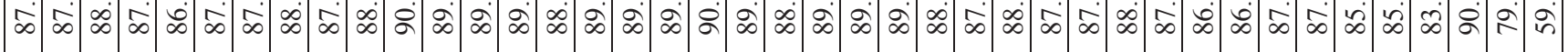

m.

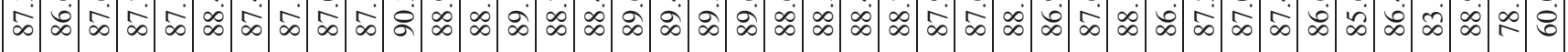

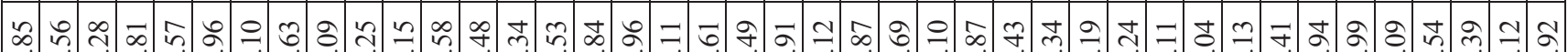

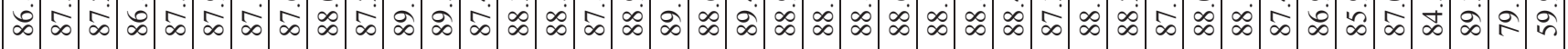

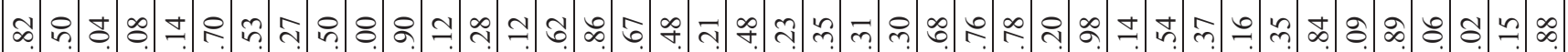

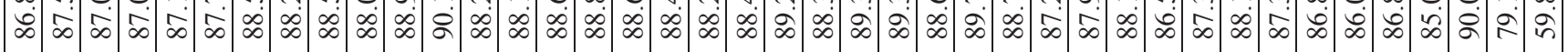

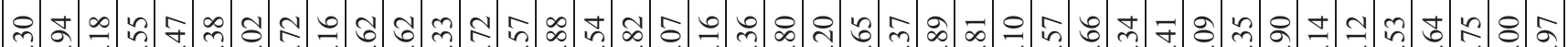

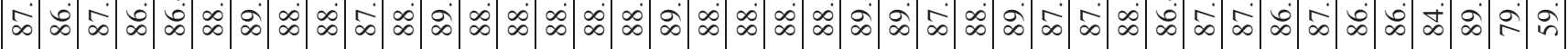

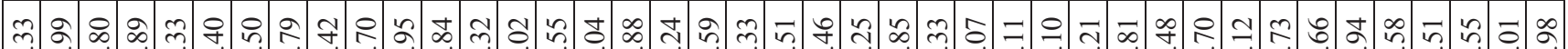

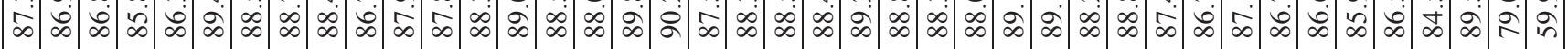
пี ㄱ. $\bar{\infty}$.

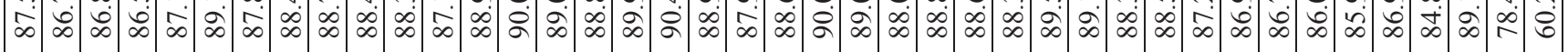

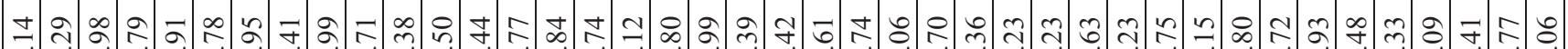

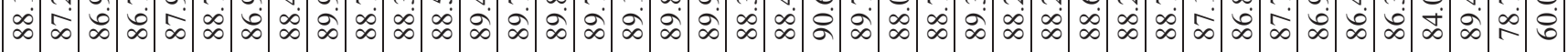

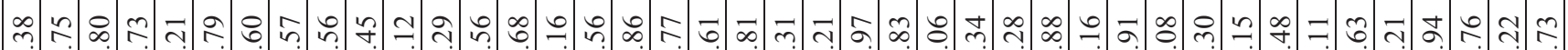

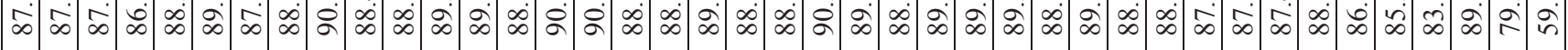

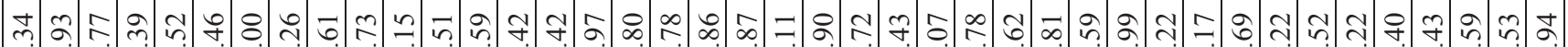

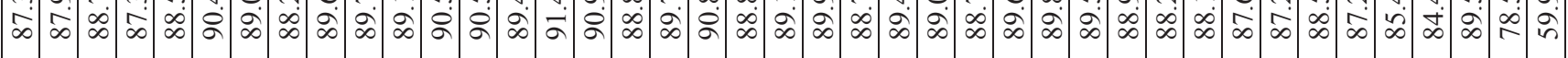

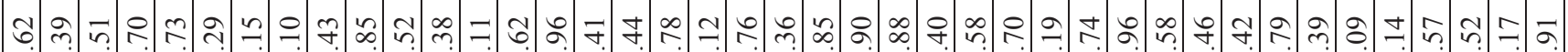

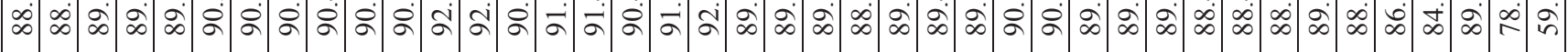

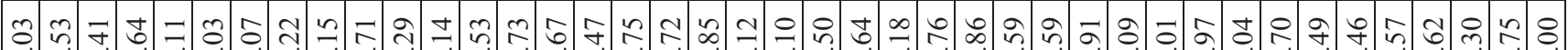

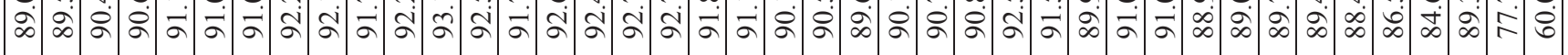

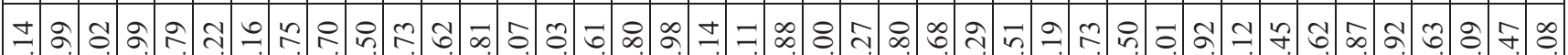

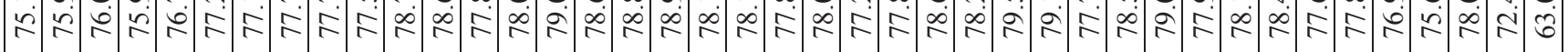
我

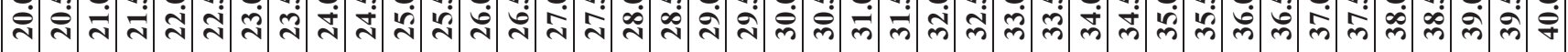


ส

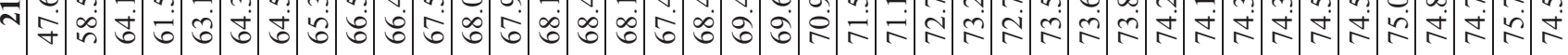
$\infty \approx \infty=0$

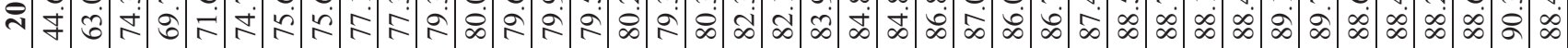

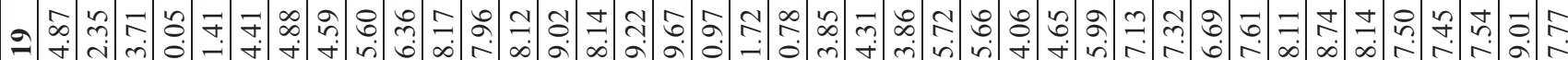
fंतु

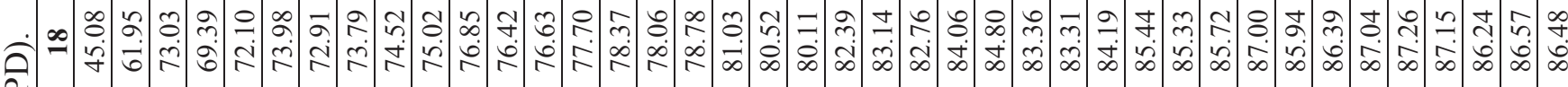

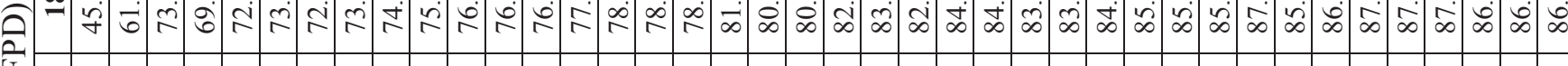
I =

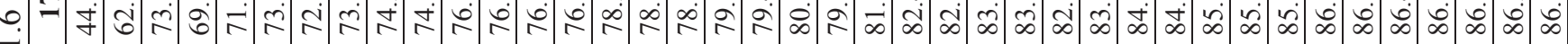
ส

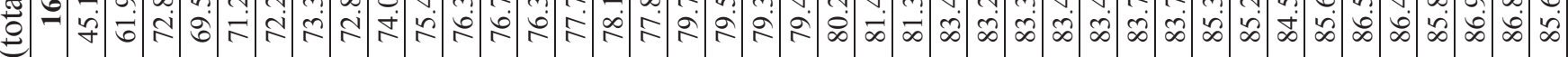

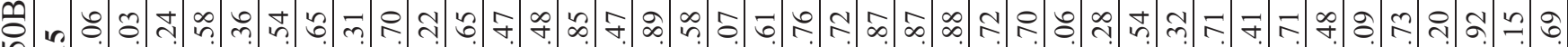

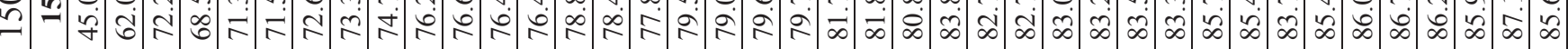

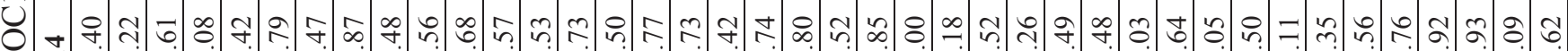

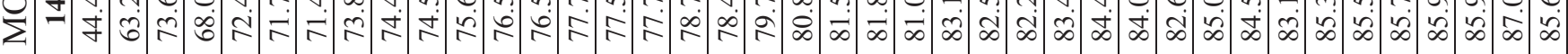
馬

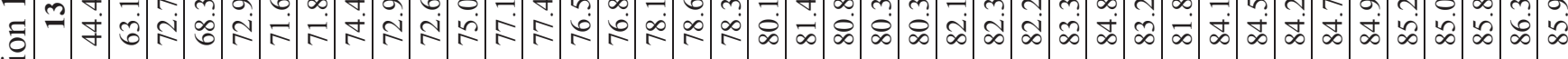

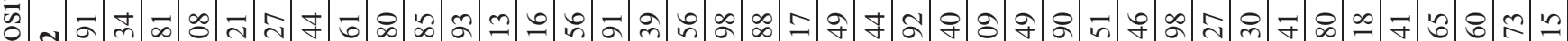

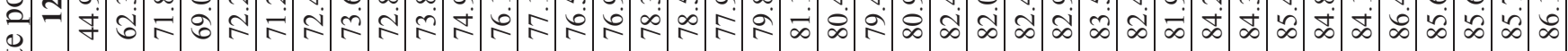

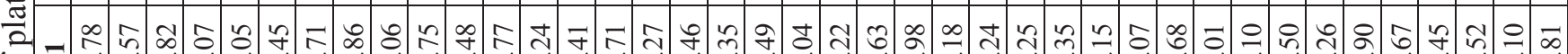

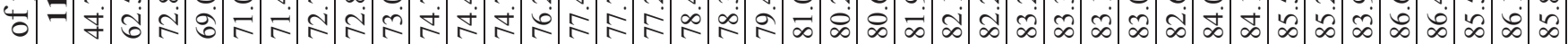
烍 ㄷำ

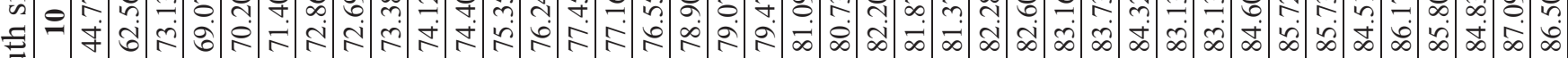

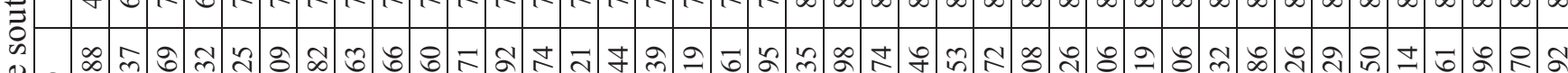

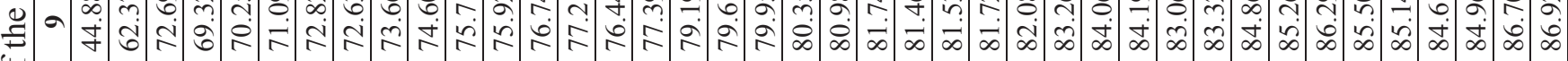

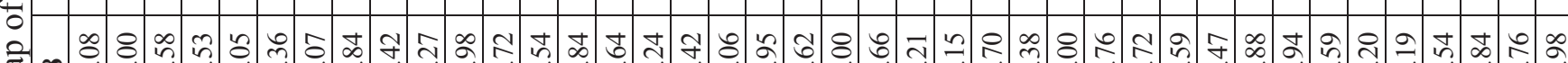

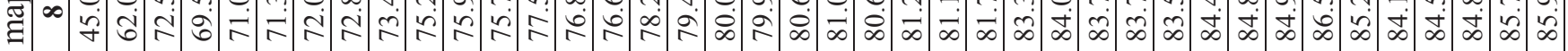
О

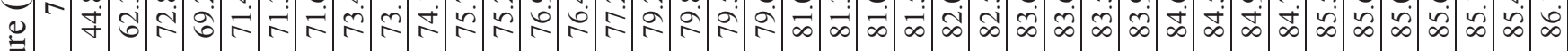

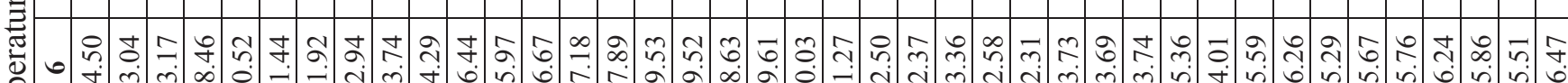

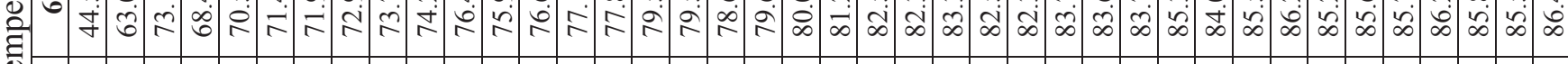

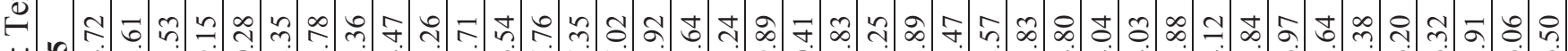
光

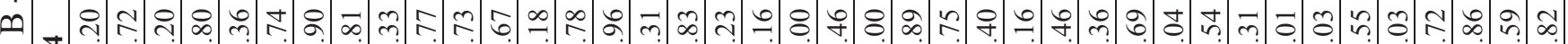

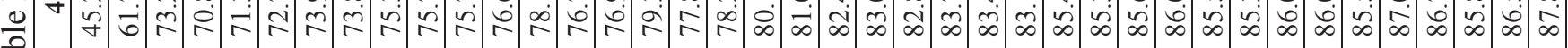
बै

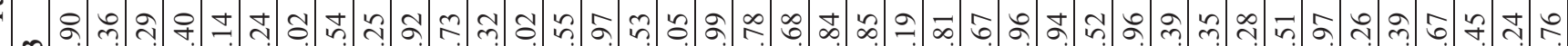

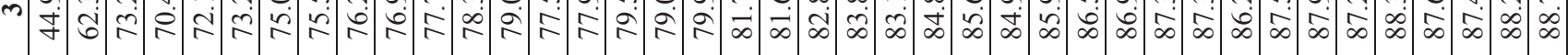

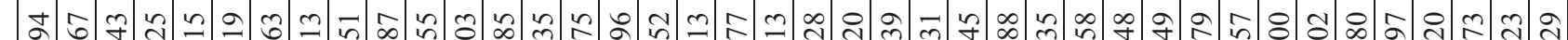

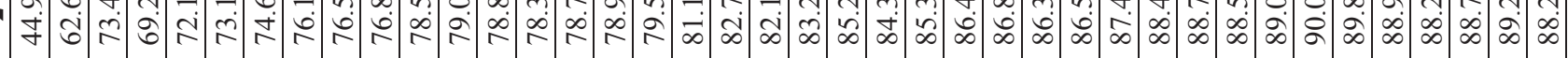
궁요

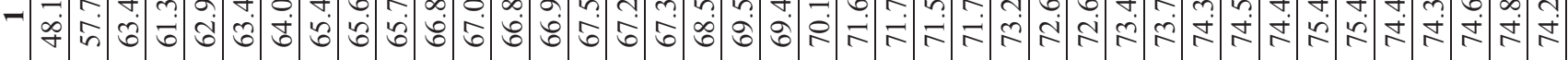
ㅇ.

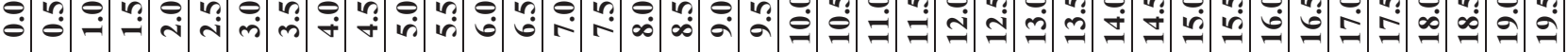


ก.

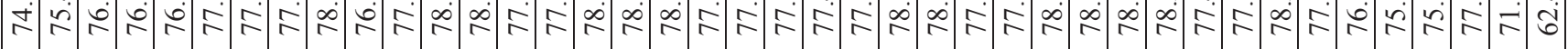

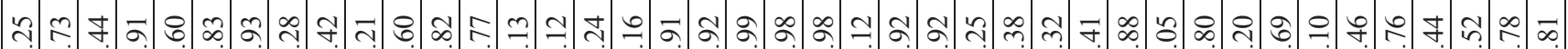

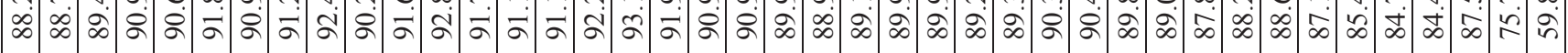

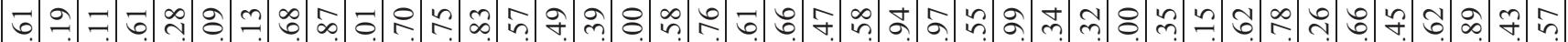

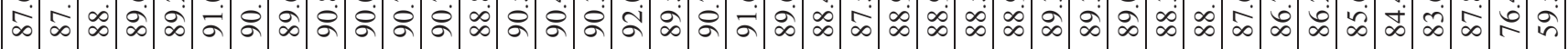

我

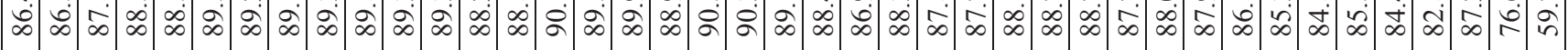

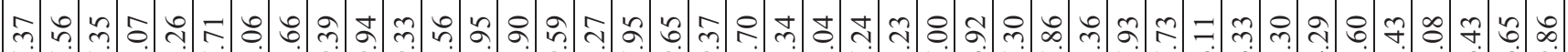

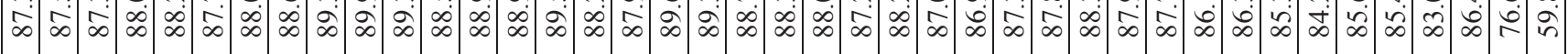

+.

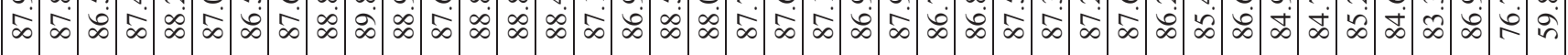

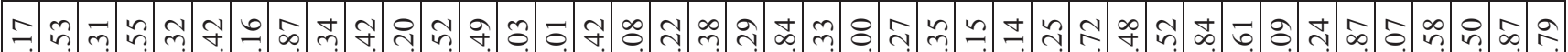

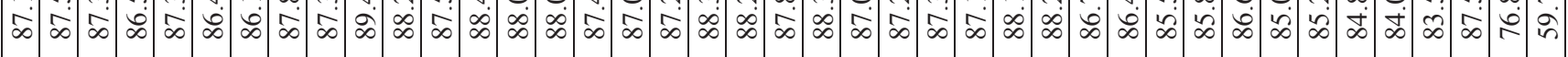

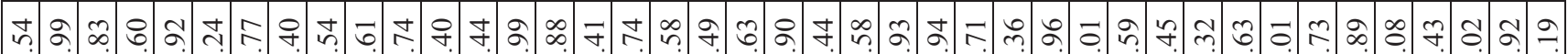

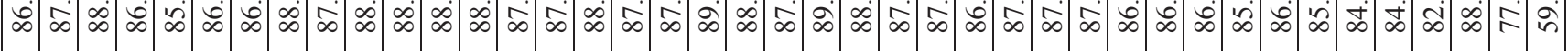

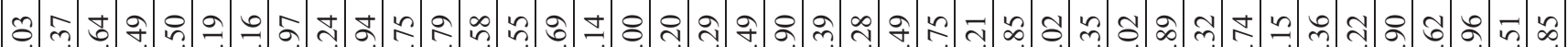

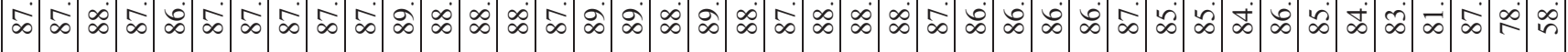

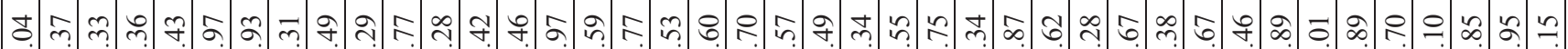

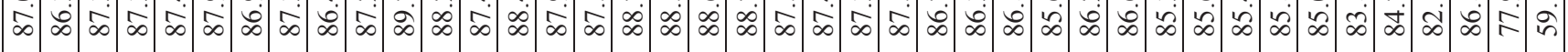

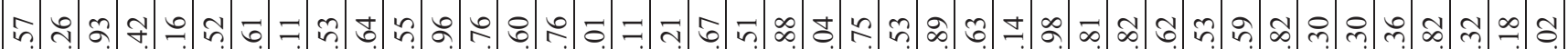

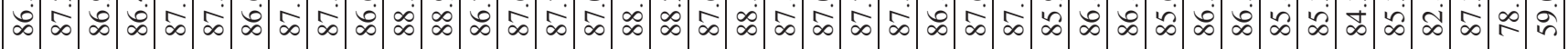

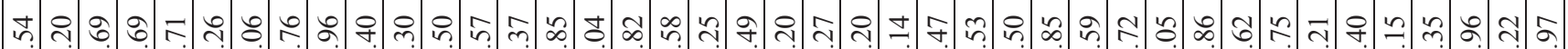

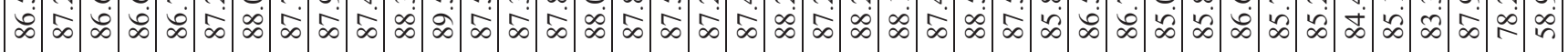

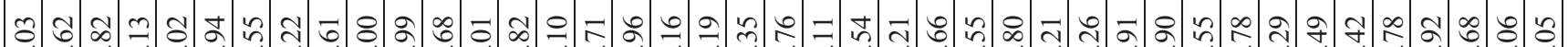

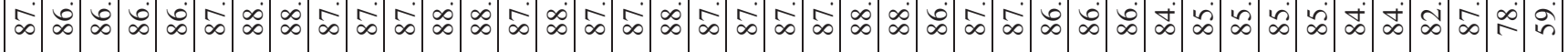

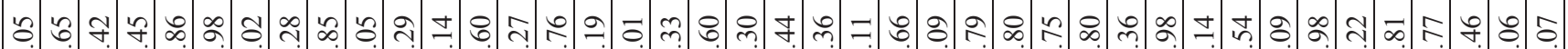

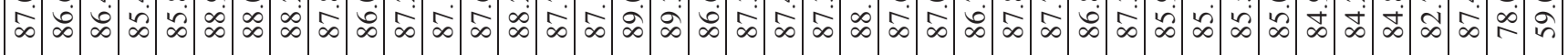

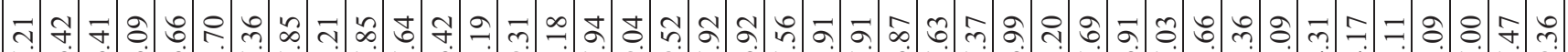

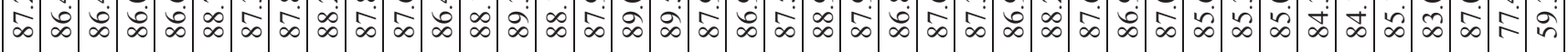
๘

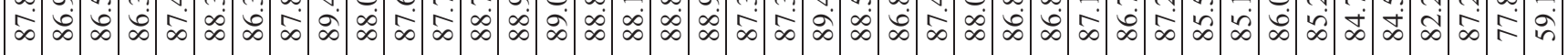
?

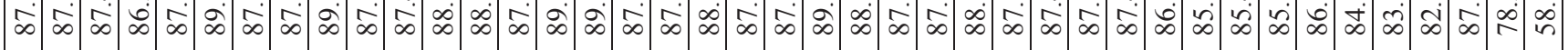

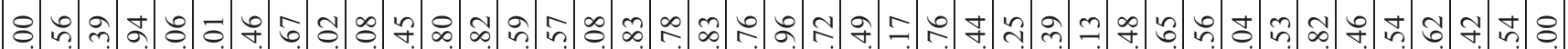

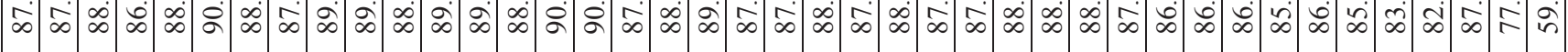

m.

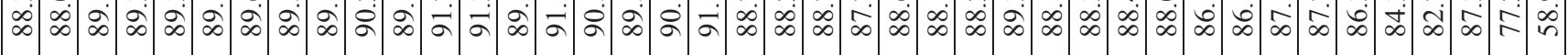

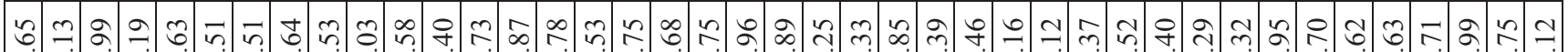

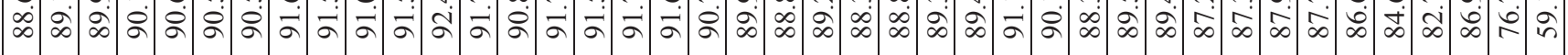

क)

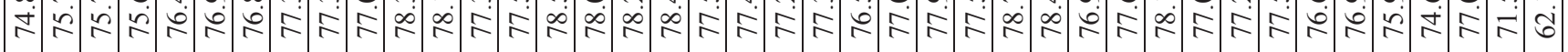
我

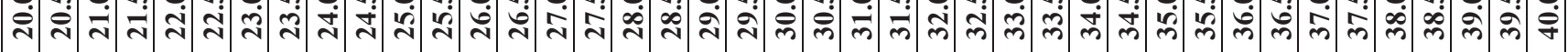


- งกำ

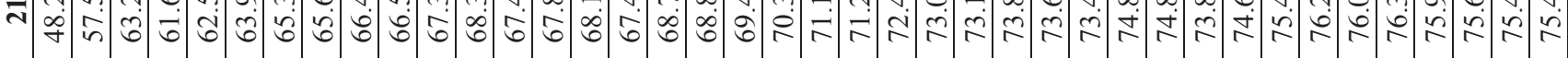

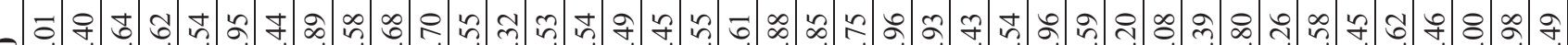
ते 字

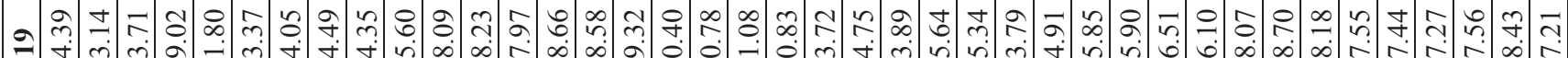

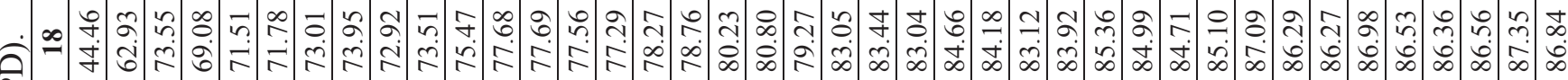

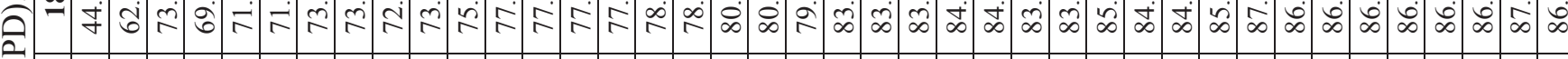
不

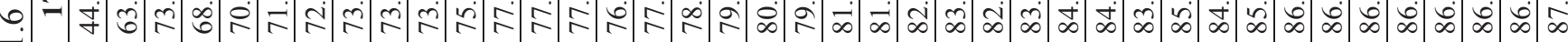

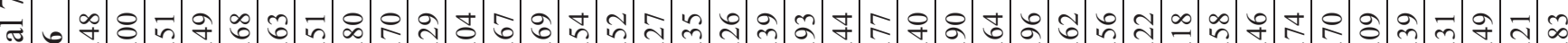

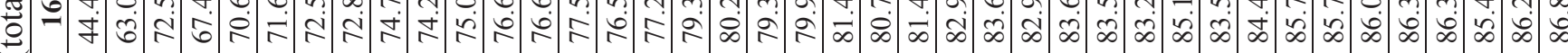

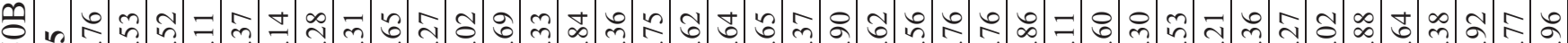

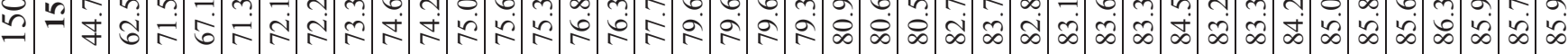
ป ஏ

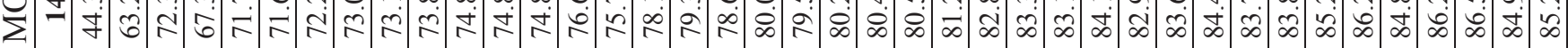

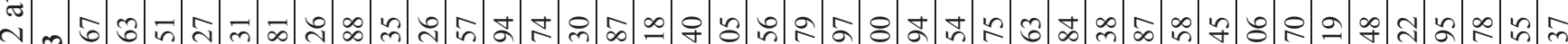

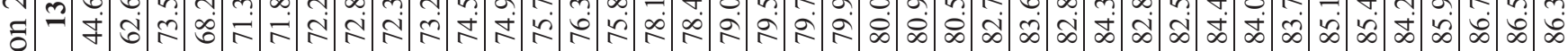
:

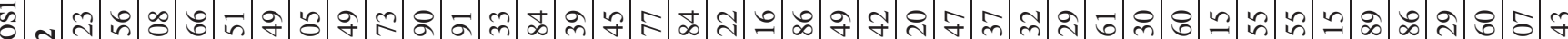

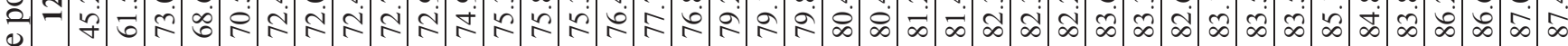

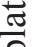

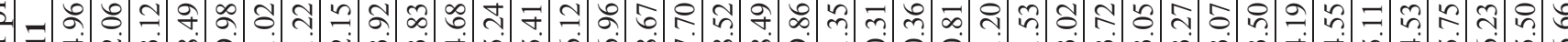
- $=$ 过

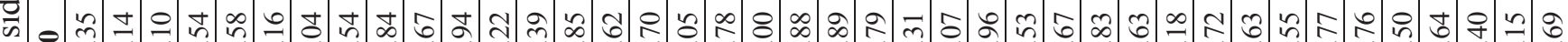

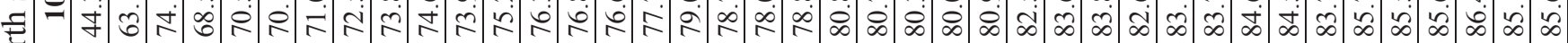
व

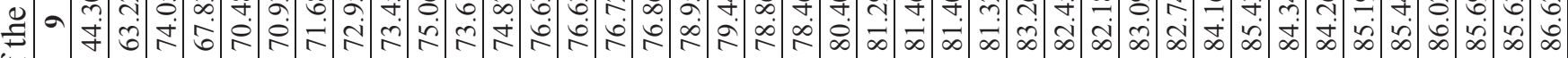

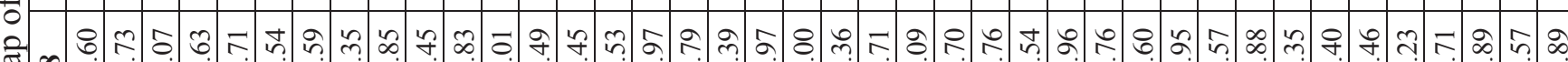

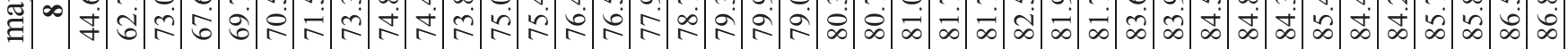

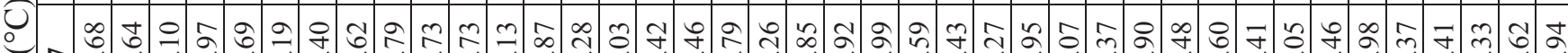

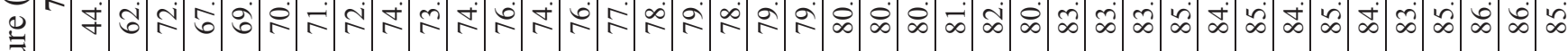

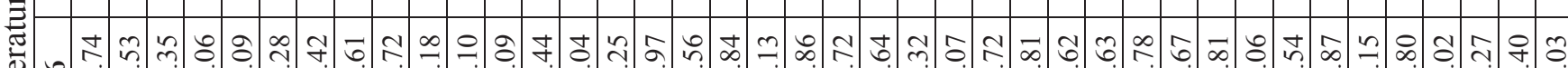
馬

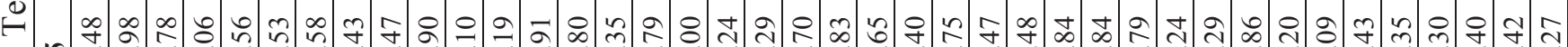

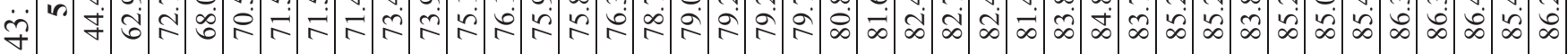
๓ -

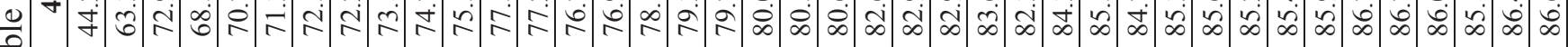
䒕

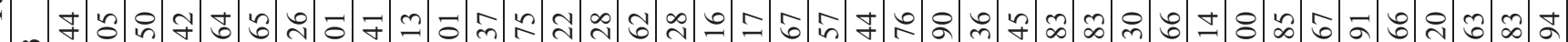

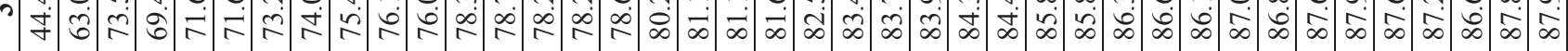

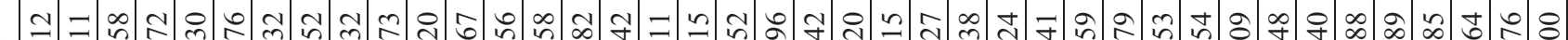
N

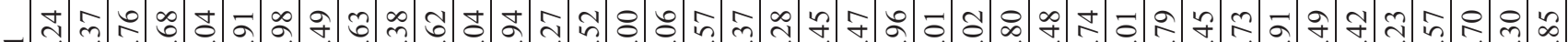

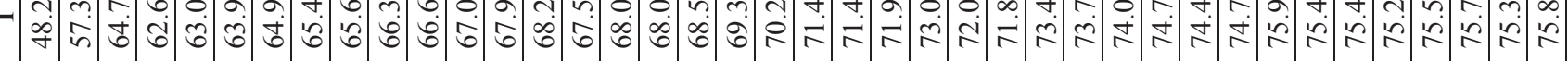

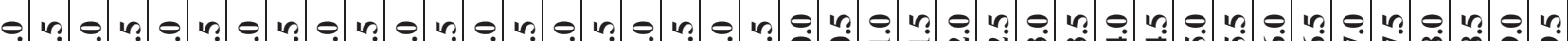

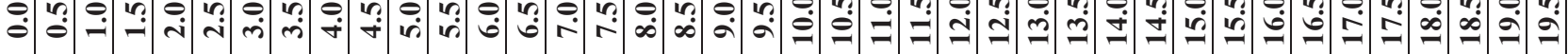




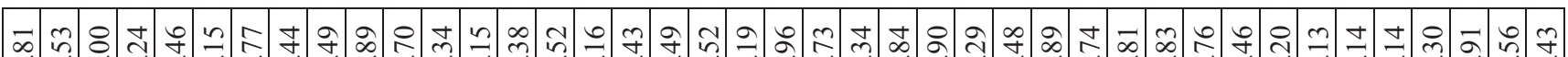

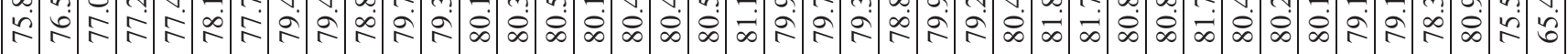

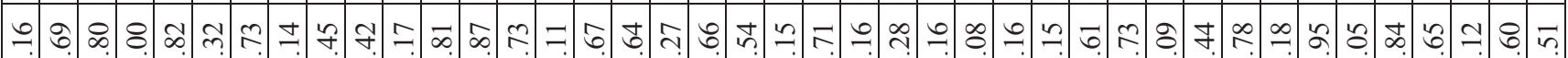

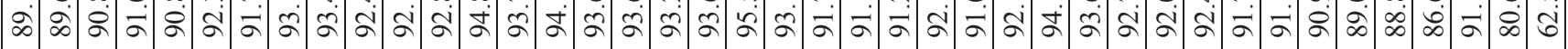

तิ

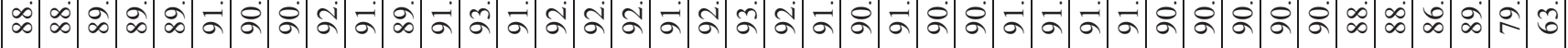

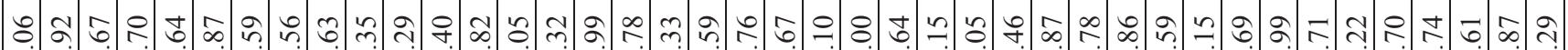

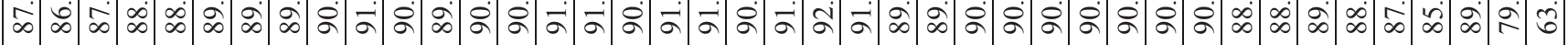

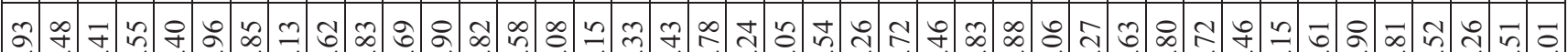

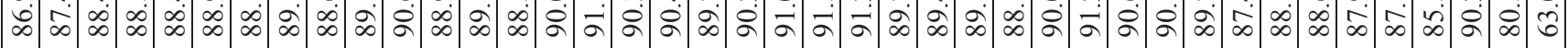

m.

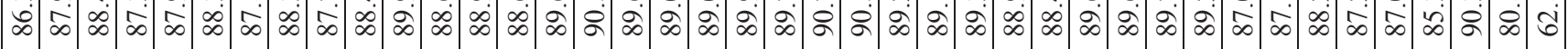

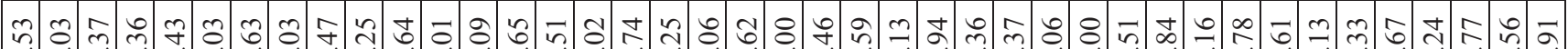

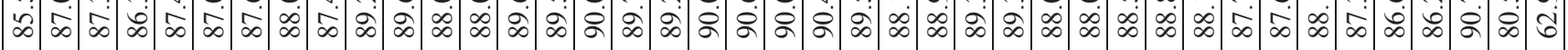

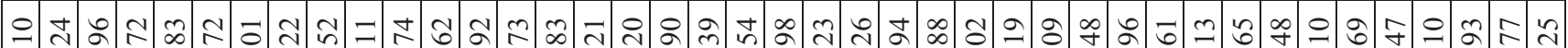

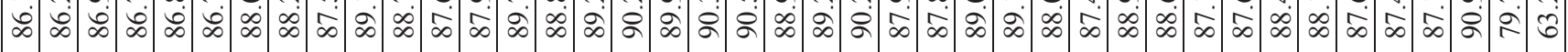

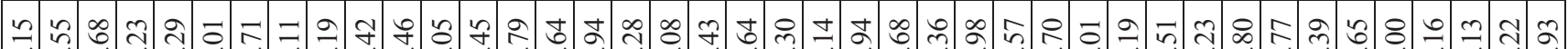

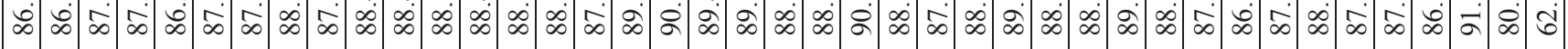

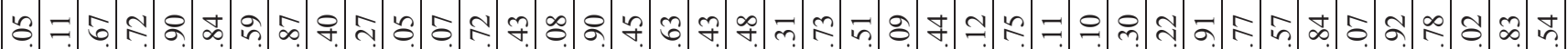

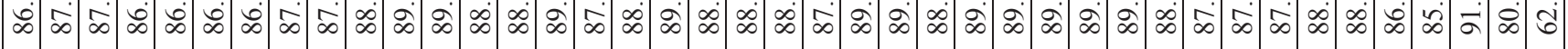

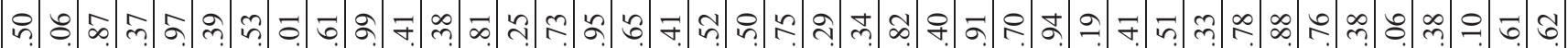

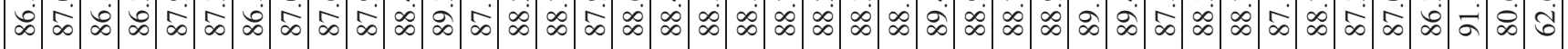

m

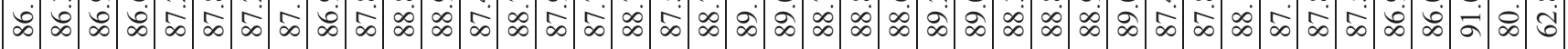

m: $\& \infty$ ๆ

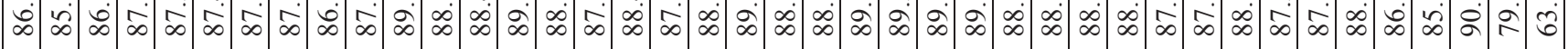

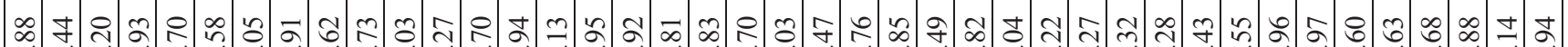

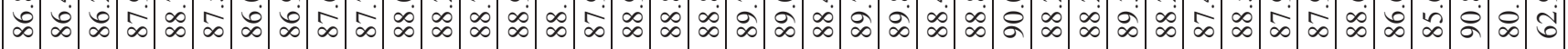
Ұ

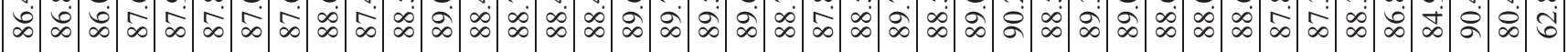
ㄷำ

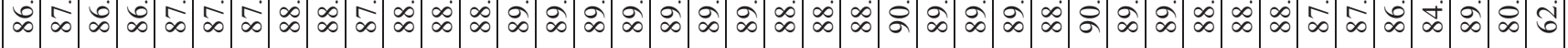

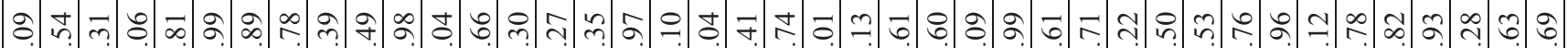

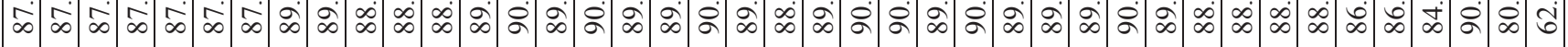

กิ ช. ป

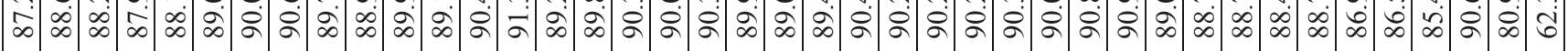

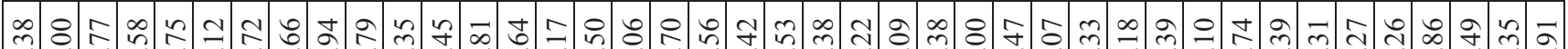

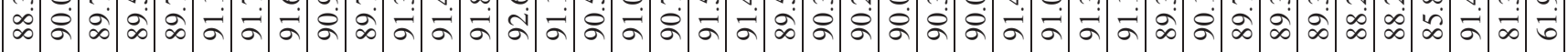

สิ f

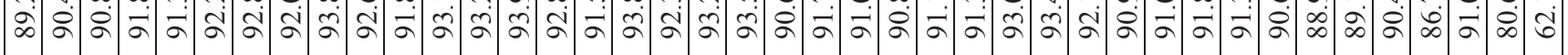
๑.

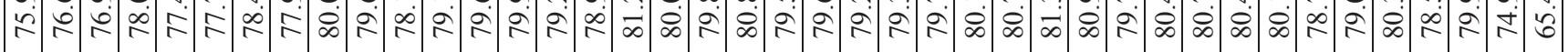




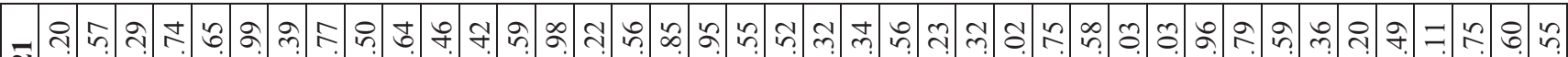
古 की

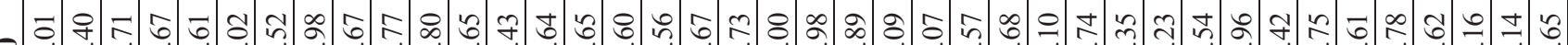
สิ

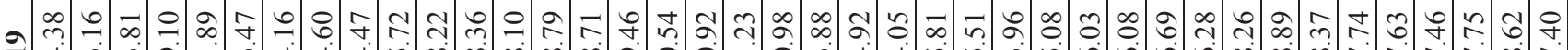

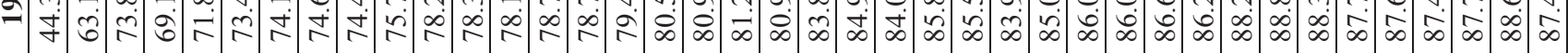

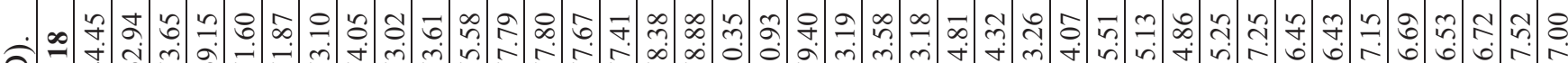

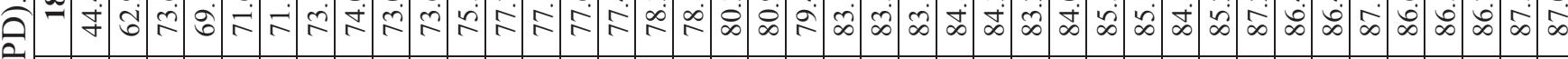

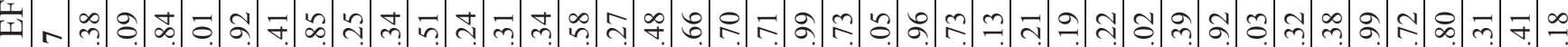

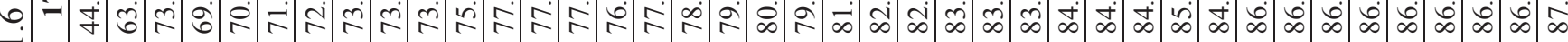

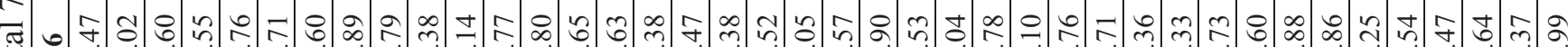

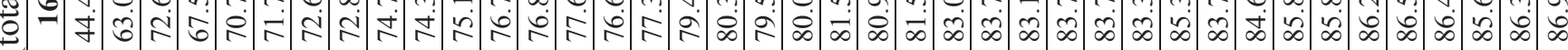

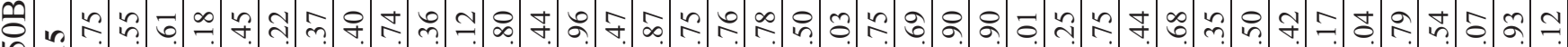

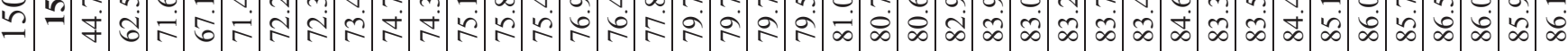

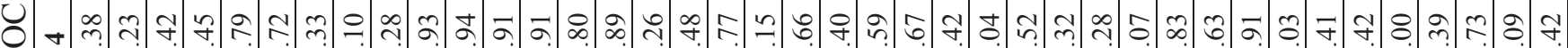

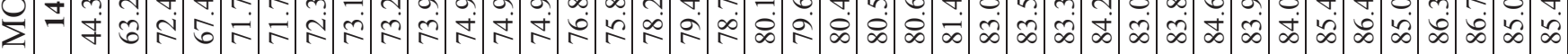
ते

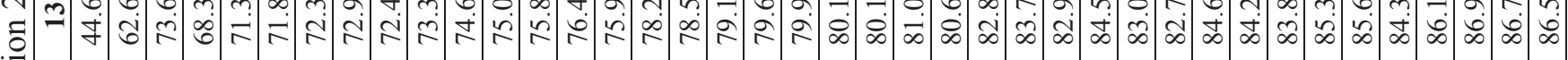
:

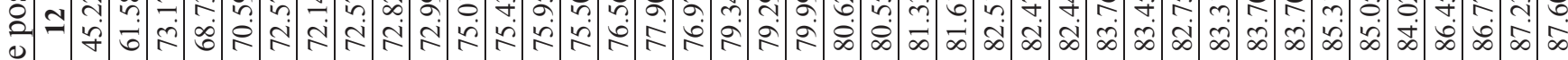
范 ב-

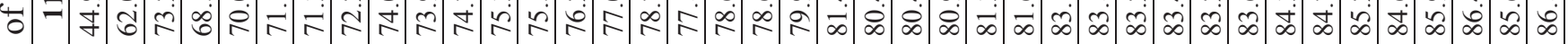
:

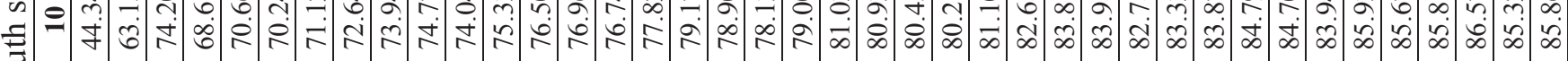

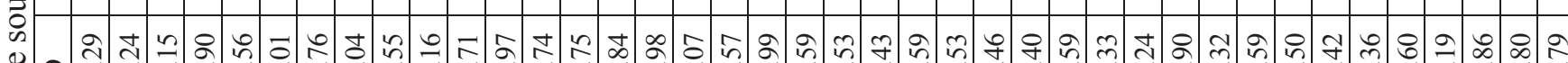
च्व

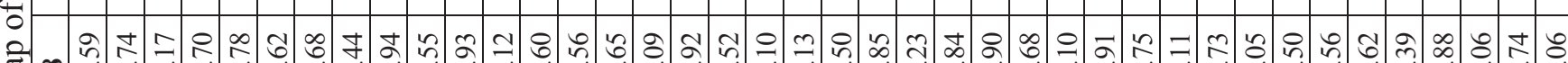

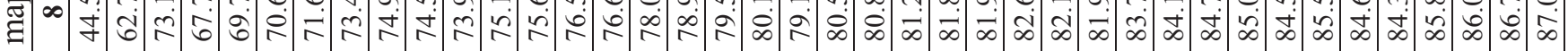

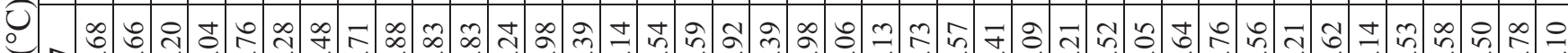

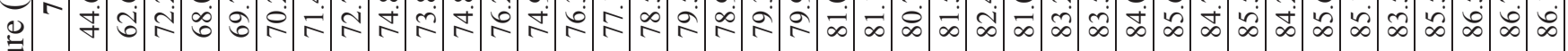

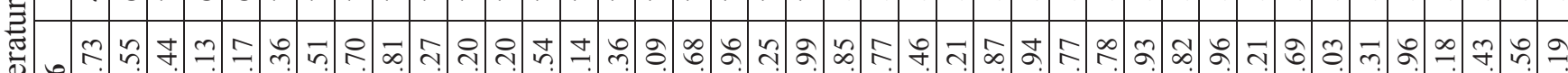

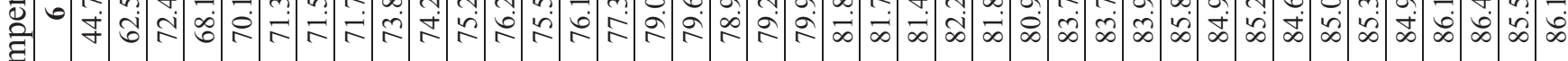

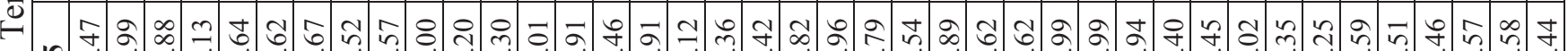

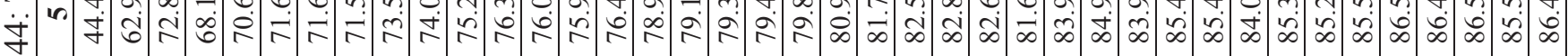
๑ -

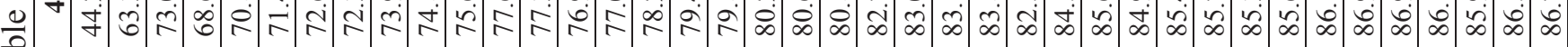
F ร $\&$ \&

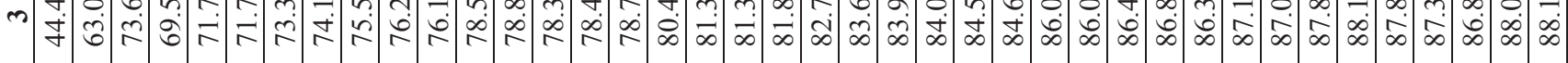
그유

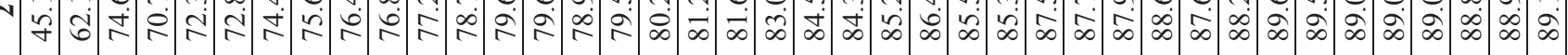

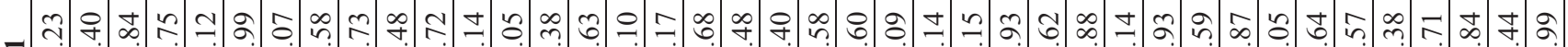
- 采 $\because$ 急 


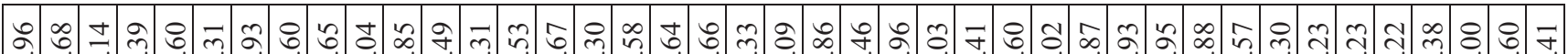

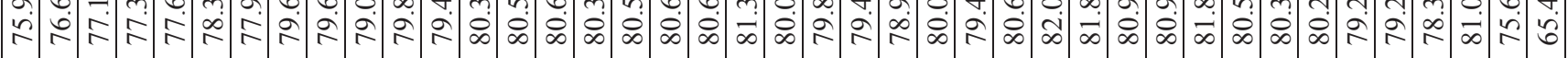
m.

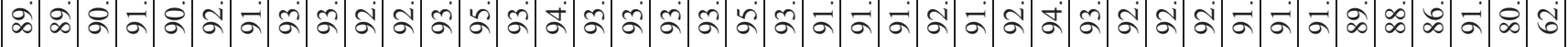

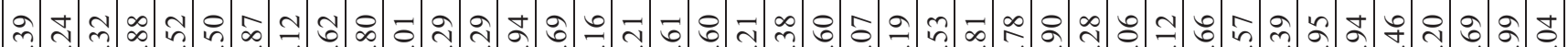

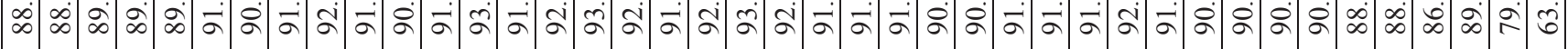

กุ 응

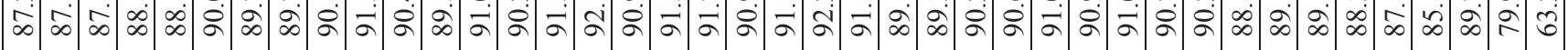

ร)

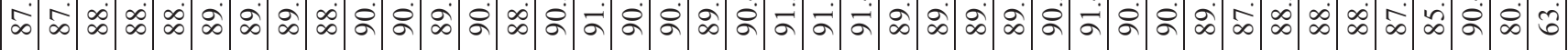

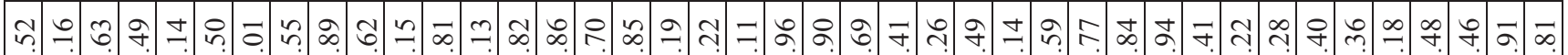

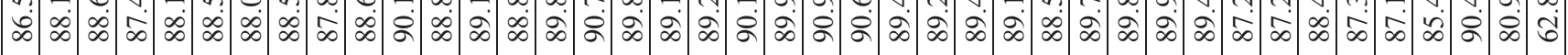

은

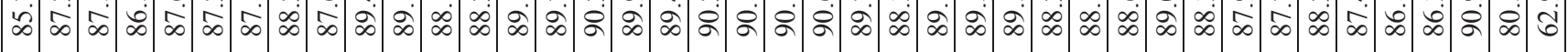

द t.

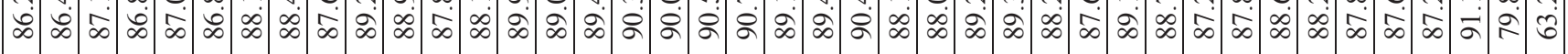

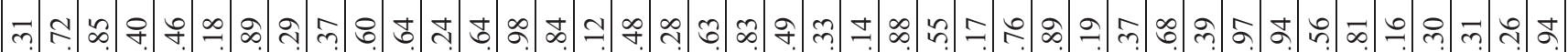

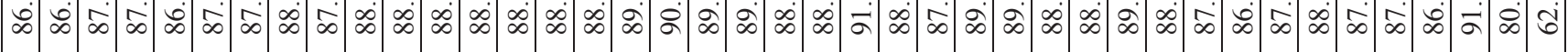

สิ

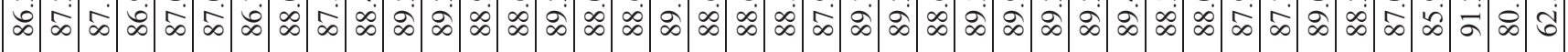
ธง

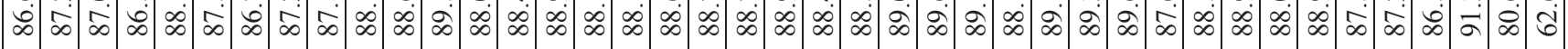

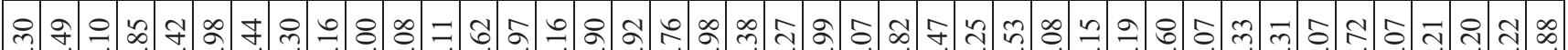

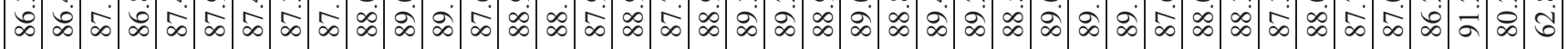

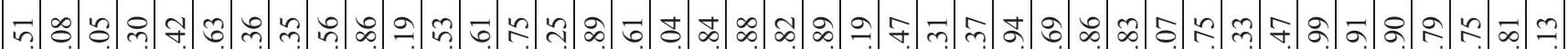

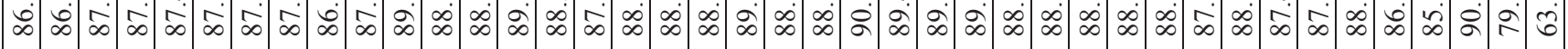
不

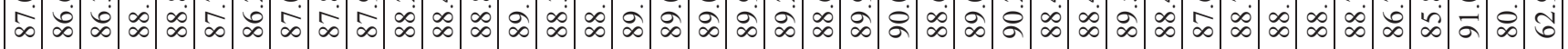

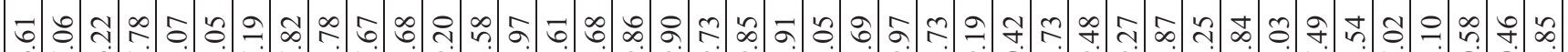

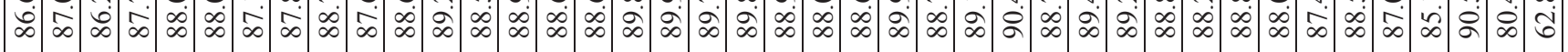

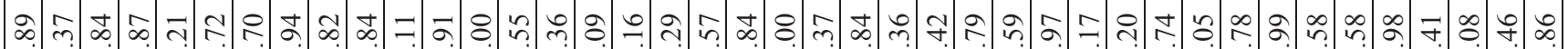

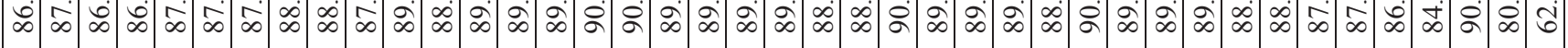

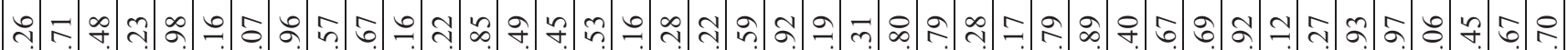

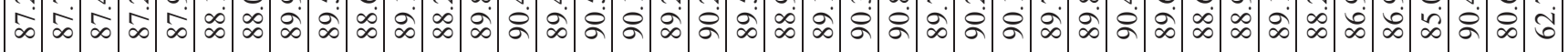
7.

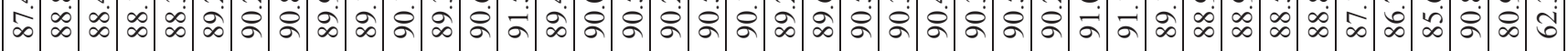

$\infty$ 궁유.

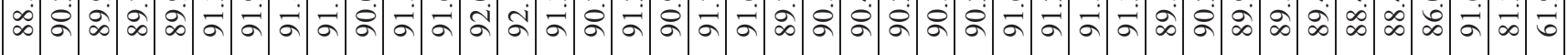

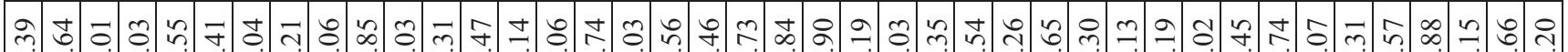

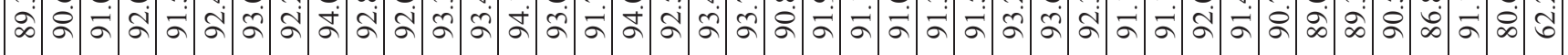
으의

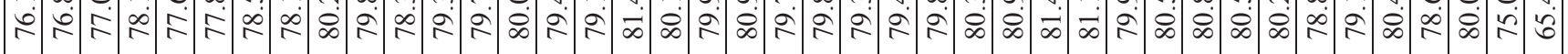

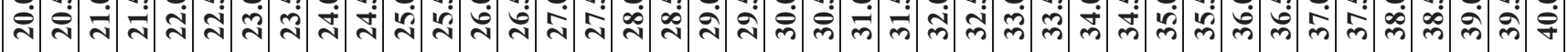


- $\infty$ क

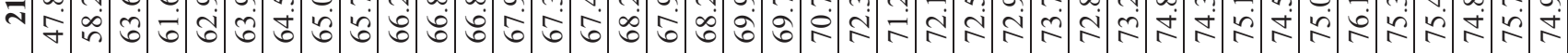
穴

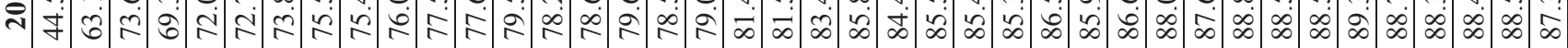

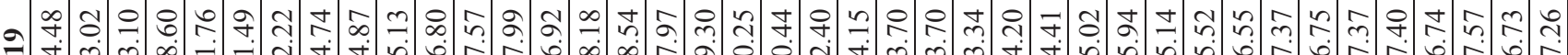
fं

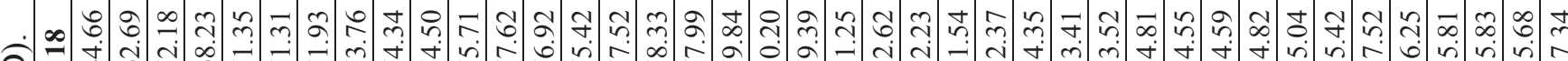

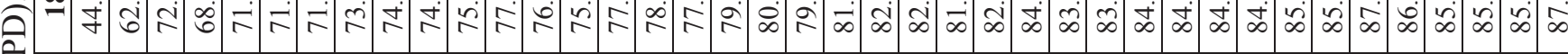
敋

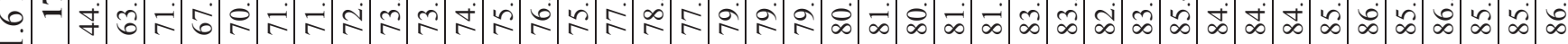
그 ㄱำ

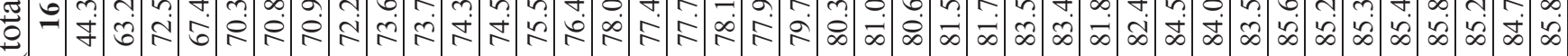

ตै ๓ กิ

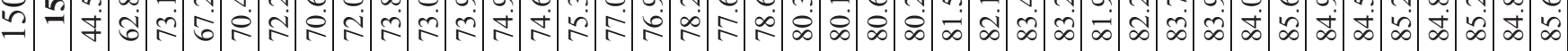

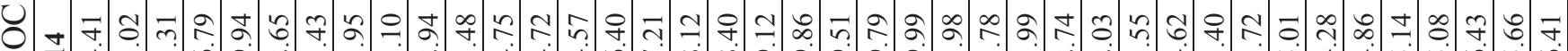

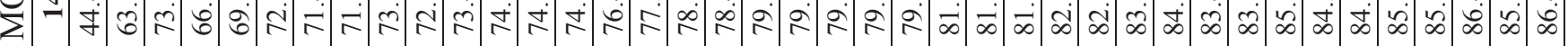

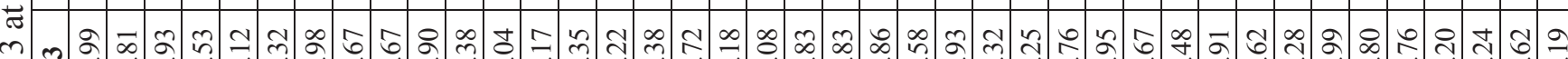

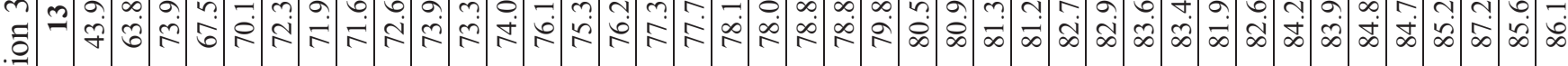
⿹ ก

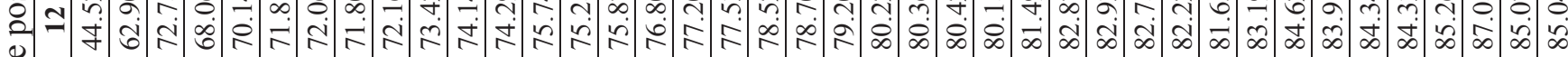
荧 ㄱ苛 =

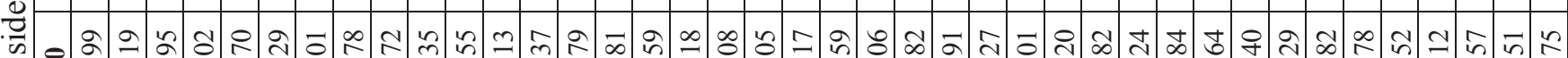

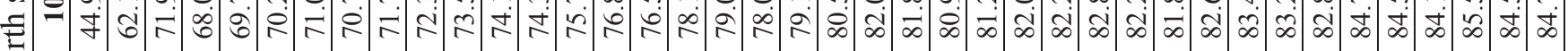

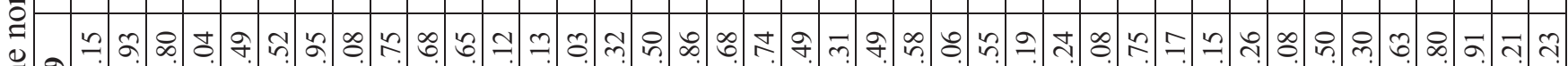

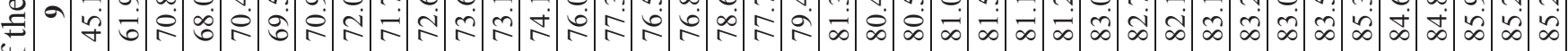

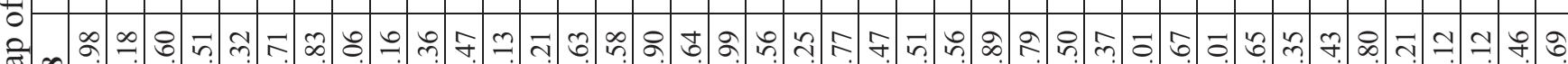

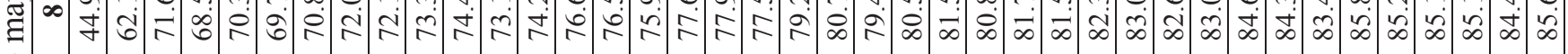
0 ⿹ 矛

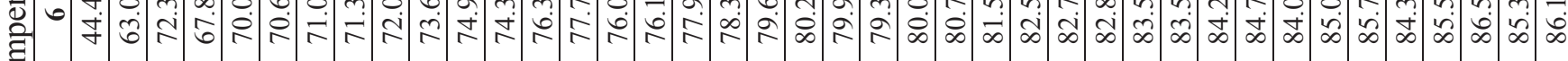

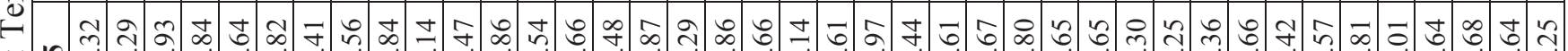

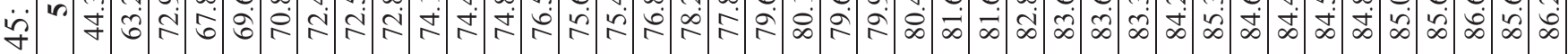

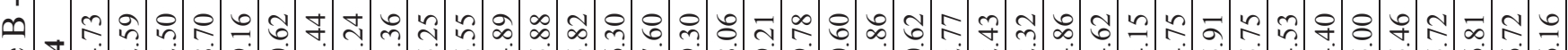

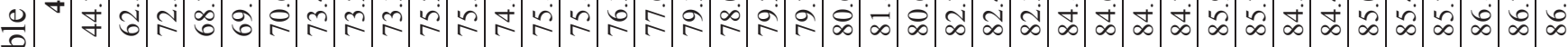
$\frac{\pi}{0}$

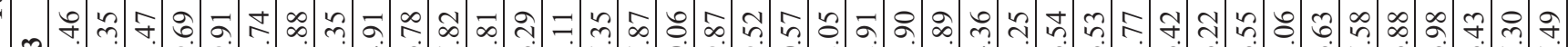

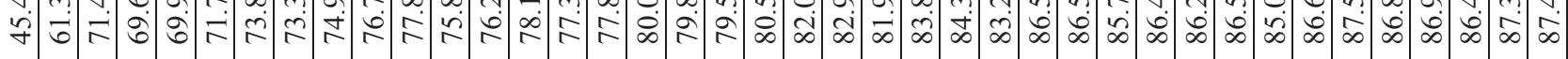
兄

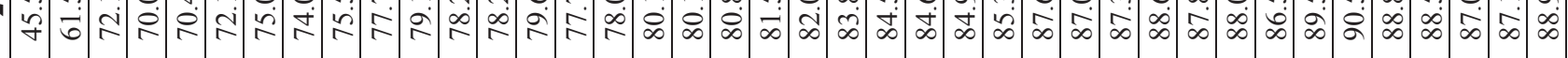

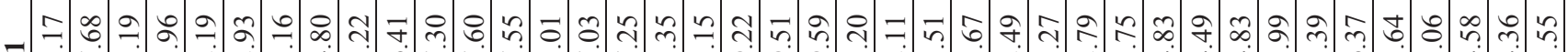

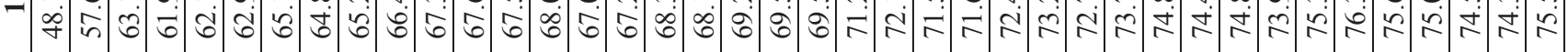
$\because$ 急 


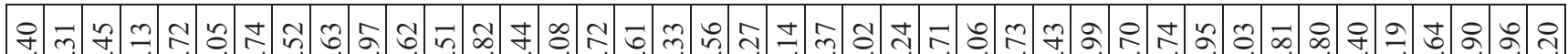

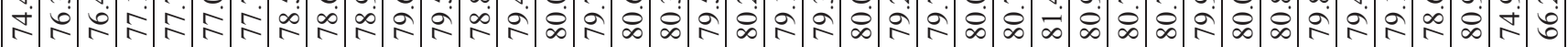

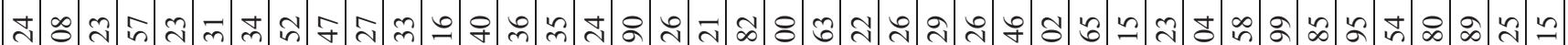

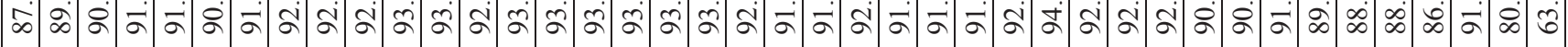

๑ ๆ இ கे

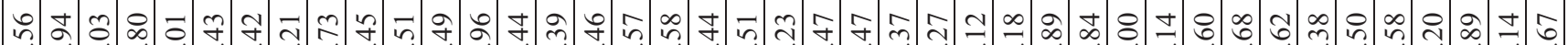

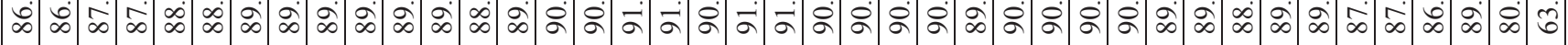

के

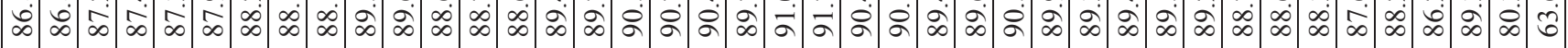

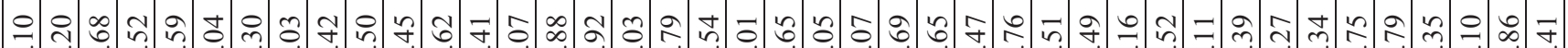

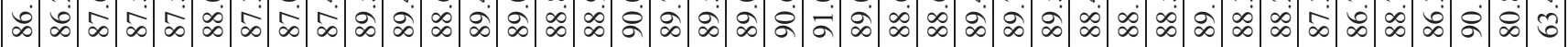

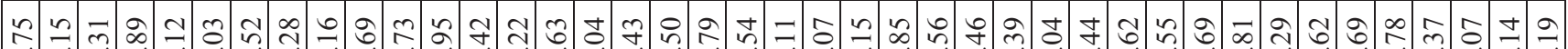

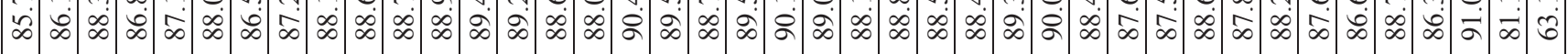

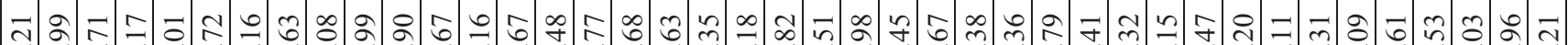

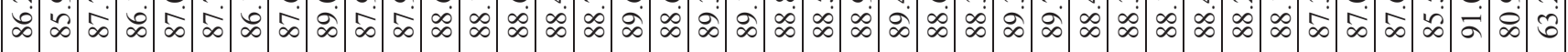

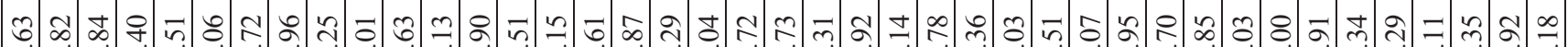

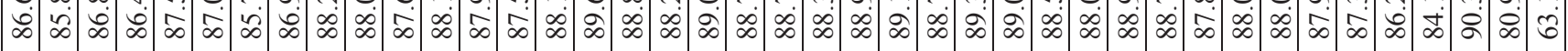

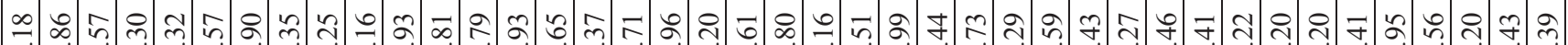

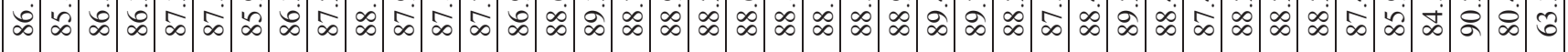

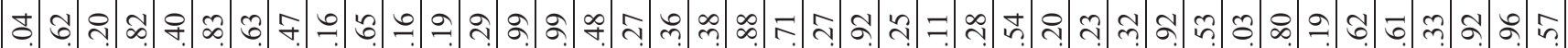

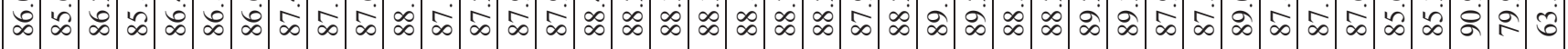

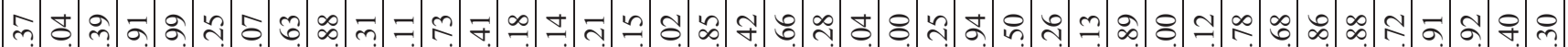

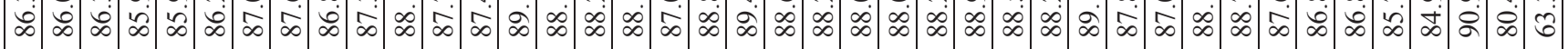

t)

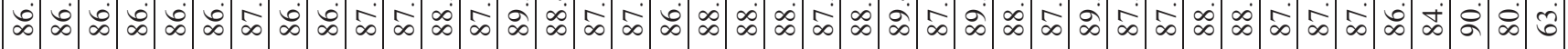

ᄀ

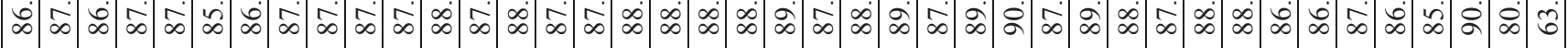

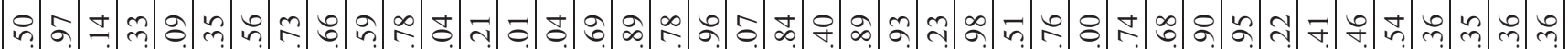

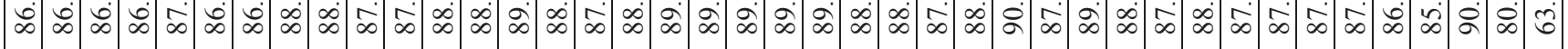
ปे

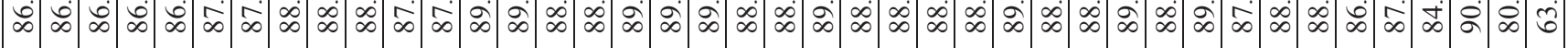

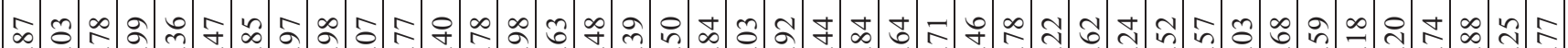

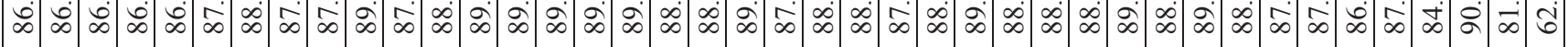

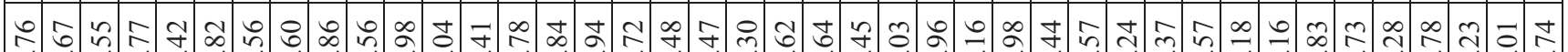

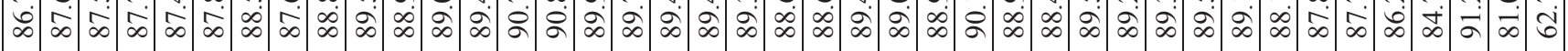

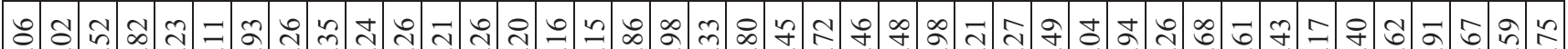

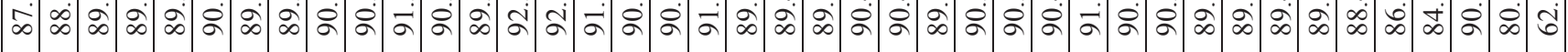

ชర

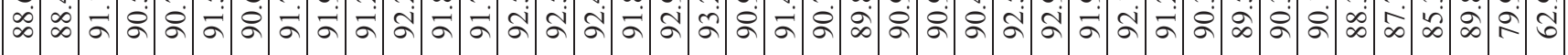

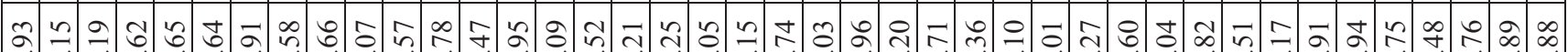

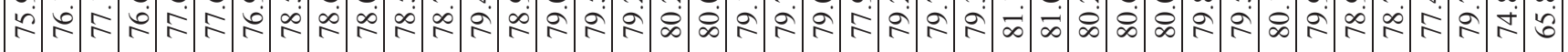




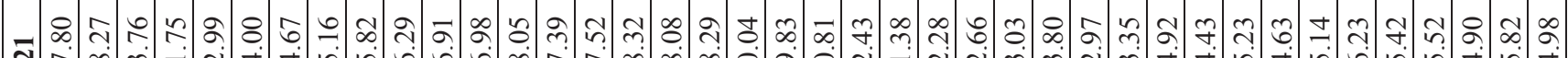

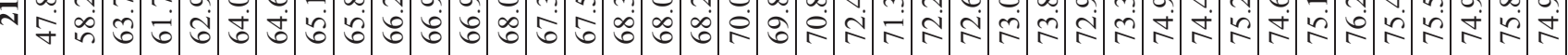
-

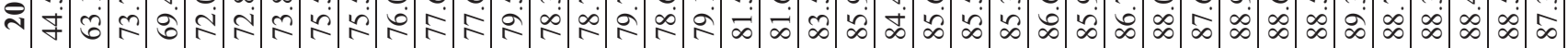

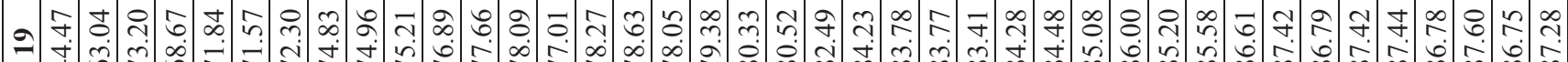
舟

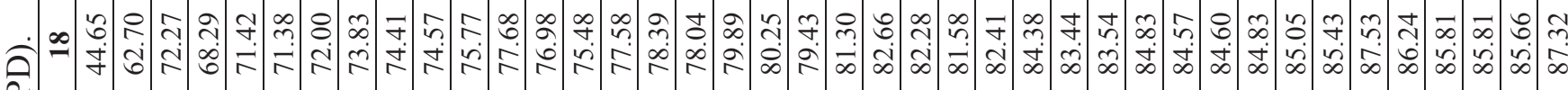

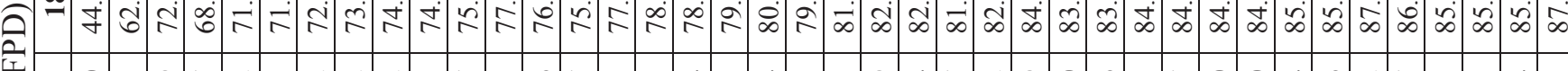

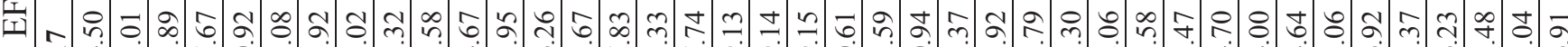

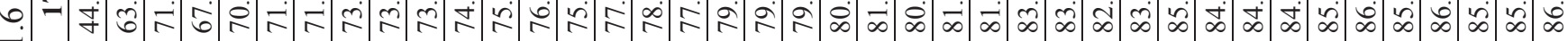
ส

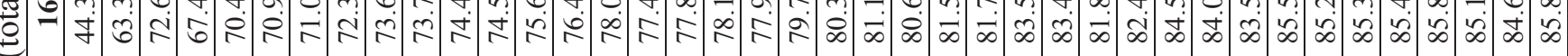

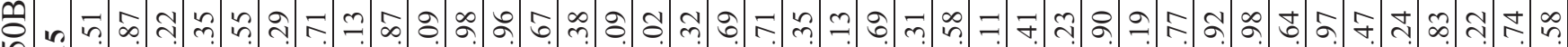

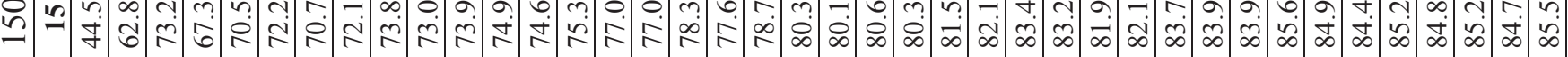

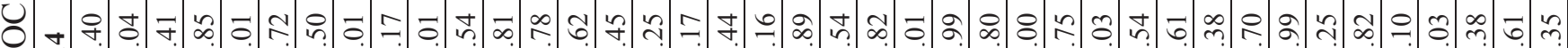
$\sum=$ J

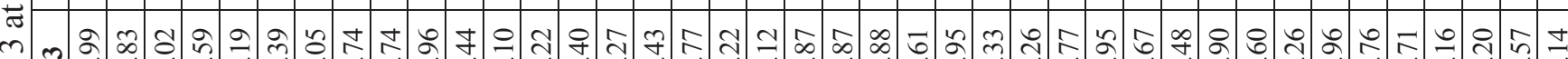

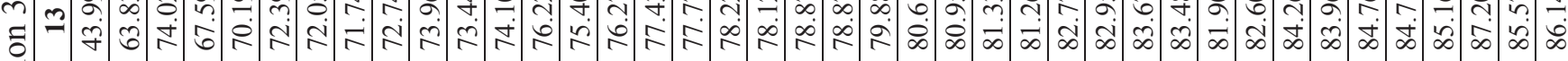
"艹

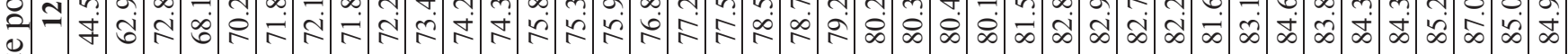
节

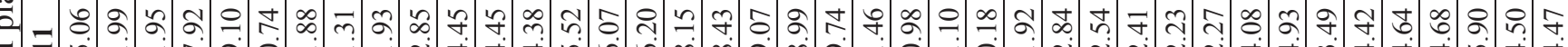

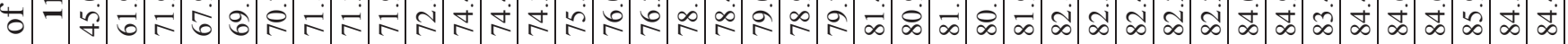
ต

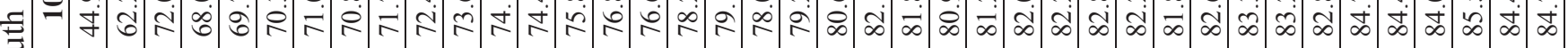

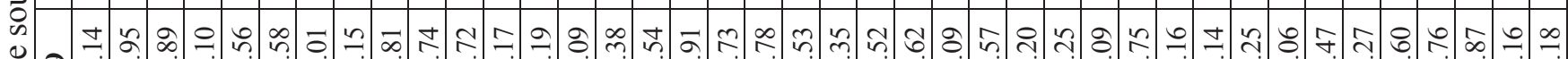
चの

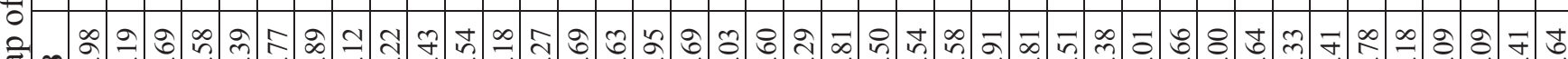

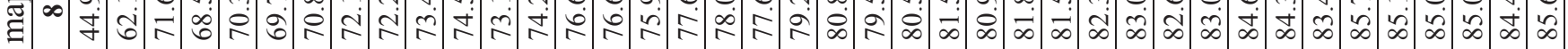

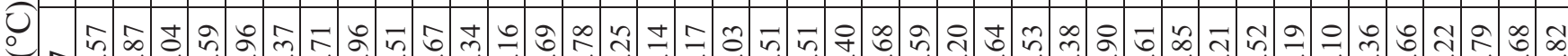
D

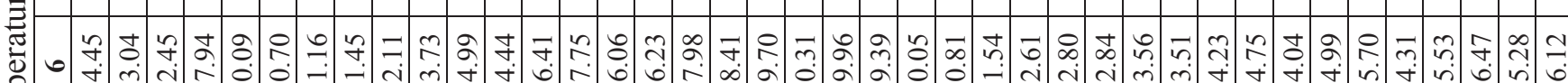

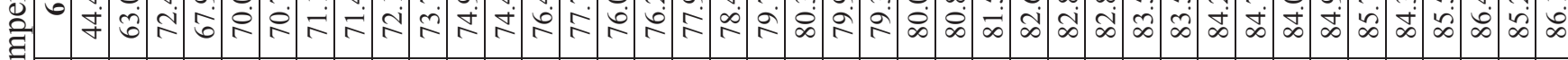

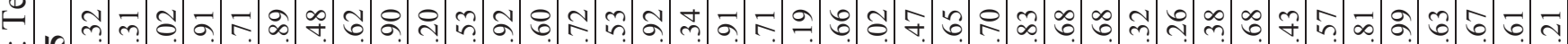

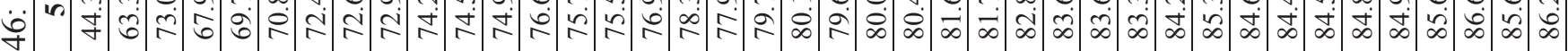

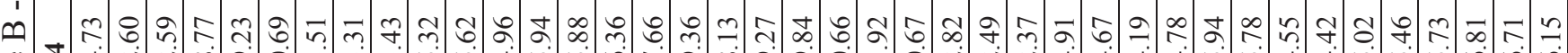

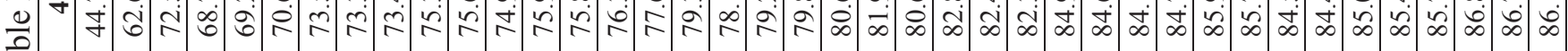

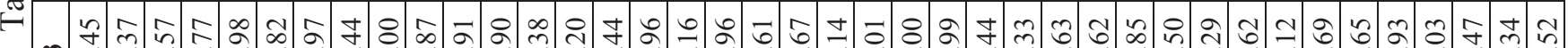

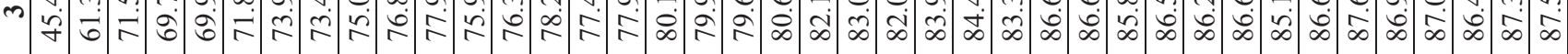

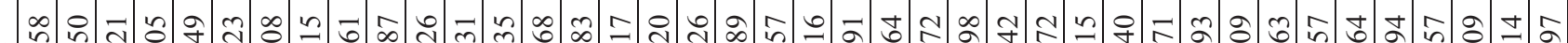

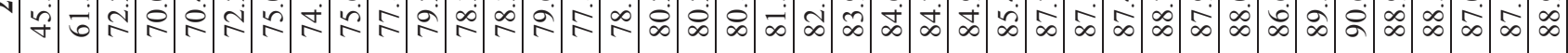

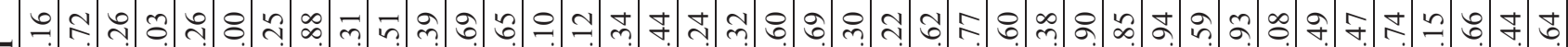

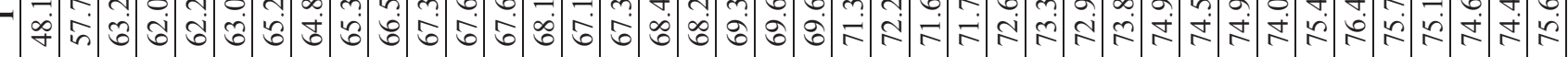

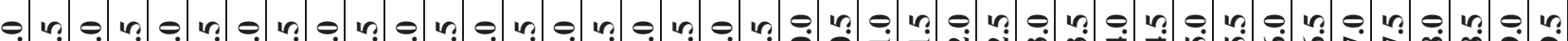

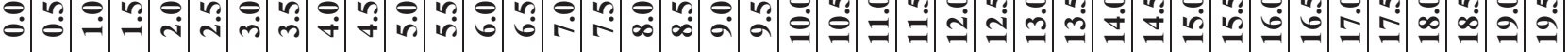




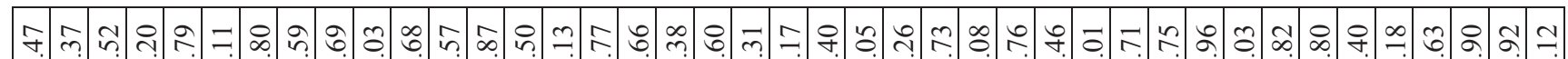

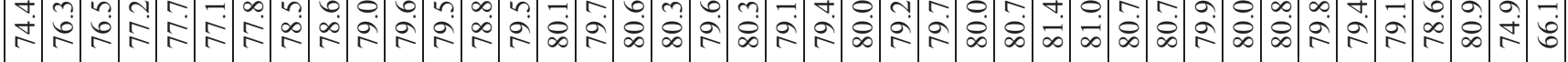
ปุ

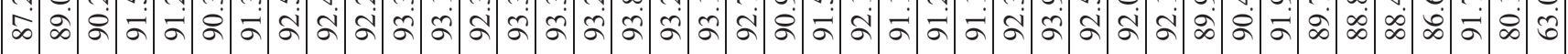

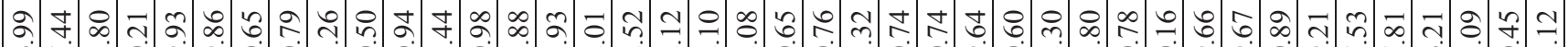

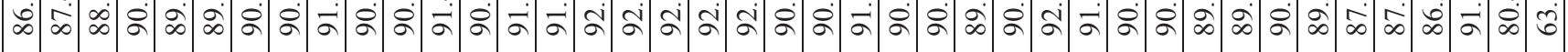

กิ

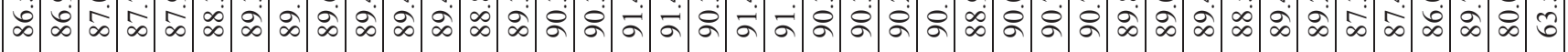

‡

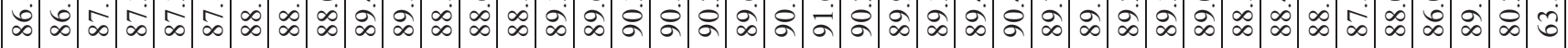

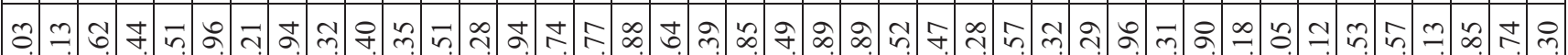

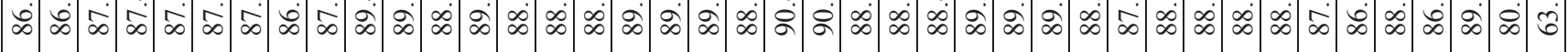

b유.

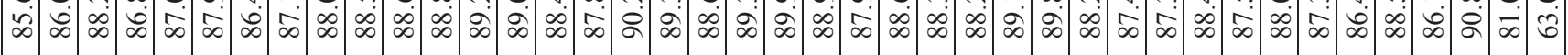

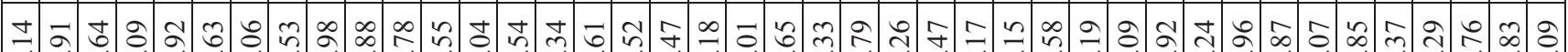

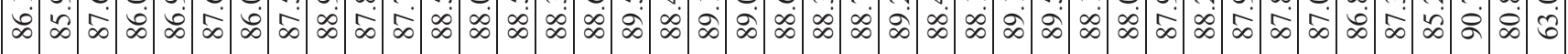

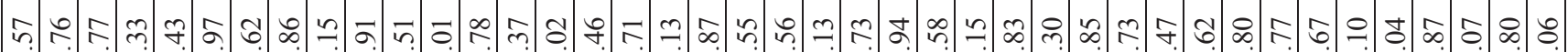

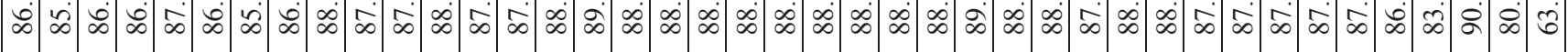

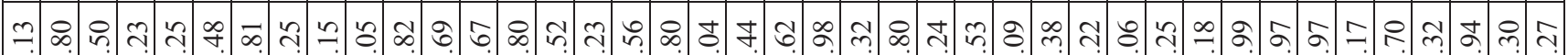

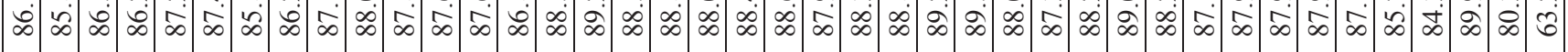

Әำ

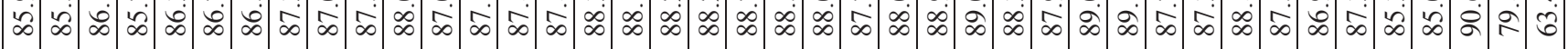

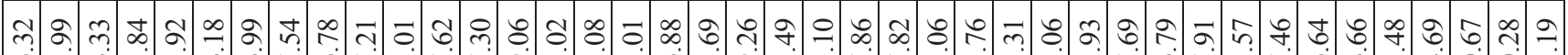

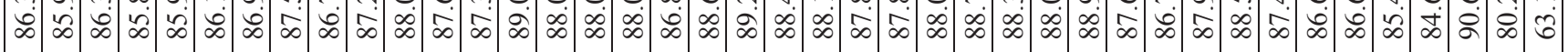

ฉ

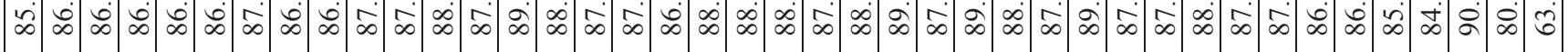

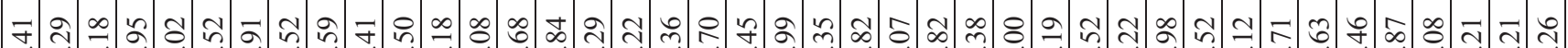

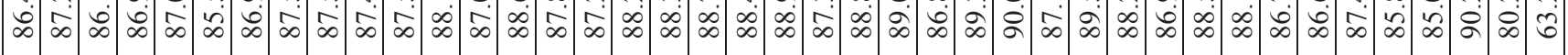

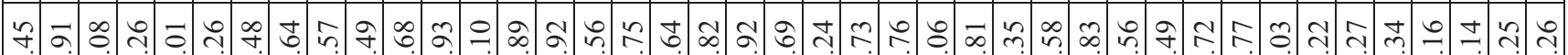

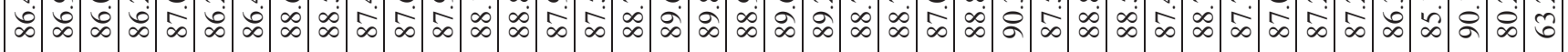

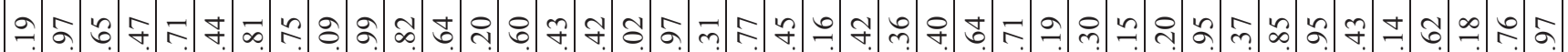

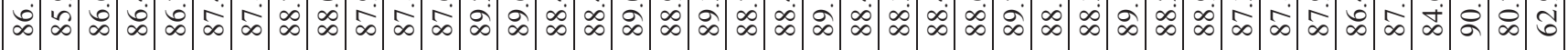

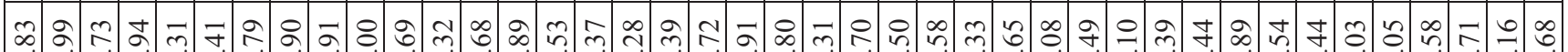

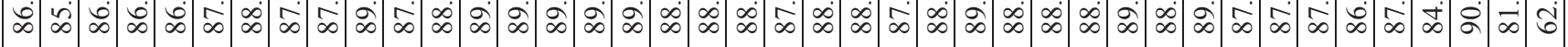

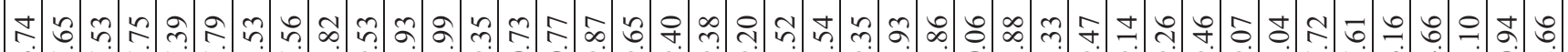

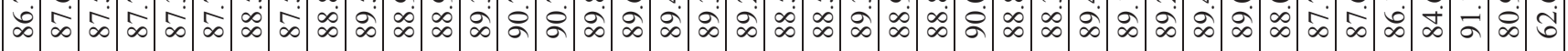

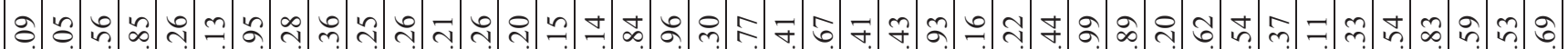

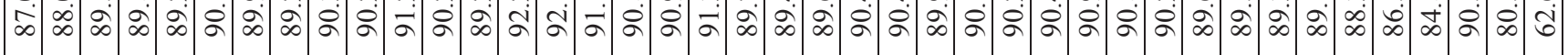

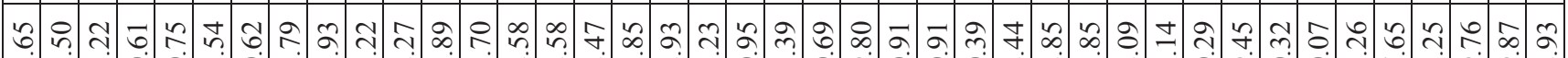

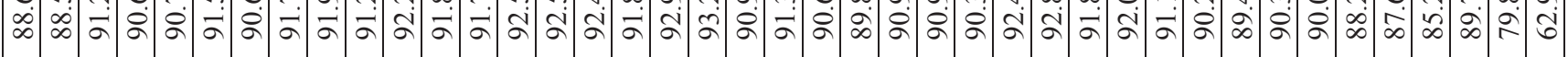

ชุ

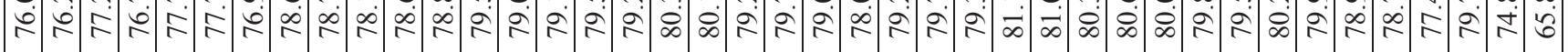

我

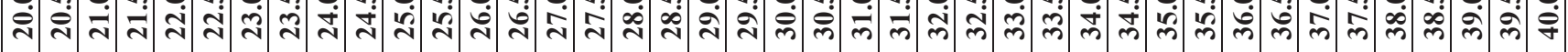




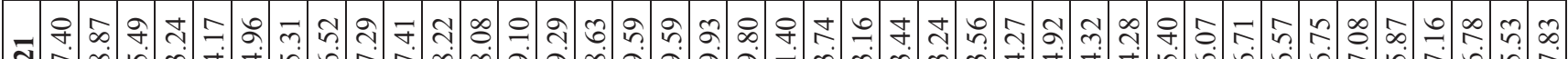

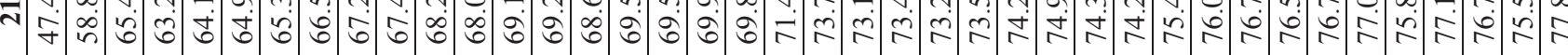
- $\tilde{\infty}$ సิ $\dot{\gamma}$ 过

a

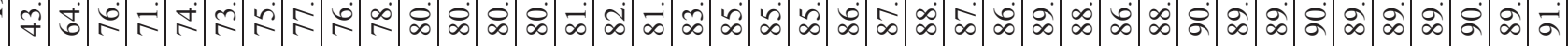

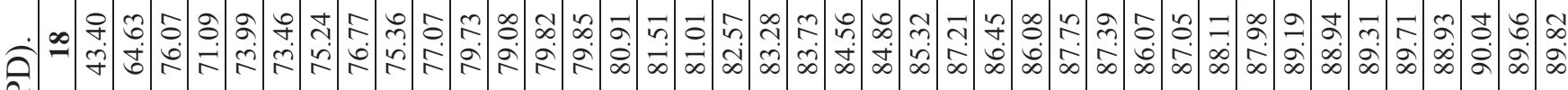
-

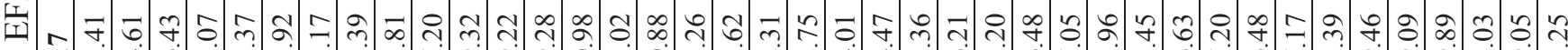

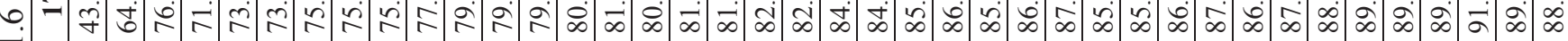
ส

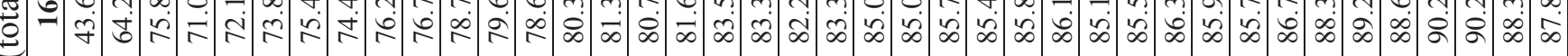

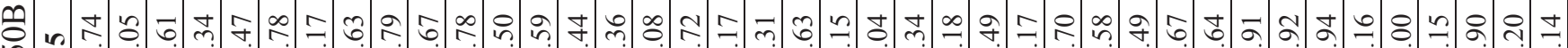

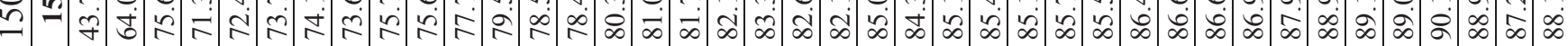

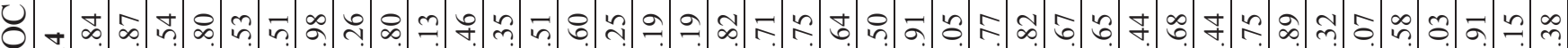

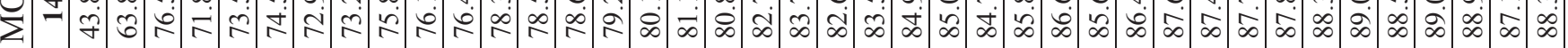
䒕

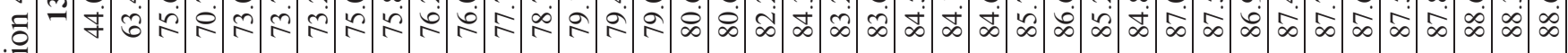
⿹

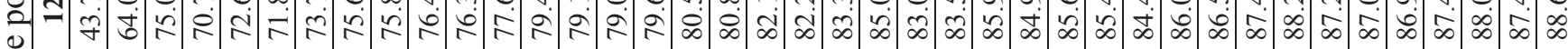

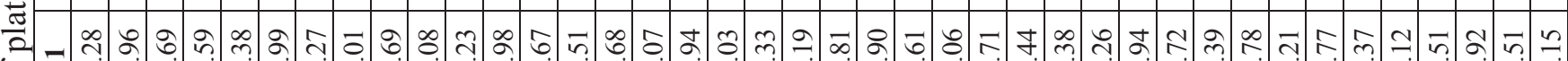

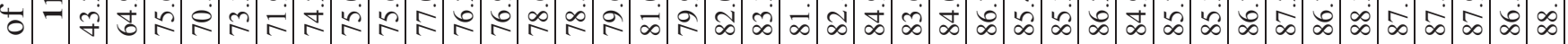

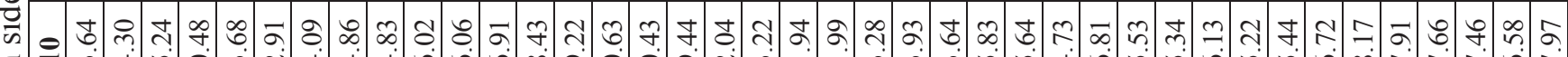
F = ป สิ

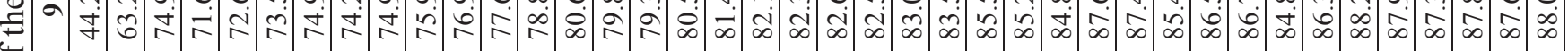
สิ

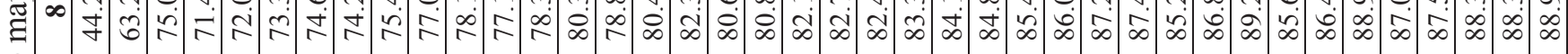

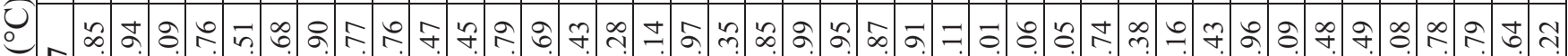
⿹

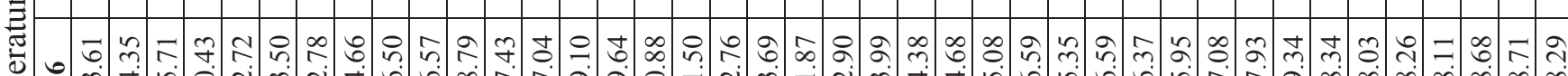

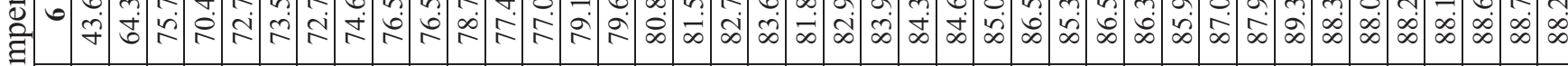
•.

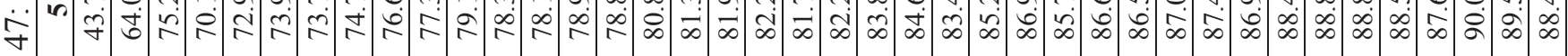

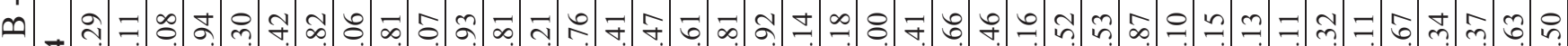

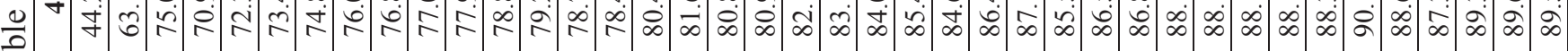

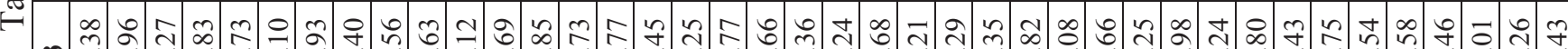

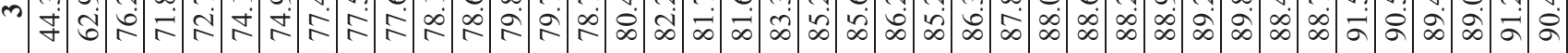

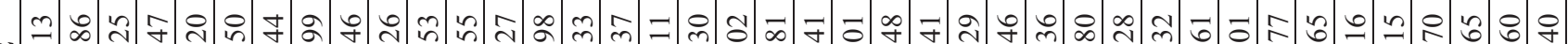

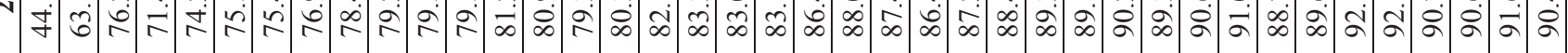
-

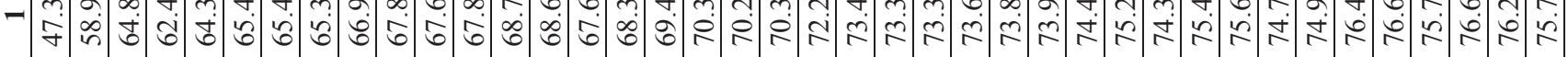

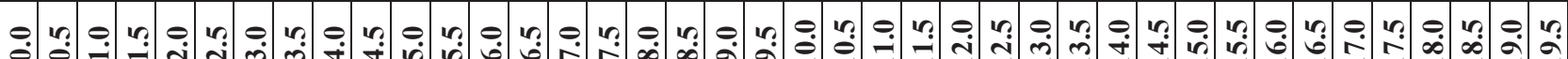




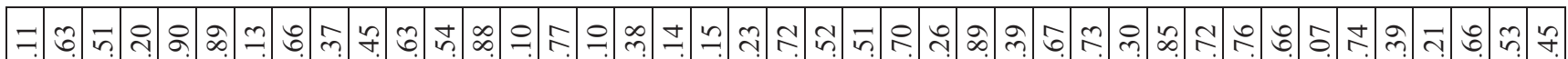

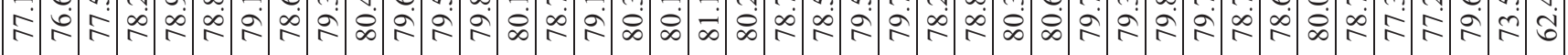

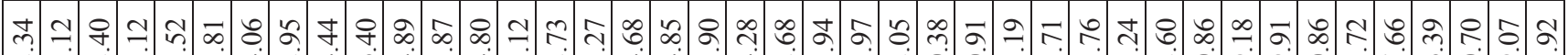

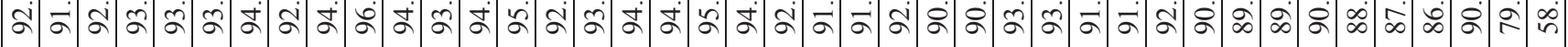

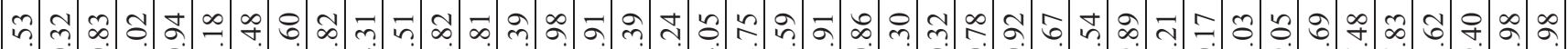

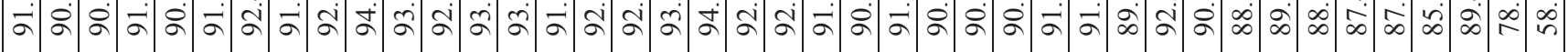

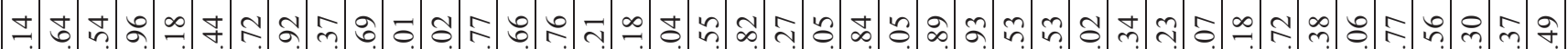

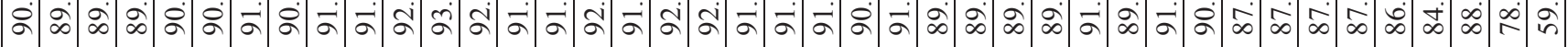
ஆ

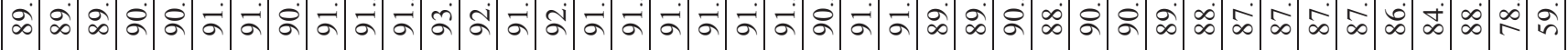
:

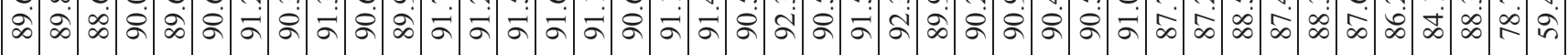

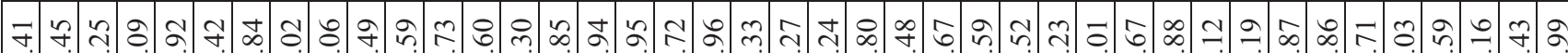

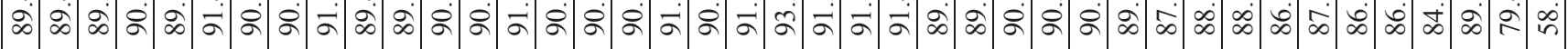

б. ำ

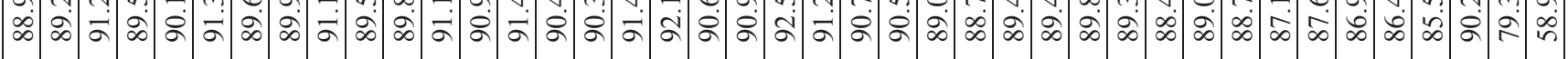

\&

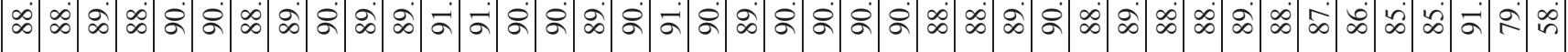

तิ

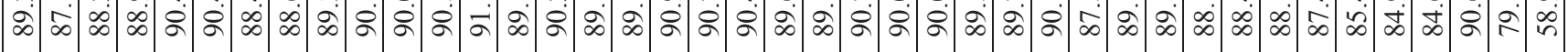

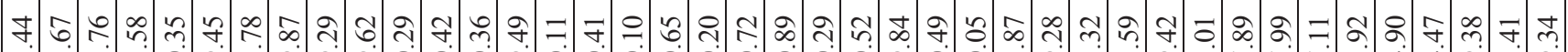

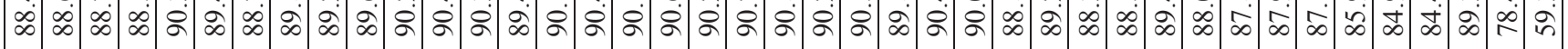

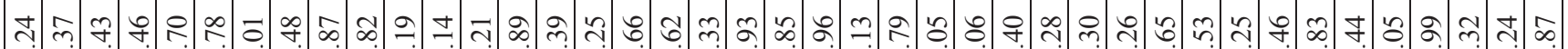

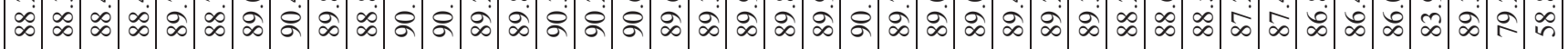

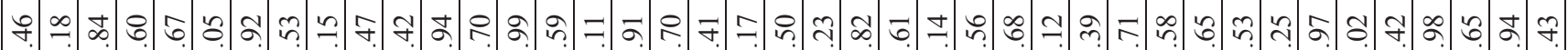

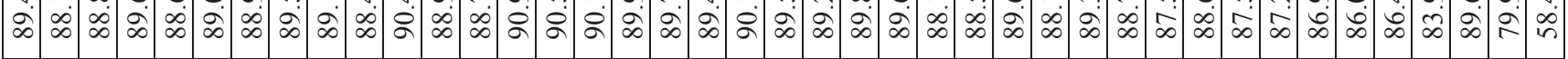

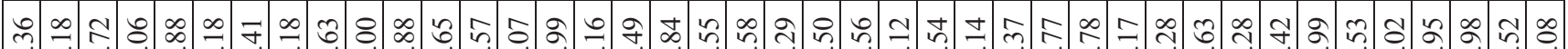

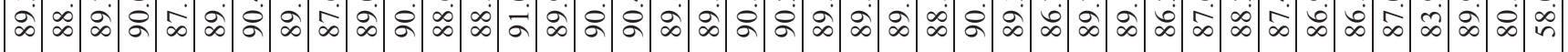
ศ.

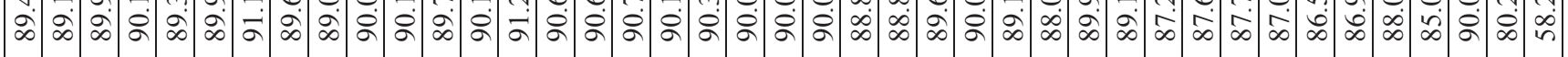

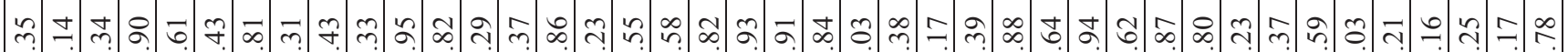

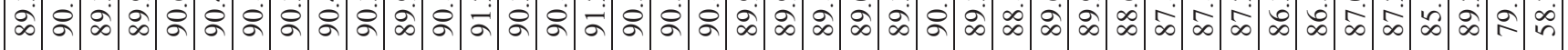
๙

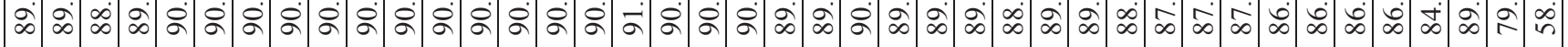

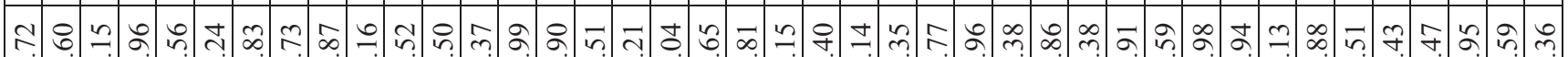

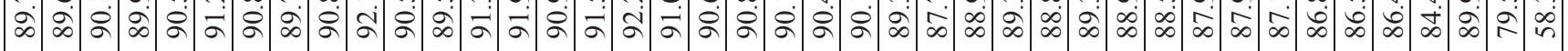

ఈ గุ

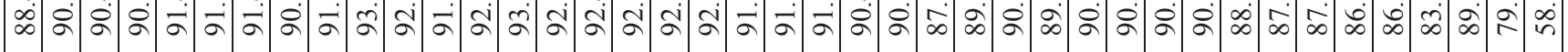

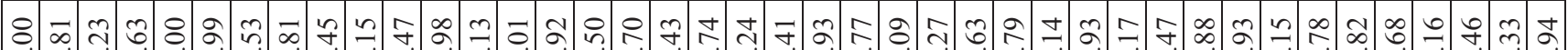
ळ बे बं

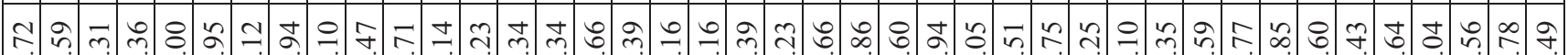

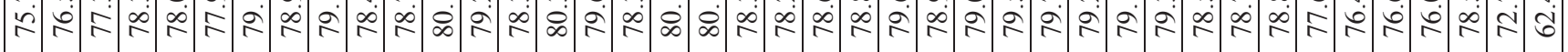
过

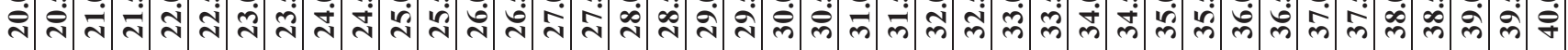




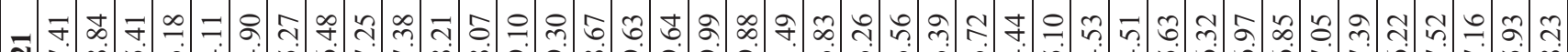

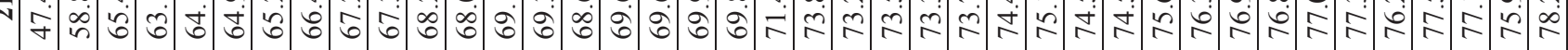

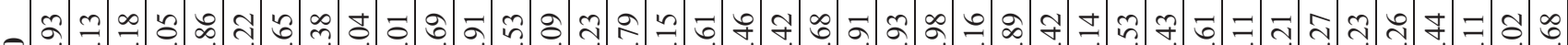

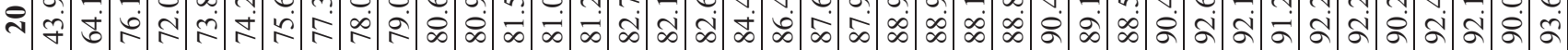

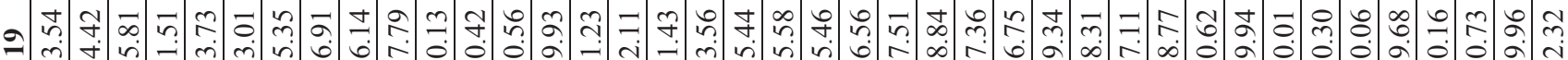

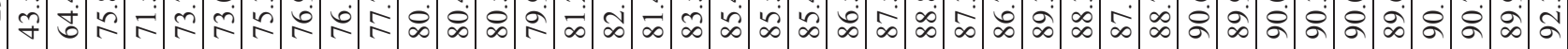

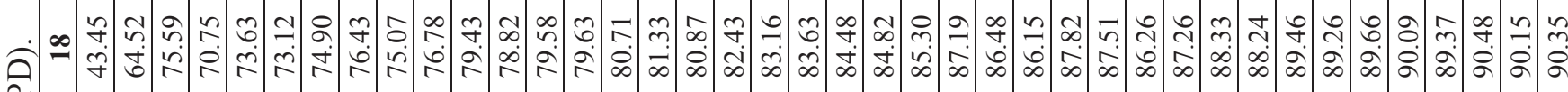

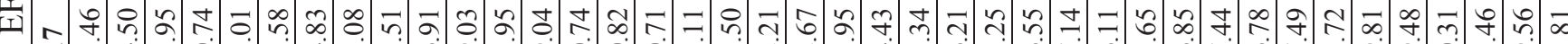

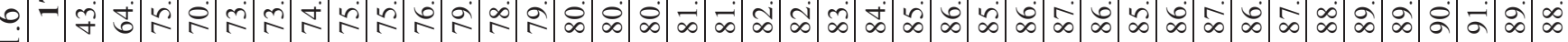
न

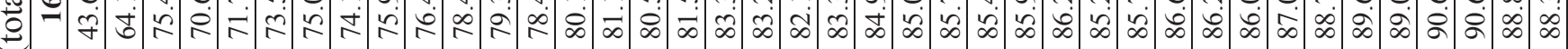

畢

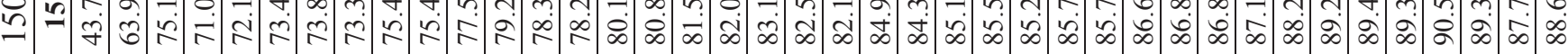

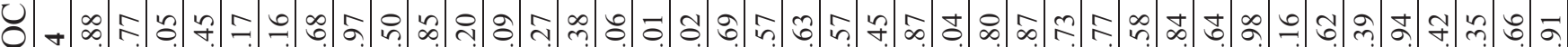

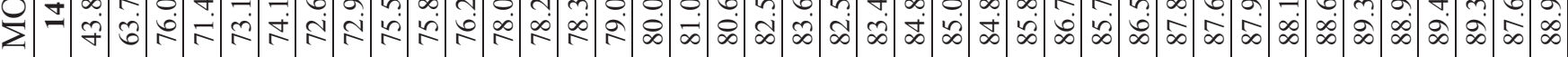
t⿹

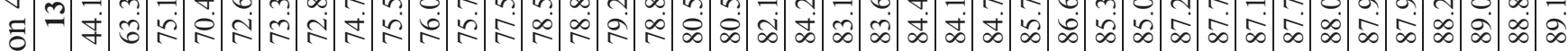
ป

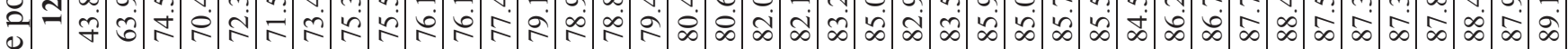
政 岁= 西 0 a

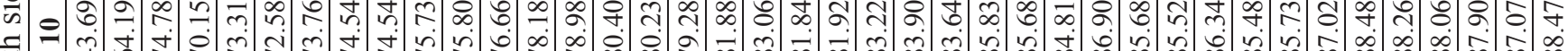

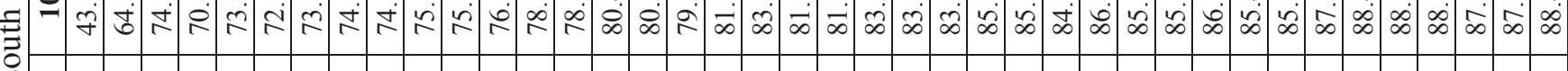

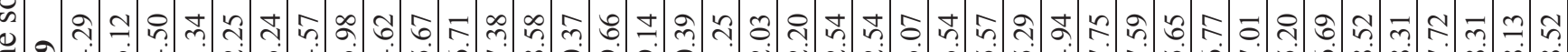

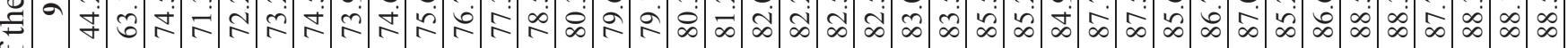
年

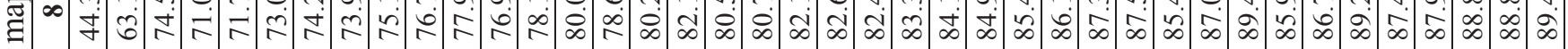

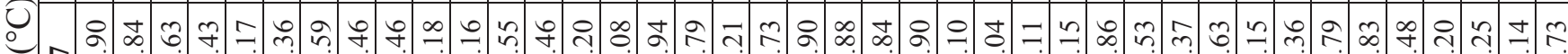
D f ๑ 节 fं $\bigoplus$ 苾

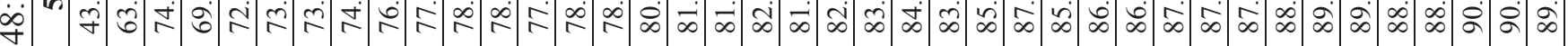
ص -

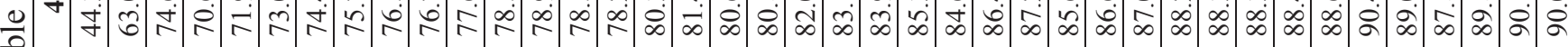
ت

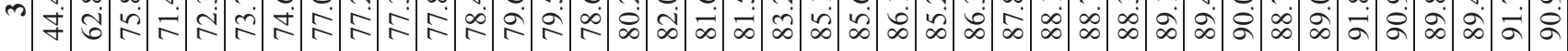

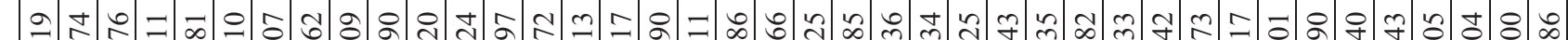

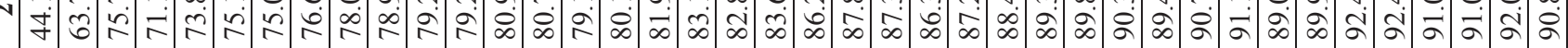
mे

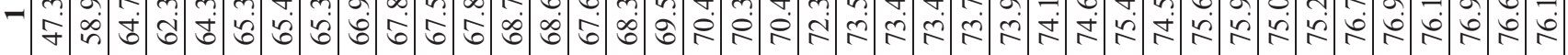
- $n$ o n 0 n

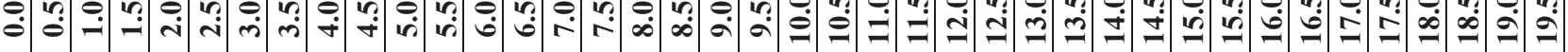


$4 \infty$

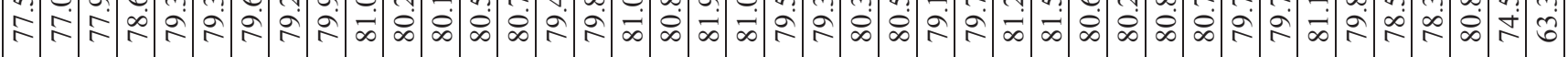
$\infty$ t

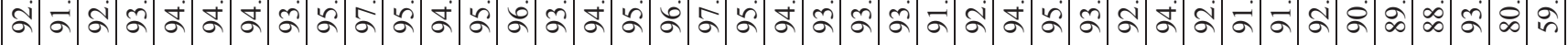

ఫ。

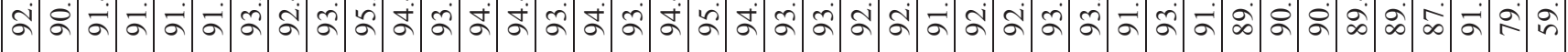

억

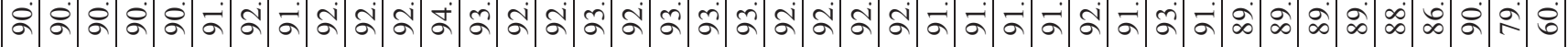

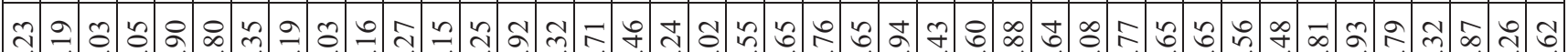

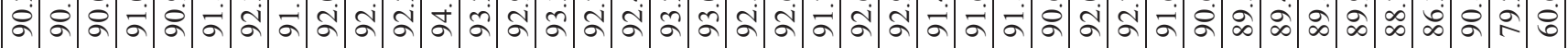

สุ ஓं ฌ ฉ

○

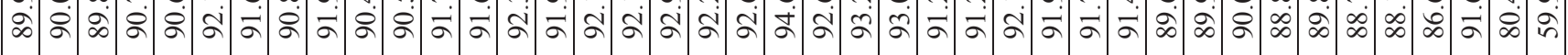

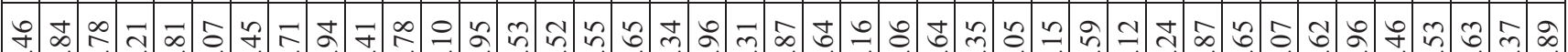

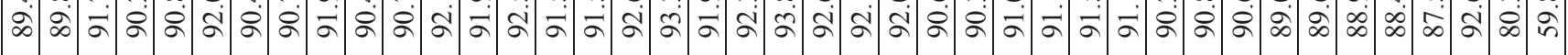

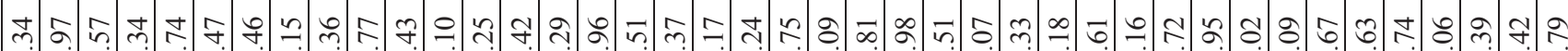

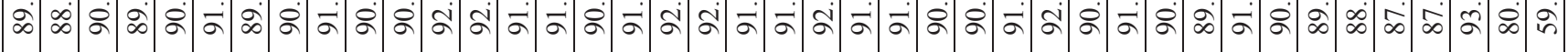

Б) f.

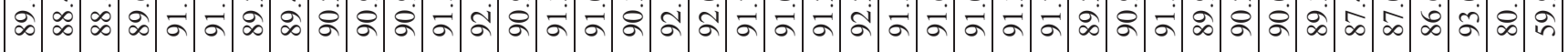

๙ิ กิ กิ สิ

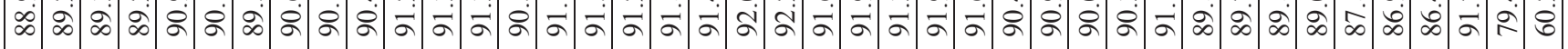

ミ

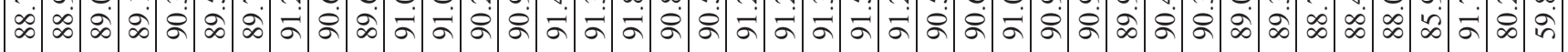

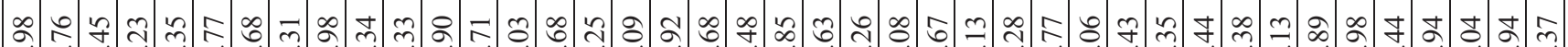

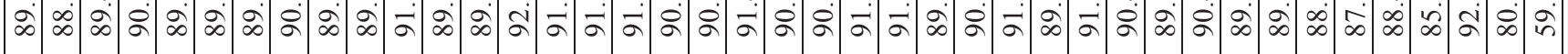

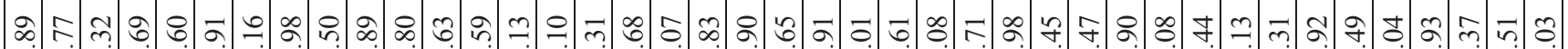

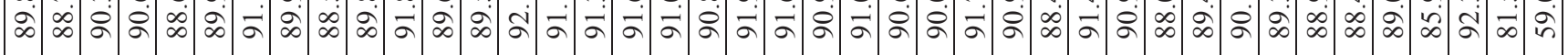

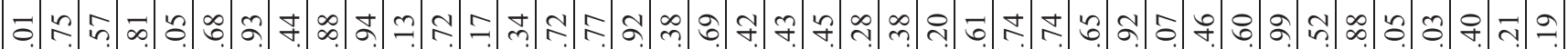

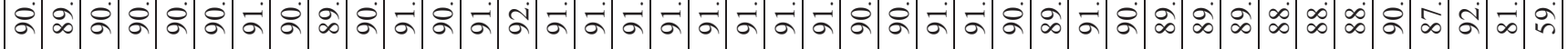

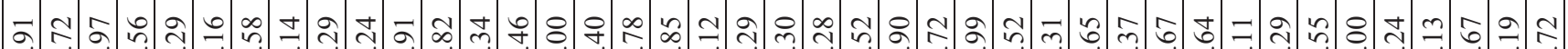

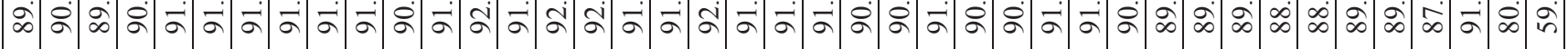

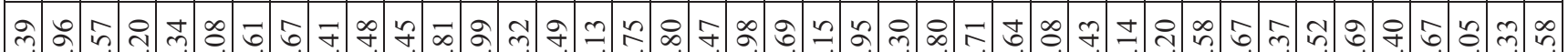

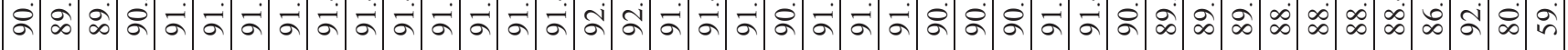

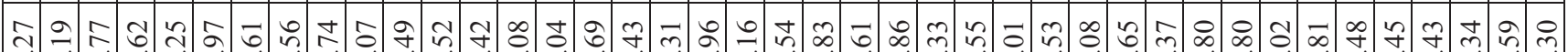

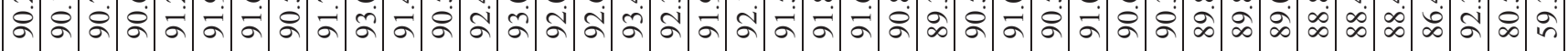

¿.

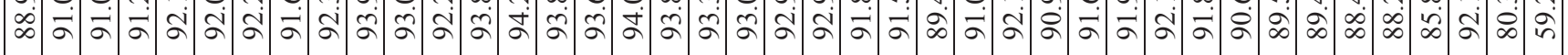

กี่

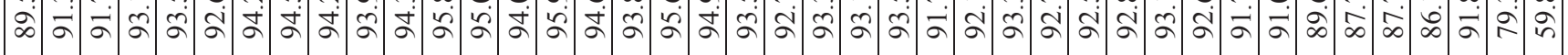
$\because$ t)

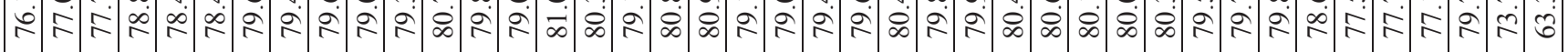

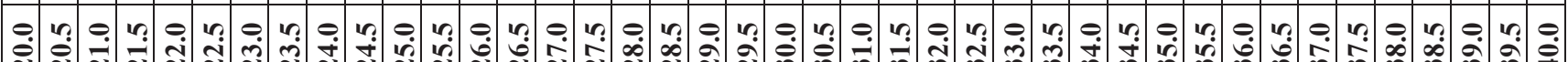

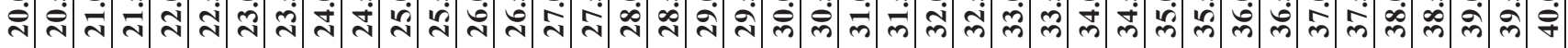


-

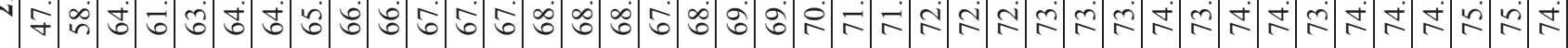
ปิ

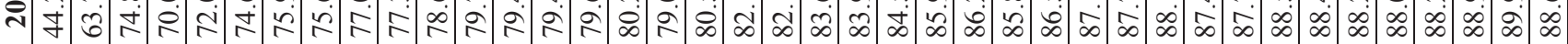

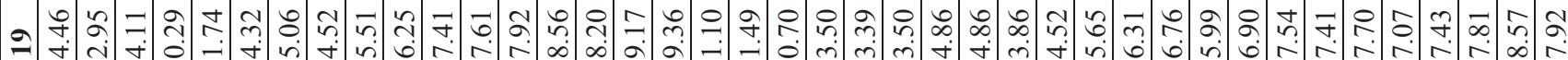

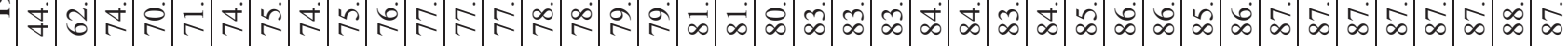

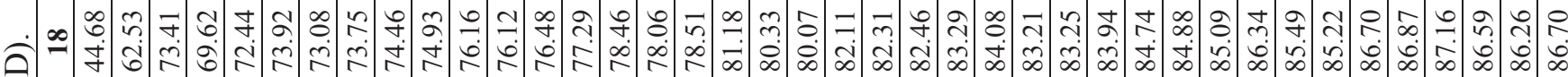

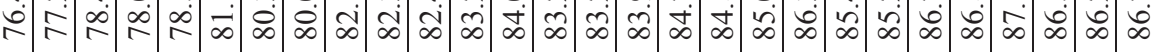

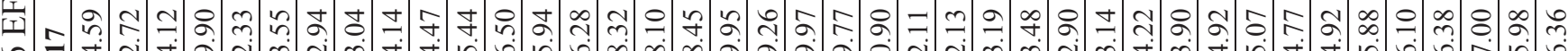

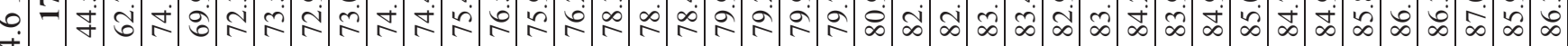

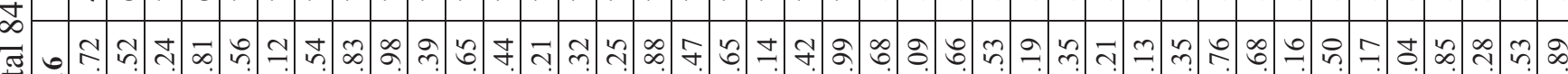

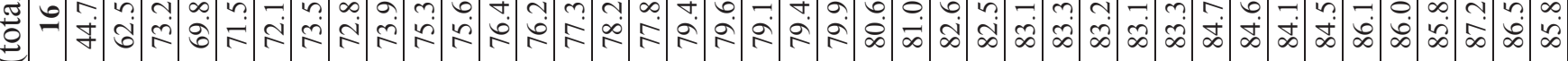

ఇ

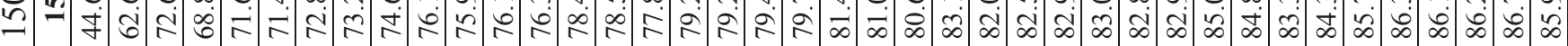

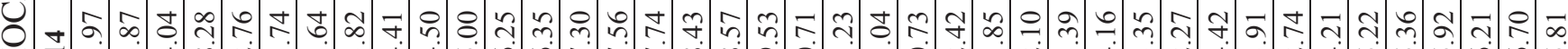

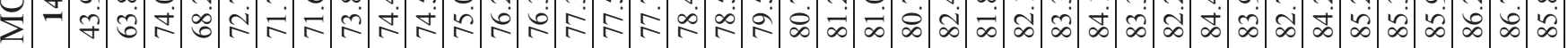
च

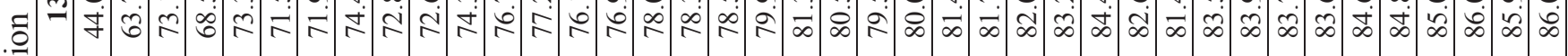

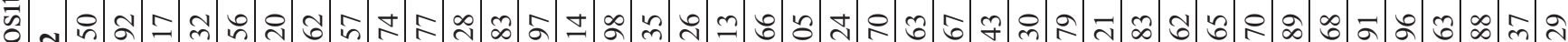

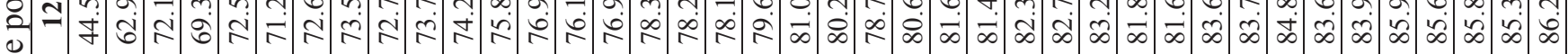

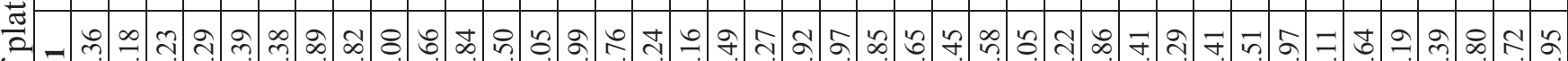

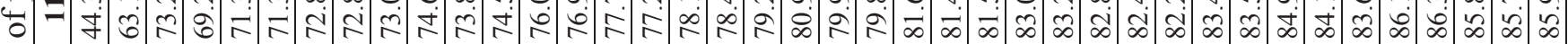
俈

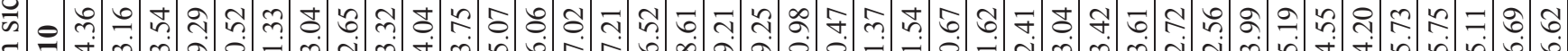

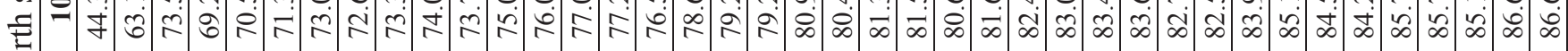
子

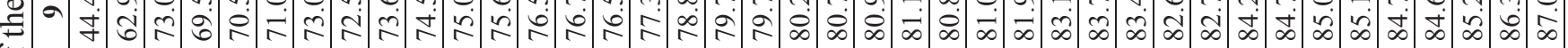
ค $\infty$ 舟

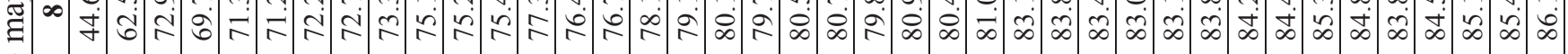
ర0 ⿹ 矛 青

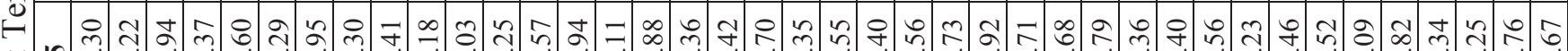

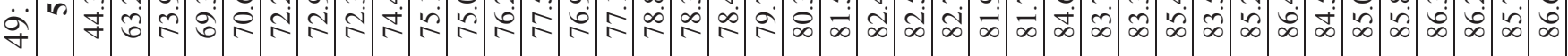

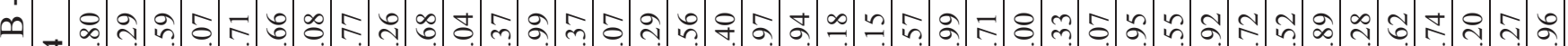

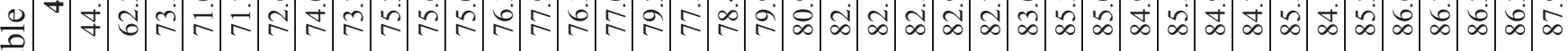

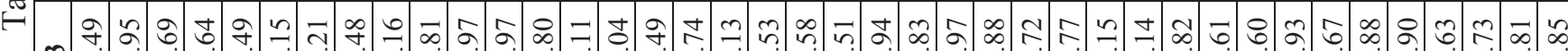

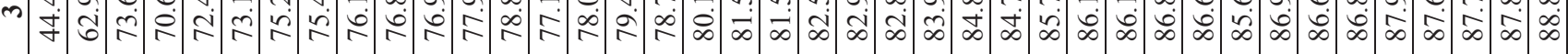

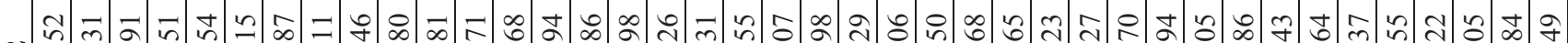

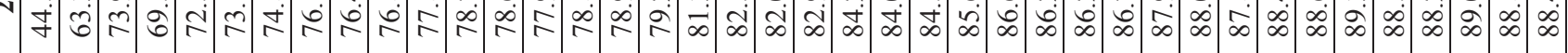
- పా

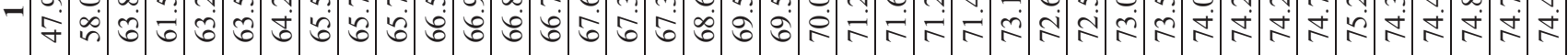
-

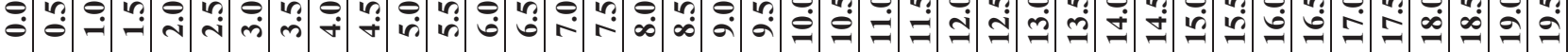




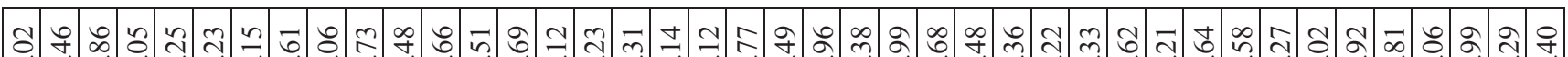

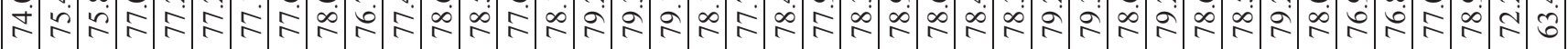

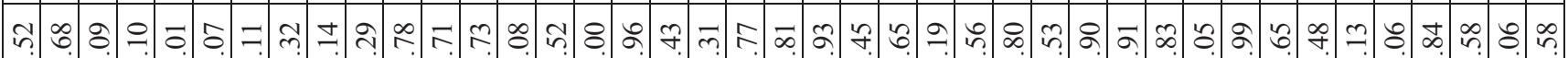

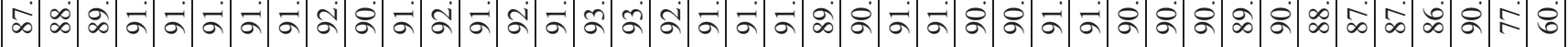

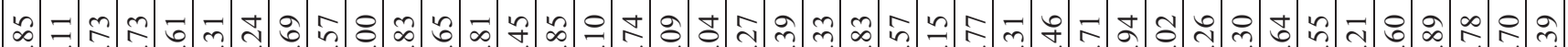

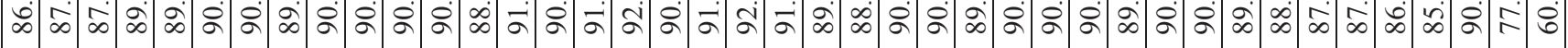

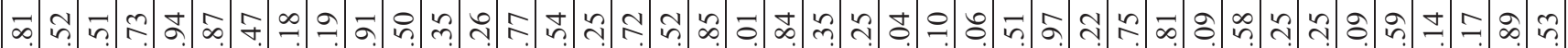

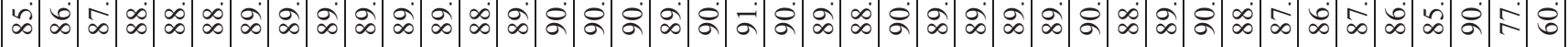

๒

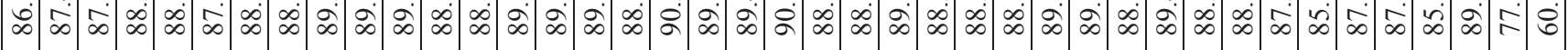

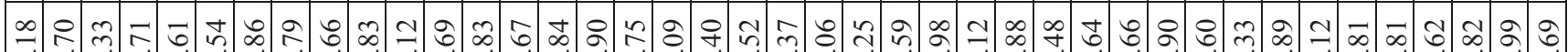

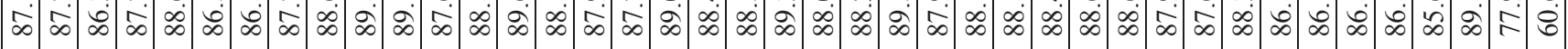

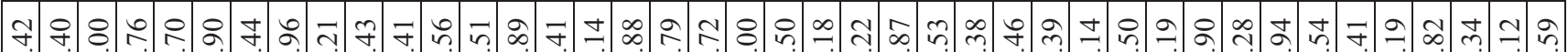

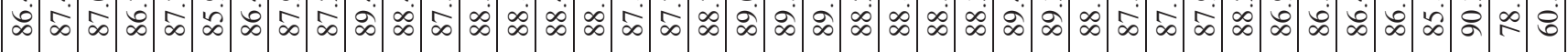

হ.

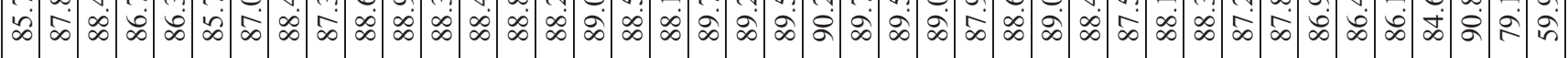

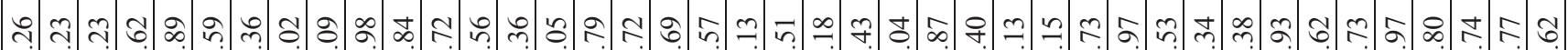

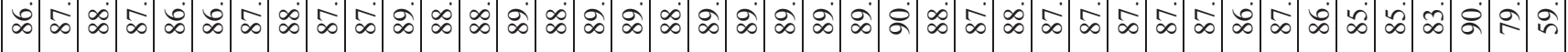

तิ

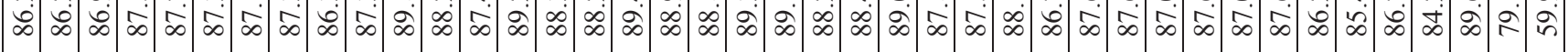

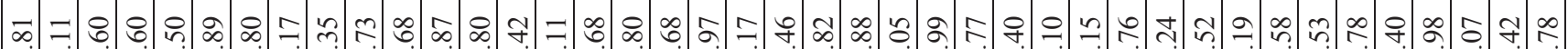

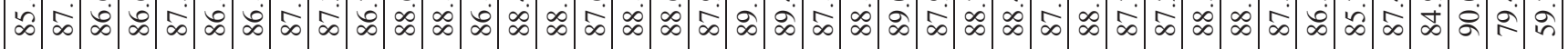

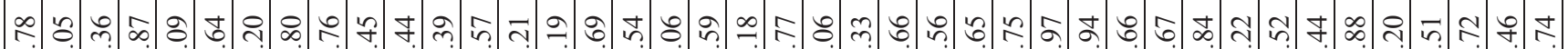

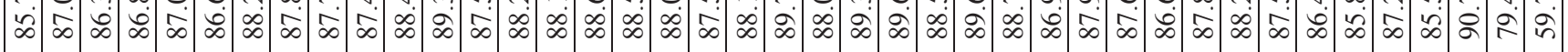

ํำ

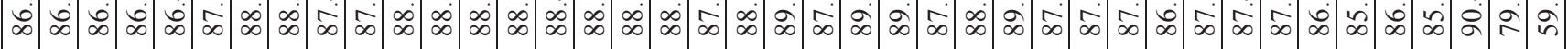

กิ

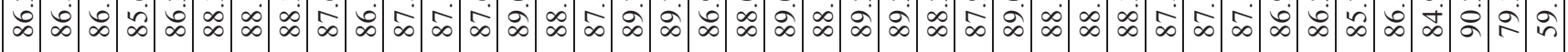

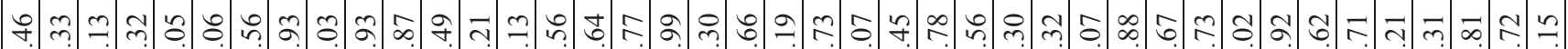

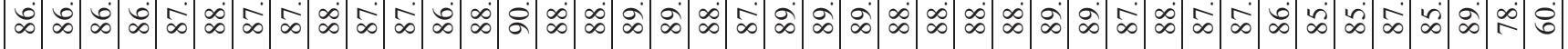
ప t)

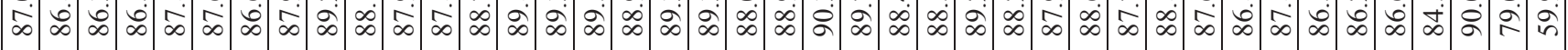
m.

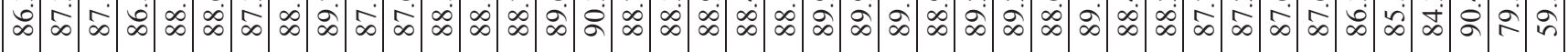

ণ

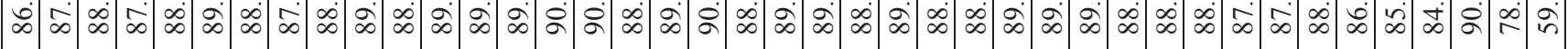

দก

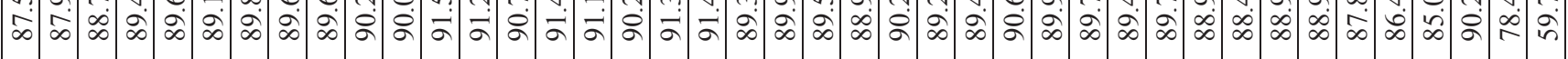

$\infty$ ఇ

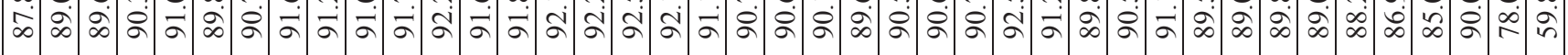

กิ

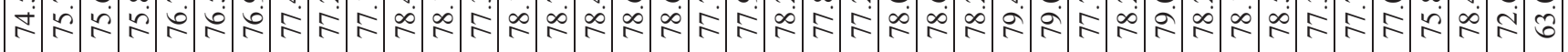

我

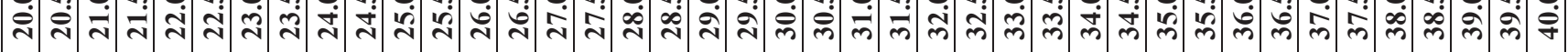




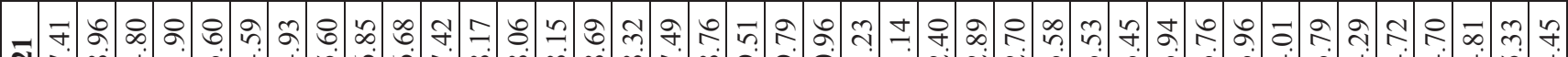

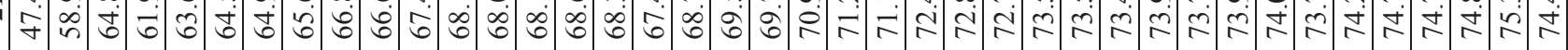

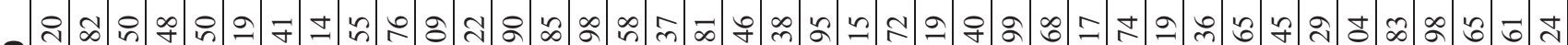

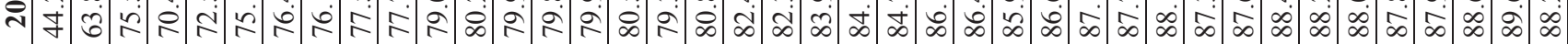

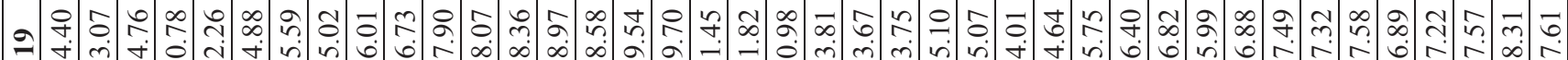

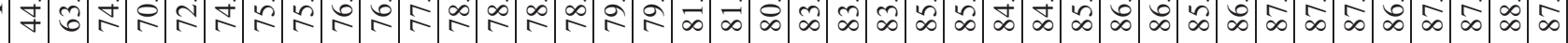

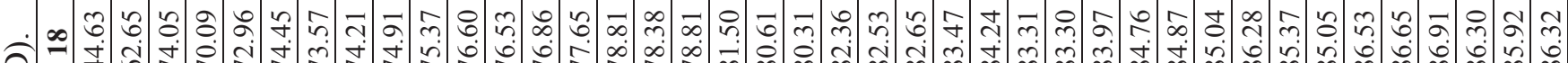

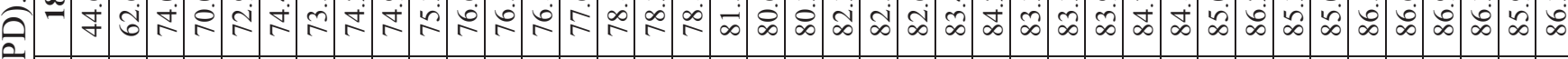

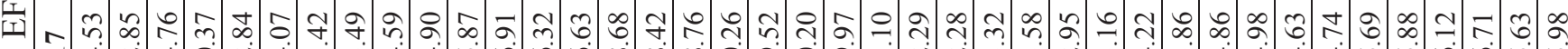

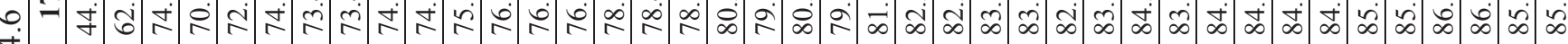

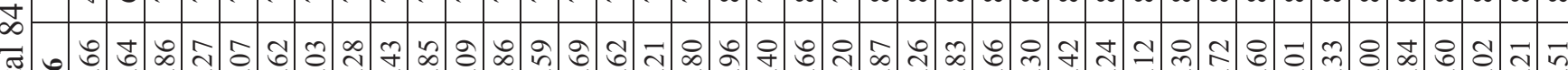

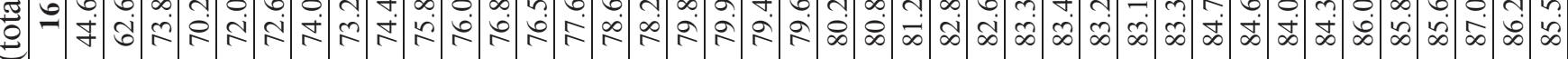

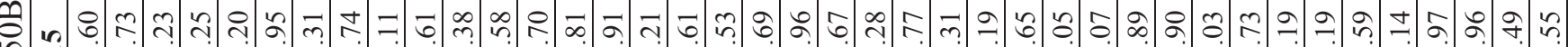

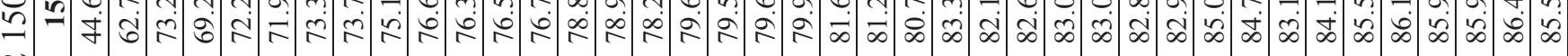

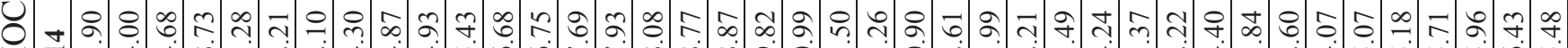

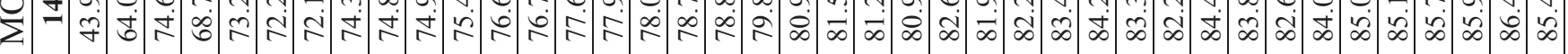

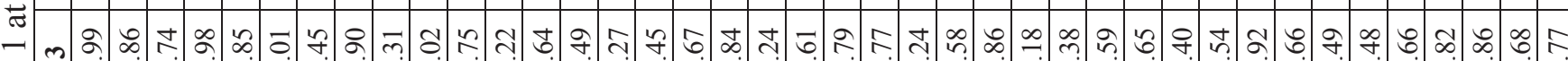
.

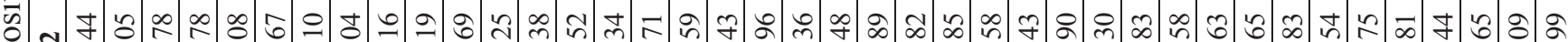

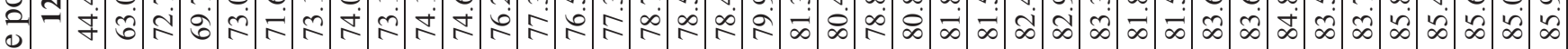

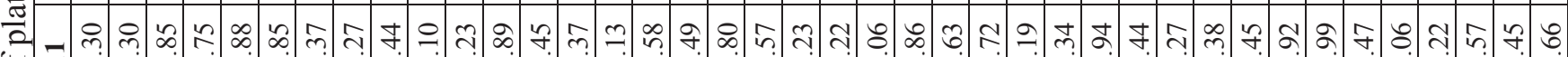

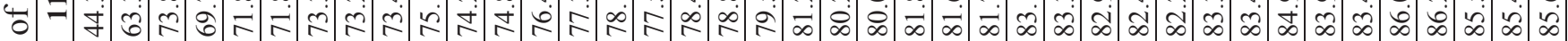

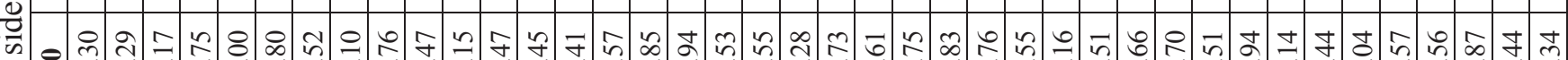

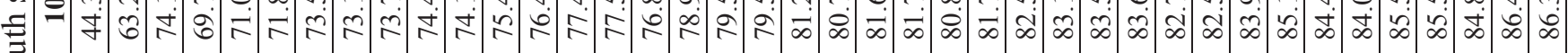
舟

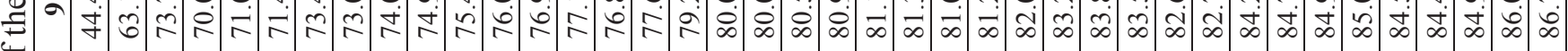

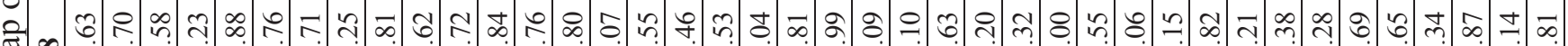

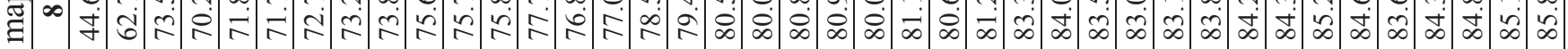

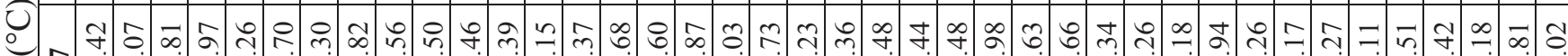

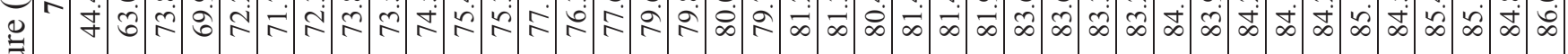
产

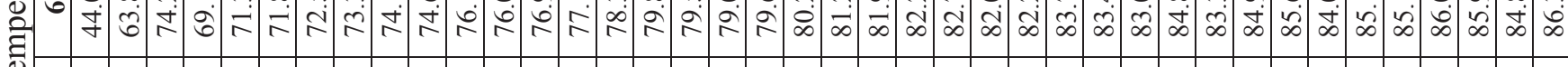

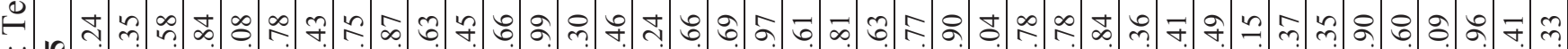
仓ं

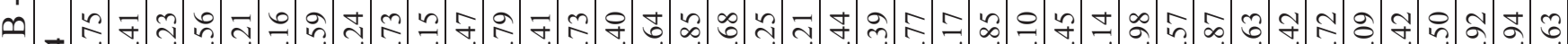

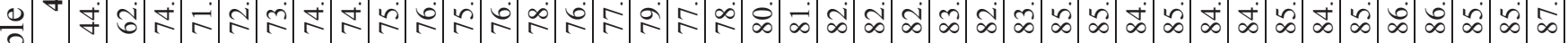
न

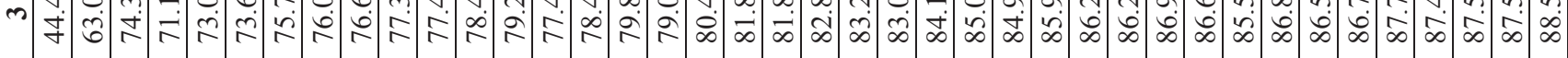

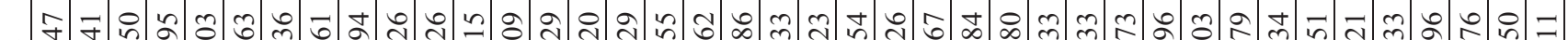

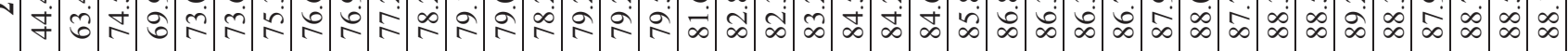

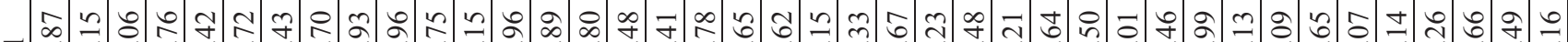

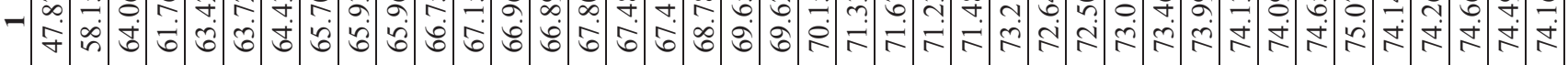

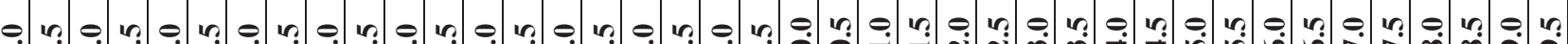

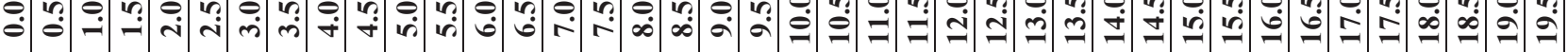




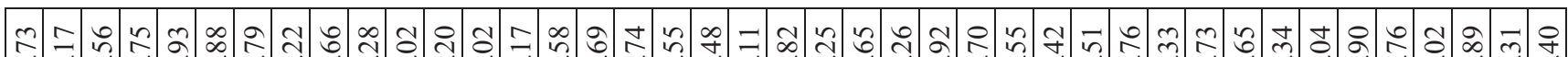

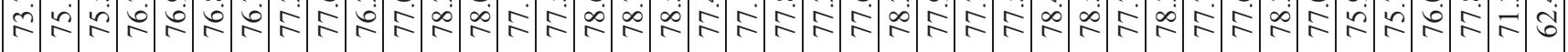

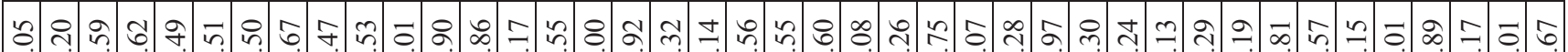

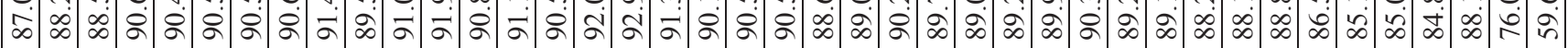

f

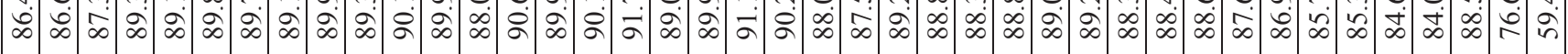

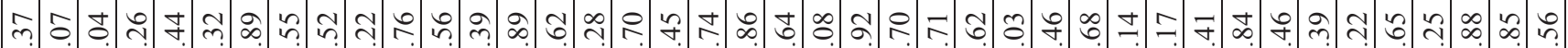

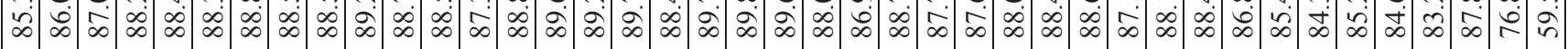

ปุ

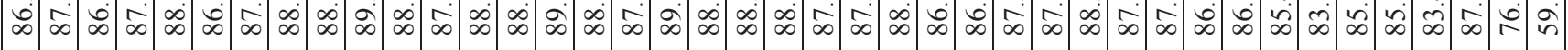

શิ กิ

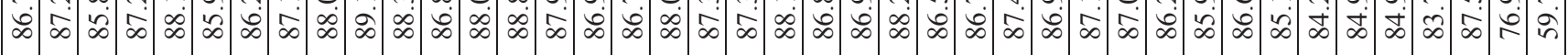

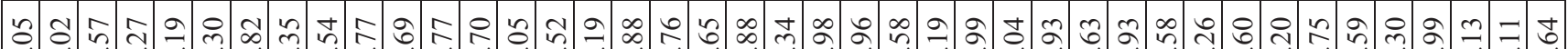

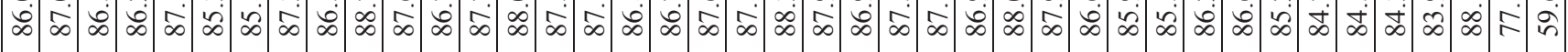

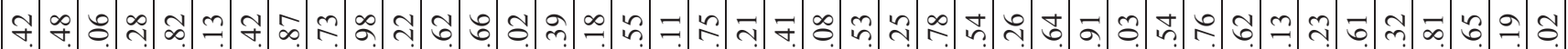

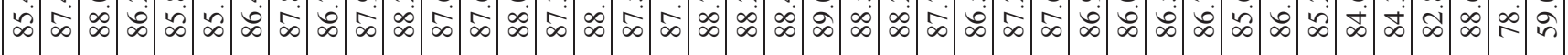

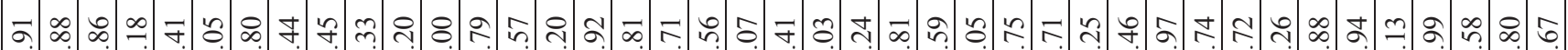

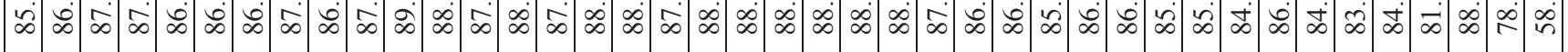

๙

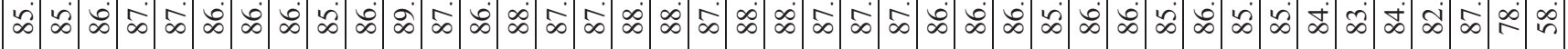

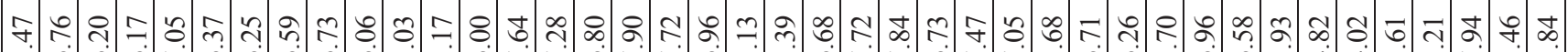

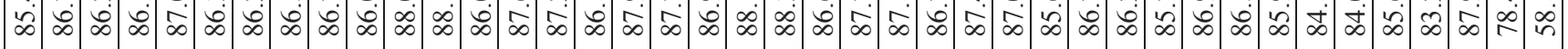

子

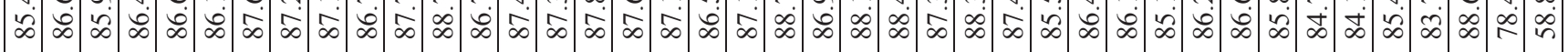

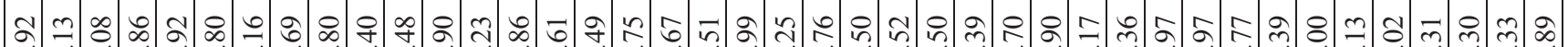

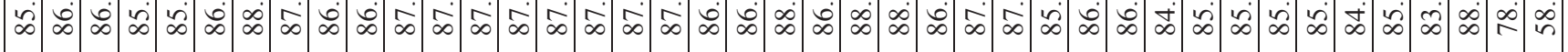

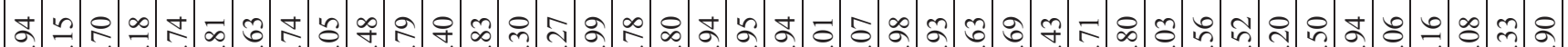

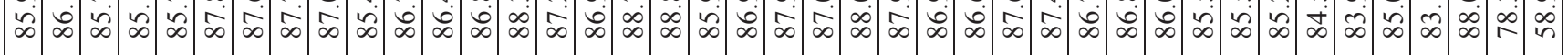

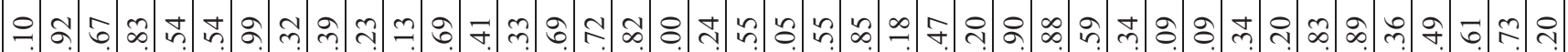

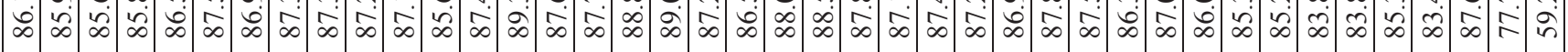

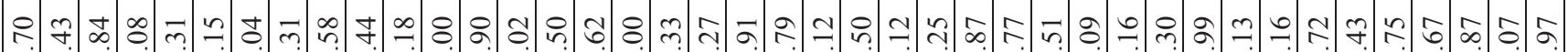

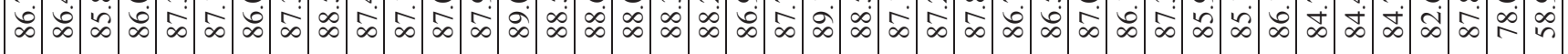

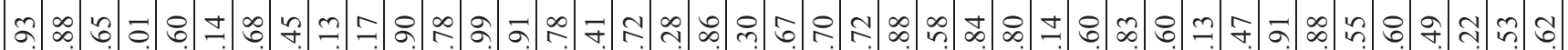

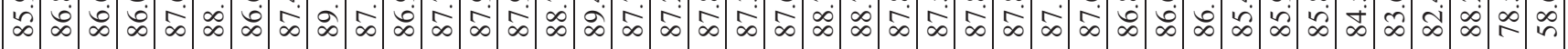

$\infty$ ○.

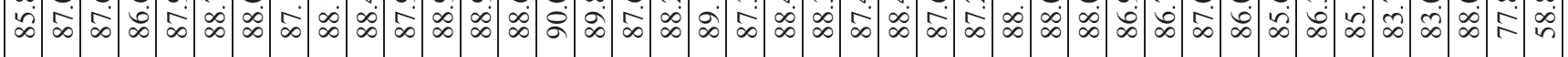

$\because$ น กิ

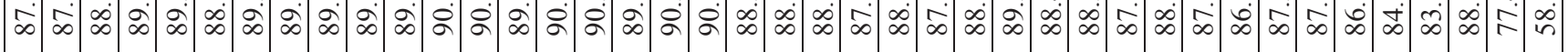

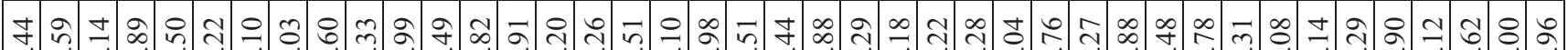

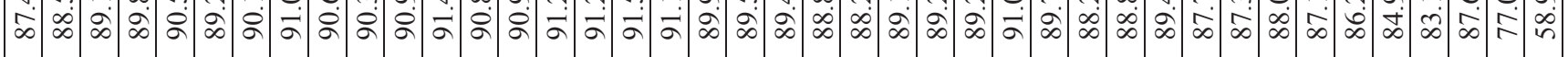

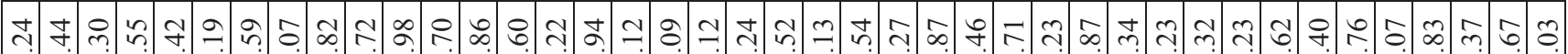

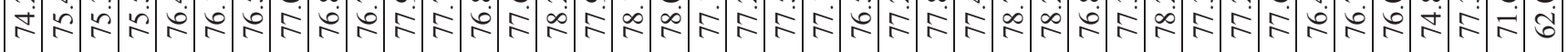
我

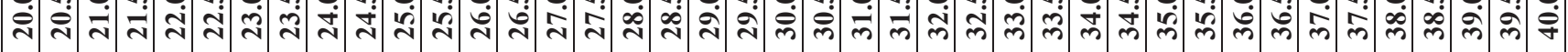




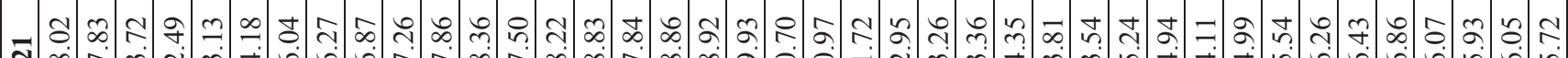

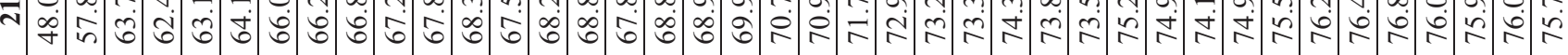

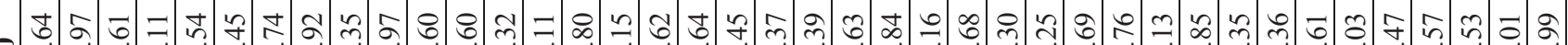
సิ $\dot{f}$ i

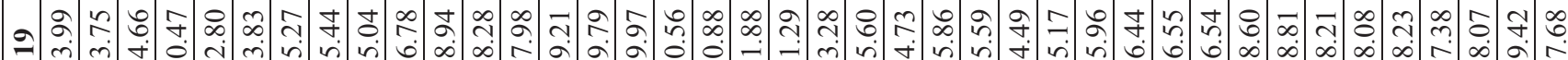
f)

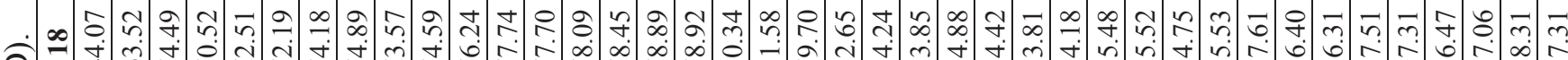

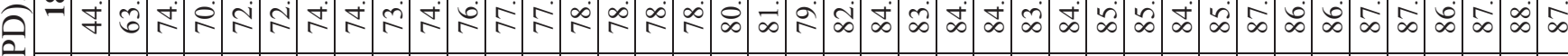

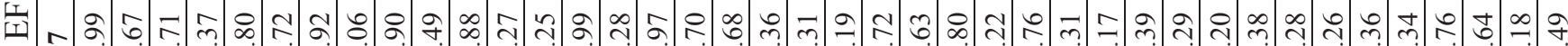

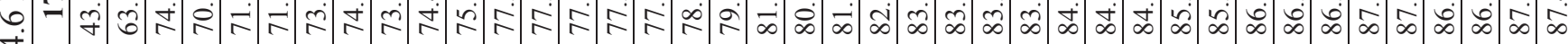
莎 严

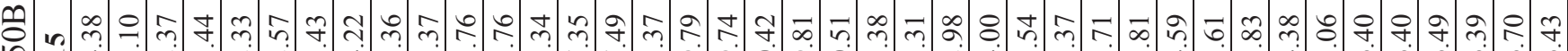

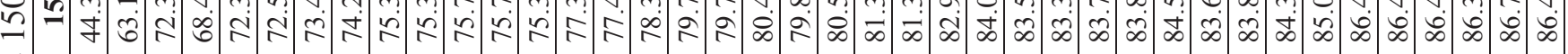

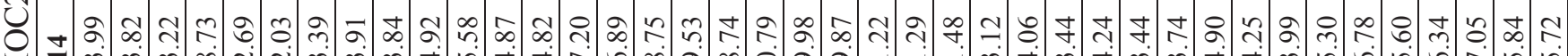

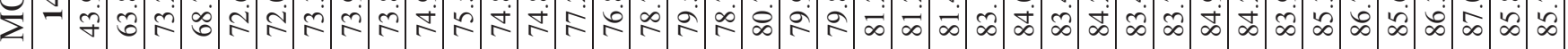

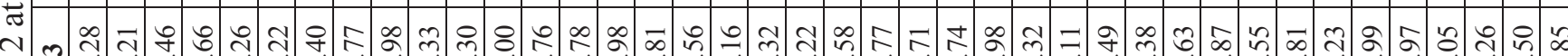

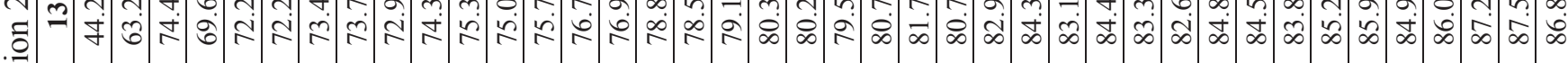

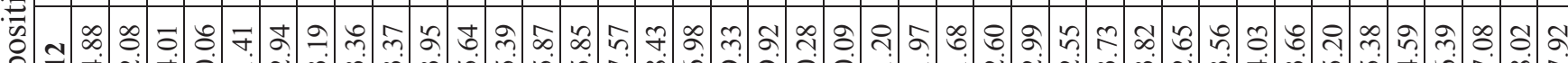

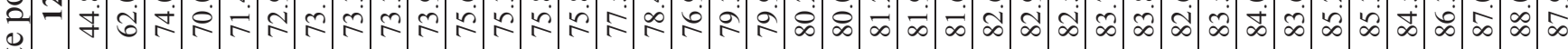

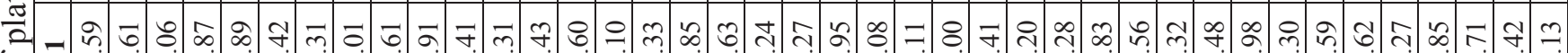

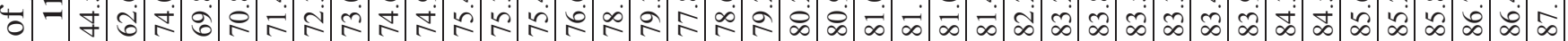

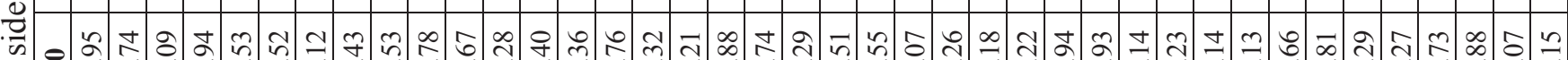

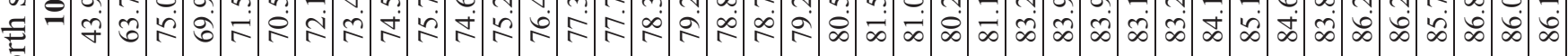

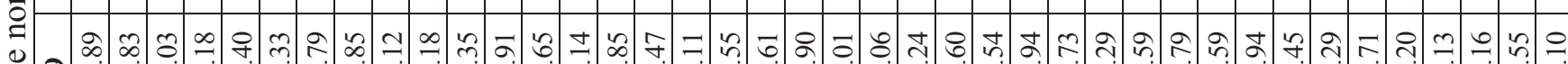
चの

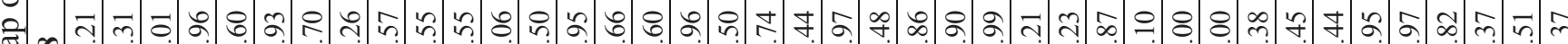

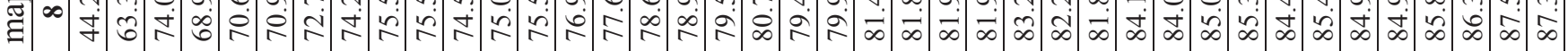

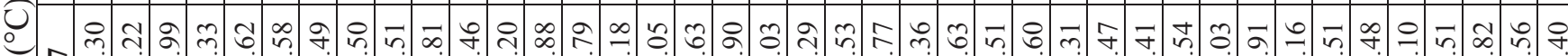

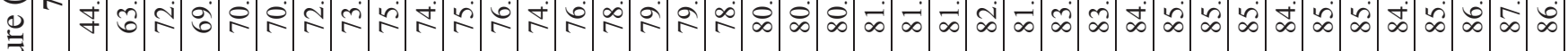
苟

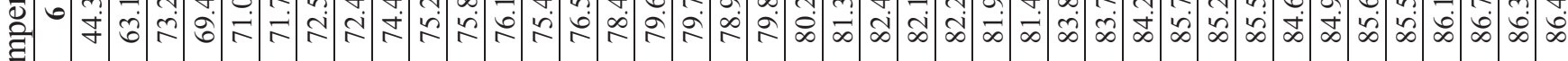

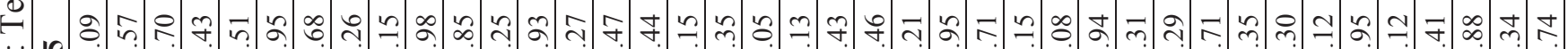

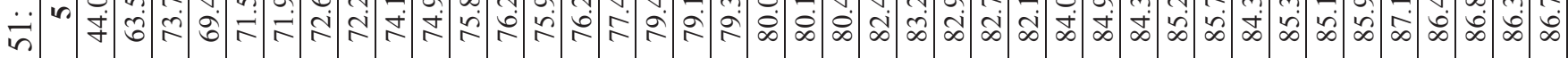

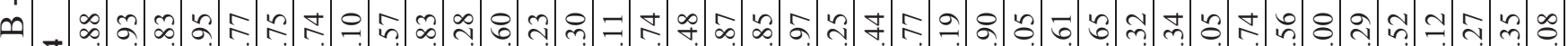

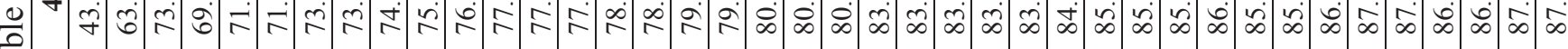
疋

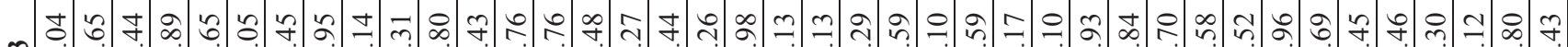

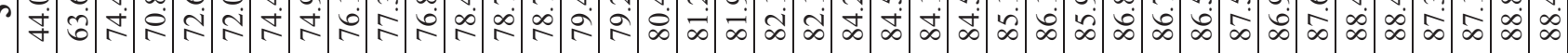
ㅇำ Иิ

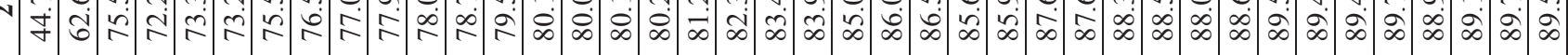

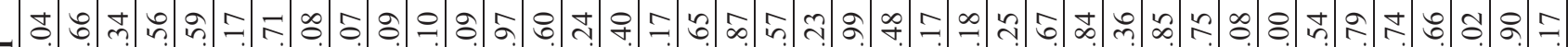

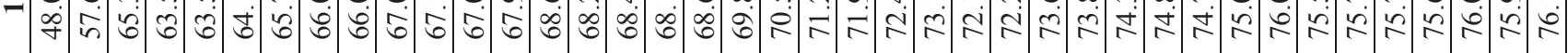
- 


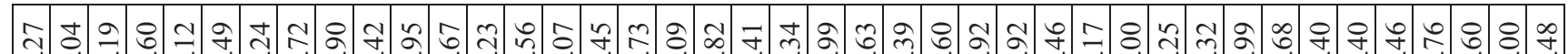

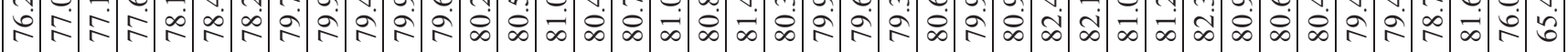
ว.

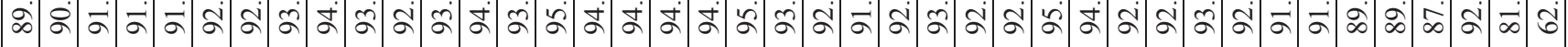

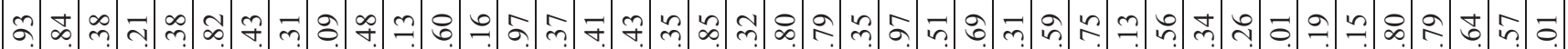

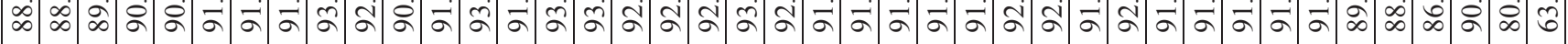

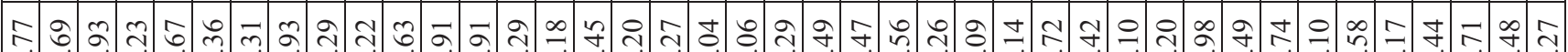

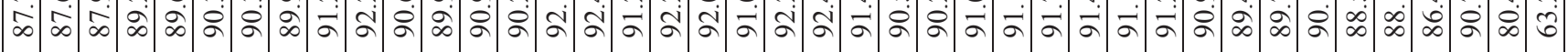

ব)

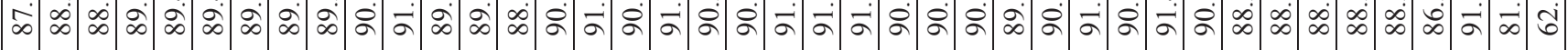

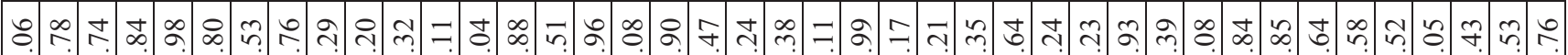

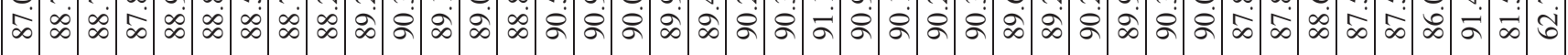

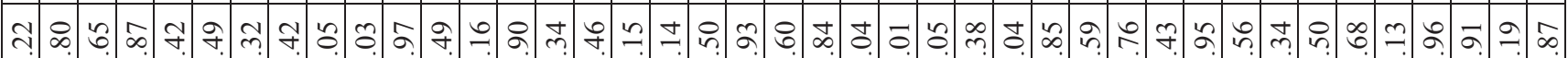

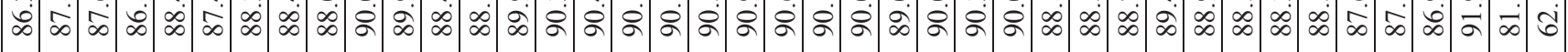

₹ \& สำ

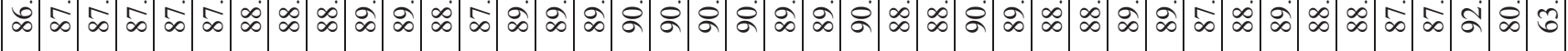

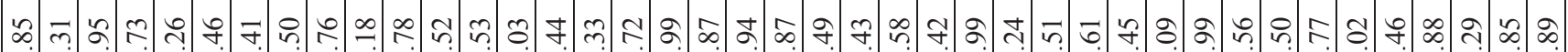

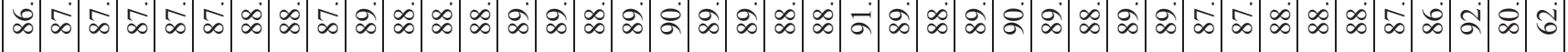

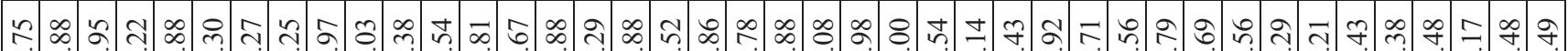

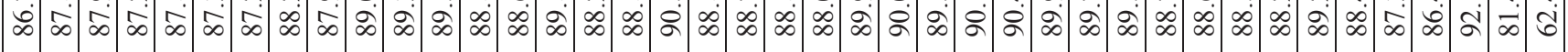

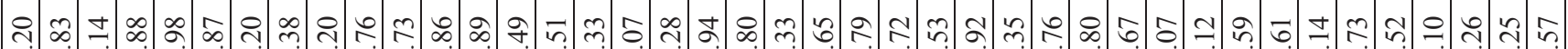

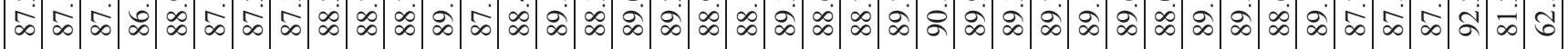

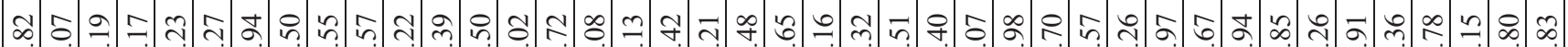

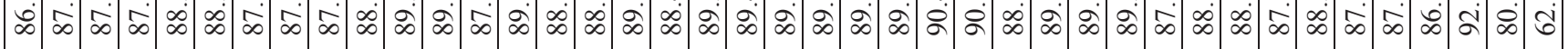

ஜ

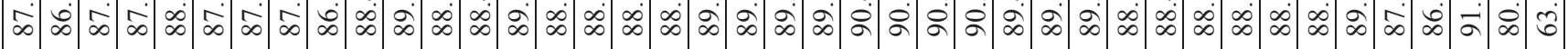
角

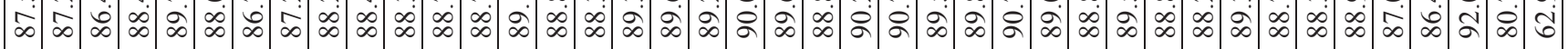
?

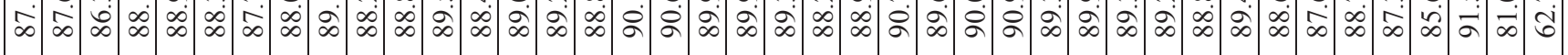

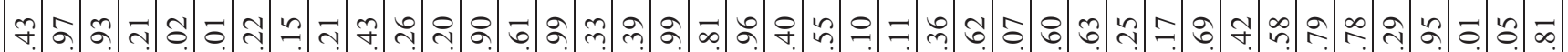

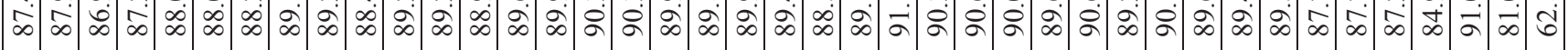

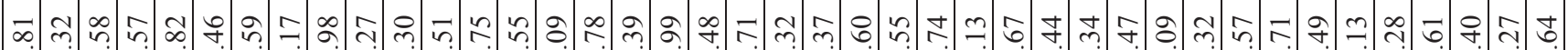

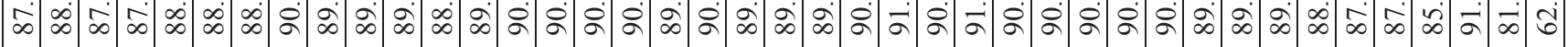

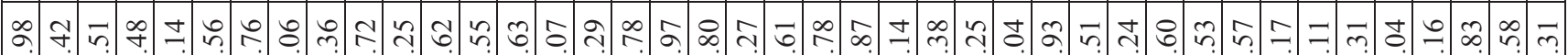

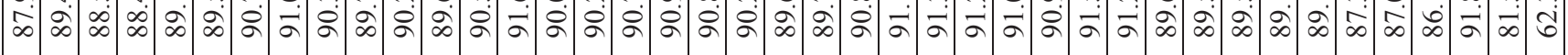

ๆ

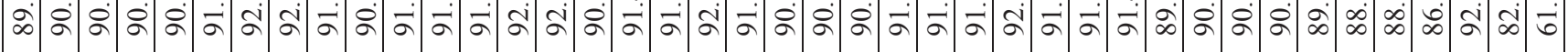

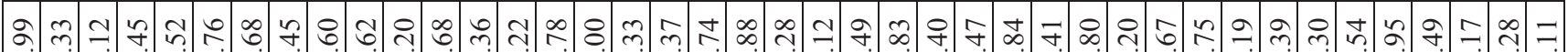

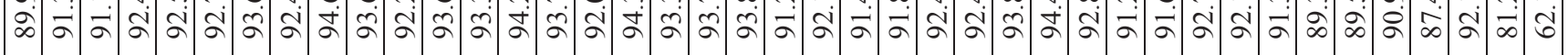

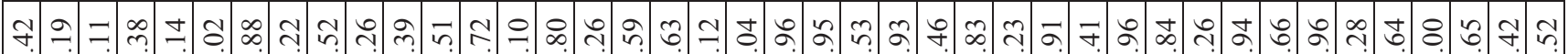

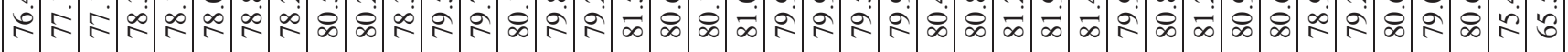

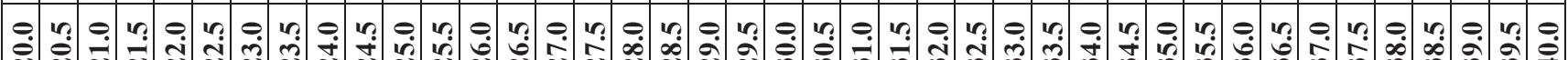

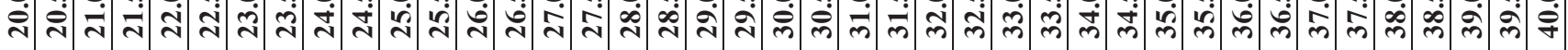


=

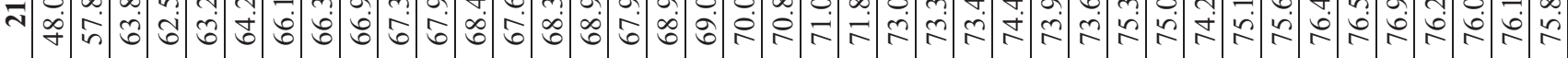
- t)

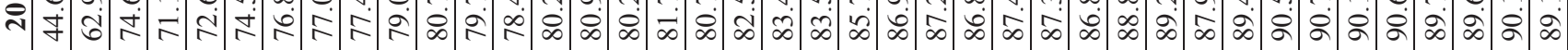

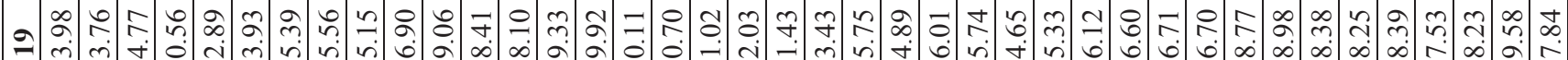
f)

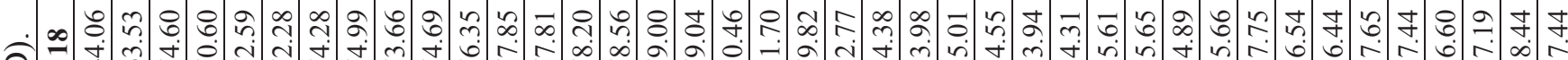

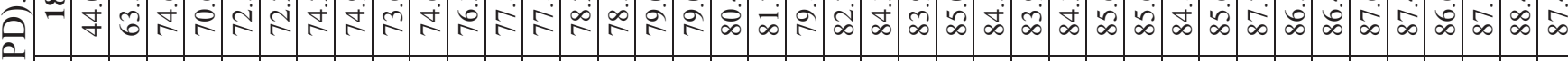

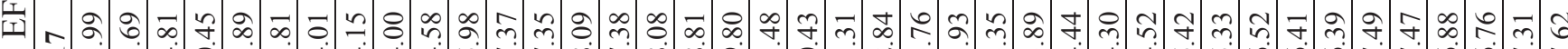

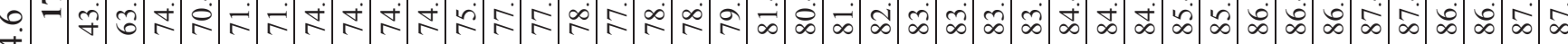

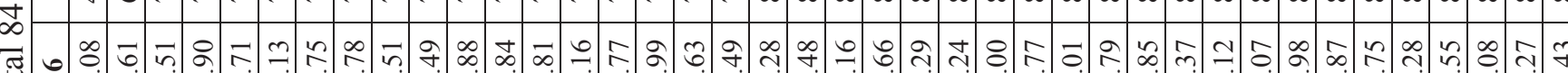

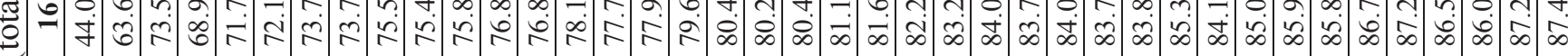

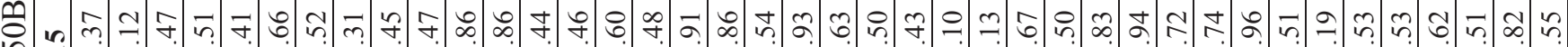

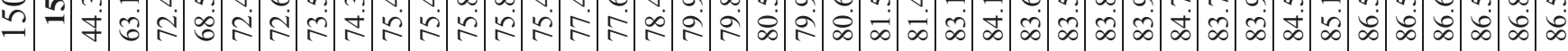

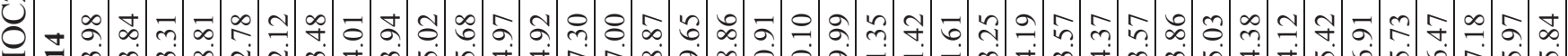

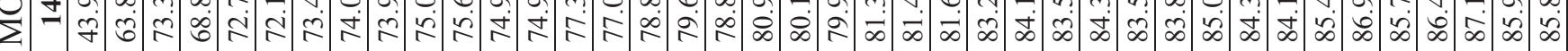
彳

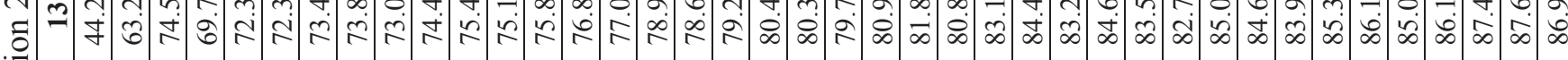
勿

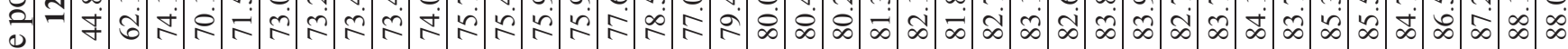

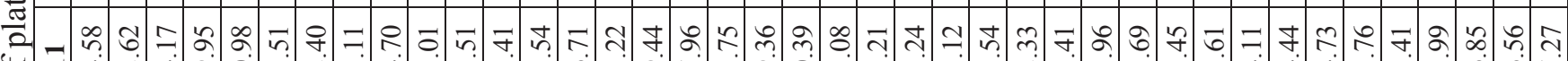

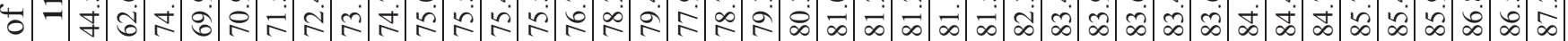

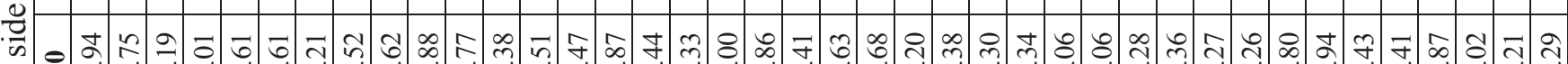

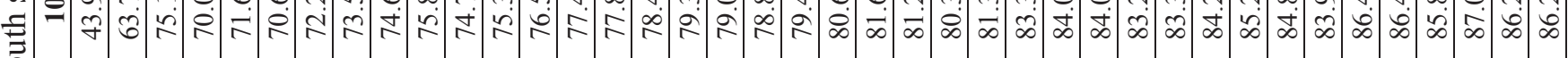

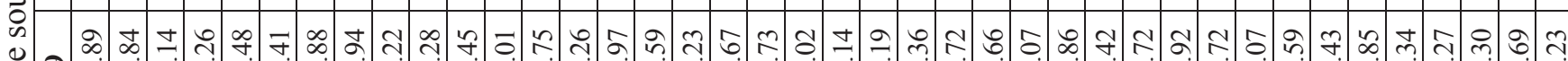
I ㅇำ

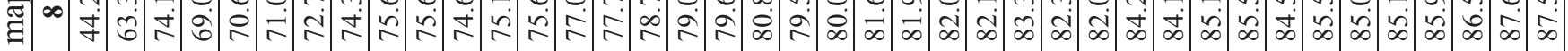
0

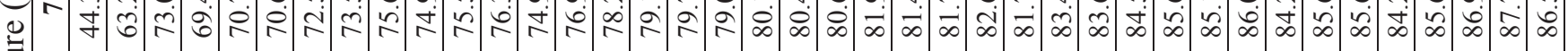

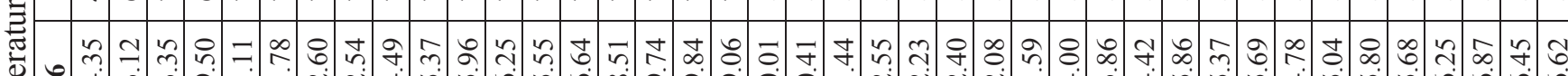

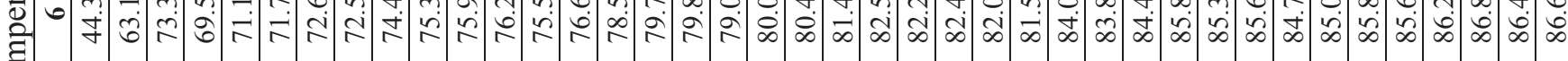
•

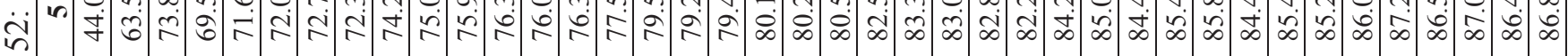
๓

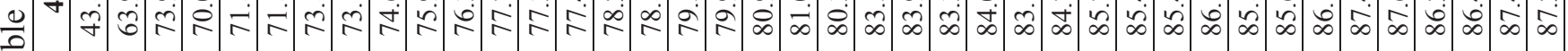
๑

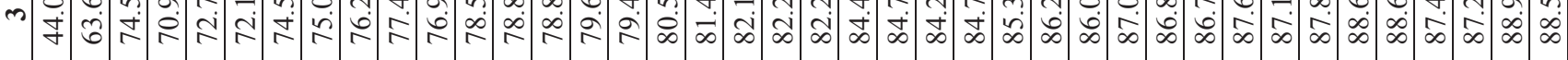
ㄴำ

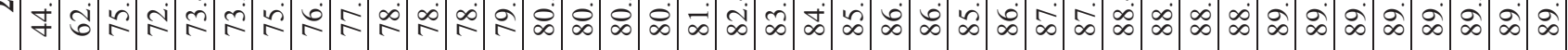
-

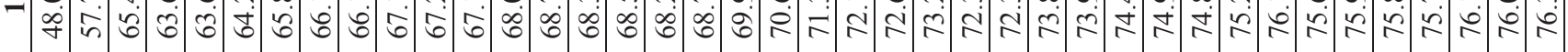
舟 


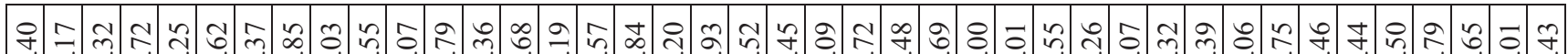

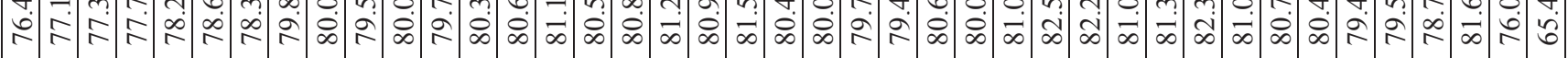

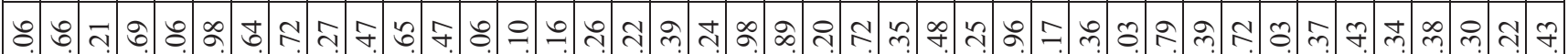

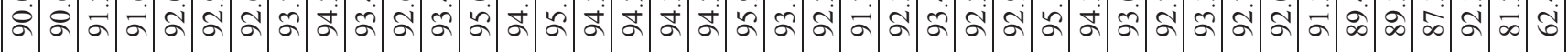

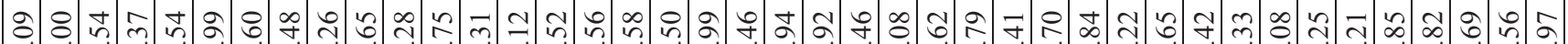

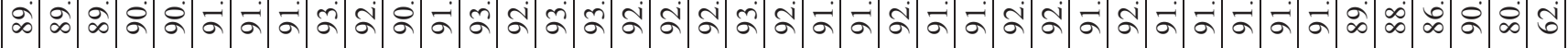

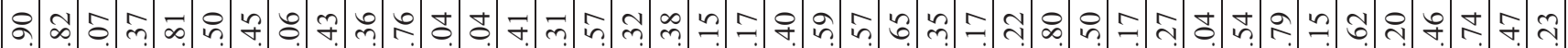

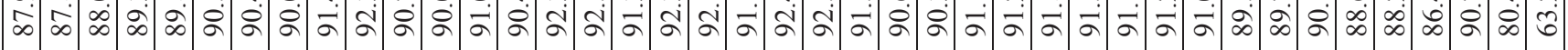

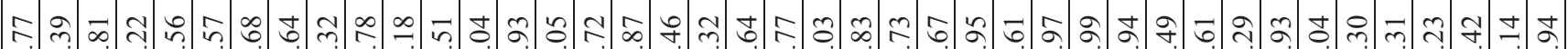

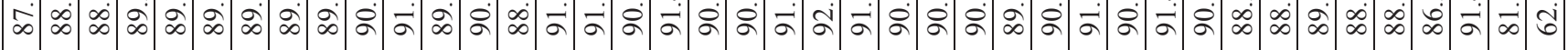

$\rightarrow$ \&

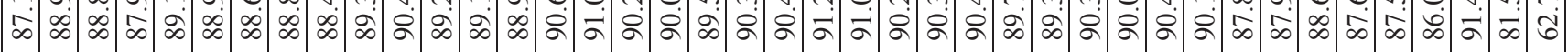

m

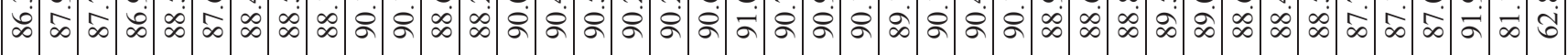

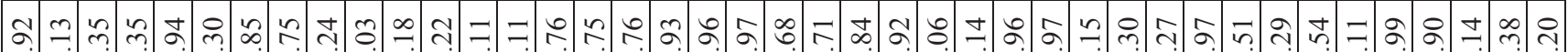

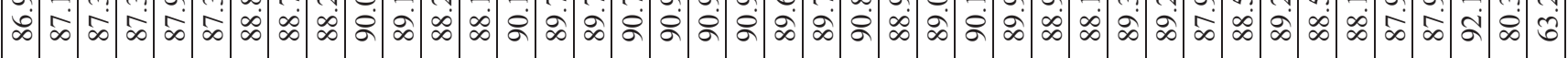

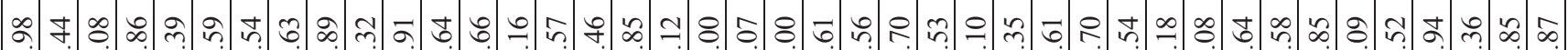

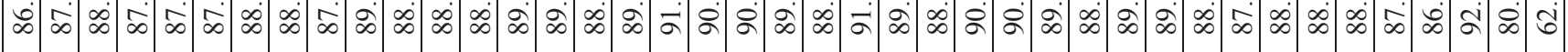

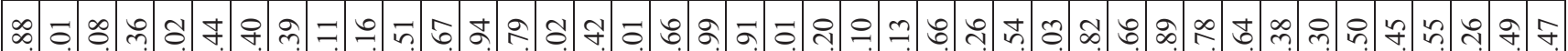

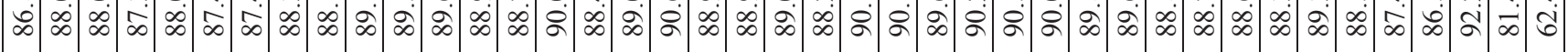

每

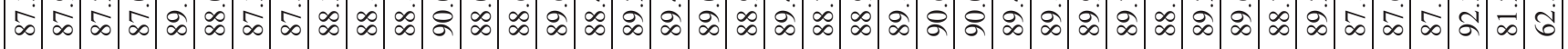

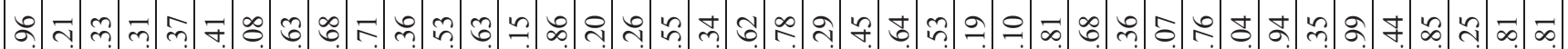

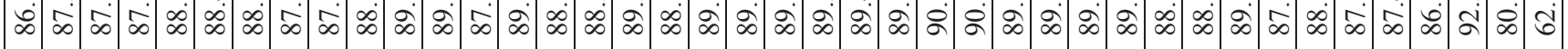

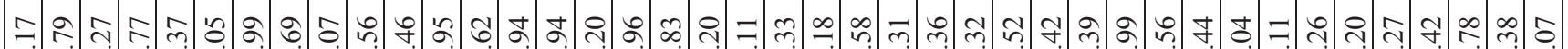

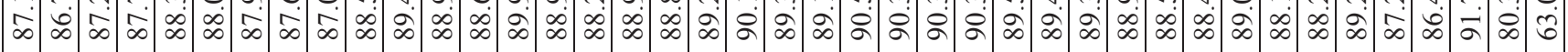

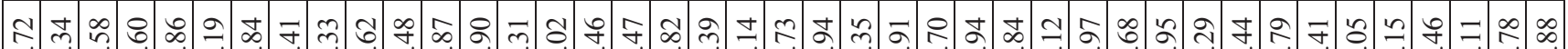

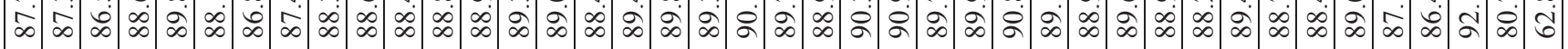

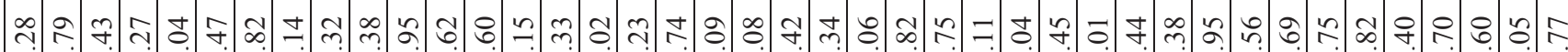

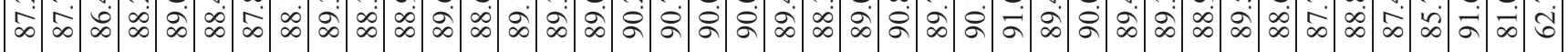

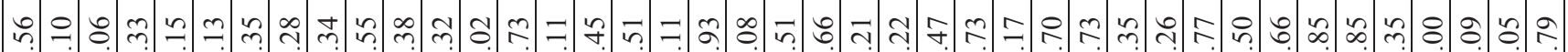

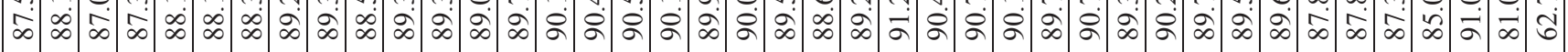

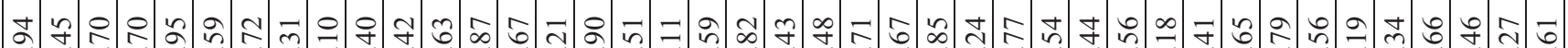

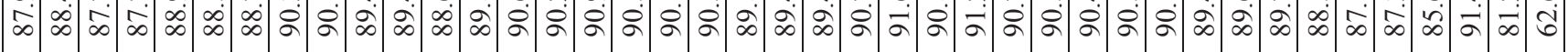
ㄱํ유

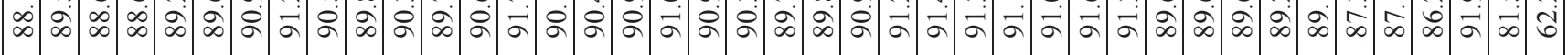

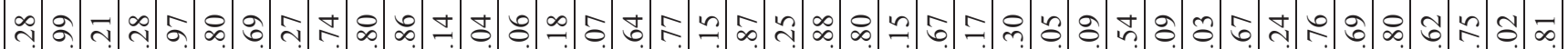

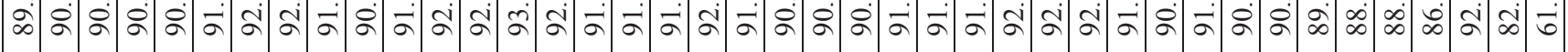

군

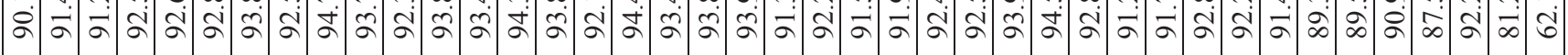
约每

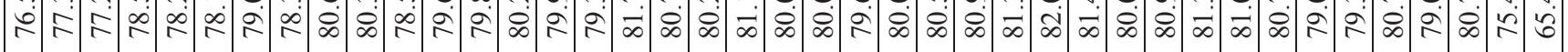

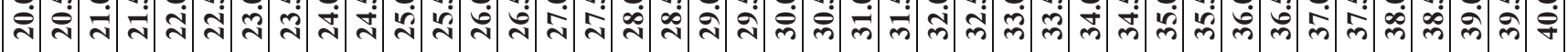




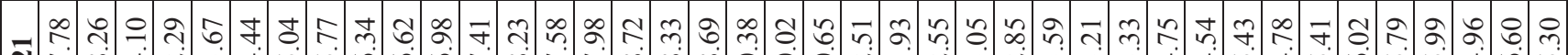

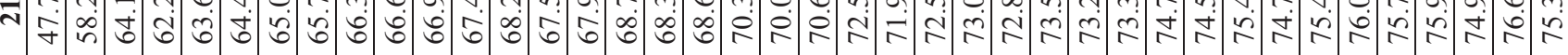
- กิ่ี่ స

๑ ఫ

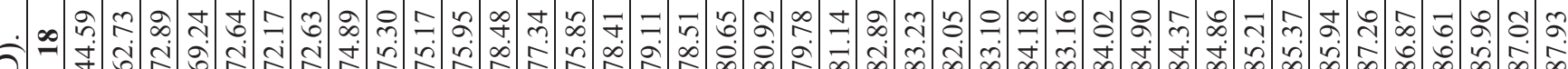
(2) 武 f

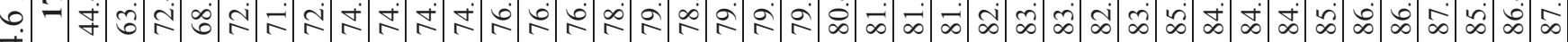

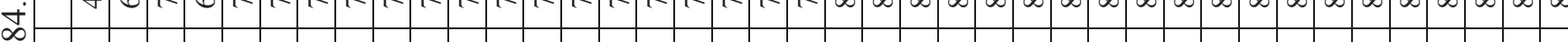
च ๑

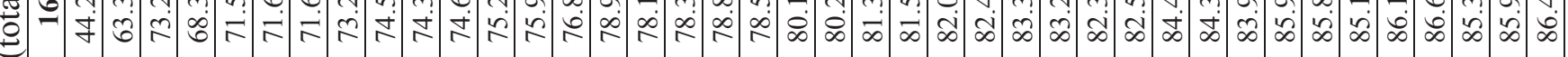

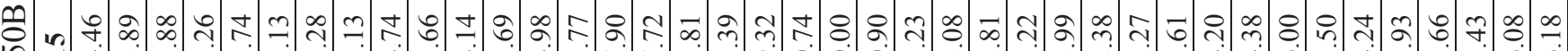

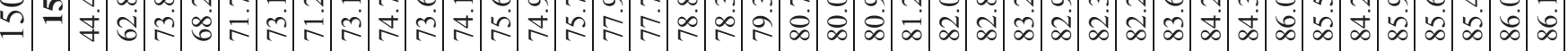
○ t

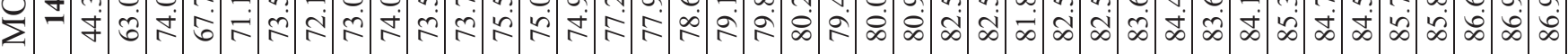

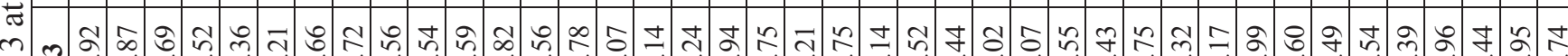

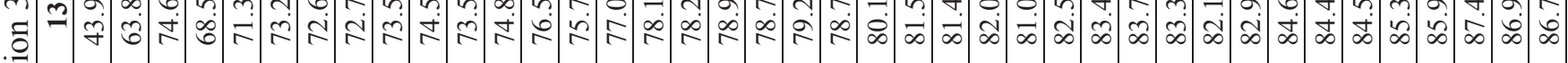
:

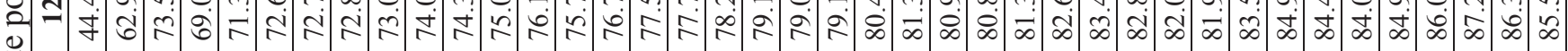

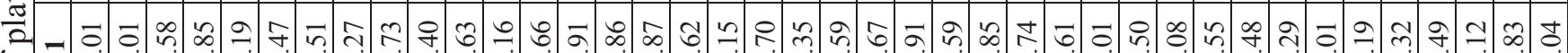
苛 =

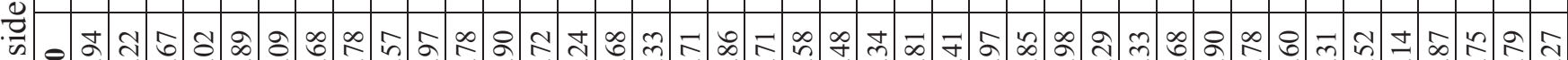

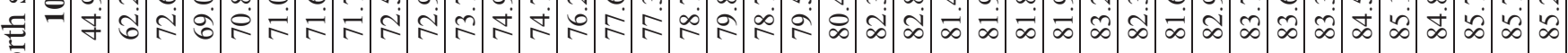

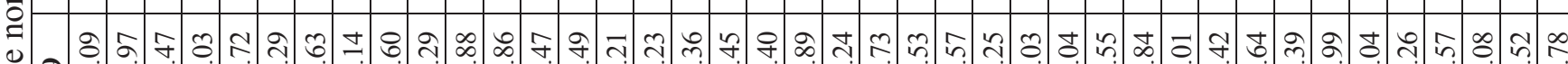

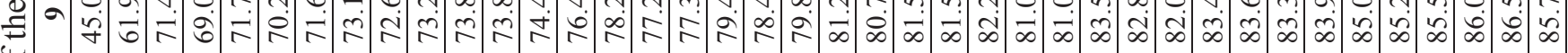
*

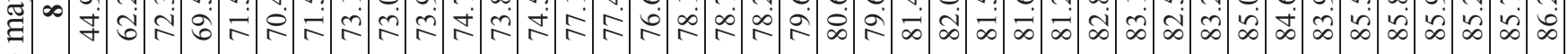

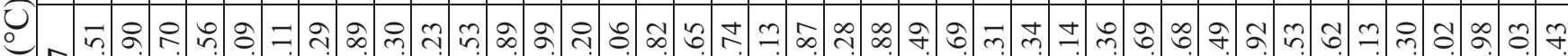
⿹

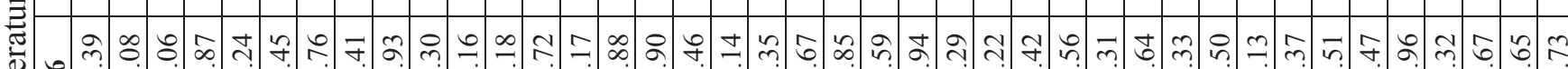

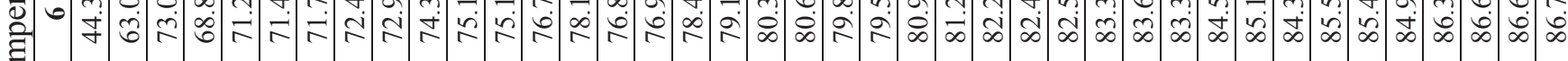

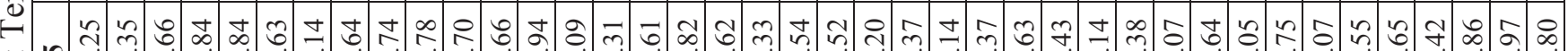
in

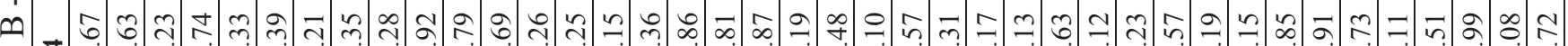

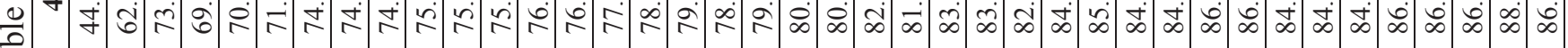
$\frac{\pi}{\pi}$

- Ұ

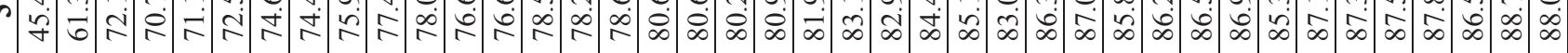
苟

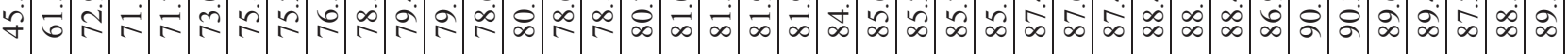

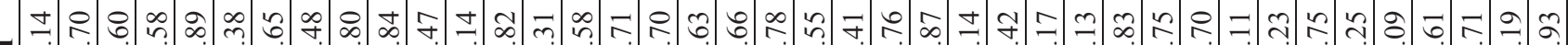

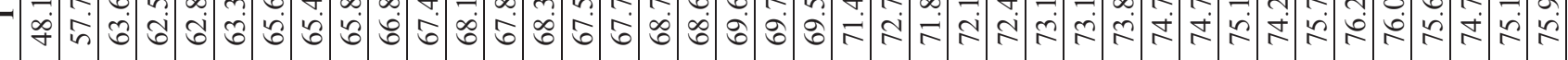

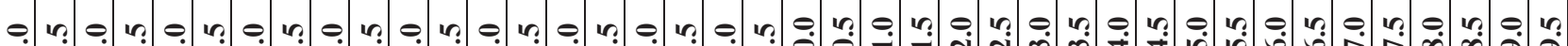

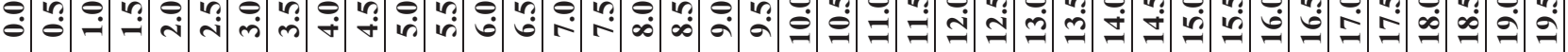




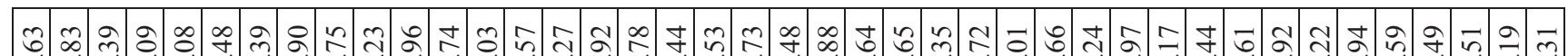

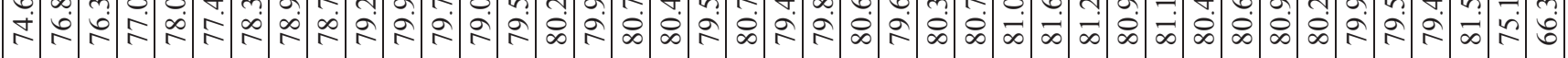

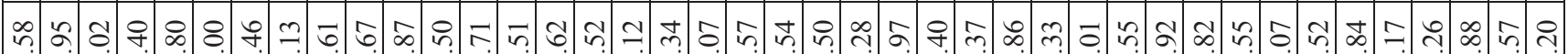

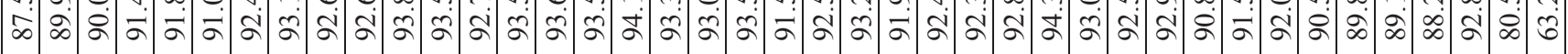

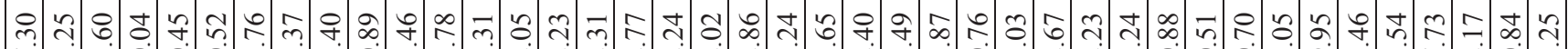

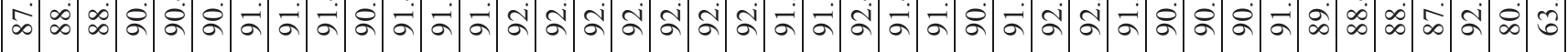

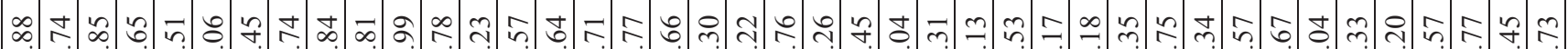

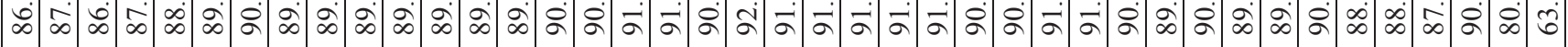

สุ

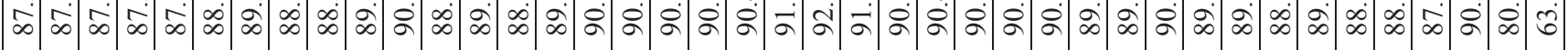

ᄀ.

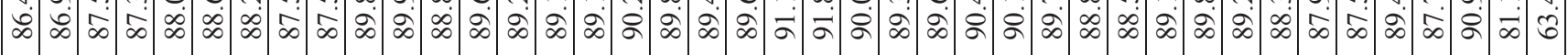

วิ

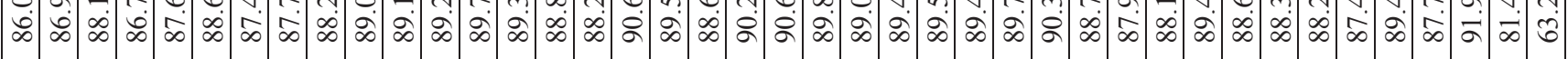

กิ

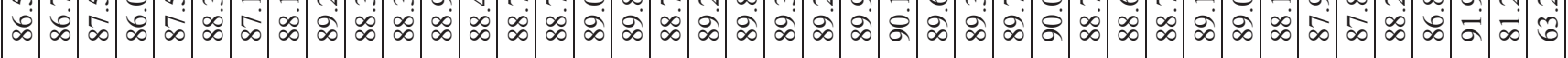

\&:

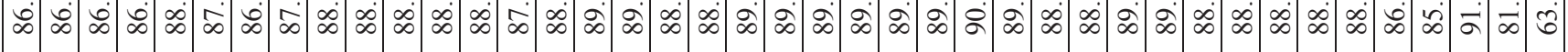

n! t

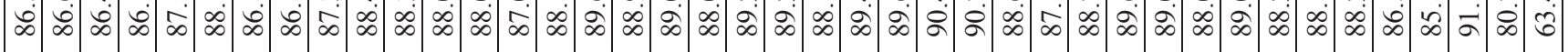

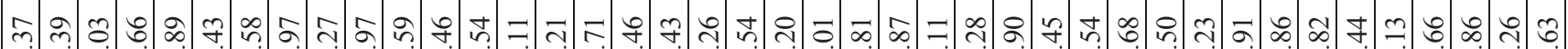

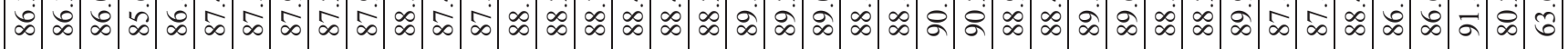
궁

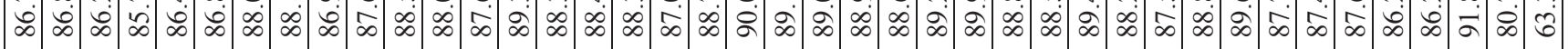
กㅊำ

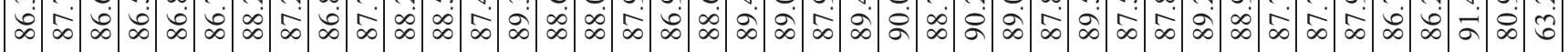

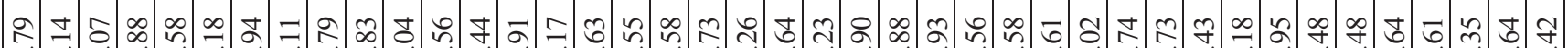

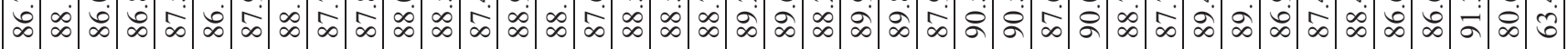

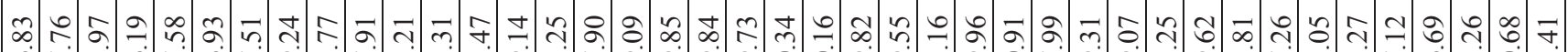

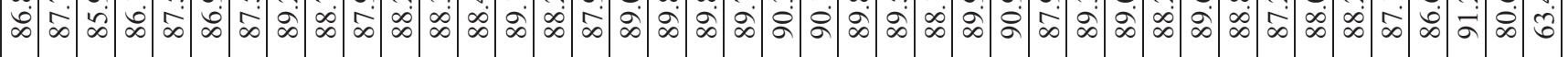
И̊

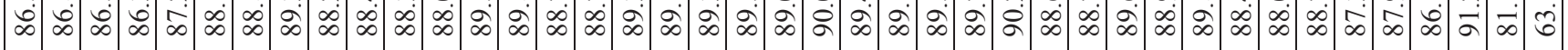

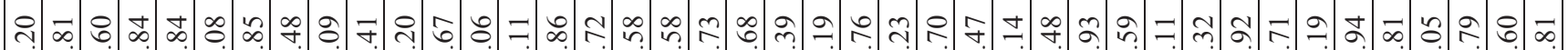

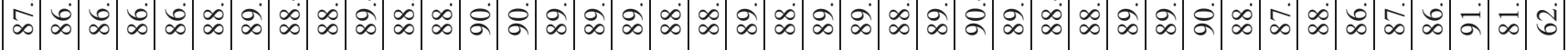

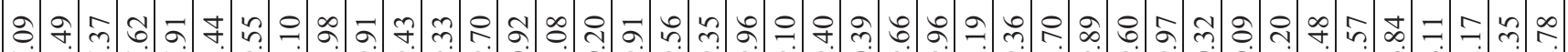

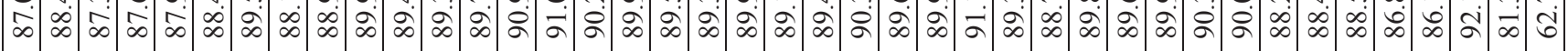

我

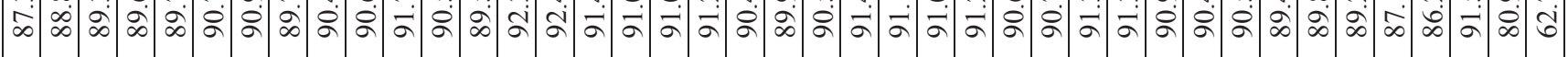

๙

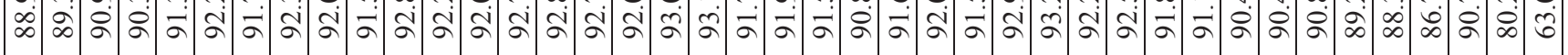

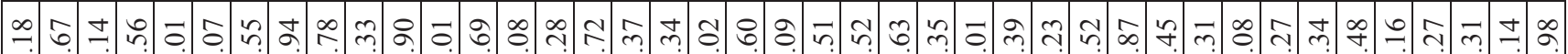

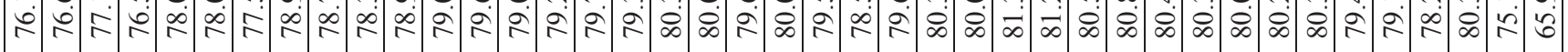
我

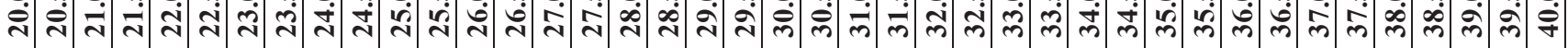


- 수유. ה年

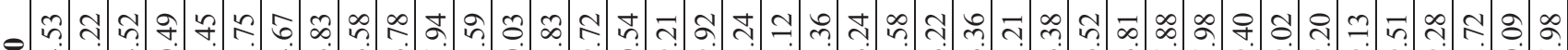
సै

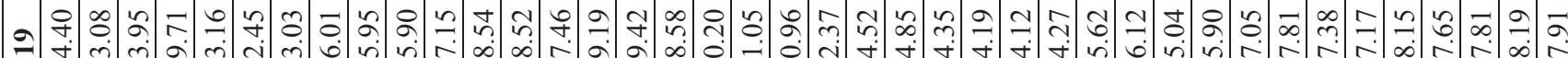
舟

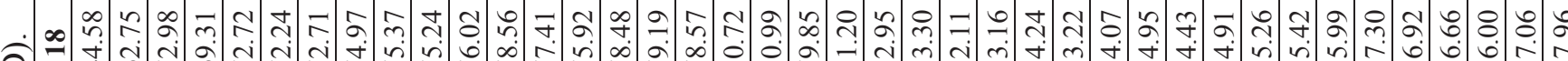

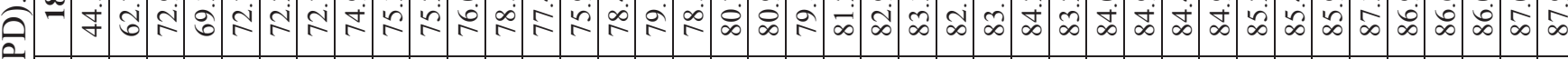
牙 ᄀำ - = 声 古

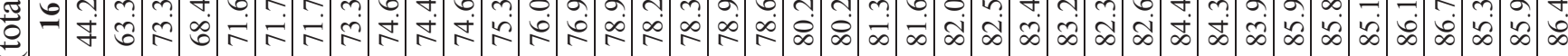

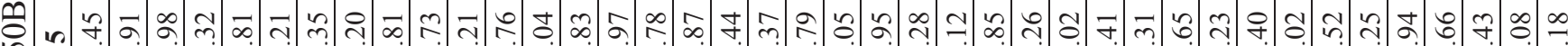

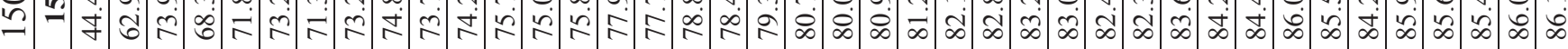
○

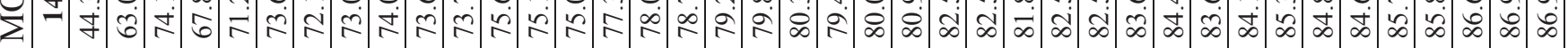

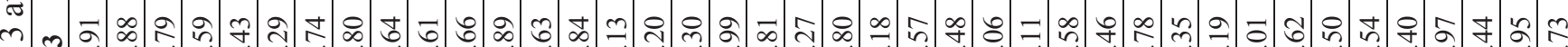

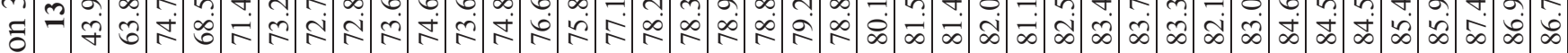
苟

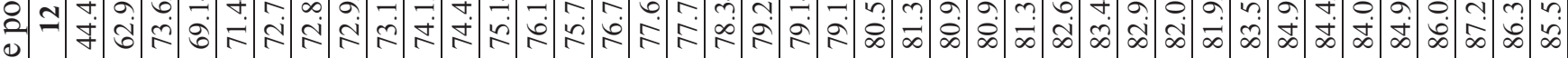

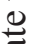

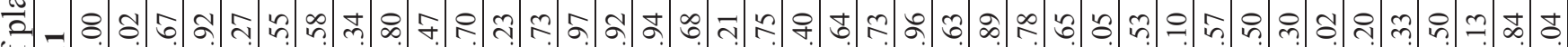

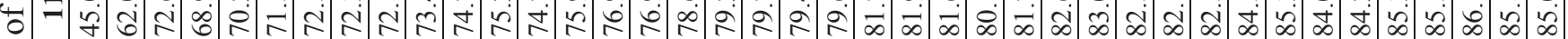

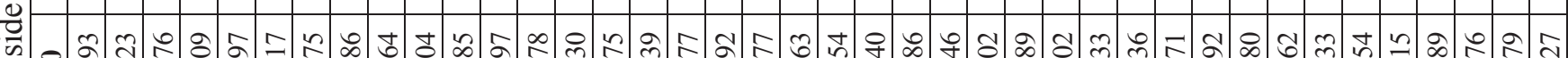

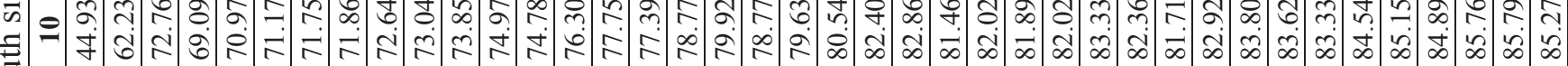

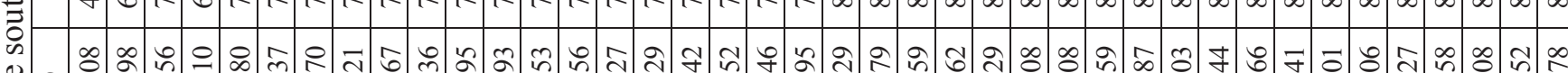

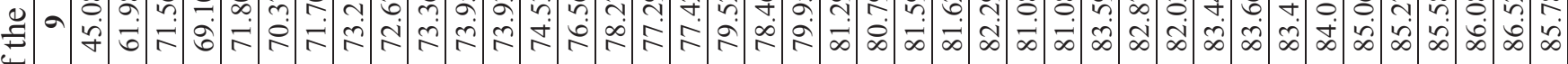

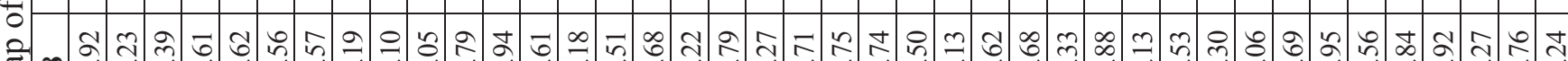

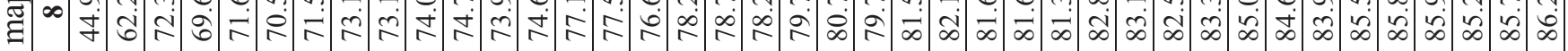

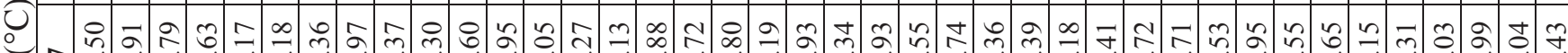
⿹

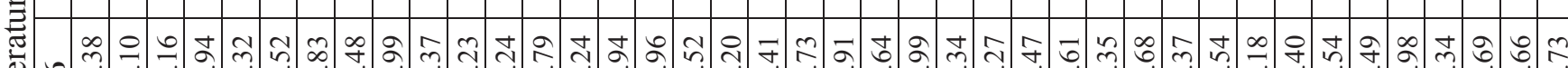
青 过 •

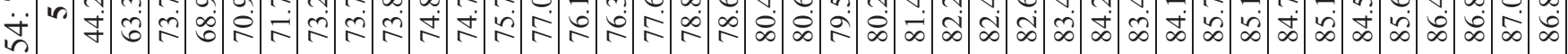

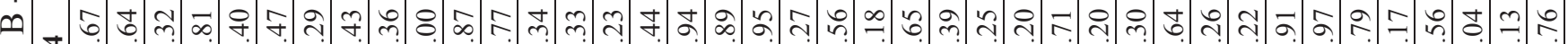

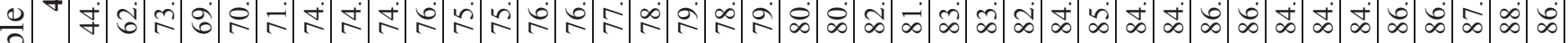
ब

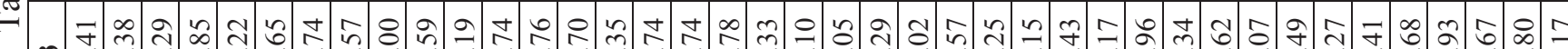

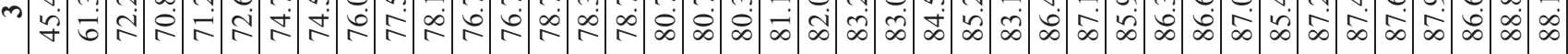

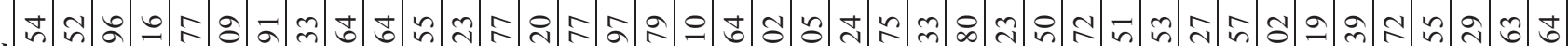

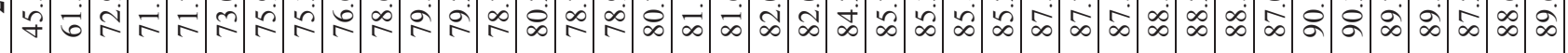

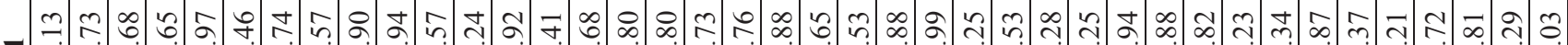

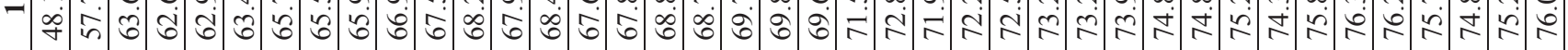

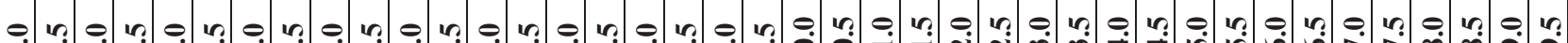

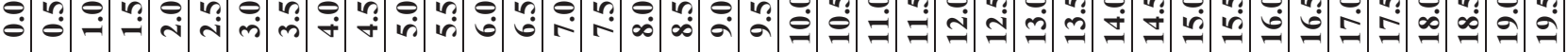




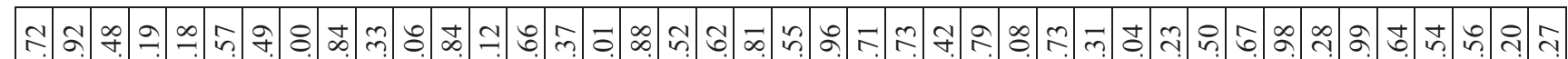

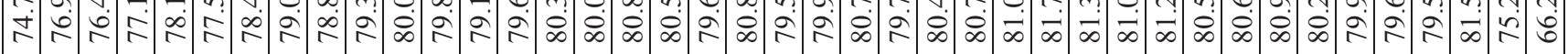

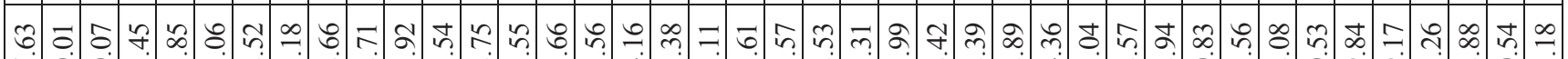

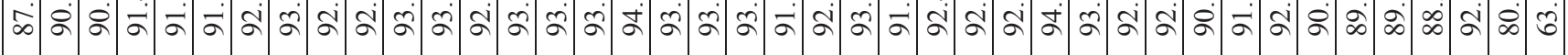

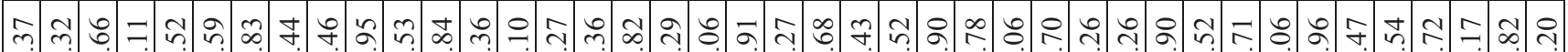

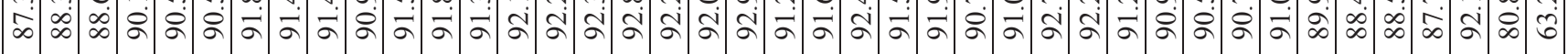

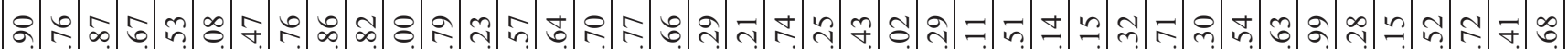

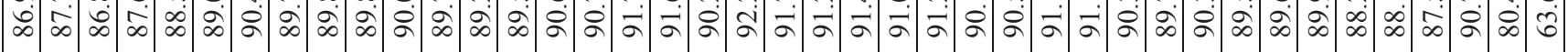

สุ

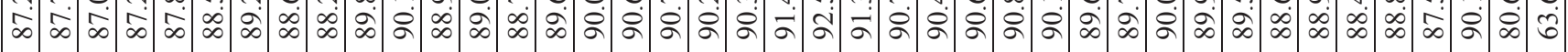

Ұ. ล. f.

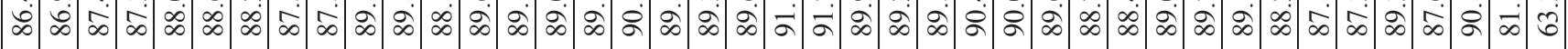

๘)

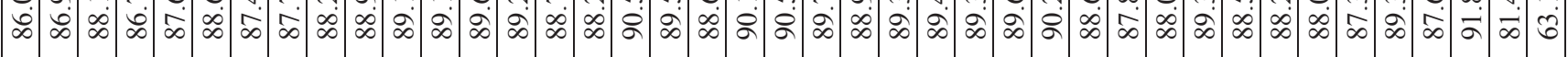

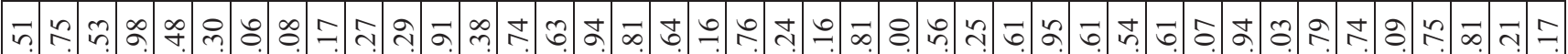

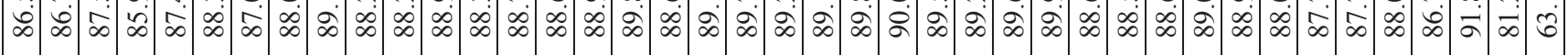

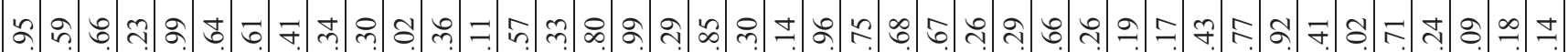

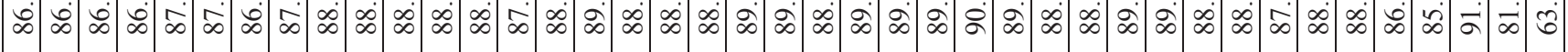

пี

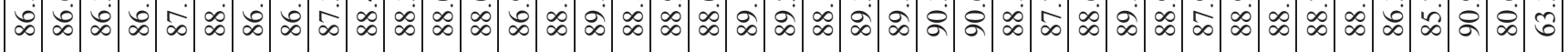

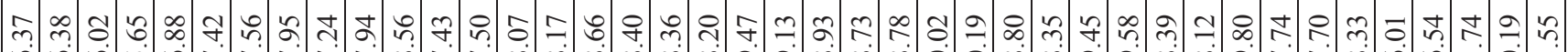

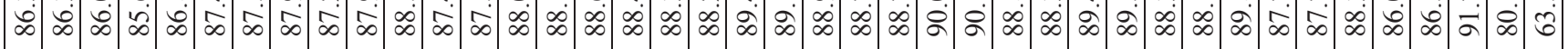

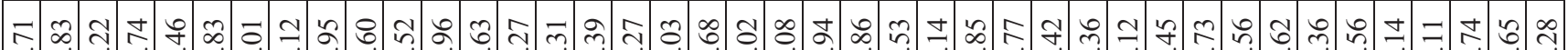

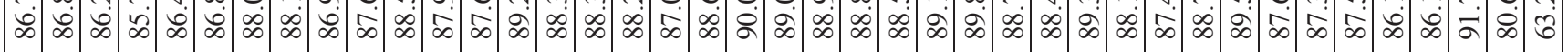

กำ

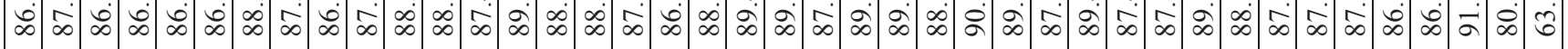

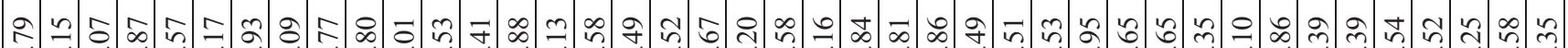

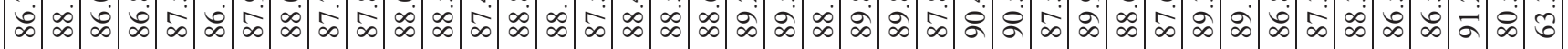

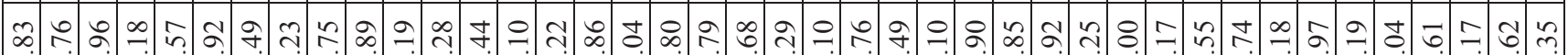

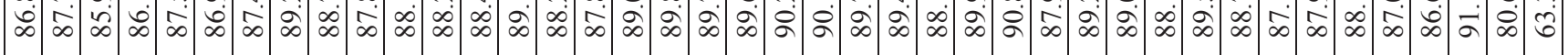

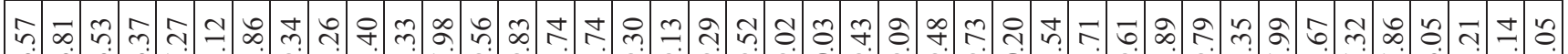

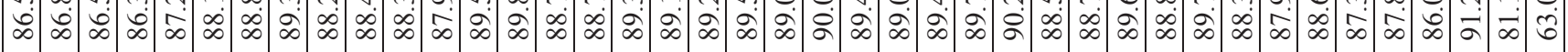
సฺ

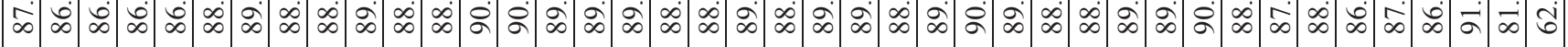

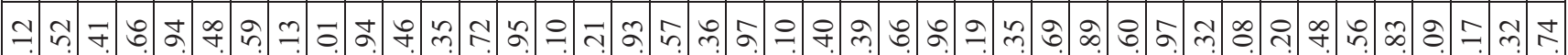

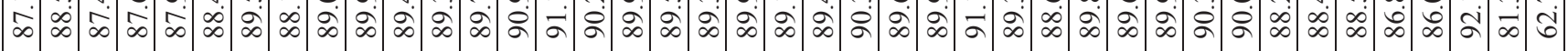

f.

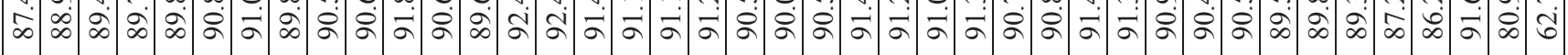

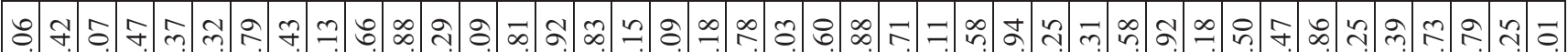

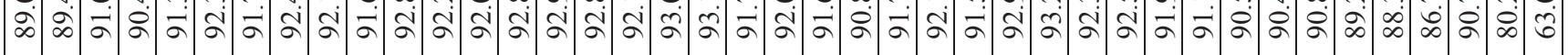

$\stackrel{\infty}{\curvearrowright}$ กฺ

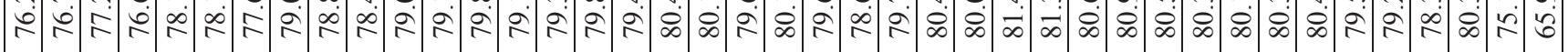

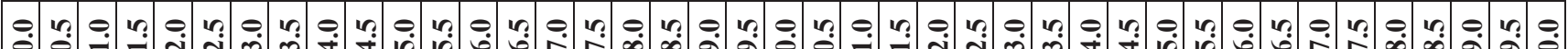

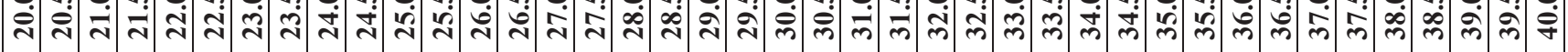




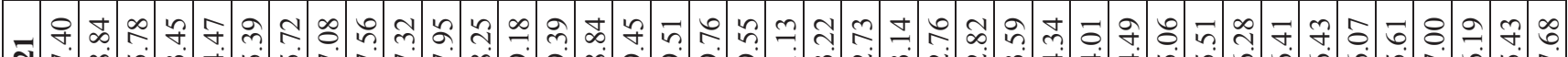

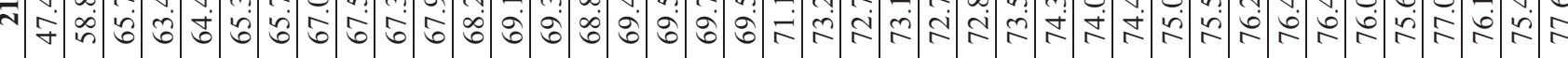
$\infty$ 乎

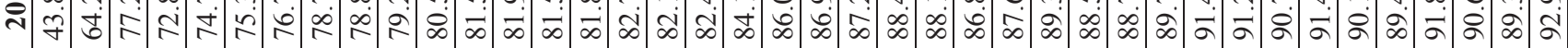

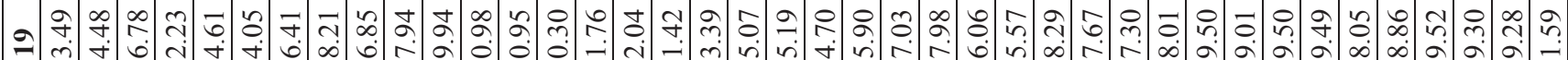

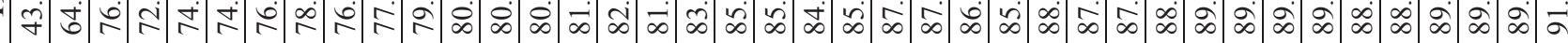

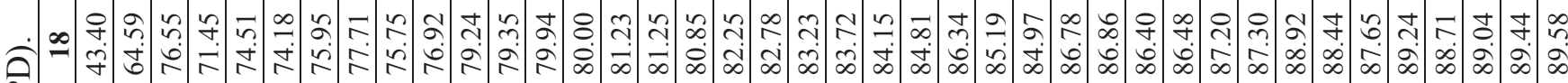
f

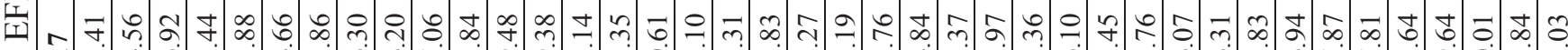

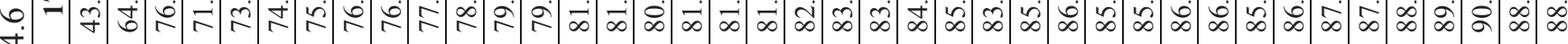

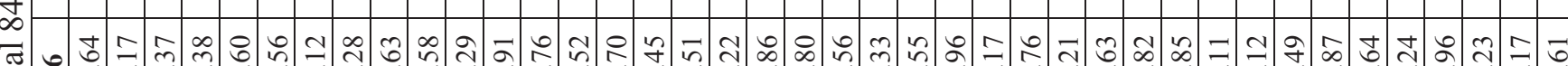

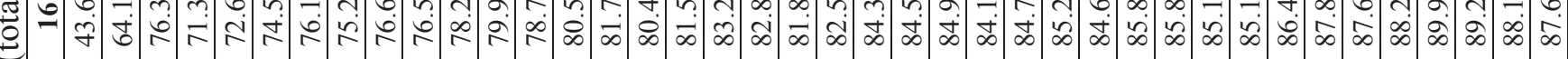

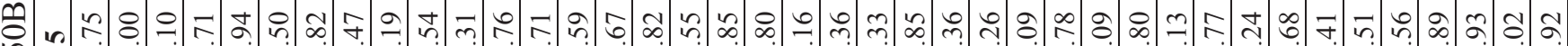

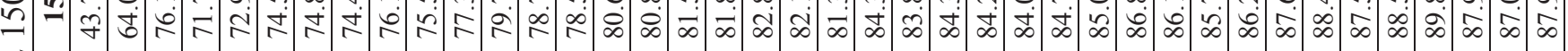

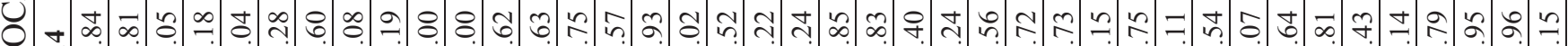

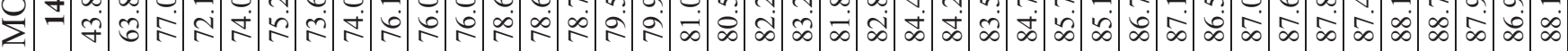
矛

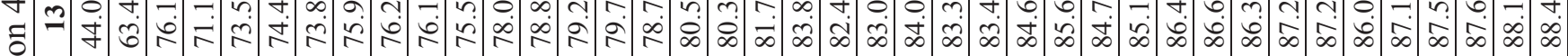
矛

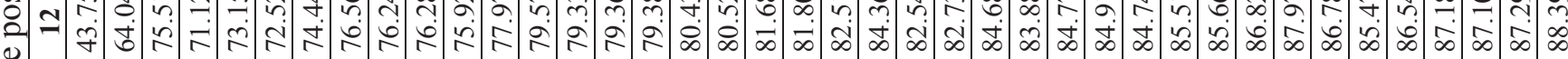
菊

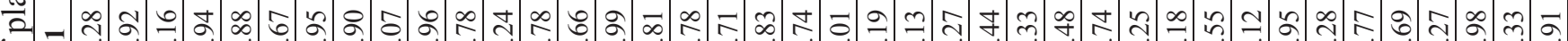
苛 =

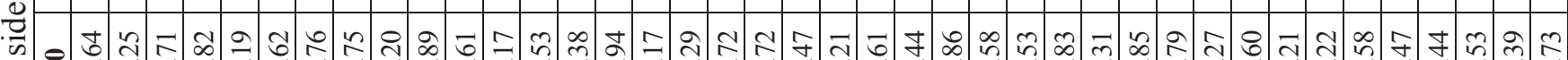
F

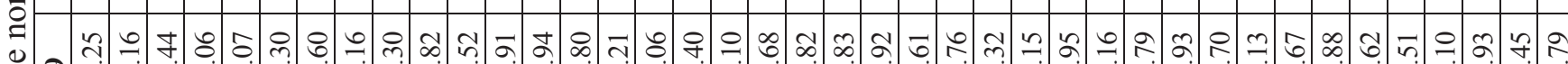

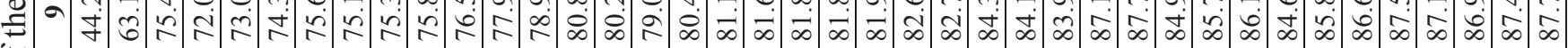
의

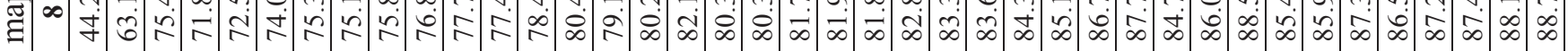
ర

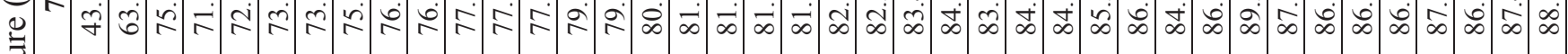

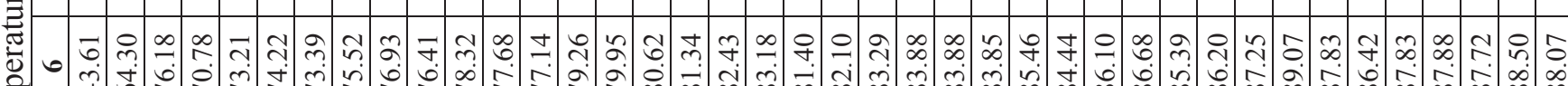

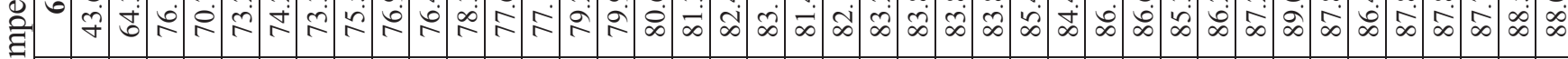

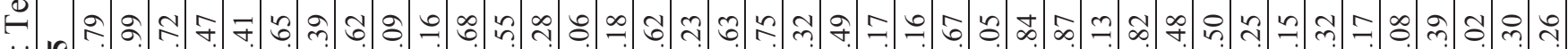

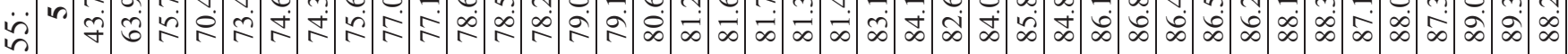

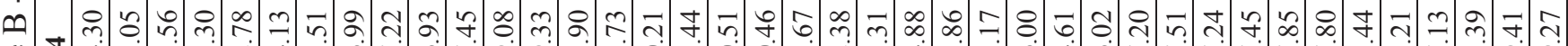

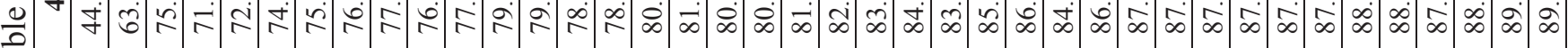
苛

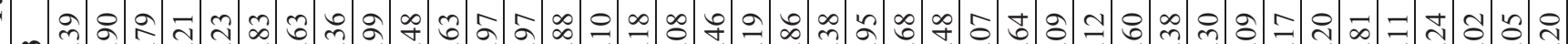
莳 J

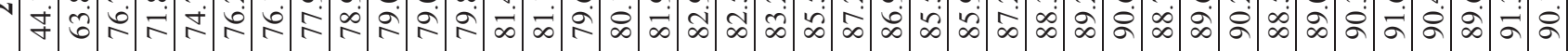
$-\infty$ ఏ

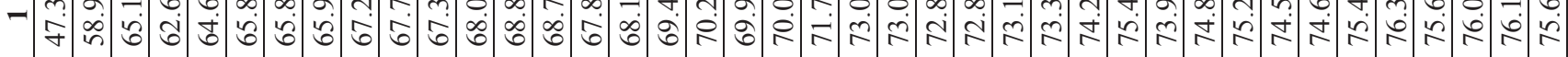
e n

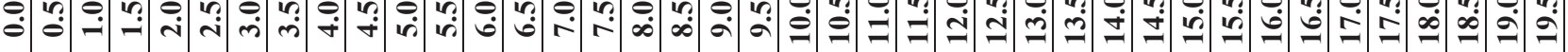




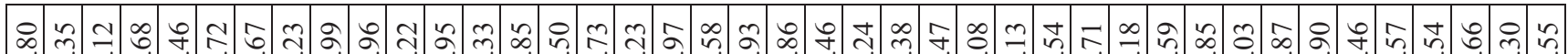

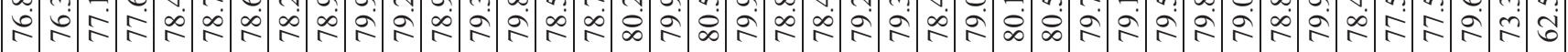

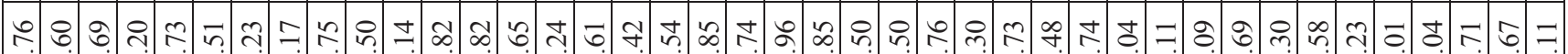

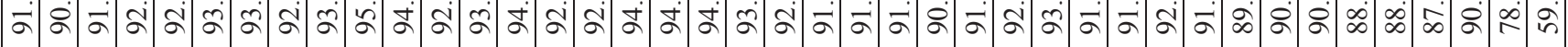

ลิ㇒

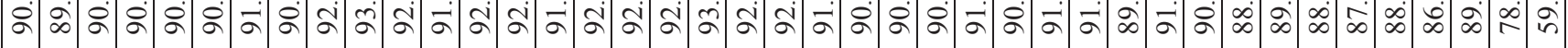

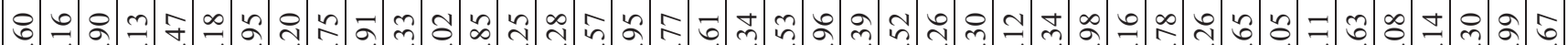

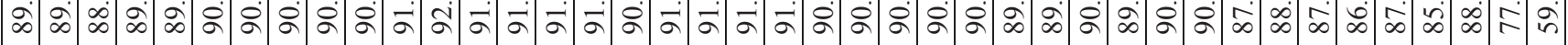

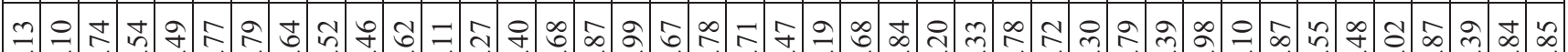

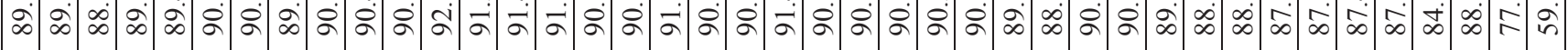

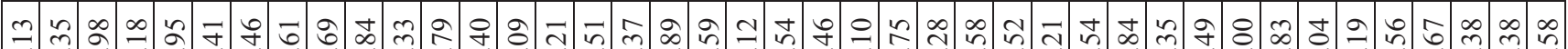

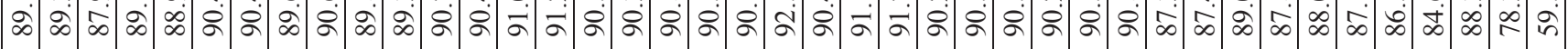

$\infty$ ล

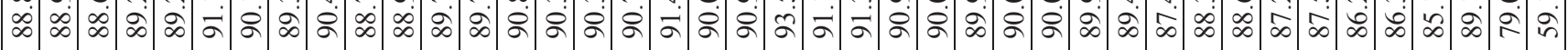

운

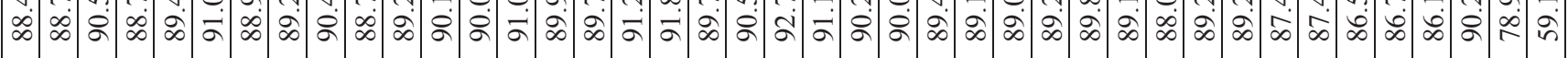

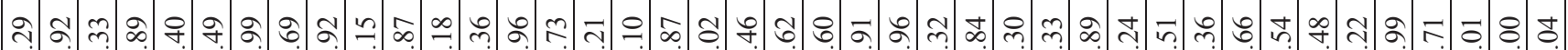

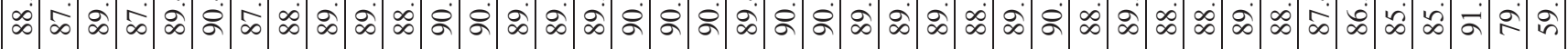

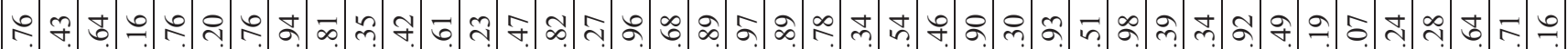

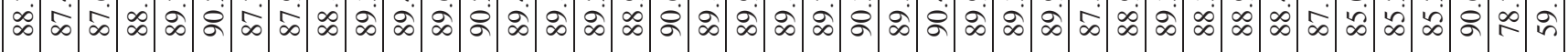

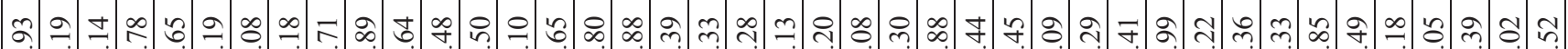

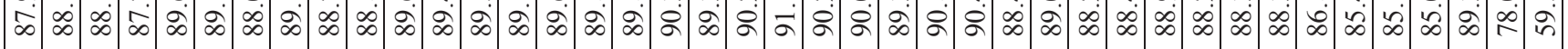

ఇ

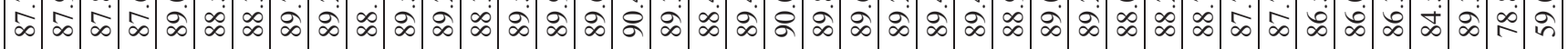

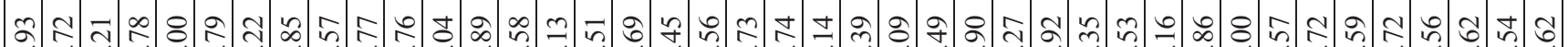

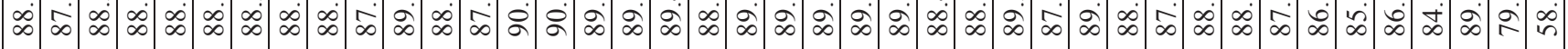

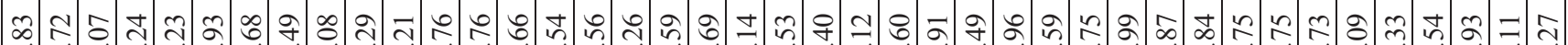

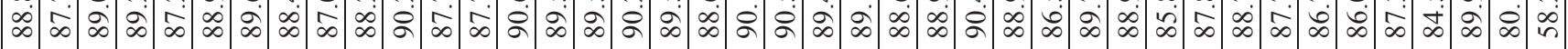
t

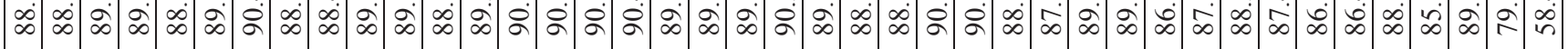
ळ

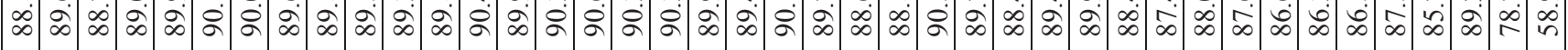

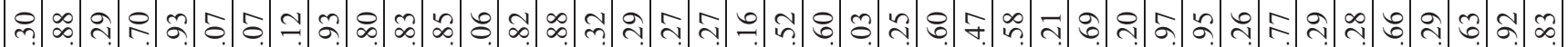

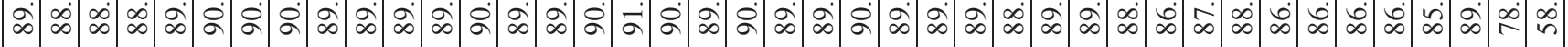

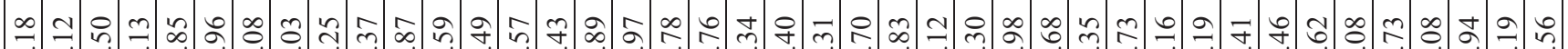

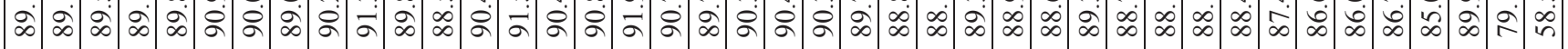

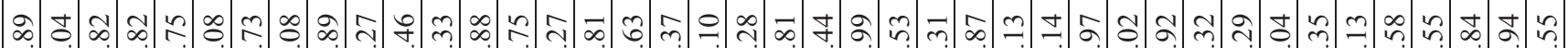

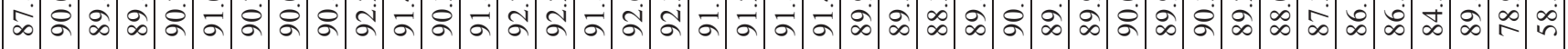

f œ

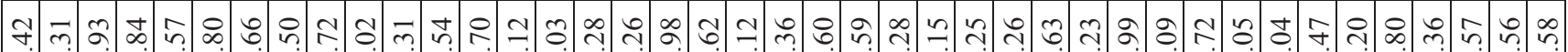

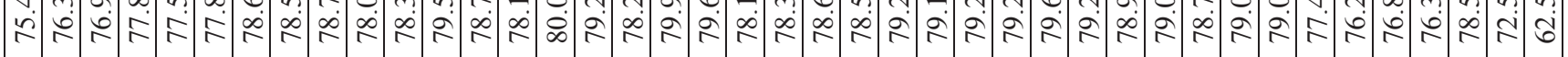


- च $\vec{\infty}$ b

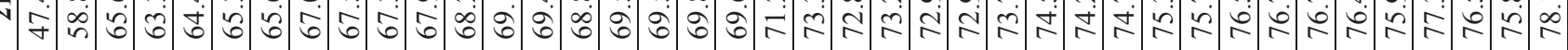

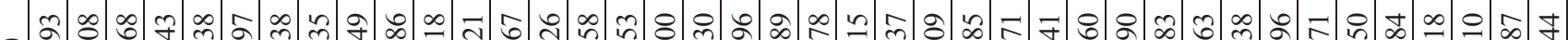

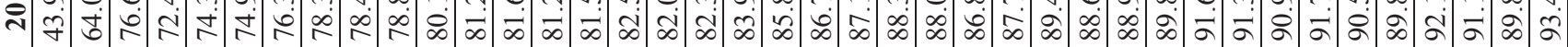

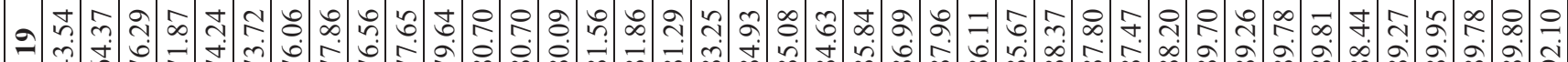

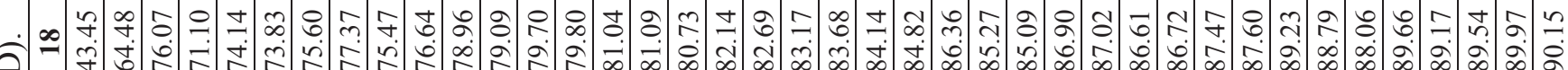
âf

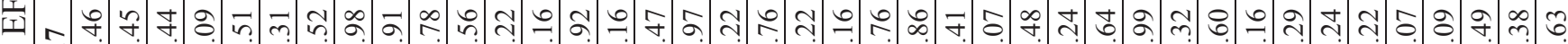

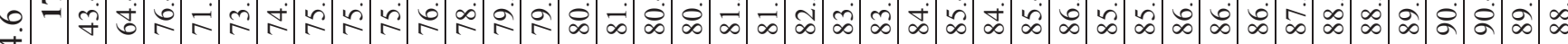

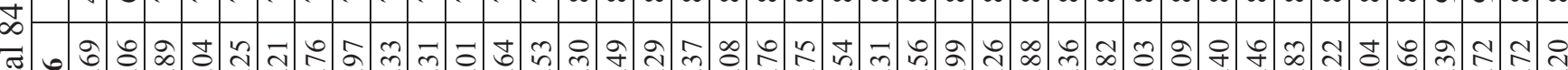

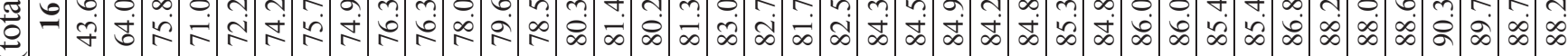
象

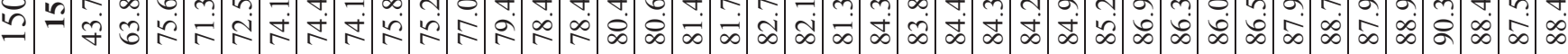
రำ

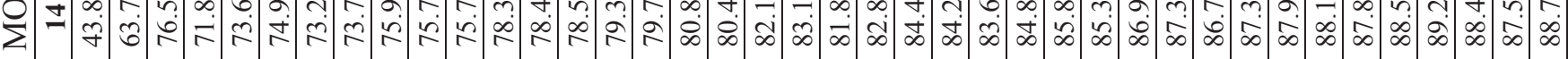
t

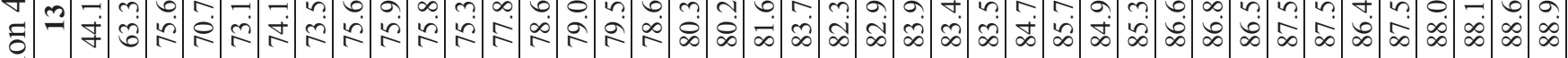
:

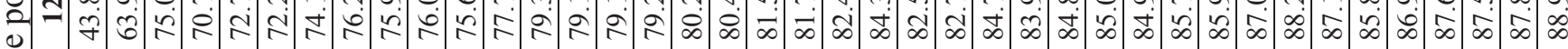
节

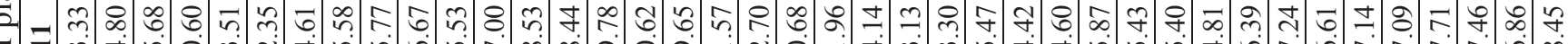

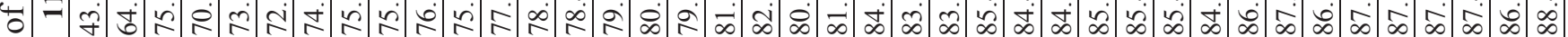
D.

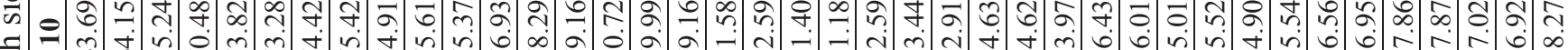

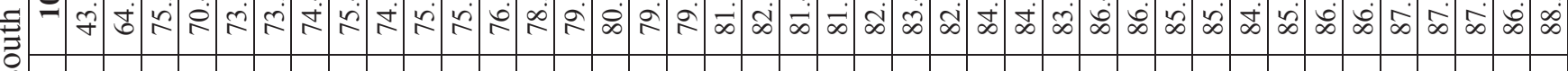

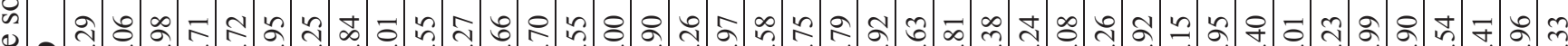

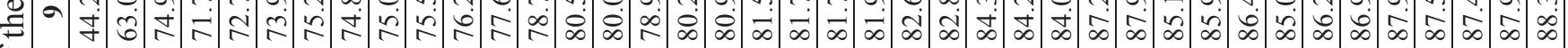

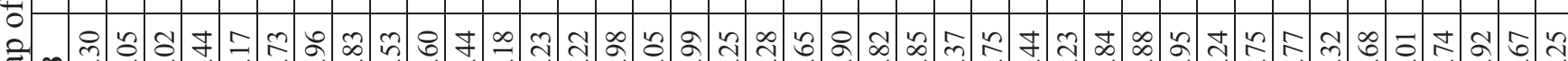

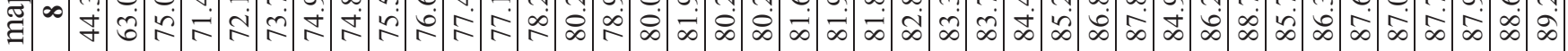

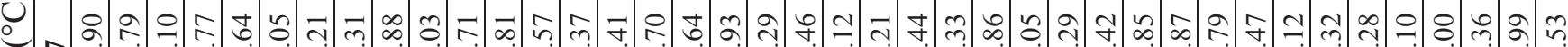

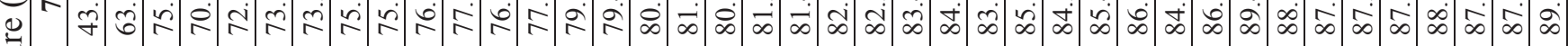
氙 :

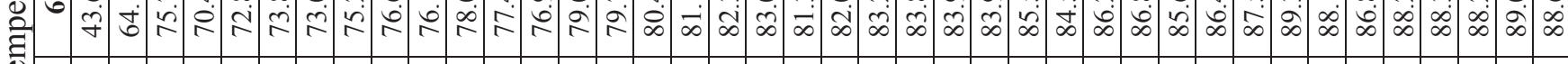

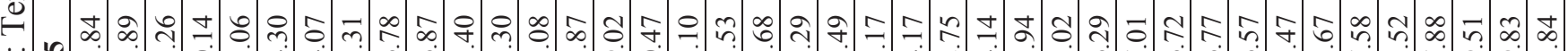

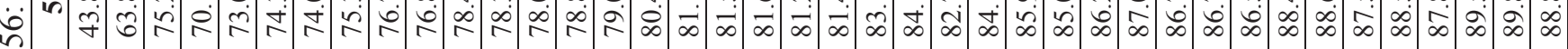

๑ t)

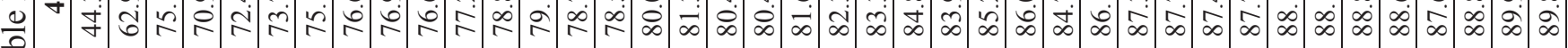

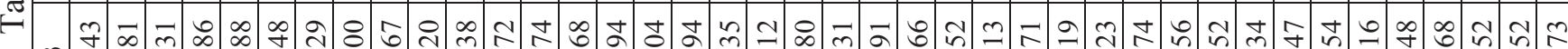

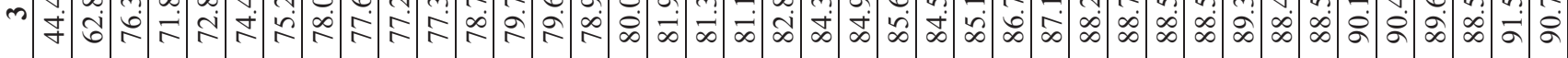

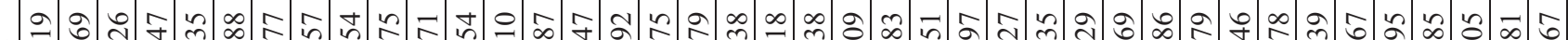

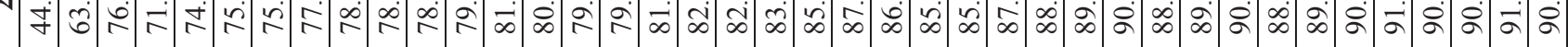

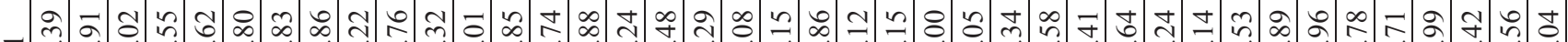

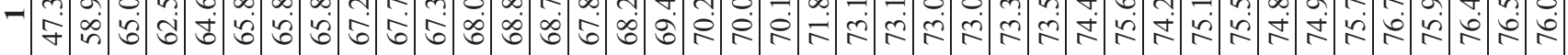
- n

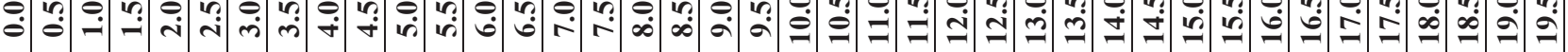




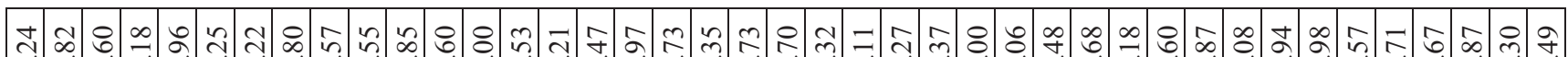

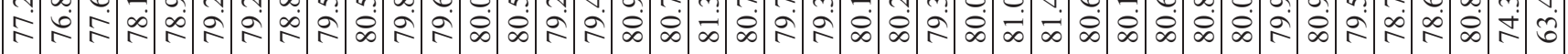

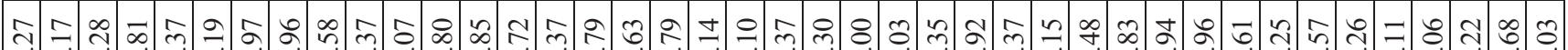

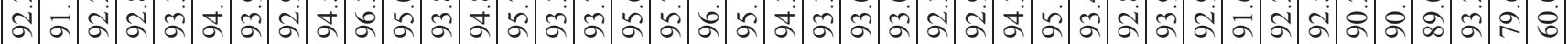

ஸี Ұฉ

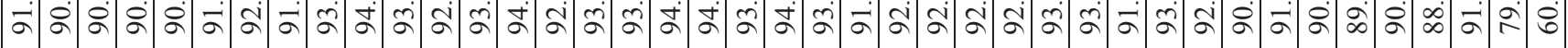

तิ $\infty$ iి

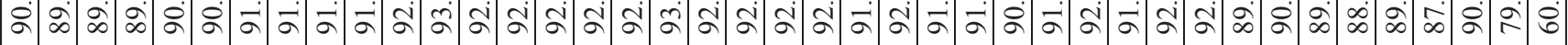

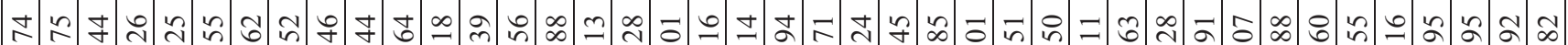

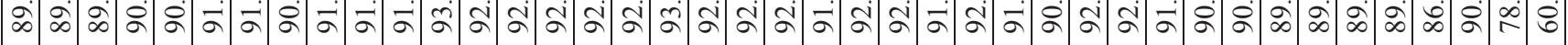

ఇ. ळ

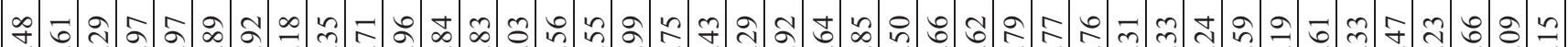

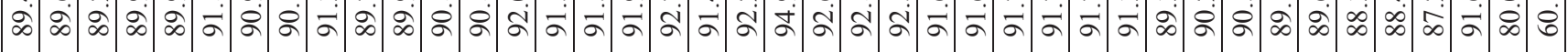

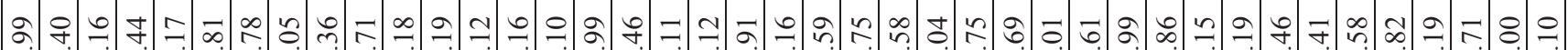

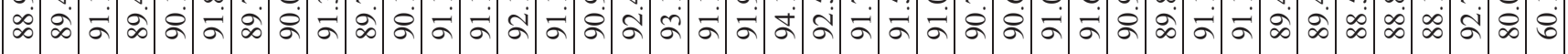

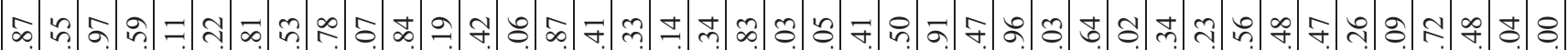

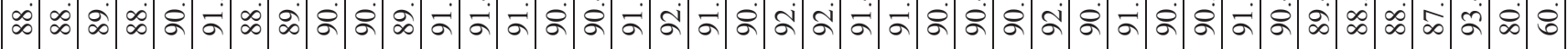

m.

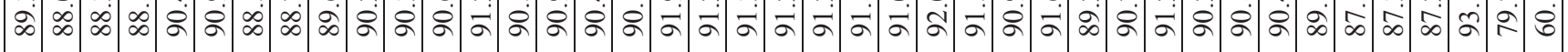

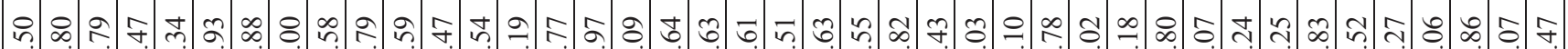

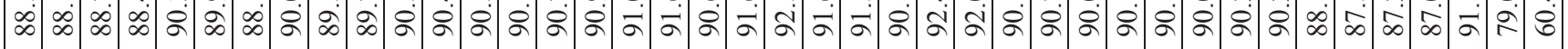

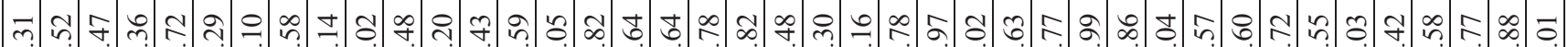

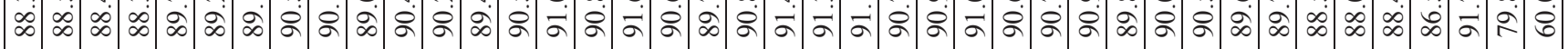

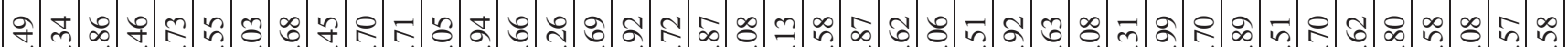

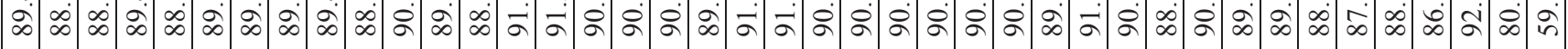

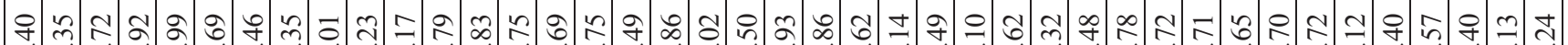

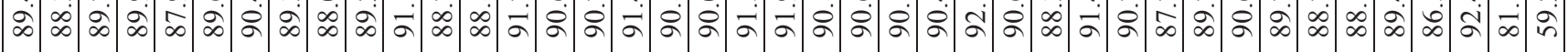
กิ

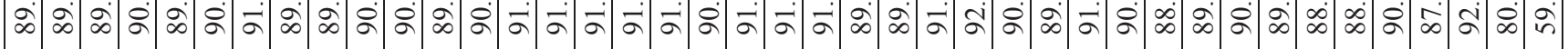
Ұ กิ

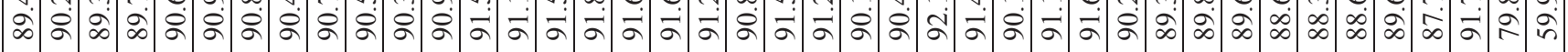

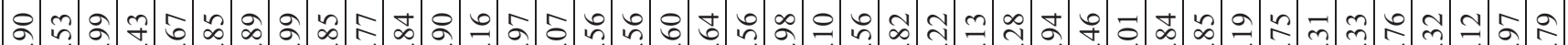

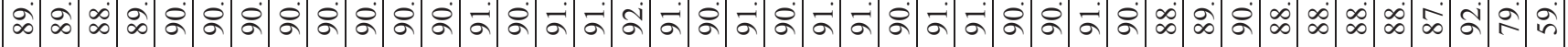

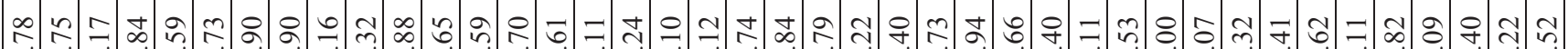

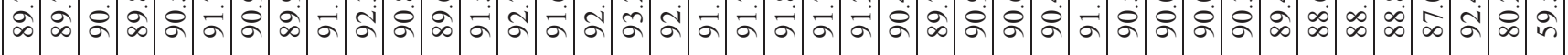

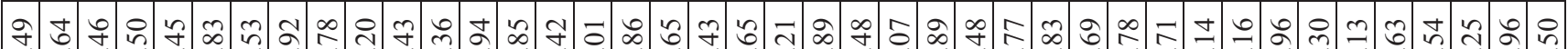

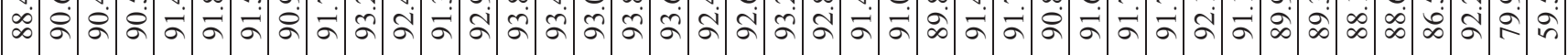

ব)

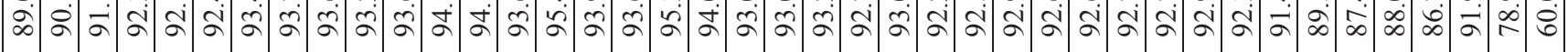

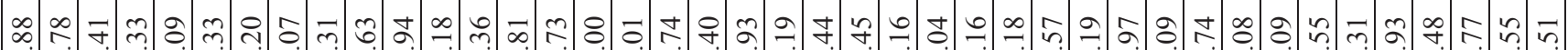

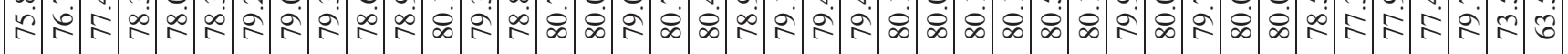

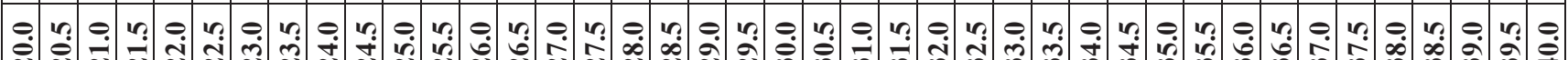

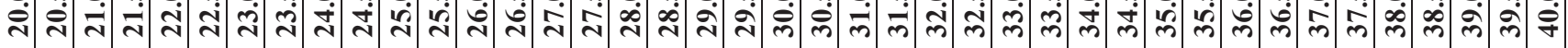




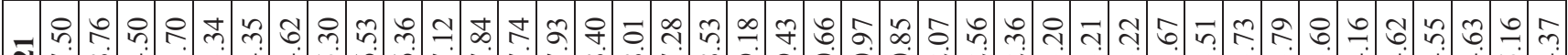

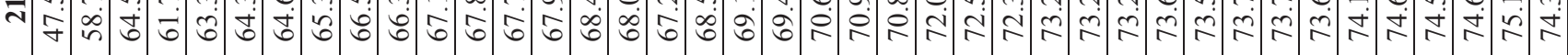
m. సै

- Й

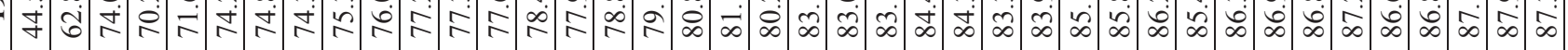

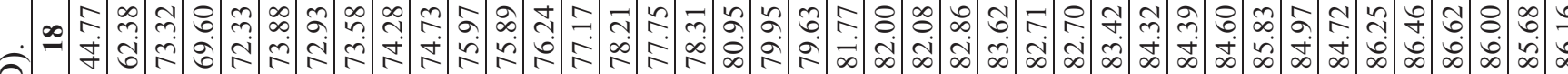

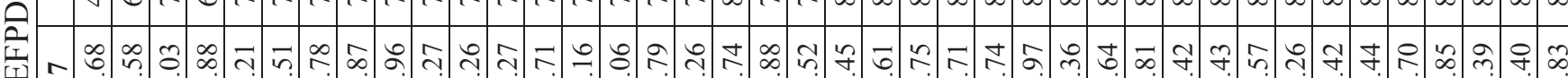
II = J J

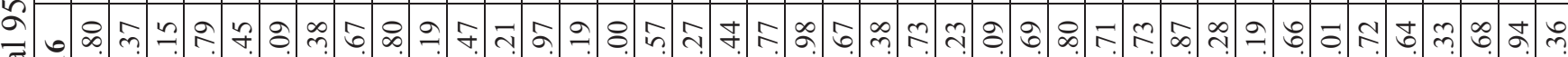

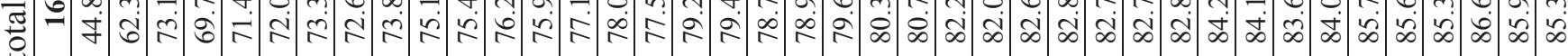

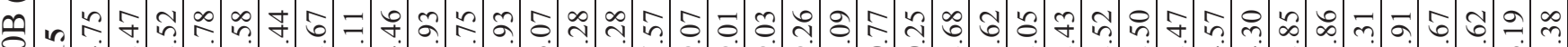

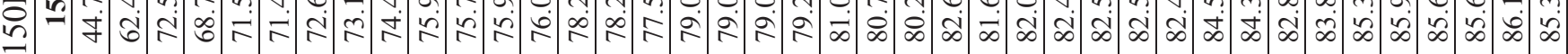

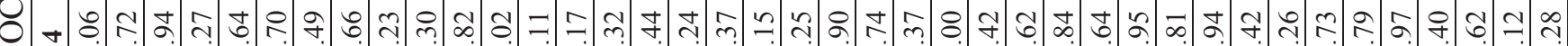

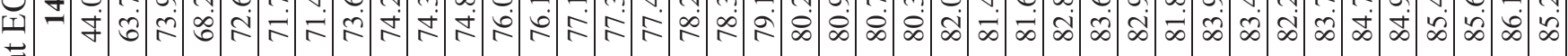

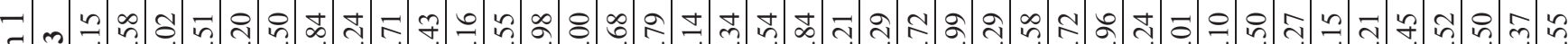

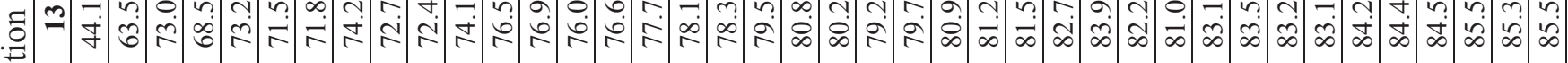

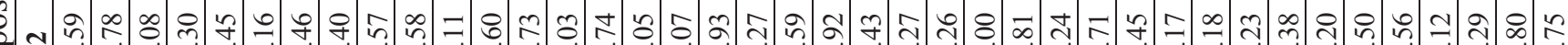

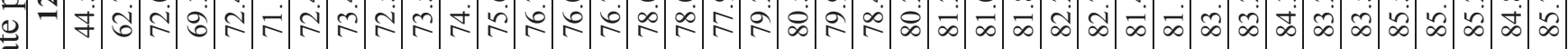

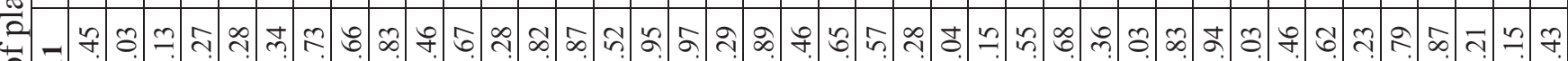

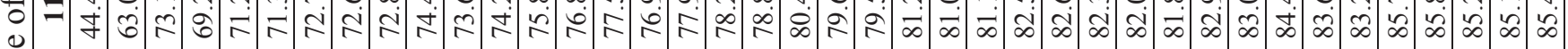

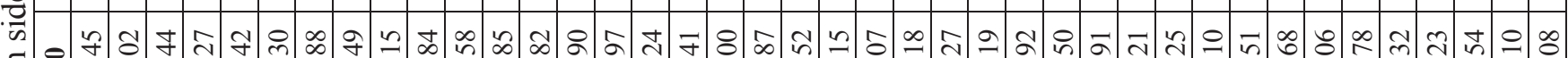

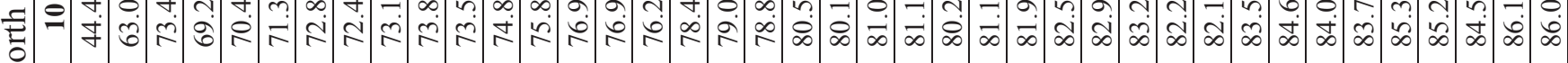
ฮ a

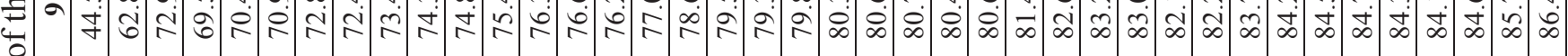

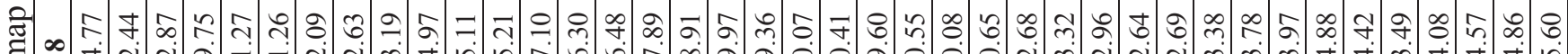

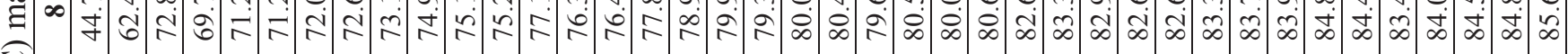

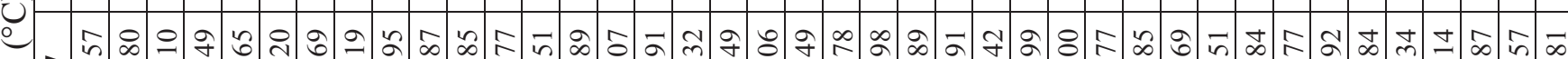
⿹ J

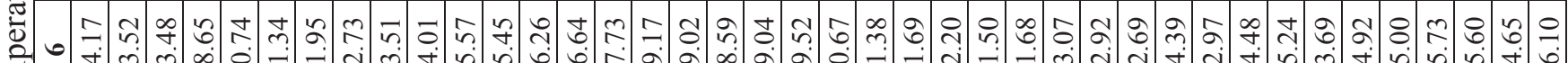
च्च

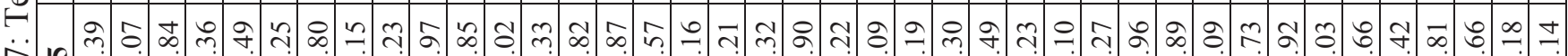

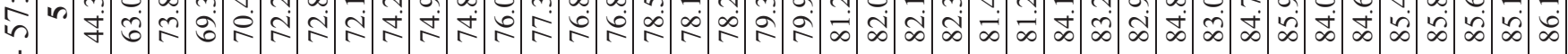
0 +

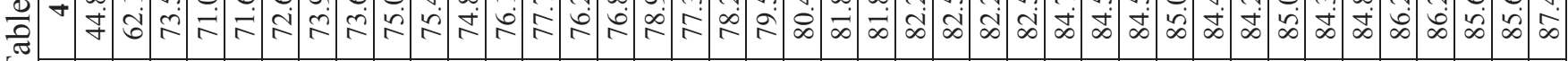
m

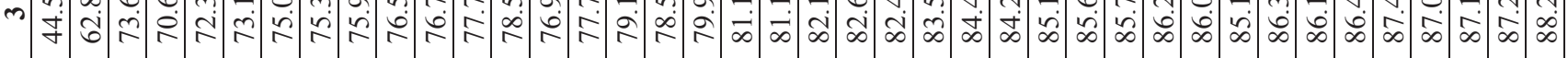
-

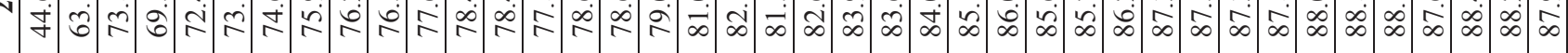

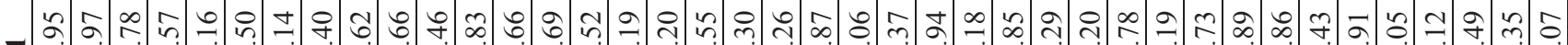

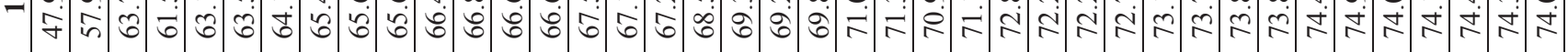
$\because$ 急 


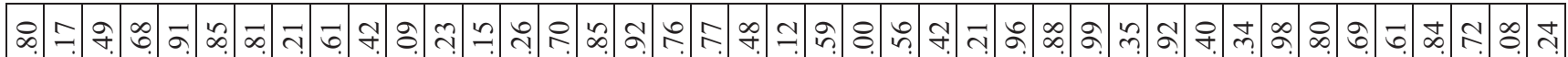

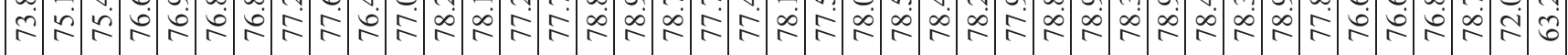

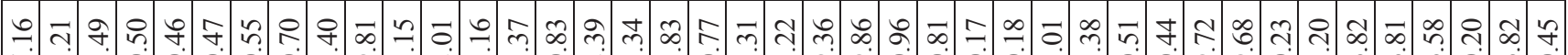

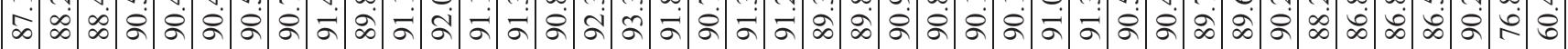

กิธ

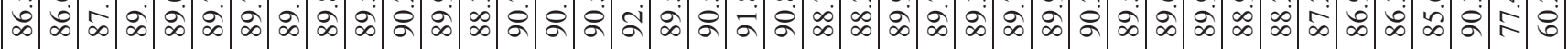
子

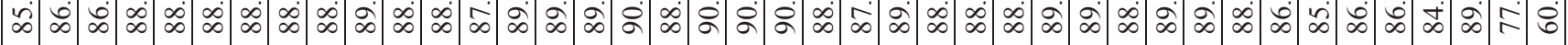

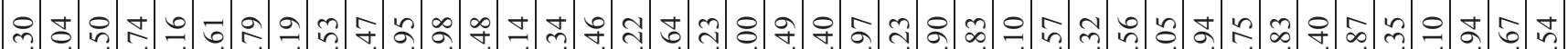

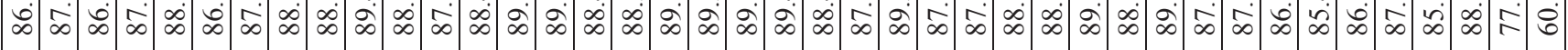

๘

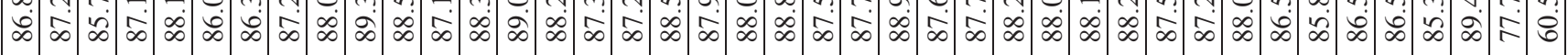

$\infty$ \&

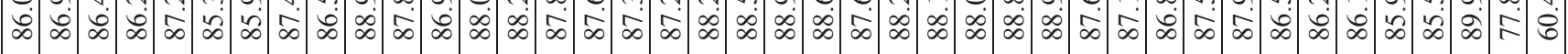

ๆ

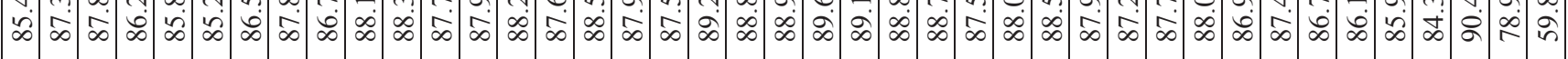

⿹

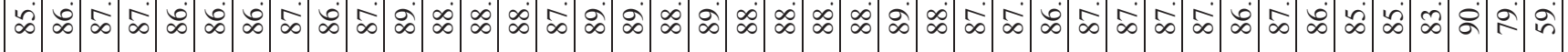

๙ூ.

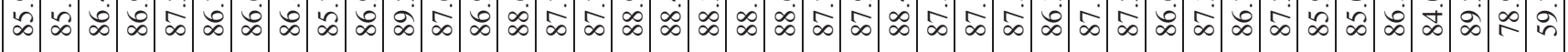

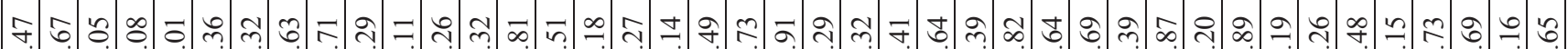

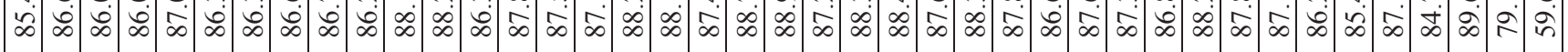

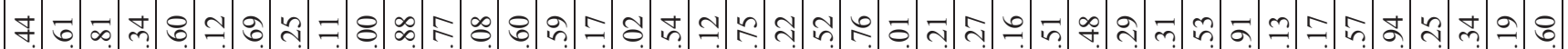

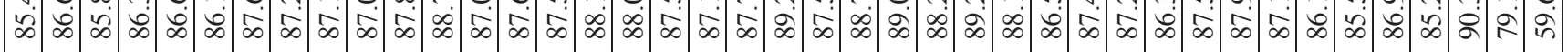

Кำ

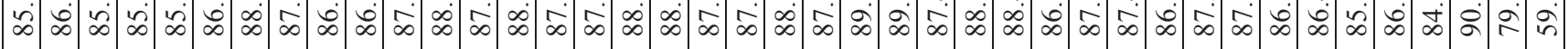
$\approx=$ nิ

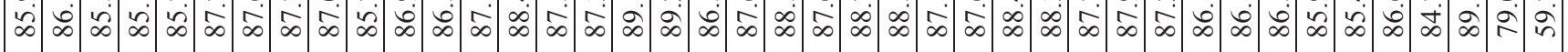

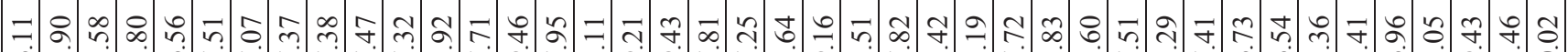

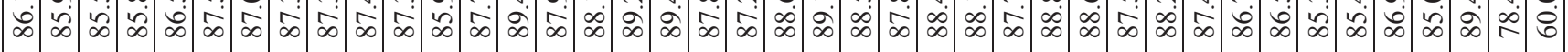

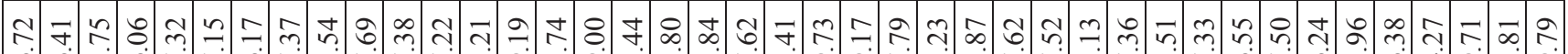

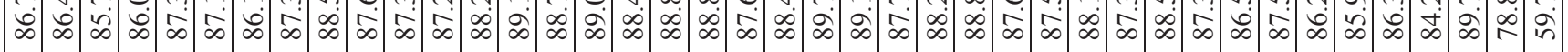
ร.

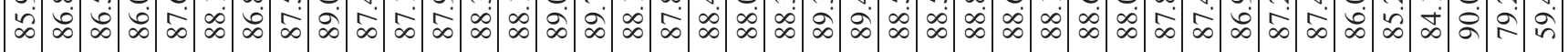

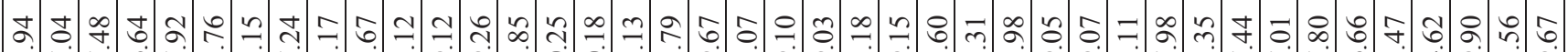

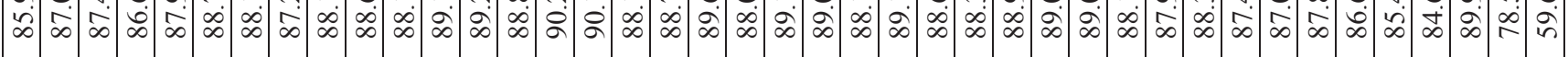

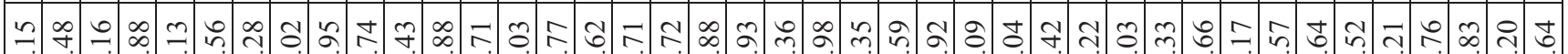

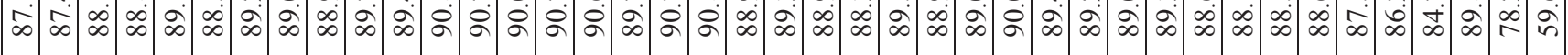

ที

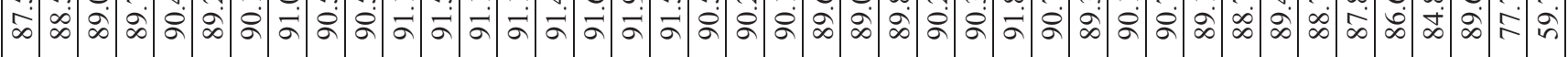

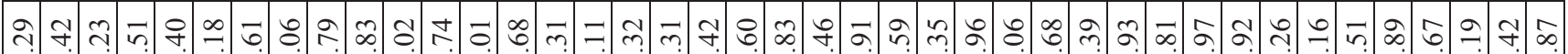

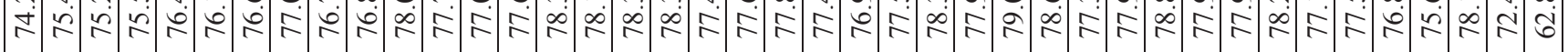




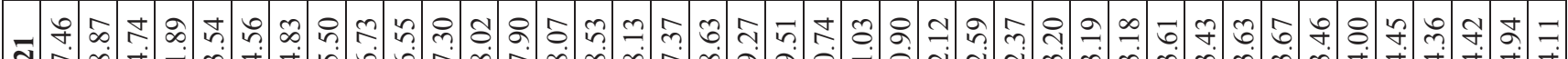

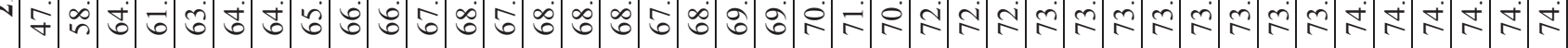
సి

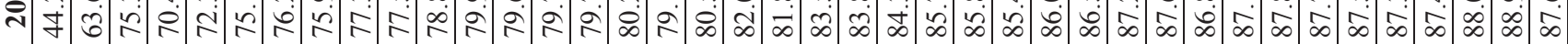

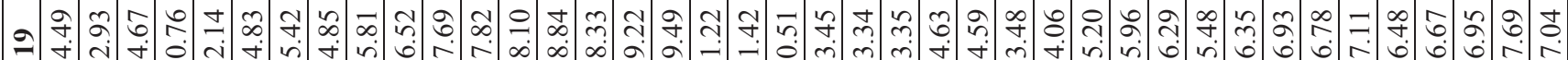
舟

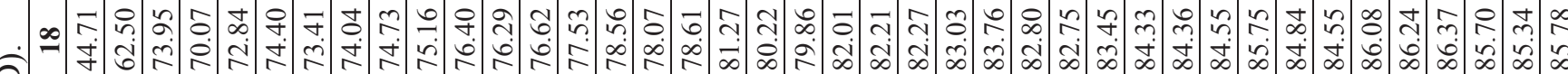

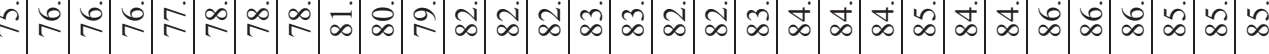

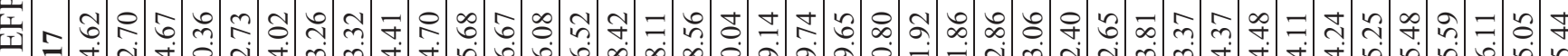

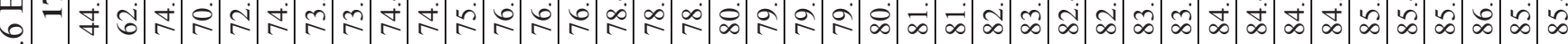

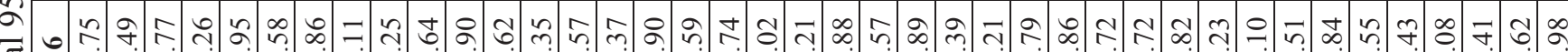
严

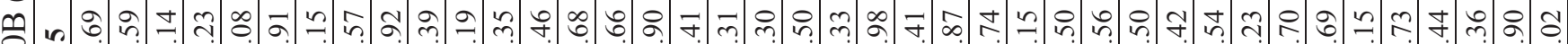

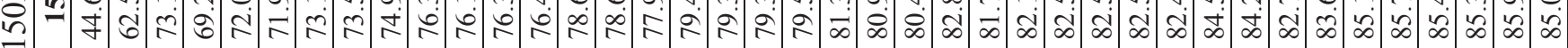

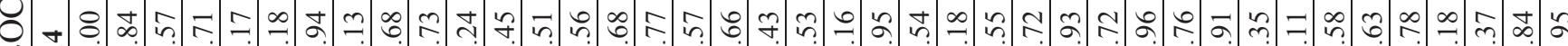
II $=$ 过 $-\infty$ -

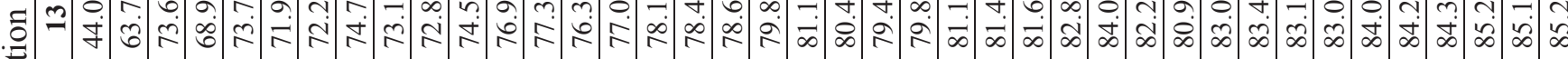
ح

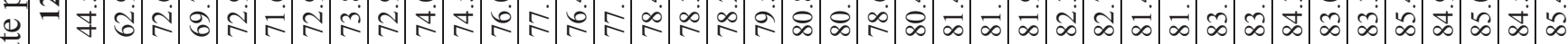

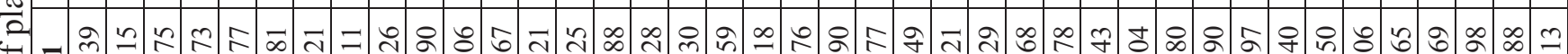

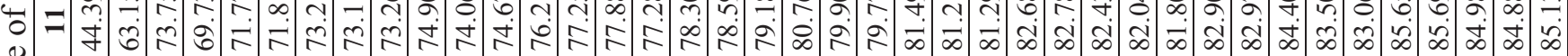

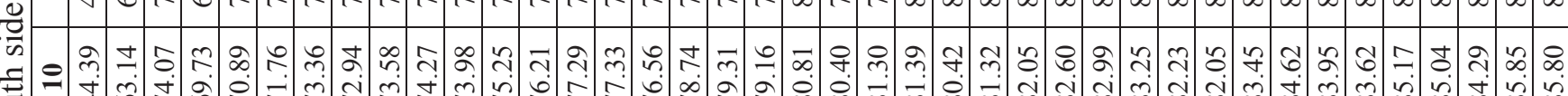

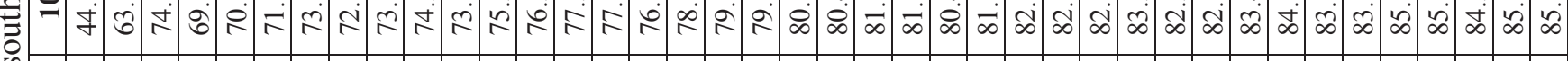
ક

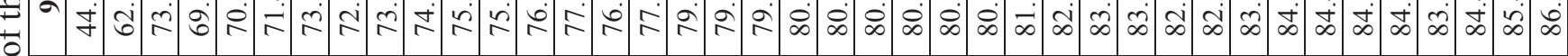

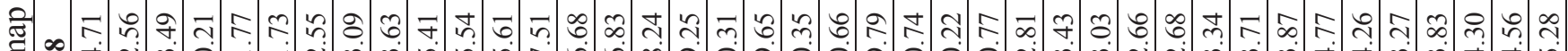

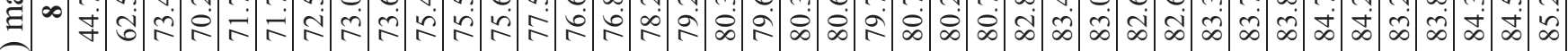

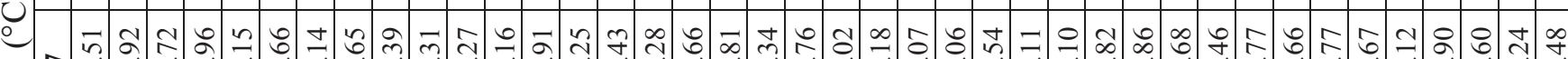

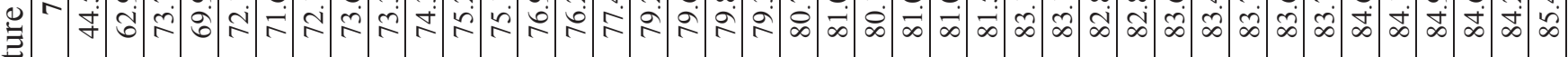
矛 च्च

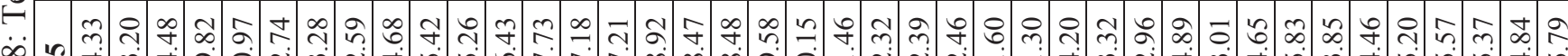

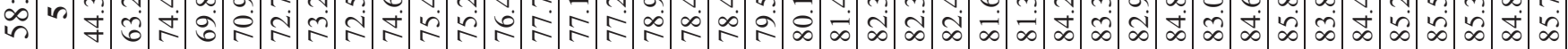
๑

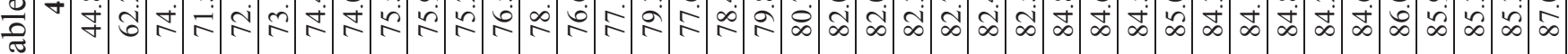

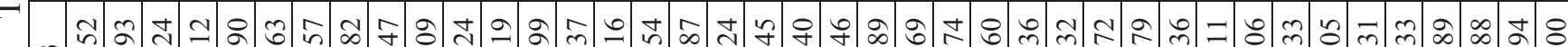

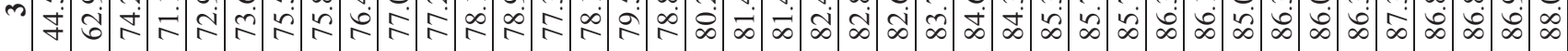
뉴유

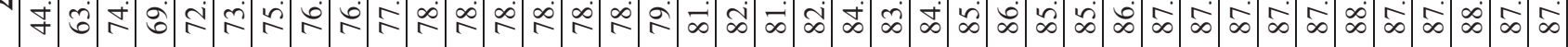
-

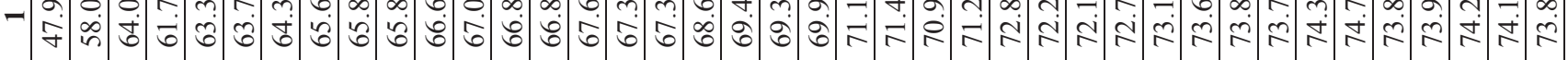

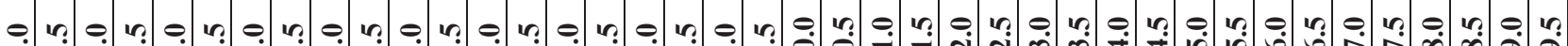

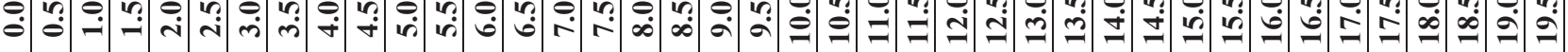




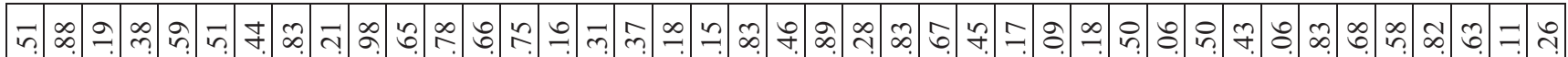

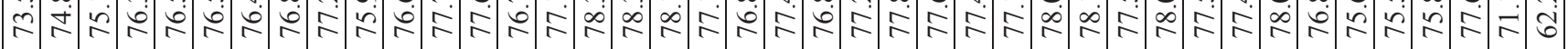
5)

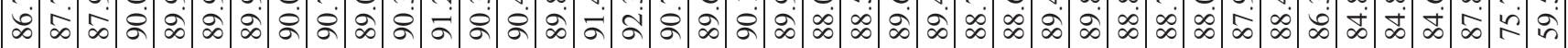

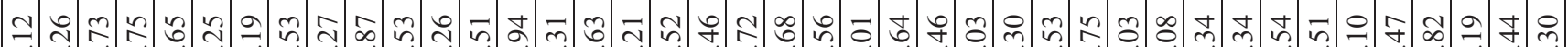

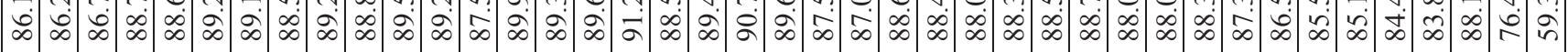

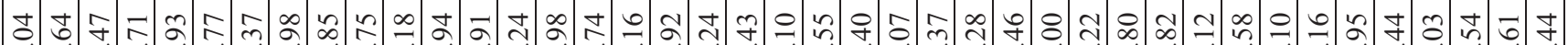

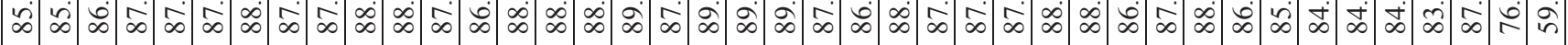

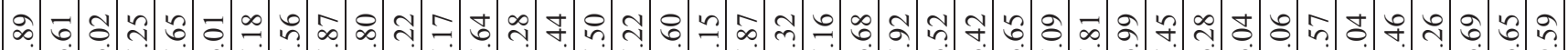

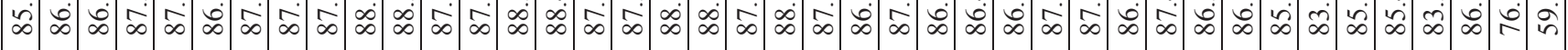

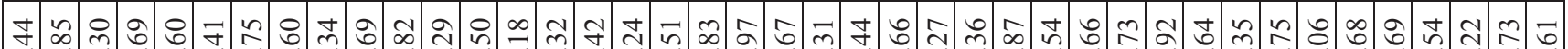

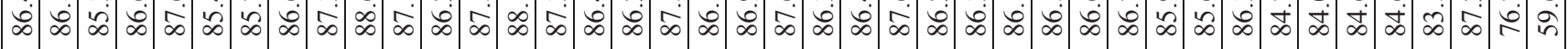

군

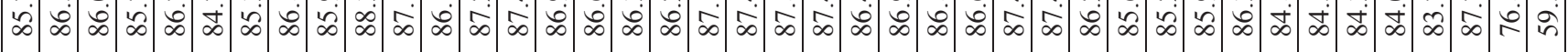

ஓे

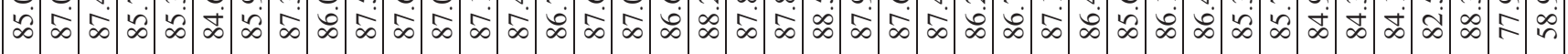

ก

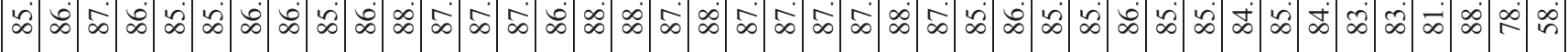

$\infty$ f. ప

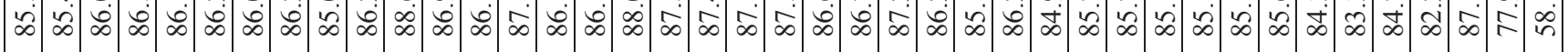

J

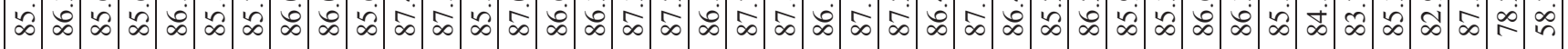

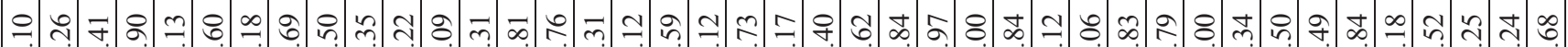

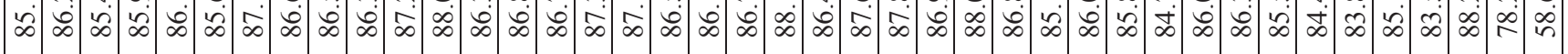

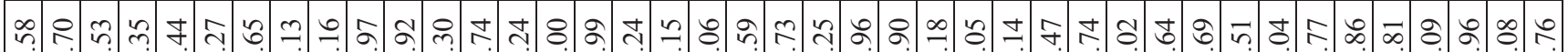

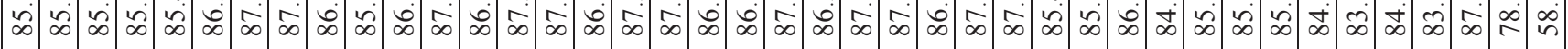

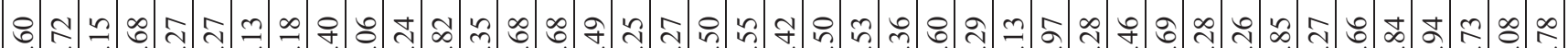

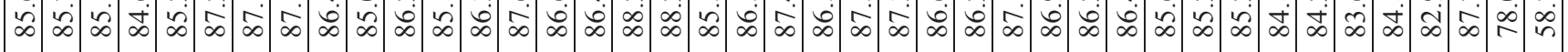
을

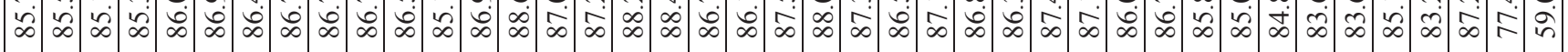

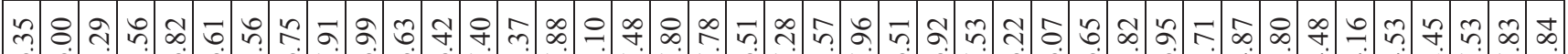

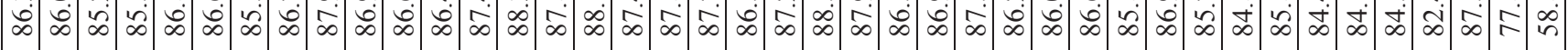

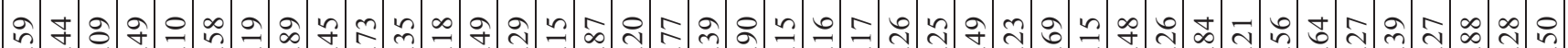

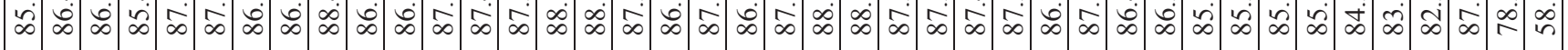

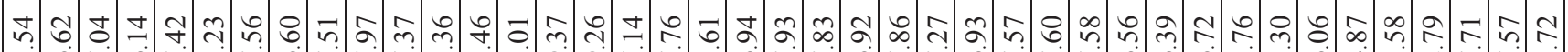

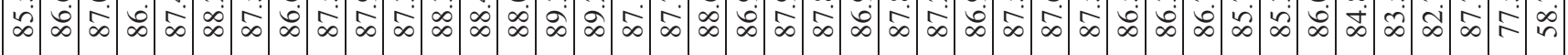

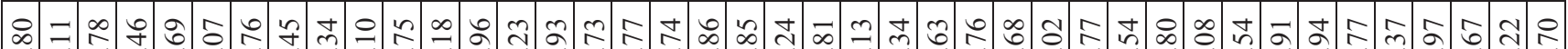

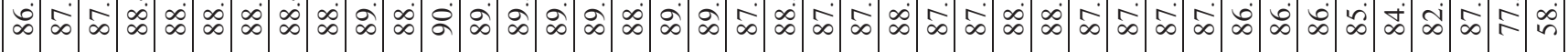

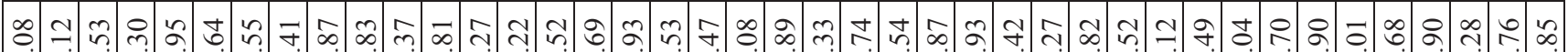

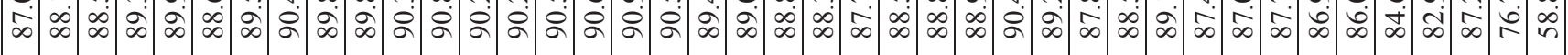

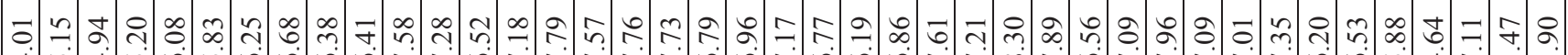

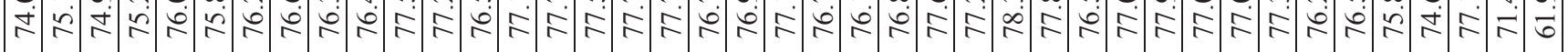




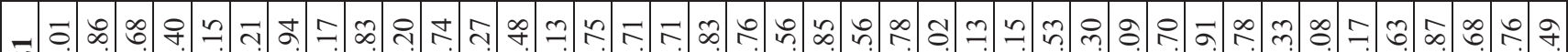

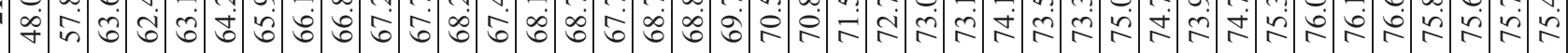

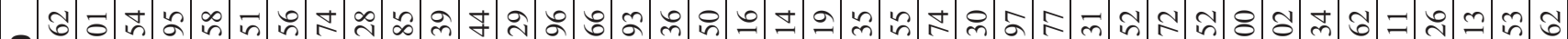

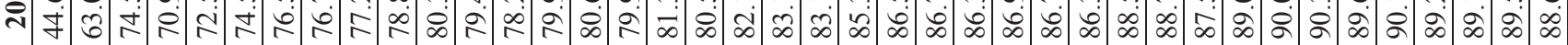

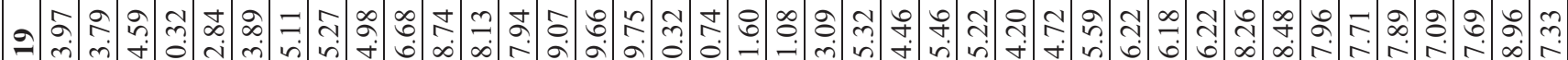
fकी

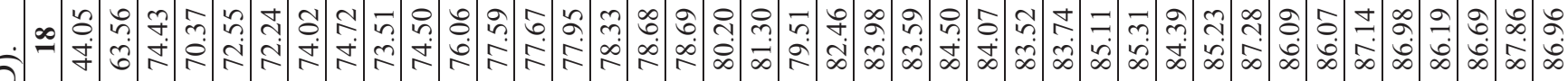

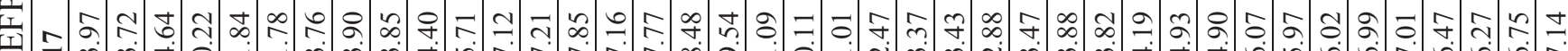

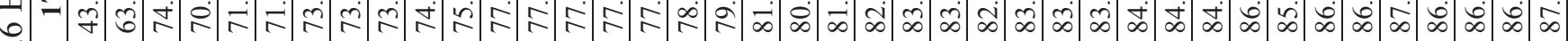

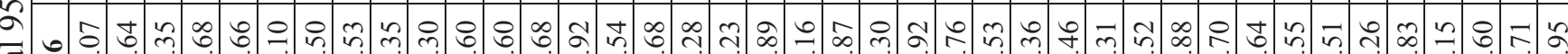
馬 ๑ ๓

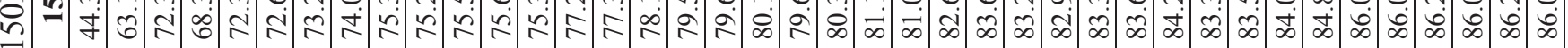

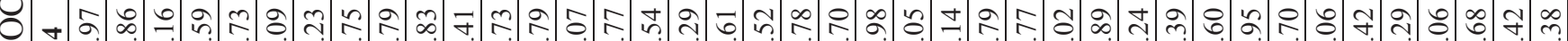

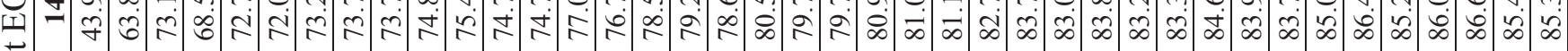

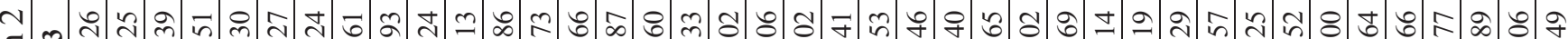

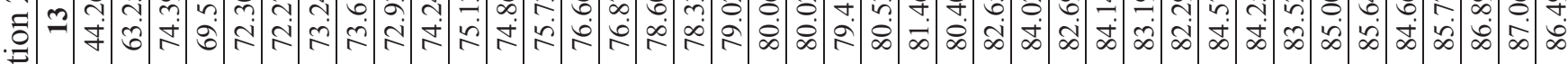

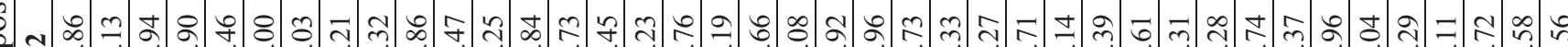
Q च

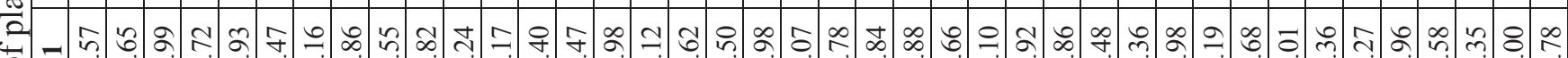

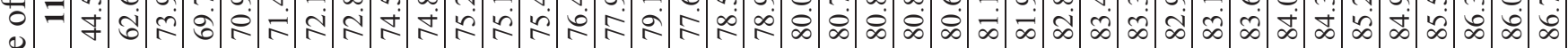

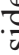

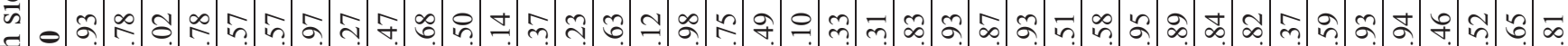
声

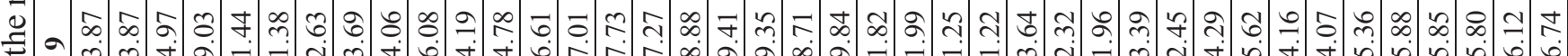

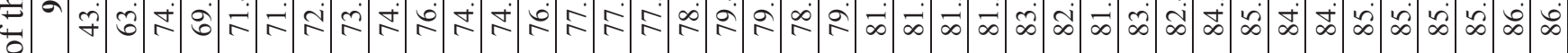

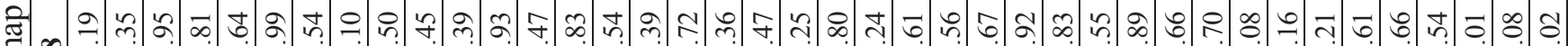

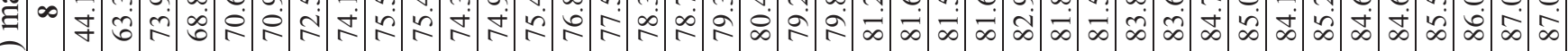
० 可

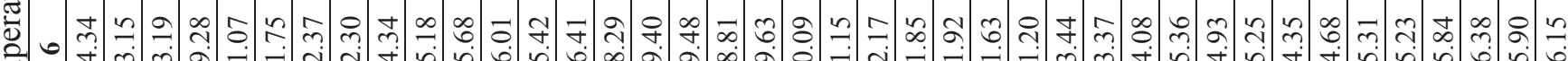

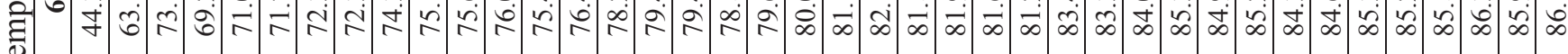

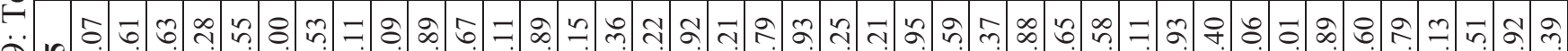

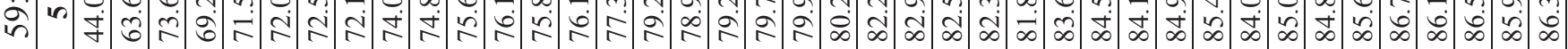

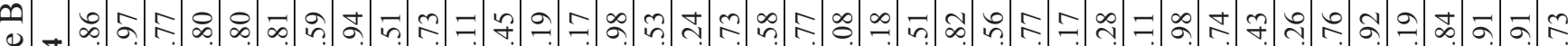

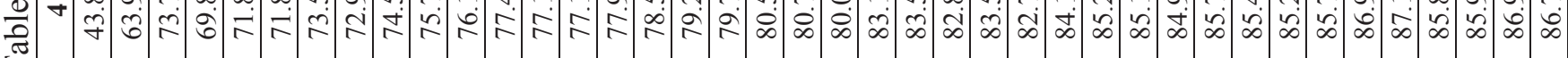

그의.

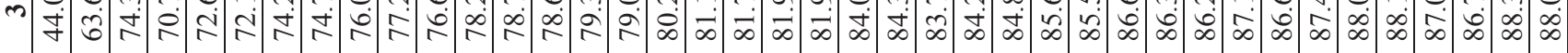

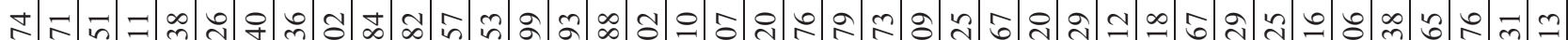

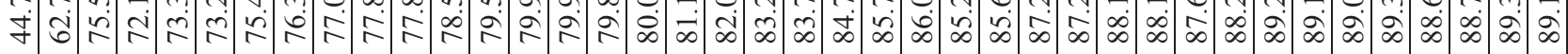

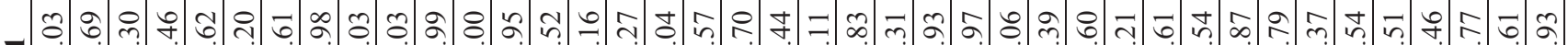

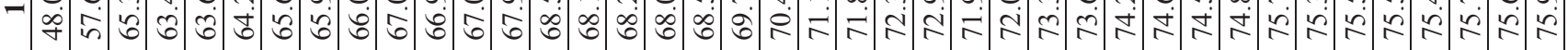

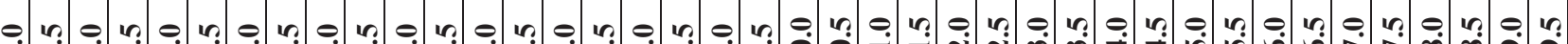

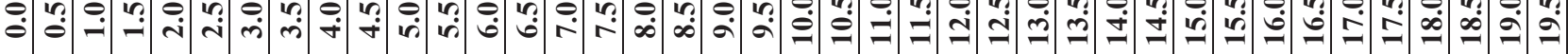


ชุ

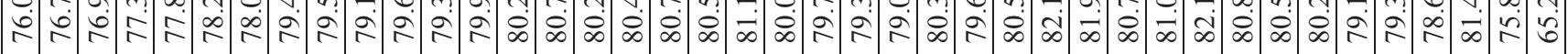
กิ

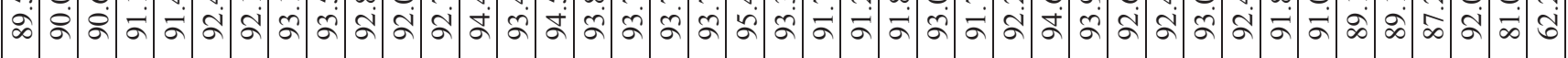

น กิ

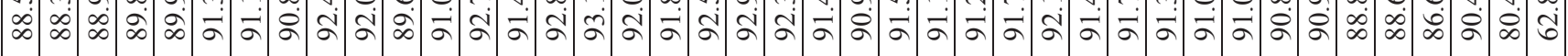

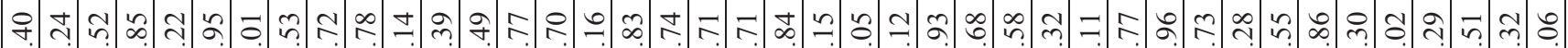

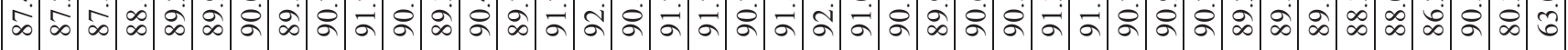

กิ

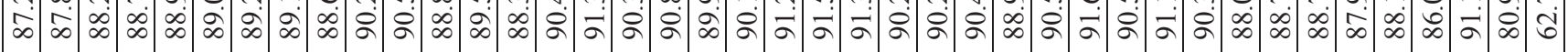

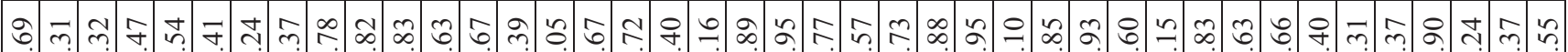

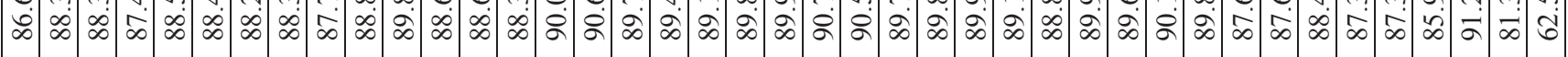

$\infty$ ก.

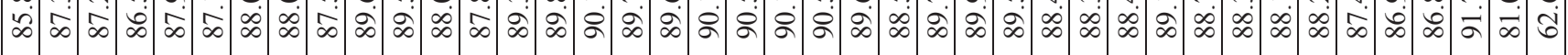

भ

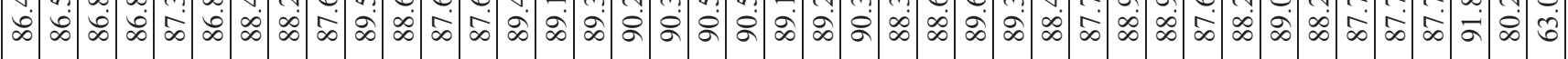

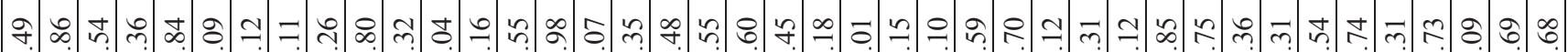

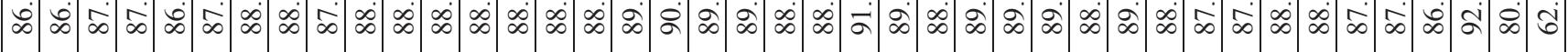

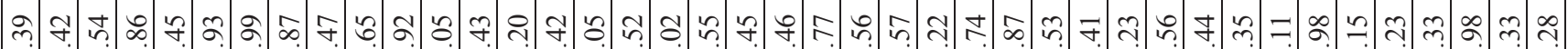

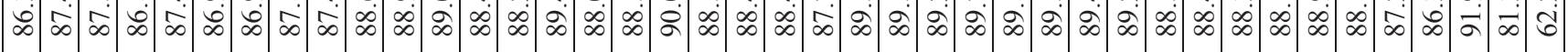

‡

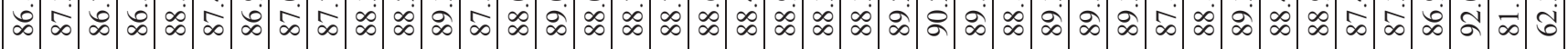

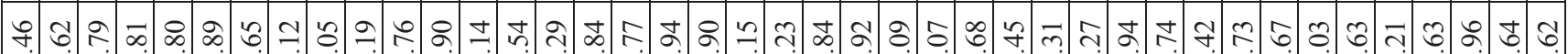

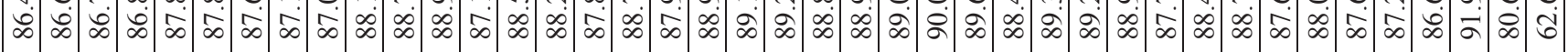

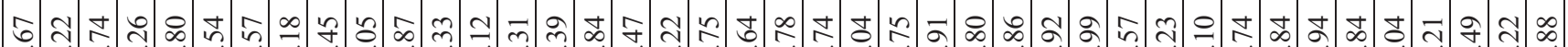

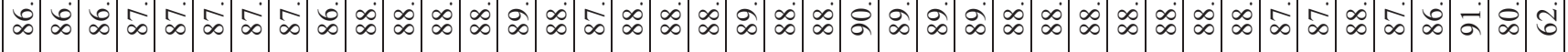
ง

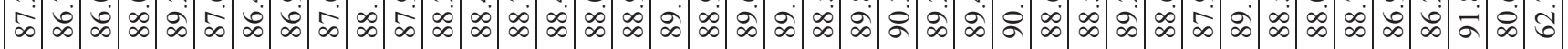

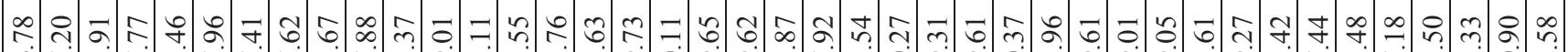

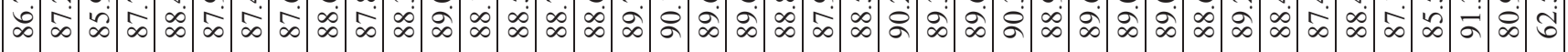

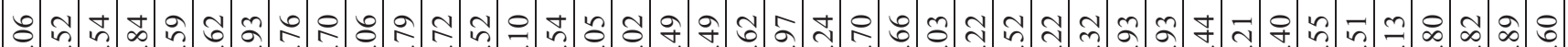

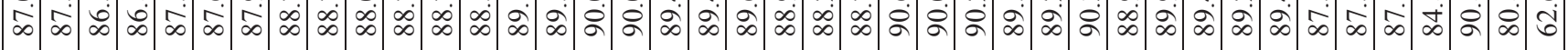

$q \sim$ ᄀ

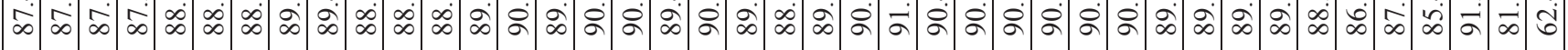

ธุ

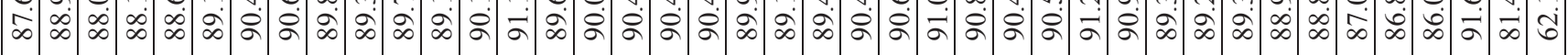

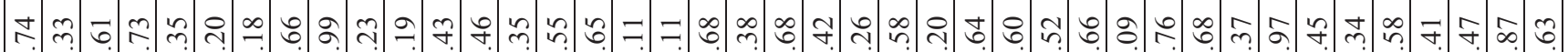

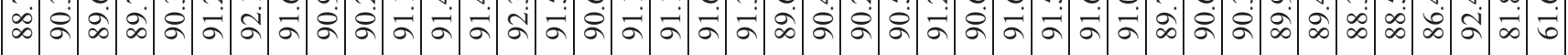

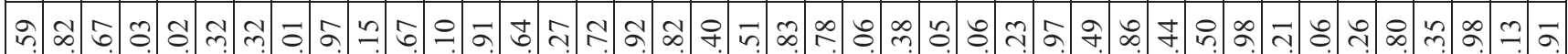

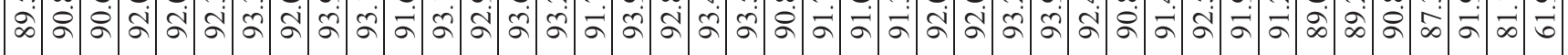

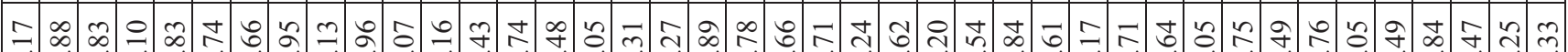

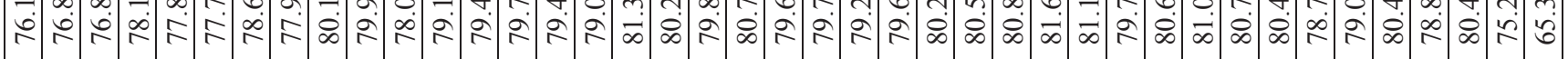


- ప.

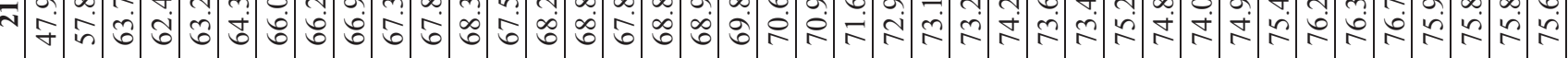

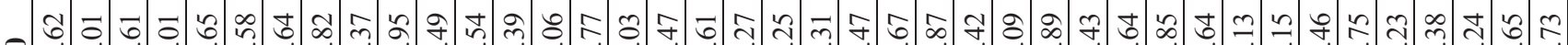

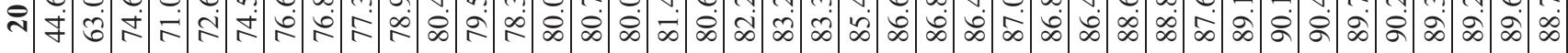

-

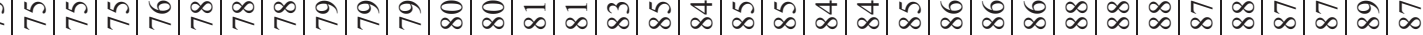
(-)

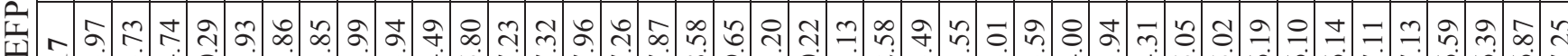

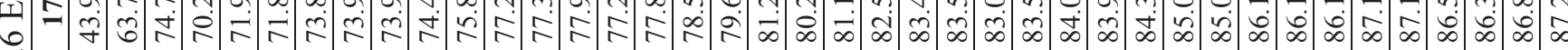

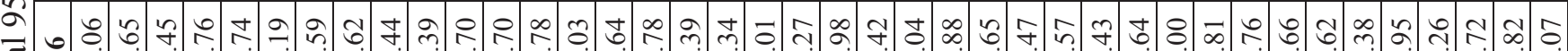

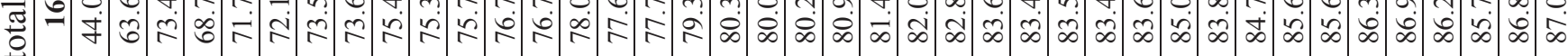
๑ ๓

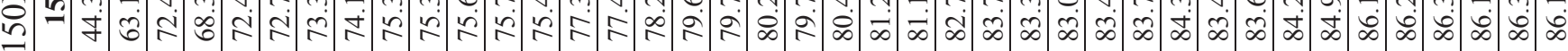

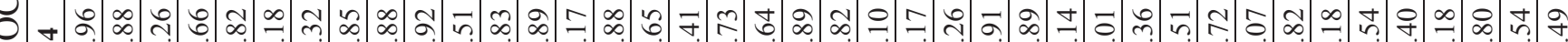

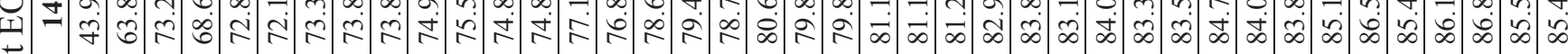
八

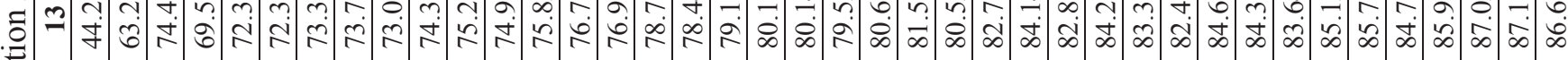
¿

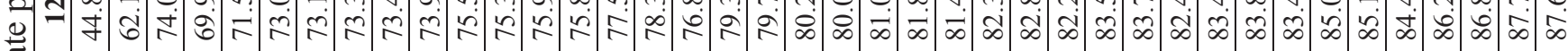
元 =

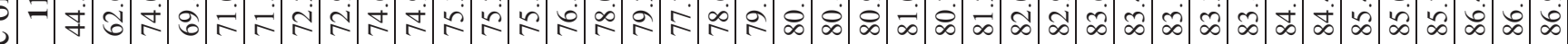

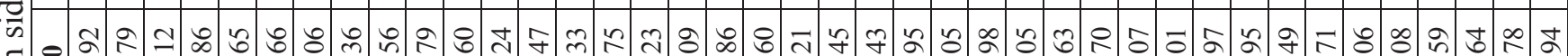

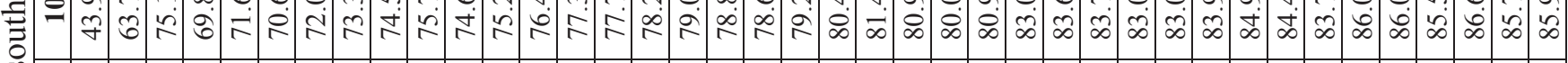
I

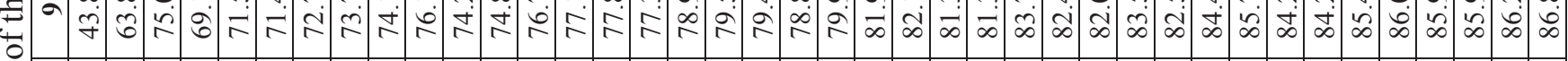

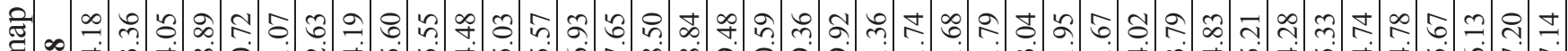

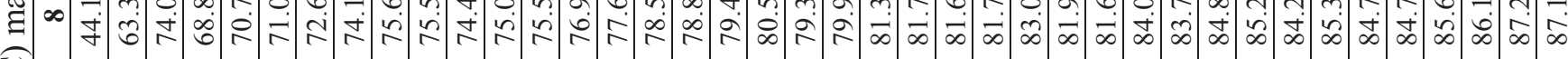
ర

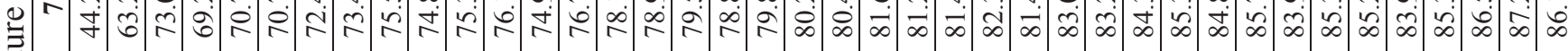
ॠ

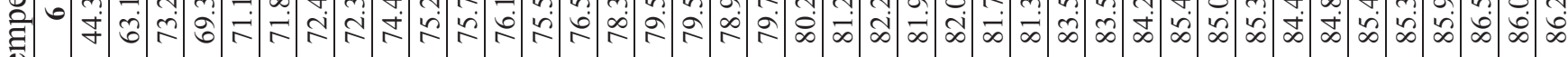

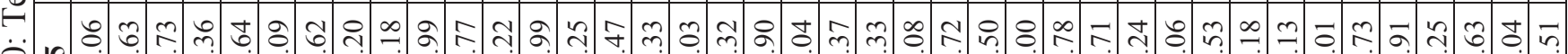

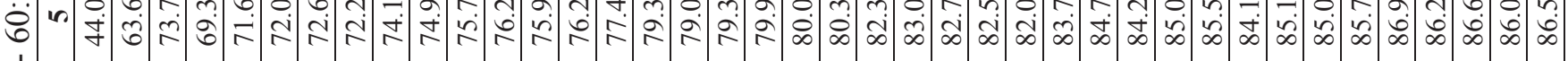

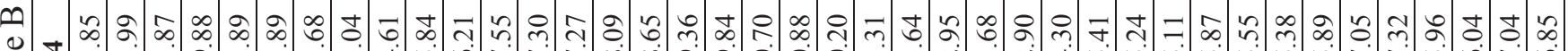
光 F ప

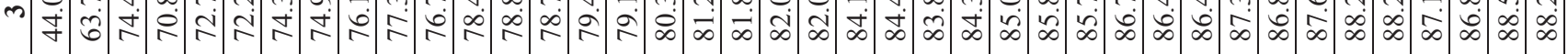

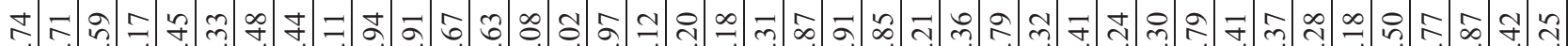

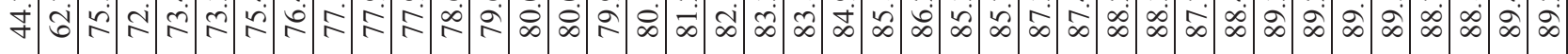

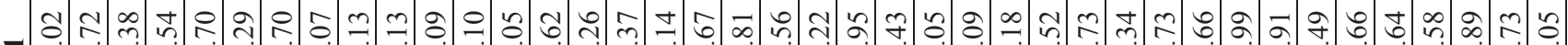

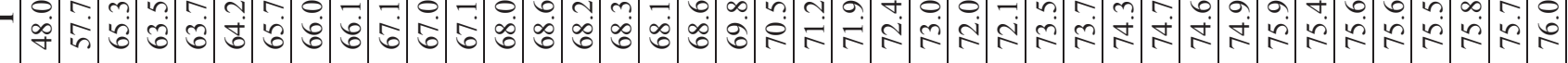
$\because$ 急 


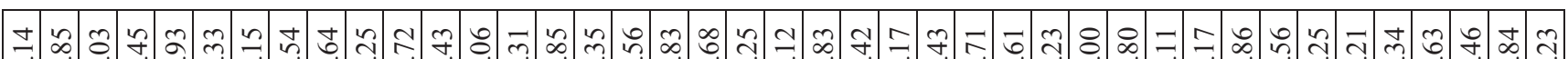

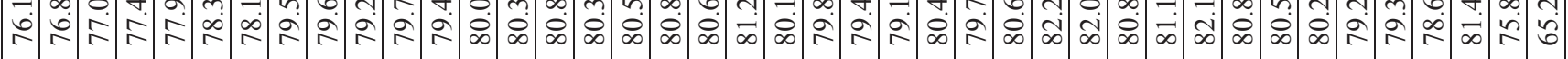

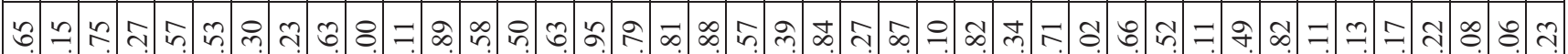

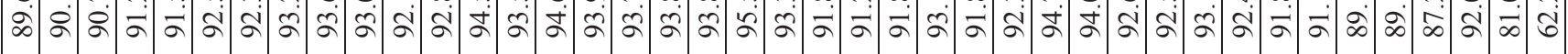

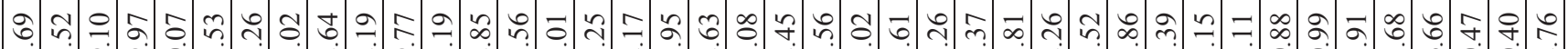

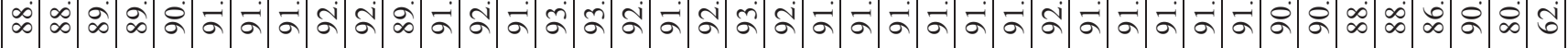

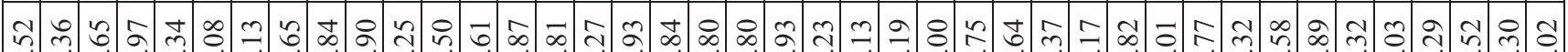

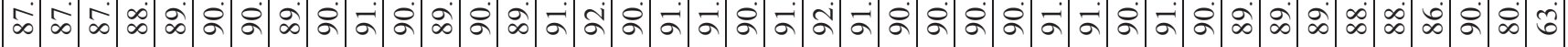

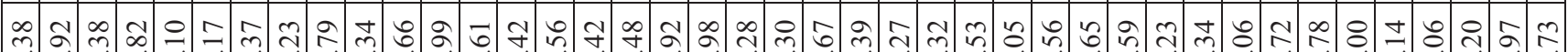

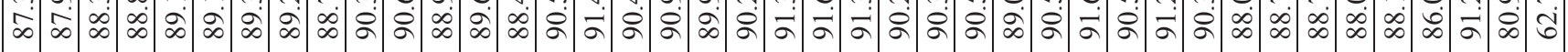

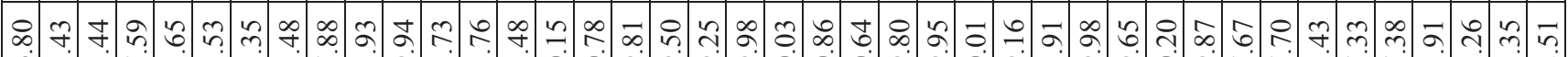

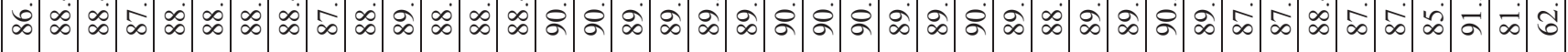

$\infty$ 守

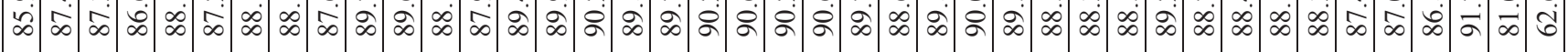

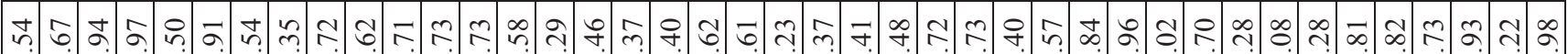

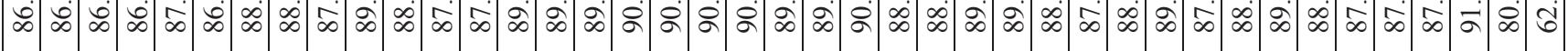

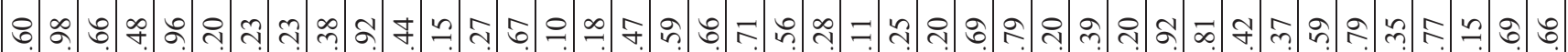

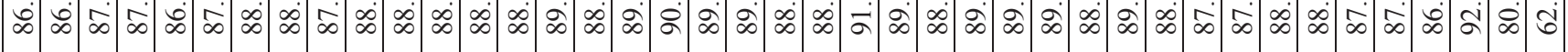

пี

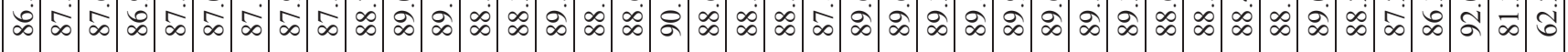
ஃ 유.

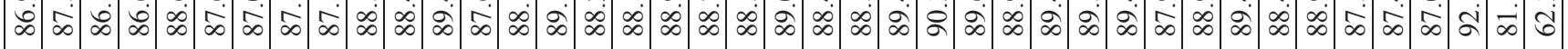

Иกำ

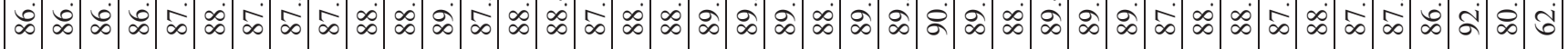

ఫ

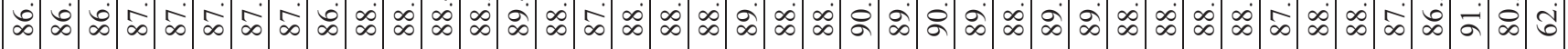

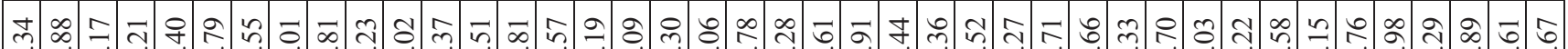

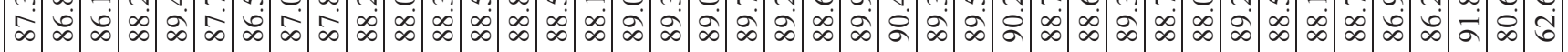
\&.

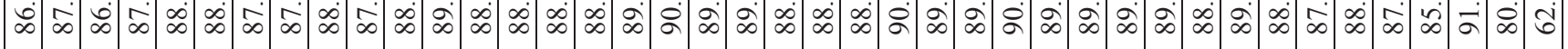

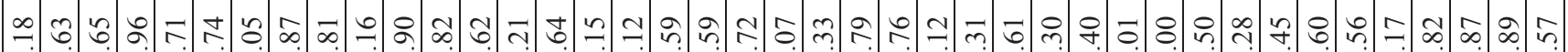

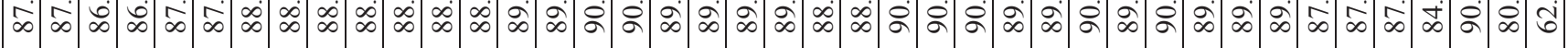

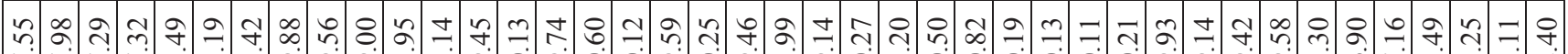

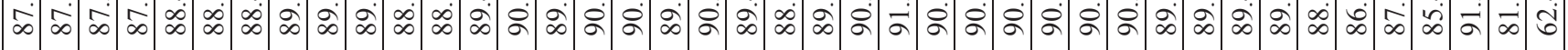
ఇ

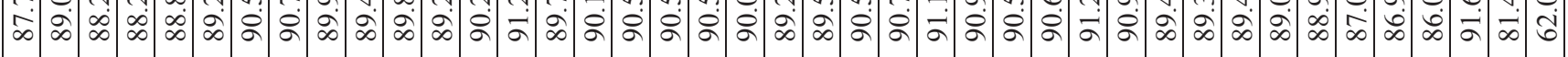

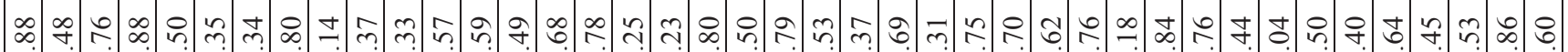

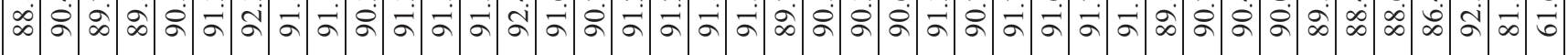

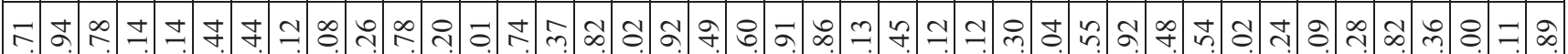

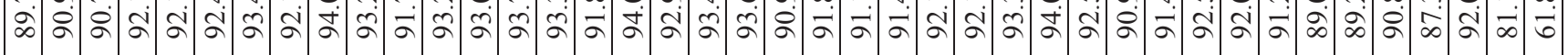

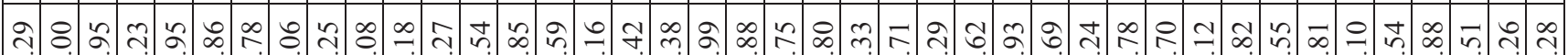

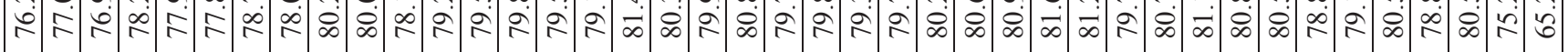

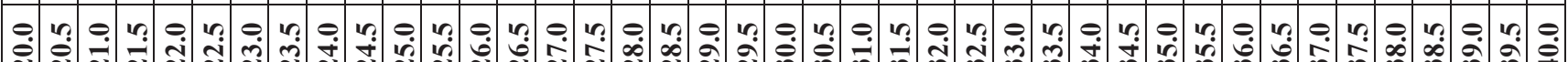

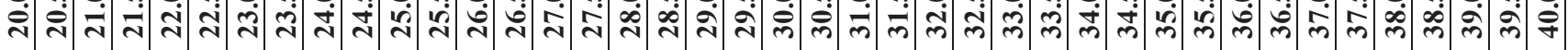




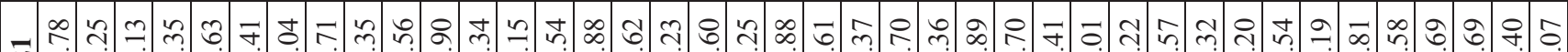

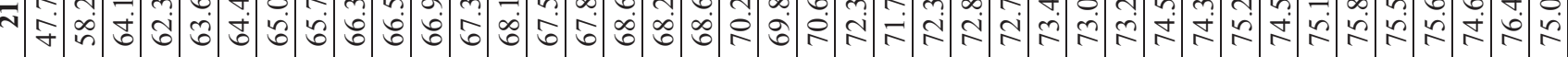

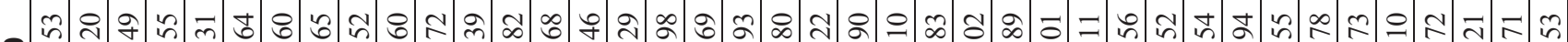

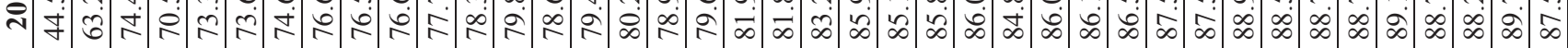

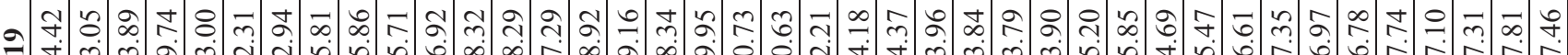
卉

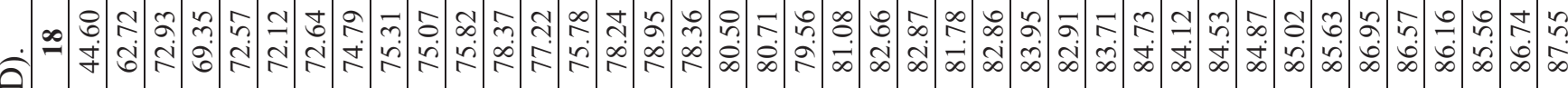

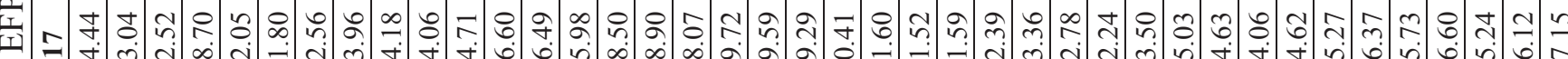

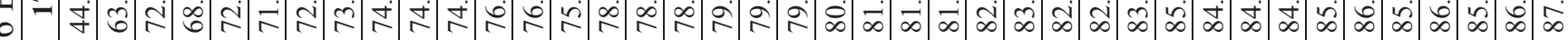

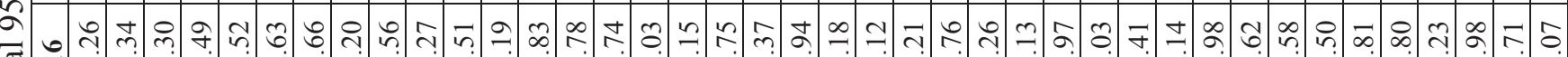

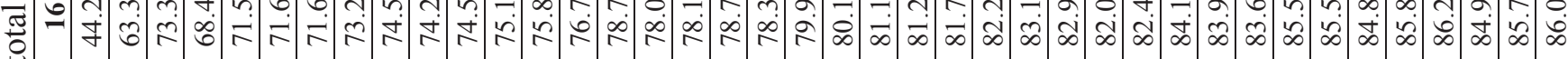

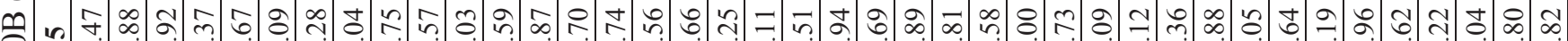

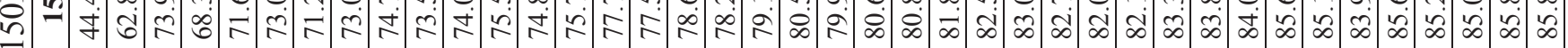
- m

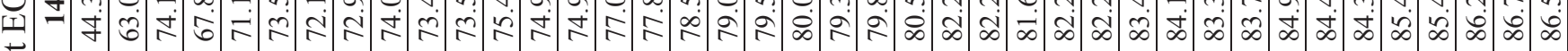

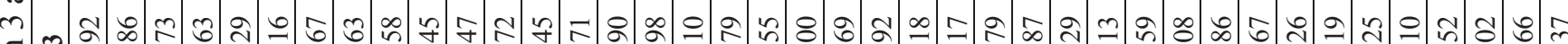

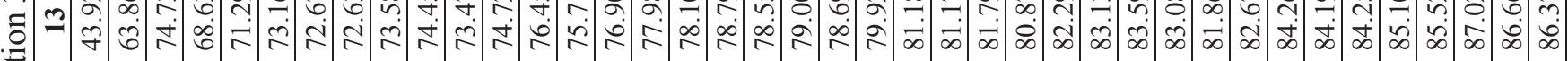

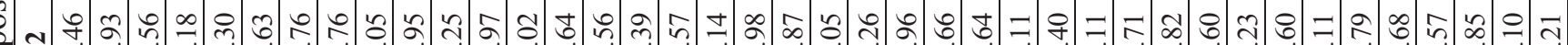

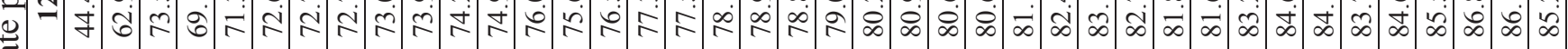

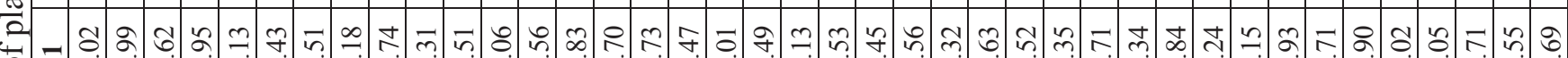

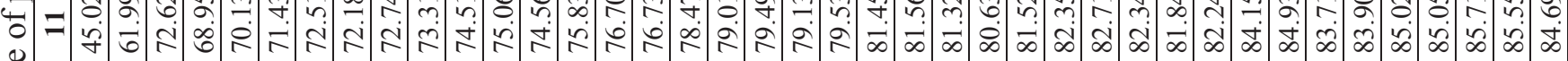
$\frac{\overrightarrow{0}}{n}$

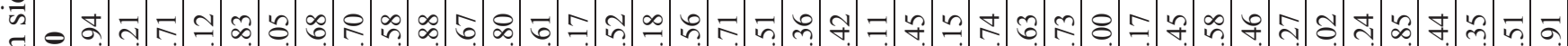

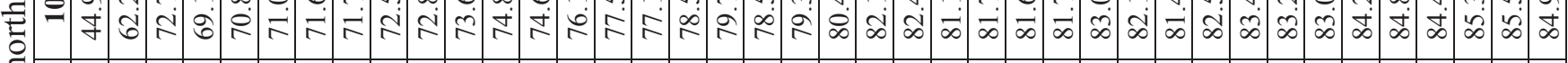
๑ จ

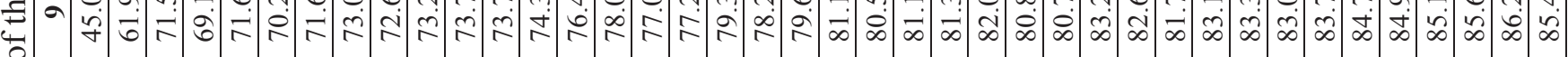

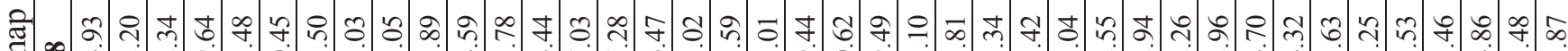

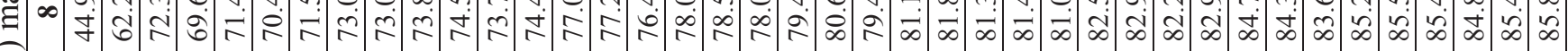

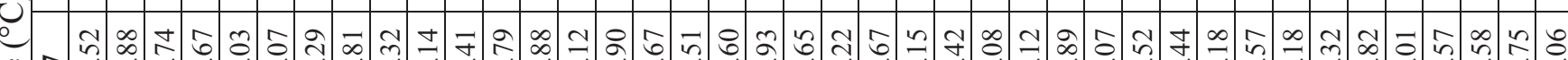

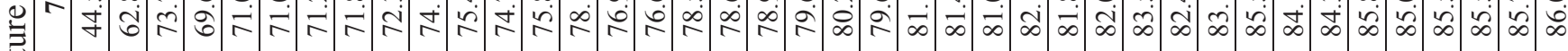
๑ च्च ㄱำ

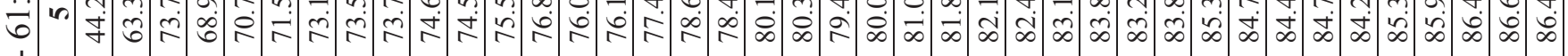

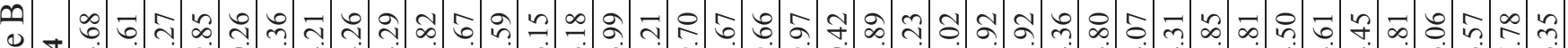

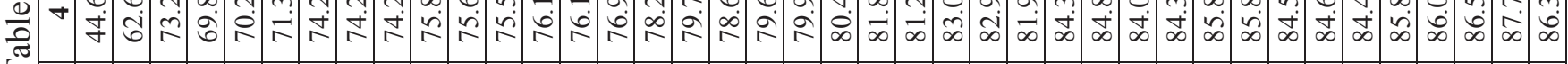

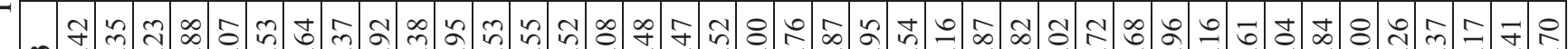

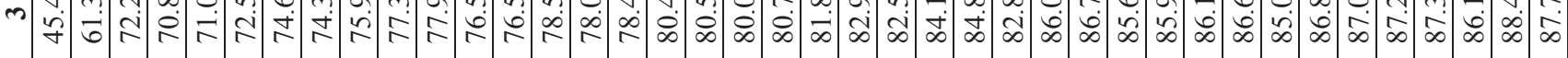

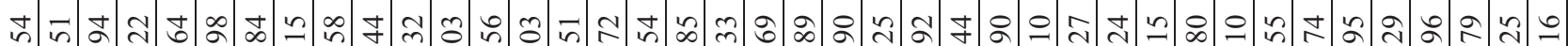

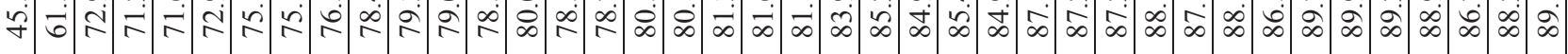
- ఫ

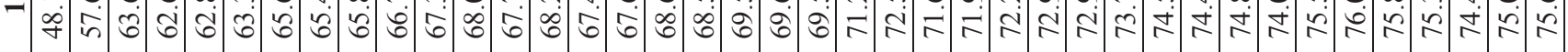
e. 0 .

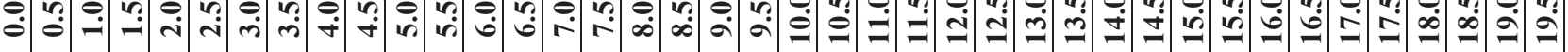


f

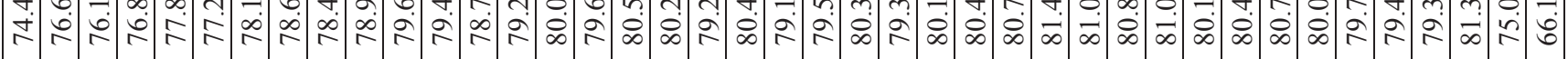

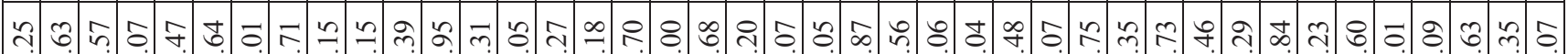

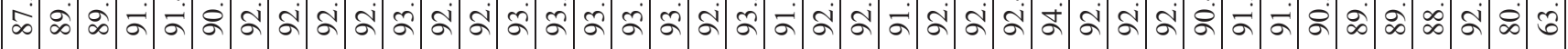

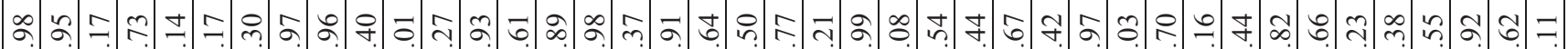
ळ

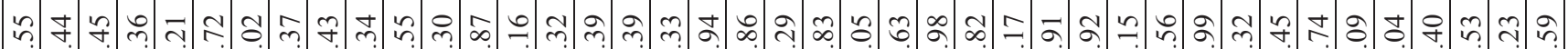

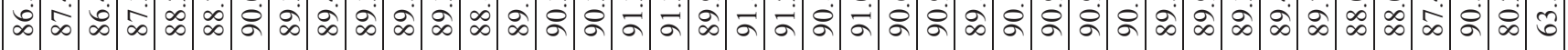

ஓ)

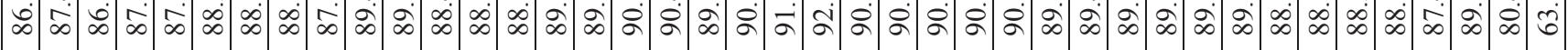

จ

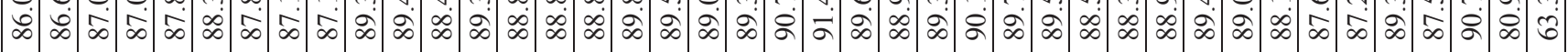

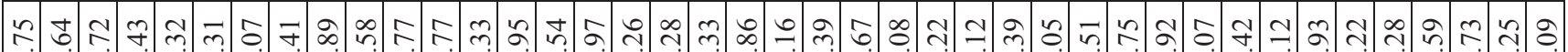

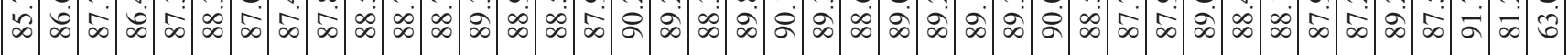

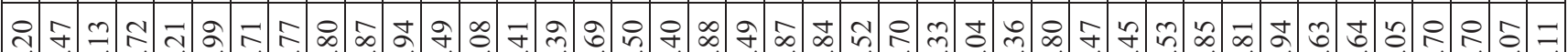

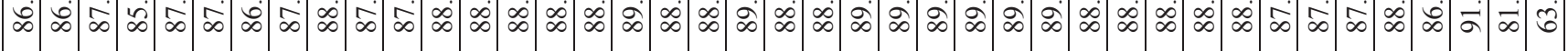

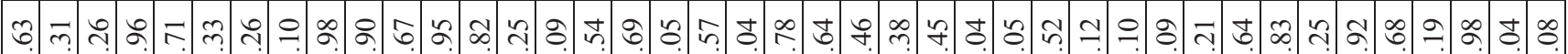

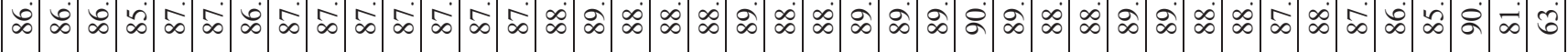

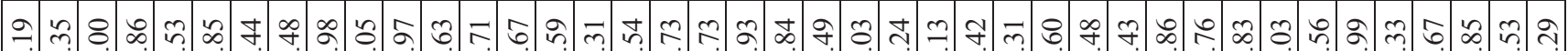

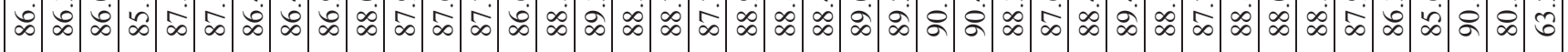
๘ 으

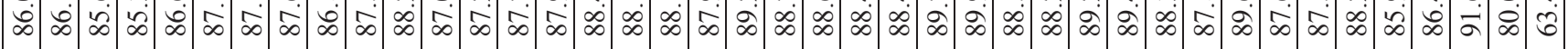

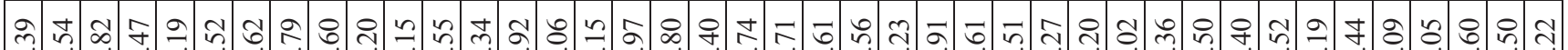

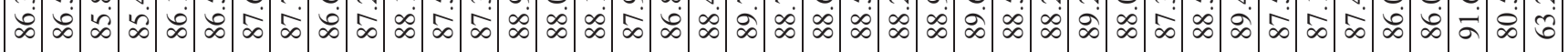

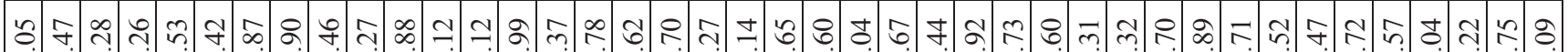

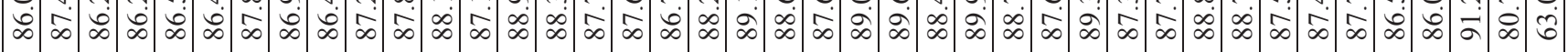

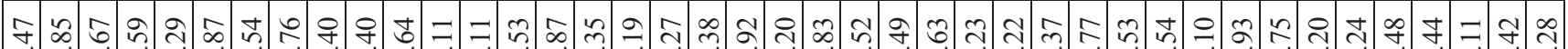

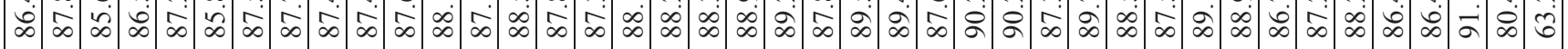

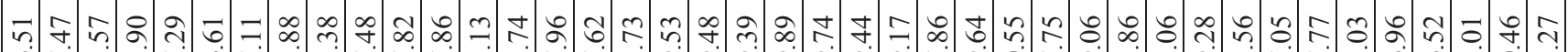

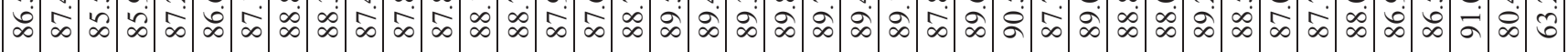
ปุ ก

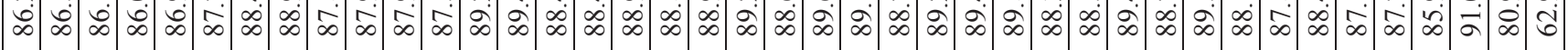

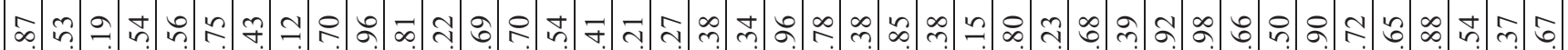

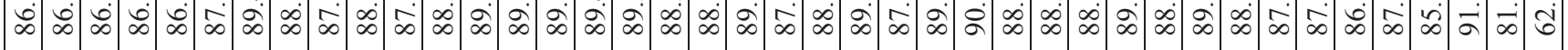
두 $\infty$ c

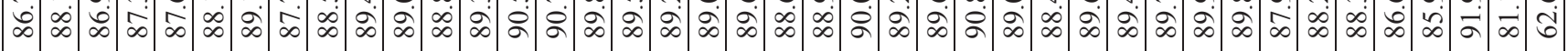

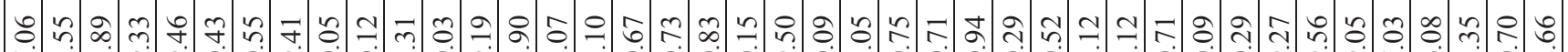

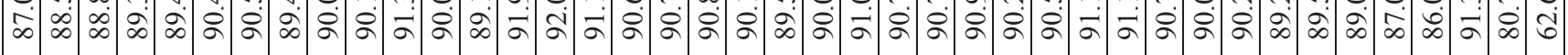

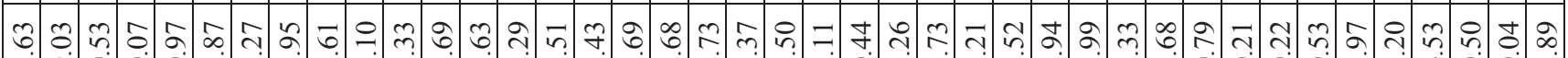

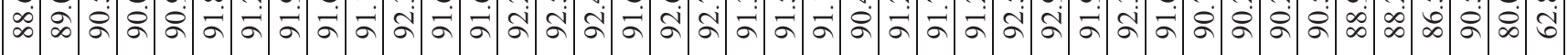

๑) ๆ $\infty$ ก

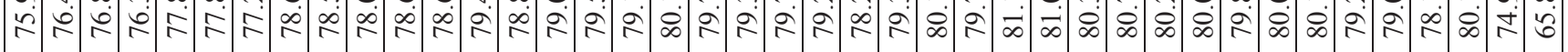




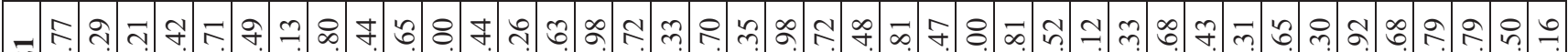

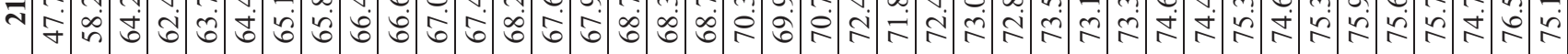

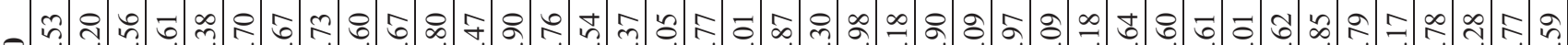

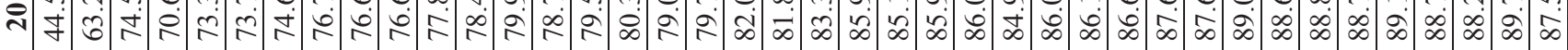

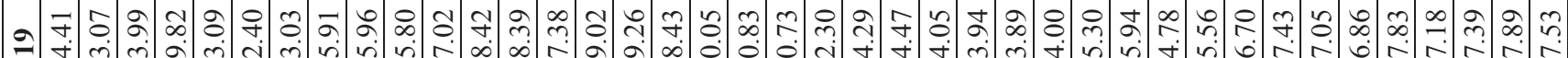

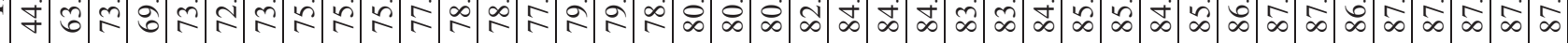

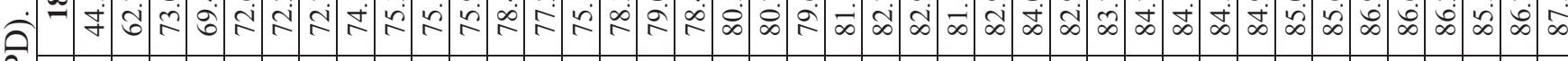

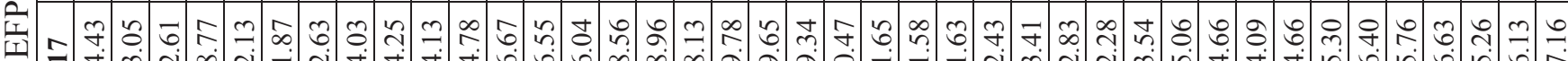

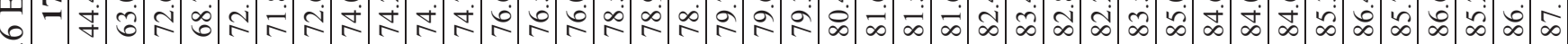

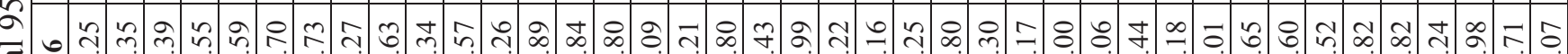

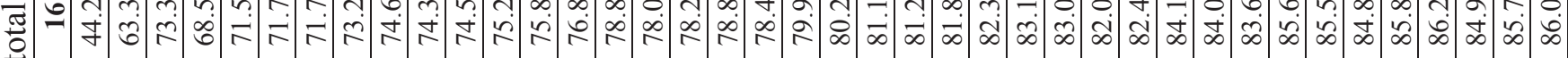
ص n f

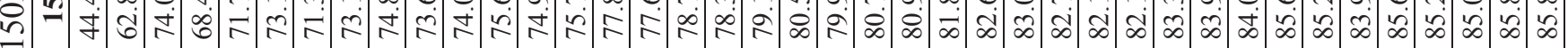

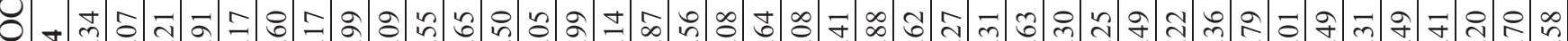

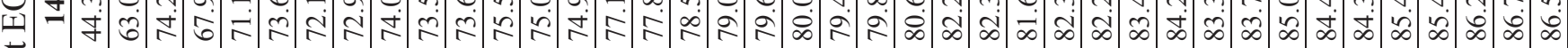

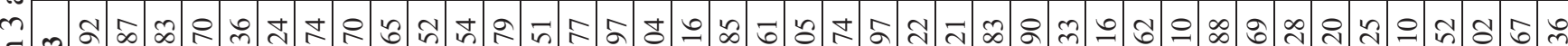

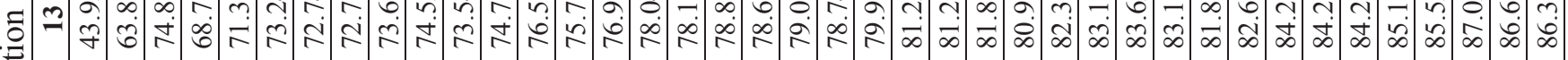

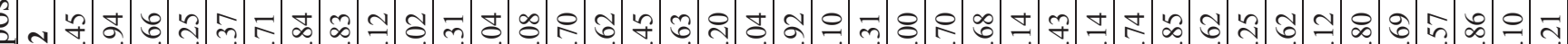
d) 范

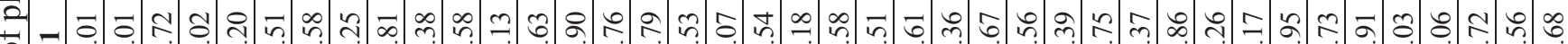

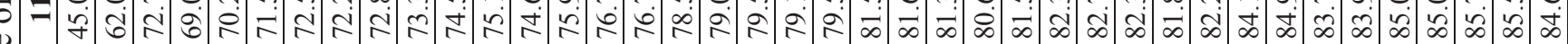

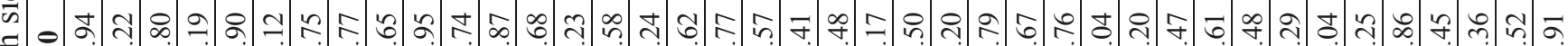
馬 I

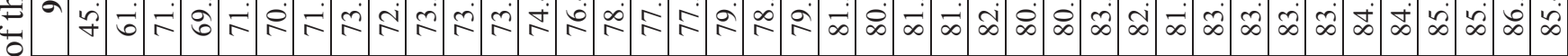

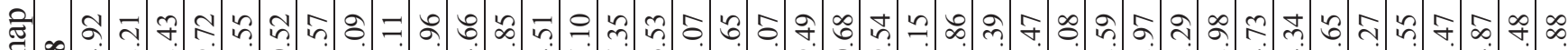

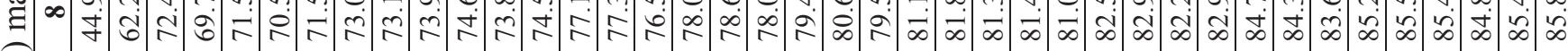

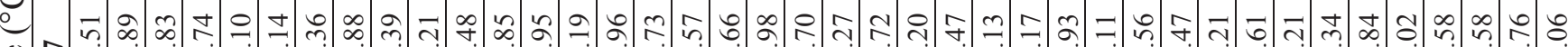

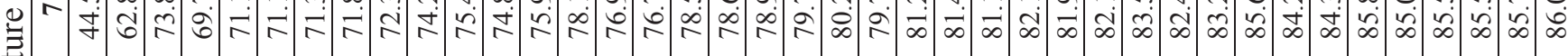

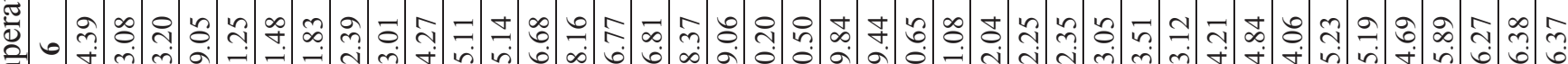

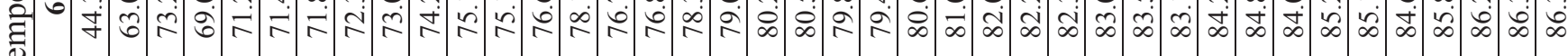
$\because$ ปิ

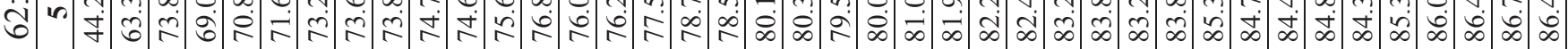

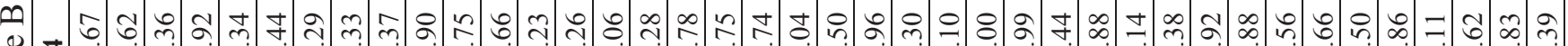

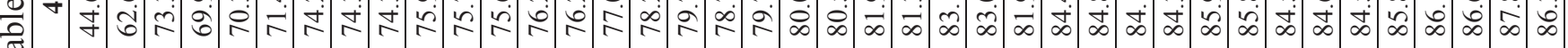

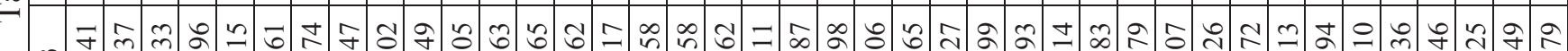

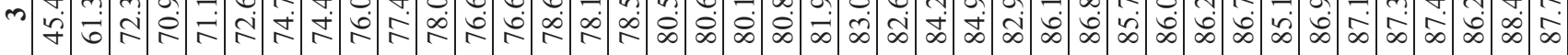

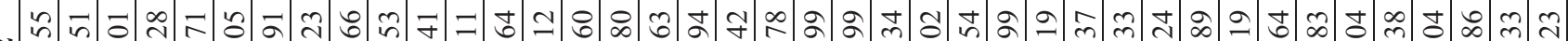

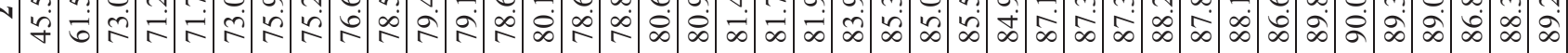

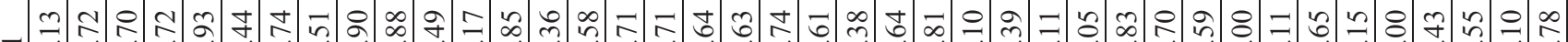

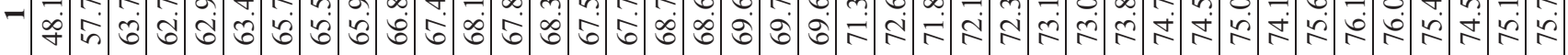
- n 0 n 0 n 0 n 0 n 0 n 0 n 0 n 0 n 0 n 0 .

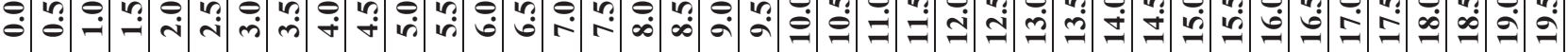


দี तี

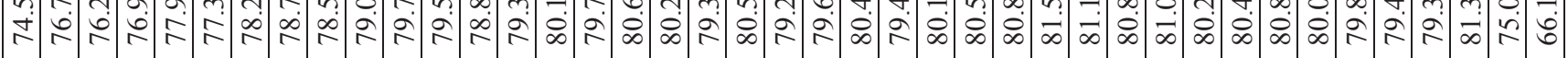
อ

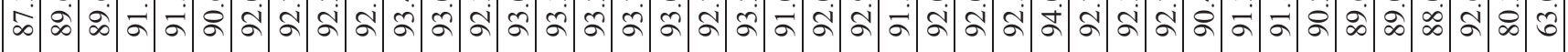

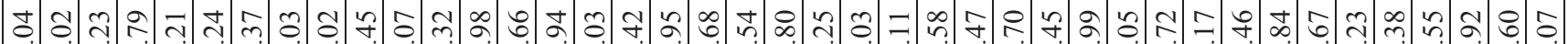

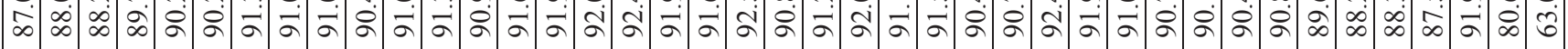

ڤ f f

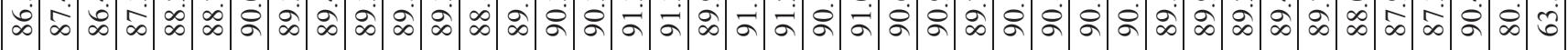

\&. ․

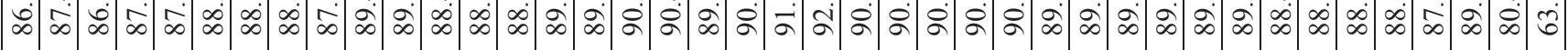

ஓे

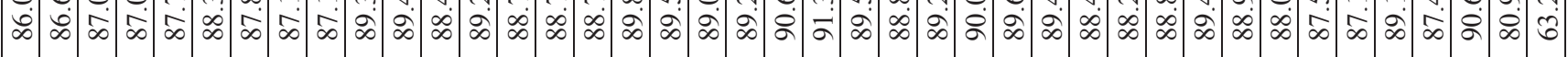

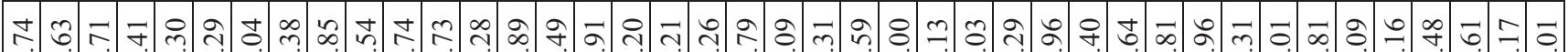

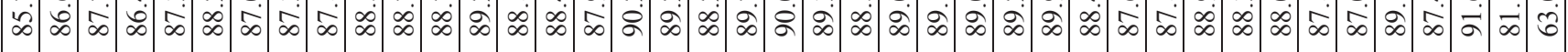

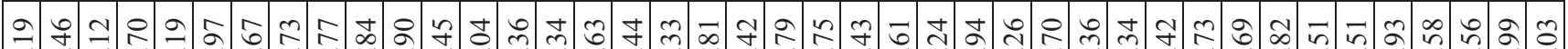

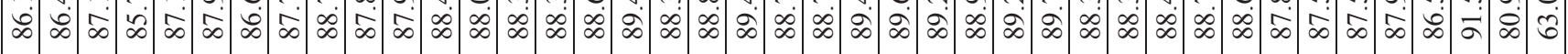

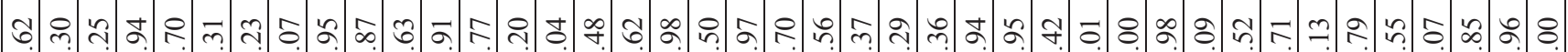

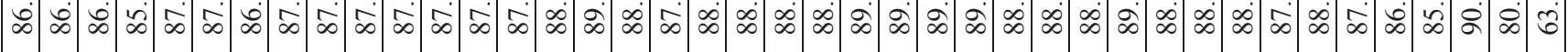

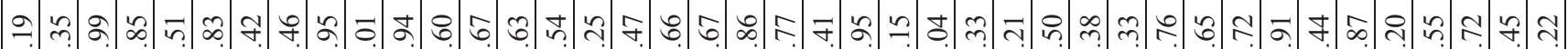

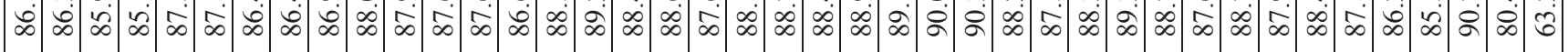

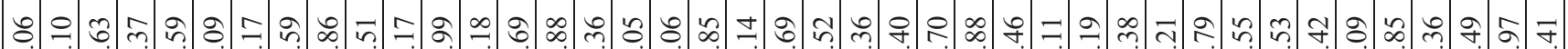

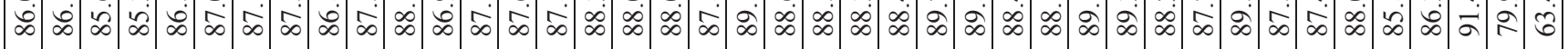

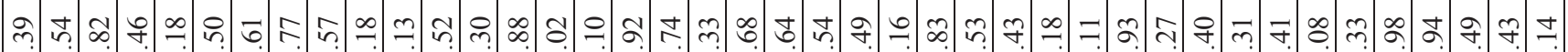

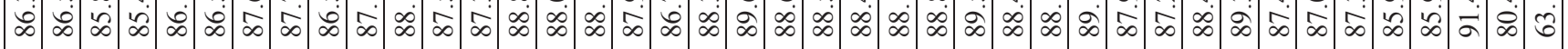

๕. ๆ. กิ กิ กิ

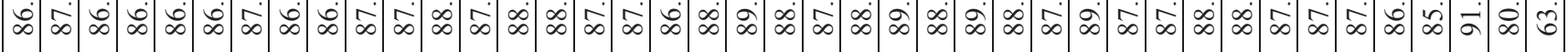

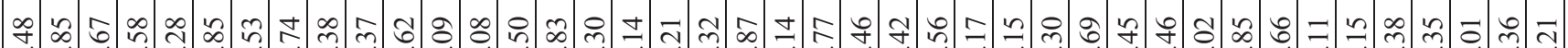

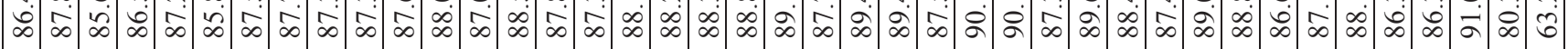
ที ๆ.

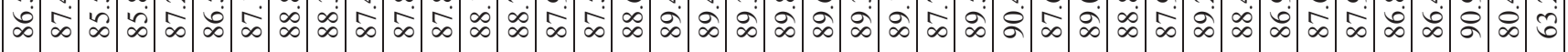

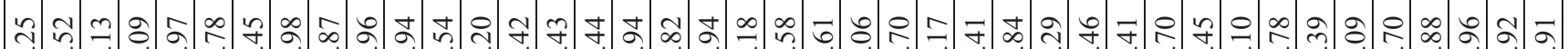

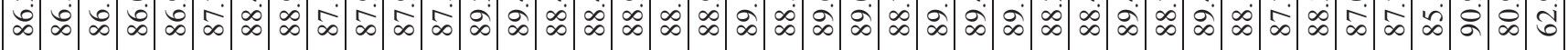

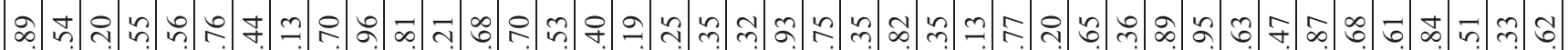

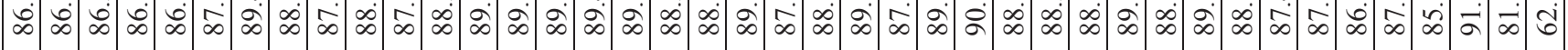
๑ สิ

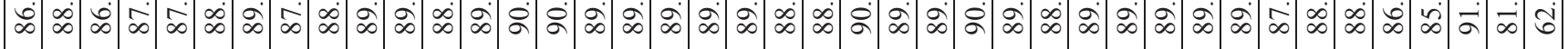

ป t

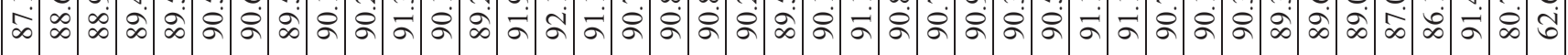

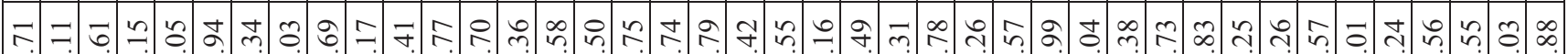

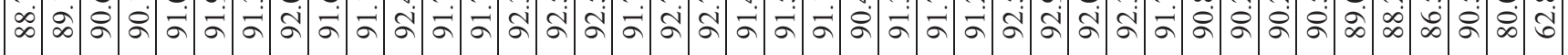
๖ กิ

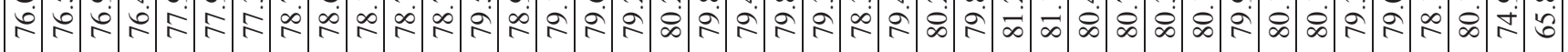




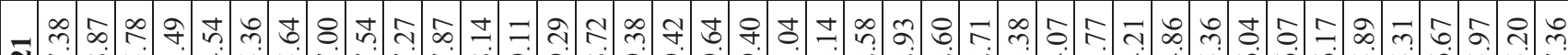

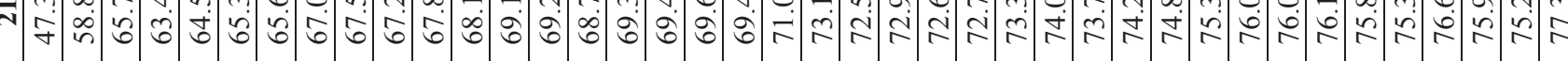
† సి సి

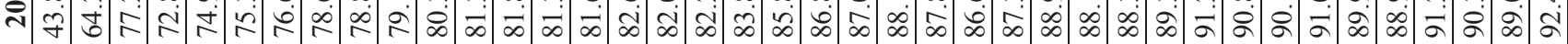

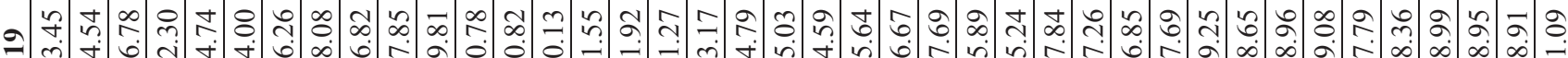
F

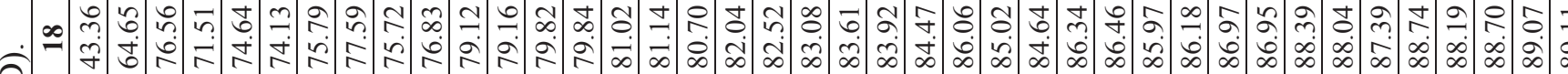
公

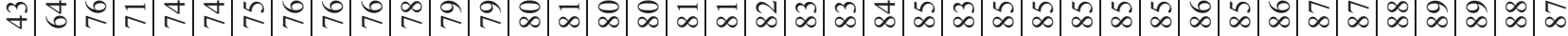
そ)

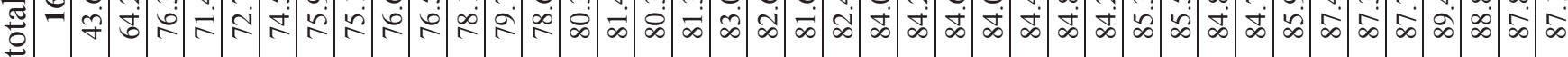

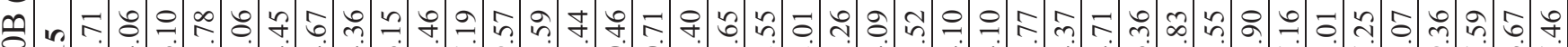

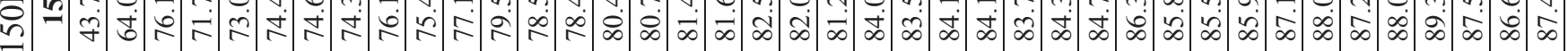

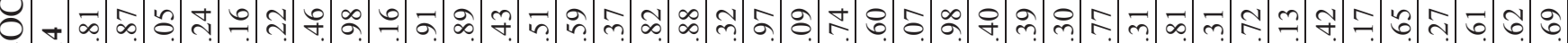

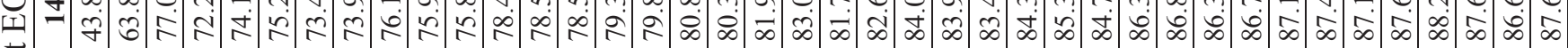
†

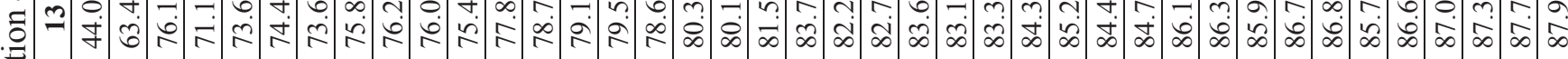

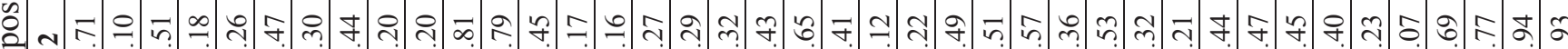
D) 는

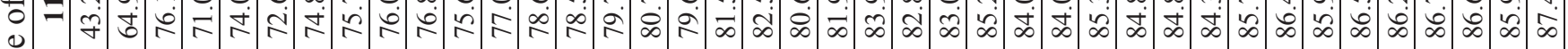
च चี ส

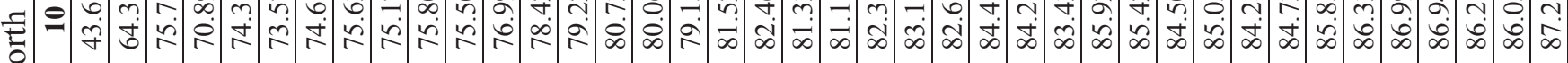

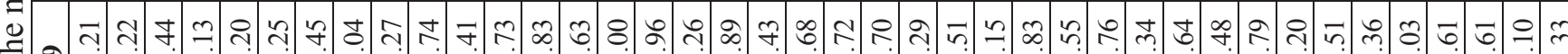

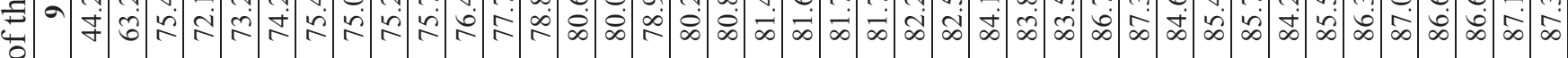

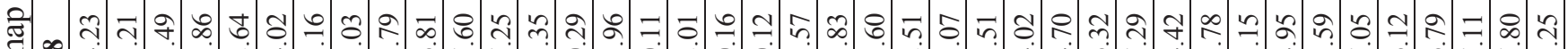

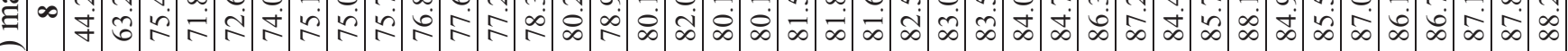

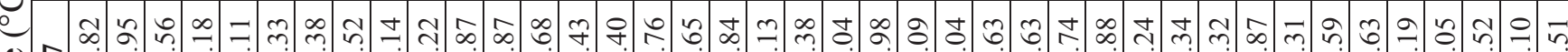

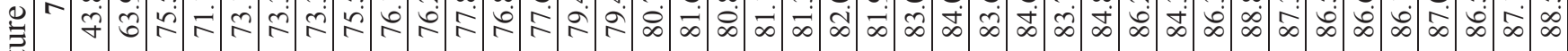
๑ 青守

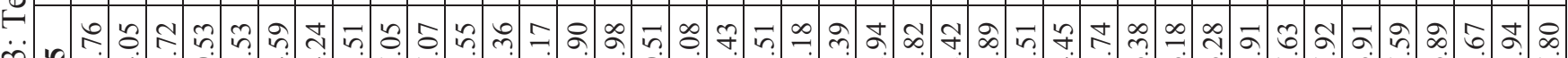

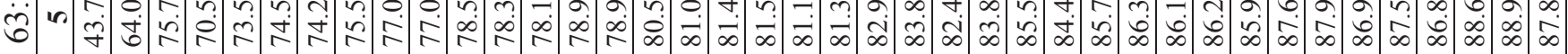

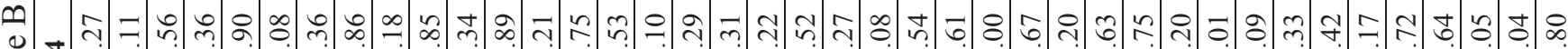

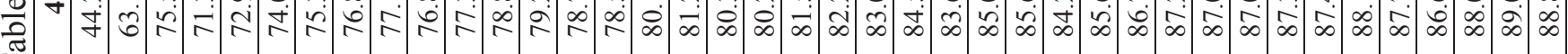

-

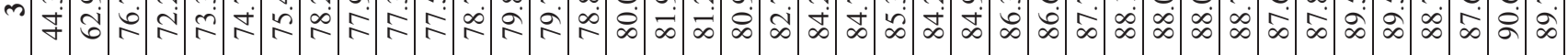
의 웅

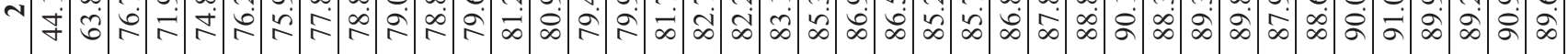
-

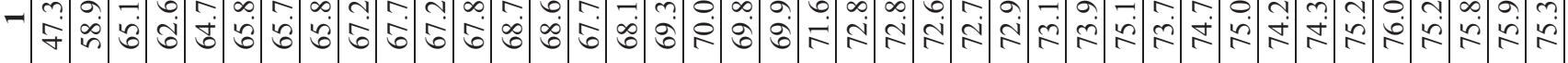
。

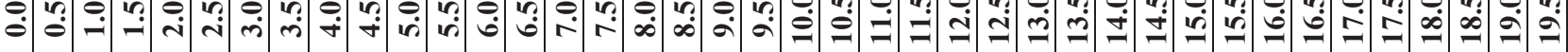




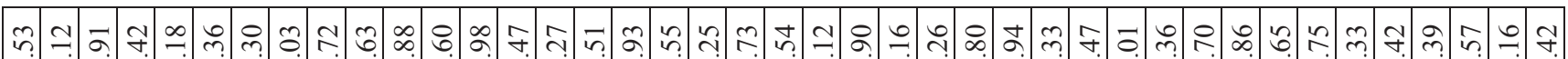

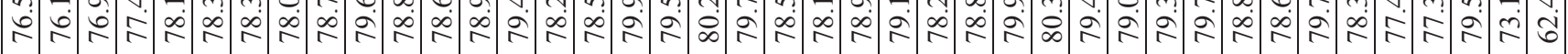
舟

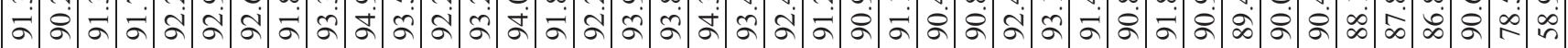

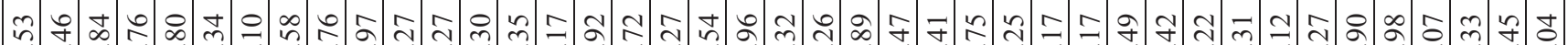

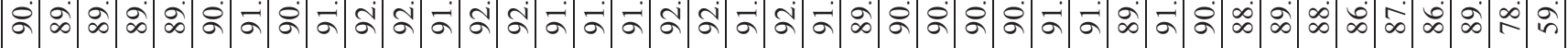

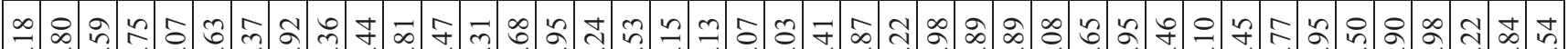

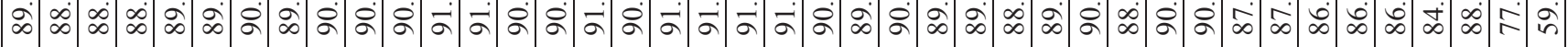
ㄱำ

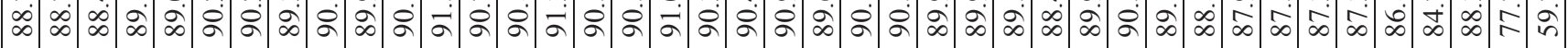

ㄱ.

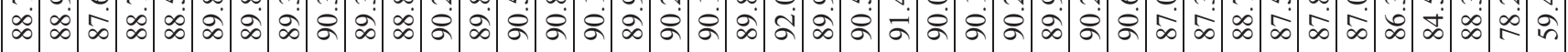

Ұ

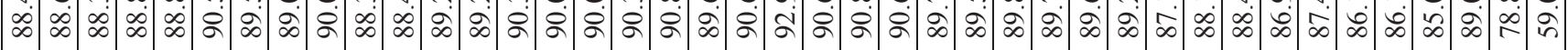

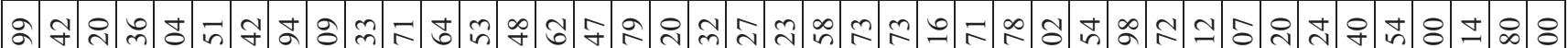

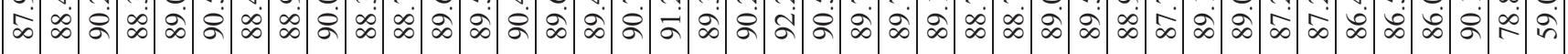

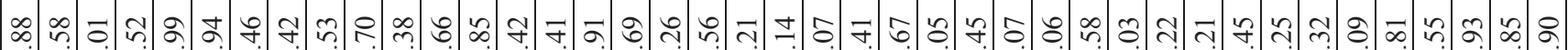

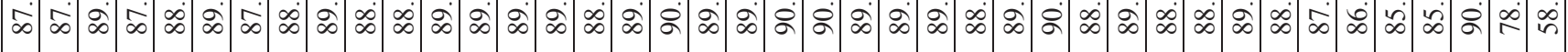

๓ి

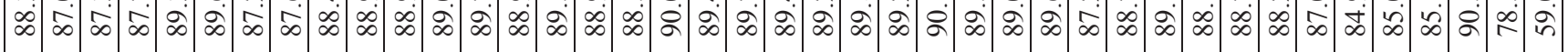

กิ

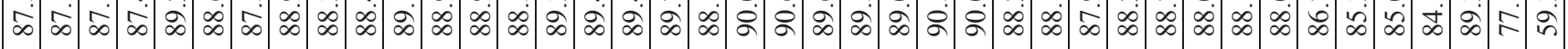

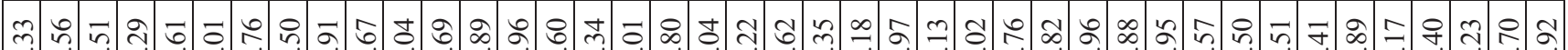

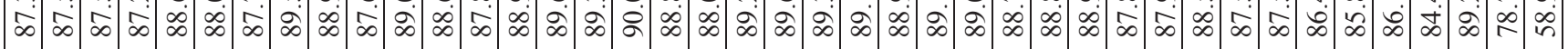

กิ

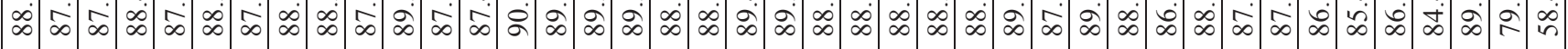

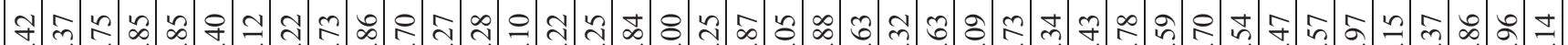

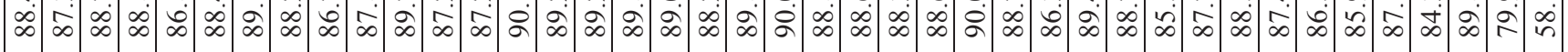
กิ

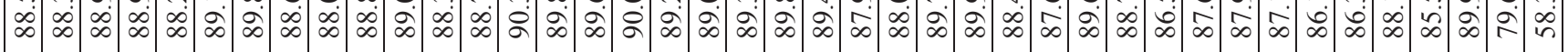

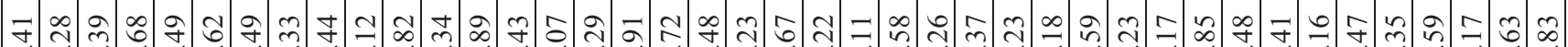

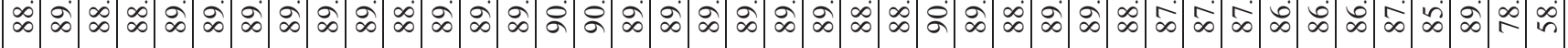

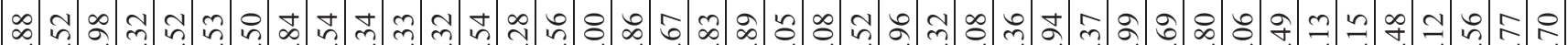

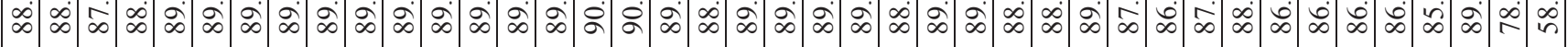

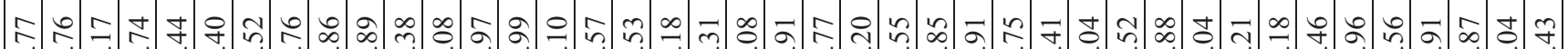

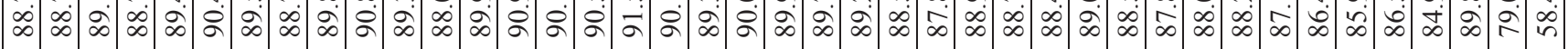

ج่

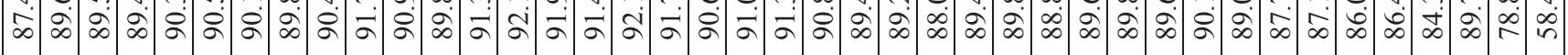

๘

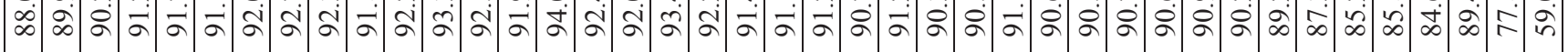

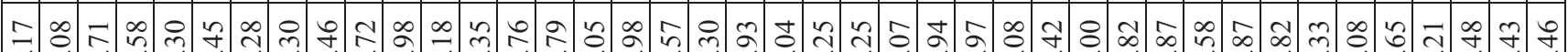

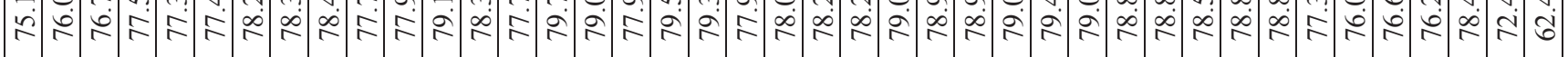




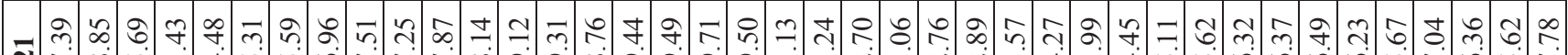

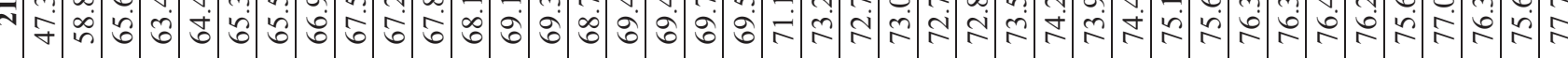

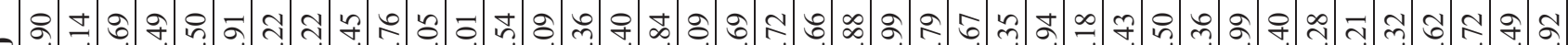

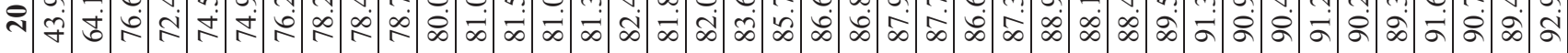

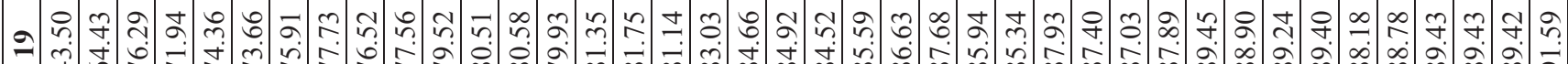

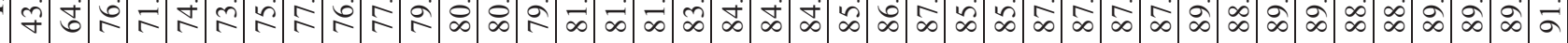

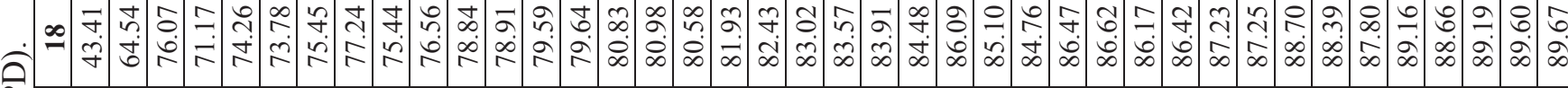

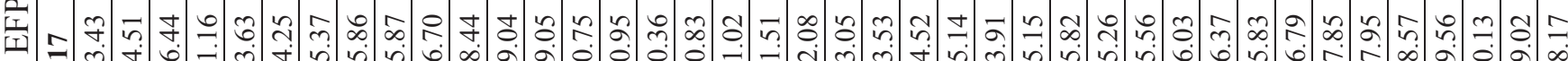

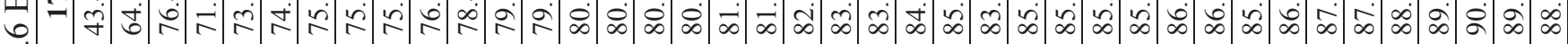

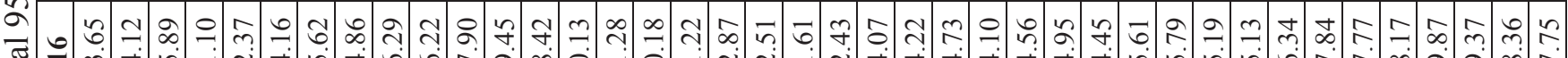

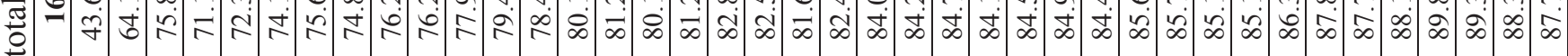
ஜ

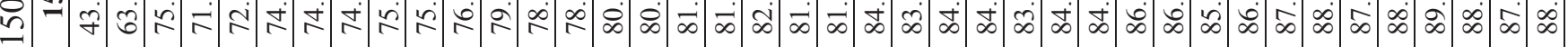
○

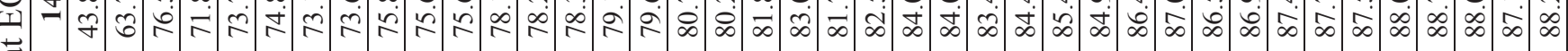
ఫ

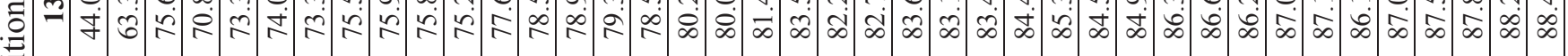
ఏ ₹

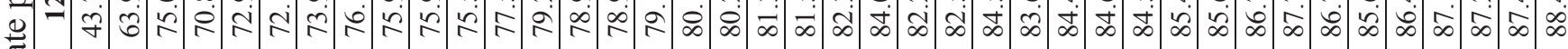

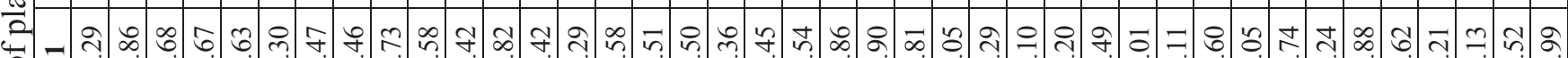

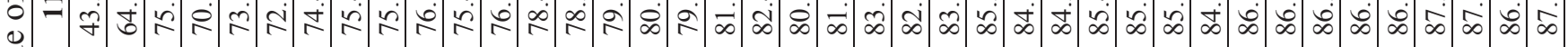

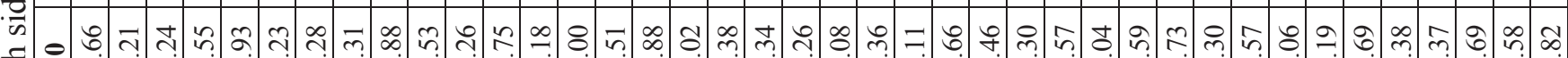

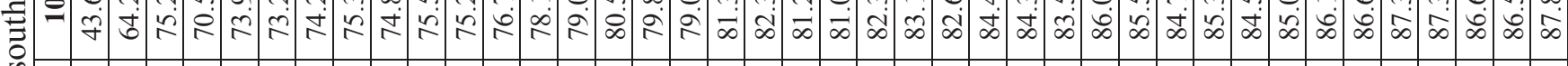
¿

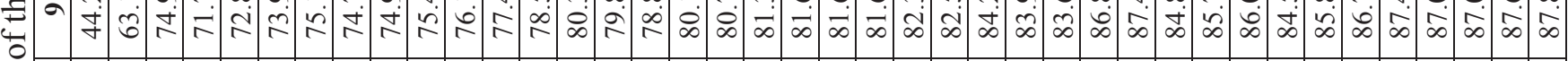

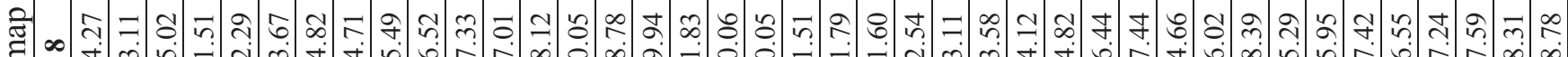

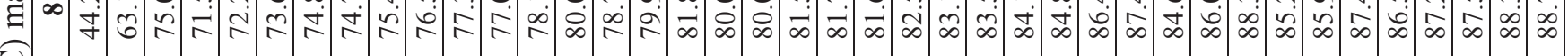
ల

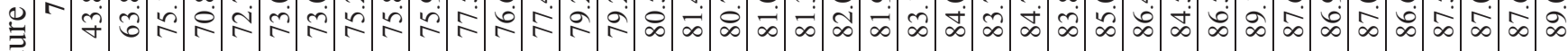

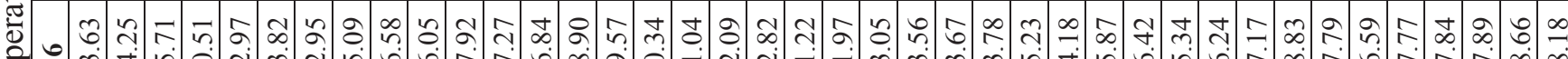
चี

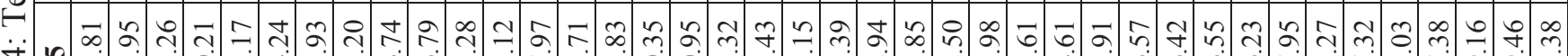

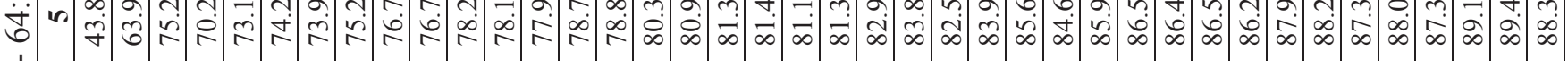

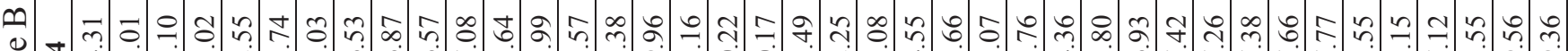

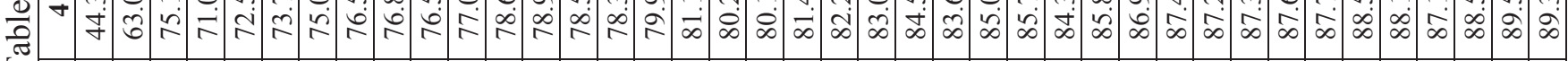

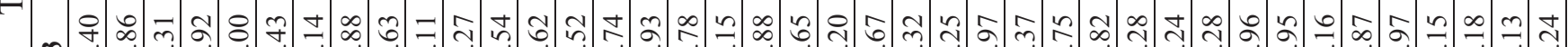
$m$ 㨁

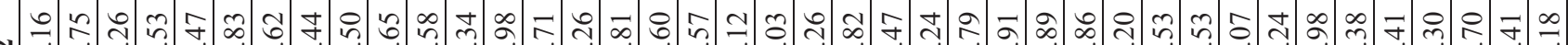

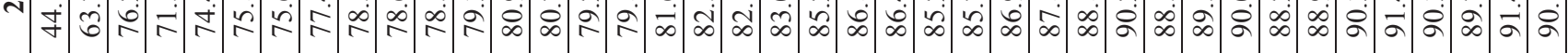

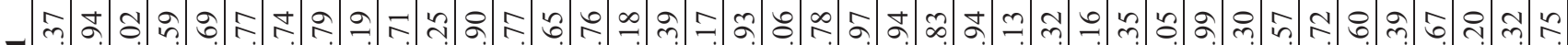

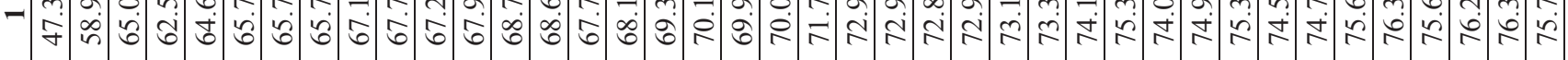

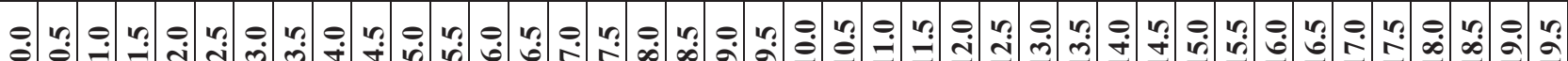


$\therefore \infty m$

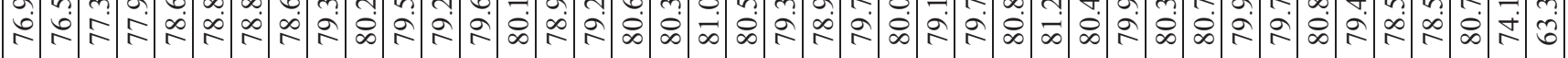

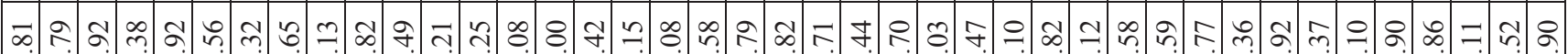

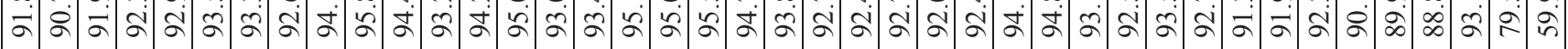

\&) f

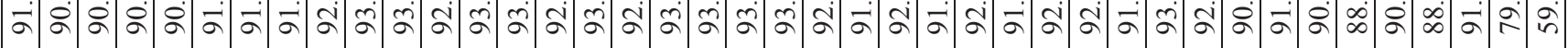

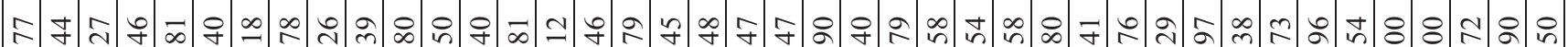

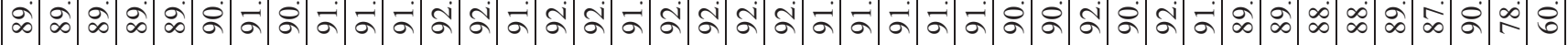
mి

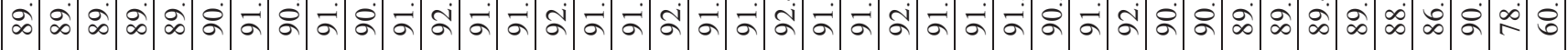

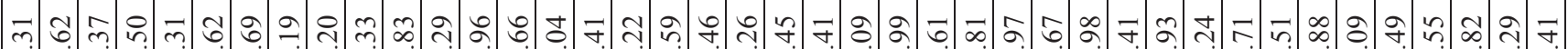

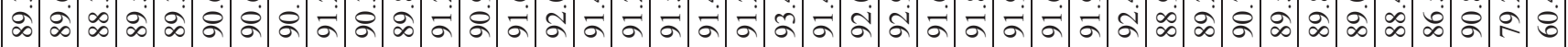

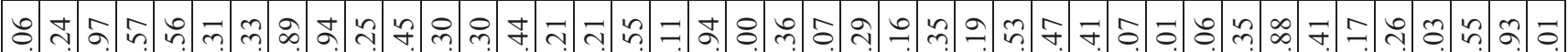

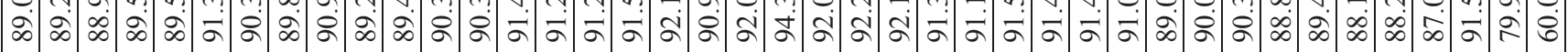

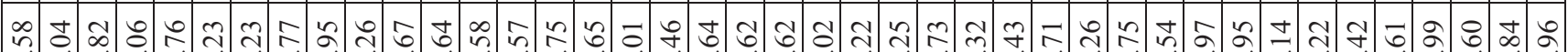

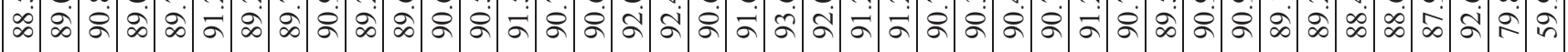

ఫ위

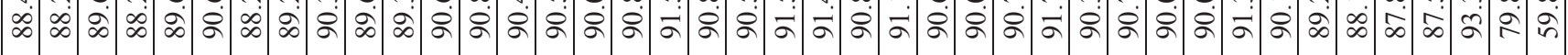

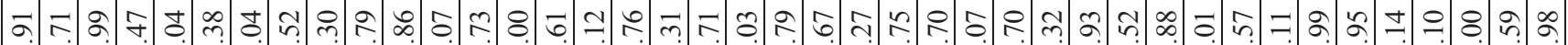

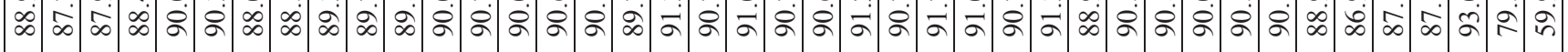

手孝

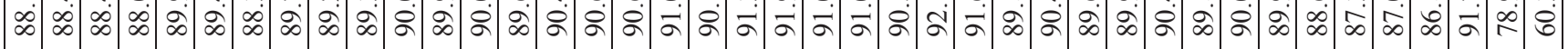

긍

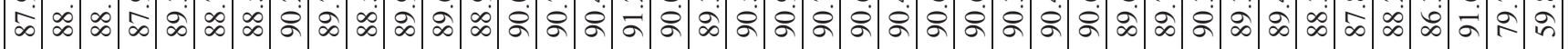

의

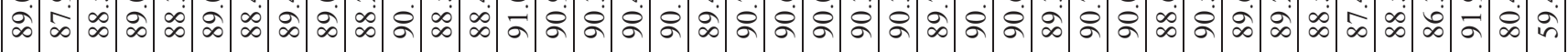

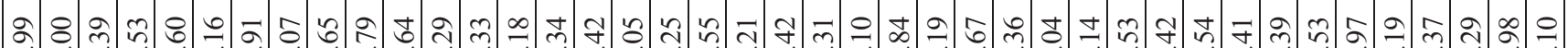

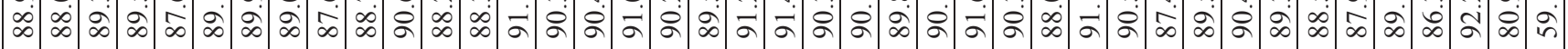
$=\&$ t

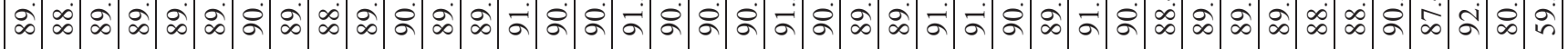

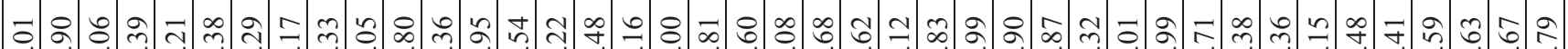

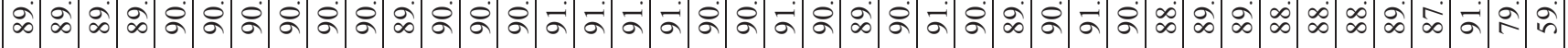
舟 $\tilde{b}$ t

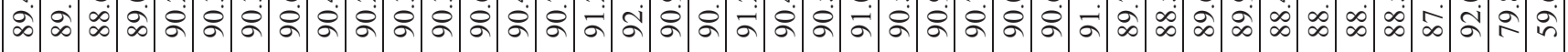
mำ

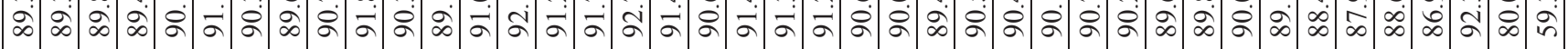

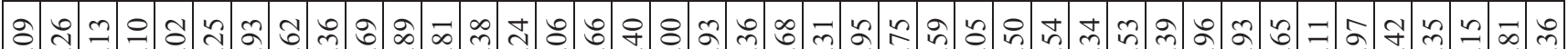

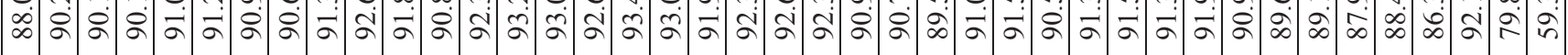

ช.

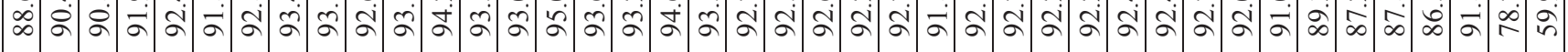

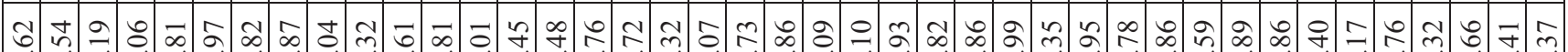

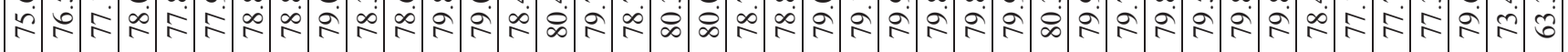

